\title{
Oligomer modulator anle138b and related compounds in neurodegeneration and beyond
}

\author{
Dissertation \\ for the award of the degree \\ "Doctor rerum naturalium" \\ of the Georg-August-Universität Göttingen \\ within the doctoral program Chemistry \\ of the Georg-August University School of Science (GAUSS)
}

\author{
submitted by \\ Sergey Ryazanov \\ from Saint Petersburg, Russia
}

Göttingen 2019 


\section{Thesis Committee}

Prof. Dr. Christian Griesinger

NMR-based structural biology, Max Planck Institute for biophysical chemistry, Göttingen

Prof. Dr. Ulf Diederichsen

Institut für Organische und Biomolekulare Chemie, Georg-August-Universität, Göttingen

Dr. Andrei Leonov

NMR-based structural biology, Max Planck Institute for biophysical chemistry, Göttingen

\section{Members of the Examination Board}

Reviewer: Prof. Dr. Christian Griesinger

NMR-based structural biology, Max Planck Institute for biophysical chemistry, Göttingen

Second Reviewer: Prof. Dr. Ulf Diederichsen

Institut für Organische und Biomolekulare Chemie, Georg-August-Universität, Göttingen

\section{Further members of the Examination Board}

Prof. Dr. Mathias Bähr

Dept. of Neurology, University Medical Center Göttingen, Göttingen

Prof. Dr. Henning Urlaub

Bioanalytical Mass Spectrometry, Max Planck Institute for biophysical chemistry, Göttingen

Prof. Dr. Tiago Fleming Outeiro

Dept. of Experimental Neurodegeneration, University Medical Center Göttingen, Göttingen

Dr. Stefan Glöggler

NMR Signal Enhancement Group, Max Planck Institute for biophysical chemistry, Göttingen

Date of the oral examination: $13^{\text {th }}$ of January 2020 


\section{Affidavit}

I hereby declare that I prepared this thesis titled Oligomer modulator anle138b and related compounds in neurodegeneration and beyond on my own and with no other sources and aids than quoted.

Place, Date

Signature 



\section{Publication list}

The thesis is based on the following publications:

1) Wagner J*, Ryazanov S*, Leonov A*, Levin J*, Shi S*, Schmidt F, Prix C, Pan-Montojo F, Bertsch U, Mitteregger-Kretzschmar G, Geissen M, Eiden M, Leidel F, Hirschberger T, Deeg AA, Krauth JJ, Zinth W, Tavan P, Pilger J, Zweckstetter M, Frank T, Bähr M, Weishaupt JH, Uhr M, Urlaub H, Teichmann U, Samwer M, Bötzel K, Groschup M, Kretzschmar H, Griesinger C, Giese A. (2013) Anle138b: a novel oligomer modulator for disease-modifying therapy of neurodegenerative diseases such as prion and Parkinson's disease. Acta Neuropathol. 125(6), 795-813.

* Equal contribution

2) Levin J, Schmidt F, Boehm C, Prix C, Bötzel K, Ryazanov S, Leonov A, Griesinger C, Giese A. (2014) The oligomer modulator anle138b inhibits disease progression in a Parkinson mouse model even with treatment started after disease onset. Acta Neuropathol. 127(5), 779-780.

3) Deeg AA, Reiner AM, Schmidt F, Schueder F, Ryazanov S, Ruf VC, Giller K, Becker S, Leonov A, Griesinger C, Giese A, Zinth W. (2015) Anle138b and related compounds are aggregation specific fluorescence markers and reveal high affinity binding to $\alpha$-synuclein aggregates. Biochim. Biophys. Acta 1850(9), 1884-1890.

4) Wagner J, Krauss S, Shi S, Ryazanov S, Steffen J, Miklitz C, Leonov A, Kleinknecht A, Göricke B, Weishaupt JH, Weckbecker D, Reiner AM, Zinth W, Levin J, Ehninger D, Remy S, Kretzschmar HA, Griesinger C, Giese A, Fuhrmann M. (2015) Reducing tau aggregates with anle138b delays disease progression in a mouse model of tauopathies. Acta Neuropathol. 130(5), 619-631.

5) Heras-Garvin A, Weckbecker D, Ryazanov S, Leonov A, Griesinger C, Giese A, Wenning GK, Stefanova N. (2019) Anle138b modulates $\alpha$-synuclein oligomerization and prevents motor decline and neurodegeneration in a mouse model of multiple system atrophy. Mov Disord. 34(2), 255-263.

6) Wegrzynowicz M, Bar-On D, Calo' L, Anichtchik O, Iovino M, Xia J, Ryazanov S, Leonov A, Giese A, Dalley JW, Griesinger C, Ashery U, Spillantini MG. (2019) Depopulation of dense $\alpha$-synuclein aggregates is associated with rescue of dopamine neuron dysfunction and death in a new Parkinson's disease model. Acta Neuropathol. 138(4), 575595. 
The thesis is based on the following patents:

1) Giese A, Bertsch U, Kretzschmar H, Habeck M, Hirschberger T, Tavan P, Griesinger C, Leonov A, Ryazanov S, Weber P (2010) New drug for inhibiting aggregation of proteins involved in diseases linked to protein aggregation and/or neurodegenerative diseases. $P C T$ Int. Appl. WO2010000372A2.

2) Giese A, Schmidt F, Griesinger C, Leonov A, Ryazanov S (2017) Water-soluble derivatives of 3,5-diphenyl-diazole compounds. PCT Int. Appl. WO2017102893A1.

Other publication:

1) Shi S, Wagner J, Mitteregger-Kretzschmar G, Ryazanov S, Leonov A, Griesinger C, Giese A (2015) Quantitative Real-Time Quaking-Induced Conversion Allows Monitoring of Disease-Modifying Therapy in the Urine of Prion-Infected Mice. J. Neuropathol. Exp. Neurol. 74(9), 924-933.

2) Fellner L, Kuzdas-Wood D, Levin J, Ryazanov S, Leonov A, Griesinger C, Giese A, Wenning GK, Stefanova N. (2016) Anle138b Partly Ameliorates Motor Deficits Despite Failure of Neuroprotection in a Model of Advanced Multiple System Atrophy. Front. Neurosci. 10, 99.

3) Draycheva A, Bornemann T, Ryazanov S, Lakomek NA, Wintermeyer W. The bacterial SRP receptor, FtsY, is activated on binding to the translocon. (2016) Mol. Microbiol. 102(1), 152-167.

4) Turriani E, Lázaro DF, Ryazanov S, Leonov A, Giese A, Schön M, Schön MP, Griesinger C, Outeiro TF, Arndt-Jovin DJ, Becker D. (2017) Treatment with diphenyl-pyrazole compound anle138b/c reveals that $\alpha$-synuclein protects melanoma cells from autophagic cell death. Proc. Natl. Acad. Sci. U. S. A. 114(25), E4971-E4977.

5) Martinez Hernandez A, Urbanke H, Gillman AL, Lee J, Ryazanov S, Agbemenyah HY, Benito E, Jain G, Kaurani L, Grigorian G, Leonov A, Rezaei-Ghaleh N, Wilken P, Arce FT, Wagner J, Fuhrmann M, Caruana M, Camilleri A, Vassallo N, Zweckstetter M, Benz R, Giese A, Schneider A, Korte M, Lal R, Griesinger C, Eichele G, Fischer A. (2018) The diphenylpyrazole compound anle $138 \mathrm{~b}$ blocks $\mathrm{A} \beta$ channels and rescues disease phenotypes in a mouse model for amyloid pathology. EMBO Mol. Med. 10(1), 32-47. 
6) Reiner AM, Schmidt F, Ryazanov S, Leonov A, Weckbecker D, Deeg AA, Griesinger C, Giese A, Zinth W. (2018) Photophysics of diphenyl-pyrazole compounds in solutions and $\alpha$ synuclein aggregates. Biochim. Biophys. Acta, Gen. Subj. 1862(4), 800-807.

7) Bartels M, Weckbecker D, Kuhn PH, Ryazanov S, Leonov A, Griesinger C, Lichtenthaler SF, Bötzel K, Giese A. (2019) Iron-mediated aggregation and toxicity in a novel neuronal cell culture model with inducible alpha-synuclein expression. Sci. Rep. 9(1), 9100.

8) Brendel M, Deussing M, Blume T, Kaiser L, Probst F, Overhoff F, Peters F, von UngernSternberg B, Ryazanov S, Leonov A, Griesinger C, Zwergal A, Levin J, Bartenstein P, Yakushev I, Cumming P, Boening G, Ziegler S, Herms J, Giese A, Rominger A. (2019) Latestage Anle138b treatment ameliorates tau pathology and metabolic decline in a mouse model of human Alzheimer's disease tau. Alzheimer's Res. Ther. 11(1), 67.

9) Ghio S, Camilleri A, Caruana M, Ruf VC, Schmidt F, Leonov A, Ryazanov S, Griesinger C, Cauchi RJ, Kamp F, Giese A, Vassallo N. (2019) Cardiolipin Promotes Pore-Forming Activity of Alpha-Synuclein Oligomers in Mitochondrial Membranes. ACS Chem. Neurosci. 10(8), 3815-3829.

10) Camilleri A, Ghio S, Caruana M, Weckbecker D, Schmidt F, Kamp F, Leonov A, Ryazanov S, Griesinger C, Giese A, Cauchi RJ, Vassallo N. (2019) Tau-induced mitochondrial membrane perturbation is dependent upon cardiolipin. Biochim. Biophys. Acta, Biomembr., 1862(2), 183064.

Other patent:

1) Becker D, Jovin TM, Griesinger C, Leonov A, Ryazanov S, Giese, A, Outeiro TF, Lazaro DF, Schön MP, Schön M. (2018) Phenyl-heterocycle-phenyl derivatives for use in the treatment or prevention of melanoma. PCT Int. Appl. WO2018206778A1. 



\section{Acknowledgement}

I am deeply grateful to Prof. Dr. Christian Griesinger for providing me an opportunity to work on this exciting project, as well as for his constant support, bright ideas, fruitful discussions, motivation and immense faith in me.

I would also like to thank Prof. Dr. Ulf Diederichsen for being my co-supervisor and for good advises during my thesis and Prof. Dr. Mathias Bähr, Prof. Dr. Henning Urlaub, Prof. Dr. Tiago Fleming Outeiro, Dr. Stefan Glöggler for agreeing to be members of my examination committee.

My special thanks to Dr. Andrei Leonov for his supervision, unfailing support and collegiality and team work in the chemistry lab.

I would like to thank Pinar Karpinar, Dr. Min-Kyu Cho and Dr. Hai-Young Kim for introduction to the world of molecular biology and NMR, for their help in the bio-labs, support and discussions on the $\alpha$-synuclein project.

I wish to thank all colleagues and collaborators whose contribution made my $\mathrm{PhD}$ thesis possible:

Prof. Dr. Armin Giese, Dr. Jens Wagner, Dr. Matthias Habeck, Dr. Felix Schmidt, Dr. Johannes Levin, Dr. Song Shi, Dr. Daniel Weckbecker for excellent collaboration, fruitful discussions, hundreds of emails and unbending faith in anle138b.

Prof. Dr. Markus Zweckstetter, Prof. Dr. Adam Lange, Prof. Dr. Marina Bennati, Dr. Tom Jovin, Dr. Donna Arndt-Jovin, Dr. Dorothea Becker, Dr. Eric Kemen for collaboration and fruitful discussions within the anle138b project.

Dr. Ulrike Teichmann, Kirsten Kiel, Dr. Jens Pilger, Dr. Sebastian Kügler, Prof. Dr. Jochen Weishaupt, Dr. Tobias Frank, Dr. Bettina Göricke for pharmacokinetic studies. I really enjoyed meetings with Ulrike and Kirsten at 5 a.m. in the animal facility.

Kerstin Overkamp and Gerhard Wolf for help with HPLC and LC-MS.

Dr. Stefan Becker, Karin Giller, Melanie Wegstroth for preparing high quality $\alpha$-synuclein samples. 
Dr. Ashutosh Kumar for solid state NMR and discussions.

Prof. Dr. Henning Urlaub for help with high-resolution mass-spectroscopy.

Prof. Dr. Tiago Fleming Outeiro and his team for LTP recordings.

Prof. Dr. Roland Benz and his team for BLM measurements.

Dr. Dietmar Riedel for electron microscopy images.

Dr. Avishay Pelah for his assistance with AFM.

Prof. Dr. Stefan Eimer, Prof. Dr. Jörg Schulz and Dr. Aaron for testing DPP compounds in invertebrate animal models.

Dr. Vladimir Belov and Jens Schimpfhauser for assistance with large scale synthesis of anle138b.

Prof. Dr. Maria Grazia Spillantini and team as well as Prof. Dr. Uri Ashery and team for the anle138b study in the MI2 transgenic mouse model.

Dr. Francisco Pan-Montojo for testing anle138b in the rotenone mouse model.

Prof. Dr. Nadia Stefanova and her team for MSA studies.

I am grateful to all co-authors of the listed publications for their contribution in the anle138b project.

Mrs. Silberer Siegrid and Mrs. Petra Breiner for helping with all paper work.

Many warm thanks to Edith, Sebastian, Manuel, Mate, Volker, Fabian, Eibe, Florian and Kris for nice and friendly atmosphere in the chemistry lab.

I also shared my work and joy with former and present colleagues in NMR department Supriya, Leif, Philip, Christophe, Luigi, Edward, Lucas, Risa, JP, Carolina, Dirk, Saskia, Hessam, David, Korvin, Nils, Donghan, Guo-Wei, Uwe.

I would also like to thank all my friends in Göttingen, in particular Dima, Karine, Michi, Albena, Shamil, Olex, Geoffrey, Csaba, Judit, Ricardo, Lucas, Caio, Masha, Sandra, Ilya, Ric, Rafa, Vesta, Anna, Vadim, Sergey, Katya and Timur.

For sport motivation, I send greetings to our football and ski communities.

I am deeply grateful to my parents and my brother Aleksey for their love and support! Katya and Maksim, thank you for everything! I love you so much! 


\section{Table of contents}

Publication list

Acknowledgements

Table of contents

Summary

1. Introduction

1.1. History of Parkinson's disease

1.2. Epidemiology, symptoms and pathology in Parkinson's disease $\quad 7$

1.2.1. Epidemiology

1.2.2. Symptoms

1.2.3. Pathology 8

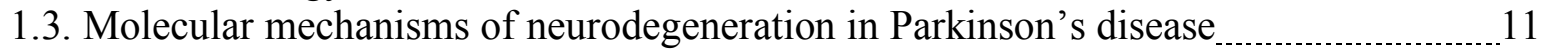

1.3.1. Genes implicated in Parkinson's disease 11

1.3.2. Impairment in the Ubiquitin-Proteasomal System $\ldots$

1.3.3. Mitochondrial dysfunction and oxidative stress $\ldots 14$

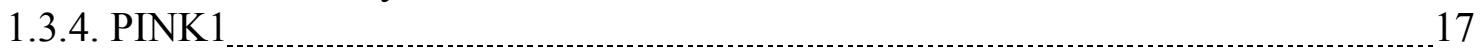

1.3.5. DJ-1

1.3.6. LRRK2 18

1.3.7. Overview $\ldots \ldots$

1.4. $\alpha$-Synuclein and its role in Parkinson's disease $\quad 20$

1.4.1. Introduction 20

1.4.2. Synuclein family

1.4.3. $\beta$-Synuclein and $\gamma$-synuclein $\ldots \ldots$

1.4.4. Synucleinopathies

1.4.5. Structure and aggregation of $\alpha$-synuclein $\quad 25$

1.4.6. Post-translational modifications of $\alpha$-synuclein $\quad 41$

1.4.7. Interactions with lipids $\quad 54$

1.4.8. Structure and toxicity of $\alpha$-synuclein aggregates $\ldots \ldots$

1.5. Medical treatment and clinical trials for Parkinson's disease $\ldots \ldots$

1.5.1. Introduction $\ldots$

1.5.2. Levodopa in the treatment of Parkinson's disease $\ldots . . .78$

1.5.3. Additional classes of drugs for treatment of Parkinson's disease $\quad 83$

1.5.4. Surgery and Transplantation $\quad 90$

1.5.5. Management of Parkinson's disease $\quad 90$

1.5.6. Overview

1.6. In search of the neuroprotection from cells to human

1.6.1. Introduction $\quad 92$

1.6.2. Animal models of Parkinson's disease $\ldots$

1.6.3. Disease-modifying strategies for Parkinson's disease $\quad 124$

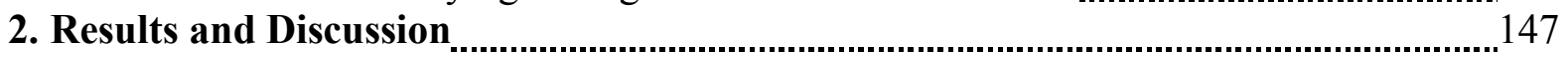

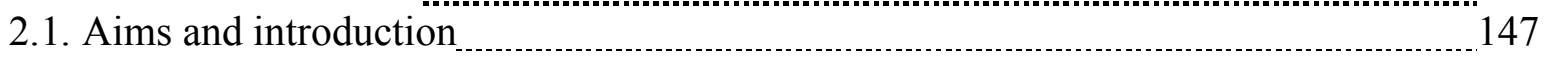

2.2. Screening of aggregation inhibitors $\ldots$

2.2.1. SIFT assay

2.2.2. Primary SIFT and cell culture screening 150

2.3. Design and synthesis of the focused library of DPP-related compounds _.............................

2.3.1. DPP scaffold and overview of focused library $\ldots 153$

2.3.2. Contribution of fragment-guided approach in DPP design $\quad 156$ 
2.3.3. General synthetic schemes 156

2.3.4. Synthesis of pyrazole- and 2-pyrazoline-based DPPs 159

2.3.5. Synthesis of isoxazole-, imidazole-, 1,2,4-oxadiazole-, pyrrole-based DPP analogs 166

2.3.6. Synthesis of complementary DPP compounds 169

2.3.7. Synthesis DPP compounds: key findings and discussion 172

2.4. DPP compounds and prion protein in vitro and in vivo

2.4.1. Prion protein in molecular SIFT assay 173

2.4.2. Inhibition of $\mathrm{PrP}^{\mathrm{Sc}}$ propagation by DPP compounds in SIFT assay $\ldots 175$

2.4.3. SAR studies and validation of DPP compounds in SIFT assay $\ldots \ldots \ldots \ldots \ldots \ldots \ldots \ldots \ldots \ldots \ldots \ldots . . .177$

2.4.4. Antiprion activity of DPP compounds in cell culture model $\quad 179$

2.4.5. Antiprion activity of DPP compounds in PMCA 182

2.4.6. Antiprion activity of DPP compounds in animal models of prion disease $\quad 187$

2.4.7. Therapeutic effect of anle138b in prion mice after onset of disease

2.4.8. Comparison of in vivo activity of anle $138 \mathrm{~b}$ with other published compounds .... 194

2.4.9. DPP derivatives and prion: Key findings and discussion $\ldots \ldots \ldots$

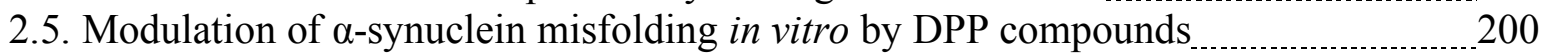

2.5.1. $\alpha$-Synuclein in molecular SIFT assay 200

2.5.2. Inhibition of $\alpha$-synuclein aggregation by DPP compounds in SIFT assay _........ 201

2.5.3. Inhibition of $\alpha$-synuclein aggregation by DPP compounds in ThioT assay 205

2.5.4. Examination of $\alpha$-synuclein aggregation in presence of DPPs

by TEM and AFM

2.5.5. Investigation of $\alpha$-synuclein aggregation in presence of DPPs by DLS $\quad 210$

2.5.6. Secondary structure of DPP-stabilized $\alpha$-synuclein oligomers by CD $\ldots \ldots \ldots \ldots \ldots . . . . . .213$

2.5.7. Characterization of DPP-stabilized $\alpha$-synuclein oligomers by

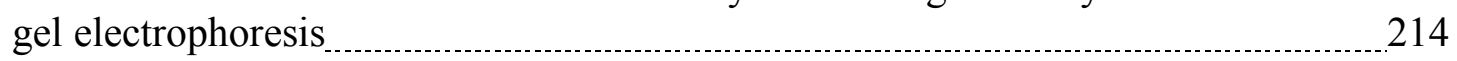

2.5.8. NMR investigation of $\alpha$-synuclein aggregation in presence of DPP compounds

2.5.9. $\alpha$-Synuclein aggregation in presence of DPP compounds under reducing conditions

2.5.10. Interactions of DPP compounds with monomeric $\alpha$-synuclein studied by NMR

2.5.11. Introduction to NMR STD experiments 233

2.5.12. Interactions of DPP compounds with $\alpha$-synuclein studied by STD NMR _....... 235

2.5.13. Interactions of sery166a with oligomeric $\alpha$-synuclein studied by STD NMR

2.5.14. Characterization of DPP-stabilized $\alpha$-synuclein oligomers

by solid state NMR

2.5.15. Comparison of DPP-stabilized oligomers with toxic oligomers

2.5.16. Inhibition of $\alpha$-synuclein aggregation by DPP compounds in vitro:

key findings and discussion

2.6. DPP compounds and $\alpha$-synuclein forms in electrophysiological studies $\quad 252$

2.6.1. Introduction 252

2.6.2. Effect of $\alpha$-synuclein on electrophysiological properties of planar lipid bilayer $\ldots$

2.6.3. Electrophysiological properties of DPP-stabilized oligomers $\ldots \ldots$

2.6.4. Inhibition of pore formation by DPP compounds $\quad 256$

2.6.5. Effect of DPP compounds and $\alpha$-synuclein on LTP

2.7. Effect of DPP compounds in in vivo models of Parkinson's disease _........................ 262

2.7.1. Introduction 262 
2.7.2. DPP compounds in non-mammalian $\alpha$-synuclein models 263

2.7.3. Why anle138b in vivo? 266

2.7.4. Therapeutics effect of anle138b in mouse models of Parkinson's disease ${ }_{2} 266$

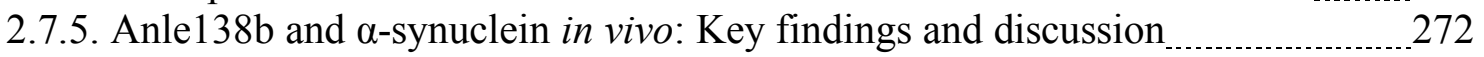

2.8. Formulation development and pharmacokinetic of anle $138 \mathrm{~b}$ and

other DPP compounds $\quad 277$

2.8.1. Introduction

2.8.2. DPP compounds in mice $\quad 277$

2.8.3. Pharmacokinetic properties of anle138b in mice $\quad 280$

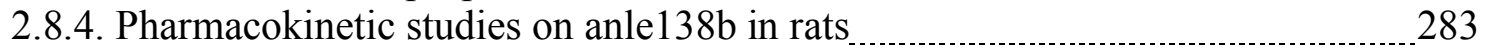

2.8.5. Optimization of oral formulation in rats $\quad 285$

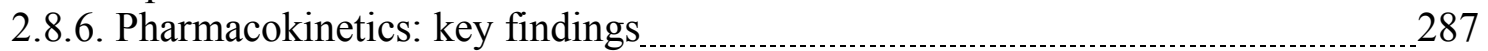

2.9. Development of anle138b prodrug 288

2.9.1. Introduction: Prodrug approach $\ldots$

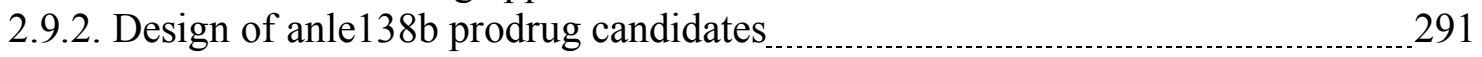

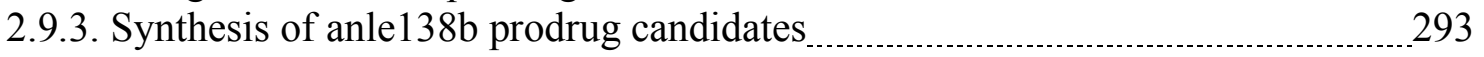

2.9.4. Physical and pharmacokinetic properties of anle138b prodrug candidates _......... 297

2.9.5. Prodrug: Summary $\quad 300$

2.10. Anle138b: Modulation of protein misfolding in other diseases _ _ _ 302

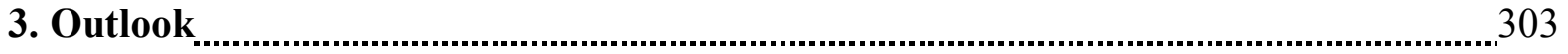

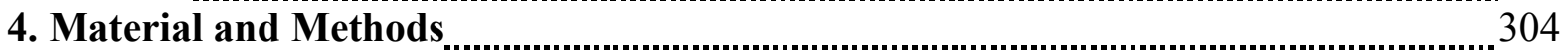

4.1. Materials I

4.1.1. Instruments and consumables $\quad 304$

4.1.2. Reagents and materials

4.1.3. E. coli strain and E. coli cell culture media $\quad 306$

4.2. Methods I

4.2.1. Protein biochemistry

4.2.2. Protein NMR

4.2.3. Other spectroscopic methods 311

4.2.4. Electrical recording of planar lipid bilayer and LTP experiments $\quad 312$

4.2.5. DPP compounds in in vivo models of Parkinson's disease _......................... 313

4.2.6. Pharmacokinetic studies with DPP compounds and prodrugs $\quad 315$

4.2.7. Experiments with the prion protein 319

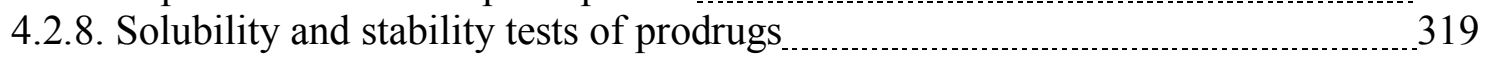

4.3. Materials and Methods Chemistry

4.3.1. Materials and Methods

4.3.2. Experimental Procedures and characterization data of compounds $\quad 321$

4.3.3. Experimental Procedures and characterization data of prodrug compounds ....... 373

5. Bibliography $\ldots$

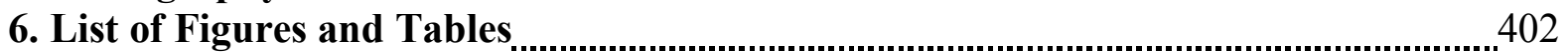

7. Abbreviations

8. Appendix 413

8.1. "Prion mice" library of DPP compounds tested for anti-prion activity in vivo ............. 413

8.2. Backbone assignment of wt $\alpha$-synuclein at $15^{\circ} \mathrm{C}$, pH 7.4, $\mathrm{PBS} \quad 416$ 


\section{Summary}

Neurodegenerative diseases including Alzheimer's disease, Parkinson's disease, Creutzfeldt-Jakob disease are characterized by a progressive deterioration of brain function as a result of a gradual loss of nerve cells. Due to the fact that age is the dominant risk factor for these diseases and life expectancy is constantly increasing, neurodegenerative disorders are considered as one of the serious future challenges for health care system. To date there is no treatment option capable of slowing, preventing or reversing the progressive death of neurons in brain. Therefore, a development of disease-modifying drugs with neuroprotective and/or neurorestorative properties has a highest priority of pharmaceutical research.

In majority of the neurodegenerative disorders, the misfolding and aggregation of specific protein(s) in the brain are the permanent histopathological hallmarks. Among the proposed mechanisms of neurotoxicity, protein misfolding may probably compromise a central cascade that leads to loss of neurons. Based on growing evidence indicating that pathological oligomers rather than large fibrillar deposits constitute the key neurotoxic species, we set out to discover small molecules interfering with pathological oligomerization.

The main goal of my thesis was to design, synthesize and assess novel compounds that can modulate in vitro and in vivo the aggregation/misfolding of amyloidogenic proteins, including prion protein, $\alpha$-synuclein and others.

In the initial phase of the project, a high-throughput screening campaign, which was conducted by Prof. Armin Giese, identified a novel class of anti-prion compounds, termed 3,5-DiPhenyl-Pyrazoles. In an attempt to optimize the DPP lead structure and to get insight into the details of structure-activity relationship, we designed and synthesized a focused library of 150 DPP-related compounds. We utilized a fragment-based structure design and an iterative approach that comprised several rounds of structure optimization and synthesis on a basis of in vitro and in vivo testing and SAR analysis. Additionally, we proposed a flexible synthetic scheme allowing straightforward synthesis of the desired compounds.

By using a range of in vitro prion protein aggregation assays including SIFT assay, PMCA and RML-infected SMB cell model, we assessed the efficacy of DPP compounds as inhibitors of prion protein misfolding. Our in vitro findings support the pronounced antiprion activity of novel compounds sharing the DPP-based core structure. In PMCA assay and in the 
cell-based model, compound anle138b shows the highest anti-prion activity against various prion stains, including RML, sCJD and vCJD. On the basis of the results from the SIFT, cell culture and PMCA assay, 38 DPP-related compounds were tested in vivo in RML prioninfected mice model. Following oral administration, anle138b and other DPP compounds strongly inhibited the accumulation of $\mathrm{PrP}^{\mathrm{Sc}}$, neuronal degeneration and disease progression. Regarding the mode of action of anle138b, several lines of evidences from in vitro and in vivo studies support the mechanism associated with direct modulation of prion protein aggregation at the oligomer level.

The second part of my thesis describes the property of selected DPP compounds to interfere with $\alpha$-synuclein aggregation and toxicity. Here, we conducted the SIFT screening assay on $\alpha$-synuclein. We were able to identify a set of highly potent inhibitors of $\alpha$-synuclein aggregation and, moreover, we performed a detailed analysis of structure-activity relationships within our focused library of DPP compounds. Next, the molecular mechanism underlying the modulation of $\alpha$-synuclein misfolding by DPP compounds was studied by various biochemical and biophysical approaches. By using ThioT aggregation assay, electron microscopy, atomic force microscopy and dynamic light scattering, we showed that watersoluble compounds (sery166a, anle138c, anle145c, sery139 and others) prevent fibrillization of $\alpha$-synuclein in vitro by stabilization of small globular heterogeneous oligomeric species. CD data suggests that sery166a stabilizes $\alpha$-synuclein in predominantly unfolded conformation accompanied a low degree of local secondary structure ordering in comparison to the monomeric $\alpha$-synuclein. To elucidate the mode of inhibition, we applied a highresolution NMR spectroscopy. HSQC-based NMR binding studies showed a lack of direct binding of all tested compounds to monomeric $\alpha$-synuclein. By using STD NMR experiments, we demonstrated that the DPP compounds preferentially interact with oligomeric species, while the affinity to monomeric $\alpha$-synuclein is weak. It's, therefore, envisioned that the mechanism of aggregation inhibition by DPP compounds might be associated with the interactions between small ligands and oligomeric forms that, in turn, lead to stabilization of specific off-pathway oligomers and, ultimately, blocking of the fibril formation.

Considering that the oligomer-induced membrane permeabilization represents a potential toxicity mechanism associated with $\alpha$-synuclein misfolding, we investigated DPP compounds and compound-stabilized $\alpha$-synuclein oligomers using electrophysiological studies in planar lipid bilayers. Our analysis of the electrophysiological data reveals that DPP compound-stabilized oligomers do not adversely affect the membrane stability. Remarkably, 
the addition of DPP compounds either in solution (sery166a, anle138c) or inside the lipid bilayer strongly interferes with membrane permeabilization induced by toxic oligomeric forms of $\alpha$-synuclein. Finally, the DPP-stabilized $\alpha$-synuclein oligomers do not alter the magnitude of long-term potentiation (LTP) induced in rat hippocampal slices.

In vivo, the water-soluble compound anle $145 \mathrm{c}$ that efficiently modulates the aggregation of $\alpha$-synuclein in vitro was found to be active in non-mammalian $\alpha$-synuclein models. In mammals, anle138b is neuroprotective in several mouse models of synucleinopathies. Of particular significance is the finding that anle $138 \mathrm{~b}$ prevents the formation of toxic oligomers and improves the behavioral, neuropathological and biochemical outcomes in all different animal models based on toxins (Sub-acute MPTP and low-dose intragastric rotenone, the latter being considered also a spreading model), spreading (Lewy body seeding mouse model) and overexpression (Thy1-A30P $\alpha$-synuclein; 1-120 $\alpha$ synuclein, PLP- $\alpha$-synuclein). Our findings provide compelling evidence that anle138b directly modulates the aggregation of $\alpha$-synuclein at the oligomer level in vivo as shown by sucrose gradient centrifugation assay and super-resolution imaging $d$ STROM. It also is noteworthy that with prolonged treatment, anle $138 \mathrm{~b}$ has no apparent toxicity in therapeutic doses.

In a further part of my thesis I discuss the pharmacokinetic (PK) properties of DPP molecules. Our pharmacokinetic studies have demonstrated that anle138b and other DPP compounds have in general a good oral bioavailability and readily cross the blood-brainbarrier. In case of anle138b, we showed that this molecule has a favorable pharmacokinetic profile in mice and rats. Moreover, we have developed and characterized in PK experiment the special anle138b-containing dry food pellets, which provides convenient way to conduct efficacy studies in mice. Finally, we have identified and tested optimized anle138b formulation V34, which is based on pharmaceutically acceptable excipients.

In the last part of my thesis I describe the development of an anle138b prodrug. Due to low aqueous solubility of anle138b, the oral application of this compound requires the dissolution in appropriate excipients. In order to improve the formulation protocol and potentially anle138b's bioavailability, we designed and synthesized several water-soluble anle138b prodrugs. Among three candidates, the prodrug sery433 showed favorable physicochemical (good stability and high solubility in water) and pharmacokinetic profiles. The comparative pharmacokinetic study in rats with sery433 and anle138b, which were applied orally at the same dose, demonstrated a significant increase of anle138b systemic exposure in the case of prodrug sery433. The mechanism of biotransformation of sery433 in 
vivo is presumably associated with the enzymatic cleavage of the phosphate moiety by the membrane-bound intestinal phosphatase. Collectively, sery433 is a promising water-soluble anle138b prodrug for further preclinical development.

Viewed together, our observations and the results from many other studies strongly support the notion that interference with the protein misfolding by small molecules may represent the attractive strategy in development of disease-modifying therapy. The validity of the aggregation inhibition as a target for drug design was recently corroborated by positive results of the clinical trials of Aducanumab, a monoclonal antibody against a conformational epitope found on misfolded amyloid beta, in early Alzheimer's Disease. Anle138b modulates the oligomer formation of different proteins by targeting structure-dependent epitopes, which are common in various types of pathological aggregates. The efficacy and preclinical data that were collected for the oligomer modulator anle138b strongly supports the selection of this molecule as a candidate for clinical trials in CJD and synucleinopathies. A phase 1 clinical trial with anle138b is scheduled for Q1 2020. 


\section{Introduction}

\subsection{History of Parkinson's disease}

Parkinson's disease was likely known to the ancients. Some symptoms of Parkinson's disease were mentioned in several sources from Egypt, India and China. An Ayurvedic medical treatise from the $10^{\text {th }}$ century B.C. describes symptoms closely related to Parkinson's disease. Moreover, this manuscript contains a treatment protocol based on a medication derived from a tropical legume called Mucuna pruriens (Figure 1.1), which is rich in LDOPA. Limited citations in the literature before $19^{\text {th }}$ century could be explained by a fact that Parkinson's disease was very rare disorder several centuries ago because not that many people lived into their 60 -s or 70 -s.

A strong impulse to the scientific investigation was given by English physician James Parkinson who published his "Essay on the shaking palsy" in 1817. This work is considered as a medical classic because of the detailed description of development and clinical symptoms of the disease. Interestingly, J. Parkinson has created his book based solely on his experience with just 6 patients, in addition 3 out of 6 patients were "casually met on the street" or "seen at a distance". He did recognize a variety of the symptoms associated with the different stages of the disease. For example, concerning early symptoms he writes; "The first symptoms perceived are, a slight sense of weakness, with a proneness to tremble in some particular part: ... but most commonly of the hands and arms." He also was able to describe many others characteristic features: resting tremor, abnormal gait, masked face, sleep disturbance, diminished muscle strength, inability to swallow and paralysis. J. Parkinson was probably the first physician that assumes Parkinson's disease to be a nervous disorder in which the brain is affected. He also recognizes the age as a main risk of the disease. The essay was well received in the medical community and 4 decades later in 1861 Jean-Martin Charlot and his colleagues confirmed the contribution of James Parkinson by introducing the term "Parkinson's Disease".

In the middle of $19^{\text {th }}$ century a detailed clinical description of Parkinson's disease was completed by Jean-Marie Charcot (Figure 1.1) and Alfred Vulpian who have worked together at the hospital in Paris. Among other advances, they made a distinction between rigidity, weakness and bradykinesia ${ }^{15}$. In addition, J.-M. Charcot was one of the first who tried to develop and evaluate new therapies for Parkinson's disease. 

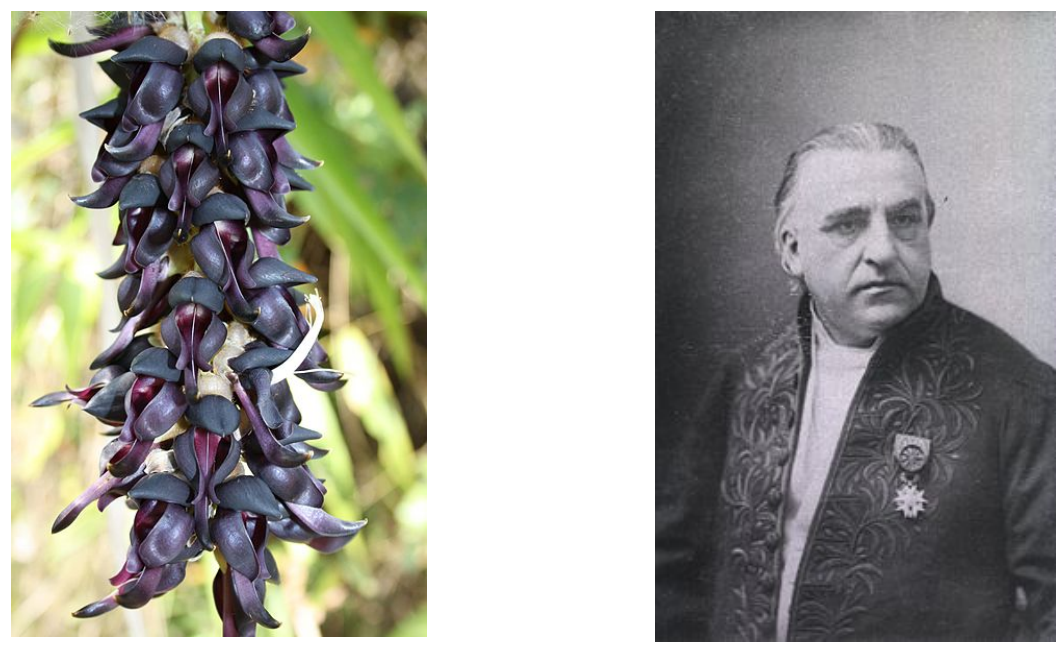

Figure 1.1. Photo of a tropical legume Mucuna pruriens (left), J.-M. Charcot (right).

(C) "Mucuna pruriens" by Agong1 is licensed under CC BY-SA 3.0. (C) Portrait of J.-M. Charcot, Source: NIH / U.S. National Library of Medicine.

Over the past quarter of the century, there has been an explosion in number of publications in the field of Parkinson's disease (Fig. 1.2). Accumulated knowledge and continuous research give us insights into the possible pathological mechanisms that might lead to disease initiation, development and progression. One of the most striking discoveries was identification and characterization of genes responsible for familiar forms of the disease, especially mutations of SNCA ( $\alpha$-synuclein) gene.

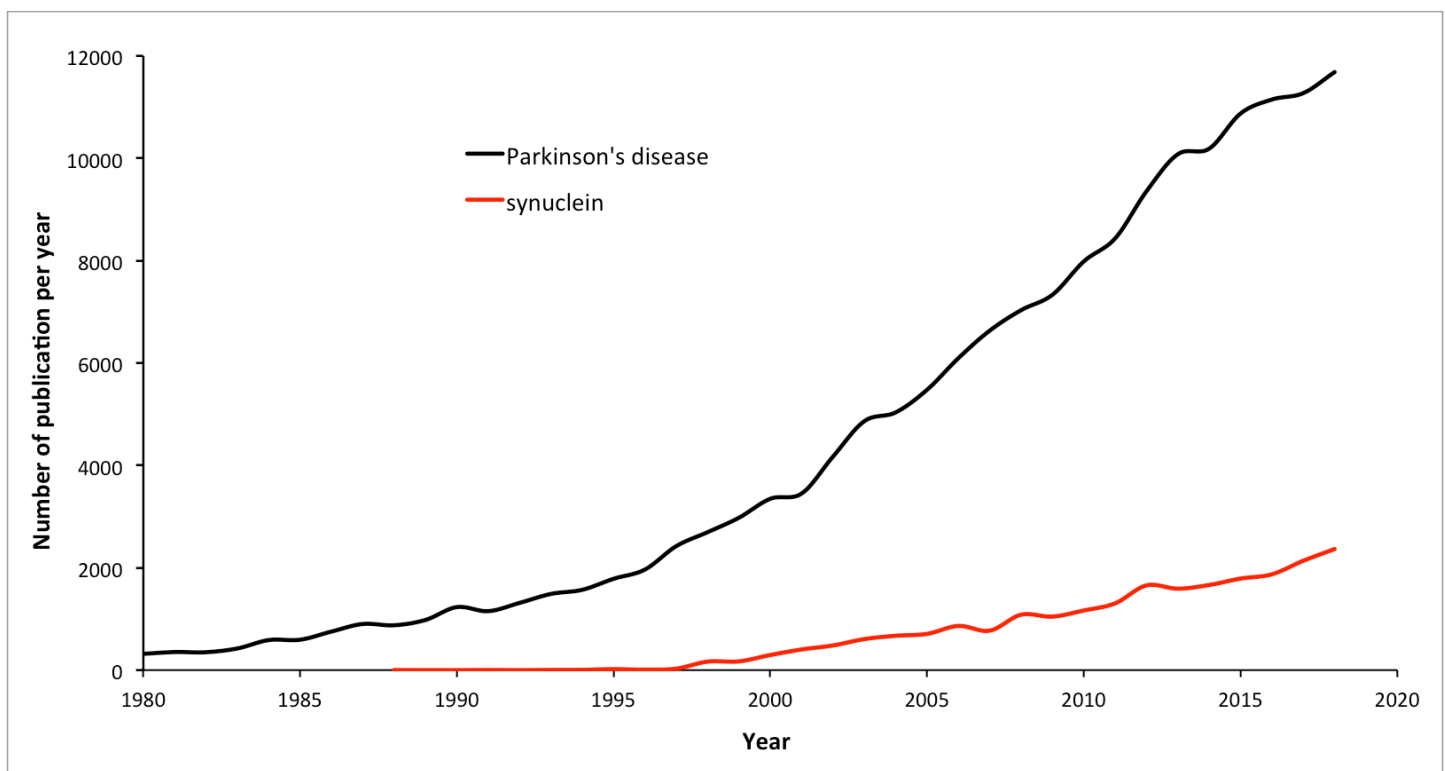

Figure 1.2. Scientific literature. The number of publications per year from 1960 to 2018 that contain "Parkinson's disease" (black) and "synuclein" (red) in the topic (search performed through SciFinder). 
Parkinson's disease is not curable at present. Available drugs such as L-DOPA, COMT-inhibitors, and dopamine agonist just improve the motor symptoms and are less effective during the late phases. Our understanding of molecular causes of the disorder including protein aggregation, oxidative stress, mitochondrial dysfunction, inflammation and others could give us an opportunity to develop new neuroprotective or neurorestorative therapy.

\subsection{Epidemiology, symptoms and pathology in Parkinson's disease}

\subsubsection{Epidemiology}

A descriptive epidemiology operates with a data concerning prevalence, incidence, survival and distribution by age and sex for certain disease. Most cases of Parkinson's disease are idiopathic, i.e. without an obvious family history. Reported incidence rate (annual rate of new cases) for Parkinson's disease varies from 5 to $25^{*} 10^{-5}$ in general population ${ }^{16}$. Another important parameter is a prevalence rate, in case of Parkinson's disease reported values range from $57 / 10^{5}$ in China to $371.5 / 10^{5}$ in Sicily ${ }^{17}$. All incident and prevalence studies demonstrated a strong positive correlation between rate and age. For example, in one study the incident rate was estimated $1 / 10^{5}$ between $0-39,5 / 10^{5}$ between $40-49$ and $174 / 10^{5}$ between $70-79$ years of age ${ }^{17}$. The similar age dependent trend is observed for the prevalence; in Rotterdam 1.4\% and 4.3\% of population have Parkinson's disease over 65 and 85 years of age, respectively ${ }^{18}$. The lifetime risk was estimated at $3.7 \%$ for women and $4.4 \%$ for men in Minnesota ${ }^{19}$. The accumulated data indicates a slightly higher risk of Parkinson's disease in the males. The risk of disorder is also linked to geography, but independent of racial and ethnic background.

\subsubsection{Symptoms}

There is a long list of symptoms associated with Parkinson's disease. The appearance and development of different symptoms can significantly vary from patient to patient. First signs of disease typically begin appearing between the ages 50 and 60; they progress slowly and often are not immediately recognized. Parkinson's disease is the movement disorder with the three cardinal features: resting tremor, rigidity and bradykinesia/hypokinesia ${ }^{20}$. In addition, almost all patients experience a range of non-motor symptoms that could be diagnosed on very early as well as on late stages of the disease (Table 1.1) ${ }^{21}$. A variety of 
motor symptoms likely reflect neuropathological changes in nigrostriatal system. Moreover, a good correlation between non-motor disturbances and neuropathology of regions within the central and peripheral nervous system is observed ${ }^{22}$. The cardinal motor symptoms become apparent when about $80 \%$ of striatal dopamine and $50 \%$ of nigral neurons are $\operatorname{lost}^{23}$.

Table 1.1. Motor and non-motor symptoms of Parkinson's disease (source ${ }^{15,20,21,24-26}$ ).

\begin{tabular}{|c|c|c|}
\hline Symptom & Stage & Frequency \\
\hline \multicolumn{3}{|l|}{ Motor symptoms: } \\
\hline - resting tremor & Early stage & frequent \\
\hline - bradykinesia/hypokinesia & Early stage & frequent \\
\hline - rigidity & Early stage & frequent \\
\hline - postural and gate abnormalities & Advanced stage & frequent \\
\hline - speech and swallowing disorders & Variable & frequent \\
\hline - neuro-ophthalmological abnormalities & Variable & - \\
\hline - respiratory disturbances & Variable & - \\
\hline \multicolumn{3}{|l|}{ Non-motor symptoms: } \\
\hline \multicolumn{3}{|l|}{ - cognitive: } \\
\hline cognitive decline & Variable & $80-90 \%$ \\
\hline dementia & Variable & $50 \%$ \\
\hline \multicolumn{3}{|l|}{ - neurobehavioral: } \\
\hline depression & Variable & $40 \%$ \\
\hline apathy & Variable & $50-55 \%$ \\
\hline anxiety & Variable & $40-50 \%$ \\
\hline - $\quad$ sleep disorders & Variable & $60-98 \%$ \\
\hline - autonomic disturbances & Variable & Up to $90 \%$ \\
\hline \multicolumn{3}{|l|}{ - $\quad$ sensory abnormalities } \\
\hline olfaction & Early stage & $90 \%$ \\
\hline pain & Early stage & frequent \\
\hline
\end{tabular}

\subsubsection{Pathology}

The microscopical examinations of brains from patients with Parkinson's disease reveal a neuronal cell loss, especially of pigmented neurons in the substantia nigra and locus ceruleus. Additionally, neurodegeneration is readily observed in the basal nucleus of Maynert, dorsal motor nucleus of the vagus and olfactory system ${ }^{27}$. Degeneration of dopaminergic neurons that are producing a neurotransmitter dopamine in the substantia nigra leads to a depletion of dopamine in the basal ganglia and, consequently, to a dysfunction of the extrapyramidal motor system. The motor dysfunction becomes apparent when dopamine level in the striatum is reduced by about $70 \%$ to $80 \%$. 
The histological hallmark of Parkinson's disease is a protein accumulation in form of intracytoplasmic Lewy bodies (LBs) and dystrophic Lewy neurites (LNs) (Figure 1.3) that are present in different parts of the brain and in many organs (adrenals, retina, heart, skin) ${ }^{28}$. Heinrich Lewy first discovered these pathological neuronal inclusions in the brains of Parkinson's disease patients in $1912^{29}$. Later similar inclusions were detected in neurons of sibstantia nigra by Tretiakoff, who also introduced the term "Lewy body" in recognition of the seminal contribution of Heinrich Lewy ${ }^{30}$. Two types of LBs are known: brainstem (classical) and cortical (Figure 1.3). Brainstem LBs are spherical, intracytoplasmic, eosinophilic inclusions with a dense core and a peripheral pale-stained halo. The classical LBs are of various sizes ranging from 8-30 $\mu \mathrm{m}$ in diameter. Cortical LBs are eosinophilic structures with irregular shape, quite often missing prominent central core or halo. Ultrastructually, all LBs are composed of 7- to 27-nm filaments that are densely packed in the core of LBs and radically arranged on periphery forming the halo. Abnormal proteinous deposits in neuronal projections are referred to Lewy neurites. Under microscopic examination, LNs are observed in various forms ranging from coarse and rounded to elongated or serpentine structures. Similar to LBs, LNs are composed of densely packed filaments ${ }^{28}$.
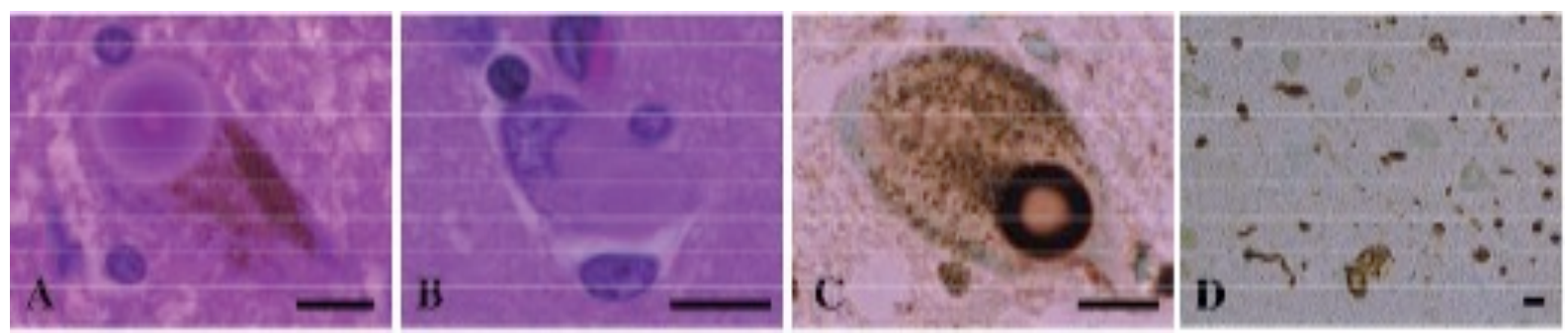

Figure 1.3. Lewy bodies and Lewy neurites. (A) Classical LB in a pigmented neuron in the substantia nigra. (B) Cortical LB in the temporal cortex. (C) Typical LB labeled with anti-asynuclein. (D) Lewy neurites labeled with anti-a-synuclein. Figure reprinted from Neuropathology, Wakabayashi et al. ${ }^{31}$ with permission from John Wiley and Sons, copyright (C) (2007).

Several years ago Braak and colleagues published a study describing a staging of the Lewy pathology in Parkinson's disease based on large autopsy series ${ }^{22}$. They suggested that the development of Lewy pathology is ordered and consists of 6 stages (Figure 1.4). Their findings provide compelling evidence that the pathological changes begin in the lower brain stem ( $\mathrm{dmX}$, intermediate reticular zone and anterior olfactory nucleus) in stage 1 ; further in 
stage 2 caudal raphe nuclei, gigantocellular reticular nucleus and ceruleus-subceruleus complex are affected. The neuropathology on stages 1 and 2 could be correlated with some of early non-motor symptoms (olfactory and autonomic). During stages 3 and 4 Lewy pathology is developed in regions of the upper and midbrain, in particular in the pars compacta of the substantia nigra and amygdala. Remarkably, these stages are associated with an appearance of the motor symptoms. In terminal stages 5 and 6 Lewy bodies are detected in neocortical areas of the brain, and might lead to the cognitive and intellectual decline. In view of these findings, LBs are considered to be a good marker of disease progression.

A

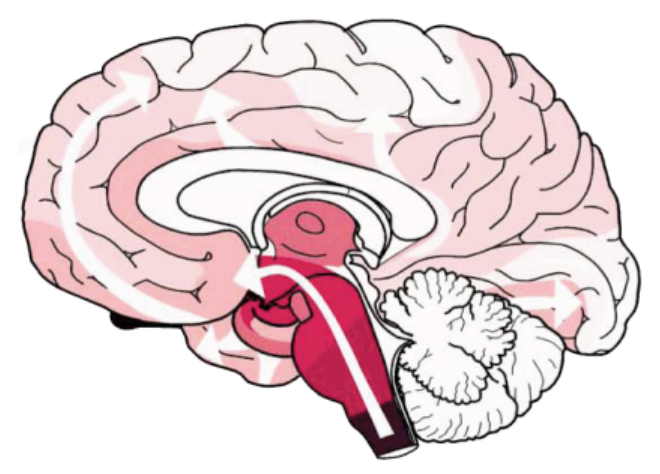

B

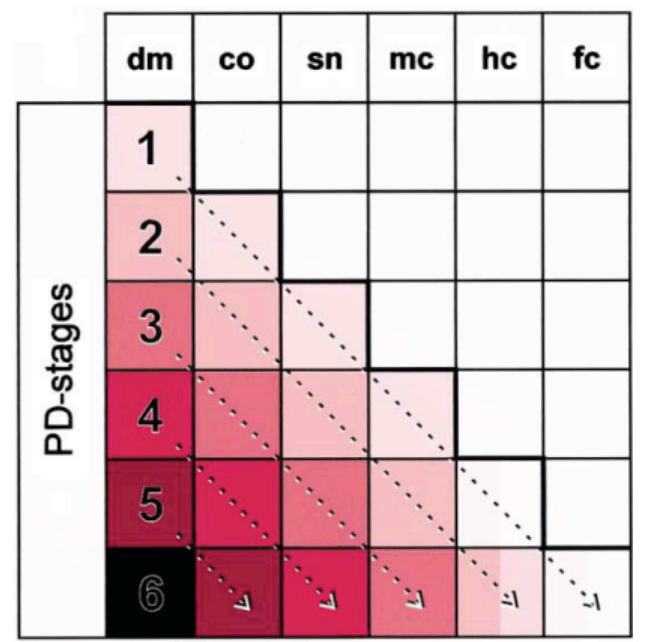

Figure 1.4. Progression of neuronal Lewy pathology. (A) Development of pathology (see white arrows). (B) Staging of Lewy pathology. Figure reprinted from Neurobiology of Aging, Braak et al. ${ }^{22}$ with permission from Elsevier, copyright (c) (2003)

To date more than 70 molecules were identified as a component of LBs and $\mathrm{LNs}^{31}$. In 1997 a Goedert's lab reported a major breakthrough in characterization of LBs with the discovery of protein $\alpha$-synuclein to be a main component of $\mathrm{LBs}^{32}$. At the same time a missense mutation in the $\alpha$-synuclein gene was shown to cause autosomal dominant heritable early-onset Parkinson's disease ${ }^{33}$. These two findings called attention to a role of $\alpha$-synuclein in Parkinson's disease and triggered extensive research activities in $\alpha$-synuclein-related field (Figure 1.2). Whereas $\alpha$-synuclein is considered as a major structural element of LBs and LNs, the composition of these neuronal inclusions is very complex and therefore much effort has been invested in defining of other essential components and its potential role in pathogenesis. The experimental findings reported from these multiple studies are summarized and classified below ${ }^{31}$ : 
1) structural elements of the LB fibrils (different forms of $\alpha$-synuclein)

2) $\alpha$-synuclein-binding proteins (agrin, synrhilin-1, tau, 14-3-3 protein)

3) synphilin-1-binding proteins (NUB1, parkin, prolylisomerase Pin 1, SIAH-1)

4) components of the ubiquitin-proteasome system (ubiquitin, ubiquitin-activating enzyme (E1), ubiquitin ligase (E3), proteasome subunits and others)

5) proteins implicated in cellular responses (molecular chaperon, heat-shock proteins, $\alpha \mathrm{B}$-crystallin, superoxide dismutase 1 and 2 and others)

6) kinases (calcium ${ }^{2+} /$ calmodulin-dependent protein kinase II, cyclin-dependent kinase 5, G-protein-coupled receptor kinase 5, leucine-rich repeat kinase)

7) cytoskeletal proteins (microtubule-associated proteins, neurofilament, tubulin)

8) cell cycle proteins (cyclin B, retinoblastoma protein)

Colocalization of various proteins listed above within LBs suggests a complex molecular pathway combining protein aggregation, post-translational modification and degradation that lead to neurodegeneration and toxicity. Plausible toxic mechanisms and a role of LBs are discussed in details below.

\subsection{Molecular mechanisms of neurodegeneration in Parkinson's disease}

\subsubsection{Genes implicated in Parkinson's disease}

The majority of cases of Parkinson's disease are considered to be idiopathic, however, $5-10 \%$ of cases are associated with familiar form of Parkinson's disease with recessive or dominant modes of inheritance. Based on genome-wide association studies mutations in three genes were identified as a cause of autosomal dominant form of Parkinson's disease (Table $1.2)^{32,33}$. In case of autosomal recessive Parkinson's disease pathological mutations were found in four genes. Linkage data has also identified several loci for which the causative genes are not discovered or functions of these genes are not fully established (Table 1.2). The intensive investigations in the field of genetics of Parkinson's disease have led to advances in our understanding of possible pathological molecular pathways that could be common for familiar as well as for sporadic forms of Parkinson's disease. At the same time, these discoveries provide us an opportunity to develop new model systems and to find new therapeutic targets. 
Table 1.2. Genetic Forms of Parkinson's disease $\mathrm{d}^{34,35}$

\begin{tabular}{|l|l|l|l|l|}
\hline Locus & Gene/protein & $\begin{array}{l}\text { Inheritance } \\
\text { pattern }\end{array}$ & \multicolumn{1}{|c|}{ Typical phenotype } & Function of protein \\
\hline $\begin{array}{l}\text { PARK1 } \\
\text { and } \\
\text { PARK4 }\end{array}$ & o-synuclein & AD* & Earlier onset & Synaptic protein \\
\hline PARK2 & parkin & AR* (mostly) & Early onset, slow progression & Ubiquitin-protein ligase \\
\hline PARK3 & Unknown & AR & Classic PD & - \\
\hline PARK5 & UCH-L1 & Unclear & Classic PD & $\begin{array}{l}\text { Ubiquitin carboxyl- } \\
\text { terminal hydroxylase }\end{array}$ \\
\hline PARK6 & PINK1 & AR & Early onset, slow progression & Mitochondrial kinase \\
\hline PARK7 & DJ-1 & AR & Early onset, slow progression & Oxidative stress protection \\
\hline PARK8 & LRRK-2 & AD & Classic PD & Kinase \\
\hline PARK9 & ATP13A2 & AR & - & Lysosomal protein \\
\hline PARK10 & Unknown & Unclear & Classic PD & - \\
\hline PARK11 & Unknown & Unclear & Classic PD & - \\
\hline PARK13 & HtrA2/Omi & AD & - & Protease \\
\hline
\end{tabular}

*AD, autosomal dominant; AR, autosomal recessive; PD, Parkinson's disease

\subsubsection{Impairment in the Ubiquitin-Proteasomal System (UPS)}

The ubiquitin-proteasomal system is the main system in the eukaryotic cells responsible for the degradation of soluble proteins. The general degradation pathway is shown in the Figure 1.5. On first step monomeric 76-amino acid polypeptide, known as ubiquitin, is activated by the ubiquitin-activating enzyme (E1). Ubiquitin is then transferred to the ubiquitin-conjugating enzyme (E2). On next step, the ubiquitin protein ligase (E3) recognizes unwanted proteins (mutated/damaged/misfolded/short-lived) and catalyzes the transfer of ubiquitin from E2 enzyme by the formation of isopeptide bond between Cterminus of ubiquitin and lysine residue of the substrate protein. The cycle of ubiquitination is repeated several times until a polyubiquitine chain linked through K29 and K48 is formed. The polyubiquitinated proteins are transferred to the $26 \mathrm{~S}$ proteasome for degradation, resulting in formation of small peptides. The polyubiquitine chain is cleaved by deubiquitinating enzymes (DUB) to produce the free monomeric ubiquitin ${ }^{36}$.

A growing number of evidence suggests that dysfunction of UPS may contribute to the pathogenesis of Parkinson's disease. In case of sporadic form of Parkinson's disease, significant alterations of proteasome system were observed in the brains of patients. Postmortem comparison of brain tissues reveals $40 \%$ reduction in content of proteasomal $\alpha$ subunit in substantia nigra and moreover the proteasomal enzymatic activities were reduced by $44 \%-55 \%{ }^{37,38}$. In contrast to degenerated regions, the UPS activities are not disturbed in 
other brain regions such as cerebellum or cortex. Furthermore, alterations in the levels of proteasome regulators were reported, for example, mRNA expression of PA700 regulator and ATPase subunit is reduced in substantia nigra of Parkinson's disease patients ${ }^{39}$.

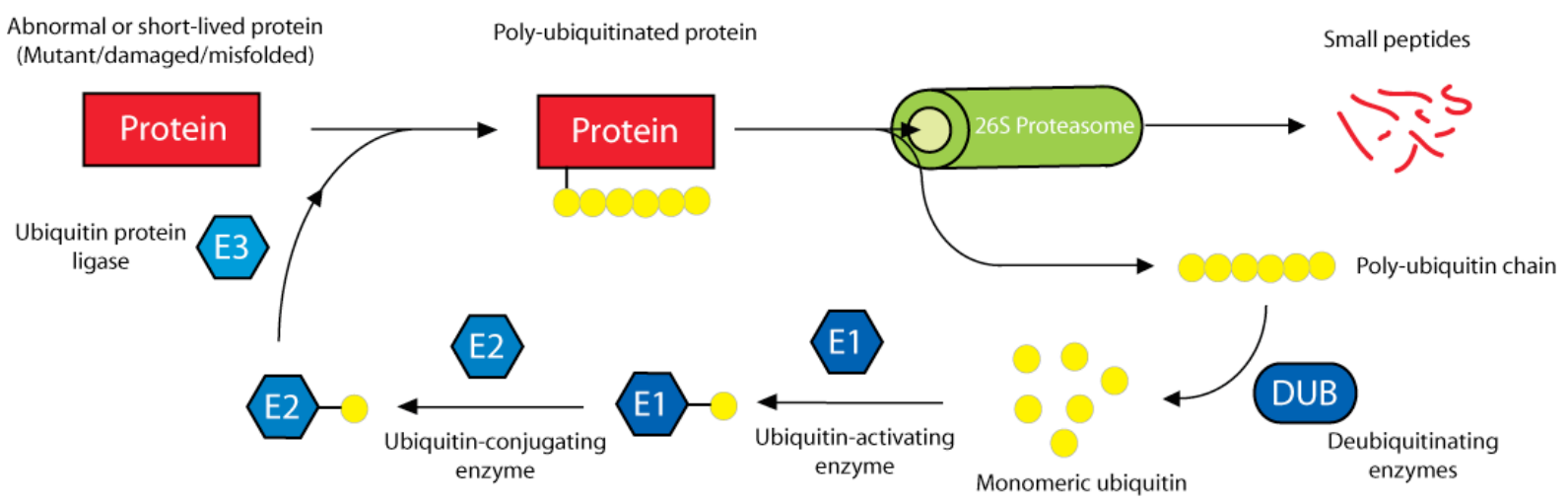

Figure 1.5. Ubiquitin-Proteasomal System

Probably the most compelling evidence supporting the role of UPS in pathogenesis is the association of parkin $^{40}$ and $U C H-L 1^{41}$ with familiar forms of Parkinson's disease. Both proteins are the part of UPS machinery: parkin has been shown to be the E3 ubiquitin ligase $^{42}$; UCH-L1 (ubiquitin carboxyl-terminal hydrolase L1) belongs to a family of deubiquitinating enzymes ${ }^{43}$.

Parkin is a 465 amino acid protein that is expressed in cytoplasm, nucleus and Golgi apparatus. As E3 ubiquitin ligase, parkin provides substrate selectivity and catalyzes a transfer of ubiquitin from E2 enzyme to proteins targeted for degradation by the UPS. Most mutations in parkin are thought to be loss-of-function and give rise to alterations of parkin properties like solubility, localization, expression levels, selectivity and enzymatic activity. Therefore, all kinds of parkin inactivation may lead to the toxic protein accumulation followed by aggregation and proteolytic stress as a consequence of the defective UPSmediated degradation. Due to this fact, a significant number of protein substrates of parkin were identified in order to understand their possible role in neurodegeneration. According to the literature, parkin can ubiquitinate a variety of diverse and widely distributed substrates including symphilin-1, the parkin-associated endothelin receptor-like receptor (Pael-R), CDCrel-1, a rare O-glycosylated isoform of $\alpha$-synuclein, p38 subunit of the aminoacyl-tRNA synthetase complex and others ${ }^{34}$. But since many of these substrates were discovered using in vitro or cell culture experiments the additional work needs to be done to confirm relevance in vivo. In search of possible mechanism, it was demonstrated that overexpression of parkin 
substrates such as $\mathrm{CDCrel}-1^{44}$, Pael- $\mathrm{R}^{45}$, p38 subunit ${ }^{46}$ and syphilin- $1 / \alpha$-synuclein ${ }^{47}$ in cell culture results in formation of cytoplasmic inclusions and/or cell death. At the same time, overexpression of parkin can protect against dopamine-mediated toxicity and toxicity induced by overexpression of some proteins, including Pael- $\mathrm{R}^{45}$, the $\mathrm{p} 38$ subunit ${ }^{46}$, mutated $\alpha$-synuclein. Furthermore, experiments with parkin knockout mice revealed an unexpected role of parkin in the regulation of normal mitochondrial function ${ }^{48}$, later this data was also confirmed in Drosophila model of Parkinson's disease ${ }^{49}$. Collectively, the properties of parkin allow us to assume that parkin can be a multipurpose neuroprotective agent.

Ubiquitin C-terminal L1 (UCH-L1) is the second protein that is involved in the UPS and probably implicated in the pathogenesis of Parkinson's disease. UCH-L1 is a highly abundant (1-2\% of the soluble proteins in the brain) 230 -amino acid protein that is expressed exclusively in neurons ${ }^{43}$. The function of UCH-L1 is poorly understood, but it is known to act as deubiquitinating enzyme hydrolyzing polyubiquitin chains, and additionally, as dimerization-dependent ubiquitin ligase $\mathrm{e}^{50}$. It has been demonstrated that mutations in UCHL1 lead to impairment of the de-ubiquitinating activity and levels of free ubiquitin in vitro and in vivo ${ }^{51}$. These results imply that UCH-L1 may modulate the overall efficiency of UPS. Interestingly, UCH-L1 can promote the accumulation of $\alpha$-synuclein in cultured cells probably due to the alternative $\alpha$-synuclein ubiquitination where poly-ubiquitin chains are linked through $\mathrm{K} 63^{50}$.

Additional evidence for the importance of the UPS in Parkinson's disease pathogenesis comes from laboratory studies. Thus, injections of proteasome inhibitors into the rat brain lead to a preferential degeneration of dopaminergic neurons in combination with the formation of Lewy body-like inclusions ${ }^{52}$. Also, a knockout mouse that lack the $26 \mathrm{~S}$ proteasome genes recapitulates many characteristics of Parkinson's disease, including nigrostriatal degeneration, inclusion body formation, and motor dysfunction ${ }^{53}$.

\subsubsection{Mitochondrial dysfunction and oxidative stress}

\subsubsection{Sporadic Parkinson's disease and mitochondria}

Mitochondria play a key role in many cellular processes including energy metabolism, calcium homeostasis, cellular quality control pathways and cell death regulation. Several lines of evidence suggest that mitochondrial dysfunction and oxidative stress are involved in the pathogenesis of Parkinson's disease. The notion of mitochondrial dysfunction was also supported by discovery of several genes that are linked to familiar form of 
Parkinson's disease and play a role in regulation of mitochondria function, morphology, and dynamics $^{54,55}$.

Mitochondria produce most of the energy in form of ATP that it's needed for the cell to function. The key process of ATP synthesis is called oxidative phosphorylation that consists of electron transport chain (ETC) and ATP synthase. It's well known that due to the ETC, mitochondria are exposed to a highly oxidative environment including harmful reactive oxygen species (ROS) ${ }^{56}$. Normally, the oxidative state is strictly controlled by mitochondrial defense system. But the failure in some mitochondrial pathways may result in oxidative stress characterized by increased concentrations of ROS and free radicals that are known to be toxic. Currently mitochondrial dysfunction is associated with many human diseases through different pathways including deficiency in ETC, generation of free radical and ROS, mitochondrial permeability transition, excitotoxicity, inflammation and others ${ }^{57}$.

Postmortem analysis of brain tissues from patients with sporadic Parkinson's disease revealed an increased level of oxidative stress, which causes oxidative damage of lipids, proteins and DNA especially in substantia nigra ${ }^{58}$. It has been suggested that oxidative stress in dopaminergic neurons can be initiated by mitochondrial dysfunction, increased dopamine metabolism, and impaired antioxidant defense pathways. The mitochondrial impairment was also supported by findings of decrement in the complex-I activity in substantia nigra ${ }^{59}$ as well as disturbances in complex-I, II+III, and IV located in the muscles of Parkinson's disease patients $^{60,61}$. Furthermore, a selective decrease in a number of subunits in complex-I was demonstrated in the affected brains ${ }^{62}$.

\subsubsection{Mitochondrial toxins}

Some of the most compelling evidence for the important role of mitochondria in neurodegeneration has come through discoveries of mitochondrial toxins like MPTP (1methyl-4-phenyl-1,2,3,6-tetrahydropyridine), rotenone, paraquat and maneb (Figure 1.6) in combination with epidemiological studies. The first incident of MPTP-induced parkinsonism was reported in the 1980s for a group of young addicts that were applying a homemade drug from meperidine family ${ }^{63}$. Later studies have demonstrated that the drug mixtures contain a by-product 1-methyl-4-phenyl-1,2,3,6-tetrahydropyridine (MPTP, Figure 1.6) that induces Parkinson's disease-specific pattern of nigrostriatal degeneration in human ${ }^{64}$, non-humanprimates $^{65}$, and mice ${ }^{66}$. Much effort has been invested in defining the molecular mechanism of MPTP-induced neurotoxicity. As it stands now, MPTP crosses the blood-brain barrier; in the brain MPTP is converted to MPP+ by monoamine oxidase, and then MPP+ is selectively 
transported to dopaminergic neurons by the dopamine transporter. In neuronal cells MPP+ is localized in mitochondria, where it inhibits complex-I of the electron transport chain and leads to increased oxidative stress, decreased energy production and neurodegeneration ${ }^{67}$. The epidemiological studies provided additional evidences that impairment of ETC could be an important cause of the development of the Parkinson's disease. For instance, several reports have implicated a role of chemicals that widely used in the agriculture in an increased risk of Parkinson's disease ${ }^{68}$. The disease has been reported to occur at high rates among population that was exposed to herbicide paraquat ${ }^{69}$, insecticide rotenone, and fungicide maneb $^{70}$. Laboratory studies have demonstrated that paraquat and rotenone are complex-I inhibitors $^{71}, 72$, while maneb binds to complex-III ${ }^{73}$. In rodents, the application of these compounds induced pathological features that closely resemble sporadic Parkinson's disease, including selective nigrostriatal degeneration, dopamine depletion, and formation of Lewybody-like cytoplasmic inclusions ${ }^{74}$ (toxin-induced animal models will be discussed in details below).<smiles>CN1CC=C(c2ccccc2)CC1</smiles><smiles>[X]c1ccc(-c2ccccc2)cc1</smiles><smiles></smiles><smiles>C=C(C)[C@H]1Cc2c(ccc3c2O[C@@H]2COc4cc(OC)c(OC)cc4[C@H]2C3=O)O1</smiles><smiles>CC(C)(C)SC(=S)NCCNC(=S)SC(C)(C)C</smiles>

MPTP $\mathrm{MPP}^{+}$ paraquat

rotenone maneb

Figure 1.6. Mitochondrial toxins.

\subsubsection{Mitochondria and familiar forms of Parkinson's disease}

The relevance of mitochondrial abnormalities in Parkinson's disease was further supported by the identification of the genes that are linked to familiar Parkinson's disease. Further studies of the gene products have shown that some of them are involved in pathways regulating mitochondrial integrity, cellular stress response and quality control systems. To date, mutations of five genes are directly or indirectly implicated in mitochondrial dysfunction, including parkin, PINK1, DJ-1, LRRK2 and $\alpha$-synuclein. 


\subsubsection{PINK1}

Mutations in PINK1 (PTNE-induced kinase-1) are the second most common cause of autosomal recessive Parkinson's disease. PINK1 is a 581-amino acid, ubiquitously expressed in the brain protein with highly conserved serine-threonine kinase domain and, notably, a mitochondrial targeting sequence at its $\mathrm{N}$-terminus ${ }^{75}$. Accordingly, under overexpression conditions PINK1 is localized in the mitochondrial membrane in cultured cells ${ }^{75,76}$.

To date, most of known pathological mutations in PINK1 are localized in the putative kinase domain, and considered to be a loss-of-function due to the impaired kinase activity ${ }^{75}$, 77. Accumulating evidences suggest that PINK1 plays important role in maintaining the proper mitochondrial function. For example, overexpression of PINK1 in neurons was shown to be protective against mitochondrial dysfunction and apoptosis under various stress conditions $^{75,78}$. Furthermore, deletion of PINK1 or expression of loss-of-function mutants in vivo models lead to mitochondrial abnormalities comprising decreases in mitochondrial membrane potential, complex I and IV activities, ATP production, increases in ROS production, abnormal mitochondrial morphology, impaired calcium homeostasis and mitochondrial trafficking. Thus, the deletion of PINK1 in Drosophila has been shown to induce mitochondrial morphological defects, locomotor deficits, degeneration of muscle and dopaminergic neurons, and male sterility ${ }^{79}, 80$. Significant mitochondrial defects have been also observed in PINK1-deficient mice ${ }^{81}$. In contrast, the overexpression of PINK1 in primary neurons as well as in mice is protective against MPP+/MPTP toxicity ${ }^{82}$. Based on these studies it was suggested that PINK1 probably prevents the mitochondrial dysfunction by phosphorylation of mitochondrial proteins under cellular stress, while the mutations in PINK1 may abolish the kinase activity and, consequently, the protective function. To corroborate this theory two PINK1 substrates were identified: TNF receptor-associated protein $1^{83}$ (TRAP1) and mitochondrial serine protease HtrA2 ${ }^{84}$ (also known as Omi). Interestingly, both putative PINK1 substrates are associated with mitochondria. It has been demonstrated that TRAP1 is a mitochondrial chaperone that could be phosphorylated by PINK1 in vitro and in cell models. In cells, phosphorylated TRAP1 prevents the release of cytochrome $\mathrm{c}$ from the mitochondria under oxidative stress ${ }^{83}$. Second binding partner for PINK1 is a mitochondrial serine protease called HtrA2/Omi, which is involved in apoptotic pathway and in protection against cellular stress. It has been shown that phosphorylation of HtrA 2 that is modulated by expression of PINK1 increases the HtrA2 protease activity and protects the cells against PD-causing toxins ${ }^{84,}{ }^{85}$. Collectively, it is plausible that the 
interactions of PINK1 with TRAP1 and HtrA2 play an important role in the protection of the mitochondria against stressor, however mechanisms remain to be formally demonstrated.

\subsubsection{DJ-1}

Mutations in the gene encoding protein DJ-1 are very rare and cause an autosomal recessive early onset Parkinson's disease ${ }^{86}$. Apart from the mutations, the implication of DJ-1 in Parkinson's disease is also supported by detection of insoluble forms of DJ-1 in the brains of sporadic patients ${ }^{87}$. DJ-1 is a conserved 189-amino acid protein that is abundantly expressed in most of mammalian tissues and brain $^{88}$. Several studies reported that DJ-1 could be found in the cytosol, mitochondria and nuclear compartment ${ }^{89}, 90$. Although the exact physiological function of DJ-1 is not fully established, DJ-1 is considered to be an atypical peroxiredoxin-like peroxidase (catalyzes a reaction: $\mathrm{ROOR}^{\prime}+2 \mathrm{e}^{-}+2 \mathrm{H}^{+} \rightarrow \mathrm{ROH}+\mathrm{R}^{\prime} \mathrm{OH}$ ) with a catalytic site around Cys106 that is a principal target for sulfinic acid formation ${ }^{91}$. It has been also hypothesized that DJ-1 may function as a sensor of oxidative stress or as an anti-oxidant protein. In cells, overexpression of DJ-1 protects against oxidative stress whereas a deletion of DJ-1 promotes the cell death under oxidative conditions ${ }^{92}, 93$. Furthermore, protective properties of DJ-1 have been also demonstrated against proteasomal inhibition and endoplasmic reticulum stress ${ }^{94}$. In search of a mechanism, it has been shown that the oxidized form of DJ-1 is translocated to mitochondria, where it has increased neuroprotective activity ${ }^{91}$. Studies in DJ-1 knock-out mice revealed an increased susceptibility of animals to the toxicity induced by MPTP and paraquat; additionally, a twofold increase in hydrogen peroxide was observed that could be an indication of failure in regulation of mitochondrial $\operatorname{ROS}^{95,96}$. Several experimental observations have implicated DJ1 in dopamine signaling and the cell death pathway ${ }^{97,}{ }^{98}$. Altogether, DJ-1 may be a crucial component of the system involved in detection as well as in protection against a wide range of stress conditions.

\subsubsection{LRRK2}

Mutations in the LRRK2 gene are found in the majority of autosomal dominant lateonset forms of Parkinson's disease ${ }^{99}$. LRRK2 is $280 \mathrm{kDa}$, multidomain protein (2527 amino acids) from ROCO protein family with unknown physiological function. Most of its pathological mutations are considered to be due to gain-of-function, although the effect of these mutations on LRRK2 function is unclear. Immunohistochemical studies of LRRK2 
overexpression in cellular culture have shown that LRRK2 is localized in the cytoplasm and membranes including the outer mitochondrial membrane ${ }^{100}$, in the patients LRRK2 was detected in LBs and $\mathrm{LNs}^{101}$. Purified LRRK2 protein shows a kinase activity in vitro that is significantly increased for several disease-causing LRRK2 mutations (I2020T, G2019S) ${ }^{102}$. These findings imply that increased or abnormal phosphorylation of LRRK2 substrates may be involved in neurodegeneration, although no putative LRRK2 substrate has been identified so far. Collectively, our knowledge about LRRK2 protein is limited; therefore there are many issues to be resolved about LRRK2 in order to understand its role in neurodegeneration.

\subsubsection{Overview}

To date the exact mechanisms of neurodegeneration for Parkinson's disease are still unknown but the discovery of genes causing familiar forms of the disease suggests several molecular pathways that probably contribute to neuronal death (Fig 1.7). One of the key events is the abnormal accumulation/aggregation of misfolded proteins that could be associated with impairment of the degradation system, abnormal protein conformation or modification (UPS, Parkin, UCH-L1, $\alpha$-synuclein). The second permanent hallmark is the mitochondrial dysfunction in combination with the oxidative stress and ATP depletion. Several genes (PINK1, LRRK-2, DJ-1) are implicated in mitochondrial function, integrity and protection from stressors. These discoveries give us an opportunity to understand on molecular level the pathogenesis in Parkinson's disease and consequently to develop diseasemodifying therapy which in turn depending on their activity would also support or refute the importance of those toxicity mechanisms that they target. 


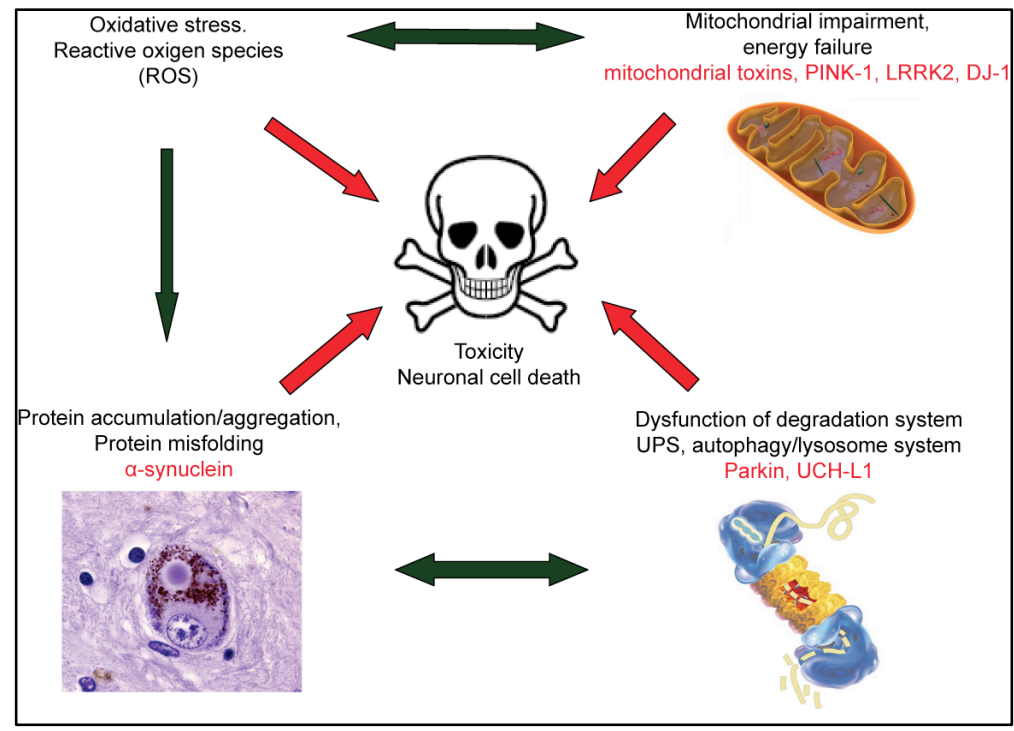

Figure 1.7. Possible mechanisms of neurodegeneration in Parkinson's disease

\section{4. $\alpha$-Synuclein and its role in Parkinson's disease}

\subsubsection{Introduction}

In 1988 Scheller and colleagues have published a report describing a new protein that was found in two species, namely Torpedo and rats ${ }^{103}$. Based on available data, this new protein was classified as neuron-specific protein with the cellular localization to the nuclear envelope and presynaptic nerve terminals and with a possible role in regulation of nuclear and synaptic events. On the basis of the subcellular localization, the isolated protein was named synuclein, where parts of the name syn/nucle originated from synapse and nucleus. Few years later, a central part of $\alpha$-synuclein (residues 61-95), so called non-amyloid-beta component of Alzheimer's disease (NAC), was identified as a second major component of amyloid plaques of Alzheimer's disease patients and these findings triggered the speculations about a possible role of synuclein in $A \beta$ pathology. However, following studies have failed to reproduce this data and, therefore, there is no consensus regarding the link between $\alpha$ synuclein and Alzheimer's disease ${ }^{104,105}$.

Groundbreaking discoveries that strongly supported a causal role for $\alpha$-synuclein in Parkinson's disease were published in 1997. First of all, it's the identification of a single point mutation in the $\alpha$-synuclein gene (A53T) causing the autosomal dominant early-onset Parkinson's disease ${ }^{33}$. Second crucial finding is recognition of fibrillar $\alpha$-synuclein as a main component of Lewy bodies ${ }^{32}$. Expanding research has further corroborated the notion that $\alpha-$ synuclein is a key player contributing to pathology of Parkinson's disease. This chapter is 
addressing the biophysical and biochemical properties of $\alpha$-synuclein and also underlying molecular mechanisms of $\alpha$-synuclein-induced toxicity.

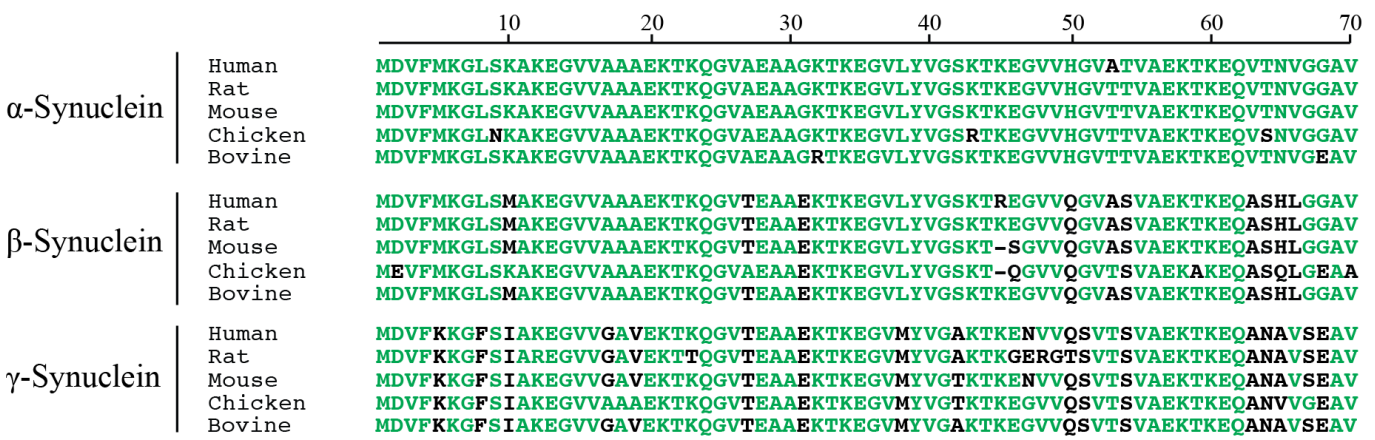

\begin{tabular}{l|l}
$\alpha$-Synuclein & $\begin{array}{l}\text { Human } \\
\text { Rat } \\
\text { Mouse } \\
\text { Chicken } \\
\text { Bovine }\end{array}$ \\
$\beta$-Synuclein & $\begin{array}{l}\text { Human } \\
\text { Rat } \\
\text { Mouse } \\
\text { Chicken } \\
\text { Bovine }\end{array}$ \\
$\gamma$-Synuclein & $\begin{array}{l}\text { Human } \\
\text { Rat } \\
\text { Mouse } \\
\text { Chicken } \\
\text { Bovine }\end{array}$
\end{tabular}

80
90

Figure 1.8. Synuclein sequences. Alignment and relationships of $\alpha-, \beta-, \gamma$-synuclein sequences from five species. Green color-code indicates identity with rat $\alpha$-synuclein.

\subsubsection{Synuclein family}

Synucleins are small, soluble, heat-resistant proteins with a high degree of sequence conservation (Fig. 1.8) and, structurally, are classified as natively unfolded ${ }^{104,106}$. So far, the synuclein gene has been only discovered in vertebrates. The synuclein protein family includes three members, namely, alpha-synuclein ( $\alpha$-synuclein), beta-synuclein ( $\beta$-synuclein) and gamma-synuclein ( $\gamma$-synuclein) (Fig 1.8). All family members show high sequence conservation of N-terminus coding for amphipathic $\alpha$-helices that are structurally similar to lipid-binding domains of class $\mathrm{A}_{2}$ apolipoproteins, while C-terminus is significantly less conserved and highly enriched in acidic residues ${ }^{107,108}$. Despite extensive efforts, the exact physiological function for any of synuclein proteins remains elusive, but several lines of evidence point the role of $\alpha$-synuclein in regulation of synaptic function. As it stands now, just $\alpha$-synuclein is implicated in pathogenesis of neurodegenerative diseases, collectively referred as synucleinopathies. The morphological hallmark of these diseases is a formation of Lewy body-like pathology in a subset of neurons or glia with fibrillar $\alpha$-synuclein as a major component. 


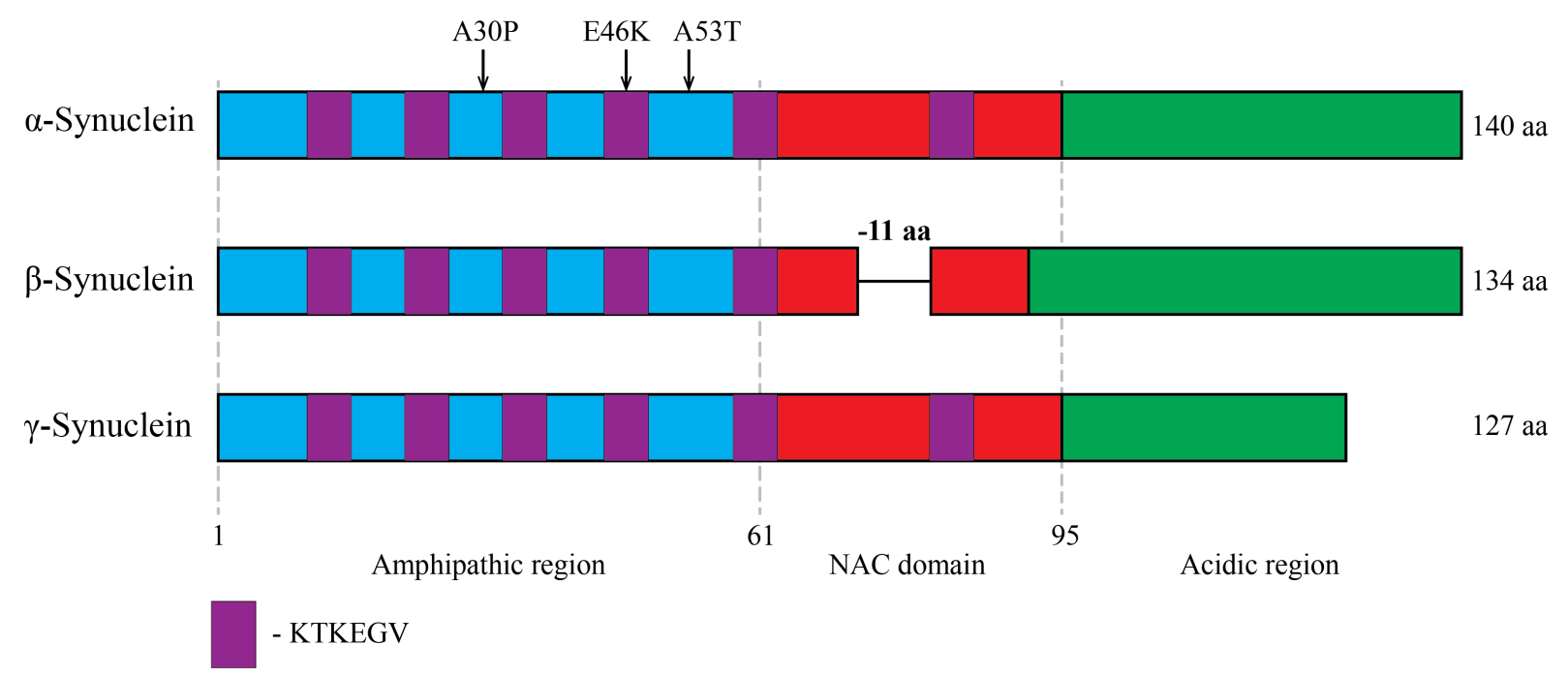

Figure 1.9. Human synuclein domain organization. Proteins domain organization and missense mutations of $\alpha$-synuclein associated with familiar Parkinson's disease.

\subsection{3. $\beta$-Synuclein and $\gamma$-synuclein}

Despite the fact that $\alpha$-synuclein is of major interest, the knowledge about $\beta$ - and $\gamma$ synuclein properties might provide us a valuable link between structure and observed behavior for the whole protein family. Both $\beta$ - and $\gamma$-synuclein have been found in human, $\beta$ synuclein gene $(S N C B)$ is mapped to chromosome $5 \mathrm{q} 35$ and encodes 134-amino-acid protein, while $\gamma$-synuclein gene (SNCG) is localized to chromosome 10q23 and coding 127-aminoacid protein (Fig 1.8 and 1.9) ${ }^{105,107,108}$. The tissue expression profile of $\gamma$-synuclein is distinct from one observed for $\alpha$ - and $\beta$-synuclein. Thus, $\gamma$-synuclein is mainly expressed in peripheral nervous system and retina (primary sensory neurons, sympathetic and motor neurons), also in smaller concentrations is present in the brain, heart, colon, and testis. $\beta$ Synuclein, similar to $\alpha$-synuclein, is primarily detected in the brain tissues with the widespread distribution in different areas including neocortex, hippocampus, striatum, thalamus and cerebellum ${ }^{105,107}$. Despite extensive efforts, the exact physiological functions of any synuclein proteins are still unknown.

All three human synuclein proteins share well conserved sequence and domain organization. Human $\beta$ - and $\gamma$-synuclein are shorter than 140-amino-acid $\alpha$-synuclein, namely 134 and 127 residues respectively, intrinsically disordered in aqueous solution and are divided in three domains (Fig. 1.9) ${ }^{107,109}$. N-terminus is most conserved region and this domain has a characteristic pattern of imperfect hexameric (KTKEGV) repeats that are involved in formation of amphipathic helix upon binding to the lipid bilayer ${ }^{107}$. Indeed, all members of synuclein protein family interact directly with lipids and membranes. In the 
lipid-bound state, that in turn might be crucial for physiological function, the N-terminus that is unfolded in solution adopts an $\alpha$-helical structure ${ }^{110}$. The central domain, referred as NAC region, is hydrophobic and associated with the amyloidogenic properties. The less conserved C-terminus is highly enriched in acidic residues (aspartate, glutamate) and, therefore, has a significant negative charge. There are some indications that the $\mathrm{C}$-terminal domain is involved in chaperone-like activity. Remarkably, the NAC region of $\beta$-synuclein is missing 11 amino acids and this deletion seems to be responsible for significantly reduced propensity of $\beta$-synuclein to form amyloid fibrils. Moreover, several in vitro studies have demonstrated that $\beta$-synuclein significantly inhibits the aggregation of $\alpha$-synuclein and, thereby suggesting a possible protective role against $\alpha$-synuclein-induced toxicity ${ }^{110}$. At present no substantial link between Parkinson's disease and $\beta$ - or $\gamma$-synuclein has been identified, as supported by the absence of $\beta$ - or $\gamma$-synuclein in Lewy bodies, lack of toxicity in animal models and other findings. In complementary experiments addressing the aggregation properties of these proteins in vitro, $\alpha$-synuclein is prone to form amyloid fibrils at most, while $\beta$ - and $\gamma$ synuclein aggregate significantly slower, in case of $\beta$-synuclein the aggregation occurs just in presence of metal ions ${ }^{110,111}$. Interestingly, $\gamma$-synuclein has been recognized as one of the breast cancer markers, since the $\gamma$-synuclein expression levels are directly correlating with the disease progression and metastatic potential of the breast tumors ${ }^{105}$.

\subsubsection{Synucleinopathies}

\subsubsection{Introduction}

Synucleinopathies are a class of devastating neurodegenerative disorders in which $\alpha$ synuclein misfolding and formation of intracellular Lewy body-like pathology are the permanent histopathological hallmarks. In addition to Parkinson's disease, the most prevalent among synucleinopathies, three other diseases, such as dementia with Lewy bodies (DLB), multiple system atrophy (MSA) and pure autonomic failure (PAF), are classified as synucleinopathies. Remarkably, until now the exact mechanisms of toxicity remain unresolved for all synuclein-related disorders, moreover the disease-modifying therapy is also not available. 


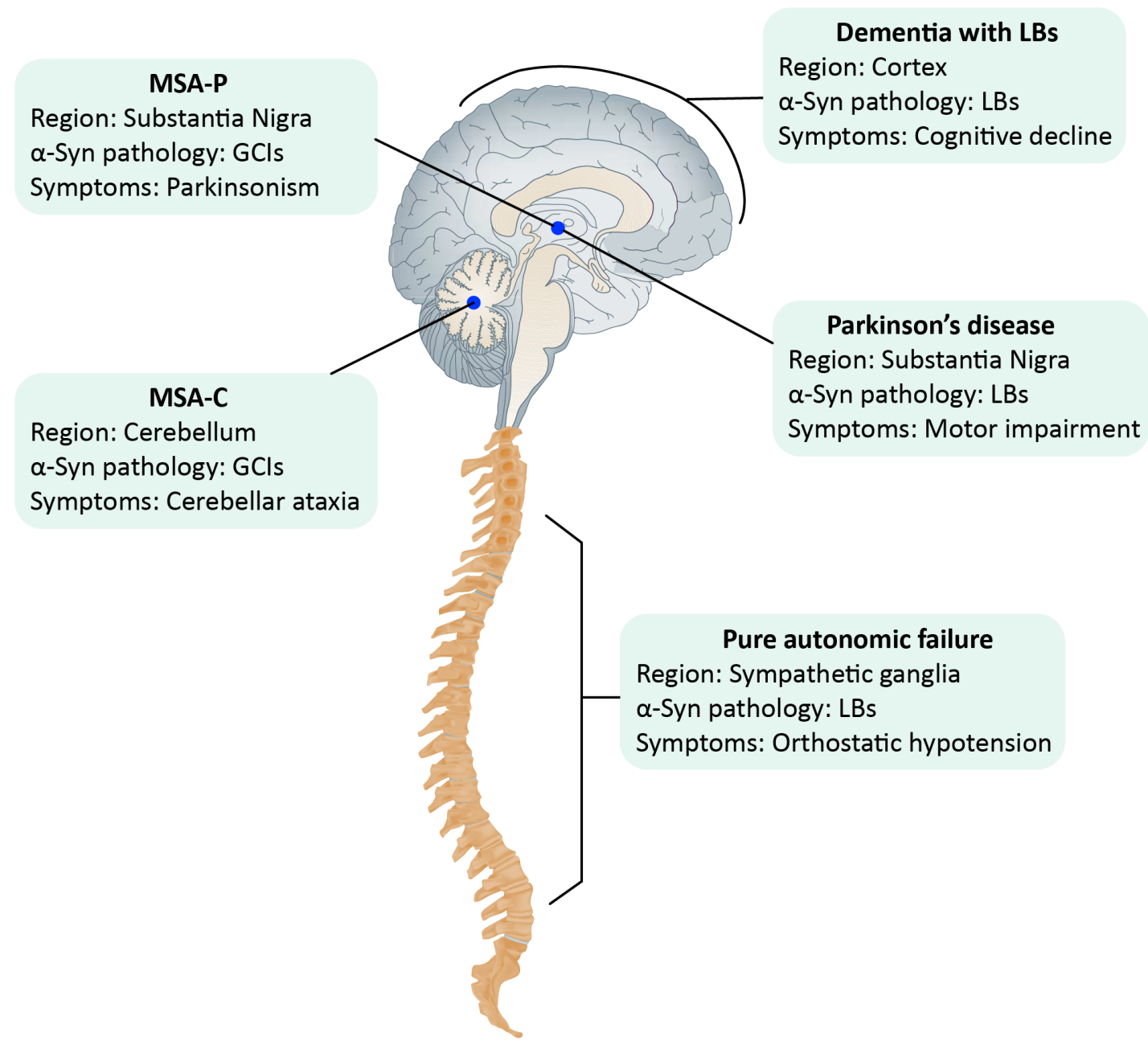

Figure 1.10. Overview of synucleinopathies and key hallmarks.

Spinal cord image designed by brgfx / Freepik.

\subsubsection{Dementia with Lewy bodies}

Dementia with Lewy bodies is a progressive neurodegenerative disease with the sporadic appearance and the age as a main risk factor ${ }^{105,112}$. DLB is quite often misdiagnosed with actual Alzheimer's disease because its clinical signs and symptoms are quite similar. DLB patients show diverse clinical phenotypes that are represented by a range of neuropsychiatric symptoms as well as motor impairments dominated by bradykinesia and rigidity. Classical histopathological markers are degeneration and neuronal Lewy body-like pathology in the cortical and limbic regions accompanied by a frequent extending to brainstem and nigral areas ${ }^{105,112}$. A fact that burden of cortical $\alpha$-synuclein pathology shows a good correlation the severity of phenotype further supports the implication of protein misfolding in neurodegeneration. 


\subsubsection{Multiple system atrophy}

Among synucleinopathies, MSA is a most severe and rapidly progressive neurodegenerative disease that could be pharmacologically poorly controlled. Two motor subtypes of MSA are known, namely MSA-P that associated with parkinsonian phenotype (prevalent symptoms: progressive akinesia, rigidity, autonomic failure) and MSA-C showing cerebellar ataxia phenotype characterized by cerebellar oculomotor dysfunction, ataxic gait, limb kinetic ataxia and autonomic failure as dominating symptoms ${ }^{105,112}$. In simple words, MSA patient suffer from problems with movement, muscle control, blood pressure regulation, heart rate and bladder function. In contrast to other synucleinopathies, unique histopathological hallmark of MSA is predominant formation of $\alpha$-synuclein-positive cytoplasmic inclusions in oligodendrocytes (argyrophilic fibrillar inclusions) and glial cells (glial cytoplasmic inclusions) in addition to neurons. The pathological pattern is variable with the frequent appearance in the nigrostriatal, olivopontocerebellar and autonomic neuronal systems $^{112}$. No disease-modifying therapy for MSA is currently available.

\subsubsection{Pure Autonomic failure}

Pure autonomic failure (PAF), or Bradburg-Eggleston symptom, is a rare, late onset, slowly progressive disease characterized by neurodegeneration of autonomic nervous system $^{113}$. Since there are no genetic and environmental factors identified, PAF is considered primarily as a sporadic disease. Based on research findings, the classical signs of pathology are defined as $\alpha$-synuclein-positive inclusions and lesions in the peripheral autonomic nervous system, in particular, in sympathetic and parasympathetic ganglia and pre- and postganglionic autonomic neurons ${ }^{105,113}$. The common clinical symptoms include the orthostatic hypotension (high blood pressure) and cerebral hypoperfusion (low blood flow through an organ), leading to patient complaints regarding muscles pain, headache, gastrointestinal problems, urinary and sexual dysfunction ${ }^{113}$. Overall, the available therapy allows a good symptomatic control, however, further investigations of underlying mechanisms and development of novel therapeutic approaches are of particular interest.

\subsubsection{Structure and aggregation of $\alpha$-synuclein}

\subsubsection{Structural properties of $\alpha$-synuclein: Domains}

Human $\alpha$-synuclein is a small (140-amino acids; 14,5 kDa; Fig. 1.8 and 1.9), presynaptic, intrinsically disordered protein characterized by high charge and heat stability. While being encoded by $S N C A$ gene that is located on chromosome $4 \mathrm{q} 22.1, \alpha$-synuclein is 
predominantly expressed in the brain making up to $1 \%$ of the total protein content and enriched in the presynaptic terminals ${ }^{105}$. To date, four $\alpha$-synuclein isoforms have been identified in human, whereas the dominating isoform (full transcript) is 140 residues long and three other isoforms are the products of alternative splicing and composed of 98-, 112- and 126 -amino acid residues ${ }^{114}$. As it stands now, the exact physiological function of $\alpha$-synuclein is still not fully understood and proposed hypotheses are summarized in the chapter below.

Coming back to the structural properties of $\alpha$-synuclein, the protein sequence, as briefly described in the chapter 1.4.3, is divided in three domains: the C-terminal domain, the NAC region and the N-terminal domain (Fig. 1.9). The N-terminus (1-60 residues) is a wellconserved region and contains five imperfect hexameric repeats (KTKEGV) that upon binding to the lipid bilayer are involved in formation of amphipathic $\alpha$-helices with structural similarity to lipid-binding domains of class A2 apolipoproteins ${ }^{109,115}$. In the lipid-bound state, that in turn might be crucial for physiological function, the N-terminus being unfolded in solution adopts $\alpha$-helical structure ${ }^{4}$. Importantly, all three missense disease-associated mutations are located in the N-terminal domain (Fig 1.9) and might contribute to the toxicity due to the alterations of membrane binding or aggregation propensity. The central domain (61-95 residues), known as NAC region, contains a high proportion of hydrophobic amino acids and is essential for the amyloidogenic properties of $\alpha$-synuclein ${ }^{104}$. Thus, the NAC domain is located in the center the core of $\alpha$-synuclein amyloid fibrils that is comprised of eight $\beta$-strands (30-110 residues) ${ }^{116}$. Upon aggregation, the dramatic conformational changes are observed for the NAC region; eventually, this domain that is initially unstructured in monomeric $\alpha$-synuclein adopts a $\beta$-sheet rich conformation in the fibrils. Furthermore, the recent understanding of fibrillation/aggregation mechanism stresses the importance of the intermolecular NAC-mediated interactions in formation of aggregation-prone conformations $^{109}$. The C-terminal domain (96-140 residues) is unfolded, enriched in acidic residues (glutamate, aspartate) and possesses a strong negative charge (-8 net charge $)^{109}$. In addition to its proposed chaperone activity, the C-terminus is supposed to be involved in stabilization of the monomeric form of $\alpha$-synuclein and, thereby, inhibiting $\alpha$-synuclein misfolding and aggregation. The underlying principle for these autoinhibitory properties is not fully clear, but two factors, namely a shielding of the NAC region by C-terminus and/or a repulsion of negative charges, have been proposed to disfavor the intermolecular interactions ${ }^{110,117}$. In contrast to other two domains, the experimental findings provide strong 
evidence that C-terminus remains highly dynamic and unstructured irrespective of measurement conditions and binding partners.
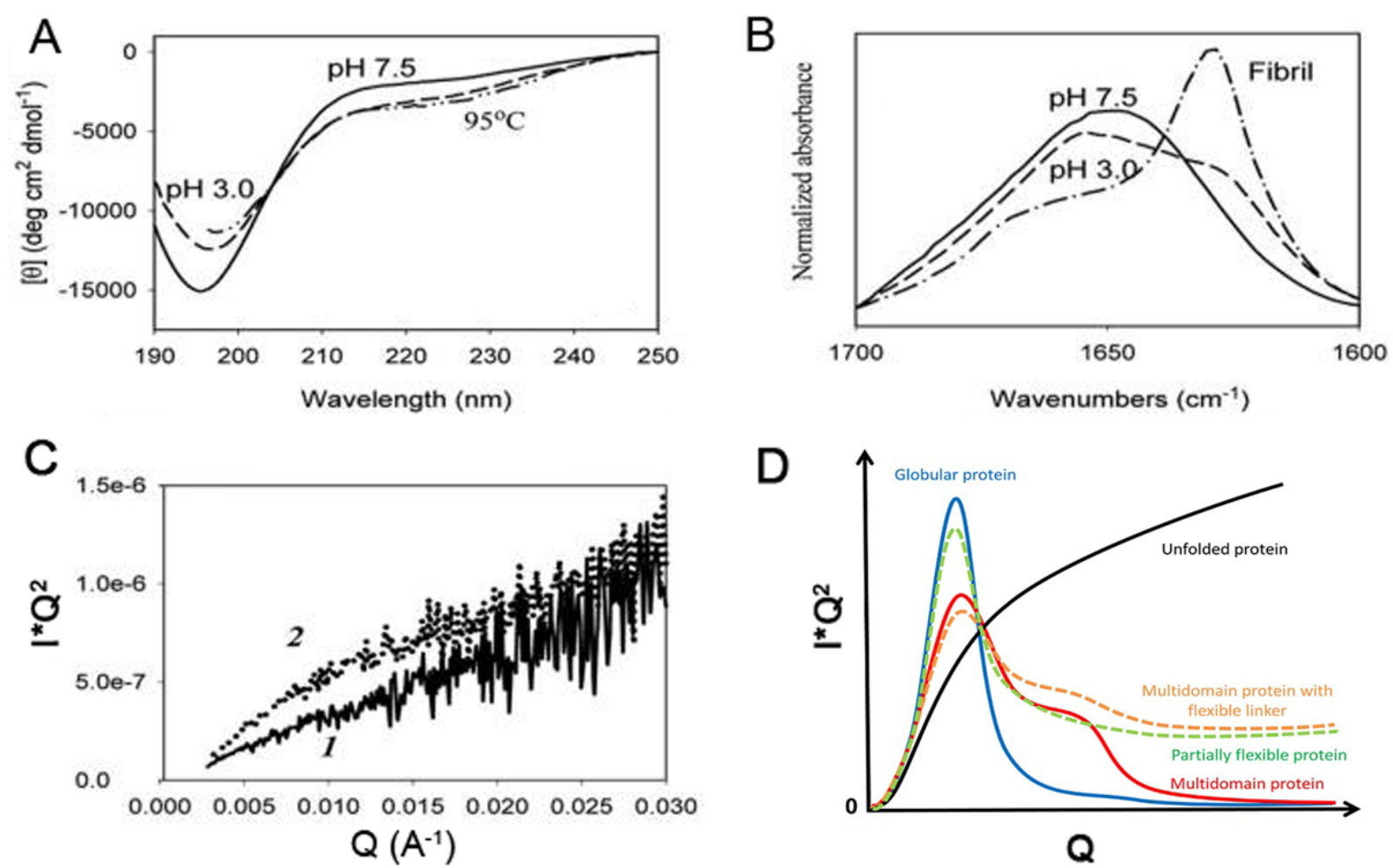

Figure 1.11. Spectral properties of $\alpha$-synuclein. (A) Far-UV CD spectra. (B) FTIR spectra (C) Kratky plot representation of SAXS analysis, 1-pH 7.5 (D) Schematic representation of typical Kratky plots. Figures A, B, C republished with permission of EUREKA SCIENCE (FZC), from Uversky and Eliezer ${ }^{115}$; permission conveyed through Copyright Clearance Center, Inc. Copyright (c) (2009)

\subsubsection{Structural properties of $\alpha$-synuclein: Conformational behavior}

It's generally accepted that $\alpha$-synuclein is an intrinsically disordered protein, meaning that it predominantly exists in solution as natively unfolded monomer, adopts a random-coil conformation and lacks a well-defined three-dimensional structure. From the theoretical point of view, most of natively unfolded proteins including $\alpha$-synuclein are characterized by low overall hydrophobicity, high net charge and low sequence complexity ${ }^{109}$. The interpretation of experimental data collected by various biophysical techniques revealed that $\alpha$-synuclein is unfolded in solution; more compact than pure random coil, and could be described as dynamic, highly flexible and heterogeneous ensemble of unfolded or partially folded conformations $^{104,109,115,117-119}$. Importantly, the structure of $\alpha$-synuclein is very sensitive to conditions/cofactors and such conformational modulation might be in turn important for its 
physiological function. In line with this thought, a concept of functional misfolding has been proposed $^{120}$. According to this concept, on one hand, the unfolded proteins contain preformed binding elements that are essential for the biological function and, on the other hand, the high conformational flexibility might facilitate the involvement of such preformed binding elements in unwanted intermolecular interactions. Based on these notions, it has been hypothesized that misfolding of the polypeptide chains facilitates the formation of non-native intramolecular interactions that, in turn, results in shielding or sequestering of the preformed binding elements. In case of $\alpha$-synuclein, in vitro and in-cell NMR spectroscopy studies in combination with calculations have demonstrated that $\alpha$-synuclein populates conformations with the transient long-range interaction between $\mathrm{N}$ - and C-terminus of the protein in vitro ${ }^{110}$, 117, 121. Along this concept of functional misfolding, one can speculate that undesirable intermolecular interactions of $\alpha$-synuclein are precluded due to the shielding of NAC region and intramolecular interactions of $\mathrm{C}$ - and $\mathrm{N}$-terminus with each other.

In 1996, first biophysical data describing the structural properties of $\alpha$-synuclein in solution was published ${ }^{122}$. This study provided evidence that $\alpha$-synuclein existed under physiological conditions in elongated or unfolded conformations. The authors came such conclusion based on measurement for $\alpha$-synuclein of Stokes radius ( $34 \AA$ ) that turned to be much larger than expected for globular proteins and reduced sedimentation rate $\left(\mathrm{s}_{20, \mathrm{w}}=1.7 \mathrm{~S}\right)$ in comparison with globular proteins of the same molecular weight. Subsequent studies confirmed the unfolded nature of $\alpha$-synuclein ${ }^{109,115}$. Thus, a far-UV CD spectrum (Fig. 1.11) recorded for monomeric $\alpha$-synuclein in aqueous buffer at $\mathrm{pH} 7.5$ showed patterns typical of the unfolded polypeptide chain, namely a negative band at $195 \mathrm{~nm}$ in combination with the absence of the signals in the 210-230 nm region. Interestingly, a small shift of the $195 \mathrm{~nm}$ band towards long wavelength plus small signal at $222 \mathrm{~nm}$ might be associated with the presence of a low populated helical structural elements. In line with the results from CD spectroscopy, the FTIR spectra of monomeric $\alpha$-synuclein demonstrated a broad peak with the maximum around $1650 \mathrm{~cm}^{-1}$ (Fig. 1.11) what would be expected for highly disordered proteins, approximately $70 \%$ of the sequence according to calculations. To further investigate the biophysical properties of $\alpha$-synuclein, several techniques have been applied to measure a range of hydrodynamic parameters. For instance, in the size-exclusion chromatography $\alpha$ synuclein is eluted in a single peak with the retention volume that corresponds to molecular mass of $\sim 63 \mathrm{kDa}$ in case of the globular protein. Since the calculated mass of $\alpha$-synuclein is $16 \mathrm{kDa}$, the significant increase of the elution time and derived molecular weight might be 
explained by unfolded protein structure that results in an increased hydrodynamic radius and, consequently, the slower migration on the gel filtration column ${ }^{118}$. The recognition of $\alpha$ synuclein as unfolded protein is also corroborated by small-angle X-ray scattering (SAXS) studies $^{109}$. By using various models and calculation protocols for analysis of SAXS data, it has been established that monomeric $\alpha$-synuclein is essentially disordered in aqueous solution, adopts a random coil conformation that is more compact than an ideally unfolded polypeptide chain. According to SAXS, the calculated radius of gyration, known as an indicator of the level of compaction in the structure, at neutral $\mathrm{pH}$ is found to be $40 \pm 1 \AA$ that is significantly lower than a predicted value for ideal random coil (52 $\AA$ ), but at the same time larger than for a globular protein with this molecular weight. Furthermore, the form of the Kratky plot that is generated based of SAXS data for $\alpha$-synuclein (Fig. 1.11) points towards random coil conformation with certain degree of compactness. Briefly, the Kratky plots allow the estimation of the globularity and conformational behavior of macromolecules; for example, the scattering curve for globular structures has a clear peak, while in case of chain-like, disordered structures, a curve rises monotonically (Fig. 1.11). Collectively, these spectroscopic findings support the hypothesis that monomeric $\alpha$-synuclein predominantly exists under physiological conditions in vitro as unfolded monomer with certain of compactness. This view is confirmed by NMR data that is discussed in next chapter.

\subsubsection{Structural properties of $\alpha$-synuclein: $N M R$}

Together with X-ray spectroscopy, high-resolution nuclear magnetic resonance spectroscopy (NMR) is a powerful technique that is widely applied for structural, dynamic and functional investigation of small and macromolecules at the atomic level. One of the main advantages of NMR over X-ray is a possibility to measure the samples under easily controlled, physiological conditions ( $\mathrm{pH}$, temperature, interaction partners) in solution phase without tedious crystallization procedure. Moreover, NMR spectroscopy represents a unique tool for structural characterization of various molecules and complexes that are impossible to crystallize. Therefore, unfolded proteins are a vivid example of such macromolecules suitable for NMR investigation, while the sample preparation for X-ray or EM in this case is not feasible.

The first in-depth NMR study describing the structural and dynamic properties of $\alpha$ synuclein in free and micelle-associated forms was published in $2001^{123}$. Following this publication, many aspects of $\alpha$-synuclein structural biology have been investigated by high- 
resolution NMR spectroscopy, including analysis of structural properties and conformational behavior; evaluation of impact of missense mutations, post-translational modifications and basic environmental factors; insight into mechanisms of misfolding/aggregation and others.

In agreement with the results obtained from other spectroscopic techniques, NMR data confirmed the predominantly unfolded and highly dynamic nature of monomeric $\alpha$ synuclein in aqueous solution ${ }^{115,117,123,124}$. Based on series of NMR measurements of $\left({ }^{15} \mathrm{~N}\right.$ ${ }^{13} \mathrm{C}$ )-labeled and ${ }^{15} \mathrm{~N}$-labeled monomeric $\alpha$-synuclein, Eliezer et $a l .{ }^{123}$, in addition to the first backbone assignment, reported a number of NMR-derived parameters that supported the lack of the well-defined secondary structure for monomeric $\alpha$-synuclein in solution. For instance, the profile of the resonance dispersion in different type of NMR spectra is different for unfolded and folded proteins. In case of $\alpha$-synuclein, the resonance dispersion in $\mathrm{H}-{ }^{15} \mathrm{~N}$ heteronuclear single quantum coherence (HSQC) spectra is characteristic for unstructured peptides, namely, a low degree of the dispersion $(1 \mathrm{ppm})$ in the proton dimension combined with a relatively large spread $(23 \mathrm{ppm})$ for the nitrogen whose chemical shifts are primarily sequence-dependent and therefore the same for intrinsically disordered and globular proteins. In addition to the small chemical shift range, the narrow line width, that is typical for a highly mobile backbone in the unfolded state or in flexible loops, was observed in HSQC spectra. The analysis of $\mathrm{CO}$ and $\mathrm{C}^{\alpha}$ chemical shifts has revealed a high degree of the dispersion for $\mathrm{CO}$ and low degree for $\mathrm{C}^{\alpha}$ that is consistent with the lack of secondary structure, since $\mathrm{CO}$ resonances are highly intrinsically dispersed and dependent on the local amino acid sequence, while $\mathrm{C}^{\alpha}$ chemical shifts are mainly determined by local secondary structure.

Browne et al. ${ }^{123}$ have also determined for monomeric $\alpha$-synuclein $\mathrm{C}^{\alpha}$ secondary chemical shifts that are considered to be a reliable indicator of the protein secondary structures. Briefly about the method, an empirical correlation between the secondary chemical shifts, that are defined as a difference between observed experimental chemical shifts and calculated shifts for the random coil conformation, and the protein backbone conformation has been found for different atoms of the backbone. $\mathrm{C}^{\alpha}$ secondary chemical shifts are largely dependent on the $\phi$ and $\psi$ backbone torsion angles and, furthermore, these angles, being depicted on so called Ramachandran plot, form special patterns that are characteristic for secondary structural elements in the protein. According to experimental data, average values of $\mathrm{C}^{\alpha}$ secondary chemical shifts for $\alpha$-helix and $\beta$-sheet are $2.6 \mathrm{ppm}$ (downfield) and -1.4 ppm (upfield), respectively; for unfolded elements these values are close to 0 . The profile of the $\mathrm{C}^{\alpha}$ secondary chemical shifts measured for monomeric $\alpha$-synuclein corroborates the predominantly disordered structure of this protein due to very low average 
values (Fig 1.12). However, a certain preference towards the positive values for N-terminus and NAC region, especially 6-37 residues, argues for the presence of transient helical structures. In contrast to the first 100 amino acids showing preference for helical structure, the last 40 residues of $\alpha$-synuclein in $\mathrm{C}$-terminal domain have very small $\mathrm{C}^{\alpha}$ secondary chemical shifts and, therefore, could be described as random coil. Remarkably, the protein region (residues 1-100) that exhibits the local helical preference is also known to be involved in the formation of the amphipathic helix upon binding to lipid bilayer and, probably, this "preformed helical structure" in solution might be crucial for the $\alpha$-synuclein function. In a follow-up NMR study focused on relaxation parameters, $\alpha$-synuclein has been also characterized in solution as a highly dynamic and flexible protein.

A

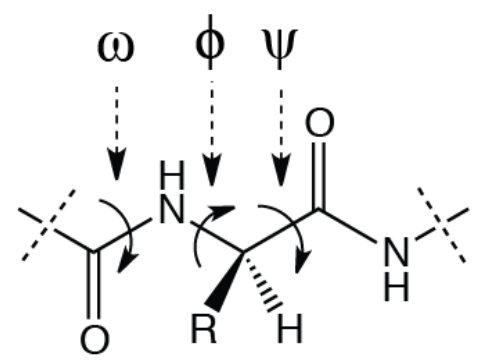

B

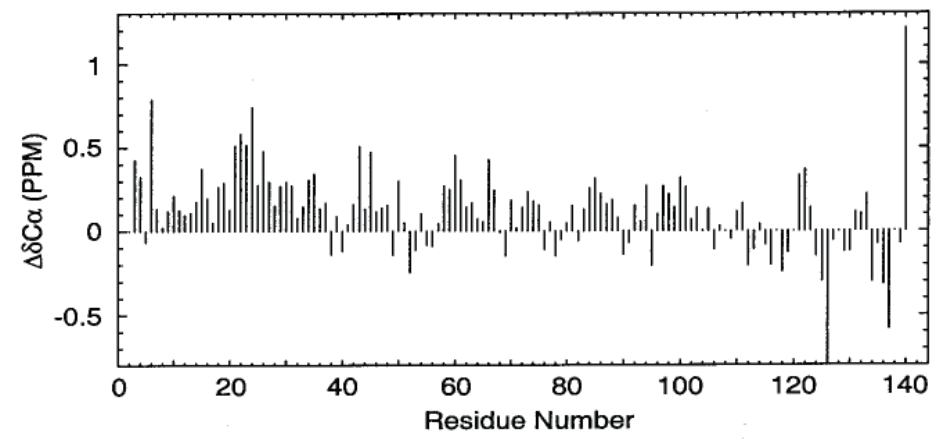

Figure 1.12. $\mathbf{C}^{\boldsymbol{\alpha}}$ secondary chemical shifts of $\boldsymbol{\alpha}$-synuclein. (A) Dihedral angles in a single amino acid. (B) $\mathrm{C}^{\alpha}$ secondary chemical shifts of free $\alpha$-synuclein (Figure $\mathbf{B}$ reprinted from Journal of Molecular Biology, Eliezer et al. ${ }^{123}$ with permission from Elsevier, copyright (C) (2001)

A next remarkable step in determining $\alpha$-synuclein properties was the discovery of long-range intermolecular interactions that provides a rationale for a greater compactness of $\alpha$-synuclein in comparison with the ideal random coil conformation. The detection of such transient contacts was possible due to the implementation of an elegant NMR technique that is based on paramagnetic relaxation enhancement (PRE) induced by a site-specific labeling with the paramagnetic nitroxide radicals. In theory, the paramagnetic nitroxide radicals induce the broadening of the NMR signal for the protons located in the close proximity to the radical (up to $25 \AA$ ). This effect is related to an increase in transverse relaxation rate and is electron-proton distance dependent $\left(r^{-6}\right)^{117}$. Experimentally, two spectral sets, paramagnetic and diamagnetic, are recorded, then the corresponding signal intensities are compared with each other and a resulted profile of the resonances broadening allows the analysis of the 
distance restraints and the long-range contacts. In two pioneering studies by Bertoncini et $a l .{ }^{117}$ and Dedmon et al. ${ }^{124}$, a range of $\alpha$-synuclein single cysteine mutants (A18C, Q24C, S42C, Q62C, S87C, A90C, N103C and A140C) has been generated and labeled with the nitroxide spin label MTSL (Fig 1.13). In addition to the PRE effect around the site of spin labeling, the analysis of the ${ }^{1} \mathrm{H}-{ }^{15} \mathrm{~N}$ HSQC spectra revealed a local signal broadening that extended significantly beyond the distances expected for the random coil (Fig 1.13). Specifically, the introduction of the spin labels in the N-terminal region leads to signal decay in the C-terminus and broad paramagnetic effect in N-terminal domain. When MTSL is attached to the cysteine in the C-terminal domain, the resonances of the residues in the Cterminus and in the NAC region exhibit the intensity decay. The PRE effect of the nitroxide radical placed into the NAC region is seen for almost all amino acids of the C-terminus. Based on these observations, the concept of long-range interaction has been proposed for $\alpha$ synuclein $^{117,124}$ and also corroborated in in-cell NMR spectroscopy in A2780 and SK-N-SH cells ${ }^{121}$. According to this concept, monomeric $\alpha$-synuclein in solution populates an ensemble of highly dynamic and structurally diverse conformers that are stabilized by intramolecular long-range interaction of the C-terminal domain with both NAC domain and N-terminus. The contacts between $\mathrm{N}$ - and C-terminus are considered to be mainly electrostatic, while Cterminal/NAC region interactions are presumably of hydrophobic nature. Furthermore, by applying several calculation approaches for interpretation of the PRE data, the ensemble of native-state conformations of $\alpha$-synuclein in solution has been generated (Fig 1.13) ${ }^{117}$. Despite a broad distribution of conformers, the majority of calculated structures are lacking secondary structure elements and significantly more compact than random coil. Addressing the underlying mechanisms of $\alpha$-synuclein structural behavior, the intermolecular long-range interactions have been proposed to play a key role in stabilization of the native structure of monomeric $\alpha$-synuclein and, thus, preventing the misfolding and aggregation. In particular, the hydrophobic integrations between residues 85-95 of the NAC region and C-terminus (110-130), leading to a shielding of the amylodogenic NAC domain, as well as contacts of the C-terminal tail with the N-terminus are considered to be pivotal. Interestingly, the similar PRE data collected for $\alpha$-synuclein under aggregation prone condition demonstrated a release of intramolecular long-range interactions and, consequently, a formation of fully unfolded conformations that are supposed to be involved in oligomerization and aggregation ${ }^{117}$.

The last NMR technique that I would like to describe as a tool for structural studies is based on the measurement of residual dipolar coupling (RDC) ${ }^{110,117}$. Without going deep into rather difficult NMR theory describing RDCs, RDCs are observed in case of incomplete 
averaging of spatial anisotropic dipolar couplings that could be achieved by partial alignment of the molecules in magnetic field using alignment media such as phage particles that align in the magnetic field. Residual dipolar couplings are dependent on the distance between two nuclei and the angle of the corresponding bond to the external magnetic field ${ }^{110,117}$. When measuring one-bond N-H RDCs, the distance between the two covalently bound nuclei is fixed and the RDC analysis provides exclusively information about the orientation that can be used for structure determination. In the case of $\alpha$-synuclein, one-bond N-H RDCs have been measured for several types of alignment media, including bacteriophage Pf1 suspensions, $n$ octyl-penta(ethylene glycol)/octanol, and stretched polyacrylamide gels ${ }^{110,117}$. Collectively, the RDC-derived structural information for $\alpha$-synuclein is consistent with the previously reported spectroscopic studies. Thus, RDC data on $\alpha$-synuclein confirmed such structural features such as the domain organization, intermolecular long-range interactions, more extended conformation of C-terminus and overall preference for helical structure in the Nterminal domain ${ }^{110,117}$. 


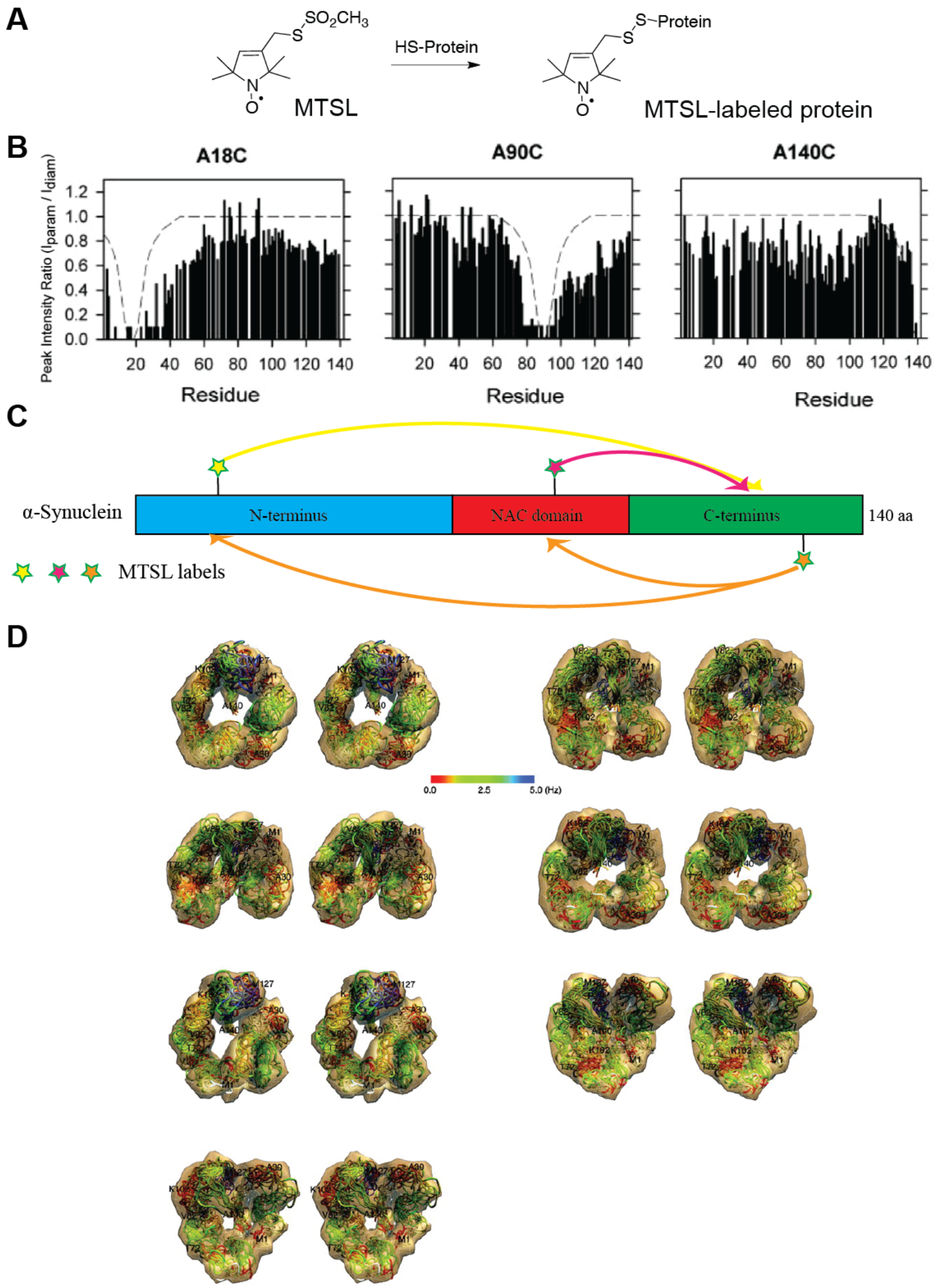

Figure 1.13. PRE data on MTSL-labeled $\alpha$-synuclein and its native state conformations. (A) MTSL labeling. (B) (C) PRE of amide protons in three spin-labeled $\alpha$-synuclein mutants. (D) Representation of the native state of free $\alpha$-synuclein calculated based on PRE data; shown are seven most populated clusters. Figures $\mathbf{B}$ and $\mathbf{D}$ reprinted from Bertoncini et al. ${ }^{117}$ Copyright (C) (2005) National Academy of Sciences. 


\subsubsection{4. $\alpha$-Synuclein aggregation: Folding vs. Aggregation}

Groundbreaking discoveries mentioned in previous chapters strongly supported a causal role for $\alpha$-synuclein misfolding and aggregation in Parkinson's disease. The crucial finding is the recognition of fibrillar $\alpha$-synuclein as a main component of Lewy bodies ${ }^{32}$. Consistent with in vivo data, the propensity of monomeric $\alpha$-synuclein to form amyloid fibril has been demonstrated in vitro ${ }^{125}$. To that end, multiple studies have focused on various aspects of $\alpha$-synuclein aggregation with a main goal to elucidate the biochemistry and biophysics of $\alpha$-synuclein misfolding and aggregation and also to establish the link to $\alpha$ synuclein-induced toxicity ${ }^{105,109,114,115}$.

In structural biology, the folding of the protein is one the crucial steps of the protein life cycle in which the linear polypeptide chain adopts a three-dimensional native conformation that is pivotal for the biological function. According to the modern interpretation of the energy landscape theory, the native folded state of each protein is solely encoded by its amino acid sequence and represents a structure with the minimum conformational energy ${ }^{126}$. During the folding the polypeptide chain adopts its lowest energy structure by a trial and stochastic process that samples a very limited conformational space and hence only a very small number of allowed conformations. Schematically, this process could be described in a form of "energy funnel" as part of the scheme of the energy landscape for protein folding and aggregation ${ }^{126}$ (Fig. 1.14). Importantly, the folding of the proteins is considered as a competing pathway to aggregation that is also leading to compaction of the polypeptide chain and formation of thermodynamically stable polymeric states ${ }^{126}$. If one compares these two pathways, it's generally believed that, in case of the folding, the transformation is controlled by intramolecular tertiary interactions between side chains that shied the peptide backbone and hydrophobic patches. Whereas the aggregation, more specifically amyloid fibril formation, is associated with primordial propensity of the polypeptide chain to form polymeric species stabilized by hydrogen bonds between parallel or anti-parallel organized $\beta$-strands ${ }^{126}$. Thus, amyloid formation is predominantly driven by the secondary and intermolecular interactions of the backbone, whilst the side chains are sensing and defining experimental conditions favoring for aggregation. In this way, the factors, which reduce tertiary interaction and promote secondary interactions, facilitate the shift of equilibrium towards aggregation pathways and other way around for conditions stabilizing intramolecular contacts ${ }^{109}$. Remarkably, the amyloid fibrils and other aggregated species including certain types of oligomers and protofibrils are characterized by higher stability and lower energy levels in comparison with the native state and folding 
intermediates. Nevertheless, a broad heterogeneous range of highly dynamic, structurally diverse, often unstable and rapidly interconverting oligomeric species is also commonly

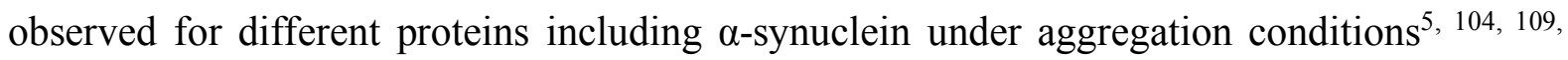
127.

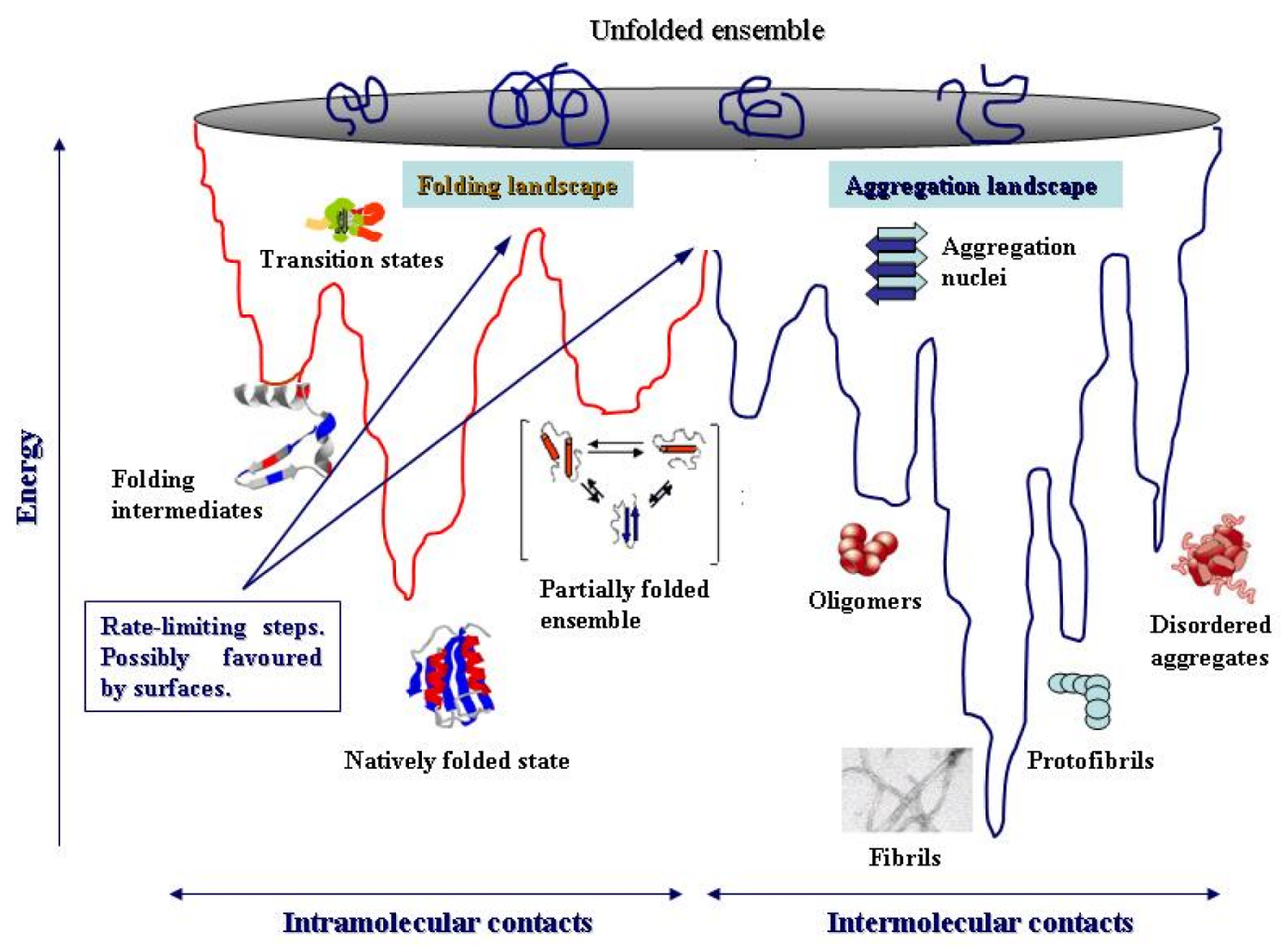

Figure 1.14. Schematic energy landscape for protein folding and aggregation. Figure adapted from Stefani ${ }^{126}$ licensed under CC BY-NC-SA 3.0. Copyright (c) (2008)

\subsubsection{5. $\alpha$-Synuclein aggregation: Multiple pathways of $\alpha$-Synuclein aggregation}

The aggregation of $\alpha$-synuclein is a process that was observed both in vivo and in vitro $^{32,125}$. For example, the fibrillar $\alpha$-synuclein is a main component of the Lewy bodies in patients with Parkinson's disease. Furthermore, the agitation of aqueous buffer solution containing micromolar concentrations of $\alpha$-synuclein at $37^{\circ} \mathrm{C}$ results in almost complete conversion of monomeric protein into insoluble amyloid fibrils within several days ${ }^{128,}{ }^{129}$. Despite being a point of intense research, understanding of various aspects of $\alpha$-synuclein aggregation in vitro and especially in vivo still remains unresolved. Overall, the structural and mechanistic studies aimed at elucidating details of $\alpha$-synuclein aggregation pathways and 
properties of corresponding intermediates are complicated and poorly reproducible due to a strong dependence on experimental conditions, plausible existence of several multistep pathways and, in particular, inherent heterogeneity and transient nature of various species that might be in dynamic equilibrium with each other.

The results from biophysical and biochemical studies of $\alpha$-synuclein aggregation in cell-free systems have supported the hypothesis that amyloid fibril formation is a nucleationdependent process characterized by initial lag phase, followed by exponential growth that reaches finally the plateau (Fig. $1.15 \mathrm{~A})^{129,}{ }^{130}$. Based on experimental data, it has been hypothesized that at early stages of aggregation the initially unfolded monomeric $\alpha$-synuclein undergoes the conformational transformation into pre-molten globular like conformations possessing highly aggregation-prone properties ${ }^{128,129}$. On next step, this partially folded $\alpha$ synuclein starts to form various transient oligomeric species that are often considered to be an amyloidogenic nucleus with the seeding property. Considering that structurally diverse $\alpha-$ synuclein oligomers or heterogeneous mixtures of such intermediates were observed under different experimental conditions, it's very likely that $\alpha$-synuclein fibrillization could occur through multiple pathways, in which most of intermediates are in dynamic equilibrium with each other (Fig 1.15 B) 5, 104, 109, 127 . Importantly, the formation of fibril nuclei during the lag phase is a rate-limiting step of whole fibril formation. During the exponential growth phase the fibril nucleus starts to grow through further addition of monomeric protein and thus to assemble first to protofilaments and eventually to mature fibrils ${ }^{128}$. In addition to a primary nucleation process, the secondary nucleation events, mainly fibril fragmentation, have a significant impact on kinetic of self-templated aggregation ${ }^{131}$. When a plateau phase is reached, the concentration of monomeric protein and oligomeric species is almost completely depleted and the sample predominantly consists of insoluble amyloid fibrils. Although in most cases the fibrils are the final product of $\alpha$-synuclein aggregation, it has been demonstrated that incubation of $\alpha$-synuclein under certain conditions in vitro could yield a variety of end state products, including soluble or insoluble amorphous aggregates and

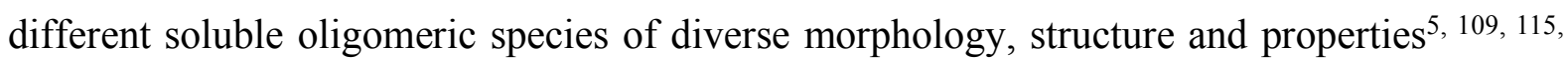
127. Despite ongoing discussion and limited information regarding $\alpha$-synuclein misfolding in vivo, increasing evidence implicates $\alpha$-synuclein oligomers as a crucial factor in aggregation kinetic and pathogenesis of Parkinson's disease (discussed in details below). 
A

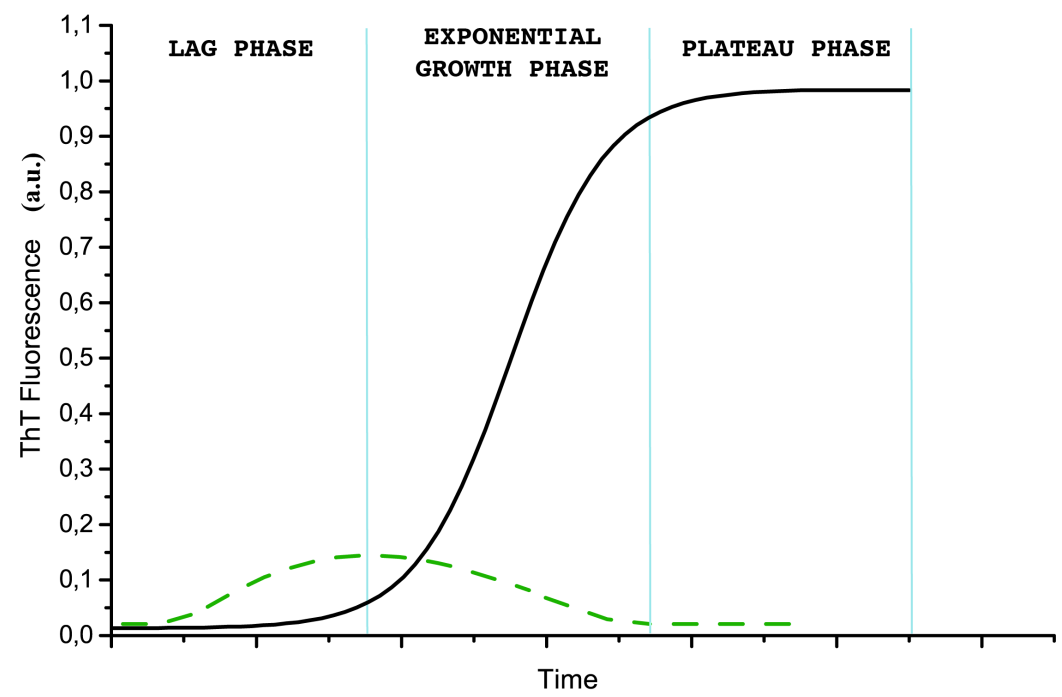

B

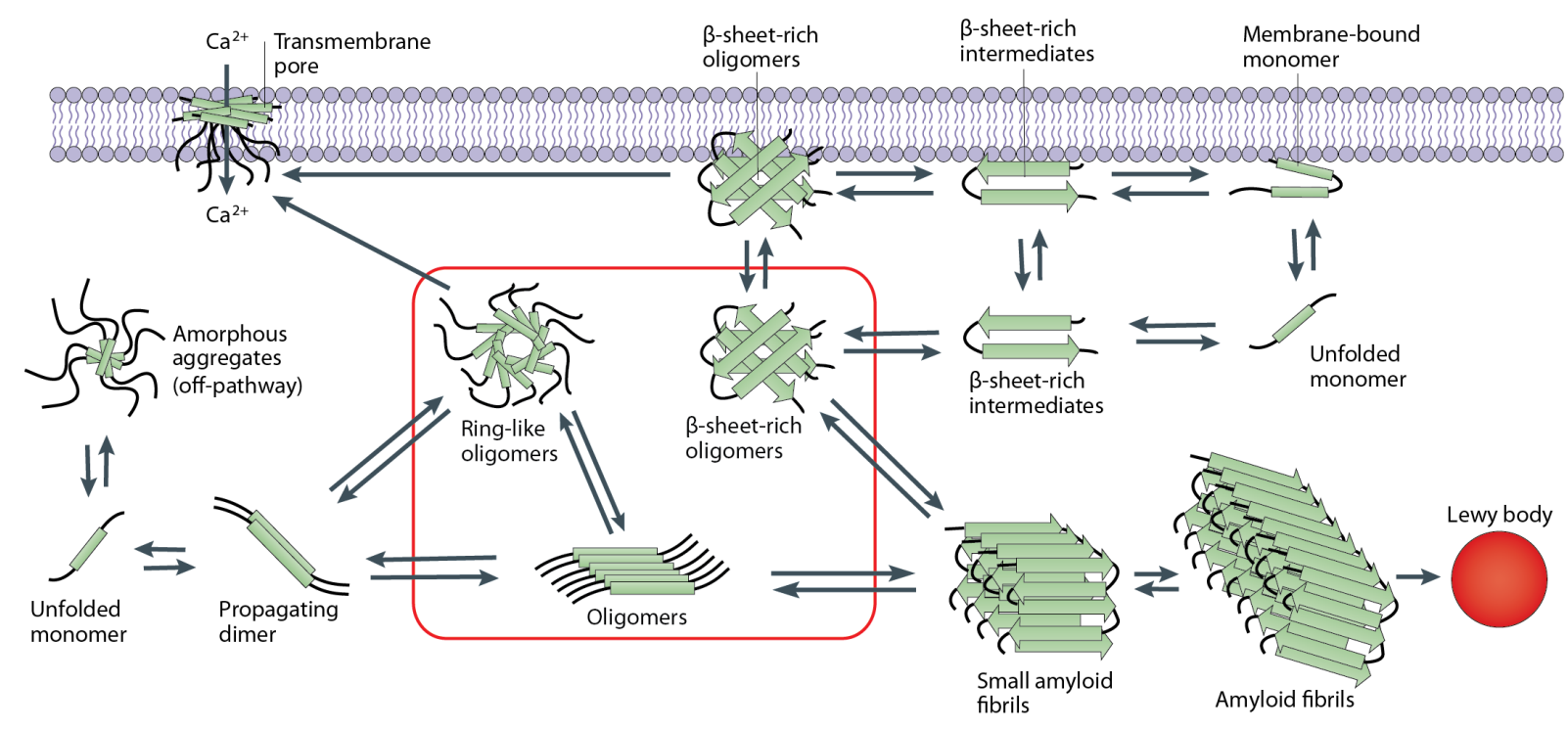

Figure 1.15. $\alpha$-Synuclein aggregation pathways. (A) Main phases of $\alpha$-synuclein fibrillization. Green dashed line represents a population of intermediates. (B) Schematic model of $\alpha$-synuclein misfolding. Soluble oligomers are highlighted in red box.

\subsubsection{6. $\alpha$-Synuclein aggregation: Factors promoting aggregation}

It's generally recognized that $\alpha$-synuclein aggregation and factors promoting $\alpha$ synuclein misfolding might play a crucial role in pathogenesis of Parkinson's disease. To date, multiple studies in vitro and in vivo have identified a wide range of environmental conditions and interacting partners that significantly accelerate $\alpha$-synuclein aggregation ${ }^{109}$, 115. While it may be possible that different factors can facilitate the aggregation by distinct mechanisms, there is a reasonable likelihood that at least in part the promoting of partially 
folded aggregation-prone conformations of monomeric $\alpha$-synuclein can constitute a common, shared mechanism. The list of factors promoting aggregation/fibrillization of monomeric $\alpha-$ synuclein in vitro ${ }^{104,109,115,129}$ :

1) Increased protein concentration ${ }^{104}$

Mechanism: elevated $\alpha$-synuclein concentration leads to increased population of partially folded aggregation-prone conformations. With higher concentration, the lag time of fibril formation is reduced, while the rate of the fibril elongation is increased.

2) Increased temperature ${ }^{129}$

Mechanism: with the temperature increase the hydrophobic interactions are facilitated and, $\alpha$-synuclein reversibly adopts more compact conformations with significant amount of $\beta$-sheet structure. As a result, the rate of fibril formation is strongly accelerated at higher temperature.

3) Low $\mathrm{pH}^{129}$

Mechanism: at neutral $\mathrm{pH} \alpha$-synuclein carries high net charge ( $\mathrm{pI} 4.7$ ), at lower $\mathrm{pH}$ the negative charge is neutralized and this promotes intramolecular interactions and partial folding to more compact conformations characterized by significant amount of $\beta$-sheet structure. These structural changes in monomeric protein are reversible and concentration independent. The intermolecular interactions are also facilitated at lower $\mathrm{pH}$ due to the suppression of electrostatic repulsion between negatively charged molecules. Thus in vitro, the lowering of $\mathrm{pH}$ leads to faster fibrillization including the shorter lag time and accelerated rate of fibril growth.

4) Certain types of liposomes (interaction with lipids are discussed in separate chapter)

5) Macromolecular crowding (agents: PEG, polysaccharides, inert proteins) ${ }^{109,115}$

Mechanism: The $\alpha$-synuclein fibrillization is significantly accelerated in the presence of various crowding agents in concentration-dependent manner. At high concentrations the crowding agents mimic the crowding environment of the living cells where the concentration of macromolecules could reach $400 \mathrm{mg} / \mathrm{ml}$. In theory, under crowding conditions a so-called "excluded volume effects" phenomenon is observed that first of all results in increased concentration of the target protein and second promotes the folding and protein-protein interaction. Thus, it has been demonstrated that $\alpha$-synuclein fibrillization is significantly accelerated in the presence of various crowding agents in concentration-dependent manner, perhaps as a consequence of partial folding to aggregation-prone conformations and "increased" protein concentration.

6) Mechanical impact (stirring, shaking, sonication) 
Mechanism: the accelerated rate of fibrillization due to the mechanical impact (stirring, shaking, sonication) is related to a large extent to additional kinetic energy of the system, increasing probability of protein-protein collision events and formation of additional nucleation centers by breaking apart big aggregates. Another possible explanation includes the enlargement of water-air and stirring bar-solution surfaces that are hydrophobic and might potentially accelerate the nucleation process.

7) Anions and salts ${ }^{109,115}$

Mechanism: mechanism is quite similar to the one described for experimental conditions of lower $\mathrm{pH}$. Briefly, the various ions compensate the net charge of the protein and at high concentrations induce a dehydration of the protein surface, which result in partial folding, masking the electrostatic intramolecular and intermolecular repulsion and consequently lead to a higher rate of aggregation.

8) Polycations and polyanions (glycosaminoglycans, proteoglycans, dextran sulfate, spermine, polyethylenimine, poly-Lys, poly-Arg) $)^{104,115}$

Mechanism: similar to anions and salts.

9) Pesticides/Herbicides (paraquat, rotenone, maneb) ${ }^{109,115}$

Mechanism: paraquat and rotenone have been shown to interact with $\alpha$-synuclein, to stabilize amyloidogenic conformations and thereby to accelerate the fibrillization in vitro in a concentration-dependent manner.

10) Metal ions (Al, $\mathrm{Ca}, \mathrm{Cu}, \mathrm{Fe}, \mathrm{Mg}, \mathrm{Mn}, \mathrm{Zn})^{109}$

Mechanism: the incubation of various mono-, di- and trivalent metal ions with $\alpha$ synuclein in aqueous solution dramatically promotes both oligomerization and fibril formation. By using various spectroscopic techniques, the formation of partially folded $\alpha$ synuclein intermediates with increased amount of the secondary structure and amyloidogenic properties was observed. Furthermore, these conformational changes and aggregation rates are correlating with the cation charge density and are supposed be mediated by direct binding of the cations to $\alpha$-synuclein that in turn neutralizes the net negative charge of $\alpha$-synuclein, reduces electrostatic repulsion and, thereby, facilitates folding and intramolecular interactions. For example, the addition of $2.5 \mathrm{mM} \mathrm{AlCl}_{3}$ to $\alpha$-synuclein promotes the intramolecular folding as evident by spectral data from far-UV, CD and ANS fluorescence measurements and at the same time the lag time of fibrillization with $\mathrm{AlCl}_{3}$ is 3 times shorter. Remarkably, most of metal cations bind to C-terminus of $\alpha$-synuclein non-specific manner with a low affinity; however, $\mathrm{Cu}^{2+}$ and $\mathrm{Fe}^{2+}$ interact with nanomolar affinity. In vitro experiments have also demonstrated that metal-induced effects on structural properties of 
monomeric $\alpha$-synuclein, oligomers and amyloid fibrils are variable and dependent on the nature of metal ion. So far, the addition of $\mathrm{Al}^{3+}, \mathrm{Ca}^{2+}, \mathrm{Cu}^{2+}, \mathrm{Fe}^{2+}, \mathrm{Fe}^{3+}$ ions to $\alpha$-synuclein is shown to promote accumulation of oligomeric intermediate, however, the structural properties of generated oligomers are often ion-specific. It's noteworthy that there is also clinical evidence supporting the implication of heavy metals in pathogenesis of Parkinson's disease, for instance, elevated levels of $\mathrm{Fe}, \mathrm{Cu}$ and $\mathrm{Al}$ in the brain and, epidemiologically, the long-term exposure to heave metals is considered as a risk factor for Parkinson's disease.

11) Organic solvents ${ }^{104}$

Mechanism: the addition of various organic solvents to $\alpha$-synuclein in aqueous buffer leads to increased population of partially folded aggregation-prone conformations of monomers followed by rapid oligomerization and finally amyloid fibrils formation. An additional hydrophobic surface and dehydration of the protein, provided by organic solvent, have been hypothesized to contribute to observed conformational changes and aggregation kinetic.

\subsubsection{Post-translational modifications of $\alpha$-synuclein}

\subsubsection{Introduction}

The post-translational modifications play a crucial role in modulation and regulation of function, properties and life cycle of many proteins. To date, more than 300 posttranslational modifications have been discovered, whereas phosphorylation, glycosylation, oxidation, ubiquitination, truncation, acetylation are the most common types. In case of $\alpha$ synuclein, multiple studies have focused on identification of post-translational modifications targeting $\alpha$-synuclein with following investigation of their impact on structure/function of $\alpha$ synuclein and implication in pathogenesis of Parkinson's disease ${ }^{105}, 109,132-135$. Viewed together, the accumulated experimental data has demonstrated that $\alpha$-synuclein undergoes multiple post-translational modifications in vivo and in vitro including phosphorylation, oxidation, nitration, ubiquitination, SUMOylation, acetylation, truncation, cross-linking and glycation, importantly, some of them are considered as potentially pathological events or a diagnostic marker of the disease. In this part, the most prominent $\alpha$-synuclein posttranslational modifications are discussed with a main focus on corresponding impact on structural properties and aggregation behavior of modified $\alpha$-synuclein in relation with functional consequences. 


\subsubsection{Phosphorylation}

A brief analysis of amino acid sequence of human $\alpha$-synuclein reveals multiple putative phosphorylation sites, namely 4 serines $(9,42,87,129), 4$ tyrosines $(39,125,133$, 136) and 10 threonines $(22,33,44,54,59,64,72,75,81,92)$ that are all highly conserved residues, excluding S87. Multiple studies of $\alpha$-synuclein appearance and its phosphorylation state in the human-derived samples have demonstrated that in vivo a dominating site of phosphorylation is S129 for cytosolic as well as for insoluble forms of $\alpha$-synuclein ${ }^{136-138}$. Additionally to S129, S87 is a second common phosphorylation product detected both in brains of patients and in animal models of Parkinson's disease ${ }^{139}$, ${ }^{140}$. So far the phosphorylation of threonines has not been observed, while tyrosine modifications of $\alpha$ synuclein at Y39 or Y125 have been found in the human brain tissues, but at quite low levels ${ }^{140}$. Several lines of experimental evidence are clearly emphasizing the implication of S129 and, to a lesser extent, S87 phosphorylation in $\alpha$-synuclein-related neurotoxicity. In support of this view, it has been shown that $90 \%$ of $\alpha$-synuclein in Lewy bodies is phosphorylated at S129 and also the amount of insoluble S129 phosphorylated $\alpha$-synuclein is correlating with the severity of brain pathology and gradually increasing with the disease progression $^{136-138}$. Remarkably, the progressive accumulation of S129 phosphorylated $\alpha$ synuclein from $5 \%$ to $30-100 \%$ of total $\alpha$-synuclein is observed in cytosolic and membrane fractions derived from certain brain regions or in the blood plasma ${ }^{136,138}$. Moreover, S129 phosphorylation leads to significant changes in subcellular localization, for instance, elevated $\alpha$-synuclein levels in the nuclei in animal models and increased membrane association (SDSsoluble fraction) in the patient brain tissues ${ }^{136,140}$. Thus, at late stages of the disease, S129 phosphorylated $\alpha$-synuclein might represent a significant or even dominated form of the protein and, therefore, might contribute to $\alpha$-synuclein toxicity and misfolding. Phosphorylation of S87 is also associated with synucleinopathies, mainly due to the accumulation in the brains of Parkinson's disease patients and the relevant animal models with high degree of colocalization with Lewy bodies ${ }^{139,140}$. Among four tyrosines, Y39 and Y125 appear to be phosphorylated in the human brain ${ }^{140}$. Importantly, the level of Y125 modification is reduced during aging and in synucleinopathies, thereby suggesting a lack of toxicity or even a potential neuroprotective role ${ }^{133}$. Recently discovered Y39 phosphorylation has been hypothesized to be involved in $\alpha$-synuclein degradation by proteasome and autophagy pathways ${ }^{141}$.

To date, many kinases have been shown to phosphorylate monomeric as well as fibrillar $\alpha$-synuclein (Table 1.3$)^{135,140}$. It is noteworthy that several kinases including CK-1, 
CK-2, PLK-1, PLK-2, PLK-3 have been identified as a components of Lewy bodies ${ }^{31}$. The accumulated experimental data from various in vitro and in vivo systems have corroborated the notion that phosphorylation of $\alpha$-synuclein has a significant impact on aggregation and toxicity $93,97,120-123$. However, these results are, in some part, mixed and conflicting to one another, possibly due to the internal features and peculiarities of different test systems and approaches.

Table 1.3. $\alpha$-Synuclein phosphorylation and kinases. (literature ${ }^{140}$ ).

\begin{tabular}{|c|l|l|}
\hline Residue & \multicolumn{1}{|c|}{ Kinase } & \multicolumn{1}{c|}{ Experimental system } \\
\hline Y39 & c-Abl & in vitro/cellular model/transgenic mice model/rat viral model \\
\hline \multirow{2}{*}{ S87 } & CK-1 & in vitro/cellular models/transgenic mice models/rat viral model \\
\cline { 2 - 3 } & Dyrk1A & in vitro/cellular models \\
\hline \multirow{4}{*}{ Y125 } & Fyn & in vitro/cellular models \\
\cline { 2 - 3 } & Src & in vitro/cellular models/Drosophila \\
\cline { 2 - 3 } & c-Abl & in vitro/cellular model/transgenic mice model/rat viral model \\
\hline \multirow{4}{*}{ S129 } & CK-1, CK-2 & in vitro/cellular models/transgenic mice models \\
\cline { 2 - 3 } & PLK-1, $-2,-3,-4,-5$ & in vitro/cellular models/yeast/transgenic mice models/rat viral model \\
\cline { 2 - 3 } & Grk2, Grk5 & in vitro/cellular models/Drosophila \\
\hline
\end{tabular}

The effect of S129 phosphorylation has been evaluated in several model systems under various conditions. In vitro, S129 phosphorylated $\alpha$-synuclein by CK-1 kinase is unable to form amyloid fibrils and oligomers as evident by ThioT assay and unchanged concentration of monomeric protein after $72 \mathrm{~h}$ of incubation ${ }^{142}$. However, other studies have demonstrated contradicting results, for example, the accelerated fibrillization rate of CK-1 phosphorylated $\alpha$-synuclein or similar aggregation kinetic in case of PLK-2 mediated modification ${ }^{140}$. Addressing the underlying molecular mechanisms of reduced aggregation propensity of S129 phosphorylated $\alpha$-synuclein it has been shown that the S129 modified monomeric $\alpha$-synuclein in solution exhibits increased conformational flexibility and adopts extended conformations that are lacking long-range interactions observed for unmodified $\alpha-$ synuclein. For instance, the hydrodynamic radius measured by NMR is $35.5 \AA, 28.2 \AA$ and $36.4 \AA$ for S129 phosphorylated $\alpha$-synuclein, monomeric $\alpha$-synuclein under physiological and denatured conditions, respectively ${ }^{142}$. It seems plausible that these structural changes and aggregation properties might be attributed to additional negative charge on C-terminus contributed by phosphate moiety. Remarkably, complimentary experiments provide strong evidence that the widely applied phosphorylation-mimicking mutants based on aspartic or glutamic acids are not reproducing the properties of S129 phosphorylated protein. Thus, the 
aggregation propensity of S129E mutant is similar to wild type $\alpha$-synuclein, while S129 phosphorylated protein is not forming fibrils under similar conditions and, furthermore, the long-range interactions within monomeric protein are disturbed by phosphorylation, but not by mimicking mutations ${ }^{142}$. Due to the crucial role of $\alpha$-synuclein-membrane interactions for biological function, in vitro binding and NMR studies have demonstrated similar lipidbinding properties for wild type and S129 phosphorylated $\alpha$-synuclein ${ }^{142}$; nevertheless the impact on aggregation of membrane-bound modified protein is still unknown.

Despite being a point of intensive research, conclusions regarding toxicity and misfolding of S129 phosphorylated $\alpha$-synuclein in cellular and animal models are, in large part, conflicting to one another ${ }^{132,135,140,143}$. In cellular models, the S129 phosphorylation or expression of mimicking mutants predominantly results in elevated levels of aggregates and oligomers, whereas toxicity readouts are inconsistent ${ }^{140}$. In invertebrates, the phospho-S129dependent neurotoxicity and aggregation profile also vary dramatically. For instance, the expression of S129D mutant or co-expression of wild type $\alpha$-synuclein with GRK-2 kinase in Drosophila have been shown to exacerbate the neurodegeneration, while the inclusions formation was similar to wild type $\alpha$-synuclein ${ }^{143}$. On the other hand, the expression of S129A mutant leads to 5 -fold increase in $\alpha$-synuclein aggregations accompanied by a lack of neuronal death. Opposite results have been reported for the C. elegans model, S129 phosphorylation reduces the $\alpha$-synuclein-related toxicity ${ }^{132}$. In line with previously described models, several studies in viral vector-infected rats didn't provide a conclusive results regarding pathological role of S129 phosphorylation and correlation between aggregation and neurodegeneration ${ }^{135,140,143}$. For instance, S129D mutant is less toxic, but more aggregation prone as S129A mutant according to one study, however, another report claimed similar phenotypes for all three constructs. On the contrary, the co-expression of wild type $\alpha$ synuclein with PLK-2 in rats leads to increased levels of S129 phosphorylated $\alpha$-synuclein that in turn ameliorates the pathological changes including formation of inclusions, neurodegeneration and behavioral alterations. Collectively, the understanding of the implication of S129 phosphorylation in biological function and pathogenesis of $\alpha$-synuclein is still elusive and obviously additional experimental studies are required to establish the role of phosphorylation and the feasibility of therapeutic targeting.

The elevated phosphorylation of S87 has been found in CNS of transgenic $\alpha$ synuclein mice models and Parkinson's disease patients, although the levels were much lower than S129 modification ${ }^{139}$. To date, two kinases CK-1 and Dyrk1A have been shown to phosphorylate $\alpha$-synuclein at S87 in vitro and in vivo (Table 1.3$)^{140}$. Similar to S129 
modification, S87 phosphorylated $\alpha$-synuclein is more flexible (hydrodynamic radius $34.7 \AA$ ) as the wild type form and fails to form amyloid fibrils in vitro biochemical assay ${ }^{139}$. However, results from cellular and animal models are not consistent ${ }^{133,140}$. For example, S87 phosphorylation of $\alpha$-synuclein by Dyrk1A in the cultured cells leads to increased toxicity and inclusion formation ${ }^{140}$. On the other hand, expression of the S87E mutant in a viral rat model results in weaker phenotype as observed by reduction of aggregation, neurodegeneration and locomotor abnormalities ${ }^{133}$. Overall, the understanding of the physiological or pathological role of $\alpha$-synuclein phosphorylation at S87 remains of interest, two observations might be important pieces of the puzzle, namely an inaccessibility of S87 residue in the amyloid fibrils and this suggests that phosphorylation occurs before aggregation. Secondly, high levels of immunoreactivity of S87 phosphorylated $\alpha$-synuclein in the membrane fraction have been reported, which in turn could indicate a change of subcellular localization, although weaker binding to the lipid bilayer is observed in vitro ${ }^{139}$.

Among four tyrosines of $\alpha$-synuclein, the phosphorylation of Y39 and Y125 has been identified in the human brain ${ }^{140,141}$. In vitro, $\alpha$-synuclein can be phosphorylated at three tyrosines (Y125, Y133, Y135) located in C-terminus by Syk kinase, while Src-family kinases (Fyn, Src, Lyn, Frg) are selectively modifying Y125 (Table 1.3) ${ }^{140}$. In various systems, the phosphorylation of C-terminal tyrosines and specifically Y125 leads to inhibition of oligomer and fibril formation and reduced toxicity. In this way, the protective role of Y125 phosphorylation has been demonstrated in Drosophila model as reflected by reduced amount of oligomers and increased neuronal survival. Recently, Mahul-Mellier et al ${ }^{141}$ have reported that $\alpha$-synuclein is a substrate for c-Abl kinase, whereas Y125 and Y39 are the modification sites. The results from various test systems argued for a possible involvement of c-Abl-driven phosphorylation in $\alpha$-synuclein degradation by proteasome and autophagy pathways.

Collectively, it's clear that the different types of $\alpha$-synuclein phosphorylation might exert various effects on toxicity, aggregation, localization, turnover and other aspect of $\alpha$ synuclein-related biology. However, the available results are mixed and often contradicting, therefore, additional studies addressing the underlying molecular mechanisms and its pathological significance are required.

\subsubsection{Truncation}

Truncation represents another common type of $\alpha$-synuclein post-translational modification. Several truncated $\alpha$-synuclein isoforms have been detected in the human brain, importantly some of them are LB-specific or disease-associated ${ }^{109,133}$. The majority of 
truncated $\alpha$-synuclein isoforms are lacking a part of the C-terminus (truncation between 115135), although $\alpha$-synuclein species cleaved within the $\mathrm{N}$-terminus have been also identified $^{109}$. In vitro, all C-terminally truncated $\alpha$-synuclein isoforms (for example, 1-87, 1 $102,1-110,1-119,1-120)$ exhibit a dramatic increase in aggregation rate and additionally a seeding effect on fibrillization of full-length $\alpha$-synuclein ${ }^{135}$. These aggregation-prone properties of truncated $\alpha$-synuclein lacking parts of acidic C-terminus might be related to the reduction of the net negative charge and, consequently, to the suppression of electrostatic repulsion between negatively charged molecules and facilitation of the intermolecular interactions and aggregation. To date, five proteases, namely $20 \mathrm{~S}$ proteasome, cathepsin $\mathrm{D}$, calpain I, neurosin and matrix metalloproteinase 3, have been shown to be involved in degradation of $\alpha$-synuclein either in vitro or in vivo ${ }^{133}$. The implication of the last three enzymes in Parkinson's disease is also supported by a detection of them as a component of Lewy bodies. In vitro, $20 \mathrm{~S}$ proteasome cleaves monomeric wild type $\alpha$-synuclein both in solution and in lipid-bound state, however, the kinetics is significantly slower in presence of liposomes ${ }^{144}$. The analysis of the $20 \mathrm{~S}$ proteasome-mediated degradation revealed the formation of three C-terminally truncated products (1-119, 1-110, 1-83) for which an accelerated aggregation rate has been demonstrated in concomitant experiment ${ }^{144}$. $\alpha$ Synuclein is also a substrate for a lysosomal degradation pathway. Thus, cathepsin D, which is a lysosomal aspartic protease, is involved in proteolytic processing of $\alpha$-synuclein in neuronal cell culture and in cell lysates. Although exact cleavage sites by cathepsin D are not known, there is a reasonable likelihood that the C-terminus is cleaved in the cells as evident by increased formation of insoluble inclusion composed of the truncated $\alpha$-synuclein ${ }^{133}$. In contrast to the previously described proteolytic pathways, calpain I- (calcium-dependent, nonlysosomal cysteine protease) and neurosin-mediated cleavages generate in vitro $\alpha$ synuclein isoforms that are modified within NAC region and, importantly, exhibit significantly reduced aggregation propensity ${ }^{133,145}$. Calpain I also is capable to degrade the fibrillar forms of $\alpha$-synuclein by truncations within the C-terminal region ${ }^{133}$. Remarkably, the biological relevance of the calpain I driven degradation of $\alpha$-synuclein is supported by observation of similar truncation products in the Drosophila and mice models of Parkinson's disease as well as in the brains of patients ${ }^{145}$. Since calpain I and neurosin proteolytic pathways produce $\alpha$-synuclein isoforms with impaired aggregation properties, these proteases might be involved in a protective response. Matrix metal proteinase 3, known as zinc mediated endopetidase, has been shown to generate 1-78, 1-91, 1-93 truncated forms of $\alpha$ synuclein in cellular model ${ }^{146}$. At the same time, various types of stress, including oxidative 
stress, trigger the accumulation of matrix metal proteinase 3 in the dopaminergic neurons, and thereby, $\alpha$-synuclein turnover is potentially affected ${ }^{146}$. Despite the fact that our knowledge about $\alpha$-synuclein degradation is limited, the available data from in vivo and in vitro studies supports the hypothesis that the various types of $\alpha$-synuclein truncations might play a pivotal role both in protection against $\alpha$-synuclein misfolding and toxicity as well as in triggering and enhancement of it.

\subsubsection{Ubiquitination}

A significant part of $\alpha$-synuclein in Lewy bodies is mono- or diubiquitinated at multiple lysine residues and beside that ubiquitinated $\alpha$-synuclein is detected in the blood plasma and soluble brain fractions ${ }^{38,137}$. In vivo, polyubiquitination of lysine 48 and to lesser extent lysine 29 is associated with targeting proteasomal degradation by $26 \mathrm{~S}$ proteasome complex as described in the chapter 1.3.2. While the mono- or diubiquitination of lysines 6 , 10, 12 have been shown for aggregated $\alpha$-synuclein ${ }^{133}$. To date, four E3 ubiquitin ligases have been shown to attach ubiquitin to $\alpha$-synuclein, namely, parkin that is targeting $\mathrm{O}$ glycosylated form, next, Siah-1 and Siah-2 that are able modify wild type protein, and Cterminal U-box domain of co-chaperone Hsp70-interacting protein (CHIP) that may act on oligomeric $\alpha$-synuclein species $^{133}$. The results from several in vitro and in vivo systems implicated the monoubiquitination of $\alpha$-synuclein at one or several lysines as a pathological modification leading to $\alpha$-synuclein aggregation and toxicity. For instance, Siah-mediated $\alpha$ synuclein modification in cellular (Hela and PC12) and Drosophila models results in $\alpha$ synuclein accumulation/aggregation and cytotoxicity ${ }^{133}, 147,148$. Biochemically, the profile of mono- and diubiquitination has certain similarities to one observed in the patients. In contrast to Siah ligase, parkin and CHIP might exert a protective role against $\alpha$-synuclein-related toxicity by targeting monomeric and misfolded $\alpha$-synuclein to proteasomal degradation (the role of parkin is discussed in details in chapters 1.3 .2 and 1.6.2. $)^{133}$. The aggregation experiments with selectively ubiquitinated $\alpha$-synuclein for a long time were elusive, that is why properties of heterogeneous mixtures containing $\alpha$-synuclein modified at different lysines in addition to intact protein have been initially investigated. In these in vitro studies, the non-uniform ubiquitination promotes the fibrillization and enhances the cytotoxicity of formed aggregates ${ }^{148}$. To evaluate the individual properties of selectively ubiquitinated $\alpha-$ synuclein, Meier et al have applied an elegant semisynthetic strategy based on a disulfidedirected ubiquitination of single cysteine mutants, and in this way, they have generated and purified nine $\alpha$-synuclein-ubiquitin conjugates for following mutants: K6C, K10C, K12C, 
$\mathrm{K} 23 \mathrm{C}$, K32C, K34C, K46C, K96C ${ }^{149}$. The analysis of the secondary structure of ubiquitinated $\alpha$-synuclein mutants with far-UV CD spectroscopy has demonstrated that the covalent modifications do not alter the random-coil structure of $\alpha$-synuclein moiety and native folding of ubiquitin. From aggregation standpoint, the ubiquitination leads to inhibition of fibrillization for all modification sites, but with significant variation. For instance, among N-terminal ubiquitination sites, mutants $\mathrm{K} 10 \mathrm{C}$ and $\mathrm{K} 23 \mathrm{C}$ form fibrils slightly slower than wild type, when K6C, K12C and K21C conjugates exhibit significantly slower aggregation rate. Remarkably, the attachment of ubiquitin within NAC region, specifically $\mathrm{K} 32 \mathrm{C}, \mathrm{K} 34 \mathrm{C}, \mathrm{K} 42$, complete blocks the fibrillization in vitro, while C-terminal K96C construct displays increased oligomer formation in absence of the mature amyloid fibrils ${ }^{149}$. Therefore, it is reasonable to assume that the modifications of the residues within NAC region and close proximity interfere with the intermolecular interactions, which are involved in formation of aggregation-prone intermediates.

\subsubsection{Oxidation}

The oxidative modifications of biomolecules have a significant impact on their properties and function. It's well known that oxidative damage is increasing with age, and furthermore, is a one of the common neuropathological features of Parkinson's disease. Thus, postmortem analysis of brain tissues from patients with sporadic Parkinson's disease revealed an increased level of oxidative stress, which causes oxidative damage of lipids, proteins and DNA especially in substantia nigra ${ }^{58}$ as well as accumulation of redox active metals $(\mathrm{Fe}, \mathrm{Cu}$, $\mathrm{Mn})$. Despite the fact that oxidized $\alpha$-synuclein has not been detected in the human-derived samples so far, the potential products of $\alpha$-synuclein oxidation have been generated and investigated in vitro. Among the amino acid residues within $\alpha$-synuclein sequence, methionines (residues: 1, 5, 116, 127) and tyrosines (residues: 39, 125, 133, 136) are susceptible to oxidation by biologically relevant oxidants including $\mathrm{H}_{2} \mathrm{O}_{2},{ }^{\bullet} \mathrm{OH}$, superoxide radical $\left(\mathrm{O}_{2}{ }^{\bullet-}\right)$, hypochlorite $(\mathrm{HOCl})$, peroxynitrite $\left(\mathrm{ONOO}^{-}\right)$. The oxidation of methionine might potentially result in formation of two products, under mild condition this is a methionine sulfoxide that could further covert to methionine sulfone in presence of strong oxidizing agents (Fig 1.16). Remarkably, the formation of methionine sulfoxide is reversible process, in which methionine sulfoxide reductase is involved as an enzyme catalyzing the counter reaction. From the structural point of view, the significant changes of the secondary structure might occur for the protein with oxidized methionine(s) due to strong increase of 
A

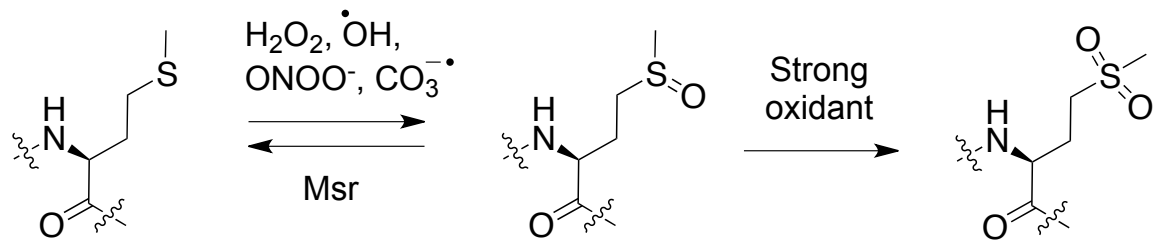

Methionine

Methionine sulfoxide
Methionine sulfone

B<smiles>CC(C)CC(Cc1ccc(O)cc1)C(N)=O</smiles>

Tyrosine<smiles>CC(=O)C(Cc1ccc(Cl)cc1)NCC(=O)O[N+](=O)[O-]</smiles><smiles>CC(=O)C(N)Cc1ccc(O)c(O)c1</smiles>
OH<smiles>[3H][Na]</smiles><smiles></smiles><smiles>[3H]C</smiles><smiles>CC(=O)C(N)Cc1ccc(O)c([N+](=O)[O-])c1</smiles>

3-Hydroxytyrosine

3-Nitrotyrosine<smiles>[Y]C(=O)C(N)Cc1ccc(O)c(-c2cc(CC(N)C(C)=O)ccc2O)c1</smiles>

Figure 1.16. Oxidative and nitrative modifications. (A) Methionine oxidation pathway. (B) Tyrosine nitration, oxidation and dimerization.

polarity of corresponding residue(s). Coming back to $\alpha$-synuclein, the incubation of $\alpha$ synuclein in $1 \%$ aqueous solution of hydrogen peroxide leads to oxidation of all four methionines, whereas M5 is most vulnerable to oxidation and M1, M116, M127 exhibit similar kinetic. The biophysical analysis of this fully oxidized $\alpha$-synuclein by FTIR, far-UV spectroscopy and SEC HPLC revealed that the modified protein is more disordered and extended than wild type. Essentially, full and also partial methionine oxidation leads to dramatic inhibition of the fibril formation in vitro. The fibrillization rate has a clear correlation with the number of methionine sulfoxides, namely the fully oxidized protein displays a lowest propensity to generate amyloid fibrils. The prolonged incubation of fully oxidized $\alpha$-synuclein induces the oligomerization of the protein producing relatively stable soluble oligomeric species with 8-11.6 nm radius of gyration, presumably off-pathway. To probe potential cytotoxicity, the dopaminergic and GABAergic neuronal cultures have been treated with the oligomers composed of oxidized $\alpha$-synuclein and, in turn, these preparations have been shown to be nontoxic to neurons. 
The tyrosine residues are also susceptible, but to a lesser extent than methionines, to oxidative modifications including formation of 3-hydroxytyrosine and dimerization to 3,3dityrosine (Fig. 1.16). From mechanistic perspective, the crucial reaction is a formation of tyrosine radical that further reacts with various substrates (Fig. 1.16). Thus, the exposure of $\alpha$-synuclein to oxidizing mixtures in vitro, like peroxynitrite $/ \mathrm{CO}_{2}$, myeloperoxidase $/ \mathrm{H}_{2} \mathrm{O}_{2} /$ nitrite, $\mathrm{CytC} / \mathrm{H}_{2} \mathrm{O}_{2}$, results in formation of complex mixtures containing 3,3-dityrosine dimerized $\alpha$-synuclein in addition to nitrated and methionine oxidized $\alpha$-synuclein. Subsequent incubation of such mixtures generates soluble heterogeneous oligomers characterized by increased stability. In case of $\mathrm{CytC} / \mathrm{H}_{2} \mathrm{O}_{2}$ system, it has been demonstrated that tyrosines 133 and 136 exhibit highest reactivity in the crosslinking.

Lastly, several lines of evidence also implicate a range of metal ions in catalysis of oxidative damage of $\alpha$-synuclein. For instance, the presence of $\mathrm{Cu}$ (II) or Fe (III) triggers the $\alpha$-synuclein oxidation in vitro that, in turn, induces accelerated oligomerization in combination with reduced fibrillization. For transition metals, the generation of hydroxyl radical via metal-catalyzed Haber-Weiss/Fenton reactions might constitute one of the main pathways involved in oxidative stress and consequent protein modification.

\subsubsection{Nitration}

Nitration of biomolecules, similarly to oxidative damage, can potentially cause profound effects on biochemistry of corresponding substrates. To date, several biologically relevant reactive nitrogen species are known, including nitric oxide (NO), nitrogen dioxide $\left(\mathrm{NO}_{2}\right)$, dinitrogen trioxide $\left(\mathrm{N}_{2} \mathrm{O}_{3}\right)$ and finally most important peroxynitrite $\left(\mathrm{ONOO}^{-}\right)$. In vivo, the inflammatory processes are considered as a main source of reactive oxygen and nitrogen species. Thus, the generation of peroxynitrite $\left(\mathrm{ONOO}^{-}\right)$, as one of the major and powerful nitration reagent, is predominantly based on reaction of superoxide $\left(\mathrm{O}_{2}{ }^{\bullet-}\right)$ with nitric oxide (NO) that in turn is synthesized by inducible nitric oxide synthase in activated macrophages ${ }^{150}$. Within the protein amino acids, the tyrosines are vulnerable to nitration in position 3 of aromatic ring (Fig. 1.16). Following clinical observations support the implication of this modification in Parkinson's disease: the amount of nitrated biomolecules is increasing with an age and, furthermore, the significant proportion of $\alpha$-synuclein in Lewy bodies has 3-nitrotyrosine residues (Fig. 1.16) ${ }^{132}$. In agreement with this line of thought, the nitration exacerbates $\alpha$-synuclein toxicity in cellular and animal models ${ }^{132,151,152}$. For 
instance in cells and primary neurons, an exposure to monomeric or aggregated forms (oligomers or fibrils) of nitrated $\alpha$-synuclein or to nitrative insults (reactive nitrogen species or overexpression of nitric oxide synthase) leads to cytotoxicity and abnormal $\alpha$-synuclein accumulation to much greater degree than unmodified $\alpha$-synuclein ${ }^{132,151,152}$. Moreover, the inoculation of nitrated $\alpha$-synuclein to the rat brain induces the degeneration of dopaminergic neurons in substantia nigra and motor impairments as detected by Rotarod test ${ }^{152}$.

In vitro, the incubation of recombinant wild type $\alpha$-synuclein with different nitration agents results in unselective nitration of one or several tyrosine residues, namely Y39, Y125, Y133, Y136 134, 151, 153. In addition to heterogeneous mixtures of nitrated $\alpha$-synuclein, the products of methionine oxidation and 3,3-dityrosine crosslinking have been consistently detected. Uversky et $a l^{153}$ archived the full nitration of all four tyrosines by treatment of $\alpha$ synuclein with a big excess of tetranitromethane as confirmed by mass spectroscopy and absorbance data. To probe potential effects of such excessive modification on structural and aggregation properties of $\alpha$-synuclein the spectrum of biophysical methods has been applied $^{153}$. Briefly, the analysis of CD, FTIR spectra and ANS fluorescence data for modified $\alpha$-synuclein revealed a formation of partially folded conformations with increased $\beta$-sheet secondary structure. The findings from SAXS and SEC measurements suggested that fully nitrated $\alpha$-synuclein has an $\mathrm{R}_{\mathrm{g}}$ of $69 \pm 1 \AA$ (unmodified $\alpha$-synuclein $41 \AA$ ) and is prone to a self-association to dominant octamer and less populated monomers, dimers and tetramers. Under aggregating condition, the fully nitrated $\alpha$-synuclein failed to form mature amyloid fibrils, but instead the formation of heterogeneous mixture of oligomeric species was observed. Importantly, the binding of $\alpha$-synuclein to membranes, which is considered as a crucial factor for biological function, is significantly reduced in case of full and partial tyrosine nitration ${ }^{151,153}$. The main drawback of this approach, namely the non-uniform nitration and consequently heterogeneous sample composition, does not allow one to evaluate the impact of the selective nitration of one or several tyrosine residues. To overcome these limitations, Lashuel and colleagues ${ }^{151}$ have recently developed an elegant, semisynthetic approach to generate a selectively nitrated $\alpha$-synuclein, specifically at tyrosines 39 or 125 . Following experiments focused on aggregation properties of these constructs showed that the propensity to form amyloid fibrils is declining in a series: wild type, nitro-Y39, nitro-Y125 and fully nitrated $\alpha$-synuclein. Remarkably, the fibrillization process for mono-nitrated $\alpha$ synuclein at initial stage is characterized by formation of significant populations of amorphous oligomers that are further assembled to short fibrils morphologically distinct from wild type $\alpha$-synuclein ${ }^{151}$. 


\subsubsection{SUMOylation}

SUMOylation, known as attachment of small ubiquitin-like modifier to proteins by isopeptide bond to lysine, is a highly dynamic and reversible post-translational modification that is involved in regulation of cellular distribution, function and metabolism of corresponding target protein ${ }^{154}$. Despite the fact that SUMOylated $\alpha$-synuclein has not been detected in human brain so far, there are several lines of evidence supporting the implication of SUMO in $\alpha$-synuclein biochemistry and Parkinson's disease. First, SUMO is colocalized with Lewy bodies and $\alpha$-synuclein-positive inclusions as demonstrated by immunohistochemical studies on Parkinson's disease brain tissues ${ }^{154}$. Second, evaluation of animal models showed increased total levels of SUMO and also elevated SUMO immunoreactivity around $\alpha$-synuclein-positive deposits ${ }^{154}$. In vitro experiments in cell-free system and HEK293 cells have demonstrated that $\alpha$-synuclein is a substrate for SUMOylation $^{155}$. Moreover, purified SUMOylated $\alpha$-synuclein fails to form amyloid fibril upon prolonged incubation and also inhibits the fibrillization of wild type $\alpha$-synuclein in dose dependent manner. At elucidating the main modification site, Orbitrap mass analysis revealed two lysines 96 and 102 as preferential conjugation residues within $\alpha$-synuclein sequence. Importantly, there are also several studies showing the evidences of cytoprotective properties of SUMO against $\alpha$-synuclein-induced toxicity ${ }^{154,155}$. The experimental evidence indicates that the SUMOylation might substantially change the $\alpha$-synuclein properties, biochemistry and, consequently, neurotoxicity, possibly by modulation of aggregation propensity, solubility and degradation pathways.

\subsubsection{Glycation and glycosylation}

Glycosylation, known as enzymatic attachment of oligosaccharides or polysaccharides to biomolecules, is another important post-translational protein modification that is involved in regulation of functional and structural properties of corresponding substrate. There are two major types of protein glycosylation, namely N-glycosylation that targets nitrogen atom of arginine or asparagine and O-glycosylation of hydroxyl group of serine, threonine or tyrosine. To date, O-glycosylated form of $\alpha$-synuclein was found in the human brain, however there is no additional information available regarding the physiological function and structural properties of O-glycosylated $\alpha$-synuclein ${ }^{135,156}$.

In contrast to glycosylation, a glycation is non-enzymatic reaction of reducing sugars (glucose, fructose) or dicarbonyl compounds (glyoxal, methylglyoxal) with the random amino group of the lysine side chains of protein $^{135}$. Chemically, the formation of Schiff's 
bases, Maillard reaction and Amadori rearrangement are considered as the core reactions leading to formation of early glycation products that further might irreversibly transform to advanced glycation end-products (AGEs) in multistep process comprising condensation, cyclization, fragmentation, oxidation, cross-linking and others steps (Fig. 1.17) ${ }^{135,156}$. Several lines of evidence from in vivo and in vitro studies have linked glycation, $\alpha$-synuclein and Parkinson's disease. For instance, an increased level of glycation is observed in the brains of Parkinson's disease patients ${ }^{156}$. Considering that $\alpha$-synuclein is co-localized and also crosslinked with AEGs in Lewy bodies, it has been suggested that $\alpha$-synuclein might be a target for pathology-related glycation ${ }^{156}$. Experiments in vitro and in cellular models, in which various $\alpha$-synuclein containing AGEs and related aggregation products were generated and characterized, have confirmed this assumption ${ }^{109,135,156}$. Thus, in the test tube $\alpha$-synuclein can form AGEs with D-ribose, glyoxal, methylglyoxal and, remarkably, these modifications do not alter the conformational properties of monomeric $\alpha$-synuclein ${ }^{156}$. However, the modified $\alpha$-synuclein showed increased propensity to form stable oligomeric species and globular-like aggregates, and failed to generate mature amyloid fibrils. In case of D-ribose glycation, the preaggregated modified $\alpha$-synuclein is toxic to SH-SY5Y cells, as evident from LDH release assay and generation of reactive oxygen species ${ }^{109,156}$.

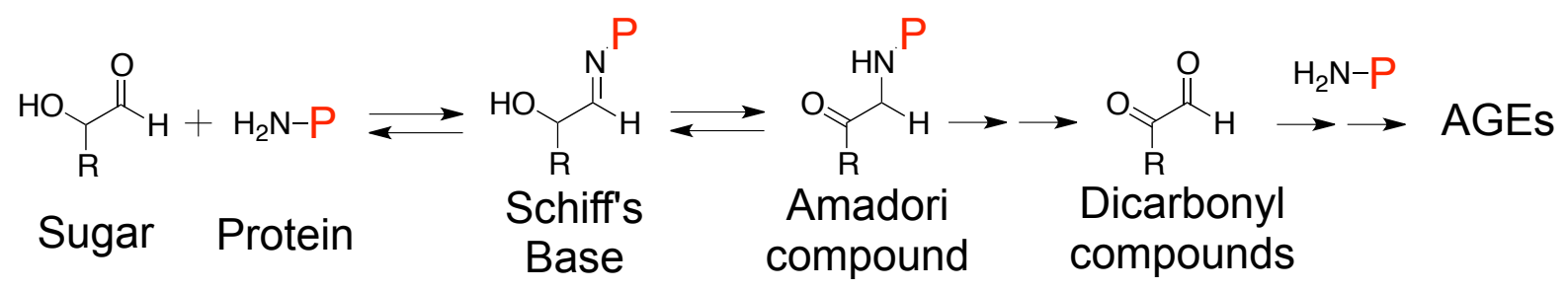

Figure 1.17. Schematic pathway of AGEs formation.

\subsubsection{Tissue transglutaminase cross-linking}

Transglutaminase is an enzyme that catalyzes the formation of intermolecular, rarely intramolecular, isopeptide bond between lysine and glutamine side chains and, thus, generates cross-linked, insoluble, high molecular weight protein polymers. Whereas transglutaminases are involved in several physiological processes like blood coagulation or regulation of cytoskeleton, there are also evidences arguing for possible link between tissue transglutaminase, $\alpha$-synuclein and Parkinson's disease ${ }^{135,157}$. Thus, increased levels of tissue transglutaminase brain expression in combination with tissue transglutaminase specific profile of cross-linking within Lewy bodies have been demonstrated in Parkinson's disease 
patients $^{135,158}$. Follow-up studies have shown that $\alpha$-synuclein is substrate for tissue transglutaminase both in vitro and in cellular models ${ }^{135,157,158}$. For example, $\alpha$-synuclein is cross-linked within 2 hours in presence of tissue transglutaminase in vitro and, in turn, these modifications are inducing fast aggregation of $\alpha$-synuclein ${ }^{158}$. Based on detailed analysis, it has been assumed that intermolecular cross-linking facilitates the misfolding and aggregation of $\alpha$-synuclein, while intramolecular modifications stabilize monomeric protein and thereby inhibit oligomerization/fibrillization. So far, several intramolecular modification sites have been identified, including Gln59-Lys60, Gln109-Lys32, Gln99-Lys58 ${ }^{135}$. The experimental results from cellular models have corroborated the notion that tissue transglutaminase catalyzes $\alpha$-synuclein cross-linking and triggers its aggregation. For instance, finding from COS7 cells that were transfected with $\alpha$-synuclein and tissue transglutaminase suggested the direct interactions of corresponding proteins leading to $\alpha$-synuclein cross-linking and conversion to detergent insoluble aggregates ${ }^{158}$. Moreover, the tissue transglutaminase has been shown to stimulate the formation of $\alpha$-synuclein positive cytoplasmic inclusions in HEK293T cells ${ }^{158}$.

\subsubsection{Interactions with lipids}

\subsubsection{1. $\alpha$-Synuclein and membranes: from physiological function to toxicity}

Mounting evidence from in vivo and in vitro studies strongly suggests a possible implication of $\alpha$-synuclein membrane interactions both in presumable physiological function(s) and pathological pathways including aggregation. Among the hypothetic $\alpha-$ synuclein physiological functions, including regulation of neurotransmitter release; synaptic function and plasticity; lipid metabolism; vesicle trafficking and recycling; mitochondrial fission and fusion; SNARE complex assembly, the membrane binding of $\alpha$-synuclein can substantially contribute to all of them. On the other hand, the abnormal interactions with lipids might exert the deleterious effects in a number of crucial cellular processes listed above by various mechanisms either by loss-of-function or gain-of-function. In addition to already mentioned aspects, findings also imply that various lipids constitute an important factor that affects the aggregation properties of $\alpha$-synuclein, and thereby contribute to modulation of biological or misfolded $\alpha$-synuclein state. Remarkably, experimental findings that have been accumulated through a variety of in vitro biophysical studies, have established many structural and functional features of $\alpha$-synuclein/membrane interactions. The relevance of in vitro data has been also confirmed by in vivo studies both in cellular models and in the animal models. 
A
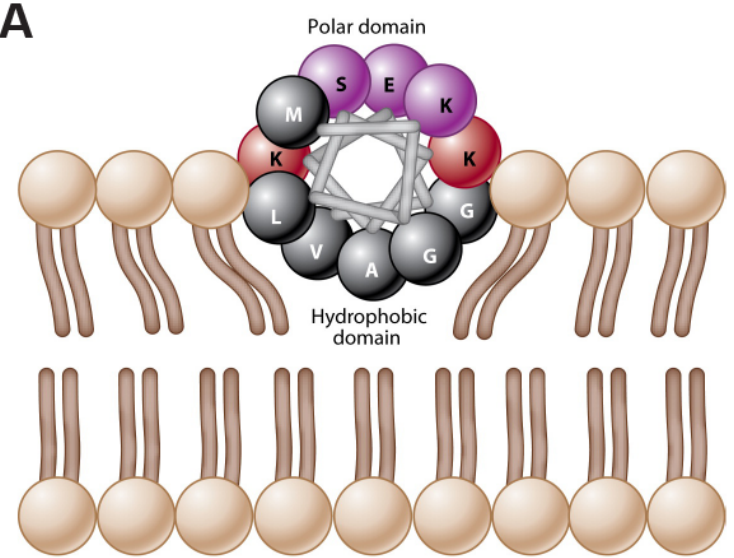

B

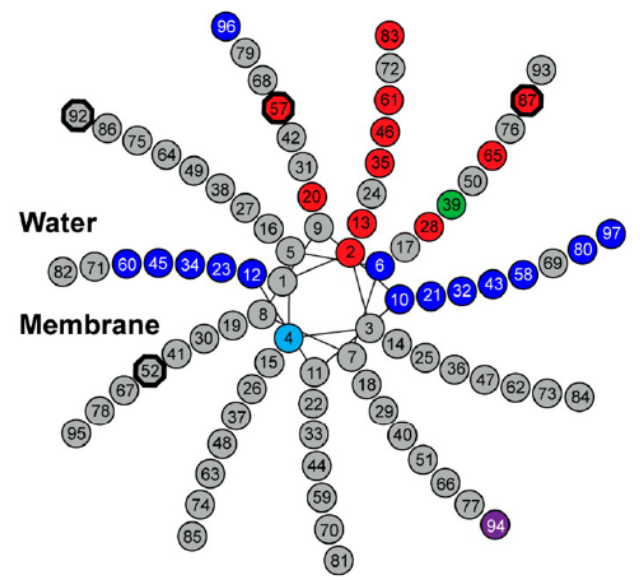

C
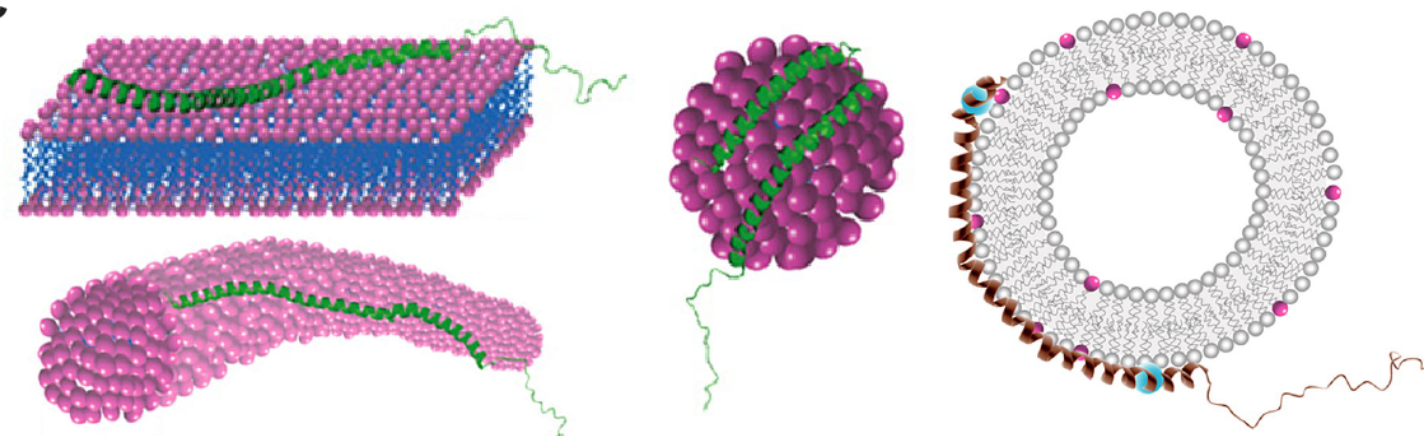

D

E
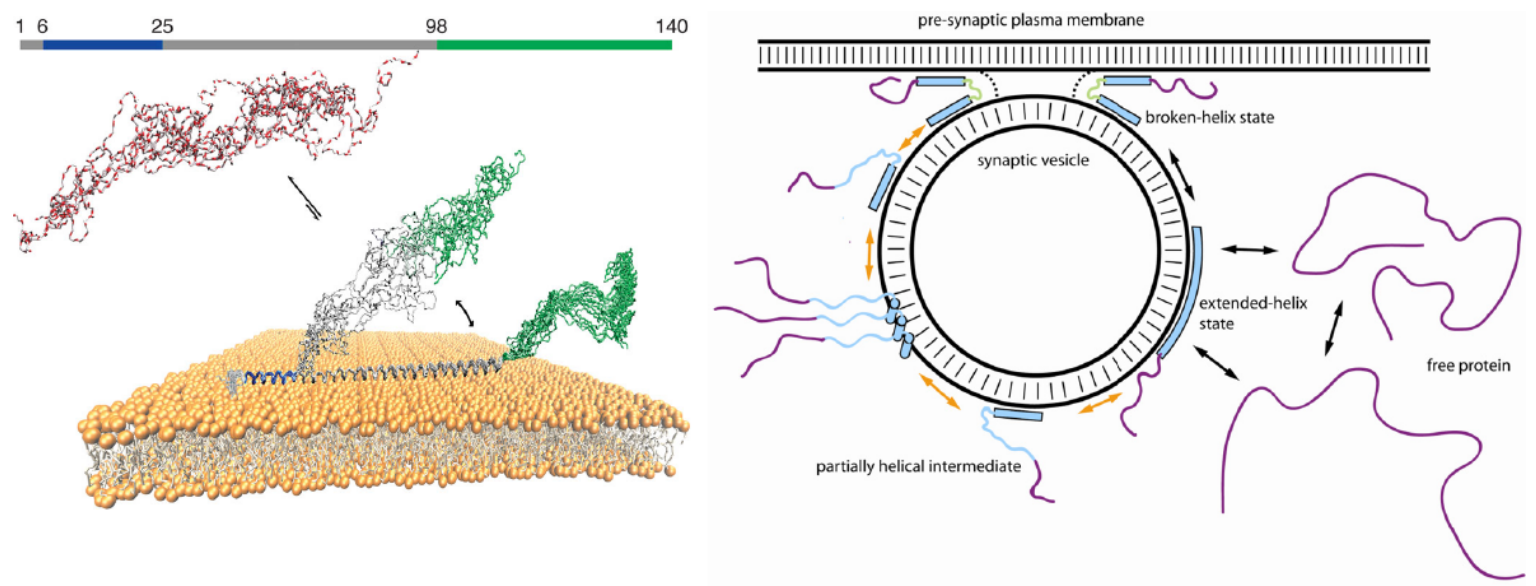

Figure 1.18. $\alpha$-Synuclein folding and interactions with membranes. (A) Edmundson helix wheel for membrane-bound $\alpha$-synuclein. (B) Helical wheel diagram for $\alpha$-synuclein extended $\alpha$-helix. (C) Broken and extended helical $\alpha$-synuclein structures upon lipid or SDS surfaces binding. (D) Schematic mode of $\alpha$-synuclein interactions with the membranes. (E) Schematic model of equilibrium between helical, disordered and aggregated states of $\alpha$-synuclein and potential role of the broken helix state in membrane fusion. Figures A republished with permission of Annual Reviews, Inc., from Auluck et $a l .{ }^{4}$; permission conveyed through Copyright Clearance Center, Inc. (c) (2010); Figure B adapted with permission from Jiang et al. ${ }^{9}$, ( ) (2015) American Chemical Society; Figure C adapted from Ferreon et al. ${ }^{12}$, (C) (2009); Figure D reprinted with permission from Springer Nature: Nature Communications, Fusco et al. ${ }^{13}$, (C) (2014); Figure E reprinted from Dikiy and Eliezer ${ }^{14}$ with permission from Elsevier, (c) (2012) 


\subsubsection{Lipids and artificial model systems}

Before discussing in details the $\alpha$-synuclein/membrane interactions I would like to describe briefly the structure and properties of common lipids and available model membranes. Lipids are a large class of organic molecules like fatty acids, detergents, soaps, sterols, glycerides, phospholipids, glycolipids, certain vitamins and others. In vivo, lipids have diverse functions including energy storage, regulatory functions, membrane building blocks and many others. All living cells are surrounded by the lipid bilayer composed of the membrane lipids that could be divided in three main classes: phospholipids

\section{Zwitterionic phospholipids}<smiles>CCCCCC(=O)OC(COP(=O)([O-])OCCNC)C(=O)OCC(C)CC</smiles>

Phosphatidylcholine (PC)

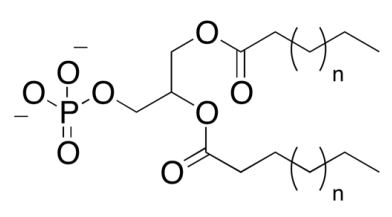

Phosphatidic acid (PA)<smiles>CCCCCC(=O)OC(COP(=O)([O-])OC1C(O)C(O)C(O)C(O)C1O)CC(=O)O</smiles>

Phosphatidylinositol (PI)

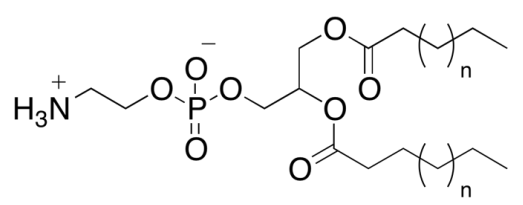

Phosphatidylethanolamine (PE)<smiles>CCCCCC(=O)OC(COC(=O)CCCC)COP(=O)([O-])OCC([NH3+])CC</smiles>

Phosphatidylserine (PS)<smiles>CCCCCC(=O)OC(COC(=O)CCCC)COP(=O)([O-])OCC(O)CO</smiles>

Phosphatidylglycerol (PG)

\section{Sphingomyelin}

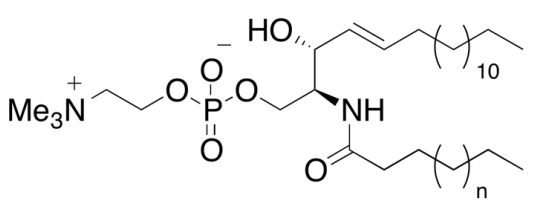

\section{Glucocerebrosides}<smiles>CCC/C=C/C(O)C(COC1OC(O)[C@H](O)C(O)C1O)NC(=O)CCCCC</smiles>

\section{Cholesterol}

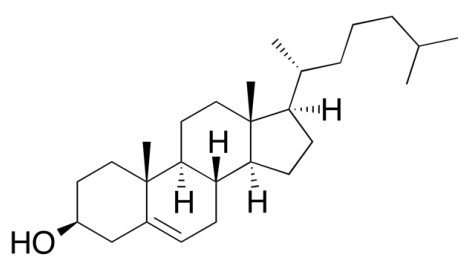

SDS<smiles>CCCCCCCCCCCCOS(=O)(=O)O[Si]</smiles>

Figure 1.19. Lipids.

(phoshoglycerides and sphingomyelin), glycolipids (for instance, glucocerebrosides) and sterols (for instance, cholesterol) (Fig. 1.19). The majority of membrane lipids are composed of the polar head group that is normally exposed to polar environment like aqueous solution, and the hydrophobic tail that constitutes the hydrocarbon interface and might contain one or two fatty acids (saturated or unsaturated), sphingosine or other apolar functions. Due to this amphipathic nature of the membrane lipids and related molecules, they exhibit the propensity 
to self-associate or aggregate in polar environment providing diverse assembles of various size and shape like tubular, disk or spherical micelles, liposomes and bilayers (Fig. 1.18 C). Depending on the selection of lipids and experimental conditions it's possible to generate the quite defined population of certain structures, for instance, sodium dodecyl sulfate (SDS, Fig. 1.19) readily forms micelle assembles that are considered as a membrane-mimetic environment, while phospholipids preferentially assemble to lipid bilayer structures like liposomes or flat bilayers. In the laboratory, the most common artificial model systems of the lipid bilayer are small or large unilamellar liposome vesicles (SUVs or LUVs), black lipid membranes and supported lipid bilayers.

\subsubsection{Monomeric membrane-bound $\alpha$-synuclein}

As discussed in the previous chapter, all synuclein family members show high sequence conservation of the N-terminus coding for amphipathic $\alpha$-helices that are structurally similar to lipid-binding domains of class $\mathrm{A}_{2}$ apolipoproteins, while their $\mathrm{C}$ terminus is significantly less conserved and highly enriched in acidic residues ${ }^{107,}{ }^{108}$. In $\alpha$ synuclein, N-terminal and NAC regions contain seven imperfect 11-residues repeats (XKTKEGVXXXX) that might undergo the structural transition upon binding to lipid bilayer from unfolded to $\alpha$-helical conformation (Fig 1.9 and 1.18). In theory, the driving force for membrane-binding amphipathic $\alpha$-helix formation is an energetically favorable distribution of hydrophobic and hydrophilic residues into lipophilic membrane-exposed domain and polar water-exposed domain (Fig. 1.18 A). In line with theoretical prediction, multiple studies using various approaches and methods such as NMR, EPR, fluorescence spectroscopy, X-ray, circular dichroism, neutron reflectometry have established that $\alpha$-synuclein binds to sodium dodecyl sulfate (SDS) micelles and to negatively charged phospholipid bilayers of varying shape, sizes and composition, and these interactions are attributed to a formation of the $\alpha$ helical conformation covering approximately first 100 residues of $\alpha$-synuclein, while the Cterminal region remains unfolded and highly dynamic (Fig. 18 A-C) ${ }^{9}, 12,13,159-162$. According to current models of membrane-bound $\alpha$-synuclein, the $\mathrm{N}$-terminal domain, up to residue $\sim 100$, forms an extended amphipathic $\alpha$-helix, in which nine glutamates and aspartates are water exposed, the hydrophobic amino acids form hydrophobic domain that is inserted into hydrocarbon interface, and finally 13 positively charged lysines are interacting with the anionic lipid head groups within the interfacial region (Fig. 18 B; glutamates and aspartates are shown in red, lysines in blue $)^{4}, 9,159$. This model and experimental findings clearly 
emphasize the important role of both electrostatic and hydrophobic interactions in $\alpha$-helix formation and preferential binding to negatively charged membranes.

Soon after the discovery that membrane-bound $\alpha$-synuclein forms helical conformation, increasing number of investigations aimed to extend and deepen the knowledge about these interactions. Several NMR and CD studies have demonstrated that $\alpha-$ synuclein in presence of SDS micelles adopts two antiparallel curved $\alpha$-helices, V3-V37 and K45-T92, connected by an ordered extended linker or, alternatively, 1 to 100 helical residues with a short brake around 43-44 amino acids (Fig. 1.18C) ${ }^{160,162}$. What's more from NMR studies in micelles, the $\alpha$-synuclein $\alpha$-helix has an unusual periodicity $11 / 3$, meaning 3 turns for every 11 amino acids, while a standard periodicity is $18 / 5^{159}$. Follow-up cross-relaxation studies revealed a penetration of the $\alpha$-helix into the micelles, represented by protein interactions with the $\mathrm{C} 3-\mathrm{C} 4$ carbon atoms of the $\mathrm{SDS}^{159}$. Furthermore, the data support a lack of tertiary structure for micelle-bound $\alpha$-synuclein. Single molecular FRET experiments, acquired with SDS micelles of different shapes, have confirmed the formation of broken $\alpha-$ helix upon $\alpha$-synuclein binding to spherical micelles, while the existence of an extended helical structures have been observed upon binding to cylindrical micelles (Fig. 1.18C) ${ }^{12}$.

Experiments with liposomes and supported bilayers are also consistent with a binding mode observed in micelles, in which $\alpha$-synuclein undergoes a conversion to a helical conformation within its $\mathrm{N}$-terminus (up to 100 residues), while the last 40 residues remain disordered and highly flexible ${ }^{13,160}$. Evaluation of NMR and fluorescence spectroscopy data has corroborated the notion that the extended $\alpha$-helix is a preferential arrangement in case of the lipid bilayers with a small curvature (Fig. 1.18C, D) $9,12,14,160$. Nevertheless, there are also indications of a broken $\alpha$-helix structure for liposome-bound $\alpha$-synuclein, thereby suggesting the inherited multistate folding that, in turn, might be essential for biological function ${ }^{12,14}$. To probe a potential specificity in protein-lipid binding, $\alpha$-synuclein has been brought into contact with liposomes of different phospholipid compositions and vesicle sizes. In such studies, $\alpha$-synuclein exhibits a strongest binding to the negatively charged liposomes containing PA or PG lipids, the binding affinity: $\mathrm{PA}>\mathrm{PG}=\mathrm{PS}>\mathrm{PE}>\mathrm{PC}$, and to highcurvature vesicles ${ }^{161}$. Recently, a combination of solid state and solution NMR spectroscopy was employed to uncover molecular details about behavior of membrane-bound $\alpha$ synuclein $^{13,163}$. Taken together, these studies have suggested two binding modes, called SL1 and SL2. In SL1 mode, first $25 \mathrm{~N}$-terminal residues strongly bind to the membrane in form of $\alpha$-helix and these interactions are characterized by a low specificity to certain phospholipids and could be considered as an "anchoring" of $\alpha$-synuclein. Whereas, the SL2 helix is 
potentially responsible for recognition of membrane characteristics and, thus, modulating the affinity and the selectivity (Fig. 1.18D). Of note, $\alpha$-synuclein also displays binding to the liposomes containing cholesterol, sphingomyelin and gangliosides that are known to be important components of synaptic vesicle membranes, lipids rafts and caveolae ${ }^{4}$.

Considering that the familiar mutations of $\alpha$-synuclein can potentially influence protein/membrane interactions, a number of studies have focused on these issues ${ }^{4,163,164}$. And indeed, every mutation, A30P or A53T or E46K, has a significant and, at the same time, mutant-specific impact on $\alpha$-synuclein membrane interactions. As demonstrated by several independent experiments utilizing different techniques, the affinity to the lipid bilayers is changing in following order: $\mathrm{A} 30 \mathrm{P}<\mathrm{A} 53 \mathrm{~T} \approx \mathrm{Wt}<\mathrm{E} 46 \mathrm{~K}^{4}, 163-165$. The $\mathrm{A} 30 \mathrm{P}$ mutant has the weakest binding that is associated with destabilization of the $\alpha$-helix by proline residue. Regarding two other mutants, A53T has minor effects, while E46K exhibits stronger affinity probably due to additional positive charge of the lysine. To further investigate the properties of the membrane-bound $\alpha$-synuclein, Bodner et al. ${ }^{163}$ have applied the concept of SL1/SL2 binding modes to three main familiar forms. Based on NMR data, the comparison of these mutants to wild type $\alpha$-synuclein revealed an increased SL1/SL2 ratio for A30P and A53T (wild type - 0.49; A30P - 1.2; A53T - 0.73; E46K - 0.49), while E46K showed a similar ratio, but increase in total population of SL1 mode as a consequence of a higher fraction of membrane-bound protein. In this model, the SL1 mode is considered as the potentially aggregation-prone set of conformations, because of intermolecular interactions in which unfolded and environment-exposed NAC region of the protein might be involved. In this way, the mutations of $\alpha$-synuclein causing the shift of SL1/SL2 equilibrium might induce the formation of toxic aggregated species and also disturb the $\alpha$-synuclein membrane-related physiological functions.

\subsubsection{Consequences of membrane binding}

Several lines of evidence suggested that $\alpha$-synuclein/membrane interactions constitute an important factor that affects both lipid bilayer properties and $\alpha$-synuclein aggregation behavior. Based of research in vitro findings, the fibrillization of $\alpha$-synuclein can be modulated by various lipid-related conditions like lipid composition, type of lipid bilayer, ratio protein/lipids and so on ${ }^{4,161,166-169}$. Viewed together, the fibril formation is inhibited in a concentration-dependent manner by negatively charged liposomes (like PA/PC, PS/PC, PG/PC) at high lipid/ $\alpha$-synuclein ratios ${ }^{161,164,166}$. Furthermore, the tendency to prevent $\alpha$ synuclein fibrillization has also associated with a smaller size of vesicles and a higher content 
of negatively charged phospholipids ${ }^{161,166}$. Since all mentioned factors facilitate $\alpha$-synuclein binding to membranes in $\alpha$-helical conformation, it is reasonable to assume that such interaction trap the protein on the membrane and inhibit aggregation to amyloid fibrils. However, the opposite situation has been observed at low lipid/ $\alpha$-synuclein ratio, as evident by accelerated fibrillization ${ }^{161}, 166$. Such inverse behavior might be attributed to following mechanisms: significant increase of the effective concentration on a limited membrane surface; formation of intermediate, aggregation-prone conformations (for example, SL1 binding mode); enhanced probability of intermolecular interactions. Aside from the synthetic phospholipids, also physiologically relevant synaptosome membranes isolated from the rat brains promote the $\alpha$-synuclein fibrillization in vitro (SUVs, lipid/ $\alpha$-synuclein ratio = $1 / 20)^{168}$. In complementary experiments, the role of the fatty acids and, specifically, polyunsaturated fatty acids has been evaluated due to the high abundance of the last ones in brain phospholipids ${ }^{167}$. As shown in these studies, the exposure of $\alpha$-synuclein to unsaturated acids either in free form or as a component of phospholipids triggers the oligomerization of the protein and the amount of oligomeric species correlates with the number of double bonds and the length of the carbon chain ${ }^{167}$. Overall, the lipid-induced $\alpha$-synuclein oligomerization is considered as a potentially pathological process; however, several observations have implicated the membrane-bound $\alpha$-synuclein species as a part of SNARE complex assembly pathway $^{169}$. According to FRET and cross-linking studies, these $\alpha$-synuclein oligomers are composed of 8 and more protein molecules adopting $\alpha$-helical confirmation. Importantly, FRET-derived interaction map suggested the formation of relatively homogeneous population of multimers characterized by quite defined intermolecular contacts ${ }^{169}$.

In addition to the drastic impact on $\alpha$-synuclein behavior, $\alpha$-synuclein/membrane interactions also have significant influence on lipid bilayer properties. Multiple studies have shown that monomeric and aggregated $\alpha$-synuclein can induce various types of membrane disruption or rearrangement ${ }^{4}, 161,165,170,171$. In case of phospholipid micelles, the addition of monomeric $\alpha$-synuclein leads to a flattening and distortion of the surface curvature ${ }^{4}$. On the other hand, it has been hypothesized that the binding of $\alpha$-synuclein to the small vesicles, which are characterized by a topologically stressed bilayer/water interface, can stabilize such curved membranes by additional ordering of the lipid acyl chains. It's therefore envisioned that $\alpha$-synuclein seems to be essential for regulation and preservation of the synaptic vesicle reserve pool ${ }^{164}$. The interactions with the negatively charged liposomes may cause an increase of membrane mobility and water penetration and, moreover, a modest permeabilization of the vesicles has been detected at high protein concentrations ${ }^{161}$. In 
addition to the already mentioned membrane model systems, the impact of $\alpha$-synuclein binding has been also assessed in planar supported lipid bilayers, for which specific biophysical methods could be applied. Based on the epifluorescence microscopy data, the addition of $\alpha$-synuclein to the supported lipid bilayers containing variable composition of $\mathrm{PA} / \mathrm{PC}$ and $\mathrm{PG} / \mathrm{PC}$ induces a lipid demixing represented by a formation of PA- or PG- and PC-rich regions ${ }^{170}$. In line with the previous results, $\alpha$-synuclein preferentially binds to PA or PG enriched clusters. Another study from the same group has shown concentrationdependent distortion of the PG/PC lipid bilayer by $\alpha$-synuclein that was attributed to lipid tubule formation ${ }^{165}$. More recently, Hähl et al ${ }^{171}$ have applied the X-ray reflectometry method that allows in situ characterization of lipid interfaces with Angstrom resolution. Thus, the evaluation of experimental data indicates a clear reduction of PS/PC bilayer thickness upon incubation with $\alpha$-synuclein accompanied by the changes in the lipid packing.

To summarize, $\alpha$-synuclein/membrane interactions are diverse and still not fully understood. While it be very likely that $\alpha$-synuclein binding to certain types of membranes is required for its biological function, but it also seems to be involved in $\alpha$-synuclein-related toxicity.

\subsubsection{5. $\alpha$-Synuclein/membrane interactions in vivo}

The association of $\alpha$-synuclein with the various types of membranes has been also demonstrated in vivo. From analysis of a rat brain homogenate, $\alpha$-synuclein is found in the cytosolic and membrane-bound forms and distributed $85 \%$ and $15 \%$, respectively ${ }^{172}$. Remarkably, the lipid-associated fraction can trigger the aggregation of soluble $\alpha$-synuclein, arguing for accumulation of the species with the seeding propensity ${ }^{172}$. Work in the cellular and yeast models has also contributed substantially to our understanding of $\alpha$-synuclein distribution and behavior inside the living cells (Fig. 1.20) $)^{4}, 164,173$. These studies have

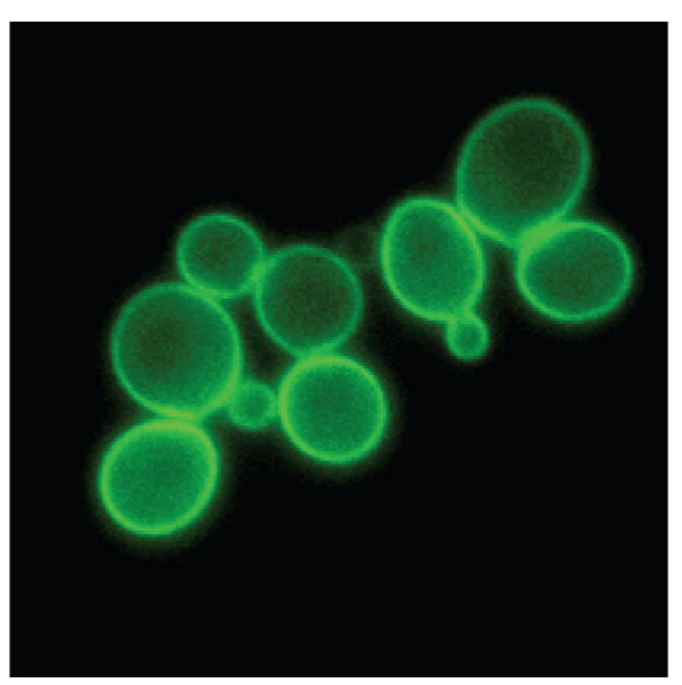

uncovered that $\alpha$-synuclein, in addition to dominated, cytosolic form, readily associates with the plasma membrane. Concurrently, several other

Figure 1.20. $\alpha$-Synuclein subcellular localization in yeast. $\alpha$-Synuclein is labeled with green fluorescence protein. Figures republished with permission of Annual Reviews, Inc., from Auluck et al. ${ }^{4}$; permission conveyed through Copyright Clearance Center, Inc. (C) (2010) 
lipid-related subcellular localizations have been demonstrated for $\alpha$-synuclein, in particular, the membranes of mitochondria, endoplasmic reticulum and synaptic vesicles; lipid droplets; polyunsaturated fatty acids and membranes rich of lipid rafts ${ }^{164,169,173}$. Recently, $\alpha$-helical oligomeric $\alpha$-synuclein has been detected in synaptic vesicles, more specifically, in presynaptic plasma membrane-bound pool, where this $\alpha$-synuclein complex presumably has a function of a chaperone for SNARE-complex assembly ${ }^{169}$. Considering so diverse distribution of $\alpha$-synuclein within the cells, it is reasonable to assume that the association of $\alpha$-synuclein with the membranes might play an important role both in physiological functions as well as pathological pathways.

\subsubsection{6. $\alpha$-Synuclein/membrane interactions from function to toxicity}

In this chapter, I would like to summarize briefly possible $\alpha$-synuclein functions and toxicity pathways in context of lipid and membrane interactions ${ }^{4,14,164,169,173 .}$

Putative $\alpha$-synuclein physiological functions:

1) Regulation of the synaptic vesicle pools and neurotransmitters release (direct binding to synaptic vesicles, stabilization of topologically stressed membranes, regulation of membrane microdomains);

2) Regulation of fusion and budding of various vesicles and membranes (formation of broken helix that can function like an anchor as depicted on Fig. 1.18 E);

3) Chaperone for SNARE complex assembly by binding to the N-terminus of vSNARE protein synaptobrevin-2;

4) Regulation of dopamine transporters in the synapsis and dopamine homeostasis ( $\alpha$-synuclein might be involved in endocytosis of dopamine transporters);

5) Antioxidant function (protection of unsaturated fatty acids and other biomolecules against peroxidation by reactive oxygen species);

6) Regulation of lipids metabolism (inhibition of phospholipase D that converts the phosphatidylcholine to the phosphatidic acid);

7) Regulation of the vesicle trafficking within the cell (for example, ER to Golgi transport);

Putative $\alpha$-synuclein toxicity pathways:

1) Dysregulation of one or several pathways listed above due to posttranslational modifications, abnormal concentrations, disturbed localization and aggregation state of $\alpha$-synuclein; 
2) Membrane- or PUFAs-induced misfolding and aggregation of $\alpha$-synuclein leading to formation of toxic species;

3) Permeabilization of plasma and organelle membranes by distortion of the lipid bilayer or by pore formation;

4) Mitochondrial dysfunction represented by morphological changes, oxidative stress, cytochrome $\mathrm{C}$ release, energy metabolism failure, inhibition of complex I activity, calcium homeostasis, mitochondrial repair and transport alterations.

5) Spreading and transmission of misfolded $\alpha$-synuclein in prion-like manner;

\subsubsection{Structure and toxicity of $\alpha$-synuclein aggregates}

\subsubsection{1. $\alpha$-Synuclein amyloid fibrils}

Soon after the discovery that fibrillar $\alpha$-synuclein is a main component of Lewy bodies, the structure and the properties of $\alpha$-synuclein amyloid fibrils, generated mainly from recombinant protein in vitro, have been the focus of many studies. A combination of multiple spectroscopic techniques, like solution and solid state NMR, EM, cryo-EM, CD, EPR, X-ray and electron diffraction, provides valuable information regarding secondary structural elements, domain organization, dynamics and other aspects ${ }^{174-179}$. The $\alpha$-synuclein conversion from the unfolded monomeric state to $\beta$-sheet-rich fibrillar structure is evident from the CD and FTIR spectrum showing typical $\beta$-sheet pattern ${ }^{115}, 176$. Complementary electron microscopy and atomic force microscopy studies have shown the formation of unbranched amyloid fibrils that are morphologically similar to the patient-derived filaments ${ }^{174,176}$. According to these studies, the fibrils have 5-12 $\mathrm{nm}$ total width, up to several microns length, composed of two filaments that could arrange to straight or twisted fibrils. Furthermore, the synthetic and native filaments have the same appearance after $\mathrm{H} 3 \mathrm{C}$ antibodies decoration as measured by the immunoelectron microscopy. $\alpha$-Synuclein fibrils isolated from the human brain have been shown to have polymorphic structures, with fibril widths of 5 and $10 \mathrm{~nm}^{180}$.

Next piece of structural information is provided by X-ray and electron refraction studies $^{176}$. Thus, the investigation of $\alpha$-synuclein amyloid fibrils revealed a cross $\beta$-structure with the perpendicular orientation of $\beta$-strands to fiber axis. Additionally, the $\beta$-sheet folding is characterized by interstrand distance of $0.47 \mathrm{~nm}$ (meridional reflection) and intersheet space of 1-1.1 nm (equatorial reflection).

Several polymorphs of $\alpha$-synuclein amyloid fibrils were also intensively studied by solid state NMR and EPR spectroscopy. According to EM and NMR data, each type of fibrils 
exhibits specific morphological properties as well as organization of the secondary structure elements. The findings from these studies support the formation of $\beta$-sheet rich core composed of several $\beta$-strands ${ }^{174,175}$ and, moreover, several structural models of the fibril organization have been proposed ${ }^{174,175,179,181,182}$. For instance, Tuttle et al. ${ }^{182}$ have suggested that the core of pathogenic $\alpha$-synuclein fibrils has a $\beta$-serpentine arrangement with a Greekkey $\beta$-sheet topology, in which the residues from 44 to 97 are involved.

A

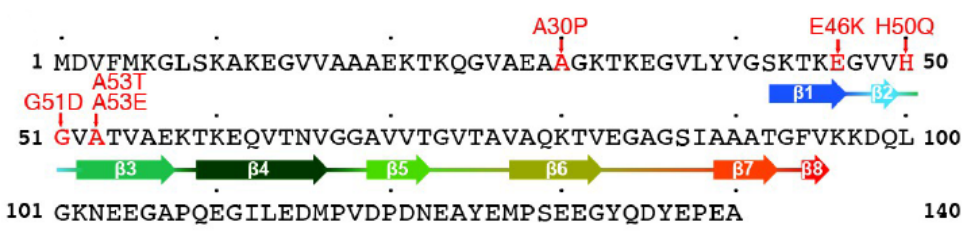

B

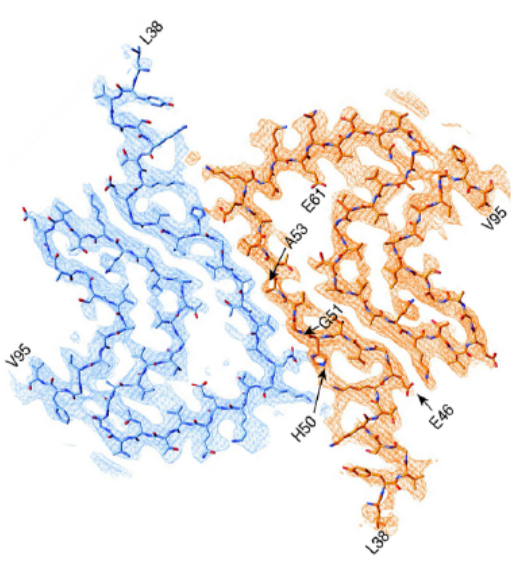

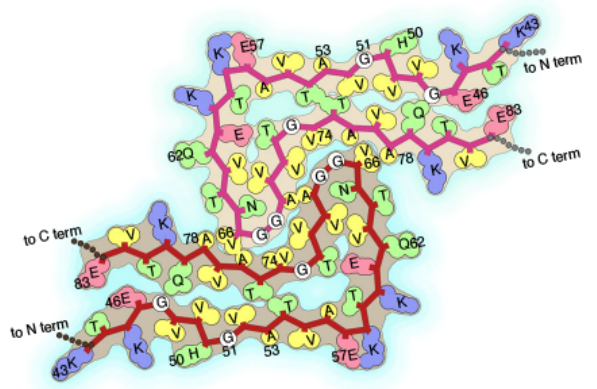

Polymorph 1

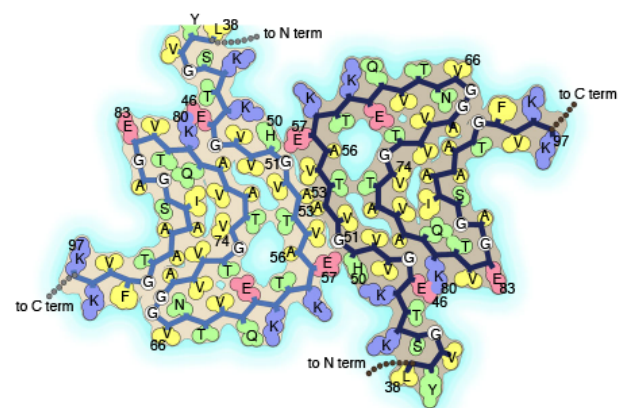

Polymorph 2

Figure 1.21. Structure of $\alpha$-synuclein fibrils. (A) Sequence of $\alpha$-synuclein and organization of $\beta$-sheet regions (left). Structural model of 1-121 $\alpha$-synuclein fibrils reported by GuerreroFerreira et al. ${ }^{116}$ (right). (B) Structural model of full-length $\alpha$-synuclein fibrils reported by Li et al. ${ }^{183}$. Figure $\mathbf{A}$ reprinted from Guerrero-Ferreira et al. ${ }^{116}$ licensed under CC BY 4.0, copyright (C) (2018). Figure B adapted from Li et al. ${ }^{183}$ licensed under CC BY 4.0, copyright (C) (2018).

Recently, two cryo-EM groups have published near-atomic structures of several $\alpha$ synuclein fibril polymorphs ${ }^{116,183}$. In 2018 , the structure of cytotoxic $\alpha$-synuclein fibrils of Cterminal truncated $\alpha$-synuclein 1-121 was determined by cryo-EM at an overall resolution of $3.4 \AA^{116}$. In this study, $\alpha$-synuclein fibrils were formed by two protofilaments each of $5 \mathrm{~nm}$ in diameter. According to proposed structural model, the core of protofilament is composed of single chain arranged eight in-register parallel $\beta$-strands from residue 41 to 95 (Fig. 1.21). Two months later Li et al ${ }^{183}$. reported the cryo-EM structures of two fibril polymorphs from 
full-length recombinant $\alpha$-synuclein. As shown in Figure 1.21, each protofilament has a conserved kernel consisting of a bent $\beta$-arch motif. Remarkably, two studied fibril polymorphs share the same $\beta$-sheet rich kernel structure ${ }^{183}$. At the same time the polymorphism is determined by different interaction interface between two protofilaments. In case of first polymorph, the inter-protofilament interface includes the residues 47-56, while in the second polymorph the fibril structure is stabilized by interaction of residues $68-78$ (Fig. 1.21). Notably, the structure of 1-121 $\alpha$-synuclein fibrils is almost identical to the structure of polymorph 2 (Fig 1.21). In line with solid state NMR data, both cryo-EM studies have proposed that the polypeptide chain adopts the Greek-key topology. The relevance of the proposed models should still be confirmed by structural investigations of the human derived fibrillar aggregates.

\subsubsection{2. $\alpha$-Synuclein oligomers: generation, structure and properties}

As already discussed above, $\alpha$-synuclein readily aggregates under various conditions following multiple pathways. In addition to mature amyloid fibrils, $\alpha$-synuclein oligomeric species have been observed in vitro and in vivo. It has been demonstrated that incubation of monomeric and fibrillar $\alpha$-synuclein under certain conditions in vitro could yield a variety of end state products, including soluble or insoluble amorphous aggregates and different soluble oligomeric species of diverse morphology, structure and properties (Fig 1.15 B, Table 1.4). In this context, it's very likely that $\alpha$-synuclein misfolding could occur through multiple pathways, in which most of intermediates are in dynamic and complex equilibrium with each other (Fig 1.15 B). Despite ongoing discussion and limited information regarding $\alpha$-synuclein misfolding in vivo, increasing evidence implicates $\alpha$-synuclein oligomers as a main pathological element. Currently, the structural and functional investigation of oligomeric species is an extremely challenging task due to the inherent multiplicity of the $\alpha$-synuclein aggregation process depending on conditions (protein concentration and modification, temperature, mechanical impact, interaction partners, buffer conditions and so on) and, furthermore, instability, transient existence, variability and heterogeneity of oligomeric structures itself. However, much effort was put into producing and elucidating the structure and properties of $\alpha$-synuclein oligomers, the data is summarized in the Table 1.4. 
Table 1.4. $\alpha$-Synuclein oligomers: Preparation, structure and properties

\begin{tabular}{|c|c|c|c|c|c|c|}
\hline 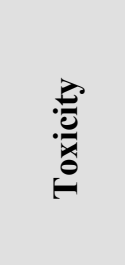 & 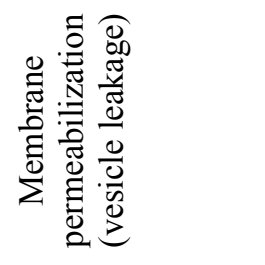 & 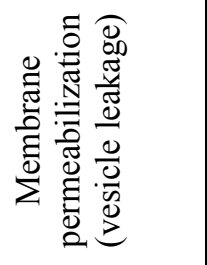 & & 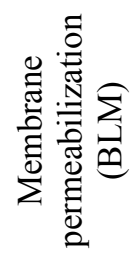 & & 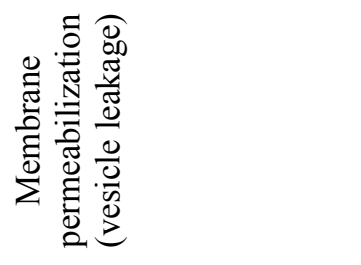 \\
\hline 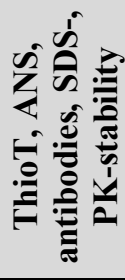 & 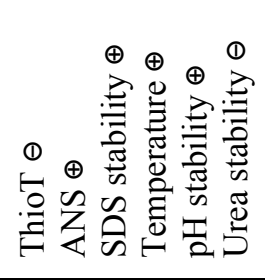 & 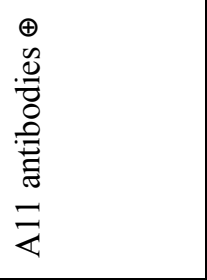 & 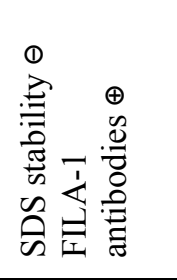 & 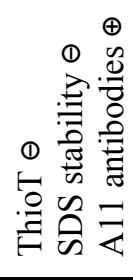 & $\begin{array}{l}\stackrel{1}{1} \\
\stackrel{0}{\circ} \\
\stackrel{0}{\mid}\end{array}$ & 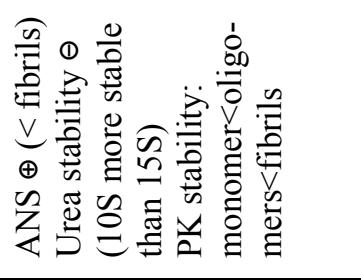 \\
\hline 吾 & $\mathscr{0}$ & & & ธี & & \\
\hline 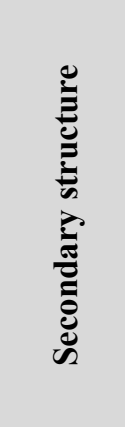 & 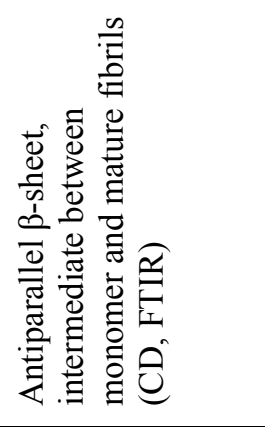 & 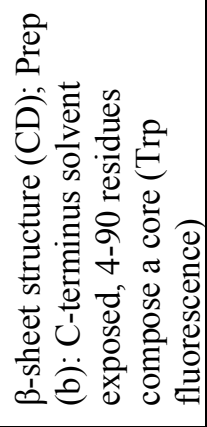 & & 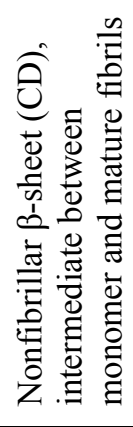 & 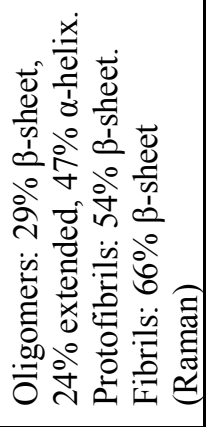 & 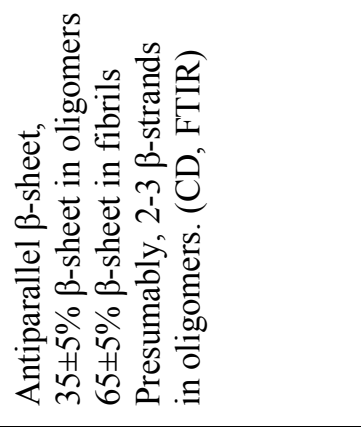 \\
\hline 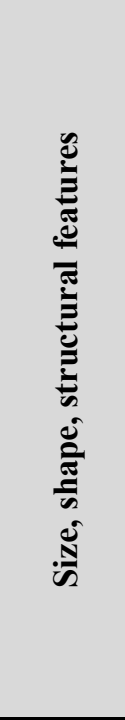 & 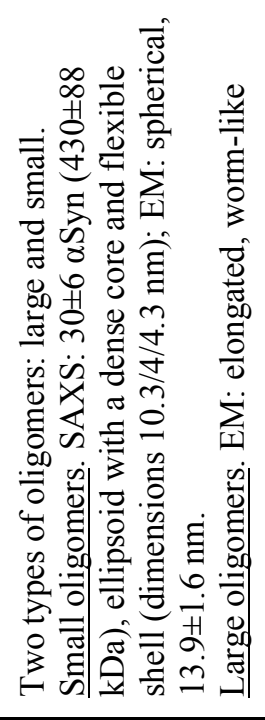 & 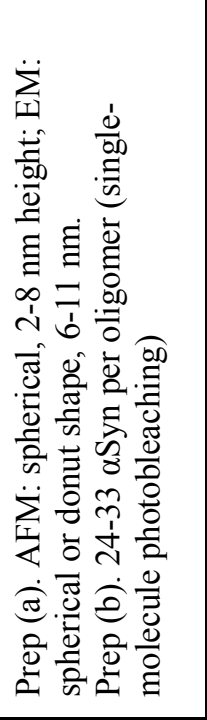 & 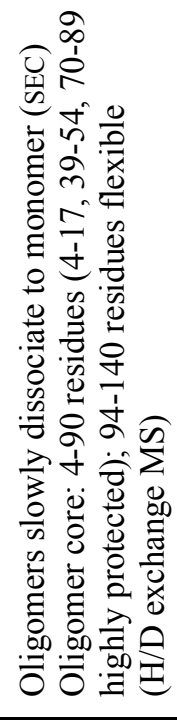 & 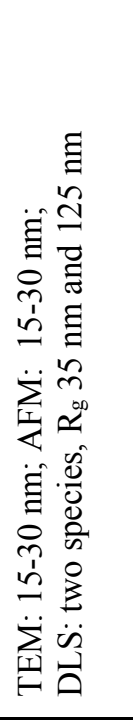 & 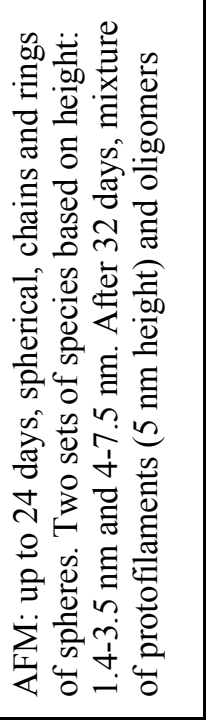 & 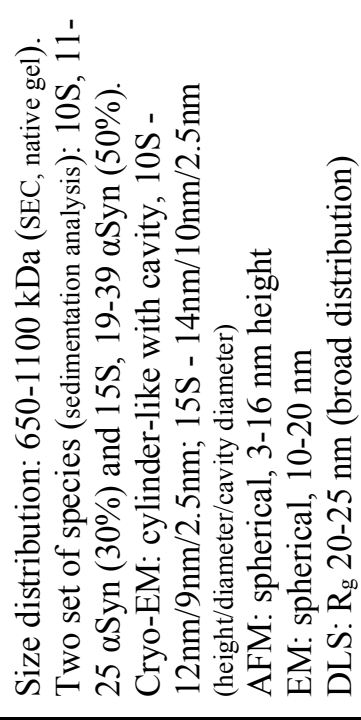 \\
\hline 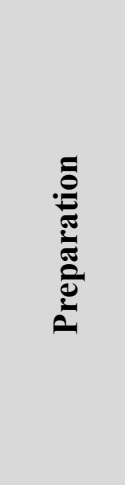 & 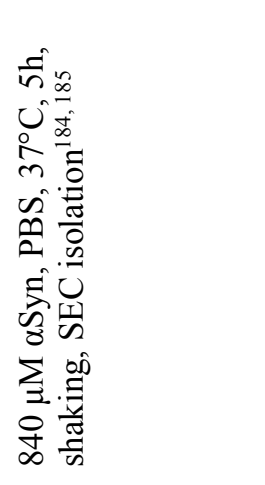 & 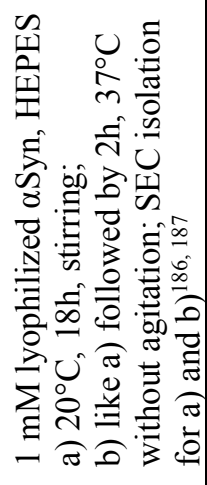 & 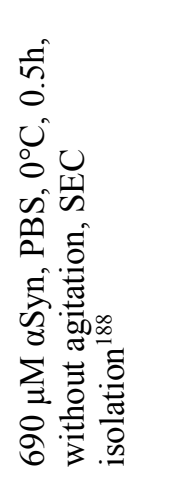 & 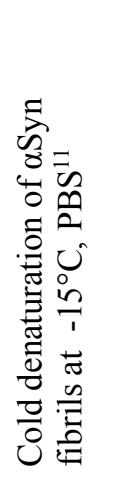 & 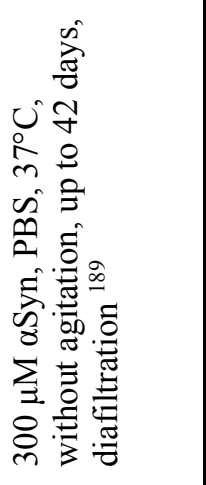 & 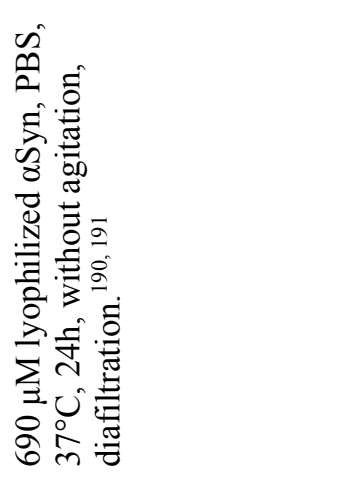 \\
\hline
\end{tabular}


Table 1.4. $\alpha$-Synuclein oligomers: Preparation, structure and properties (continued)

\begin{tabular}{|c|c|c|c|c|c|c|}
\hline 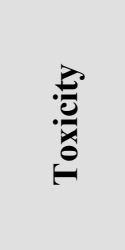 & 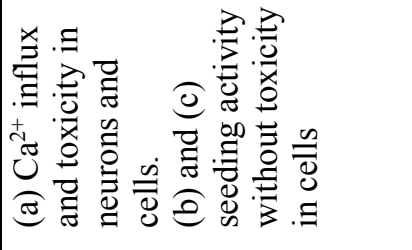 & 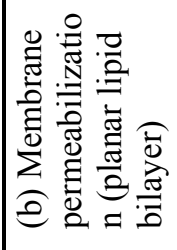 & 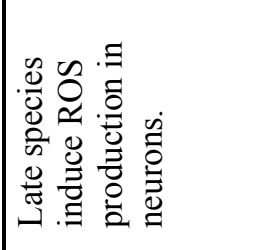 & 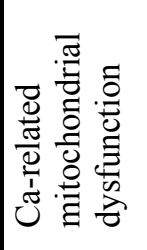 & 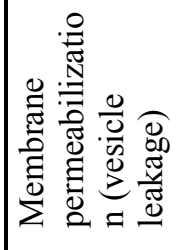 & 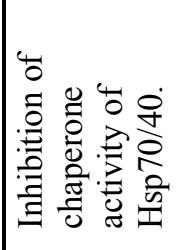 \\
\hline 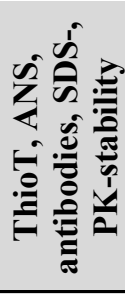 & 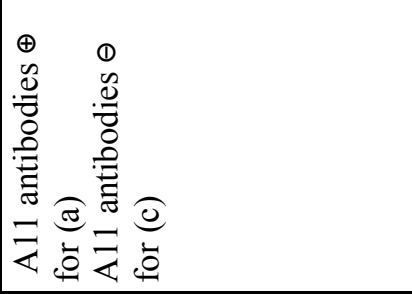 & 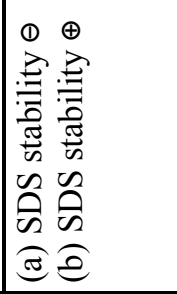 & 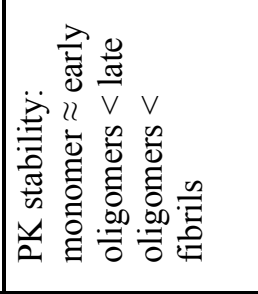 & $\begin{array}{l}0 \\
\stackrel{0}{0} \\
\stackrel{0}{9} \\
\vec{F}\end{array}$ & $\begin{array}{l}0 \\
\stackrel{0}{0} \\
\stackrel{0}{3} \\
\qquad\end{array}$ & 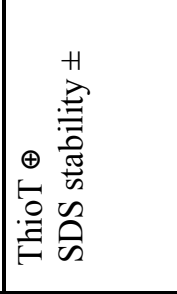 \\
\hline 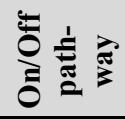 & $\tilde{\sigma}$ & ธี & & $\tilde{0}$ & ธี & 㫕 \\
\hline 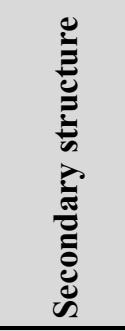 & & & 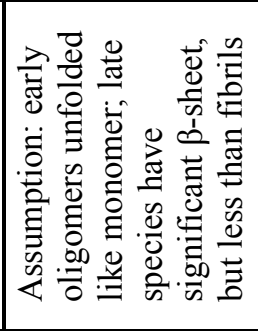 & & & 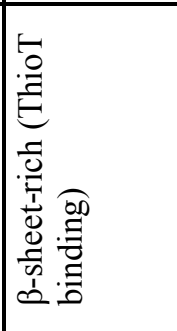 \\
\hline 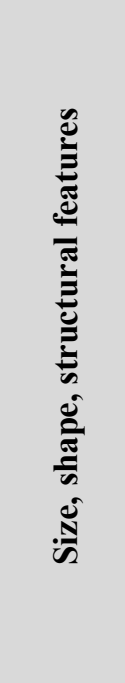 & 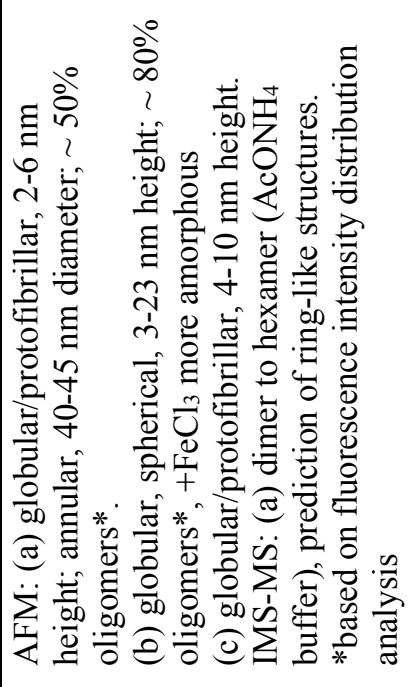 & 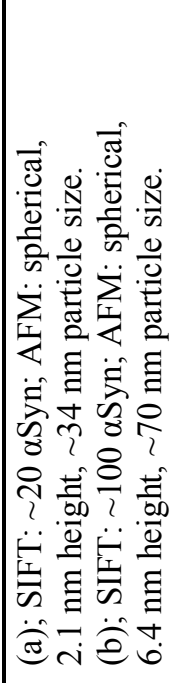 & 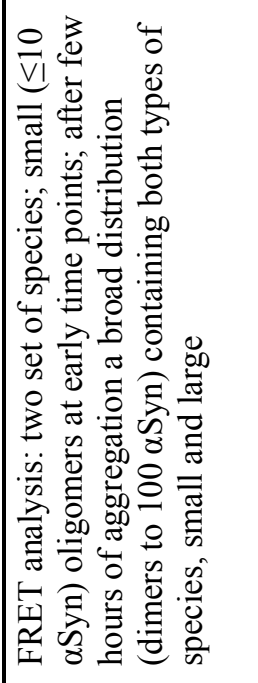 & 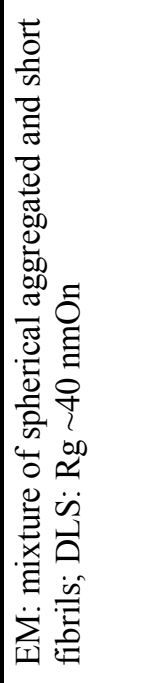 & 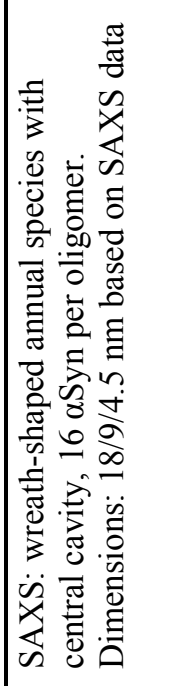 & 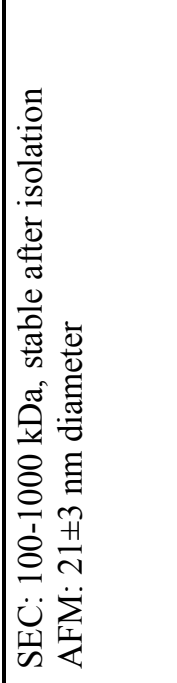 \\
\hline 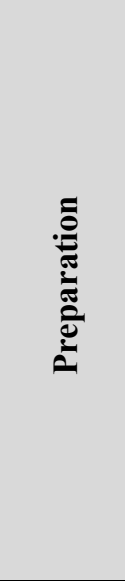 & 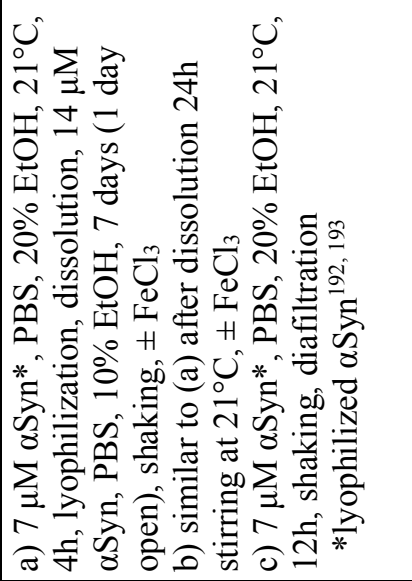 & 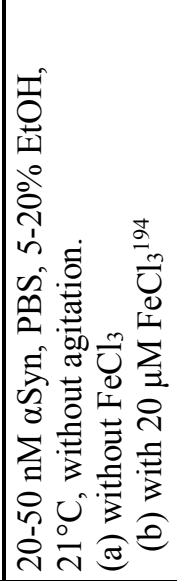 & 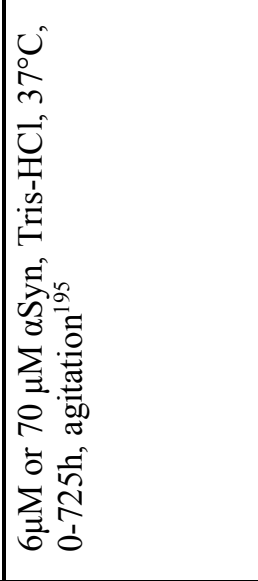 & 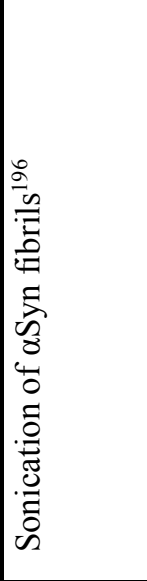 & 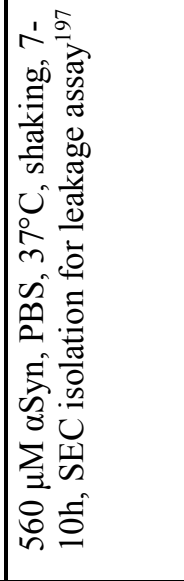 & 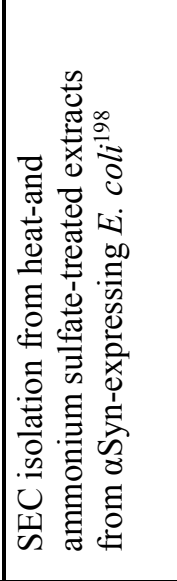 \\
\hline
\end{tabular}


Table 1.4. $\alpha$-Synuclein oligomers: Preparation, structure and properties (continued)

\begin{tabular}{|c|c|c|c|c|}
\hline 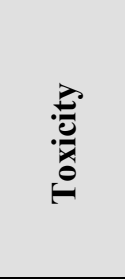 & \multirow{5}{*}{ 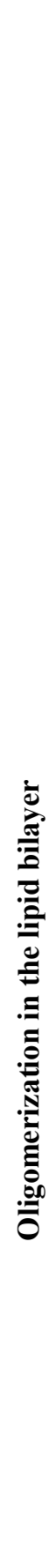 } & 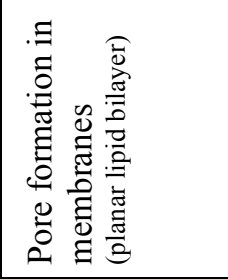 & 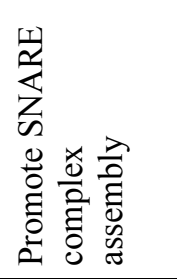 & \\
\hline 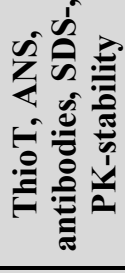 & & 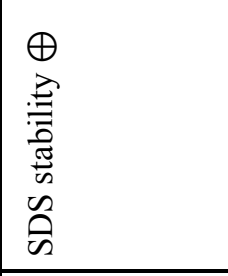 & 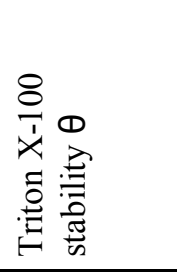 & 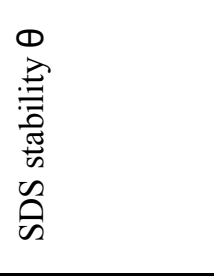 \\
\hline 吾光 & & & & \\
\hline 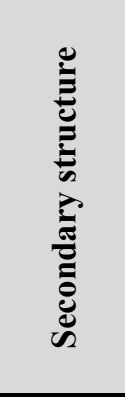 & & & 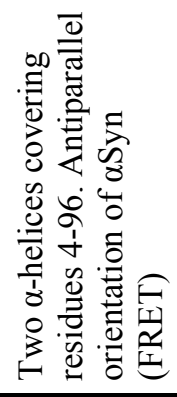 & 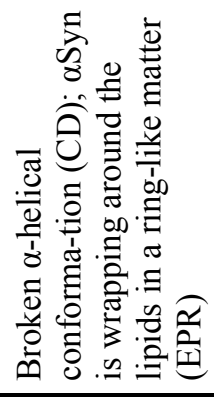 \\
\hline 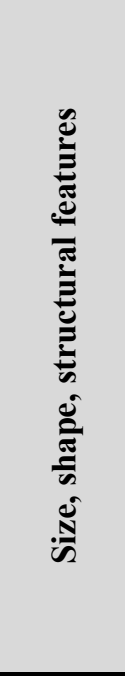 & & 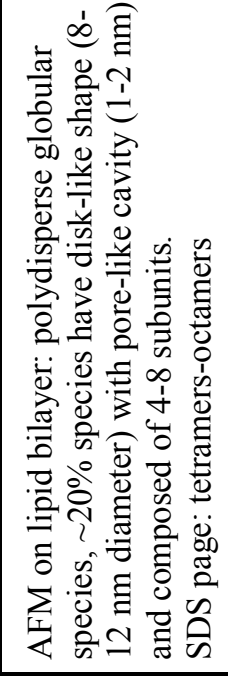 & 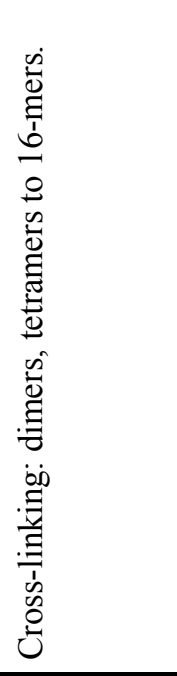 & 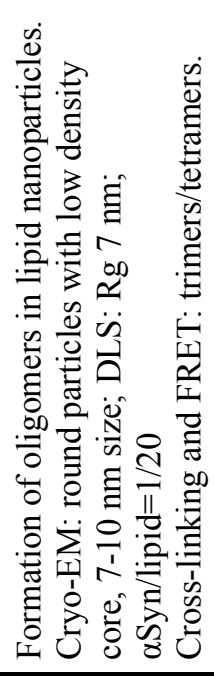 \\
\hline 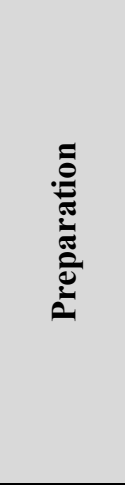 & & 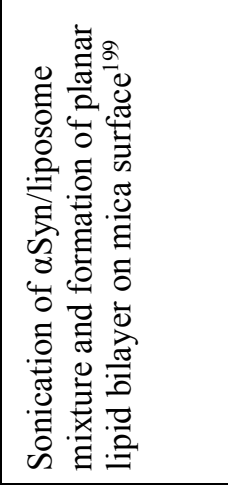 & 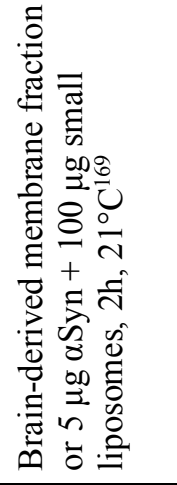 & 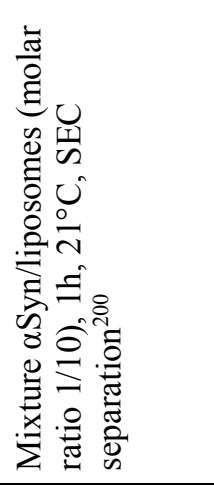 \\
\hline
\end{tabular}


Collectively, $\alpha$-synuclein oligomers may be composed from 2-4 protein molecules to up to 100. The size of aggregates varies from 6-30 nm in EM and 2-20 nm height/10-50 nm diameter in AFM. The morphology of species is also very diverse, including spherical, globular, amorphous, worm-, pore-, cylinder-like species, chains and ring of spherical subunits, elongated protofibrils and others. Based on CD, Raman and FTIR measurements, the majority of $\alpha$-synuclein oligomers adopt $\beta$-sheet-rich (antiparallel) conformations that are intermediate between monomer and mature fibrils, on the other hand, presumably physiological and functional $\alpha$-helical oligomers have been detected on lipid bilayer. Several types of $\alpha$-synuclein multimeric structures, especially so-called off-pathway aggregates, that are not seeding fibrillization of monomeric protein, show relatively good stability during isolation, storage and detergent treatment. However, the biological relevance of such offpathway structures is unclear. For further structural and biochemical characterization the following approaches are frequently applied: immunoblotting with conformation or epitopespecific antibodies, DLS, SAXS, fluoresce-based spectroscopy, solution and solid state NMR, MS, cross-linking, EPR and others. To date, the high-resolution structures of $\alpha$-synuclein oligomers are still missing and, therefore, remain an important goal that could potentially provide us better understanding of toxicity mechanisms as well as a base for drug design.

\subsubsection{Toxic mechanisms of $\alpha$-synuclein: Introduction}

It is increasingly accepted that $\alpha$-synuclein misfolding probably comprises a central cascade leading to neurodegeneration and cytotoxicity, which in turn are induced by prime suspects - oligomeric $\alpha$-synuclein species. Multiple studies have focused on $\alpha$-synuclein toxicity in various in vitro and in vivo systems with diverse experimental approaches, including $\alpha$-synuclein overexpression, application of oligomers generated from recombinant protein, design and expression of specific $\alpha$-synuclein mutants, detection and characterization of $\alpha$-synuclein misfolded species formed by endogenous protein and others ${ }^{2,127,201}$. Despite a number of technical and fundamental challenges related to $\alpha$-synuclein oligomers generation, detection, characterization, isolation and so on, current research findings point towards a hypothesis that $\alpha$-synuclein species induce the toxicity through a variety of possible mechanisms (Fig. 1.22) $^{2}$. Although the exact mechanism(s) of oligomer toxicity is still under debate, there are little evidences of deleterious effects that are associated with monomeric protein alone. Also the formation of fibrillar $\alpha$-synuclein aggregates is currently considered as a cellular defensive pathway "to trap" the potentially toxic soluble oligomeric species into insoluble Lewy body like intracellular inclusions ${ }^{2,127,201}$. On the other hand, it has been 
proposed that amyloid fibrils are in any case harmful and could contribute to cellular toxicity as a passive provider of monomeric and oligomeric species due to the slow dissociation and, secondly, as an agent involved in a prion-like cell-to-cell transmission triggering $\alpha$-synuclein misfolding in the healthy cells, so called seeding activity ${ }^{2,201}$. Due to the strong momentum of "toxic oligomers" hypothesis, I give an overview in this chapter over the discovered pathological pathways that are linked to $\alpha$-synuclein oligomerization, including data from several $\alpha$-synuclein overexpression models, in which protein misfolding has not been directly evaluated, although the formation of aggregated species is reasonable to assume.

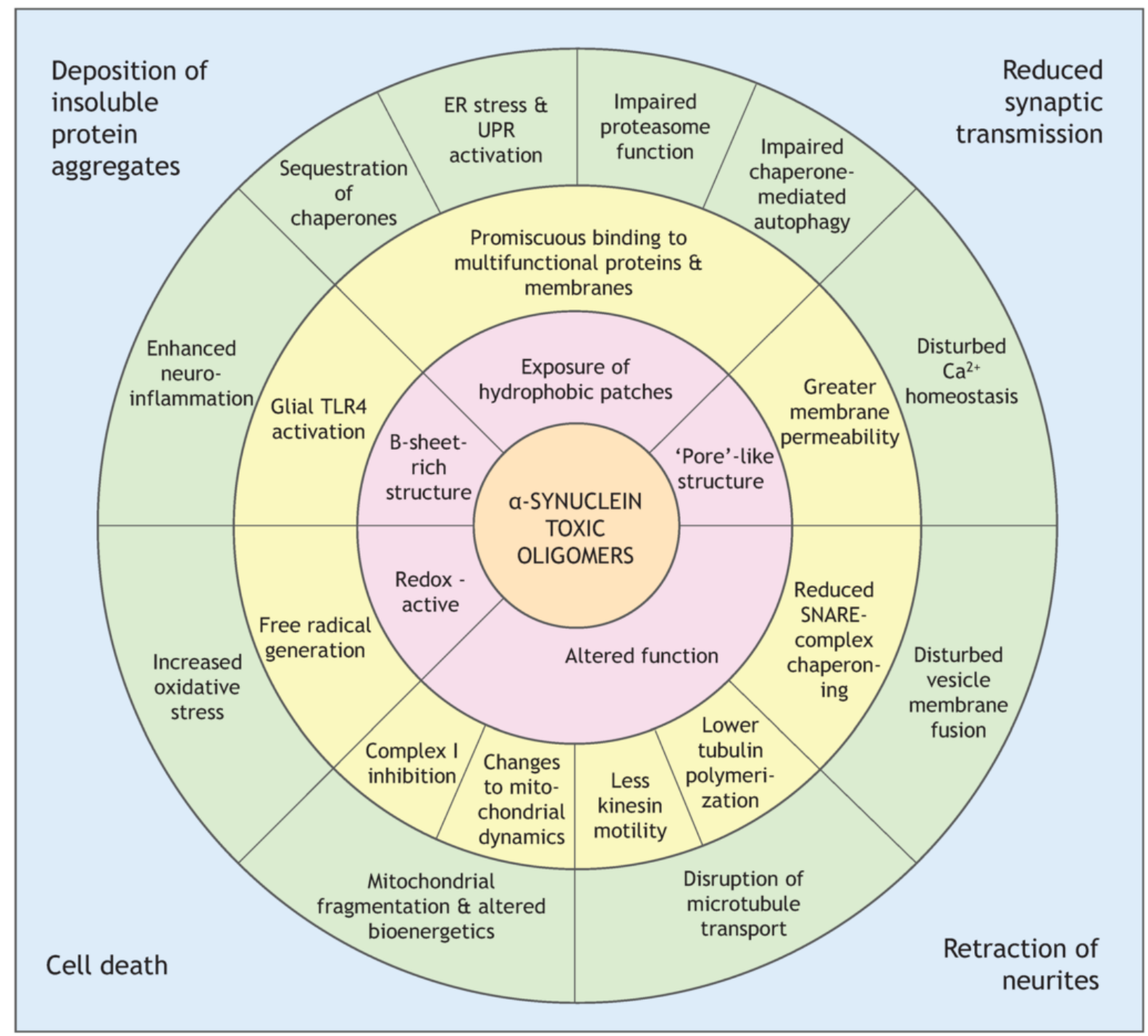

Figure 1.22. Putative toxicity pathways induced by misfolded $\alpha$-synuclein. Figure adapted from Roberts and Brown ${ }^{2}$ licensed under CC BY 4.0, copyright (C) (2015). 


\subsubsection{Toxic mechanisms of $\alpha$-synuclein: Membrane permeability}

Among all putative toxicity mechanisms, which have been proposed in conjunction with $\alpha$-synuclein misfolding, a permeabilization of the lipid bilayer by oligomeric $\alpha$-synuclein was one of the first ${ }^{202,203}$. To date, numerous studies have shown that application of various exogenous $\alpha$-synuclein oligomers to artificial membranes (liposomes or flat lipid bilayers) or to the living cells causes an abnormal flux of small molecules or ions across the membranes ${ }^{6}$, 11, 192-194, 199, 204, 205. Potentially, the permeabilization of plasma/organelle membranes or cytoplasmic vesicles can lead to severe disruption of the cell homeostasis, failure of many crucial cellular processes, release of harmful molecules (ROS) and eventually to cell death.

In vitro, $\alpha$-synuclein oligomers generated from recombinant protein exhibit a strong binding to diverse phospholipid bilayers, including those that mimic natural membranes ${ }^{186,202 \text {, }}$ 206, 207. Despite the relatively unselective binding to lipids, significant oligomer-induced permeabilization is predominantly observed in case of the highly negatively charged membranes ${ }^{186,206}$. For instance, the mixing of liposomes with prefibrillar $\alpha$-synuclein, so called vesicle leakage assay, triggers a fast, concentration-dependent permeabilization of vesicles as represented by influx or efflux of molecules across membrane. The deleterious effects of oligomeric $\alpha$-synuclein on membrane integrity have been also registered in electrophysiological studies ${ }^{6,11}$. Thus, single-channel electrophysiology measurements have demonstrated a discrete stepwise increase in bilayer conductance and also events of channel opening and closing upon addition of oligomerized $\alpha$-synuclein. Importantly, monomeric and fibrillar $\alpha$-synuclein might also cause a membrane leakage, but much higher concentrations are required ${ }^{186,202}$. As an example, following preps, $0,04 \mu \mathrm{M}$ oligomers $/ 1,8 \mu \mathrm{M}$ fibrils $/ 5 \mu \mathrm{M}$ monomer (all concentrations is calculated based on monomeric $\alpha$-synuclein), produce the similar leakage of POPG LUVs ${ }^{186}$.

Addressing the underlying molecular mechanisms of oligomer-induced membrane permeabilization, two possible scenarios have been proposed based on experimental data ${ }^{6,199}$, 205. First, $\alpha$-synuclein oligomers upon binding to membrane form distinct transmembrane pores or, in other words, pathological membrane channels ${ }^{6,}$ 199, 203. The observations supporting this hypothesis: i) discrete step-like changes of bilayer conductance; ii) size selectivity for molecules passing across membrane; iii) in cryo-EM and AFM, ion-channellike structures resembling pore-forming bacterial toxins; iv) pore-like annular complex with central channel-like cavity (low resolution structure from SAXS). According to the alternative hypothesis, the interactions of $\alpha$-synuclein oligomers with the membranes alter the structure, composition and properties of the lipid bilayer and cause the leakage due to an impairment of 
membrane integrity ${ }^{186,205,206}$. In support of the non-channel mechanism, reports, mainly from Subramaniam's lab, are consistently showing that $\alpha$-synuclein oligomers cause the structural alteration of membranes like a thinning on lipid bilayer, lipid packing defects, increased lipid flip-flop. Moreover, the membrane permeabilization is significantly reduced in case of wellpacked or cholesterol-stabilized lipid bilayers ${ }^{186}$. In this context, the experimental findings, including the strong correlation of leakage and bilayer physical properties, poor reproducibility of electrophysiological studies and formation of well-defined pores from diverse populations of oligomers, argue in favor of disruption mechanism. Since these two hypotheses are still under debate, additional experimental data is required to come up with a detailed mechanism.

The evidence of membrane permeabilization by $\alpha$-synuclein in vivo is limited. In several overexpression-based cellular models, Feng et a ${ }^{204}$ have observed a misfolding of $\alpha-$ synuclein in conjunction with the cytotoxicity and an increased membrane conductance reminiscent of the leak channels. Of note, the detected channels are non-selective and prodromal to the cell death. In the other studies, the addition of pore-forming $\alpha$-synuclein oligomeric species to SH-SY5Y elicits a fast influx of extracellular $\mathrm{Ca}^{2+}$ inside the cells and eventual cytotoxicity ${ }^{192,193}$. Additionally, $\mathrm{Ca}^{2+}$ flux or cytochrome $\mathrm{C}$ release across the mitochondrial membrane has been shown for isolated organelles treated with prefibrillar $\alpha-$ synuclein $^{208}$.

\subsubsection{Toxic mechanisms of $\alpha$-synuclein: Mitochondrial dysfunction}

General aspects of mitochondrial dysfunction in Parkinson's disease are discussed above in the chapter 1.3.3, while here the results addressing the link between $\alpha$-synuclein misfolding and mitochondria are summarized. Overall, the mitochondrial malfunction leads to depleted energy supply, which is especially important for neuronal cells, dysregulation of cellular homeostasis and activation of various death pathways. Main arguments supporting the link between mitochondrial impairment and $\alpha$-synuclein rely on following observations ${ }^{2}$, 55, 105, 201, 209, 210: (i) $\alpha$-synuclein accumulation in mitochondria of neurons derived from substantia nigra and striatum of affected human brains; (ii) interaction of $\alpha$-synuclein with mitochondrial membrane in cellular and animal models; (iii) prominent mitochondrial abnormalities in Parkinson's disease patients and $\alpha$-synuclein-based models, including decreased complex I activity, morphological changes, reduced mitochondrial membrane potential, abnormal $\mathrm{Ca}^{2+}$ homeostasis and other. Thus, $\alpha$-synuclein, being overexpressed in mice, C. elegans, cellular and neuronal cultures, has been shown to induce a failure of 
mitochondrial function(s) frequently followed by the cell death ${ }^{55,209-211}$. For instance, analysis of SH-SY5Y cells expressing $\alpha$-synuclein revealed the formation of small $\alpha$-synuclein

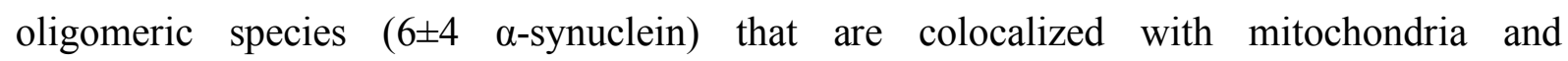
lysosomes $^{211}$. Moreover, the mitochondria have abnormal morphology, smaller size and increased fragmentation. In complementary experiments with wild type $\alpha$-synuclein and A53T mutant in PC12 cells and cultured neurons, massive mitochondrial pathology, which is characterized by reduced size and total number of mitochondria and pronounced cellular toxicity, has been observed ${ }^{209}$. Despite the fact that $\alpha$-synuclein has been found in mitochondria, an elevated autophagic removal of mitochondria has been proposed as a main mechanism of cellular toxicity. The results from $\alpha$-synuclein-based C. elegans model are in line with the cellular data, namely $\alpha$-synuclein binding to mitochondrial outer membrane and eventual mitochondrial fragmentation ${ }^{210}$. Based on in vitro data, showing the reduced fusion of lipid vesicles in presence of $\alpha$-synuclein, Kamp et al ${ }^{210}$ have proposed that fragmentation of mitochondria in worms is mainly attributed to suppression of mitochondrial fusion by bound $\alpha$-synuclein. In contrast, the binding of $\alpha$-synuclein to mitochondria has not been detected in yeast; nevertheless the strong defects in mitochondrial function are prominent in this model as well ${ }^{210}$.

Since the evaluation of the $\alpha$-synuclein aggregation status in the overexpression models is still extremely challenging and technically difficult, several studies have characterized the effect of exogenous prefibrillar $\alpha$-synuclein generated from the recombinant protein in vitro. Thus, recent work has shown that incubation of isolated mitochondria with recombinant $\alpha$-synuclein oligomers results in severe membrane perturbation and cytochrome C release, presumably by the pore-like mechanism ${ }^{208}$. Luth et al ${ }^{196}$ have also confirmed the deleterious $\mathrm{Ca}^{2+}$-mediated effects of oligomeric species on isolated mitochondria, namely abnormal swelling, inhibition of complex I activity, depolarization, $\mathrm{Ca}^{2+}$ disbalance and cytochrome $\mathrm{C}$ release. However, it has been hypothesized that the interactions of misfolded $\alpha-$ synuclein with mitochondrial membrane trigger the opening of permeability transition pore. Of note, the mitochondrial permeability transition pore is a large channel that is normally closed, but could be opened in response to $\mathrm{Ca}^{2+}$ accumulation inside the organelle, moreover, opening leads to failure of mitochondrial function and oxidative stress ${ }^{55}$.

Collectively, these findings support the hypothesis that misfolding of $\alpha$-synuclein can induce derangements in mitochondrial function(s) leading to activation of cellular death pathways and eventually cell death. Furthermore, the oxidative stress that is one of the 
consequences of mitochondrial failure can further promote $\alpha$-synuclein aggregation and thus close a pathological feedback loop.

\subsubsection{Toxic mechanisms of $\alpha$-synuclein: Impairment of degradation pathways}

Certain aspects of the cellular role of ubiquitin-proteasomal system and its potential contribution in Parkinson's disease are recounted in the chapter 1.3.2. Here, the interplay between various degradation pathways and $\alpha$-synuclein is briefly described.

From cellular biology textbooks, the degradation system that is represented by two main pathways, namely ubiquitin-proteasomal system (UPS) and autophagy/lysosomal system (ALS), is involved in clearance of unwanted or harmful proteins and intracellular components, including entire organelles. The dysregulation or impairment of clearance machinery lead to proteolytic stress, accumulation of the toxic species, failure of diverse cellular processes, activation of the death pathways and eventually cell death. UPS, which is mainly involved in degradation of soluble proteins, performs its function by several multisubunit complexes, termed the proteasomes (Fig. 1.5). Among various cellular proteasomes, 26S ubiquitin-independent, 20S and 26S ubiquitin-independent proteasomes are the best characterized. In case of autophagy/lysosomal system (ALS), cellular organelles, known as lysosomes, are the key degradation factories that are able to disassemble all types of intracellular and extracellular materials, including insoluble protein deposits ${ }^{212}$. Depending on a substrate delivery mechanism to the lysosome, ALS is subdivided into heterophagy, in which extracellular or membrane-bound components are transported to lysosome with implication of endocytosis (membrane-associated targets) or phagocytosis (extracellular targets); macroautophagy (often equivalent to autophagy) that is based on engulfment of intracellular soluble/insoluble substrates or entire organelles and formation of autophagosomes; and finally chaperone-mediated autophagy (CMA) that targets soluble proteins containing KFERQ-like recognition motif (Fig. 1.23) 3 ,212.

Several lines of evidence supporting dysfunction of degradation systems come from analysis of both patient-derived material and various cellular or animal models of Parkinson's disease $^{3,105}$. Much data points to the possibility that $\alpha$-synuclein and especially aggregated forms substantially contribute to impairment of almost all degradation pathways. It has been shown that $\alpha$-synuclein is a substrate for UPS as well as for ALS ${ }^{3,105,212}$. According to published data, monomeric, cytosolic $\alpha$-synuclein undergoes the cleavage by UPS and CMA, while membrane-bound $\alpha$-synuclein is transported to lysosomes via endocytosis ${ }^{3,105,212}$. In case of aggregated and insoluble $\alpha$-synuclein, the autophagy is considered as a major 


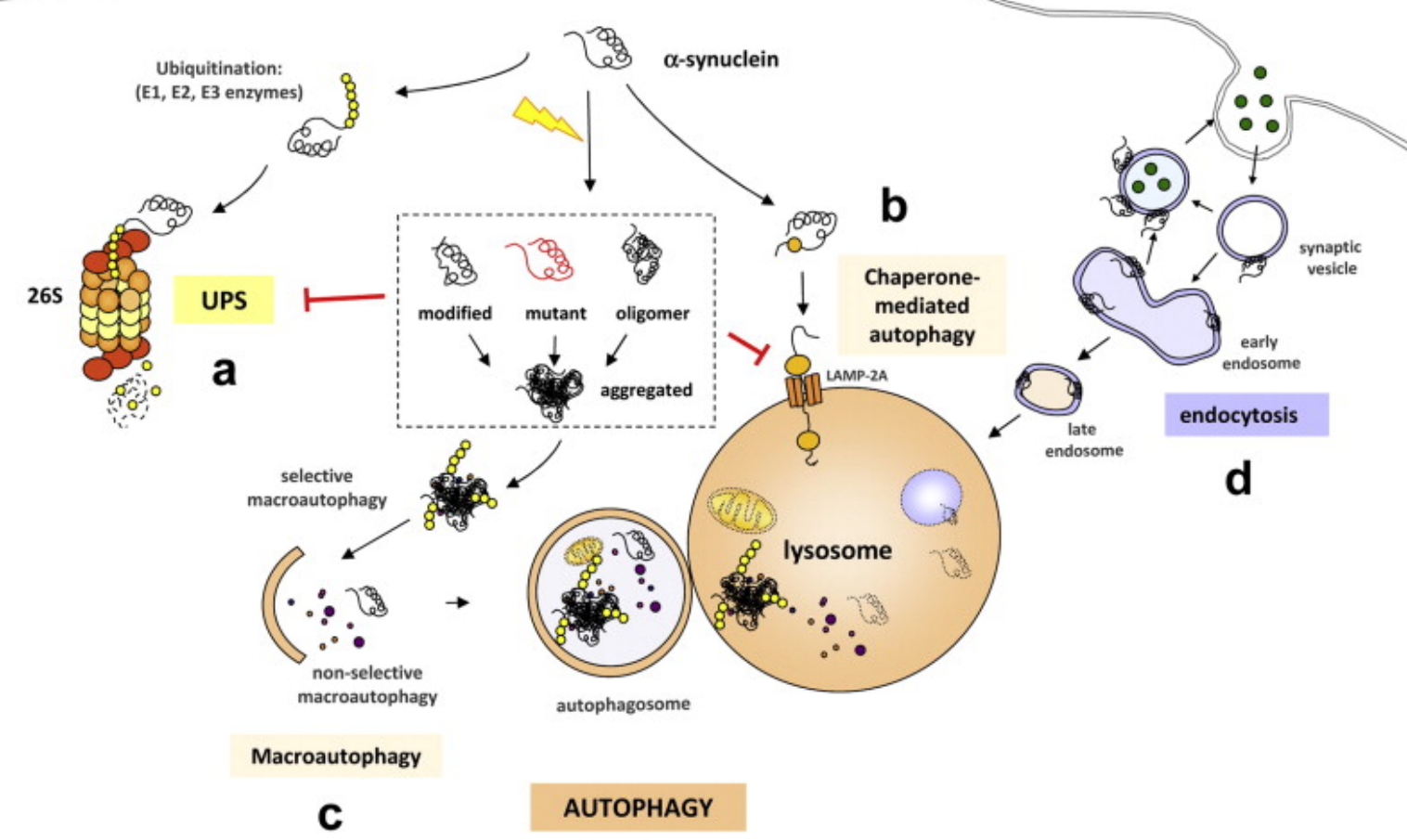

Figure 1.23. Degradation of monomeric and misfolded $\alpha$-synuclein. Figure reprinted from Experimental Neurology: Martinez-Vicente and Vila ${ }^{3}$, copyright (c) (2013) with permission from Elsevier.

clearance mechanism. Remarkably, the post-translational modifications and the cellular localization of $\alpha$-synuclein might also be involved in regulation its turnover ${ }^{213}$.

Studies in $\alpha$-synuclein-based models have aimed at elucidating details of UPS dysfunction and potential pathological pathways. Thus, the evaluation of aged transgenic mice has uncovered the UPS malfunction as evident by reduced 20S-mediated proteolytic activity and declining levels of both $19 \mathrm{~S}$ subunit and polyubiquitinated proteins ${ }^{214}$. Similar phenotypes have been shown in cellular models overexpressing $\alpha$-synuclein, specifically, suppression of the UPS enzymatic activity, accumulation of misfolded $\alpha$-synuclein and structural defects in the proteasomes ${ }^{215-217}$. Furthermore, the experiments are consistent with the crucial role of oligomeric species in UPS dysfunction ${ }^{216,217}$. Thus, it has been demonstrated that in mice and in cells $\alpha$-synuclein oligomers are targeted to ubiquitindependent $26 \mathrm{~S}$ proteasomal system by means of their aberrant conformation. However, these oligomeric species turn to be resistant to the clearance and instead form a complex with the $26 \mathrm{~S}$ proteasome by binding to $19 \mathrm{~S}$ cap $^{217}$. Importantly, these interactions result in strong inhibition of $26 \mathrm{~S}$ proteasomal activity. In case of monomeric $\alpha$-synuclein and $20 \mathrm{~S}$ proteasomes, no alterations have been observed. The deleterious impact of $\alpha$-synuclein aggregation on UPS has been also shown in cells and neurons exposed to the seeding amounts 
of amyloid fibrils ${ }^{215}$. These experiments have provided strong evidence of UPS and ALS dysfunction, impaired clearance of $\alpha$-synuclein inclusion, interactions of aggregated $\alpha$ synuclein with $20 \mathrm{~S}$ proteasome and eventual cellular toxicity. Although the oligomerization pathway has not been addressed in this study, it seems plausible that $\alpha$-synuclein oligomers might also contribute to the observed pathological changes.

Evidence, that various forms of $\alpha$-synuclein, being substrates for ALS, could also interfere with certain lysosomal pathways, is accumulated from in vitro and in vivo studies ${ }^{3}$, 201. Experiments, acquired under $\alpha$-synuclein overexpression conditions, have shown an activation of $\mathrm{ALS}^{209,211,215}$. Insoluble and oligomeric $\alpha$-synuclein inhibits of autophagosomes clearance in cellular models and in vitro ${ }^{3,215}$. The reduced ALS activity, in relation with $\alpha-$ synuclein oligomers, is supported by fluorescence microscopy data demonstrating accumulation of multimeric $\alpha$-synuclein on lysosomal membranes and sequestration inside lysosomes $^{211,213}$. Beside macroautophagy, there are also indications that the CMA pathway is impaired by $\alpha$-synuclein aggregation and post-translational modification ${ }^{213}$. For instance, it has been found that S129E $\alpha$-synuclein (phosphorylation mimicking mutant) after recognition by cytosolic chaperons is transported to the lysosomes where it binds to lysosomal-associated membrane protein A2 (LAMP 2A), but translocation to lysosomes, the last step of CMA, is inefficient. The similar issues with reduced translocation are proposed for dopamine-modified $\alpha$-synuclein and oligomeric forms of nitrated $\alpha$-synuclein. Moreover, the strong association of abnormal $\alpha$-synuclein with LAMP 2A is supposed to block the function of the lysosomes ${ }^{215}$.

Viewed together, the various forms of $\alpha$-synuclein might cause the dysfunction of UPS and CMA pathways that leads to proteolytic stress and eventual activation of the macroautophagy. If abnormal $\alpha$-synuclein also disrupts the macroautophagy and lysosomal function, there is a general failure of all degradation systems in cells followed by further accumulation of toxic species and finally cell death. Importantly, current research points towards the oligomeric species as the prime suspects interfering with UPS and ALS.

\subsubsection{Toxic mechanisms of $\alpha$-synuclein: ER stress}

Several observations have highlighted $\alpha$-synuclein misfolding as a factor potentially contributing to a dysfunction of endoplasmic reticulum (ER) and ER-Golgi trafficking. Postmortem brain analysis of patients with Parkinson's disease revealed accumulation of oligomeric $\alpha$-synuclein in ER and evidence of ER stress represented by upregulation of ER stress factors (PERK phosphorylation, increased ER chaperons) ${ }^{201,} 218$. Importantly, the protein accumulation in ER could activate the unfolded protein response, whereas chronic ER 
stress or prolonged unfolded protein response might lead to ROS generation, mitochondrial dysfunction, abnormal $\mathrm{Ca}^{2+}$ homeostasis, activation of apoptotic pathways and eventually cell death. Another aspect that might contribute to ER pathology is $\alpha$-synuclein-related alterations in vesicle trafficking between ER and Golgi.

To date, several studies in cellular and animal models have established a possible link between $\alpha$-synuclein and ER stress ${ }^{201,218-220}$. For example in cellular and yeast models, the application of misfolded $\alpha$-synuclein or $\alpha$-synuclein expression leads to upregulation of ER stress markers and ER morphological changes ${ }^{2}$, 201. In case of the yeast model, an impairment of ER-Golgi vesicle trafficking is observed in addition to ER stress and, moreover, this phenotype correlates with accumulation of prefibrillar $\alpha$-synuclein oligomers ${ }^{219}$. Results from transgenic mice have also corroborated the notion that $\alpha$-synuclein exerts detrimental effects on $\mathrm{ER}^{218,220}$. Thus, A53T $\alpha$-synuclein transgenic mice model shows an induction of ER stress and unfolded protein response that is accompanied by accumulation of $\alpha$-synuclein oligomers in ER, and neurological defects ${ }^{220}$. Importantly, the buildup of these oligomeric species in ER is age-dependent and correlates with the pathological changes. Structurally, $\alpha$-synuclein oligomers are SDS-stable and interact with ER chaperons, A11 and FILA-1 antibodies. Overall, the accumulation of misfolded $\alpha$-synuclein in ER results in significant ER stress, activation of unfolded protein response and progressive cell death both in animal and cellular models, however, exact toxicity pathways remain unknown.

\subsubsection{Toxic mechanisms of $\alpha$-synuclein: Others}

In addition to the toxicity pathways described above, application of misfolded $\alpha$ synuclein or $\alpha$-synuclein expression have been shown to induce destabilization of cytoskeleton and generation of $\operatorname{ROS}^{2}, 195,201,221$. With respect to the cytoskeleton, microtubule destabilization, reduced tubulin polymerization, inhibition of tau activity have been reported in cellular models based on $\alpha$-synuclein overexpression ${ }^{2,201}$. Evidence of elevated ROS levels comes from a demonstration that application of recombinant $\alpha$-synuclein oligomers to primary neuronal culture triggers rapid mitochondrial-independent cytosolic ROS production in dose-dependent manner ${ }^{195,221}$. Interestingly, recipient cells readily internalize all types of $\alpha$-synuclein species, including monomer, various oligomers and amyloid fibrils. Another possible reason of ROS generation might be associated with activation of microglia. 


\subsection{Medical treatment and clinical trials for Parkinson's disease}

\subsubsection{Introduction}

In contrast to other neurodegenerative diseases, physicians could offer to Parkinson's disease patients a relatively effective symptomatic therapy, which is based mainly on dopamine replacement with levodopa (L-3,4-dihydroxyphenylalanine, L-dopa) or dopamine agonists in combination with adjuvant drugs that improve pharmacokinetic properties of levodopa (L-dopa) (Fig 1.24). However, in most patients, the long-term L-dopa therapy has limitations and side effects including motor and non-motor complications, reduced respond to the drug and others. To date there is no treatment option capable of slowing, preventing or reversing the progressive death of dopaminergic neurons in substantia nigra. Therefore, a development of disease-modifying drugs with neuroprotective and/or neurorestorative properties is an urgent pharmacological challenge. This chapter describes in some details the available treatment for Parkinson's disease as well as a next generation of treatment approaches, also summarizes most important published results of previous clinical trials.<smiles>NC(Cc1ccc(O)c(O)c1)C(=O)O</smiles>

L-DOPA<smiles>NCCc1ccc(O)c(O)c1</smiles>

Dopamine

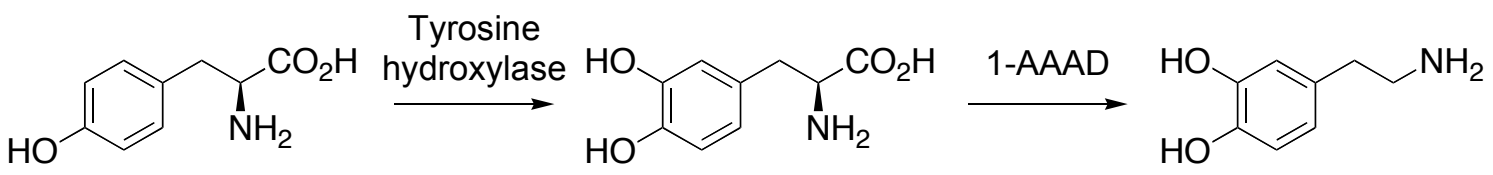

Figure 1.24. L-Dopa and dopamine biosynthesis.

\subsubsection{Levodopa in the treatment of Parkinson's disease}

\subsubsection{Landmarks in the history of levodopa}

The history of L-dopa starts in 1910 when it was first isolated from the bean of Vicia faba by Italian Torquato Torquati followed by a structure determination two years later by Markus Guggenheim. The identification of L-dopa decarboxylase enzyme in 1938 gave an initial insight in the biological role of L-dopa as a possible precursor to biological catecholamines. A number of important discoveries were made in the late 1950s, among them a detection of dopamine in the human brain (1957), an excitatory effect of L-dopa in rodents that was related to increase of dopamine level (1957-1958) and probably most important finding - a discovery of dopamine deficiency in the parkinsonian brain by Hornykiewicz in 
1960. After several clinical trials in the 1960s that demonstrated the efficacy of L-dopa in improving motor disabilities in patients with Parkinson's disease the US Food and Drug administration approved L-dopa as a treatment for Parkinson's disease in 1970. Thereafter, Ldopa remains the gold standard in the symptomatic treatment for Parkinson's disease.

\subsubsection{Dopamine turnover and mechanism of $L$-dopa action}

In the healthy brain, the biosynthesis of endogenous dopamine in substantia nigra starts from L-tyrosine that is converted to L-dopa by the enzyme tyrosine hydroxylase followed by decarboxylation of L-dopa to dopamine catalyzed by aromatic L-amino acid decarboxylase (AAAD) in dopaminergic terminals (Fig 1.8). Newly synthesized dopamine is packed into vesicles for storage and protection against oxidation by monoamino oxidase (MAO-A). Depolarization of nigrostriatal neuron induces the fusion of synaptic vesicles with cell membrane and subsequent release of dopamine into the synaptic cleft. In the synapse dopamine can stimulate postsynaptic dopamine receptors, allowing the signal to propagate. The action of dopamine is terminated by metabolism or by reuptake back into presynaptic neuron via selective dopamine transporter. In Parkinson's disease patients the progressive degeneration of dopaminergic neurons producing dopamine leads to a depletion of dopamine and, consequently, to a dysfunction of the extrapyramidal motor system. The motor dysfunction becomes apparent when the dopamine level in the striatum is reduced by about $70 \%$ to $80 \%$. The main goal of symptomatic treatment is to restore the dopamine levels in the brain in order to facilitate the signal transmission in remaining neurons, thus improving the locomotor performance.

The simplest solution is an application of dopamine, but various pharmacokinetic studies have demonstrated a very poor blood brain barrier permeability of dopamine itself. This problem was overcome by a logical use of L-dopa that could be considered as a dopamine prodrug (prodrug - an inactive or partially active drug that is metabolically changed in the body to an active drug). The current model of L-dopa action involves a number of crucial steps ${ }^{222}$ :

1) Gastrointestinal absorption

2) Passage across blood-brain barrier

3) Neuronal uptake

4) Enzymatic conversion to dopamine

5) Synaptic release of dopamine 
A number of large-scale, long-term clinical trials have demonstrated the efficacy of levodopa to maintain symptomatic control, to reduce mortality and to extend the life span of PD patients. Although levodopa relieves all major symptoms and restores the dopamine levels in the brain, there is no strong evidence from clinical trials (ELLDOPA, FIRST-STEP) supporting disease-modifying or neuroprotective effects of L-dopa treatment in the Parkinson's disease patients ${ }^{223}$.

\subsubsection{Side effects and limitations of L-dopa therapy}

More than 50 years of clinical experience with L-dopa treatment revealed a number of limitations and side effects. Major drawbacks of the therapy are development of levodopaassociated motor fluctuations and dyskinesia; a "wearing off" effect, a short drug half-life, "on-off" fluctuations and freezing.

Thus, approximately $70 \%$ of patients with Parkinson's disease after 6 years of L-dopa treatment show motor complications ${ }^{224}$. Clinical trials clearly establish that motor complications are L-dopa dose-dependent and mainly occur at the later stages of Parkinson's disease. During first years of L-dopa application a long duration response and good symptomatic control are observed and presumably are related to proper dopamine processing, storage and release. In later Parkinson's disease the reduction of the number of dopaminergic neurons leads to impaired dopamine turnover and signaling. For instance, due to the small number of functional dopaminergic neurons the conversion of L-dopa to dopamine is limited and dopamine is stored in non-neuronal cells. All these factors cause the "wearing off" effect and a short, unpredictable and inappropriate response to L-dopa (Fig 1.25) ${ }^{223}$.

The risk of developing L-dopa related dyskinesias is estimated to be $10 \%$ annum and mainly associated with postsynaptic mechanisms. Considering that in the healthy brain dopamine receptors in the striatum are tonically innervated it has been suggested that pulsatile stimulation with high levels of neurotransmitter initiates the downstream dysfunction of basal ganglionic neurons $\mathrm{s}^{225}$. There is also some evidence linking the degree of striatum neurodegeneration with the onset of dyskinesia. In addition, the good correlation between dyskinesia symptoms and increased L-dopa plasma levels highlights the crucial role of levodopa therapy in development of dyskinesia (Fig 1.25) 223 .

Another important limitation of pure L-dopa formulation is the short half-life of Ldopa that is varied from 36 to 96 minutes in chronically treated Parkinson's disease patients $^{222}$. However, several approaches improving efficacy, bioavailability of L-dopa and 
reducing the side effects have been developed, and the most widely applied options are summarized in next chapter.

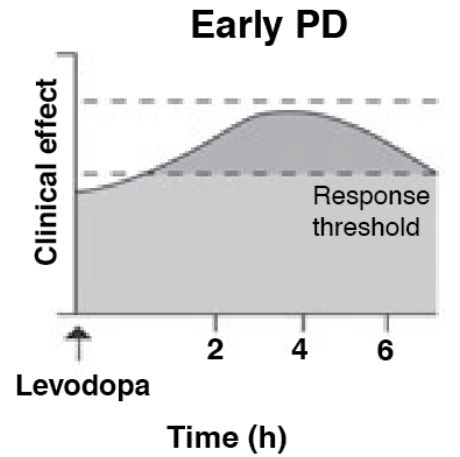

Good symptom control

Smooth, extended duration of target clinical response

Low incidence of dyskinesias

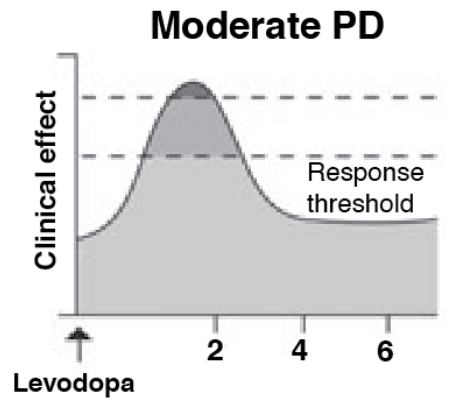

Time (h)

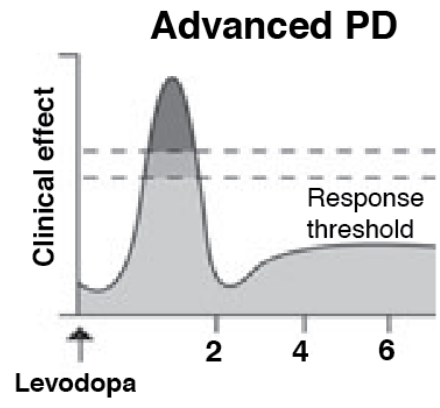

Time (h)

Risk of complications Inadequate symptom control

Diminished duration of target clinical response

Increased incidence of dyskinesias
Short duration of target clinical response

'ON' time is associated with dyskinesias

Figure 1.25. Change in levodopa response over the course of Parkinson's disease. 'ON' time refers to periods of adequate control of PD symptoms in response to medication. Figure adapted from European Journal of Neurology, Schapira et al. ${ }^{223}$ with permission from John Wiley and Sons, copyright (c) (2009).

\subsubsection{Optimizing L-dopa therapy}

To date, several approaches aimed to improve the pharmacological and therapeutic properties of L-dopa therapy have been developed. Keeping in mind the short half-life of Ldopa, many researches concentrated their effort to optimize L-dopa metabolic profile. It has been shown that orally applied L-dopa has the total bioavailability of $35 \%$ and just $1 \%$ of Ldopa can reach the brain $^{226}$. The disadvantageous pharmacokinetic properties are mainly related to the extensive peripheral metabolism (Fig. 1.26) by following enzymes (ranked by importance):

1) Decarboxylases; where: intestine, liver, heart, plasma

2) Catechol-O-methyltransferase (COMT); where: gut, liver

3) Tyrosine aminotransferase

4) Various oxidation, for example by enzyme tyrosinase

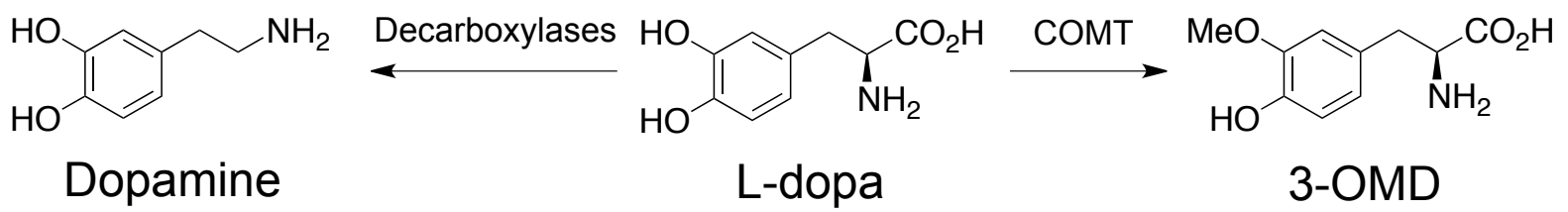

Figure 1.26. Main L-dopa metabolic pathways. 
Thus, experiments in the dogs highlighted the importance of L-dopa metabolism after oral application, in view of the fact that $50 \%$ over half of L-dopa is decarboxylated to dopamine during absorption in the gut $^{227}$. A first significant success in L-dopa optimization belongs to Austrian neurologist Walter Birkmayer, how was the first in 1966 testing combined medication L-dopa with benserazide (Fig. 1.27), an AAAD inhibitor. In pioneering work of Birkmayer, the concomitant administration demonstrated better therapeutic efficacy and tolerability of L-dopa in the patients. Following clinical trials for L-dopa/benserazide oral treatment showed significantly improved levodopa pharmacokinetic profile, namely, prolonged peripheral half-life, 10-fold increased CNS availability, reduction of required Ldopa dose by $60-80 \%$ and related side effects ${ }^{224}$. Presently, benserazide is partially substituted by another AAAD inhibitor carbidopa. The underlying principle for predominant peripheral decarboxylase inhibition by benserazide or carbidopa is associated with an inability of both molecules to cross the blood-brain barrier and therefore the desired levodopa decarboxylation in CNS is not perturbed.

After the development of the combined L-dopa/benserazide medication, a substantial activation of the second COMT metabolic pathway (Fig. 1.26) leading to formation of 3-OMethyldopa (3-OMD) was revealed in the Parkinson's disease patients ${ }^{226}$. Catechol-Omethyltransferase is localized in many tissues, however L-dopa is primarily methylated by COMT in the gut, blood and liver ${ }^{223}$. In addition to direct impact on levodopa half-life, the accumulation of 3-OMD reduces the levodopa bioavailability because 3-OMD is known to be a competitive inhibitor of active transport of L-dopa in the intestine and across the blood brain barrier ${ }^{226}$. These findings stimulated the development of selective and suitable for clinical use COMT inhibitors. On the market the first effective COMT inhibitor tolcapone was released in 1997 by Roche, followed by entacapone in 1998 from Novartis and Orion (Fig. 1.27). Clinical studies demonstrated that concomitant medication L-dopa/benserazide plus tolcapone or entacapone further improved levodopa pharmacokinetic profile. Thus, the levodopa half-live and bioavailability were increased by $50 \%$ and 2 -fold respectively, also significant reduction of "off" time and required L-dopa dose were reported ${ }^{228}$. Of note, tolcapone was restricted in some countries due to its hepatic toxicity and several lethal cases. In 2003 a triple combination tablet Stalevo ${ }^{\circledR}$ (L-dopa, carbidopa and entacapone) from Novartis was approved by FDA and currently Stalevo ${ }^{\circledR}$ is a standard therapy for Parkinson's disease patients with motor fluctuations ${ }^{223}$.

At the same time, considerable effort has been invested in the development and optimization of L-dopa formulation. The major aims are the reduction of dopamine level 
fluctuation, more constant dopaminergic stimulation and, consequently, improved motor control and delayed motor complications. One of the approaches involves the administration of viscous levodopa gel via per-cutaneous gastrostomy tube to the jejunum. The clinical tests have shown that this formulation protocol reduces the "off" time and risk of dyskinesias ${ }^{229}$. In addition, several other approaches allowing continuous delivery have been developed ${ }^{224,229}$ :

1. Sustained-release formulations of L-dopa

2. Once-daily L-dopa pills

3. Transdermal formulations

4. Slow metabolized L-dopa prodrugs

In conclusion, L-dopa is still the gold standard for Parkinson's disease therapy. Significant progress in improving pharmacological and therapeutic properties of L-dopa therapy was achieved by introducing AAAD and COMT inhibitors and new formulation techniques. However, in most patients, the long-term L-dopa therapy has limitations and side effects including motor and non-motor complications.

A<smiles>NC(CO)C(=O)NNCc1ccc(O)c(O)c1O</smiles><smiles>CC(N)(Cc1ccc(O)c(O)c1)C(=O)O</smiles>

carbidopa
B<smiles>Cc1ccc(C(=O)c2cc(O)c(O)c([N+](=O)[O-])c2)cc1</smiles><smiles>CCNC(=O)/C(C#N)=C/c1cc(O)c(O)c([N+](=O)[O-])c1</smiles>

Figure 1.27. Drugs for concomitant therapy. (A) AAAD inhibitors. (B) COMT inhibitors.

\subsubsection{Additional classes of drugs for treatment of Parkinson's disease}

\subsubsection{Monoaminooxidase B inhibitors}

Monoamino oxidases (MAO) are the crucial mitochondrial enzymes that are involved in the oxidative metabolism of monoamines (Fig. 1.28) including many neurotransmitters, such as serotonin, dopamine, norepinephrine and others. Two main forms MAO-A and MAO$\mathrm{B}$ of $\mathrm{MAO}$ are known ${ }^{230}$. Although the two MAO isoforms $\mathrm{A}$ and $\mathrm{B}$ are structurally overlapping, isoenzymes represent different sets of properties, notably the substrate specificity and the tissue distribution. Thus, MAO-A is mainly expressed in brain (catecholaminergic neurons), liver, gut, while MAO-B is predominantly localized in CNS (serotonergic, histaminergic neurons and glial cells) and thrombocytes ${ }^{231}$. Regarding substrate specificity, both isoforms could perform deamination of dopamine, tryptamine and tyramine, at the same time MAO-A has higher specificity to serotonin and noradrenaline; conversely, 
MAO-B is more selective for benzylamine and phenylethylamine substrates ${ }^{231,232}$. Furthermore, it has been demonstrated that substrate specificity of MAO also depends on substrate and enzyme concentrations and other factors.

Coming back to Parkinson's disease, the involvement of MAO-B in disease is supported by observations that the oxidative deamination by MAO-B is the main catabolic pathway for dopamine in the brain and MAO-B activity is elevated in the substantia nigra of Parkinson's disease patients ${ }^{232}$. These findings allow to hypothesized that MAO-B could contribute to the initiation and progression of disease due to the depletion of dopamine, induction of oxidative stress and generation of toxic species. Therefore, the selective inhibition of MAO-B could result in stabilization of dopamine levels and reduction of the oxidative stress.

A

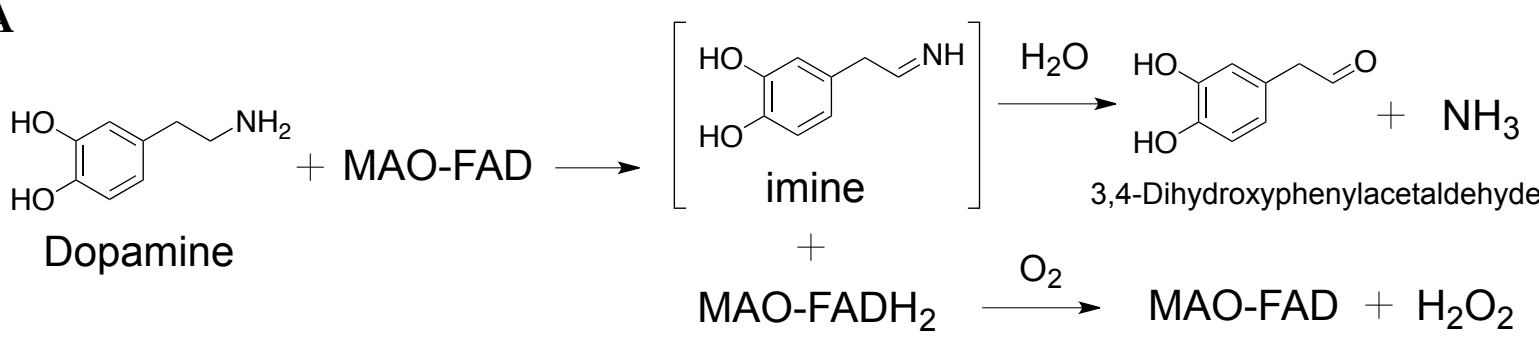

B<smiles>C#CCN(C)C(Cc1ccccc1)C(=O)O</smiles>

selegiline<smiles>C#CCNC1CCc2ccccc21</smiles>

rasagiline<smiles>NCCNC(=O)c1ccc(Cl)cn1</smiles>

lazabemide

Figure 1.28. Monoamino oxidases. (A) Dopamine metabolism by MAO. (B) MAO-B inhibitors.

To date, a large number of MAO inhibitors of different classes were developed and some of them approved as a drug 232 . In case of Parkinson's disease, the effective drug should possess following properties: high selectivity to MAO-B and good blood-brain penetration. Most published scientific research findings and clinical experience are referred to three MAOB inhibitors, rasagiline, selegiline and lazabemide (Fig. 1.28).

Historically, selegiline was the first MAO-B inhibitor approved for treatment of Parkinson's disease. Selegiline is a relatively selective irreversible MAO-B inhibitor. The mechanism of irreversible inhibition is based on the covalent modification of the FAD moiety of MAO-B by propargylamine group of selegiline ${ }^{230}$. Thus, the irreversible MAO-B inhibition leads to a long duration of the pharmacological effects, because the biosynthesis of new 
enzyme is a limiting step. Rasagiline is a second-generation irreversible MAO-B inhibitor with a propargylamine scaffold and, compare to selegiline, rasagiline represents higher MAOB selectivity, better pharmacokinetic/metabolic properties and minor side effect ${ }^{232}$. In patients the oral administration of both drugs leads to effective MAO-B inhibition in the different tissues resulting in stabilization of dopamine levels and symptomatic benefits, mainly improvements in motor function. Currently, rasagiline is approved as effective monotherapy in early Parkinson's disease, providing the delayed need for dopaminergic treatment and improving United Parkinson's Disease Rating Scale (UPDRS) scores. In advance Parkinson's disease an adjunct use of MAO-B inhibitors with levodopa has been shown to extend the "on" time for patients with wearing off fluctuations ${ }^{233}$.

Lazabemide represents another class of selective, but reversible MAO-B inhibitors. Clinical studies with lazabemide revealed similar to the irreversible MAO-B inhibitors pharmacological effects. Thus, patients treated with lazabemide experienced less worsening of UPDRS scores and demonstrated reduced risk of reaching the end point of needing levodopa ${ }^{233}$.

In addition to the symptomatic action, MAO-B inhibitors in clinical trials have also provided evidence for a neuroprotective effect that discussed in details below.

\subsubsection{Dopamine agonists}

Understanding of the biological processes related to dopamine function as the neurotransmitter, its turnover and depletion in nigrostriatal tract of Parkinson's disease patients, as well as identification of dopamine receptors provided the basis for development of dopamine agonists. Dopamine agonists are the molecules that could directly stimulate postsynaptic dopamine receptors in the striatum, and therefore compensating the dopamine depletion and improving motor function.

According to a simplified model of neuronal projections in basal gangliathalamocortical circuit (Fig. 1.29) there are two dopamine-associated pathways: direct pathway in which dopamine activates D1 receptors and indirect pathway mediated by D2 receptors ${ }^{1,234}$. D1 subfamily of dopamine receptors consists of two members; D1 and D5 that are expressed exclusively in postsynaptic dopamine-receptive cells. While D2, D3 and D4 belong to D2 subfamily and localize postsynaptically on dopamine-sensing cells as well as presynaptically on dopaminergic cells ${ }^{235}$. Functionally, the stimulation of D1 class receptors leads to activation of adenylyl kinase activity, synthesis of second messenger cAMP and a moderate stimulatory effect on locomotor activity. In case of D2 class, activation of 
D2/D3/D4 receptors induces the inhibition of adenylyl kinase activity. Notably, the presynaptic activation of D2-like receptors is a part of a negative feedback mechanism that modulates the release and synthesis of dopamine and reduces the locomotor response, whereas the postsynaptic stimulation results in activation of locomotor function $^{234,235}$. The described mechanism is significantly simplified and the physiological role and signaling pathways of dopamine receptors, dopamine itself and interactions with other messengers are

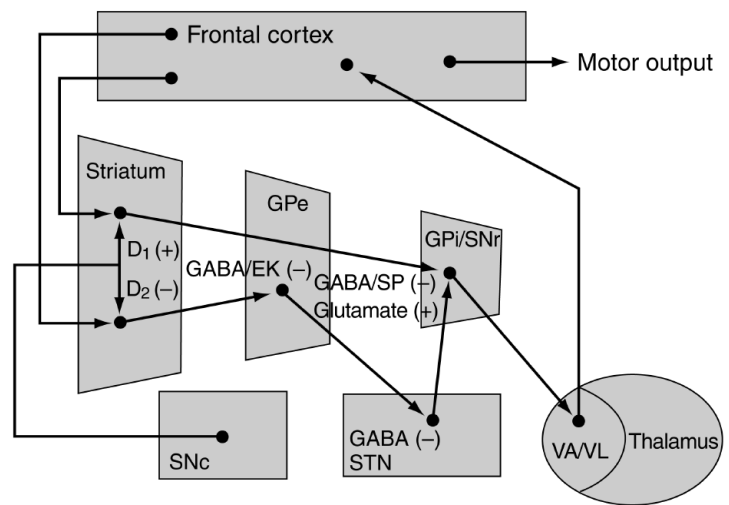

Figure 1.29. Simplified model of basal ganglia-thalamocortical circuit.

Figure reprinted from Pharmacotherapy, Chen et al. ${ }^{1}$ with permission from John Wiley and Sons, copyright @ (2007). considerably more complicated and detailed discussion is beyond the scope of this thesis.

A growing understanding of complex pathways of dopamine-related signaling and its role in Parkinson's disease have led to development and clinical application of dopamine receptor agonist including apomorphine, bromocriptine, pergolide and other compounds (Fig. 1.30; Table 1.5) ${ }^{1,234}$. Theoretically, the dopamine antagonists as a dopaminergic treatment have several advantages over L-dopa therapy ${ }^{234,236 .}$

1) Direct stimulation of dopamine receptors independently from the population of the degenerating nigrostriatal neurons

2) Longer half-life and, consequently, more sustained and stable stimulation of dopamine receptors and reduced risk for developing motor complications

3) Improved bioavailability and pharmacokinetic properties

4) Possible neuroprotective effects due to the antioxidative and antiapoptotic properties

5) No metabolic transformation is required

6) Variety of administration protocols

As mentioned before, the experimental findings provide the strong evidence that locomotor activity is primarily mediated by D1, D2, and, to a lesser degree, D3 receptors. Most of dopamine agonists have a high selectivity to D2 and D3 receptors (Table 1.5), while the rest of endogenous dopamine can provide D1 receptor stimulation. The prolonged stimulation of D1 receptors with agonists is not desirable, because it might lead to the development of dyskinesias and, presumably, to the desensitization of these receptors. 
Table 1.5. Properties of available dopamine agonists ${ }^{234}$

\begin{tabular}{|c|c|c|c|c|c|c|}
\hline \multirow{2}{*}{$\begin{array}{c}\text { Dopamine } \\
\text { agonist }\end{array}$} & \multirow{2}{*}{ Ergot? } & \multicolumn{3}{|c|}{ Dopamine receptors selectivity } & \multirow{2}{*}{$\mathbf{t}_{1 / 2}, \mathbf{h}$} & \multirow{2}{*}{ Administration } \\
\hline & & D1 & D2 & D3 & & \\
\hline Apomorhine & No & ++ & ++ & ++ & 0.5 & Subcutaneous \\
\hline Ropinirole & No & 0 & +++ & +++ & 6 & Oral \\
\hline Pramipexole & No & 0 & +++ & ++++ & 10 & Oral \\
\hline Bromocriptine & Yes & - & ++ & ++ & $6-8$ & Oral \\
\hline Pergolide & Yes & + & ++++ & +++ & 24 & Oral \\
\hline Cabergoline & Yes & 0 & +++ & +++ & 65 & Oral \\
\hline Lisuride & Yes & + & +++ & +++ & 2 & Oral \\
\hline
\end{tabular}

$+\rightarrow++++$ : Increasing agonism. -: Antagonism

At present, the clinical trials have shown that dopamine agonist treatment is effective as a monotherapy in early and mild-to-moderate Parkinson's disease, in particular, providing symptomatic relief, delaying the need of levodopa and reducing the risk of developing motor complications $^{234}$. In patients with advanced Parkinson's disease dopamine agonists are commonly applied as adjuncts to levodopa treatment. Thus, clinical trials using levodopa/dopamine agonist formulations have demonstrated clinical benefits including improved motor function, reduced "off" time and lowering of the required L-dopa dose $\mathrm{e}^{1,236}$.

A<smiles>CN1CCc2cccc3c2[C@H](Cc2ccc(O)c(O)c2-3)C1</smiles>

Apomorhine<smiles>CCCN(CCC)CCc1cccc2c1CC(=O)N2</smiles>

Ropinirole<smiles>CCCN[C@H]1CCc2nc(N)sc2C1</smiles>

Pramipexole

B<smiles></smiles>

Bromocriptine<smiles>CCCN1C[C@H](CSC)C[C@H]2c3cccc4[nH]cc(c34)C[C@H]21</smiles>

Pergolide<smiles>C=CCN1CC(C(=O)N(CCCN(C)C)C(=O)NCC)CC2c3cccc4[nH]cc(c34)C[C@H]21</smiles>

Cabergoline<smiles>CCN(CC)C(=O)NC1CC2c3cccc4[nH]cc(c34)C[C@H]2N(C)C1</smiles>

Lisuride

Figure 1.30. Dopamine agonists. (A) Non-ergot derived dopamine agonists. (B) Ergot derived dopamine agonists. 
Almost all dopamine agonist possesses a wide range of side effects related to peripheral and central dopaminergic stimulation. The peripheral dopaminergic stimulation results in nausea, vomiting, dizziness, bradycardia and others. While central side effects comprise of hallucinations, psychosis, confusion and others. In general, non-ergot derived compounds are considered to be more safe than ergot derivatives that have been shown to increase the risk of tissue fibrosis ${ }^{1,237,238}$. Currently, the most frequently prescribed dopamine agonists are non-ergot pramipexole and ropinirole ${ }^{236}$. However, potential issues with personal tolerability and adverse effects for certain dopamine agonist could be solved by slow titration and switching to other dopamine agonists.

\subsubsection{Amantadine and Anticholinergics}

In the late 1960s amantadine (Fig. 1.31) was first time reported to provide the antiparkinsonian effects that were later confirmed in several clinical trials ${ }^{239}, 240$. Multiple studies have demonstrated that amantadine is beneficial both as monotherapy and as a combination with dopaminergic agents ${ }^{240}$. Thus, the patients treated with amantadine alone or in addition to dopaminergic treatment have better symptomatic control compared to the placebo group. In addition, amantadine is one of the best antidyskinetic drugs currently available on the market. Although, amantadine is in the clinical practice for more than 40 years the precise mechanism of action is not fully established. Several lines of evidences implicate dopaminergic as well as nondopaminergic mechanisms in the pharmacological properties. The concept of dopaminergic mechanism is supported by observations that presynaptically amantadine may stimulate the release of neurotransmitters from dopaminergic terminals (amphetamine-like action) and inhibit the dopamine reuptake. Furthermore, postsynaptically amantadine exhibits the dopamine D2 receptor agonist properties ${ }^{237}$, 240 . In support of nondopaminergic mechanisms, amantadine has been shown to be a potent glutamatergic N-methyl-D-aspartate (NMDA) receptor antagonist ${ }^{241}$. First of all, the inhibition of NMDA receptors results in inhibition of acetylcholine release and, consequently, in suppressing of the cholinergic hyperactivity observed in striatum of Parkinson's disease patients. Second, the NMDA blockage may reduce the glutamate excitotoxicity and the glutaminergic hyperstimulation that presumably contribute to neuronal death and development of dyskinesia ${ }^{242}$. The patients receiving adamantine experience a set of side effects including confusion, dizziness, hallucinations and others. ${ }^{238}$ 
A<smiles>NC12CC3CC(C1)C3C2</smiles>

Amantadine<smiles>[B]N1CCCC1OCc1ccccc1</smiles>

Benzatropine

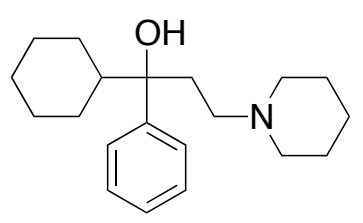

Trihexyphenidyl<smiles>CN(C)CCOC(c1ccccc1)c1ccccc1</smiles>

Diphenhydramine

Figure 1.31. Non-dopaminergic drugs. (A) Amantadine. (B) Anticholinergic agents.

The oldest anticholinergic agents, in form of naturally occurring alkaloids, were the first antiparkinsonism medication that was described in the $19^{\text {th }}$ century. Modern synthetic anticholinergics (benzatropine, trihexyphenidyl, diphenhydramine; Fig. 1.31) have been shown to be modest effective in mitigating some locomotor symptoms of Parkinson's disease, both as monotherapy as well as adjunct treatment ${ }^{243,244}$. Although the exact mechanism of action is still elusive, experimental findings support the hypothesis that anticholinergics restore the dopaminergic-cholinergic balance in the striatum. In healthy brain dopamine tonically inhibits acetylcholine neurons in striatum, whereas the development of dopamine deficiency leads to cholinergic hyperactivity that contributes to the tremor of Parkinson's disease $^{1,244}$. In addition, some of these compounds can also inhibit the dopamine reuptake in dopaminergic neurons ${ }^{243}$. Currently, anticholinergic drugs are not considered as first-line drugs due to the quite modest efficacy on one hand and a wide range of serious adverse effects (memory difficulty, confusion, constipation, dementia and others) and a frequent intolerability on the other hand 236,243 .

\subsubsection{Therapy of non-motor symptoms}

As it has been already discussed above in the chapter summarizing symptomatic features of Parkinson's disease, the non-motor symptoms, mainly psychiatric, cognitive, autonomic and pain problems, are increasingly recognized in clinical practice. Compare to locomotor disturbances, the majority of non-motor symptoms are significantly varying form patient to patient, and therefore the individual approaches should be applied in each case, taking into consideration multiple factor (age, severity, lifestyle, drug interactions). Without going into specific details, the list of drugs for prevention and treatment of non-motor symptoms includes antidepressants (depression, anxiety), neuroleptics (psychosis), gastroprokinetic/antihypertensive agents (autonomic dysfunction) and other $237,238,245$. 


\subsubsection{Surgery and Transplantation}

Surgical treatment of Parkinson's disease is available in two forms: ablative and stimulation approaches. The ablative neurosurgical therapies (like thalamotomy, pallidotomy) are invasive procedures that sever small areas of the brain in order to equilibrate the signaling disbalances that are caused by pathophysiological changes. Thus, thalamotomy has been shown to be effective in mitigating tremor, dyskinesias, and, moreover, the benefit may be observed for several years ${ }^{237}$ 246. Deep brain stimulation (DBS) is a basis of the stimulation approach. Although DBS is approved as a therapy, the exact mechanism of action is not fully understood. In the clinic, application of DBS for treatment of Parkinson's disease has been proven to be effective in managing motor symptoms and complications, specifically tremor, motor fluctuations, gait abnormalities and others ${ }^{236,246}$. The beneficial effects of DBS are considered to be reversible and dependent on the brain area where DBS devices, generating high frequency pulses, are implanted. Thus, the stimulation of the ventral intermediate nucleus is performed to specifically reduce tremor. One important concern about surgery, the neurologists for each patient should perform very careful and individual selection. For example, the DBS of subthalamic nucleus is not effective in the patients characterized by weak response to L-dopa ${ }^{237,246}$.

So far the tissue transplantation compensating dopamine-producing cells has been performed on a small groups of patients and on animals in laboratory conditions ${ }^{237}$. Previous and running clinical studies have reported potential therapeutic effects ${ }^{247}$, however, considerably more research is required in evaluation and development of these technologies before it becomes available for wide range of human patients.

\subsubsection{Management of Parkinson's disease}

After the diagnosis of Parkinson's disease the physicians relying on analysis of the individual multiple factors suggest the most suitable treatment strategy. According to a number of available treatment guidelines, some kind of diamond-shaped paradigm (Fig 1.32) for pharmacological intervention is accepted in the field ${ }^{1}$. Thus, during the early stages of the disease patients require the monotherapy; on next stages dose optimization and adjunctive agents are applied and eventually the number of drugs is reduced due to the side effects and lack of response. Figure 1.32 illustrates the simplified scheme (treatment of non-motor symptoms is excluded) of the management of Parkinson's disease ${ }^{1,237}$. 


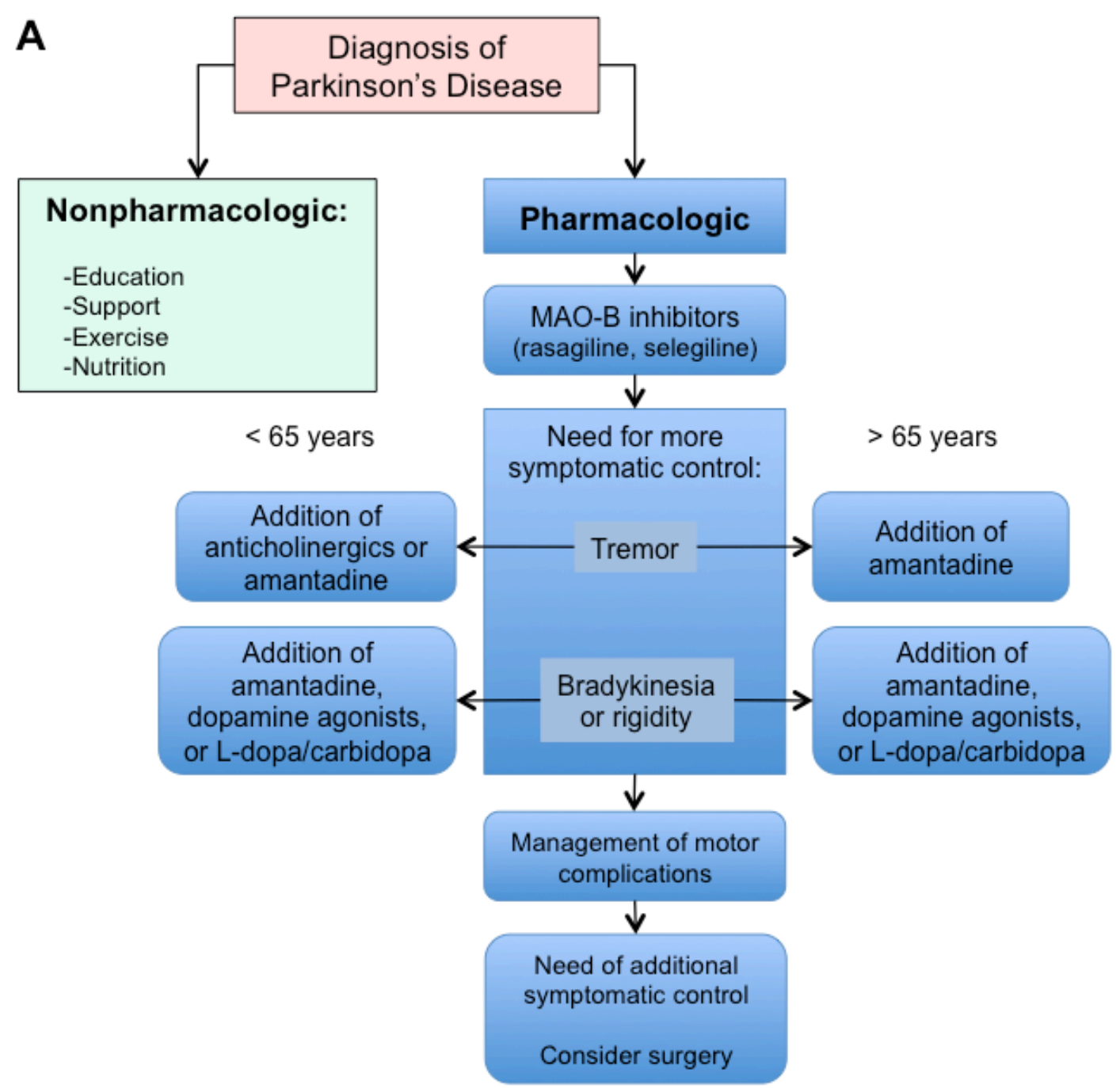

B

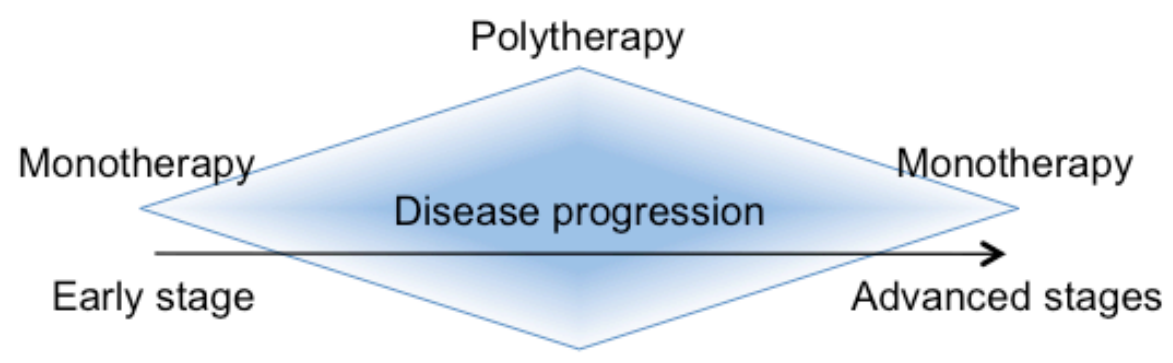

Figure 1.32. Management of Parkinson's disease. (A) Simplified scheme of the management. (B) Diamond-shaped paradigm for pharmacological intervention. Figure A adapted with additional color-coding from Pharmacotherapy, Chen et al. ${ }^{1}$ with permission from John Wiley and Sons, copyright @ (2007). 


\subsubsection{Overview}

Despite the fact that there is no clear understanding on the molecular level of the pathological mechanisms underlying the nigrostriatal neurodegeneration, the currently available therapeutic approaches provide effective symptomatic control that is translated to good patient's quality of life and sustainable functional status. According to the available treatment guidelines, during early phases of Parkinson's disease the monotherapy (MAO-B inhibitors) and nonpharmacological procedure are recommended, while at later stages of the disease concomitant therapies with dopaminergic drugs as a core are required. It's important to mention that careful and individual management of pharmacological intervention is highly desirable in order to optimize long-term therapeutic benefits and avoid adverse effects. To date, L-dopa is still the gold standard for Parkinson's disease therapy; however, the long-term L-dopa application is associated with a variety of motor and non-motor complications and decaying drug response. Keeping in mind the symptomatic nature of all available approaches, the development of the disease-modifying therapies is of high interest. In the next chapter, most promising strategies and drug candidates with potential neuroprotective effects are discussed based on clinical and preclinical experience.

\subsection{In search of the neuroprotection from cells to human}

\subsubsection{Introduction}

Continuously advancing knowledge of pathological processes and changes that occur during Parkinson's disease led to significant progress in development of new methods and approaches with a goal of neuroprotective treatment/drug for Parkinson's disease. With this regard, for a successful breakthrough in finding neuroprotective therapy the deep understanding of many crucial aspects of drug development and clinical trials is required. In this chapter I attempt to overview currently available animal models and the diseasemodifying strategies that are in preclinical or clinical stages.

\subsubsection{Animal models of Parkinson's disease}

\subsubsection{From cells to primates: Strength and Limitations}

It's extremely hard to overestimate the contribution of different cellular and animal models in the investigation of the etiology, pathology and molecular mechanisms related to Parkinson's disease. Analysis of the enormous volume of experimental data from different in vivo and in vitro models and patients has clearly indicated that Parkinson's disease is very 
complex with many different pathological processes. Over the last several decades, many various models, that recaputilate almost all hallmarks of the Parkinson's disease, have been generated $^{66,248,249}$. In addition to the great importance of insight into molecular mechanisms, the modeling of the pathological process is a valuable tool to test and compare novel therapeutic compounds and approaches.

What is an ideal model of Parkinson's disease? To date the widely accepted view is that the ideal model should reproduce following manifestations ${ }^{66,250}$ :

- progessive and age dependent profile

- neurodegeneration in the nigrostriatal pathway and other relevant part of the brain

- locomotor dysfunction (rigidity, tremor, akinsesia, motor performance and others)

- formation and spreading of Lewy pathology

- pathologial features (oxidative stress; inflammation; mitochondrial impairment, degradation system failure)

- response to dopaminergic treatment

So far, there are no such models that fully reproduce all preclinical and clinical manifestations of Parkinson's disease. Due to the multifactorial nature of Parkinson's disease all available models resemble just one or several certain pathological features depending on the genetic or toxin-based approaches. Historically, the first available models were the toxinbased models, in which enviromental or synthetic neurotoxins are applied in vivo and in vitro systems resulting in neurotoxicity ${ }^{251}$. However, the progess in gene manipulation techniques and the discovery of many disease-associated genes has resulted in the develoment of a variety of genetic models in in vivo as well as in vitro systems ${ }^{248}$. Recently, a third type of Parkinson's disease model was published, in which the neurodegeneration and Lewy pathology are triggered by intracerebral inoculation of Lewy body extracts or recombinant $\alpha$ synuclein fibrils ${ }^{252}$. These models could be referred as seeding, spreading or propagation models.

Until now, genetically modified or toxin-treated rodent species like mice and rats are most frequently used models of Parkinson's disease. However, the cellular and, particularly, small organism models also find a wide application due to the low cost, fast response and feasibility for high-throughput screening. Thus, significant progress has been achieved in mimicking disease mechanisms in zebrafish (Danio rerio), fruit flies (Drosophila melanogaster), nematodes (Caenorhabditis elegans) and anurans (frog and toad) ${ }^{249}$. The main focus of the cellular models is the investigation of molecular functions and mechanisms related to certain genes, proteins or organic molecules, but the conclusions from such in vitro 
studies has to be verified in animal models, preferably in mammals. Therefore, in this chapter I will briefly summarize the properties of available neurotoxin, genetic and seeding models of Parkinson's disease in the mammals and in some of non-traditional model organisms.

It's also important to mention about an impressive progress in development of diverse tests and approaches that provide reliable assessment and evaluation of pathological changes on behavioral, anatomical and biochemical levels ${ }^{251}$. Thus, the battery of the behavioral tests includes activity monitoring, clinical ratings, motor skills tasks, cognitive tests, tremor or rotations recording and many others. However, the postmortem analysis of the tissues with biochemical, histological, microscopy/spectroscopy techniques represents the most essential approach extensively applied in almost all laboratories. Furthermore, despite many limitations, application and development of in vivo imaging techniques, namely positron emission tomography (PET), magnetic resonance imaging (MRI) and fluorescence/luminescence-based methods, allow scientists to collect valuable information about the animal model, but at the same time bring researchers closer to the clinical situation.

\subsubsection{Toxin-induced models}

As mentioned before, the toxin-induced models are the most widely used animal models of Parkinson's disease. To date, a number of various toxin-based animal models have been generated and utilized for investigation of pathological mechanisms and therapeutic approaches $^{248,253}$. Most of widely applied toxins could be divided in three classes (Fig. 1.6): neurotoxins (MPTP, 6-hydroxydopamine (6-OHDA)), pesticides/herbicides (paraquat, maneb, rotenone) and UPS inhibitors (lactacystin, MG-132). The neurotoxic properties of these compounds and related pathological events have been investigated in a variety of organisms including monkeys, mice, rats, fruit flies, C. elegans and others as well as in different exposure and administration protocols ${ }^{248,249,253}$.

The following sequence of events is implicated in a general mechanism of toxininduced pathology. After peripheral or intracerebral administration of the given toxin, the parent compound or its metabolic products are selectively (not for all toxins) transported through DAT to dopaminergic neurons, where translocation to the mitochondria occurs. In mitochondria, the toxin or its metabolite inhibit the electron transport chain complex I resulting in the failure of mitochondrial functions. In addition to the mitochondrial collapse, a range of other pathophysiological processes has been implicated in toxicity of certain toxins, namely, microglia activation, oxidative stress, upregulation of NADPH-oxidase (NADPH 
generating superoxide from molecular oxygen), elevation of reactive oxygen species (ROS) level and other ${ }^{66}$.

The majority of toxin-induced animal models have been shown to develop obvious clinical and pathologic phenotypes that resemble many features of late-stage Parkinson's disease in human. For example, the administration of toxins to mammalian species leads to a selective loss of dopaminergic neurons, depletion of dopamine levels, locomotor abnormalities and formation of $\alpha$-synuclein-positive inclusions ${ }^{66,254}$. However, there are several crucial drawbacks of using these models. First of all, the relevance of the models is limited due the irreversible, acute and not progressive nature of the observed phenotypes. Furthermore, the formation of $\alpha$-synuclein-positive inclusions in the brain is often very limited and at the same time structurally different from the typical Lewy body inclusions. Overall, the toxin-induced animal models are suitable for testing therapeutic candidates preventing motor symptoms, mitochondrial dysfunction and oxidative stress at late stages of Parkinson's disease.

\section{MPTP}

After a tragic discovery that accidental MPTP (Fig. 1.6) administration led to development of acute parkinsonism in humans MPTP became one of the most widely used toxins to mimic the pathological phenotype in animals. In humans as well as in the animals, MPTP treatment causes severe degeneration of dopaminergic neurons, oxidative stress, dopamine depletion and inflammation ${ }^{63}$, 248. It has been shown that the lesions of norepinephrinergic and serotonergic neurons might also occur ${ }^{255}$. The proposed mechanism of MPTP-induced toxicity is discussed above in chapter "Mitochondrial toxins". The weak points of MPTP-based model are a very fast neuronal death and an absence of Lewy body pathology, although there are several reports demonstrating $\alpha$-synuclein-positive inclusions. Furthermore, the behavioral evaluation of MPTP-treated animals has revealed a weak correlation of the symptoms with the degree of neurodegeneration and dose of MPTP 253 . With this respect, the MPTP-based primate models among other animals most closely recapitulate the behavioral and symptomatic features of human Parkinson's disease.

Currently, the MPTP-induced phenotypes are described in many species including primates, rodents, invertebrates and others (Table 1.6 $)^{249}$. 253. Importantly, a different susceptibility to MPTP toxin is observed in different animals, for example, rats are resistant to MPTP. In addition, such factors like gender, age, body weight and exposure protocols have a significant impact on pathophysiological and behavioral effects. For instance, depending on 
the applied MPTP administration protocols, namely, single dose (30 mg/kg MPTP), acute (4 MPTP injections every $2 \mathrm{~h}$ during one day), subacute/subchronic (daily MPTP injections during one week) or chronic (repeated treatments over 4-5 weeks) the neuronal loss of 20$30 \%, 50 \%, 24 \%$ and $50-80 \%$ has been shown in mice, respectively ${ }^{253}$.

Table 1.6. Properties of available MPTP-based animal models

\begin{tabular}{|c|c|c|c|c|}
\hline Species & $\begin{array}{c}\text { Behavioral } \\
\text { symptoms }\end{array}$ & Neuropathology & $\alpha$-Synuclein & Disadvantages \\
\hline Primates & $\begin{array}{l}\text { - Motor impairment } \\
\text { - Good response to } \\
\text { dopaminergic treatment }\end{array}$ & $\begin{array}{l}\text { - Severe loss of } \\
\text { dopaminergic neurons } \\
\text { - Dopamine deficiency } \\
\text { - Extra-nigral pathology }\end{array}$ & $\begin{array}{l}\alpha \text {-Synulein } \\
\text { positive } \\
\text { inclusions }\end{array}$ & $\begin{array}{l}\text { Nonprogressive } \\
\text { cell death }\end{array}$ \\
\hline Rat & \multicolumn{4}{|c|}{ Weak response to MPTP toxin } \\
\hline Mice & $\begin{array}{l}\text { Minor motor } \\
\text { impairments }\end{array}$ & $\begin{array}{l}\text { - Severe loss of } \\
\text { dopaminergic neurons } \\
\text { - Dopamine deficiency }\end{array}$ & $\begin{array}{l}\text { Increased } \alpha- \\
\text { Synulein } \\
\text { immunoreactivity }\end{array}$ & $\begin{array}{l}\text { - Nonprogressive } \\
\text { cell death } \\
\text { - No typical LBs }\end{array}$ \\
\hline Frog & Rigidity, tremor, akinesia & Dopamine deficiency & Unknown & $\begin{array}{l}\text { No permanent cell } \\
\text { death }\end{array}$ \\
\hline Zebrafish & $\begin{array}{l}\text { - Increased } \\
\text { depigmentation } \\
\text { - Respiratory difficulties } \\
\text { - Swimming impairment }\end{array}$ & $\begin{array}{l}\text { Loss of monoaminergic } \\
\text { neurons }\end{array}$ & Unknown & $\begin{array}{l}\text { - Nonprogressive } \\
\text { cell death } \\
\text { - No } \alpha \text {-Synulein } \\
\text { aggregation }\end{array}$ \\
\hline Nematode & $\begin{array}{l}\text { - Reduced motility } \\
\text { - Increased mortality }\end{array}$ & $\begin{array}{l}\text { Loss of dopaminergic } \\
\text { neurons }\end{array}$ & Unknown & $\begin{array}{l}\text { Nonprogressive } \\
\text { cell death }\end{array}$ \\
\hline
\end{tabular}

\section{Rotenone}

Rotenone (Fig. 1.6) is a highly lipophilic compound that is applied in the agriculture as herbicide and insecticide. Rotenone has been shown to be a potent inhibitor of complex I of mitochondrial electron transport chain. As a consequence of high lipophilicity, rotenone readily crosses the blood brain barrier and the cell membranes ${ }^{254}$. Thereby, the distribution of rotenone in the brain is not specific for certain neurons and it doesn't require dopamine transporters $^{248}$. Similar to other toxins, the administration of rotenone in different species leads to various phenotypes (Table 1.7). Until now, the rat model with systemic chronic exposure is the most widely used rotenone-based animal model. In rats, chronic exposure that is achieved by intravenous injections or osmotic pumps allows to reproduce almost all neurochemical, neuropathological, anatomical and behavioral features of Parkinson's disease $^{253,254}$. Thus, various studies have demonstrated that rats, chronically treated with rotenone, develop a selective neurodegeneration of the nigrostriatal dopaminergic system in combination with oxidative stress, dopamine depletion, formation of $\alpha$-synuclein-positive 
cytoplasmic inclusions, inflammation and proteolytic stress ${ }^{72,256}$. At higher doses, rotenone also causes the death of serotoninergic, noradrenergic and cholinergic neurons ${ }^{254}$. Behaviorally, the rats develop a number of parkinsonism symptoms including bradykinesia, rigidity, tremor and others, which can be ameliorated by levodopa. One of the main advantages of the rotenone-lesioned rat model is a progressive nature of neurodegeneration and Lewy body pathology ${ }^{256}$. At the same time, however, the major limitation of this model is related to high individual variability to the chronic rotenone exposure ${ }^{253}$.

Table 1.7. Properties of available rotenone-based animal models

\begin{tabular}{|c|c|c|c|c|}
\hline Species & $\begin{array}{l}\text { Behavioral } \\
\text { symptoms }\end{array}$ & Neuropathology & $\alpha$-Synuclein & Disadvantages \\
\hline Primates & \multicolumn{4}{|c|}{ No established models } \\
\hline Rat & $\begin{array}{l}\text { - Motor impairment } \\
\text { - Response to } \\
\text { dopaminergic } \\
\text { treatment }\end{array}$ & $\begin{array}{l}\text { - Progressive loss of } \\
\text { dopaminergic neurons } \\
\text { - Dopamine deficiency } \\
\text { - Extra-nigral pathology }\end{array}$ & $\begin{array}{l}\text { Progressive Lewy } \\
\text { body pathology }\end{array}$ & $\begin{array}{l}\text { High variation of } \\
\text { clinical } \\
\text { phenotype }\end{array}$ \\
\hline $\begin{array}{c}\text { Mice } \\
\text { (low dose) }\end{array}$ & $\begin{array}{l}\text { - Progressive motor } \\
\text { dysfunction (rotarod) } \\
\text { - Motor impairments } \\
\text { correlate with degree } \\
\text { of neuronal loss }\end{array}$ & $\begin{array}{l}\text { - Selective loss of } \\
\text { dopaminergic neurons in } \\
\text { the substantia nigra } \\
\text { - No systemic complex-I } \\
\text { inhibition in the brain } \\
\text { and muscles }\end{array}$ & $\begin{array}{l}\text { - } \alpha \text {-Synuclein } \\
\text { pathology in ENS } \\
\text { and CNS } \\
\text { - Progressive spread } \\
\text { of pathology from } \\
\text { ENS to the brain }\end{array}$ & \\
\hline $\begin{array}{c}\text { Mice } \\
\text { (high dose) }\end{array}$ & $\begin{array}{l}\text { - Dose- and time- } \\
\text { dependent decline of } \\
\text { locomotor activity } \\
\text { (rotarod) }\end{array}$ & $\begin{array}{l}\text { - Progressive loss of TH- } \\
\text { positive neurons in the } \\
\text { substantia nigra }\end{array}$ & $\begin{array}{l}\text { Increased } \alpha \text { - } \\
\text { Synuclein } \\
\text { immunoreactivity }\end{array}$ & $\begin{array}{l}\text { High variation of } \\
\text { clinical } \\
\text { phenotype }\end{array}$ \\
\hline Flies $^{257}$ & $\begin{array}{l}\text { - Dose-dependent } \\
\text { locomotor } \\
\text { impairments } \\
\text { - Response to L-dopa }\end{array}$ & $\begin{array}{l}\text { - Selective loss of } \\
\text { dopaminergic neurons in } \\
\text { all brain regions } \\
\text { - Dopamine deficiency }\end{array}$ & Unknown & Fast cell death \\
\hline Nematode $^{258}$ & Unknown & $\begin{array}{l}\text { Loss of dopamine } \\
\text { neurons in a } \\
\text { concentration-dependent } \\
\text { manner }\end{array}$ & Unknown & Fast cell death \\
\hline
\end{tabular}

Almost all attempts to induce neurodegeneration with rotenone in mice and monkeys were unsuccessful. However, several years ago two rotenone-based mice models were published. In one model, chronic systemic rotenone exposure (orally, 30-100 mg/kg) over 2 months results in progressive loss (ca. 70\%) of TH-positive neurons in the substantia nigra and increased $\alpha$-synuclein immunoreactivity in surviving neurons. Additionally, this mice model is characterized by dose- and time-dependent decline of locomotor activity evaluated by rotarod test $\mathrm{t}^{259}$. 
The second mouse model is based on chronic intragastric administration of rotenone at low dose $(5 \mathrm{mg} / \mathrm{kg} \text {, daily in drinking water, } 1.5-3 \text { months })^{260}$. This mouse model is characterized by a fundamentally different and potentially advantageous mechanism of pathology development and progression. Under this treatment regime, rotenone has been shown to induce the accumulation and aggregation of $\alpha$-synuclein in the enteric nervous system (ENS) (neurons of duodenum and ileum) at the first stage. Over the course of the experiment sequential spreading of $\alpha$-synuclein from ENS to the brain through synaptically connected nervous systems is observed. Thus, the $\alpha$-synuclein pathology from ENS expands to central nervous system centers in the spinal cord and the brainstem (dorsal motor nucleus of the vagus and intermediolateral nucleus in the spinal cord) and finally after 3 months (but not after 1.5 months) an increased amount of intracellular $\alpha$-synuclein aggregates is detected in the substantia nigra. Moreover, the spread of $\alpha$-synuclein pathology is accompanied by $\alpha$ synuclein phosphorylation, inflammation, time-dependent motor dysfunction (rotarod) and selective loss of dopaminergic neurons in the substantia nigra. Importantly, due to the low dose and intragastric application of rotenone, the direct toxic effect of rotenone is confined to the gut without systemic complex-I inhibition in the brain and muscles, thus differing from classical rotenone models. Overall, this new rotenone model recapitulates the essential features of PD pathology in humans such as $\alpha$-synuclein accumulation and progressive spread of pathology from ENS to the brain and, therefore, represents a valuable tool for testing new therapeutic approaches and for validation of the enteric system spreading hypothesis.

\section{6-Hydroxydopamine}

Administration of 6-hydroxydopamine (6-OHDA) (Fig. 1.6) is a basis of the next classic and frequently used toxin-based animal model of Parkinson's disease. Mechanistically, after entering the neurons via dopamine transporter, 6-OHDA is oxidized generating hydrogen peroxide and paraquinone, both of which are toxic for neurons ${ }^{253}$. To date, 6-OHDA-related phenotypes have been described for a wide range of various animal species including mammals (monkey, cat, dog, rat, mice), invertebrates (worm, fly) and others (Table 1.8) 249, 261, 262. Among the other animals, the rat model is considered to be a 'gold standard' for evaluation of the behavioral, biochemical and neuropathological effects of 6OHDA $^{248,253}$. Since 6-OHDA couldn't cross the blood brain barrier, the administration of the toxin is performed by intracerebral injection into certain regions of the brain. In most protocols, 6-OHDA injections are unilateral (in one hemisphere) and, therefore, offer certain 
advantages, namely, an internal control based on the second unaffected hemisphere and asymmetric behavioral phenotype ${ }^{248}$.

Table 1.8. Properties of available 6-OHDA-based animal models

\begin{tabular}{|c|c|c|c|c|}
\hline Species & $\begin{array}{c}\text { Behavioral } \\
\text { symptoms }\end{array}$ & Neuropathology & $\alpha-S y n u c l e i n$ & Disadvantages \\
\hline Monkey $^{261}$ & $\begin{array}{l}\text { - Behavioral or body } \\
\text { asymmetries } \\
\text { - Amphetamine- } \\
\text { induced rotation }\end{array}$ & $\begin{array}{l}\text { - Complete or partial } \\
\text { unilateral lesions of } \\
\text { dopaminergic neurons }\end{array}$ & $\begin{array}{l}\text { No Lewy bodies } \\
\text { pathology }\end{array}$ & $\begin{array}{l}\text { - Rapid loss of } \\
\text { neurons } \\
\text { - No } \alpha \text {-synuclein } \\
\text { aggregation }\end{array}$ \\
\hline Rat & $\begin{array}{l}\text { - Asymmetric } \\
\text { behavioral phenotype } \\
\text { - Amphetamine- } \\
\text { induced rotation } \\
\text { - Reduced motor } \\
\text { activity (rotarod) }\end{array}$ & $\begin{array}{l}\text { - Rapid and selective } \\
\text { loss of dopaminergic } \\
\text { neurons } \\
\text { - Dopamine depletion }\end{array}$ & $\begin{array}{l}\text { No Lewy bodies } \\
\text { pathology }\end{array}$ & $\begin{array}{l}\text { - Rapid loss of } \\
\text { neurons } \\
\text { - No } \alpha \text {-synuclein } \\
\text { aggregation }\end{array}$ \\
\hline Mice $^{262}$ & $\begin{array}{l}\text { Reduced motor } \\
\text { activity (rotarod) }\end{array}$ & $\begin{array}{l}\text { - Selective loss of } \\
\text { dopaminergic neurons } \\
\text { - Dopamine depletion }\end{array}$ & $\begin{array}{l}\text { No Lewy bodies } \\
\text { pathology }\end{array}$ & $\begin{array}{l}\text { - No } \alpha \text {-synuclein } \\
\text { aggregation } \\
\text { - Not progressive }\end{array}$ \\
\hline Frog & $\begin{array}{l}\text { - Motor and cognitive } \\
\text { symptoms correlated } \\
\text { with degree of } \\
\text { neurodegeneration }\end{array}$ & $\begin{array}{l}\text { Loss of } \\
\text { catecholaminergic } \\
\text { neurons in a dose- } \\
\text { dependent manner }\end{array}$ & Unknown & Acute model \\
\hline Nematode & Unknown & $\begin{array}{l}\text { - Selective, rapid loss } \\
\text { of dopamine neurons }\end{array}$ & Unknown & Acute model \\
\hline
\end{tabular}

In rats, the 6-OHDA-induced phenotype is highly reproducible and dependent on the site of injection and dose of 6-OHDA. Thus, the 6-OHDA injection to the substantia nigra or medial forebrain buddle results in a rapid death of dopaminergic neurons with subsequent loss of TH-positive terminals in the striatum and dopamine depletion ${ }^{253}$. Whereas, a neuropathology that has been demonstrated after inoculation of 6-OHDA into striatum, to certain degree mimics the retrograde pathological process occurring in human, meaning the initial loss of TH-positive terminal in striatum followed by progressive loss of dopaminergic neurons in the substantia nigra ${ }^{248}$. Due to the unilateral toxin application the rats develop asymmetric behavior, which is quantified by rotation tests and step motor test ${ }^{248,253}$. The rotational test allows estimating the degree of neurodegeneration with a reasonable accuracy. The main limitations of the model are related to the very rapid loss of neurons and to an absence of Lewy body pathology. Overall, the 6-OHDA-based systems could be considered as a reliable model of late stages of Parkinson's disease as well as a well-established platform for testing symptomatic treatments. 
Table 1.9. Properties of available paraquat-based animal models

\begin{tabular}{|c|c|c|c|c|}
\hline Species & $\begin{array}{l}\text { Behavioral } \\
\text { symptoms }\end{array}$ & Neuropathology & $\alpha$-Synuclein & $\begin{array}{l}\text { Disadvanta- } \\
\text { ges }\end{array}$ \\
\hline Primates & \multicolumn{4}{|c|}{ No established models } \\
\hline Rat & $\begin{array}{l}\text { - Behavioral } \\
\text { abnormalities } \\
\text { - Loss of sense of } \\
\text { smell }\end{array}$ & $\begin{array}{l}\text { - Progressive loss of } \\
\text { dopaminergic neurons } \\
\text { - Striatal mitochondrial } \\
\text { dysfunction }\end{array}$ & Unknown & $\begin{array}{l}\text { High variation } \\
\text { of clinical } \\
\text { phenotype }\end{array}$ \\
\hline Mice & $\begin{array}{l}\text { - Reduced motor } \\
\text { activity }\end{array}$ & $\begin{array}{l}\text { - Selective and dose- } \\
\text { dependent loss of } \\
\text { dopaminergic neurons } \\
\text { in the substantia nigra }\end{array}$ & $\begin{array}{l}\text { - Up-regulation of } \alpha \text { - } \\
\text { synuclein } \\
\text { - Formation Lewy } \\
\text { body-like inclusions in } \\
\text { dopaminergic neurons } \\
\text { of substantia nigra }\end{array}$ & $\begin{array}{l}\text { High variation } \\
\text { of clinical } \\
\text { phenotype }\end{array}$ \\
\hline Flies ${ }^{263}$ & $\begin{array}{l}\text { - Dose-dependent } \\
\text { motor impairments } \\
\text { - Dose-dependent } \\
\text { lifespan reduction } \\
\text { - Response to L-dopa }\end{array}$ & $\begin{array}{l}\text { - Selective loss of } \\
\text { dopaminergic neurons } \\
\text { - Increased catalase } \\
\text { activity }\end{array}$ & Unknown & \\
\hline Zebrafish & $\begin{array}{l}\text { Altered swimming } \\
\text { behavior }\end{array}$ & $\begin{array}{l}\text { Loss of dopamine } \\
\text { neurons in a dose- } \\
\text { dependent manner }\end{array}$ & Unknown & \\
\hline
\end{tabular}

\section{Paraquat}

The results of epidemiological studies suggested that exposure to an herbicide called paraquat (Fig. 1.6) is associated with an increased risk of developing Parkinson's disease in human. Several animal models addressing the toxicity of paraquat have been established in various species including rats, mice, Drosophila and others (Table 1.9)248, 249, 254, 263. In mammals, paraquat crosses the blood brain barrier via L-amino acid transporter; the passive transport of toxin across the blood brain barrier is not possible. In general, the administration of rotenone leads to an MPTP-like phenotype, namely, a selective loss of dopaminergic neurons, oxidative stress, locomotor abnormalities and formation of $\alpha$-synuclein-positive inclusions ${ }^{248}$. In contrast to the MPTP models, the exposure to paraquat causes up-regulation of $\alpha$-synuclein and progressive formation of $\alpha$-synuclein positive Lewy body-like inclusions in dopaminergic neurons of the substantia nigra pars compacta. For example, in rats the ip injections of paraquat result in progressive loss of TH-positive neurons, behavioral abnormalities and striatal mitochondrial dysfunction ${ }^{264,265}$. Although paraquat is structurally similar to MPP+, several lines of evidence support a different mechanism of toxicity. Thus, it has been demonstrated that paraquat might cause oxidative stress and mitochondrial dysfunction not by inhibition of mitochondrial complex I (weak inhibition of respiratory 
chain by paraquat is observed in vivo), but rather by redox cycling, which generates ROS and eventually leads to oxidative modifications of biomolecules ${ }^{254}$. Furthermore, paraquat interferes with the normal redox cycle of glutathione by shifting the equilibrium to the oxidized form of glutathione and subsequently reducing the efficacy of intracellular antioxidant defense $\mathrm{e}^{248}$. The main drawbacks of this model are controversial in vivo results and not fully established mechanism of action.

\section{LPS}

Neuroinflammation in the brain that is represented by activated microglia or astrocytes is one of the common features of Parkinson's disease in humans. Normally, the microglial cells are activated as a response to some changes of brain microenvironment. When activated, microglia starts to produce pro-inflammatory molecules (ROS, chemokines, nitric oxide and others) that are involved in clearing of toxic species ${ }^{253}$. On the other side, the dysregulation of microglia activation and permanent neuroinflammation could lead to oxidative damage of neurons specifically in case of the dopaminergic neurons that are vulnerable to oxidative stress. The inflammation-mediated neurodegeneration was investigated in rodent models that are based on application of a bacterial endotoxin lipopolysaccharide (LPS) ${ }^{266}$. So far, several protocols for LPS intoxication in rodents have been developed including chronic and acute intracerebral injections; acute systemic intraperitoneal injection. Generally, LPS exposure induces rapid microglia activation and neuroinflammation, followed by delayed, progressive, selective degeneration of dopaminergic neurons and dopamine deficiency ${ }^{266}$. Thus, in rats the chronic infusion of LPS to substantia nigra via an osmotic pump (2 weeks) leads to progressive neuronal loss, namely $10 \%$ loss after 2 weeks and $60 \%$ loss after 10 weeks. In case of systemic acute exposure to LPS in mice, the reduction in the number of dopaminergic neurons of $23 \%$ and $43 \%$ was observed after 7 and 10 months, respectively. Of note, there is no Lewy body pathology detected in the brains of LPS-lesioned animals ${ }^{266}$. Collectively, the LPS-based animal models are useful tools to investigate the role of the inflammation in neurodegeneration and to validate novel therapeutic strategies.

\subsubsection{Genetic models: Introduction}

As it has been already discussed above, about $10 \%$ of Parkinson's disease cases are associated with genetic defects of certain genes. To date, more than 10 genes have been implicated in familiar forms of Parkinson's disease. Based on these discoveries and an assumption that both familial and sporadic types of Parkinson's disease share similar pathological mechanisms, a wide range of different genetic animal models have been 
developed. Similar to the toxin-induced models, there is no ideal genetic animal model of Parkinson's disease that fully recapitulates the key clinical and neuropathological features of the disorder. However, the intensive investigation of gene-related phenotypes have led to advances in our understanding of possible pathological molecular pathways that could be common for familiar as well as for sporadic forms of Parkinson's disease. At the same time, these models provide us a useful platform for finding new therapeutic targets and for testing novel treatment approaches.

The available genetic animal models could be classified into 3 main groups according to the type of genes and delivery methods. The major group is represented by overexpression or knockout models (etiologic models), in which the genetic manipulations are focused on the genes associated with the familiar forms of Parkinson's disease. Second, the animal models rely on the deletion of the genes that are involved in development and function of dopaminergic neurons. And the third group of genetic animal models is based on overexpression of the genes using the viral vectors that can transduce the cells. In addition to different genes and delivery methods, a variety of model organisms has been used for in vivo investigation of the function of genes and proteins that are implicated in Parkinson's disease. For instance, the genetic models of Parkinson's disease have been developed in mice, rats, anurans, Drosophila melanogaster, C. elegans, yeast and others. Currently, the mouse is the preferred species for genetic models due to established protocols for genetic manipulations and relatively short lifespan (2 years). Therefore, in this chapter I predominantly focus on the genetic models of Parkinson's disease in mouse, but some applications of other species are also shortly summarized.

\subsubsection{Genetic etiologic models}

\section{$\alpha-S y n u c l e i n$}

In a pioneering work describing a genotype analysis of Italian kindred with autosomal dominant early-onset familiar form of Parkinson's disease, a single substitution of a nuclear base pair at position 209 from $\mathrm{G}$ to $\mathrm{A}$ in PARK1 gene has been shown to result in expression of mutated $\alpha$-synuclein carrying the A53T mutation. Few years later, two additional mutations (A30P and E46K) in the PARK1 gene have been linked to familiar Parkinson's disease. Moreover, genomic multiplications (duplication/triplication) of the wild type $\alpha$-synuclein gene (PARK1) have been demonstrated to cause hereditary early-onset Parkinson's disease. Clinically, $\alpha$-synuclein-related familiar forms of Parkinson's disease are characterized by early onset and, more aggressive progression and clinical pathology. The presence of $\alpha$ - 
synuclein in Lewy bodies and neurites as a main component is clearly emphasizing the link between sporadic and familiar forms of the disease, as well as the importance of $\alpha$-synuclein as one of the key factor implicated in pathological pathways. All these crucial findings therefore clearly facilitated the development of $\alpha$-synuclein-related genetic animal models that were supposed to provide us insight into the possible pathological molecular pathways and new therapeutic targets.

To date, the genetic models based on overexpression of wild type or pathogenic mutant forms of $\alpha$-synuclein have been generated in various species including mice, Drosophila melanogaster, C. elegans, yeast, zebrafish and others (Table 1.10). Overall, many $\alpha$-synuclein-related models exhibit a number of key pathological features of Parkinson's disease, namely presence of aggregated forms of $\alpha$-synuclein, functional abnormalities in the nigrostriatal system, behavioral phenotype, but at the same time, limited neuronal loss is generally observed. The relevance of the connection between $\alpha$-synuclein and observed pathology corroborated by the findings shows that higher expression levels result in more severe phenotype and that mutant forms of $\alpha$-synuclein are more toxic than wild type protein.

In invertebrates, the $\alpha$-synuclein overexpression models have been developed in Drosophila melanogaster and C. elegans. Despite absence of clear $\alpha$-synuclein orthologous genes in $C$. elegans, several lines of transgenic worms expressing wild type or mutant $\alpha$ synuclein under control of various promoters have been generated and phenotypically characterized $^{249,267,268}$. Of note, the utilization of tissue specific promoters allows scientist to investigate function or impact of the protein in certain types of the cell, in which selective gene expression is initiated. In general, varying from promoter to promoter the overexpression of wild type or mutant (A30P or A53T) $\alpha$-synuclein in C. elegans leads to loss of dopaminergic neurons, accumulation of $\alpha$-synuclein often without formation of inclusions; locomotor decline, mitochondrial abnormalities, dopamine deficiency and impaired foodsensing behavior. For instance, a transgenic C. elegans line expressing various $\alpha$-synuclein constructs (wild type, A30P, A53T) in 4 pairs of dopaminergic neurons under control of the dopamine transporter promoter (dat-1) shows significant and non-progressive loss of dopaminergic neurons and corresponding markers including dopamine, diffuse $\alpha$-synuclein accumulation without formation of fibrils, motor deficits and abnormal food-sensing behavior that could be rescued by dopamine ${ }^{267,269}$. The C. elegans models based on pan-neuronal (expression in almost all types of neurons) or motorneuron-specific overexpression exhibit mild neurodegeneration, motor defects, in some cases certain signs of mitochondrial dysfunction and massive $\alpha$-synuclein accumulation without formation of fibrillar aggregates 
or inclusions ${ }^{267,268}$. Remarkably, a comparison of the phenotypes is clearly demonstrating an increased toxicity of mutant forms of $\alpha$-synuclein and, therefore, supporting the relevance of this model, but at the same time the lack of aggregation could be considered as a drawback.

Table 1.10. Properties of available $\alpha$-synuclein non-mammalian models

\begin{tabular}{|c|c|c|c|}
\hline 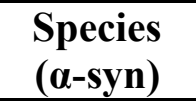 & $\begin{array}{l}\text { Behavioral } \\
\text { symptoms }\end{array}$ & Neuropathology & $\alpha$-Synuclein \\
\hline $\begin{array}{c}\text { Drosophila } \\
\text { (WT, A30P, } \\
\text { A53T) }\end{array}$ & $\begin{array}{l}\text { - Dopamine-responsive } \\
\text { locomotor deficits (climbing } \\
\text { test) } \\
\text { - Response to L-dopa } \\
\text { - Early mortality }\end{array}$ & $\begin{array}{l}\text { - Selective and progressive } \\
\text { loss of dopaminergic neurons } \\
\text { - Mitochondrial dysfunction } \\
\text { - Impaired ER-Golgi } \\
\text { trafficking }\end{array}$ & $\begin{array}{l}\text { - } \alpha \text {-Synuclein-positive } \\
\text { widespread inclusions } \\
\text { - Oligomerization and } \\
\text { pathological } \\
\text { posttranslational } \\
\text { modification of } \alpha \text { - } \\
\text { synuclein }\end{array}$ \\
\hline $\begin{array}{c}\text { C. elegans } \\
(\mathrm{WT}, \mathrm{A} 30 \mathrm{P}, \\
\text { A53T) }\end{array}$ & $\begin{array}{l}\text { - Locomotor deficits } \\
\text { - Response to dopamine } \\
\text { - Impaired food-sensing } \\
\text { behavior }\end{array}$ & $\begin{array}{l}\text { - Non-progressive loss of } \\
\text { dopaminergic neurons } \\
\text { - Dopamine depletion } \\
\text { - Mitochondrial abnormalities }\end{array}$ & $\begin{array}{l}\text { Massive } \alpha \text {-synuclein } \\
\text { accumulation without } \\
\text { formation of fibrillar } \\
\text { aggregates or inclusions }\end{array}$ \\
\hline $\begin{array}{c}\text { Zebrafish } \\
\text { (WT) }\end{array}$ & $\begin{array}{l}\text { - Mild phenotype with } \\
\text { respect to morphology and } \\
\text { lethality (CREST3 enhancer) } \\
\text { - High rate of lethality and } \\
\text { morphological abnormalities } \\
\text { (Huc promoter) }\end{array}$ & $\begin{array}{l}\text { - Moderate neuronal loss } \\
\text { - Progressive axonopathy } \\
\text { - Abnormal mitochondrial } \\
\text { morphology and transport } \\
\text { - UPS inhibition }\end{array}$ & $\begin{array}{l}\text { Abundant } \alpha \text {-synuclein- } \\
\text { positive inclusions (Huc } \\
\text { promoter) }\end{array}$ \\
\hline
\end{tabular}

Transgenic Drosophila models relying on pan-neuronal or site-specific $\alpha$-synuclein overexpression are the first models, in which the formation of $\alpha$-synuclein-positive widespread inclusions was found ${ }^{268}$. In most of the Drosophila models, the expression of different $\alpha$-synuclein constructs is driven by the GAL4/UAS system containing different promoters $^{270}$. Although there is no ideal model of Parkinson's disease, $\alpha$-synuclein transgenic flies recapitulate a number of key pathological features of Parkinson's disease, namely agedependent and selective loss of dopaminergic neurons, accompanied by progressive and widespread $\alpha$-synuclein deposition and dopamine-responsive locomotor deficits (climbing test $)^{269-271}$. For example, the age-dependent neurodegeneration in $\alpha$-synuclein transgenic flies is illustrated by a progressive loss of TH-positive dorsomedial neurons as follows: $5 \%, 15 \%$ and $70 \%$ loss at day 5, 10 and 20 respectively $^{272}$. Furthermore, the pathological posttranslational modifications (phosphorylation of S129 and ubiquitination) of $\alpha$-synuclein that are prominent in human Parkinson's disease have been found in these Drosophila models ${ }^{272}$. Several studies have also demonstrated a reduced lifespan and retina degeneration. Similar to C. elegans, the phenotypic outcome remarkably depends on the $\alpha$-synuclein construct being 
expressed, namely the pathogenic mutants are associated with more severe pathological as well as behavioral phenotypes. Multiple studies have focused on the possible mechanisms of $\alpha$-synuclein toxicity; so far, mitochondrial dysfunction, oxidative stress, impaired ER-Golgi trafficking, oligomerization and posttranslational modification of $\alpha$-synuclein have been implicated in pathogenesis ${ }^{269,271}$.

Recently, a new genetic model of Parkinson's disease has been developed in zebrafish. In this model, the application of GAL4/UAS system with a tissue-specific promoter leads to a robust expression of human wild type $\alpha$-synuclein in peripheral sensory neurons and coexpression of GFP for in vivo imaging. In this study, a mild phenotype with respect to morphology and lethality is observed, whereas in vivo time-lapse imaging has revealed a progressive axonopathy, moderate neuronal loss and abnormal mitochondrial morphology and impaired mitochondrial transport in $\alpha$-synuclein-expressing axons ${ }^{273}$. The second zebrafish model that is based on neuronal expression of human wild type $\alpha$-synuclein under control of Huc promoter. This genetic zebrafish line develops a severe phenotype characterized by a high rate of lethality and morphological abnormalities that are associated with dysfunction and death of crucial neurons. Addressing the underlying mechanisms of neurotoxicity, the authors have demonstrated a formation of abundant $\alpha$-synuclein-positive inclusions and inhibition of UPS in $\alpha$-synuclein-expressing neurons ${ }^{274}$.

Several arguments, including the recognition of $\alpha$-synuclein as one of the key factor implicated in the pathological processes and anatomical and physiological similarities between mice and humans, are strongly corroborating the notion that transgenic $\alpha$-synuclein mouse models are potentially the most relevant models of Parkinson's disease. Thus, much effort has been invested in development and phenotypic characterization of $\alpha$-synuclein-based overexpression models. Due to the particular importance of such transgenic models for investigation of mechanism/s of $\alpha$-synuclein-induced neuropathology and validation of novel therapeutic approaches, the overview of properties as well as advantages/disadvantages of published models is more comprehensive.

According to the literature, $\alpha$-synuclein transgenic mice are among the most frequently used genetic animal models of Parkinson's disease. Currently, numerous lines of mice expressing wild type or mutant forms of $\alpha$-synuclein under control of various promoters have been reported (Table 1.11) 255, 268, 275-277. Similar to other species, the behavioral and neuropathological phenotypes significantly depend on form of $\alpha$-synuclein and type of promoter being used for overexpression. Soon after development of transgenic mouse model overexpressing human wild type $\alpha$-synuclein under control of PDGF $\beta$ or Thy1 promoters in 
2000, a variety of promoters, including PrP, PLP, TH, CaMKII $\alpha$, were utilized to drive the expression of wild type and mutant forms of $\alpha$-synuclein.

Table 1.11. Properties of available $\alpha$-synuclein transgenic mouse models

\begin{tabular}{|c|c|c|c|}
\hline $\begin{array}{c}\text { Promoter/ } \\
\text { a-Syn }\end{array}$ & $\begin{array}{l}\text { Behavioral } \\
\text { symptoms }\end{array}$ & Neuropathology & $\alpha-S y n u c l e i n$ \\
\hline $\begin{array}{l}P D G F \boldsymbol{B} / \\
\text { WT } \alpha-S y n\end{array}$ & $\begin{array}{l}\text { - Mild motor deficits (12 } \\
\text { months of age) }\end{array}$ & $\begin{array}{l}\text { - No loss of dopaminergic } \\
\text { neurons } \\
\text { - 25-50\% dopamine depletion } \\
\text { - Reduced TH levels in striatum }\end{array}$ & $\begin{array}{l}\text { - } \alpha \text {-Synuclein/ubiquitin-positive } \\
\text { widespread inclusions including } \\
\text { SN ( } 3 \text { months of age) } \\
\text { - Increased S129 phosphorylation } \\
\text { of } \alpha \text {-synuclein }\end{array}$ \\
\hline $\begin{array}{c}\text { PDGF } \boldsymbol{\beta} / \\
\text { A53T } \alpha-\text { Syn }\end{array}$ & $\begin{array}{l}\text { - Progressive motor } \\
\text { deficits }\end{array}$ & $\begin{array}{l}\text { - No loss of dopaminergic } \\
\text { neurons }\end{array}$ & $\begin{array}{l}\text { - Limited amount of } \alpha \text {-synuclein- } \\
\text { positive inclusions } \\
\text { - } \alpha \text {-Syn oligomeric aggregates }\end{array}$ \\
\hline $\begin{array}{c}\boldsymbol{P r P} / \\
\text { WT } \alpha-\text { Syn } \\
\text { A30P } \alpha-\text { Syn }\end{array}$ & No & $\begin{array}{l}\text { No for WT } \alpha \text {-Synuclein } \\
\text { Very limited for A30P } \alpha \text { - } \\
\text { Synuclein }\end{array}$ & $\begin{array}{l}\text { Increased } \alpha \text {-synuclein levels, but } \\
\text { no aggregation }\end{array}$ \\
\hline $\begin{array}{c}\boldsymbol{P r P} / \\
\text { A53T } \alpha-S y n\end{array}$ & $\begin{array}{l}\text { - Severe motor } \\
\text { phenotype with } \\
\text { paralysis ( } 8 \text { months of } \\
\text { age } \\
\text { - Early mortality }\end{array}$ & $\begin{array}{l}\text { - Pathology in the spinal cord, } \\
\text { brain stem and cortex } \\
\text { - Astrogliosis and } \\
\text { mitochondrial abnormalities } \\
\text { - Intact substantia nigra }\end{array}$ & $\begin{array}{l}\text { - LB-like fibrillar cytoplasmic } \\
\text { inclusions ( } 6-8 \text { months of age) } \\
\text { throughout central nervous system }\end{array}$ \\
\hline $\begin{array}{c}\text { Thy1/ } \\
\text { WT } \alpha-\text { Syn }\end{array}$ & $\begin{array}{l}\text { - Early onset impaired } \\
\text { coordination and motor } \\
\text { performance (1-2 } \\
\text { months) } \\
\text { - Non-motor symptoms }\end{array}$ & $\begin{array}{l}\text { - Degeneration of motor } \\
\text { neurons in the spinal cord } \\
\text { - Gliosis } \\
\text { - No loss of dopaminergic } \\
\text { neurons }\end{array}$ & $\begin{array}{l}\text { - LB-like } \alpha \text {-synuclein inclusions in } \\
\text { many brain regions (incl. } \mathrm{SN} \text { ) }\end{array}$ \\
\hline $\begin{array}{c}\text { Thyl/ } \\
\text { A30P } \alpha-S y n\end{array}$ & $\begin{array}{l}\text { - Progressive motor } \\
\text { impairments }\end{array}$ & $\begin{array}{l}\text { - Degeneration of motor } \\
\text { neurons in the spinal cord } \\
\text { - Astrogliosis and abnormal } \\
\text { neuronal morphology } \\
\text { - Intact substantia nigra }\end{array}$ & $\begin{array}{l}\text { - Age-dependent ( } 8 \text { months onset) } \\
\alpha \text {-synuclein pathology in the spinal } \\
\text { cord, brainstem } \\
\text { - Pathological } \alpha \text {-synuclein } \\
\text { posttranslational modifications }\end{array}$ \\
\hline $\begin{array}{c}\text { Thyl/ } \\
\text { A53T } \alpha-S y n\end{array}$ & \multicolumn{3}{|c|}{$\begin{array}{l}\text { Similar phenotype to A30P/Thy } 1 \text { model, but more severe. Motor phenotype - onset } 1 \text { month of } \\
\text { age, } \alpha \text {-synuclein pathology - onset } 3 \text { month of age; Intact substantia nigra }\end{array}$} \\
\hline $\begin{array}{c}\boldsymbol{T H} / \\
\text { WT } \alpha-S y n\end{array}$ & No & - Microglial activation & $\begin{array}{l}\text { Abnormal accumulation of } \alpha- \\
\text { synuclein in dopaminergic neurons } \\
\text { (9-12 months of age) }\end{array}$ \\
\hline $\begin{array}{l}\boldsymbol{T H} / \\
\text { A53T } \alpha-S y n, \\
\text { A30P } \alpha-S y n\end{array}$ & $\begin{array}{l}\text { Progressive motor } \\
\text { impairments }\end{array}$ & $\begin{array}{l}\text { - Dopamine deficiency } \\
\text { - Mitochondrial dysfunction } \\
\text { - No neuronal loss }\end{array}$ & No $\alpha$-synuclein pathology \\
\hline $\begin{array}{c}\boldsymbol{T H} / \\
\text { A30P, A53T } \\
\alpha-\text { Syn }\end{array}$ & $\begin{array}{l}\text { Severe progressive } \\
\text { motor phenotype }\end{array}$ & $\begin{array}{l}\text { - Nigrostriatal pathology } \\
\text { - Dysregulation of dopamine } \\
\text { turnover } \\
\text { - Inflammation }\end{array}$ & Occasional $\alpha$-synuclein inclusions \\
\hline $\begin{array}{l}\text { CaMKIIL/ } \\
\text { WT } \alpha-S y n\end{array}$ & $\begin{array}{l}\text { Progressive motor and } \\
\text { learning skill } \\
\text { impairments ( } 7-8 \\
\text { months of age) }\end{array}$ & $\begin{array}{l}\text { - Abnormal neuronal } \\
\text { morphology } \\
\text { - Disturbed neurogenesis } \\
\text { - 20-30\% loss TH-positive } \\
\text { neurons }\end{array}$ & No $\alpha$-synuclein pathology \\
\hline
\end{tabular}


Overexpression of human wild type $\alpha$-synuclein under control of PDGF $\beta$ promoter (pan-neuronal promoter) in mice results in robust expression of the transgene in many areas of the brain $276,278,279$. Thus, the accumulation of $\alpha$-synuclein has been observed in neocortex, limbic system and olfactory regions. In these transgenic mice first signs of pathology were prominent at 2-3 months of age and characterized by a formation of intraneuronal $\alpha$ synuclein/ubiquitin-positive inclusions in neocortex, hippocampus and substantia nigra. Despite minimal neurodegeneration in the brain, a range of pathological changes has been demonstrated including nerve terminal degeneration in the basal ganglia, reduced levels and activity of tyrosine hydroxylase and progressive transcriptional dysregulation. Behaviorally, 1-year old transgenic animals developed mild-to-moderate motor deficits (rotarod) that were correlated with dopamine deficiency. For instance, 12-months-old transgenic mice exhibited $50 \%$ reduction of $\mathrm{TH}$-positive terminals in the striatum, at the same time the rotarod performance of these animals dropped by $40 \%$. The second transgenic model relying on PDGF $\beta$ promoter is based on the expression of A53T mutant of human $\alpha$-synuclein ${ }^{276}$. The overall phenotype has been found to be more severe in comparison with wild type $\alpha$ synuclein. Thus, these transgenic mice showed accumulation of A53T $\alpha$-synuclein in synapses and neurons, development of progressive motor impairments and neurodegeneration. Despite the expression of comparable levels of A53T $\alpha$-synuclein, very limited amount of $\alpha$-synuclein-positive inclusions were found, while the proportion of oligomeric $\alpha$-synuclein species was significantly increased in the A53T model, thereby suggesting a role of $\alpha$-synuclein oligomers in neuronal toxicity.

Several groups have developed the transgenic mouse models, in which the panneuronal promoter PrP controls the expression of wild type or mutant forms of human $\alpha-$ synuclein $^{275,276,280-282}$. Analysis of the expression profile revealed a robust (3-15 fold) and broad expression in all brain regions, brainstem and spinal cord. $\alpha$-Synuclein was detected at high concentrations mainly in the neurons, but also glial cells contained low levels of the protein. From many transgenic lines expressing wild type, A53T or A30P $\alpha$-synuclein under control of PrP promoter, only A53T mice displayed a severe phenotype with dramatic motor impairment, pathological protein accumulation and premature lethality ${ }^{280-282}$. One of the essential features of A53T mice is a progressive and widespread $\alpha$-synuclein pathology characterized by formation of $\alpha$-synuclein-positive fibrillar cytoplasmic inclusions with first appearance at 6-8 months of age, notably, before onset of locomotor symptoms. Abnormal $\alpha-$ synuclein accumulation that is mainly observed in neuronal cell bodies and neurites of midbrain, cerebellum, brainstem and spinal cord was followed by neurodegeneration of 
certain neuronal populations and astrogliosis in the affected areas. The motor impairments including partial paralysis, bradykinesia, ataxia, gait disturbances, dystonia were developed starting from 8 months of age and rapidly progressed leading to severe paralysis and death, presumably the dramatic paralysis is associated with the spinal cord pathology. The relevance of A53T mice is also corroborated by the presence of ubiquitin in $\alpha$-synuclein-positive inclusions and also by signs of mitochondrial dysfunction that are known to be part of the pathology in human Parkinson's disease. One of the major disadvantages of this A53T/PrP model is an intact substantia nigra and nigrostriatal dopaminergic system. Thus, no experimental evidences of neurodegeneration, $\alpha$-synuclein pathology and dopamine or tyrosine hydroxylase deficiency were found in these transgenic animals. Although the A53T overexpression model shows this quite severe phenotype, a similar expression of wild type or A30P form of $\alpha$-synuclein does not lead to pathological changes in mice up to 24 months of age $^{282}$. Just A30P animals exhibited limited $\alpha$-synuclein accumulation without significant proportion of detergent insoluble $\alpha$-synuclein and co-localization with ubiquitin.

The next type of transgenic mouse model is based on utilization of central neuronal system specific promoter called Thy1. Both wild type and mutant $\alpha$-synuclein (A53T or

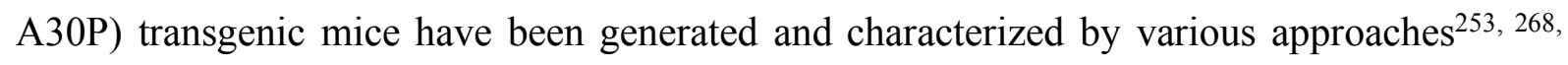
275, 279, 283, 284. In general, the $\alpha$-synuclein gene expression under transcriptional control of Thy1 promoter led to high expression levels in the neurons of central nervous system, in some mouse lines including substantia nigra. Phenotypically, these transgenic animals showed early-onset progressive motor and sensorimotor abnormalities, the severity of which was correlated with the $\alpha$-synuclein expression levels. Furthermore, the behavioral impairments were accompanied by progressive $\alpha$-synuclein pathology, neurodegeneration of certain neuronal populations and several others pathological changes. In 2002, the publication describing the phenotype of transgenic mice expressing full-length human $\alpha$-synuclein was published $^{276,279}$. These animals developed progressive $\alpha$-synuclein pathology as evidenced by the formation of LB-like PK-resistant inclusions and an age-dependent increase of S129 hyperphosphorylated/oxidized/ubiquitinated $\alpha$-synuclein in affected brain regions. $\alpha$ synuclein deposits have been found in many subcortical regions, olfactory bulb, spinal cord, brainstem and in some cases in the substantia nigra. Despite quite late appearance of inclusions, the animal displayed early-onset ( 2 months of age) progressive behavioral phenotype characterized by impaired coordination and motor performance, sensorimotor dysfunction and, notably, non-motor symptoms including olfactory deficits and problems with digestion. Regarding neurodegeneration, no apparent signs of neuronal cell death or 
dopaminergic system damage have been found in the brain, but at the same time the denervation of neuromuscular junctions and axonal degeneration in spinal roots were observed. Thy1-based models relying on expression of mutant forms of human $\alpha$-synuclein (A53T or A30P) have similar, but more severe phenotype compared to wild type, and in most transgenic lines the A53T mutant is more toxic than A30P. The A30P/Thy1 model have been developed by Haass et $a l^{268,283}$, the homozygous animals with a high expression levels developed age-dependent ( 8 months onset) $\alpha$-synuclein pathology in the spinal cord, brainstem and also at the terminal stage in the cortex. The comprehensive investigation of the brain tissues revealed a remarkable spectrum of pathological markers highly reminiscent of abnormalities found in the human brain of Parkinson's disease patient. Namely, the $\alpha$ synuclein deposits were PK-resistant and ThioT S positive and had the immunoreactivity to S129 phosphorylated/oxidized/nitrated $\alpha$-synuclein and ubiquitin. Based of electron microscopy, $\alpha$-synuclein inclusions contained the characteristic 9- to $12-\mathrm{nm}$ filaments. The most affected brain areas also exhibited astrogliosis and abnormal neuronal morphology. Behaviorally, A30P/Thy-1 mice developed progressive motor phenotype; first symptoms were unsteady gait, tail posture, progressive deterioration of rotarod performance followed by the paralysis at the end stage. In contrast to human disease, the striatum and substantia nigra were spared from the pathology. Thus, the dopamine and metabolite levels were normal, no loss of dopaminergic neurons and no formation of LB-like $\alpha$-synuclein pathology were observed. These results argued for a regional specificity of $\alpha$-synuclein-induced toxicity in these mice and reasons for this selectivity remain to be clarified. Mice expressing A53T $\alpha-$ synuclein mutant under control of Thyl promoter showed a phenotype similar to the A30P/Thy1 model, but in more aggressive form ${ }^{275}$, 284. Upon examination, the LB-like pathology, that was found in several brain regions, brainstem and spinal cord, was accompanied by astrogliosis and microglia activation. The first $\alpha$-synuclein inclusions were apparent starting from 3 months of age and, remarkably, in older animals the pronounced immunostaining for ubiquitin was detected in affected regions. The behavioral studies revealed early onset (1 month) progressive motor impairments that were linked to a loss of motor neurons and denervation of neuromuscular junctions. Despite the more severe phenotype compared to other $\alpha$-synuclein/Thy1 models, the A53T/Thy1 mice also didn't develop the pathology in the nigrostriatal system, perhaps, due to the low $\alpha$-synuclein expression levels. Altogether, the transgenic mice models expressing different forms of $\alpha-$ synuclein under control of the Thy1 promoter recapitulate several essential features of early 
stages of Parkinson's disease and, therefore, they could be considered as a valuable tool to study $\alpha$-synuclein-related mechanisms of toxicity, and corresponding phenotypes.

In order to achieve a sustainable $\alpha$-synuclein concentration in the nigrostriatal system, transgenic mouse models expressing wild type or mutant forms of $\alpha$-synuclein under control of TH promoter have been generated $255,275,276,285$. Of note, $\mathrm{TH}$ promoter provides a selective gene expression in the dopaminergic neuron. The available transgenic models, expressing wild type $\alpha$-synuclein using TH promoter, exhibited a relatively mild phenotypes that might be explained by quite low gene expression levels. Upon examination, abnormal accumulation of $\alpha$-synuclein was observed in the neurons of the substantia nigra, but behavioral phenotype and neuronal loss were absent. In contrast to wild type $\alpha$-synuclein model, mice expressing A53T or A30P $\alpha$-synuclein mutant didn't develop the $\alpha$-synuclein pathology, however, progressive motor impairment, dopamine deficiency and mitochondrial dysfunction have been found in these transgenic models. Most severe behavioral and neurological phenotypes among TH-based mouse models have been shown in the animals expressing A53T/A30P double mutants ${ }^{276}$. Although the $\alpha$-synuclein pathology was occasional, the A53T/A30P models displayed progressive motor dysfunction and nigrostriatal pathology characterized by neuronal cell loss, dysregulation of dopamine turnover, inflammation and morphological changes in dopaminergic terminals. Several lines of evidence implied the truncated versions of $\alpha$-synuclein in the pathogenesis of human Parkinson's disease. Therefore, Spillantini et $a l .{ }^{285}$ have developed a mouse model, in which the 1-120 $\alpha$-synuclein construct is expressed in the brain under control of the TH promoter. The immunostaining analysis revealed $\alpha-$ synuclein localization in the nigrostriatal pathway and olfactory bulb. First $\alpha$-synucleinpositive inclusions were observed in the olfactory bulb in 3 months old animals, while in the substantia nigra the $\alpha$-synuclein pathology was found in at least 1-year-old mice; structurally, $\alpha$-synuclein deposits consisted of mixed granular and fibrillar material. Synaptic alterations, reduced dopamine release, dopamine deficiency and mild neurodegeneration contributed to nigrostriatal pathology in this transgenic model. Behaviorally, the aged animals (18 months) showed progressive reduction in spontaneous locomotor activity. Overall, the TH-based genetic model can recapitulate certain aspects of early stages of Parkinson's disease specifically in the nigrostriatal system and, consequently, they could be considered as a relevant test system for mechanistic/fundamental and pharmacological studies.

Another available transgenic mouse model of Parkinson's disease, in which the substantia nigra has a high level of $\alpha$-synuclein expression, is based on tetracycline-regulated tet-off system expressing human wild type $\alpha$-synuclein under the control of 
calcium/calmodulin-dependent protein kinase II $\alpha$ promoter ${ }^{286}$. The immunostaining and Western blot analysis of the brain tissues demonstrated a robust and widespread transgene expression profile covering midbrain and forebrain regions including, importantly, the substantia nigra. An interesting feature of this conditional model is the possibility to switch off the transgene transcription by oral application of doxycycline and, thereby, being able to investigate the underlying mechanisms of toxicity and disease progression. In these transgenic mice, despite prominent $\alpha$-synuclein accumulation in axons, dendrites and synaptic terminals, no evidences of LB-like inclusions or $\alpha$-synuclein insoluble aggregates were found. Although $\alpha$-synuclein pathology was absent, the investigation of the substantia nigra and other brain regions showed abnormal neuronal morphology, disturbed neurogenesis, significant number of degenerated neurons and $20-30 \%$ loss of TH-positive neurons. Furthermore, the 7-8 months old mice exhibited progressive motor and learning skill impairments accompanied by mild changes in the dopamine turnover and DAT binding. Of note, the turning off of the $\alpha-$ synuclein expression in aged animals didn't reverse the behavioral phenotype, but rather slowed down its progression rate. Aside from the lack of LB pathology, this conditional model mimics several key pathological events including neurodegeneration of dopaminergic neurons and progressive motor dysfunction and certainly needs to be included in the list of relevant model systems.

To summarize this part describing the $\alpha$-synuclein-based transgenic mouse models, the various mouse lines expressing $\alpha$-synuclein under control of different promoters have been generated and extensively characterized. Similar to other models, there is no ideal $\alpha$-synuclein model that fully recapitulates all pathological and behavioral features of complex human Parkinson's disease. The relevance of various $\alpha$-synuclein transgenic mice is supported by observations of pathological changes highly reminiscent of abnormalities in human brain including LB-like pathology, $\alpha$-synuclein post-translational modifications, locomotor impairments, dopamine deficiency and many others. Importantly, most of these phenotypic alterations occur in an age-dependent manner and in correlation with $\alpha$-synuclein concentration. Similar to human situation, the expression of the mutant forms of $\alpha$-synuclein in many instances results in more aggressive and severe phenotypes. The main drawbacks of these models are that very often neuronal loss is relatively mild or absent and, what is more important, no pathological changes are found in substantia nigra. Overall, the $\alpha$-synuclein transgenic models could be considered as good systems to investigate early stages of Parkinson's disease and to get insight into details of $\alpha$-synuclein function and its involvement in pathological processes. Aside from the fundamental studies, such animal models are the 
excellent tools for development and testing of therapeutic approaches that are aiming to stop or delay the progression of the disease at early stages.

Development and optimization of recombinant viral vectors and their in vivo delivery have facilitated an extensive application of this technique in many fields of biology and medicine, including animal disease models and gene therapy. The viral models have certain advantages over classical transgenic models, namely: 1) various mammalian species from rodent to non-human primates; 2) the local transgene delivery allows specific targeting of certain regions of interest; 3) control and regulation of expression levels by gene dose adjustment; 4) delivery into the brain by stereotactic injections, in case of unilateral injection the second hemisphere represents a reliable internal control and also asymmetric behavioral tests provide valuable information; 5) no overexpression during development; 6) fast model development and optimization due to the relatively easy manipulations with the virus. Main drawbacks are related to the moderate reproducibility of the results and technically difficult virus injections. Coming back to $\alpha$-synuclein-based animal models of Parkinson's disease, the viral vector-mediated models have been developed in mammals, including mice, rats and nonhuman primates, in which the $\alpha$-synuclein transgenes were delivered by stereotactic injections of viral vectors in substantia nigra. Considering the big brain volume and relatively low expenses, currently rats are the most commonly used animal species for viral animal models of Parkinson's disease.

At present, two vector systems, namely lentiviral vector (RNA as genetic material) and adeno-associated virus (rAAV, single-stranded DNA as genetic material), have been successfully implemented as an efficient gene delivery approach resulting in high transduction rates and a long-lasting neuronal gene expression. For instance, the injection of HIV-1-derived recombinant lentiviral vector into the substantia nigra resulted in $\alpha$-synuclein expression in up to $50 \%$ of dopaminergic neurons ${ }^{287}$. Furthermore, AAV vectors exhibited even higher transducing efficiency that is reaching up to $80-90 \%$ of dopaminergic neurons ${ }^{287}$. Similar to the mouse transgenic models, the toxicity of various human $\alpha$-synuclein constructs comprising wild type, A30P, A53T, S129D, S129A and others delivered by viral vectors into the nigrostriatal pathway have been comprehensively characterized in rats $255,287,288$.

In general, the rats injected with the virus vectors encoding human wild type or mutant forms of $\alpha$-synuclein in the substantia nigra showed high levels of $\alpha$-synuclein expression and developed progressive, robust phenotypes characterized by abnormal $\alpha$-synuclein accumulation/aggregation, loss of dopaminergic neurons, striatal denervation, depletion of dopamine and other markers, disturbed neuronal morphology and, in some cases, behavioral 
phenotype $^{288}$. Thus, multiple studies utilizing the rAAV-based delivery approach have demonstrated a rapid neuronal loss up to $80 \%$ accompanied by formation of $\alpha$-synuclein deposits. For example, Kirik et al. have demonstrated $23 \%$ and $55 \%$ reduction of TH-positive neurons in the substantia nigra after unilateral injection of rAAV coding wild type or A53T mutant $\alpha$-synuclein at 3 and 8 weeks, respectively ${ }^{288}$. Furthermore, a significant reduction of dopamine levels $(27 \%$ at 3 weeks, $41 \%$ at 8 weeks) and $\mathrm{TH}$ activity (40-50\% at all time points) in substantia nigra were observed for both $\alpha$-synuclein constructs. Importantly, the animals in which the neuronal loss exceeded 50-60\% exhibited impaired behavior in apomorphine rotation (at 8 weeks) and paw-reaching (at 24 weeks) tests ${ }^{288}$. Another example is a rat model created by Kügler et al., in which intranigral injections of rAAV vectors encoding wild type or A30P mutant $\alpha$-synuclein were used to induce the pathological phenotype $^{289}$. Histological examinations that were done at 2, 4, 8 and 14 weeks after injections demonstrated a long-lasting progressive degeneration of dopaminergic neurons, axonal pathology and formation of PK-resistant intraneuronal $\alpha$-synuclein inclusions in both groups, although wild type $\alpha$-synuclein resulted in more severe phenotype. For instance, the expression of wild type $\alpha$-synuclein produced $43 \%, 50 \%, 56 \%$ and $70 \%$ loss in VMAT2positive cells in substantia nigra at 2, 4, 8 and 14 weeks post-injections, respectively, while at the same time points A30P animals showed 22\%, 27\%, 31\% and 39\% neurodegeneration. The evaluation of the brain tissues by immunohistochemistry revealed a formation of PK resistant $\alpha$-synuclein aggregates as early as 2 weeks post-injections for wild type $\alpha$-synuclein rats and at 4 weeks for A30P group. The number of $\alpha$-synuclein aggregates was twice higher in the animals expressing wild type form in comparison with A30P rats. These observations highlighted the correlation of the neurotoxicity with the plaque load.

In line with data from AAV-based rat models, similar neuropathological changes were found in the substantia nigra after transduction by lentiviral vectors expressing wild type or mutant (A30P or A53T) forms of $\alpha$-synuclein. In rats, Lo Bianco et al. reported 35\%, 33\% and $24 \%$ loss of dopaminergic neurons in the substantia nigra at 6 months after lentiviral injections for wild type, A30P and A53T $\alpha$-synuclein constructs correspondingly $287,288$. Despite various extent of neurodegeneration, the LB-like pathology, confirmed by the formation of cytoplasmic granular $\alpha$-synuclein-positive inclusions both in neurites and cell bodies of substantia nigra neurons, was similar in all groups of animals. Overall, viral vectormediated rat models have emerged as a valuable system for studying of mechanisms of $\alpha$ synuclein-related neurotoxicity and evaluation of therapeutic approaches. These models mimic certain pathological events associated with early stages of Parkinson's disease in a 
progressive and dose-dependent manner, however, the relatively high expression levels and weak behavioral phenotypes are one of the main limitations.

\section{LRRK2}

Mutations in the leucine-rich repeat kinase 2 (LRRK2, PARK8) gene are the most common cause of late-onset autosomal dominant inherited form of Parkinson's disease. To date, at least 6 mutations are associated with Parkinson's disease, whereas G2019S and $\mathrm{R} 1441 \mathrm{C} / \mathrm{G} / \mathrm{H}$ has been shown to be most frequent ${ }^{290}$. Clinically, LRRK2-associated Parkinson's disease is indistinguishable from idiopathic form. LRRK2 is a multi-domain protein and, importantly, the pathogenic mutations are located in GTPase (R1441C/G/H) and kinase (G2019S, I2020T) domains ${ }^{255}$. Although the exact mechanism of LRRK2-related toxicity is unknown, several lines of evidence support the hypothesis that mutations in PARK8 gene belong to the gain-of-function class. Thus, an enhanced kinase activity has been demonstrated for LRRK2 G2019S mutant. Identification of eukaryotic initiation factor 4E binding protein (4E-BP) as a potential substrate for LRRK2 supports a hypothesis that LRRK2 mutants may induce the toxicity by hyperphosphorylation of certain proteins that play a crucial role in different cellular processes. In case of 4E-BP that is involved in protection system against oxidative and unfolded protein stresses, the hyperphosphorylation by LRRK2 mutant results in inactivation of 4E-BP and subsequently in reduction of protein translation $^{271}$.

Different types of genetic LRRK2 models based on overexpression, knock-in (KI) and knockout (KO) approaches have been developed for various species, including mice, Drosophila melanogaster, C. elegans, yeast, zebrafish (Table 1.12) $66,250,271,291$. In invertebrates (Drosophila melanogaster, C. elegans), the overexpression of wild type or pathogenic mutant forms of LRRK2 result in age-dependent selective loss of dopaminergic neurons, reduction of locomotor activity and early mortality. The locomotor impairments in these models could be reversed by dopamine replacement treatment, while the exposure to environmental toxins or the expression of pathogenic mutant forms of LRRK2 amplify the observed phenotypes ${ }^{66,271}$. The investigation of LRRK2 function in KO invertebrate models has demonstrated that LRRK2 plays a minor role in development and function of dopaminergic neurons and, thereby, supporting the gain-of-function principle ${ }^{250}$.

In contrast to invertebrate models, available LRRK2 mouse models have been shown to develop mild variable phenotypes ${ }^{250,255,291}$. Overall, the overexpression of mutant LRRK2 leads to mild nigrostriatal pathology, dopamine dysregulation, abnormal phosphor-tau levels and weak behavioral phenotypes ${ }^{291}$. For instance, transgenic LRRK2-S2019S mice exhibit a 
mild loss of dopaminergic neurons in substantia nigra and late-onset locomotor abnormalities. The mice model based on the LRRK2-R1441G mutant shows progressive, age-dependent locomotor dysfunction that could be restored by dopaminergic treatment, but without significant neurodegeneration ${ }^{250,255}$. The relevance and possible application of these models are limited due to the absence of prominent neurodegeneration and Lewy body pathology, and high variability of phenotypes. Perhaps, the LRRK2 rodent models may mimic the early stages of Parkinson's disease and therefore, it could be a useful tool to identify and investigate mechanisms of pathological processes preceding the death of dopaminergic neurons, interactions with the environmental toxins and effects of LRRK2 inhibitors.

Table 1.12. Properties of available LRRK2 animal models

\begin{tabular}{|c|c|c|c|c|}
\hline Species & $\begin{array}{c}\text { Behavioral } \\
\text { symptoms }\end{array}$ & Neuropathology & $\alpha$-Synuclein & Disadvantages \\
\hline $\begin{array}{c}\text { Mice } \\
(\mathrm{G} 2019 \mathrm{~S}, \\
\mathrm{R} 1441 \mathrm{C}, \\
\mathrm{I} 2020 \mathrm{~T})\end{array}$ & $\begin{array}{l}\text { - Mild motor } \\
\text { dysfunction } \\
\text { - Response to L-dopa }\end{array}$ & $\begin{array}{l}\text { - Minimal or mild } \\
\text { neurodegeneration } \\
\text { - Increased Tau } \\
\text { phosphorylation } \\
\text { - Altered nigrostriatal } \\
\text { dopaminergic } \\
\text { neurotransmission }\end{array}$ & $\begin{array}{l}\text { No } \alpha \text {-Synuclein } \\
\text { pathology }\end{array}$ & $\begin{array}{l}\text { - High variability } \\
\text { of phenotypes } \\
\text { - Subtle neuronal } \\
\text { loss }\end{array}$ \\
\hline $\begin{array}{l}\text { Zebrafish } \\
\text { (deletion of } \\
\text { WD40 } \\
\text { domain) }\end{array}$ & $\begin{array}{l}\text { - Locomotor defects } \\
\text { - Response to L-dopa }\end{array}$ & $\begin{array}{l}\text { Loss of dopaminergic } \\
\text { neurons }\end{array}$ & Unknown & \\
\hline $\begin{array}{c}\text { Drosophila } \\
\text { (WT, G2019S, } \\
\text { Y1699C, } \\
\text { G2385R) }\end{array}$ & $\begin{array}{l}\text { - Locomotor } \\
\text { impairments } \\
\text { - Response to L-dopa } \\
\text { - Early mortality }\end{array}$ & $\begin{array}{l}\text { Progressive loss of } \\
\text { dopaminergic neurons }\end{array}$ & $\begin{array}{l}\text { No } \alpha \text {-Synuclein } \\
\text { pathology }\end{array}$ & \\
\hline $\begin{array}{l}\text { C. elegans } \\
\text { (G2019S, } \\
\text { R1441C) }\end{array}$ & $\begin{array}{l}\text { - Locomotor deficits } \\
\text { - Response to L-dopa }\end{array}$ & $\begin{array}{l}\text { Age-dependent } \\
\text { neurodegeneration }\end{array}$ & Unknown & \\
\hline
\end{tabular}

\section{Parkin}

Mutations in the parkin gene are the most common cause of familiar juvenile autosomal-recessive Parkinson's disease. As discussed in details in the chapter about ubiquitin proteasome system (UPS), parkin is a 465-amino acid E3 ubiquitin ligase that is an important part of the UPS responsible for protein degradation. Therefore, a loss of parkin function may lead to toxic protein accumulation followed by aggregation and proteolytic stress as a consequence of the defective UPS-mediated degradation. To date, more than one hundred disease-causing mutations in the parkin gene have been identified and in most cases these mutations result in loss of parkin E3 ubiquitin ligase activity. 
Keeping in mind the loss-of-function mechanism, several $\mathrm{KO}$ and parkin-null mice and Drosophila models have been generated and extensively characterized (Table 1.13). Thus, the KO and null-parkin Drosophila models exhibit reduced lifespan, male sterility, mitochondrial defects, oxidative stress, muscles degeneration and severe behavioral phenotype characterized by L-dopa responsive flying and climbing disabilities ${ }^{49,} 271,292$. For these fly models, the degeneration of a certain subset of dopaminergic neurons in the brain has been demonstrated, but the total neuronal loss is limited ${ }^{292}$. The knockdown of parkin gene in the zebrafish induces a death of dopaminergic neurons (approx. 20\%), dysfunction of mitochondrial respiratory chain and increased sensibility to MPP+ toxicity ${ }^{293}$. In contrast, the parkin knockout mouse models exhibit quite mild dopamine-related phenotypes without neurodegeneration, substantial behavioral abnormalities and Lewy body pathology, thereby potentially mimicking the early stages of the disease ${ }^{248,294}$. For instance, mice models based on the deletion of exon 3 in parkin show subtle nigrostriatal alterations, namely increased extracellular dopamine levels, changes in dopamine release and synaptic function in striatum, but in absence of neurodegeneration. Furthermore, mild sensorimotor and locomotor abnormalities, reduced body weight, signs of mitochondrial dysfunction and oxidative stress have been reported for this parkin model ${ }^{294}$. Of note, evaluation of $\mathrm{KO}$ parkin models in $C$. elegans didn't reveal distinct phenotypes.

\section{PINK1}

Mutations in PINK1 are the second most frequent cause of early-onset familiar Parkinson's disease with autosomal recessive inheritance. PINK1 is 581 amino acids PTENinduced putative serine/threonine kinase 1 with mitochondrial targeting sequence ${ }^{75}, 77$. Available experimental results, which are at length summarized in the chapter 1.3.4, support the hypothesis that PINK1 is primarily involved in regulation of mitochondrial trafficking and morphology. Considering that the disease causing mutations in PINK1 result in loss of function, a number of KO and PINK1-null animal models have been generated and phenotypically characterized. To date, PINK1-deficient animal models have been developed in mice, Drosophila melanogaster, C. elegans, zebrafish and, remarkably, many of these models exhibit phenotypes that are similar to the parkin-related models (Table 1.13) 66, 249, 271, 293. Thus, PINK1 mutant Drosophila flies show apoptotic muscle degeneration, mild loss of dopaminergic neurons, male sterility, disturbed mitochondrial morphology, reduced lifespan and locomotor dysfunction (climbing and flying defects) ${ }^{271}$. Furthermore, knockout of PINK1 in mice leads to age-dependent mitochondrial dysfunction, disturbances in dopamine neurotransmission and hypersensitivity to toxins, but at the same time no neurodegeneration, 
dopamine deficiency and behavioral abnormalities are observed ${ }^{250} 66$. Zebrafish and $C$. elegans loss-of function models of PINK1 have a close phenotypic profile comprising mild defects in neuronal morphology, impaired mitochondrial function (reduced number, morphology) and increased sensitivity to neurotoxins ${ }^{249}$. In addition to phenotypic similarities of parkin- and PINK1-deficient models, the hypothesis of a common PINK1/parkin pathway that is involved in regulation of mitochondrial function is supported by observations that parkin overexpression reduces the pathology in PINK1-null Drosophila models. Since PINK1 has no effect on parkin-null phenotype in flies, it seems plausible that parkin in this pathway functioning downstream of PINK1271.

\section{DJ-1}

Several cases of early-onset familiar Parkinson's disease with autosomal recessive inheritance have been associated with mutations in DJ-1 gene ${ }^{86}$. It has been already mentioned in the chapter 1.3 .5 , DJ-1 is a multifunctional protein that is ubiquitously expressed in the different tissues including brain and localized in cytosol, mitochondria and intermediate space ${ }^{90}$. Functionally, DJ-1 is considered to possess antioxidant, peroxidase and redox-sensitive chaperone functions, and therefore DJ-1 has been implicated in cellular response to oxidative stress and, furthermore, to proteasome and mitochondrial dysfunction ${ }^{93}$, 250. Relying on observation that the mutations in DJ-1 result in loss of function, a number animal models mimicking DJ-1 deficiency have been generated in mice, Drosophila melanogaster, zebrafish (Table 1.13) 250,271,293. Two orthologs DJ-1 $\alpha$ and DJ-1 $\beta$ to the human DJ-1 gene have been found in Drosophila. While a selective silencing of neither DJ-1 $\alpha$ nor DJ-1 $\beta$ gene lead to phenotype, the flies with the acute knockdown of both genes show mild neurodegeneration, mitochondrial dysfunction (decreased ATP level, defects in spermatogenesis) and increased susceptibility to oxidative stress inducing agents (paraquat, rotenone $)^{270,271}$. The main drawbacks of this DJ-1 Drosophila models are related to the high variation of observed phenotypes and the lack of prominent neurodegeneration. The evaluation of the DJ-1-deficient zebrafish model has revealed an absence of neurodegeneration, but the sensitivity of dopaminergic neurons to hydrogen peroxide and proteasome inhibition is significantly increased ${ }^{293}$. Several DJ-1 KO mouse models have been reported. Similar to PINK1 and parkin, DJ-1-null mice show very mild pathological and behavioral phenotype that, in most cases, is represented by disturbed dopamine neurotransmission, signs of mitochondrial dysfunction and hypersensitivity to MPTP toxicity $95,97,250$. In behavioral tests the reduced locomotor activity of DJ-1 mutant mice after 
methamphetamine stimulation has been demonstrated. Interestingly, the overexpression of DJ-1 ameliorates the MPTP-induced toxicity in mice.

Overall, PINK1, parkin and DJ-1 loss of function animal models recapitulate some features (dysfunction of mitochondria and dopamine neurotransmission) of early stages of Parkinson's disease and, therefore, these models are useful tool to study the mechanisms of the processes related to mitochondria that precede neurodegeneration and Lewy body pathology and to test therapeutic approaches with disease-modifying potential.

\section{HtrA2/Omi}

Mutations in HtrsA2/Omi (PARK13) have been linked to a rare familiar form of Parkinson's disease. HtrsA2/Omi is a mitochondrial enzyme with serine protease function that is involved in regulation of apoptosis ${ }^{66}$. To date, HtrsA2/Omi deficiency animal models have been developed in mice, Drosophila melanogaster 66 , 270, 295. In Drosophila, the deletion of HtrsA2/Omi doesn't cause any phenotype; the mutant flies are viable, fertile without signs of neurodegeneration and mitochondrial pathology $y^{270}$. On the other hand, the transgenic mice expressing HtrA2/Omi-S276C mutant with reduced protease activity develop a severe phenotype characterized by progressive locomotor defects, striatal neurodegeneration and death by 40 days of age ${ }^{295}$. Due to the contradictive findings in different models, understanding the detailed HtrsA2/Omi-related mechanisms remains an important goal, thereby limiting the application of these models.

Table 1.13. Major animal models of autosomal recessive Parkinson's disease

\begin{tabular}{|c|c|c|c|c|}
\hline Gene and species & $\begin{array}{c}\text { Motor } \\
\text { deficits }\end{array}$ & $\begin{array}{c}\text { Neuronal } \\
\text { loss } \\
\end{array}$ & $\begin{array}{c}\text { Mitochondrial } \\
\text { dysfunction }\end{array}$ & $\begin{array}{l}\text { LB pathology/ } \\
\text { DA deficiency }\end{array}$ \\
\hline $\begin{array}{l}\text { Parkin (PARK2) } \\
\text { - Nematode } \\
\text { - Fly } \\
\text { - Zebrafish } \\
\text { - Mice }\end{array}$ & $\begin{array}{c}- \\
+ \\
\mathrm{ND} \\
\pm\end{array}$ & $\begin{array}{l}- \\
+ \\
+ \\
-\end{array}$ & $\begin{array}{c}- \\
+ \\
\mathrm{ND} \\
\pm\end{array}$ & $\begin{array}{l}-1- \\
-1- \\
-1- \\
-1-\end{array}$ \\
\hline $\begin{array}{l}\text { PINK-1 (PARK6) } \\
\text { - Nematode } \\
\text { - Fly } \\
\text { - Zebrafish } \\
\text { - Mice } \\
\end{array}$ & $\begin{array}{l}- \\
+ \\
- \\
-\end{array}$ & $\begin{array}{l}+ \\
+ \\
+ \\
-\end{array}$ & $\begin{array}{l}+ \\
+ \\
+ \\
+\end{array}$ & $\begin{array}{l}-1- \\
-1- \\
-1- \\
-1-\end{array}$ \\
\hline $\begin{array}{l}\text { DJ-1 (PARK7) } \\
\text { - Fly } \\
\text { - Zebrafish } \\
\text { - Mice }\end{array}$ & $\begin{array}{c}- \\
\mathrm{ND} \\
+\end{array}$ & $\begin{array}{l}+ \\
- \\
-\end{array}$ & $\begin{array}{c}+ \\
\text { ND } \\
\pm\end{array}$ & $\begin{array}{l}-1- \\
-1- \\
-1-\end{array}$ \\
\hline
\end{tabular}

ND, not determined; LB, Lewy body; DA, dopamine; \pm , variable reports 


\section{UCH-L1}

In 1998, a missense mutation in the UCH-L1 (PARK5) gene has been associated with rare inherited form of Parkinson's disease in German family. UCH-L1 stands for ubiquitin carboxyl-terminal hydrolase L1 enzyme that is a part of UPS machinery and is responsible for recycling and stabilization of ubiquitin ${ }^{41,66}$. Despite ongoing discussion regarding loss or gain of function mechanism for UCH-L1-I93M mutant, in vitro experiments with recombinant UCH-L1 variants have demonstrated $50 \%$ reduction of hydrolase activity for I93M mutant ${ }^{66}$. The deletion of UCH-L1 in mice does not lead to neuropathology, while the overexpression of UCH-L1-I93M mutant causes age-dependent behavioral and pathological phenotype, thereby supporting the gain of function mechanism. Overall, the UCH-L1-I93M mutant mice show significant loss of dopaminergic neurons in substantia nigra, striatal dopamine depletion, increased sensitivity to MPTP-induced toxicity and mild locomotor abnormalities ${ }^{296}$. Although this mice model recapitulates certain features of Parkinson's disease, still much effort has to be invested in establishing of underlying mechanisms of UCH-L1-related toxicity and its potential relevance to Parkinson's disease.

\subsubsection{Nurr1 and Pitx3 genetic models}

\section{NURR1}

Nurr1 is an orphan nuclear receptor that plays important role in differentiation and development of dopaminergic neurons. During development Nurr1 as a transcription factor is involved in regulation of expression of dopaminergic neurons markers like tyrosine hydroxylase, AADC and dopamine transporter. Recent reports have demonstrated that Nurr1 is also expressed in adult brain and is essential for the proper function and maintenance of mature dopaminergic neurons ${ }^{297}$. Thus, Nurr1 might be implicated in regulation of genes linked to mitochondrial respiratory activity and to response to oxidative stress and excitotoxicity. Interestingly, experimental findings provide evidence of in vivo interactions between $\alpha$-synuclein and Nurr1, namely, $\alpha$-synuclein overexpression significantly downregulates Nurr1 protein and also its mRNA ${ }^{297}$.

Currently, two fundamentally different types of Nurr1 mouse models have been developed $294,297,298$, the first being a mouse model based on heterozygous $\left(\mathrm{Nurr}^{+/-}\right)$or homozygous (Nurrl ${ }^{-/-}$) Nurrl-deficiency. While $\mathrm{Nurr}^{-/-}$mice are not viable due to agenesis of dopaminergic neurons in substantia nigra, the heterozygous $N u r r 1^{+/-}$mice exhibit a progressive behavioral and pathological phenotype ${ }^{294,298}$. Thus, comparison of $\mathrm{Nurr}^{+/-}$ animals with a control group revealed significant age-dependent loss of TH-positive 
dopaminergic neurons ( $23 \%$ at 17 months, $31 \%$ at 17 moths of age) in substantia nigra and twofold depletion of striatal dopamine level. Behaviorally, Nurrl ${ }^{+/-}$mice show impaired locomotor activity and rotarod performance 298 . Additionally, an increased sensitivity to various stress conditions including MPTP, proteasome inhibitor lactacystin and methamphetamine has been observed in $\mathrm{Nurr}^{+/-}$mice. The second type of Nurr1-deficient mice is based on a conditional knockout of Nurr1 in the adult brain. The evaluation of this model has demonstrated that the knockout of Nurr1 in the adult mice leads to progressive dopaminergic pathology associated with neuronal loss, dopamine deficiency in striatum and locomotor abnormalities ${ }^{297}$. These findings support the hypothesis that, besides the important role in differentiation and development of dopaminergic neurons, Nurr1 is essential for functional maintenance and survival of midbrain dopaminergic neurons in the adult brain. Collectively, Nurr1 loss-of-function mice models recapitulate certain features of early and middle stages of Parkinson's disease and, therefore might be suitable for testing therapeutic approaches.

\section{PITX3}

Like Nurr1, pituitary homeobox 3 (Pitx3) is one of the developmental transcription factors that are involved in development and/or maintenance of midbrain dopamine neurons. Based on research findings, Pitx 3 has been implicated in regulation of expression of tyrosine hydroxylase, brain derived neurotrophic factor, glial cell line derived neurotrophic factor and aldehyde dehydrogenase $2^{299}$. The link to Parkinson's disease is supported by the fact that polymorphism of the Pitx3 gene is a risk factor for Parkinson's disease. To date, Pitx3 knockout has been realized in so-called aphakia mice ${ }^{294}$. These Pit $x 3^{-/-}$mice show complete and selective absence of dopaminergic neurons in substantia nigra, depletion of dopamine levels in the striatum and levodopa-responsive sensorimotor impairments ${ }^{253,294}$. The neuropathological changes occur early during post-natal development and notably without signs of Lewy body pathology. Interestingly, the population of dopaminergic neurons in the ventral midbrain is intact. Overall, Pitx $3^{-/-}$mice could be considered as a good model to investigate the late stages of the disease and to test symptomatic or neurorestorative therapy.

\subsubsection{Seeding/Spreading $\alpha$-synuclein models}

An increasing number of studies has converged to support the so-called prion-like hypothesis related to self-propagating aggregation and spreading properties reminiscent of prions found for many proteins including $\alpha$-synuclein, $\mathrm{A} \beta$, Tau that are involved in several common neurodegenerative diseases. For a long time, the animal models of prion diseases 
were unique experimental systems in which the pathological cascades were triggered by inoculation of infectious materials. In the past few years, the protein misfolding propagation animal models utilizing synthetic recombinant or in vivo derived $\alpha$-synuclein aggregates as a seeding agent have been developed and phenotypically characterized ${ }^{300-303}$. In rodents and primates, the exposure of the CNS of transgenic as well as wild type animals to different forms of misfolded $\alpha$-synuclein can significantly accelerate the formation, accumulation and spreading of Lewy body-like pathology accompanied by other characteristic abnormalities comprising neurodegeneration, inflammation, motor dysfunction and dopamine depletion. One of the most remarkable features of these models is a progressive retrograde transmission/propagation of $\alpha$-synuclein pathology between interconnected brain regions far beyond injection sites, thereby recapitulating the highly stereotypical pattern of Lewy body like accumulation and proliferation in human Parkinson's disease.

In a pioneering work, Luk et al. ${ }^{301}$ have demonstrated that unilateral injections of either brain homogenates derived from old symptomatic animal or artificial $\alpha$-synuclein fibrils into neocortex and striatum of A53T $\alpha$-synuclein transgenic mice exacerbate the behavioral and pathological phenotype. Thus, the first intracellular Lewy body-like inclusions were detected by 30 dpi close to the site of inoculation; at 90 dpi abundant $\alpha$-synuclein deposits were observed throughout the CNS including striatum, neocortex, frontal cortex, thalamus, hypothalamus, brain stem nuclei. In the control group, $\alpha$-synuclein deposition was absent up to $90 \mathrm{dpi}$. In addition to age-dependent accumulation and spreading of Lewy body-like pathology, the inoculated mice exhibited a significant increase in amounts of detergentinsoluble $\alpha$-synuclein, phosphorylated $\alpha$-synuclein and ubiquitin-positive inclusions. Furthermore, in LB-injected animals the onset of motor symptoms (100 dpi vs. 175 dpi control) and lifespan (204 days vs. 316 days) were dramatically reduced in comparison with the control animals. Considering the importance of the nigrostriatal system, the examination of the substantia nigra revealed the presence of $\alpha$-synuclein pathology and reduced THimmunoreactivity in animals that had received aggregated material. These findings for the first time provided support for the hypothesis that aggregated forms of $\alpha$-synuclein could trigger protein misfolding and its self-propagation along connected neuronal networks in vivo and, thereby, leading to earlier onset of neurological symptoms and death ${ }^{301}$.

As described in the follow-up study from the same scientific group, the intrastriatal inoculation of recombinant murine $\alpha$-synuclein fibrils to the wild type! mice also induces the pathological progressive accumulation of endogenous $\alpha$-synuclein with distinct pattern ${ }^{300}$. For instance, first $\alpha$-synuclein-positive inclusions were detected at $30 \mathrm{dpi}$ around the injection 
site, in striatum and olfactory bulb; analysis of the brain material at 90 and 180 dpi revealed spreading of Lewy body-like pathology to other brain regions mainly following neuronal connections. Furthermore, immunohistochemical analysis of the brain tissues from mice injected with $\alpha$-synuclein fibrils showed a progressive loss of TH-positive neurons in the substantia nigra (15\%, $90 \mathrm{dpi} ; 35 \%, 180 \mathrm{dpi})$ accompanied by Lewy body-like pathology in the remaining dopaminergic neurons, dopamine deficiency (up to 50\% reduction of dopamine levels), time-dependent reduction of DAT levels. Behaviorally, the animals developed modest motor dysfunction at 180 dpi assessed by rotarod and wire hang tests ${ }^{300}$.

In line with the results described above, the different $\alpha$-synuclein fractions isolated from postmortem Parkinson's disease brains with were assayed for their propensity to trigger the pathological alterations after inoculation into substantia nigra or striatum of wild type mice or macaque monkeys ${ }^{302}$. Experimentally, two $\alpha$-synuclein samples were purified from the nigral brain tissues by sucrose gradient fractionation that is known to provide separation under mild conditions. The first $\alpha$-synuclein fraction contained mainly soluble or finely granular $\alpha$-synuclein, while the second fraction was predominantly Lewy body-like insoluble $\alpha$-synuclein aggregates as confirmed by a presence of hyperphosphorylated $\alpha$-synuclein, ubiquitin and p62 and filamentous ThioS-positive structures. In mice, single inoculation of Lewy body fraction led to a set of pathological changes including cytoplasmic $\alpha$-synuclein accumulation with PK resistance and hyperphosphorylation; progressive neurodegeneration characterized by loss of dopaminergic neurons in substantia nigra and TH-positive striatal terminals starting from 4 months after injection and astrogliosis. In line with the results obtained by Luk et al. ${ }^{301}$, patient-derived $\alpha$-synuclein aggregates also trigger the spread of pathology from the site of injection to interconnected brain regions and, importantly, significantly smaller amount of protein material is required to induce similar phenotype observed in case of recombinant $\alpha$-synuclein fibrils. To further investigate the in vivo effect of human-isolated $\alpha$-synuclein fractions, Vila et al. ${ }^{302}$ have injected the corresponding samples into substantia nigra or striatum of macaque monkeys. In agreement with mice data, monkeys receiving the Lewy body-like $\alpha$-synuclein fraction to neurodegeneration showed $\alpha$-synucleindependent progressive nigrostriatal degeneration as assessed by neuropathological examination and PET scans. For instance, the stereological counting of TH-positive neurons in substantia nigra revealed $40 \%$ neuronal loss at 14 months post injection, while PET experiments on living animals demonstrated $\sim 34 \%$ reduction in striatal dopaminergic innervation at 9 months post injection. The profile of $\alpha$-synuclein pathology that is observed in monkeys is reminiscent of the alterations documented in mice, such as abnormal 
cytoplasmic $\alpha$-synuclein accumulation in form of diffuse cytoplasmic inclusion that are formed from endogenous $\alpha$-synuclein, PK resistant and containing phosphorylated $\alpha$ synuclein. Importantly, also in monkeys, $\alpha$-synuclein deposits were found in several brain regions distant from inoculation site and these findings further support the hypothesis that protein misfolding could spread along connected neuronal networks in vivo by a cell-to-cell transmission mechanisms. In contrast to spreading mice models from Luk et al. ${ }^{301}$, both animal species exposed to patient-derived $\alpha$-synuclein aggregates didn't develop the behavioral phenotype. Regarding controls, no deleterious effects were observed in mice and monkeys when soluble $\alpha$-synuclein fraction or control buffer were applied as inoculum ${ }^{302}$.

To further investigate the propensity of misfolded $\alpha$-synuclein to initiate $\alpha$-synuclein aggregation and consequent spreading, several studies have been conducted to examine the possible transmission of $\alpha$-synuclein pathology from the peripheral neuronal system to the brain. In one rat model, the high levels of wild type $\alpha$-synuclein expression in the medulla oblongata (the lower part of the brain stem) have been achieved after injection of rAVV vector to the vagus nerve ${ }^{303}$. The analysis of the brain tissues at 4,8 and 18 weeks after transduction revealed a progressive, caudo-rostral spreading of exogenous $\alpha$-synuclein that followed a distinct stereotypical pattern from the site of injection to the interconnected brain regions including contralateral pons, midbrain and forebrain. This protein propagation probably comprises a central cascade that leads to the observed neurological abnormalities and increased amount of $\alpha$-synuclein deposits. In another study, the monomeric or oligomeric form of recombinant human $\alpha$-synuclein was injected into the olfactory bulb of wild type mice. Few hours after inoculation, human $\alpha$-synuclein was detected in several brain regions (frontal cortex, anterior olfactory nucleus, striatum and others) that are known to have the neuronal connections with the olfactory bulb ${ }^{303}$.

Collectively, these in vivo results stress the importance of $\alpha$-synuclein transfer along the neuronal pathway and plausible contribution of such spreading to the disease progression. From therapeutic standpoint, the seeding models could be considered as valuable and relevant models for development of novel disease-modifying therapies that are targeting cell-to-cell spread and transfer of pathogenic $\alpha$-synuclein species. The weak points of such systems are associated with partially mixed results published by different groups, probably due to the variation of the inoculated material and/or injection site. 


\subsubsection{Disease-modifying strategies for Parkinson's disease}

\subsubsection{Development of therapy: Ongoing challenges}

Despite a significant progress in understanding of the pathogenesis of Parkinson's disease and in development of various model systems, there are still many obstacles and limitation that must be overcome both in preclinical stage and in clinical studies.

In case of preclinical development of new therapies for Parkinson's disease, following challenges/limitations/restrictions should be carefully considered:

1) Variety of putative mechanisms of toxicity

2) Lack of a well-defined drug target(s)

3) Lack of animal model(s) that recapitulates the majority of pathological features of Parkinson's disease

4) Difficulties of the drug delivery to the brain

The clinical evaluation of drug-candidates or new therapeutic approaches also posits numerous challenges and inherent limitations that are listed below:

1) Late diagnosis of the disease and, therefore, small time window for intervention

2) Lack of reliable biomarkers and imaging techniques

3) Clinical heterogeneity among patients with Parkinson's disease

4) Limitations of clinical measurements of disease progression

5) Lack of verified design of clinical trials that can differentiate symptomatic versus disease-modifying treatment

6) Potential requirement in multi-therapy

7) Long-term treatment (low drug toxicity, minimum side effects)

8) Frequent overlapping with other brain diseases

\subsubsection{Potential drug targets and disease-modifying strategies}

It is increasingly accepted that the pathogenesis of Parkinson's disease is manifold, and several mechanisms have been implicated as crucial to neurodegeneration, as discussed at length in the chapters 1.3 and 1.4.8.3-1.4.8.8. Among the proposed mechanisms of neurotoxicity, $\alpha$-synuclein misfolding may probably induce a central cascade that leads to loss of neurons and, therefore, a modulation of various aspects of $\alpha$-synuclein biochemistry and turnover is considered as a promising therapeutic target (Fig. 1.33). Beside $\alpha$-synucleinassociated approaches, a range of the pathological processes, which are not directly linked to $\alpha$-synuclein and could be primary or secondary, may also contribute substantially to 
dysfunction and death of neurons and, therefore, the therapeutic targeting of these pathways might be neuroprotective. In this regard, following pathophysiological pathways are currently in focus: neuroinflammation, microglia activation, oxidative stress, apoptosis, excitotoxicity, calcium dysregulation and others (Fig. 1.33) 105, 132, 233, 242, 304-306. Importantly, all these processes may contribute to neurodegeneration synergistically being a part of complex cascades/equilibriums or forming a vicious cycle, like $\alpha$-synuclein misfolding-mitochondrial failure- $\alpha$-synuclein misfolding. To that end, the development of combination therapy approaches or multitarget drug molecules that could arrest or slowdown the progression of several pathological processes underlying Parkinson's disease may represent a promising strategy in search of neuroprotective agent(s). This is also very much in line with FDA roadmap for developing combination therapies for Alzheimer's disease ${ }^{307}$.

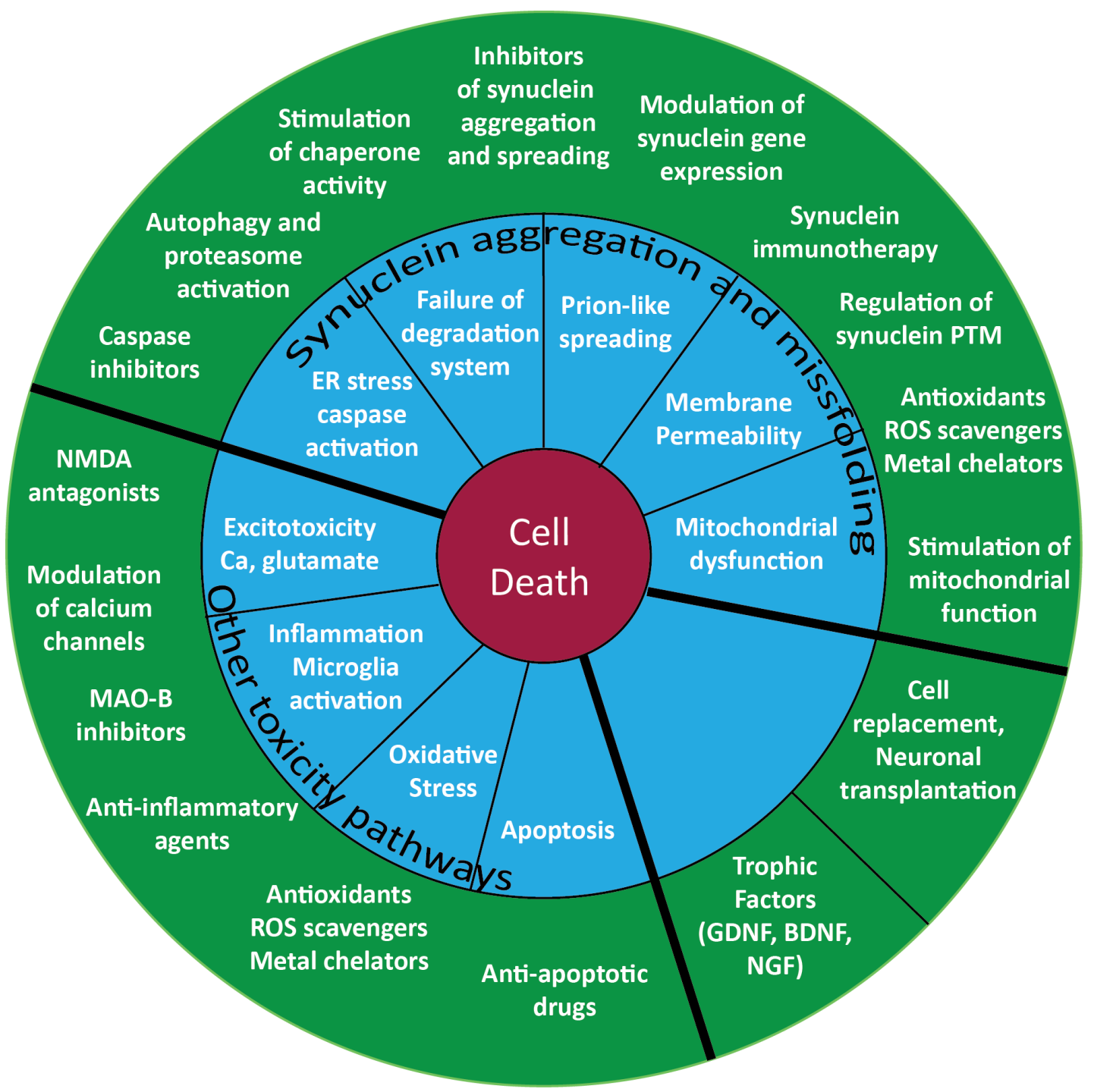

Figure 1.33. Pathogenic mechanisms and corresponding therapeutic strategies.

Pathogenic mechanisms in blue; potential therapeutic approaches in green. 


\subsubsection{Disease-modifying strategies: $\alpha$-Synuclein}

Although the exact physiologic function of $\alpha$-synuclein and molecular/structural details of $\alpha$-synuclein-related toxicity remain unknown, there is an increasing body of evidence from clinical and preclinical studies supporting a hypothesis that targeting of $\alpha$ synuclein misfolding and accumulation may constitute a promising disease-modifying strategy. In this context, two possible concepts have been suggested ${ }^{5,127,132,304}$. The first approach is based on direct modulation of various aspects of $\alpha$-synuclein biochemistry. A second strategy is focused on the downstream effects of $\alpha$-synuclein-mediated toxicity, and aims to ameliorate the impairments of various cellular pathways, thus improving the function and survival of neurons.

\subsubsection{Disease-modifying strategies: $\alpha$-Synuclein as a direct target}

Given a complexity of $\alpha$-synuclein misfolding, aggregation and turnover, a number of experimental approaches that might interfere with the $\alpha$-synuclein-mediated toxicity have been developed and applied mainly in the animal models, except an antibody treatment having reached a clinical phase. Collectively, the therapeutic approaches aiming to reduce $\alpha-$

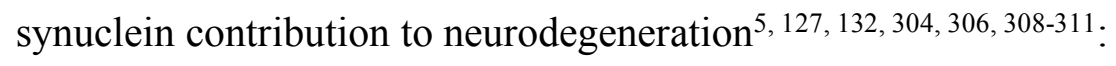

1) Targeting $\alpha$-synuclein gene expression

2) Modulation of cellular $\alpha$-synuclein levels

3) Clearance of toxic $\alpha$-synuclein species

4) Inhibition of $\alpha$-synuclein misfolding

5) Prevention of cell-to-cell prion-like spreading of $\alpha$-synuclein aggregates

6) Modulation of $\alpha$-synuclein pathological post-translational modifications

7) Stabilization of "native" state of $\alpha$-synuclein

\subsubsection{Disease-modifying strategies: Targeting $\alpha$-synuclein gene expression}

In synucleinopathies including Parkinson's disease the reduction of $\alpha$-synuclein synthesis by a modulation of the gene expression might represent the simplest and most obvious approach. So far, several studies have demonstrated the beneficial effect of $\alpha$ synuclein gene downregulation in several animal models. An experiment, in which a mouse model with the conditional and controlled expression of A53T $\alpha$-synuclein has been evaluated, might be considered as a proof-of-principle. Thus, switching off $\alpha$-synuclein expression for 3 months in 9-month-old A53T mice results in almost complete stop of $\alpha$ synuclein pathology progression and even a partial clearance of $\alpha$-synuclein deposits in 
certain brain regions ${ }^{132}$. From the therapeutic standpoint, the modulation of $\alpha$-synuclein synthesis could be achieved by oligonucleotide-based approaches, namely, inhibitory RNAs, such as short hairpin RNA (shRNA) and related micro-RNA (miRNA), or small interfering RNAs $^{105,132}$. For instance, miRNA-7 and miRNA-15 are considered as promising candidates. Being predominantly expressed in the CNS, these miRNAs exhibit a high affinity to 3'untranslated region of $\alpha$-synuclein mRNA and significantly suppress the levels of $\alpha$-synuclein mRNA and $\alpha$-synuclein protein itself in the cultured neurons ${ }^{105,312}$. Interestingly, analysis of MPTP-based cellular and mouse models revealed a significant reduction of miRNA-7 that in turn might lead to increased $\alpha$-synuclein expression levels ${ }^{105}$. Recently, two other miRNAs, miRNA-34b and miRNA-34c, have been implicated as potential suppressors of $\alpha$-synuclein synthesis $^{313}$. The second promising technique that has been shown to knock down the $\alpha$ synuclein gene expression is based on application of chemically modified siRNAs targeting the $\alpha$-synuclein transcript. In support of this view, significant reduction of $\alpha$-synuclein expression has been successfully accomplished by brain infusions of corresponding siRNAs in rat and in non-human primate models ${ }^{132}$. Collectively, the gene regulation might be considered as a potential therapy for protein misfolding diseases, however, there is still a long way from development to the patients. For instance, Spanish company nLife is conducting the pharmacokinetic and pharmacodynamics studies (brain infusions, intravenous and intranasal applications) on candidate molecule NLF-PD-1233 that targets specific neuronal population and downregulates the expression of $\alpha$-synuclein.

\subsubsection{Disease-modifying strategies: Modulation of $\alpha$-synuclein extracellular and} intracellular levels and clearance

The second treatment concept from the list above is referring to the modulation of $\alpha$ synuclein extracellular and intracellular levels. There are two fundamentally different approaches to achieve this goal: 1) stimulate the degradation pathways, including proteasomal, lysosomal, proteolytic systems; 2) passive or active immunization.

So far, much effort has been put into development and elucidation of various $\alpha$ synuclein-directed immunotherapies both in the animal models and recently in the clinical trials $^{127,132,304,306,311,314}$. There is substantial evidence suggesting that both active and passive immunization could target various $\alpha$-synuclein species and eventually reduce the levels of extracellular $\alpha$-synuclein as well as the cell-to-cell transmission of misfolded protein ${ }^{132}$. The concept of active immunization, in other words vaccination with antigen(s), has demonstrated promising results in transgenic mice model, namely, a reduction of cerebral $\alpha$-synuclein and 
amelioration of neuropathology and neuronal survival ${ }^{132,311}$. Considering a good safety and tolerability in first-in-human study, the Austrian company AffiRiS has successfully conducted a phase I studies with AFFITOPE vaccines (AFFiRiS official website), phase 2 trial is planned. Concurrently, the passive immunization with antibodies that could specifically target monomeric or pathological $\alpha$-synuclein species and stimulate its clearance also shows promise in preclinical studies. For instance, $\alpha$-synuclein transgenic mice treated with a monoclonal anti- $\alpha$-synuclein antibody, called 9E4, exhibit significantly improved phenotype, including reduced accumulation and spreading of $\alpha$-synuclein pathology, and improved behavioral readouts ${ }^{315}$. Moreover, the passive immunization by application of 9E4 and related antibodies that recognizes C-terminal region $\alpha$-synuclein (9E4 - residues 118-126) strongly abolishes the majority of pathological alterations in cell-based and transgenic mice models ${ }^{315}$. Remarkably, much experimental data points to possibility that these antibodies are able to preclude the formation and the spreading of $\alpha$-synuclein oligomers and other misfolded species. Following the promising results in the lab studies, PRX002 monoclonal antibody, the humanized analog of 9E4, has entered the clinical phase. In healthy volunteers, infusions of PRX002 have been shown to be safe and well-tolerated and, moreover, it could dramatically lower the $\alpha$-synuclein blood levels. The phase II is currently ongoing (estimated completion date December 2019, NCT03100149, clinicaltrials.gov). The paradigm of immunization can be also extended to an application of single-chain fragment variable ( $\mathrm{scFv}$ ) antibodies that might have advantages due to improved pharmacokinetic properties and/or efficient delivery protocols, such as recombinant AAV systems ${ }^{132,316,317}$. To date, several conformationspecific scFvs against monomeric and oligomeric $\alpha$-synuclein have been generated and tested in vitro and in vivo ${ }^{316,317}$. Thus, the encouraging results, in particular a reduction of $\alpha$ synuclein aggregation and related cytotoxicity, have been obtained from the cellular model coexpressing $\alpha$-synuclein and corresponding scFv.

The next possible approach to modulate the levels of $\alpha$-synuclein relies on a stimulation of the cellular degradation systems, including proteasomal and lysosomal pathways. On the one hand, the activation of degradation machinery could be achieved by elevation of the levels of appropriate enzymes involved in protein clearance either by gene therapy or by exogenous delivery. On the other hand, it is a pharmacological intervention in the regulatory mechanisms of proteasomal and lysosomal pathways.

In case of "protein" therapy, following candidates are so far of major interest: heat shock proteins (Hsps), neurosin, glucocerebrosidase (GCase), parkin, cathepsin $\mathrm{D}^{132,} 212$. Being molecular chaperones, HSPs play an essential role in recognition and correction of 
protein misfolding and unwanted aggregation and that, in turn, leads to proper clearance of harmful proteins. Hsp70, which is probably the most studied Hsp in context of Parkinson's disease, has been shown to exert the protective response to $\alpha$-synuclein-mediated toxicity in various animal models including transgenic mice and Drosophila ${ }^{318}$. Remarkably, Hsp70 also has a propensity to inhibit $\alpha$-synuclein aggregation and disassemble the amyloid fibrils in vitro $^{318}$. Two lysosomal enzymes, glucocerebrosidase and cathepsin $\mathrm{D}$, have been proposed to stimulate the lysosomal activity and eventual $\alpha$-synuclein degradation ${ }^{132,212}$. Thus, the experiments in dopaminergic cells co-expressing synuclein and lysosomal protease cathepsin $\mathrm{D}$ have uncovered the induction of $\alpha$-synuclein clearance ${ }^{319}$. In agreement with this line of thought, stimulation of lysosomal pathway by GCase overexpression utilizing the rAAVbased brain delivery ameliorates the neuropathological and behavioral alterations in $\alpha$ synuclein transgenic mice model ${ }^{132}$. Furthermore, neurosin, an important trypsin-like serine protease in CNS, has been shown to be capable of cleaving $\alpha$-synuclein in NAC region both in vitro and in vivo ${ }^{320}$. From the therapeutic point, experiments in $\alpha$-synuclein-based cellular and animal models are consistent with the protective potential of neurosin to inhibit $\alpha$ synuclein accumulation and related neuropathological changes ${ }^{320}$. Finally, several studies have auspiciously demonstrated beneficial effects of parkin in context of UPS activation as discussed in details in chapter 1.3.2.

There is a growing number of studies showing that pharmacological activation of protein degradation systems might be beneficial for protein misfolding diseases. In case of $\alpha-$ synucleinopathies, several molecules that are supposed to stimulate the activity of protein clearance pathways have been identified as having the neuroprotective potential based on efficacy in animal models ${ }^{132,212,304}$. The properties and outcome of several such agents are summarized in Table 1.14128, 202, 294306, 308-311.

\subsubsection{Disease-modifying strategies: Modulation of $\alpha$-synuclein pathological post-}

\section{translational modifications}

There is substantial evidence suggesting a possible implication of post-translational modification of $\alpha$-synuclein in the development and progression of Parkinson's disease by altering the properties, biochemistry and turnover of $\alpha$-synuclein. The majority of $\alpha$-synuclein modifications are described in details in the chapter 1.4.6. In context of therapeutic application, a modulation of presumably pathological $\alpha$-synuclein modification may constitute a promising treatment approach ${ }^{5}$ 127. Despite still limited understanding of pathological pathways, in which modified $\alpha$-synuclein is involved, the modulation of 
following post-translational modification, including phosphorylation (kinase inhibitors or phosphatase activators), oxidation/nitration (antioxidant compounds), C-terminal truncations, has been proposed as a possible pharmacological target. To date, there are no published investigations aimed to evaluate the effect of such modulators, excluding antioxidants, in $\alpha$ synuclein-based animal models of Parkinson's disease.

Table 1.14. Pharmacological activation of protein degradation systems.

\begin{tabular}{|c|c|c|}
\hline Agent & Mechanism of action & Effect in preclinical studies \\
\hline Glucocorticoids & $\begin{array}{l}\text { Stimulation of expression } \\
\text { of proteasomal components }\end{array}$ & - Neuroprotective in MPTP mice model \\
\hline Carenoxolone & $\begin{array}{c}\text { Chemical induction of } \\
\text { Hsp70 }\end{array}$ & $\begin{array}{l}\text { - Neuroprotection and improved motor } \\
\text { function in rotenone rat model } \\
\text { - Reduction of } \alpha \text {-synuclein toxicity and } \\
\text { aggregation in neuroglioma cells }\end{array}$ \\
\hline $\begin{array}{l}\text { Rapamycin } \\
\text { Latrepirdine }\end{array}$ & $\begin{array}{l}\text { Induction of mTOR- } \\
\text { dependent autophagy }\end{array}$ & $\begin{array}{l}\text { - Rapamycin is neuroprotective in MPTP } \\
\text { mice model and AVV- } \alpha \text {-synuclein } \\
\text { treated rat model } \\
\text { - Latrepirdine reduces } \alpha \text {-synuclein } \\
\text { accumulation in cells and wild-type mice }\end{array}$ \\
\hline Thehalose & $\begin{array}{c}\text { TOR-independent } \\
\text { stimulation of autophagy }\end{array}$ & $\begin{array}{l}\text { - Reduced } \alpha \text {-synuclein aggregation and } \\
\text { improved } \alpha \text {-synuclein clearance in } \\
\text { cellular models } \\
\text { - Improved neuropathology and } \\
\text { behavioral phenotype in } \alpha \text {-synuclein } \\
\text { transgenic mice model and AVV- } \alpha \text { - } \\
\text { synuclein treated rat model }\end{array}$ \\
\hline Nilotinib & $\begin{array}{l}\text { Inhibition of cAbl tyrosine } \\
\text { kinase and activation of } \alpha \text { - } \\
\text { synuclein autophagic } \\
\text { clearance }\end{array}$ & $\begin{array}{l}\text { - Rescue of neuronal cell loss and motor } \\
\text { deficits in MPTP-mice model } \\
\text { - Increased } \alpha \text {-synuclein clearance, } \\
\text { improved neuropathology and behavioral } \\
\text { phenotypes in transgenic and lentiviral } \\
\text { gene transfer animal models }\end{array}$ \\
\hline
\end{tabular}

\subsubsection{Disease-modifying strategies: Modulation of $\alpha$-synuclein misfolding}

Although the exact mechanisms of neurodegeneration in Parkinson's disease are not fully established, the modulation of $\alpha$-synuclein misfolding by small organic drug-like compounds or by relatively big macromolecules or biomolecules represents an attractive therapeutic approach with an obvious disease-modifying potential. Following this line of 
thought, the cytoprotective effect of the modulation of $\alpha$-synuclein misfolding could be associated with several modes of action. First of all, active compounds can inhibit $\alpha$-synuclein aggregation pathways that are leading to formation of potentially toxic species. A second plausible mechanism constitutes a remodeling of already existing toxic aggregates to harmless $\alpha$-synuclein structures as a result of binding of therapeutic agent. Given that certain oligomeric and fibrillar $\alpha$-synuclein species are resistant to degradation inside the cells, the molecules that could interact with such highly resistant structures, thereby facilitating the clearance of those, may also constitute the promising therapy. Finally, the emerging paradigm implies the prion-like spreading of $\alpha$-synuclein in progression and development of Parkinson's disease. Therefore, beneficial therapeutic effects could be expected for compounds that arrest or slow down the process of prion-like spreading by stabilization of less propagation-prone structure(s), in other words reducing the infectivity of amyloid seeds; by reduction of number of amyloid seeds, or by restricting corresponding templated conformational changes.

To date, various compounds and big macromolecules or biomolecules have been shown to interfere with the $\alpha$-synuclein aggregation pathway in vitro and rarely in vivo (Table $1.15,1.16$; citations are listed in the table). Initially, the focus of many studies was mainly inhibition of amyloid fibrils formation; however, during the last years the interest was shifting towards the reduction of oligomerization, since increasing evidence points to $\alpha$-synuclein oligomers as main toxic suspects. At the same time, in-depth studies, comprising the characterization of mechanism of action for known and novel compounds, the evaluation of stabilized protein structures and further optimization/screening aggregation inhibitor, encounter a number of fundamental and technical problems, similar to the analysis of native oligomeric species. Among these difficulties: the inherent multiplicity of $\alpha$-synuclein aggregation process depending on conditions, the structural and morphological heterogeneity of the aggregated mixtures, lack of well-defined secondary structure, poor stability of species during analysis and isolation, potentially complex mechanisms of action, lack of cellular environment in case of in vitro studies and so on. What's more, there are no good experimental techniques available that allow scientists to investigate the details of $\alpha$-synuclein aggregation in vivo, and thus limiting the translation from tube to cells and animal studies. Despite all these issues, the modulation of $\alpha$-synuclein misfolding remains the extremely attractive targets for therapeutic development.

According to the published studies focused on investigation of $\alpha$-synuclein aggregation inhibitors, following set of biophysical techniques is frequently applied: 
1) Morphological studies: electron and fluorescence microscopy, AFM;

2) Monitoring of amyloid fibril formation: ThioT, static light scattering, SIFT, solubility tests

3) Analysis of size and shape of stabilized species: DLS, SAXS, SANS;

4) Sample composition: SEC, SDS-PAGE, PICUP, ELISA, BiFC;

5) Determination of secondary structure: CD, FT-IR, NMR;

6) Analysis of drug-protein interaction and structural elements: NMR, MS, SDS-RCS, UV spectroscopy and immunoblotting with conformation or epitope-specific antibodies;

7) Stability assays: sarkosyl, SDS, PK, urea treatment;

As it has been already mentioned, many molecules have been shown to inhibit $\alpha$ synuclein fibrillization or alter the aggregation pathways (Table 1.15 and 1.16, citations are listed in the table) and even destabilize the preformed amyloid fibrils in vitro. Upon examination, the active molecules could be divided in several classes of organic compounds: a) natural and synthetic polyphenols (dopamine, tannic acid, hydroquinone); b) polyphenolic flavonoids (baicalein, morin); c) catechin-related compounds that are also polyphenolic (examples: (+)-catechin, EGCG, theaflavin); d) antibiotics (tetracycline, rifampicin); e) porphyrins (PcTS, hemin); f) aldehydes and ketones (PQQ, tanshinone, HNE, glyoxal); g) chaperones (mannitol, scyllo-inositol, Hsp70); h) conjugated aromatic dye-like compounds (congo red, lacmoid); i) $\alpha$-synuclein-related peptides; j) others ("molecular tweezers" CLR01, PAMAM, nicotine). In general, one can conclude that inhibition of $\alpha$-synuclein aggregation by various agents frequently occurs by arresting the amyloid fibril formation and redirecting $\alpha$-synuclein association toward diverse heterogeneous soluble oligomeric structures. Overall, the inhibitor-stabilized species are lacking well-defined morphology and secondary structure and exhibit diverse properties and cytotoxicity. The key aspects of $\alpha$-synuclein aggregation inhibition for each compound are summarized in the table 1.15 .

Among all compounds, the modulation of $\alpha$-synuclein aggregation by dopamine s21-329 $^{2}$ and baicalein ${ }^{330-334}$ is probably studied in most detailed manner. Thus, both compounds prevent in dose-dependent manner the formation of amyloid fibrils in favor of formation of soluble heterogeneous spherical oligomeric species $325,327,331,333$. Both baicalein- and dopamine-stabilized oligomers are stable during SEC analysis and isolation and SDS-stable. Based on MS and SEC-UV analysis, it has been demonstrated that dopamine as well as baicalein are bound to monomeric and oligomeric $\alpha$-synuclein, presumably as covalent adduct via Schiff base formation or Michael addition to lysines, however, several labs were not able to detect the covalent modifications ${ }^{322,} 328,331,333$. The structural investigations of the 
generated oligomers with CD, FT-IT have revealed no significant changes of secondary structure, which is the random coil, in comparison with monomeric $\alpha$-synuclein ${ }^{328,331}$. In case of dopamine, MD simulations have suggested ${ }^{125}$ YEMPS $^{129}$ region and E83 potential binding sites $^{323,335}$. Alternative mechanisms of action emphasize the potential involvement of methionines and inhibitors oxidation in stabilization these off-pathway oligomers ${ }^{324,} 336$. Cytotoxicity data for dopamine-induced aggregates is controversial, both toxic and non-toxic species have been found in cellular models ${ }^{322,336,337}$. While baicalein has been shown to reduce $\alpha$-synuclein aggregation, including oligomerization, and $\alpha$-synuclein-related toxicity in the cellular models ${ }^{334}$. Moreover, the baicalein-stabilized oligomeric species are characterized by reduced toxicity following extracellular application.

Many compounds that are listed in the table 1.15 have been also tested in the rodent models of Parkinson's disease. Various animal studies have shown the protective effects, including neuroprotection, amelioration of behavioral phenotypes and normalization of corresponding biomarkers, for almost 20 compounds (baicalein, baicalin, caffeic acid, ceftriaxone, curcumin, EGCG, ferulic acid, gallic acid, nicotine, pramipexole, rifampicin, porinirole, rosmarinic acid, selegiline). However, all mentioned compounds exhibit therapeutic properties just in the toxin-based (MPTP, rotenone, 6-OHDA) rodent models, which, in turn, have minimal connection with $\alpha$-synuclein misfolding and aggregation. Therefore, the efficacy of these candidates to reduce the $\alpha$-synuclein pathology and related phenotypes has to be evaluated in the well-established $\alpha$-synuclein transgenic/viral/seeding animal models. One example is study conducted in $\alpha$-synuclein transgenic mice (mThy $1-\alpha-$ synuclein transgenic mice), in which the beneficial effect of the treatment with the aggregation inhibitor mannitol has been observed ${ }^{338}$. According to presented data, a daily intraperitoneal application of mannitol $(1 \mathrm{~g} / \mathrm{kg})$ for 4 weeks leads to significant reduction of $\alpha$ synuclein accumulation in the hippocampus and substantia nigra as evidenced by immunohistochemistry and analysis of the soluble and insoluble $\alpha$-synuclein. For instance, the levels of soluble and insoluble $\alpha$-synuclein are decreased 3 and 2 fold, respectively. Furthermore, the mannitol treatment also ameliorates the neuropathological phenotype, in particular, almost complete protection of $\mathrm{TH}-$ and NeuN-positive neurons in several brain regions $^{338}$. Second promising candidate is a small molecule NPT200-11 ${ }^{339}$, which was developed by Neuropore Therapies Inc. Together with initial lead compound NPT100-18 ${ }^{340}$, NPT200-11 interferes in vitro with oligomerization $\alpha$-synuclein on membrane by interaction with C-terminus. Both compounds were tested in two $\alpha$-synuclein transgenic mouse models, mThy1- $\alpha$-synuclein and mThy1- $\alpha$-synuclein E57K ${ }^{339}$, 340. Thus, the administration of 
NPT200-11 by intraperitoneal injections leads to significant reduction of $\alpha$-synuclein brain pathology, reduced CNS inflammation and improved behavioral phenotype. Therefore, these findings strongly support the notion that modulation of $\alpha$-synuclein misfolding and aggregation by small molecules may represent an attractive strategy in development of disease-modifying therapy. 
Table 1.15. Inhibitors of $\alpha$-synuclein aggregation (alphabetically)

\begin{tabular}{|c|c|c|c|c|c|c|c|c|c|}
\hline 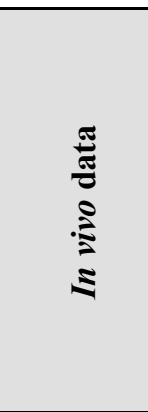 & & 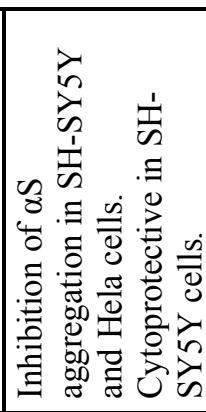 & 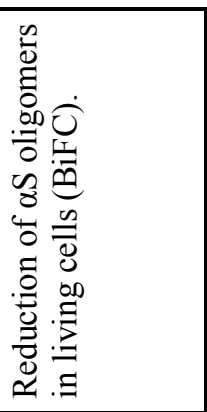 & & 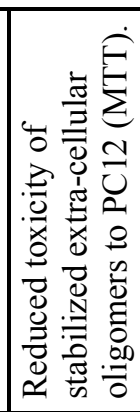 & & 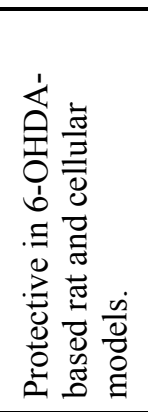 & 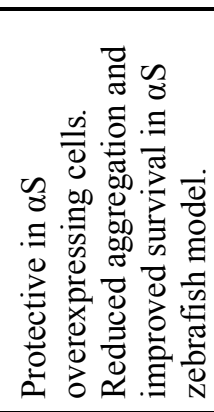 & \\
\hline 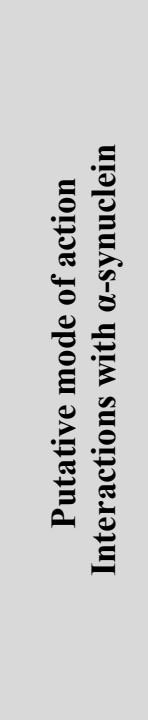 & 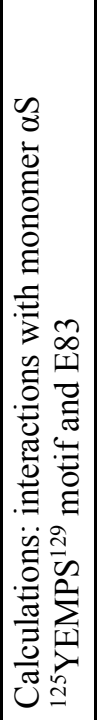 & 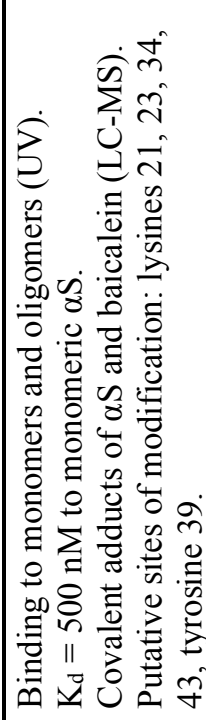 & 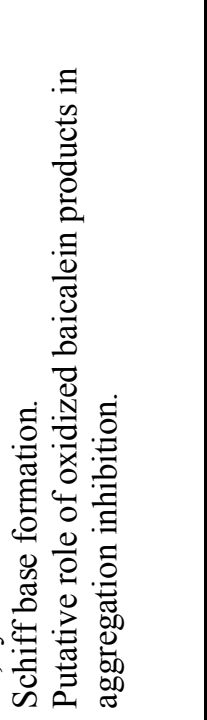 & & 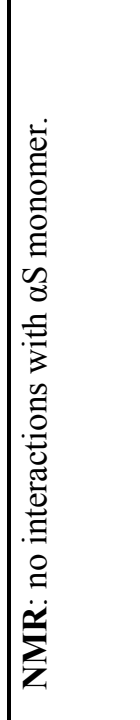 & & 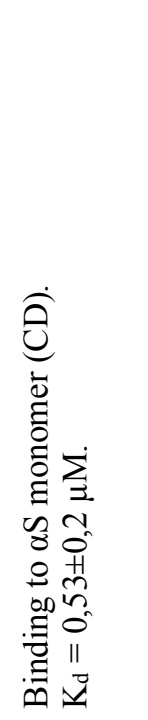 & 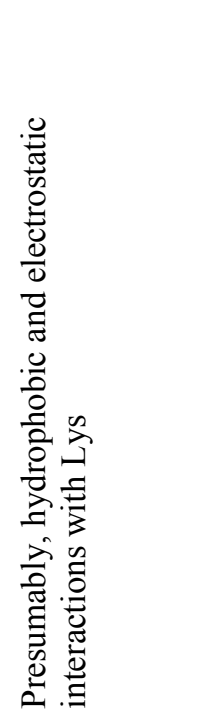 & 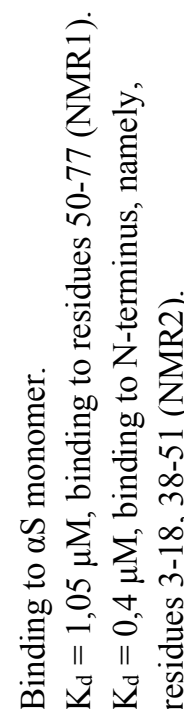 \\
\hline 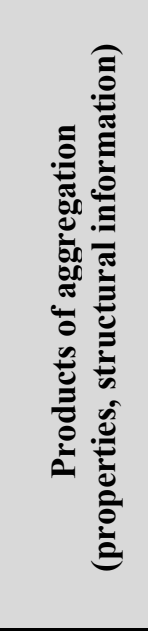 & 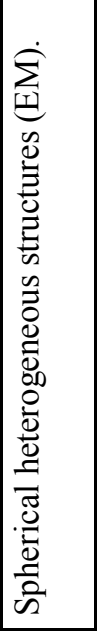 & 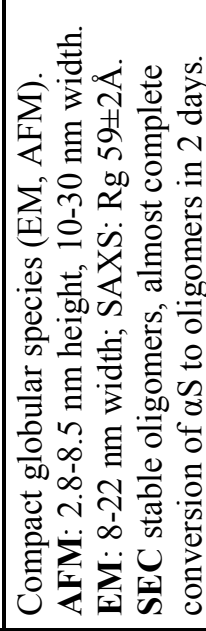 & 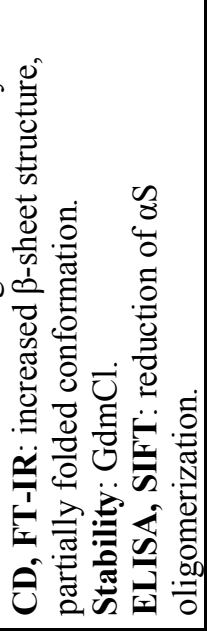 & & 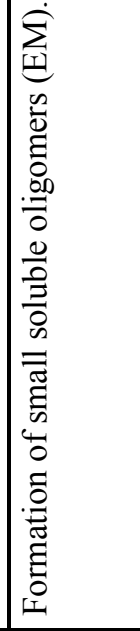 & 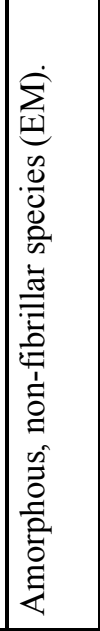 & 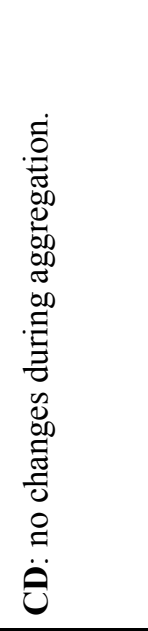 & 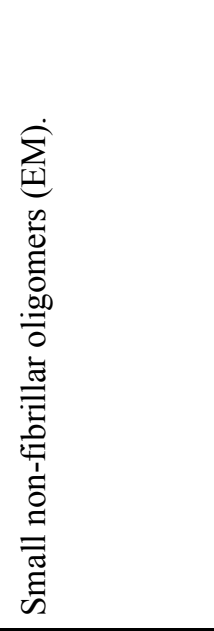 & 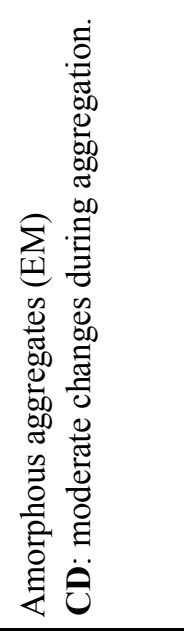 \\
\hline 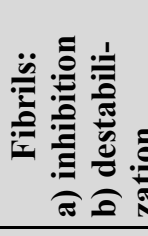 & 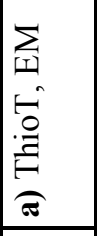 & 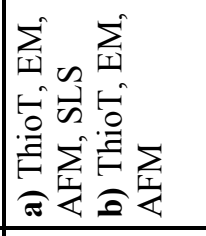 & & 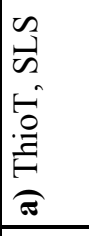 & 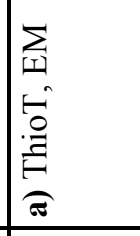 & 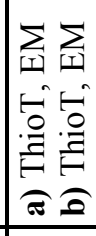 & $\stackrel{\theta}{\text { 己 }}$ & 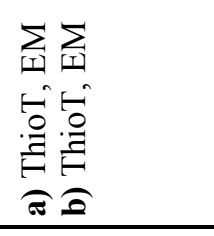 & 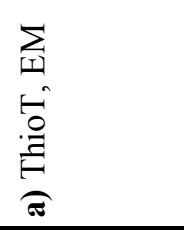 \\
\hline 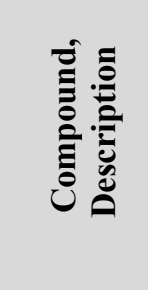 & 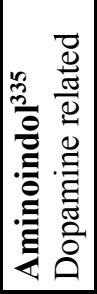 & 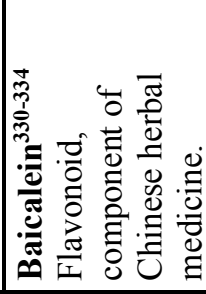 & & 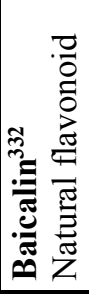 & 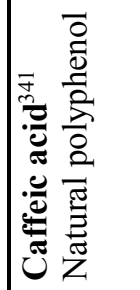 & 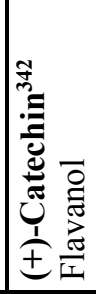 & 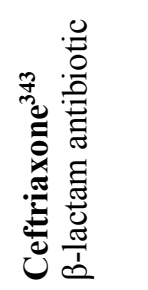 & 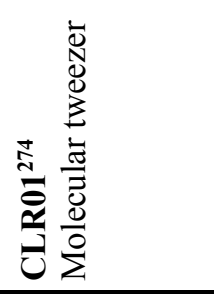 & 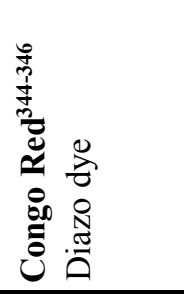 \\
\hline
\end{tabular}


Table 1.15. Inhibitors of $\alpha$-synuclein aggregation (continued)

\begin{tabular}{|c|c|c|c|c|c|c|c|c|}
\hline 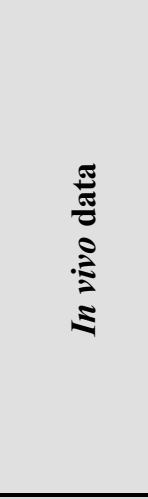 & 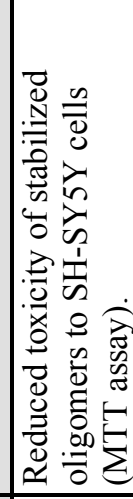 & 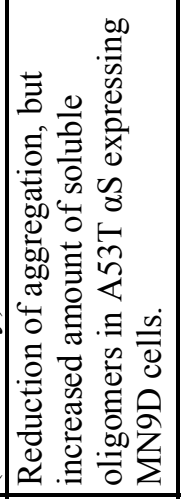 & & & & 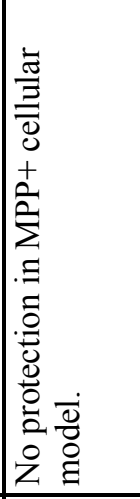 & 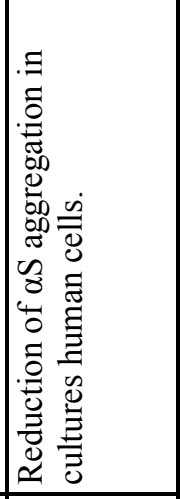 & 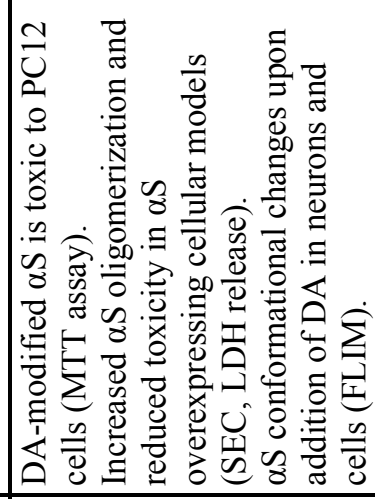 \\
\hline 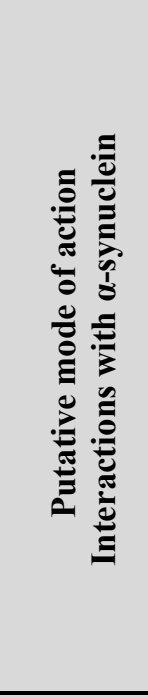 & 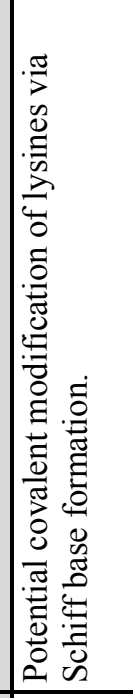 & 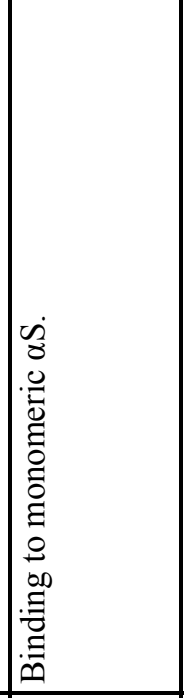 & 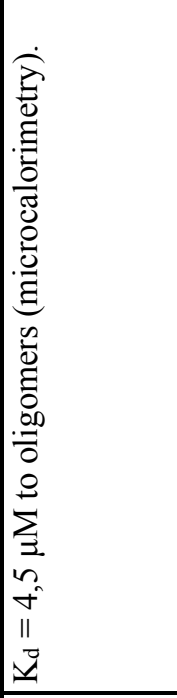 & & 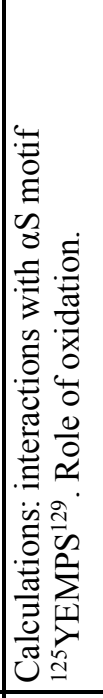 & 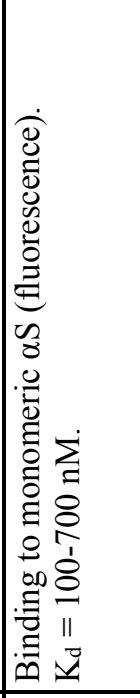 & 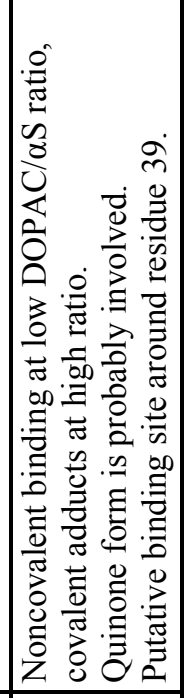 & 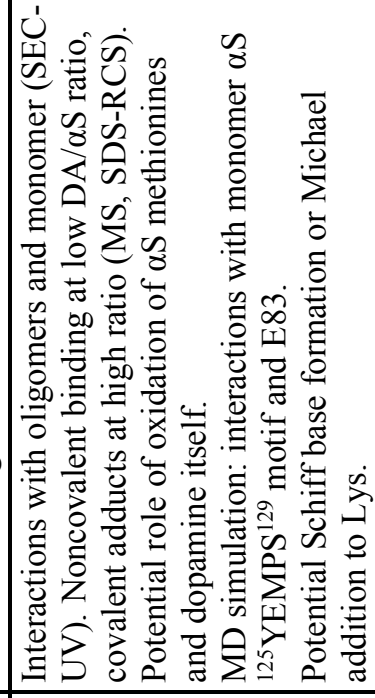 \\
\hline 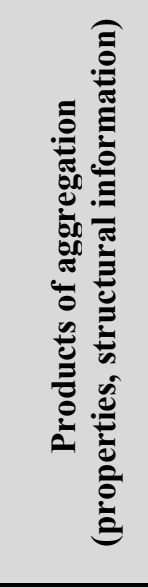 & 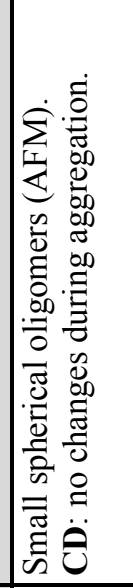 & 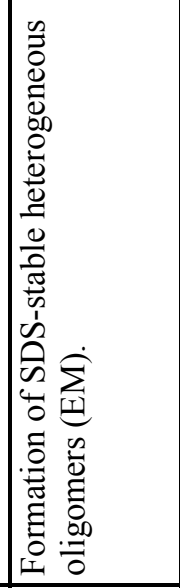 & 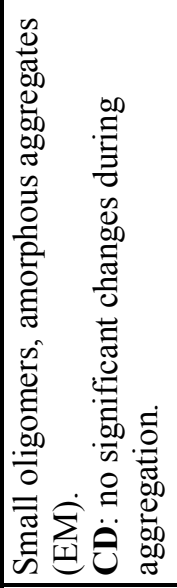 & 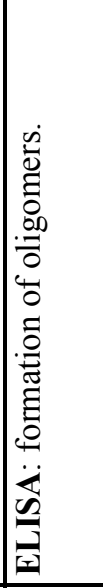 & 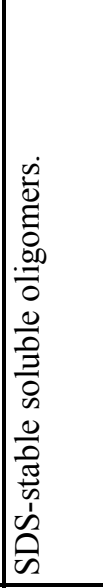 & 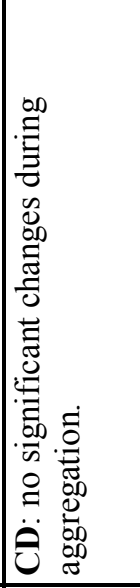 & 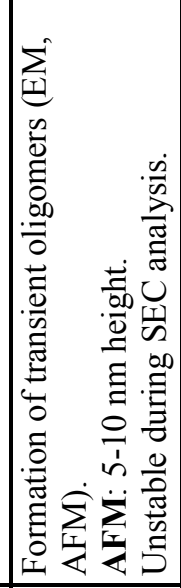 & 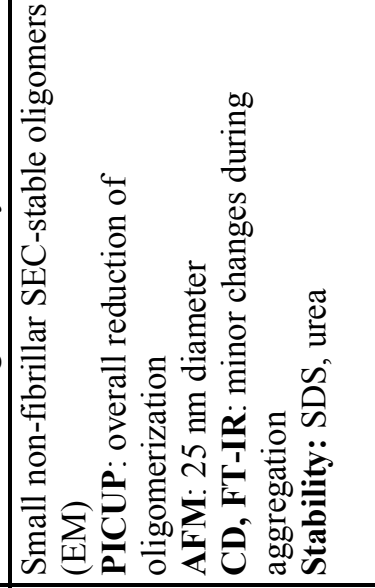 \\
\hline 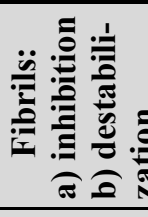 & $\frac{\sqrt[1]{0}}{3}$ & 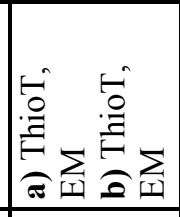 & $\begin{array}{l}\bar{\rho} \\
\stackrel{\partial}{\vec{F}} \\
\tilde{\sigma}\end{array}$ & 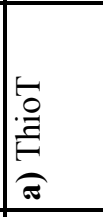 & 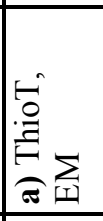 & 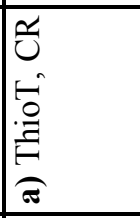 & 竞 & 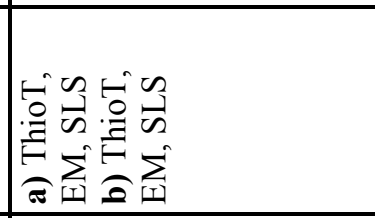 \\
\hline 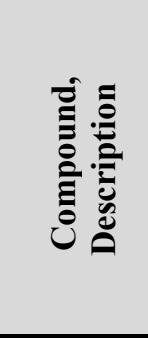 & 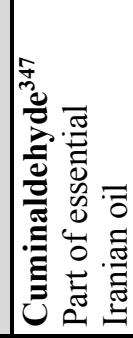 & 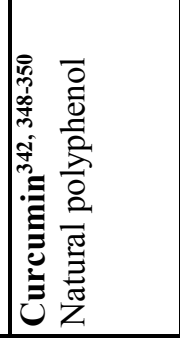 & 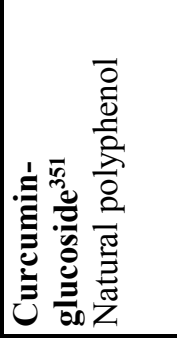 & 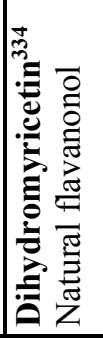 & 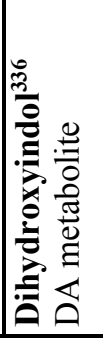 & 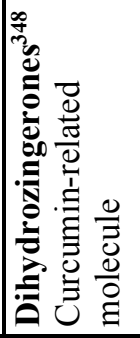 & 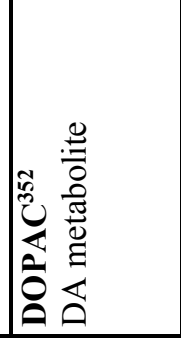 & 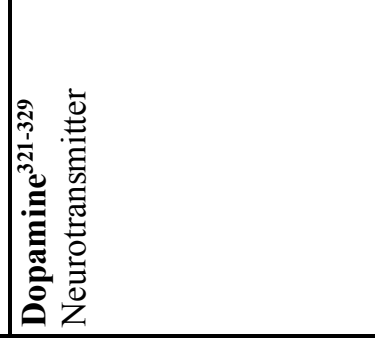 \\
\hline
\end{tabular}


Table 1.15. Inhibitors of $\alpha$-synuclein aggregation (continued)

\begin{tabular}{|c|c|c|c|c|c|c|c|c|c|}
\hline 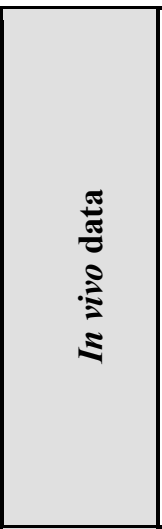 & 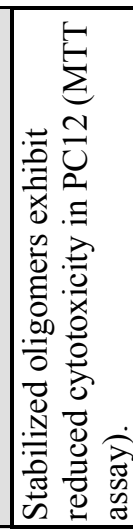 & 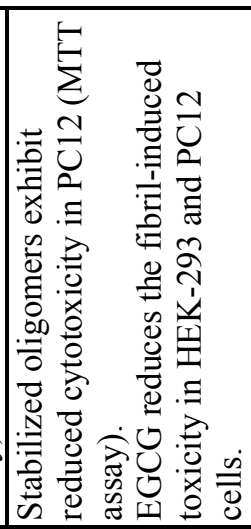 & & & & 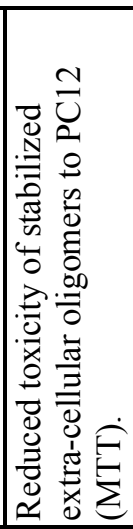 & & & \\
\hline 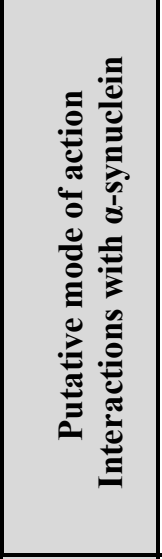 & 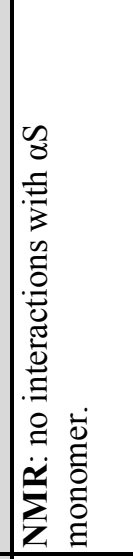 & 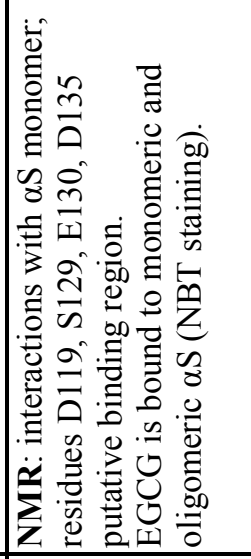 & 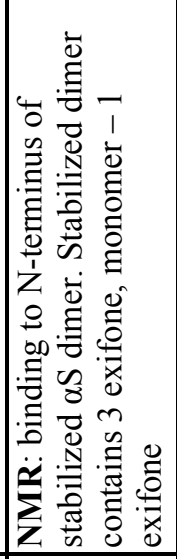 & & & 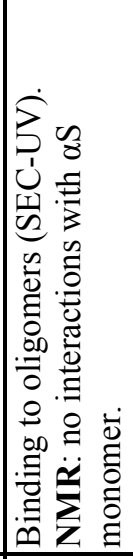 & & 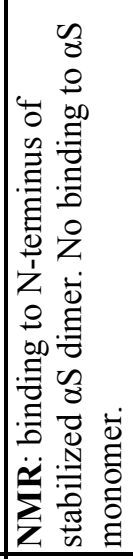 & \\
\hline 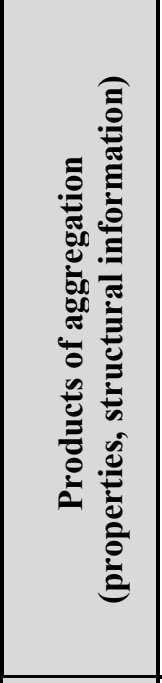 & 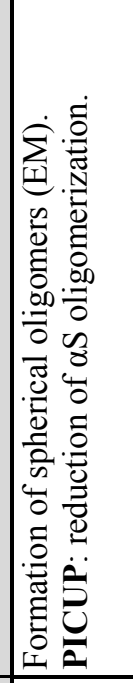 & 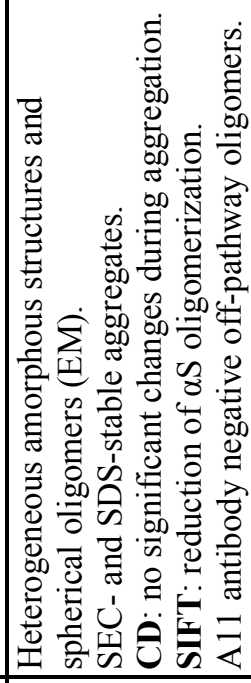 & 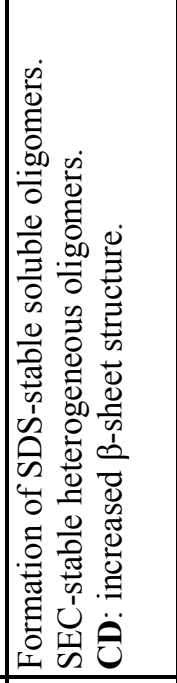 & 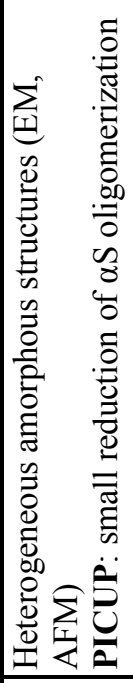 & 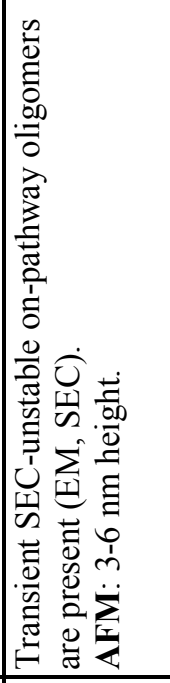 & 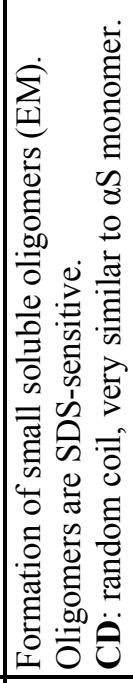 & 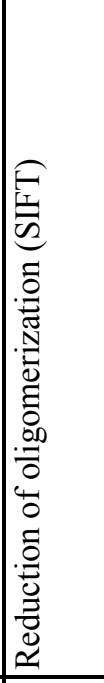 & 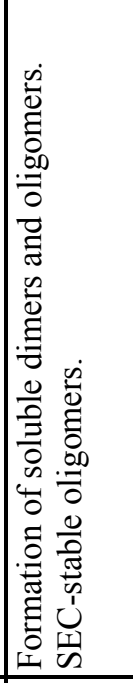 & 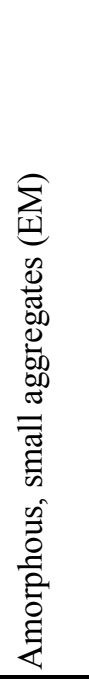 \\
\hline 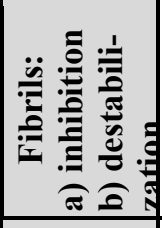 & 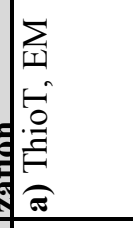 & 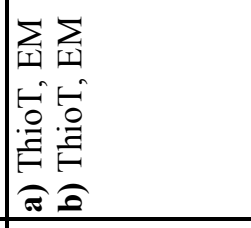 & 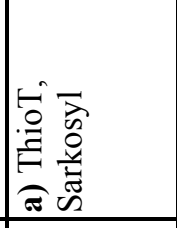 & 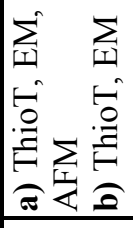 & 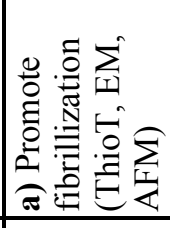 & 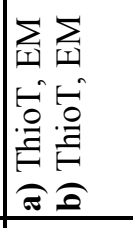 & & 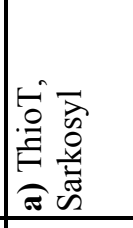 & 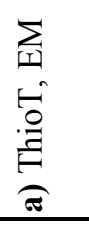 \\
\hline 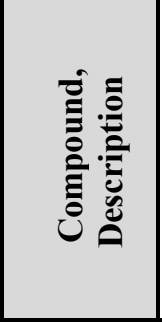 & 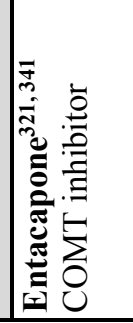 & 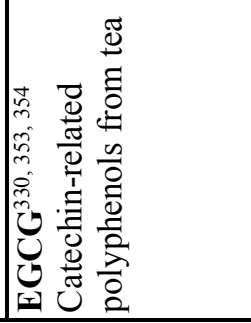 & 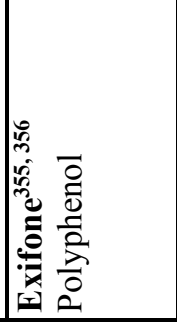 & 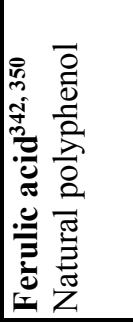 & 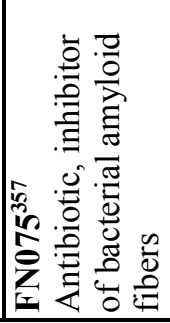 & 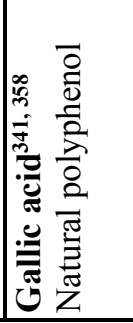 & 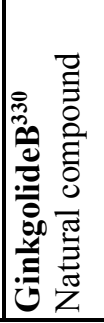 & 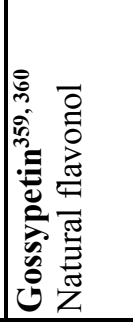 & 㟒起 \\
\hline
\end{tabular}


Table 1.15. Inhibitors of $\alpha$-synuclein aggregation (continued)

\begin{tabular}{|c|c|c|c|c|c|c|c|c|c|c|}
\hline 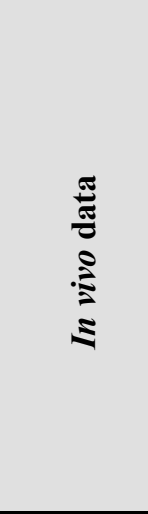 & 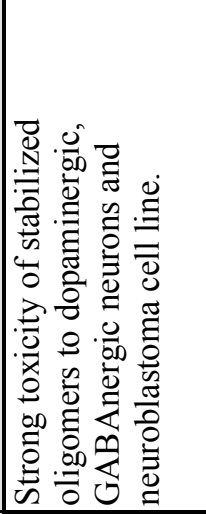 & & & & & & 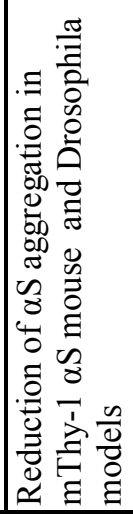 & & & 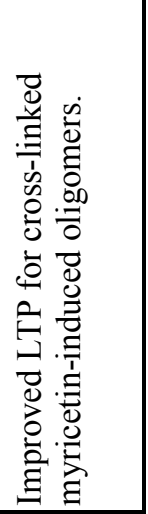 \\
\hline 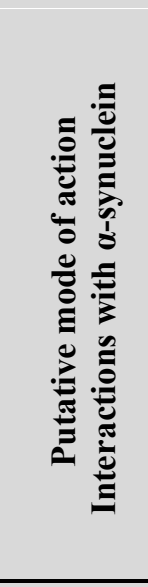 & 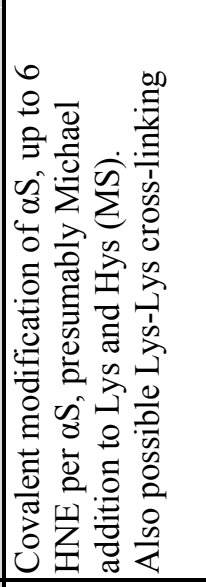 & 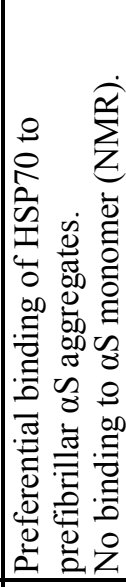 & 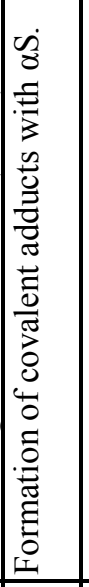 & 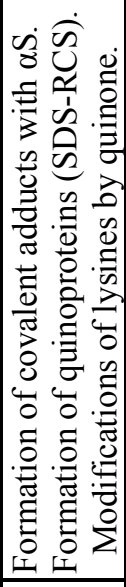 & 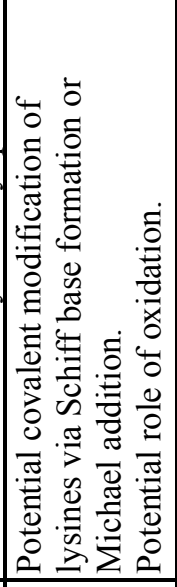 & 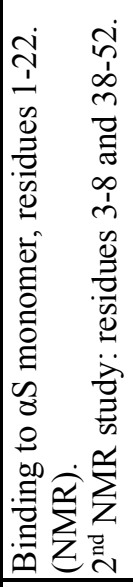 & & 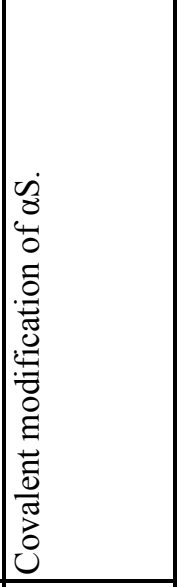 & & 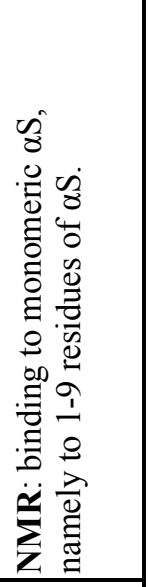 \\
\hline 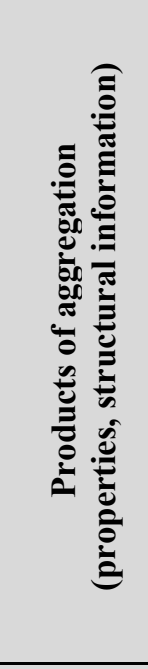 & 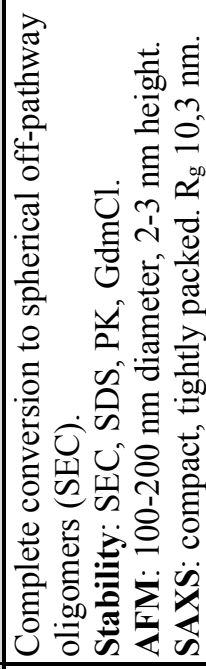 & 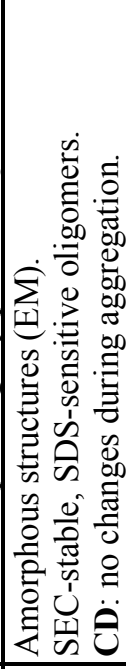 & 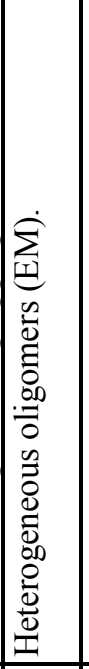 & 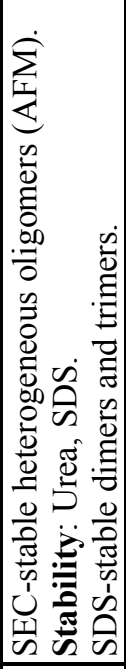 & 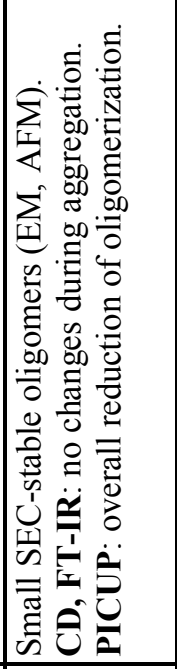 & 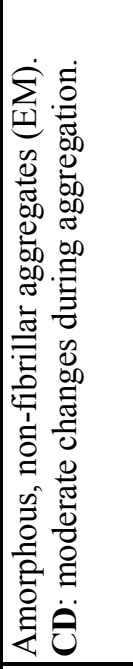 & 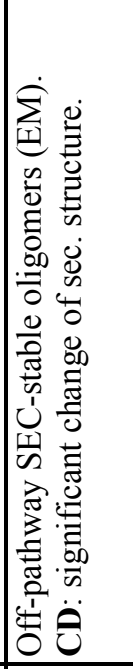 & 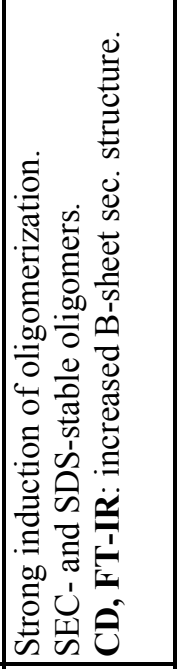 & & 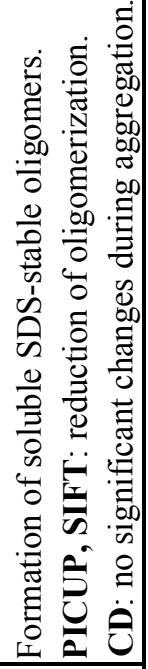 \\
\hline 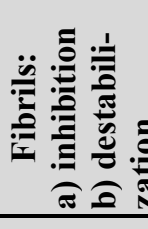 & 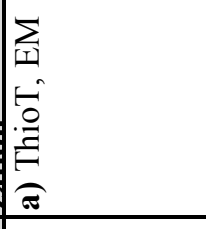 & 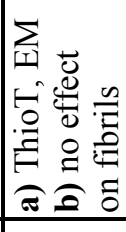 & 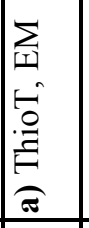 & 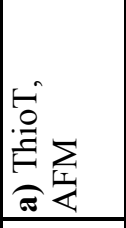 & 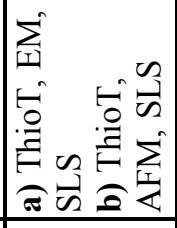 & 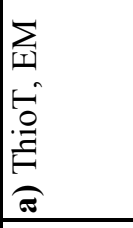 & 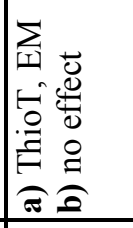 & 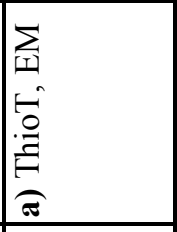 & 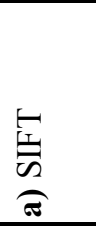 & 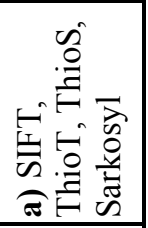 \\
\hline 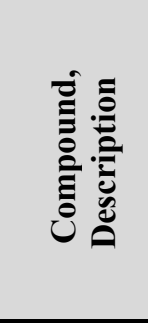 & 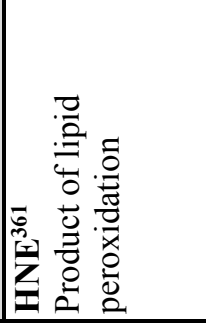 & 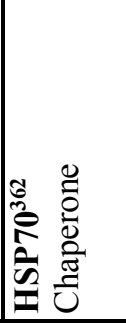 & 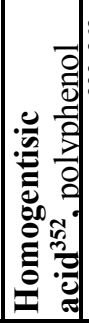 & 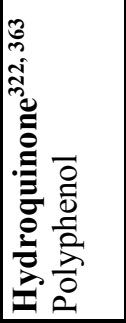 & 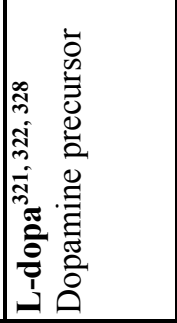 & 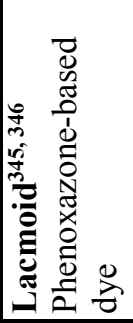 & 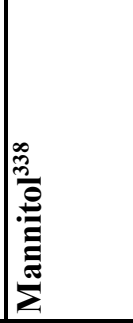 & 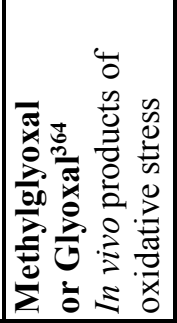 & 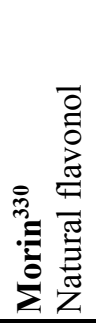 & 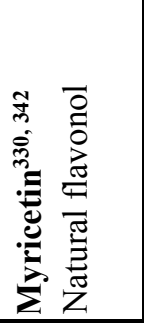 \\
\hline
\end{tabular}


Table 1.15. Inhibitors of $\alpha$-synuclein aggregation (continued)

\begin{tabular}{|c|c|c|c|c|c|c|c|c|c|c|}
\hline 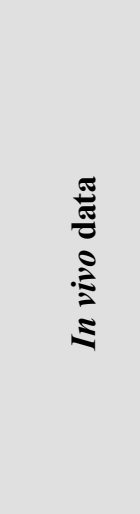 & & & & & 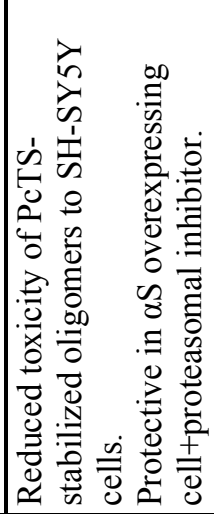 & 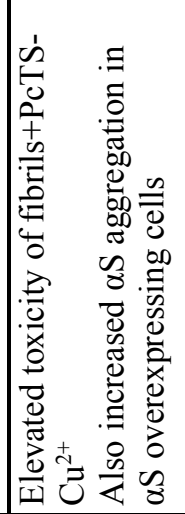 & 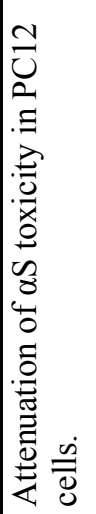 & 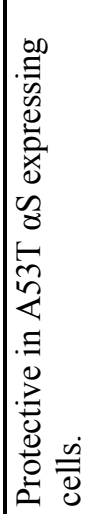 & 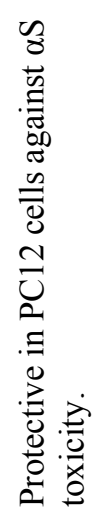 & \\
\hline 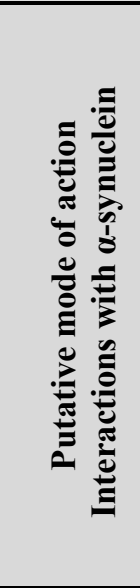 & & & & & 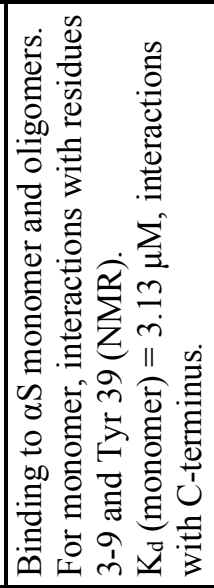 & 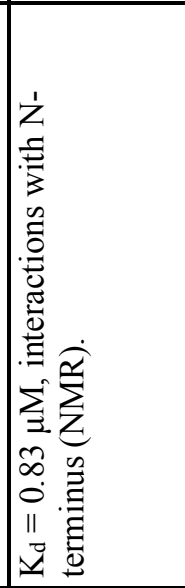 & 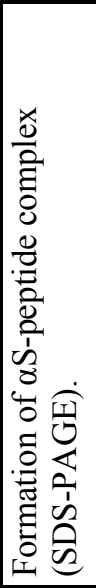 & 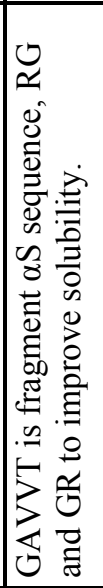 & 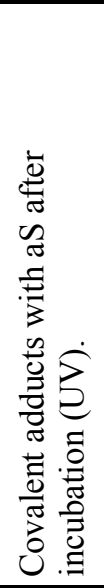 & \\
\hline 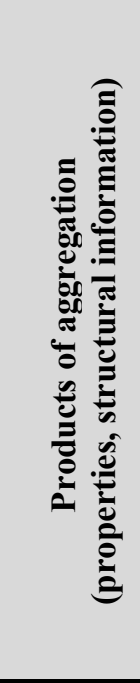 & 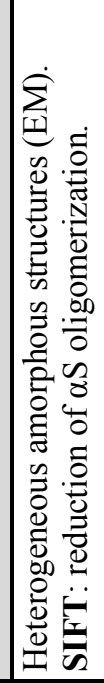 & 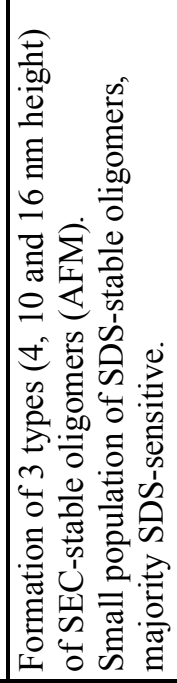 & 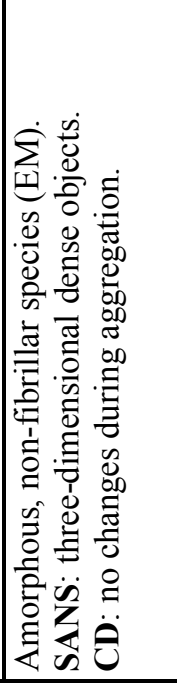 & & 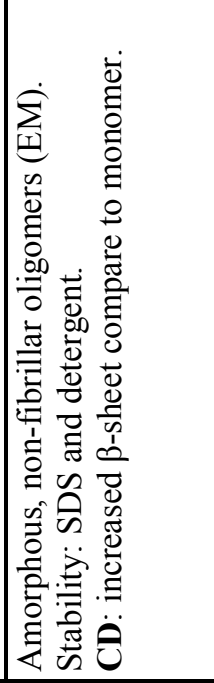 & & 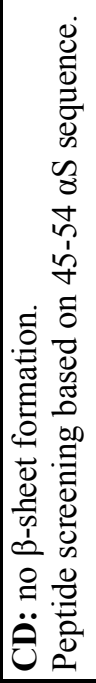 & 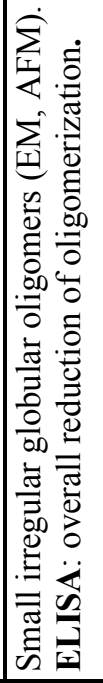 & 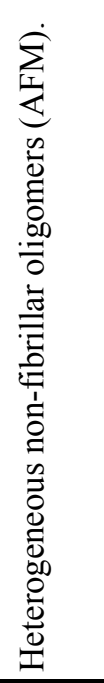 & 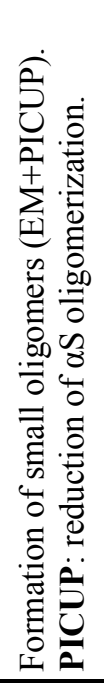 \\
\hline 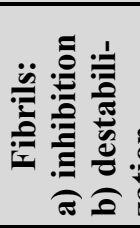 & 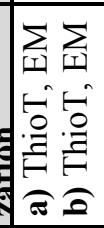 & 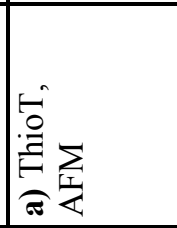 & 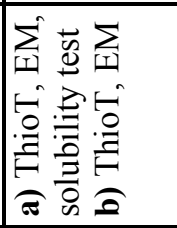 & 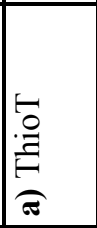 & 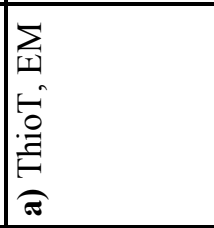 & 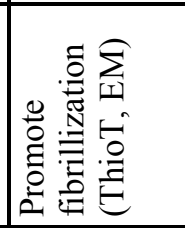 & 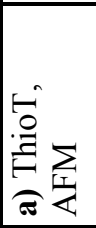 & 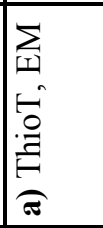 & 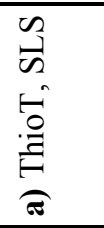 & 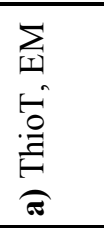 \\
\hline 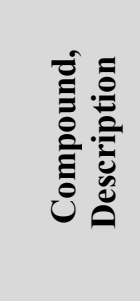 & 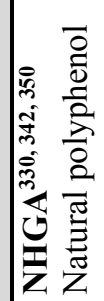 & 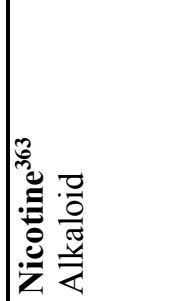 & 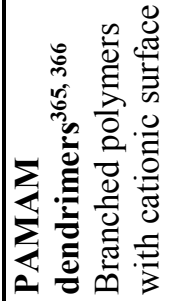 & 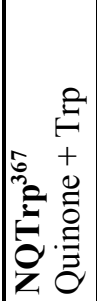 & 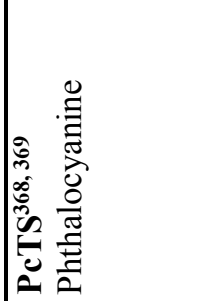 & 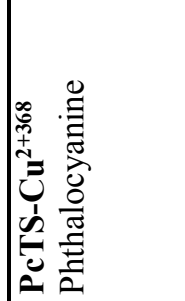 & 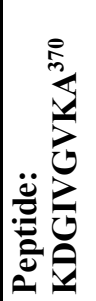 & 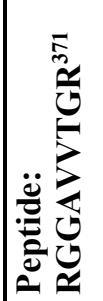 & 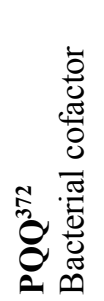 & 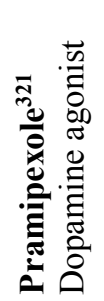 \\
\hline
\end{tabular}


Table 1.15. Inhibitors of $\alpha$-synuclein aggregation (continued)

\begin{tabular}{|c|c|c|c|c|c|c|c|c|c|c|c|}
\hline 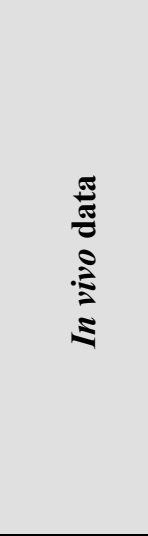 & 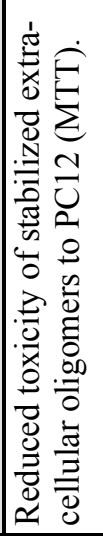 & 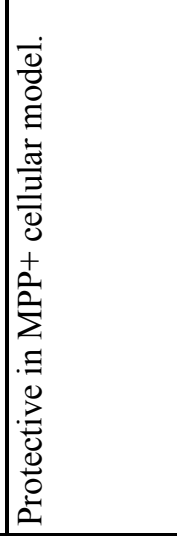 & & 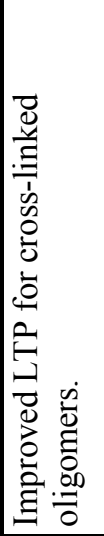 & & & 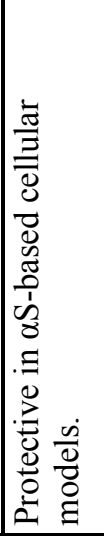 & & & 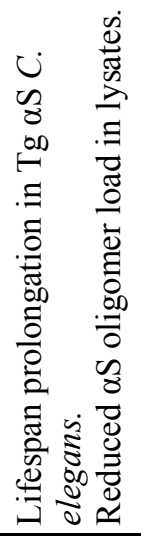 & \\
\hline 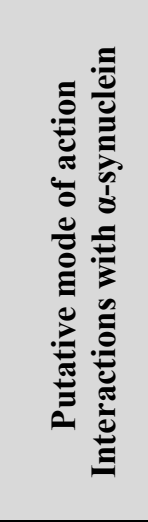 & 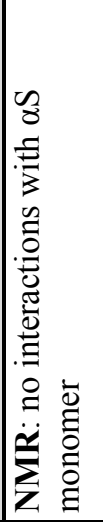 & 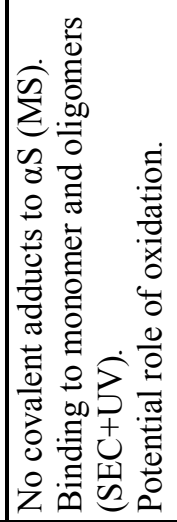 & & & & 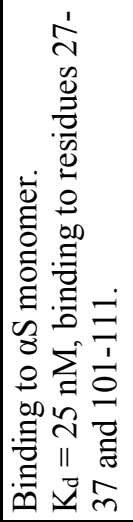 & & 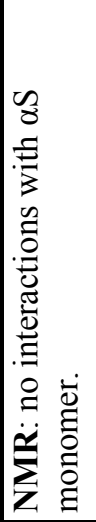 & & 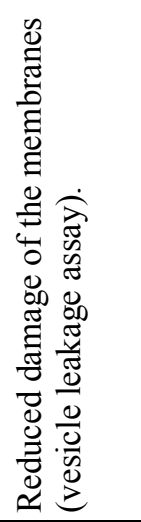 & \\
\hline 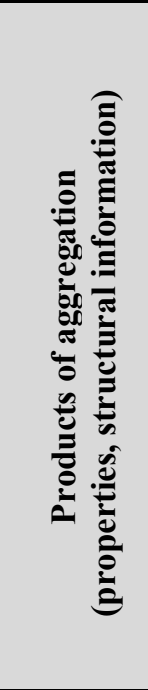 & 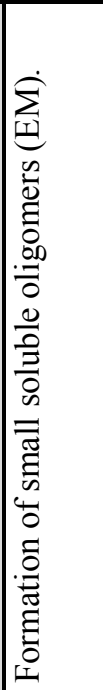 & 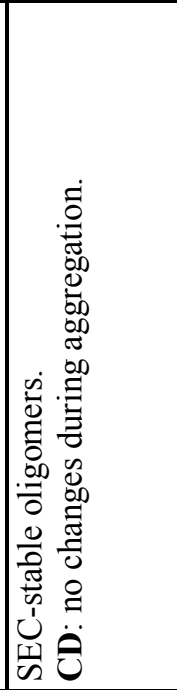 & 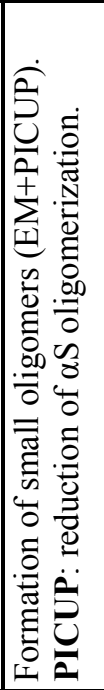 & 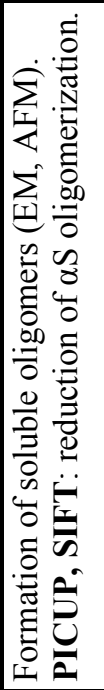 & 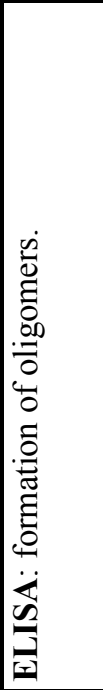 & 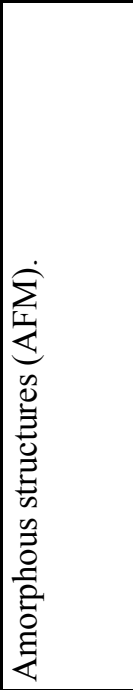 & 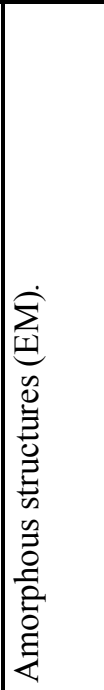 & 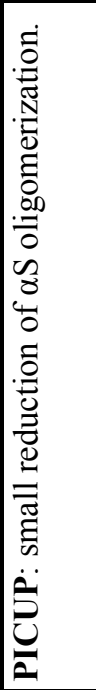 & 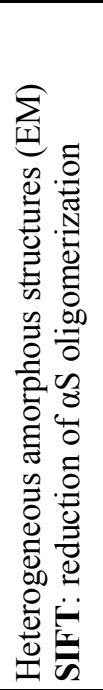 & 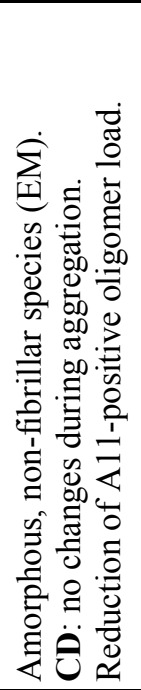 & 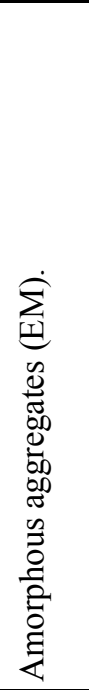 \\
\hline 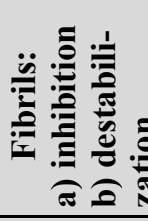 & 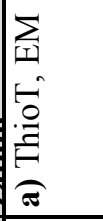 & 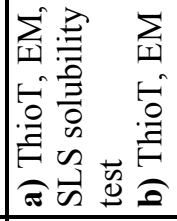 & 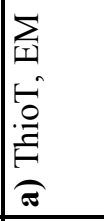 & 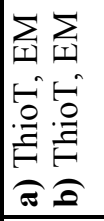 & $\begin{array}{l}\overline{0} \\
\stackrel{\theta}{\vec{E}} \\
\bar{\sigma}\end{array}$ & 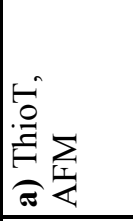 & 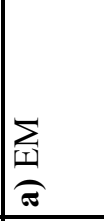 & 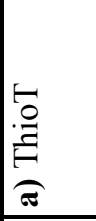 & 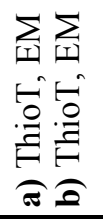 & 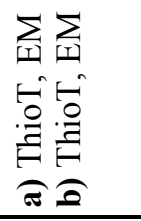 & 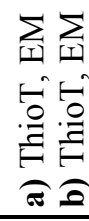 \\
\hline 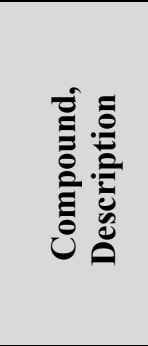 & 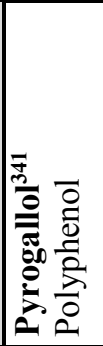 & 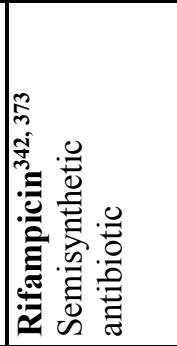 & 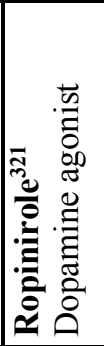 & 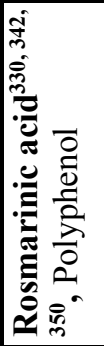 & 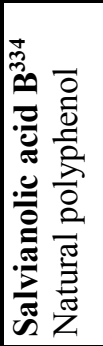 & 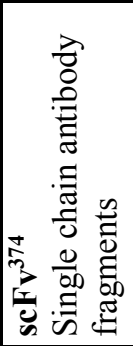 & 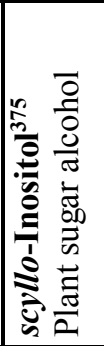 & 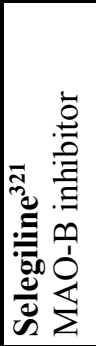 & 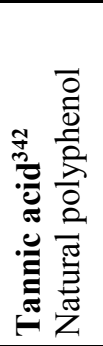 & 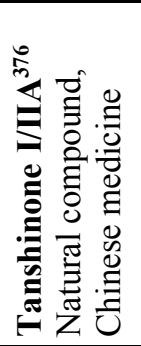 & 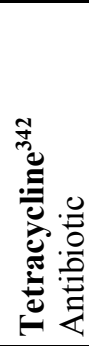 \\
\hline
\end{tabular}


Table 1.15. Inhibitors of $\alpha$-synuclein aggregation (continued)

\begin{tabular}{|c|c|c|c|c|c|c|c|}
\hline 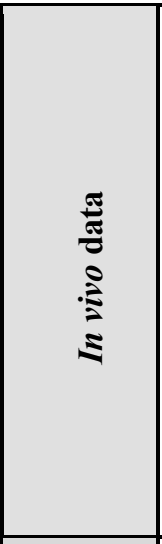 & 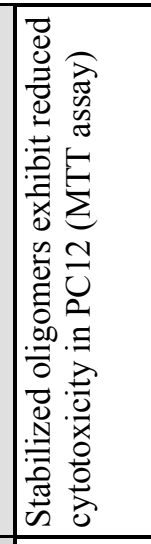 & 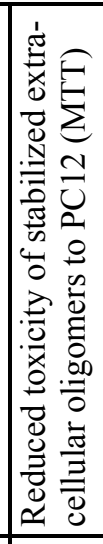 & & & 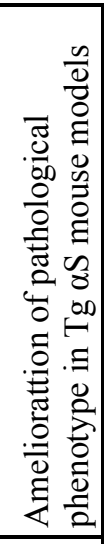 & & 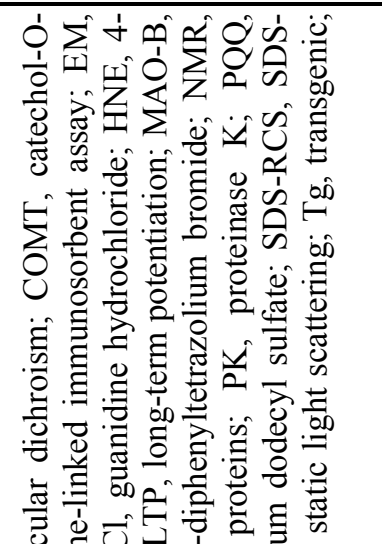 \\
\hline 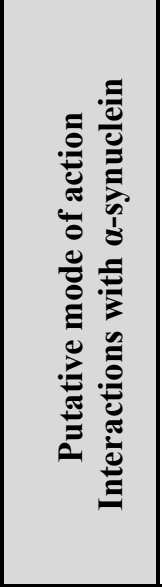 & & 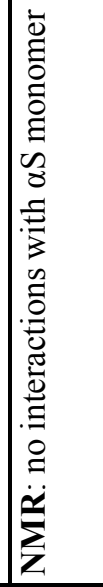 & 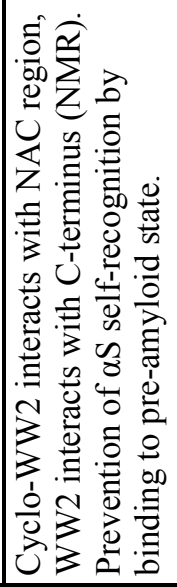 & 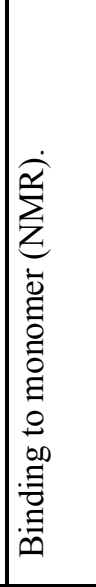 & 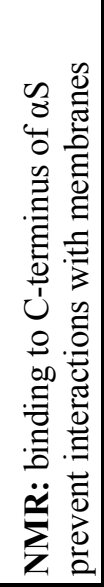 & & 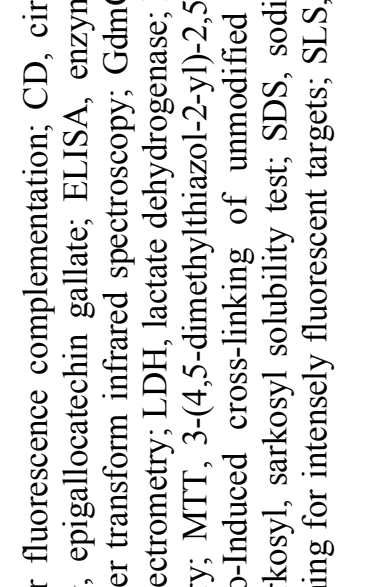 \\
\hline 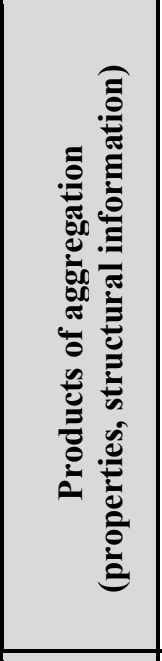 & 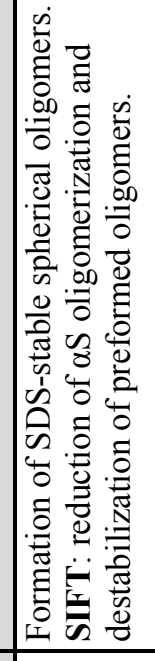 & 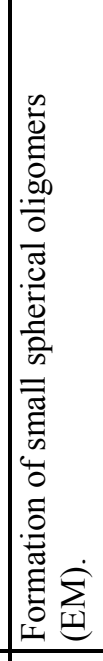 & 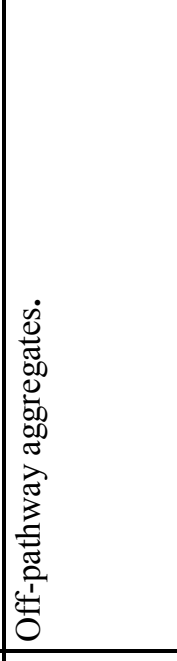 & 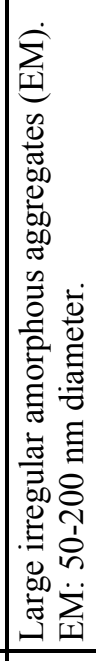 & & 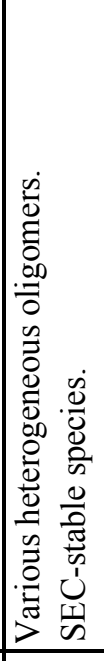 & 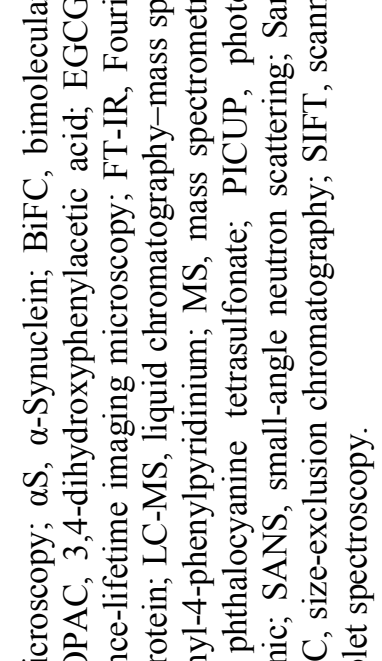 \\
\hline 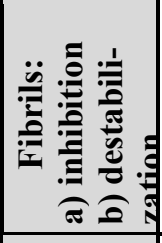 & 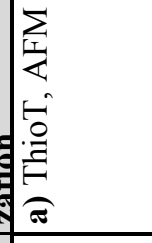 & 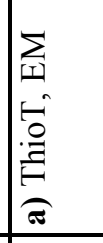 & 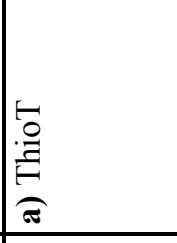 & 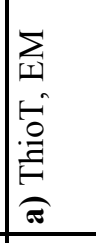 & 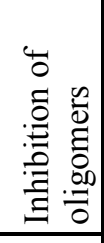 & 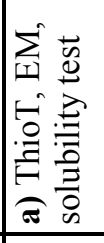 & 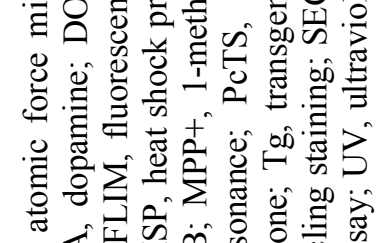 \\
\hline 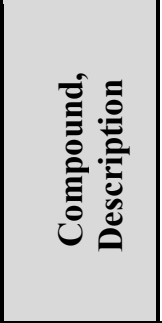 & 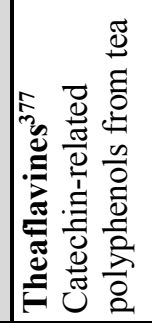 & 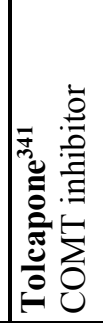 & 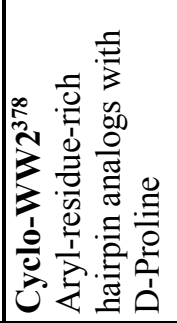 & 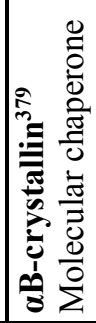 & 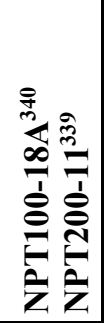 & 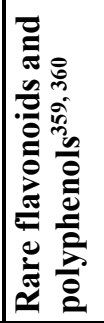 & 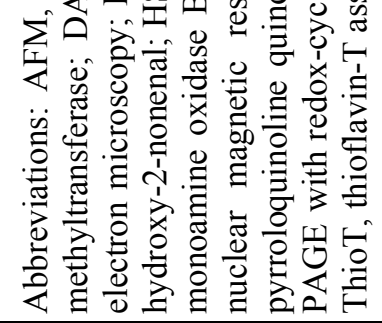 \\
\hline
\end{tabular}


Table 1.16. Chemical structures of inhibitors listed in table 1.15. (alphabetically)

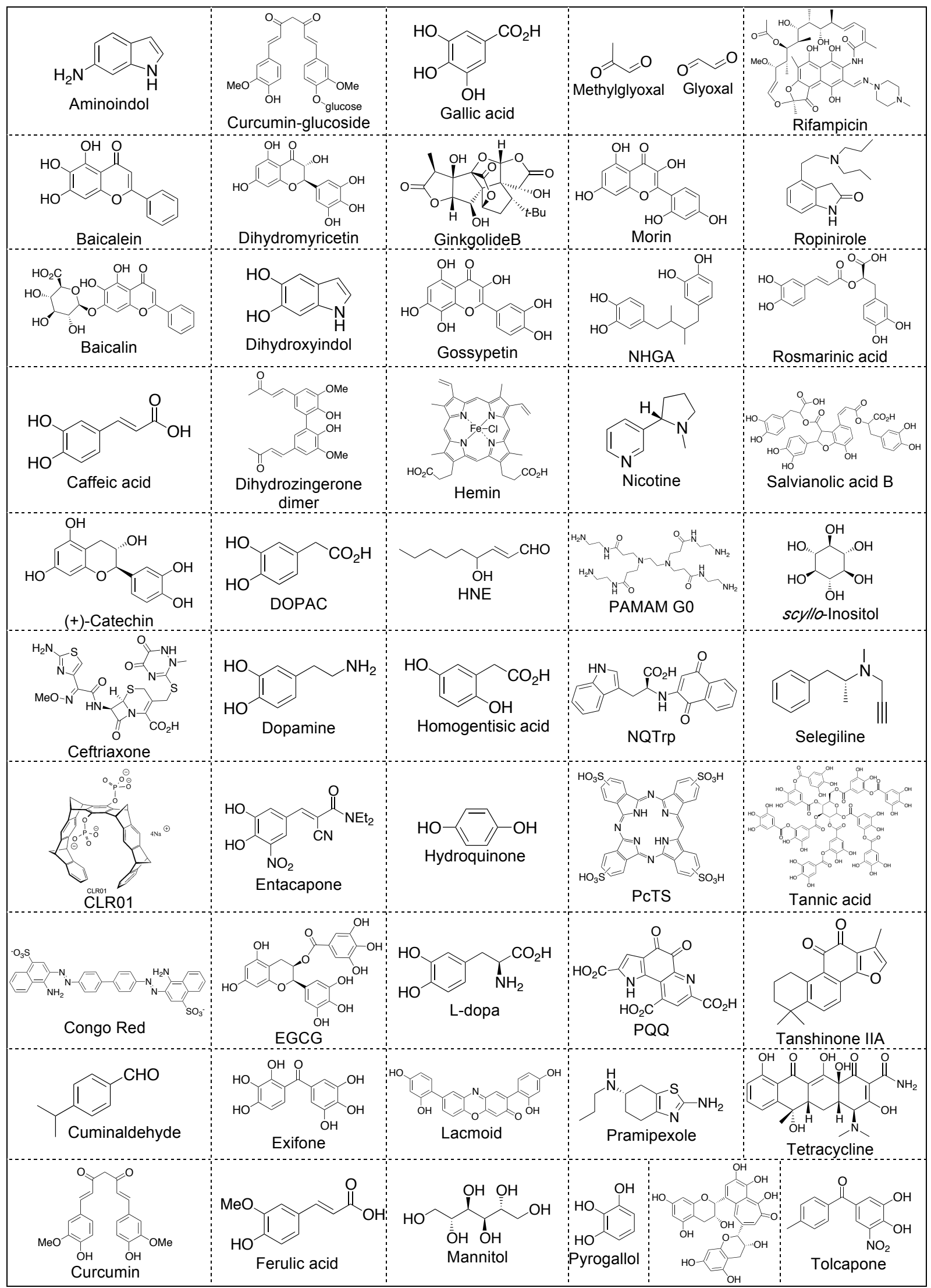




\subsubsection{Disease-modifying strategies: $\alpha$-Synuclein downstream toxicity}

Beside $\alpha$-synuclein-associated approaches, a range of the pathological processes, which are potentially downstream of $\alpha$-synuclein misfolding, may also contribute substantially to dysfunction and death of neurons and, therefore, the therapeutic targeting of these pathways might be neuroprotective. In this regard, following pathophysiological pathways are currently in focus: mitochondrial dysfunction, ER stress, neuroinflammation, microglia activation, oxidative stress, apoptosis, excitotoxicity, calcium dysregulation and others (Fig. 1.33) ${ }^{105,132 \text {, }}$ 233, 242, 304, 305. Accordingly, the drugs capable of improving the function of altered physiological pathways, reducing the level of toxic species like ROS or inhibiting the cascades involved in neuronal cell death may have a potential to emerge as a diseasemodifying therapeutic option for Parkinson's disease. However, it's worth to mention that the clinical trials evaluating a number of promising candidates have failed so far to demonstrate the neuroprotective effects, despite the positive data in preclinical studies. The main characteristics of potential neuroprotective drugs, some aspects of underlying molecular mechanisms as well as outcome from preclinical and clinical studies are summarized in Table $1.17^{233,242,305,306,308-311,314,380-384}$.

\subsubsection{Disease-modifying strategies: Restorative therapies}

Fundamentally different and potentially advantageous treatment approaches involve the use of a cell transplantation to replace the degenerated neurons or application of trophic factors and related neuroimmunophilin ligands to prevent the cell death and stimulate regeneration. Despite mixed and inconclusive results of cell replacement therapy in Parkinson's disease that have been generated for almost 30 years starting with a first transplantation of nigral tissue in 1987, this approach is still considered a promising 380,385 . Thus, following facts support this view, namely, an effective reinnervation of grafted dopaminergic neurons, a clinical benefit for a small number of patients, a current work on optimization of transplant protocol and patient selection and, finally, development of novel sources of transplantable dopaminergic neurons ${ }^{385}$. Regarding a possible sources of the transplant tissue, four candidates have been proposed: human embryonic stem cells, induced pluripotent stem cells, induced neuronal cells and induced dopaminergic cells ${ }^{385}$. The detailed discussion of protocols and types of transplantable dopaminergic neurons is beyond the scope of this thesis (for detailed review ${ }^{309}, 385$ ), but it is noteworthy to mention a new European multicenter project called TRANSEURO that is focused on optimization of the major aspects 
of neuronal transplantation in context of Parkinson's disease and evaluation of fetal midbrain transplants to patients with early Parkinson's disease ${ }^{385}$.

Similar to the cell replacement therapy, the application of trophic factors and related neuroimmunophilin ligands has been shown to provide neuroprotection and even restoration of neuronal function in various toxin-based animal models. Among the tested candidates in preclinical studies, two trophic factors, glial-derived neurotrophic factor (GDNF) and neuroturin, and two neuroimmunophilin ligands, FK506 and GPI-1485, have been selected for clinical trials based on efficacy, safety and tolerability profile $242,304,305,314$.

GDNF is a potent neurotrophic factor for dopaminergic neurons that is known to enhance the neuronal survival. For instance, GDNF-based treatment of MPTP-lesioned mice and monkeys has been shown to reverse the neurodegenerative phenotype and restore the motor behavior ${ }^{242,380}$. Neuroturin is another GDNF-related neurotrophic factor that following viral vector delivery exerts robust beneficial effects in 6-OHDA rat and MPTP monkey models ${ }^{242}$. Despite significant limitations of trophic factor-based therapy with respect to delivery protocols and poor blood-brain barrier penetration, several clinical trials have been performed testing intraventricular and intraputaminal infusions of GDNF as well as gene transfer approaches with viral vectors, like AAV2-GDNF and AAV2-neuroturin ${ }^{242,304,305,314 .}$ Unfortunately, all completed clinical studies have failed to provide benefit in comparison to control group and revealed a high risk of troublesome side effects. Nevertheless, further development and optimization of delivery protocols may still have a potential to be translated to disease-modifying therapy. To that end, a next clinical trial with modified AAV2-GDNF treatment in advanced Parkinson's disease is currently ongoing (NCT01621581, www.clinicaltrials.gov)

An alternative to neurotrophic factor treatment is the application of the neuroimmunophilin ligands that can potentially provide the trophic-like effects. To date, two molecules, immunosuppressant FK506 and related derivative GPI-1485 lacking immunosuppressing activity, have attracted a considerable interest $\mathrm{t}^{242,305,382}$. Thus, these neuroimmunophilin ligands have shown to be neuroprotective in several toxin-based models of Parkinson's disease. Results from follow-up clinical trials suggest that both drugs lack disease-modifying effects in Parkinson's disease patients ${ }^{242,305}$. 
Table 1.17. Assessment of potential neuroprotective drug candidates

\begin{tabular}{|c|c|c|c|c|c|c|c|c|c|c|c|c|}
\hline 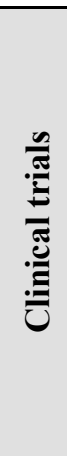 & & 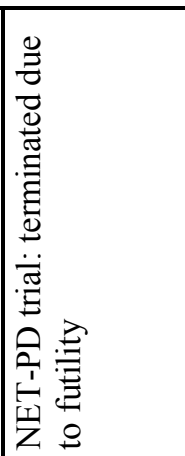 & 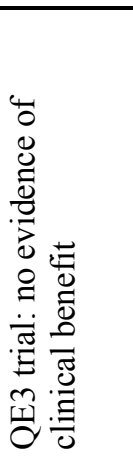 & & 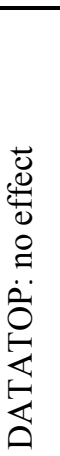 & 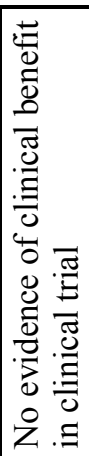 & 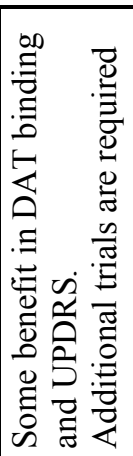 & 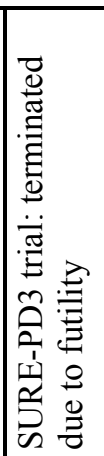 & & 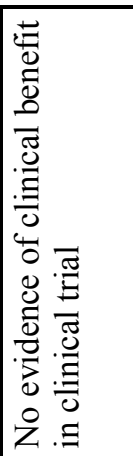 & 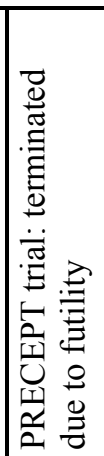 & 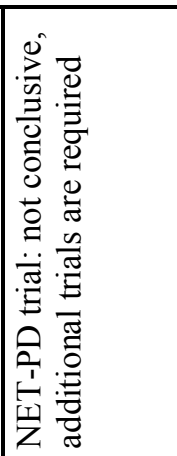 \\
\hline 忩 & & + & + & & + & 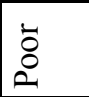 & ¿̈ & $\begin{array}{l}\ddot{\circ} \\
\circ \\
\circ\end{array}$ & & + & + & + \\
\hline 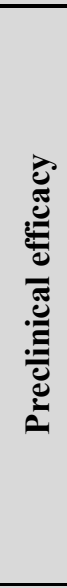 & 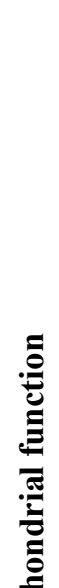 & 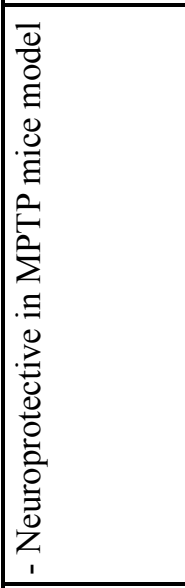 & 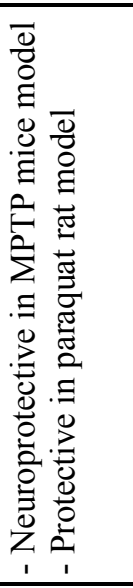 & 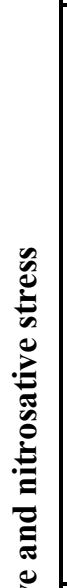 & 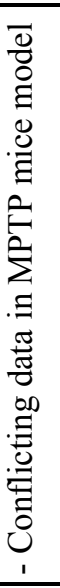 & 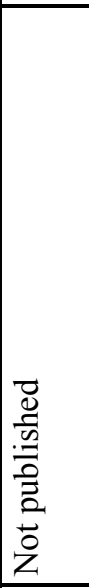 & 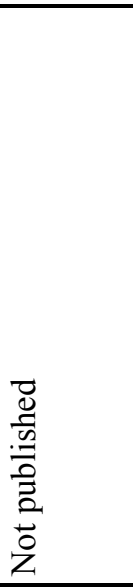 & 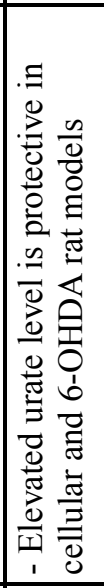 & 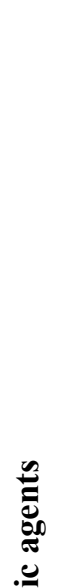 & 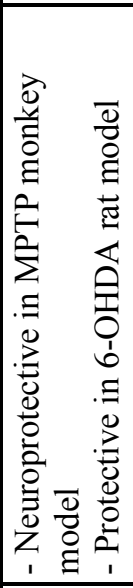 & 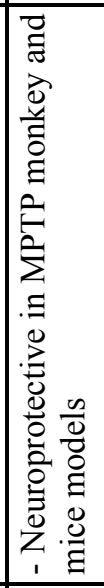 & 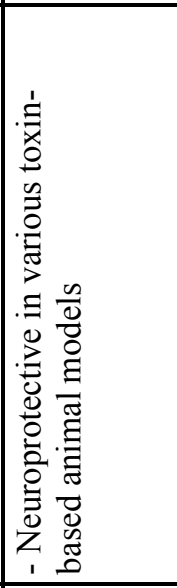 \\
\hline 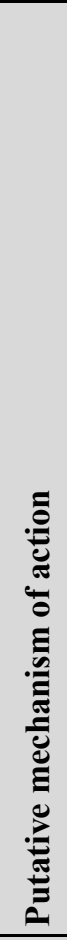 & 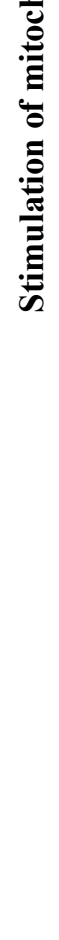 & 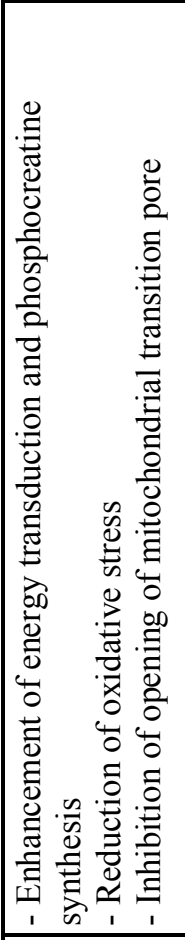 & 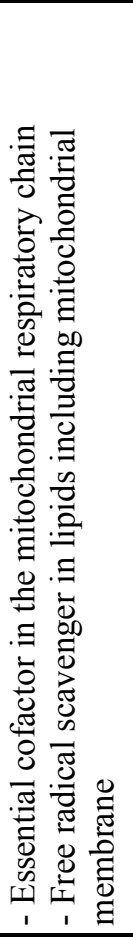 & 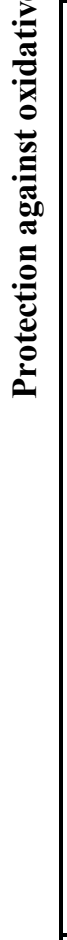 & 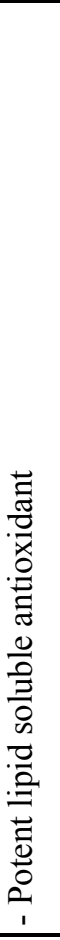 & 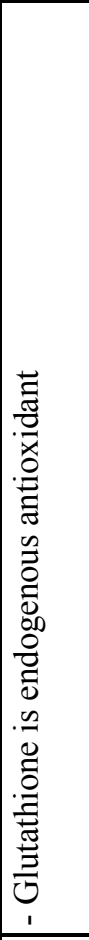 & 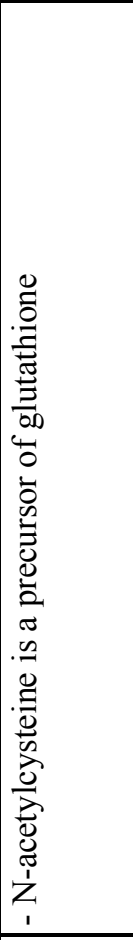 & 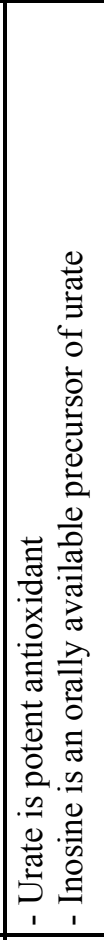 & & 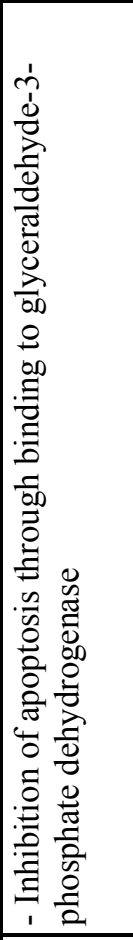 & 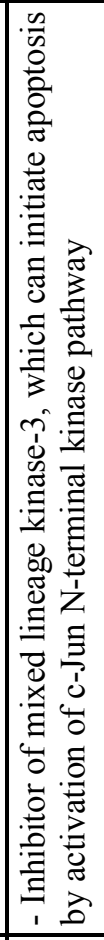 & 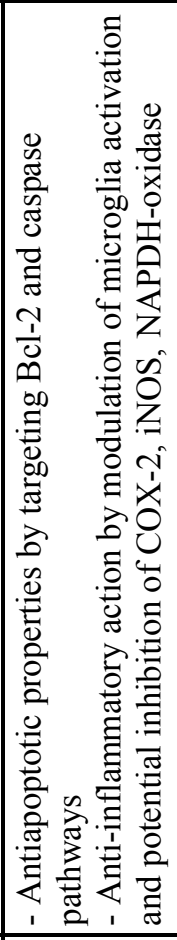 \\
\hline $\begin{array}{l}\vec{E} \\
\stackrel{5}{*}\end{array}$ & & 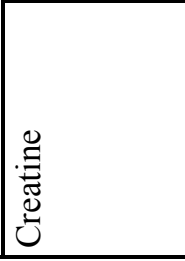 & 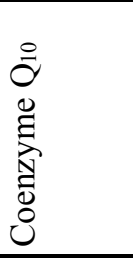 & & 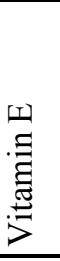 & 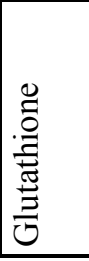 & 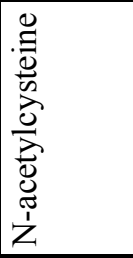 & 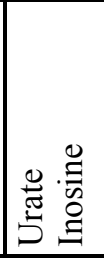 & & 先 & 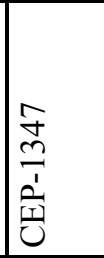 & 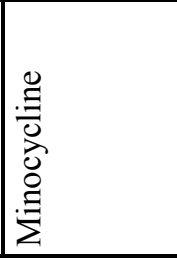 \\
\hline
\end{tabular}


Table 1.17. Assessment of potential neuroprotective drug candidates (continued)

\begin{tabular}{|c|c|c|c|c|c|c|c|c|c|c|c|c|c|c|c|c|}
\hline 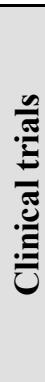 & & 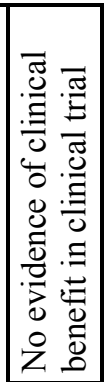 & & 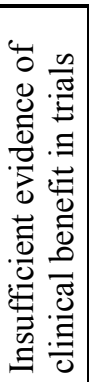 & 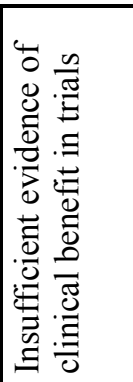 & 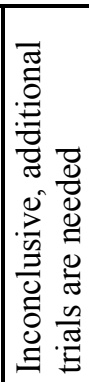 & & 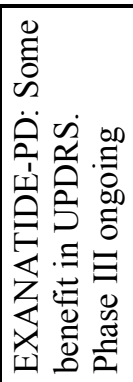 & 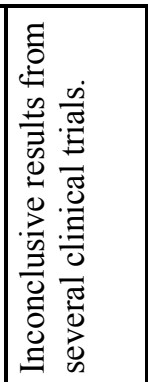 & 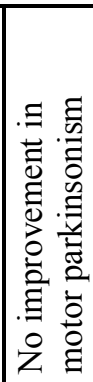 & 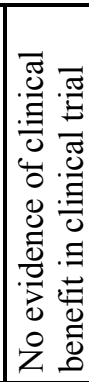 & 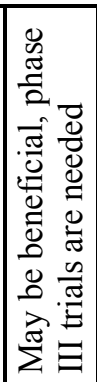 & 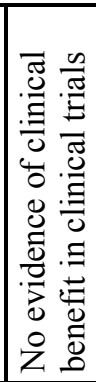 & & 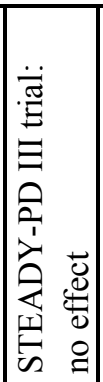 & 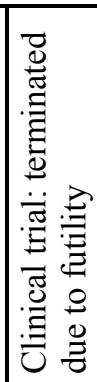 \\
\hline$\underline{\mu}$ & & + & & + & + & + & & + & +++ & + & + & $\overline{0}$ & + & & & + \\
\hline & $\mid$ & 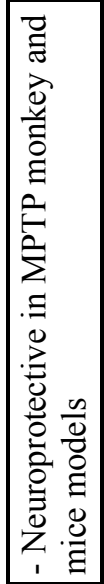 & $\stackrel{0}{0}$ & 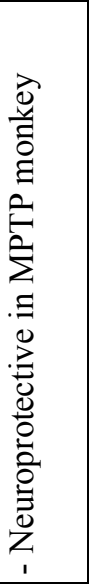 & 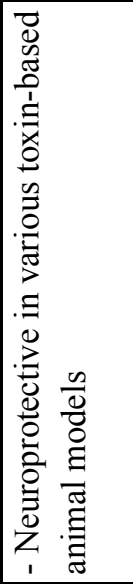 & 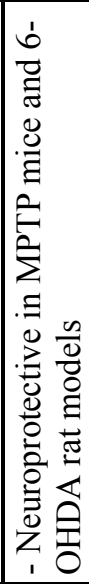 & : & 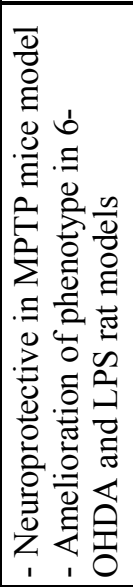 & 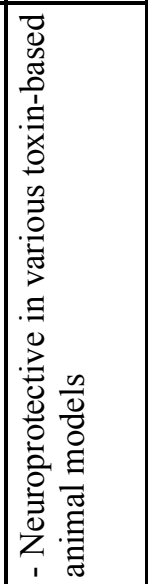 & 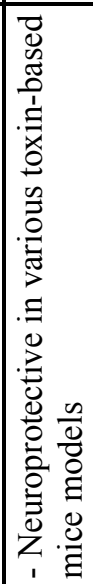 & 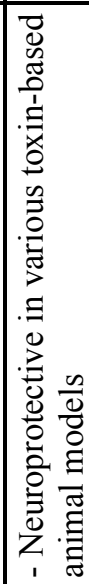 & 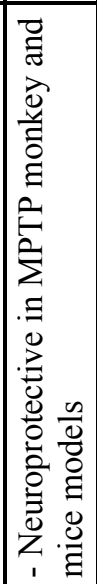 & 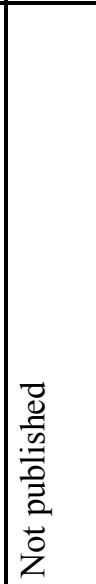 & 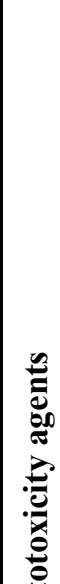 & 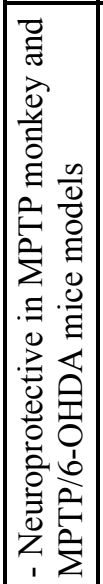 & 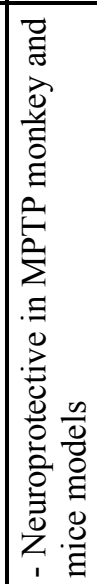 \\
\hline 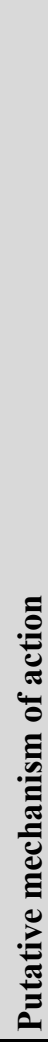 & |⿹ & 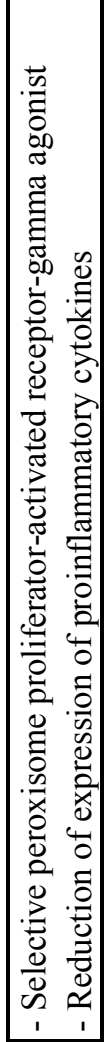 & 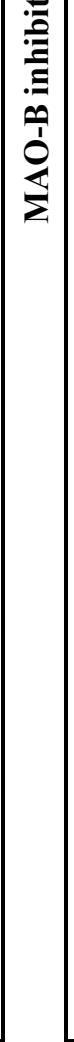 & 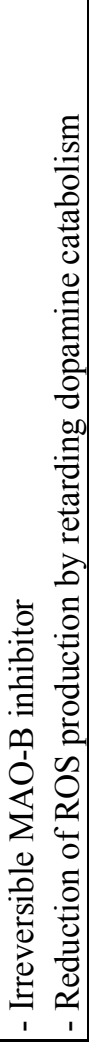 & 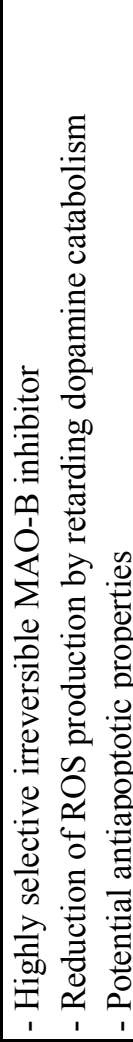 & 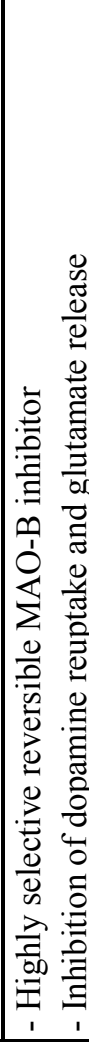 & 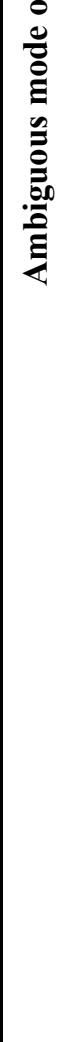 & 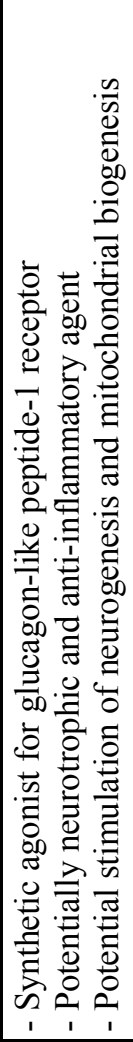 & 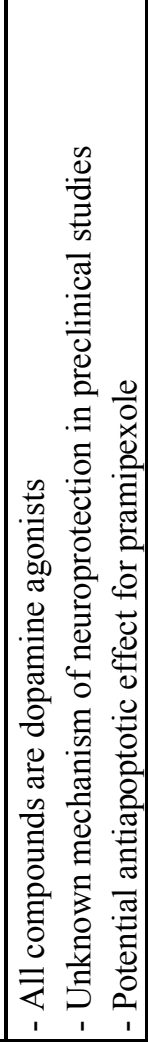 & 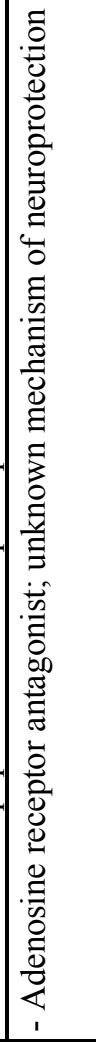 & 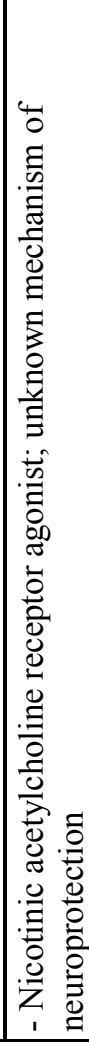 & 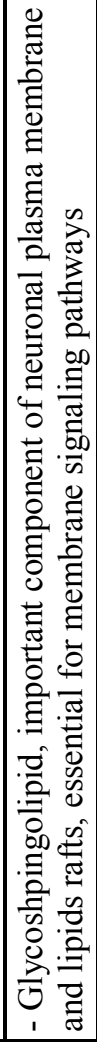 & 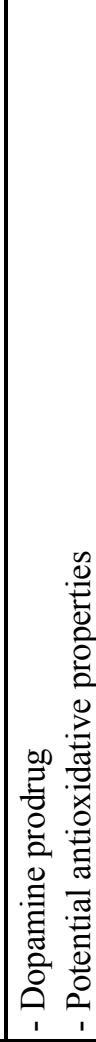 & 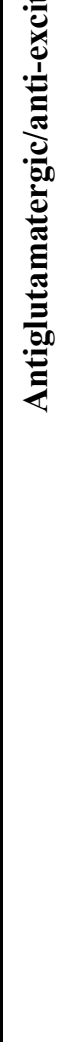 & 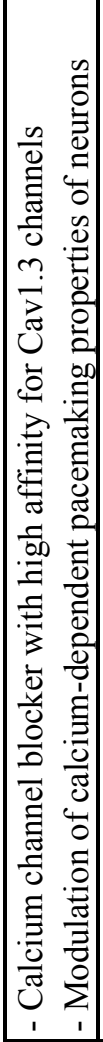 & 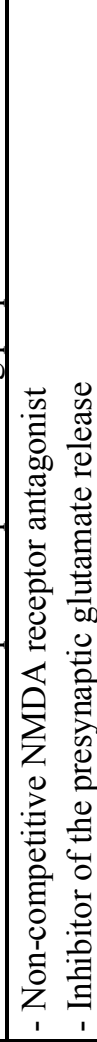 \\
\hline & & 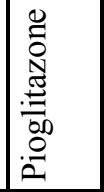 & & 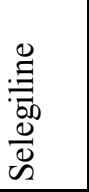 & 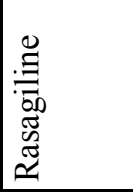 & 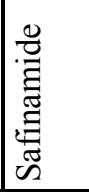 & & 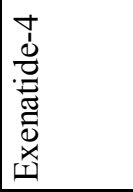 & 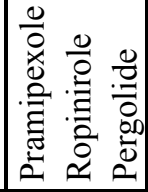 & & 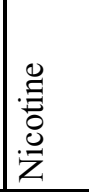 & 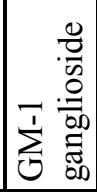 & & & 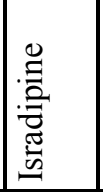 & $\begin{array}{l}\stackrel{0}{\circ} \\
\stackrel{N}{=}\end{array}$ \\
\hline
\end{tabular}




\section{Results and Discussion}

\subsection{Aims and introduction}

It is increasingly accepted that the pathogenesis of Parkinson's disease and other protein misfolding diseases is manifold, and several mechanisms have been implicated as crucial to neurodegeneration, as discussed at length in the chapters 1.3 and 1.4.8.3-1.4.8.7 for Parkinson's disease. Among the proposed mechanisms of neurotoxicity, protein misfolding may probably compromise a central cascade that leads to loss of neurons. Therefore, a modulation of various aspects of biochemistry and turnover of $\alpha$-synuclein or other amyloidogenic proteins is considered as a promising therapeutic target ${ }^{132,306,386}$. Moreover, the targeting of protein misfolding by small organic drug-like compounds is consistently recognized as an attractive therapeutic approach with an obvious disease-modifying potential ${ }^{109,306}$. Based on growing evidence indicating that pathological oligomers rather than large fibrillar deposits constitute the key neurotoxic species ${ }^{127}$, we set out to discover small molecules interfering with pathological oligomerization and eventually to provide a therapeutic strategy for causal disease-modifying treatment of devastating protein-misfolding diseases for which only symptomatic treatment is available so far.

The main goal of my thesis was to design, synthesize and assess novel compounds that can modulate in vitro and in vivo the aggregation/misfolding of amyloidogenic proteins, including prion protein, $\alpha$-synuclein, amyloid-beta peptide, Tau protein, islet amyloid polypeptide and others. The optimization of initial hits relied on structure activity relationship in a series of diverse in vitro assays. The most promising leads were then used in low throughput assays, i.e. mouse models of neurodegenerative diseases. Whether the applied selection criteria were always justified, or lead to overlooking active compounds will be discussed with examples. In addition to the evaluation of the compound efficacy, we also put much effort into elucidating the underlying molecular mechanisms and potential mode(s) of action of most active substances. Moreover, promising candidates have entered 
pharmacokinetic and metabolic studies, in combination with the development of prodrugs and appropriate administration protocols with the goal to improve the bioavailability. For therapeutic compounds, broad efficacy for many aggregating proteins is desirable due to comorbidity and aggregation of more than one protein in one disease such as in Alzheimer's disease.

The results obtained in this multidisciplinary and multicenter project are divided in 10 chapters. To the topics that are described in my thesis I devoted approximately two-thirds of the time of my $\mathrm{PhD}$. For every study that was conducted in collaboration, the contribution of colleagues and my direct personal contribution are stated. 


\subsection{Screening of aggregation inhibitors}

\subsubsection{SIFT assay}

A novel screening platform based on scanning of intensively fluorescent targets (SIFT) has been selected by our collaborator Prof. Armin Giese in this project as one of the core biophysical technique for investigation of the aggregation of disease-associated amyloid proteins and, more importantly, for identification of small drug-like compounds interfering with protein misfolding ${ }^{387}$. In a pioneering work, Prof. Armin Giese (LMU, Munich), who was involved in development and implementation of a novel SIFT-based screening platform, has initiated a systemic high-throughput screening of molecules that interfere with the interaction of $\mathrm{PrP}^{\mathrm{C}}$, cellular prion protein, and $\mathrm{PrP}^{\mathrm{Sc}}$, disease-associated, $\beta$-sheet-rich, scrapie isoform of prion protein ${ }^{7}$.

The molecular SIFT assay clearly represents one of the most advanced and sensitive high-throughput screening approaches for investigation of protein aggregation and search for compounds interfering with this process. Here, I would like to describe briefly the main principle and advantages of this assay. The SIFT assay has been developed as a combination of fluorescence correlation spectroscopy, dual-color labeling schemes and dual-color scanning set-up (Fig. 2.2.1). The use of conventional fluorescence correlation spectroscopy

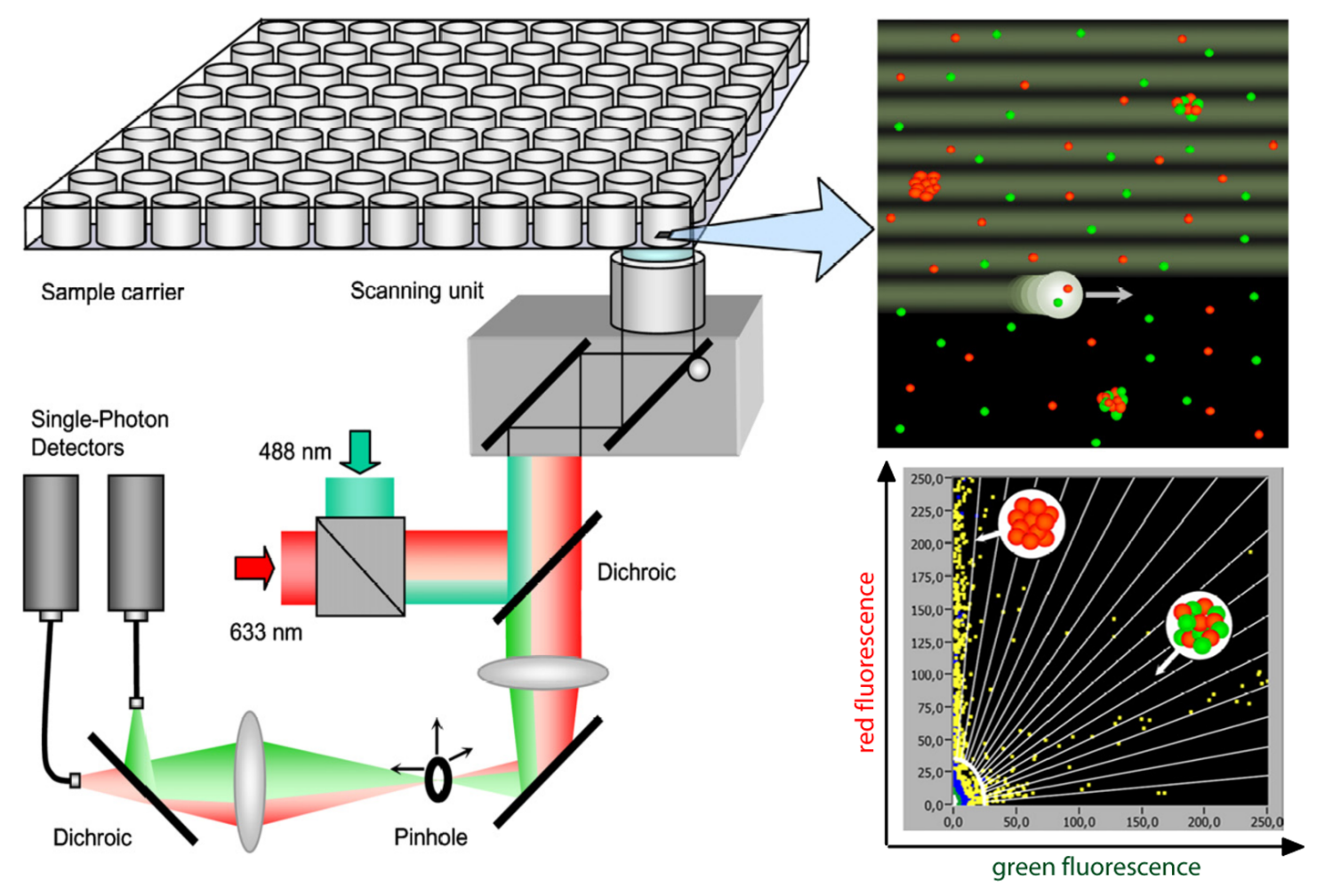

Figure 2.2.1. Principle of aggregation analysis by dual-color SIFT. Figure was kindly provided by Prof. Dr. A. Giese. 
allows the analysis of the fluorescence fluctuations generated by diffusion of labeled species through an open detection volume. Given the heterogeneity and complexity of the protein aggregation, implementation of the scanning set-up and dual-color labeling provides significant improvement of detection efficiency, reproducibility and analysis ${ }^{7,387}$. As shown in Figure 2.2.1, the SIFT recording can be done in highly automated manner with multiwell plates. The aggregation assay with the SIFT measurement is normally performed on a mixture of target protein that is labeled with two different fluorescence dyes or antibody(s). Following various incubation conditions, the sample is analyzed by the scanning procedure, in which laser focus is moved through the sample and fluorescence from two corresponding fluorophores is recorded simultaneously by two single-photon detectors with the single molecule sensitivity. If the protein in the sample is aggregated or oligomerized, the generated multimeric species are intensively labeled, because they are composed of many single labeled monomers. When such aggregated particle(s) occurs in the laser focus, a burst of high fluorescence intensity is detected (Fig. 2.2.1). Remarkably, the evaluation of the SIFT data can provide useful information regarding the molecular composition of the target particle(s), based on signal intensity of specific fluorophore, as well as general description of the sample, like size and relative populations of various species. Furthermore, the step-by-step improvement of the hardware and experimental protocols for this assay system allows nowadays working at very low protein concentrations $(2-6 \mathrm{nM})$ in small sample volumes (20 $\mu \mathrm{l})$ and with high throughput capacity (up to 1000 samples per day) ${ }^{7}$. Currently, the SIFT assay is established with some setup variation for three proteins, prion protein, $\alpha$-synuclein and Tau. Given the described advantages, SIFT method clearly represents a highly attractive approach for high-throughput screening of anti-aggregative compounds.

\subsubsection{Primary SIFT and cell culture screening}

Prof. Armin Giese (LMU, Munich) has initiated a first round of high-throughput screening of antiprion agents ${ }^{7}$. As a base for primary screening, the combination of molecular SIFT assay for inhibition of the prion protein and a prion-infected cell culture assay has been selected, and two commercially available libraries (DIVERSet1 and DIVERSet2; 10000 compounds each) of chemically diverse drug-like compounds, provided by Chembridge Corp., have been tested.

One of the reasons to test compounds in the prion-related cellular assay is the lack of appropriate cell-based high-throughput screening methods for other disease-relevant amyloid proteins. Similar to the SIFT assay, a high-throughput prion cell screening system, which was 
established in the laboratory of Prof. M. H. Groschup, is suitable for the rapid testing of large compound libraries in various cell lines infected with prion strains. Experimentally, the antiprion activity of drug-like molecules was determined by compound application to scrapieinfected cell lines (ScN2a, SMB[RC040] and SMB[22F]) followed by protease K digestion and immuno-dot plot detection of $\mathrm{PK}$ resistant prion protein $\left(\mathrm{PrP}^{\mathrm{Res}}\right)$ in cell lysates ${ }^{388}$.

In the first phase of the project, a library of 10,000 chemically diverse drug-like compounds was tested for inhibition of prion protein aggregation in the molecular SIFT screening assay. Based on the evaluation of the primary hits from SIFT assay and the cellular antiprion model, $N$-benzylidene-benzohydrazide (Fig. 2.2.2)<smiles>O=C(NN=Cc1ccccc1)C1=CCCC=C1</smiles>

N-benzylidene-benzohydrazide

Figure 2.2.2. $N$-benzylidenebenzohydrazide scaffold. derivatives were identified as a new lead structure with anti-prion activity providing a proof of concept of the screening strategy ${ }^{7}$. However, $\mathrm{N}$ benzylidene-benzohydrazides contain a Schiff's base like structure ( $=\mathrm{N}-\mathrm{NH}-\mathrm{CO}-)$ that may result in low stability and in rapid metabolism in vivo. In order to identify additional lead structures with more favorable pharmacological properties, the screening campaign was extended to 10,000 additional compounds, which were tested in SIFT and in a microtiter plate based high-throughput anti-prion cell culture assay (mouse neuroblastoma [ScN2a] and scrapie mouse brain [SMB] cells infected with Rocky Mountain Laboratory scrapie strain). As a result of the data evaluation a SAR-map was generated (Fig. 2.2.3). This analysis identified a group of highly active compounds belonging to the chemical compound class of 3,5-DiPhenyl-Pyrazole (DPP) derivatives (Fig. 2.2.3). This group includes five neighboring clusters (termed DPP_1 through DPP_5) containing structurally similar compounds that show high activity in both assays. The prototypical compounds representing these clusters are displayed on Figure 2.3 and, in fact, all of these chemical structures could be classified as 3,5-Diphenyl-pyrazole (DPP) derivatives. To confirm the antiprion activity in vivo, one of the DPP compounds from the initial screening, namely 10353_F11 (Fig. 2.2.3), was tested in the prion-infected mice model. Thus, a 2 weeks treatment with the compound 10353_F11 significantly prolonged the survival (by 11 days) after intracerebral inoculation with RML prion strain ${ }^{10}$. Notably, the protective effect of 10353_F11 was observed following an oral administration, which suggests favorable pharmacokinetic properties of DPP compounds and blood-brain barrier (BBB) penetration On this basis, DPP scaffold has been selected as a core structural element and we have synthesized a focused library of DPP-related compounds for 
further testing in vitro, in cell culture, and in vivo for to their effects on the aggregation of disease-relevant amyloid proteins, therapeutic efficacy in animal models and other potential applications. Although originally the "DPP" abbreviation was introduced for 3,5-Diphenylpyrazole, in my thesis I use DPP abbreviation for DiPhenyl-Pentocycles. Below in the text I will apply the term "DPP" to denote the class of chemical compounds represented by Markush formula in Figure 2.3.1.

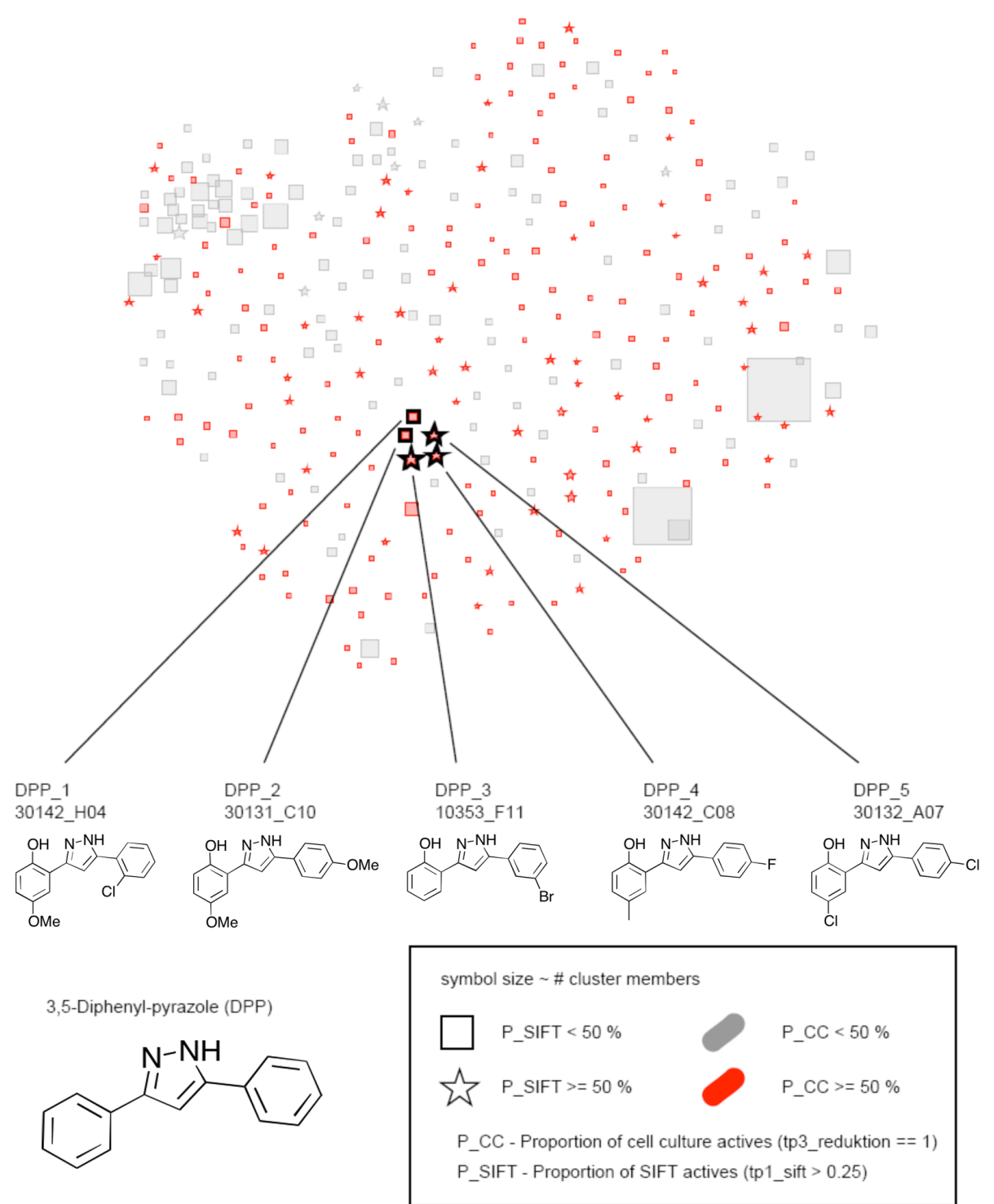

Figure 2.2.3. Structure-Activity-Relationships (SAR)-Map and DPP scaffold. SARmap is generated for substances of the library screened for antiprion activity using the SIFT and cellular assays. The SAR-Map shows clusters of structurally similar compounds (represented by stars or boxes) built from the 837 hit compounds of the primary cell culture screening campaign of the substance libraries DIVERSet 1 and 2. Symbols are arranged according to their structural similarity. The sizes, shapes and colors of the symbols have been assigned based on cluster-specific properties. The figure is adopted from J. Wagner, S. Ryazanov et al ${ }^{10}$, copyright (C) 2013) 


\subsection{Design and synthesis of the focused library of DPP-related compounds}

\subsubsection{DPP scaffold and overview of focused library}

As mentioned in the previous chapter, the initial high-throughput screening combined with the pilot treatment in the prion-infected mice model revealed the DPPs as particular promising lead compounds in terms of both anti-aggregation activity and pharmacokinetic properties. In an attempt to optimize the DPP lead structure and to get insight into the details of structure-activity relationship (SAR), we designed and synthesized a focused library of DPP-related compounds. Regarding the design of new compounds, we utilized an iterative approach that comprised several rounds of structure optimization and synthesis on a basis of in vitro and in vivo testing and SAR analysis. Moreover, the development of compounds was guided by several additional considerations including the structural properties of published aggregation inhibitors, the metabolic and chemical stability, pharmacokinetic issues and the affordable synthetic approach. In parallel, we have attempted to identify substituents and structural elements that are crucial for the biological activities, brain penetration and sustainable bioavailability.

In order to achieve the stated objectives and goals, we have synthesized and examined the focused library composed of approximately 150 DPP-related compounds. Here, I would like to acknowledge the contribution of my colleague Dr. Andrei Leonov. Together with him we performed the synthesis of all compounds, thus, the compounds with abbreviations anle and sery were prepared by Andrei Leonov and me, respectively. With some exceptions, the vast majority of synthesized DPP-based analogues are covered by the Markush structure that is shown in figure 2.3.1. In our SAR study, we sought to explore several series of compounds, which differ in their substitution at 4 main positions, including the replacement of $R_{1}$ and $R_{2}$ substituents in the flanking phenyl rings, the variation of central heterocyclic ring and the introduction of the $\mathrm{R}_{3}$ substituent into the heterocyclic ring. Despite the fact that

Markush structure of DPP-related library

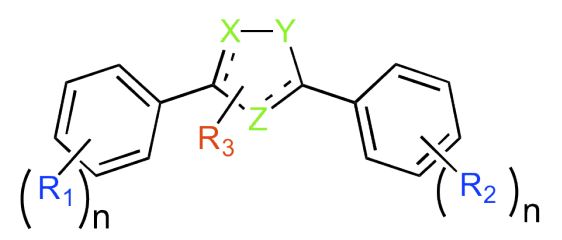

\section{Substitution}

$\mathrm{R}_{1}, \mathrm{R}_{2}$ selected from:

1) $\mathrm{H}$

2) Halogens: $\mathrm{F}, \mathrm{Cl}, \mathrm{Br}$, I

3) $\mathrm{OH}, \mathrm{O}-\mathrm{Alkyl}$, Methylenedioxy

4) $\mathrm{NO}_{2}, \mathrm{NH}_{2}, \mathrm{NMe}_{2}$, $\mathrm{n}=0,1,2,3$
$X, Y, Z$ selected from: C, N, O

$\mathrm{R}_{3}$ selected from: $\mathrm{H}, \mathrm{Br}, \mathrm{OH}$, Alkyl, substituted Alkyl

Figure 2.3.1. Markush structure of DPP-related compounds. Overview of focused library of DPP-related compounds represented as Markush structure. 
our focused library includes the DPP-related molecules covering relatively diverse sets of $\mathrm{R}_{1}$ and $\mathrm{R}_{2}$ substituents, we introduced another level of classification comprising 4 main scaffolds (Fig. 2.3.2A):

1) Compounds with $\mathrm{OH}$ groups;

2) Compounds with OMe groups;

3) Compounds with Methylenedioxy (MD) moiety;

4) Compounds with amino or $\mathrm{NMe}_{2}$ groups.

In our central ring exploration, we selected, successfully synthesized and tested DPP analogues bearing six different five-membered heterocycles containing 1, 2 or 3 heteroatoms, at least one of which is nitrogen (Fig. 2.3.2B).

Given that the lead optimization often requires the broad variation of the structural elements, we tried to ensure the adequate chemical diversity. As discussed below, we also performed the fragment-based compound selection on a basis of structure-activity information for described aggregation inhibitors and corresponding physicochemical properties of molecules of interest.

A

Scaffold 1

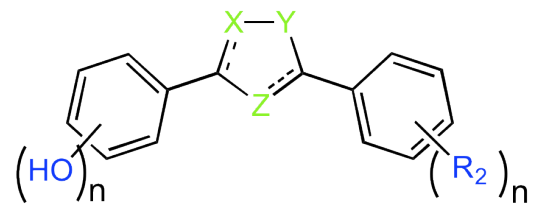

Scaffold 2

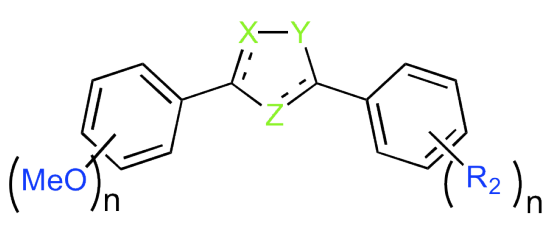

Scaffold 3

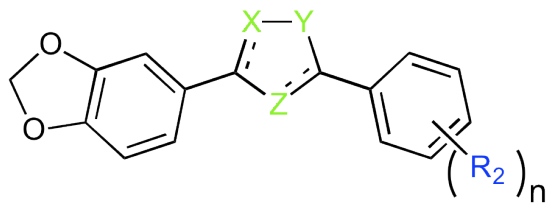

Scaffold 4

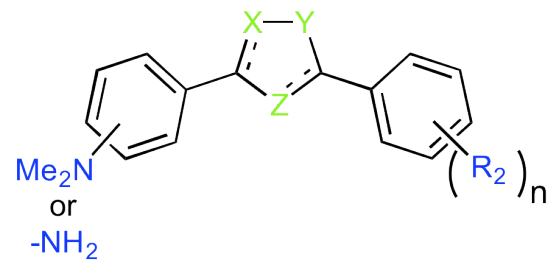

B<smiles>Cc1cc(C)[nH]n1</smiles><smiles>Cc1cc(C)on1</smiles>

pyrazole

isoxazole<smiles>CC1=NNC(C)C1</smiles>

2-pyrazoline<smiles>Cc1c[nH]c(I)c1</smiles>

1H-pyrrole<smiles>Cc1c[nH]c(I)n1</smiles>

imidazole<smiles>Cc1noc(C)n1</smiles>

1,2,4-oxadiazole

Figure 2.3.2. Overview and classification DPP-related compounds. (A) Four main scaffolds within the focused library represented as Markush structure. (Selection of $\mathrm{R}_{2}, \mathrm{X}$, $\mathrm{Y}, \mathrm{Z}$ and $\mathrm{n}$ is shown in figure 2.3.1) (B) Heterocyclic ring systems included in the SAR study. 


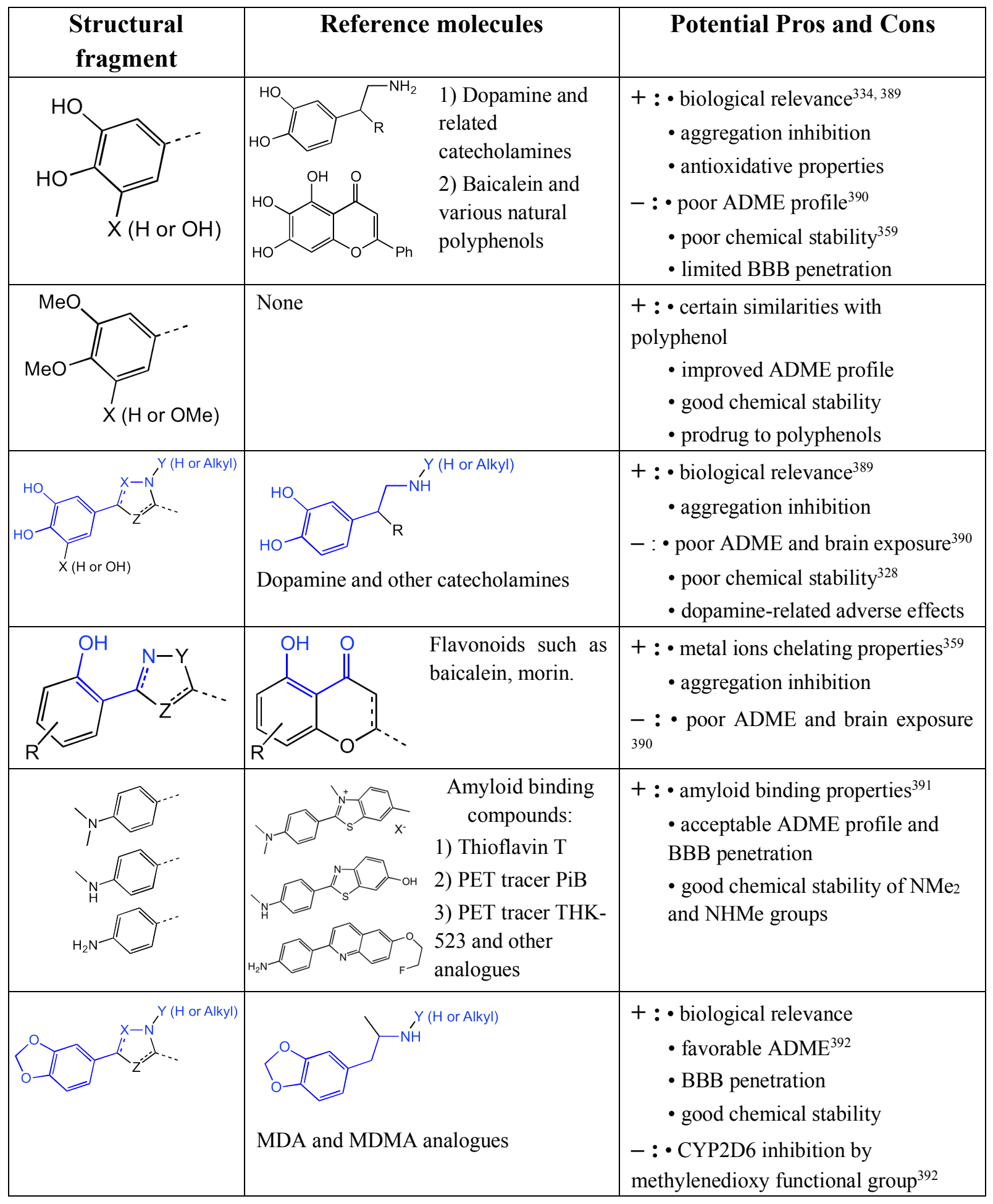

Table 2.3.1. Fragment-based design of DPP compounds. The table summarizes the key structural fragments, which were widely applied for DPP lead optimization. Corresponding theoretical cons and pros for each type of modifications are listed. 


\subsubsection{Contribution of fragment-guided approach in DPP design}

An early objective of design of the novel DPP compound was to modify and optimize the general DPP lead structure. As mentioned above, the majority of generated structures is covered by 4 main scaffolds (Fig. 2.3.2A). To that end, among the DPP analogues examined, the design of many novel molecules was realized by application of simplified fragment-based approach. Thus, we sought to introduce into the DPP lead structure several types of structural fragments or moieties. There are several reasons behind this; first, we included structural fragments that are frequently present in potent anti-amyloidogenic compounds or amyloid interacting molecules, such as various polyphenols, flavonoids, thioflavin $\mathrm{T}$, catecholamines (Table 2.3.1). Secondly, we introduced the structural elements of molecules that are biologically active in the brain, like dopamine, methylenedioxyphenethylamines (MDA, MDMA). Finally, we designed the DPP derivatives with the aim of improving BBB penetration, chemical stability and ADME (absorption, distribution, metabolism and excretion) profile. An example of this lead optimization might be the protection of free $\mathrm{OH}$ groups by methyl or methylene groups potentially leading to favorable ADME profile and refined chemical stability. The key structural fragments, which we applied in our compound design, together with corresponding theoretical advantages and disadvantages for each type of modifications are summarized in table 2.3.1.

\subsubsection{General synthetic schemes}

In combination with the design of a focused library of DPP-related compounds, the corresponding synthetic schemes and analytical methods were developed and successfully applied. In this chapter, I would like to summarize the main synthetic approaches and provide an overview of key intermediates that allowed us to afford the majority of proposed DPP analogues. It should be also noted that almost all reaction schemes have been previously published, however, we have performed a substantial optimization of the reaction conditions to ensure short, reliable and versatile synthetic routes that provide the desired compounds in gram scale and high purity, preferentially without chromatographic methods of the isolation and purification. 
Starting Compounds

Key Intermediates

DPP analogous<smiles>[R][R]c1ccc(C(=O)Oc2cccc(C(C)=O)c2)cc1</smiles><smiles>[R]c1ccc(-c2cc(-c3ccccc3)no2)cc1</smiles><smiles>[R]c1ccc(-c2cc(-c3cc[R]cc3)n[nH]2)cc1</smiles><smiles>[R][R]1cccc(C=O)c1</smiles><smiles>CCCCCCCCC</smiles><smiles>[R]c1ccc(C=CC(=O)c2cc[R]cc2)cc1</smiles><smiles>C[Te]</smiles><smiles>[R]CCC[R]</smiles><smiles>[R][R]c1ccc(C(=O)CC(C[N+](=O)[O-])c2ccc([R])cc2)cc1</smiles><smiles>[R20]OC(=O)c1ccc[R]([R])c1</smiles>

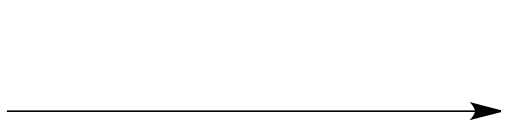<smiles>[R]c1ccc(-c2noc(-c3ccc([R])cc3)n2)cc1</smiles><smiles>[R][R]1cccc(C(=O)CBr)c1</smiles><smiles>[R]c1ccc(-c2cnc(-c3ccccc3)[nH]2)cc1</smiles>

Figure 2.3.3. Main synthetic scheme. Synthesis of key synthetic intermediates and corresponding preparation of various DPP compound subtypes differing in the central heterocyclic ring.

The main synthetic schemes representing the synthesis the vast majority of DPP derivatives are shown in figure 2.3.3. To afford the desired products, we proposed the following approach, in which inexpensive, commercially available starting compounds, like simple acetophenones, benzaldehydes, benzoic acids and its derivatives, were converted to 
the key intermediates or directly to DPP-related compounds. In the next step, cyclocondensation of the intermediate compounds provided the corresponding DPP molecules. Moreover, several synthetic schemes also involved the preparation of an alternative starting material for cyclocondensation. For example, chalcones could be brominated to corresponding 2,3-dibromopropane-1-one from which isoxazole and pyrazolebased compounds could be generated. Additionally, the synthesis of certain DPP candidates was achieved by conversion of one DPP compounds to another that involves either the transformation of the central heterocyclic ring, for instance the preparation of pyrazoles from 2-pyrazolines, or the modification of functional groups in the flanking phenyl rings. In our studies, there are 3 major types of substituent transformations in the flanking phenyl rings (Fig. 2.3.4). One of those is a reaction of methoxy derivatives with the strong Lewis acid boron tribromide yielding the desired phenols and polyphenols (Fig. 2.3.4 A). Alternatively, the phenols were obtained by hydrogenolysis of appropriate $O$-benzyl ethers (Fig. 2.3.4 B). Second, the synthesis of DPP compounds bearing free amino groups was accomplished by using the reduction of corresponding nitro groups in presence of iron powder/ammonium chloride or sodium sulfide (Fig. 2.3.4 C). Among the synthesized DPP compounds, the introduction of the protective groups was applied just in few special cases that are discussed below.

A

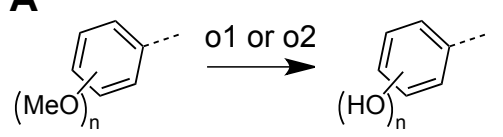

B

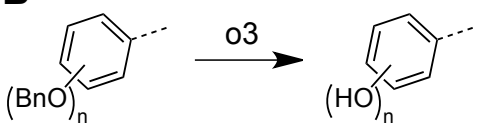

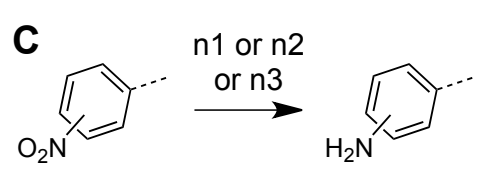

Figure 2.3.4. General synthesis of DPP compounds bearing hydroxy or amino groups. Reaction conditions: (o1) $\mathrm{BBr}_{3}, \mathrm{CH}_{2} \mathrm{Cl}_{2},-78^{\circ} \mathrm{C}$ and $\mathrm{RT}$; (o2) $\mathrm{AlBr}_{3}$, EtSH, RT; (o3) $\mathrm{H}_{2}, \mathrm{Pd} / \mathrm{C}, \mathrm{AcOH}, \mathrm{RT}$ (n1) Fe, $\mathrm{NH}_{4} \mathrm{Cl}, \mathrm{EtOH}, \mathrm{H}_{2} \mathrm{O}, 60^{\circ} \mathrm{C}$; (n2) $\mathrm{Na}_{2} \mathrm{~S}$, dioxane, $\mathrm{H}_{2} \mathrm{O}, 80^{\circ} \mathrm{C}$; (n3) $\mathrm{Fe}, \mathrm{AcOH}, 100^{\circ} \mathrm{C}$.

Next, I would like to emphasize the main advantages of the proposed synthetic approach: 1) the majority of compounds have been produced by up to 3-step synthesis; 2) use of cheap and available starting materials; 3) different heterocyclic systems are accessible through multiple synthetic pathways, which eventually provide a flexibility with respect to suitable functional groups and availability of starting materials; 4) possibility to produce several DPP derivatives from a single intermediate product; 5) in general, good yields and simple isolation protocols. Collectively, the proposed synthetic scheme has emerged as 
highly efficient and flexible approach to generate the focused library of diverse DPP compounds.

\subsubsection{Synthesis of pyrazole- and 2-pyrazoline-based DPPs}

Among DPP compounds included in the focused library, a majority of designed molecules have as a central heterocyclic ring the 3,5-substituted pyrazole moiety (Fig. 2.3.5). As described above, we sought to explore the diverse substitution on flanking aromatic rings $\mathrm{A}$ and $\mathrm{B}$ by introduction of the functional groups that are independently selected from the key structural elements, which are summarized in table 2.3.1, and other substituents listed in figure 2.3.1. Depending on the type of substitution and the availability of the starting material, six different methods for the preparation of pyrazole-based compounds were developed mainly by adapting the published procedures.

Based on our research findings, the method 1, shown in figure 2.3.5, proved to be most efficient and straightforward route to obtain pyrazole-based DPP compounds just in two steps. Thus, this approach involved the Claisen condensation of acetophenones with methyl benzoates in presence of $\mathrm{NaH}$ and DMSO, as described by Anselme ${ }^{393}$ to afford the corresponding 1,3-diketones. Among examined protocols for this condensation, the $\mathrm{NaH} / \mathrm{DMSO}$-based approach was optimal regarding both yield and product purity in comparison with other conditions, such as different bases (EtONa, $\mathrm{NaOH}, t \mathrm{BuOK}$ ) and solvents (ethanol, methanol, THF, benzene). On next step, the desirable pyrazoles were prepared in high yields by reaction 1,3-diketones with hydrazine ${ }^{394}$. By utilizing this method, we were able to obtain pyrazoles bearing on ring A and B the following functional groups: halogens, OAlkyl, methylenedioxy, $\mathrm{CF}_{3}, \mathrm{NMe}_{2}$ and fused heterocyclic rings. Importantly, both reactions are scalable and the isolation is predominantly performed by recrystallization. Method 1 is not suitable for synthesis of compounds containing $\mathrm{NO}_{2}, \mathrm{NH}_{2}, \mathrm{NHAlkyl}, \mathrm{OH}$ groups.

As shown in figure 2.3.5, the method 2 also relies on the formation of pyrazoles from 1,3-diketones. However, the corresponding 1,3-diketones were synthesized through the formation of PFP esters of benzoic acid in the first step, which were then condensed with acetophenones in the presence of HMDS and $n$ - $\mathrm{BuLi}^{395}$. The main advantage of this method is flexibility, good yields and application for benzoic acids with diverse functionality. The drawbacks are associated with the inability to scale up and higher costs. 


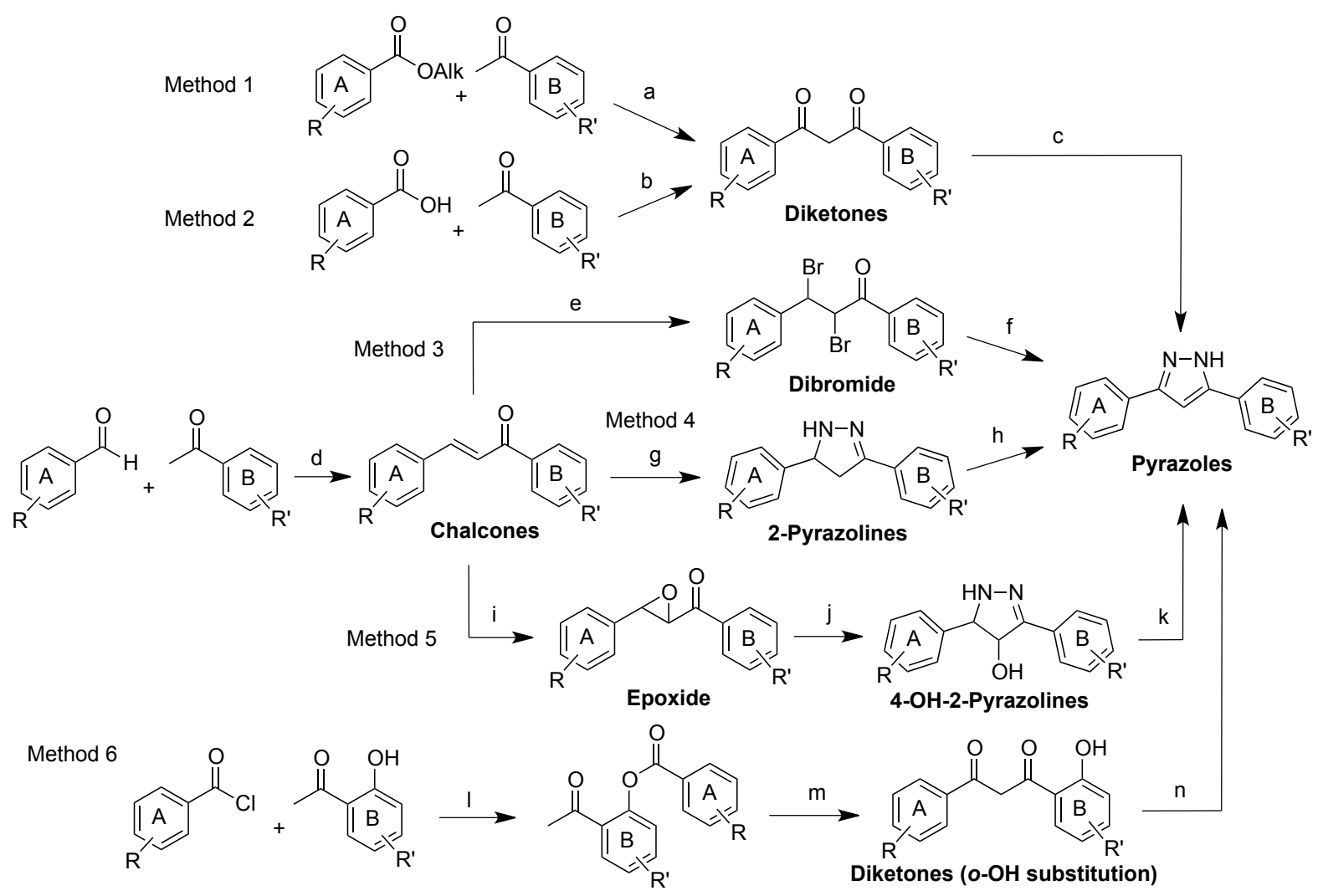

Figure 2.3.5. Methods of synthesis of pyrazole-containing DPP compounds.

Reaction conditions: (a) NaH, THF, DMSO; (b) (1) PFP, DCC, dioxane, RT; (2) HMDS, $n \mathrm{BuLi}$, cyclohexane, $0^{\circ} \mathrm{C}$, w/o isolation; (c) $\mathrm{N}_{2} \mathrm{H}_{4} \bullet \mathrm{H}_{2} \mathrm{O}$, EtOH, reflux; (d) $\mathrm{Ba}(\mathrm{OH})_{2} \bullet 8 \mathrm{H}_{2} \mathrm{O}, \mathrm{NaOH}, \mathrm{MeOH}$, RT; (e) $\mathrm{Br}_{2}, \mathrm{CHCl}_{3}$, $0^{\circ} \mathrm{C}$; (f) $\mathrm{N}_{2} \mathrm{H}_{4} \bullet \mathrm{H}_{2} \mathrm{O}$, EtOH, reflux; (g) $\mathrm{N}_{2} \mathrm{H}_{4} \bullet \mathrm{H}_{2} \mathrm{O}, \mathrm{H}_{2} \mathrm{O}$ or EtOH, reflux; (h) $\mathrm{MnO}_{2}, \mathrm{CHCl}_{3}$, RT; (i) $\mathrm{H}_{2} \mathrm{O}_{2}$, $\mathrm{NaOH}, \mathrm{EtOH}, \mathrm{H}_{2} \mathrm{O}$, RT; (j) $\mathrm{N}_{2} \mathrm{H}_{4} \bullet \mathrm{H}_{2} \mathrm{O}$, EtOH, reflux; (k) PTSA, toluene, reflux; (l) DMAP, pyridine, RT; (m) $\mathrm{KOH}$, pyridine, $50^{\circ} \mathrm{C}$.

In case of other three methods of pyrazole synthesis, we utilized chalcones as the first intermediate (Fig. 2.3.5). The chalcones were readily prepared in high yields via aldol condensation of benzaldehydes and acetophenones under basic catalysis $\mathrm{Ba}(\mathrm{OH})_{2} / \mathrm{NaOH}$ $(1: 1)$ in methanol ${ }^{396}$. In the next step, the chalcones were brominated ${ }^{397}$ or epoxidated $^{398}$ to produce the corresponding dibromides (method 3) or epoxides (method 5), respectively, as described before (Fig. 2.3.5). While dibromides were treated with hydrazine to give desired pyrazoles in one step ${ }^{399}$, the epoxide-based route required two steps, including the formation of 4-OH-pyrazolines in reaction with hydrazine ${ }^{398}$ followed by aromatization with PTSA in refluxing toluene to provide the desired pyrazole ${ }^{400}$. In the method 4 , chalcones were reacted with hydrazine to obtain 2-pyrazoline ${ }^{401}$, which were further oxidized to corresponding pyrazoles by $\mathrm{MnO}_{2}{ }^{402}$ in chloroform. In summary, the chalcone-based synthesis of pyrazoles is experimentally simple, scalable, cheap, and applicable to a variety of functional groups, including $\mathrm{NO}_{2}$, as a precursor of $\mathrm{NH}_{2}$. With respect to the yields, the majority of intermediate 
products (chalcones, epoxides) and DPP-related compounds (2-pyrazolines, 4-OHpyrazolines) can be isolated without chromatography in good to high yields. However, the final step of synthesis, namely the formation of the pyrazole ring, is frequently not very efficient as evident from moderate yields and the need for additional purification steps. In our study, we utilized chalcone-based synthetic routes for derivatives containing a nitro group that can be transformed conventionally into a free amino group (Fig. 2.3.4B).

A small group of pyrazoles in our focused library has an ortho-hydroxyl group on one of the wing phenyl rings (A or B). These DPP derivatives were synthesized in three steps starting from ortho-hydroxyl acetophenones that were treated with selected benzoyl chlorides to give corresponding benzoate ${ }^{403}$ (Fig. 2.3.5, Method 6). Baker-Venkataraman rearrangement of this intermediate, using $\mathrm{KOH}$ in pyridine, gave rise to the requisite 1,3diketones $^{403}$ that were subsequently converted to pyrazoles under standard conditions, namely using hydrazine in refluxing ethanol. In our hand, this approach to synthesized DPP compounds with ortho-hydroxyl group on one of the wing phenyl rings proved superior to other schemes.

The structures of all synthesized compounds, applied methods and experimental details are listed in the tables 2.3.2 (1,3-diketones, pyrazoles), 2.3.3 (chalcones, dibromides, epoxides) 2.3.4 (pyrazoline-based DPPs). 
Table 2.3.2. Synthesized 1,3-diketones and 3,5-substituted pyrazoles.

\begin{tabular}{|c|c|c|c|c|}
\hline Name & $\operatorname{Ring} \mathbf{A}^{1}$ & Ring $B^{1}$ & Method & Yield \\
\hline \multicolumn{5}{|c|}{ 1,3-Diketones } \\
\hline anle125 & $\mathrm{H}$ & $3,4-\mathrm{MD}^{2}$ & $\mathrm{a}$ & $62 \%$ \\
\hline anle136a & $3-\mathrm{F}$ & $3,4,5-\mathrm{OCH}_{3}$ & $\mathrm{a}$ & $95 \%$ \\
\hline anle137a & $3,5-\mathrm{F}$ & $3,4,5-\mathrm{OCH}_{3}$ & $\mathrm{a}$ & $70 \%$ \\
\hline anle138b & $3-\mathrm{Br}$ & $3,4-\mathrm{MD}^{2}$ & $\mathrm{a}$ & $87 \%$ \\
\hline anle142a & $3-\mathrm{Br}$ & $3,4,5-\mathrm{OCH}_{3}$ & $\mathrm{a}$ & $99 \%$ \\
\hline anle143a & $3,4-\mathrm{OCH}_{3}$ & $3,4,5-\mathrm{OCH}_{3}$ & $\mathrm{a}$ & $29 \%$ \\
\hline anle145a & $3,5-\mathrm{Br}$ & $3,4,5-\mathrm{OCH}_{3}$ & $\mathrm{a}$ & $90 \%$ \\
\hline anle186a & $3-\mathrm{F}$ & $3,4-\mathrm{MD}^{2}$ & $\mathrm{a}$ & $89 \%$ \\
\hline anle197a & $3-\mathrm{I}$ & $3,4-\mathrm{MD}^{2}$ & $\mathrm{a}$ & $22 \%$ \\
\hline anle232a & $3,4-\mathrm{MD}^{2}$ & $3,4-\mathrm{MD}^{2}$ & $\mathrm{a}$ & $71 \%$ \\
\hline anle233a & $3-\mathrm{OCH}_{3}$ & $3,4-\mathrm{MD}^{2}$ & $\mathrm{a}$ & $68 \%$ \\
\hline anle234a & $2-\mathrm{Br}$ & $3,4-\mathrm{MD}^{2}$ & $\mathrm{a}$ & $82 \%$ \\
\hline anle236a & $3-\mathrm{OCF}_{3}$ & $3,4-\mathrm{MD}^{2}$ & $\mathrm{a}$ & $83 \%$ \\
\hline anle246a & $3,5-\mathrm{Br}$ & $3,4-\mathrm{MD}^{2}$ & $\mathrm{a}$ & $80 \%$ \\
\hline anle253a & $3-\mathrm{Br}$ & $4-\mathrm{N}\left(\mathrm{CH}_{3}\right)_{2}$ & $\mathrm{a}$ & $94 \%$ \\
\hline anle254a & $3-\mathrm{Cl}$ & $4-\mathrm{N}\left(\mathrm{CH}_{3}\right)_{2}$ & $\mathrm{a}$ & $85 \%$ \\
\hline sery148 & $3,4-\mathrm{OCH}_{3}$ & $3,4-\mathrm{OCH}_{3}$ & $\mathrm{a}$ & $100 \%$ \\
\hline sery150 & $3,4-\mathrm{OCH}_{3}$ & $4-\mathrm{OCH}_{3}$ & $\mathrm{a}$ & $67 \%$ \\
\hline sery157 & $3-\mathrm{F}$ & $3,4-\mathrm{OCH}_{3}$ & $\mathrm{a}$ & $92 \%$ \\
\hline sery219b & $2-\mathrm{F}$ & $3,4-\mathrm{OCH}_{3}$ & $\mathrm{a}$ & $30 \%$ \\
\hline sery $219 \mathrm{e}$ & $3,5-\mathrm{F}$ & $3,4-\mathrm{OCH}_{3}$ & $\mathrm{a}$ & $88 \%$ \\
\hline sery $220 \mathrm{c}$ & $2,5-\mathrm{F}$ & $3,4-\mathrm{OCH}_{3}$ & $\mathrm{a}$ & $68 \%$ \\
\hline sery230a & $2,3-\mathrm{OCH}_{3}$ & $3-\mathrm{F}$ & $\mathrm{a}$ & $67 \%$ \\
\hline sery $255 \mathrm{a}$ & $3-\mathrm{Br}$ & $3,4-\mathrm{OCH}_{3}$ & $\mathrm{a}$ & $74 \%$ \\
\hline sery $256 a$ & $4-\mathrm{OCH}_{3}$ & $3-\mathrm{Br}$ & $\mathrm{a}$ & $60 \%$ \\
\hline sery $257 a$ & $5-\mathrm{Cl}-2-\mathrm{OCH}_{3}$ & $3-\mathrm{Br}$ & $\mathrm{a}$ & $75 \%$ \\
\hline sery 258 & $2-\mathrm{OCH}_{3}$ & $3-\mathrm{F}$ & $\mathrm{a}$ & $73 \%$ \\
\hline sery 259 & $5-\mathrm{Cl}-2-\mathrm{OCH}_{3}$ & $3-\mathrm{F}$ & $\mathrm{a}$ & $90 \%$ \\
\hline sery269a & $4-\mathrm{Cl}$ & $4-\mathrm{OCH}_{3}$ & $\mathrm{a}$ & $84 \%$ \\
\hline sery 282 & 4-Cl & $2-\mathrm{OH}$ & $\mathrm{m}$ & $42 \%$ \\
\hline sery $287 a$ & $3-\mathrm{OCH}_{3}$ & $4-\mathrm{Cl}$ & $\mathrm{a}$ & $34 \%$ \\
\hline sery $287 \mathrm{~b}$ & $3-\mathrm{OCH}_{3}$ & $3-\mathrm{Cl}$ & $\mathrm{a}$ & $30 \%$ \\
\hline sery290a & $3,4-\mathrm{OCH}_{3}$ & $2-\mathrm{OH}$ & $\mathrm{m}$ & $73 \%$ \\
\hline sery292a & $3-\mathrm{Br}$ & naphthalen-2-yl ${ }^{3}$ & $\mathrm{a}$ & $73 \%$ \\
\hline sery294a & $3,5-\mathrm{F}$ & $3,4-\mathrm{MD}^{2}$ & $\mathrm{a}$ & $85 \%$ \\
\hline sery297a & $3,5-\mathrm{Cl}$ & $3,4-\mathrm{MD}^{2}$ & $\mathrm{a}$ & $98 \%$ \\
\hline sery $312 \mathrm{a}$ & $3-\mathrm{Br}$ & 2,3-dihydrobenzofuran-5-yl & $\mathrm{a}$ & $88 \%$ \\
\hline sery $315 \mathrm{a}$ & $3-\mathrm{Br}$ & 2,3-dihydro-1,4-benzodioxin-6-y ${ }^{3}$ & $\mathrm{a}$ & $89 \%$ \\
\hline sery316a & $2,5-\mathrm{F}$ & $3,4-\mathrm{MD}^{2}$ & $\mathrm{a}$ & $89 \%$ \\
\hline sery335a & $3-\mathrm{Cl}$ & $3,4-\mathrm{MD}^{2}$ & $\mathrm{a}$ & $87 \%$ \\
\hline sery336b & $3-\mathrm{OBn}$ & $3,4-\mathrm{MD}^{2}$ & $\mathrm{a}$ & $46 \%$ \\
\hline sery $342 b$ & $2-\mathrm{Cl}-5-\mathrm{OBn}$ & $3,4-\mathrm{MD}^{2}$ & $\mathrm{a}$ & $87 \%$ \\
\hline sery378a & 4-Br & $3,4-\mathrm{MD}^{2}$ & $\mathrm{a}$ & $76 \%$ \\
\hline sery $388 \mathrm{a}$ & $3-\mathrm{OCH}_{2} \mathrm{CH}_{2} \mathrm{~F}$ & $3,4-\mathrm{MD}^{2}$ & $\mathrm{a}$ & $54 \%$ \\
\hline sery393a & 2-Br-pyridin-6-yl ${ }^{3}$ & $3,4-\mathrm{MD}^{2}$ & $\mathrm{a}$ & $79 \%$ \\
\hline sery397a & 2-Br-pyridin-4-yl ${ }^{3}$ & $3,4-\mathrm{MD}^{2}$ & $\mathrm{a}$ & $74 \%$ \\
\hline sery397b & 3-Br-pyridin-5-yl & $3,4-\mathrm{MD}^{2}$ & $\mathrm{a}$ & $82 \%$ \\
\hline sery401a & imidazo[1,2-a]pyridin-7-yl $^{3}$ & $3-\mathrm{Br}$ & $\mathrm{a}$ & $49 \%$ \\
\hline sery $428 \mathrm{a}$ & $3-\mathrm{Br}$ & benzofuran-6-yl ${ }^{3}$ & $\mathrm{a}$ & $60 \%$ \\
\hline sery $455 a$ & $3,4-\mathrm{OCH}_{3}$ & $4-\mathrm{OBn}$ & $\mathrm{a}$ & $56 \%$ \\
\hline sery456a & $3-\mathrm{OBn}$ & $3,4-\mathrm{OCH}_{3}$ & $\mathrm{a}$ & $63 \%$ \\
\hline
\end{tabular}


Table 2.3.2. Synthesized 1,3-diketones and 3,5-substituted pyrazoles (continued).

\begin{tabular}{|c|c|c|c|c|}
\hline Name & $\operatorname{Ring} \mathbf{A}^{1}$ & Ring $\mathbf{B}^{\mathbf{1}}$ & Method & Yield \\
\hline \multicolumn{5}{|c|}{ Pyrazoles } \\
\hline anle126 & $\mathrm{H}$ & $3,4-\mathrm{MD}^{2}$ & $\mathrm{c}$ & $70 \%$ \\
\hline anle136b & $3-\mathrm{F}$ & $3,4,5-\mathrm{OCH}_{3}$ & $\mathrm{c}$ & $88 \%$ \\
\hline anle136c & $3-\mathrm{F}$ & $3,4,5-\mathrm{OH}$ & ol/anle136b & $100 \%$ \\
\hline anle137b & $3,5-\mathrm{F}$ & $3,4,5-\mathrm{OCH}_{3}$ & $\mathrm{c}$ & $100 \%$ \\
\hline anle137c & $3,5-\mathrm{F}$ & $3,4,5-\mathrm{OH}$ & ol/anle137b & $100 \%$ \\
\hline anle138b & $3-\mathrm{Br}$ & $3,4-\mathrm{MD}^{2}$ & $\mathrm{c}$ & $89 \%$ \\
\hline anle138c & $3-\mathrm{Br}$ & $3,4-\mathrm{OH}$ & o1/sery255b & $87 \%$ \\
\hline anle142b & $3-\mathrm{Br}$ & $3,4,5-\mathrm{OCH}_{3}$ & $\mathrm{c}$ & $92 \%$ \\
\hline anle142c & $3-\mathrm{Br}$ & $3,4,5-\mathrm{OH}$ & o1/anle142b & $68 \%$ \\
\hline anle143b & $3,4-\mathrm{OCH}_{3}$ & $3,4,5-\mathrm{OCH}_{3}$ & $\mathrm{c}$ & $69 \%$ \\
\hline anle143c & $3,4-\mathrm{OH}$ & $3,4,5-\mathrm{OH}$ & ol/anle143b & $81 \%$ \\
\hline anle145b & $3,5-\mathrm{Br}$ & $3,4,5-\mathrm{OCH}_{3}$ & $\mathrm{c}$ & $99 \%$ \\
\hline anle145c & $3,5-\mathrm{Br}$ & $3,4,5-\mathrm{OH}$ & ol/anle145b & $100 \%$ \\
\hline anle186b & $3-\mathrm{F}$ & $3,4-\mathrm{MD}^{2}$ & $\mathrm{c}$ & $86 \%$ \\
\hline anle197b & $3-\mathrm{I}$ & $3,4-\mathrm{MD}^{2}$ & $\mathrm{c}$ & $75 \%$ \\
\hline anle $232 b$ & $3,4-\mathrm{MD}^{2}$ & $3,4-\mathrm{MD}^{2}$ & $\mathrm{c}$ & $71 \%$ \\
\hline anle233b & $3-\mathrm{OCH}_{3}$ & $3,4-\mathrm{MD}^{2}$ & $\mathrm{c}$ & $79 \%$ \\
\hline anle234b & $2-\mathrm{Br}$ & $3,4-\mathrm{MD}^{2}$ & $\mathrm{c}$ & $60 \%$ \\
\hline anle236b & $3-\mathrm{OCF}_{3}$ & $3,4-\mathrm{MD}^{2}$ & $\mathrm{c}$ & $90 \%$ \\
\hline anle236c & $3-\mathrm{OCF}_{3}$ & $3,4-\mathrm{OH}$ & o1/anle236b & $100 \%$ \\
\hline anle246b & $3,5-\mathrm{Br}$ & $3,4-\mathrm{MD}^{2}$ & $\mathrm{c}$ & $95 \%$ \\
\hline anle253b & $3-\mathrm{Br}$ & $4-\mathrm{N}\left(\mathrm{CH}_{3}\right)_{2}$ & $\mathrm{c}$ & $85 \%$ \\
\hline anle254b & $3-\mathrm{Cl}$ & $4-\mathrm{N}\left(\mathrm{CH}_{3}\right)_{2}$ & $\mathrm{c}$ & $85 \%$ \\
\hline anle270 & 2,2-difluoro-1,3-benzodioxol-5-yl ${ }^{3}$ & $3-\mathrm{Br}$ & $\mathrm{b}$ & $64 \%$ \\
\hline sery 85 & $3-\mathrm{F}$ & $3,4-\mathrm{OH}$ & o1/sery161 & $95 \%$ \\
\hline sery106 & $\mathrm{H}$ & $3,4-\mathrm{OH}$ & o1/anle126 & $90 \%$ \\
\hline sery149 & $3,4-\mathrm{OCH}_{3}$ & $3,4-\mathrm{OCH}_{3}$ & $\mathrm{c}$ & $100 \%$ \\
\hline sery 156 & $3,4-\mathrm{OCH}_{3}$ & $4-\mathrm{OCH}_{3}$ & $\mathrm{c}$ & $90 \%$ \\
\hline sery159a & $3,4-\mathrm{OH}$ & $3,4-\mathrm{OH}$ & o1/sery149 & $85 \%$ \\
\hline sery161 & $3-\mathrm{F}$ & $3,4-\mathrm{OCH}_{3}$ & $\mathrm{c}$ & $95 \%$ \\
\hline sery $221 \mathrm{~b}$ & $2-\mathrm{F}$ & $3,4-\mathrm{OCH}_{3}$ & $\mathrm{c}$ & $87 \%$ \\
\hline sery221c & $3,5-\mathrm{F}$ & $3,4-\mathrm{OCH}_{3}$ & $\mathrm{c}$ & $81 \%$ \\
\hline sery221d & $2,5-\mathrm{F}$ & $3,4-\mathrm{OCH}_{3}$ & $\mathrm{c}$ & $20 \%$ \\
\hline sery $230 \mathrm{~b}$ & $2,3-\mathrm{OCH}_{3}$ & $3-\mathrm{F}$ & $\mathrm{c}$ & $79 \%$ \\
\hline sery230c & $2,3-\mathrm{OH}$ & $3-\mathrm{F}$ & o1/sery230b & $96 \%$ \\
\hline sery $255 b$ & $3-\mathrm{Br}$ & $3,4-\mathrm{OCH}_{3}$ & $\mathrm{c}$ & $98 \%$ \\
\hline sery $256 \mathrm{~b}$ & $4-\mathrm{OCH}_{3}$ & $3-\mathrm{Br}$ & $\mathrm{c}$ & $63 \%$ \\
\hline sery $257 b$ & $5-\mathrm{Cl}-2-\mathrm{OCH}_{3}$ & $3-\mathrm{Br}$ & $\mathrm{c}$ & $85 \%$ \\
\hline sery260a & $4-\mathrm{OH}$ & $3-\mathrm{Br}$ & $\mathrm{o} 2 /$ sery $256 \mathrm{~b}$ & $97 \%$ \\
\hline sery $260 \mathrm{~b}$ & $5-\mathrm{Cl}-2-\mathrm{OH}$ & $3-\mathrm{Br}$ & $\mathrm{o} 2 /$ sery $257 \mathrm{~b}$ & $51 \%$ \\
\hline sery261a & $2-\mathrm{OCH}_{3}$ & $3-\mathrm{F}$ & $\mathrm{c}$ & $90 \%$ \\
\hline sery $261 b$ & $5-\mathrm{Cl}-2-\mathrm{OCH}_{3}$ & $3-\mathrm{F}$ & $\mathrm{c}$ & $98 \%$ \\
\hline sery263a & $2-\mathrm{OH}$ & $3-\mathrm{F}$ & o2/sery260a & $59 \%$ \\
\hline sery $263 \mathrm{~b}$ & 5-Cl-2-OH & $3-\mathrm{F}$ & $\mathrm{o} 2 /$ sery $261 \mathrm{~b}$ & $60 \%$ \\
\hline sery $269 b$ & $4-\mathrm{Cl}$ & $4-\mathrm{OCH}_{3}$ & $\mathrm{c}$ & $83 \%$ \\
\hline sery $275 a$ & $3-\mathrm{Cl}$ & $3-\mathrm{NO}_{2}$ & $\mathrm{f}$ & $67 \%$ \\
\hline sery $275 b$ & $3-\mathrm{Cl}$ & $3-\mathrm{NH}_{2}$ & $\mathrm{n} 2 /$ sery275a & $67 \%$ \\
\hline sery278a & $4-\mathrm{Cl}$ & $3-\mathrm{NO}_{2}$ & $\mathrm{f} / \mathrm{g}+\mathrm{h}$ & $60 / 54 \%$ \\
\hline sery $278 \mathrm{~b}$ & $4-\mathrm{Cl}$ & $4-\mathrm{NO}_{2}$ & $\mathrm{f}$ & $50 \%$ \\
\hline sery $279 b$ & $3,4-\mathrm{OCH}_{3}$ & $4-\mathrm{OH}$ & $\mathrm{o} 3 /$ sery $455 \mathrm{~b}$ & $71 \%$ \\
\hline sery280a & $4-\mathrm{Cl}$ & $3-\mathrm{NH}_{2}$ & n1/sery278a & $28 \%$ \\
\hline
\end{tabular}


Table 2.3.2. Synthesized 1,3-diketones and 3,5-substituted pyrazoles (continued)

\begin{tabular}{|c|c|c|c|c|}
\hline sery $280 \mathrm{~b}$ & $4-\mathrm{Cl}$ & $4-\mathrm{NH}_{2}$ & $\mathrm{n} 1 /$ sery $278 \mathrm{~b}$ & $22 \%$ \\
\hline sery 283 & $4-\mathrm{Cl}$ & $2-\mathrm{OH}$ & $\mathrm{c}$ & $46 \%$ \\
\hline sery 289 & $3-\mathrm{OCH}_{3}$ & $4-\mathrm{Cl}$ & $\mathrm{c}$ & $65 \%$ \\
\hline sery $290 \mathrm{~b}$ & $3,4-\mathrm{OCH}_{3}$ & $2-\mathrm{OH}$ & $\mathrm{c}$ & $86 \%$ \\
\hline sery $292 b$ & $3-\mathrm{Br}$ & naphthalen-2-yl ${ }^{3}$ & $\mathrm{c}$ & $88 \%$ \\
\hline sery294b & $3,5-\mathrm{F}$ & $3,4-\mathrm{MD}^{2}$ & $\mathrm{c}$ & $81 \%$ \\
\hline sery $297 b$ & $3,5-\mathrm{Cl}$ & $3,4-\mathrm{MD}^{2}$ & $\mathrm{c}$ & $81 \%$ \\
\hline sery301 & $3-\mathrm{OCH}_{3}$ & $3-\mathrm{Cl}$ & $\mathrm{c}$ & $76 \%$ \\
\hline sery $302 c$ & $3-\mathrm{NO}_{2}$ & $3,4-\mathrm{MD}^{2}$ & $\mathrm{j}+\mathrm{k}$ & $72 \%$ \\
\hline sery308 & $3-\mathrm{NH}_{2}$ & $3,4-\mathrm{MD}^{2}$ & $\mathrm{n} 1 /$ sery302c & $31 \%$ \\
\hline sery309 & $2-\mathrm{NO}_{2}$ & $4-\mathrm{Cl}$ & $\mathrm{b} / \mathrm{j}+\mathrm{k}$ & $48 / 25 \%$ \\
\hline sery $310 \mathrm{~b}$ & $3-\mathrm{OH}$ & $3,4-\mathrm{OCH}_{3}$ & o3/sery $456 \mathrm{~b}$ & $90 \%$ \\
\hline sery $312 b$ & $3-\mathrm{Br}$ & 2,3-dihydrobenzofuran-5-y1 ${ }^{3}$ & $\mathrm{c}$ & $82 \%$ \\
\hline sery $315 b$ & $3-\mathrm{Br}$ & 2,3-dihydro-1,4-benzodioxin-6-yl ${ }^{3}$ & $\mathrm{c}$ & $69 \%$ \\
\hline sery316b & $2,5-\mathrm{F}$ & $3,4-\mathrm{MD}^{2}$ & $\mathrm{c}$ & $83 \%$ \\
\hline sery319 & $2,3-\mathrm{F}$ & $3,4-\mathrm{MD}^{2}$ & $j+k$ & $50 \%$ \\
\hline sery $320 \mathrm{a}$ & $4-\mathrm{Cl}$ & $4-\mathrm{OH}$ & o1/sery $269 \mathrm{~b}$ & $92 \%$ \\
\hline sery $320 \mathrm{~b}$ & $3-\mathrm{OH}$ & $4-\mathrm{Cl}$ & o1/sery289 & $82 \%$ \\
\hline sery $320 \mathrm{c}$ & $3-\mathrm{OH}$ & $3-\mathrm{Cl}$ & o1/sery301 & $67 \%$ \\
\hline sery $335 b$ & $3-\mathrm{Cl}$ & $3,4-\mathrm{MD}^{2}$ & $\mathrm{c}$ & $83 \%$ \\
\hline sery339a & $3-\mathrm{OBn}$ & $3,4-\mathrm{MD}^{2}$ & $\mathrm{c}$ & $74 \%$ \\
\hline sery339b & $3-\mathrm{OH}$ & $3,4-\mathrm{MD}^{2}$ & o3/sery339a & $78 \%$ \\
\hline sery $342 \mathrm{c}$ & 2-Cl-5-OBn & $3,4-\mathrm{MD}^{2}$ & $\mathrm{c}$ & $84 \%$ \\
\hline sery344 & $2-\mathrm{Cl}-5-\mathrm{OH}$ & $3,4-\mathrm{MD}^{2}$ & o3/sery342c & $93 \%$ \\
\hline sery352 & $3-\mathrm{Br}-5-\mathrm{NO}_{2}$ & $3,4-\mathrm{MD}^{2}$ & $\mathrm{f}$ & $55 \%$ \\
\hline sery357 & $3-\mathrm{Br}-5-\mathrm{NH}_{2}$ & $3,4-\mathrm{MD}^{2}$ & n3/sery352 & $47 \%$ \\
\hline sery369 & $3-\mathrm{OCH}_{2} \mathrm{CH}_{2} \mathrm{~F}$ & $3,4-\mathrm{MD}^{2}$ & $\mathrm{c}$ & $82 \%$ \\
\hline sery $378 b$ & $4-\mathrm{Br}$ & $3,4-\mathrm{MD}^{2}$ & $\mathrm{c}$ & $75 \%$ \\
\hline sery381a & $3-\mathrm{Cl}$ & $4-\mathrm{NO}_{2}$ & $\mathrm{f}$ & $25 \%$ \\
\hline sery $381 \mathrm{~b}$ & $3-\mathrm{Br}$ & $4-\mathrm{NO}_{2}$ & $\mathrm{f}$ & $27 \%$ \\
\hline sery 383 & $3-\mathrm{Cl}$ & $4-\mathrm{NH}_{2}$ & n2/sery381a & $75 \%$ \\
\hline sery384 & $3-\mathrm{Br}$ & $4-\mathrm{NH}_{2}$ & n3/sery381b & $70 \%$ \\
\hline sery $393 b$ & 2-Br-pyridin-6-yl ${ }^{3}$ & $3,4-\mathrm{MD}^{2}$ & $\mathrm{c}$ & $85 \%$ \\
\hline sery398a & 2-Br-pyridin-4-yl ${ }^{3}$ & $3,4-\mathrm{MD}^{2}$ & $\mathrm{c}$ & $85 \%$ \\
\hline sery398b & 3-Br-pyridin-5-yl ${ }^{3}$ & $3,4-\mathrm{MD}^{2}$ & $\mathrm{c}$ & $82 \%$ \\
\hline sery $401 \mathrm{~b}$ & imidazo[1,2-a]pyridin-7-yl $1^{3}$ & $3-\mathrm{Br}$ & $\mathrm{c}$ & $78 \%$ \\
\hline sery415 & $\begin{array}{l}\text { 1-MOM-1 } H \text {-benzimidazol-6-yl/ } \\
\text { 1-MOM-1 } H \text {-benzimidazol-5-yl }\end{array}$ & $3-\mathrm{Br}$ & $\mathrm{b}$ & $49 \%$ \\
\hline sery416b & $3-\mathrm{Cl}$ & $\begin{array}{l}\text { 1-MOM-1 } H \text {-benzimidazol-6-yl/ } \\
\text { 1-MOM-1H-benzimidazol-5-yl }\end{array}$ & $a+c$ & $70 \%$ \\
\hline sery417 & 1H-benzimidazol-5-yl ${ }^{4}$ & $3-\mathrm{Br}$ & o1/sery415 & $54 \%$ \\
\hline sery418 & 1H-benzimidazol-5-yl ${ }^{4}$ & $3-\mathrm{Cl}$ & o1/sery416b & $60 \%$ \\
\hline sery $428 b$ & $3-\mathrm{Br}$ & benzofuran-6-yl ${ }^{3}$ & $\mathrm{c}$ & $55 \%$ \\
\hline sery $455 \mathrm{~b}$ & $3,4-\mathrm{OCH}_{3}$ & 4-OBn & $\mathrm{c}$ & $76 \%$ \\
\hline sery $456 \mathrm{~b}$ & $3-\mathrm{OBn}$ & $3,4-\mathrm{OCH}_{3}$ & $\mathrm{c}$ & $86 \%$ \\
\hline
\end{tabular}

Table 2.3.2. Synthesized 1,3-diketones and 3,5-substituted pyrazoles. The structures of synthesized 1,3-diketones and 3,5-substituted pyrazoles are listed, including the yields, preparation methods and, where required, starting materials. The reaction conditions are shown in figures 2.3.4 and 2.3.5. In "method" column, $x+y$ - two steps; $x / y$ - two methods were independently applied; x/compound - method/starting material. ${ }^{1}$ Ring labeling corresponds to figure 2.3.5. ${ }^{2} 3,4$-MD states for 3,4-Methylenedioxy. ${ }^{3}$ The full name of the substituent. ${ }^{4}$ The mixture of isomers. 


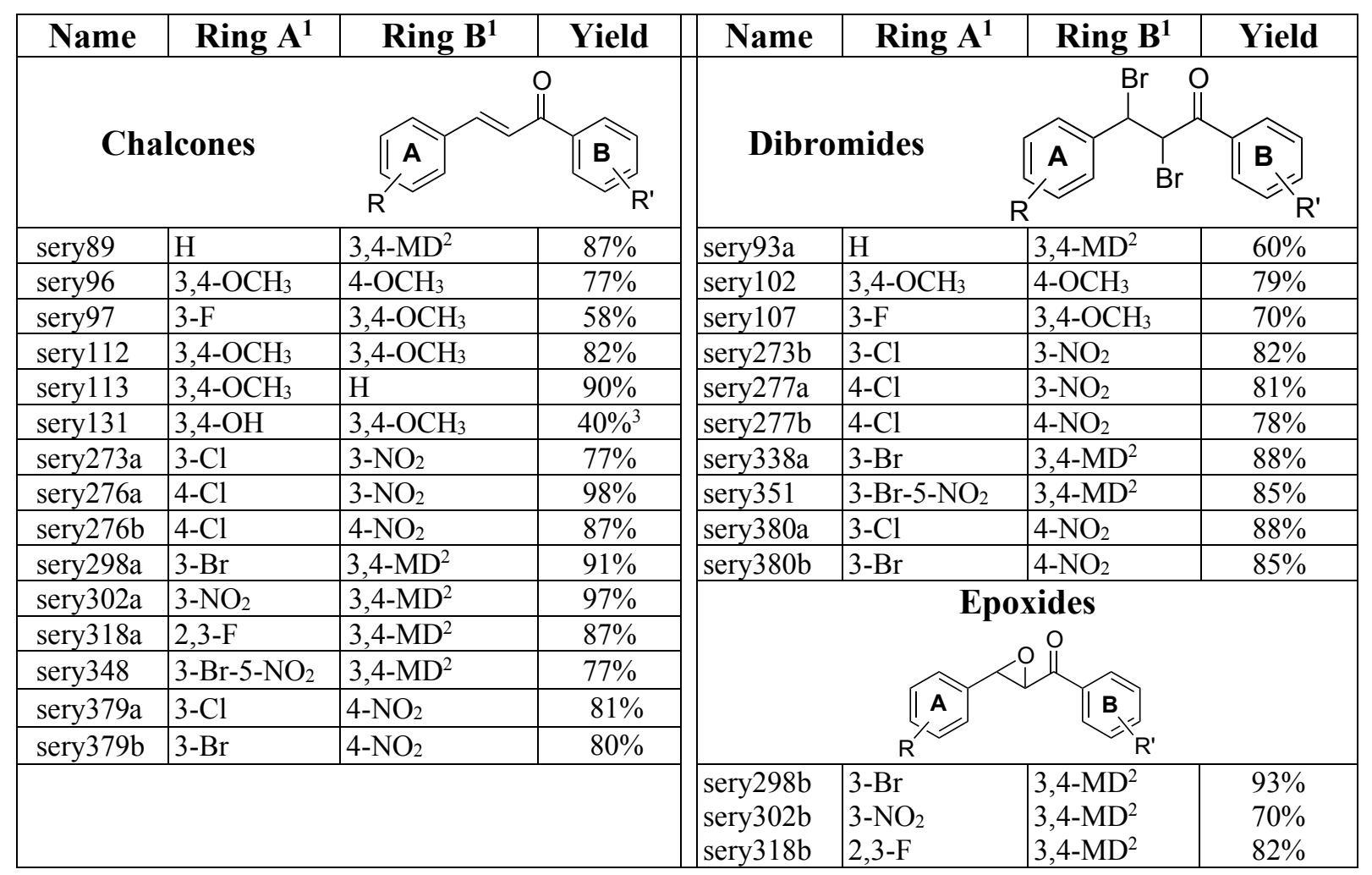

Table 2.3.3. Synthesized chalcones, dibromides and epoxides. Listed compounds were prepared as shown in figure 2.3.5. ${ }^{1}$ Ring labeling corresponds to figure 2.3.5. ${ }^{2} 3,4$-MD states for 3,4-Methylenedioxy. 3 synthesized from corresponding THP-protected 3,4dihydroxybenzaldehyde.

\begin{tabular}{|c|c|c|c|c|c|c|c|}
\hline Name & $\operatorname{Ring} \mathbf{A}^{1}$ & Ring $\mathbf{B}^{1}$ & Yield & Name & $\operatorname{Ring} \mathbf{A}^{1}$ & $\operatorname{Ring} B^{1}$ & Yield \\
\hline \multicolumn{2}{|c|}{ 2-Pyrazolines } & & & \multicolumn{4}{|c|}{ 4-OH-2-Pyrazolines } \\
\hline sery 132 & $3,4-\mathrm{OCH}_{3}$ & $3,4-\mathrm{OCH}_{3}$ & $90 \%$ & anle $127 \mathrm{~b}$ & $\mathrm{H}$ & $3,4-\mathrm{MD}^{2}$ & $56 \%$ \\
\hline sery133 & $3,4-\mathrm{OCH}_{3}$ & $4-\mathrm{OCH}_{3}$ & $85 \%$ & sery300a & $3-\mathrm{Br}$ & $3,4-\mathrm{MD}^{2}$ & $51 \%$ \\
\hline sery 135 & $3,4-\mathrm{OCH}_{3}$ & $\mathrm{H}$ & $82 \%$ & \multirow{4}{*}{\multicolumn{4}{|c|}{ 1-Acetyl-2-Pyrazolines }} \\
\hline sery 136 & $\mathrm{H}$ & $3,4-\mathrm{MD}^{2}$ & $85 \%$ & & & & \\
\hline sery 144 & $3,4-\mathrm{OH}$ & $3,4-\mathrm{OCH}_{3}$ & $53 \%$ & & & & \\
\hline \multirow[t]{5}{*}{ sery 145} & $3-\mathrm{F}$ & $3,4-\mathrm{OCH}_{3}$ & $62 \%$ & & & & \\
\hline & & & & anle129 & $\mathrm{H}$ & $3,4-\mathrm{MD}^{2}$ & $74 \%$ \\
\hline & & & & sery105 & $\mathrm{H}$ & $3,4-\mathrm{OH}$ & $37 \%^{3}$ \\
\hline & & & & sery108 & $3,4-\mathrm{OCH}_{3}$ & $4-\mathrm{OCH}_{3}$ & $58 \%$ \\
\hline & & & & sery115 & $3-\mathrm{F}$ & $3,4-\mathrm{OCH}_{3}$ & $70 \%$ \\
\hline
\end{tabular}

Table 2.3.4. List of synthesized 2-pyrazoline-based compounds. Listed compounds were prepared as shown in figure 2.3.5. ${ }^{1}$ Ring labeling corresponds to figure 2.3.5. ${ }^{2} 3,4$-MD states for 3,4-Methylenedioxy. ${ }^{3}$ Method o1 from anle129. 
2.3.5. Synthesis of isoxazole-, imidazole-, 1,2,4-oxadiazole-, pyrrole-based DPP $\underline{\text { analogs }}$

As discussed above, the majority of DPP compounds in our focused library are mainly related to 1,3-substituted pyrazole and pyrazoline scaffolds. However, to ensure the adequate coverage of the central heterocycle ring, we designed and synthesized a number of DPP analogs by replacing pyrazole with isoxazole, imidazole, pyrrole and 1,2,4-oxadiazole. Despite the fact that the proposed ring modifications do not cover all possible heterocyclic structures, the evaluation of SAR for proposed DPP analogs is very helpful in understanding the structural determinants of activity and pharmacological/pharmacokinetic properties.
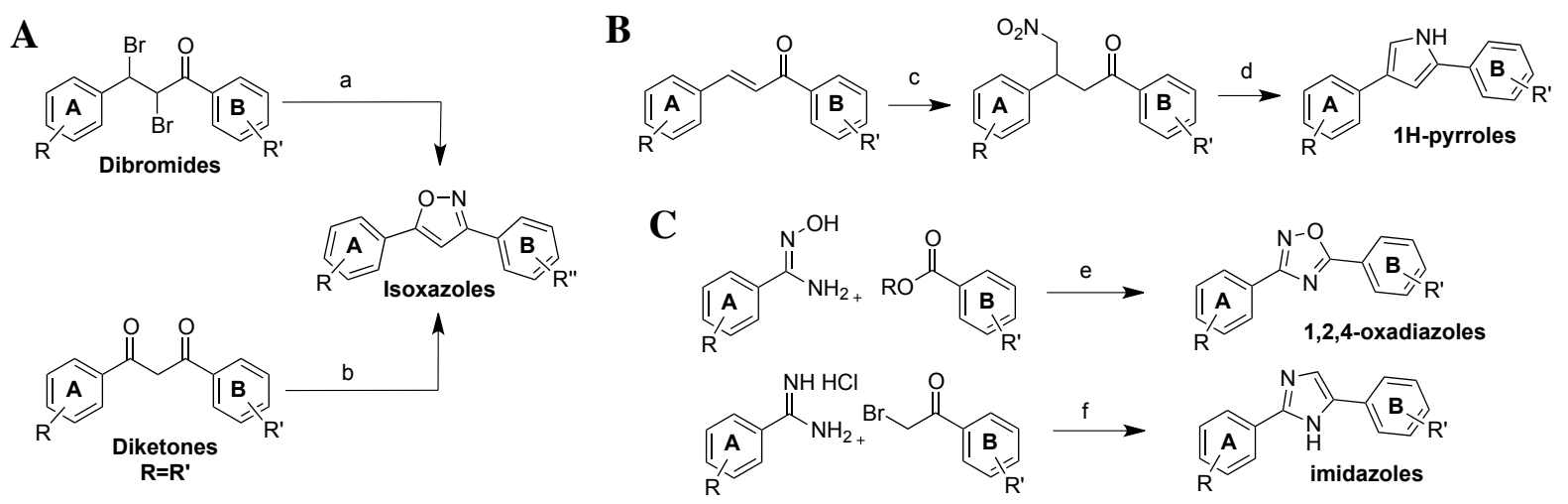

Figure 2.3.6. Methods of synthesis of DPP analogs bearing isoxazole, pyrrole, imidazole and 1,2,4-oxadiazole heterocycles.

Reaction conditions: (a) $\mathrm{NH}_{2} \mathrm{OH} \bullet \mathrm{HCl}, \mathrm{NaOH}, \mathrm{H}_{2} \mathrm{O}$, EtOH, reflux; (b) $\mathrm{NH}_{2} \mathrm{OH} \bullet \mathrm{HCl}$, pyridine, reflux; (c) $\mathrm{CH}_{3} \mathrm{NO}_{2}, \mathrm{Et}_{2} \mathrm{NH}, \mathrm{MeOH}$, reflux; (d) (1) $\mathrm{KOH}, \mathrm{MeOH}$, THF, RT; (2) $\mathrm{H}_{2} \mathrm{SO}_{4}, \mathrm{MeOH}$, RT (3) $\mathrm{NH}_{4} \mathrm{OAc}, \mathrm{AcOH}$, $100{ }^{\circ} \mathrm{C}$; (e) $t \mathrm{BuOK}, \mathrm{EtOH}$, reflux; (f) $\mathrm{NaHCO}_{3}, \mathrm{H}_{2} \mathrm{O}$, THF, reflux.

Similar to the preparation of pyrazole-based DPP compounds, the corresponding analogues with one of the alternative central heterocyclic rings were synthesized from commercially available starting materials in 1-4 steps by established methods. Importantly, by utilizing of the proposed general synthetic scheme (Fig. 2.3.3.) many of the DPP compounds listed below were prepared just in 1-2 steps from common intermediates.

For the preparation of a series of isoxazole-based compounds two methods proved to be suitable (Fig. 2.3.6 A). The first method is more general and is based on the reaction of corresponding dibromides with hydroxylamine hydrochloride in the presence of sodium hydroxide in refluxing water/ethanol mixture to provide the desired products in good yields $^{397}$. The second approach involves the reaction of 1,3-diketones with hydroxylamine hydrochloride in pyridine ${ }^{404}$ (Fig 2.3.6). Whereas the conversion of 1,3-diketones to isoxazoles proceeds with higher yields, the main advantage of the dibromide-based route is the formation of a single isomer, when the flanking aromatic rings have different substitution. 
Therefore, the synthesis of isoxazoles from 1,3-diketones is preferable just in case of similar substitution in phenyl rings $\mathrm{A}$ and $\mathrm{B}$. In order to prepare compounds with free $\mathrm{OH}$ groups, we utilized the conventional $\mathrm{BBr}_{3}$-based method as shown on Figure 2.3.4 A. The list of structures and further experimental information are summarized in the Table 2.3.5.

The synthesis of desired pyrroles was accomplished using a 2-step route starting from chalcones as described by M. J. Hall ${ }^{405}$ (Fig. 2.3.6B). Thus, chalcones were first reacted with nitromethane under basic conditions to afford the corresponding nitro 1,3-diaryl-4-nitobutan-1-ones. These intermediates were next employed in two-step procedure that includes the formation of 1-keto-4-dimethylacetals via Nef reaction and subsequent condensation with ammonium acetate in acetic acid to give 2,4-diaryl- $1 H$-pyrroles in moderate to good yields (Fig. 2.3.6 and Table 2.3.5). Of note, the described preparation of pyrroles from corresponding nitro derivatives does not require the isolation and purification of generated intermediates.

In case of 1,2,4-oxadiazole and $1 H$-imidazole-based DPP compounds, we applied the established 1-step synthetic schemes (Fig. 2.3.6 C, Table 2.3.5). Thus, $1 H$-imidazoles were produced by condensation of aromatic amidines with phenacyl bromides in presence of sodium bicarbonate in THF/water mixture ${ }^{406}$. These compounds were obtained in high yields; however, the purification by column chromatography was required. A series of 1,2,4oxadiazoles was synthesized by reaction of methyl/ethyl benzoates with aromatic amidoximes, which are readily accessible from corresponding nitriles ${ }^{407}$. The general procedure involves refluxing of amidoxime, methyl benzoate and potassium tert-butoxide, as a base, in ethanol followed by simple isolation and recrystallization.

Given that we were interested in the preparation of phenolic and polyphenolic compounds, the desired DPP analogs, namely isoxazole, imidazole, pyrrole and 1,2,4oxadiazole, bearing hydroxyl groups in flanking phenyl rings were produced by a conventional $\mathrm{BBr}_{3}$-based method as shown on Figure 2.3.4 A. In line with the results of the $\mathrm{BBr}_{3}$ deprotection of pyrazole-based DPPs, the corresponding phenolic DPP compounds with different heterocyclic systems were obtained in good yields from appropriate substrates (Table 2.3.5). 


\begin{tabular}{|c|c|c|c|c|}
\hline Name & $\operatorname{Ring} \mathbf{A}^{1}$ & $\operatorname{Ring} \mathbf{B}^{1}$ & $\begin{array}{l}\text { Method/ } \\
\text { Substrate }\end{array}$ & Yield \\
\hline \multicolumn{5}{|c|}{ Isoxazoles } \\
\hline sery93b & $\mathrm{H}$ & $3,4-\mathrm{MD}^{2}$ & $a /$ sery93a & $53 \%$ \\
\hline sery103 & $3,4-\mathrm{OCH}_{3}$ & $4-\mathrm{OCH}_{3}$ & $\mathrm{a} /$ sery 102 & $43 \%$ \\
\hline sery109 & $3-\mathrm{F}$ & $3,4-\mathrm{OCH}_{3}$ & a/sery107 & $60 \%$ \\
\hline sery117 & $3-\mathrm{F}$ & $3,4-\mathrm{OH}$ & o1/sery109 & $65 \%$ \\
\hline sery118 & $3,4-\mathrm{OH}$ & $4-\mathrm{OH}$ & o1/sery103 & $68 \%$ \\
\hline sery 137 & $3,4-\mathrm{OCH}_{3}$ & $3,4-\mathrm{OCH}_{3}$ & b/sery149 & $89 \%$ \\
\hline sery $158 \mathrm{~b}$ & $3,4-\mathrm{OH}$ & $3,4-\mathrm{OH}$ & o1/sery137 & $93 \%$ \\
\hline sery $338 \mathrm{~b}$ & $3-\mathrm{Br}$ & $3,4-\mathrm{MD}^{2}$ & $\mathrm{a} /$ sery338a & $62 \%$ \\
\hline \multicolumn{5}{|c|}{ 1,2,4-Oxadiazoles } \\
\hline anle128b & $3,4-\mathrm{MD}^{2}$ & $\mathrm{H}$ & $\mathrm{e}$ & $50 \%$ \\
\hline sery95 & $3,4-\mathrm{OH}$ & $\mathrm{H}$ & o1/anle128b & $54 \%$ \\
\hline sery128 & $3,4-\mathrm{MD}^{2}$ & $3-\mathrm{F}$ & $\mathrm{e}$ & $25 \%$ \\
\hline sery129 & $3,4-\mathrm{MD}^{2}$ & $3,4-\mathrm{MD}^{2}$ & $\mathrm{e}$ & $38 \%$ \\
\hline sery139 & $3,4-\mathrm{OH}$ & $3,4-\mathrm{OH}$ & o1/sery129 & $90 \%$ \\
\hline sery140 & $3,4-\mathrm{MD}^{2}$ & $3-\mathrm{F}$ & o1/sery128 & $60 \%$ \\
\hline sery 180 & $3,4-\mathrm{OCH}_{3}$ & $3,4-\mathrm{OCH}_{3}$ & $\mathrm{e}$ & $44 \%$ \\
\hline sery182 & $3,4-\mathrm{OCH}_{3}$ & $3-\mathrm{F}$ & $\mathrm{e}$ & $34 \%$ \\
\hline sery $185 a$ & $3,4-\mathrm{OH}$ & $3-\mathrm{F}$ & o1/sery182 & $80 \%$ \\
\hline sery 266 & $3,4-\mathrm{OCH}_{3}$ & $\mathrm{H}$ & $\mathrm{e}$ & $46 \%$ \\
\hline \multicolumn{5}{|c|}{ Imidazoles } \\
\hline anle131b & $\mathrm{H}$ & $3,4-\mathrm{MD}^{2}$ & $\mathrm{f}$ & $71 \%$ \\
\hline anle132 & $\mathrm{H}$ & $3,4-\mathrm{OH}$ & o1/anle131b & $75 \%$ \\
\hline anle237 & $3,4-\mathrm{MD}^{2}$ & $3,4-\mathrm{MD}^{2}$ & $\mathrm{f}$ & $73 \%$ \\
\hline sery152 & $\mathrm{H}$ & $3,4-\mathrm{OCH}_{3}$ & $\mathrm{f}$ & $84 \%$ \\
\hline sery165 & $3,4-\mathrm{MD}^{2}$ & $3,4-\mathrm{OCH}_{3}$ & $\mathrm{f}$ & $82 \%$ \\
\hline sery166a & $3,4-\mathrm{OH}$ & $3,4-\mathrm{OH}$ & o1/sery165 & $89 \%$ \\
\hline sery267a & $3,4-\mathrm{OCH}_{3}$ & $3,4-\mathrm{OCH}_{3}$ & $\mathrm{f}$ & $72 \%$ \\
\hline sery 345 & $3,4-\mathrm{MD}^{2}$ & $3-\mathrm{Br}$ & $\mathrm{f}$ & $85 \%$ \\
\hline \multicolumn{5}{|c|}{ Pyrroles } \\
\hline anle130b & $\mathrm{H}$ & $3,4-\mathrm{MD}^{2}$ & $\mathrm{~d}$ & $54 \%$ \\
\hline sery160 & $3,4-\mathrm{OCH}_{3}$ & $\mathrm{H}$ & $\mathrm{d}$ & $45 \%$ \\
\hline sery166b & $3,4-\mathrm{OH}$ & $\mathrm{H}$ & o1/sery160 & $73 \%$ \\
\hline sery 167 & $3,4-\mathrm{OCH}_{3}$ & $3,4-\mathrm{OCH}_{3}$ & $\mathrm{~d}$ & $39 \%$ \\
\hline
\end{tabular}

Table 2.3.5. Synthesized DPP compounds bearing isoxazole, 1,2,4-oxadiazole, imidazole and pyrrole heterocycles. The structures of the synthesized 3,5-substituted isoxazoles, 3,5-substituted 1,2,4-oxadiazoles, 2,4-substituted $1 \mathrm{H}$-imidazoles and 2,4substituted $1 H$-pyrroles are listed, including the yields, preparation methods and, where required, starting materials. The reaction conditions are shown in figures 2.3.4 and 2.3.6. ${ }^{1}$ Ring labeling corresponds to figure 2.3.6. ${ }^{2}$ 3,4-MD stands for 3,4-Methylenedioxy. 


\subsubsection{Synthesis of complementary DPP compounds}

In addition to the series of DPP compounds presented above, our complementary SAR effort was directed at exploring the effect of substitution in the central heterocyclic ring. Regarding the selection of novel functional groups, preferable heterocyclic system and position of attachment, we took the following considerations into account. First of all, we focused mainly on modifications of pyrazole-based DPP compounds due to their promising biological potency and simplicity of chemical synthesis. Next, having identified anle138b as one of the most potent lead structure, we considered accessing the corresponding anle138brelated analogs with additional substituents in the pyrazole ring, while keeping the substitution on the phenyl rings intact. Herein, the characterization of the structural determinants in biological activity of anle138b-based compounds was an important goal for us.

In this context, we synthesized five DPP compounds, which were modified at position 4 of the central heterocyclic ring, including four pyrazoles and one isoxazole. We introduced a bromine atom at this position for three compounds, namely anle134, sery330 and sery197. As shown in figure 2.3.7 A and B, corresponding brominated analogues are accessible in one step according to the published procedure ${ }^{408}$. Experimentally, the selected substrates were reacted with $\mathrm{N}$-bromosuccinimide in chloroform or in acetic acid to provide the desired

A
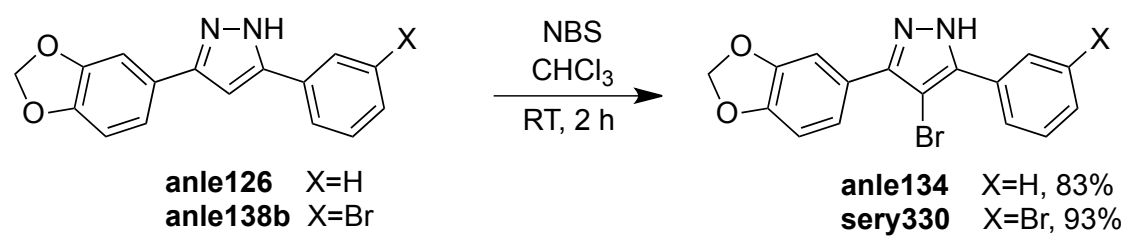

B<smiles>COc1ccc(-c2cc(-c3ccc(OC)c(OC)c3)on2)cc1</smiles>
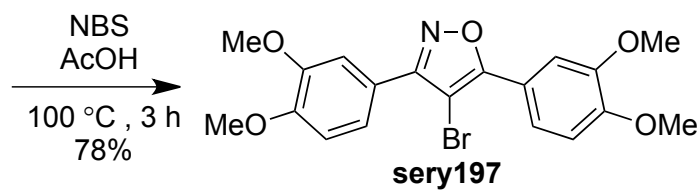

C<smiles>[PbH2]OC1C(c2ccc3c(c2)OCO3)=NNC1c1ccccc1</smiles>

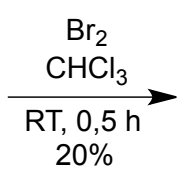<smiles></smiles>

$\mathbf{D}$

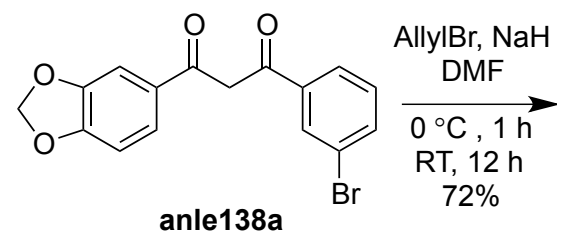<smiles>CC=CCC(C(=O)c1cccc(Br)c1)C(=O)c1ccc2c(c1)OCO2</smiles>

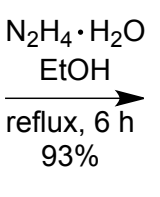

sery 375

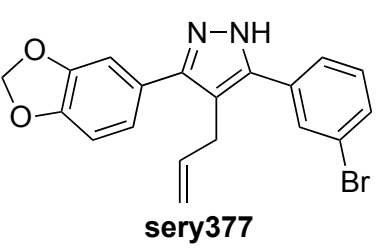

sery 377

Figure 2.3.7. Synthesis of 3,4,5-substituted pyrazoles and isoxazole. Synthesis of DPP compounds (anle134, sery330, sery197, anle127c, sery377) bearing bromine or hydroxyl or allyl in position 4 of central heterocyclic ring. 
brominated-DPPs in high yields (Fig. 2.3.7 A and B). Next, we prepared one compound anle137c with the hydroxyl group in position 4 of the pyrazole ring. The synthetic procedure involved the treatment of corresponding 4-hydroxy-pyrazoline anle127b with bromine in chloroform (Fig. 2.3.7C). Although the formation of many side products was observed under the applied conditions, it was possible to isolate the desirable compounds in $20 \%$ yield. The last example of modifications of position 4 in pyrazole ring is an introduction of the allyl group. The corresponding compound sery 377 was prepared in two steps starting from 1,3diketone anle138b that was alkylated by allyl bromide in the presence of sodium hydride as a base (Fig. 2.3.7D). In the second step, the obtained 1,3-diketone sery375 was readily converted to pyrazole sery 377 by condensation with hydrazine hydrate in refluxing ethanol.

In line with the strategy discussed above, our parallel SAR effort was directed on exploring the diverse modifications of the pyrazole ring on nitrogen atom. The list of designed and synthesized compounds is shown on figure 2.3.8. Hence, we prepared $N$ acylated and $N$-alkylated pyrazole-based DPP analogues. The $N$-acyl derivative sery 153 was prepared according to the published procedure involving the reaction of sery 149 with acetyl chloride in the presence of $\mathrm{Et}_{3} \mathrm{~N}^{409}$. To obtain $N$-alkyl pyrazole-based DPPs, we utilized two different approaches. According to the first route, the corresponding pyrazole substrates were reacted with appropriate alkylating agent such as methyl iodide or tosylated 2-fluoroethanol, to afford mixtures of isomer (Fig. 2.3.8 B, C). The separation of the mixture by column chromatography provided the desired products. We utilized this approach to obtain sery363a, sery363b, sery392a, and sery392b. It is noteworthy to mention that these DPP analogues appear to be a particular promising starting point for the development of novel PET tracers. For instance, $\left[{ }^{11} \mathrm{C}\right]$ sery $363 \mathrm{a} / \mathrm{b}$ could be readily prepared by methylation of anle $138 \mathrm{~b}$ with $\left[{ }^{11} \mathrm{C}\right]$ methyl iodide. Concurrently, the second candidate compound sery $363 \mathrm{a} / \mathrm{b}$ is suitable for radiolabeling with ${ }^{18} \mathrm{~F}$ isotope. In addition to the design and conventional synthesis of the described PET tracer candidates, we have also developed the corresponding protocols that are easy adoptable to radiochemical synthesis. Details of PET tracer development are not discussed in my thesis.

Coming back to the synthesis of $N$-alkyl pyrazole-based DPPs, the second synthetic approach is based on the reaction of 1,3-diketones with alkyl hydrazines (Fig. 2.3.8 D, E). The experimental conditions worked quite well for pyrazole formation, but, as expected, the mixtures of two isomers were obtained. Therefore, the main weak point of this procedure is a relatively tedious separation of isomers by several rounds of column chromatography. As shown on figure 2.3.8 D and E, 1,3-diketones anle138a and sery397a reacts readily with 
A

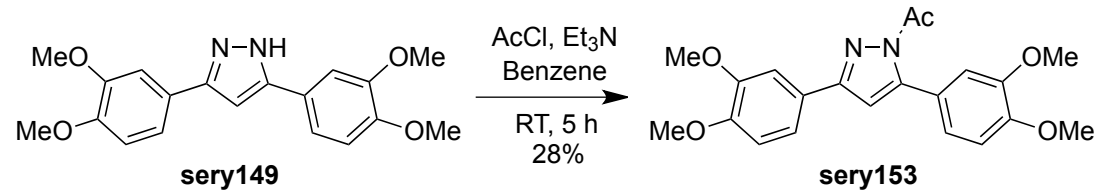

B

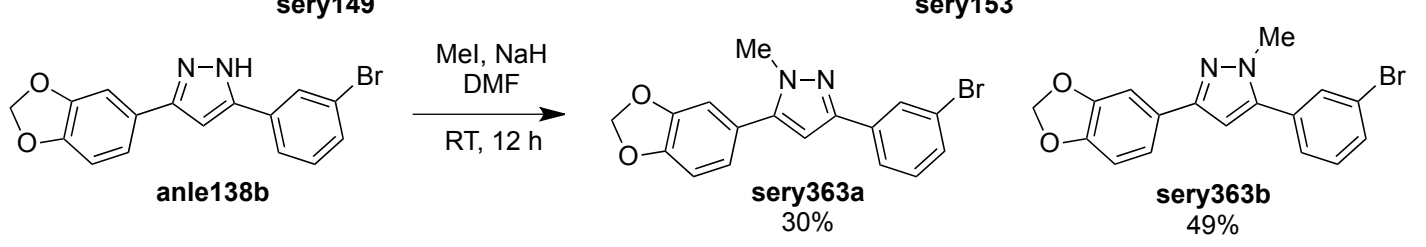

C
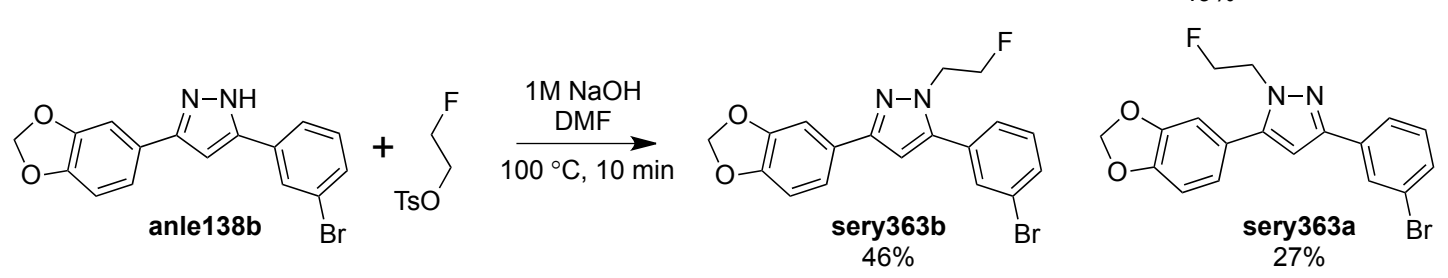

D

anle138a

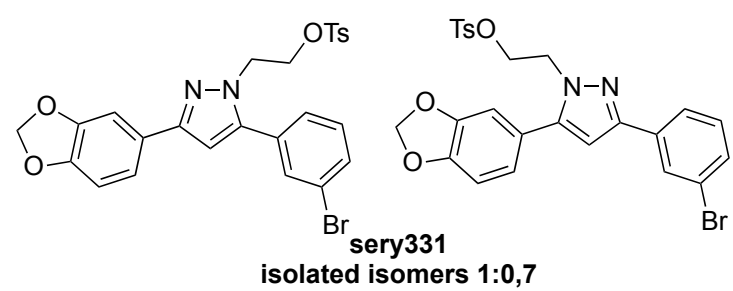<smiles>OCCn1nc(-c2ccc3c(c2)OCO3)cc1-c1cccc(Br)c1</smiles>

$\mathrm{OH} \quad \mathrm{HO}$

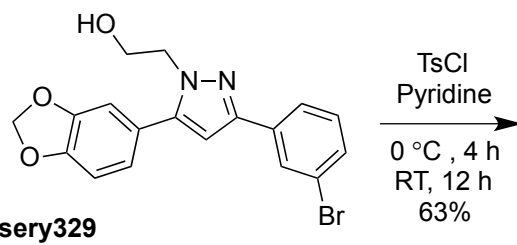
mixture of isomers 1:1

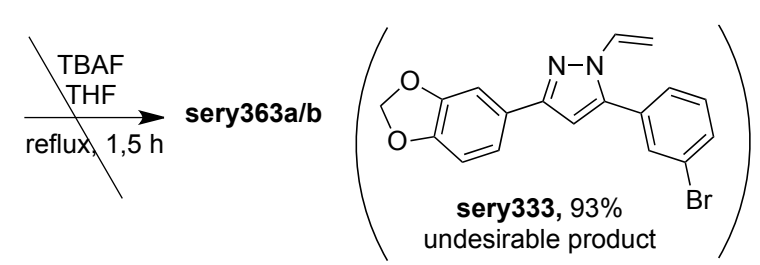

$\mathbf{E}$<smiles>Cn1nc(-c2ccc3c(c2)O[C@H](C(C)(C)C)O3)cc1-c1ccnc(Br)c1</smiles>

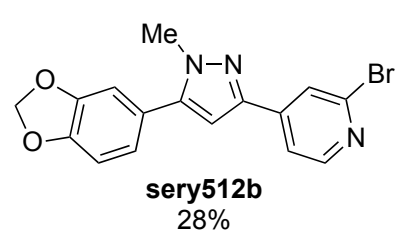

F<smiles>Nc1ccc(-c2cc(-c3cccc(Br)c3)[nH]n2)cc1</smiles>

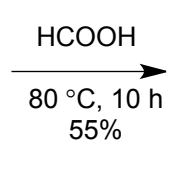<smiles>O=CNc1ccc(-c2cc(-c3cccc(Br)c3)[nH]n2)cc1</smiles>

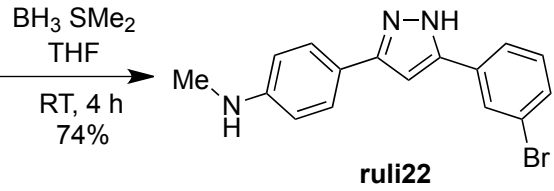

Figure 2.3.8. Synthesis of N-substituted pyrazoles and ruli22.

methyl hydrazine and 2-hydroxyethyl hydrazine, respectively, to afford corresponding $N$ alkyl pyrazoles sery512a/b and sery329, which were used in further studies as a mixture of isomers in the ratio $1: 1$. In our initial synthesis of already mentioned sery $363 \mathrm{a} / \mathrm{b}$ compounds, sery329 was tosylated in pyridine, but we were unsuccessful in our attempts to exchange OTs group with fluorine atom (Fig. 2.3.8D). Instead of the fluorine-containing target product, the product of the elimination reaction, namely sery333, was isolated in high yield (Fig. 2.3.8D).

The last synthetic scheme (Fig. 2.3.8 F) shows the preparation of ruli22 that was considered a valuable precursor for radiochemical synthesis of anle $253 \mathrm{~b}$, which exhibited an 
excellent anti-amyloidogenic activity in our biological assays. The previously suggested methods of pyrazole synthesis are not compatible with secondary amine functional groups. Therefore, we developed a two-step scheme starting from available compound sery384 bearing the free amino groups. Thus, the sery384 was first reacted with formic acid ${ }^{410}$; the resulting formamide ruli16 was further reduced by borane dimethylsulfide ${ }^{411}$ yielding the desirable product ruli22. The synthesis of ruli22 was performed by R. Linder.

\subsubsection{Synthesis DPP compounds: key findings and discussion}

For many years small organic heterocyclic compounds are of immense interest for pharmaceutical industry. Currently, the majority of new drugs, up to $90 \%$, contain one or several heterocyclic rings ${ }^{412}$. In this study we designed and synthesized the focused library of more than 150 diverse heterocyclic compounds falling under proposed Markush structure (Fig. 2.3.1). To ensure the chemical diversity, we selected six different 5-membered heterocycles as a central ring, including pyrazole, imidazole, pyrazoline, isoxazole, pyrrole and oxadiazole. The substitution of the phenyl rings was also broadly diversified.

In general, the synthesis of desired DPP derivatives was accomplished by using published routes and protocols. From the perspective of medical chemistry, the main achievement of this study is a design of general synthetic schemes that have certain advantages, which are: flexibility, small number of steps, cheap and available starting materials, high yield and simple isolation protocols. For anle138b, a bulk production protocol (250 g per synthesis) was developed.

All compounds were isolated in a high purity and at least in $50 \mathrm{mg}$ quantity. All novel structures were characterized spectroscopically and chemically. 


\subsection{DPP compounds and prion protein in vitro and in vivo}

\subsubsection{Prion protein in molecular SIFT assay}

Based on the positive outcome of the high-throughput screening campaign in search for compounds with anti-prion activity that was initiated by Prof. Armin Giese, we selected the molecular SIFT assay as the core in vitro technique to evaluate the activity of novel DPP compounds from the focused library. In this context, I would like briefly to highlight the specific aspects and advantages of prion-related SIFT assay.

As discussed in the chapter 2.2.1, the application of SIFT-based technology provides the rapid, reliable and efficient approach for investigation of protein aggregation and screening for compounds interfering with this process at the molecular level. In contrast to other aggregating proteins, the evaluation of prion protein in SIFT assay has a slightly different underlying principle. For instance, $\alpha$-synuclein SIFT is performed on the mixture of $\alpha$-synuclein constructs labeled with two different fluorophores. Upon incubation, the formation of the large aggregated/oligomerized $\alpha$-synuclein species is shown by the presence of highly labeled multimeric particle that are predominantly composed of both green and red labeled- $\alpha$-synuclein (Fig. 2.2.1). In case of prion protein, the SIFT assay allows detection of

A

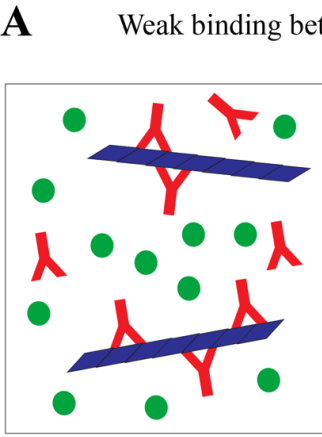

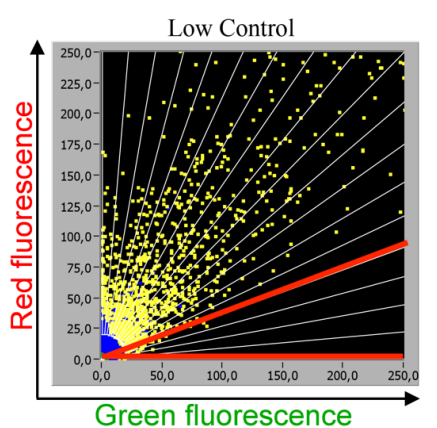

B Strong binding between $r$ PrP and $\operatorname{PrPSc}$
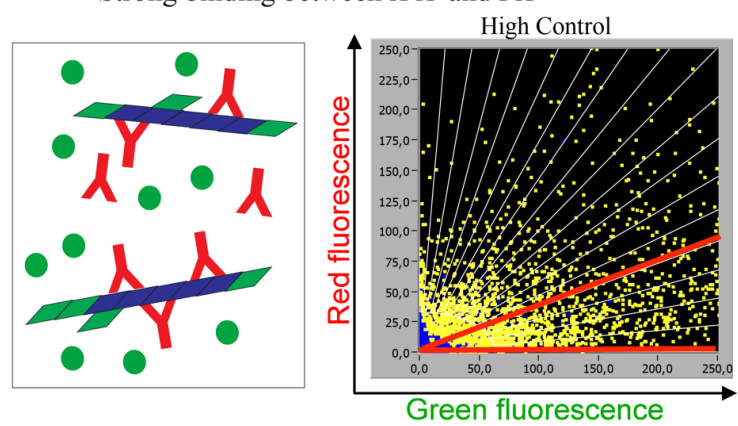

- Recombinant mouse PrP (labeled with green fluorophore)

- Green-labeled mouse PrP bound to human $\mathrm{PrPS}^{\mathrm{Sc}}$

- Human $\mathrm{PrP}^{\mathrm{Sc}}$ aggregates (no labeling)

Y - Red-labeled L42 antibodies against human PrP

Figure 2.4.1. Schematic representation of the SIFT assay on prion protein. Schematic representations and typical 2D fluorescence intensity distribution histograms for situations of weak (A) and strong (B) interactions between $\mathrm{rPrP}$ and $\mathrm{PrP}^{\mathrm{Sc}}$. Lack of binding between $\mathrm{rPrP}$ and $\mathrm{PrP}^{\mathrm{Sc}}$ leads to minimal detection of aggregates inside of sector separated by red lines on 2D histograms (low control). In case of strong interactions (high control), the formation of highly labeled ternary species that consist of $\mathrm{rPrP}, \mathrm{PrP}^{\mathrm{Sc}}$ and $\mathrm{L} 42$ antibody is confirmed by detection of high-intensity events in corresponding sector. Two-dimensional fluorescence intensity distribution histograms were kindly provided by Prof. Dr. A. Giese. 
binding events between monomeric recombinant prion protein $(\mathrm{rPrP})$ and human-derived misfolded beta-sheet-rich isoform of prion protein, also known as scrapie isoform $\left(\mathrm{PrP}^{\mathrm{Sc}}\right)^{7}$. Due to the fact that prion SIFT is focused on the evaluation of interactions, the corresponding experimental setup has significant differences in comparison to those of $\alpha$-synuclein both regarding labeling scheme and data analysis. In this way, the assay mixture includes $r P r P$ labeled with green fluorophore, red-labeled monoclonal antibodies L42 and human-derived $\mathrm{PrP}^{\mathrm{Sc}}$. Importantly, the monoclonal antibodies L42 selectively recognize the human prion protein and, thereby, under applied conditions L42 binds just to $\operatorname{PrP}^{\mathrm{Sc}}$, since $\operatorname{rPrP}$ has the mouse sequence. Thus, in the mixture of these three components, $\mathrm{PrP}^{\mathrm{Sc}}$ is indirectly labeled with red fluorophore of L42 antibodies, while $\operatorname{rPrP}$ is carrying the green fluorophore. When there are weak interactions between two prion isoforms, the formation of prion protein aggregates that are composed of both isoforms is inhibited and eventually the reduced SIFT signal of the particles with high fluorescence intensity in both green and red channels is observed (Fig. 2.4.1 A). Alternatively, the binding of $\mathrm{rPrP}$ to $\mathrm{PrPSc}^{\mathrm{S}} \mathrm{L} 42$ complex results in formation of ternary assemblies with many red and green labels (Fig. 2.4.2. B) ${ }^{7}$.

For quantitative evaluation of the $\mathrm{rPrP} / \mathrm{PrPSc}^{\mathrm{Sc}}$ association in the SIFT assay, 2D fluorescence intensity histograms are divided into several sectors, and the color distribution of the generated prion species is analyzed according to this layout. In our study, we monitored the signals of green-labeled aggregates inside of the sector that is separated by red lines of 2D histograms (Fig. 2.4.1). The presence of strong SIFT signal in this area is attributed to formation of high molecular misfolded $\mathrm{rPrP}$ species as a result of the strong $\mathrm{rPrP} / \mathrm{PrPSc}$ interaction. The corresponding high control is shown on figure 2.4.1B. When the binding of $\mathrm{rPrP}$ to $\mathrm{PrP}^{\mathrm{Sc}}$ is altered by any means, the reduction of the signal intensity within the marked sector can be observed. For instance, the addition of known inhibitor of $\operatorname{PrP}^{\mathrm{Sc}}$ propagation, cationic lipid DOSPA, to our assay mixture leads to expected change in color distribution in 2D SIFT histograms, namely strong reduction of fluorescence signal inside of the marked sector (Fig. 2.4.A) ${ }^{7}$. Collectively, the described above prion-based SIFT assay provides the platform for efficient screening of molecules interfering with the key pathological event in prion propagation. 


\subsubsection{Inhibition of $\mathrm{PrP}^{\mathrm{Sc}}$ propagation by DPP compounds in SIFT assay}

To evaluate the anti-prion activity of DPP compounds from the focused library, a primary screening of more than 70 novel DPP derivatives (library "Prion SIFT assay") in prion SIFT assay was done in the lab of Prof. A. Giese (Scheme 2.1). In this experiment, I was contributing with the selection of compounds, data evaluation and extraction of SAR information.

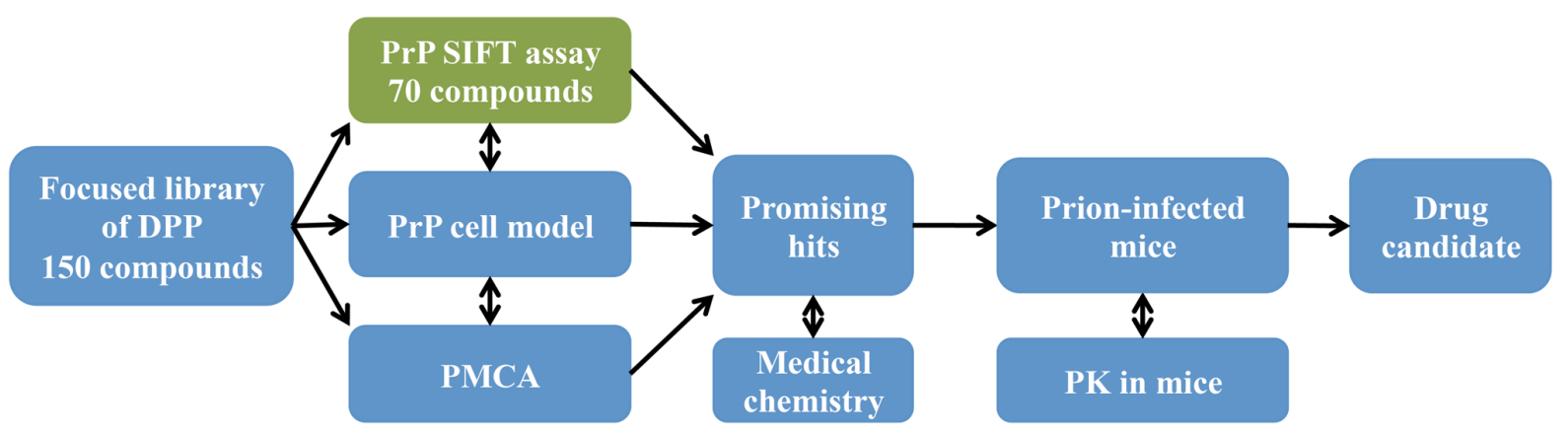

Scheme 2.1. Key steps of the anti-prion drug candidate discovery. Current step in green.

The main objectives of the primary SIFT screening were a confirmation of antiprion properties of compounds represented by DPP scaffold, identification of most promising inhibitors and determination of the major structural determinants of inhibitory activity. To ensure an adequate structural diversity for the initial SIFT assay, we selected the DPP compounds that are representing three main scaffolds (scaffolds 1, 2 and 3, Fig. 2.3.2A) and, moreover, are bearing relatively diverse sets of $\mathrm{R}_{1}$ and $\mathrm{R}_{2}$ substituents. The anti-prion activities of the tested DPP molecules are depicted in the figure 2.4.2, while the corresponding structures can be found in Table 2.5.1 and in the synthesis part.

From our SIFT-based screening efforts of more than 70 novel DPP molecules, we confirmed the high potency of DPP analogs to interfere with the $\mathrm{rPrP} / \mathrm{PrP}{ }^{\mathrm{Sc}}$ association and aggregation. As demonstrated in figure 2.4.2, more than one quarter of tested compounds (21 compounds) showed the inhibitory activity higher than 50\%. Moreover, SIFT data also suggest that approximately half of the compounds was notable for moderate antiprion activity, meaning $10-50 \%$ of inhibition in comparison to high control samples. Finally, 19 DPP compounds out of 74 were found to be inactive in prion SIFT assay. Remarkably, among the new DPP variants examined, we have identified several promising hits that appear to be highly potent inhibitors of $\mathrm{rPrP} / \mathrm{PrP}^{\mathrm{Sc}}$ interactions. For instance, sery166a, sery166b, anle145c, anle142c and sery85 almost completely abolished the SIFT signals associated with prion protein misfolding and propagation. Herein, it should also be noted that primary SIFT 
screening was performed with relatively high concentration of DPP compounds $(100 \mu \mathrm{M})$ and, therefore, the solubility properties might lead to significant underestimation of SIFT activity of poor soluble molecules.

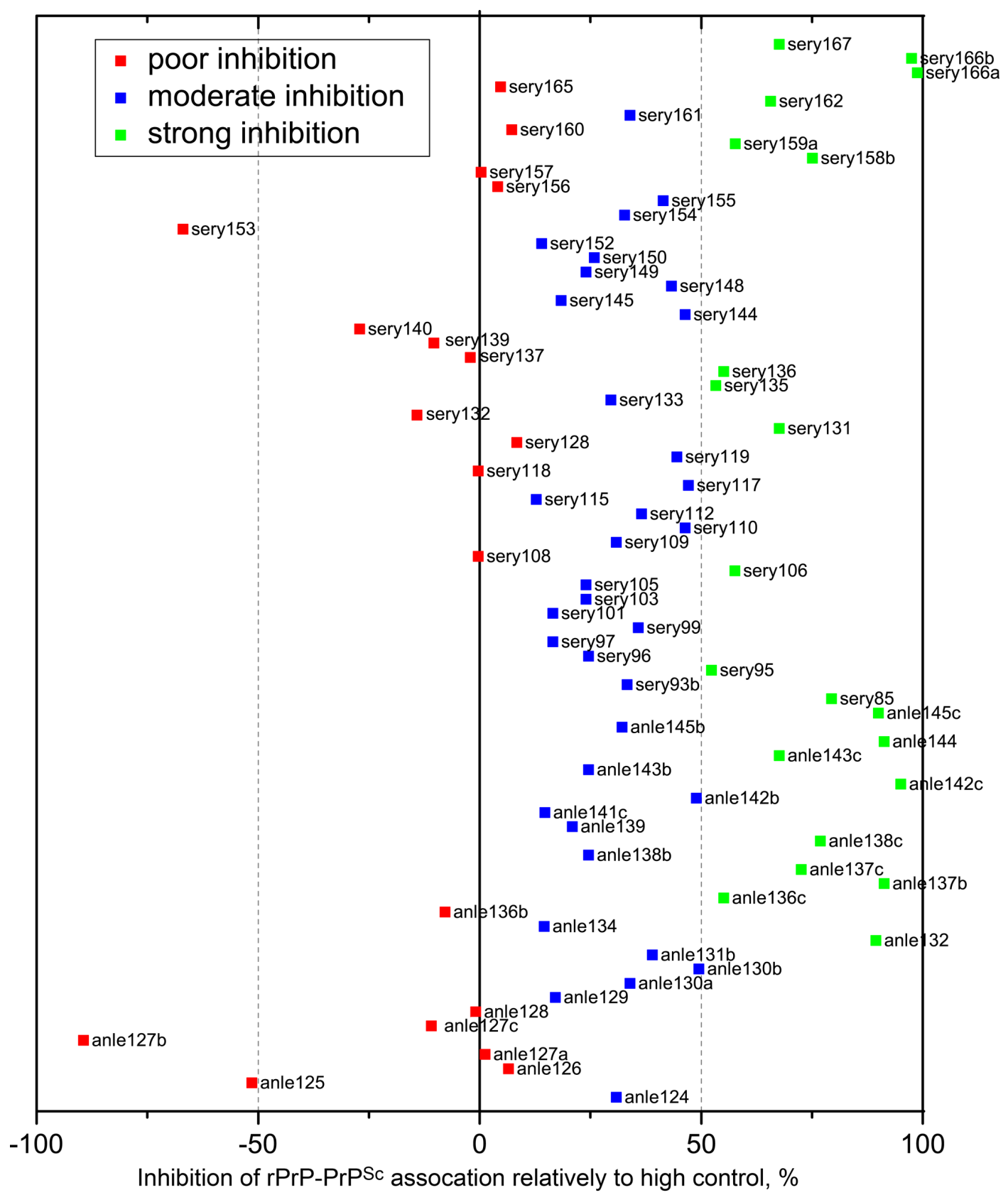

Figure 2.4.2. Results of primary SIFT screening of DPP compounds. Inhibitory activity of DPP compounds from "Prion SIFT" library against $\mathrm{rPrP}-\mathrm{PrP} \mathrm{Pc}^{\mathrm{Sc}}$ association and related aggregation. DPP compounds are classified according to their activity into three groups: strong ( $>50 \%$, green labels), moderate $(10-50 \%$, blue labels) and poor $(<10 \%$, red labels) inhibition activity in SIFT assay. Inhibition is calculated relatively to high controls; compound concentration $100 \mu \mathrm{M}$. 


\subsubsection{SAR studies and validation of DPP compounds in SIFT assay}

In order to evaluate more fully the SAR and validate the promising hits from initial SIFT screening, the dose response analysis was carried out for several active molecules by performing SIFT assay on dilution series. Upon examination, the sustainable inhibition of $\mathrm{rPrP} / \mathrm{PrP}^{\mathrm{Sc}}$ association by DPP compounds in concentration-dependent manner was confirmed in second round of SIFT. Characterization of inhibitory activity in dilution series revealed the $50 \%$ effective concentration $\left(\mathrm{EC}_{50}\right)$ values in the range of $10-20 \mu \mathrm{M}$ for most active DPP analogs. To illustrate these results, representative dose response curves are shown in figure 2.4.3A together with corresponding chemical structures of tested compounds from "Prion SIFT assay" library (Scheme 2.1.). Remarkably, the compassion of determined EC $_{50}$ values with the results of primary SIFT screening indicated a relevant correlation between antiprion activities determined in these two experiments. Thus, all selected compounds, including anle138c, anle142c, anle143c, sery85, that displayed strong inhibition in primary SIFT study also showed high activity based on $\mathrm{EC}_{50}$ values calculated according SIFT assay on dilution series.
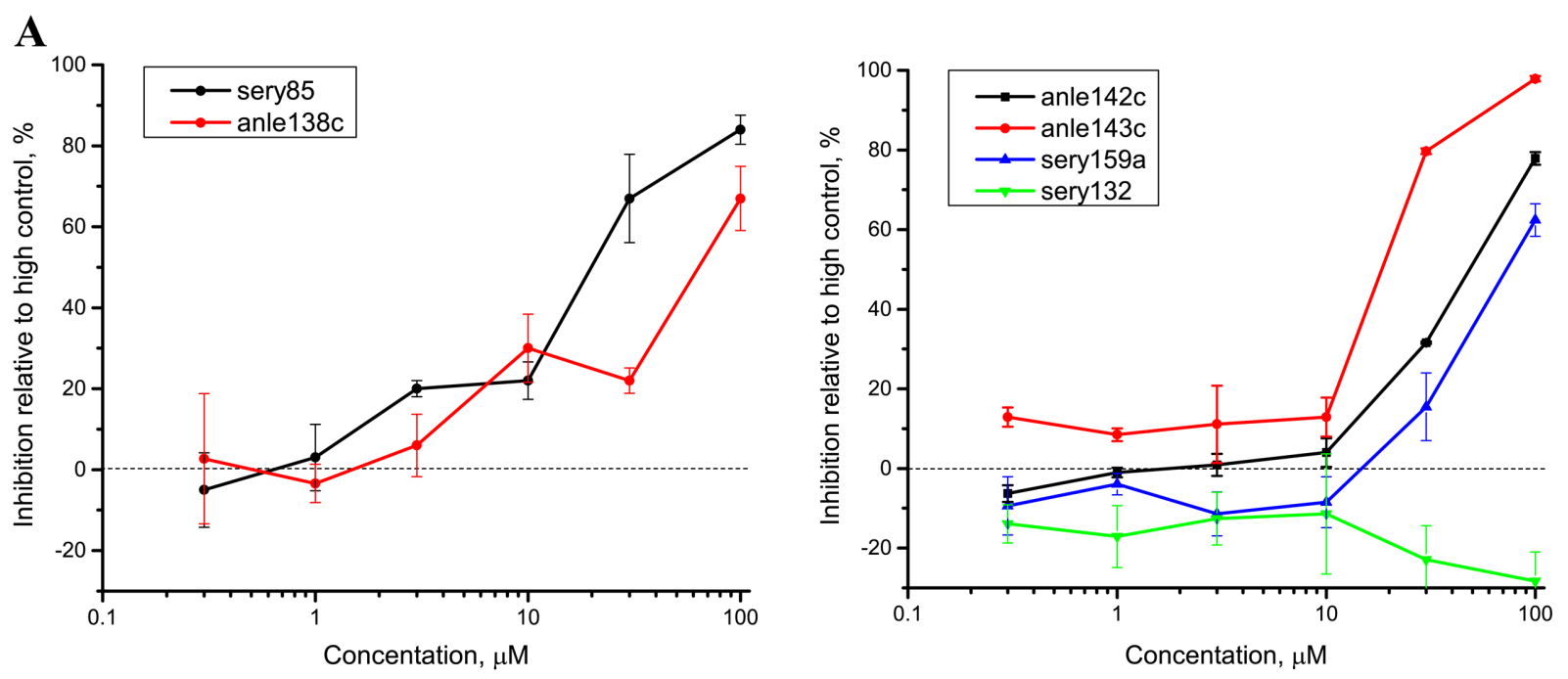

B

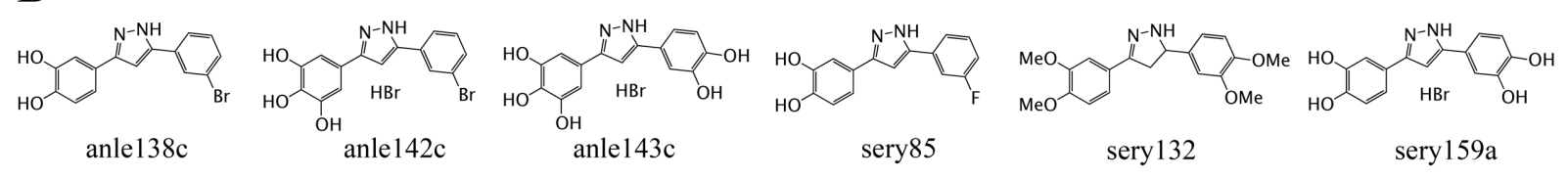

Figure 2.4.3. Concentration-dependent inhibitory activity of DPP compounds in

SIFT assay. (A) Concentration-dependent inhibition of $\mathrm{rPrP}-\mathrm{PrP} \mathrm{P}^{\mathrm{Sc}}$ association by selected DPP compounds in molecular SIFT assay. Corresponding dose-response curves obtained for 6 concentrations. (B) Chemical structures of selected DPP compounds. Error bars in (A, B) indicate standard error. 
The analysis of the combined data of primary and complementary SIFT assay on inhibition of $\mathrm{rPrP} / \mathrm{PrP}^{\mathrm{Sc}}$ association/aggregation for more than 70 diverse DPP-related compounds allowed us to establish certain aspects of structure-activity relationship:

1) The crucial structural requirement for DPPs to be a potent inhibitor in vitro SIFT assay is a presence of vicinal dihydroxyphenyl moiety, also as part of 3,4,5-trihydroxyphenyl function.

2) The activity shows correlation with a number of hydroxyl groups and increases as follows: hydroxy $<<$ dihydroxy $<$ trihydroxy $\leq$ two dihydroxy $<$ dihydroxy+trihydroxy.

3) The replacement of one or several hydroxyl groups with alkoxy groups abolishes the activity in SIFT assay.

In this context, there are several possible explanations of the significant decline of activity in case of DPP compounds bearing alkoxy groups. First, these modifications are undesirable and, therefore, compounds are inactive. Second, the solubility of alkoxy derivatives might play an important role. In fact, the solubility of DPP compounds with alkoxy groups is typically below $10 \mu \mathrm{M}$ in aqueous buffers. Considering the results of the SIFT assay on dilution series, in which even highly active and soluble molecules, like sery159a or anle143c, displayed significant effect starting from $5-10 \mu \mathrm{M}$, it is reasonable to assume that the poor solubility might be the main reason of misleading low activity in SIFT for poor soluble DPP analogs.

Collectively, the evidence reported above strongly supports the pronounced antiprion activity of novel compounds sharing the DPP-based core structure. Thus, $\sim 25 \%$ of the tested molecules were classified as primary hit showing the inhibitory activity higher than $50 \%$. We found that the SIFT activity distribution for compounds from "Prion SIFT assay" library is ultimately shifted towards antiprion properties. Those findings led us to speculate that the DPP scaffold and derived SAR information represent a good starting point for further lead optimization and investigation of the underlying mechanisms of pathological processes associated with prion protein misfolding and aggregation. It is noteworthy that the molecular SIFT assay has emerged as a valuable screening platform for studying $\mathrm{rPrP} / \mathrm{PrP}^{\mathrm{Sc}}$ interactions as well as for the fast and efficient method of identification and subsequent optimization of antiprion compounds. 


\subsubsection{Antiprion activity of DPP compounds in cell culture model}

Following the primary SIFT screening and validation of promising compounds in SIFT assay on dilution series, we were interested in the investigation of the antiprion activities of DPP compounds in more biologically relevant cellular model (Scheme 2.2). For this purpose we generated a "Prion cell model" library of DPP compounds (Fig. 2.4.5), which was tested in a well-established prion-infected cell model ${ }^{413}$ in collaboration with the laboratory of Prof. M. Groschup (Friedrich-Löffler-Institute, Greifswald-Insel Riems, Germany). It should be noted that the rational selection of compounds was an important step. Thus, in order to ensure an optimal selection following parameters were taken into consideration: inhibitory activity in SIFT assay, SAR information, chemical diversity and physical-chemical properties. Altogether, "Prion cell model" library of 24 DPP compounds was generated and transferred to our collaborating partner.

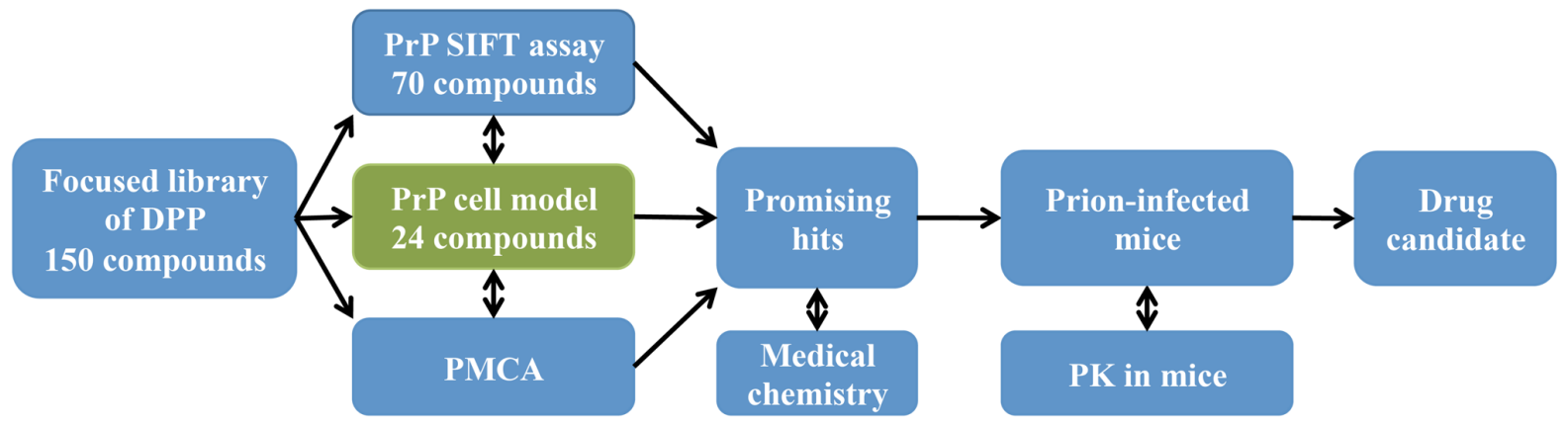

Scheme 2.2. Key steps of the anti-prion drug candidate discovery. Current step in green.

In this paragraph I would like to briefly describe the cellular model, the experimental setup and potential readout. To date, there is a number cell systems, in which the propagation and multiplication of prions was demonstrated and characterized ${ }^{388}$. For our study, a mouse brain-derived cell line SMB infected with Rocky Mountain Laboratory scrapie strain has been chosen. Previous reports have demonstrated that a stable and consistent propagation and accumulation of the RML prions can be achieved in these cells ${ }^{413}$. Among other readouts, the accumulation of detergent-insoluble and PK-resistant $\mathrm{PrP}^{\mathrm{Sc}}$ in $\mathrm{SMB}$ cells, which can be measured by dot plot assay, represents an attractive marker allowing to quantify the propagation of prions and assess the corresponding inhibitory effect of small molecules. In previous studies, the prion-infected SMB cell system was successfully utilized as a screening platform for search of antiprion compounds ${ }^{7}$.

Figure 2.4.4 shows the results obtained with RML-infected SMB cell cultures in presence of selected DPP compounds. In this experiment, the inhibition of $\mathrm{PrP}^{\mathrm{Sc}}$ by small 
molecules was calculated as a reduction of amount of PK-resistant $\mathrm{PrP}^{\mathrm{Sc}}$ in presence of inhibitor in comparison to non-treated control cells. Thus, the relative amount of PK-resistant $\mathrm{PrP}^{\mathrm{Sc}}$ in mock-treated cells was set at $100 \%$.

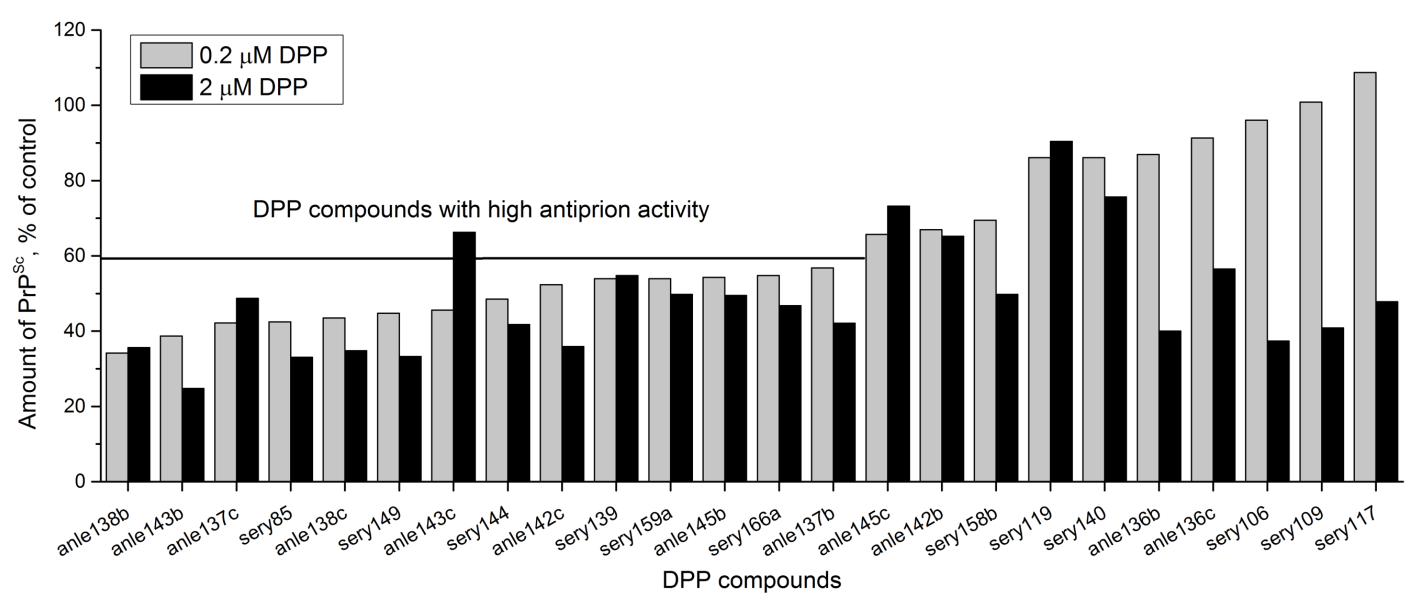

Figure 2.4.4. Inhibition of $\operatorname{PrP}^{\mathrm{Sc}}$ accumulation in $\mathrm{SMB}$ cells infected with RML scrapie strain. Quantification of detergent-insoluble and PK-resistant $\mathrm{PrP}^{\mathrm{Sc}}$ after 3 days of incubation in presence of DPP compounds in concentrations of 2 or $0.2 \mu \mathrm{M}$ from "Prion cell model" library. The compounds are ranked according to antiprion activity at concentration of $0.2 \mu \mathrm{M}$. Anle $138 \mathrm{~b}$ is a most active compound at $0.2 \mu \mathrm{M}$ concentration. Inhibition was calculated relative to amount $\mathrm{PrP}^{\mathrm{Sc}}$ in the untreated RML cells $(100 \%)$.

As shown in this study, the majority of selected DPP compounds exhibit a pronounced antiprion activity at both tested concentrations. Thus, the reduction of amount of PK-resistant $\mathrm{PrP}^{\mathrm{Sc}}$ by more than 40-50\% was observed for 13 out of 24 compounds (Figure 2.4.4 and 2.4.5). At the same time, compounds anle136c, anle136b sery106, sery109, sery117 can efficiently interfere with the accumulation of $\mathrm{PrP}^{\mathrm{Sc}}$ just at high concentration of $2 \mu \mathrm{M}$, while at low concentration $(0.2 \mu \mathrm{M})$ the activity against $\mathrm{PrP}^{\mathrm{Sc}}$ is marginal. Several compounds, for instance sery119, sery140, were found to be inactive in this cellular model.

Despite the fact that the number of compounds in the "Prion cell model" library is limited, the available data allows us to derive valuable SAR information. In line with the prion SIFT assay, the high antiprion activity was observed for compounds bearing several free hydroxy groups, for example anle137c, sery 85 , anle138c and others. Moreover, several potent molecules are substituted with several methoxy groups, namely anle143b, sery149, anle145b. In addition to favorable hydroxy and methoxy groups, the introduction of bromine or fluorine in a meta position of phenyl ring is associated with a high antiprion activity. That can be illustrated by comparison of potent compounds sery 85 and anle138c with sery106 that exhibits low activity and, structurally, is lacking substituents in one of the phenyl rings. One 
of the most important findings of this screening was the identification of a highly potent

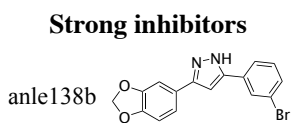

Average and poor inhibitors compound, called anle138b. In this cellular model, anle138b exhibits the strongest inhibition of $\mathrm{PrP}^{\mathrm{Sc}}$ at low concentration within the "Prion cells" library. This striking effect of anle $138 \mathrm{~b}$ is mainly determined by the presence of methylenedioxy moiety (Fig. 2.4.5). It is also of interest to compare the effect of the central heterocyclic ring. Among four different heterocycles, including pyrazole, imidazole, oxadiazole and isoxazole, compounds with the pyrazole ring appear to be particular promising leads. At the same time, the introduction of isoxazole and oxadiazole rings is considered as unfavorable, for example in a series of compounds: sery85 $>>$ sery106 $>$ sery117 (Fig. 2.4.5).

Collectively, the results of screening in the RML-infected SMB cell cultures have corroborated the notion that DPP core structure represents a promising class of molecules in the search for antiprion drugs. Moreover, the
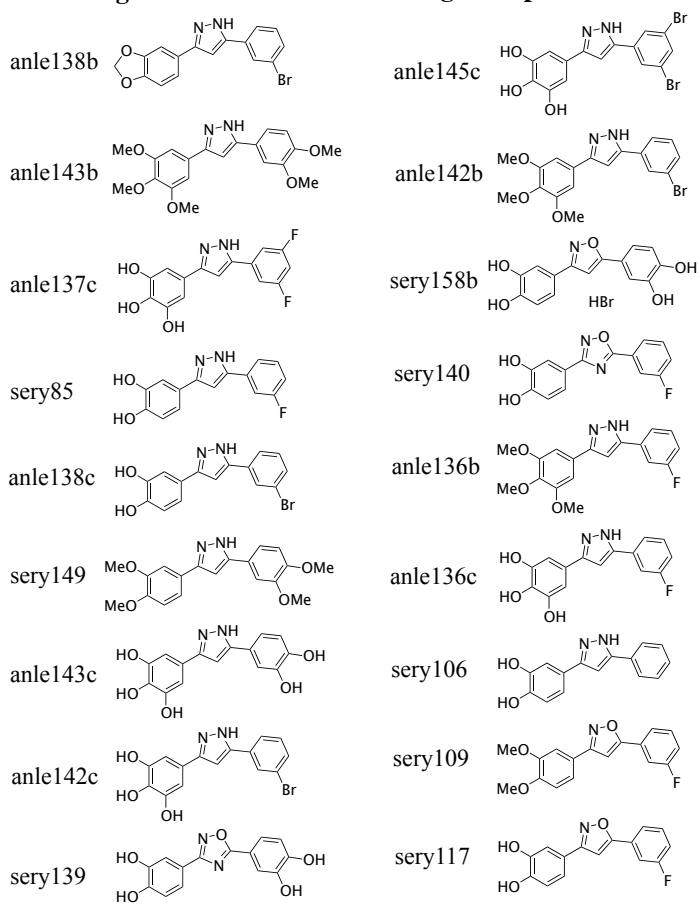

sery106

Sery109

sery 117
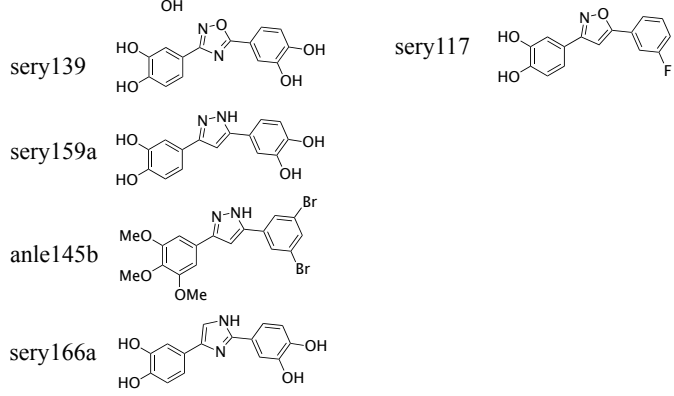

anle137b

Figure 2.4.5. Structures of DPP compounds tested in SMB cells. The compounds are classified and ranked according to their antiprion activity in SMB cells. utilization of the cell-based systems provides an important tool for identification of additional potent molecules that are not recognized under in vitro conditions of the SIFT screening system since in the in vivo assays hydrophobic compounds will accumulate in the membranes of the cell while in the in vitro assay system, there are no such membranes. In this context, the compound anle138b is one of such striking examples. The explanation for the relatively low effect of this compound in the SIFT assay in comparison to cell-based model might be attributed to a very high lipophilicity and poor solubility of anle138b in aqueous solutions. 


\subsubsection{Antiprion activity of DPP compounds in PMCA}

In addition to SIFT assay and cell-based model, a set of DPP compounds was also tested in a cell-free conversion assay that is called Protein Misfolding Cyclic Amplification (PMCA) in the lab of Prof. A. Giese (Scheme 2.3). In this experiment, I was contributing with the selection of compounds, data evaluation and extraction of SAR information.

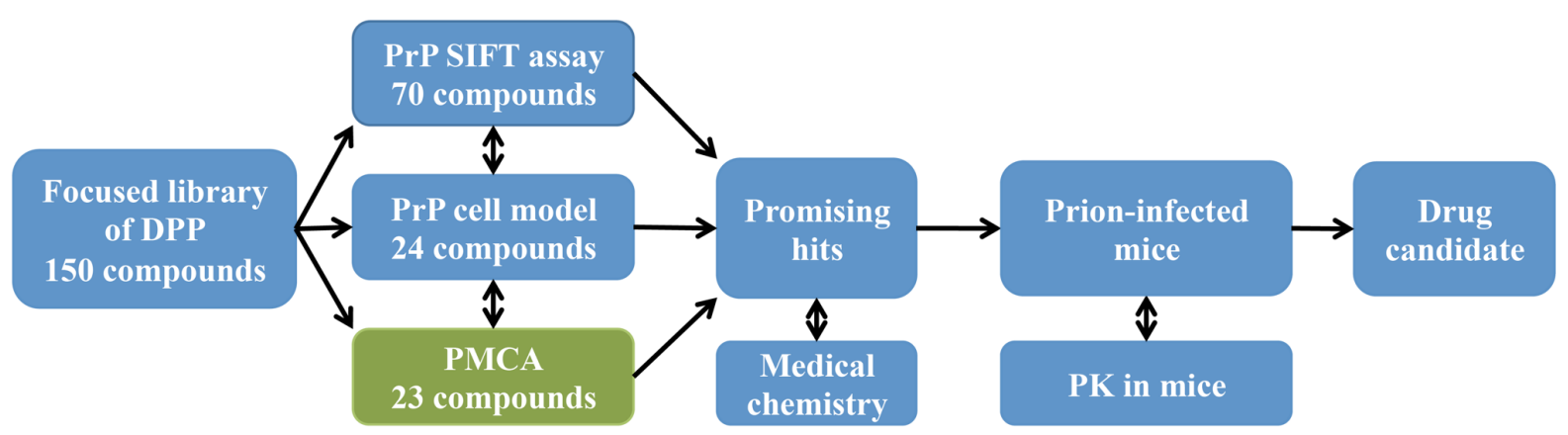

Scheme 2.3. Key steps of the anti-prion drug candidate discovery. Current step in green.

This assay was initially developed in the Soto's lab and was published in $2001^{414}$. It's widely accepted that the application of PMCA technology provides the rapid, reliable and efficient approach for detection of $\mathrm{PrP}^{\mathrm{Sc}}$ species and investigation of $\mathrm{PrP}^{\mathrm{Sc}}$ propagation. Some of the main advantages of PMCA are listed below ${ }^{415}$ :

1) Possibility to generate and amplify the infectious prions

2) Indefinite and strain-specific amplification of prions, including human-derived strains

3) High sensitivity of detection

4) Use of the cell lysates

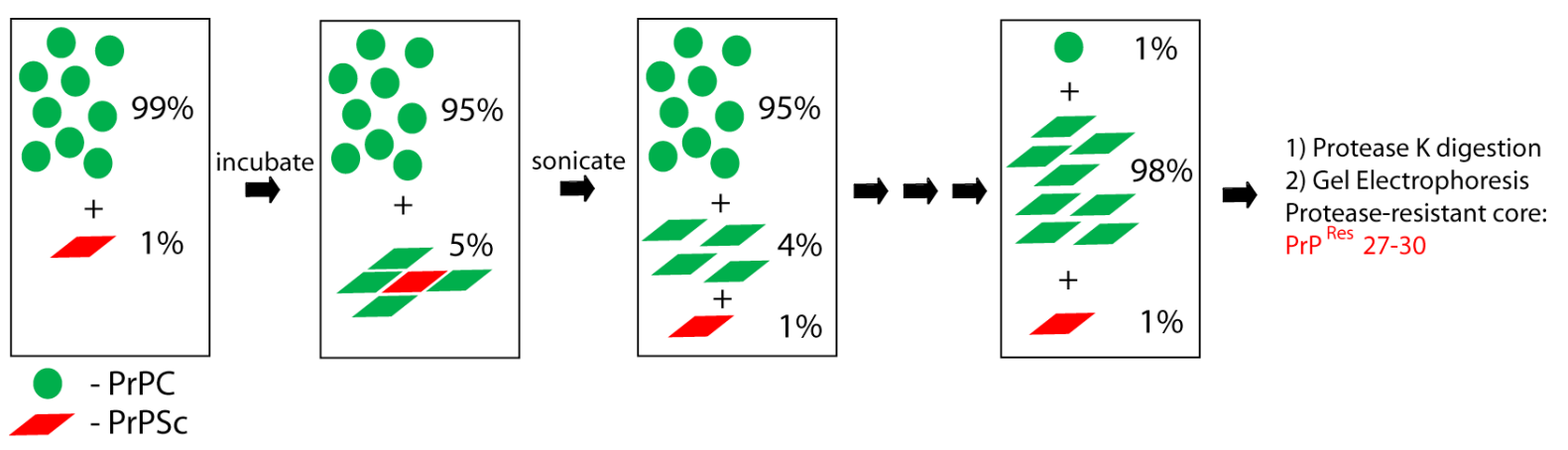

Figure 2.4.6. Schematic representation of the PMCA assay. 
Fundamentally, the PMCA assay is based on the nucleation-dependent polymerization mechanisms of the prion misfolding, whereas small amounts of $\mathrm{PrP}^{\mathrm{Sc}}$ aggregates are the templates for conversion of $\mathrm{PrP}^{\mathrm{C}}$ into the infection forms. An example of the experimental setup is schematically shown in Figure 2.4.6. The starting sample consists of a small amount of infectious material and a big excess of $\operatorname{PrP}^{\mathrm{C}}$. During the incubation period $\operatorname{PrP}^{\mathrm{Sc}}$ nuclei trigger the conformational conversion of $\mathrm{PrP}^{\mathrm{C}}$, which eventually leads to formation of new $\mathrm{PrP}^{\mathrm{Sc}}$ prions that are characterized by similar strain properties and species specificity. On the next step, the $\mathrm{PrP}^{\mathrm{Sc}}$ aggregates are broken down in smaller nuclei by sonication. The repetition of these incubation-sonication cycles results in exponential amplification of the $\mathrm{PrP}^{\mathrm{Sc}}$ prions. After the protease $\mathrm{K}$ digestion the amount of $\mathrm{PrP}^{\mathrm{Sc}}$ can be determined by dot plot or western blot.
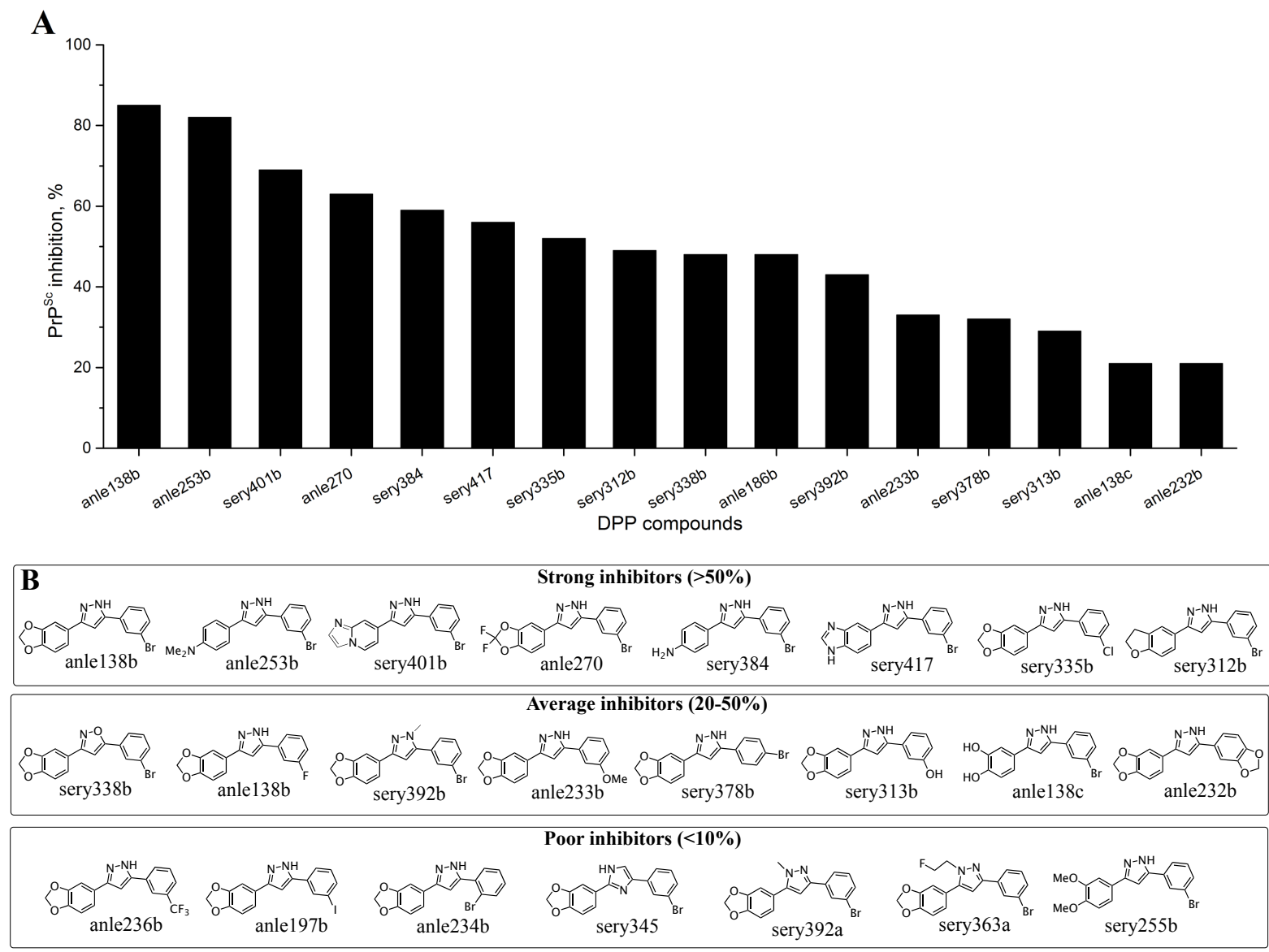

Figure 2.4.7. Inhibition of prion propagation by DPP compounds in PMCA assay. (A) The effect of DPP compounds ("PMCA" library on propagation of RML prion strain. $\%$ inhibition states for percentage of inhibition relative to high control. All compounds were tested at $10 \mu \mathrm{M}$ concentrations. (B) The chemical structures of all tested compounds; the compounds are ranked according inhibition of propagation of RML prion strain under PMCA conditions. 
Similar to the cell-based assay, we performed a rational selection of most promising compounds from the focused library for PMCA test. We tried to include in the "PMCA" library the compounds that appear to be particular promising candidates, based on available experimental data. At the same time, our goal was to ensure the chemical diversity and the optimal variation of substituents. At the end, 23 compounds were selected for the PMCA test.

The analysis of the amplification of $\mathrm{PrP}^{\mathrm{Sc}}$ (RML prion strain) under PMCA conditions revealed that the majority of selected DPP compounds exhibit a pronounced antiprion activity (Figure 2.4.7). Thus, the reduction of amount of PK-resistant $\mathrm{PrP}^{\mathrm{Sc}}$ by more than $50 \%$ was observed for 8 out of 23 compounds, 20-50\% inhibitory activity was found for another 8 compounds. Several compounds were found to be inactive in the PMCA assay (Figure 2.4.7B). In line with the data generated in prion-infected cell model, the results from PMCA assay further confirmed that the class of DPP compounds represents a promising lead in the search of antiprion drugs. It is remarkable finding that among the examined molecules in prion-infected cells and PMCA the compound anle138b shows the highest activity in interfering with propagation of $\mathrm{PrP}^{\mathrm{Sc}}$ in both models. On the basis of data from PMCA experiment, the SAR analysis was performed and the main conclusions of SAR are summarized below:

1) The effect of the substituents in the left-hand phenyl ring:

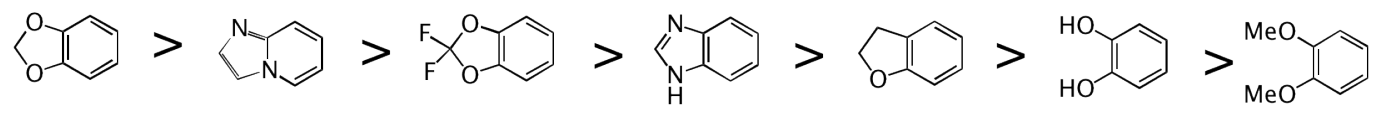

Methylenedioxy substituent is associated with the highest antiprion activity. Introduction of nitrogen or carbon atoms instead of oxygen atoms in this heterocyclic ring is not favorable. Surprisingly, the compounds bearing hydroxy or methoxy substituent, which are quite potent in SIFT and cell-based assays, are not able to interfere with the $\mathrm{PrP}^{\mathrm{Sc}}$ amplification under PMCA conditions.

2) The effect of the substituents in the right-hand phenyl ring:

Variation in meta position

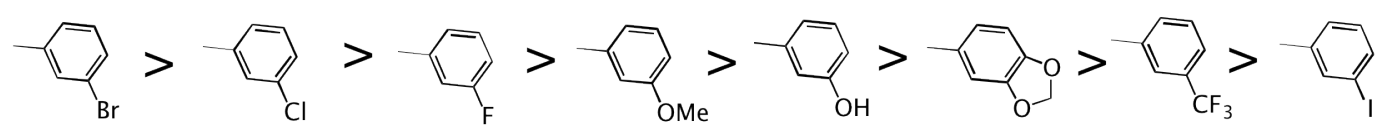

Variation of bromine position

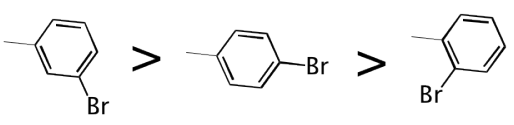


In the series of compounds substituted in the meta position of the right-hand phenyl ring, the introduction of halogens, namely $\mathrm{Br}>\mathrm{Cl}>\mathrm{F}>>\mathrm{I}$, is associated with the high antiprion activity. Among the halogens, the bromine is considered as a most beneficial. All other groups in meta-position, like $\mathrm{OMe}, \mathrm{OH}, \mathrm{CF}_{3}$, resulting in reduced activity. By activity comparison of anle138b, sery $378 \mathrm{~b}$ and anle $234 \mathrm{~b}$, the meta-bromine substitution is clear winner over ortho- and para-substituted analogs. Thus, sery $378 \mathrm{~b}$ exhibits the reduced activity, in case of anle234b the activity is abolished.

3) The effect of the central heterocycle ring:

Variation of central heterocycle

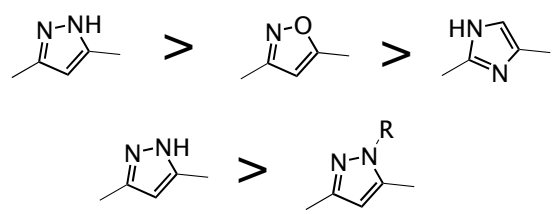

Among the variants examined, the compounds containing pyrazole as a central heterocyclic ring show the highest potency. The replacement of the pyrazole ring with isoxazole (sery338b) leads to reduced activity and in case of imidazole derivative sery 345 the activity is abolished. Moreover, the consequent introduction of simple substituents in pyrazole ring, like methyl, 2-fluoroethyl, is found to be unfavorable (Fig. 2.4.7).

Our findings in PMCA assay in combination with the data from cell-based model suggested that anle138b shows the highest anti-prion activity against RML prion stains. Encouraged by the potency of anle138b against murine RML prions, we decided to test anle138b in the PMCA assay using the range of murine (RML, ME7, 301C) and, what is more important, human prion stains, including SCJD and vCJD. It is important to highlight that PMCA assay constitutes the unique experimental approach to test the efficacy of small molecules with authentic human prions and human brain tissues and to support the relevance of the finding in context of human disease. Additionally, the efficacy of anle138b in PMCA was characterized by dose-response curves for human strain vCJD and for murine prion strain RML.

As shown on Figure 2.4.8, anle138b strongly inhibits the propagation of several nonhuman prion strains and, what is more important; the amplification of human strains SCJD and VCJD in the presence of anle138b is also significantly reduced under PMCA conditions. Remarkably, the correlation of antiprion activities for different strains is further supported by the PMCA data generated for compound anle234b, which failed to inhibit not just the RML prions, but also all other strains (Fig. 2.4.8 A, B). Of particular significance is a comparison 
of $\mathrm{EC}_{50}$ values derived from dose-response curves for human and non-human prion strains (Fig. 2.4.8 C, D). This experiment has established that anle138b inhibits equally good the propagation of both prion strains in dose-dependent manner with $\mathrm{EC}_{50}$ values around $7 \mu \mathrm{M}$ $\left(\mathrm{EC}_{50} \mathrm{RML}=7.3 \mu \mathrm{M}, \mathrm{EC}_{50 \mathrm{VCJD}}=7.1 \mu \mathrm{M}\right)$. These intriguing findings provide forcible arguments for further developed of anle $138 \mathrm{~b}$ as a therapeutic option for human prion disease.
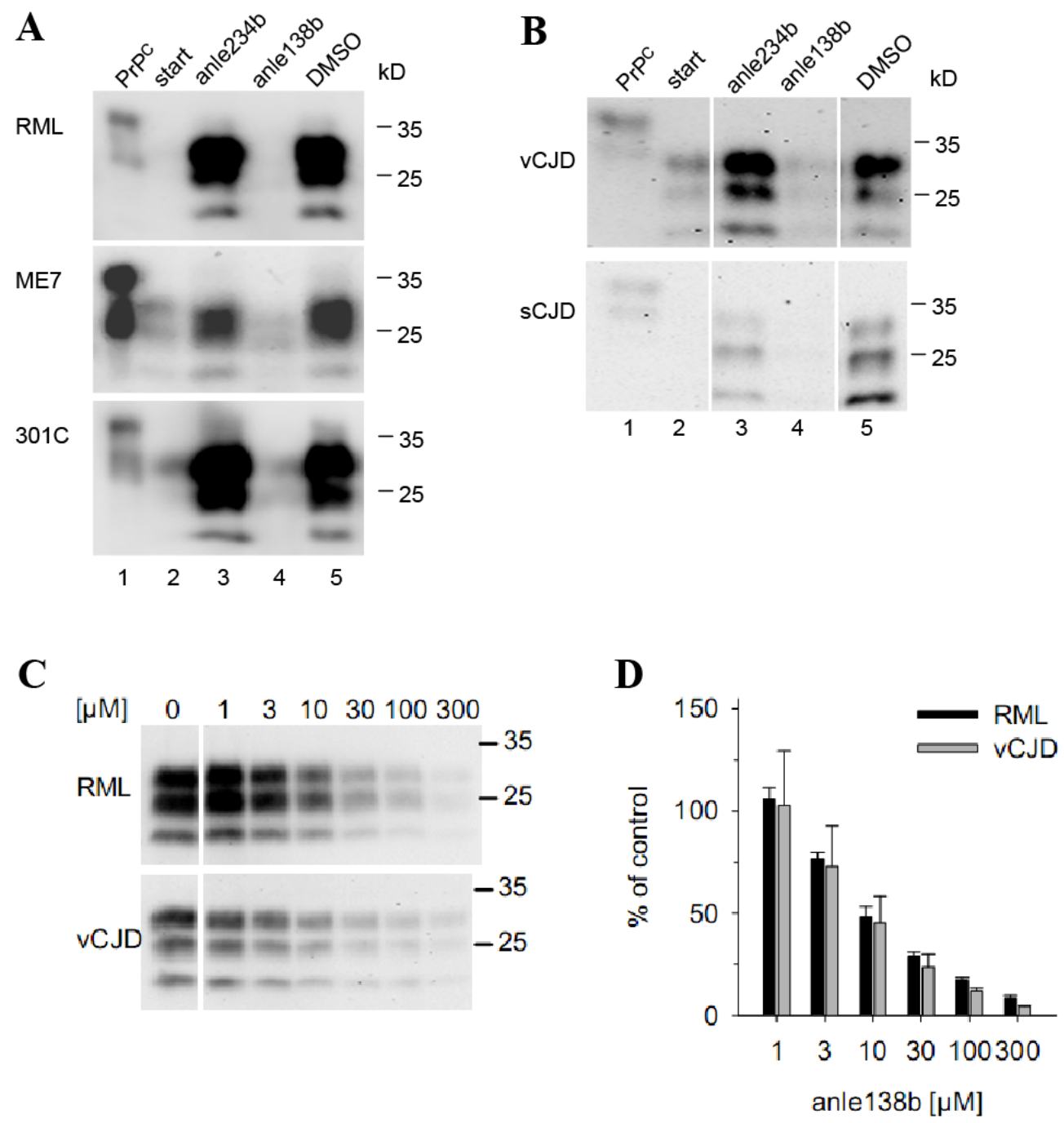

Figure 2.4.8. Inhibition of propagation of different prion strains by anle138b PMCA assay. (A, B)* The effect of anle138b on propagation of mouse-adapted scrapie strains (RML, ME7) and mouse-adapted BSE strain (A); human vCJD and SCJD (B) prion strains. (C)* The dose-dependent effect of anle138b on propagation of RML and VCJD (D) The densitometrical quantification of the amount of $\mathrm{PrP}^{\mathrm{Sc}}$ in PMCA with different concentrations of anle138b. The amount of $\mathrm{PrP}^{\mathrm{Sc}}$ is normalized to the control reaction without compound. Error bars in (D) indicate standard error. Figures are reproduced from J. Wagner, S. Ryazanov, et al. ${ }^{10}$ Copyright (C) 2013.

*In all gels, $0.5 \%(\mathrm{w} / \mathrm{v})$ normal brain homogenates from C57/BL6 and human brain, respectively, were loaded directly in the last lane without proteolysis as a reference (indicated as $\mathrm{PrP}^{\mathrm{C}}$ on the top). All other samples were treated with $50 \mu \mathrm{g} / \mathrm{ml}$ PK. "Start" (lane 1 of each gel) indicates samples containing infected brain homogenate before PMCA. 


\subsubsection{Antiprion activity of DPP compounds in animal models of prion disease}

In chapters 2.4.6-2.4.7, I would like to summarize the results of several studies focused on evaluation of the therapeutic effect of DPP compounds in mouse model of prion disease (Scheme 2.4). Almost all experiments that are described in this chapter were performed in the laboratory of Prof. A. Giese. My scientific contribution in this part of the project includes the discussions on a selection of compounds, a design of the treatment schemes and evaluation of the generated data in combination with SAR analysis. Moreover, I was involved in in vitro part of the pharmacokinetic studies that are described in details in chapter 2.8. Parts of the text and the figures in this chapter were adopted with some modifications from our publication J. Wagner, S. Ryazanov, et al. ${ }^{10}$.

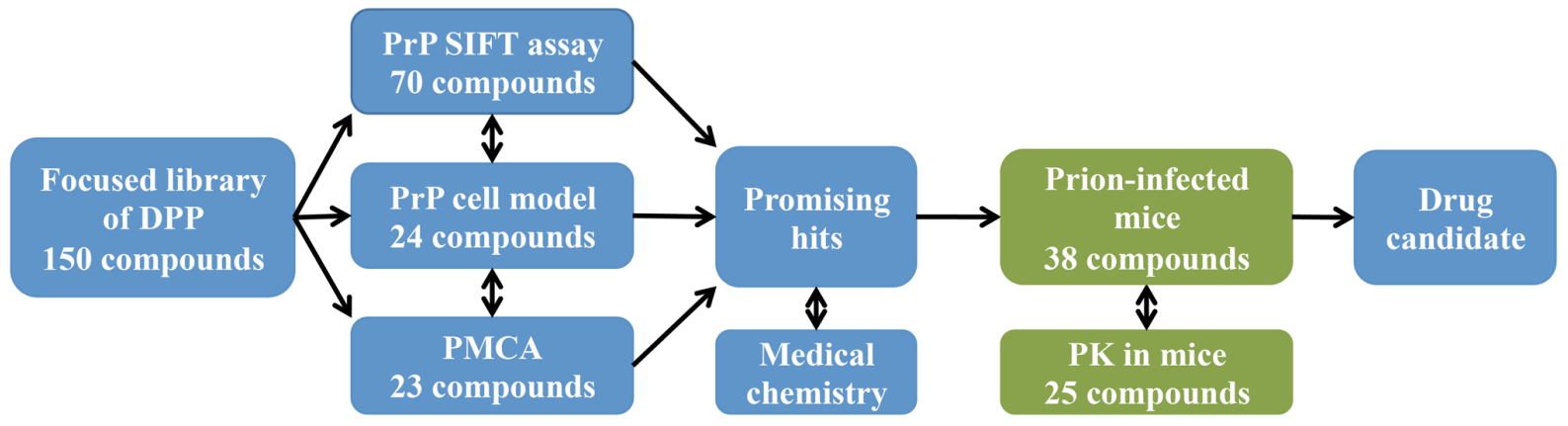

Scheme 2.4. Key steps of the anti-prion drug candidate discovery. Current step in green.

On the basis of the results from the SIFT, cell culture and PMCA assay, 38 DPPrelated compounds were selected and combined in "Prion mice" library for in vivo testing in RML prion-infected mice model. The list of these compounds, the therapeutic effects in the prion-infected mice and the detailed experimental summary are shown in Figure 2.4.9 and Figure 8.1 in Appendix.

The assessment of selected DPP derivatives was done in the RML-based prion model, as this provides an authentic neurodegenerative disease model with a well-defined and relatively rapid time course $\mathrm{e}^{416}$. Among other readouts, the levels of $\mathrm{PrP}^{\mathrm{Sc}}$ accumulation in the brain and in the spleen were predominantly analyzed for estimation of the therapeutic efficacy. The majority of compounds were given orally and, in addition to therapeutic efficacy, brain concentration was determined for a number of compounds (Fig. 2.4.9). The figure 2.4.10 summarizes the effect of various compounds in regard to prion propagation in vitro (PMCA) and in vivo in prion-infected mice, as well as compound concentrations achieved in the mouse brain $4 \mathrm{~h}$ after oral application of $1 \mathrm{mg}$ compound in DMSO/ peanut butter. In figure 2.4 .9 , for all compounds other than anle138b, only the part of the molecule 
(R1, R2, or R3) that differs from this lead structure is displayed. Collectively, anle138b showed the highest anti-prion activity both prion-infected mouse model and in vitro PMCA assay (Fig. 2.4.9 and 2.4.10).

$\begin{array}{llll}\text { PrPsc } & \text { conc. brain } \\ \text { [nmol/g] } \\ \text { sery417 }\end{array}$

Figure 2.4.9. Structure-activity relationship for selected DPP-derivatives from "Prion mouse" library.

The figure is adopted from J. Wagner, S. Ryazanov, et al. ${ }^{10}$ Copyright (c) 2013.

\# Inhibition relative to high control, $10 \mu \mathrm{M}$ compound, RML prion strain

* Relative inhibition of $\mathrm{PrP}^{\mathrm{Sc}}$ accumulation normalized to DMSO-treated group ( $0 \%$ inhibition) and $\mathrm{PrP}^{\mathrm{Sc}}$ level at start of treatment (100\% inhibition)

a $\mathrm{PrP}^{\mathrm{Sc}}$ level in brain 120 days after i.c. infection and treatment for 40 days with $1 \mathrm{mg}$ compound

$\mathrm{b} \mathrm{PrP}^{\mathrm{Sc}}$ level in spleen determined 35 days after i.p. infection followed by 34 days of treatment with $1 \mathrm{mg}$ compound

${ }^{\mathrm{c}} \mathrm{PrP}^{\mathrm{Sc}}$ level in brain at 106 days after i.c. infection and treatment for 24 days of treatment 
Systematic variation of anle $138 \mathrm{~b}$ structure revealed a well-defined structureactivity relationship (SAR). Regarding the 5phenyl (R3), bromine in meta-position led to the highest inhibitory activity, whereas the change of bromine location to para- or orthoposition in the 5-phenyl ring (R3) reduced or abolished the activity, respectively. Testing different halogen atoms in the meta-position revealed a bell-shaped correlation with the size of the substituent. Regarding the central five-membered ring (R2), pyrazole was associated with highest activity. For the substituent at the 3-phenyl (R1), SAR was more complex, as modifications in this

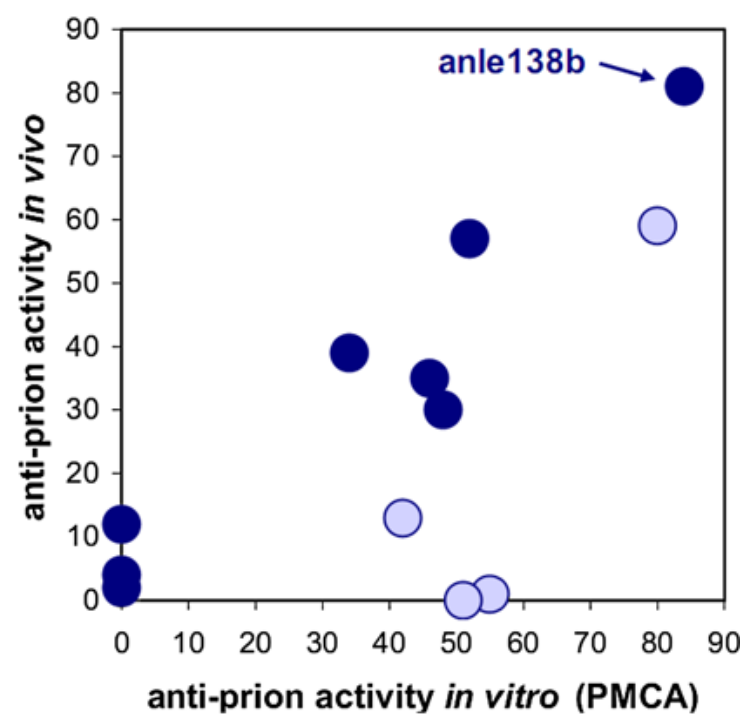

Figure 2.4.10. Correlation between anti-prion activity in vitro and in vivo.

Levels of compound in the brain:

Dark blue dots $>15 \mathrm{nmol} / \mathrm{g}$

Light blue dots $<15 \mathrm{nmol} / \mathrm{g}$ position strongly affected bioavailability in the brain. 1,3-Benzodioxole as in anle138b was associated with best bioavailability and inhibitory activity in vitro, thus, resulting in highest activity in vivo. Notably, when brain levels are considered, there is a strong correlation between anti-prion activity in vitro and in vivo (Fig. 2.4.10) indicating that the therapeutic effect in vivo is based on direct targeting of $\mathrm{PrP}^{\mathrm{Sc}}$. Of particular significance of the present study is the finding that the combination of data from in vitro prion amplification PMCA assay with pharmacokinetic properties of the lead compounds allows the prediction of in vivo efficacy with the relatively good accuracy. 


\subsubsection{Therapeutic effect of anle $138 \mathrm{~b}$ in prion mice after onset of disease}

Of note, the parts of text and the figures in this chapter were adopted with modifications from our publication J. Wagner, S. Ryazanov, et al. ${ }^{10}$

As discussed in chapter 2.4.6, SAR information for 38 compounds from "Prion mice" library was primarily derived through the effect of treatment for 40 days [80-120 days post infection (dpi)] on $\mathrm{PrP}^{\mathrm{Sc}}$ accumulation, which provides rapid and reliable biochemical quantification and thus allowed testing of a large number of compounds in vivo. To further investigate the mode of action and therapeutic potency, we chose anle138b, which had in our SAR study the highest therapeutic efficacy in vivo. To be useful for treatment in humans, a compound should also be effective when given after signs and symptoms of disease are detectable. Thus, we analyzed the effect on clinical outcome (i.e. motor performance, weight loss and survival) for treatment started at different time points after intracerebral infection (Fig. 2.4.11). Without treatment, at about 80 dpi subtle clinical signs can be observed ${ }^{416}$ and $\mathrm{PrP}^{\mathrm{Sc}}$ is detectable in the brain (Fig. 2.4.11C), at 120 dpi obvious signs of disease are present (Fig. 2.4.11B, C). Even start of treatment with anle138b after onset of disease at 120 dpi resulted in a substantially prolonged survival and preservation of body weight (Fig. 2.4.11A, B).

Analysis of mice at different time points during treatment revealed that anle138b strongly inhibited accumulation of $\mathrm{PrP}^{\mathrm{Sc}}$ (Fig. Fig. 2.4.11C, D) and neuronal cell death (Fig. 2.4.11E) even when start of treatment was in the symptomatic phase. Interestingly, the number of pyknotic nuclei indicating apoptotic neuronal cell death seemed not to depend on the absolute amount of $\mathrm{PrP}^{\mathrm{Sc}}$ but correlated with the rate of $\mathrm{PrP}^{\mathrm{Sc}}$ amplification. This is in line with published data ${ }^{417}$ and provides an explanation for the strong therapeutic effect found also in the late-treatment group. 

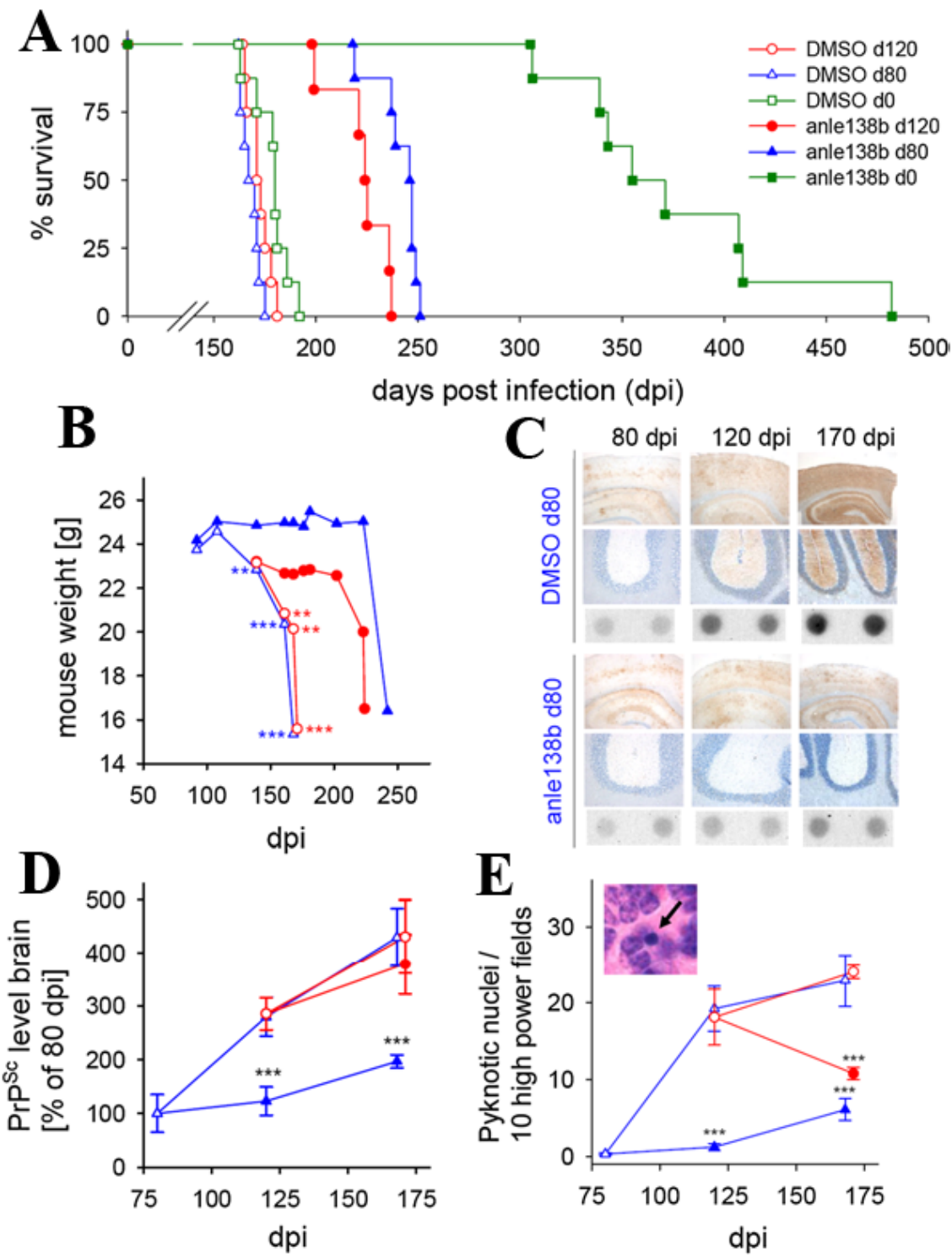

Figure 2.4.11. Influence of daily anle138b treatment on $\mathrm{PrP}^{\mathrm{Sc}}$ accumulation and prion pathology of mice infected with RML scrapie. (A) Survival curves of mice treated orally with anle138b beginning at 0,80 or 120 days post i.c. prion infection. (B) Control mice showed progressive weight loss starting after $100 \mathrm{dpi}$. Treatment with anle138b from $80 \mathrm{dpi}$ onwards prevents weight loss for $\sim 100$ days. Treatment from 120 dpi inhibits further weight loss for $\sim 70$ days. (C) Immunohistochemistry (upper row cortex/hippocampus, middle row cerebellum) and dot blot analysis (lower row) showed that anle138b treatment inhibits $\operatorname{PrP}^{\mathrm{Sc}}$ accumulation in comparison to DMSO-treated control animals. (D) Quantification of brain $\mathrm{PrP}^{\mathrm{Sc}}$ levels at different time points shows highly significant inhibition of $\mathrm{PrP}^{\mathrm{Sc}}$ accumulation in mice treated from 80 dpi onwards. $\mathrm{PrP}^{\mathrm{Sc}}$ accumulation is also reduced in animals treated from 120 dpi. (E) Histological analysis reveals a significantly reduced number of pyknotic nuclei both in mice treated from 80 and 120 dpi onwards. Inset shows an example of a pyknotic granule cell nucleus (arrow). Error bars in (D, E) indicate standard error. The figure is adopted from J. Wagner, S. Ryazanov, et al. ${ }^{10}$ Copyright (c) 2013. 
Regarding the mode of action, these findings in combination with the fact that $\operatorname{PrP}^{\mathrm{C}}$ expression is unaffected by anle $138 \mathrm{~b}^{10}$ indicate that anle138b directly blocks $\mathrm{PrP}^{\mathrm{Sc}}$ amplification and reduces neurotoxicity in vivo. Notably, we observed a significant change in size distribution of aggregates indicating a modulation of the abundance of different oligomeric species. To characterize oligomer formation in vivo, we used sucrose-gradient centrifugation, as this method, which is well established in the prion field ${ }^{418}$, has several advantages compared to other methods for analysis of size distribution of aggregates: (i) it allows direct analysis of brain homogenates, which would be difficult by gel filtration, (ii) there is no purification step, which might modify the aggregation state, required prior to analysis, and (iii) it allows to detect and quantify all potential particle sizes (i.e. monomers, oligomers, fibrils) simultaneously. Analysis of brain homogenates by sucrose-gradient centrifugation assay reveals a strong reduction of high molecular weight species and also a shift towards smaller oligomer size for low molecular weight oligomers (Fig. 2.4.12A, B). Thus, mice treated with anle138b show a strong reduction of high molecular weight species (fractions 7-12). Also small molecular weight oligomers (fractions 3-4) are reduced and show a shift towards smaller size (fraction 2) indicating that anle138b blocks aggregation at the level of small oligomers. DMSO-treated mice are indistinguishable from terminally ill untreated mice (Fig. 2.4.12A, B).
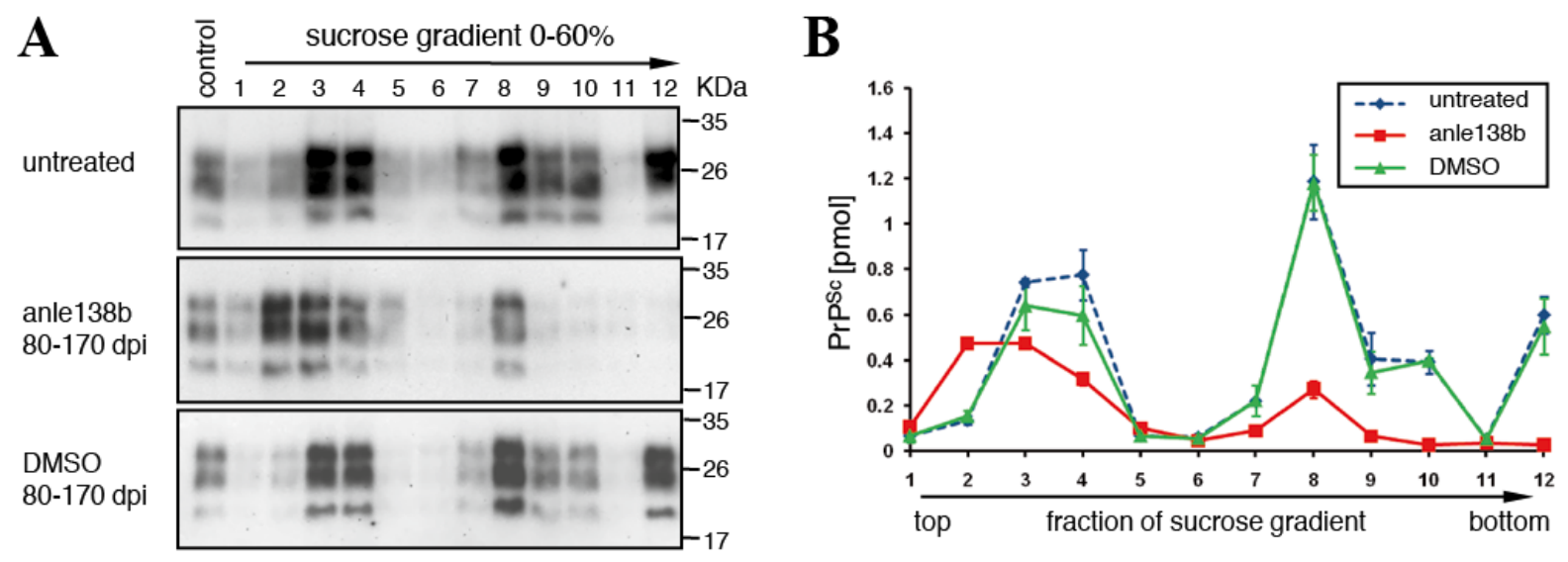

Figure 2.4.12. Size modulation of PrPSc oligomers by anle138b and inhibition of various human and non-human prion strains in PMCA. (A, B) Size distribution of $\mathrm{PrP}^{\mathrm{Sc}}$ aggregates was analyzed by sucrose-gradient centrifugation. Error bars in (B) indicate standard error. The figure is adopted from J. Wagner, S. Ryazanov, et al ${ }^{10}$ Copyright (C) 2013. 
Table 2.4.1. Comparison of in vivo activity of anle138b with other published compounds.

\begin{tabular}{|c|c|c|c|c|c|c|c|}
\hline Compound & Mouse & $\begin{array}{l}\text { Prion } \\
\text { strain }\end{array}$ & $\begin{array}{l}\text { Start of the } \\
\text { treatment, } \\
\text { application }\end{array}$ & $\begin{array}{l}\text { Dose, } \\
\mathrm{mg} / \mathrm{kg}\end{array}$ & $\begin{array}{l}\text { Control } \\
\text { Median/ } \\
\text { Mean* } \\
\text { survival, } \\
\text { days }\end{array}$ & $\begin{array}{l}\text { Therapy } \\
\text { Median/ } \\
\text { Mean* } \\
\text { survival, } \\
\text { days }\end{array}$ & $\Delta$, days \\
\hline \multirow{3}{*}{ Anle $138 b^{10}$} & \multirow{3}{*}{$\mathrm{C} 57 \mathrm{BL} / 6$} & \multirow{3}{*}{ RML, i.c. } & $0 \mathrm{dpi}$, oral & \multirow{3}{*}{250} & 180 & 355 & 175 \\
\hline & & & $80 \mathrm{dpi}$, oral & & 168 & 242 & 74 \\
\hline & & & $120 \mathrm{dpi}$, oral & & 172 & 224 & 52 \\
\hline \multirow{7}{*}{ IND24 ${ }^{419}$} & \multirow{7}{*}{ FVB } & \multirow{3}{*}{ RML, i.c. } & $1 \mathrm{dpi}$, oral & \multirow{3}{*}{210} & $118^{*}$ & $204 *$ & 86 \\
\hline & & & $74 \mathrm{dpi}$, oral & & $118^{*}$ & $192 *$ & 74 \\
\hline & & & 90 dpi, oral & & $118^{*}$ & $118^{*}$ & $\mathbf{0}$ \\
\hline & & \multirow{2}{*}{$\begin{array}{l}\text { ME7, i.c. } \\
\text { ME7, i.c. }\end{array}$} & $1 \mathrm{dpi}$, oral & \multirow{2}{*}{210} & $126^{*}$ & 214* & 88 \\
\hline & & & $60 \mathrm{dpi}$, oral & & $126^{*}$ & $158^{*}$ & 32 \\
\hline & & \multirow{2}{*}{$\begin{array}{l}\text { 22L, i.c. } \\
\text { 22L, i.c. }\end{array}$} & $1 \mathrm{dpi}$, oral & \multirow{2}{*}{210} & $111^{*}$ & $164 *$ & 53 \\
\hline & & & $60 \mathrm{dpi}$, oral & & $111^{*}$ & $126^{*}$ & 15 \\
\hline Aryl amide $1^{420}$ & FVB & RML, i.c. & $1 \mathrm{dpi}$, oral & 25 & 115 & 213 & 98 \\
\hline \multirow{2}{*}{ Aryl amide $37^{420}$} & Tg4053 & RML, i.c. & $1 \mathrm{dpi}$, oral & 200 & 51 & 119 & 68 \\
\hline & Tg1014 & sCJD, i.c. & $1 \mathrm{dpi}$, oral & 200 & 78 & 76 & -2 \\
\hline \multirow{2}{*}{ CompoundB $^{421}$} & Tga20 & RML, i.c. & $0 \mathrm{dpi}$, oral & 300 & 63 & 174 & 111 \\
\hline & ICR & RML, i.c. & 0-187 dpi, oral & 300 & 156 & 270 & 120 \\
\hline \multirow{2}{*}{${ }_{423}^{\text {Amphoterin } \mathrm{B}^{422,}}$} & \multirow[t]{2}{*}{ C57BL/6 } & \multirow{2}{*}{$\begin{array}{l}\text { C506M3, } \\
\text { i.c. }\end{array}$} & 0 dpi, i.p. & 2.5 & 171 & 223 & 52 \\
\hline & & & 80 dpi, i.p. & 2.5 & 158 & 196 & 38 \\
\hline \multirow{2}{*}{ MS- $8209^{422,423}$} & \multirow{2}{*}{$\begin{array}{l}\text { C57BL/6 } \\
\text { C57BL/6 }\end{array}$} & \multirow{2}{*}{$\begin{array}{l}\text { C506M3, } \\
\text { i.c. }\end{array}$} & 0 dpi, i.p. & 2.5 & 171 & 215 & 44 \\
\hline & & & 80 dpi, i.p. & 25 & 158 & 221 & 63 \\
\hline Curcumin $^{424}$ & $\mathrm{C} 57 \mathrm{BL} / 6$ & 139A, i.c. & $100 \mathrm{dpi}$, oral & 50 & $196^{*}$ & $208^{*}$ & 12 \\
\hline \multirow{2}{*}{$\operatorname{PcTS}^{425}$} & RML & RML, i.c. & $0-28$ dpi, ip & 25 & $174.7 *$ & $171.1 *$ & -3.6 \\
\hline & Tg7 & $263 \mathrm{~K}$, i.c. & $0-28$ dpi, ip & 25 & $46.3^{*}$ & $49.6^{*}$ & 3.3 \\
\hline $\mathrm{PPS}^{426}$ & $\operatorname{Tg} 7$ & $263 \mathrm{~K}$, i.c. & 10-38 dpi, ip & 20 & & & $\mathbf{0}$ \\
\hline Comp $59^{427}$ & CD-1 & 139A, i.p. & $0 \mathrm{dpi}$, ip & 10 & 170 & 215 & 45 \\
\hline Trimipramine ${ }^{427}$ & CD-1 & 139A, i.p. & 0 dpi, ip & 10 & 170 & 210 & 40 \\
\hline Fluphenazine ${ }^{427}$ & CD-1 & 139A, i.p. & $0 \mathrm{dpi}, \mathrm{ip}$ & 10 & 170 & 205 & 35 \\
\hline Pravastatin $^{428}$ & C57BL/6 & 139A, i.c. & $0 \mathrm{dpi}$, oral & 200 & $177^{*}$ & $194 *$ & 17 \\
\hline Simvastatin $^{429}$ & C57BL/6 & RML, i.c. & $41 \mathrm{dpi}$, oral & 20 & $148^{*}$ & $167^{*}$ & 19 \\
\hline $\mathrm{Na}_{2} \mathrm{CaCDTA}^{430}$ & $\mathrm{Balb} / \mathrm{c}$ & M1000 & 7 dpi, ip & $\begin{array}{l}200 \mathrm{ul} \\
0.2 \mathrm{M}\end{array}$ & $169^{*}$ & $189^{*}$ & 20 \\
\hline \multirow{2}{*}{ LIN5044 ${ }^{431}$} & Tga20 & RML, i.c. & 49 dpi, i.p. & 20 & 67 & 81 & 14 \\
\hline & Tg81 & $263 \mathrm{~K}$, i.c. & 20 dpi, i.p. & 20 & 69 & 78 & 9 \\
\hline Astemizole $^{432}$ & C57BL/6 & RML, i.c. & 20-50 dpi, ip & 3 & 149 & 155 & 6 \\
\hline Tacrolimus $^{433}$ & C57BL/6 & RML, i.p. & Simptoms, ip & 5 & $26^{*}$ & $35^{*}$ & 9 \\
\hline
\end{tabular}




\subsubsection{Comparison of in vivo activity of anle $138 \mathrm{~b}$ with other published compounds}

Whereas none of published compounds has shown efficacy against human CJD in clinical trials, several therapeutic studies have reported the antiprion efficacy in the mice models of prion disease. As described in the previous chapter, the oral treatment with anle $138 \mathrm{~b}$ results in the greatly extended survival of intracerebrally inoculated mice. In order to provide a broad overview of drug candidates, I tried to summarize and compare the effect of anle138b and alternative compounds in the prion mouse models (Table. 2.4.1). Upon literature analysis, I set several selection parameters, namely: 1) mice model; 2) intracerebral or intraperitoneal prion infection; 3) start of the treatment after inoculation; 4) oral or i.p. drug application. As shown in the Table 2.4.1, anle138b treatment of prion-infected mice model even in late-stage treatment experiments demonstrates one of the largest prolongations of survival that has been found for drug-like compounds tested so far. In addition to anle138b, two alternative classes of antiprion compounds 2-aminothiazoles ${ }^{419}$ and aryl amides $^{420}$, which were developed in the laboratory of S. Prusiner, appear to be particular promising leads. In these studies, screening efforts in the prion-infected cell models, subsequent SAR optimization and pharmacokinetic studies gave rise to several candidates, for instance ID24, that can significantly extend the survival of the prion-infected mice ${ }^{419}$.

\subsubsection{DPP derivatives and prion: Key findings and discussion}

Many details of our findings and results of development of DPP-related compounds as a potential therapeutic option for prion disease are discussed in length in the chapters 2.4.1-2.4.7. Herein, I would like to highlight and discuss the most important aspects of anle $138 \mathrm{~b}$ project in a context of the prion protein.

To date, there is no treatment available for the prion disease that can delay or modify the course of this fatal and devastating brain disorder ${ }^{434,435}$. The prion disease affects not just human, but also animals. In case of human prion diseases, the sporadic form of CreutzfeldtJakob disease (sCJD) accounts for majority of case $\mathrm{s}^{436}$. In addition to SCJD, two forms of prion disease, which are associated with the intake of prion-infected food and tissues, have been characterized, namely kuru disease and variant Creutzfeldt-Jakob disease (vCJD) ${ }^{436,437}$. In animal world, prion disease is found in sheep (scrapie), deer (chronic wasting disease) and cattle (bovine spongiform encephalopathy) ${ }^{437}, 438$. Neuropathological hallmark of the prion disease is spongiform pathology in the brain ${ }^{434,}{ }^{436}$. It's generally accepted that all forms of this disease share a similar pathological mechanism, in which a key pathological step is a conformational change of the cellular form of the prion protein $\operatorname{PrP}^{\mathrm{C}}$ into beta-sheet rich, $\mathrm{PK}$ - 
resistant and infectious form, termed $\mathrm{PrP}^{\mathrm{Sc}} 434,438$. The indispensable property of $\mathrm{PrP}^{\mathrm{Sc}}$ is the ability to self-propagate, which leads to indefinite and strain-specific amplification of prions $^{438}$. The development of the small molecules that can interfere with these pathological events was one of the main goals of the project and my thesis.

According to the literature, several fundamentally different and potentially advantageous approaches are being pursuit currently in the field of prion therapeutics ${ }^{435}, 439$. The first approach is based on interference with the replication of prion by small drug-like compounds or biomolecules ${ }^{439,440}$. Alternative strategy involves the reduction of the amount of the prion protein for instance by increasing the clearance and degradation of all form of the prion protein ${ }^{435}$. The third concept is a selective targeting of the toxic prion species by antibodies $^{435}$. Despite the fact that all strategies have a rational background, we consider the targeting of prion propagation as most promising. Aside from the mode of action, it very important to keep in mind that prion disease affects the brain and therefore the selected compounds should have appropriate pharmacokinetic profile, especially in regards to the blood-brain penetration, metabolism and long-term toxicity.

Collectively, our findings indicate that the class of DPP-related compounds exhibits strong antiprion properties in vitro and in vivo. At the start of the project after the initial discovery of DPP scaffold as a result of screening campaign, one of the main challenges was the design of focused library of compounds with the favorable medical chemical properties. The second important aspect in the development and optimization of the compounds was the selection of appropriate screening models and protocols. In addition to in vitro experiments, the data obtained in pharmacokinetic and efficacy studies in vivo provide the key insight into pharmacological properties of DPP compounds and allow the discovery of highly active new lead compound anle138b and the speculation regarding the plausible mode of action.

In this study we have developed an attractive strategy of screening and follow-up optimization of compounds in vitro, which ensures rational selection of compounds targeting prion toxicity for further in vivo experiments. Here, we employed the combination of 3 different in vitro and cell-based assays. First, quick and sensitive SIFT assay ${ }^{7,387}$ that is the system for screening of compounds interfering with the process of $\mathrm{PrP}^{\mathrm{Sc}}$ misfolding at the molecular level. Second, a prion-infected SMB cell-based model ${ }^{413}$, in which the accumulation of detergent-insoluble and PK-resistant $\mathrm{PrP}^{\mathrm{Sc}}$ represents an attractive marker allowing to assess and to quantify the propagation of prions and corresponding effect of small molecules. Another important element of the screening strategy is the cell-free PMCA assay $^{414}, 415$. Among the advantages of this technique are indefinite and strain-specific 
amplification of authentic prions, including human-derived strains, high sensitivity and use of the lysates. From my perspective, the use of the brain homogenates in PMCA, in contrast to SIFT and cell-based assay, is particular attractive, because samples contain soluble cell components, which might refine the biological relevance of the amplification conditions and also improve the solubility of the drug-like compounds. Altogether, the combination of several screenings may provide a reliable platform for identification of anti-prion compounds, validation of the various hits and investigation of the strain specificity.

The screenings of focused library of DPP-related compounds in prion SIFT assay confirmed the general antiprion activity of the DPP structures (Fig. 2.4.2). Furthermore, for the promising hits from the initial SIFT screening, the dose response analysis was carried out by performing SIFT assay on dilution series. Upon examination, the sustainable inhibition of $\mathrm{rPrP} / \mathrm{PrP}^{\mathrm{Sc}}$ association by DPP compounds in concentration-dependent manner was confirmed in a second round of SIFT screening. Characterization of inhibitory activity in dilution series revealed the $50 \%$ effective concentration $\left(\mathrm{EC}_{50}\right)$ values in the range of 10-20 $\mu \mathrm{M}$ for most active DPP analogs (Fig. 2.4.3). From the massif of SIFT activities, we derived the valuable SAR information that supports high antiprion activity of compounds bearing vicinal hydroxy groups (sery85, sery166a, anle138c, anle145c) and moderate activity of compounds substituted with methylenedioxy and methoxy groups. Based on results from SIFT assay, we speculate that a mechanism of inhibition of the prion misfolding by DPPrelated compounds might be driven by specific interference of small molecules with the interaction events between $\mathrm{PrP}^{\mathrm{C}}$ and $\mathrm{PrP}^{\mathrm{Sc}}$ at the molecular level.

In case of SMB cell-based model, similar to SIFT assay the majority of selected DPP compounds exhibit a pronounced antiprion activity at both tested concentrations (Figure 2.4.4 and 2.4.5). One of the most important findings of this screening was the identification of a highly potent compound, called anle138b. Structurally, this striking effect of anle138b is mainly determined by the presence of methylenedioxy moiety (Fig. 2.4.5).

The antiprion properties of anle138b, as a frontrunner, and related DPP compounds are further confirmed in PMCA assay. We found that anle138b strongly inhibits the propagation of several non-human prion strains and, what is more remarkable; the amplification of human strains SCJD and VCJD is also significantly reduced under PMCA conditions in dose-dependent manner with $\mathrm{EC}_{50}$ values around $7 \mu \mathrm{M}\left(\mathrm{EC}_{50} \mathrm{RML}=7.3 \mu \mathrm{M}\right.$, $\left.\mathrm{EC}_{50 \mathrm{vCJD}}=7.1 \mu \mathrm{M}\right)$.

Clearly, the development of DPP compounds is not the first study aiming to identify the compounds that inhibit the $\mathrm{PrP}^{\mathrm{Sc}}$ amplification. Several groups have reported the results 
of the screening campaign as well as evaluation of specific compounds ${ }^{432,439-441}$. The most interesting and advanced studies are summarized in Table 2.4.1. In efforts to identify novel inhibitors of prion misfolding, the promising hits in these studies were tested in vitro by the utilization of just one specific screening system that could be either the prion-infected cell models $^{419,420}$ or PMCA assay ${ }^{442}$ or FRET-based methods ${ }^{432}$ and also in silico screening ${ }^{431}$. Based on our research findings, the combination of the several assays might represent an efficient drug-discovery strategy, which is schematically shown in Figure 2.4.13. In case of anle138b development, this approach has several clear advantages, which are: (i) reduced the number of false negative hits, since in vitro assays are done at various conditions; (ii) rational selection of most promising candidates based on data from at least two in vitro assay; (iii) good correlation of combined PMCA/PK data with in vivo efficacy; (iv) PMCA validation of promising hit on human strains of prion disease.

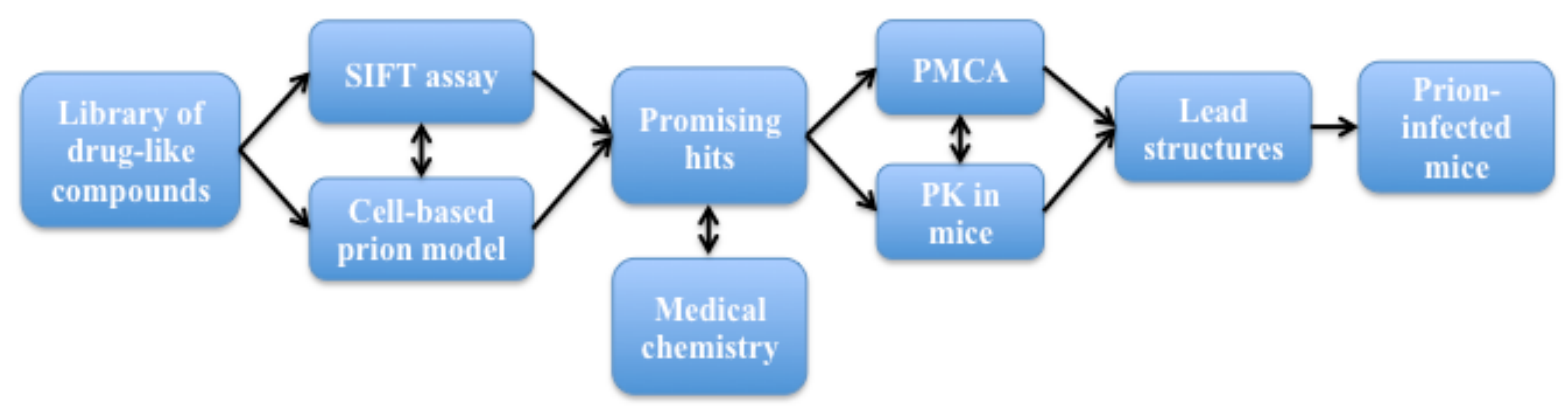

Figure 2.4.13. Drug-discovery strategy against prion disease.

The evaluation of anle138b and related compounds in the prion-infected mice in combination of pharmacokinetic experiments confirmed the therapeutic value of this class of compounds. Thus, the majority of tested DPP derivatives in prion-infected mice can reduce the accumulation of $\mathrm{PrP}^{\mathrm{Sc}}$ either in the brain or in the spleen (Fig. 2.4.9 and 2.4.11, Fig. 8.1 appendix). Moreover, several candidates, including anle138b, can significantly extend the survival. Since anle138b was recognized as a most potent compound, the comprehensive in vivo evaluation was performed under various conditions (Fig. 2.4.11, 2.4.12, Table 2.4.1). Collectively, our studies have demonstrated that anle138b treatment of RML-infected mice leads to remarkable amelioration of pathological signs. Thus, the antiprion efficacy of anle $138 \mathrm{~b}$ is confirmed by prolongation of survival even in case of the delayed treatment on day 120 post infection, reduction of $\mathrm{PrP}^{\mathrm{Sc}}$ levels in spleen and brain, improved motor performance and body weight development. Regarding the mode of action of anle138b, several lines of evidences from in vitro and in vivo studies support the mechanism associated 
with direct modulation of aggregation at the oligomer level. In particular, the analysis of brain homogenates by sucrose-gradient centrifugation assay revealed a strong reduction of high molecular weight species and also a shift towards smaller oligomer size for low molecular weight oligomers (Fig. 2.4.12A, B) as a result of anle138b treatment. In the SIFT assay anle138b and other DDP compounds interfere with $\mathrm{PrP}^{\mathrm{C}} / \mathrm{PrP}^{\mathrm{Sc}}$ interactions. Importantly, anle138b does not alter the level of normal $\mathrm{PrP}^{\mathrm{C}}$ in mice. On this basis, we propose that the therapeutic effect of anle138b in vivo is associated with direct targeting of pathological aggregation at the level of oligomer.

Recently, anle138b has been tested in transgenic prion mouse models by two independent groups ${ }^{419,443}$. These studies have demonstrated that anle138b did not extend the survival of $\operatorname{Tg}(\mathrm{PrP}-\mathrm{A} 116 \mathrm{~V})$ mice and $\mathrm{Tg} 1014$ mice infected with sCJD prions. $\mathrm{Tg}(\mathrm{PrP}-$ A116V) mice is the animal model of so called Gerstmann-Sträussler-Scheinker syndrome, an extremely rare, fatal neurodegenerative disease belonging to genetic form of prion disease. Gerstmann-Sträussler-Scheinker syndrome is caused by the point mutations in PRPN gene encoding the prion protein. The oral treatment of $\operatorname{Tg}(\mathrm{PrP}-\mathrm{A} 116 \mathrm{~V})$ mice with the anle138bcontaining food pellets leads to significant reduction of $\mathrm{PrP}^{\mathrm{Sc}}$ pathology in the brain, however the onset of symptoms and the survival were the same in treated and in placebo groups ${ }^{443}$. The lack of clinical benefits can be explained by following considerations. In the $\operatorname{Tg}(\mathrm{PrP}-$ A116V) mouse model, prion protein with the A116V mutation was used ${ }^{443}$. This is a very special mutation that results in the formation in an aberrant topological form of PrP termed Ctm-PrP, which has a transmembrane topology ${ }^{444}$. This form is different from $\mathrm{PrP}^{\mathrm{Sc}}$ and has little connection with prion-like mechanisms but causes pathology by a completely different pathway. So, it is likely that a compound that affects pathological aggregation has no effect on the pathology caused via Ctm-PrP resulting from the A116V mutation. Efficacy of anle138b was also tested in Tg1014 mice inoculated with SCJD brain homogenate. The transgenic Tg1014 mouse line is characterized by expression of chimeric mouse/human $\operatorname{PrP}$ transgenes at four times higher level than endogenous prion protein in wild type mice ${ }^{419}$. Due to the expression of the chimeric transgene, these mice are susceptible to sCJD prions. The oral administration of anle $138 \mathrm{~b}$ in the liquid diet to $\operatorname{Tg} 1014$ mice failed to extend the survival $^{419}$. There are several potential explanations of anle138b inactivity in Tg1014 model. First, anle138b is not as effective for the specific human prion strain used in this study, therefore it might be interesting to perform PMCA assay with this SCJD prion strain and anle138b. Secondly, the transgenic models with significant overexpression of prion protein are not ideally suited for testing therapeutics such as anle138b because: i) overexpression of 
$\operatorname{PrP}$ makes neurons more susceptible to the toxicity of $\mathrm{PrP}^{\mathrm{Sc}}$ so that much lower levels of $\mathrm{PrP}^{\mathrm{Sc}}$ already cause neurodegeneration, and ii) inoculation of $\mathrm{PrP}^{\mathrm{Sc}}$ to overexpressing mice leads to aggressive phenotypes lacking a nice dose-dependent incubation time (Prof. Armin Giese, personal communication). Finally, the systemic anle138b exposure might be not optimal in case of the liquid food formulation used in this study.

Viewed together, the efficacy and preclinical data that were collected for the oligomer modulator anle138b strongly supports the selection of this molecule as a candidate for clinical trials in CJD. 


\subsection{Modulation of $\alpha$-synuclein misfolding in vitro by DPP compounds}

\subsection{1. $\alpha$-Synuclein in molecular SIFT assay}

The majority of the compounds from the focused DPP library was tested in molecular SIFT assay ${ }^{445}$, in which the inhibition activity against $\alpha$-synuclein oligomerization for each molecule could be determined in rapid and well-standardized manner. In addition to the description of the molecular SIFT assay in the chapter 2.2.1, I would like to provide additional information regarding the experimental setup and potential readout. Thus, all experiments were conducted with a mixture of Alexa-488- and Alexa-647-labeled $\alpha$-synuclein at final protein concentration around $20 \mathrm{nM}$. Due to the extremely low concentration of $\alpha-$ synuclein, at which almost no oligomers are observed during long incubation, the aggregation of $\alpha$-synuclein was triggered by addition of $1 \% \mathrm{DMSO}$ and/or $10 \mu \mathrm{M} \mathrm{FeCl}_{3}$, as described previously ${ }^{194}$. Of note, the effects of various organic solvents and metal ions on $\alpha$-synuclein aggregation have been extensively evaluated with the SIFT assay previously and, moreover, the generated oligomeric species have been explicitly characterized by biophysical and
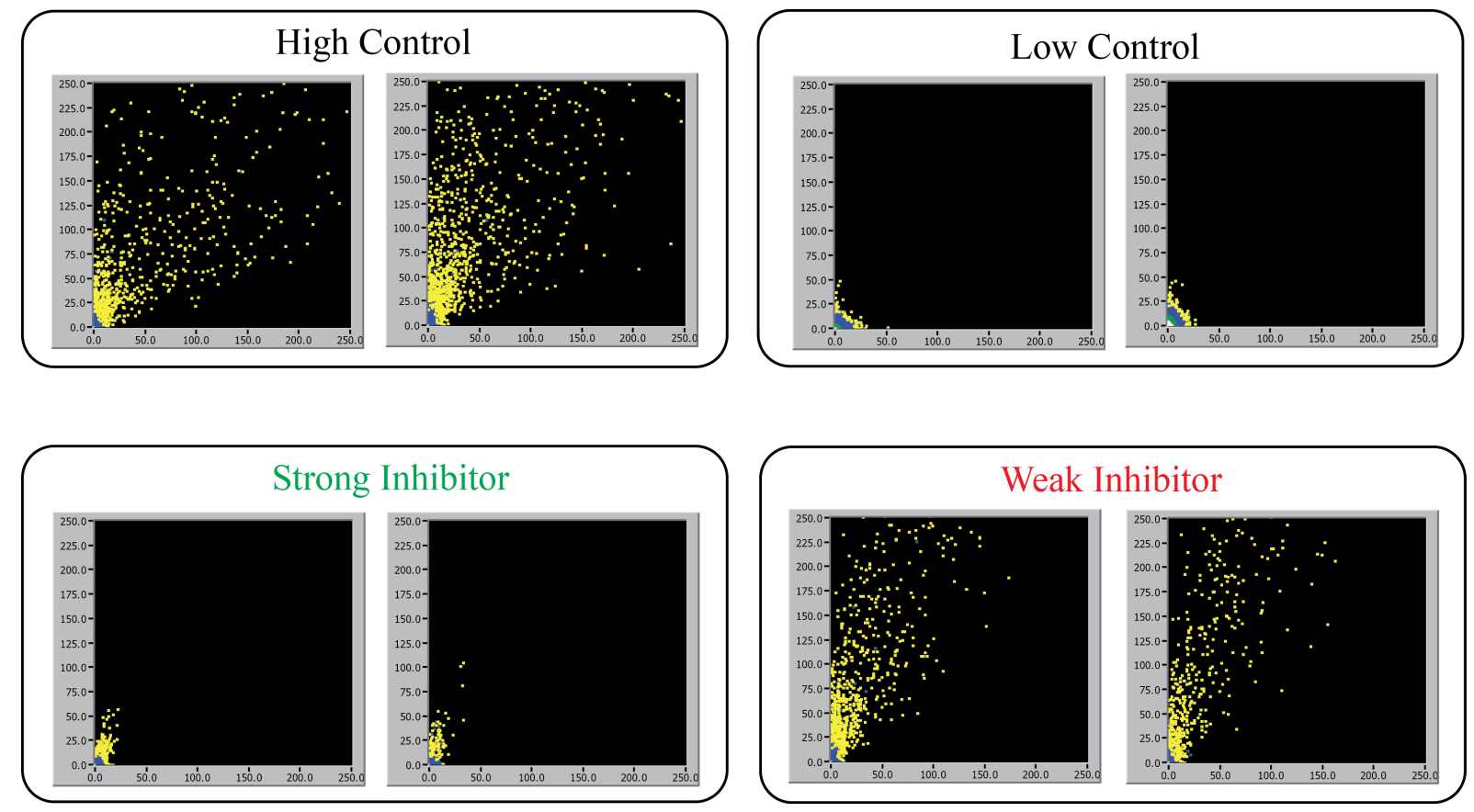

Figure 2.5.1. Examples of two-dimensional intensity distribution histograms recorded in molecular SIFT assay. Upper row: typical SIFT histograms for high (left) and low (right) controls. In case of high control, the formation of highly labeled multimeric species is evident. Lower row: typical SIFT histograms obtained for active inhibitor of aggregation (left) and weak inhibitor of aggregation (right). Vertical axis intensity of red fluorescence (photons/bins); horizontal axis - intensity of green fluorescence (photons/bins). 
electrophysiological methods ${ }^{194}$. Evaluation of SIFT data in two-dimensional intensity distribution histograms was performed by summing up the numbers of photons from high intensity bins using a two-dimensional SIFT software module (Evotec-Technologies). For threshold setting, non-aggregated reference samples were used (low control). The photon count in bins above the threshold was considered as aggregate signal. Typical twodimensional intensity histograms obtained from SIFT-based $\alpha$-synuclein aggregation assay are presented on Figure 2.5.1. Qualitatively, the formation of the large aggregated $\alpha$-synuclein species is evident for a "high control" sample, as suggested by the presence of many yellow spots corresponding to highly labeled multimeric particles, and, in contrast, the aggregation is minimal in the "low control" sample (Fig. 2.5.1). Accordingly, the SIFT assay can determine the antiaggregation activity of selected compounds (Fig. 2.5.1).

Given that newly synthesized DPP compounds were generated in small libraries and immediately tested in the SIFT assay, the collection of SIFT data consists of several blocks and final "big" screening of selected molecules. Therefore, the experimental conditions might be slightly different from one data set to another due to the continuous optimization of the SIFT assay in the group of Prof. Armin Giese. Thus, the initial SIFT measurements were performed following DMSO-induced or $\mathrm{DMSO} / \mathrm{FeCl}_{3}$-induced $\alpha$-synuclein aggregation that, in turn, leads to formation of specific types of oligomeric species ${ }^{194}$. For later studies, the SIFT analysis has been done under simultaneous induction of $\alpha$-synuclein by addition of $1 \%$ DMSO and $10 \mu \mathrm{M} \mathrm{FeCl}_{3}$. Overall, the antiaggregation activity of our DPP compounds showed good correlation and reproducibility regardless of the experimental conditions.

\subsubsection{Inhibition of $\alpha$-synuclein aggregation by DPP compounds in SIFT assay}

In our SIFT screening, the majority of DPP compounds from the focused library have been analyzed for their ability to interfere with the aggregation of $\alpha$-synuclein in the lab of Prof. A. Giese. In this study, I performed several SIFT measurements, travelling to Munich, the selection of compounds, data evaluation and extraction of SAR information.

As mentioned above, the collection of SIFT data consists of several blocks and the final "big" screening of selected DPP molecules. The candidates for the "big" screening SIFT that covers approximately 120 DPP-related compounds have been selected based on data set generated in several independent preliminary measurements with consecutive libraries of 2050 compounds. In general, all SIFT studies were performed at two inhibitor concentrations (1 and $10 \mu \mathrm{M}$ ) and, additionally, for several compounds the dose response curves were obtained. Overall, the accumulated SIFT data strongly support a potent inhibitory activity of DPP 
compounds on $\alpha$-synuclein aggregation in dose-dependent manner. Moreover, our results also suggest that a number of DPP compounds, including sery 85 , anle138c, sery166a, anle145c, sery166b and many others, could almost completely block the formation of aggregated species in comparison with high controls (Fig. 2.5.2 and Table 2.5.1). Of note, the inhibitory potency of DPP compounds was wide-ranging, an example of poor antiaggregation activity, sery330, is presented on the Figure 2.5.2. The dose response analysis carried out for several active molecules revealed $\mathrm{IC}_{50}$ values in nanomolar range; results of corresponding SIFT evaluation of sery85 are shown in figure $2.5 .2 \mathrm{C}$.

$\mathbf{A}$

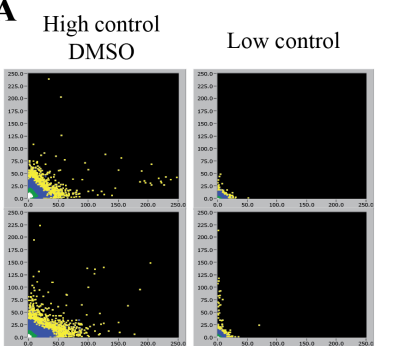

anle138c

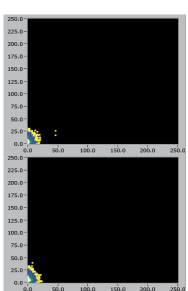

anle138c

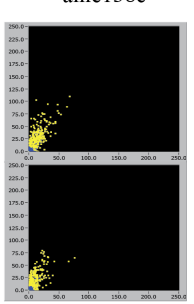

sery $166 \mathrm{a}$

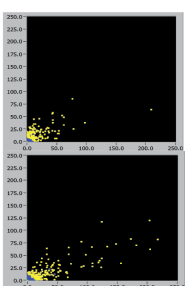

anle145c
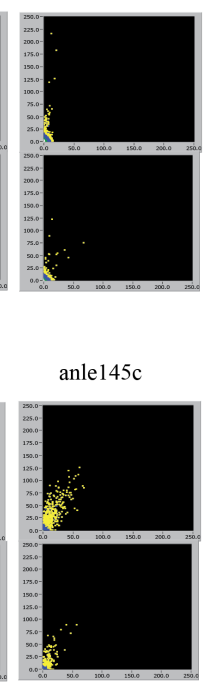

sery $166 b$
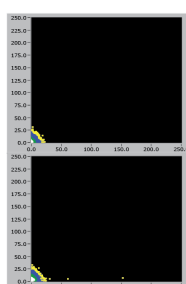

sery $166 \mathrm{~b}$

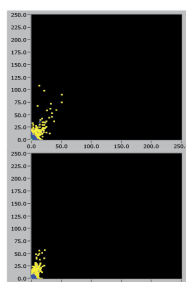

sery 85
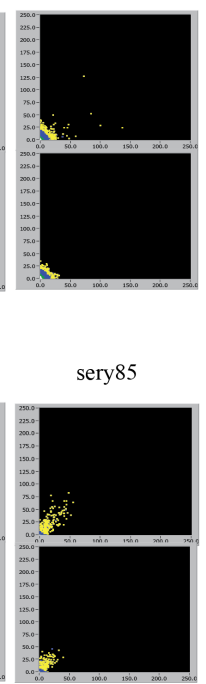

sery 330

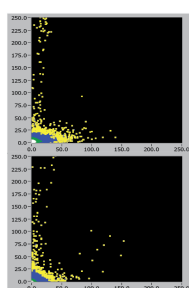

sery 330

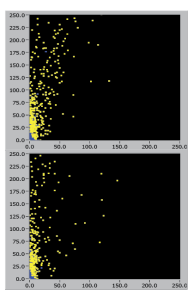

high control

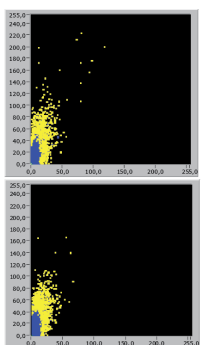

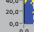

C
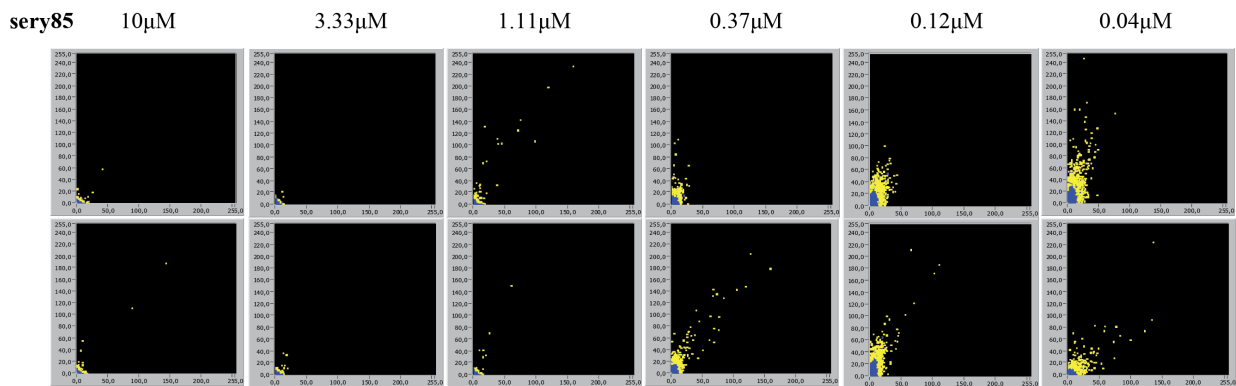

Figure 2.5.2. Inhibition of $\alpha$-synuclein aggregation in SIFT assay. (A) Representative SIFT histograms showing control and inhibitory effect of selected compounds on DMSOinduced $\alpha$-synuclein aggregation. (B) Similar SIFT histograms for $\mathrm{DMSO} / \mathrm{Fe}^{3+}$-induced aggregation. (C) Concentration dependent inhibition of DMSO-induced $\alpha$-synuclein aggregation by sery 85 . 
Analysis of collected SIFT data on inhibition $\alpha$-synuclein aggregation for more than 120 diverse DPP-related compounds (Table 2.5.1) allows us to establish important aspects of structure-activity relationship:

1) The crucial structural requirement for DPPs to be a potent aggregation inhibitor in vitro is a presence of vicinal dihydroxyphenyl moiety.

2) The activity shows correlation with a number of hydroxyl groups and increases as follows: hydroxy $<<$ dihydroxy $<$ trihydroxy $\leq$ two dihydroxy $<$ dihydroxy+trihydroxy.

3) Regarding the central five-membered ring (R2), pyrazole is associated with highest activity, although the correlation is relatively weak.

4) Substitution in ortho-positions of phenyl rings in majority cases reduces or abolishes the activity.

5) Introduction of the substituents, such as halogens, $\mathrm{NH}_{2}, \mathrm{OH}, \mathrm{OMe}, \mathrm{CF}_{3}$, in metaposition of one of the phenyl rings tends to increase the antiaggregation activity in comparison with unsubstituted compound.

6) High antiaggregation activity is also associated with amino-groups in para-position to pyrazole central ring.

To conclude, we have tested more than 120 DPP and related compounds in regard of interfering with $\alpha$-synuclein aggregation in molecular SIFT assay. Based on this screening campaign, we were able to identify a set of highly potent inhibitors and, moreover, we performed the detailed analysis of structure-activity relationships within our focused library. A direct comparison of $\alpha$-synuclein and prion protein antiaggregation activity for DPP compounds clearly suggests a strong correlation in context of SAR findings. It is noteworthy that the molecular SIFT assay has emerged as a valuable screening platform for studying $\alpha$ synuclein oligomerization itself as well as for the fast and efficient identification and subsequent optimization of aggregation inhibitors. 


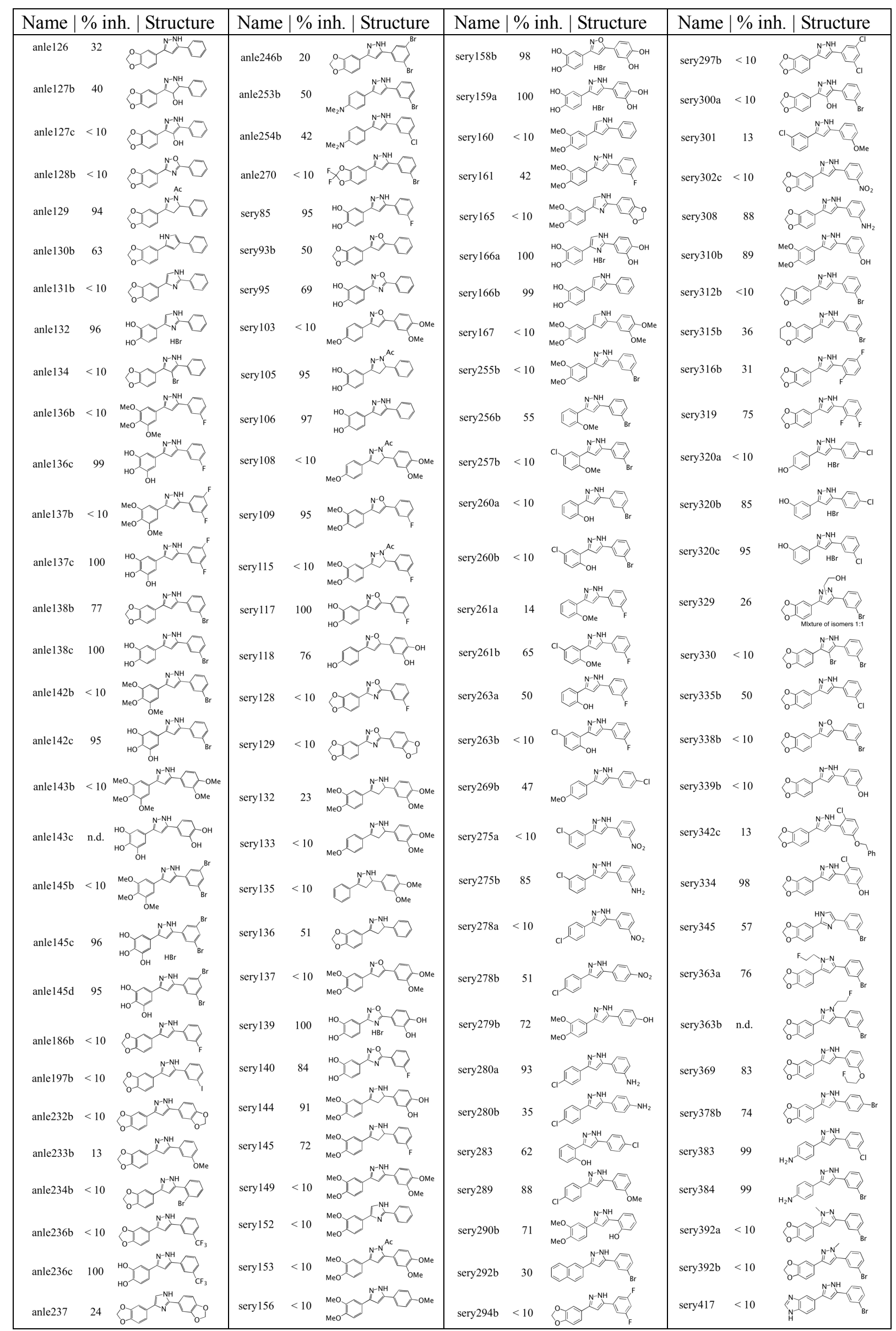


FTable 2.5.1. Activity of DPP compounds in SIFT assay. The table summarizes the effect of DPP compounds from focused library on $\mathrm{DMSO} / \mathrm{Fe}^{3+}$-induced $\alpha$-synuclein aggregation. \% inh. states for percentage of inhibition relative to high control. All compounds were tested at $10 \mu \mathrm{M}$ concentrations.

\subsubsection{Inhibition of $\alpha$-synuclein aggregation by DPP compounds in ThioT assay}

On the basis of the molecular SIFT assay, several DPP compounds, which were classified as a potent inhibitors of $\alpha$-synuclein oligomerization in the screening campaign, have been selected and tested for their ability to interfere with the amyloid fibril formation in well-established thioflavin $\mathrm{T}$ (ThioT) fluorescence assay ${ }^{446}$. Thio $\mathrm{T}$ is a fluorescent dye that specifically recognizes the beta-sheet rich amyloid structures including $\alpha$-synuclein fibrils. Upon fibril binding, ThioT displays a significantly enhanced fluorescence in combination with a characteristic red shift of spectrum and, thereby, allows sensitive and, what is more important, quantitative monitoring of the amyloid formation ${ }^{446}$.

In agreement with the nucleated polymerization mechanism, the aggregation of monomeric $\alpha$-synuclein, being monitored in ThioT assay at physiological $\mathrm{pH}, 37^{\circ} \mathrm{C}$ and mild stirring, is described by a characteristic sigmoidal shaped curve (Fig 1.15A and 2.5.4) comprising 3 stages, lag phase, exponential growth phase and plateau phase. In complementary ThioT assay, we tested 5 selected compounds (anle138c, anle138b, anle143c, anle145c and sery166b) in stoichiometric concentrations to protein and, additionally, the compound sery166a was evaluated with regard to dose-dependency. For compound selection, we focused on the following parameters: high activity in the SIFT assay, good solubility in aqueous solutions and structural diversity, namely, the variation of a number of hydroxyl groups, central heterocycle and configuration of other substitution. In line with the SIFT data, DPP compounds, including anle138c, anle143c, anle145c, sery166a and sery166b (Fig. 2.5.3), showed almost complete inhibition of $\alpha$-synuclein amyloid fibril formation as

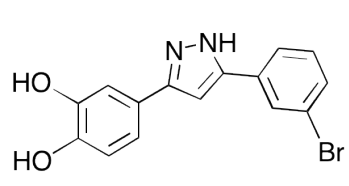

anle138c
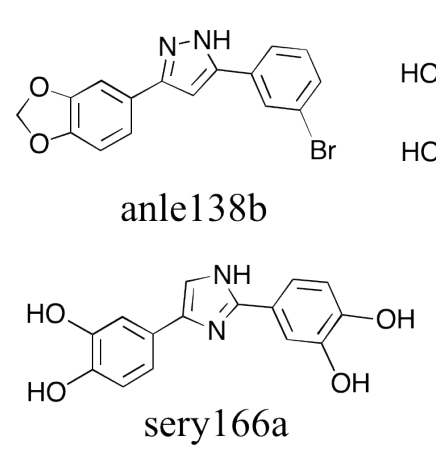

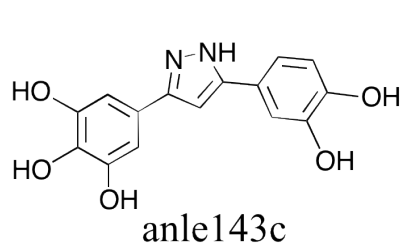

anle $143 \mathrm{c}$

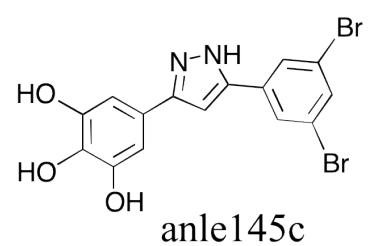

anle $145 \mathrm{c}$<smiles>Oc1ccc(-c2c[nH]c(-c3ccccc3)c2)cc1[Mg]</smiles>

Figure 2.5.3. Chemical structures of DPP compounds tested in ThioT assay. 
measured by ThioT assay (Fig. 2.5.4). The lack of fibrils was confirmed by TEM. Moreover, analysis of $\alpha$-synuclein aggregation in presence of sery166a revealed that the observed effect is concentration-dependent. Remarkably, the experiments with variable sery166a concentrations established at the same time that this compound had significant impact on both the amount of fibrils and the lag time of fibrillization (Fig. 2.5.4). We also determined a moderate inhibitory effect of anle138b in this assay (Fig. 2.5.5). However, the characterization of such extremely low soluble compounds, like anle138b, in aqueous samples posits numerous fundamental and technical challenges. Among these limitations: low compound solubility, as consequence a poor control of soluble fraction; compound precipitation that eventually could seed the protein aggregation. In addition, attempts to decrease $\alpha$-synuclein concentrations keeping reasonable protein/compound ratio lead to significantly prolonged lag times (up to several weeks), reduced sensitivity and poor reproducibility. Thereby, the generated data should be interpreted with caution.

Collectively, we demonstrated that DPP compounds showing potent inhibitory activity on $\alpha$-synuclein aggregation in SIFT assay are also capable to prevent $\alpha$-synuclein fibrillization in vitro in dose-dependent manner. The fact that we observed the good correlation between two aggregation assays confirms the efficiency and relevance of SIFT-based screening platform.

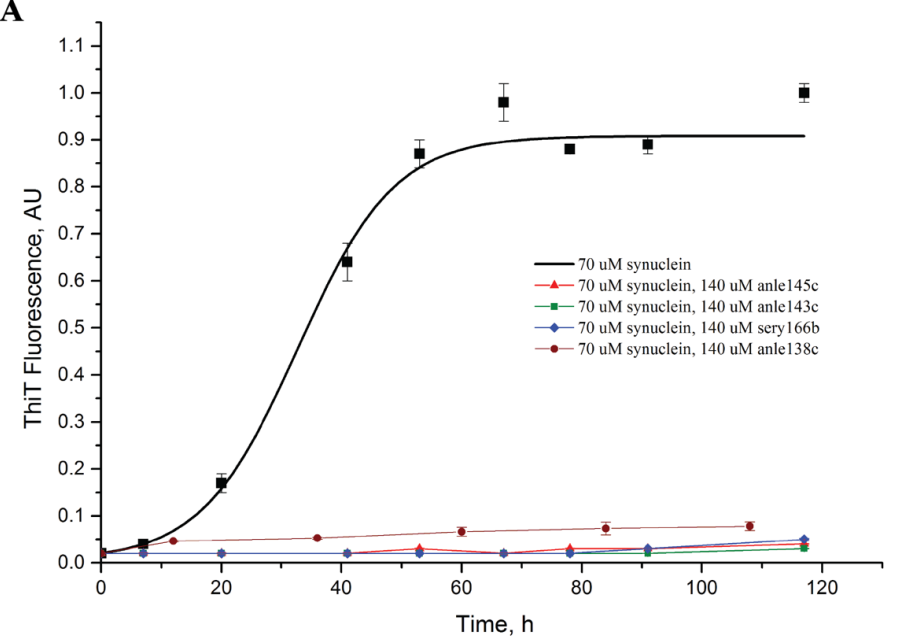

B

Figure 2.5.4. DPP compounds inhibit $\alpha$-synuclein fibrillization in ThioT assay.

(A) Kinetic of $\alpha$-synuclein fibrillization in presence of DPP compounds, including anle138c, anle143c, anle145c, sery166b. (B) Kinetic of $\alpha$-synuclein fibrillization in presence of sery166a at various concentration. Error bars in $(\mathbf{A}, \mathbf{B})$ indicate standard error.

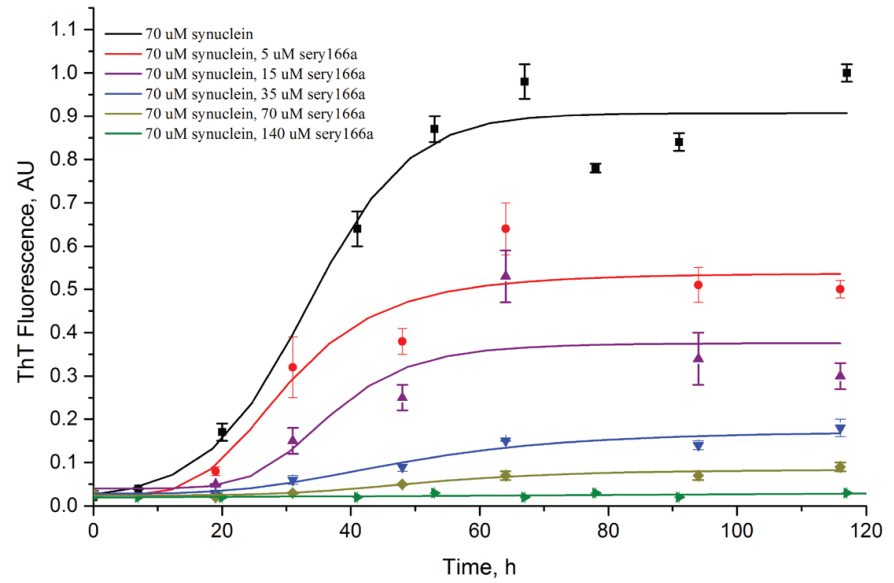




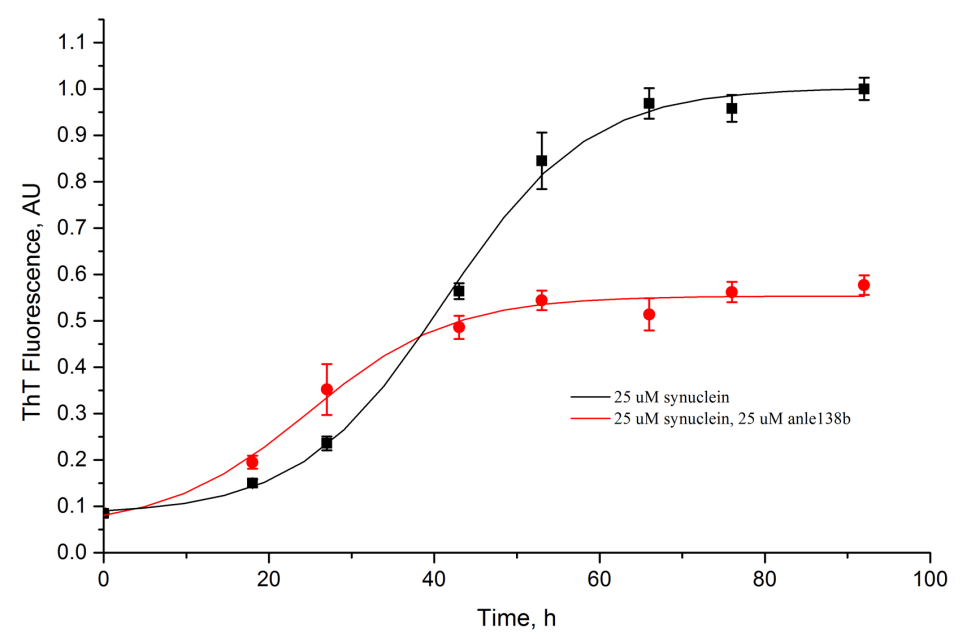

Figure 2.5.5. anle138b moderately inhibits $\alpha$-synuclein fibrillization in ThioT assay. Error bars indicate standard error.

\subsubsection{Examination of $\alpha$-synuclein aggregation in presence of DPPs by TEM and AFM}

To characterize the effect of DPP compounds on $\alpha$-synuclein aggregation and to determine the morphological changes occurring with the samples during the long-term incubation, we used atomic force microscopy (AFM) and transmission electron microscopy (TEM). I performed AFM recordings with assistance of Dr. Avishay Pelah, TEM images were taken by Dr. Dietmar Riedel.

In line with the results from ThioT assay, EM and AFM images also supported the ability of DPP compounds to prevent the formation of amyloid fibrils. Thus, TEM micrographs of $\alpha$-synuclein samples incubated without test compound showed the assembly of monomeric $\alpha$-synuclein into the long unbranched mature fibrils starting from 28-40 h at 37 ${ }^{\circ} \mathrm{C}$ with stirring. The fibrils are normally up to $1-2 \mu \mathrm{m}$ long and distributed relatively uniformly on a surface of the films (Fig. 2.5.6C). In contrast to these control images, the evaluation of the $\alpha$-synuclein samples co-incubated with sery166a or anle138c revealed almost complete inhibition of $\alpha$-synuclein fibrillization as demonstrated by absence of typical fibrillar structures (Fig. 2.5.6A and B). Remarkably, the fibril formation is arrested by addition of DPP compounds for almost one week. Concurrently, TEM micrographs of the incubated samples containing inhibitors showed the formation of complex mixtures containing multi-size globular oligomeric species and dense amorphous structures (Fig. 2.5.6A and $\mathrm{B})$. 
A

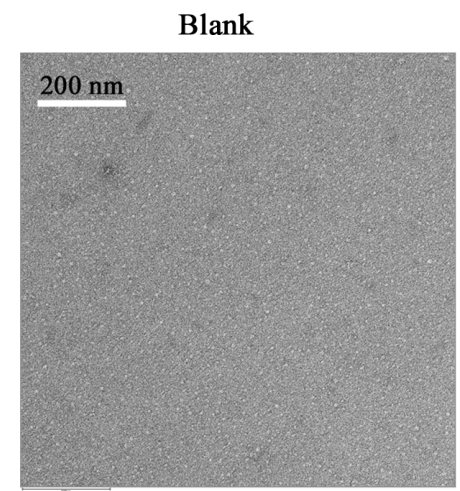

$60 \mathrm{~h}$

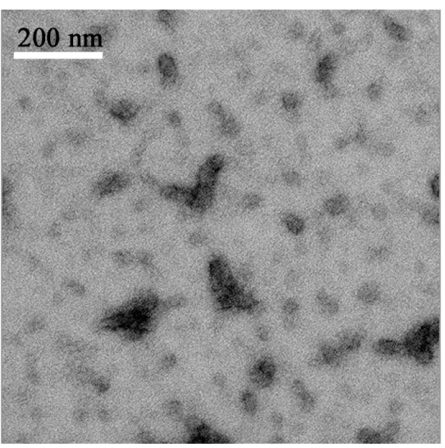

B

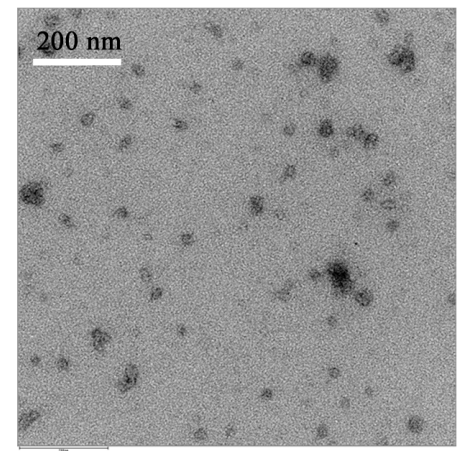

C

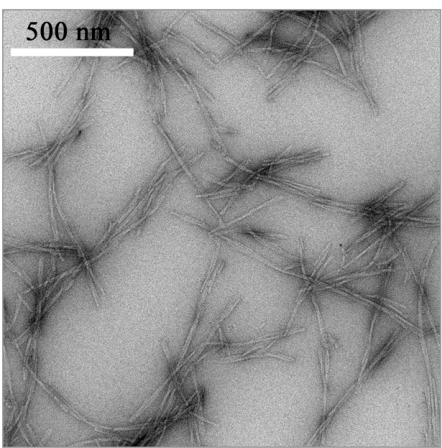

$24 \mathrm{~h}$

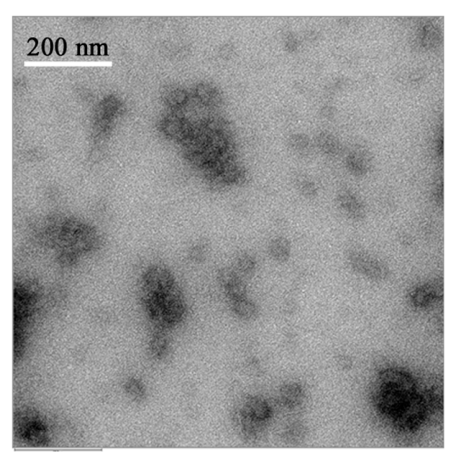

$114 \mathrm{~h}$
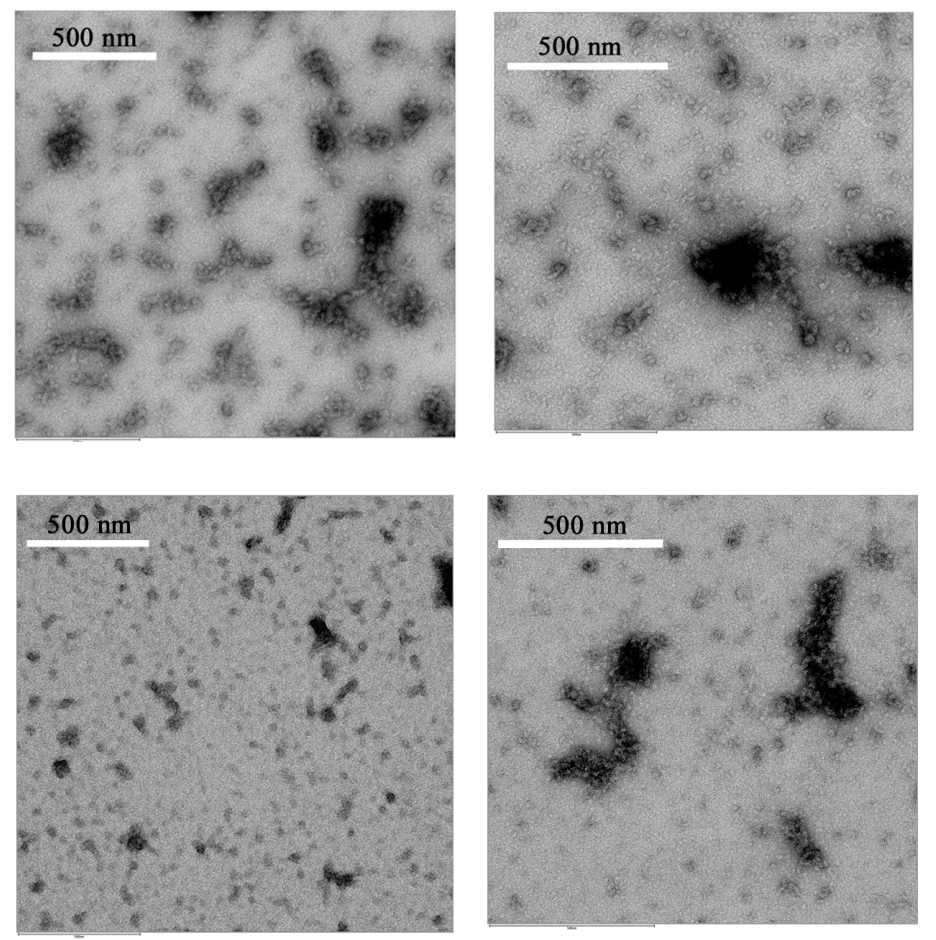

$41 \mathrm{~h}$

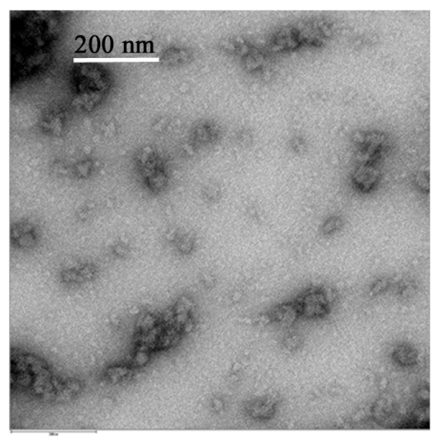

$150 \mathrm{~h}$
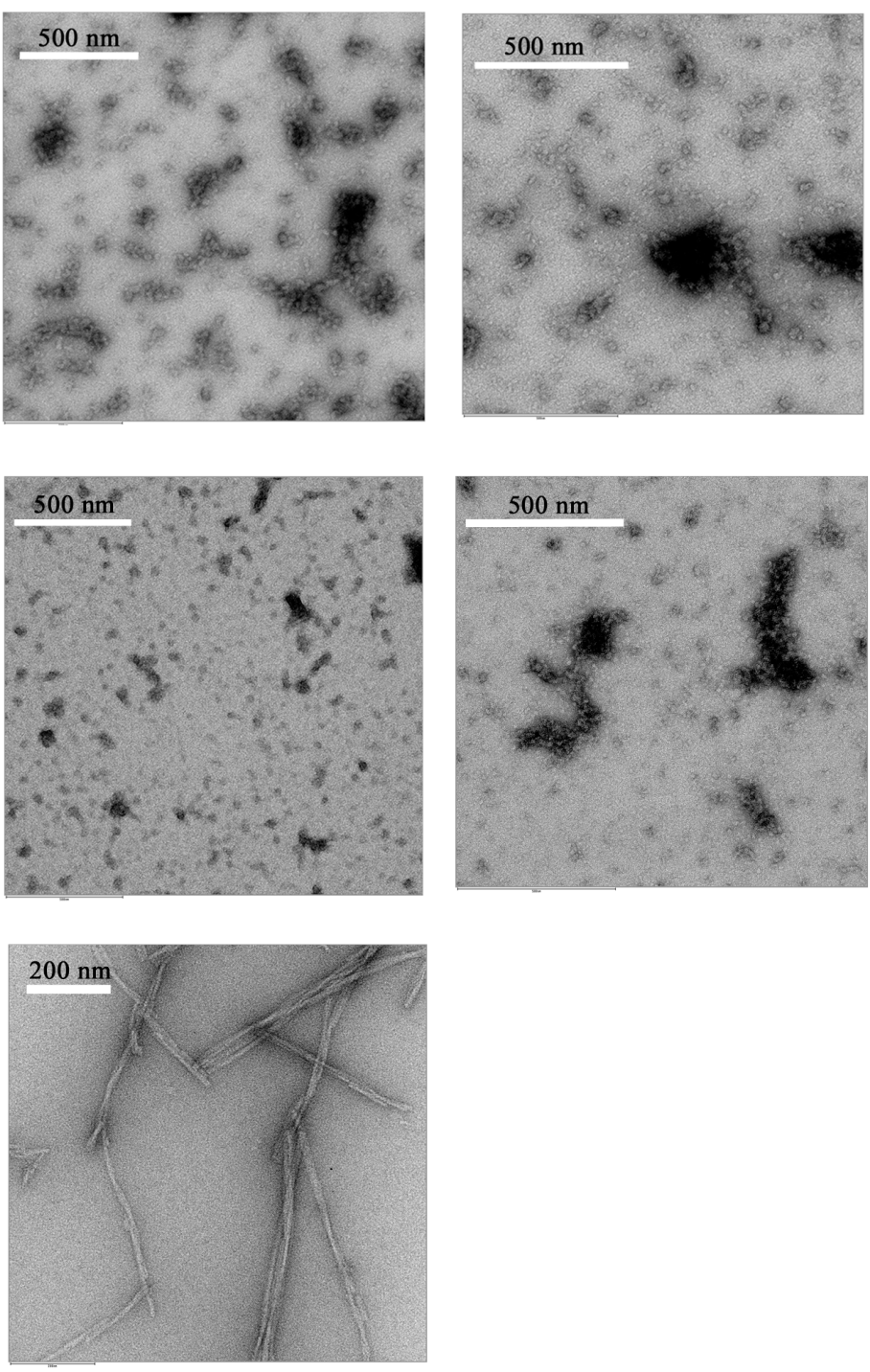

Figure 2.5.6. Representative TEM images. (A) TEM images of $\alpha$-synuclein samples incubated in presence of sery166a at various time points. (B) TEM images of $\alpha$ synuclein samples incubated in presence of anle138c for 4-5 days. (C) TEM images of amyloid fibril formed in control samples after 5 days of incubation. 
In general, the population of these globular oligomers is heterogeneous with diameters in the range of 25-55 nm. The dense amorphous structures (big black structures characterized by undefined shape and dense central core) are presumably composed of numerous oligomeric subunits associated with each other.

We also tried to characterize the time course by collecting TEM images at different time points during incubation with sery166a. Although the quantitative analysis of TEM data is problematic, it is evident that the amount of various $\alpha$-synuclein species is constantly increasing during the aggregation experiment. Additionally, at early time points, mainly the single globular oligomeric species are observed, while for a later time points, the appearance of dense amorphous structures is more frequent (Fig. 2.5.6A).

Further evidence that DPP compounds prevent $\alpha$-synuclein fibrillization and induce the formation of nonfibrillar oligomeric species was obtained from AFM imaging. To that end, we examined the $\alpha$-synuclein co-incubated for 5 days with anle138c at $37{ }^{\circ} \mathrm{C}$ with stirring. Consistent with the TEM data, AFM images also showed the presence of spherical oligomeric species with size varying in ranges of 2-10 $\mathrm{nm}$ (in height) and 20-80 $\mathrm{nm}$ (in width), while no fibrils were detected (Fig. 2.5.7).

Collectively, the AFM and TEM data confirms the inhibition of $\alpha$-synuclein fibril formation by selected DPP compounds. Importantly, co-incubation of $\alpha$-synuclein with DPP compounds produces the small globular heterogeneous oligomeric species with size in the

\section{A}
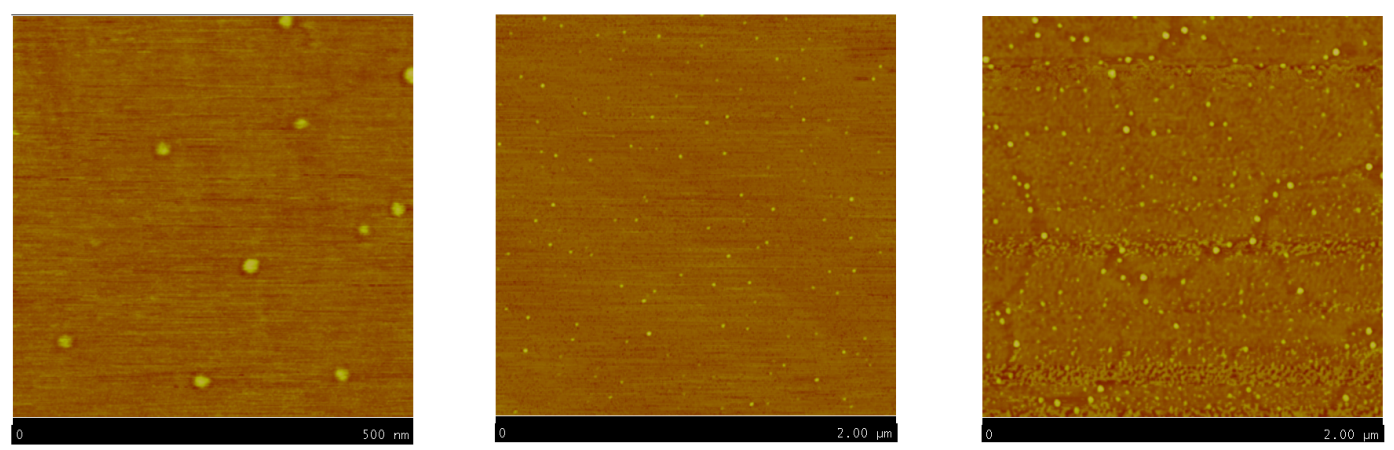

B
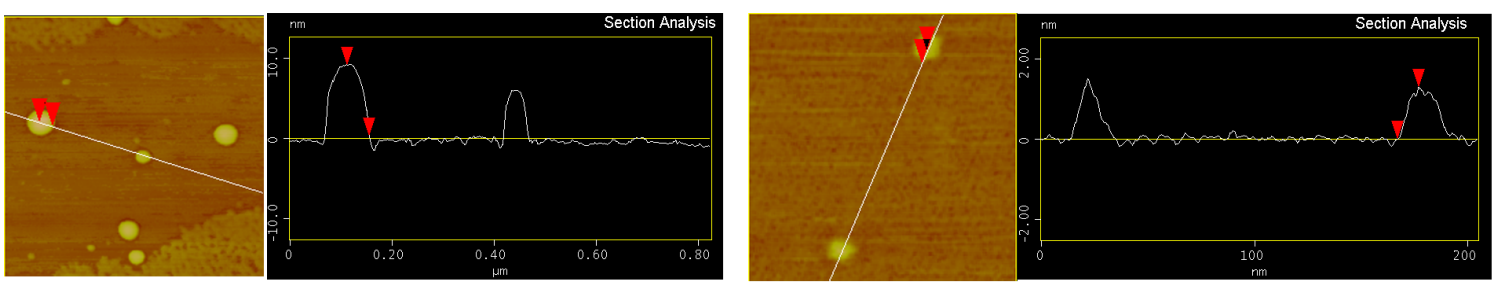

Figure 2.5.7. AFM images. (A) AFM images of $\alpha$-synuclein samples incubated in presence of anle138c for $72 \mathrm{~h}$. (B) Analysis of corresponding AFM images. 
range of 20-80 $\mathrm{nm}$ in width. Over the long incubation time, the increasing population of such oligomers has a propensity to associate with each other and built up the relatively big amorphous structures. TEM and AFM studies have uncovered that DPP compounds do not stabilize $\alpha$-synuclein solely in monomeric state, but also induce the formation of relatively heterogeneous oligomeric structures.

\subsubsection{Investigation of $\alpha$-synuclein aggregation in presence of DPPs by DLS}

Whereas the microscopical investigation provided evidence that DPP compounds prevent fibril formation, but at the same time promote the formation of oligomeric species, we performed the analysis of the correspoding samples by dynamic light scattering to determine the size of the generated structures in solution. DLS is a noninvasive spectroscopy technique that allows to estimate the hydrodynamic radius of particles in the solution based on determination of translational diffusion coefficient and corresponding calculations using the Stokes-Einstein equation ${ }^{447}$. Importantly, the hydrodynamic radius is not describing the size of the particle itself, but rather the size of the object including the surrounding solvent or ions and potentially further molecules that altogether, as complex, have the same translational diffusion coefficient. Importantly, the intensity size distribution plots, as one of the ways to represent and evaluate the scattering data, provide information regarding the relative intensity of light scattered by the corresponding particles and, since big particle scatter much more light, the intensity of the signal is proportional to $\mathrm{d}^{6}$. For instance, $10 \mathrm{~nm}$ particles will generate 1000000 smaller signal than $100 \mathrm{~nm}$ particles on intensity size distribution plots.

To better characterize the time course of the oligomer formation and possible transformations, we measured and analyzed the DLS data for $\alpha$-synuclein samples coincubated with selected soluble DPP compounds (Fig. 2.5.8). First of all, we determine the hydrodynamic diameter of monomeric $\alpha$-synuclein in physiological buffer. Consistent with the previously published data ${ }^{448}$, we measured the hydrodynamic diameter in range of 7-9 nm for freshly purified $\alpha$-synuclein monomer. When $\alpha$-synuclein is incubated in the presence of one of the DPP compounds, namely anle138c, anle145c, anle143c and sery166a, the analysis of the scattering data revealed a fast formation of oligomeric species for all sample and at all time points. The intensity size distribution plots for each compound and various time points are presented on Figure 2.5.8. Although intensity size distribution plots showed some variation among different compounds, the DLS data suggest that the stabilized oligomeric species are quite heterogeneous and the maximum of hydrodynamic diameter is varying in ranges of 70-150 nm, irrespective of DPP compound. The smallest and homogeneous 
oligomeric species (70-100 $\mathrm{nm}$ in diameter) are observed in case of sery166a and anle143c, while the presence of anle138c or anle145c leads to oligomeric population characterized by more broad size distribution with the maximum around $100 \mathrm{~nm}$. Remarkably, the patterns of size distribution plots exhibit little changes during the incubation and these transformations are mainly attributed, first, to a slow consecutive shift of the oligomeric signal towards bigger sizes and, second, to gradual reduction of monomer signal (although the accurate quantitative analysis of populations of various species is not possible).

Overall, DLS data showed a good correlation with the result of the microscopical studies both in terms of the size of the stabilized oligomers and their appearance at early time points. Moreover, DLS constitutes a valuable approach to compare the populations of various oligomeric species in solution, in contrast to AFM and TEM. Such comparison with onpathway $\alpha$-synuclein oligomers is discussed in details below. 

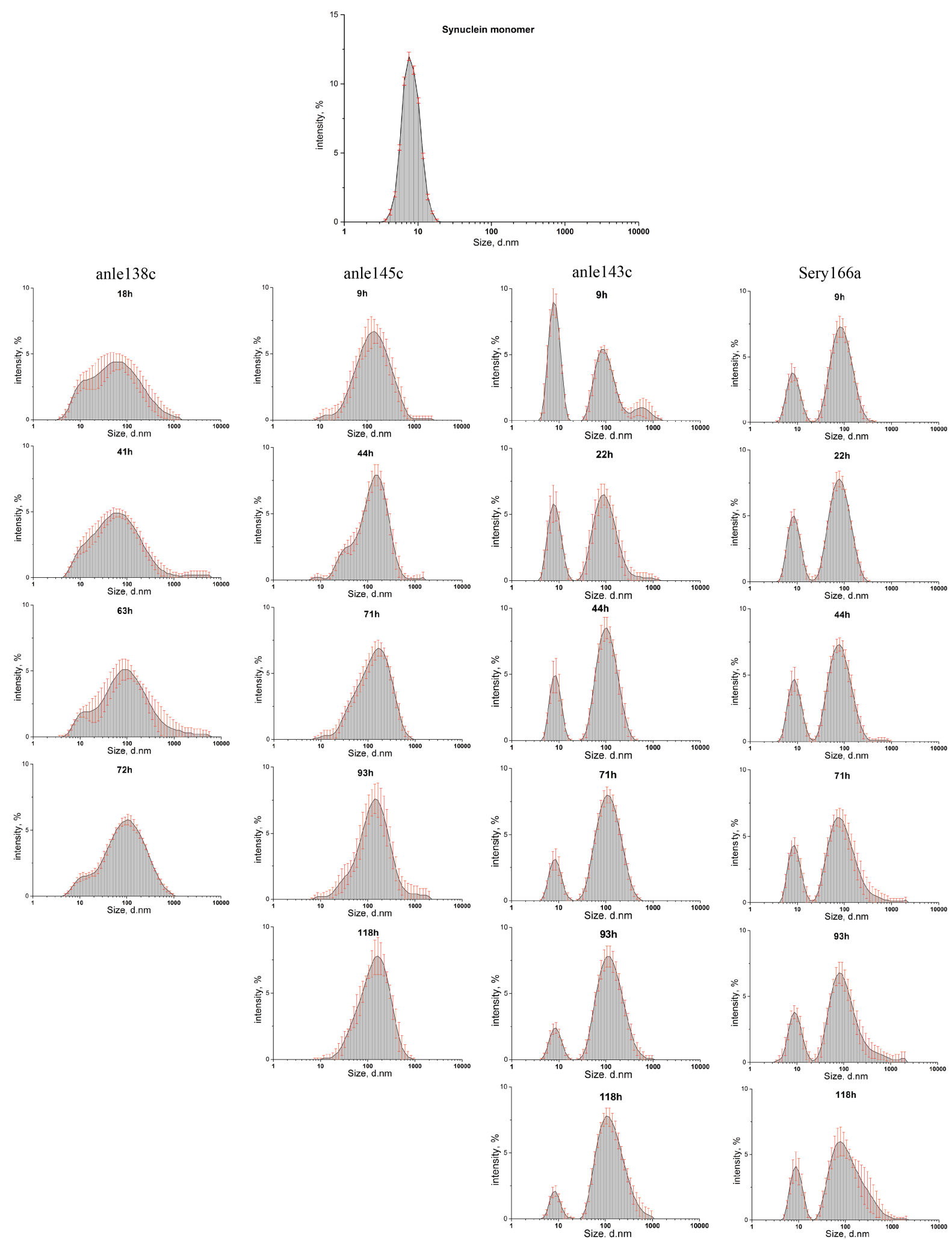

Figure 2.5.8. DLS measurements. Representative intensity size distribution plots for monomeric $\alpha$-synuclein and $\alpha$-synuclein co-incubated with 4 DPP compounds (anle138c, anle143c, anle145c and sery166a) at various time points. Error bars indicate standard error. 


\subsubsection{Secondary structure of DPP-stabilized $\alpha$-synuclein oligomers by CD}

In order to probe the effect of DPP compounds on $\alpha$-synuclein secondary structure and the time course of corresponding conformational changes, we employed the circular dichroism spectroscopy (CD) and recorded the CD spectra of $\alpha$-synuclein incubated alone or in presence of sery166a. In agreement with previously published data ${ }^{109}$, CD spectra of freshly prepared monomeric $\alpha$-synuclein show characteristic features typical for disordered or random coil conformation of the polypeptide chain, especially a major negative band in the 195 to $200 \mathrm{~nm}$ region and an absence of additional bands around 210 to $230 \mathrm{~nm}$ (Fig. 2.5.9). It's also well known that the amyloid fibril formation is associated with $\alpha$-synuclein conformational transition from unfolded state to $\beta$-sheet secondary structure ${ }^{176}$. As illustrated in figure 2.5.9A, the incubation of $\alpha$-synuclein alone at $37{ }^{\circ} \mathrm{C}$ with stirring results in gradual loss in random coil and concomitant increase of $\beta$-sheet structure as evident from the appearance of a broad negative band at about $220 \mathrm{~nm}$. In contrast, the analysis of CD data, which was recorded for $\alpha$-synuclein samples incubated with sery166a, (protein:compound ratio $=1: 1$ ) over $142 \mathrm{~h}$, revealed that sery166a stabilizes the essentially unfolded conformation and interferes with the formation of $\beta$-sheet structure (Fig. 2.5.9B). On the other hand, sery166a also induces a small, but noticeable degree of ordering of local secondary structure, as evident from ellipticity intensity changes of the minima at $196 \mathrm{~nm}$. Remarkably, the CD spectra measured within time intervals from 27 and $147 \mathrm{~h}$ strongly supported a conformational stability of the generated species. Overall, CD data suggests that sery166a stabilizes $\alpha$-synuclein in predominantly unfolded conformation accompanied a low degree of local secondary structure ordering in comparison to the monomeric $\alpha$-synuclein. Of note, we
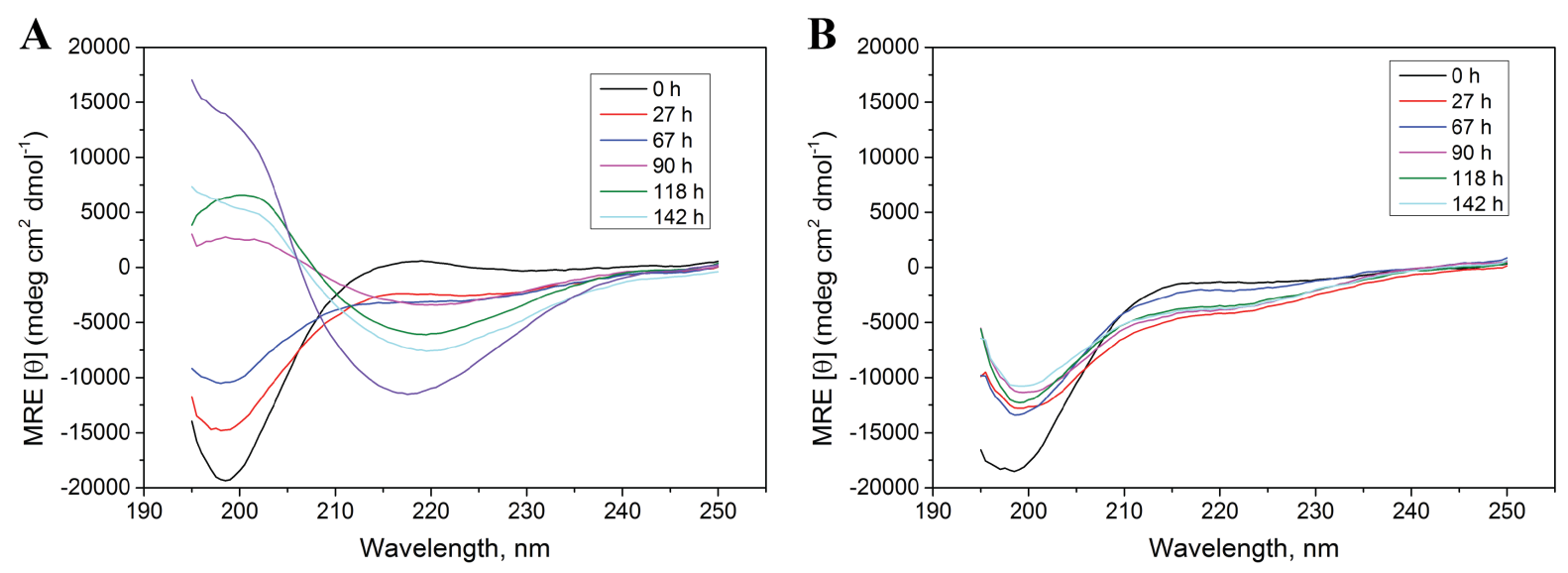

Figure 2.5.9. Far-UV CD spectral data. (A) Far-UV CD spectra of $\alpha$-synuclein $(100$ $\mu \mathrm{M})$ incubated alone. (B) Far-UV CD spectra of $\alpha$-synuclein $(100 \mu \mathrm{M})$ incubated in presence of sery166a $(100 \mu \mathrm{M})$. 
performed the CD measurements with the samples containing $2 \%$ of ethanol by volume, since DMSO, which was used in previously described experiments as organic solvent for dissolving DPP compounds showed a very strong contribution to CD spectra and, thus, interfered with data recording and interpretation.

\subsubsection{Characterization of DPP-stabilized $\alpha$-synuclein oligomers by gel electrophoresis}

Following our efforts to characterize the effects of DPP compounds on $\alpha$-synuclein aggregation and the properties of generated species, we also applied a gel electrophoresis approach, as one of the most common and widely used technique to analyze the composition of complex protein mixtures in regard of main components and protein molecular weight estimation. Despite the fact that these techniques have certain limitations that are associated with the use of detergents (SDS-PAGE) and questionable correlation of the behavior of unfolded proteins with the calibration standards composed of globular proteins, the gel electrophoresis investigation of $\alpha$-synuclein aggregated samples provided valuable pieces of information. Thus, the time-resolved analysis of $\alpha$-synuclein incubated in presence sery $166 \mathrm{a}$ over $110 \mathrm{~h}$ revealed no significant changes in the sample composition based on denaturing polyacrylamide gels visualized by Coomassie Blue staining (Fig. 2.5.10A). Figure 2.5.10A

A

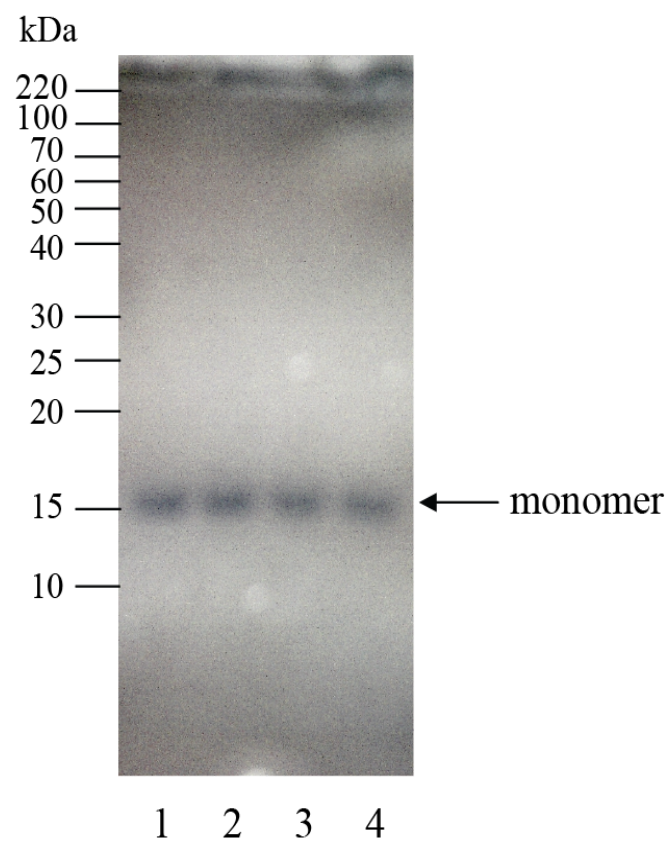

B

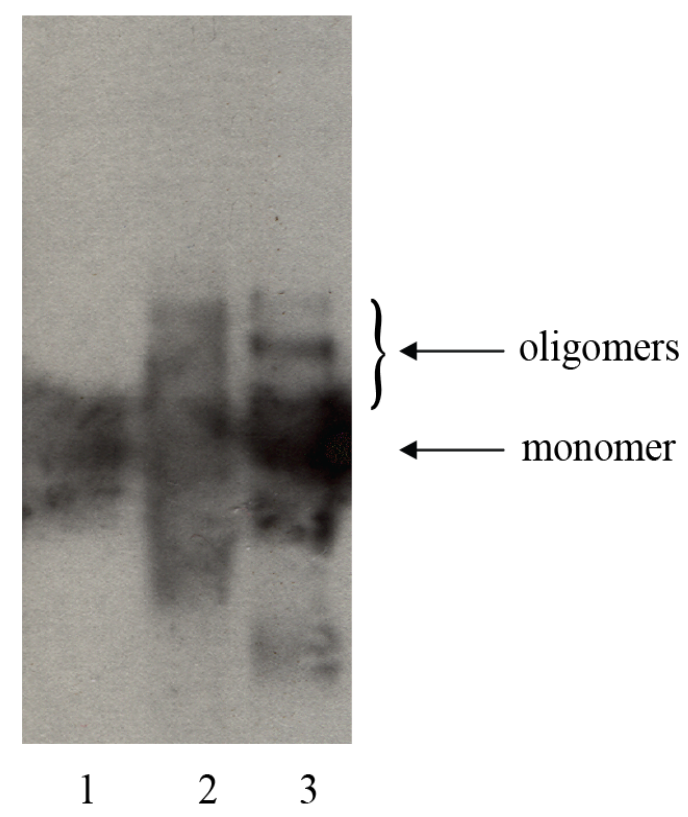

Figure 2.5.10. SDS-PAGE and Native-PAGE analysis. (A) Time-resolved SDS-PAGE analysis of $\alpha$-synuclein incubated in presence of sery $166 \mathrm{a}$; lane $1, \alpha$-synuclein monomer; lane 2, 3, 4, a-synuclein incubated in presence of sery166a for $48 \mathrm{~h}, 72 \mathrm{~h}, 110 \mathrm{~h}$, respectively. (B) Native-PAGE analysis of $\alpha$-synuclein incubated in presence of sery166a; lane 1, $\alpha$-synuclein monomer; lane 2, 3, $\alpha$-synuclein incubated in presence of sery166a for $72 \mathrm{~h}$, lane $32 \mathrm{x}$ loaded material. 
demonstrates that all samples, including monomeric $\alpha$-synuclein (lane 1) and aggregated samples (lanes $2,3,4$ ), are composed of relatively similar amounts of monomeric $\alpha$-synuclein that migrates with an apparent molecular mass of $15 \mathrm{kDa}$. These results further corroborate the notion that DPP compounds, in this experiment - sery166a, prevent the formation of amyloid fibrils, since almost complete disappearance of monomeric protein is observed for fibrillated sample (data not shown). Moreover, the lack of the bands in the region of higher molecular weights, whilst one could expect such signals for compound-stabilized oligomers, suggests that such oligomeric species are sensitive to SDS treatment at high temperature and undergo the dissociation back to monomer under denaturing conditions.

Next, we examined a mixture of $\alpha$-synuclein co-incubated with sery166a for $72 \mathrm{~h}$ at 37 ${ }^{\circ} \mathrm{C}$ on non-denaturing native polyacrylamide gel electrophoresis and Western blot analysis. In theory, the application of the non-denaturing experimental protocols preserves the native conformation of the proteins and precludes the decomposition of the protein complexes including various types of self-associated assemblies ${ }^{449}$. As shown in Figure 2.5.10 B, freshly prepared monomeric $\alpha$-synuclein migrates as a single band (lane 1). On the other hand, the aggregated sample contains, in addition to the dominated band of monomeric $\alpha$-synuclein, immunoreactive signals from higher molecular weight structures, presumably compoundstabilized oligomers (Fig. 2.5.10, lane 2 and 3, lane 3 contains 2x loaded material). Thus, we demonstrated that DPP compounds interfere with $\alpha$-synuclein fibrillation and induce the formation of SDS-sensitive oligomers that could be detected by native-PAGE/western blot analysis.

\subsubsection{NMR investigation of $\alpha$-synuclein aggregation in presence of DPP compounds}

To obtain further insight into the effect of soluble DPP compounds on aggregation of $\alpha$-synuclein we employed NMR spectroscopy. NMR spectroscopy represents a powerfully tool for structural analysis of various molecules and corresponding processes in solution. In this project, we have recorded the NMR data, namely time series of $1 \mathrm{D}{ }^{1} \mathrm{H}$ spectra and $2 \mathrm{D}{ }^{1} \mathrm{H}$ ${ }^{15} \mathrm{~N}$ HSQC spectra, for ${ }^{15} \mathrm{~N}$-labeled $\alpha$-synuclein co-incubated with one of the DPP compounds, sery166a, anle138c and anle145c. Data Evaluation from 1D and 2D experiments allows us to investigate the kinetic of $\alpha$-synuclein aggregation and the sample composition at various time points under applied conditions. Moreover, the analysis $2 \mathrm{D}{ }^{1} \mathrm{H}-{ }^{15} \mathrm{~N}$ HSQC spectra could help us to describe the effect of DPP compounds on $\alpha$-synuclein in residue-specific manner and potentially to address the underlying molecular mechanisms of aggregation inhibition. Experimentally, the aggregation assays have been done directly in the $5 \mathrm{~mm}$ NMR tube with 
the small magnetic stirring bar. All samples, composed of $70 \mu \mathrm{M}{ }^{15} \mathrm{~N}$-labeled $\alpha$-synuclein, 70$140 \mu \mathrm{M}$ compound, $3 \%$ of DMSO- $\mathrm{d}_{6}, 10 \% \mathrm{D}_{2} \mathrm{O}$ in phosphate buffer, were kept at $37{ }^{\circ} \mathrm{C}$ under constant stirring. The $1 \mathrm{D}{ }^{1} \mathrm{H}$ spectra were recorded at $25{ }^{\circ} \mathrm{C}(15$ minutes $) ; 2 \mathrm{D}{ }^{1} \mathrm{H}^{-15} \mathrm{~N}$ HSQC spectra were recorded at $15^{\circ} \mathrm{C}$ (2-3 hours).

Based on previously described biophysical data and also physical properties of DPP candidates, we selected three DPP compounds, anle138c, anle145c and sery166a, for NMRbased analysis. First, the series of $1 \mathrm{D}{ }^{1} \mathrm{H}$ spectra were acquired for $\alpha$-synuclein samples coincubated with/without corresponding compound for several days. The monitoring of $\alpha-$ synuclein aggregation in absence of DPP inhibitors by 1D proton NMR showed almost complete disappearance of the protein signals after $40 \mathrm{~h}$ of incubation and that is mainly attributed to formation amyloid fibrils (Fig. 2.5.11 A) with the kinetic, which is similar to one observed in ThioT-based aggregation assay (Fig. 2.5.4). Why amyloid fibrils do not show the proper spectra in solution? The observed loss of the signal intensity for the fibrils and other high molecular weight species is associated with a significant increase of the molecular weight of such protein complexes leading to a slower tumbling in solution, longer correlation times, fast relaxation and eventually strong broadening of the NMR signals beyond detection. Thus, the HSQC or 1D experiment is only sensitive to the monomer concentration. This is further supported by the almost uniform decrease of the signals during aggregation. Moreover, the amyloid fibrils have a low solubility. Thus, NMR spectroscopy can be considered as an accurate and robust tool to monitor the sample composition during the aggregation assays, mainly the levels of the remaining monomeric protein. 
A

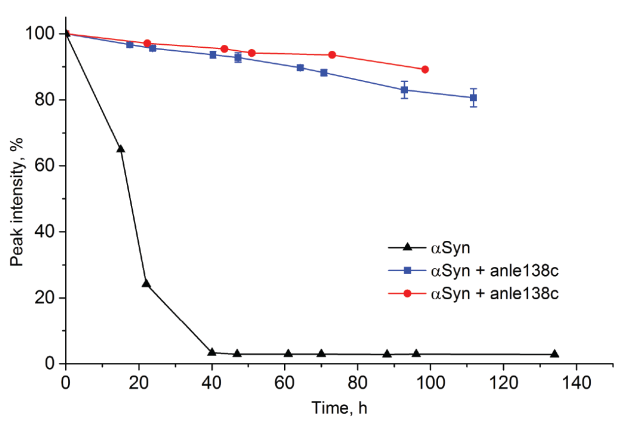

B

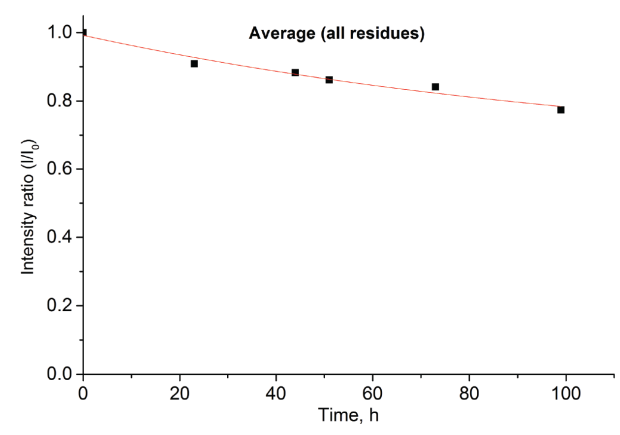

C

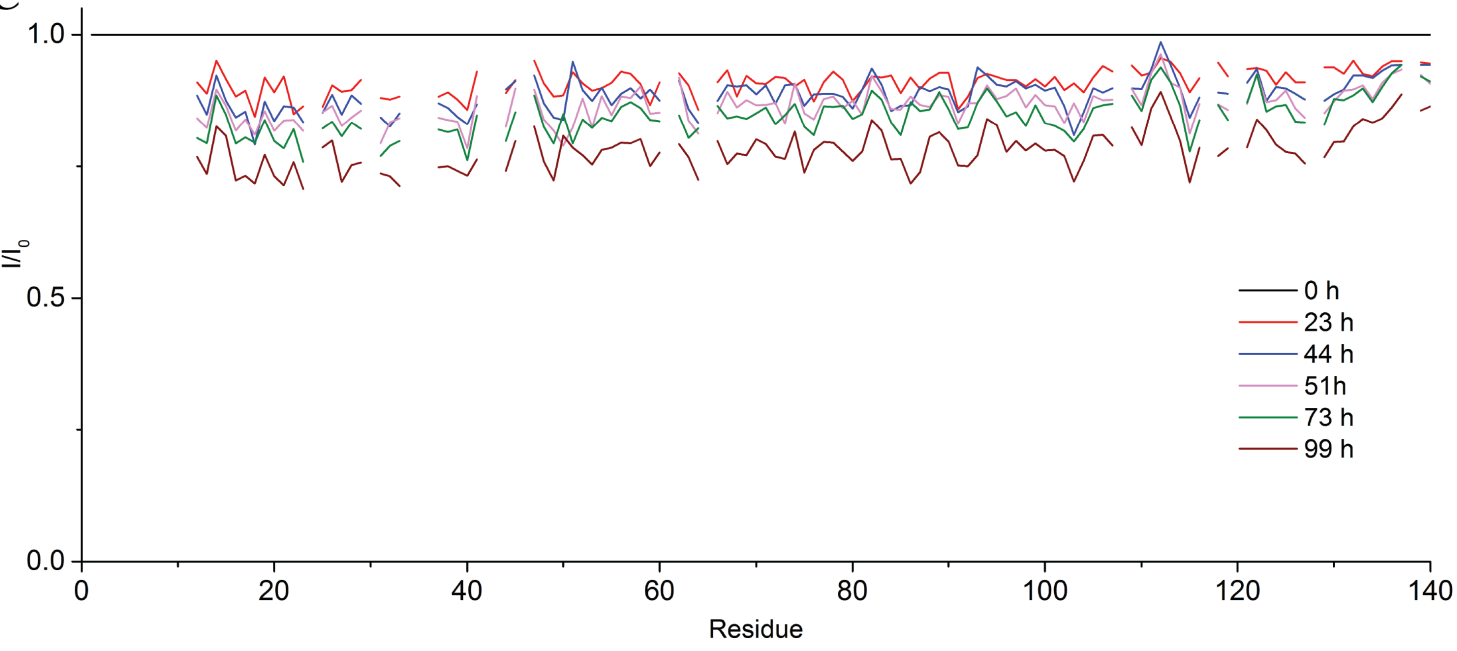

D
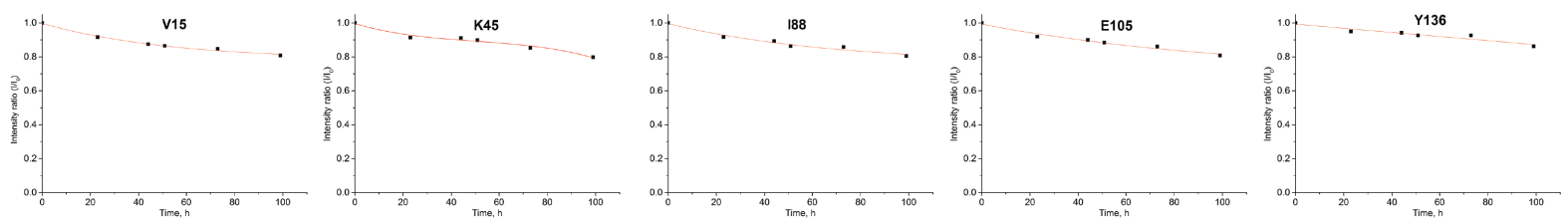

Figure 2.5.11. NMR investigation of $\alpha$-synuclein aggregation in presence of anle138c.

(A) Decay of $\alpha$-synuclein $(70 \mu \mathrm{M})$ monomer signal intensity in aliphatic region of $1 \mathrm{D}{ }^{1} \mathrm{H}$ spectra in absence and presence of anle138c (140 $\mu \mathrm{M}, 2$ independent experiments). (B) Average $\mathrm{H}-\mathrm{N}$ peak intensity decay curve in $2 \mathrm{D}{ }^{1} \mathrm{H}-{ }^{15} \mathrm{~N}$ HSQC spectra of ${ }^{15} \mathrm{~N}$-labeled $\alpha$ synuclein $(70 \mu \mathrm{M})+$ anle138c $(140 \mu \mathrm{M})$. (C) Changes of $\mathrm{H}-\mathrm{N}$ signal intensity in $2 \mathrm{D}{ }^{1} \mathrm{H}$ ${ }^{15} \mathrm{~N}$ HSQC spectra of ${ }^{15} \mathrm{~N}$-labeled $\alpha$-synuclein $(70 \mu \mathrm{M})+\operatorname{anle138c}(140 \mu \mathrm{M})$. (D) Representative $\mathrm{H}-\mathrm{N}$ peak intensity decay curves for selected residues in $2 \mathrm{D}^{1} \mathrm{H}^{1}{ }^{15} \mathrm{~N}$ HSQC spectra of ${ }^{15} \mathrm{~N}$-labeled $\alpha$-synuclein $(70 \mu \mathrm{M})+\operatorname{anle138c}(140 \mu \mathrm{M})$. Overlapping and modified residues were excluded from analysis. Where possible, the exponential decay fit was applied. Error bars indicate in (A) standard error. 
A

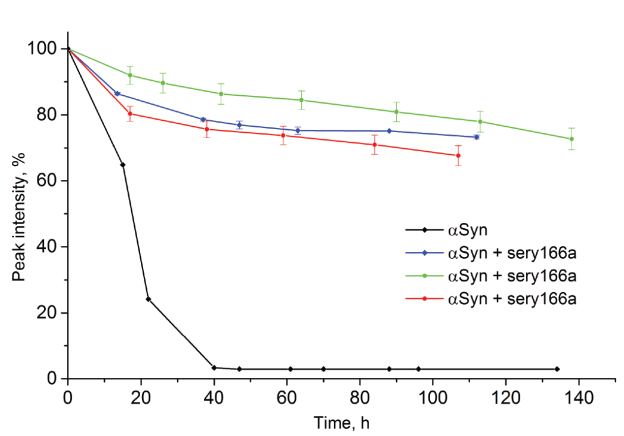

C$$
1.0
$$

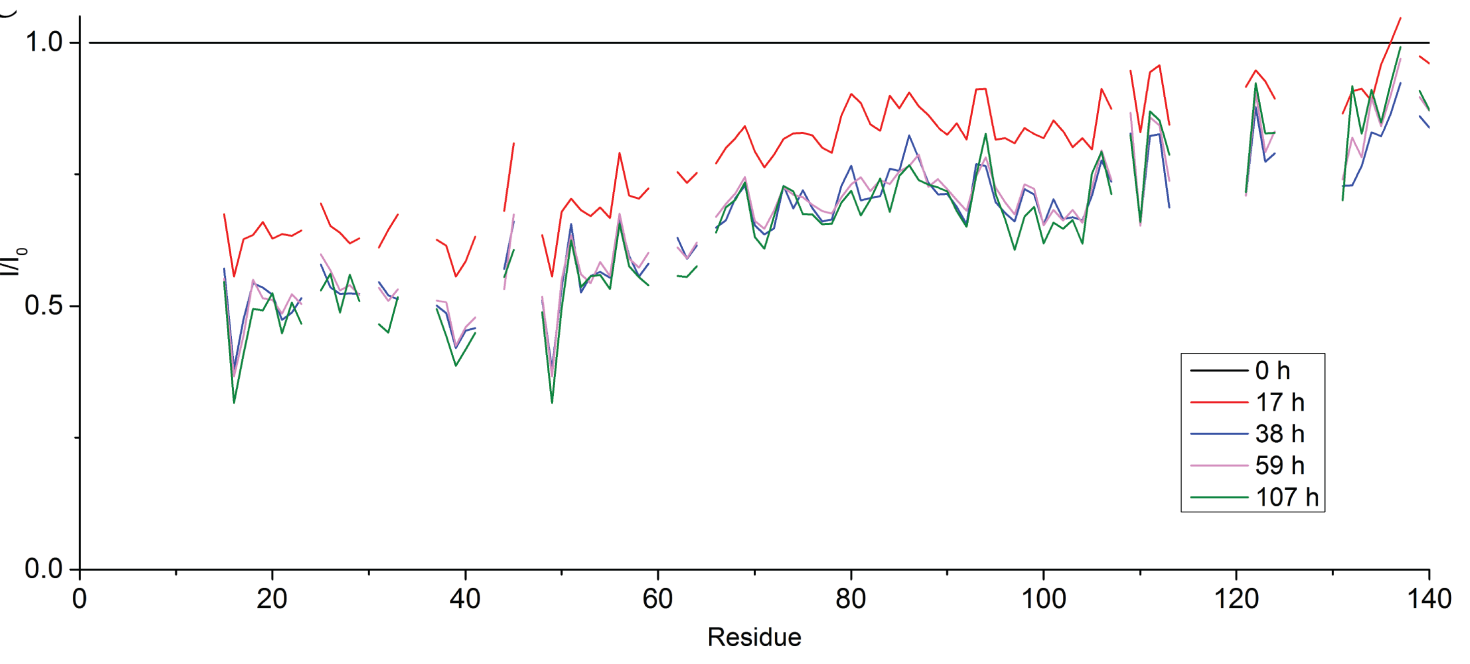

B

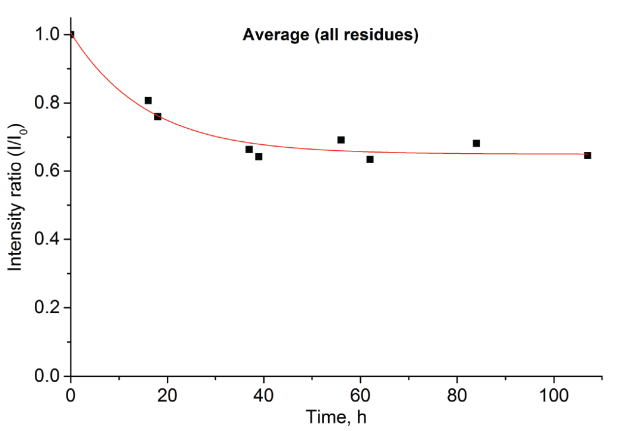

D

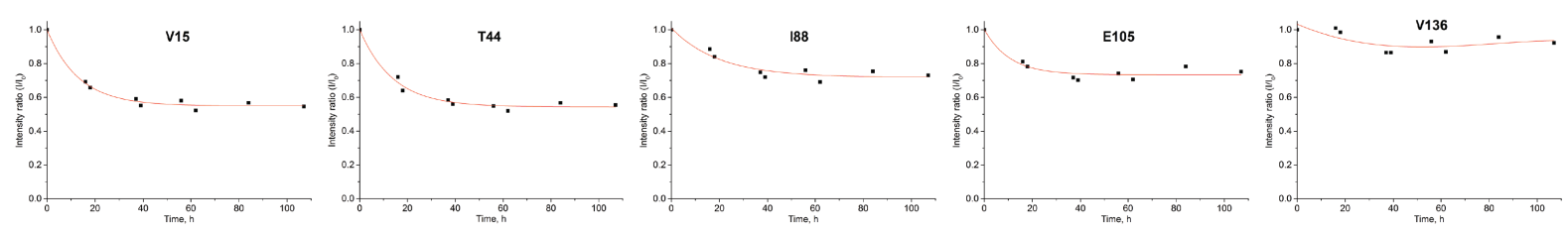

Figure 2.5.12. NMR investigation of $\alpha$-synuclein aggregation in presence of sery166a.

(A) Decay of $\alpha$-synuclein $(70 \mu \mathrm{M})$ monomer signal intensity in aliphatic region of $1 \mathrm{D}{ }^{1} \mathrm{H}$ spectra in absence and presence of sery $166 \mathrm{a}(140 \mu \mathrm{M}, 3$ independent experiments). (B) Average $\mathrm{H}-\mathrm{N}$ peak intensity decay curve in $2 \mathrm{D}{ }^{1} \mathrm{H}-{ }^{15} \mathrm{~N}$ HSQC spectra of ${ }^{15} \mathrm{~N}$-labeled $\alpha$ synuclein $(70 \mu \mathrm{M})+\operatorname{sery} 166 \mathrm{a}(140 \mu \mathrm{M})$. (C) Changes of $\mathrm{H}-\mathrm{N}$ signal intensity in $2 \mathrm{D}{ }^{1} \mathrm{H}$ ${ }^{15} \mathrm{~N}$ HSQC spectra of ${ }^{15} \mathrm{~N}$-labeled $\alpha$-synuclein $(70 \mu \mathrm{M})+\operatorname{sery} 166 \mathrm{a}(140 \mu \mathrm{M})$. (D) Representative $\mathrm{H}-\mathrm{N}$ peak intensity decay curves for selected residues in $2 \mathrm{D}{ }^{1} \mathrm{H}-{ }^{15} \mathrm{~N}$ HSQC spectra of ${ }^{15} \mathrm{~N}$-labeled $\alpha$-synuclein $(70 \mu \mathrm{M})+\operatorname{sery} 166 \mathrm{a}(140 \mu \mathrm{M})$. Overlapping and modified residues were excluded from analysis. Where possible, the exponential decay fit was applied. Error bars indicate in (A) standard error. 
Coming back to DPP compounds, the investigation of $\alpha$-synuclein co-incubated with three selected compounds by $1 \mathrm{D}{ }^{1} \mathrm{H}$ spectra is shown in figures 2.5.11A-2.5.13A. For each compound the protein signal intensity decay curves have been obtained when analyzing the time series of $1 \mathrm{D}^{1} \mathrm{H}$ spectra recorded over 4-5 days. In contrast to the fast loss of the protein signal in case of $\alpha$-synuclein alone (black curves in figures 2.5.11A-2.5.13A), all tested DPP compounds prevented the complete depletion of the signal intensity, thereby confirming the fibrillization inhibitory activity (colored curves in figures 2.5.11A-2.5.13A). Although the fibril formation was inhibited in presence of DPP compounds, we still observed a gradual decay of protein signal for all samples with some compound-specific variation. Thus, in case of anle138c and anle145c the remaining signals of the monomeric $\alpha$-synuclein after $100 \mathrm{~h}$ incubation are in the range $80-85 \%$ and $75-80 \%$, respectively (Fig. 2.5.11A and 2.5.13A). For experiments with sery166a we observed the strongest signal intensity reduction among tested molecules to approximately $70 \%$ of the initial levels at $100 \mathrm{~h}$ time point (Fig. 2.5.12A). Taking into account the substantial evidence from microscopy, DLS and gel electrophoresis studies suggesting the formation of oligomeric species in presence of DPP compounds, the most plausible explanation of NMR data, namely the signal intensity loss, is that $\alpha$-synuclein oligomers induced by DPP compounds are also invisible in NMR spectra due to their high molecular weight, similar to amyloid fibrils. Following this line of thought, evidence from NMR studies strongly suggested that $\alpha$-synuclein co-incubated with DPP compounds for 4-5 days stays mainly in the monomeric form. On the other hand, the changes in NMR spectra indicated the formation of high molecular weight oligomers and, moreover, provided quite accurate quantification of the oligomeric fraction, which under applied condition is varying between 0 and $30 \%$ of total protein amount.

To obtain more detailed information, in particular, a residue specific analysis, about $\alpha$ synuclein aggregation pathways in presence of DPP compounds, we extended the NMR experiments to the time series of $2 \mathrm{D}{ }^{1} \mathrm{H}-{ }^{15} \mathrm{~N}$ HSQC spectra and analyzed the changes of the cross-peaks from the backbone amide nuclei during incubation of uniformly $\mathrm{N}^{15}$-labeled $\alpha$ synuclein and selected DPP inhibitors (anle138c, anle145c and sery166a). First, we analyzed the chemical shift changes of the assigned peaks in $2 \mathrm{D}{ }^{1} \mathrm{H}-{ }^{15} \mathrm{~N}$ HSQC spectra that are induced both by addition of DPP compounds to freshly prepared monomeric $\alpha$-synuclein and by prolonged incubation of corresponding samples under aggregation promoting conditions. Overall, no significant chemical shift perturbations of N-H cross peaks in $2 \mathrm{D}{ }^{1} \mathrm{H}^{-15} \mathrm{~N}$ HSQC spectra were observed in both types of NMR experiments, thereby, suggesting weak interactions of DPP compounds with monomeric protein. However, the analysis of incubated 
A

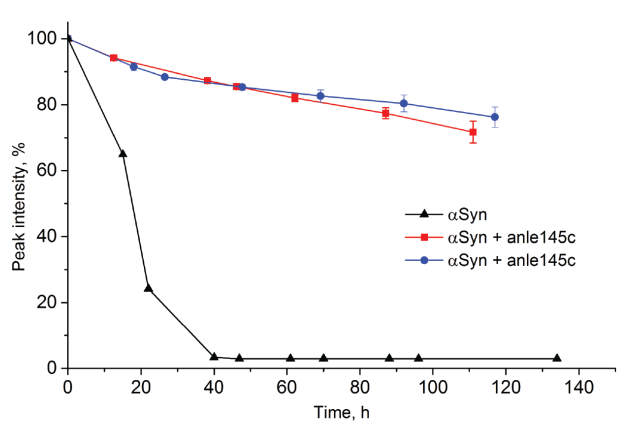

B

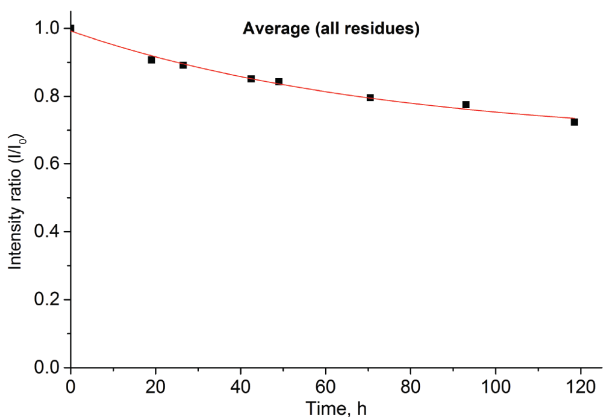

C

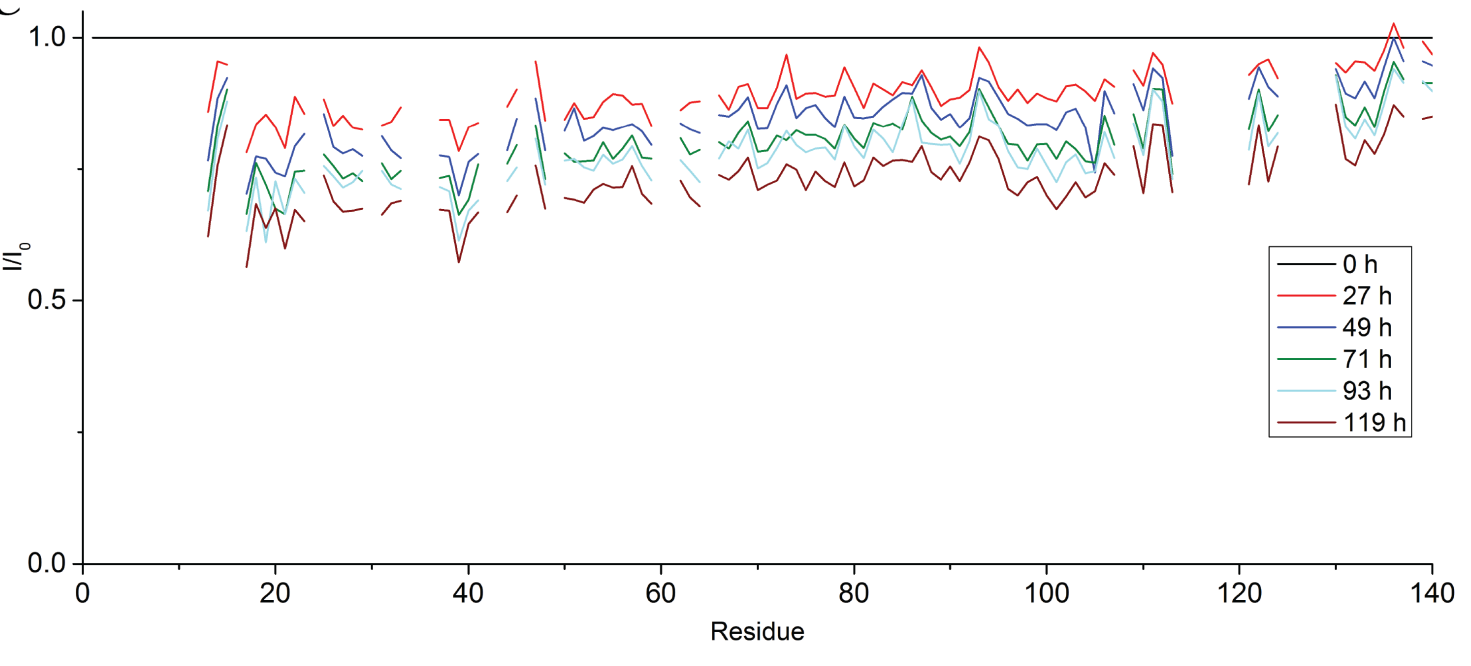

D

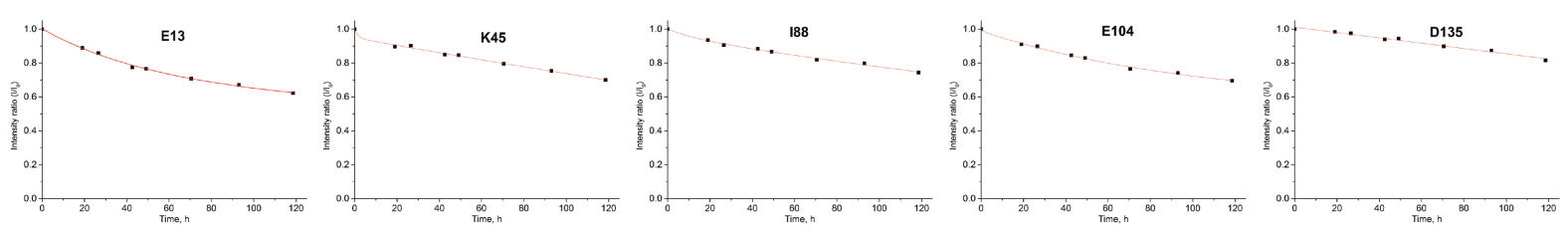

Figure 2.5.13. NMR investigation of $\alpha$-synuclein aggregation in presence of anle145c. (A) Decay of $\alpha$-synuclein $(70 \mu \mathrm{M})$ monomer signal intensity in aliphatic region of $1 \mathrm{D}{ }^{1} \mathrm{H}$ spectra in absence and presence of anle145c (140 $\mu \mathrm{M}, 2$ independent experiments). (B) Average $\mathrm{H}-\mathrm{N}$ peak intensity decay curve in $2 \mathrm{D}{ }^{1} \mathrm{H}-{ }^{15} \mathrm{~N}$ HSQC spectra of ${ }^{15} \mathrm{~N}$-labeled $\alpha$ synuclein $(70 \mu \mathrm{M})+$ anle145c $(140 \mu \mathrm{M})$. (C) Changes of $\mathrm{H}-\mathrm{N}$ signal intensity in $2 \mathrm{D}{ }^{1} \mathrm{H}$ ${ }^{15} \mathrm{~N}$ HSQC spectra of ${ }^{15} \mathrm{~N}$-labeled $\alpha$-synuclein $(70 \mu \mathrm{M})+\operatorname{anle145c}(140 \mu \mathrm{M})$. (D) Representative H-N peak intensity decay curves for selected residues in $2 \mathrm{D}^{1} \mathrm{H}^{-15} \mathrm{~N}$ HSQC spectra of ${ }^{15} \mathrm{~N}$-labeled $\alpha$-synuclein $(70 \mu \mathrm{M})+$ anle145c $(140 \mu \mathrm{M})$. Overlapping and modified residues were excluded from analysis. Where possible, the exponential decay fit was applied. Error bars indicate in (A) standard error.

samples revealed a gradual oxidation affecting all four methionine residues of $\alpha$-synuclein. Among methionine residues, methionines M1 and M5 are more susceptible to chemical oxidation leading to formation of corresponding methionine sulfoxide. Regarding DPP compounds, sery166a-containing samples showed highest oxidation rate, while much less oxidation was observed for anle138c and anle145c. In this part, we excluded from analysis the 
NMR data for those residues, for which the strong effect of these chemical modifications was detected in $2 \mathrm{D}{ }^{1} \mathrm{H}-{ }^{15} \mathrm{~N}$ HSQC spectra. The aspects of protein oxidation as well as the comprehensive NMR investigation of interactions between monomeric $\alpha$-synuclein and several DPP compounds are discussed at length in following chapter.

Similar to $1 \mathrm{D}{ }^{1} \mathrm{H}$ spectra, we analyzed the signal intensity decay of the cross-peaks from the backbone amide nuclei in $2 \mathrm{D}^{1} \mathrm{H}^{-15} \mathrm{~N}$ HSQC spectra during incubation. As shown in figures 2.15.11-2.15.13B, the signal intensity decay curves that are plotted based on intensities averaged over all residues follow an exponential decay curve and overall show overall a good correlation with 1D NMR data for all three selected compounds, anle138c, anle145c and sery166a. For instance, the remaining signals of the monomeric $\alpha$-synuclein after $100 \mathrm{~h}$ incubation with anle145c are close to $80 \%$ according to $1 \mathrm{D}$ data as well as to $2 \mathrm{D}$ ${ }^{1} \mathrm{H}-{ }^{15} \mathrm{~N}$ HSQC data averaged over all residues (Fig. 2.5.13A and 2.5.13B). The evidence reported above supports the formation of $\alpha$-synuclein oligomers in presence of DPP compounds and describes the kinetic of the oligomerization and the levels of remaining monomeric protein.

To obtain the information about the behavior of each $\mathrm{N}-\mathrm{H}$ cross peak during aggregation assay, we performed a residue specific analysis of the time series of ${ }^{1} \mathrm{H}-{ }^{15} \mathrm{~N}$ HSQC spectra for three selected compounds (2.15.11-2.15.13 C and D). As shown in figures 2.15.11-2.15.13C, the amide cross-peak intensities displayed gradual decay profiles that are suggesting the formation of high molecular weight oligomers. However, we observed some variation of the patterns depending on the applied ligand. Thus, the evaluation of the signal intensity decay that is induced upon incubation of $\alpha$-synuclein with anle138c showed the similar degree of intensity loss for the whole polypeptide chain (Fig. 2.15.11 C and D). The intensity decay curves for selected residues are plotted in figure 2.15.11D and they also support the notion of uniform kinetic of the signal decay in case on anle138c. Analysis of the graphs obtained in experiments with anle145c revealed slight differences from anle138c pattern. As demonstrated in figure 2.15.13C, the alterations in peak intensities are not equal within the whole protein. In this regard, two broadly distinct domains can be identified with the first covering residues 1-70 and the second domain from residues 71 to 140 . The Nterminal half of the $\alpha$-synuclein exhibits a higher degree of peak intensity loss (approx. 30\% at $93 \mathrm{~h}$ ) in comparison with the C-terminal 71-140 residues, for which estimation is approx. $20 \%$ at $93 \mathrm{~h}$. The intensity decay curves for selected residues further support these findings (Fig. 2.15.13D). Similar, but much more pronounced, changes are observed in the timeresolved patterns of intensity for amide resonances for $\alpha$-synuclein incubated in presence of 
sery166a. Thus, for N-terminal region (residues 1-60) the line bordering is most significant and close to $50 \%$ after $38 \mathrm{~h}$ of incubation (Fig. 2.15.12 C). According to observed signal decay, the C-terminal region can be further subdivided into two domains exhibiting different degree of signal loss. Hence, the intensity decay for residues in the middle of $\alpha$-synuclein sequence (residues $60-105$ ) was in a range of $25-35 \%$ after $38 \mathrm{~h}$ of incubation, whereas the last 35 amino acids showed the smallest average signal decay. The intensity decay curves for selected residues from corresponding domains also demonstrate the variation of the signal loss (Fig. 2.15.12D).

The comparison of the NMR data for three DPP compounds highlights several important differences. First of all, it is a variation of amount of generated oligomers, specifically, our results indicate an order of activity to induce oligomerization: sery166a $>$ anle145c $>$ anle138c. The second point is associated with the compound-dependent pattern of signal intensity decay in $2 \mathrm{D}^{1} \mathrm{H}^{-15} \mathrm{~N}$ HSQC experiments. In this regard, the different profiles of intensity changes may be attributed to formation of various types of oligomers for each compound. For instance, the interpretation of the ${ }^{1} \mathrm{H}-{ }^{15} \mathrm{~N}$ HSQC data measured for sery166a, in which the stronger signal loss of N-terminal region is observed (Fig. 2.15.12C), supports the notion that C-terminal region of these protein assemblies is much more flexible than the residues in N-terminal domain. It's therefore conceivable for sery166a situation that the N-terminal region of protein might be involved in formation of oligomeric core that is supposed to be more structured and less flexible, while C-terminus in oligomers is flexible and structurally similar to unfolded monomer. Concerning anle138c and anle145c, the decay of the intensity for amide resonances is quite uniform, arguing for different structural organization of oligomeric species, in which all residues constitute the oligomeric core and, in accordance, are less flexible and have longer correlation times. On the other hand, the different patterns of signal intensity decay might also be explained by the formation of heterogeneous mixtures characterized by a broad distribution of the oligomeric assemblies. In this way, the different compounds are capable to modulate the populations of certain oligomeric structures. In other words, DPP inhibitors might induce the similar oligomers, but the population of these species is different for each compound and that translates to different profile of the signal decay.

Collectively, our NMR findings in combination with other biophysical studies confirm the fact that DPP compounds preclude the formation of $\alpha$-synuclein fibril and at the same time induce the assembly of oligomeric species. Due to the high molecular weight of these $\alpha$ synuclein oligomers, we observe the gradual loss of the signal intensity of monomeric protein 
in time course $1 \mathrm{D}{ }^{1} \mathrm{H}$ and $2 \mathrm{D}{ }^{1} \mathrm{H}^{-15} \mathrm{~N}$ HSQC NMR experiments. Notably, the applied NMR characterization represents a powerful tool to explore the aggregation pathways on residuespecific basis and in time-resolved and quantitative manner.

2.5.9. $\alpha$-Synuclein aggregation in presence of DPP compounds under reducing $\underline{\text { conditions }}$

In an attempt to clarify a potential effect of oxidation of anti-aggregation activity of DPP compounds, we have performed a number of experiments, in which the aggregation assays have been done in presence of a reducing agent, namely $5 \mathrm{mM}$ sodium thiosulfate. Collectively, we have analyzed the $\alpha$-synuclein samples co-incubated with anle138c, anle145c or sery166a by ThioT assay and, moreover, we extended the characterization of aggregation mixtures containing anle145c by DLS and NMR investigations. According to the literature, the oxidation of one or several methionines in $\alpha$-synuclein has been shown to reduce the propensity of the protein to fibrillize $\mathrm{e}^{450,451}$, as discussed in chapter 1.6.3.8. Furthermore, there are evidences supporting an implication of anti-amyloidogenic compounds, mainly polyphenolic structures including dopamine, EGCG, flavonoids and others, as a cause of these oxidation protein modifications ${ }^{324}, 333$. The mechanisms of methionine oxidation are primarily related to a generation of reactive oxygen species and hydrogen peroxide during self-oxidation of polyphenolic moiety of corresponding molecules ${ }^{333}$. Therefore, we would like to investigate and to compare $\alpha$-synuclein aggregation pathways in presence of selected DPP compounds both under reducing and non-reducing conditions.

In order to perform the aggregation assays and corresponding biophysical studies under reducing conditions, we selected sodium thiosulfate at $5 \mathrm{mM}$ concentration as an internal reducing agent. Previously published studies have demonstrated a negligible effect of this compound on $\alpha$-synuclein aggregation. Moreover, we substituted DMSO that was used as the organic component in previous experiments with ethanol due to the potential contribution of DMSO to oxidation. We have chosen ethanol taking into account following facts: first, a relatively high boiling point and, eventually, low evaporation during long incubation at $37^{\circ} \mathrm{C}$; second, no redox activity; third, water solubility, chemical stability and availability in fully deuterated form as required for NMR studies. 
To probe the effect of selected DPP compounds on $\alpha$-synuclein aggregation under reducing conditions, we first monitored the kinetic of fibril formation via ThioT assay. In line with the previous results, the incubation of $\alpha$-synuclein alone under reducing conditions and stirring at $37^{\circ} \mathrm{C}$ led to a rapid assembly of amyloid fibrils within two days (Fig. 2.5.14 A). On the other hand, the addition of one of the DPP molecules (anle138c, anle145c, sery166a) resulted in nearly complete inhibition of $\alpha$-synuclein fibrillogenesis. These experiments, which were acquired in presence of reducing agent ( $5 \mathrm{mM}$, sodium thiosulfate), are consistent with a role of DPP compounds as potent inhibitors of $\alpha$-synuclein fibril formation (Fig. 2.5.14 A). However, high levels of DPP compounds are required in order to achieve a similar inhibitory activity in comparison with the non-reducing conditions that were applied in complementary assays. Thus, in absence of $\mathrm{Na}_{2} \mathrm{~S}_{2} \mathrm{O}_{3}$, DPP compounds, namely anle138c, anle145c and sery166a, are capable to block fibril formation at equimolar mixtures of $\alpha$ synuclein and compounds, meaning inhibitor/protein ratio around 1. Whereas the sustained inhibitory effect of selected DPP compounds under reducing conditions was observed at inhibitor/protein ratio in range from 2 to 3 (Fig. 2.5.14). For instance, sery166a precluded $\alpha-$ synuclein fibrillization at 2.8:1 $\alpha$-synuclein:sery166a ratio, while at lower compound concentration a partial inhibition was observed (data not shown). Overall, ThioT data

A

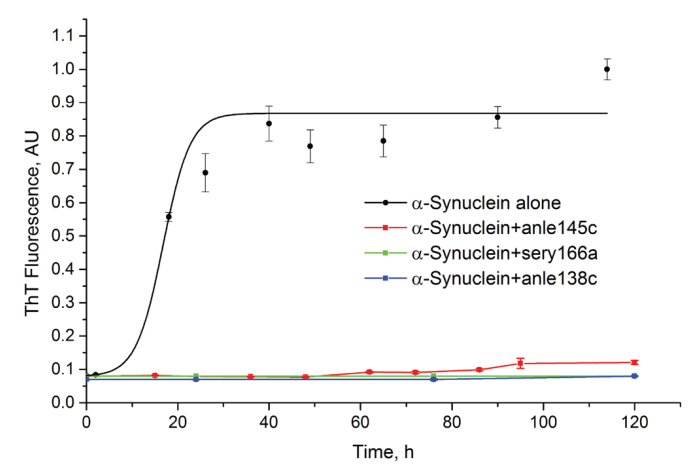

B

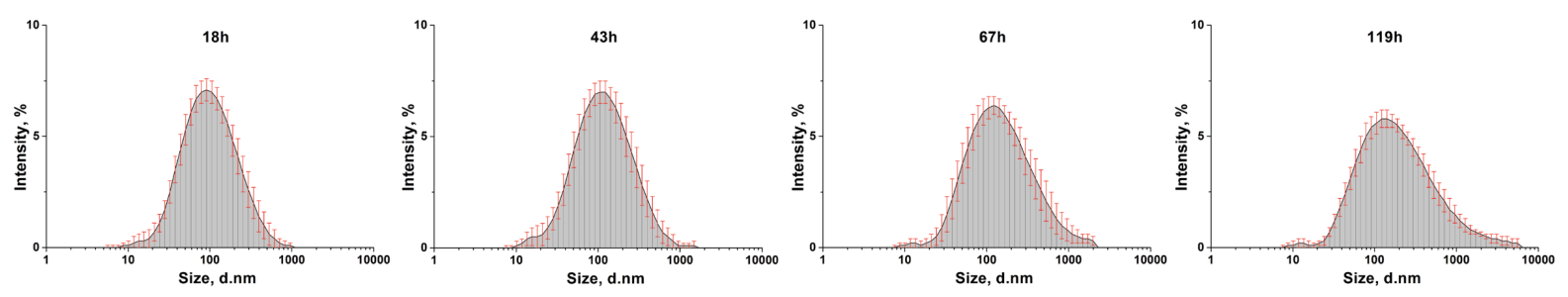

Figure 2.5.14. Inhibition of $\alpha$-synuclein aggregation by DPP compounds under reducing conditions. (A) Kinetic of $\alpha$-synuclein $(50 \mu \mathrm{M})$ fibrillization in presence of DPP compounds, including anle138c $(100 \mu \mathrm{M})$, anle145c $(140 \mu \mathrm{M})$, sery166a $(140 \mu \mathrm{M})$, in ThioT assay. (B) DLS analysis; representative intensity size distribution plots for $\alpha$ synuclein $(50 \mu \mathrm{M})$ co-incubated with anle145c $(140 \mu \mathrm{M})$ at various time points. Error bars indicate in $(\mathbf{A}, \mathbf{B})$ standard error. 
confirmed the inhibitory activity of DPP compounds on $\alpha$-synuclein aggregation also under reducing conditions. The observations that compounds exhibited the reduced activity in presence of $\mathrm{Na}_{2} \mathrm{~S}_{2} \mathrm{O}_{3}$ suggest potential minor contributions of redox processes targeting both compounds and protein to aggregation inhibition.

To further characterize the $\alpha$-synuclein aggregation pathway in presence of anle $145 \mathrm{c}$ under reducing conditions, we investigated the composition of incubated mixtures by DLS and compared with anle145c-related DLS data, previously discussed in chapter 2.5.5. Experimentally, we performed aggregation assay under similar conditions described above for ThioT measurements, specifically, $50 \mu \mathrm{M} \alpha$-synuclein, $140 \mu \mathrm{M}$ anle145c, $5 \mathrm{mM} \mathrm{Na}_{2} \mathrm{~S}_{2} \mathrm{O}_{3}, 3 \%$

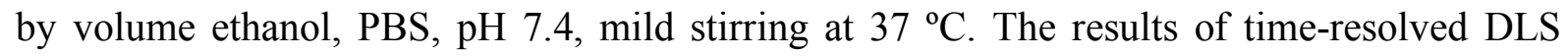
measurements are presented in figure 2.5.14B. Viewed together, DLS intensity size distribution plots at various time points showed a formation of high molecular weight oligomeric species that are characterized by a broad size distribution with a maximum approximately around 100-130 $\mathrm{nm}$ of hydrodynamic diameter. Over the course of the experiments, a slight shift of the oligomeric peak in direction of increasing size of particle accompanied by considerably increased heterogeneity of sizes of generated protein assemblies are apparent as demonstrated in figure 2.5.14B. Consistent with the DLS results obtained for anle145c aggregation assays under non-reducing conditions, the analysis of DLS measurements, which acquired in presence of $\mathrm{Na}_{2} \mathrm{~S}_{2} \mathrm{O}_{3}$, strongly suggested the formation of anle145c-stabilized $\alpha$-synuclein oligomers that are characterized by a similar size distribution centered at 100-150 $\mathrm{nm}$ of diameter. On the contrary, anle145c in the presence of reducing agent favored the formation of less homogeneous mixtures of oligomers as evident from a comparison of the peak shapes (fig. 2.5.14B and 2.5.8).

In order to provide more insights into the effects of anle145c on $\alpha$-synuclein aggregation under reducing conditions and also to confirm the lack of methionine oxidation we employed NMR-based approach that is similar to one discussed in details above. Thus, we acquired the time series of $1 \mathrm{D}{ }^{1} \mathrm{H}$ spectra and 2D ${ }^{1} \mathrm{H}_{-}{ }^{15} \mathrm{~N}$ HSQC spectra to obtain data on kinetic of $\alpha$-synuclein aggregation and the sample composition at various time points in combination with the residue-specific information. The investigation of $\alpha$-synuclein coincubated with anle145c in presence of reducing agent by $1 \mathrm{D}{ }^{1} \mathrm{H}$ spectra is shown in figure 2.5.15A. Analysis of the protein signal intensity decay curves from the time series of $1 \mathrm{D}{ }^{1} \mathrm{H}$ spectra revealed a gradual decay of protein signal intensity corresponding to monomeric $\alpha$ synuclein that, in turn, is very similar to experimental finding collected in absence of $\mathrm{Na}_{2} \mathrm{~S}_{2} \mathrm{O}_{3}$ (Fig. 2.5.15A and 2.5.13A). These results are consistent with fibrillization inhibitory activity 
of anle145c and also are supporting the formation of high molecular weight oligomers that are invisible in $1 \mathrm{D}{ }^{1} \mathrm{H}$ spectra. Remarkably, no significant changes were observed regarding the amounts of generated oligomers with and without the reducing agent, suggesting that anle145c-induced pathway might be similar regardless of the applied redox conditions. For example, the remaining signals of the monomeric $\alpha$-synuclein after $100 \mathrm{~h}$ incubation with and without addition of $\mathrm{Na}_{2} \mathrm{~S}_{2} \mathrm{O}_{3}$ are in the range $70-75 \%$ and $75-80 \%$, respectively (Fig. 2.5.13A and 2.5.15A).

A

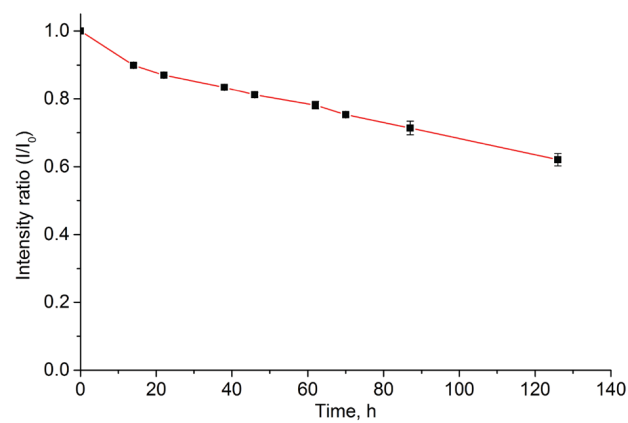

B

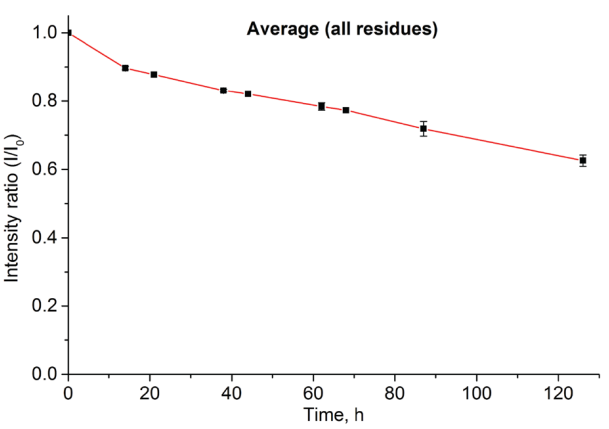

C

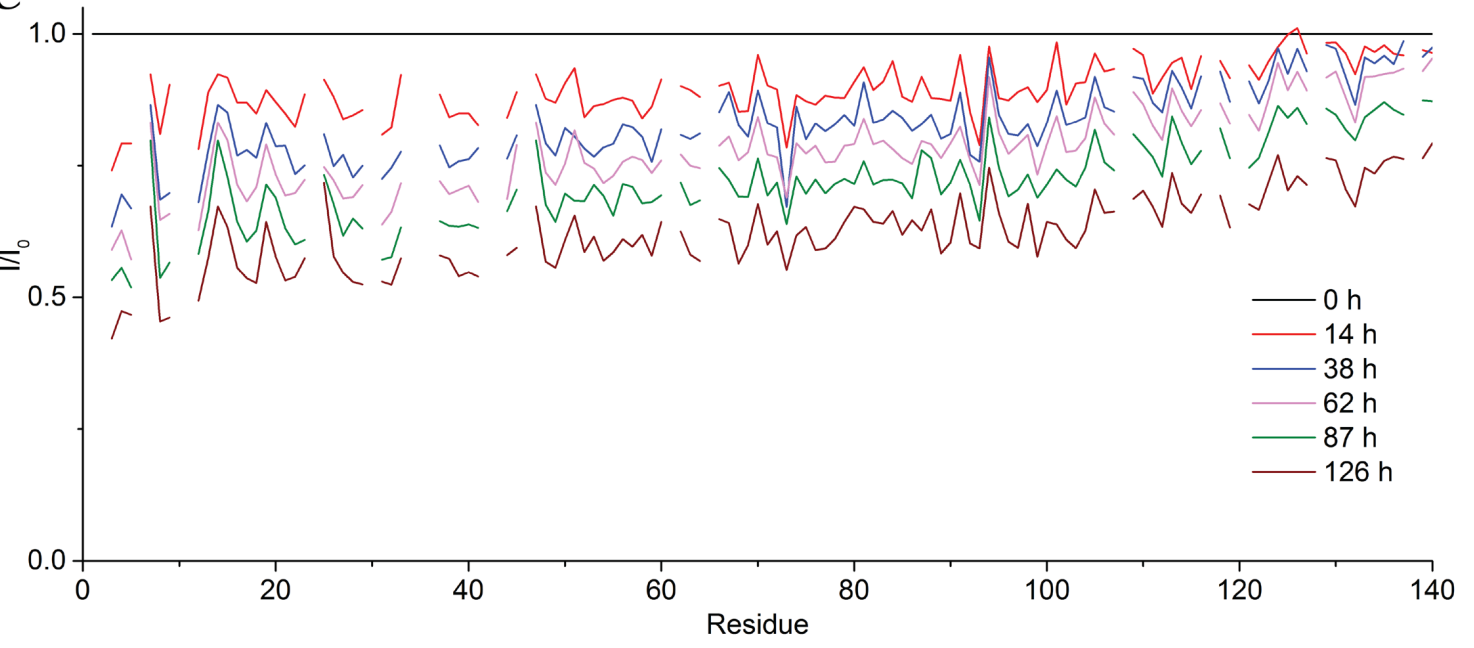

D

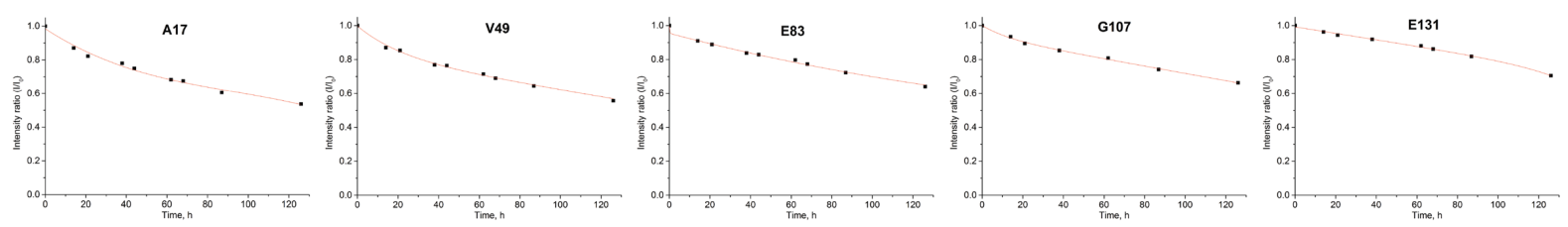

Figure 2.5.15. NMR investigation of $\alpha$-synuclein aggregation in presence of anle145c under reducing conditions. (A) Decay of $\alpha$-synuclein monomer signal intensity in aliphatic region of $1 \mathrm{D}{ }^{1} \mathrm{H}$ spectra in absence and presence of anle145c (2 independent experiments). (B) Average $\mathrm{H}-\mathrm{N}$ peak intensity decay curve in $2 \mathrm{D}^{1} \mathrm{H}_{-}{ }^{15} \mathrm{~N}$ HSQC spectra of ${ }^{15} \mathrm{~N}$-labeled $\alpha$-synuclein $(50 \mu \mathrm{M})+\operatorname{anle} 145 \mathrm{c}(140 \mu \mathrm{M})$. (C) Changes of $\mathrm{H}-\mathrm{N}$ signal intensity in $2 \mathrm{D}{ }^{1} \mathrm{H}-{ }^{15} \mathrm{~N}$ HSQC spectra of ${ }^{15} \mathrm{~N}$-labeled $\alpha$-synuclein $(50 \mu \mathrm{M})+$ anle $145 \mathrm{c}(140$ $\mu \mathrm{M})$. (D) Representative H-N peak intensity decay curves for selected residues in $2 \mathrm{D}{ }^{1} \mathrm{H}$ ${ }^{15} \mathrm{~N}$ HSQC spectra of ${ }^{15} \mathrm{~N}$-labeled $\alpha$-synuclein $(50 \mu \mathrm{M})+\operatorname{anle145c}(140 \mu \mathrm{M})$. Where possible, the exponential decay fit was applied. 
Similar to $1 \mathrm{D}{ }^{1} \mathrm{H}$ spectra, we analyzed the signal intensity decay of the cross-peaks from the backbone amide nuclei in $2 \mathrm{D}^{1} \mathrm{H}^{15} \mathrm{~N} \mathrm{HSQC}$ spectra during incubation of $\alpha$-synuclein in presence of anle145c and reducing agent. As shown in figures 2.5.15B, the signal intensity decay curves that are plotted based on intensities averaged over all residues showed time dependent decline of remaining amide resonances and, notably, exhibited a good correlation with 1D NMR data (Fig. 2.5.15A) and HSQC data previously summarized for experiments under non-reducing conditions (Fig. 2.5.15B). Importantly, a residue specific analysis of the time series of ${ }^{1} \mathrm{H}-{ }^{15} \mathrm{~N}$ HSQC spectra has confirmed the prevention of the oxidative methionine modifications during whole course of aggregation. This finding implies that the partial oxidation of methionines in $\alpha$-synuclein observed in previous studies is not the main mechanism of aggregation inhibition and oligomer formation. Moreover, the resonance intensity attenuation profiles from 2D ${ }^{1} \mathrm{H}^{15} \mathrm{~N}$ HSQC experiments showed little differences between reducing and non-reducing aggregation assays (Fig. 2.5.13C and 2.5.15C). This statement is correct as for overall pattern of alterations, meaning the higher degree of peak intensity loss for residues in $\mathrm{N}$-terminus of $\alpha$-synuclein and smaller reduction of signal intensity of C-terminal residues, as well as for the kinetic of observed decline of the resonance intensity. The intensity decay curves for selected residues further support these findings (Fig. 2.5.15D). All together, the NMR findings strongly suggest that the time-dependent attenuation in signal intensity of $\alpha$-synuclein induced by anle $145 \mathrm{c}$ under reducing conditions, seen in Figure 2.5.15, correlates with the changes observed in absence of reducing agent, seen in Figure 2.5.13, and, thereby, this data points to a similar mechanisms of aggregation inhibition and formation of analogous oligomeric species under both experimental conditions. The comparison of DLS data also supports this conclusion.

\subsubsection{Interactions of DPP compounds with monomeric $\alpha$-synuclein studied by NMR}

NMR is also an optimal technique to study the protein-protein and protein-ligand interaction at atomic resolution. Therefore, we performed high-resolution NMR studies that were mainly based on two-dimensional ${ }^{1} \mathrm{H}-{ }^{15} \mathrm{~N}$ HSQC-NMR titration experiments with monomeric $\alpha$-synuclein and selected DPP compounds. Additionally, our data set includes ${ }^{1} \mathrm{H}$ ${ }^{15} \mathrm{~N}$ HSQC-NMR binding studies recorded for mixtures of $\alpha$-synuclein and DPP ligand at highest possible concentrations with regard to the latter. The main goal of these NMR studies was to demonstrate possible interactions of DPP compounds with monomeric $\alpha$-synuclein and describe the corresponding interaction interfaces and binding affinities. With this respect, the analysis of the chemical shift perturbations and the peak intensity profile of the backbone 
amide resonances of protein, in our case $\alpha$-synuclein, in presence of single or increasing concentrations of the ligand can provide the valuable information about interaction sites and parameters of binding. In this context, the chemical shifts perturbations of amide protons of the protein backbone upon ligand addition points towards the changes of chemical environment as a result of direct binding and related conformational transition. Moreover, the changes of the signal intensities, mainly resonance broadening, could also indicate the binding events that affect the exchange rates between free and bound forms of the protein. However, in addition to exchange phenomena the decay of the signal intensity could be triggered by other processes, including pre-equilibrium conformational change of the protein before ligand binding; or structural rearrangement of the protein/ligand complex after binding; or protein dimerization/oligomerization before or after binding ${ }^{452}$.

In an attempt to obtain insight into the nature of $\alpha$-synuclein-DPP compounds interactions and propose a possible mechanism(s) of anti-aggregation activity, we measured and analyzed ${ }^{1} \mathrm{H}-{ }^{15} \mathrm{~N}$ HSQC spectra for following samples: ${ }^{15} \mathrm{~N}$-labeled monomeric $\alpha$ synuclein in presence of sery166a (ligand-to-protein ratio $=25$ ), sery139 (ligand-to-protein ratio $=25$ ), anle138c (ligand-to-protein ratio $=2$ ), anle145c (ligand-to-protein ratio $=2$ ). Moreover, we performed ${ }^{1} \mathrm{H}-{ }^{15} \mathrm{~N}$ HSQC-NMR titration experiments with ${ }^{15} \mathrm{~N}$-labeled monomeric $\alpha$-synuclein $(50 \mu \mathrm{M})$ and various concentrations of either sery139 $(0-1 \mathrm{mM})$ or anle137c $(0-200 \mu \mathrm{M})$. All NMR measurements were acquired at $15{ }^{\circ} \mathrm{C}$ in $50 \mathrm{mM}$ phosphate buffer ( $\mathrm{pH}$ 7.4) with addition of DMSO- $\mathrm{d}_{6}$ (3-5 vol. \%). The selection of compounds was based on water solubility properties, structural diversity and, most importantly, the high aggregation inhibition activity. Thus, all compounds included in the NMR binding studies are potent inhibitors of $\alpha$-synuclein fibrillization and do have the solubility of 100-200 $\mu \mathrm{M}$ in case of anle138c, anle145c and anle137c, while for sery139 and sery166a the concentration of $1 \mathrm{mM}$ could be achieved, which translates to high ligand-to-protein molar ratios required for titration studies. 
A

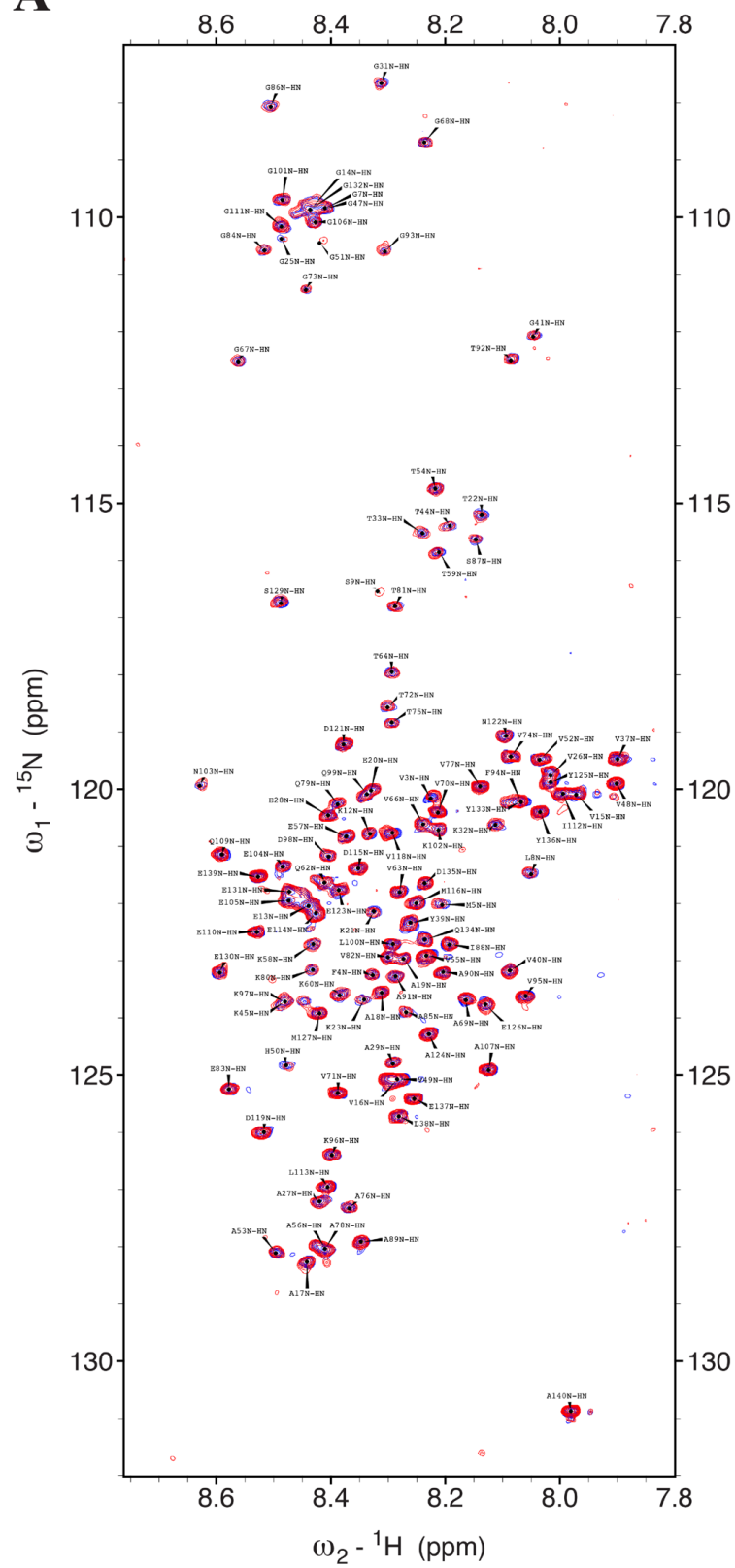

B

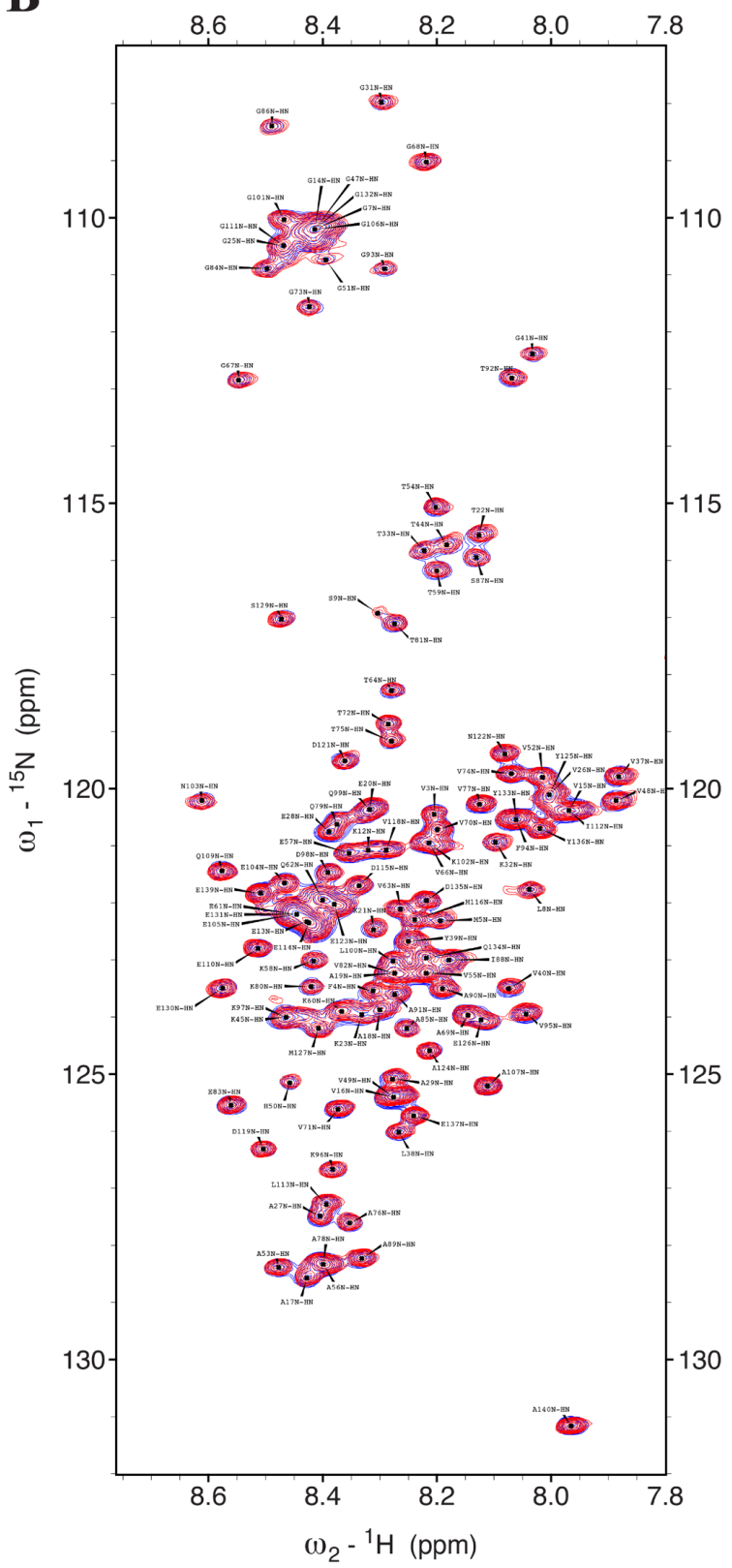

Figure 2.5.16. ${ }^{1} \mathrm{H}-{ }^{15} \mathrm{~N}$ HSQC spectra of $\alpha$-synuclein in the presence of sery166a and sery139. (A) Overlay of the $2 \mathrm{D}{ }^{1} \mathrm{H}-{ }^{15} \mathrm{~N}$ HSQC spectra of ${ }^{15} \mathrm{~N}$-labeled $\alpha$-synuclein $(40 \mu \mathrm{M})$ in presence (blue) and absence (red) of sery 166a (1 mM) acquired at $900 \mathrm{MHz}$ (B) Overlay of the $2 \mathrm{D}^{1} \mathrm{H}^{-15} \mathrm{~N}$ HSQC spectra of ${ }^{15} \mathrm{~N}$-labeled $\alpha$-synuclein $(40 \mu \mathrm{M})$ in presence (blue) and absence (red) of sery139 $(1 \mathrm{mM})$ acquired at $600 \mathrm{MHz}$. 
First, we analyzed the ${ }^{1} \mathrm{H}_{-}{ }^{15} \mathrm{~N}$ HSQC data acquired for ${ }^{15} \mathrm{~N}$-labeled monomeric $\alpha$ synuclein in presence of sery139 or sery166a at high ligand-to-protein ratio. Under described conditions, the high ligand-to-protein ratio of 25 allows us to detect the binding event(s) and to identify the most strongly affected residues. The superimposed ${ }^{1} \mathrm{H}-{ }^{15} \mathrm{~N}$ HSQC spectra of monomeric $\alpha$-synuclein alone (red) and in presence of sery166a (blue) and sery139 (blue) are shown on figure 2.5.16A and 2.5.16B, respectively. The comparison of the HSQC spectra of $\alpha$-synuclein alone and in presence of these ligands (sery166a and sery139) revealed no significant differences in regard of chemical shifts and intensity profiles for both compounds.

A
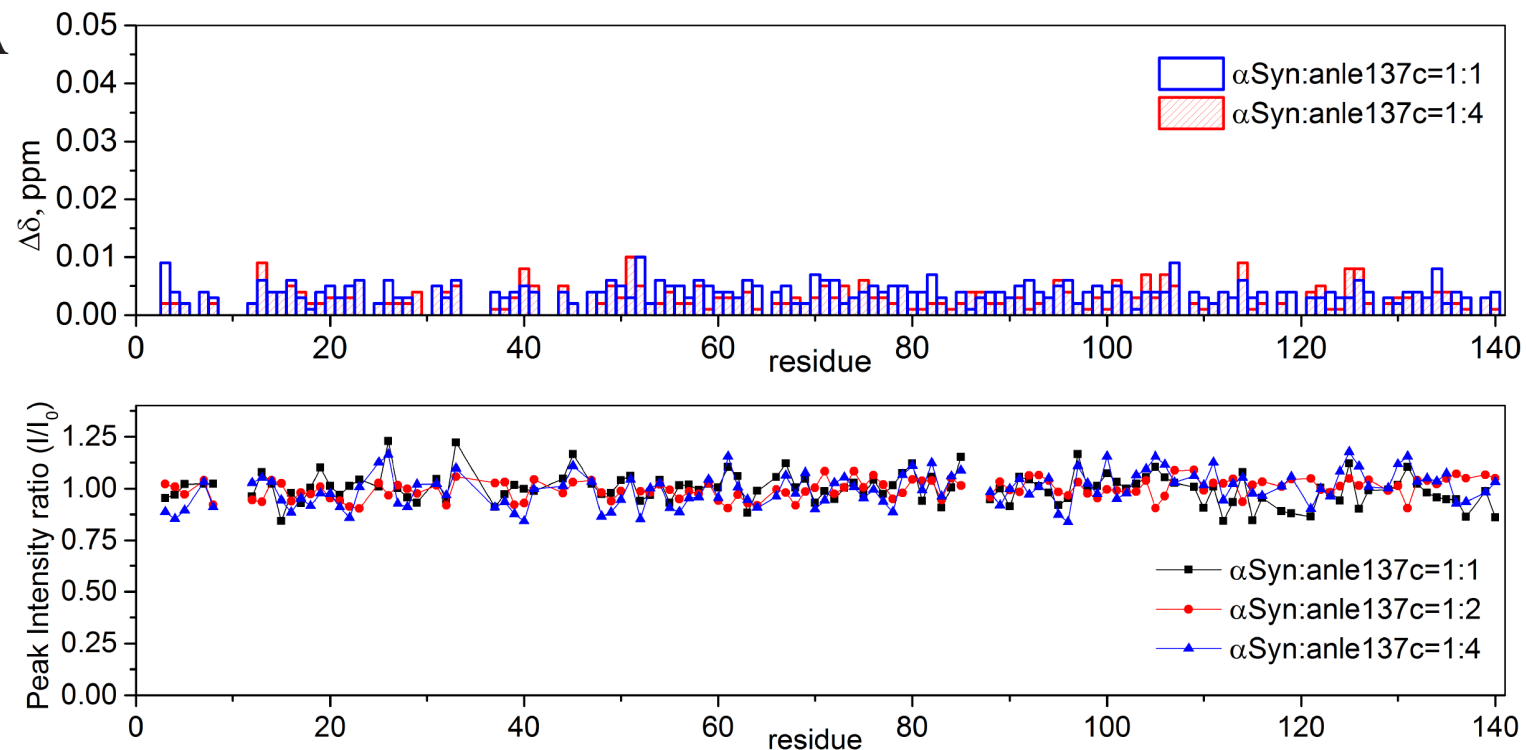

B
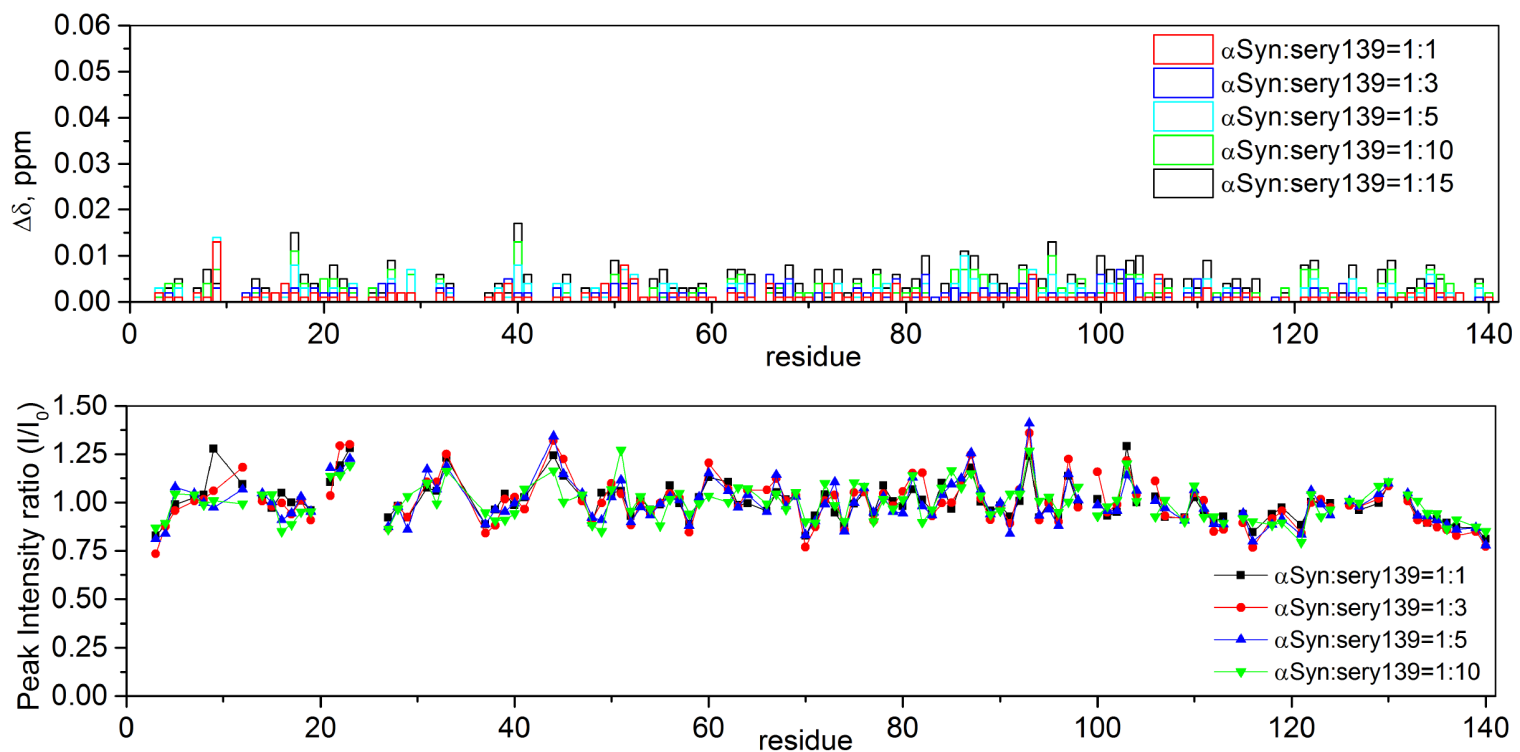

Figure 2.5.17. ${ }^{1} \mathrm{H}-{ }^{15} \mathrm{~N}$ HSQC-NMR titration experiments of $\alpha$-synuclein with anle137c and sery139. (A) Chemical shift perturbation plot and peak intensity profile of backbone amide resonances of ${ }^{15} \mathrm{~N}$-labeled $\alpha$-synuclein in presence of increasing concentrations of anle137c. (B) Chemical shift perturbation plot and peak intensity profile of backbone amide resonances of ${ }^{15} \mathrm{~N}$-labeled $\alpha$-synuclein in presence of increasing concentrations of sery 139 . 
The corresponding quantitative evaluation of the data is represented on figure 2.5.18A and 2.5.18B in form of chemical shift perturbation plots and peak intensity profiles of backbone amide resonances of $\alpha$-synuclein. Given that no significant deviation of chemical shifts and intensities of assigned resonances were observed along the polypeptide chain of $\alpha$-synuclein, these findings strongly indicate the lack of direct interactions of sery166a and sery 139 with monomeric $\alpha$-synuclein even at such high ligand-to-protein molar ratios. Of note, the higher resolution of HSQC spectra on figure 2.5.16A in comparison with $2.5 .16 \mathrm{~B}$ is mainly attributed to measurements at higher field NMR spectrometer, namely $900 \mathrm{MHz}$ and 600 MHz.

Although our NMR-based binding experiments acquired at high ligand-to-protein molar ratios failed to designate the interactions of monomeric $\alpha$-synuclein with sery $166 \mathrm{a}$ or sery139, we performed ${ }^{1} \mathrm{H}-{ }^{15} \mathrm{~N}$ HSQC-NMR titration studies with two DPP compounds (sery139 and anle137c) in order to explore the dose-dependence of residue specific changes in HSQC spectra. In line with the previously discussed findings, the residue specific analysis of the chemical shift perturbations and peak intensity changes in the titration series of HSQCs didn't provide evidences supporting the binding of DPP ligands to $\alpha$-synuclein monomers (Fig. 2.5.17A-B). Thus, the titration experiment for anle137c binding to monomeric $\alpha-$ synuclein at three different concentrations of ligand (ligand-to-protein molar ratios 1:1, 1:2 and 1:4) showed a lack of significant dose-dependent spectral changes as for the single residues as well as for the protein domains (Fig. 2.5.17A). In case of sery139, one could expect significantly stronger compound-induced changes due to increased ligand-to-protein molar ratios. However, the patterns of chemical shift and resonance intensities perturbation for each titration point, up to 15 fold molar excess of the ligand, do not support the binding of compound to the protein as evident from overall minor changes characterized by poor dose dependency (Fig. 2.5.17B). The observed deviation of chemical shifts and intensities of assigned resonances might be attributed to slight variations of $\mathrm{pH}$, measurement conditions and DMSO concentration.

Due to promising properties of anle138c and anle145c regarding the modulation of $\alpha$ synuclein misfolding in the various assays, we also included these compounds in the HSQCbased NMR binding studies despite their limited solubility in the aqueous buffers. As shown on figure $2.5 .18 \mathrm{C}$ and $2.5 .18 \mathrm{D}$, the addition of corresponding small molecules to monomeric $\alpha$-synuclein did not induce any significant changes in chemical shifts when compared with the free protein. Consistent with the NMR data for other DPP compounds mentioned above, the lack of pronounced effects of the ligands (anle138c or anle145c) on assigned resonances 
along the polypeptide chain of $\alpha$-synuclein supports the notion of absence of strong specific interactions between these small molecules and monomeric $\alpha$-synuclein.

A
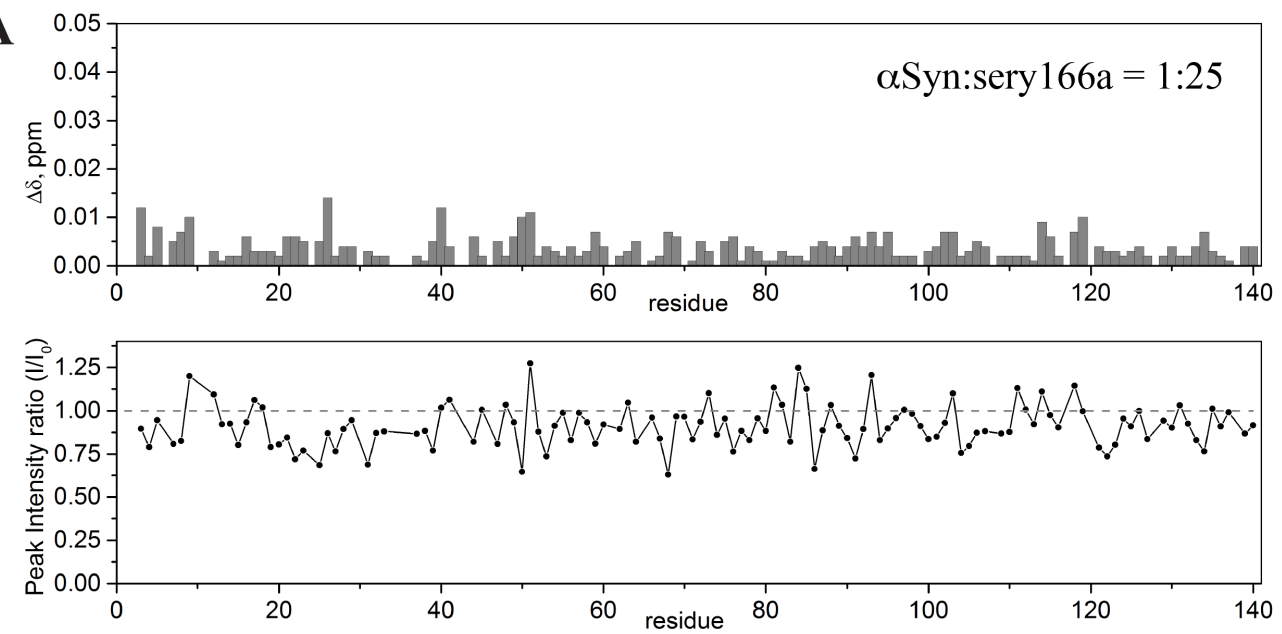

B
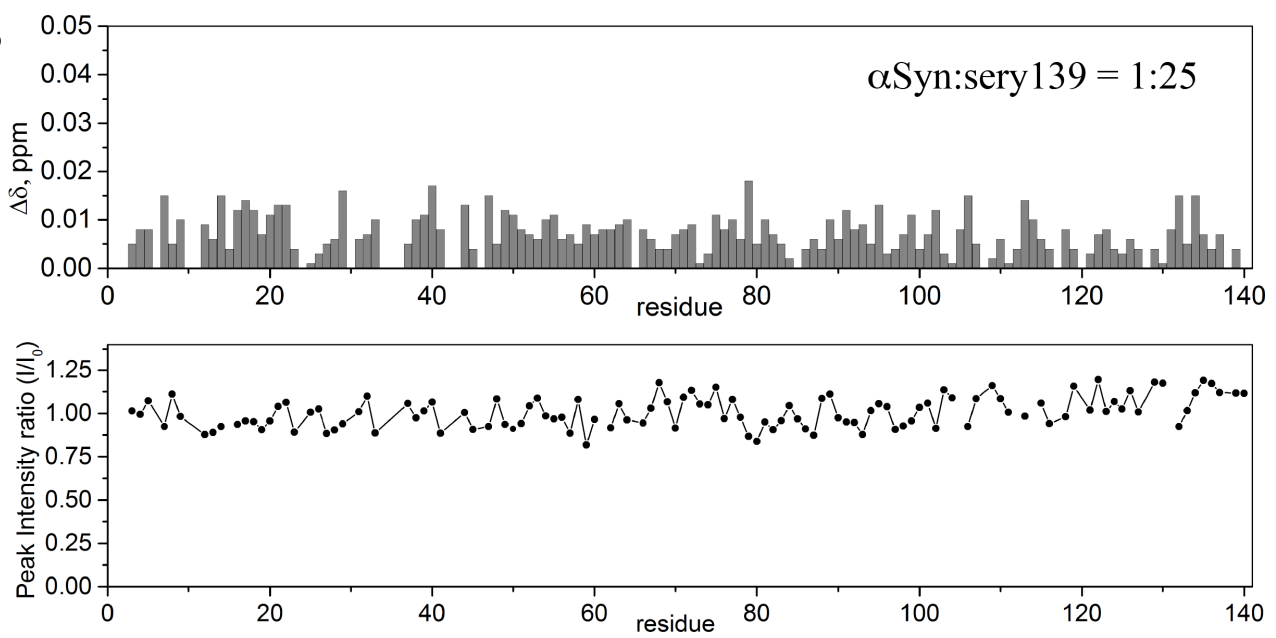

C

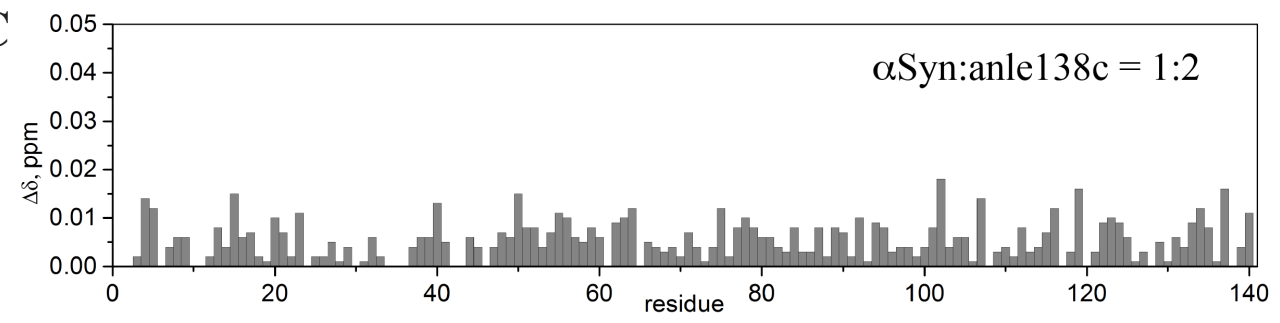

$\mathbf{D}$

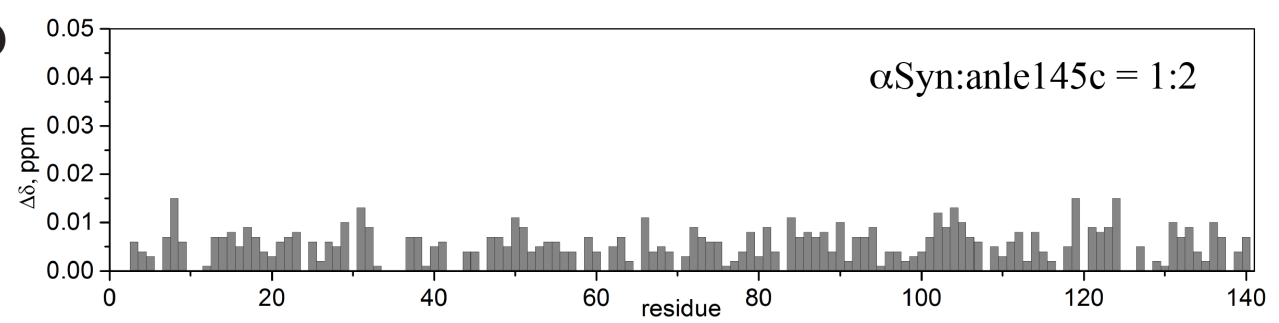

Figure 2.5.18. ${ }^{1} \mathrm{H}-{ }^{15} \mathrm{~N}$ HSQC-NMR experiments with $\alpha$-synuclein and selected DPP compounds. (A-D) Chemical shift perturbation plots and peak intensity profiles of backbone amide resonances of ${ }^{15} \mathrm{~N}$-labeled $\alpha$-synuclein in presence of sery166a (A), sery139 (B), anle138c (C) and anle145c (D) at various molar ratios. 
Overall, the summarized above results of the HSQC-based binding studies argue for no direct binding of all tested compounds to monomeric $\alpha$-synuclein. These findings allow us to speculate that the aggregation inhibitory activity of DPP molecules is not associated with the specific interactions of DPP compounds and $\alpha$-synuclein in monomeric form, although these biophysical investigations have been done only with the very soluble DPP compounds. Due to extremely low solubility, we could not use such assays for anle138b.

\subsubsection{Introduction to NMR STD experiments}

Despite the extensive HSQC-based NMR studies, the question of how DPP compounds are capable to inhibit the $\alpha$-synuclein fibrillization remains unresolved. Therefore, we extended our NMR biding studies to a fundamentally different approach that is described in details below.

Overall, there are two main types of NMR-based binding studies; first, it is direct measurements of biomolecule in presence and absence of the ligand, of note, the discussed above HSQC studies with DPP ligands utilizing the related chemical shifts mapping approach $^{453,454}$. Despite many advantages of the direct monitoring of target molecule, there are also a number of drawbacks, including requirements for specific labeling and relatively high amounts of biomolecules, long measurement times, molecular weight limit and a poor compatibility with the ligand screening campaigns. The second type of NMR-based binding studies is focused on the ligand itself and the characterization of complex formation is achieved by evaluation of changes in ligand properties that are induced upon binding to the

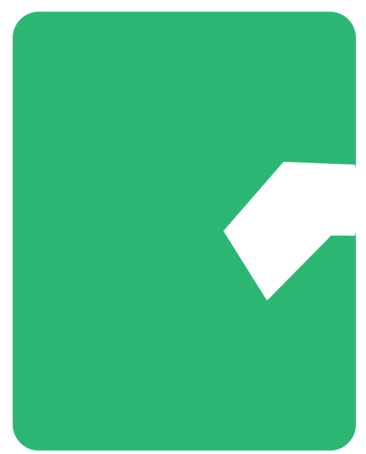

Big Protein

- slow Brownian motion;

- slow diffusion;

- long correlation time $\tau_{c}$

- fast relaxation, short $\mathrm{T} 2$

- negative NOE
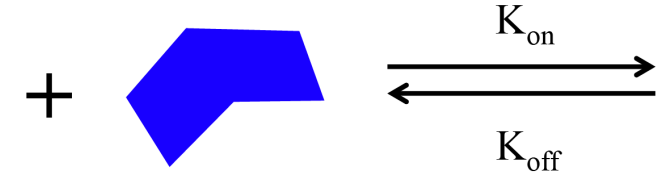

Small Ligand:

- fast Brownian motion;

- fast diffusion;

- short correlation time $\tau_{\mathrm{c}}$

- slow relaxation, long T2

- possitive or zero NOE

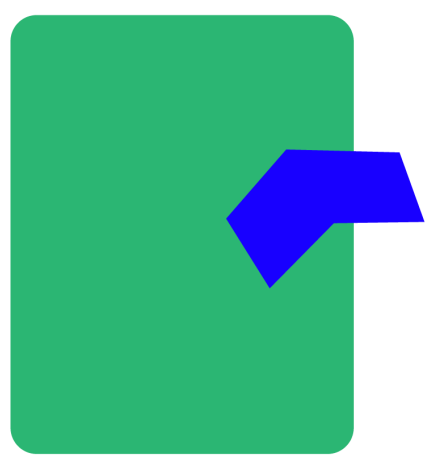

Complex of

Big Protein and Small Ligand: Small ligand starts to behave like a big protein, attaining the motional and relaxation properties of whole complex.

Figure 2.5.19. Motional and relaxation properties of small molecular weight ligands, big macromolecules and corresponding complexes. 
target biomolecule ${ }^{454,455}$. Although in majority of ligand-based or so-called "transfer" experiments it is not possible to obtain the residue-specific information about the binding site, there are also significant advantages of this NMR technique. For instance, lack of specific isotope labeling of any component, small amount of biomolecule, short measurement time and ligand screening protocols. Due to the simplicity of the measurements and following analysis, the "transfer" NMR binding studies have found wide application. To date, several types of ligand-based NMR binding methods have been developed and successfully applied, including saturation transfer differences (STD) experiments, transfer NOESY (trNOE)-related 1D and 2D experiments, NMR experiments with relaxation filter, diffusion-edited experiments, binding studies with immobilized or paramagnetic-labeled macromolecules ${ }^{454}$, 455. The underlying principle for such "transfer" techniques is based on phenomena, in which the bound small ligand starts to behave like a whole complex and, thereby, gains certain physical properties of high molecular weight structures. Thus, the free small molecular weight ligands are characterized by fast diffusion and Brownian motion; short correlation time $\left(\tau_{\mathrm{c}}\right)$, slow relaxation with long $\mathrm{T}_{2}$, and small positive or close to zero NOE rates (Fig. 2.5.19). In contrast, the high molecular weight biomolecules exhibit slow motion properties, fast relaxation with short $T_{2}$ time and strong negative NOE rates (Fig. 2.5.19). As it has been already mentioned, the interactions of the small ligand and the target macromolecule affect the listed ligand properties that become similar to the behavior of the whole complex. For instance, one can observe the broadening of the ligand signals in presence of target biomolecule due to faster $T_{2}$ relaxation. Another good example of related NMR studies is $1 D$ or $2 \mathrm{D}$ NOESY experiments, in which binding events lead to the generation of strong NOE signals for ligand resonances (same sign as diagonal, i.e. negative NOE rates), also known as a trNOE ${ }^{455}$. Similarly, the other types of ligand-based NMR experiments are able to identify the changes of the spectral properties and eventually select the interacting compounds and characterize the corresponding binding properties ${ }^{456}$.

Among the listed "transfer" techniques, we have selected STD NMR experiments ${ }^{457}$, 458 as a potentially powerful technique to study the interaction of DPP compounds with monomeric as well as oligomeric $\alpha$-synuclein. STD experiments are also utilizing the trNOE phenomenon and could be considered as a 1D steady-state NOE study. The information about the binding events in STD is derived from the detection of magnetization transfer from the biomolecule to the interacting ligand. In other words, the protein resonances are selectively saturated by irradiation of the region of the spectra lacking the signals of the ligand. In case of complex formation, the saturation from the irradiated protein protons is transferred to the 
bound ligand's protons and after dissociation could be detected on the free ligand protons in solution. Experimentally, the STD spectra are recorder for a high ligand concentration in presence of substoichiometric amounts of the protein or other high-molecular compounds, where standard ratio ligand-to-target varies in a range 1:20-1:200. The STD effect is evaluated by generation of the differences spectrum from on-resonance set of data, in which the protein proton signals are saturated, and off-resonance set of data, in which the saturation frequency is far from any signals, for instance, $30-50 \mathrm{ppm}$. The size of STD effect depends on many parameters, including the off rates, the ligand-to-target ratio, the saturation time ${ }^{455}$. Importantly, the STD-based NMR experiments provide a powerful and sensitive approach to detect and characterize interaction as in measurements with a single ligand as well as in competition studies with the several compounds. Therefore, there is a huge potential of STDbased strategy focused on ligand screening applications, SAR analysis and related structure optimization.

\subsubsection{Interactions of DPP compounds with $\alpha$-synuclein studied by STD NMR}

Considering the advantages of STD NMR binding studies, we have utilized this technique to detect and characterize the potential binding of DPP compounds to various forms of $\alpha$-synuclein. First, we selected 3 candidates taking in account following aspects: high aggregation inhibitory activity (SIFT, ThioT) and the good solubility in order to reach high ligand-to-protein molar ratios and eventually high sensitivity. According to listed requirements, sery166a, sery139 and anle137c have been chosen for STD NMR experiments (Fig. 2.5.20). In addition to strong aggregation inhibition, these compounds are also possessing the desirable solubility range in aqueous buffers, namely $1 \mathrm{mM}$ for sery $166 \mathrm{a}$ and sery $139 ; 300 \mu \mathrm{M}$ in case of anle137c.
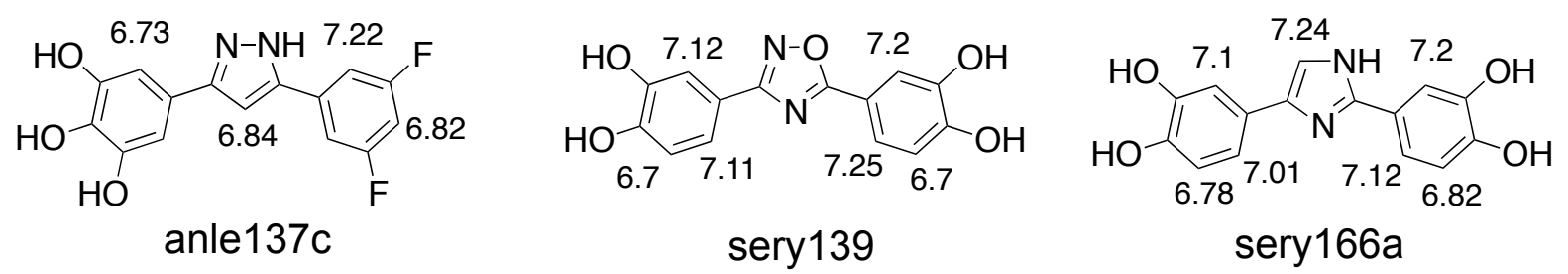

Figure 2.5.20. DPP compounds selected for STD experiments.

In an attempt to clarify the aspects of interactions between DPP compounds and $\alpha$ synuclein, we have recorded the titration series of STD spectra varying the ligand excess from 1:10 up to 1:200, while the protein concentration was constant ( $3 \mu \mathrm{M} \alpha$-synuclein for anle137c and $5 \mu \mathrm{M} \alpha$-synuclein for sery166a or sery139). Based on published STD 
experiments, we employed the shaped pulses for selective and efficient saturation of the protein resonances in aliphatic region of NMR spectra. To exclude a potential non-specific saturation of the ligands, we acquired a set of control STD measurements under similar experimental setup on samples containing just the ligand at highest possible concentration. For the data representation and analysis, the series of STD spectra were generated in combination with calculations of corresponding STD amplification factor ${ }^{455,457}$.

The STD amplification factors were calculated according to equation below, where $\mathrm{I}_{0}$ - intensity of the signal(s) in the off-resonance spectrum; $I_{s a t}$ - intensity of the signal(s) in the on-resonance spectrum; $\mathrm{I}_{0}-\mathrm{I}_{\text {sat }}$ - intensity of the signal(s) in the STD spectrum. The values of $I_{0}$ and $I_{\text {sat }}$ were obtained by integration of signals in corresponding spectra. Due to a small variation of STD effect among given protons or group of protons within a certain ligand, averaged STD amplification factors were generated.

$$
\text { STD amplification factor }=\frac{I_{0}-I_{\text {sat }}}{I_{0}} \times \text { ligand excess }
$$

Being introduced by Mayer and Meyer ${ }^{457}$, STD amplification factor clearly represents a useful tool for quantification and comparison of STD data. For instance, the use of titration

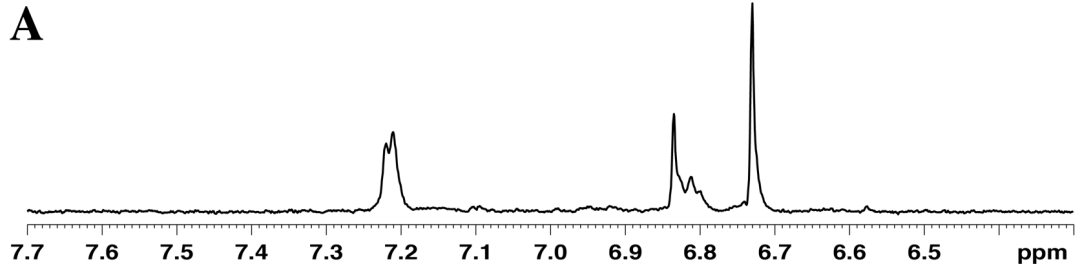

B

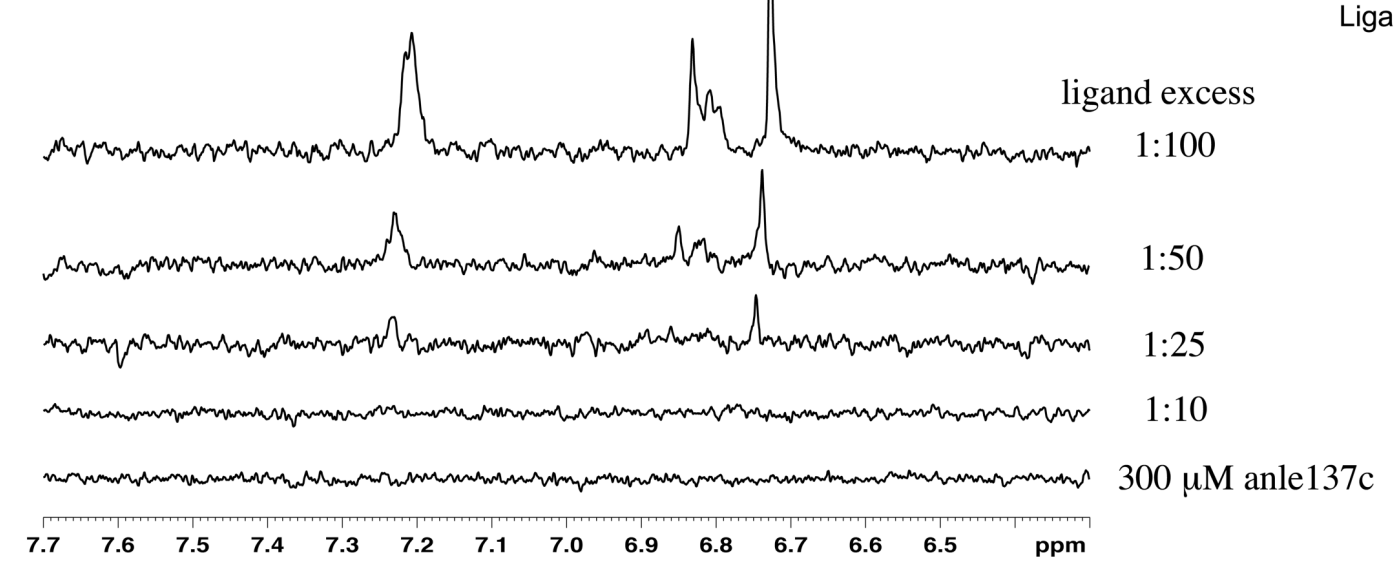

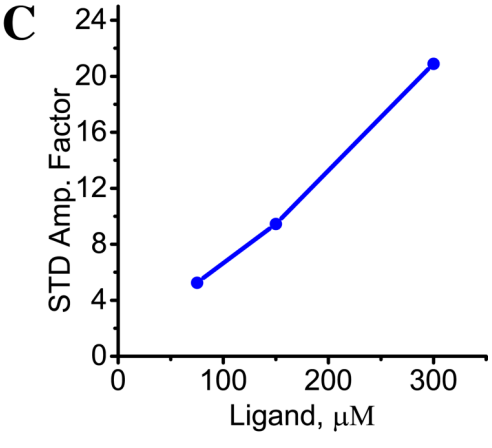

Figure 2.5.21. Characterization of anle137c- $\alpha$-synuclein interactions by STD NMR. (A) Reference 1H NMR spectrum of anle137c. (B) STD NMR spectra recorded at different anle137c: $\alpha$-synuclein ratios and for anle137c alone (irradiation at $1.5 \mathrm{ppm}, \alpha$-synuclein 3 $\mu \mathrm{M})$. (C) Averaged STD amplification factor as a function of anle137c concentration. 
plots monitoring the increase of STD amplification factor of the ligand versus the ligand concentration provides a possibility to derive ligand-receptor binding affinities and KD values $^{455,457}$. STD amplification factor clearly represents a useful tool for quantification and comparison of STD data. For instance, the use of titration plots monitoring the increase of STD amplification factor of the ligand versus the ligand concentration provides a possibility to derive ligand-receptor binding affinities and $K_{D}$ values ${ }^{455,457}$. From another point of view, STD amplification factor shows how many ligand molecules are saturated per the molecule of the target biomolecule, thereby giving information about magnitude of STD effects and sensitivity of corresponding NMR experiments. However, current research points towards inherited limitations of direct STD-based $K_{D}$ determination due to significant dependence of STD effects on lifetime of the protein-ligand complex, saturation time, relaxation properties of the ligand, rebinding events and others ${ }^{455}$.

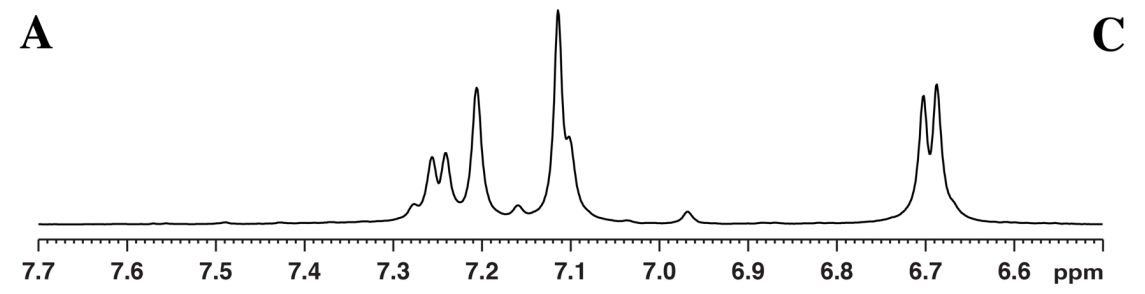

\section{B}
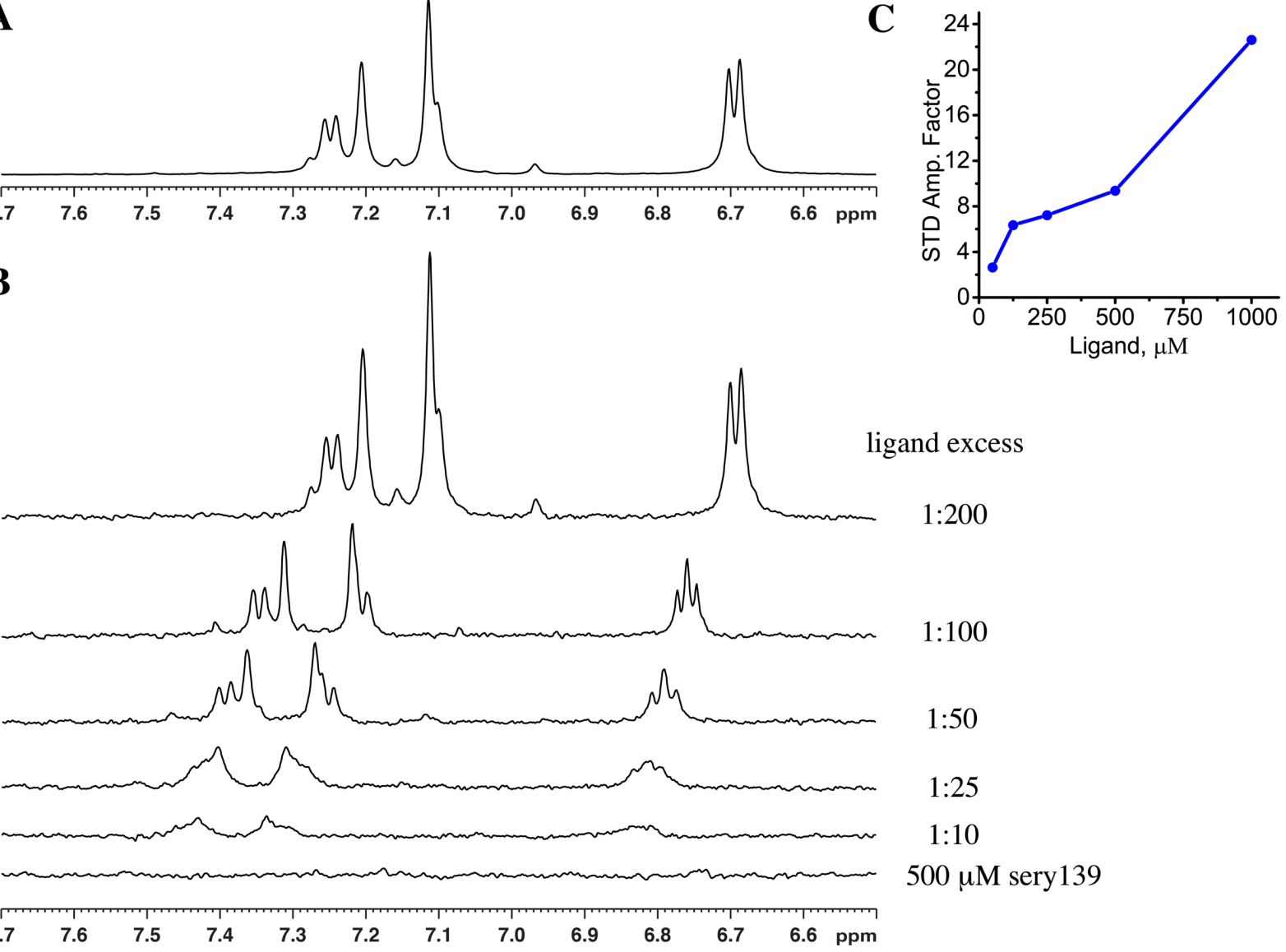

ligand excess

$1: 200$

$1: 100$

$1: 50$

$1: 25$

$1: 10$

$500 \mu \mathrm{M}$ sery 139

Figure 2.5.22. Characterization of sery139- $\alpha$-synuclein interactions by STD NMR. (A) Reference 1H NMR spectrum of sery139. (B) STD NMR spectra recorded at different sery 139: $\alpha$-synuclein ratios and for sery139 alone (irradiation at $1.2 \mathrm{ppm}, \alpha$-synuclein 5 $\mu \mathrm{M}) .(\mathbf{C})$ Averaged STD amplification factor as a function of sery139 concentration. 
Although our HSQC-based binding experiments failed to designate the interactions of monomeric $\alpha$-synuclein with sery166a and sery139, we acquired the titration series of STD spectra for three DPP compounds of interest, namely anle137c, sery139 and sery166, and monomeric $\alpha$-synuclein (Fig. 2.5.21-2.5.23). For on-resonance experiments, the selective saturation of the signals of the protein was achieved by placing the irradiation frequency in aliphatic region, where no signals of the ligands are located (1.2 ppm for STD NMR with sery139 and sery166a; 1.5 ppm for STD NMR with anle137c), whereas the off-resonance frequency was set to $50 \mathrm{ppm}$. As shown on figures 2.5.21B $-2.5 .23 \mathrm{~B}$, the lowest spectra, the selected DPP compounds at high concentrations didn't show any STD signals in absence of $\alpha$ synuclein.

Using the titration STD NMR experiments, we were able to detect the binding events between $\alpha$-synuclein and all selected DPP molecules. The titration series of STD spectra for each DPP compound are shown on figures 2.5.21-2.5.23, together with reference proton
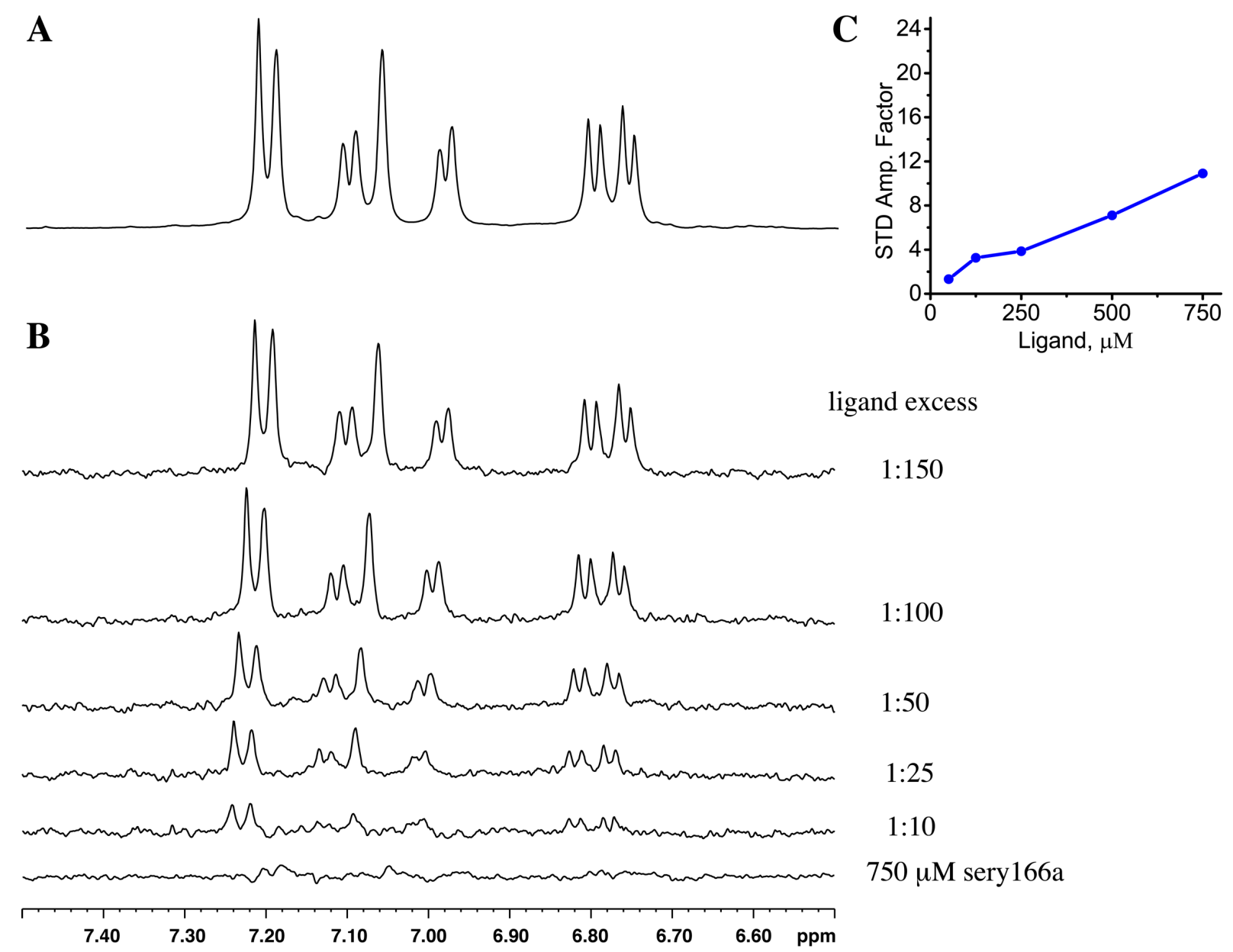

Figure 2.5.23. Characterization of sery166a- $\alpha$-synuclein interactions by STD NMR. (A) Reference 1H NMR spectrum of sery166a. (B) STD NMR spectra recorded at different sery 166a: $\alpha$-synuclein ratios and for sery166a alone (irradiation at $1.2 \mathrm{ppm}, \alpha$-synuclein 5 $\mu \mathrm{M})$. (C) Averaged STD amplification factor as a function of sery166a concentration. 
spectra of the pure ligands and binding isotherms of STD amplification factors. The presence of STD signals that associated with respective DPP ligand in the differences spectrum proves the transfer of the magnetization from the saturated biomolecule to small organic molecule, thereby confirming the interactions of $\alpha$-synuclein with sery166a, sery139 and anle137c. For instance, the STD spectra that were acquired for sery139 at different ratios to $\alpha$-synuclein show a growth of STD effect with increasing ligand concentration (Fig. 2.5.22 B). Moreover, the intensities of the signals of all ligand protons in STD are quite similar to the reference spectra of pure sery139. In theory, the strongest STD effect is expected for the protons of the ligand that are in close proximity to saturated protons of the biomolecule and, eventually one can extract information about the binding epitope. Therefore, it is feasible that all protons of the DPP ligand are relatively equally involved in interaction with $\alpha$-synuclein, since the variation of saturation efficiency between the individual or overlapped resonances is small, within $10-15 \%$. For example, among the four signals in sery 166 a spectrum, namely doublet $7.2 \mathrm{ppm}$, multiplet 7.15-7.05 ppm, doublet $7.0 \mathrm{ppm}$ and multiplet 6.85-6.7, the strongest STD effect is observed for doublet at 7.2 ppm. Hence, other signals exhibit 0.94 (7.15-7.05 ppm), $0.85(7.0 \mathrm{ppm})$ and $0.91(6.85-6.7 \mathrm{ppm})$ STD intensity normalized to degree of saturation of doublet at 7.2 ppm as calculated from STD experiment with 150-fold excess (Fig. 2.5.23B).

To further evaluate the parameters of interaction between $\alpha$-synuclein and selected DPP molecules, we calculated the averaged STD amplification factor as described in details above. Taking in account the small variation of STD effect among the individual protons of corresponding ligands, the averaged STD amplification factors were generated by averaging the calculated values of individual protons and overlapped resonances. Next, we plotted the STD amplification factors against the concentration of the ligand in order to obtain the hyperbolic dose-response curves, also known as binding isotherms, that allow to derive the values of equilibrium dissociation constants $\left(\mathrm{K}_{\mathrm{D}}\right)$. As shown on figures 2.5.21C -2.5.23C, the analysis of the binding isotherms for all tested compounds revealed almost a linear increase of STD amplification factor with the ligand concentration. To that end, the $\mathrm{K}_{\mathrm{D}}$ calculations are not possible within the available concentration range since no saturation of the binding site(s) is reached. Overall, the high absolute values of STD amplification factors determined at high ligand-to-protein ratios might be attributed to fast turnover between the bound and free state, thereby suggesting high values of rate dissociation constants and eventual weak affinity. On the other hand, increasing of the ligand concentration might have an effect on $\alpha$-synuclein state and ultimately resulting in variation of sample composition. Since, DPP compounds are able to stabilize the off-pathway oligomeric species upon incubation, as discussed in details in 
previous chapters, it is therefore envisioned that the addition of DPP compounds to monomeric $\alpha$-synuclein during titration experiments may facilitate the oligomerization of $\alpha$ synuclein. This hypothesis is also supported by DLS data, which indicates the presence of oligomerized $\alpha$-synuclein even at initial time points of aggregation. Hence, it is conceivable that the linear growth of the STD amplification factors with increasing ligand concentration might be related to the changes of the sample composition leading to gradual oligomerization and generation of novel binding sites. Collectively, the results of STD-based titration studies allow us to make several important conclusions. First, we have demonstrated that DPP compounds indeed bind to $\alpha$-synuclein and the corresponding interactions with monomeric protein are weak. Importantly, it also has been proposed that DPP compounds can interact with oligomerized forms of $\alpha$-synuclein. To explore further this hypothesis, additional STD NMR experiments with a variation of on-resonance frequencies were carried out with sery $166 \mathrm{a}$ and $\alpha$-synuclein samples of various compositions.

\subsubsection{Interactions of sery 166 a with oligomeric $\alpha$-synuclein studied by STD NMR}

Based on STD NMR titration studies, DPP compounds have been proposed to interact with the aggregated forms of $\alpha$-synuclein. Considering the importance of these interactions in context of a potential mechanism of aggregation inhibition and stabilization of off-pathway oligomeric species, we employed the STD NMR technique to uncover the details of binding mode.

This question has been addressed experimentally by using two approaches. In the first approach, we utilized the STD-based NMR experiment, in which the selective saturation of the oligomeric species was achieved. Practically, the underlying principle of this NMR study is the previously discussed findings showing that $\alpha$-synuclein oligomers formed in presence of DPP compounds have the high molecular weight and, therefore, produce the very broad signals in NMR spectrum. Hence, for on-resonance experiments we selected the position of the irradiation pulse in such way that the saturation of the broad and "invisible" signals of oligomeric protein is achieved without affecting signals of monomeric protein and ligand itself. The basis of the second approach was STD measurements of the protein samples containing different fractions of oligomeric $\alpha$-synuclein. To generate these samples, we took the advantage of the aggregation assay in presence of DPP compounds, in which the timedependent gradual formation of high-molecular weight $\alpha$-synuclein complexes is observed. As discussed in previous chapters, upon incubation of $\alpha$-synuclein with one of the watersoluble DPP molecules it is possible to obtain the protein preparations with oligomeric 
enrichment up to $20-25 \%$. The main goal of this experiment was to monitor the changes of STD effect at various sample compositions and different positions of irradiation pulse and eventually to obtain insight into the role of DPP compounds. In agreement with this line of thoughts, sery166a was selected both as the ligand for STD studies and as the aggregation inhibitor for oligomer preparation. Among the arguments for sery166a selection are the established kinetic of aggregation, high solubility and potent inhibitory activity.

Experimentally, we have measured six time points during course of aggregation, namely $0 \mathrm{~h}, 24 \mathrm{~h}, 48 \mathrm{~h}, 72 \mathrm{~h}, 96 \mathrm{~h}$ and $144 \mathrm{~h}$. With respect to variation of the irradiation frequency, for all samples, except the $144 \mathrm{~h}$ time point, we acquired four positions of saturation pulse, $1.2 \mathrm{ppm},-0.8 \mathrm{ppm},-1.3 \mathrm{ppm},-2.3 \mathrm{ppm}$ and $-4.0 \mathrm{ppm}$. In case of $144 \mathrm{~h}$ aggregated sample that is supposed to contain the highest load of oligomers we extended the STD measurements with additional four irradiation frequencies, including -7 ppm, -10 ppm, $15 \mathrm{ppm},-20 \mathrm{ppm}$. This experimental setup allows us to estimate the degree of signal broadening for oligomeric species. Of note, the aggregation assays were performed at $100 \mu \mathrm{M}$ of $\alpha$-synuclein and $100 \mu \mathrm{M}$ sery166a under standard conditions; regarding the STD probes, corresponding aliquots were collected from aggregated mixtures and adjusted to provide the following composition: $\alpha$-synuclein $10 \mu \mathrm{M}$, sery $166 \mathrm{a} 1 \mathrm{mM}$.

The results of STD experiments, which are described above, are summarized on figure 2.5.24. Taken together, our data provides strong evidence that sery166a has a high affinity to oligomeric forms of $\alpha$-synuclein. There are several experimental observations supporting these conclusions. First of all, the analysis of the STD effects of sery166a showed that the transfer of magnetization occurs even if the irradiation pulse is not affecting the signal of the monomeric protein (Fig. 2.5.24A). Among the measured experimental conditions, the selective saturation of broad and "invisible" signal of the high molecular weight oligomers was achieved when positions of the pulse were upfield relative to $-1.3 \mathrm{ppm}$. Importantly, we were able to detect interactions between sery166a and oligomeric species even for a sample containing freshly prepared monomeric $\alpha$-synuclein. We conclude, therefore, that in presence of sery166a the oligomerization process of $\alpha$-synuclein takes place or, alternatively, $\alpha$ synuclein preparations under applied conditions may contain small percentage of high molecular weight species. Next, we measured STD experiments over the course of $\alpha$ synuclein/sery166a aggregation, for which the time dependent accumulation of oligomers is expected. Figures 2.5.24A and B demonstrate that STD amplification factors for all onresonance frequencies are gradually rising with the incubation times. In other words, the intensity of the STD effect, as indication of interactions, has a clear correlation with the 
A

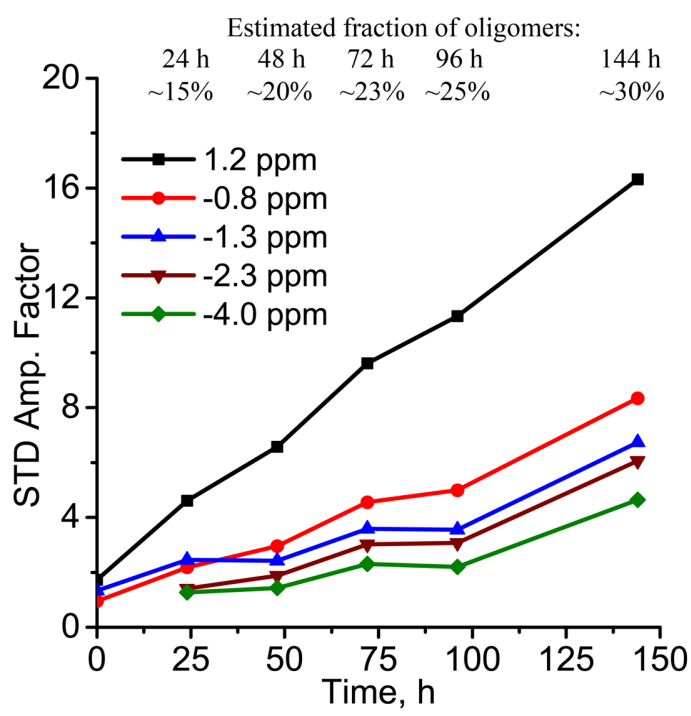

C

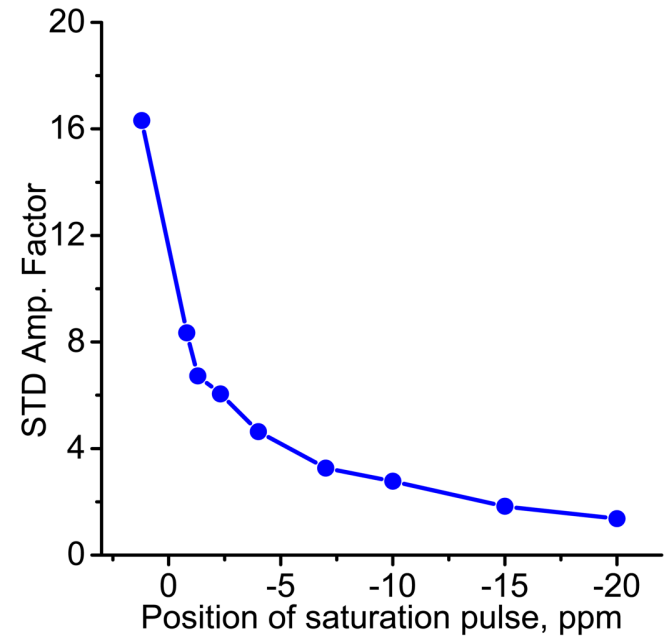

B

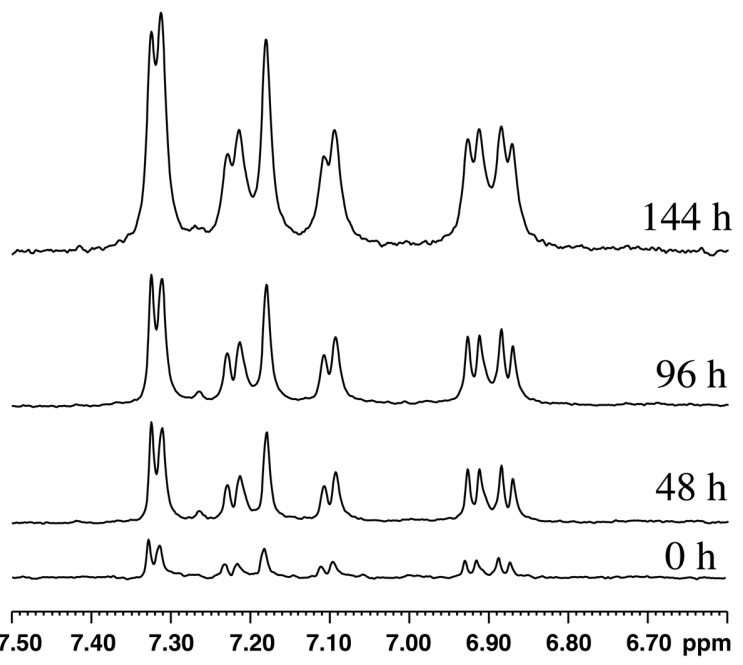

D

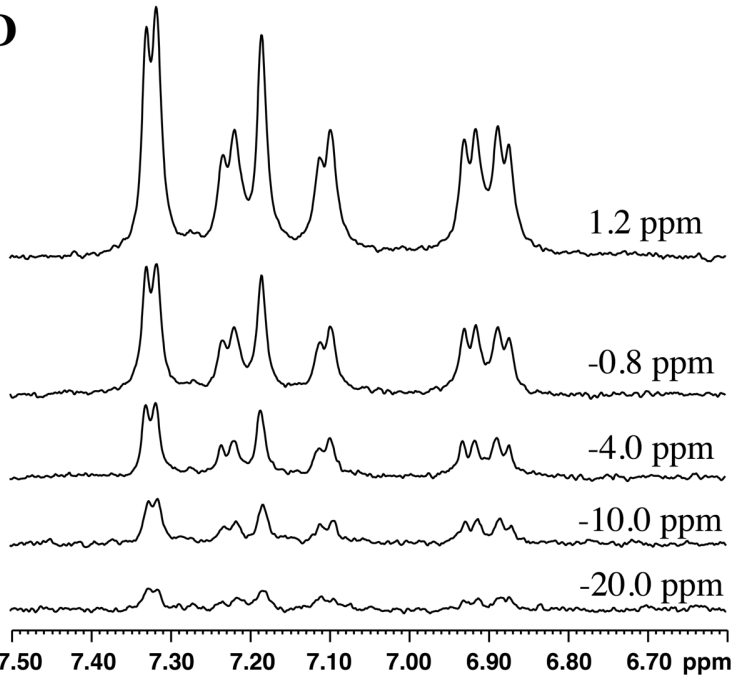

Figure 2.5.24. Characterization of sery166a- $\alpha$-synuclein interactions by STD NMR over the course of $\alpha$-synuclein aggregation in presence of sery166a. (A) Averaged STD amplification factors obtained at various irradiation frequencies as a function of aggregation time. (B) Selected STD NMR spectra recorded at different aggregation time points. (Irradiation at $1.2 \mathrm{ppm}$ ) (C) Averaged STD amplification factor obtained at various irradiation frequencies on sample aggregated for $144 \mathrm{~h}$. (D) Selected STD NMR spectra recorded at different irradiation frequencies on sample aggregated for $144 \mathrm{~h}$. (All STD probes: $\alpha$-synuclein $10 \mu \mathrm{M}$, sery $166 \mathrm{a} 1 \mathrm{mM}$ )

amount of oligomeric species in the corresponding samples. Here is our line of thinking based on this data: over the course of aggregation approximately $20-25 \%$ of monomeric $\alpha$-synuclein coverts to oligomeric state; therefore, for later time points one could expect the reduction of STD effect if sery166a interacts with monomeric protein; contrarily, we clearly detect the growth of STD effect that could be explained by binding of sery166a to newly generated oligomers and STD signal is stronger the more oligomers and, thereby, binding sites are 
present. Our finding implies that sery166a and other DPP compounds bind predominantly to oligomeric species and, presumably, these interactions constitute the central pathway that leads to stabilization of oligomeric species of $\alpha$-synuclein and, consequently, to inhibition of fibrillization process. Whether a small subpopulation of the hydrophilic DPP compound binds very tightly to the oligomer and then further DPP compounds bind weakly to this oligomer is unresolved, but quite probable since the aggregation inhibition occurs already at one digit micromolar concentrations such that the several 100 micromolar concentrations used here should lead to saturation. Thus, the compounds seem to bind to the already compound stabilized oligomers weakly.

To explore further the properties of compound-stabilized oligomers and corresponding interactions, we carried out extended STD NMR experiments on the samples, which were incubated for $144 \mathrm{~h}$ and had highest concentration of oligomeric species. First of all, we were interested how broad are the signals of $\alpha$-synuclein oligomers possessing the affinity to sery166a. We acquired the series of STD experiments by applying for on-resonance set of data the saturation pulses in a range of $1.2 \mathrm{ppm}$ to $-20 \mathrm{ppm}$. The calculated STD amplification factors are plotted as a function of irradiation frequency on figure 2.5.24C and representative STD spectra are shown on figure 2.5.24D. Given that the measured STD effects as representation of the binding events are detectable even at $-20 \mathrm{ppm}$, it seems reasonable to estimate that corresponding "invisible" $\alpha$-synuclein species interacting with sery166a have relatively high molecular weight and give broad "invisible" signals in proton NMR spectrum up to $-20 \mathrm{ppm}$. In this context, the further development and application of STD methodology can provide a theoretical possibility to perform more accurate characterization of ligandoligomer complex, including estimation of the mixture composition, affinity constants, correlation times and size of the complex. However, this work is beyond the scope of my thesis.

Overall, the take-home messages from STD-based binding studies are that the DPP compounds preferentially interact with oligomeric species, while the affinity to monomeric $\alpha-$ synuclein is weak. It's, therefore, envisioned that the mechanism of aggregation inhibition by DPPs might be associated with the interactions between small ligands and oligomeric forms that, in turn, lead to stabilization of specific off-pathway oligomers and, ultimately, blocking of the fibril formation. 
2.5.14. Characterization of DPP-stabilized $\alpha$-synuclein oligomers by solid state NMR

According to previously presented data, the structural characterization of DPP compound-stabilized off-pathway oligomers posits numerous fundamental challenges, including high molecular weight, complex composition of the oligomeric preparations, dynamic nature and conformational flexibility of corresponding protein assemblies. Therefore, solid state NMR (ssNMR) spectroscopy has attracted considerable interest as a technique that is able to investigate the structure of big protein complexes with the single residue resolution. Good examples of the ssNMR-based structural studies of various amyloid fibrils that have been done in our department ${ }^{181}$ ). Moreover, the ssNMR spectra were acquired for potentially on-pathway $\alpha$-synuclein oligomers that are produced by cold-induced dissociation of the corresponding amyloid fibrils ${ }^{11}$. Although the residue specific analysis of these samples was not possible, the evaluation of ${ }^{13} \mathrm{C}$ cross-polarization $1 \mathrm{D}$ spectra provided valuable information regarding the transformation of secondary structure in comparison with fibrillar and monomeric $\alpha$-synuclein.

In close collaboration with ssNMR group of our department, we employed the ssNMR spectroscopy to get insight into the structural properties of $\alpha$-synuclein oligomers stabilized by DPP compounds. The ssNMR measurements were performed by Dr. Ashutosh Kumar, I did the preparation and characterization (ThioT, DLS) of the corresponding samples. Thus, we generated and analyzed several samples containing anle138c-stabilized $\alpha$-synuclein oligomers both from uniformly $\mathrm{C}^{13} / \mathrm{N}^{15}$-labeled $\alpha$-synuclein. The respective oligomeric preparations we obtained by 3-4 days incubation of monomeric $\alpha$-synuclein $(70 \mu \mathrm{M})$ in presence of anle138c $(100 \mu \mathrm{M})$ under standard conditions. To follow the process of oligomerization and characterize the composition of these samples, several complimentary biophysical techniques, including ThioT assay, DSL and EM, were applied. Due to the crucial requirements of ssNMR regarding the amount of isotope-labeled material and, especially, in case of 2D experiments, the desired sensitivity was successfully achieved by consecutive centrifugal concentrations in Centricon YM-10 filters up to 60 folds and approximately up to $4 \mathrm{mM}$ total protein concentration. To ensure the lack of impact of this preparation step on appearance and properties of oligomeric species, ThioT and EM were recorded before and after the centrifugal concentration. The representative EM micrographs and ThioT fluorescence spectra showed no obvious influence of sample preparation on the morphology and properties of generated $\alpha$-synuclein assemblies (Fig. 2.5.25 B). 
First, we recorded ${ }^{13} \mathrm{C}$ cross-polarization $1 \mathrm{D}$ spectra and compared the spectral properties of anle138b-stabilized oligomers with $\alpha$-synuclein monomer, cold-induced oligomers and fibrils. As observed in figure 2.5.25A, for each $\alpha$-synuclein preparation the unique spectra were obtained arguing for significant structural differences. Before discussing the properties of anle138c-stabilized oligomers, the ssNMR-based key findings in context of other $\alpha$-synuclein preparations are briefly summarized. Consistent with the previously published data 11,181 , the spectrum of $\alpha$-synuclein fibrils contains quite sharp signals
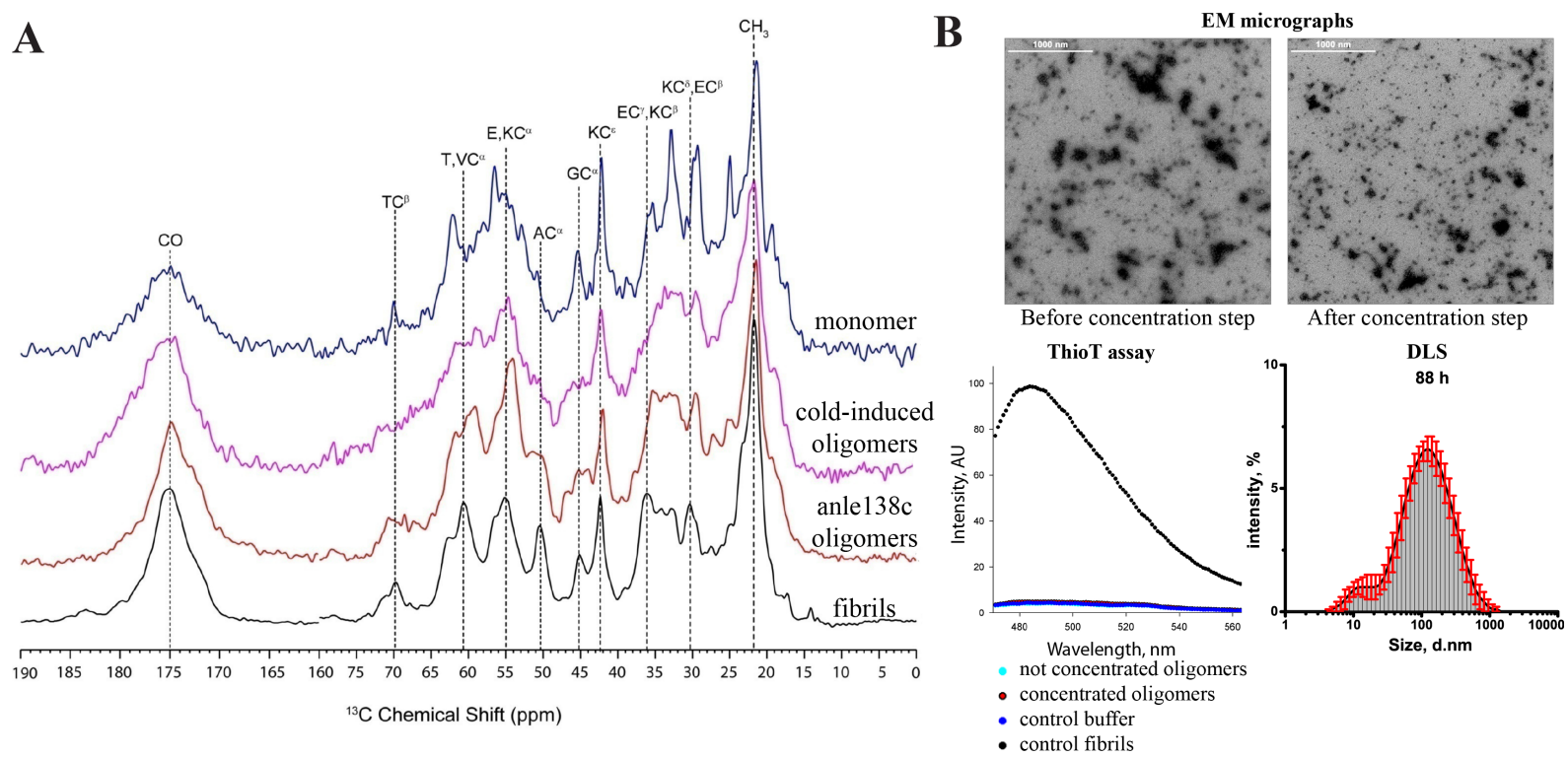

C
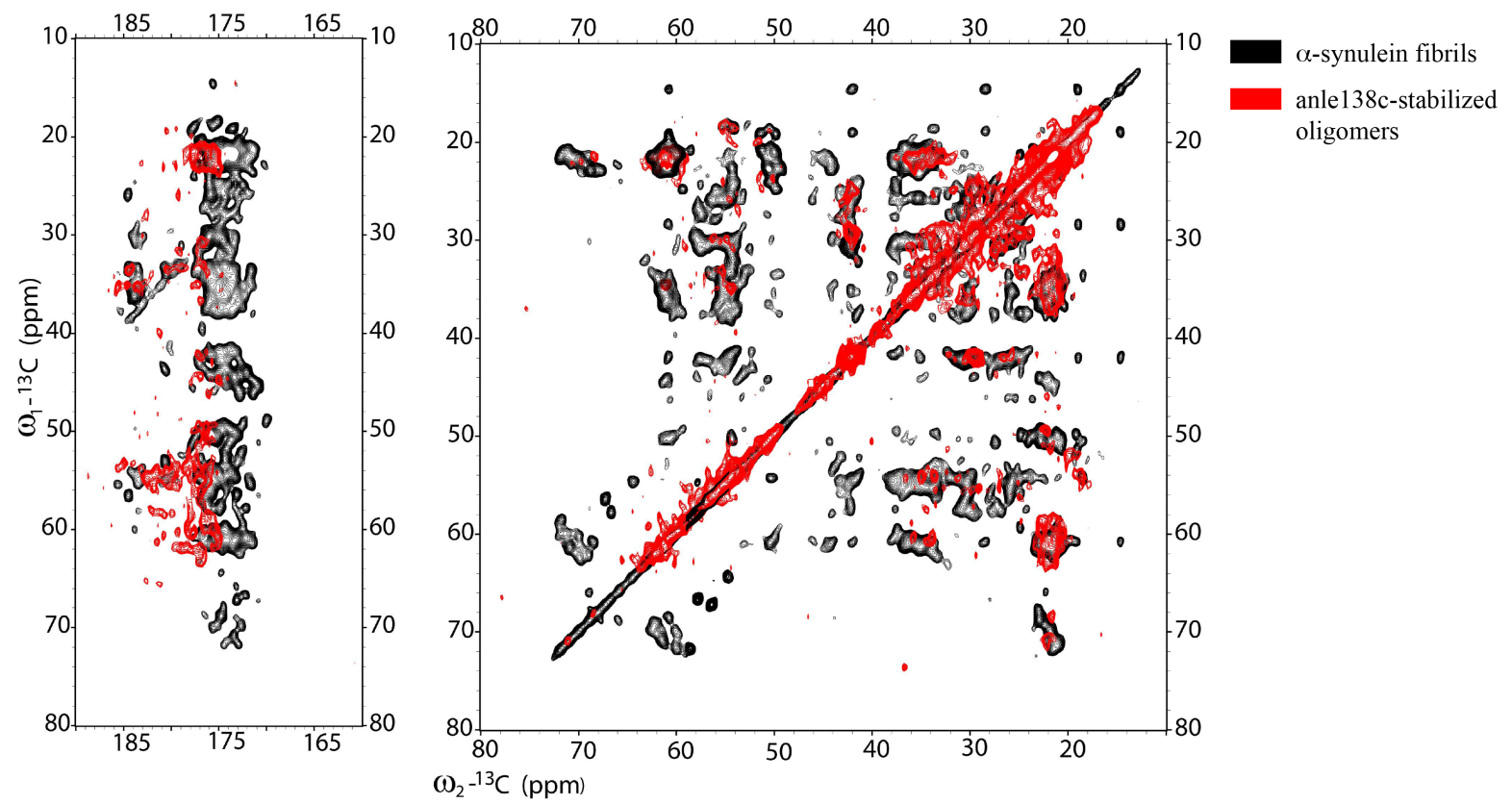

Figure 2.5.25. Characterization of anle138c-stabilized $\alpha$-synuclein oligomers by ssNMR. (A) Overplayed ${ }^{13} \mathrm{C}$ cross-polarization ssNMR spectra of $\alpha$-synuclein monomer, cold-induced oligomers, anle138c-stabilized oligomers and fibrils. (B) EM micrographs and ThioT fluorescence of anle138c-stabilized $\alpha$-synuclein oligomers before and after concentration step. (C) Overplayed ${ }^{13} \mathrm{C} 2 \mathrm{D}$ PDSD spectra of anle138c-stabilized $\alpha-$ synuclein oligomers and fibrils. 
indicating a well-ordered structure. Moreover, the analysis of chemical shifts changes of $\mathrm{C}^{\alpha}$ resonances corresponding to threonine, valine, glutamate, and lysine, namely an upfield shift for preparation composed of fibrils, confirmed the presence of $\beta$-sheet structure in the fibrillar material in comparison to monomeric protein. Regarding the $\alpha$-synuclein oligomers generated by cold-induced fibril dissociation, it has been proposed that structurally these assemblies exhibit non-fibrillar $\beta$-sheet structure that is intermediate between monomeric and fibrillar $\alpha$ synuclein. Moreover, the significant broadening of the resonances observed in the spectra of this preparation points toward the presence of heterogeneous distribution of oligomeric species. Finally, the monomers are mostly random coil and have weak tertiary interactions.

Coming back to anle138c-stabilized oligomers generated from uniformly $\mathrm{C}^{13} / \mathrm{N}^{15}$ labeled, we were able to record the good quality ${ }^{13} \mathrm{C}$ cross-polarization $1 \mathrm{D}$ spectra and, moreover, the $2 \mathrm{D}{ }^{13} \mathrm{C}$ proton-driven spin diffusion spectrum with relatively good resolution and signal-to-noise ratio (Fig. 2.5.25A and C). The evaluation of ${ }^{13} \mathrm{C}$ cross-polarization spectra and comparison with the previously described ssNMR data provide strong evidence that anle138c-stabilized oligomers are structurally different from all other $\alpha$-synuclein forms included in the study. Based on analysis of the chemical shift changes of threonine, valine, glutamate, and lysine $\mathrm{C}^{\alpha}$ resonances, it has been proposed that compound-induced oligomers are generally more structured than monomeric and cold-induced oligomers, but less than fibrils. To that end, the concomitant increase in $\beta$-sheet structure is observed in the order of: $\alpha$-synuclein monomer, cold-induced oligomers, anle138c-stabilized oligomers and fibrils. Comparison of $2 \mathrm{D}{ }^{13} \mathrm{C}$ proton-driven spin diffusion spectrum (PDSD), which is shown in figure 2.5.25C, has also corroborated the notion of significant distinctions between fibrils and compound-induced oligomers. Only few residue-types, such as hydrophobic Ala and Val and charged Glu and Lys contribute to the correlation spectrum of anle138c-stabilized oligomers. The appearance of these residues suggests that the anle138c-induced oligomers are stabilized by hydrophobic and salt-bridge interactions. Remarkably, $\alpha$-helical chemical shift, mainly from alanines, is observed in 2D PDSD spectrum of anle138c-stabilized species that was not detected in other $\alpha$-synuclein samples. Thus the compounds seem to stabilize $\alpha$-synuclein in a so far not described aggregated form that involves a significant proportion of $\alpha$-helical structure. In this context, it is also noteworthy to mention that ssNMR has emerged as a powerful technique for structural investigation and comparison of diverse aggregated protein forms. 
Taken together, these studies indicate that anle138c and most probably other DPP inhibitors are able to stabilize the unique oligomeric species that are structurally distinct from all other $\alpha$-synuclein forms measured so far. Moreover, based on all available biophysical data, it is feasible to assume that compound-stabilized oligomers might be off-pathway and, eventually, less toxic than on-pathway $\alpha$-synuclein aggregates.

\subsubsection{Comparison of DPP-stabilized oligomers with toxic oligomers}

The ssNMR-based observations that DPP compounds induce the formation of specific $\alpha$-synuclein oligomers that are structurally distinct from cold-induced oligomers highlight the importance of extensive comparison of properties among various types of generated species. Despite the fact that in $\alpha$-synuclein research field there is no clear understanding of which oligomers are most relevant and are involved in toxicity pathways, two types of promising oligomeric preparations have been developed and characterized in our department. One of these methods is cold-induced dissociation of $\alpha$-synuclein fibrils ${ }^{11,459}$, whereas the second protocol is based on incubation of triple proline (TP) $\alpha$-synuclein mutant (A30P, A56P, A76) ${ }^{269}$. Remarkably, the described proline mutations have been introduced according structure-based design and, thereby, TP mutant shows an impaired propensity to form amyloid fibril, but at the same time an increased oligomerization rate is observed upon incubation $^{269}$. What's more, the research findings reported in cited publications provide substantial arguments supporting the biological relevance of generated misfolded $\alpha$-synuclein species as evidenced by elevated toxicity in various in vitro and in vivo test models ${ }^{11,269,459}$.

Of particular significance in our project is the effect of DPP compounds on $\alpha$ synuclein oligomerization pathway in direct comparison with potentially on-pathway misfolded $\alpha$-synuclein. Thus, in addition to ssNMR data, the characterization has been achieved by the application of non-invasive approaches, including the native-PAGE and DLS analysis that are primarily provide information about the size of the generated species. In agreement with the ssNMR results, the DLS investigation also revealed significant differences between both $\alpha$-synuclein oligomeric preparations and DPP-stabilized oligomers. As shown in figure 2.5.26A, both oligomeric samples without DPP compounds are predominantly composed of monomeric protein with hydrodynamic diameter of 7-10 $\mathrm{nm}$ and oligomeric population characterized by broader size distribution with the maximum around 230 and $250 \mathrm{~nm}$ for mutant and cold-induced species, respectively. In contrast, the incubation of $\alpha$-synuclein with sery166a or other DPP compounds leads to stabilization of oligomeric species that are more heterogeneous and the maximum of hydrodynamic diameter is varying 
in ranges of 70-150 nm (Fig. 2.5.26A and 2.5.8). Based on this data, it has been hypothesized that DPP compound can modulate the aggregation of $\alpha$-synuclein and facilitate the formation of the oligomers that are significantly smaller than on-pathway misfolded species included in this study. The general assumption of the smaller sized distribution in case of compoundinduced oligomers is also confirmed by native-PAGE analysis. In this regard, the sample aggregated in presence of sery166a contains, in addition to the dominated band of monomeric $\alpha$-synuclein, immunoreactive signals from higher molecular weight structures, presumably compound-stabilized oligomers (Fig. 2.5.26B, lane 3 and 4, lane 3 contains 2x loaded material). The lane 1 in figure 2.5.26B shows the composition of aggregated TP $\alpha$-synuclein mutant. As expected, the incubation of this mutant induced the formation of oligomeric structures in the mixture with dominated monomeric state. As evident from this native-PAGE,

$\mathbf{A}$
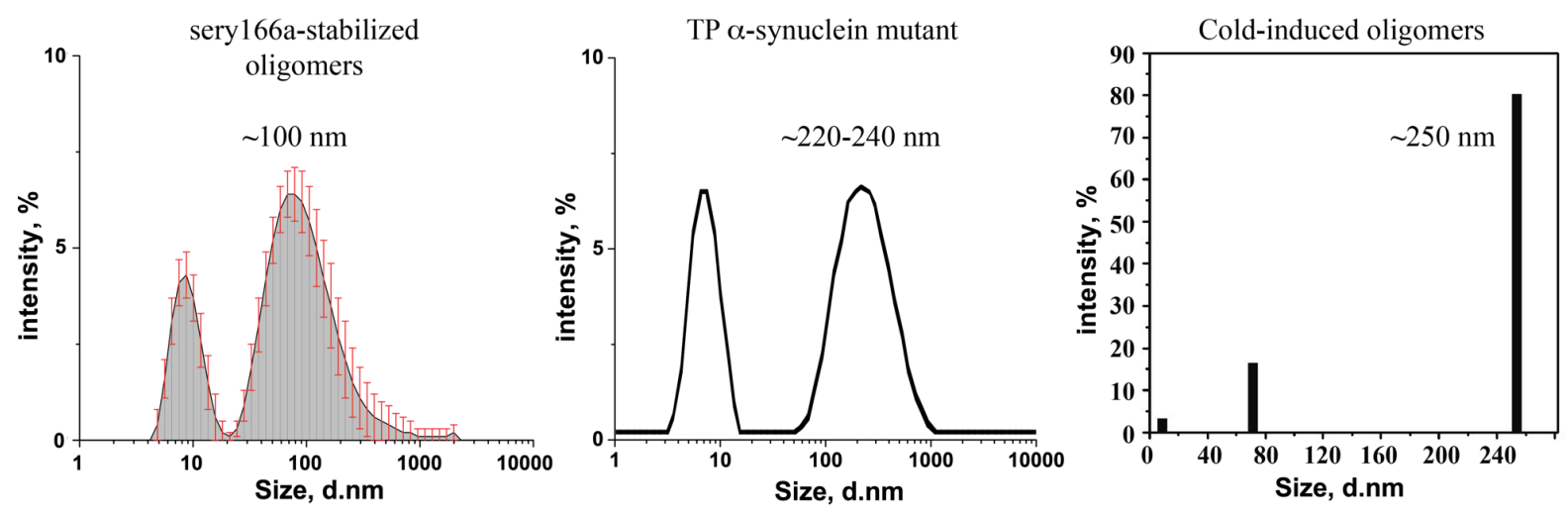

B

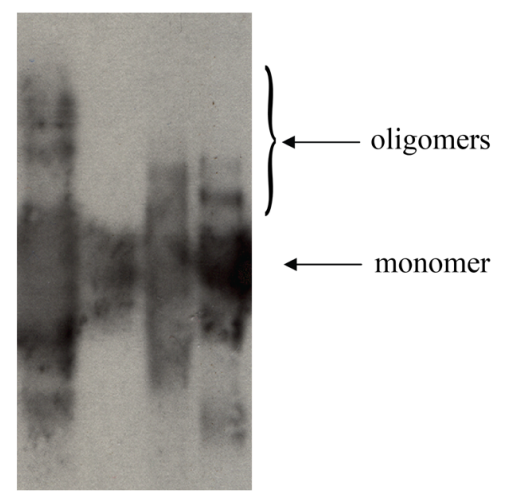

lane:

1 - TP $\alpha$-synuclein mutant aggregated for $72 \mathrm{~h}$

2 - $\alpha$-synuclein monomer

3 - $\alpha$-synuclein aggregated with sery166a for $72 \mathrm{~h}$

4 - $\alpha$-synuclein aggregated with sery 166 a for $72 \mathrm{~h}, 2 \mathrm{x}$ load

$\begin{array}{llll}1 & 2 & 3 & 4\end{array}$

Figure 2.5.26. Comparison of various oligomeric $\alpha$-synuclein species. (A) DLS analysis of sery166a-stabilized $\alpha$-synuclein oligomers, aggregated TP $\alpha$-synuclein mutant and cold-induced $\alpha$-synuclein oligomers. (72 h incubation for sery166a and TP mutant) (B) Native-PAGE analysis of aggregated TP $\alpha$-synuclein mutant and $\alpha$-synuclein incubated in presence of sery166a; lane 1, TP $\alpha$-synuclein mutant aggregated for $72 \mathrm{~h}$. lane 2, $\alpha$-synuclein monomer; lane 3, 4, $\alpha$-synuclein incubated in presence of sery 166 a for $72 \mathrm{~h}$, lane $42 \mathrm{x}$ loaded material.

DLS trace of cold denatured oligomers was kindly provided by Dr. Hai-Young Kim. 
the comparison of migration profile of two types of oligomers strongly supports the differences in the hydrodynamic size and shape. Considering that the oligomeric population of TP mutant sample shows lower mobility in direct comparison with the corresponding sery166a-stabilized species, our results suggest that DPP compounds induce the formation of oligomeric structures characterized by smaller size than on-pathways oligomeric species. Moreover, the native-PAGE analysis revealed that for all analyzed samples the population of high molecular weight species is complex, meaning both variable size and abundance of corresponding protein assemblies. Altogether, evidence from biophysical studies has consistently shown that DPP compounds stabilize $\alpha$-synuclein in new aggregate form(s) that is distinct from other on-pathways oligomeric forms.

\subsubsection{Inhibition of $\alpha$-synuclein aggregation by DPP compounds in vitro: key} findings and brief discussion

Targeting of protein misfolding by small organic drug-like compounds represents an attractive therapeutic approach and a power tool to investigate the nature of aggregation process and related mechanisms of toxicity. In our study, we demonstrated that the watersoluble DPP-based compounds effectively inhibit the formation of $\alpha$-synuclein fibrils in vitro. Experimental evidences supporting the high anti-aggregation properties of selected DPP derivatives come from various biophysical assays. Thus, by applying SIFT assay we were able to identify a set of highly potent inhibitors with the well-defined structure-activity relationship within the focused library. ThioT aggregation assay and NMR studies confirmed the arrest of $\alpha$-synuclein fibril formation by DPP compounds, such as sery166a, anle138c, anle145c and others. Based on TEM and AFM analysis, we suggest that DPP compounds suppress the assembly of amyloid fibrils and promote the formation of small globular heterogeneous oligomeric species with size in the range of $20-80 \mathrm{~nm}$ in width. This view is strongly corroborated by DLS and gel electrophoresis analysis. Thus, the evaluation of the scattering data revealed a fast formation of DPP-stabilized oligomeric species, which are quite heterogeneous and have a hydrodynamic diameter in range of $70-150 \mathrm{~nm}$, irrespective of DPP compound. Remarkably, DPP-stabilized oligomers are SDS-sensitive, but could be separated on non-denaturing native polyacrylamide gel. The observation of the low SDS-stability points towards the certain structural differences of DPP-induced oligomers in comparison to previously described $\alpha$-synuclein structures, which are generated in presence of alternative small compounds. For example, the incubation of $\alpha$-synuclein together with compounds like dopamine ${ }^{325}$, dihydroxyindol ${ }^{336}$, curcumin $^{349}$, EGCG ${ }^{354}$ or PcTS $^{368}$ leads to formation of SDS- 
stable oligomeric species. These finding implies that DPP-induced $\alpha$-synuclein oligomers are more dynamic and stabilized by relatively weak interactions. The lack of rigid, stable and well-structured core of DPP-stabilized oligomers is supported by CD data. In these regards, CD measurements suggest that sery166a stabilizes $\alpha$-synuclein in predominantly unfolded conformation accompanied with small degree of the ordering of local secondary structure in comparison to monomeric $\alpha$-synuclein. This predominantly unfolded secondary structure differs remarkably from the $\beta$-sheet rich conformations that were found in several highly toxic $\alpha$-synuclein oligomeric preparations ${ }^{11,184,186,190,459}$.

The results from liquid state NMR binding studies showed that DPP compounds have a very low affinity to monomeric $\alpha$-synuclein. It is evident from ${ }^{1} \mathrm{H}-{ }^{15} \mathrm{~N}$ HSQC-NMR titration experiments, in which the residue specific analysis of the chemical shift perturbations and peak intensity changes does not provide evidences supporting the binding of DPP ligands to monomeric $\alpha$-synuclein (Fig. 2.5.17A-B). In NMR STD-based binding experiments we demonstrated that the DPP compounds preferentially interact with the oligomeric species, while the affinity to monomeric $\alpha$-synuclein, if present, is weak. Our NMR data essentially confirms and elaborates the results of binding studies with DPP compounds and $\alpha$-synuclein, which were carried out using fluorescence techniques ${ }^{460,461}$. Collectively, our in vitro findings support the hypothesis that the mechanism of aggregation inhibition by DPPs is associated with the direct interactions between small ligands and oligomeric forms that, in turn, lead to stabilization of specific off-pathway oligomers and, ultimately, blocking of the fibril formation. Remarkably, this mechanism excludes the interference with the physiological functions of $\alpha$-synuclein by DDP compounds due to weak interactions with monomeric proteins. Therefore, DPP molecules could have a potential advantage over other compounds, for which anti-aggregation properties were associated with preferential binding to monomeric $\alpha$-synuclein ${ }^{345,354}$.

Finally, our data indicate that DPP-stabilized oligomers are structurally distinct from on-pathway oligomeric form of $\alpha$-synuclein. Our results from comparative studies, which include ssNMR, DLS, native-PAGE analysis, demonstrated the unique structural features of DPP-induced $\alpha$-synuclein oligomers such as smaller hydrodynamic radius and increased $\alpha$ helical structure.

Based on the available data, the determination of the oligomeric structure and characterization of the inhibition mechanism on atomic level with NMR or other techniques are elusive. Such structural investigations of high molecular weight species posit numerous fundamental and technical challenges. First, oligomers are heterogeneous mixtures of highly 
dynamic and constantly evolving complexes. From the perspective of liquid state NMR, the high molecular weight of such protein complexes leads to a slower tumbling in solution, longer correlation times, fast relaxation and eventually strong broadening of the NMR signals. In contrast to the hydrophilic water-soluble DPP compounds, the investigation of lipophilic DPP compounds like anle138b in vitro is challenging due to extremely low solubility in aqueous solutions (below $1 \mu \mathrm{M}$ ). To overcome these issues, we are currently expanding our research with ssNMR to study the interactions of anle138b with various form of $\alpha$-synuclein in the membranes. Moreover, we are developing the photo-crosslinking approaches in order to localize protein residues involved in interactions with small compounds.

Collectively, we show that DPP compounds can inhibit the aggregation of $\alpha$-synuclein in vitro by stabilizing specific off-pathway oligomers structurally distinct from toxic onpathway $\alpha$-synuclein species. Combined with the data from next chapter, showing that DPPstabilized oligomers do not adversely affect the structure and function of membranes, our observations strongly support the view that interference with $\alpha$-synuclein aggregation by small molecules may represent an attractive strategy for development of disease-modifying therapy. 


\subsection{DPP compounds and $\alpha$-synuclein forms in electrophysiological studies}

\subsubsection{Introduction}

As discussed in detail in the chapter 1.4.8.4, the permeabilization of the artificial membranes by oligomeric forms of $\alpha$-synuclein is considered as one of putative mechanisms of toxicity leading to neuronal dysfunction and cell death ${ }^{203}, 462$. The experimental finding provide strong evidence that application of various exogenous $\alpha$-synuclein oligomers, but not monomeric or fibrillar protein, to the artificial membranes (liposomes or flat lipid bilayers) or to the living cells causes an abnormal flux of small molecules or ions across the membranes 6 , 11, 192-194, 199, 204, 205. Addressing underlying molecular mechanisms of oligomer-induced membrane permeabilization, two possible scenarios have been proposed based on experimental data ${ }^{6}, 199,205$. The first is the so-called transmembrane pore hypothesis, supporting the insertion of oligomers into lipid bilayers leading to formation of distinct uniform pore complexes with the characteristic electrophysiological and morphological properties $^{6,199,203}$. Alternatively, the second hypothesis implicates $\alpha$-synuclein oligomers as cause of the structural unspecific alteration of membranes like a thinning on lipid bilayer, lipid packing defects, increased lipid flip-flop ${ }^{174,195,196}$. Regardless of ongoing debate about the exact mechanism of the oligomer-induced membrane permeabilization, the prevention of membrane damage by small molecules or the stabilization of harmless $\alpha$-synuclein assemblies may constitute a promising therapeutic strategy and, moreover, valuable mechanistic information can be obtained. In the following, we investigate whether the DPP compounds

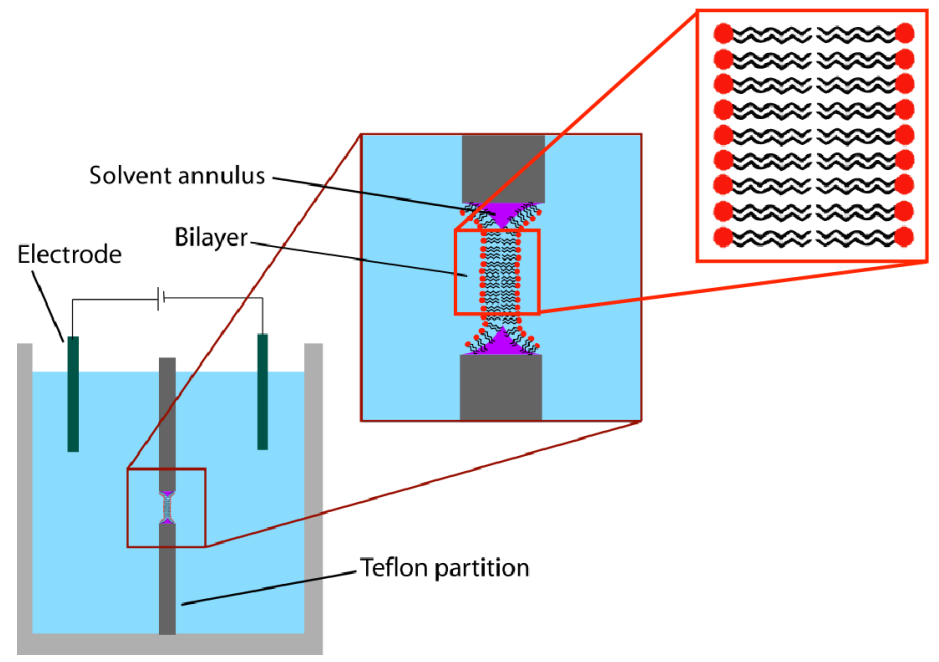

Figure 2.6.1. Schematic planar lipid bilayer electrophysiology setup. have any of these activities.

Therefore, for investigation of DPP compounds and compound-stabilized oligomers we took the advantages of the methods developed in our department in combination with the extensive external collaboration with groups of Prof. Armin Giese and Prof. Roland Benz. First of all, we had an opportunity to perform the electrophysiological studies on two different 
setups. Of particular importance is the availability of two fundamentally different and, at the same time, potentially relevant preparations of oligomeric $\alpha$-synuclein, with well-established electrophysiological properties that are briefly described below.

Next, I would like to address in few words the basic principles of the single-channel electrophysiology and provide some information about experimental setup itself. In figure 2.6.1 the schematic view of corresponding setup is shown. Two chambers are separated by non-conducting material with a small hole, wherein the planar lipid bilayer is introduced in most cases by painting techniques ${ }^{463}$. Each chamber contains an electrolytic solution and an electrode. When the membrane potential is applied, the lipid bilayer membrane acts as a capacitor preventing the flow of ions between separated compartments. Either the insertion of ion channels/permeabilizing pores or the destabilization of the membrane all result in increase of the bilayer conductance as a consequence of the enhanced flow of the ions across the lipid bilayer. Thus, the electrophysiological recording could provide valuable information about channel-like activities of the target molecules, ion-selectivity, effect of lipid composition and many other electrophysiological properties.

$\Delta$

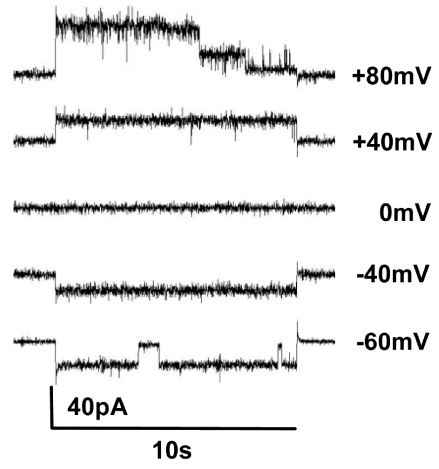

B

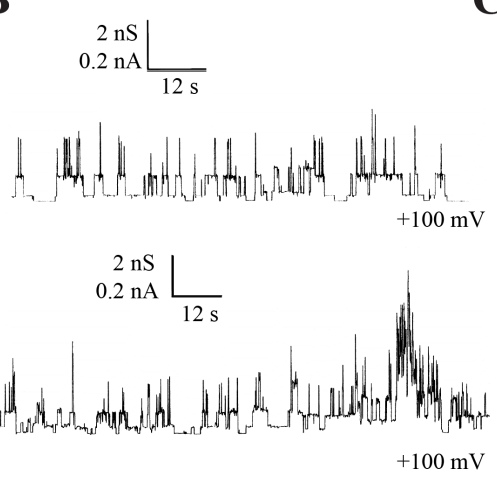

C

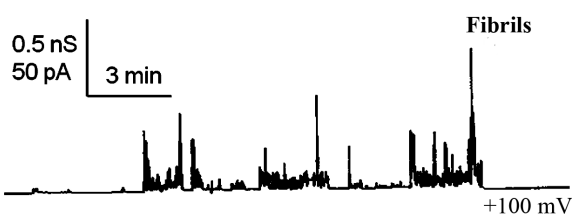

$1.33 \mathrm{nS}$

$0.2 \mathrm{nA} 1 \mathrm{~min}$

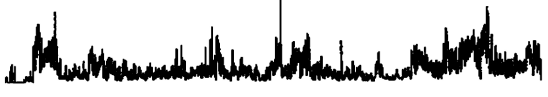

Figure 2.6.2. Pore formation by oligomeric forms of $\alpha$-synuclein in planar lipid bilayers.

(A) Current traces at various membrane voltages showing the pore formation upon addition of $\mathrm{Fe}^{3+}$-induced oligomers. Figure adapted from F. Schmidt et al. ${ }^{6}$ licensed under CC BY 4.0. Copyright (C) (2012) (B) Current traces at membrane voltages $+100 \mathrm{mV}$ showing the pore formation upon addition of the oligomers obtained by cold-induced fibril dissociation. (C) Current traces at membrane voltages $+100 \mathrm{mV}$ showing minor or negligible pore formation upon addition of $\alpha$-synuclein monomer and fibrils. Figure $\mathbf{B}$ and $\mathbf{C}$ reprinted with permission from H.-Y. Kim et al. ${ }^{11}$ Copyright (c) (2009) American Chemical Society.

\subsubsection{Effect of $\alpha$-synuclein on electrophysiological properties of planar lipid bilayer}

As mentioned before, the deleterious effects of oligomeric $\alpha$-synuclein, but not monomeric or fibrillar $\alpha$-synuclein, on membrane integrity have been measured in electrophysiological studies ${ }^{6,11}$. Based on research findings, two types of oligomeric $\alpha-$ 
synuclein intermediates demonstrated the pronounced pore formation activity leading to permeabilization of the planar lipid membranes. First sample preparation is based on coldinduced dissociation of corresponding $\alpha$-synuclein fibrils. The oligomers generated according to this protocol showed a high rate of the pore insertion in comparison with monomeric or fibrillar protein (Fig. 2.6.2 B and C). Analysis of electrophysiological recordings on bilayers composed of PC or PS/PC lipids revealed that the membrane channels formed by the coldinduced oligomers have several well-defined conductance states ${ }^{11}$. For instance, two main conductance levels were detected at $+100 \mathrm{mV}$ transmembrane potential, namely $800 \mathrm{pS}$ and $1,7 \mathrm{nS}$ in $1 \mathrm{M} \mathrm{KCl}$. Other type of preformed oligomers, which were included in the study, is generated by incubation of $\alpha$-synuclein in presence of $\mathrm{Fe}^{3+}$ ions for $72 \mathrm{~h}^{6}$. As is evident from electrophysiology, the addition of these oligomeric intermediates to planar lipid bilayers also leads to dose- and time-dependent pore formation. Upon examination, the uniform transmembrane pores were reproducibly detected and analyzed (Fig. 2.6.2 A). Obtained experimental data supports the insertion of distinct channels. Essential to comprehend the mechanism of interactions was the demonstration of the step-like changes in conductivity $(100 \mathrm{pS}$ at $+80 \mathrm{mV})$ and well-defined peaks in the distribution of conductance levels. Moreover, the generated pores showed a unidirectional incorporation and certain selectivity to the available cation ${ }^{6}$. Altogether, the mounting evidence support the notion that certain type of misfolded oligomeric intermediates of $\alpha$-synuclein, but not monomeric or fibrillar forms, may exert the deleterious effects on the integrity of the lipid bilayers as a result of the formation of uniform transmembrane pores. Importantly, the ion channel measurements with the pore-forming oligomers clearly represent the attractive model for development and investigation of small molecule capable to modulate and prevent the membrane damage.

\subsubsection{Electrophysiological properties of DPP-stabilized oligomers}

Since the interactions of potentially on-pathway oligomeric $\alpha$-synuclein with membranes are leading to permeabilization of the lipid bilayers, we decided to investigate the electrophysiological properties of membranes in presence of $\alpha$-synuclein assemblies that are formed in presence of DPP compounds. The experiments with DPP-stabilized oligomers, hydrophilic DPP compounds and anle138b-dopped membranes were performed in the laboratory of Prof. Roland Benz. For these experiments, I delivered the compounds and oligomeric preparations, and analyzed the data. 


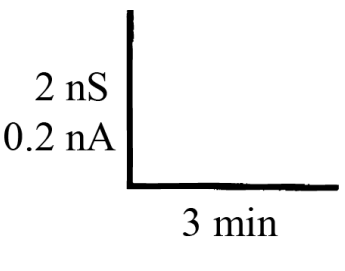

sery166a-stabilized oligomers

$+20 \mu \mathrm{l} \mathrm{cis}$

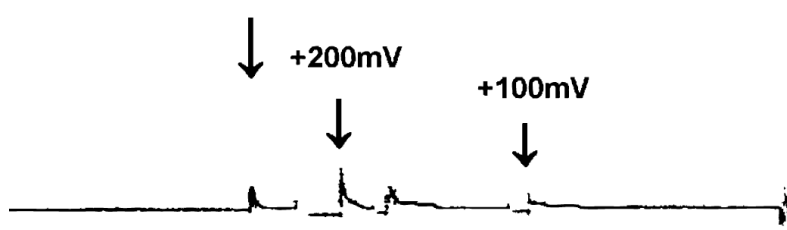

$+2 \times 10 \mu l$

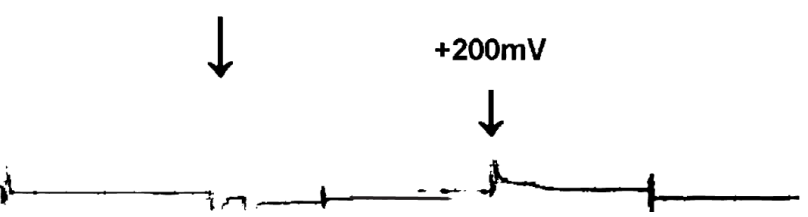

Figure 2.6.3. Lack of pore formation activity for sery166a-stabilized oligomers. (B) Representative current trace at various membrane voltages showing a lack of membrane permeabilization upon addition of sery166a-stabilized oligomers. Bilayers are formed from DiphPC/n-decane in $1 \mathrm{M} \mathrm{KCl}, 1 \%$. Arrows labeled with " $+20 \mu 1$ cis" and " $+2 \times 10 \mu l$ " represent the addition of oligomers.

The main goal of this study was to investigate the effect of DPP compounds on pore formation under different conditions. For appropriate comparison $\mathrm{Fe}^{3+}$-induced and coldinduced $\alpha$-synuclein oligomers were selected as relevant positive controls. Thus, we generated a set of samples containing $\alpha$-synuclein oligomers that are stabilized by three different DPP compounds, including sery166a, anle138c and anle145c. Due to the fact that redox reactions may constitute a factor affecting the aggregation pathways, we included the compoundstabilized oligomers obtained under reducing conditions in our measurements. The complete list of samples and experimental details are summarized in the table 2.6.1. Briefly, we incubated $\alpha$-synuclein in presence of one of inhibitors for 4-6 days with addition either DMSO $(3 \% \mathrm{v} / \mathrm{v})$ or EtOH $(3 \% \mathrm{v} / \mathrm{v})$ with $\mathrm{Na}_{2} \mathrm{~S}_{2} \mathrm{O}_{3}(5 \mathrm{mM})$. Depending on selected compound and incubation time, the oligomeric load was in a range of $15-30 \%$ in combination with the remaining monomeric protein. For electrophysiological studies the stock solutions were

Table 2.6.1. DPP-stabilized oligomers in BLM

\begin{tabular}{|c|c|c|c|c|}
\hline \multicolumn{4}{|c|}{ Preparation of compound-stabilized oligomers } & \multirow{2}{*}{$\begin{array}{c}\text { Results of } \\
\text { electrophysiological } \\
\text { recordings }\end{array}$} \\
\hline $\begin{array}{c}\text { Compound, } \\
\boldsymbol{\mu} M\end{array}$ & $\begin{array}{c}\alpha-S y n, \\
\mu M\end{array}$ & $\begin{array}{l}\text { Incubation } \\
\text { time, } h\end{array}$ & Additional components & \\
\hline sery166a, 100 & 100 & $144 \mathrm{~h}$ & $3 \% \mathrm{DMSO} v / \mathrm{v}$ & No permeabilization of membrane \\
\hline sery166a, 150 & 100 & $96 \mathrm{~h}$ & $3 \% \mathrm{DMSO} v / \mathrm{v}$ & Insignificant channel activity \\
\hline anle138c, 100 & 100 & $144 \mathrm{~h}$ & $3 \% \mathrm{DMSO} v / \mathrm{v}$ & No permeabilization of membrane \\
\hline anle138c, 100 & 50 & $110 \mathrm{~h}$ & $3 \% \mathrm{EtOH} \mathrm{v/v,} 5 \mathrm{mM} \mathrm{Na} 2 \mathrm{~S}_{2} \mathrm{O}_{3}$ & Insignificant channel activity \\
\hline anle145c, 140 & 70 & $110 \mathrm{~h}$ & $3 \% \mathrm{DMSO} v / \mathrm{v}$ & Insignificant channel activity \\
\hline anle145c, 140 & 50 & $110 \mathrm{~h}$ & $3 \% \mathrm{EtOH} \mathrm{v/v,} 5 \mathrm{mM} \mathrm{Na} 2 \mathrm{~S}_{2} \mathrm{O}_{3}$ & Insignificant channel activity \\
\hline
\end{tabular}


diluted in the cis compartment to a final concentration of $5-10 \mu \mathrm{M} / \mathrm{ml}$.

Analysis of the electrophysiological data from the painted lipid bilayers assay revealed that all sample containing DPP compound-stabilized oligomers exhibit negligible or minimal ion channel activity (Table 2.6.1). In contrast to the on-pathway oligomeric species, for which the discrete step-like fluctuation of conductance is observed, the single-channel recording at similar voltages with compound-stabilized oligomers showed no significant changes of membrane permeability, as confirmed by lack of distinct peaks and stability of the ground conductance levels. In support of this view, a representative current trace recorded for sery166a-stabilized oligomer shows striking differences in comparison with previously described electrophysiological properties of on-pathway oligomers (Fig. 2.6.2 and 2.6.3). These findings led us to speculate that $\alpha$-synuclein assemblies formed in presence of DPP compounds are not able to exert the deleterious effects on membrane organization and function and, therefore, a low cytotoxicity might be expected.

A

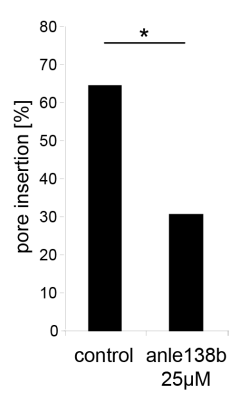

B

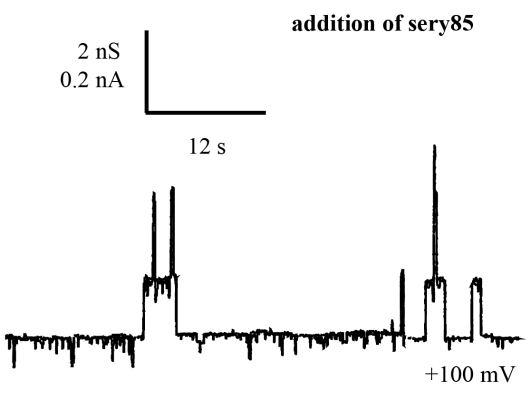

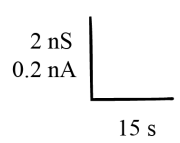

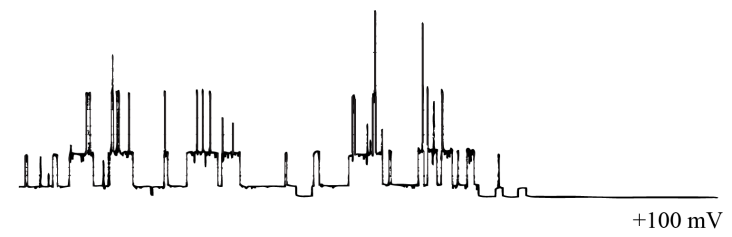

Figure 2.6.4. Complete or partial inhibition of oligomer-induced pore formation by DPP compounds. (A) Anle138b significantly reduces the membrane permeabilization induced by oligomers generated in presence of $\mathrm{Fe}^{3+}$. (B) DPP compounds prevent the pore formation induced by $\alpha$-synuclein oligomers obtained by cold-assisted dissociation of fibrils. Strong inhibition of pore formation by addition of sery85 (10-20 fold excess of compound) to oligomeric sample (left). Incorporation of lipophilic compounds (1 mM anle $138 \mathrm{~b}$ is shown) into the lipid bilayers leads to complete blocking of channel activity within few minutes after addition of oligomers (right).

\subsubsection{Inhibition of pore formation by DPP compounds}

Considering that the oligomer-induced membrane permeabilization represents a potential toxicity mechanism associated with $\alpha$-synuclein misfolding, we investigated the ability of exogenously applied DPP compounds to interfere with these processes. Experimentally, we developed two types of protocols. First, we shortly treated the poreforming oligomers with one of hydrophilic DPP compounds (sery166a, anle138c and sery85) that have reasonable solubility (higher than $10 \mu \mathrm{M}$ ) in aqueous buffer and then performed the 
ion channel measurements of derived samples. Additionally, we conducted the similar experiment with anle138b incubated with $\mathrm{Fe}^{3+}$-induced $\alpha$-synuclein oligomeric intermediates. Experiments with $\mathrm{Fe}^{3+}$ oligomers were performed by Dr. Felix Schmidt. Regarding the second approach, we investigated the impact of lipophilic DPP compounds, which are insoluble in water, but could be reconstituted in the lipid bilayers, on oligomer-induced membrane permeabilization. In turn, such electrophysiological studies with the specifically doped membranes are highly relevant to in vivo situation, where the lipophilic drugs are mainly localized in the lipid-rich compartments, including all types of biological membranes.

Table 2.6.2. Inhibition of pore formation by DPP compounds in solution or in membrane

\begin{tabular}{|c|c|}
\hline Sample preparation & \multirow{2}{*}{$\begin{array}{c}\text { Results of electrophysiological } \\
\text { recordings }\end{array}$} \\
\hline Addition of compounds to solution & \\
\hline $\mathrm{Fe}^{3+}$-induced oligomers alone & $60-70 \%$ pore detection rate \\
\hline $\mathrm{Fe}^{3+}$-induced oligomers + anle $138 \mathrm{~b}$ & Significant reduction of pore insertion \\
\hline Cold-induced oligomers alone & High frequency of pore formation \\
\hline Cold-induced oligomers + anle138c & Disappearance of pores after 5-10 minutes \\
\hline Cold-induced oligomers + sery $166 a$ & Disappearance of pores after 5-10 minutes \\
\hline Cold-induced oligomers + sery 85 & Disappearance of pores after 5-10 minutes \\
\hline \multicolumn{2}{|l|}{ Experiments with compound-doped membrane } \\
\hline Cold-induced oligomers + anle138b ( $1 \mathrm{mM}$ in membrane $)$ & \multirow{4}{*}{$\begin{array}{l}\text { For all samples, the addition of oligomeric } \\
\text { preparation leads to the channel formation that is } \\
\text { eventually disappeared within few minutes. In } \\
\text { case of anle138b, blocking occurs after } 1-2 \\
\text { minutes. }\end{array}$} \\
\hline Cold-induced oligomers + anle186b ( $1 \mathrm{mM}$ in membrane $)$ & \\
\hline Cold-induced oligomers + sery $335 \mathrm{~b}(1 \mathrm{mM}$ in membrane $)$ & \\
\hline Cold-induced oligomers + anle197b ( $1 \mathrm{mM}$ in membrane $)$ & \\
\hline
\end{tabular}

In line with the previous results, the beneficial effects, meaning the attenuation or complete blocking of the pore-forming activity of two types of on-pathway $\alpha$-synuclein oligomers, were observed upon addition of selected DPP compounds to oligomeric preparations. Thus, the analysis of the electrophysiological data that was acquired on preformed $\mathrm{Fe}^{3+}$-related oligomeric intermediates $(2.1 \mu \mathrm{M})$ incubated in presence of anle138b $(25 \mu \mathrm{M})$ clearly demonstrated a significant reduction, more than 2 fold, in the frequency of pore formation events (Fig. 2.6.4 A). In addition to anle138b, several hydrophilic DPP compounds, including sery166a, anle138c and sery85, also inhibited the channel-forming activity of cold-induced $\alpha$-synuclein oligomers. For example, the short-term treatment of these oligomers with one of selected DPP compounds leads to strong attenuation of the pore 
formation, as indicated by single channel recordings showing insignificant channel fluctuation. The representative current trace for sery 85 is shown in figure 2.6.4 B (left), the corresponding data for all compounds is summarized in the table 2.6.2. Collectively, the accumulated data for hydrophilic compounds points to the possibility that DPP compounds in solution are able to modulate the structure of toxic pore-forming oligomeric species in favor of $\alpha$-synuclein forms characterized by the minimal impact on the membrane stability.

Based on the second experimental setup, we investigated whether lipophilic DPP compounds being directly embedded in the planar lipid bilayers can prevent the pore-forming activity of oligomers obtained by cold-induced fibril dissociation. Experimentally, the doping of membranes with DPP compounds was achieved by addition of ethanolic solution of selected molecules to corresponding lipids followed by evaporation and standard procedure for bilayer formation by painting technique. We successfully applied this protocol for 4 lyophilic compounds, including anle138b, sery335b, anle197b and anle186b, and confirmed that the stability and electrophysiological properties of these membranes were within the normal range. Of note, in this study the concentration of DPP compounds was $1 \mathrm{mM}$ with respect to lipid. In line with our previous results, selected DPP compounds as a component of lipid bilayer also interfered with the pore formation. As shown in figure 2.6.4 B (right) and in table 2.6.2, at the start of the experiment, which is the addition of oligomeric samples to the cis-chamber, the significant number of pore formation events were detected for several minutes. Thereafter, we consistently observed for all doped membranes that the ion channel activity was inhibited within 2-10 minutes, as evident from restoration of conductance to its ground levels and from the lack of conductivity fluctuations. Remarkably, repetitive addition of the next oligomeric aliquot to the same chamber induced similar pattern, meaning shortterm membrane permeabilization followed by attenuation of the channel activity. Among all tested DPP molecules, anle138b is clearly the most potent inhibitor of pore formation (Fig. 2.6.4 B, right), when the time of the pore activity is compared. While the structural information regarding the modulation of the pore formation by DPP inhibitors is very limited, several modes of actions seem plausible to us. The general assumption is that after binding of oligomeric intermediates to membrane and organization of transmembrane channel, the embedded DPP compound directly interacts with the pore leading to blocking of the lipid bilayer permeability. Herein, several pathways are possible, first, DPPs are directly blocking the channel of the pore; second, pore inactivation due to the conformational transformation of the channel itself upon binding of DPP molecule; third, the complete dissociation of the pore. 
To dissect the exact mechanism(s) involved, additional experiments have to be carried out, using wide palette of biophysical and electrophysiological studies.

Overall, all tested DPP compounds either in solution or in membranes strongly interfere with the oligomer-driven membrane permeabilization. Since the alterations of the membrane integrity and function by misfolded protein aggregates is considered as one of the potential mechanisms of toxicity, the inhibition of this process by described DPP compounds may constitute the promising therapeutic strategy.

\subsubsection{Effect of DPP compounds and $\alpha$-synuclein on LTP}

To further evaluate the effect of DPP compounds on misfolding of $\alpha$-synuclein and related toxicity pathways, we utilized another fundamentally different method of electrophysiological studies. In collaboration with the group of Prof. Tiago Fleming Outeiro, we investigated the impact of compound-stabilized $\alpha$-synuclein oligomers on one of the basic mechanisms of synaptic plasticity, so called long-term potentiation (LTP).

In neuroscience, the process of learning and memory is tightly linked to the physiological phenomenon, known as synaptic plasticity 464,465 . According to textbooks, the synaptic plasticity is described as the ability of synapses to modulate their strength over time depending on the pattern of synaptic activity ${ }^{464,465}$. Although numerous forms of synaptic plasticity are described, the most basic classification includes short-lasting forms, including short-term synaptic enhancement and depression, and long-term synaptic plasticity, comprising long-term potentiation and depression ${ }^{465}$. Under physiological conditions, LTP is a long-lasting amplification of synaptic strength in response to synaptic stimulation. In other words, the stimulation of synapses leads to long-term increase in efficacy of synaptic transmission at preexisting synapses. Thus, the synapses that experienced the LTP generate stronger response to the stimuli and therefore preserve the information.

To date, the impairment of LTP has been intensively studied in context of Alzheimer's disease $^{466}$. Several groups have focused on the role of amyloid-beta misfolding in connection to significant alterations of LTP and eventual deterioration of the memory function. Herein, the emerging paradigm implicates oligomeric intermediates of amyloid-beta in dysregulation of the normal LTP as evident from electrophysiological studies that have been carried out on the brain slices from transgenic models of Alzheimer's disease ${ }^{467}$. Concurrently, the significant LTP malfunction can be induced by extracellularly applied amyloid-beta oligomers $^{468}$. 
The potential impact of various $\alpha$-synuclein forms on LTP was investigated in the group of Prof. Tiago Fleming Outeiro. Experimentally, the LTP magnitude was assessed by extracellular recording of field excitatory postsynaptic potential (fEPSP) evoked by stimulation of Schaffer-collaterals/CA1 glutamatergic fibers in hippocampal slices ${ }^{8}$. Before the electrophysiological recording the brain slices were treated with monomers, fibrils and two types of oligomers, including 4-Hydroxynonenal-induced oligomers and purified onpathway oligomers. Collectively, experimental data provide strong evidence that several different oligomeric $\alpha$-synuclein intermediates, but not monomeric or fibrillar protein, cause a sustainable decline of LTP (Fig. 2.6.5 A and C) ${ }^{8}$. Addressing the underlying mechanisms of observed neuronal dysfunction, the abolishment of LTP, which is induced by $\alpha$-synuclein oligomers, has been linked to elevated basal synaptic transmission through NMDA receptor activation accompanied by NMDA-evoked $\mathrm{Ca}^{2+}$ influx ${ }^{8}$.

Taking into account the deleterious effects of on-pathway $\alpha$-synuclein oligomers on LTP, it therefore of great interest to investigate the behavior of $\alpha$-synuclein species stabilized by DPP compounds under similar conditions. For this study we selected two DPP inhibitors,

A

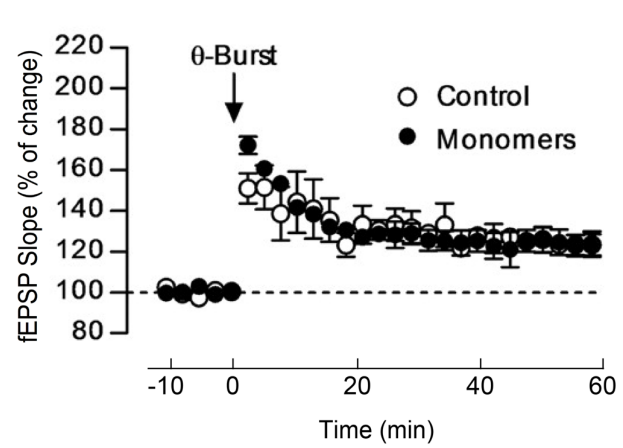

C

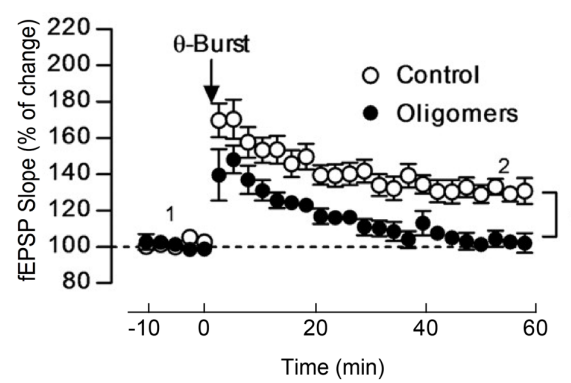

B
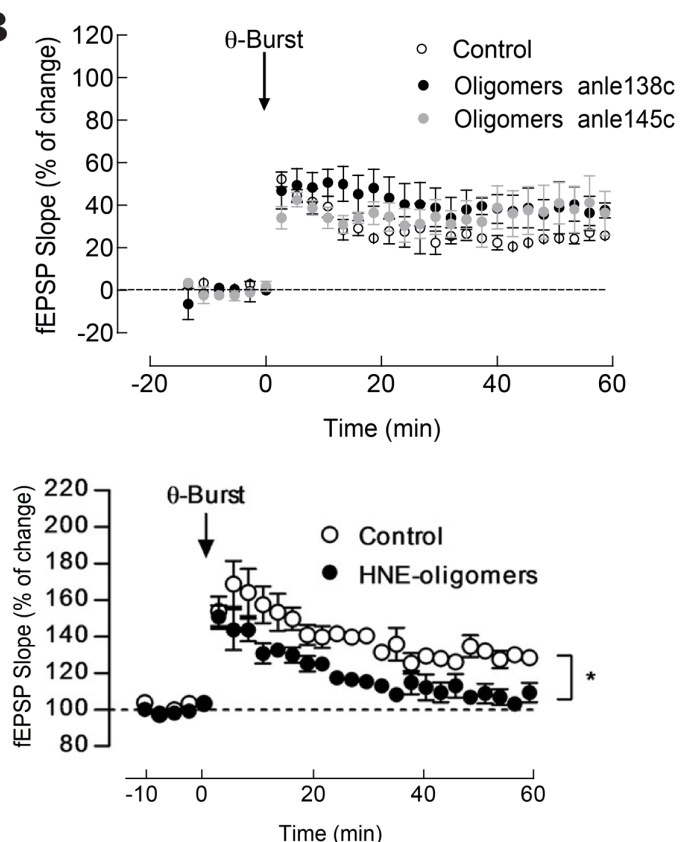

Figure 2.6.5. Effect of various $\alpha$-synuclein forms on LTP. Changes of fEPSP slope induced by theta-burst stimulation in control slices and slices pre-incubated with monomeric $\alpha$-synuclein (A), anle138c- or anle145c-stabilized oligomers (B), three types of $\alpha$-synuclein oligomeric preparations, including cold-induced oligomers, HNE-modified oligomers and unmodified oligomers $(\mathbf{C})$. Total protein concentration $500 \mathrm{nM}$, incubation time 60-90 min). The figures $\mathbf{A}$ and $\mathbf{C}$ are adopted from $\underline{M}$. J. Diógenes et al. ${ }^{8}$ licensed under CC BY-NC-SA 3.0. Copyright (C) (2012) 
namely anle138c and anle145c. The respective oligomeric preparations were obtained by $70 \mathrm{~h}$ incubation of monomeric $\alpha$-synuclein $(70 \mu \mathrm{M})$ in presence of either anle138c $(100 \mu \mathrm{M})$ or anle145c $(140 \mu \mathrm{M})$ according to the standard protocol. In contrast to electrophysiological recordings on on-pathway oligomers, pre-incubation of hippocampal slices with these compound-stabilized $\alpha$-synuclein assemblies (500 nM, $60 \mathrm{~min}$ ) had no significant impact on LTP magnitude in comparison with corresponding controls (Fig. 2.6.5 B). This conclusion is based on analysis of the changes of fEPSP slope induced by theta-burst stimulation, that is equivalent of intensive synaptic activity if described in simplistic terms. In case of impaired LTP, the changes of fEPSP slope are similar before theta-burst stimulation and 40-60 minutes after as shown in figure 2.6.5C. In control experiments, including buffer and monomeric $\alpha$ synuclein, the robust LTPs were observed as evidenced by significant increase of fEPSP slope when compared with the values before theta-burst stimulation (Fig. 2.6.5 A). Importantly, the compound-stabilized oligomeric species do not alter the magnitude of LTP (Fig. 2.6.5 B); thereby, it is another piece of evidence supporting the low neurotoxicity of DPP-stabilized $\alpha$ synuclein oligomers, herein regarding the basic neurophysiological function LTP and synaptic plasticity. Moreover, the application of DPP compounds can potentially normalize the LTP by remodeling of on-pathway oligomers. 


\subsection{Effect of DPP compounds in in vivo models of Parkinson's disease}

\subsubsection{Introduction}

As described in previous chapters, several DPP-related compounds, including anle138b, anle145c, sery166a and others, can interfere with the aggregation pathway of $\alpha$ synuclein in vitro. The evidence that DPP-based compounds are $\alpha$-synuclein oligomer modulators as in solution as well as in membranes has accumulated through the variety of biophysical and electrophysiological methods. On the basis of these studies and the results of prion-related project, several compounds were selected for testing in in vivo models of Parkinson's disease and MSA.

To date, many different animal models of $\alpha$-synuclein toxicity have been developed, as discussed in details in the introduction part of my thesis (Chapter 1.6.2). Briefly, there is no model of Parkinson's disease that fully reproduce all preclinical and clinical manifestations of Parkinson's disease. Due to the multifactorial nature of Parkinson's disease all available models resemble one or several pathological features depending on genetic, spreading or toxin-based approaches. The transgenic, innoculated or toxin-treated rodent species, like mice and rats, are most frequently used models of Parkinson's disease ${ }^{250,255}$. However, the cellular and, particularly, lower organism models also find a wide application due to the low cost, fast response, adaptation for high-throughput screening 249,469 .

In our efforts to investigate the therapeutic potential of anle138b and related DPP compounds with respect to $\alpha$-synuclein-related toxicity in vivo, an international scientific collaboration network of the labs focused on $\alpha$-synuclein research was established by active involvement of Prof. Dr. C. Griesinger and Prof. Dr. A. Giese. As of 2019, DPP-related compounds have been tested in two non-mammalian models, in seven different mice models and in one monkey model of $\alpha$-synuclein toxicity. The overview of collaboration partners and the corresponding animal models is listed below:

1) Caenorhabditis elegans model - Prof. S. Eimer;

2) Drosophila model - Prof. J. B. Schulz;

3) A30P-hum- $\alpha$-synuclein mouse model - Prof. A. Giese;

4) Low-dose intragastric rotenone mice model - Dr. F. Pan-Montojo;

5) Sub-acute MPTP mouse model - Prof. J. Weishaupt;

6) 1-120 $\alpha$-synuclein mouse model - Prof. M. G. Spillantini;

7) Lewy body seeding mouse model - Prof. E. Bezard;

8) PLP- $\alpha$-synuclein transgenic models - Prof. N. Stefanova and Prof. G. K. Wenning; 
9) Lewy body seeding monkey model - Prof. E. Bezard;

In this chapter I will briefly describe the results of in vivo experiments with anle $138 \mathrm{~b}$ and related compounds in the two non-mammalian models and in three mice models, namely A30P-hum- $\alpha$-synuclein, low-dose intragastric rotenone and 1-120 $\alpha$-synuclein mouse models. More detailed information and the results from other animal studies are published in several papers, please refer to the list of my publications.

\subsubsection{DPP compounds in non-mammalian $\alpha$-synuclein models}

In order to assess the ability of DPP-based compounds to interfere with $\alpha$-synucleinrelated toxicity in non-mammalian $\alpha$-synuclein models, we have selected two wellcharacterized models, namely Caenorhabditis elegans model ${ }^{267,269}$ based on expression of A53T $\alpha$-synuclein mutant and Drosophila melanogaster model ${ }^{269-271}$ expressing either A53T mutant or wild type $\alpha$-synuclein. The testing of compounds in Caenorhabditis elegans and Drosophila melanogaster model were done in collaboration with the laboratories of Prof. Stefan Eimer and Prof. Jörg B. Schulz, respectively. My scientific contribution in this part of the project includes a selection of compounds, a design of the treatment schemes and evaluation of the generated data.

For detailed discussion of $\alpha$-synuclein models in invertebrate models please refer to the chapter 1.6.2.4. Herein, I would like to highlight the key phenotypic features of Caenorhabditis elegans and Drosophila melanogaster models. The transgenic C. elegans lines expressing various $\alpha$-synuclein constructs (wild type, A30P, A53T) show significant and non-progressive loss of dopaminergic neurons and corresponding markers including dopamine, diffused $\alpha$-synuclein accumulation without formation of fibrils, motor deficits and abnormal food-sensing behavior that could be rescued by dopamine ${ }^{267,269}$. $\alpha$-Synuclein transgenic Drosophila flies recapitulate a number of key pathological features of Parkinson's disease, namely age-dependent and selective loss of dopaminergic neurons, accompanied by progressive and widespread $\alpha$-synuclein deposition and dopamine-responsive locomotor deficits (climbing test) ${ }^{269-271}$. Remarkably, a comparison of the phenotypes is clearly demonstrating an increased toxicity of mutant forms of $\alpha$-synuclein and, therefore, supporting the relevance of these models.

The list of selected compounds is shown in Figure 2.7.1 A. In total, 6 compounds were tested in C. elegans and 3 compounds in flies. In selection process following criteria were taken into account: i) high aggregation inhibition; ii) good solubility and appropriate chemical properties; iii) variation of the chemical space (different substitution patterns, variation of 
central heterocyclic ring). In case of $C$. elegans, the hydrophobic compounds were excluded from screen due to unfavorable exposure profile and requirement of special application $\operatorname{protocols}^{470}$.

The results of experiment in C. elegans are shown in Figure 2.7.1B. Experimentally, the test compounds were added into the nematode growth medium at final concentration of 50 $\mu \mathrm{M}$ using 1\% DMSO. The phenotypic evaluation based on analysis of the neuronal defects was done four days after reaching adulthood. Among 6 DPP-related compounds, anle145c had strongest effect on abrogation the neuronal toxicity of A30P $\alpha$-synuclein mutant. Thus, the number of the worm with pronounced neuronal defects was reduced roughly by effect of two under treatment with anle145c (Fig. 2.7.1B). All other compounds did not show significant activity in this model.

To test the effect of DPPs in the Drosophila model, the compound was blended into yeast Drosophila diet at the final concentration $250 \mu \mathrm{M}$ (anle138c, anle1445c) and $10 \mu \mathrm{M}$ (anle138b) using 3\% DMSO and the treatment started on day 1-2 post-eclosion. In both flies models expressing either A53T mutant or wild type $\alpha$-synuclein, the treatment with hydrophilic compounds, anle145c and anle138c, significantly improved the survival of flies in comparison with untreated animals (Fig. 2.7.1C, D). Remarkably, the observed survival in presence of anle145c is similar to the life-span of wild type flies (data not shown). Among three compounds, anle145c exhibited the highest activity in A53T model (Fig. 2.7.1 C). In case of the flies expressing wild type $\alpha$-synuclein, the similar therapeutic effects were observed for anle138c and anle145c (Fig. 2.7.1 D). On the contrary, treatment with anle138b did not improve significantly the survival (Fig. 2.7.1C). The reduced efficacy of anle138b is most probably linked to low solubility of the compound in water or unfavorable biodistribution in flies.

Collectively, the presented findings allowed us to speculate the effects of the DPP compounds are likely due to specific interference with toxicity mediated by $\alpha$-synuclein oligomers. Further support comes from similar findings in a fly model of AD where anle $145 \mathrm{c}$ extends the life span of flies most efficiently. Considering the limited data, this concept would have to be further confirmed by additional biochemical and phenotypical characterization of the treated animals. For example, it might be interesting to analyze $\alpha$-synuclein aggregation state in presence and in absence of anle145c by using techniques like high-resolution microscopy and sucrose gradient centrifugation. Nevertheless, given that these animals do not suffer from $\alpha$-synuclein aggregation induced neurodegeneration because they natively don't 
overexpress this protein, our focus shifted towards mammals where the described experiments were done (see below).

A
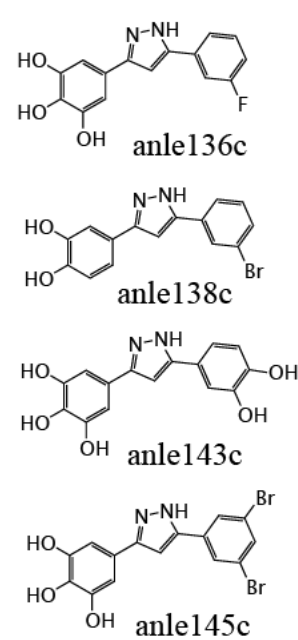

C

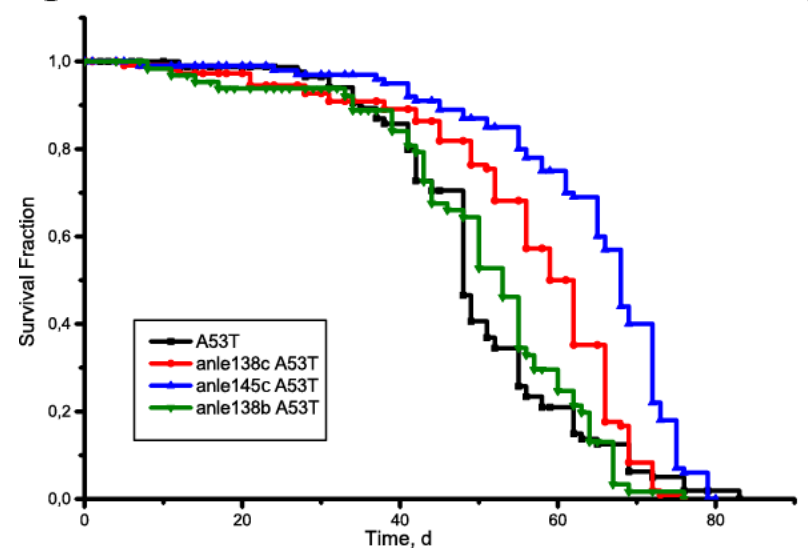

B
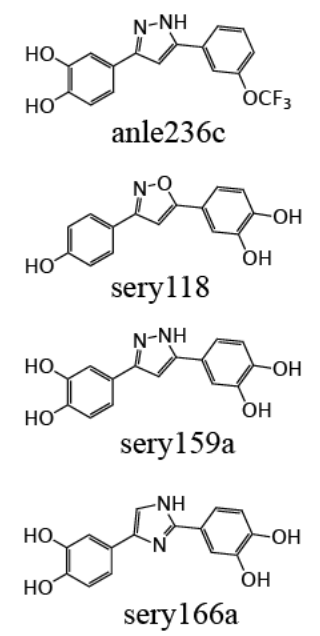

seryl66a

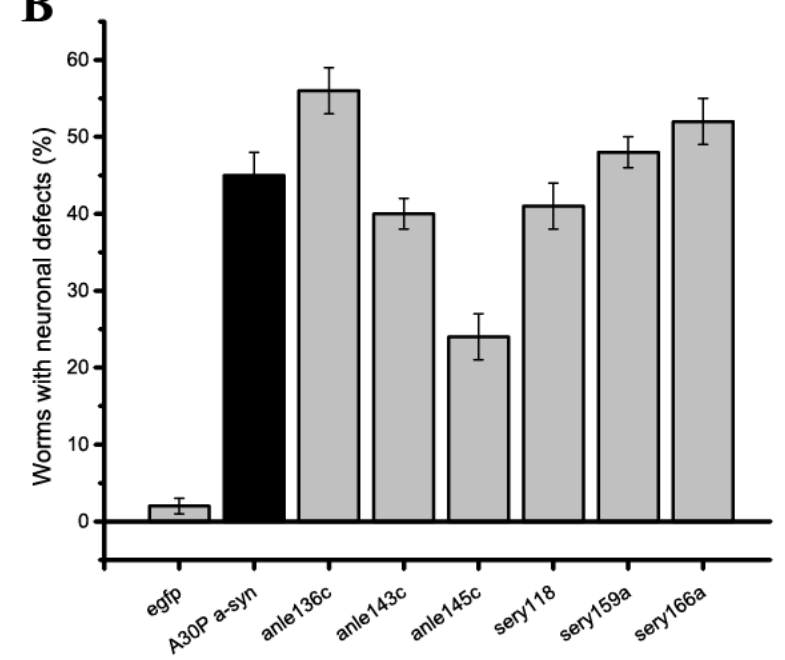

D

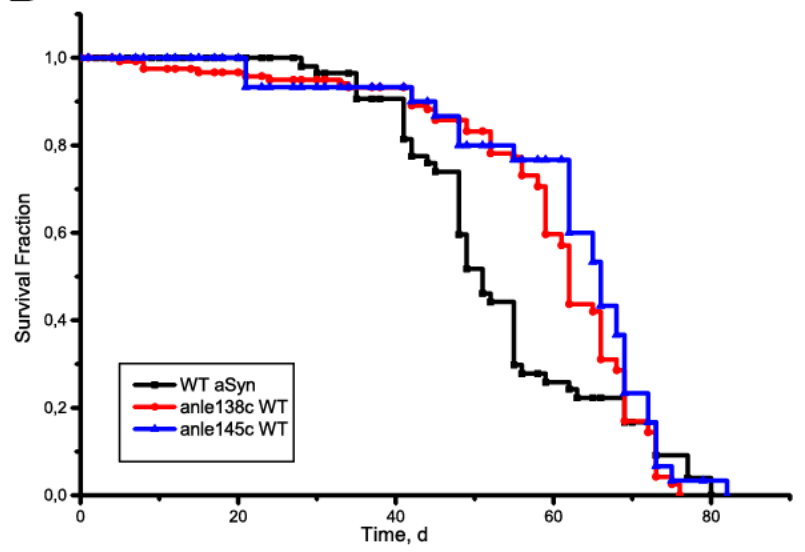

Figure 2.7.1. DPP compounds in $\boldsymbol{C}$. elegans and Drosophila $\alpha$-synuclein models. (A) The structure of compounds selected for testing in C. elegans and Drosophila $\alpha$-synuclein models. (B) Neuronal degeneration in C. elegans induced by expression of A30P $\alpha$-synuclein mutant (black bars) and effect of DPP compounds at $50 \mu \mathrm{M}$ (gray bars) on neuronal pathology; egfp group shown as a control (gray bars) (C) and (D) Treatment with selected DPP compounds of transgenic Drosophila flies expressing A53T mutant (C) or wild type (D) $\alpha$-synuclein.

Remarkably, the compound anle145c that efficiently modulates the aggregation of $\alpha$ synuclein in vitro was found to be active in both non-mammalian $\alpha$-synuclein models. This observation further supports the relevance and comparability of these two animal models. Moreover, the value of Caenorhabditis elegans and Drosophila models is also supported by increased toxicity of $\alpha$-synuclein mutants (A30P and A53T), which are linked to familiar forms of Parkinson's disease, in comparison to wild type form of the protein. 
Considering a low number of the tested compounds, SAR analysis does not make much sense and therefore follow-up screening experiments with an extended library of compounds would be required. From the screening perspective, these two animal models have certain limitation associated with the testing of compounds characterized by low solubility in water and high hydrophobicity. To overcome these problems, advanced delivery protocols, which result in improved bioavailability, can be implemented ${ }^{470}$.

\subsubsection{Why anle $138 \mathrm{~b}$ in vivo?}

In this short paragraph I would like to spotlight key arguments supporting the selection of anle138b for in vivo examination in mice models of Parkinson's disease:

- Encouraging efficacy of anle138b in the prion-infected mice. Mounting evidences in a field of neurodegenerative diseases support the hypothesis that protein misfolding and related pathological mechanisms share many common features with the prion disease $\mathrm{e}^{471,472}$.

- anle138b inhibits $\alpha$-synuclein aggregation in vitro (SIFT assay).

- anle138b ameliorates up to $100 \% \alpha$-synuclein-induced membrane permeabilization (BLM)

- favorable pharmacokinetic profile and established oral application scheme

- anle138b has no signs of toxicity at therapeutic doses and long-term treatment Considering all these arguments, anle138b was selected as a best candidate for in vivo testing in mice models of Parkinson's disease. The hydrophilic compounds such as sery166a or anle 145 c are metabolically too instable to be useful in mammals. In addition, they don't go over the blood brain barrier. Therefore, hydrophobic compounds with high metabolic stability have to be selected.

\subsubsection{Therapeutics effect of anle $138 \mathrm{~b}$ in mouse models of Parkinson's disease}

In this chapter I would like to summarize and discuss the results of several studies focused on evaluation of the therapeutic effect of anle138b in mouse models of Parkinson's disease. The data described in this chapter is the result of the work of many people. In fact, the experiments with A30P mice model were performed in the laboratory of Prof. A. Giese; data from low-dose intragastric rotenone mice model was generated in the lab of Dr. F. PanMontojo; the effect of anle138b in 1-120 $\alpha$-synuclein mouse model was assessed in laboratory of Prof. M. G. Spillantini. My scientific contribution in this part of the project includes a participation in the design of treatment regiments schemes and evaluation of the generated data. Moreover, I was involved in in vitro part of the pharmacokinetic studies that are 
described in details in chapter 2.8. The main text and the figures in the chapters 2.7.3 were adopted with some modifications from corresponding publications J. Wagner, S. Ryazanov, et $a l .{ }^{10}$ (A30P and rotenone models) and M. Wegrzynowicz, et al. ${ }^{473}$ (1-120 $\alpha$-synuclein mice).

The first model that I would like to describe is the low-dose oral rotenone model. Recent evidence suggests that $\alpha$-syn aggregation also plays a role in mediating toxicity of complex-I inhibitors ${ }^{474}$. The neuroprotective effect of anle138b in vivo was evaluated in a recently established low-dose oral rotenone model that recapitulates essential features of PD pathology in humans such as $\alpha$-syn accumulation and progressive spread of pathology ${ }^{260}$. Of note, the detailed description of low-dose oral rotenone and comparison with other toxinbased models is provided in the chapter 1.6.2.2. Due to the low dose and intragastric application of rotenone, the direct toxic effect of rotenone is confined to the gut without systemic complex-I inhibition in the brain and muscles, thus differing from classical rotenone models. Over the course of the experiment the sequential spreading of $\alpha$-synuclein from the enteric nervous system to the brain through synaptically connected neurons is observed. $\alpha-$ Synuclein pathology from neurons of duodenum and ileum expands to the central nervous system affecting the spinal cord and the brainstem and finally in 3 months an increased amount of intracellular $\alpha$-synuclein aggregates is detected in the substantia nigra. Moreover, the spread of $\alpha$-synuclein pathology is accompanied by $\alpha$-synuclein phosphorylation, inflammation, time-dependent motor dysfunction (rotarod) and selective loss of dopaminergic neurons in the substantia nigra ${ }^{260}$.

As mentioned in the introduction, the experiments with the low-dose oral rotenone model were performed in the lab of Dr. F. Pan-Montojo. In this model anle138b prevented the impairment of motor dysfunction (Fig. 2.7.2A). It is also remarkable that the therapeutic effect of anle138b was similar to effect of the procedure called hemivagotomy, which involves the resection of autonomic nerves. This surgical intervention has recently been shown to partially block the spread of $\alpha$-syn pathology from the enteric nervous system to the $\mathrm{CNS}^{475}$ (Fig. 2.7.2A). Moreover, treatment with anle138b almost completely rescued the phenotype in the 1-h stool collection gut motility test (Fig. 2.7.2B), which has previously been shown to correlate with the presence of $\alpha$-syn pathology in the enteric nervous system in this model $^{475}$. 
A

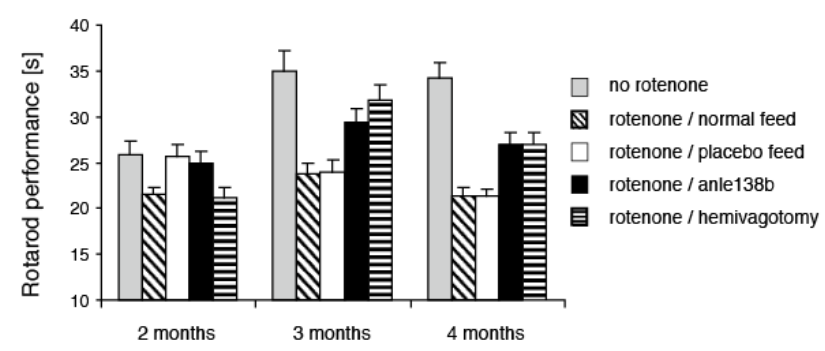

B

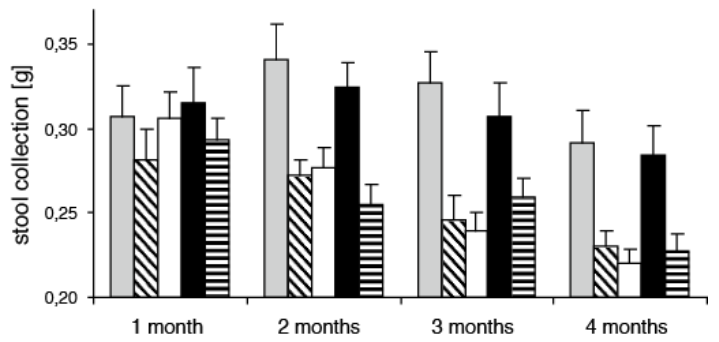

Figure 2.7.2. Effect of anle138b in low-dose intragastric mice model of Parkinson's disease. (A) Oral administration of anle138b results in a significant improvement of motor performance (rotarod) at 3 and 4 months of rotenone treatment. Effects of anle138b and hemivagotomy are similar. (B) 1-h stool collection gut motility test. Error bars in (A, B) indicate standard error. The figures are adapted from J. Wagner, S. Ryazanov, et al. ${ }^{10}$ Copyright (c) 2013.

Next, the effect of anle138b was tested in a well-established long-term transgenic $\alpha$ synucleinopathy mouse model based on neuronal expression of human A30P $\alpha$-synuclein mutant $^{283}$. This animal study was performed in the laboratory of Prof. A. Giese.

Analysis of the placebo-treated transgenic mice revealed that pathological deposition of $\alpha$-synuclein in the brain was first detected in the brain stem at the end of the first year of life, increased with time and spread to other brain areas. The formation of $\alpha$-synuclein inclusions showed correlation with signs of disease. The alterations during a prodromal phase (approximately 300-450 days of age) were represented by reduced gain of weight and increased fluctuations in motor performance during rotarod measurements (Fig. 2.7.3A, B). Of note, the signs of increased fluctuations in motor performance are considered a typical feature of human synucleinopathies ${ }^{476}$. During a clinical phase the animals showed a rapid decline in rotarod performance (Fig. 2.7.3C). In comparison to the placebo group, anle138b treatment significantly mitigated the motor phenotype (Fig. 2.7.3A) and restored the development of body weight almost to the level of wild type mice (Fig. 2.7.3B). Disease-free survival was significantly prolonged by $\sim 10$ weeks (Fig. 2.7.3C). 
$\mathbf{A}$

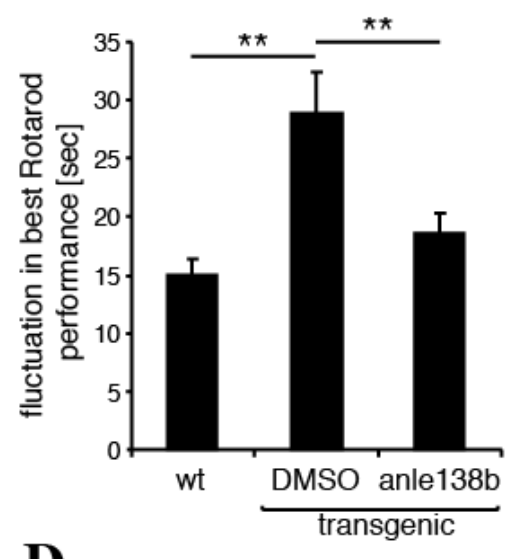

D

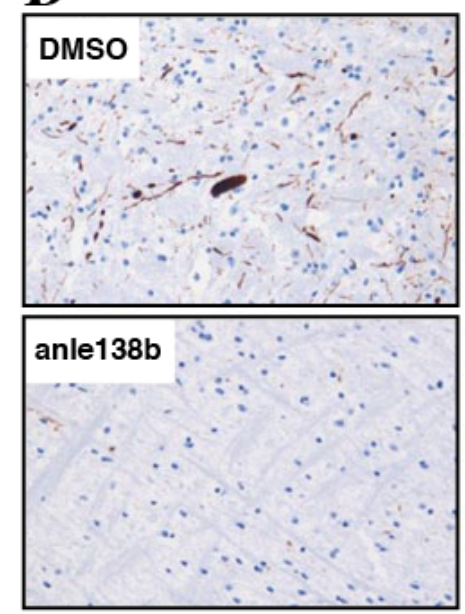

B

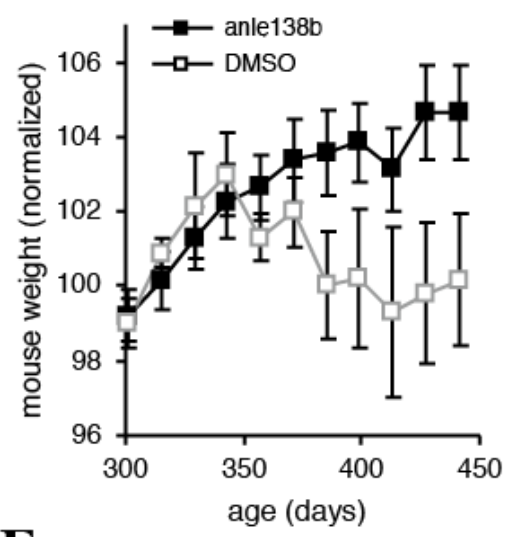

$\mathbf{E}$

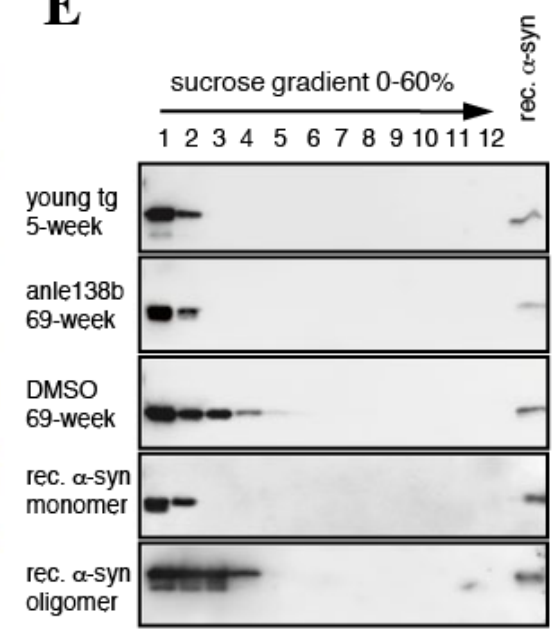

$\mathbf{C}$

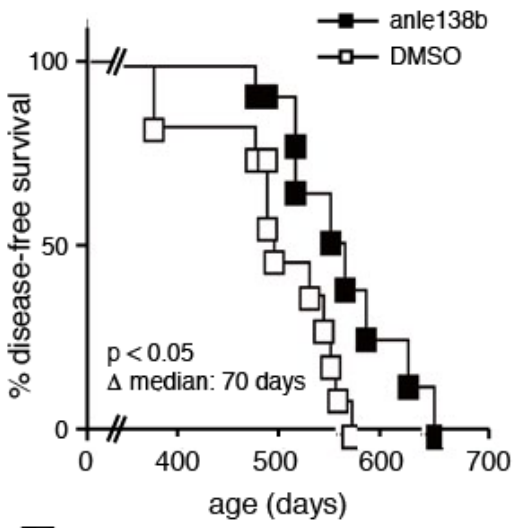

$\mathbf{F}$

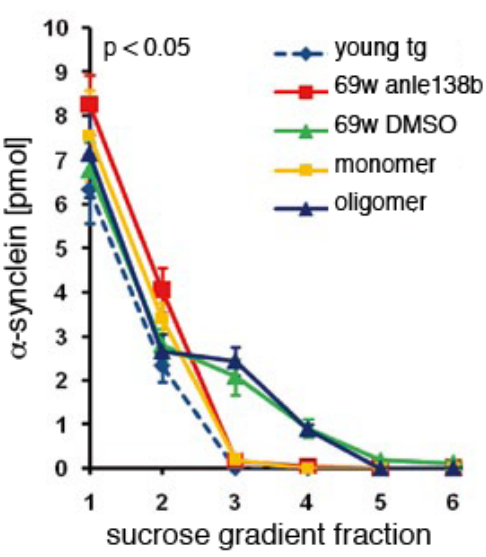

Figure 2.7.3. Effect of anle138b in A30P-hum-a-synuclein transgenic mice model of Parkinson's disease. (A) Oral administration of anle138b mitigates the fluctuations in motor performance during rotarod measurements. (B) Development of the body weight in placebo and anle138b-treated groups. (C) Kaplan-Meier evaluation of treatment with anle138b shows a significant increase in disease-free survival by 10 weeks. (D) Immunohistochemical analysis of the representative brain slices shows a significant reduction of $\alpha$-synuclein deposits in 69week-old the anle138b-treated group in comparison to matched control animals $(\mathbf{E}, \mathbf{F})$ Sucrose-gradient centrifugation shows the inhibition of $\alpha$-synuclein oligomer formation in anle138b-treated 69-week-old animals. Size distribution analysis of young A30P mice, 69week-old A30P placebo mice, recombinant monomeric $\alpha$-synuclein, $\mathrm{DMSO} / \mathrm{Fe}^{3+}$-induced $\alpha$ synuclein oligomers. Error bars in $(\mathbf{A}, \mathbf{B}, \mathbf{F})$ indicate standard error. The figures are adapted from J. Wagner, S. Ryazanov, et al. ${ }^{10}$ Copyright (C 2013. 
In addition to phenotypical rescue, the immunohistochemical analysis of the brain tissue showed a significant reduction of $\alpha$-synuclein deposits in 69 -week-old the anle138btreated group in comparison to matched control animals (Fig. 2.7.3D). In order to obtain more detailed characterization of $\alpha$-synuclein pathology, the sucrose gradient centrifugation of the brain homogenates was performed. As shown in Figure 2.7.3EF, anle138b treatment significantly reduced the amount of pathological oligomeric forms in the brain (fractions 3 and 4) to the level of young transgenic animals. It is also remarkable that the size distribution of recombinant $\alpha$-synuclein oligomers, which are induced by $\mathrm{DMSO} / \mathrm{Fe}^{3+}$, is concomitant with the properties of in vivo-formed oligomeric $\alpha$-synuclein (Fig. 2.7.3F). It was also experimentally confirmed that anle $138 \mathrm{~b}$ does not change the $\alpha$-synuclein expression levels in these mice ${ }^{10}$.

Next, the effect of anle138b was also evaluated in a recently developed transgenic mice line (MI2) expressing human truncated 1-120 $\alpha$-synuclein ${ }^{473}$. This animal study was performed in the laboratory of Prof. M. G. Spillantini.

For brief introduction, MI2 mouse model relies on overexpression of human truncated 1-120 $\alpha$-synuclein under control of TH promoter. The biological relevance of C-truncated forms of $\alpha$-synuclein in context of Parkinson's disease is supported by detection of these forms in the patient-derived samples. For instance, it was estimated that $10-25 \%$ of Lewy body of total $\alpha$-synuclein is represented by various truncated fragments ${ }^{477}$. From a view of mechanistic implication in development of Parkinson's disease, various in vitro studies have suggested that $\mathrm{C}$-truncated forms of $\alpha$-synuclein are more prone to aggregation that full length protein $^{135}$ Moreover, the increased toxicity of C-truncated forms of $\alpha$-synuclein was demonstrated in vivo ${ }^{478}$. The characterization of MI2 mice model has also confirmed the detrimental role of truncated 1-120 $\alpha$-synuclein in in vivo neurotoxicity ${ }^{473}$. Thus, MI2 mice model exhibits the phenotype associated with the $\alpha$-synuclein pathology that recapitulates many features of human Parkinson's disease. In particular, the aggregation and progressive accumulation of $\alpha$-synuclein in the dopaminergic neurons; progressive dopaminergic deficits and neuronal cell death ${ }^{473}$. Behavioral tests have shown the early symptomatic dysfunction (gait impartment) in young animals and pronounced motor deficits in old mice (Rotarod). Remarkably, the Rotarod motor impartment is observed in MI2 mice at age when a loss of dopaminergic neurons reaches $50 \%$, which has certain analogy with the human situation. Another impressive findings of this study were obtained by use of novel high-resolution microscopy technique $\mathrm{dSTROM}^{479}$. Analysis of the brain tissues with dSTROM at a near single-molecule resolution revealed the age-dependent accumulation of $\alpha$-synuclein 
aggregates with a diameter of $20-500 \mathrm{~nm}$.

A

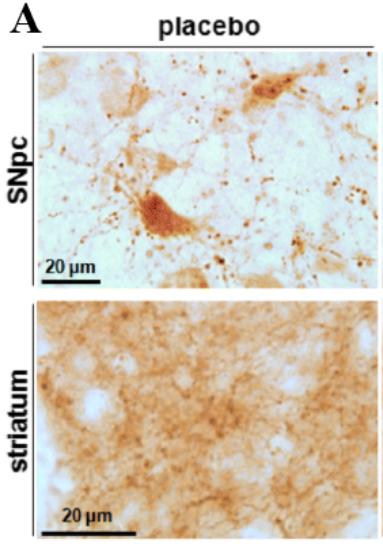

B

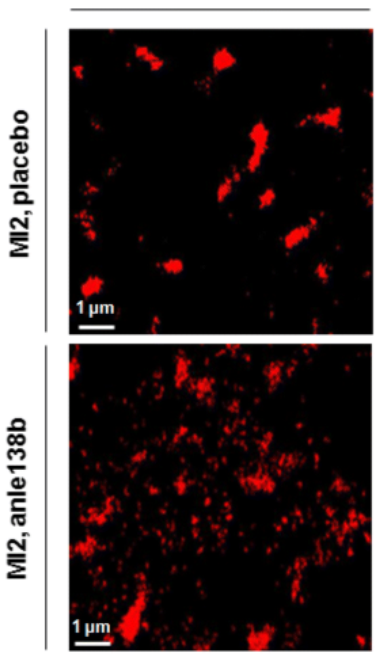

$\mathbf{C}$
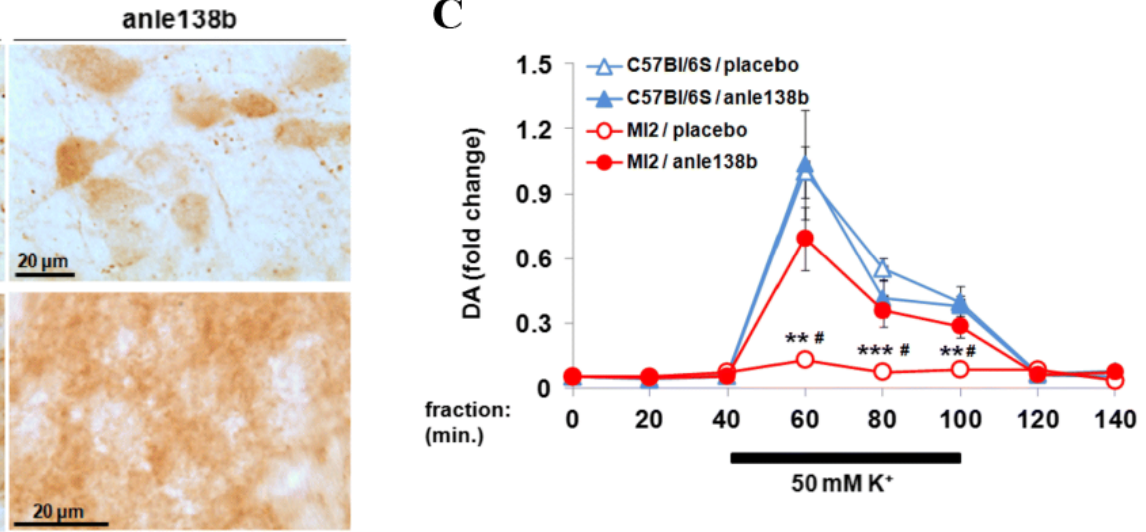

D
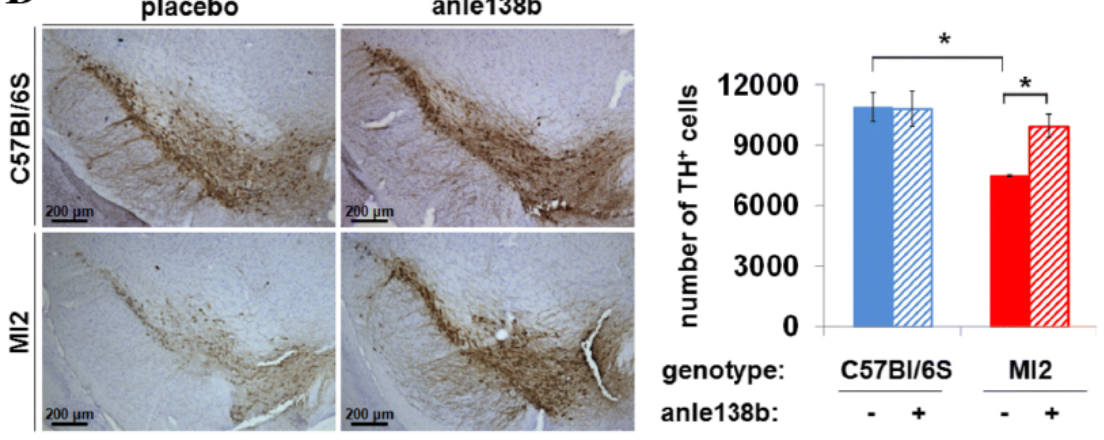

Figure 2.7.4. Effect of anle138b in transgenic 1-120 haSyn MI2 mice. (A) Anle138b reduces the accumulation of 1-120 $\alpha$-synuclein deposits in SNpc and striatum (Syn1 immunostaining). (B) Representative $d$ STORM images of 1-120 haSyn staining in the striatum of anle138b and placebo-treated 12 months old MI2 mice. (C) The effect of anle138b treatment on striatal dopamine release studied by in vivo microdialysis. Data are normalized to $\mathrm{C} 57 \mathrm{~B} 1 / 6 \mathrm{~S} /$ placebo group. (D) Anle138b reduces the nigral neurodegeneration of THpositive neurons. Left: TH immunostaining of SNpc of anle138b- and placebo-treated animals. Right: Stereological quantification of a number of TH-positive cells. Error bars in (C, D) indicate standard error. The figures are adapted from M. Wegrzynowicz, et al. ${ }^{473}$ licensed under CC BY 4.0, copyright (C) (2019)

Considering the relevance of the described phenotype, the effect of anle138b treatment was investigated in the MI2 mouse model. In order to be close to the clinical situation, the administration of anle $138 \mathrm{~b}$ was done for 3 months from 9 months of age, when neuronal loss is not significant, but the impaired dopamine release is present. In comparison to placebo 
group, anle $138 \mathrm{~b}$ treatment significantly restored the dopaminergic impairments, as evident for a restoration of the striatal dopamine release to the levels of wild type mice (Fig. 2.7.4A). The immunohistochemical analysis of the SNpc showed a significant reduction of 1-120 $\alpha$ synuclein deposits in anle138b-treated group (Fig. 2.7.4B). The effect of anle138b on 1-120 $\alpha$-synuclein pathology was further investigated by high-resolution ASTORM imaging. Herein, the comprehensive analysis of the dSTORM images not only confirmed the pronounced inhibition of 1-120 $\alpha$-synuclein aggregation by anle138b, but also showed a modulation of size distribution of 1-120 $\alpha$-synuclein aggregates (Fig. 2.7.4B). As shown in Figure 2.7.4B, $\alpha$ synuclein pathology in the anle138b-treated group is characterized by increased fraction of small non-clustered 1-120 $\alpha$-synuclein species and reduced inner density of small and large 1$120 \alpha$-synuclein aggregates. Last but not least, the administration of anle138b also attenuated the loss of TH-positive neurons and partially improved behavioral phenotype ${ }^{473}$.

Collectively, the efficacy data from three different types of mouse models of Parkinson's disease confirmed the therapeutic value of anle138b. As mentioned in the chapter 2.7.1, anle138b has been also tested in four other mice models and in one monkey model of $\alpha$ synuclein toxicity. Although the detailed discussion of all available data is beyond the scope of my thesis, I would like to emphasize the key findings of these studies. First, the oral administration of anle138b has been shown to prevent the dopaminergic cell death in the substantia nigra in a sub-acute MPTP model for Parkinson's disease ${ }^{10}$. As described in two recent publications ${ }^{480,481}$, anle138b treatment also leads to beneficial therapeutic effects in model of multiple system atrophy, which is another neurodegenerative disease characterized by oligodendroglial $\alpha$-synuclein pathology. Within our collaboration with the lab of Prof. E. Bezard, the effect of anle138b was studied in seeding model of Parkinson's disease in mice and in monkey that recapitulates several pathophysiological features of the prion-like pathways in $\alpha$-synucleinopathies ${ }^{302}$. These studies have established that the nigral dopaminergic cell loss, which is induced by inoculation of purified Lewy bodies from human patients, is rescued in the anle138b-treated mice and monkeys (unpublished data).

\subsubsection{Anle138b and $\alpha$-synuclein in vivo: Key findings and discussion}

In Parkinson's disease and other synucleinopathies, misfolding of $\alpha$-synuclein is considered as a key event involved in neurotoxic pathways leading to neurodegeneration and dopaminergic pathology $105,109,127$. Under normal conditions, $\alpha$-synuclein exits in a dynamic equilibrium between cytosolic unfolded monomeric form and $\alpha$-helical membrane-bound form. The exact physiological function of $\alpha$-synuclein is still not fully understood, but several 
studies suggested the role of this protein in vesicle trafficking, synaptic function, neurotransmitter release, and axonal transport ${ }^{482,483}$. Under pathological conditions, which are induced by so far unknown trigger(s), $\alpha$-synuclein undergoes a conformational transition to an aggregation-prone state and that eventually leads to formation of soluble oligomeric assemblies and insoluble fibrillar deposits ${ }^{5,484}$. Mounting evidences suggest that soluble oligomeric forms of $\alpha$-synuclein may constitute the main neurotoxic species, however the extract structure of the toxic oligomers and associated pathogenic pathways are not fully established 2, 127, 269. Although the exact mechanism(s) of oligomer toxicity is still under debate, it has been proposed that detrimental role of misfolded $\alpha$-synuclein might be associated with a permeabilization of plasma/organelle membranes by formation of pore-like structures, impairment of mitochondrial function ${ }^{210}$, interference with proteasomal and lysosomal protein degradation pathways ${ }^{216}$, ER dysfunction 201 and oxidative stress ${ }^{55}$. Considering the crucial role of $\alpha$-synuclein oligomerization and aggregation in pathogenic cascade, the interference and modulation of $\alpha$-synuclein misfolding has been recognized as a promising therapeutic opportunity ${ }^{127,132,311}$. To date, the inhibition of $\alpha$-synuclein aggregation has been achieved by the use of small molecules, intrabodies and immunotherapies both in vitro and in vivo ${ }^{311}$. Despite considerable progress in the field of immunotherapies, the development of small organic compounds for CNS has several advantages, including ability of drug to enter inside the cells, suitable pharmacokinetic profile, blood-brain-barrier penetration and low cost.

Here we show that DPP-related compounds and specifically anle138b are neuroprotective in several mouse models of synucleinopathies and, what's more, this therapeutic effect is associated with direct modulation of $\alpha$-synuclein aggregation on the molecular level. Of particular significance for the present study is the finding that anle $138 \mathrm{~b}$ prevents the formation of toxic oligomers and improves the behavioral, neuropathological and biochemical outcomes in all different animal models based on toxins, spreading and overexpression. In light of ongoing discussion on the relevance of one or another specific $\alpha-$ synuclein model, the evaluation of anle $138 \mathrm{~b}$ in several models recapitulating certain features of the complex disease pathogenesis allowed us to address the specific mechanisms of $\alpha$ synuclein toxicity as a target for pharmacological intervention. Remarkably, the accumulated evidences from these studies have consistently shown that the beneficial effect of anle138b is linked to direct modulation of $\alpha$-synuclein oligomers in vivo.

The neuroprotective effect of anle138b treatment was demonstrated in low-dose oral rotenone model. Under this low-dose regime, rotenone has been shown to induce the 
accumulation and aggregation of $\alpha$-synuclein in the enteric nervous system (ENS) ${ }^{260}$. At later stages this pathology spreads to the central nervous system through synaptically connected neuronal structures. The relevance of this model is confirmed by clinical findings suggesting that $\alpha$-synuclein accumulation in ENS is a permanent feature of early Parkinson's disease ${ }^{485}$. In our study, the oral administration of anle $138 \mathrm{~b}$ prevented the development of motor impairment and dysfunction of enteric nervous system as evident by 1 -h stool collection gut motility test (Fig. 2.7.2). Based in this data, it seems plausible that anle138b attenuates the toxicity of $\alpha$-synuclein in the neurons of the enteric nervous system and eventually prevents the spreading of pathology to the brain. Therefore, our findings support the potential of anle $138 \mathrm{~b}$ in treatment of non-motor disturbances and neuropathology of peripheral nervous system at early stages Parkinson's disease.

We also demonstrated the therapeutic effect of anle138b in two $\alpha$-synuclein transgenic mouse models expressing human A30P $\alpha$-synuclein or 1-120 human $\alpha$-synuclein in neurons. Altogether, these two models recapitulate many clinical and pathophysiological features of Parkinson's disease together with the progressive $\alpha$-synuclein pathology. In case of A30P model, anle $138 \mathrm{~b}$ treatment significantly reduces the accumulation of $\alpha$-synuclein deposits in the brain (Fig 2.7.2). The beneficial effect of anle138b on $\alpha$-synuclein pathology also correlates with improvement of motor performance and body weight development in comparison to untreated animals (Fig. 2.7.2). In line with results from A30P mice, anle138b rescues the phenotype of MI2 mice model, which is characterized by progressive dopaminergic deficits, neuronal cell death and consistent $\alpha$-synuclein aggregation in dopaminergic neurons. We demonstrated that anle138b dosing for 3 months to 9 months old MI2 mice restores the dopamine release back to its normal levels as observed in healthy control mice (2.7.3). Moreover, anle138b significantly reduces the neurodegeneration and the accumulation of aggregated $\alpha$-synuclein in substantia nigra and striatum (2.7.3). Collectively, our observations in A30P and MI2 mouse models have demonstrated the efficient inhibition by anle138b of $\alpha$-synuclein misfolding and aggregation in vivo, which persistently translates to neuroprotective effect on dopaminergic neurons and improvement of motor function. It is also remarkable that anle138b has a big therapeutic window as evident from the experiments in MI2 mice, in which dopaminergic function impaired by $\alpha$-synuclein misfolding can be fully recovered (2.7.3). In line with these results, the follow-up study in old A30P mice has confirmed the efficacy of anle138b treatment started in the symptomatic phase of the disease $^{486}$. From therapeutic and pharmacological standpoint, anle138b has striking advantage over other published inhibitors of $\alpha$-synuclein aggregation. First, anle138b is neuroprotective 
in 8 different animals models, while most advanced compounds like NPT200-11 ${ }^{339}$, NPT100$18 \mathrm{~A}^{340}$ or mannitol ${ }^{338}$ have been tested just in one or two models. Second, anle138b has low toxicity; excellent oral bioavailability and property to cross the blood-brain-penetration resulting in stable brain exposure.

Regarding the mode of action, our findings provide compelling evidence that anle138b directly modulates the aggregation of $\alpha$-synuclein at the oligomer level in vivo. In support of this mechanism, we demonstrated in sucrose gradient centrifugation assay a significant reduction of oligomeric fractions in the brain homogenates of old A30P mice treated with anle138b (Fig. 2.7.3). Similar results were obtained in the PLP-haSyn model of $\mathrm{MSA}^{480,481}$. In this model, which is relying on expression of $\alpha$-synuclein in oligodendrocytes, the sucrose gradient centrifugation analysis of brain homogenates derived from the mice with pronounced pathological phenotype showed a significant accumulation of $\alpha$-synuclein oligomeric species. In contrast to control group, the oligomer formation in PLP-haSyn mice is strongly inhibited by oral administration of anle $138 \mathrm{~b}^{481}$.

Super-resolution imaging, such as $d$ STROM, provides a possibility to characterize the properties of protein aggregates at a single molecular level in vivo ${ }^{479}$. In our study, $d$ STROM analysis revealed that the progressive pathological phenotype in MI2 mice is correlating with accumulation of 20-500 $\mathrm{nm} \alpha$-synuclein species. On the other hand, comprehensive $d$ STROM-based evaluation of the corresponding brain slices from anle138b-treated animals showed 1) the increased fraction of small non-clustered 1-120 $\alpha$-synuclein species, which likely represent monomeric or low molecular weight forms of $\alpha$-synuclein; 2) the reduced inner density of small and large 1-120 $\alpha$-synuclein aggregates (Fig. 2.7.3). These observations are concordant with the results of sucrose gradient centrifugation assay.

Finally, we demonstrated the neuroprotective effect of anle138b in the LB-seeding mouse model ${ }^{386}$. Expanding research continues to link the pathogenic role of $\alpha$-synuclein with prion-like properties of aggregation-prone $\alpha$-synuclein species. Like prions, misfolded $\alpha$ synuclein can induce seeding, recruitment and templating of endogenous $\alpha$-synuclein in vitro ${ }^{130}$. "Prion" paradigm is further supported by observations of cell-to-cell transmission of $\alpha$-synuclein aggregates in cell cultures ${ }^{487}$, and, more importantly, by neuropathological manifestations in vivo, including pathogenesis of Lewy body-like and fibrillar $\alpha$-synuclein in animal models ${ }^{302}$, 303; spreading of $\alpha$-synuclein pathology into the grafter neurons in patients $^{488}$ and development of dual-hit hypothesis of propagation of synucleinopathy by Braak $^{22}$. Our study in seeding mouse model of Parkinson's disease have demonstrated that anle138b can efficiently ameliorates the dopaminergic pathology induced by inoculation of 
human derived $\alpha$-synuclein aggregated form ${ }^{302}$. To the best of our knowledge, anle138b is a single example of small drug compound that can interfere with prion-like mechanisms of $\alpha$ synuclein propagation and toxicity in vivo.

Taken together, the presented data supports the hypothesis that in vivo anle138b targets the pathological cascade at the level of $\alpha$-synuclein oligomeric structures. Our observations and the results from many other studies strongly support the notion that interference with $\alpha$-synuclein oligomerization by small molecules may represent the attractive strategy in development of disease-modifying therapy. 


\subsection{Formulation development and pharmacokinetic of anle138b and other DPP compounds}

\subsubsection{Introduction}

In pharmaceutical research, pharmacokinetic studies play very important role. In simple words, pharmacokinetics describes how the organism deals with a drug or any other applied compound. More specifically, pharmacokinetics characterizes the absorption, distribution, metabolism, and elimination of the drug. The pharmacokinetic characterization is an essential part of preclinical and clinical drug development, especially in the case of biodistribution analysis, site-selective drug delivery, toxicity studies, pharmacokineticpharmacodynamic modeling, and optimization of drug formulation.

In our project, we performed the pharmacokinetic studies to evaluate the oral bioavailability of anle138b and other DPP compounds in mice and in rats. Moreover, we analyzed the biodistribution to confirm the delivery of anle $138 \mathrm{~b}$ to the brain and other organs and tissues. Additionally, we developed and characterized several anle138b administration protocols in order to support the efficacy studies in various animal models.

In these studies, our collaborators (Dr. Ulrike Teichmann and her team, Dr. Jens Wagner, Dr. Tobias Frank; Dr. Bettina Göricke, Dr. Sebastian Kügler) did the animal part of the experiments; I contributed with the study design, processing of biological samples, HPLC analysis and the data evaluation. Dr. Jens Pilger has assisted me in early phases of the project.

\subsubsection{DPP compounds in mice}

As described in the chapter 2.4.6, several DPP compounds exhibited promising therapeutic effects in a prion-infected mouse model. Since the efficacy of the treatment depends on the concentration of the corresponding compound at the site of action, we performed a pharmacokinetic study in mice with the 25 most interesting DPP compounds (Fig. 2.8.1C). In chapter 2.4.9 we propose the determination of pharmacokinetic properties of the lead molecules as one of key steps of the proposed drug-discovery strategy against prion disease (Fig. 2.4.14).

The main objective of this experiment was to determine the concentration of the DPP compounds in the brain and in the spleen. A comparative analysis of the PK parameters and the efficacy data allowed us to obtain valuable SAR information and also explain the lack of therapeutic effect of substances with poor bioavailability. Moreover, we wanted to understand 
the correlation between structural properties of DPP compounds, drug exposure and bloodbrain-barrier penetration.

In this study, we employed the same administration protocol as in the efficacy experiments in the prion-infected mice. Thus, the corresponding compounds were applied orally in the peanut butter/DMSO mixture at a single dose of $50 \mathrm{mg} / \mathrm{kg}$. The tissues were collected 4 hours after dosing. To analyze the levels of DPP compounds, we have developed a reliable extraction protocol with acetonitrile, which ensures a complete dissolution of poorly water-soluble DPP molecules.

As shown in Figure 2.8.1, the majority of tested DPP compounds exhibited a good oral bioavailability and, more importantly, an excellent blood-brain-barrier penetration. Among 21 compounds detected in the brain, the levels higher than $10 \mu \mathrm{M}$ were observed for 14 compounds including anle138b. Also in the spleen, the oral administration of DPP compounds leads to significant levels, which in many samples are exceeding $10 \mu \mathrm{M}$ concentration.

Based on pharmacokinetic data, we designate several structural elements that appear particularly important for DPP compounds to reach the brain. Thus, the compounds bearing methylenedioxy moiety in one phenyl ring in combination with nonpolar substituent(s) in another phenyl ring are orally bioavailable and can readily cross the blood-brain-barrier in mice, for example anle138b, anle186b, anle197b, anle270, sery335b and others. On the other hand, the exchange of nonpolar group like halogens with more hydrophilic substituents such as methoxy, hydroxyl and amino leads to significant reduction of brain levels, for instance anle233b, sery313b, sery383. This observation of reduced brain exposure might be associated with several factors including higher hydrophilicity, poor absorption, fast metabolism, different concentration-time profiles. Our results also indicate that the methylenedioxy moiety is a key element responsible for the favorable pharmacokinetic profile. The role of this structural element can be illustrated by comparison of anle $138 \mathrm{~b}$ with sery $312 \mathrm{~b}$, sery $401 \mathrm{~b}$ and sery417. As shown in Figure 2.8.1, the exchange of oxygen atoms in the methylenedioxy moiety to carbon or nitrogen atoms results in significant reduction of brain levels. 
$\mathbf{A}$

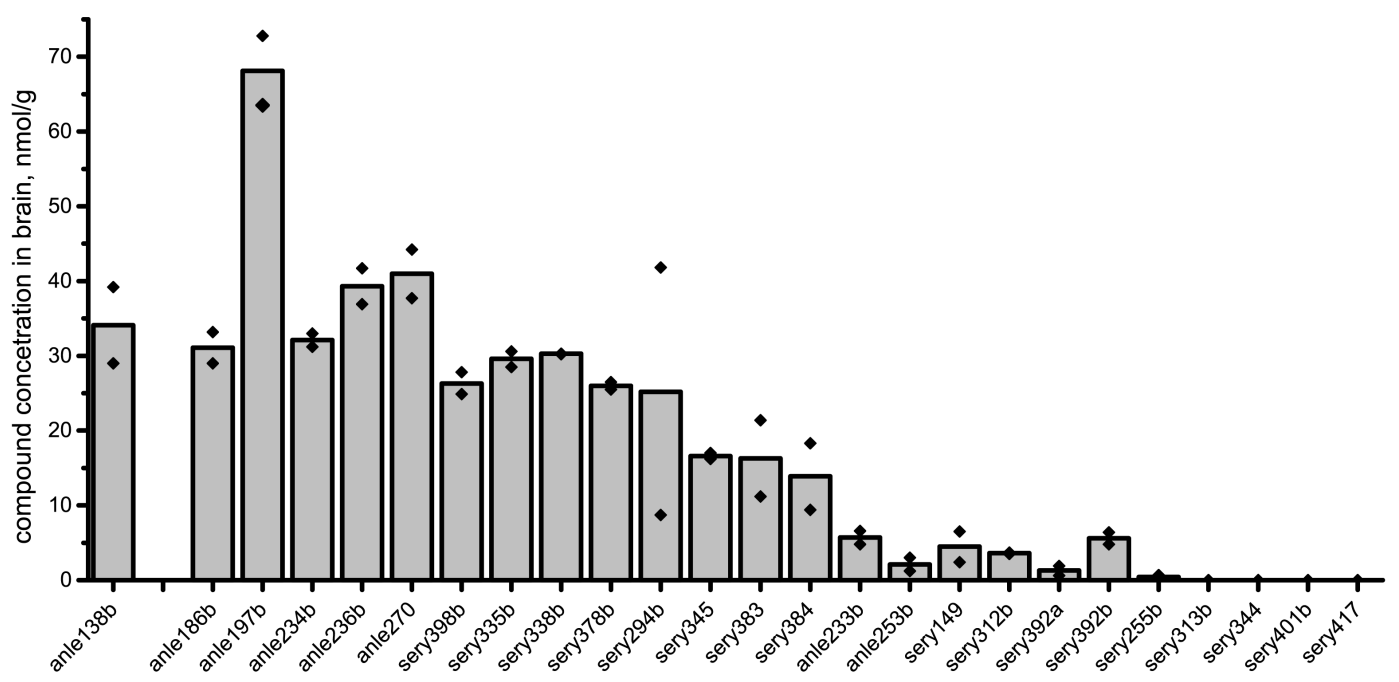

B

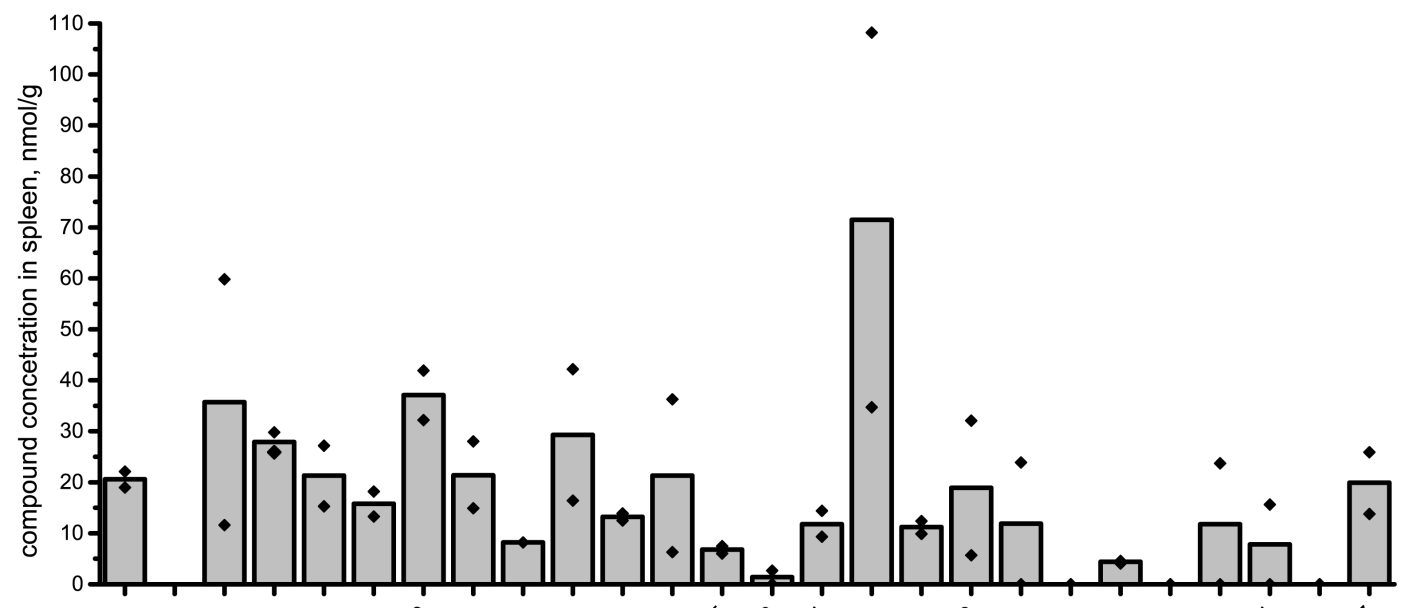

C
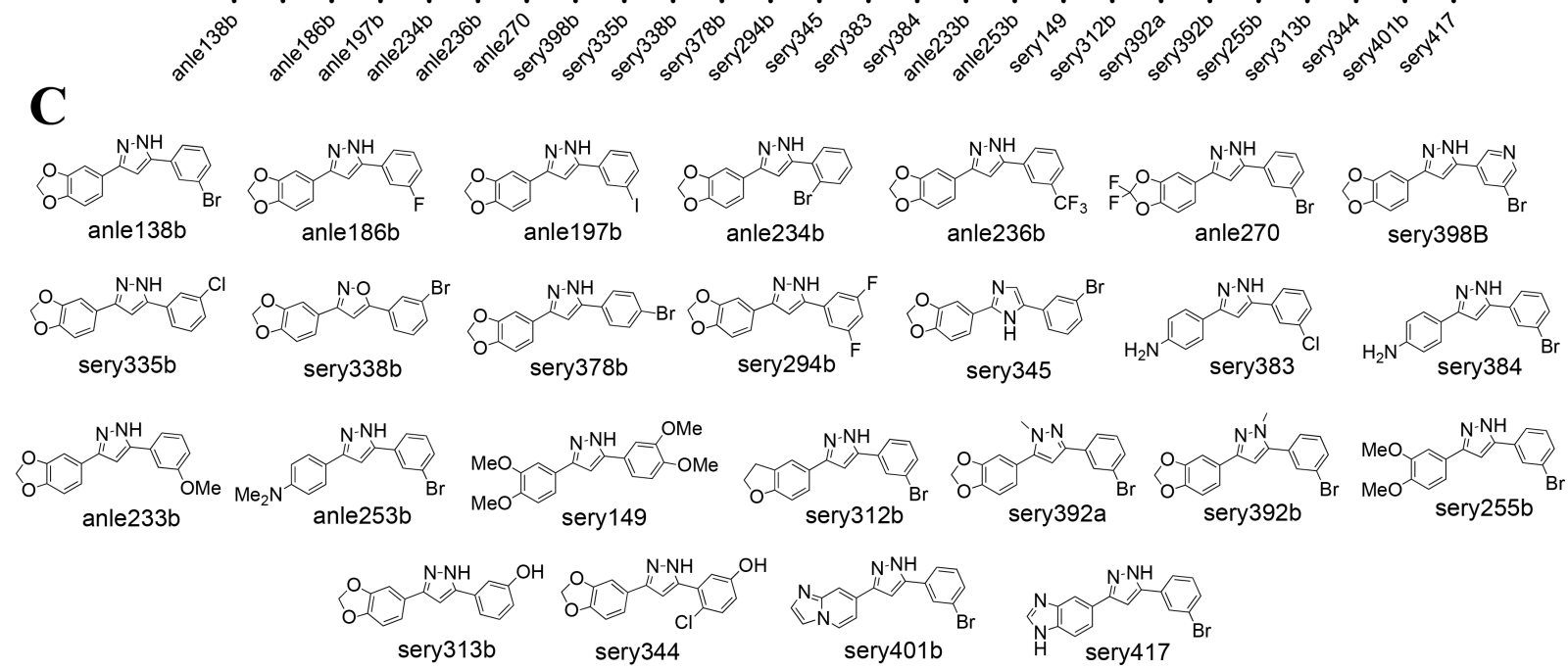

Figure. 2.8.1. PK study of DPP compounds in mice. Concentrations of DPP compounds in mouse brain (A) and spleen (B). DPP compounds were applied orally in peanut butter/DMSO at $50 \mathrm{mg} / \mathrm{kg}$ dose. Two animals per time point were sacrificed and analyzed. Bars represent a mean concentration; concentration for each individual animal is shown by dots. (C) Structures of tested DPP compounds sorted according to the chart. 
Collectively, our study in mice supported the favorable pharmacokinetic profile of DPP compounds following oral administration. Moreover, for many DPPs we observed good blood-brain-barrier penetration. From the structural perspective, the combination of methylenedioxy moiety and nonpolar substituents like halogens or $\mathrm{CF}_{3}$ group is required to achieve significant brain exposure.

\subsubsection{Pharmacokinetic properties of anle $138 \mathrm{~b}$ in mice}

Based on our efficacy data in different animal models of neurodegenerative disease, anle $138 \mathrm{~b}$ was identified as the most promising lead compounds. Therefore, we performed the pharmacokinetic analysis of the different application protocols used in various mice models. These pharmacokinetic studies provide usefully information for the analysis and evaluation of the efficacy data in relation to the drug exposure profile. Considering a study design in each animal model, the selection of formulation protocol is mainly determined by the duration of treatment and the dose. For the short treatment schemes with high doses of anle138b, the administration via gavage is most suitable. This protocol was applied in anle138b efficacy study in MPTP mouse model ${ }^{10}$. The oral formulation via gavage has several drawbacks such as a high animal stress, increased phenotypic variability and gavage is a time-consuming procedure. Therefore, long-term animal experiments require a more animal-friendly and practical administration protocol. At the initial phases of the project, anle138b was applied to prion-infected mice in a mixture with peanut butter and small amounts of $\mathrm{DMSO}^{10}$. In collaboration with the animal food manufacture SSNIFF we have developed a special anle138b-containing mouse diet, which can be used instead of normal food pellets. This special diet was used in all other mice studies.

Since pharmacokinetic profile of the drug is strongly affected by the route of administration, we have characterized the aforementioned anle138b formulation protocols in mice. In addition to anle $138 \mathrm{~b}$ concentration assessment, we have also determined several important pharmacokinetic parameters including maximum concentration $\left(\mathrm{C}_{\max }\right)$, time point when $\mathrm{C}_{\max }$ is reached $\left(\mathrm{T}_{\max }\right)$ and area under the curve (AUC). In pharmacokinetics, the AUC is a measure of the total systemic exposure to the drug. 

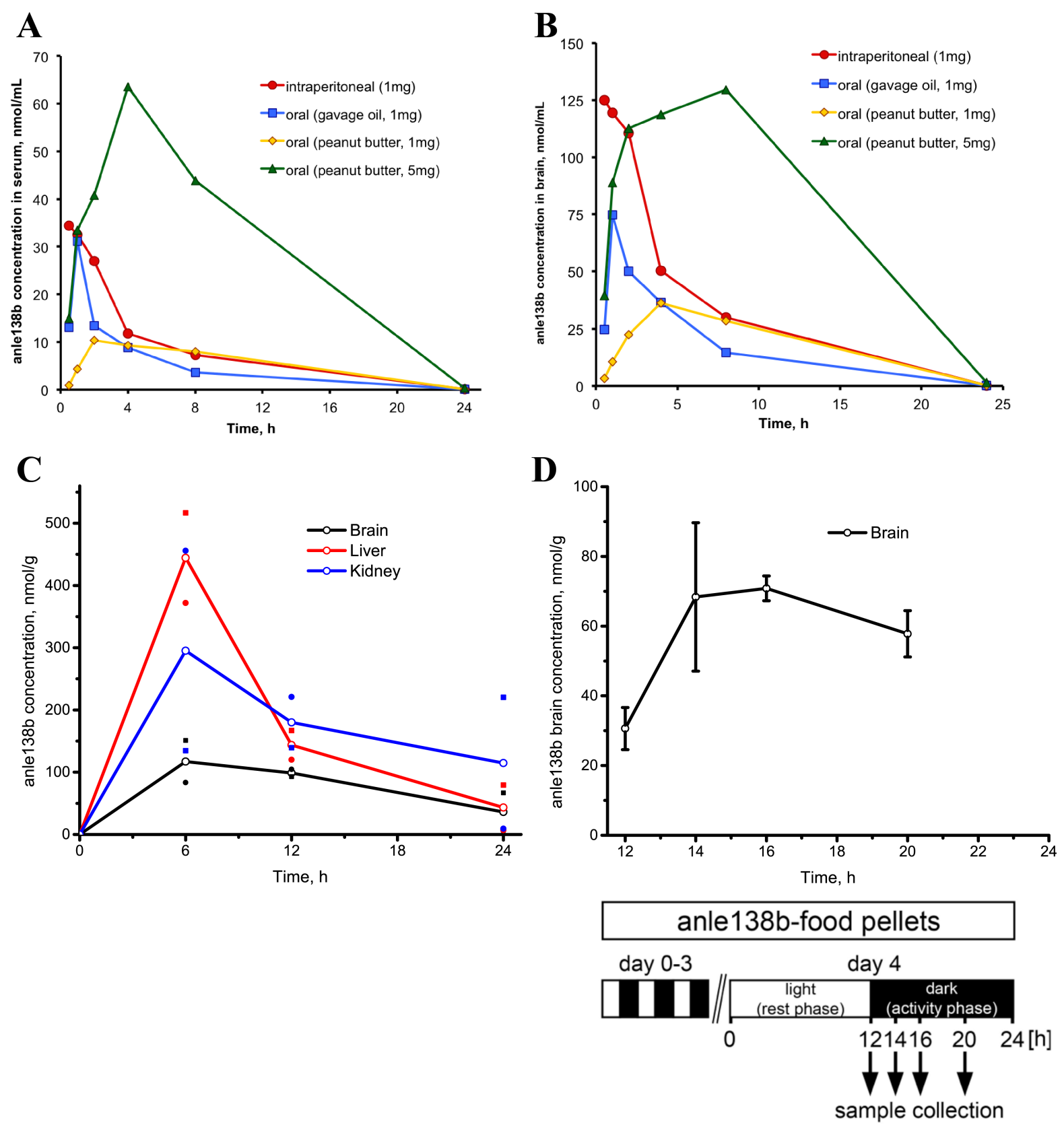

Figure 2.8.2. Pharmacokinetic analysis of anle138b in mice. Concentration-time profiles of anle138b applied by different application routes. Serum (A) and brain (B) anle138b concentration-time profile after single application of anle138b. The amount of anle138b applied per animal and the application protocol is shown in the figure inserts. (C) anle138b concentration-time profile in brain, liver and kidney after single oral application via gavage of anle138b in olive oil/DMSO at $250 \mathrm{mg} / \mathrm{kg}$ dose. Concentration for each individual animal is shown by dots (D) Concentration-time profiles of anle138b in mice fed ad libitum with special food diet ( $2 \mathrm{~g}$ anle138b/kg of pellets) on day 4 at $12 \mathrm{~h}, 14 \mathrm{~h}, 16 \mathrm{~h}$ and $20 \mathrm{~h}$ (upper). The anle $138 \mathrm{~b}$ diet was provided ad libitum for 4 days. Experimental scheme of pharmacokinetic experiment with anle138b-containing diet (lower). Error bars in (D) indicate standard error. The figures (A, B) are adapted from J. Wagner, S. Ryazanov, et al. ${ }^{10}$ Copyright (c) 2013. 
The results of several pharmacokinetic studies are summarized in Figure 2.8.2 and 2.8.3. The experiments shown on Figure 2.8.2 AB were performed in the laboratories of Prof. Manfred Uhr and Prof. Armin Giese. In line with the PK data described in the chapter 2.8.2, the pharmacokinetic analysis of the different administration protocols in mice showed that anle138b has a good oral bioavailability and good blood-brain-barrier penetration. The oral application of anle138b at high doses in peanut butter and in olive oil leads to slower absorption and prolonged exposure in different tissues and blood. At $250 \mathrm{mg} / \mathrm{kg}$ dose, anle $138 \mathrm{~b}$ is detected in the brain at very high concentration as demonstrated by anle $138 \mathrm{~b} \mathrm{C}_{\max }$ values of around $100 \mu \mathrm{M}$ (Fig. 2.8.2A, C and 2.8.3). It is remarkable that anle138b is effectively absorbed not only from the solution, but also when it is applied in crystalline form as additive to the animal diet (Fig. 2.8.2 D). Experimentally, the animals had free access to the anle138b-containing diet for 3 days, on day 4 the tissues samples were collected at different time periods during the dark phase, at which the mice are active and consume the pellets (Fig. 2.8.2 D). The pharmacokinetic analysis of these animals revealed that during the dark phase, anle $138 \mathrm{~b}$ displays a high and stable brain exposure in mice fed with anle138b-

\begin{tabular}{|c|c|c|c|c|}
\hline Matrix & $\begin{array}{l}\text { ANLE138B dose } \\
(\mathrm{mg} / \mathrm{kg})\end{array}$ & $\begin{array}{c}\mathrm{C}_{\max } \\
(\mathrm{nmol} / \mathrm{g})\end{array}$ & $\begin{array}{l}\mathrm{T}_{\max } \\
\text { (h) }\end{array}$ & $\begin{array}{c}\mathrm{AUC}_{0-24} \\
\text { (ng h/mL) }\end{array}$ \\
\hline \multicolumn{5}{|c|}{ i.p. dosing, DMSO } \\
\hline Brain & 50 & 124.8 & 0.5 & 263949 \\
\hline Serum & 50 & 34.4 & 0.5 & 65346 \\
\hline \multicolumn{5}{|c|}{ oral dosing, vegetable oil } \\
\hline Brain & 50 & 88.8 & 1 & 137275 \\
\hline Serum & 50 & 31.5 & 1 & 38633 \\
\hline \multicolumn{5}{|c|}{ oral dosing, peanut butter } \\
\hline Brain & 50 & 36.2 & 4 & 149888 \\
\hline Serum & 50 & 10.4 & 2 & 43481 \\
\hline \multicolumn{5}{|c|}{ oral dosing, peanut butter } \\
\hline Brain & 250 & 129.4 & 8 & 657944 \\
\hline Plasma & 250 & 63.5 & 4 & 248976 \\
\hline \multicolumn{5}{|c|}{ oral dosing, olive oil } \\
\hline Brain & 250 & 117.2 & 6 & 620693 \\
\hline Liver & 250 & 444.4 & 6 & 1446774 \\
\hline Kidney & 250 & 295.2 & 6 & 1399543 \\
\hline \multicolumn{5}{|c|}{ oral dosing, pellets } \\
\hline Brain & 500 & 70.8 & $4 *$ & $169939 * *$ \\
\hline
\end{tabular}

Figure 2.8.3. Pharmacokinetic analysis of anle138b in mice. Major pharmacokinetic parameters of anle138b after different application protocols in various tissues of mice (Mean values are listed). 
containing diet (Fig. 2.8.2 D). In case of this application protocol, we measured anle138b $\mathrm{C}_{\max }$ values of around $70 \mu \mathrm{M}$ (Fig. 2.8.3). In fact, the development and pharmacokinetic characterization of anle $138 \mathrm{~b}$ diet allowed us to conduct long-term mice studies in accordance with animal-friendly application protocol. For instance, all studies in mice models of Parkinson's disease, Alzheimer's disease ${ }^{489,490}$, with the exception of MPTP, were performed using this dry food diet. Additional pharmacokinetic parameters including $\mathrm{C}_{\max }, \mathrm{T}_{\max }$ and AUC are listed in Figure 2.8.3.

\subsubsection{Pharmacokinetic studies on anle $138 \mathrm{~b}$ in rats}

Considering the favorable pharmacokinetic properties on anle138b in mice, we decided to investigate the pharmacokinetic profile of anle $138 \mathrm{~b}$ in a second rodent species, rat. There are several reasons why we would like to characterize the pharmacokinetic properties of anle138b in rats. First, several rat models of Parkinson's disease are available such as viral models and they can be used for efficacy studies with anle $138 b^{287}$. Second, rat is most frequently used rodent species in preclinical toxicity studies. From the perspective of pharmacokinetics, blood is the most easily accessible sampling site to monitor the drug concentrations in the organism. In contrast to mice, rats have higher volumes of blood, which allows multiple blood sampling from a single animal during the course of experiment.

In addition to studying anle $138 \mathrm{~b}$ blood and brain exposure, we characterized the biodistribution of anle138b in various organs. Thus, we conducted a small pharmacokinetic experiment, in which three rats received a single oral application via gavage of anle138b in olive oil/DMSO at $250 \mathrm{mg} / \mathrm{kg}$ dose. At each time point (4h, $8 \mathrm{~h}$ and $12 \mathrm{~h}$ after dosing), the biological samples such as serum, brain, liver, kidney, fat, spleen lung, thymus were collected from a single animal. Herein, the interpretation of the data should be done with caution due to very low number of animals.

In line with mice PK data described in the chapter 2.8.3, the pharmacokinetic analysis of anle138b in rats showed that anle138b has a good oral bioavailability and good bloodbrain-barrier penetration following single oral administration in olive oil via gavage. At 250 $\mathrm{mg} / \mathrm{kg}$ dose, anle $138 \mathrm{~b}$ is detected in the brain and in the serum at high concentrations as demonstrated by $\mathrm{C}_{\max }$ values of around $169 \mu \mathrm{M}$ and $29 \mu \mathrm{M}$, respectively (Fig. 2.8.4 A, C). The brain-to-blood ratios of 4-6 were measured, which corroborates the excellent blood-brainbarrier penetration properties of anle138b (Fig. 2.8.4A). Due to the hydrophobicity of the molecule it seems to distribute in the various compartments of the body according to availability of lipid content or lipophilic proteins. In rats, we observe a slightly reduced 
anle 138b exposure in comparison to AUC values in mice (Fig. 2.8.3 and 2.8.4C). However, it can be explained by the limited number of time points and animals, especially in the period between formulation and first sample collection at 4 hours (Fig. 2.8.4A). As shown in Figure $2.8 .4 \mathrm{~B}$, anle $138 \mathrm{~b}$ is widely distributed in the various tissues, namely brain, liver, kidney, fat, spleen lung, thymus. The highest tissue concentration of anle138b was observed in the fat tissue (up to $500 \mu \mathrm{M}$ ), followed by the kidney and the liver (Fig. 2.8.4B). We assume that such profile of anle138b biodistribution is determined by high lipophilicity of this molecule that leads to higher concentration of anle138b in "lipophilic" tissues like fat and brain. The high levels in the liver and in the kidney are associated with process of anle138b metabolism and excretion. Additional pharmacokinetic parameters including $\mathrm{C}_{\max }, \mathrm{T}_{\max }$ and $\mathrm{AUC}$ are listed in Figure 2.8.4C.
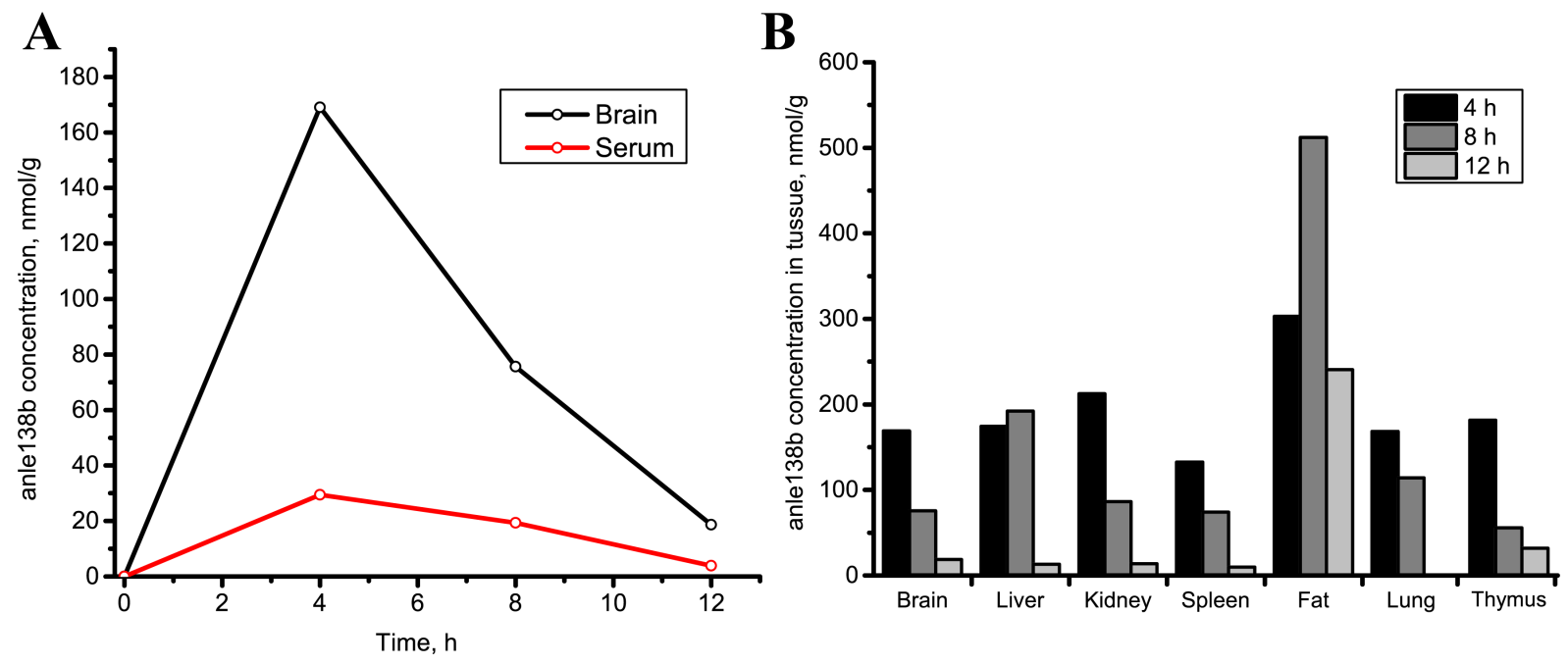

C

\begin{tabular}{ccccc}
\hline Matrix & $\begin{array}{c}\text { ANLE138B dose } \\
(\mathrm{mg} / \mathrm{kg})\end{array}$ & $\begin{array}{c}\mathrm{C}_{\max } \\
(\mathrm{nmol} / \mathrm{g})\end{array}$ & $\begin{array}{c}\mathrm{T}_{\max } \\
(\mathrm{h})\end{array}$ & $\begin{array}{c}\mathrm{AUC}_{0-12} \\
(\mathrm{ng} \mathrm{h} / \mathrm{mL})\end{array}$ \\
\hline oral dosing, olive oil & & & & \\
\hline Serum & 250 & 29.4 & 4 & 69492 \\
Brain & 250 & 169.1 & 4 & 348557 \\
Liver & 250 & 192.1 & 8 & 511893 \\
\hline
\end{tabular}

Figure 2.8.4. Pharmacokinetic analysis of anle138b in rats. anle138b concentrations were measured in rat serum and tissues after single oral application (gavage) of anle138b in olive oil with $3 \%$ DMSO at $250 \mathrm{mg} / \mathrm{kg}$ dose. (A) Serum and brain anle138b concentration-time profile after single application of anle138b. (B) anle138b concentrations in rat tissues at $4 \mathrm{~h}, 8$ $\mathrm{h}$ and $12 \mathrm{~h}$ after oral administration. (C) Major pharmacokinetic parameters of anle138b in selected tissues of rats. At each time point, the samples from one animal were collected. 


\subsubsection{Optimization of oral formulation in rats}

In all animal studies that are described in previous chapters anle $138 \mathrm{~b}$ was orally applied in the oil-based mixtures such as peanut butter, vegetable or olive oil that contained DMSO or dry food. Since the clinical use of such mixtures containing DMSO in human is not feasible, we decided to develop alternative formulations based on pharmaceutically acceptable excipients ${ }^{491}$.

At first phase of this project, the company Aeterna Zentaris conducted for us several in vitro experiments with anle $138 \mathrm{~b}$ including determination of anle $138 \mathrm{~b}$ solubility in different excipients followed by formulation optimization and dissolution studies in aqueous medium $(0.1 \mathrm{~N} \mathrm{HCl})$. As a result of these studies, three formulation compositions with the most promising properties have been selected for pharmacokinetic studies in rats (Fig. 2.8.5C). Together with Dr. Ulrike Teichmann and her team, we conducted the comparative pharmacokinetic studies of newly developed and olive oil formulations. In this experiment, the pharmacokinetics of anle138b was assessed in male wistar rats following single oral administration (gavage) of anle138b $10 \mathrm{mg} / \mathrm{kg}$ as solution in one of the formulations (V25, V32, V25 and olive oil) or as i.v. bolus at anle138b dose of $2 \mathrm{mg} / \mathrm{kg}$. Plasma samples were collected for analysis at seven time points after dosing ( $0.25 \mathrm{~h}, 0.5 \mathrm{~h}, 1 \mathrm{~h}, 2 \mathrm{~h}, 4 \mathrm{~h}, 8 \mathrm{~h}, 24 \mathrm{~h})$.

As shown in Figure 2.8.5, anle138b was detected in plasma of all animals regardless of administration protocols. Following i.v. application at $2 \mathrm{mg} / \mathrm{kg}$ dose, a mean $\mathrm{C}_{\max }$ was 4.1 $\mu \mathrm{M} / \mathrm{mL}$ and mean $\mathrm{AUC}_{0-8} 1098 \mathrm{ng} \mathrm{h} / \mathrm{mL}$ (Fig. 2.8.5B, D). In case of oral dosing, the highest $\mathrm{C}_{\max }$ and highest $\mathrm{AUC}_{0-8}$ values were observed for formulation V34 (Fig. 2.8.5A, D). Thus, anle138b systemic exposure was approximately 1.8-fold higher in V34 experimental group than in the animals receiving olive oil (Fig. 2.8.5D). It is remarkable that the use of V32 or V25 also resulted in improvement of anle138b pharmacokinetic parameters. Based on these AUCs, the absolute bioavailability of anle $138 \mathrm{~b}$ formulated in V34 was almost $50 \%$. The calculation of absolute bioavailability: AUC (V34, dose $10 \mathrm{mg} / \mathrm{kg})=2569 \mathrm{ng} * \mathrm{~h} / \mathrm{mL}$; adjusted AUC (i.v., $10 \mathrm{mg} / \mathrm{kg})=(1098 \mathrm{ng} * \mathrm{~h} / \mathrm{mL}) * 5=5490 \mathrm{ng} * \mathrm{~h} / \mathrm{mL}$; the absolute bioavailability $=$ $2569 / 5490=48 \%$. For different formulation, the absolute bioavailability is the amount of the drug in the systemic circulation (AUC) relative to i.v. application (AUC), adjusted to the same dose.

Collectively, using in vitro formulation screening we were able to develop the new anle138b formulation V34, which is characterized by optimized drug delivery performance in rats. Importantly, V34 formulation consists of pharmaceutically acceptable excipients. 


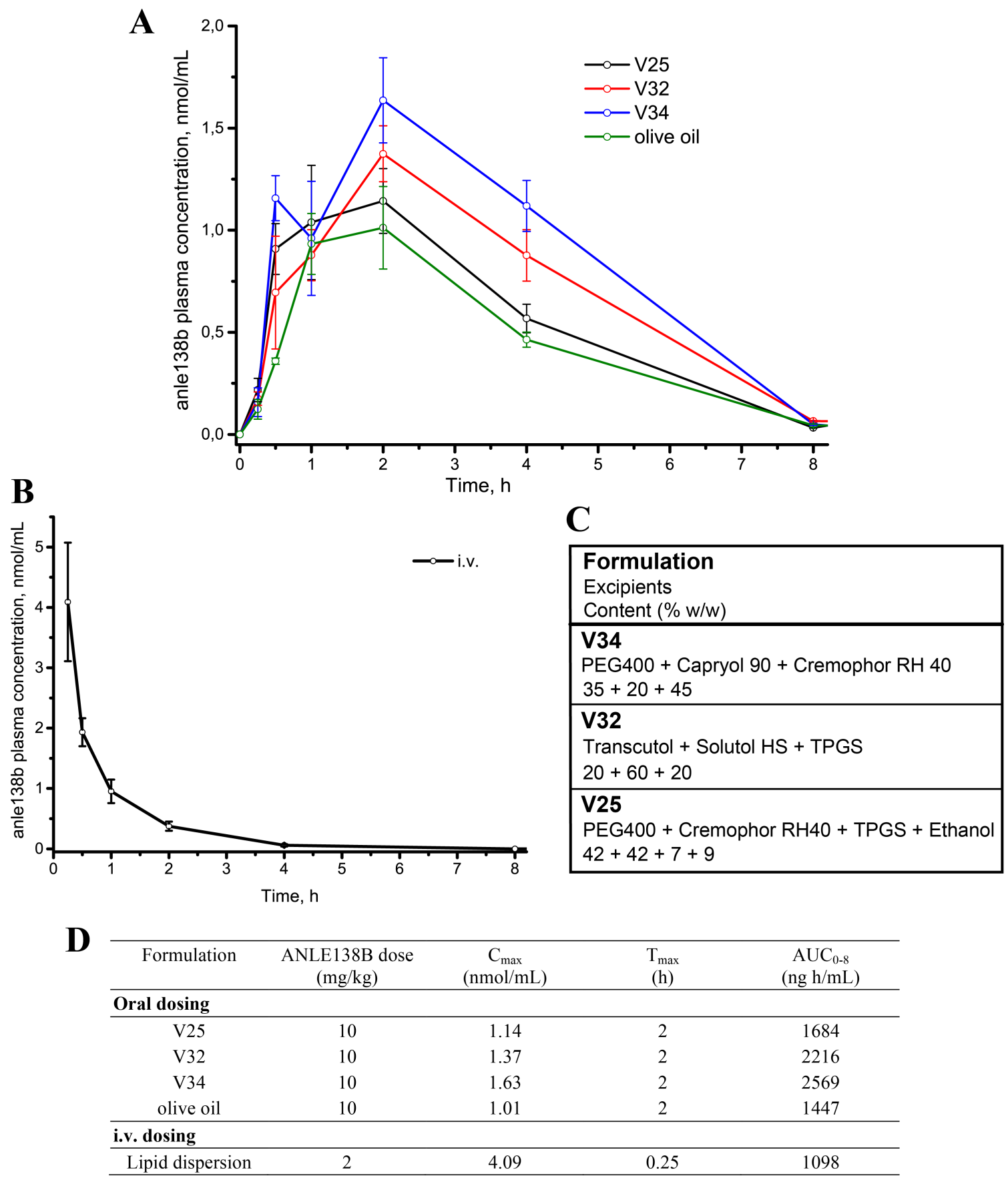

Figure 2.8.5. Comparative pharmacokinetic analysis of anle138b formulations in rats. anle $138 \mathrm{~b}$ concentrations were measured in rat plasma after single oral application (gavage) of anle $138 \mathrm{~b}$ in corresponding formulation at $10 \mathrm{mg} / \mathrm{kg}$ dose or after i.v. application of anle $138 \mathrm{~b}$ in lipid dispersion/NMP mixture at $2 \mathrm{mg} / \mathrm{kg}$ dose. (A) Plasma anle138b concentration-time profiles after single oral application of anle138b in different formulations. (B) Plasma anle138b concentration-time profile after i.v. application of anle138b. (C) Description of tested formulations. (D) Major pharmacokinetic parameters of anle138b in rat plasma following different application protocols (Mean values are listed). Error bars in (A, B) indicate standard error. 


\subsubsection{Pharmacokinetics: key findings}

Our pharmacokinetic studies have demonstrated that DPP compounds have in general a good oral bioavailability and readily cross the blood-brain-barrier. In case of anle138b, we showed that this molecule has favorable pharmacokinetic profile in mice and rats. Regardless of the administration protocol, the good oral bioavailability and high brain exposure were measured in anle138b-treated mice and rats. Moreover, we have developed the special anle138b-containing dry food, which provides convenient way to conduct efficacy studies in mice. Additionally, we have identified and tested optimized anle138b formulation V34, which is based on pharmaceutically acceptable excipients. 


\subsection{Development of anle138b prodrug}

\subsubsection{Introduction: Prodrug approach}

The development of new drugs is a very complex and long process, which involves many steps such as identification of disease and relevant drug target, high-throughput screening campaign and subsequent lead optimization, in vivo efficacy and toxicity studies, development of administration protocols and others. Unfortunately, many pharmacologically active candidates exhibit poor physicochemical and biopharmaceutical properties that are quite difficult to overcome in later stages of drug development. The design of appropriate prodrug offers an attractive approach to improve the properties of potent drug compounds and thereby enhance the developability of the promising drug candidate. Currently, pharmaceutical industry has recognized the potential of the prodrug approach that can also be implemented on early stages of the drug development. According to literature, up to $10 \%$ newly approved drugs can be classified as prodrug molecules ${ }^{492}$.

What is a prodrug? "Prodrugs are bioreversible derivatives of drug molecules that undergo an enzymatic and/or chemical transformation in vivo to release the active parent drug, which can then exert the desired pharmacological effect" ${ }^{493}$. The schematic representation of the prodrug concept is shown in Figure 2.9.1. In most cases, prodrugs consist of two parts, drug moiety and pro-moiety. The main role of the pro-moiety is to improve specific aspects of the parent drug molecule, for example low stability or poor aqueous solubility. Following the administration of the prodrug, the pro-moiety is cleaved either enzymatically and/or chemically to release the active drug at the right compartment of the body. The second, less frequent approach is based on point prodrug activation by addition of the missing fragment, leading to increase the therapeutic activity ${ }^{492}$.

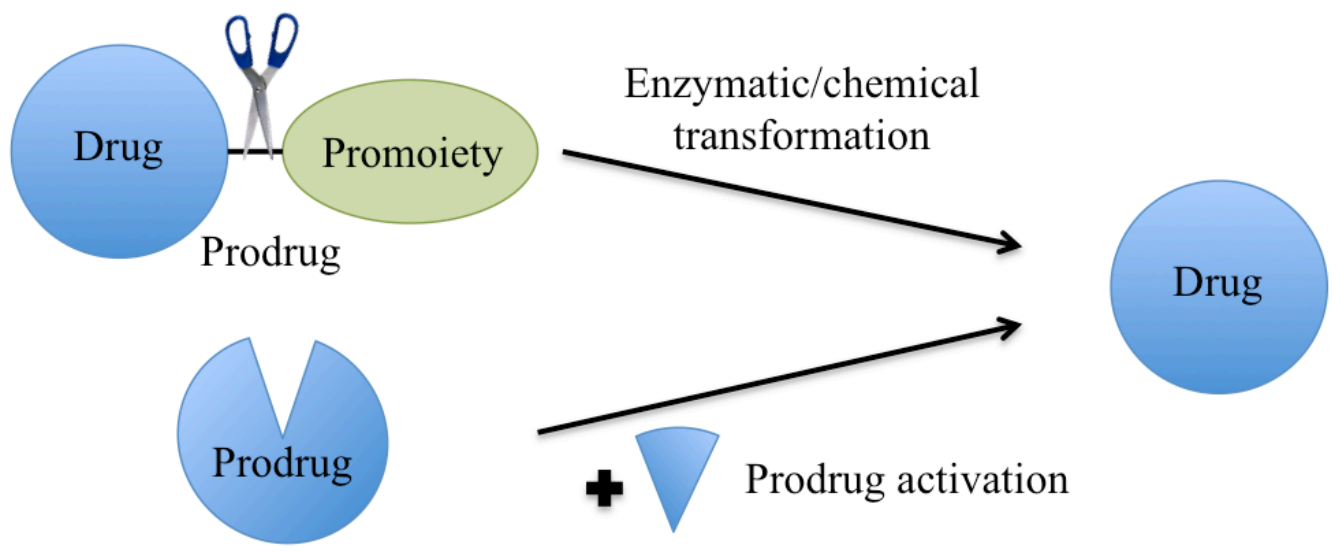

Fig. 2.9.1. Simplified illustration of the prodrug concept. 
Before active drug is reaching the corresponding biological target inside the organism, it has to overcome several barriers including formulation, adsorption, distribution, metabolism and excretion. The rational design of the prodrug molecule provides a powerful tool to improve specific properties of the parent compound and to achieve favorable physicochemical or pharmacokinetic profiles. Modern prodrug strategies can help to overcome following limitations: poor aqueous solubility, limited absorption, cellular permeation, chemical instability, unfavorable metabolism, inadequate brain penetration, toxicity, local irritation ${ }^{492}$, 493. Moreover, recent progress in the molecular biology enables the design of prodrugs capable of a targeted delivery of the drugs to specific organs or even cells. The prodrug applications for site selective therapy are especially beneficial in cancer, brain and liver diseases ${ }^{493-495}$.

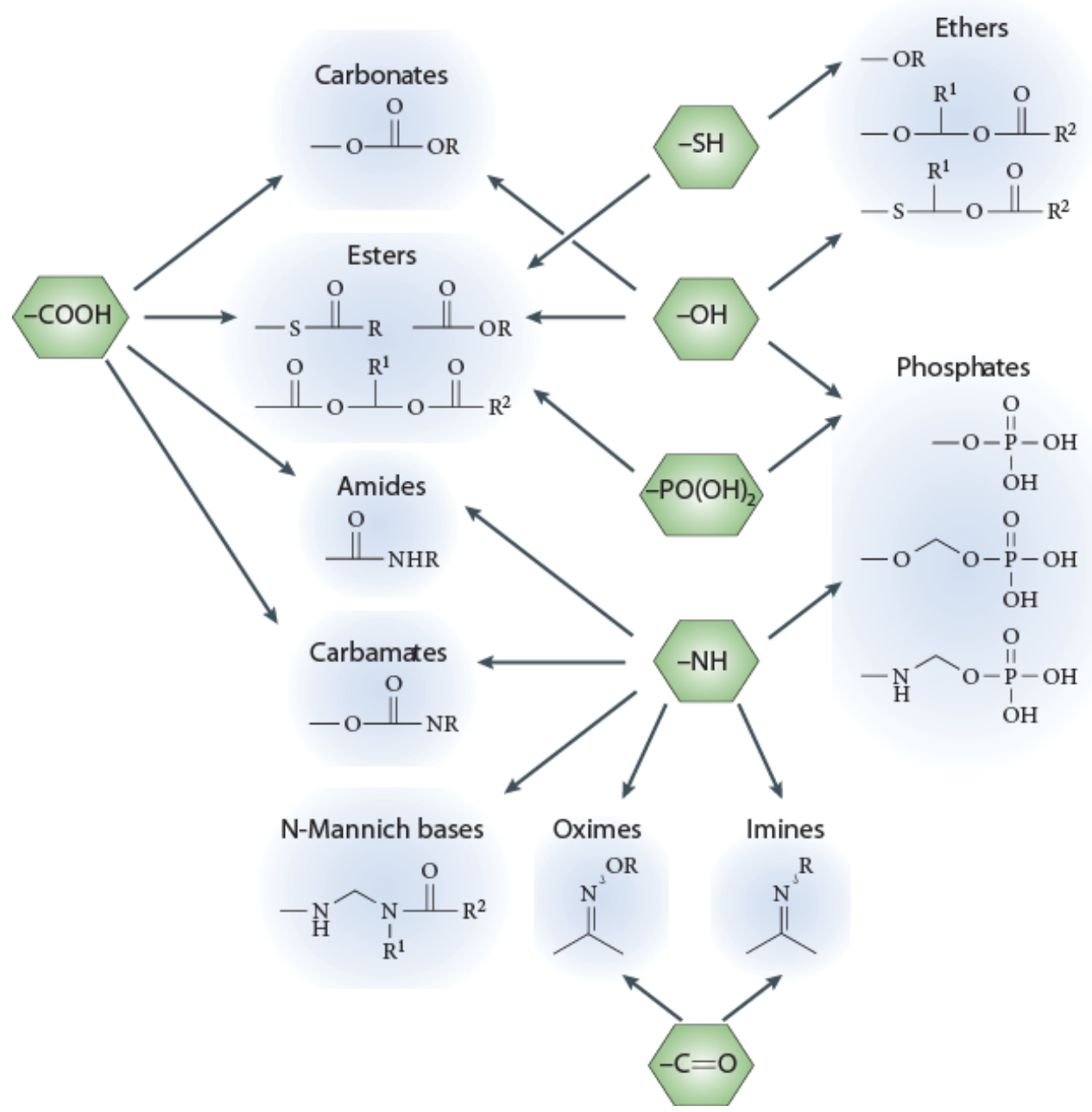

Figure 2.9.2. Common functional groups and types of chemical modifications with promoiety. Types of prodrug modifications and common functional groups of the parent drug (in green). Figure reprinted by permission from Springer Nature: Nat. Rev. Drug Discov, J. Rautio et al. ${ }^{493}$. copyright $@(2008)$. 
From the perspective of organic chemistry, the design of prodrug compound involves identification of the functional group on the parent drug that is suitable for modification. Another important step is selection of appropriate pro-moiety, which improves the weak points of the parent drug molecule or facilities the targeted delivery. Most common functional groups and types of chemical modifications are summarized in Figure 2.9.2.

Esters, being the most common prodrugs used, are usually enzymatically cleaved by ubiquitous esterases in blood, liver or other organs/tissues. In most cases, the ester prodrugs are generated in order to mask charged/polar groups $\left(-\mathrm{OH},-\mathrm{CO}_{2} \mathrm{H},-\mathrm{PO}(\mathrm{OH})_{2}\right)$ of the parent drug, increase lipophilicity and thereby enhancing the passive membrane permeabilization and oral absorption ${ }^{493}$. Good examples of this strategy are prodrugs Osetamivir and Famciclovir, for which the oral bioavailability is dramatically increased ${ }^{493}$ (Fig. 2.9.3). Additionally, the strategy of improving the lipophilicity is applied in the development of prodrugs targeting the brain ${ }^{495}$.

Another common issue is a low aqueous solubility of highly hydrophobic drugs, which leads to problems with appropriate formulation and reduced bioavailability. For such cases, an attachment of the phosphate moiety to a free hydroxyl or amino group is considered as the first choice. The advantages of phosphate pro-moiety are high polarity, good chemical stability and fast enzymatic cleavage by alkaline phosphatases present at intestinal cell surface $^{493,496}$. For example, miproxifene phosphate has 1000 fold increased solubility in water in comparison to its parent drug, which translates to significantly enhanced bioavailability 493 (Fig. 2.9.3). In addition to phosphate, the solubility of drug molecule in water can be improved by conjugation of amino acids or choline-based moieties ${ }^{493,496}$.
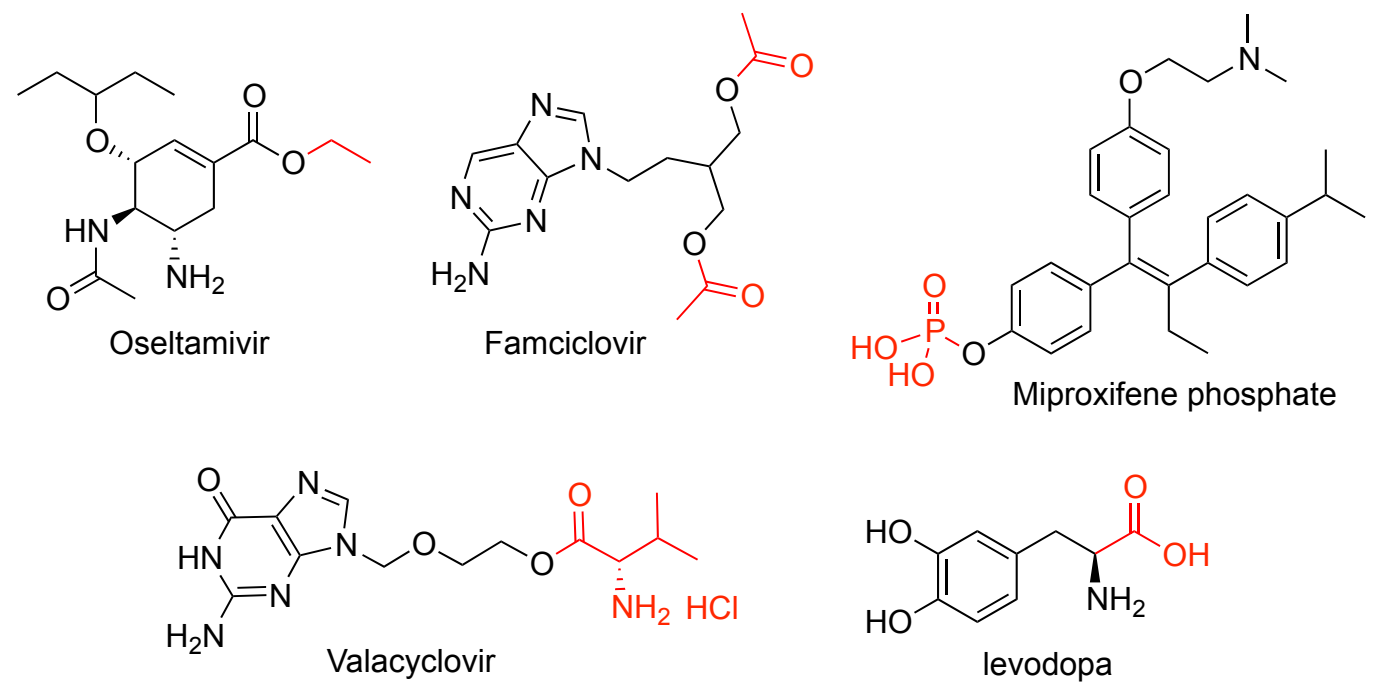<smiles>N[C@@H](Cc1ccc(O)c(O)c1)C(=O)O</smiles>

Figure 2.9.3. Examples of developed prodrugs. Pro-moiety is highlighted in red. 
An alternative approach for improving the bioavailability of poorly absorbed drugs is carrier-mediated absorption. The enhanced bioavailability of the drug is achieved by attachment of pro-moieties, which are targets of the endogenous transporters in the intestine, such as small peptides (peptide transporters (hPEPT1)) and structural analogs of $\gamma$ aminobutyric acid (monocarboxylate transporter type 1 (MCT1) ${ }^{493}$. An example of such prodrugs is the antiviral compound Valacyclovir (Fig. 2.9.3)

Among the various prodrug approaches, a site selective delivery of the drug is clearly one of the most attractive strategies, which enable the release of the active agent in the specific location. The prodrug applications for site selective therapy are especially useful in cancer, brain and liver diseases ${ }^{493-495}$. The site specificity can be achieved by several mechanisms, including transporter-mediated delivery (levodopa), passive drug enrichment in the organ, selective metabolic activation by specific enzymes, and antigen targeting ${ }^{492-495}$. Among the site selective prodrug, levodopa, a dopamine prodrug, is used in treatment of Parkinson's disease for almost 50 years (Fig. 2.9.3). In contrast to polar dopamine, levodopa is transported across the blood-brain-barrier by the neutral amino-acid transporter (LAT1). Inside the brain, a rapid decarboxylation of levodopa by aromatic amino acids decarboxylase leads to release of dopamine. Remarkably, dopamine is not able to cross the blood-brainbarrier and therefore trapped in the brain ${ }^{495}$.

Collectively, prodrug has become a powerful tool to address many important bottlenecks in the drug development. In addition to already mature approaches to improve drug solubility, bioavailability and stability; recent progress in molecular biology opens enormous prospects for the rational design of prodrugs for site selective therapies.

\subsubsection{Design of anle138b prodrug candidates}

As discussed in the previous chapter, anle138b has the very low water solubility and therefore the oral application of this compound requires the dissolution in appropriate excipients. To date, several protocols of oral dosing of anle138b have been developed that provide good bioavailability in mice and rats. However, the available dosing regiments have certain disadvantages, including application of big volumes of excipients, limited stability of formulation, tolerability of certain types of excipients in human, additional cost of excipients. 


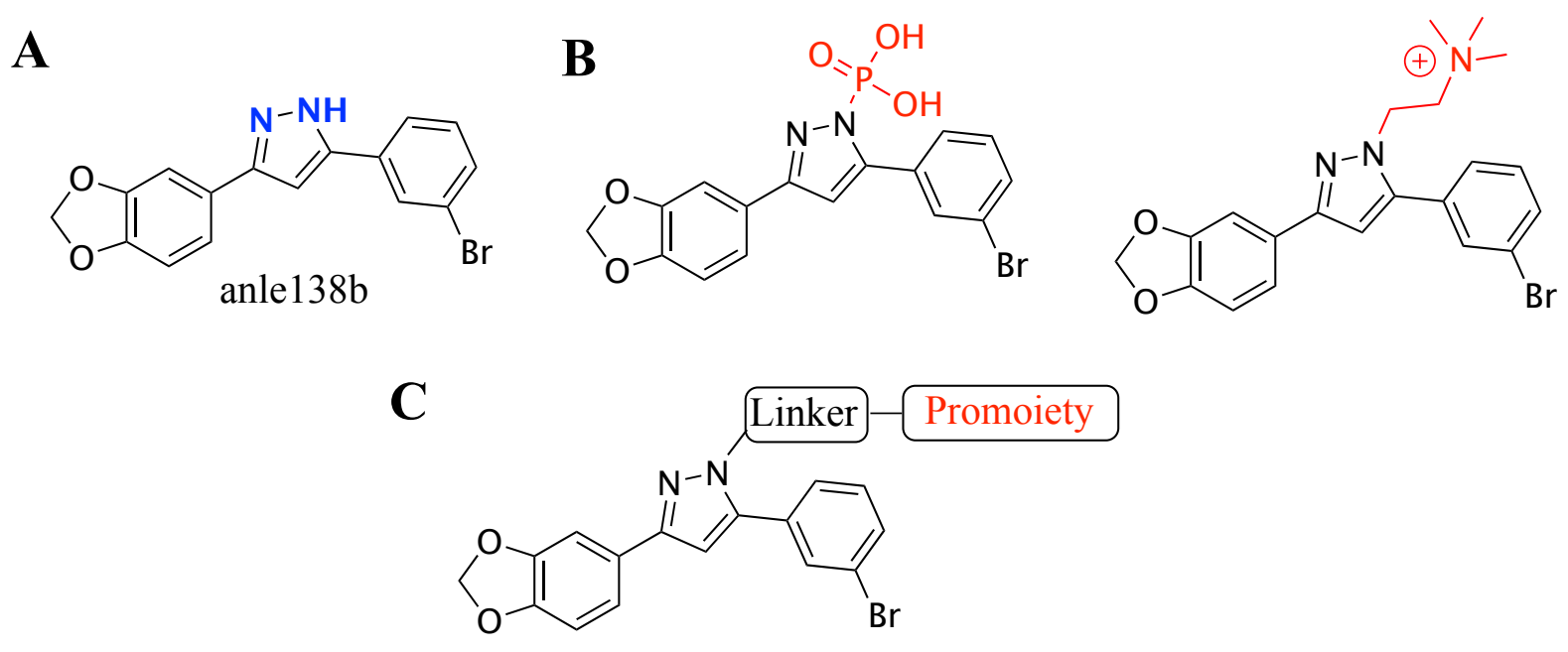

Figure 2.9.4. Design of anle138b prodrug. (A) Structure of anle138b, possible positions for modification in blue. (B) Unfavorable structures with direct attachment of pro-moieties (in red) to pyrazole ring. (C) Proposed structure of the prodrug additional linker.

In order to overcome these issues, we decided to develop a prodrug of anle138b. The main goal of this project was to improve the water solubility of the parent compound and to achieve good bioavailability in vivo after oral prodrug application in aqueous solutions. One of the possible approaches to improve the water solubility of lipophilic compounds is an attachment of the polar solubilizing pro-moieties such as phosphoric acids, amino acids and choline moiety. The evaluation of the chemical structure of anle138b revealed that the single possible position for modification is one of the nitrogen atoms of the pyrazole ring (Fig. 2.9.4A). The direct attachment of the polar pro-moiety to nitrogen atom has been considered as not appropriate due to low chemical stability of corresponding (1H-pyrazol-1yl)phosphonic acid and expected high enzymatic stability of choline-related derivatives (Figure 2.9.4B). Therefore, we decided to introduce a linker between the polar pro-moiety and the parent drug. Following extensive assessment of available reaction protocols and properties of published prodrugs, three prodrug candidates based on anle138b lead structure have been designed and synthesized (Fig. 2.9.5A). As shown in Figure 2.9.5B, the putative mechanism of phosphate prodrugs involves the enzymatic cleavage of phosphate ester bond by alkaline phosphatases followed by spontaneous elimination of the linker and release of the anle138b. In case of the choline-based prodrug, the mechanism of drug release is not well defined and can be driven by acid catalyzed hydrolysis of hemiaminal moiety or enzymatic cleavage of ether bond (Fig. 2.9.5B). 


\section{A}
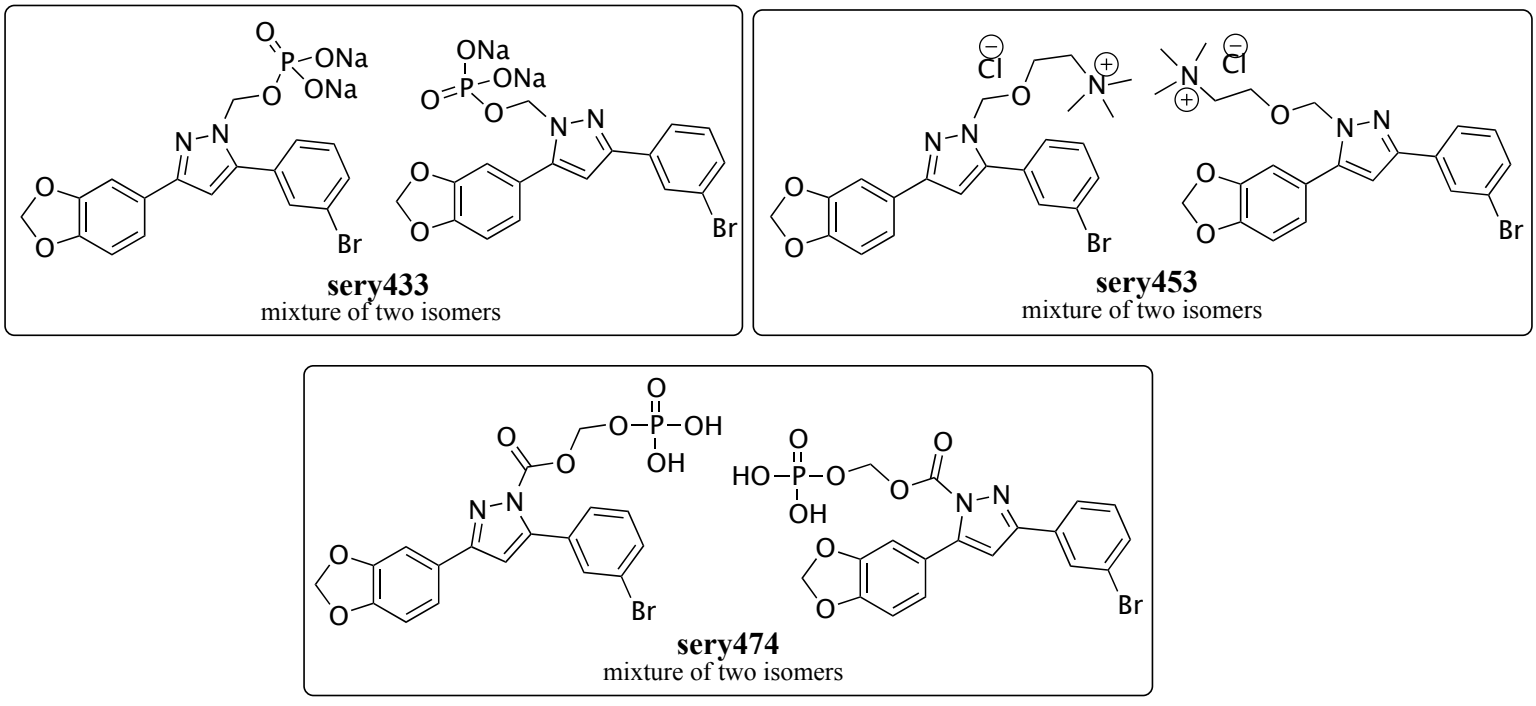

\section{B}

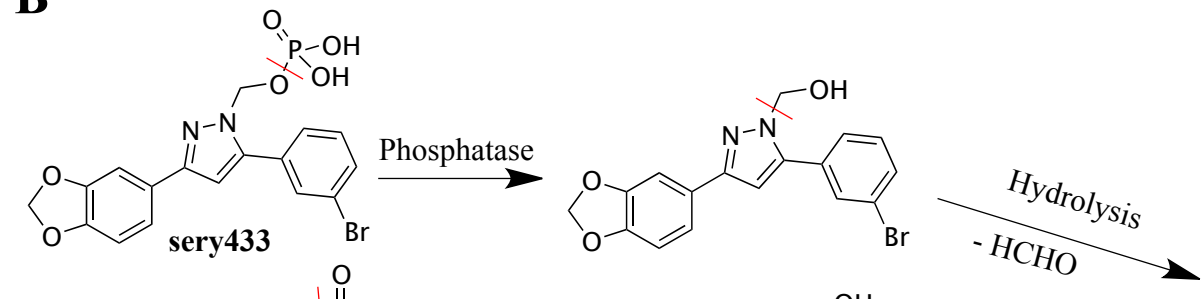

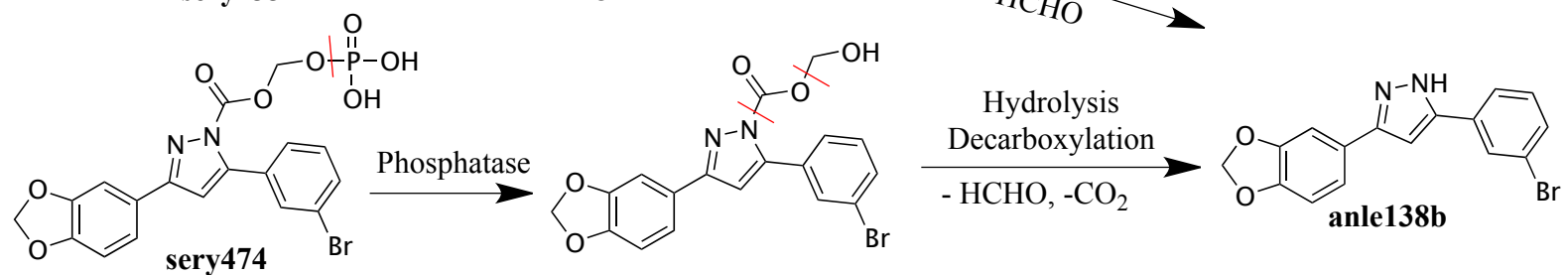

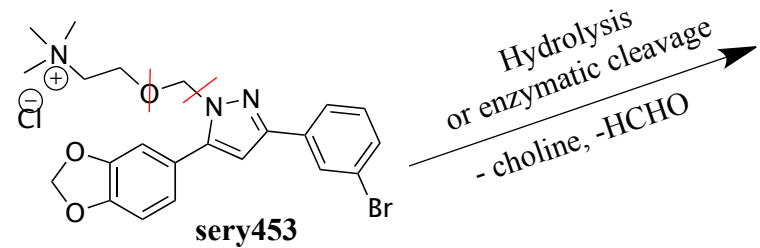

Figure 2.9.5. Anle138b prodrug candidates. (A) The chemical structures of anle138b prodrug candidates. All presented prodrugs are the mixtures of two regioisomers (approx. ratio of isomers 3:2). (B) Putative mechanisms of enzymatic and chemical transformation of prodrug candidates to anle $138 \mathrm{~b}$

\subsubsection{Synthesis of anle $138 \mathrm{~b}$ prodrug candidates}

Following the design of prodrug candidates, we have developed and optimized the synthesis of the corresponding compounds. As shown in Figure 2.9.6, three prodrug candidates, namely sery 433 , sery 474 and sery 453 , were prepared from anle138b in two or four steps. Of note, the structures, in which the position of the substituent is not defined (between two $\mathrm{N}$ atoms of the pyrazole ring), represent a mixture of two regioisomers of the $\mathrm{N}$-substituted pyrazoles (Fig. 2.9.6). 
In Figure 2.9.6A, the pyrazole sery432, which is a mixture of two regioisomers, was prepared in $82 \%$ yield from the anle138b by alkylation reaction with di-t-butyl chloromethyl phosphate ${ }^{497}$ in presence of $\mathrm{Cs}_{2} \mathrm{CO}_{3}$ in DMSO. The selective hydrolysis of di-t-butyl phosphate was achieved by treatment of sery 432 with TFA in DCM at $0^{\circ} \mathrm{C}$. The target compound sery433 was isolated in quantitative yield as a mixture of two isomers a and $\mathbf{b}$ (Fig.2.9.6A). By using NMR and HPLC analysis, we quantified the ratio of isomers (isomers $\mathbf{a} /$ isomer $\mathbf{b}=2 / 3$ ) and determined the structure of each regioisomer (Fig. 2.9.7). It should also be noted that the presented protocol allows the synthesis of sery433 on a multigram scales (up to $35 \mathrm{~g}$ ) without chromatography and additional purification steps. To develop this method, an

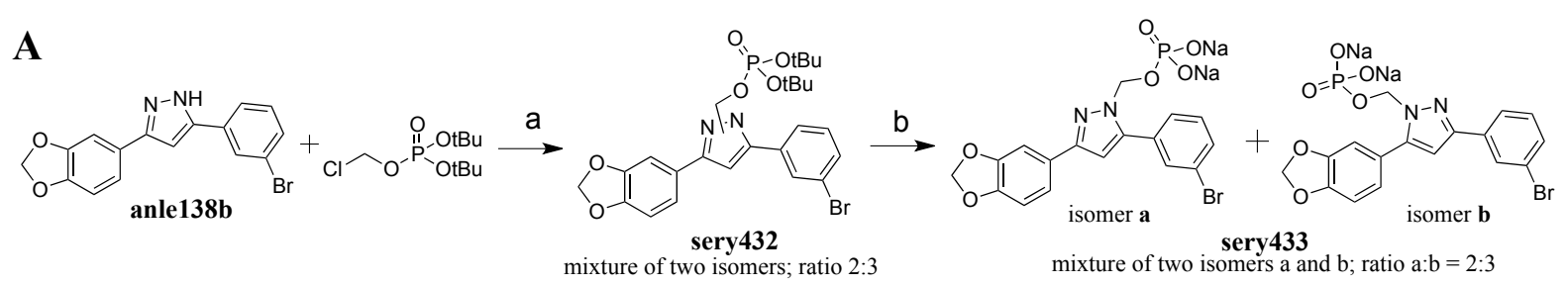

B
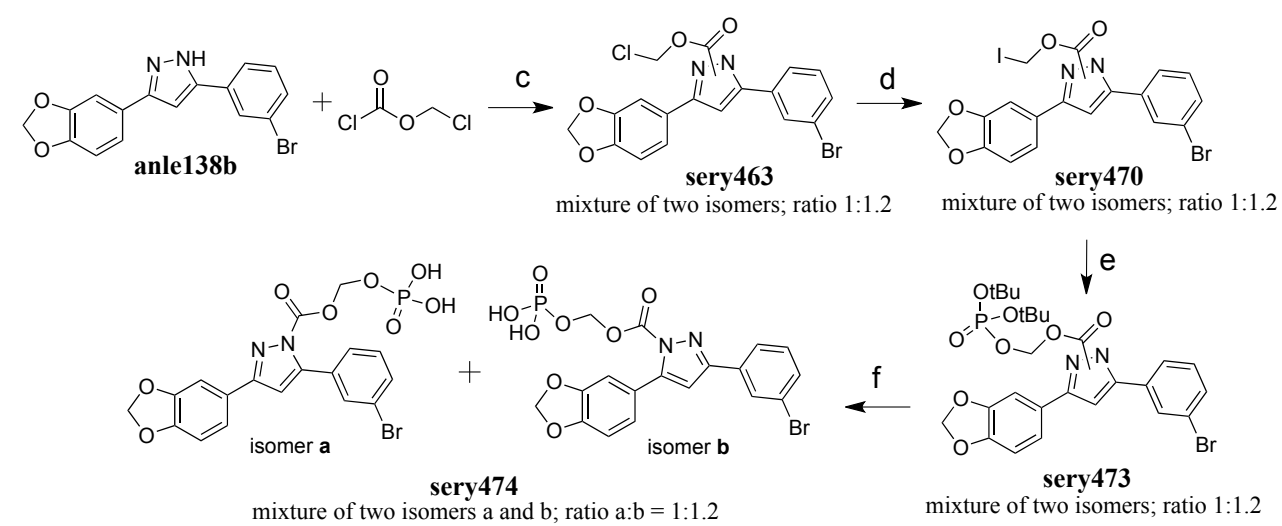

C
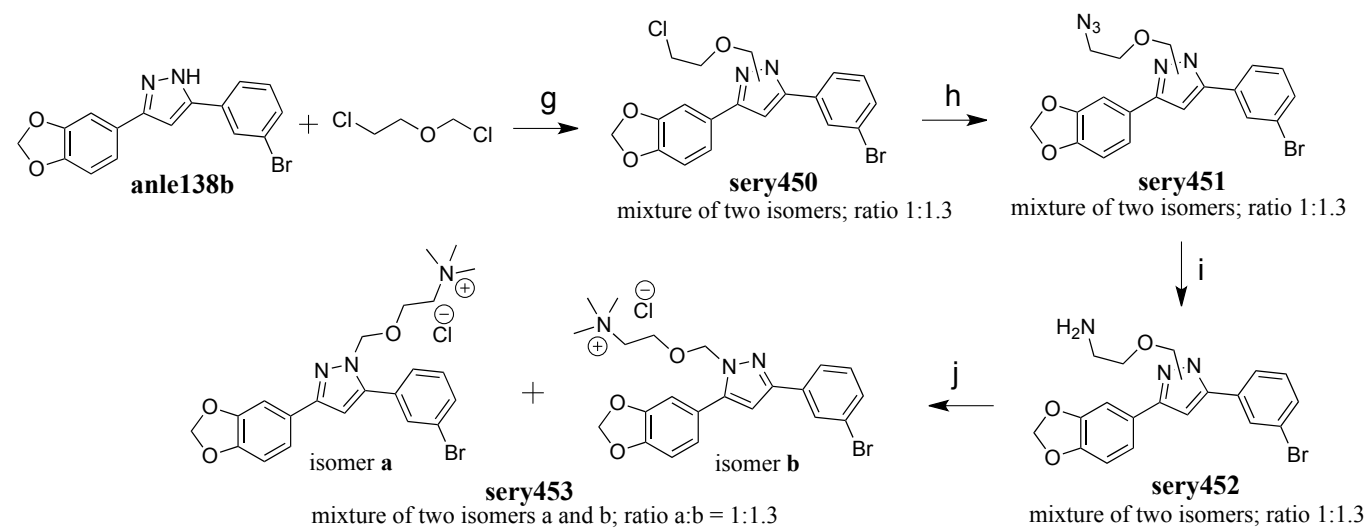

Figure 2.9.6. Synthesis of prodrug candidates. Synthesis of sery432 (A), sery474 (B), sery453 (C). The structures, in which the position of the substituent is not defined (between two $\mathrm{N}$ atoms of the pyrazole ring), represent a mixture of two regioisomers of the $\mathrm{N}$ substituted pyrazoles. Reaction conditions: (a) $\mathrm{Cs}_{2} \mathrm{CO}_{3}$, DMSO, RT, $82 \%$; (b) TFA, DCM, $0{ }^{\circ} \mathrm{C}, 98 \%$; (c) $\mathrm{Et}_{3} \mathrm{~N}, \mathrm{DCM}, \mathrm{RT}, 67 \%$; (d) NaI, acetone, $40^{\circ} \mathrm{C}, 75 \%$; (e) potassium di-tert-butylphosphate, $\mathrm{Ag}_{2} \mathrm{O}, \mathrm{ACN}, \mathrm{RT}$, $74 \%$; (f) TFA, DCM, $0^{\circ} \mathrm{C}$, not isolated due to instability; (g) NaH, DMF, RT, $96 \%$; (h) $\mathrm{NaN}_{3}, \mathrm{NaI}, \mathrm{DMF}$, $80^{\circ} \mathrm{C}, 95 \%$; (i) $\mathrm{Ph}_{3} \mathrm{P}$, water/THF, RT, 96\%; (j) MeI, $\mathrm{KHCO}_{3}$, ACN, RT; $51 \%$. 
extensive optimization of reaction conditions was performed. For anle138b alkylation reaction (Fig. 2.9.6A), cesium carbonate showed the best results among 9 different bases $\left(\mathrm{NaH}, \mathrm{Cs}_{2} \mathrm{CO}_{3}, \mathrm{NaHCO}_{3}, \mathrm{KHCO}_{3}, \mathrm{Ag}_{2} \mathrm{CO}_{3}, \mathrm{Et} 3 \mathrm{~N}, \mathrm{DBU}\right.$, pyridine/DMAP, $\mathrm{K}_{2} \mathrm{CO}_{3}$ ). Next, we performed the solvent screening for sery432 synthesis in presence of $\mathrm{Cs}_{2} \mathrm{CO}_{3}$. Among other solvents, the use of DMSO leads to fast and clean transformation followed by the simple aqueous workout. Regarding the second step of sery433 synthesis, which is the selective hydrolysis of di-t-butyl phosphate, the limited stability of sery433 under acidic conditions was the main problem. Under standard conditions of $t$-butyl ester hydrolysis with strong mineral acids we observed the formation of significant amount of anle138b. In order to optimize the conditions of this reaction, we screened different acids, solvents and temperatures. At the end, we were able to achieve $99 \%$ yield of sery 433 by treatment of sery 432 with big excess of TFA in DCM at $0^{\circ} \mathrm{C}$ for $8-10$ hours. Once the reaction is complete, it is important to remove TFA by repeated evaporation with toluene at $0^{\circ} \mathrm{C}$. The resulting solid acid form of sery 433 is washed with acetonitrile to get rid of impurities from previous alkylation reaction. Next, we have neutralized sery433 with an appropriate amount of aqueous sodium hydroxide, filtered and lyophilized the resulting solution to provide pure sery433 disodium salt (Fig. 2.9.6).

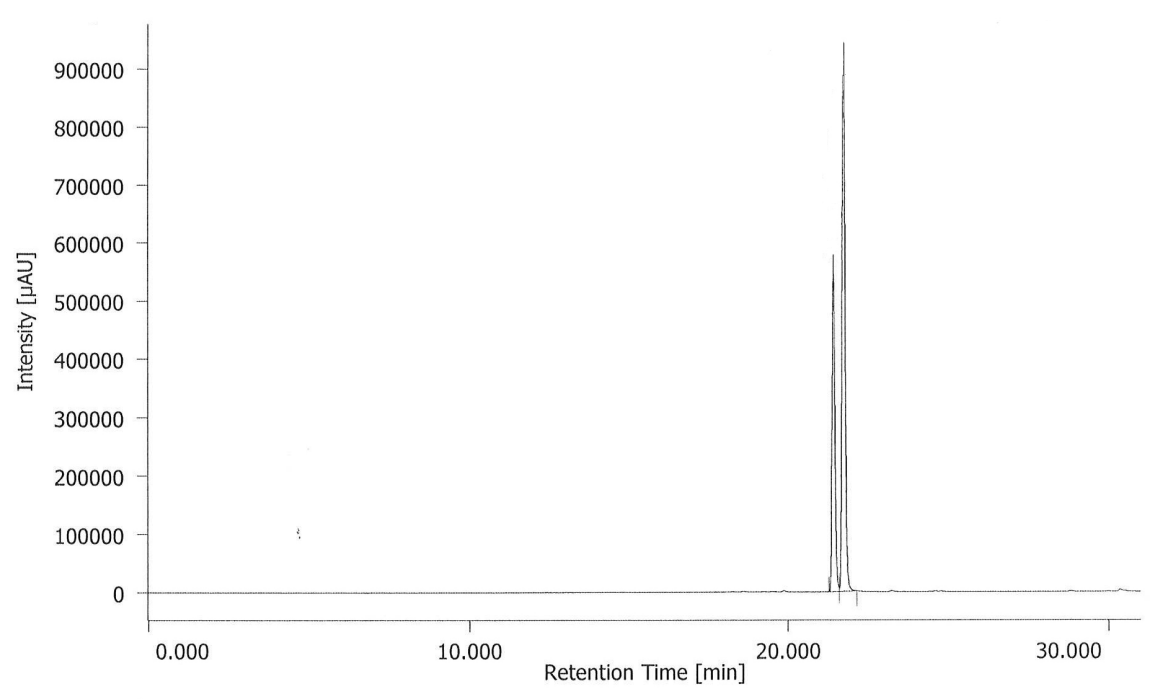

Figure 2.9.7. HPLC chromatogram of sery433. HPLC analysis shows high purity of sery433. Two peaks observed on chromatogram correspond to two regioisomers present in the sery433. Column: Nucleodur Sphinx, RP, $5 \mu \mathrm{m}$; gradient: $0-60 \%$ in $30 \mathrm{~min}(5 \mathrm{mM}$ ammonium acetate $\rightarrow$ acetonitrile). 
As shown in Figure 2.9.6B, sery474 was prepared in four step synthesis from anle138b. First, anle138b was reacted with chloromethyl chloroformate in presence of $\mathrm{Et}_{3} \mathrm{~N}$ to provide sery463 as a mixture of two regioisomers. Since we failed to directly substitute chlorine in sery463 with phosphate moiety, we exchanged the chlorine to iodine with a good yield under standard conditions of Finkelstein reaction ${ }^{498}$. On the next step, sery470 was readily converted to sery 473 via direct esterification of potassium di-tert-butylphosphate in presence of $\mathrm{Ag}_{2} \mathrm{O}$ in acetonitrile ${ }^{499}$. The acid-catalyzed hydrolysis of sery473 was done according to the mild protocol developed for sery433. The formation of sery474 in presence of TFA in $\mathrm{CDCl}_{3}$ at $0^{\circ} \mathrm{C}$ was confirmed by NMR measurements. However, we found out that the addition of any aqueous solution to organic phase leads to immediate decomposition of sery474 to anle138b. The rapid hydrolysis of sery474 even in the presence of the trace amounts of water might be explained by intramolecular catalytic activity of the phosphate group 500

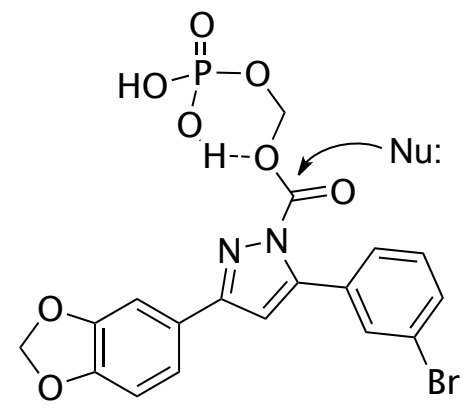

Figure 2.9.8. Putative mechanism of sery 474 hydrolysis. (Fig. 2.9.8).

The first step of the synthesis of choline-based prodrug sery453 is the alkylation of anle138b with 2-chloroethyl chloromethyl ether in DMF at room temperature with sodium hydride as a base. In line with the previous examples, sery450 was isolated as a mixture of two regioisomers, which was used in further steps without separation. The intermediate sery 451 was prepared in $95 \%$ yield by the nucleophilic displacement of chlorine with azide in presence of $\mathrm{NaN}_{3} / \mathrm{NaI}$ system according to published procedure ${ }^{501}$. The azide sery 451 was readily converted to sery452 by Staudinger reaction with $\mathrm{PPh}_{3}{ }^{502}$. Finally, sery453 was synthesized by alkylation of amino group with big excess of methyl iodide followed by anion exchange column. The exact ratio of regioisomers in sery452 mixture was determined based on NMR and LC-MS analysis.

Collectively, we have developed the synthesis of three anle $138 \mathrm{~b}$ candidates. With the optimized method, multigram quantities of compound sery433 can be prepared in excellent yield and high purity without any chromatographic purification steps. 


\subsubsection{Physical and pharmacokinetic properties of anle $138 \mathrm{~b}$ prodrug candidates}

Among other properties, a good chemical stability and high solubility in water are crucial parameters for water-soluble prodrugs. From this perspective, the development of compounds, which are unstable or insoluble in aqueous solutions, is not attractive. Therefore, we investigated the stability and solubility properties of three prodrug candidates.

In our study the stability of sery433, sery453 and sery474 in aqueous solutions was analyzed by NMR measurements. As discussed in the chapter 2.9.2, the addition of water to sery474 leads to immediate decomposition of this compound to anle138b. Based on these results, the further development of sery474 was terminated. Two other compounds showed good stability in aqueous solution according to NMR measurements. Experimentally, $5 \mathrm{mg}$ of corresponding compound was dissolved in deuterated water $(0,5 \mathrm{ml})$ and ${ }^{1} \mathrm{H}$ NMR spectra were recorded immediately after dissolution, after 12 and 24 hours incubation at room temperature. The acquired NMR spectra at different time points were identical and did not show any signals of decomposition products.

In addition to preparation of aqueous NMR samples, we also performed a very simple solubility test, in which $10 \mathrm{mg}$ of sery 453 or sery 433 were dissolved in $1 \mathrm{ml}$ of deionized water. After gentle agitation, the formation of clean solutions was observed for both compounds. Taken together, compounds sery453 and sery433 have high solubility and good stability in aqueous solutions.

Considering the favorable physical properties, two prodrug candidates, sery433 and sery453, were tested in a pilot pharmacokinetic study in mice. The main aim of this experiment was to show that the corresponding prodrug molecules being applied orally in water undergo the transformation in vivo to release the parent drug anle138b. Dr. Felix Schmidt did an animal part of experiment; I have designed the study and performed the tissue homogenization and HPLC analysis.

As shown in Figure 2.9.9, both sery433 and sery453 being applied orally in aqueous solution are metabolized in vivo to release the parent drug anle138b. The HPLC analysis of the biological samples from mice treated with sery433 revealed significant levels of anle138b in brain and in plasma 1 hour after administration (Fig. 2.9.9A). The exposure of the anle138b is increased at later time point reaching $40-50 \mathrm{nmol} / \mathrm{g}$ levels in the brain (Fig. 2.9.9A). In line with the previous pharmacokinetic studies, the anle138b brain-to-plasma ratio was estimated in a range from 5 to 10 . In mice treated with sery 453 we also observed anle138b in the brain at all measured time intervals (Fig. 2.9.9A). However, in plasma sample we measured 
anle $138 \mathrm{~b}$ just in one animal out of two per group at 1,2, and 4 hours after gavage (Fig. 2.9.9A).

A
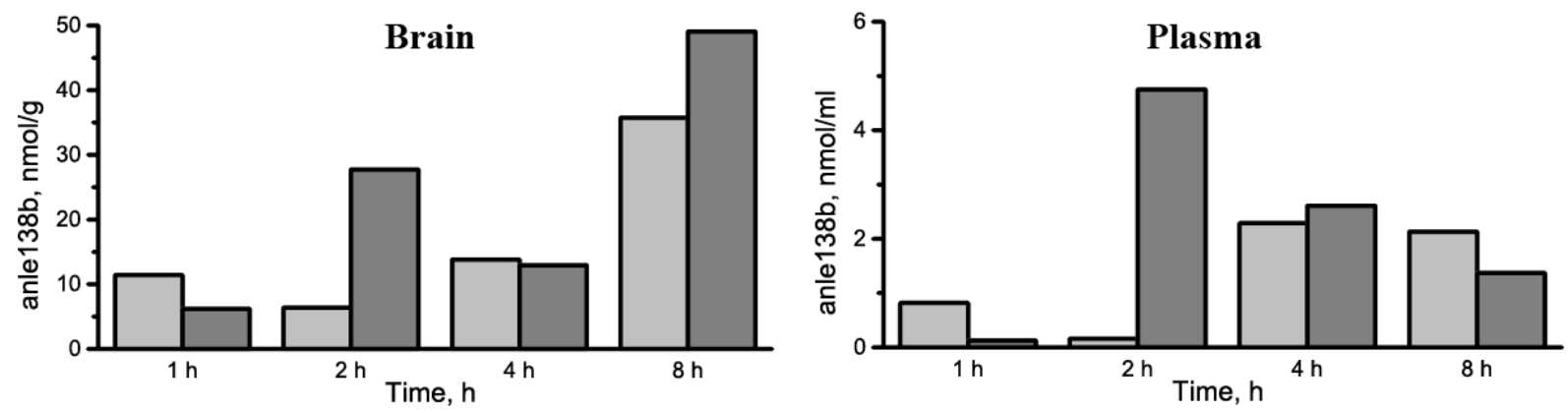

B
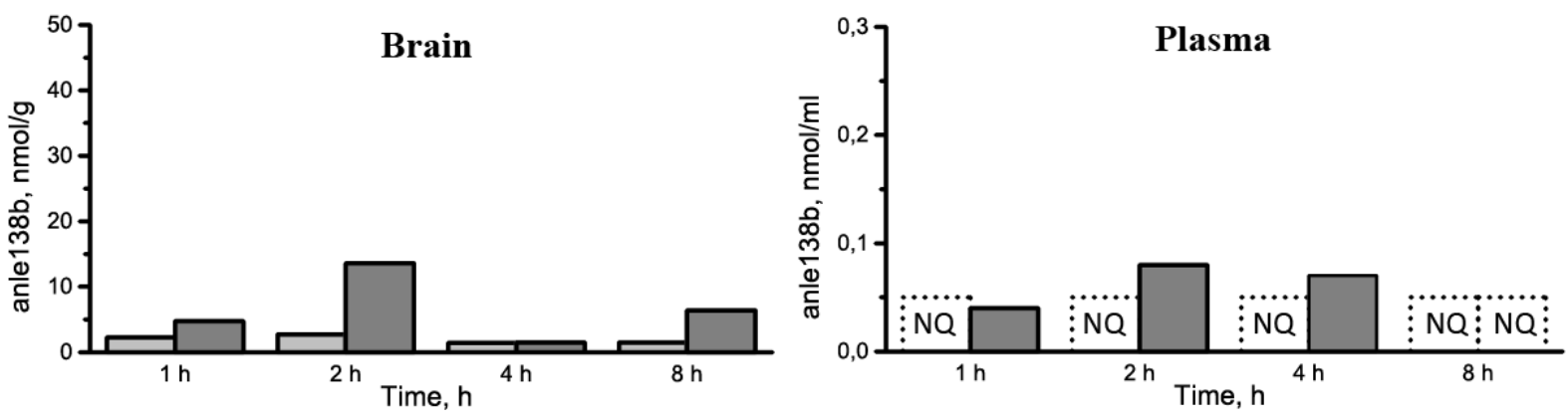

Figure 2.9.9. PK study of prodrugs in mice. Anle138b concentration in plasma and in brain at $1 \mathrm{~h}, 2 \mathrm{~h}, 4 \mathrm{~h}, 8 \mathrm{~h}$ after single oral application of prodrugs via gavage at $70 \mathrm{mg} / \mathrm{kg}$ dose in mice. Two animals per time point were sacrificed and analyzed. Anle138b concentration for each individual animal is shown. (A) anle138b concentration following sery433 application. (B) anle138b concentration following sery453 application. NQ - level below detection limit.

From presented pharmacokinetic data, it is evident that both conceived compounds sery433 and sery 453 are cleaved in vivo to provide detectable levels of anle138b in the brain. Regarding the direct comparison of sery433 and sery453, our study showed that the oral administration of sery433 leads to significantly higher anle138b exposure both in brain and in plasma than the similar dose of sery453. Thus, the plasma concentrations of anle138b were reaching $2-4 \mu \mathrm{M}$ in sery433 treated animals (Fig. 2.9.9B), while the anle138b levels did not exceed $0.1 \mu \mathrm{M}$ in sery453 treated group (Fig. 2.9.9B). Similar pharmacokinetic profiles were observed in the brain. Therefore, between these two prodrug candidates, sery 433 was selected for further evaluation and development.

To confirm the favorable pharmacokinetic properties of sery 433 observed in mice, we analyzed the anle138b exposure in rats treated orally with a high dose of sery433 (180 $\mathrm{mg} / \mathrm{kg}$ ). In this study, Dr. Sebastian Kügler did an animal part of experiment; I have designed the study and performed the tissue homogenization and HPLC analysis. In line with the 
results from the mice experiment, the prodrug sery 433 is readily transformed to anle $138 \mathrm{~b}$ also in rats. At both time periods after oral administration of sery433, high levels of anle138b were measured in the brain and in the plasma (Fig. 2.9.10). Despite the limited number of time points, the comparison of pharmacokinetic profiles in two rodent species suggests a faster metabolism of sery433 and a better of absorption of anle138b in rats (Fig. 2.9.10) then in mice (Fig 2.9.9A).

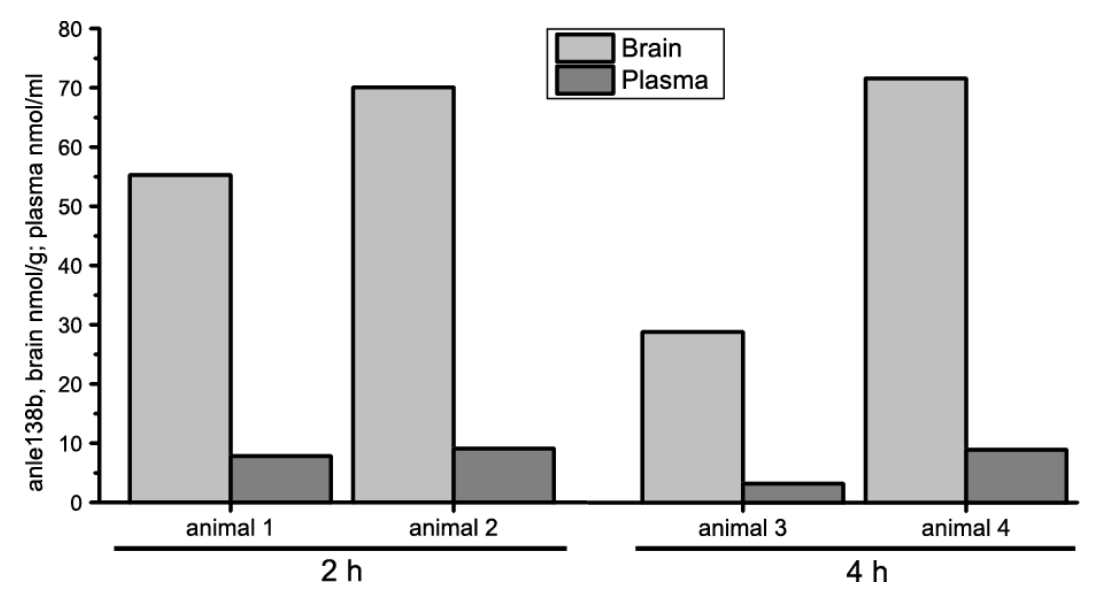

Figure 2.9.10. PK study of sery 433 in rats. Anle138b concentration in plasma (dark grey) and in brain (light gray) at $2 \mathrm{~h}, 4 \mathrm{~h}$ after single oral application of prodrugs via gavage at 180 $\mathrm{mg} / \mathrm{kg}$ dose in rats. Two animals per time point were sacrificed and analyzed. Anle138b concentration for each individual animal is shown.

As shown in Figures 2.9.9A and 2.9.10, the pharmacokinetic characteristics of sery433 lead to therapeutically useful levels of anle138b in mice and rats. Since anle138b is also orally available compound in animals, it was therefore very important to perform the comparative pharmacokinetic study with sery433 and anle138b under well-standardized conditions. The corresponding studies were conducted by the company Aptuit, Italy.

The results of this interesting experiment are shown in Figure 2.9.11. According to proposed study design, anle138b was orally applied in excipient Cremophor ${ }^{\circledR}$ at $10 \mathrm{mg} / \mathrm{kg}$ dose to three rats. During 24 hours after dosing, the blood samples were collected and analyzed later. After one week of washout period, sery433 in the aqueous solution was formulated orally at $10 \mathrm{mg} / \mathrm{kg}$ dose to the same animals. The advantage of this protocol is a low intraindividual variability due to the similar metabolism rate of a given animal in both experiments. The evaluation of the pharmacokinetic data revealed a significant improvement of anle138b exposure in sery433 group in comparison to anle138b/Cremophor ${ }^{\circledR}$ (Fig. 2.9.11). For instance, the anle138b systemic exposure, in terms of AUC (area under the curve), was approximately 4 -fold higher in case of the prodrug sery $433^{503}$. 


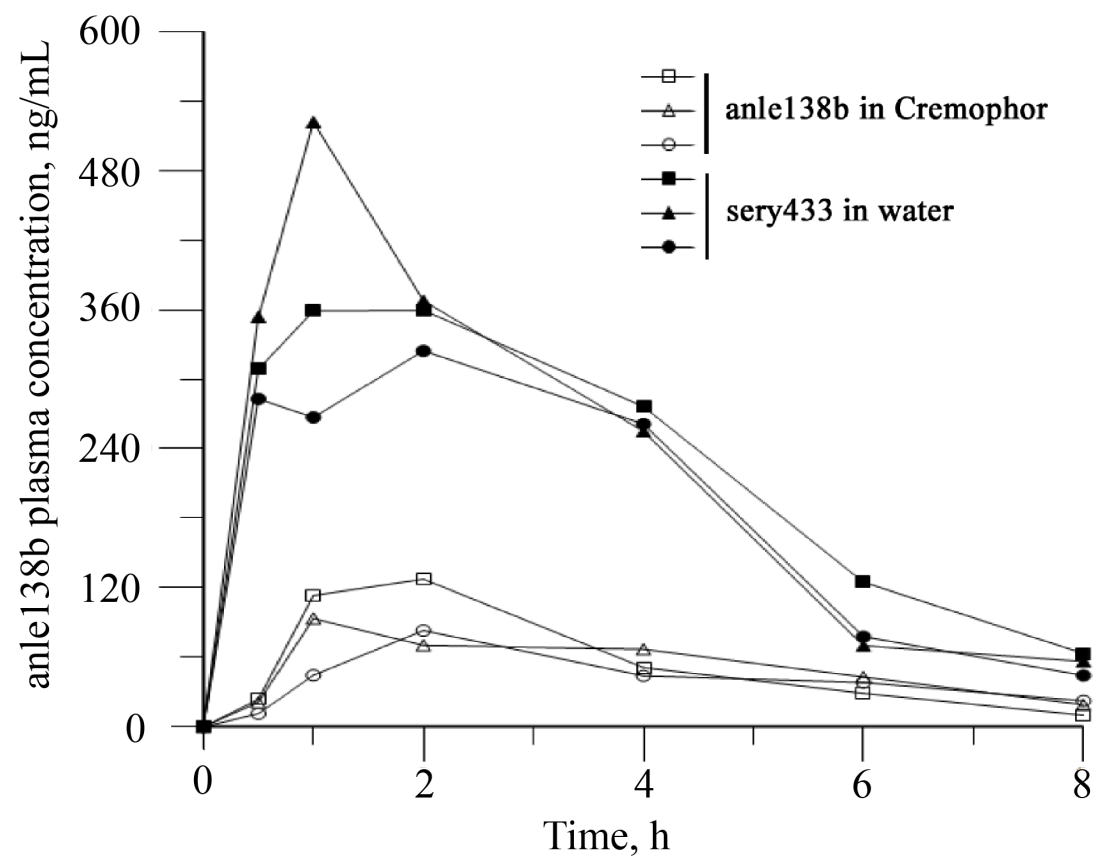

Figure 2.9.11. The comparative $P K$ study of sery433 and anle138b in rats. Plasma anle $138 \mathrm{~b}$ concentration-time profile after single oral application via gavage of anle138b in Cremophor at $10 \mathrm{mg} / \mathrm{kg}$ dose (open shapes) and sery 433 in aqueous solution at $10 \mathrm{mg} / \mathrm{kg}$ dose (filled shapes) in rats. Each trace corresponds to individual animal, for which the 8 blood samples were collected during 24 hours after dosing. Similar shapes correspond to the similar animal in both experiments. The figure is adapted from patent WO2017102893 ${ }^{503}$.

\subsubsection{Prodrug: Summary}

We have designed and synthesized several promising anle138b prodrugs. Among three candidates, sery474 exhibit low chemical stability in aqueous solutions. Two other compounds sery433 and sery453 are characterized by high solubility and high stability in water. In vivo studies in in rats and in mice provided strong indication that prodrug sery 433 has a preferred pharmacokinetic profile, which translates into high anle138b exposure in comparison to sery453. Moreover, the comparative pharmacokinetic study with sery 433 and anle138b, which were applied orally at the same dose, demonstrated a significant increase of anle138b systemic exposure in the case of prodrug sery 433.

The mechanism of biotransformation of sery433 in vivo is presumably associated with the enzymatic cleavage of the phosphate moiety by the membrane-bound intestinal phosphatase (Fig. 2.9.5). After rapid and spontaneous hydrolysis of the hemiaminal, anle138b is released in the intestine, possibly in the form of monodisperse particles, which are readily absorbed into the blood by the passive diffusion through the intestinal membrane. 
From a clinical point of view, the use of water-soluble prodrug sery 433 might have several benefits, including the lack of special excipients, the possibility to provide standard dosage forms (pills, tablets) and smaller volumes of pharmaceutically acceptable additives.

The weak point of sery433 prodrug is the fact that it is a mixture of two regioisomers. To solve this problem we are currently trying to develop a regioselective synthesis.

Collectively, sery433 is the promising water-soluble anle138b prodrug for further preclinical development. 


\subsection{Anle138b: Modulation of protein misfolding in other diseases}

As described in the previous chapters, anle138b modulates the oligomer formation in case of $\alpha$-synuclein and prion protein. In our efforts to investigate the therapeutic potential of anle138b and related DPP compounds with respect to other protein misfolding disease, several in vitro and in vivo studies were conducted in collaboration with several scientific labs.

In case of Alzheimer's disease, anle138b interferes with misfolding of Amyloid beta and Tau protein in vitro and in vivo ${ }^{489,} 490,504$. Thus, anle138b improves the behavioral, neuropathological and biochemical outcomes in transgenic APPPS $1^{490}$ mouse model and in Tau transgenic P301S (PS19) 489, 505 mouse model. In these studies, the groups of Prof. A. Fischer (DZNE, Göttingen), Prof. G. Eichele (Max Planck Institute for biophysical chemistry, Göttingen), Prof. M. Fuhrman (DZNE, Bonn), Prof. A. Giese (LMU, Munich), Prof. A. Rominger (LMU, Munich) were major collaborating partners.

Islet amyloid polypeptide (IAPP) is associated with the progression of type 2 diabetes mellitus disease. Accumulating evidence suggests that the aggregation of islet amyloid polypeptide may contribute to $\beta$-cell death in type 2 diabetes. The effect of anle138b and other DPP compounds was studied in the groups of Prof. A. J. Killian and Prof. J. W. M. Hoppener in Utrecht University. Thus, it has been demonstrated that anle138b and other DPP compounds interferes with misfolding of IAPP in vitro and in vivo. Specifically, in a preventive study anle $138 \mathrm{~b}$ treatment normalized the glucose level in obese transgenic IAPP mouse model (data not published).

Finally, several DPP compounds and especially anle138b exhibit pronounced anticancer activity in melanoma ${ }^{506,507}$. It has been demonstrated that $\alpha$-synuclein is unregulated in aggressive forms of melanoma skin cancer. Experimental evidences suggest that $\alpha$-synuclein is highly beneficial to the survival of melanoma in its advanced stages. Treatment with anle $138 \mathrm{~b}$ leads to massive melanoma cell death due to a major dysregulation of autophagy both in cell cultures and in mouse bearing $\alpha$-synuclein expressing human melanoma xenografts $^{506}$. This study was conducted in collaboration with the groups of Dr. Dorothea Becker (UMG, Göttingen), Prof. T. F. Outeiro (UMG, Göttingen) and Dr. D. J. Arndt-Jovin (Max Planck Institute for biophysical chemistry, Göttingen). 


\section{Outlook}

Here we show that anle138b modulates the oligomer formation of different proteins. However, the exact mode of interaction between the misfolded proteins and anle $138 \mathrm{~b}$ remains unclear. To elucidate the mechanisms of anle138b binding to pathological aggregates in a residue specific manner, we extend our biophysical studies with two different approaches. First, we would like to apply a high resolution solid-state NMR methods to characterize the interactions of anle138b with various forms of $\alpha$-synuclein in the lipid bilayers which was started in the thesis of my colleague Leif Antonschmidt in the NMR department. In a second approach we would like to design analogs of anle138b bearing a small photoactivatable functional group. By a combination of photoinduced cross-linking with high-resolution mass spectrometry we aim to map the anle138b/a-synuclein interactions under various conditions, which is currently under investigation by my colleague Kris Runge.

Non-invasive imaging methods such as PET and MRI can be valuable tools for diagnosis of Parkinson's disease and monitoring of disease progression. Currently, the biomarkers to detect $\alpha$-synuclein pathology are missing. Therefore, DPP compounds might represent a promising starting point for development of novel PET tracers and MRI imaging agents. These projects are currently pursued by myself and my colleague Dr. Andrei Leonov, in collaboration with the PET facility in Tübingen headed by Prof. Bernd Pichler and the company MODAG.

Finally, prodrug sery433 was licensed to the company MODAG, which is going to conduct pharmacokinetic studies of the prodrug sery433 in monkeys, as a basis for a full preclinical development program aiming to introduce this molecule in the clinic as well. Additionally, the regioselective synthesis of sery433 is an important goal for us.

During my studies, it has become clear that aggregation of proteins does not only play a role in neurodegeneration but also in cancer and further pathological processes. With the possibility of interfering with these processes we hope to get further insight into the biology of these processes as well as in the SAR of the DPP compounds. 


\section{Material and Methods}

\subsection{Materials I}

4.1.1. Instruments and consumables

Laboratory instruments and consumables used in this work in the biolab (Max Planck Institute for biophysical chemistry) are summarized in Table 4.1.

Table 4.1. Instruments and consumables in the biolab

\begin{tabular}{|c|c|}
\hline Equipment & Manufacturer and type \\
\hline $\begin{array}{l}\text { Atomic force } \\
\text { microscope }\end{array}$ & $\begin{array}{l}\text { Veeaco Digital Instruments MultiMode scanning probe } \\
\text { microscope IIIa }\end{array}$ \\
\hline Balances & Sartorius E 235 P \& AC $200 \mathrm{~S}$ \\
\hline Centrifuge & $\begin{array}{l}\text { Beckman Coulter Avanti J-20, TL-100, L-80 XP with rotors: } \\
\text { JLA 8.100, JLA-9.1000, JA 25.50 Ti, JA } 30.50 \mathrm{Ti} \text {, Ti70 } \\
\text { Eppendorf Centrifuge 5415D \& 5804 }\end{array}$ \\
\hline Concentrators & Amicon Microcon, Centricon, Centriplus \\
\hline EDTA tubes & Sarstedt Micro tube $1.3 \mathrm{ml} \mathrm{K3E}$ \\
\hline Dialysis & $\begin{array}{l}\text { Pierce Slide-A-Lyzer Dialysis Cassettes } \\
\text { Spectrum Spectra/Por cellulose ester membranes kit }\end{array}$ \\
\hline $\begin{array}{l}\text { Dynamic light } \\
\text { scattering }\end{array}$ & Malvern Zetasizer Nano ZS \\
\hline Electron microscope & Philips CM12 \\
\hline $\begin{array}{l}\text { Fluorescence } \\
\text { spectrophotometer }\end{array}$ & Varian Cary Eclipse \\
\hline Filter (syringe) & Sartorius or Millipore $0.22 \mu \mathrm{m}$ syringe filter \\
\hline FPLC & $\begin{array}{l}\text { ÄKTA basic, Amersham Pharmacia Biotech } \\
\text { Thermo Scientific POROS20 HQ anion exchange column } \\
\text { Pharmacia Biotech Superdex75 HiLoad } 120 \mathrm{ml} \text { column }\end{array}$ \\
\hline Homogenizer & $\begin{array}{l}\text { Bertin Technologies Precellys Evolution Super homogenizer, } \\
\text { Precellys tissue homogenizing tube kit } \\
\text { IKA ULTRA-TURRAX Tube drive workstation } \\
\text { IKA dispersion Tube DT-20 kit }\end{array}$ \\
\hline Incubator & $\begin{array}{l}\text { Infors Multitron HT and Braun Biotech Certomat } \\
\text { Thermo Scientific Heraeus B20 }\end{array}$ \\
\hline Lyophilization & Braun Biotech Christ Alpha 2-4 \\
\hline NMR spectrometers & $\begin{array}{l}\text { Bruker Avance } 400 \mathrm{MHz} \text { with TXI probe, } \mathrm{z} \text { axis gradient } \\
\text { Bruker DRX } 600 \mathrm{MHz} \text { with QXI probe, z axis gradient } \\
\text { Bruker Avance } 600 \mathrm{MHz} \text { with cryoprobe, z-axis gradient } \\
\text { Bruker Avance } 700 \mathrm{MHz} \text { with TXI probe, triple axis gradient } \\
\text { Bruker DRX } 800 \mathrm{MHz} \text { with TXI probe, triple axis gradient } \\
\text { Bruker Avance } 900 \mathrm{MHz} \text { with cryoprobe, z-axis gradient } \\
\text { Bruker Avance ssNMR } 600 \mathrm{MHz} \text { with MAS probe }\end{array}$ \\
\hline Sonicator & Bandelin SONOPLUS HD 2200 \\
\hline Ultrasonic device & Elma Elmasonic S60 \\
\hline $\begin{array}{l}\text { UV-Vis } \\
\text { spectrophotometer }\end{array}$ & Hewlett-Packard 8453 \\
\hline $\begin{array}{l}\text { UV-Vis CD } \\
\text { spectrophotometer }\end{array}$ & Applied Photophysics Chirascan \\
\hline
\end{tabular}




\subsubsection{Reagents and materials}

Chemical reagents and materials used in this work in the biolab (Max Planck Institute for biophysical chemistry) are summarized in Table 4.2.

Table 4.2. Chemical and materials in biolab

\begin{tabular}{|c|c|}
\hline Chemicals/reagents & Supplier \\
\hline Agar, PMSF, Streptomycine & $\begin{array}{l}\text { AppliChem, Darmstadt, } \\
\text { Germany }\end{array}$ \\
\hline Ammonium chloride $\left(>98 \%{ }^{15} \mathrm{~N}\right)$ & $\begin{array}{l}\text { Cambridge Isotope } \\
\text { Laboratories, Andover, USA }\end{array}$ \\
\hline${ }^{13} \mathrm{C}$-D-glucose $\left(>98 \%{ }^{13} \mathrm{C}\right)$ & $\begin{array}{l}\text { Spectra Stable Isotopes, } \\
\text { Columbia, USA }\end{array}$ \\
\hline $\begin{array}{l}\text { Ammonium molybdate tetrahydrate, ascorbic acid, } \\
\text { Coomassie Brillant Blue R-250, Copper chloride } \\
\text { dihydrate, Iron (II) sulfate heptahydrate, }\end{array}$ & Fluka, Neu-Ulm, Germany \\
\hline $\begin{array}{l}\text { ampicillin sodium salt, APS, dipotassium hydrogen } \\
\text { phosphate, ethidium bromide, EDTA, HEPES, } \\
\text { potassium dihydrogen phosphate, sodium acetate, } \\
\text { Streptomycin sulphate, TEMED, tryptone, yeast } \\
\text { extract, 2x YT medium }\end{array}$ & Roth, Karlsruhe, Germany \\
\hline $\begin{array}{l}\text { Acetic acid, Acetonitrile, } \alpha-\mathrm{D}(+) \text {-glucose } \\
\text { monohydrate, Ammonium sulfate, Ammonium } \\
\text { chloride, Ammonium hydrocarbonate, Boric acid, } \\
\text { Bromophenol Blue, Disodium hydrogen phosphate, } \\
\text { Ethanol, Glycerol, Glycine, Hydrochloric acid, } \\
\text { Manganese chloride dihydrate, Magnesium sulfate } \\
\text { heptahydrate, Methanol, Sodium chloride, Sodium } \\
\text { dihydrogen phosphate, Sodium hydroxide, Sodium } \\
\text { thiosulphate, TFA, Thiaminechloride hydrochloride, } \\
\text { ThioflavinT, Tris, Urea }\end{array}$ & Merck, Darmstadt, Germany \\
\hline Bench Mark protein ladder & $\begin{array}{l}\text { Invitrogen, Karlsruhe, } \\
\text { Germany }\end{array}$ \\
\hline Cobalt chloride hexahydrate & $\begin{array}{l}\text { Riedel-de Haëen, Seelze, } \\
\text { Germany }\end{array}$ \\
\hline Deuterated solvents & $\begin{array}{l}\text { DEUTERO GMBH, } \\
\text { Kastellaun, Germany }\end{array}$ \\
\hline DTT & Gerbu, Gaiberg, Germany \\
\hline $4-15 \%$ precast polyacrylamide gels & $\begin{array}{l}\text { Bio-Rad Laboratories, } \\
\text { California, USA }\end{array}$ \\
\hline SDS & Serva, Heidelberg, Germany \\
\hline Purified Mouse Anti- $\alpha$-synuclein (BD) & $\begin{array}{l}\text { BD Biosciences, Heidelberg, } \\
\text { Germany }\end{array}$ \\
\hline
\end{tabular}




\subsubsection{E. coli strain and E. coli cell culture media}

E. coli strain - Escherichia coli BL21 (DE 3)

The composition of culture media for cultivation of $E$. coli cells is summarized in Table 4.3. Prior to usage $100 \mu \mathrm{g} / \mathrm{mL}$ ampicillin was added to each medium.

Table 4.3. The composition of culture media

\begin{tabular}{l|l}
\hline Name & Chemical (amount) \\
\hline $\begin{array}{l}\text { Ampicillin } \\
\text { stock }\end{array}$ & $100 \mathrm{mg} / \mathrm{mL}$ ampicillin sodium salt \\
\hline LB medium & Tryptone $(10 \mathrm{~g})$, yeast extract $(5 \mathrm{~g}), \mathrm{NaCl}(10 \mathrm{~g}), \mathrm{H}_{2} \mathrm{O}(1 \mathrm{~L})$ \\
\hline $\begin{array}{l}\text { M9 minimal } \\
\text { medium }\end{array}$ & $\begin{array}{l}\mathrm{Na}_{2} \mathrm{HPO}_{4}(6.8 \mathrm{~g}), \mathrm{KH}_{2} \mathrm{PO}_{4}(3 \mathrm{~g}), \mathrm{NaCl}(0.5 \mathrm{~g}), \mathrm{NH}_{4} \mathrm{Cl}(1 \mathrm{~g}), \mathrm{D}- \\
\text { glucose }(4 \mathrm{~g}), \\
1 \mathrm{M} \mathrm{MgSO}(2 \mathrm{ml}), 2 \mathrm{M} \mathrm{CaCl} \\
\\
\text { hydrochloride }(0.03 \mathrm{~g}), \text { solution of trace elements }(10 \mathrm{ml}), \mathrm{H}_{2} \mathrm{O}(1 \\
\\
\mathrm{L})\end{array}$ \\
\hline $\begin{array}{l}\text { Solution of trace } \\
\text { elements }\end{array}$ & $\begin{array}{l}\mathrm{CoCl}_{2} \cdot 6 \mathrm{H}_{2} \mathrm{O}(0.08 \mathrm{~g}), \mathrm{CuCl}_{2} \cdot 2 \mathrm{H}_{2} \mathrm{O}(0.03 \mathrm{~g}), \mathrm{FeSO}_{4} \cdot 7 \mathrm{H}_{2} \mathrm{O}(0.6 \mathrm{~g}), \\
\mathrm{MnCl}_{2} \cdot 2 \mathrm{H}_{2} \mathrm{O}(0.094 \mathrm{~g}), \mathrm{H}_{3} \mathrm{BO}_{3}(0.002 \mathrm{~g}),\left(\mathrm{NH}_{4}\right)_{6} \mathrm{Mo}_{7} \mathrm{O}_{24} \cdot 4 \mathrm{H}_{2} \mathrm{O} \\
(0.025 \mathrm{~g}), \mathrm{ZnSO}_{4} \cdot 7 \mathrm{H} \mathrm{H}_{2} \mathrm{O}, \mathrm{EDTA}(0.5 \mathrm{~g}), \mathrm{H}_{2} \mathrm{O}(100 \mathrm{ml})\end{array}$ \\
\hline
\end{tabular}




\subsection{Methods I}

\subsubsection{Protein biochemistry}

\subsubsection{Expression and purification of $\alpha$-synuclein}

Preparation of $\alpha$-synuclein samples were performed according to published protocol ${ }^{508}$ with minor modifications. The coding sequence for wild type human $\alpha$-synuclein in the plasmid pT7-7 was kindly provided by the Lansbury Laboratory, Harvard Medical School, Cambridge, MA. Several batches of $\alpha$-synuclein, ${ }^{15} \mathrm{~N}$-labelled $\alpha$-synuclein and ${ }^{13} \mathrm{C},{ }^{15} \mathrm{~N}-$ labelled $\alpha$-synuclein were prepared by Dr. Stefan Becker and Karin Giller by using similar protocols.

Transformation of E. coli: $1 \mu$ plasmid DNA (pt7-7 vector containing human $\alpha-$ synuclein gene) was added to $50 \mu \mathrm{l}$ chemically competent cells. After incubation for $30 \mathrm{~min}$ on ice, the cells were subjected to a heat shock of $42{ }^{\circ} \mathrm{C}$ for $45 \mathrm{~s}$ followed by cooling for 2 minutes on ice. Pre-warmed to $42{ }^{\circ} \mathrm{C} 0.5 \mathrm{ml}$ of $2 \times$ YT medium was added and the cells was incubated for $1 \mathrm{~h}$ at $37^{\circ} \mathrm{C}$. Finally, the cells were plated onto LB-agar plates containing ampicillin $(100 \mu \mathrm{g} / \mathrm{ml})$ and incubated overnight $(>16 \mathrm{~h})$ at $37^{\circ} \mathrm{C}$.

Expression and purification of $\alpha$-synuclein: A single $E$. coli $\mathrm{BL} 21$ cell colony was incubated in $2 \mathrm{ml}$ of LB-Amp medium $\left(100 \mu \mathrm{g} / \mathrm{ml}\right.$ ampicillin) for $5 \mathrm{~h}$ at $37^{\circ} \mathrm{C}$ and $200 \mathrm{rpm}$. The pre-culture $(50 \mu \mathrm{l})$ was added to $50 \mathrm{ml}$ of LB-Amp medium $(100 \mu \mathrm{g} / \mathrm{ml}$ ampicillin) and incubated overnight at $37^{\circ} \mathrm{C}$ and $200 \mathrm{rpm}$. The pre-culture was diluted $(50 \mathrm{ml}$ in $1 \mathrm{~L} \mathrm{LB}-\mathrm{Amp}$ containing $100 \mu \mathrm{g} / \mathrm{ml}$ ampicillin) and allowed to grow at $37^{\circ} \mathrm{C}$ and $120 \mathrm{rpm}$ until an optical density $\left(\mathrm{OD}_{600}\right)$ of $0.6-0.8(2.5-3 \mathrm{~h})$. Expression of the desired gene construct was then induced with $1 \mathrm{mM}$ IPTG. Following $6 \mathrm{~h}$ of incubation at $37^{\circ} \mathrm{C}$ and $120 \mathrm{rpm}$, the cells were harvested by centrifugation at $6000 \mathrm{rpm}$ for 30 minutes (Beckman Coulter Avanti J-20, rotor JLA-9.1000).

The bacterial pellet was resuspended in $20 \mathrm{ml}$ of lysis buffer $(10 \mathrm{mM}$ Tris, $\mathrm{pH} 8.0,1$ $\mathrm{mM}$ EDTA and $1 \mathrm{mM}$ PMSF) and lysed by three freeze-thaw cycles, freezing in liquid nitrogen (2 minutes) and incubation at $70{ }^{\circ} \mathrm{C}$ water bath $(15$ minutes) and following sonication (Sonoplus HD 2200 sonifier quipped with macro tip; 3 times, $40 \mathrm{sec}$ at 40, 50 and $60 \%$ power sequentially for 6 sonication cycles on ice, 2 minutes pause between cycles). In order to denaturate host cell proteins, the cell lysate was incubated for 20 minutes at $95{ }^{\circ} \mathrm{C}$ and centrifuged at $20000 \mathrm{rpm}$ for 30 minutes at $4{ }^{\circ} \mathrm{C}$ (Beckman Coulter Avanti J-20, rotor JA$30.50)$. For precipitation of DNA, the resulting supernatant was treated with streptomycinsulfate (final concentration $10 \mathrm{mg} / \mathrm{ml}$ ) and the mixture was stirred at $4{ }^{\circ} \mathrm{C}$ for 20 minutes followed by centrifugation at $20000 \mathrm{rpm}$ for 30 minutes at $4{ }^{\circ} \mathrm{C}$ (Beckman Coulter Avanti J20 , rotor JA-30.50). Next, $\alpha$-synuclein protein was precipitated by slow addition of ammonium sulfate to a final concentration of $361 \mathrm{mg} / \mathrm{ml}$ to the supernatant from previous centrifugation at $4{ }^{\circ} \mathrm{C}$. After stirring at $4{ }^{\circ} \mathrm{C}$ for 10 minutes, a crude protein precipitate was collected by centrifugation at $20000 \mathrm{rpm}$ for 30 minutes at $4{ }^{\circ} \mathrm{C}$ (Beckman Coulter Avanti J20 , rotor JA-30.50). The protein pellet was resuspended in $10 \mathrm{ml}$ of $25 \mathrm{mM}$ Tris buffer, $\mathrm{pH}$ 7.7, and dialyzed overnight at $4{ }^{\circ} \mathrm{C}$ against $2 \mathrm{~L}$ of $25 \mathrm{mM}$ Tris buffer, $\mathrm{pH} 7.7$ (Spectrum Spectra/Por cellulose ester membrane MWCO of $10 \mathrm{kDa}$ ).

The dialyzed protein solution was filtered through a $0.22 \mu \mathrm{M}$ filter and loaded onto POROS20 HQ anion exchange column in ÄKTA Basic system equilibrated with $25 \mathrm{mM}$ Tris$\mathrm{HCl}, \mathrm{pH} 7.7$ at room temperature. The fractions containing protein (determined by SDSPAGE gel electrophoresis by running on $15 \%$ of polyacrylamide gels) were combined and 
concentrated using Amicon Centriplus YM-10 regenerated cellulose centrifugal filter (Millipore) to a final concentration of $200 \mu \mathrm{M}$. The resulting solution was dialyzed overnight against 4 L $50 \mathrm{mM}$ HEPES, $100 \mathrm{mM} \mathrm{NaCl}$ buffer, pH 7.4 (10 kDa MWCO Slide-A-Lyzer dialysis cassette, Pierce).

The dialyzed protein solution was filtered through a $0.22 \mu \mathrm{M}$ filter and purified by size exclusion chromatography on ÄKTA Basic system by using Superdex75 HiLoad $120 \mathrm{ml}$ column (Pharmacia Biotech). The elution with degassed $50 \mathrm{mM}$ HEPES, $100 \mathrm{mM} \mathrm{NaCl}$ buffer, $\mathrm{pH} 7.4$ at room temperature. The fractions containing protein (determined by SDSPAGE gel electrophoresis by running on $15 \%$ of polyacrylamide gels) were combined and concentrated using Amicon Centriplus YM-10 regenerated cellulose centrifugal filter (Millipore) to a final concentration of $300 \mu \mathrm{M}$.

The concentrated $\alpha$-synuclein solution was dialyzed overnight against the desired buffer (10 kDa MWCO Slide-A-Lyzer dialysis cassette from Pierce). Purity of the protein was assessed by SDS-PAGE and the concentration was determined with absorbance at 280 $\mathrm{nm}$ using an extinction coefficient of $5960 \mathrm{M}^{-1} \mathrm{~cm}^{-1}$. Proteins were aliquoted, flash frozen in liquid $\mathrm{N} 2$ and stored at $-80{ }^{\circ} \mathrm{C}$.

Preparations of ${ }^{15} \mathrm{~N}$ - and ${ }^{13} \mathrm{C},{ }^{15} \mathrm{~N}$-labeled $\alpha$-synuclein were performed following the same protocol as that of the non-labeled $\alpha$-synuclein with the difference that LB medium was replaced by M9 minimum medium supplemented with ${ }^{15} \mathrm{NH}_{4} \mathrm{Cl}$ or ${ }^{15} \mathrm{NH}_{4} \mathrm{Cl}$ and ${ }^{13} \mathrm{C}$-Dglucose.

\subsubsection{2. $\alpha$-Synuclein aggregation assay in presence of DPP compounds}

The concentrated $\alpha$-synuclein stock solution was filtered through a $0.22 \mu \mathrm{M}$ filter and ultracentrifuged at $40000 \mathrm{rpm}$ for $1 \mathrm{~h}$ at $4{ }^{\circ} \mathrm{C}$ (Optima L-80 XP, Rotor Ti70, Beckman Coulter). The supernatant was collected (approx. $95 \%$ of the volume) and concentration of $\alpha-$ synuclein was determined with absorbance at $280 \mathrm{~nm}$ using an extinction coefficient of 5960 $\mathrm{M}^{-1} \mathrm{~cm}^{-1}$. The concentration of $\alpha$-synuclein was adjusted by addition of appropriate volume of the buffer. The aggregation assays were performed at 70-100 $\mu \mathrm{M} \alpha$-synuclein concentration in $50 \mathrm{mM}$ sodium phosphate, $100 \mathrm{mM} \mathrm{NaCl}$ buffer ( $\mathrm{pH}$ 7.4) or in $50 \mathrm{mM}$ HEPES, $100 \mathrm{mM}$ $\mathrm{NaCl}$ buffer ( $\mathrm{pH} 7.4$ ) containing $0.01 \% \mathrm{NaN}_{3}$. DPP compounds were dissolved in appropriate amount of filtered DMSO to make a stock solution.

The DMSO stock solution of DPP compound was added to $\alpha$-synuclein solution to provide a target compound concentration with the final DMSO concentration of $3 \%$. The control samples were prepared by addition of the same amount of pure DMSO. All experiments were performed in triplicates. The resulting samples were incubated in sterile glass vials (Zinsser Analytik, Frankfurt, Germany) at $37^{\circ} \mathrm{C}$ under constant stirring (200 rpm) with sterile magnetic microbars for indicated period of time. The process of aggregation was followed by various techniques using aliquots withdrawn during the course of incubations.

\subsubsection{Thiot assay}

ThioT binding measurements were performed to monitor the fibrillation in presence and absence of DPP compounds. ThioT assay was performed according to published protocol ${ }^{508}$ with minor modifications.

During the course of aggregation the aliquots $(5 \mu \mathrm{l})$ were withdrawn from the samples and added to $2.0 \mathrm{ml}$ of $25 \mu \mathrm{M}$ ThioT solution in $50 \mathrm{mM}$ glycine buffer, $\mathrm{pH} 8.0$. Fluorescence measurements were carried out on a Cary Eclipse spectrofluorometer using $3.5 \mathrm{ml}$ quartz cuvettes (Hellma, Germany) with a $1 \mathrm{~cm}$ light-path. Fluorescence emission spectra were 
collected from 465 to $600 \mathrm{~nm}$, with an excitation at $446 \mathrm{~nm}$, an integration time of 0.1 second, and both excitation and emission bandwidths of $10 \mathrm{~nm}$. Aggregation kinetics curves were generated from ThioT fluorescence intensity at $480 \mathrm{~nm}$ at different time points. ThioT fluorescence values were normalized to the values of control samples at the end of the aggregation assay.

\subsubsection{Polyacrylamide Gel Electrophoresis}

Conventional sodium dodecyl sulfate SDS-PAGE was performed with a sample buffer containing $10 \%$ SDS. Coomasie stain was performed following the instructions from the supplier.

Native gel electrophoresis was performed by using 4-15\% precast polyacrylamide gels (Bio-Rad Laboratories, California). Native sample buffer: $62.5 \mathrm{mM}$ Tris-HCl, pH 6.8 , 40\% glycerol, $0.01 \%$ Bromophenol Blue. Running buffer: $25 \mathrm{mM}$ Tris- $\mathrm{HCl}, 192 \mathrm{mM}$ glycine $\mathrm{pH}$ 8.3. After the electrophoresis under non-denaturing conditions, the proteins were transferred onto methanol-activated PVDF membrane and blotted using the anti- $\alpha$-synuclein antibody (1:3000, BD Biosciences), following instructions from the supplier.

For polyacrylamide gel electrophoresis analysis, the samples containing $100 \mu \mathrm{M} \alpha$ synuclein, $100 \mu \mathrm{M}$ sery166a in $50 \mathrm{mM}$ sodium phosphate, $100 \mathrm{mM} \mathrm{NaCl}$ buffer (pH 7.4) and $3 \%$ DMSO were incubated as described in 4.2.1.2.

\subsubsection{Protein NMR}

\subsubsection{Liquid-State NMR Spectroscopy}

\section{$1 D^{1} \mathrm{H}$ spectra and $2 D^{1} \mathrm{H}_{-}{ }^{15} \mathrm{~N} \mathrm{HSQC}$}

NMR spectra were acquired on NMR spectrometers from Bruker AG (Karlsruhe, Germany) that are listed in the Table 4.1. The conventional $1 \mathrm{D}{ }^{1} \mathrm{H}$ spectra and $2 \mathrm{D}{ }^{1} \mathrm{H}-{ }^{15} \mathrm{~N}$ HSQC spectra were acquired at $15{ }^{\circ} \mathrm{C}$ on uniformly ${ }^{15} \mathrm{~N}$-labelled $\alpha$-synuclein. The backbone assignment of ${ }^{15} \mathrm{~N}$-labelled $\alpha$-synuclein was kindly provided by Pinar Karpinar and Dr. MinKyu Cho.

For NMR sample preparation, the concentrated $\alpha$-synuclein stock solution was filtered through a $0.22 \mu \mathrm{M}$ filter and ultracentrifuged at $40000 \mathrm{rpm}$ for $1 \mathrm{~h}$ at $4{ }^{\circ} \mathrm{C}$ (Optima L-80 XP, Rotor Ti70, Beckman Coulter). The supernatant was collected (approx. 95\% of the volume) and concentration of $\alpha$-synuclein was determined with absorbance at $280 \mathrm{~nm}$ using an extinction coefficient of $5960 \mathrm{M}^{-1} \mathrm{~cm}^{-1}$. The DPP compounds were added in given concentrations to the protein solution in DMSO- $d 6$. studies:

Composition of NMR samples for $2 \mathrm{D}{ }^{1} \mathrm{H}_{-}{ }^{15} \mathrm{~N}$ HSQC-based binding and titration

1) $\alpha$-synuclein $(50 \mu \mathrm{M})$, sery $139(0,50,150,250,500,750,1000 \mu \mathrm{M})$

2) $\alpha$-synuclein $(40 \mu \mathrm{M})$, sery $139(0,1000 \mu \mathrm{M})$

3) $\alpha$-synuclein $(50 \mu \mathrm{M})$, anle137c $(0,50,100,200 \mu \mathrm{M})$

4) $\alpha$-synuclein $(40 \mu \mathrm{M})$, sery166a $(0,1000 \mu \mathrm{M})$

5) $\alpha$-synuclein $(70 \mu \mathrm{M})$, anle138c $(0,140 \mu \mathrm{M})$

6) $\alpha$-synuclein $(70 \mu \mathrm{M})$, anle $145 \mathrm{c}(0,140 \mu \mathrm{M})$

Buffer: $50 \mathrm{mM}$ sodium phosphate, $100 \mathrm{mM} \mathrm{NaCl}, 10 \% \mathrm{D}_{2} \mathrm{O}, \mathrm{pH} 7.4,0.01 \% \mathrm{NaN}_{3}$. Samples 1, 2, 3, 4 contained 5\% DMSO- $d 6$, samples 5 and 6 contained 3\% DMSO- $d 6$.

To follow the course of $\alpha$-synuclein aggregation in presence of DPP compounds, the conventional 1D ${ }^{1} \mathrm{H}$ spectra and $2 \mathrm{D}{ }^{1} \mathrm{H}-{ }^{15} \mathrm{~N}$ HSQC spectra were acquired at $15{ }^{\circ} \mathrm{C}$ on 
uniformly ${ }^{15} \mathrm{~N}$-labelled $\alpha$-synuclein. The corresponding solutions were incubated in sterile 5 mm NMR tube at $37{ }^{\circ} \mathrm{C}$ under constant stirring (200 rpm) with sterile magnetic microbars for indicated period of time. During the course of NMR aggregation assay, the samples were taken out from incubator once or twice a day for NMR measurements (approximately 1.5-2 hours). The magnetic microbars were removed from NMR tube and put back after NMR experiments. The experiments were performed in triplicates.

Composition of NMR samples for 2D ${ }^{1} \mathrm{H}-{ }^{15} \mathrm{~N}$ HSQC-based NMR measurements during the aggregation assay:

1) $\alpha$-synuclein $(70 \mu \mathrm{M})$, sery166a $(140 \mu \mathrm{M})$

2) $\alpha$-synuclein $(70 \mu \mathrm{M})$, anle138c $(140 \mu \mathrm{M})$

3) $\alpha$-synuclein $(70 \mu \mathrm{M})$, anle $145 \mathrm{c}(140 \mu \mathrm{M})$

4) $\alpha$-synuclein $(50 \mu \mathrm{M})$, anle145c $(140 \mu \mathrm{M}), 5 \mathrm{mM} \mathrm{Na}_{2} \mathrm{~S}_{2} \mathrm{O}_{3}$

Buffer: $50 \mathrm{mM}$ sodium phosphate, $100 \mathrm{mM} \mathrm{NaCl}, 10 \% \mathrm{D}_{2} \mathrm{O}, \mathrm{pH} 7.4,0.01 \% \mathrm{NaN}_{3}$. Samples 1, 2, 3 contained 3\% DMSO-d6, samples 4 contained 3\% ethanol- $d 5$.

2D ${ }^{1} \mathrm{H}-{ }^{15} \mathrm{~N}$ HSQC experiments were recorded using $256 \times 1024$ complex incremental data points in $\mathrm{F} 1\left({ }^{15} \mathrm{~N}\right)$ and $\mathrm{F} 2\left({ }^{1} \mathrm{H}\right)$ dimensions, with 8 to 16 scans per increment and a relaxation delay of $1.2 \mathrm{~s}$. The sampled frequency space (sweep width) was $26 \mathrm{ppm}$ for the nitrogen dimension (centered in $119 \mathrm{ppm}$ ) and $10 \mathrm{ppm}$ for the proton dimension (centered in $4.7 \mathrm{ppm})$.

All NMR spectra were processed using XWINNMR 3.5 (Bruker AG, Karlsruhe, Germany) or NMRPipe ${ }^{509}$ and analyzed using Sparky 3 (T.D Goddard and D.G. Kneller, University of California, San Francisco).

The chemical shift perturbations were calculated with the following equation ${ }^{510}$ :

$$
\Delta \delta_{H N}=\sqrt{\frac{\left(\frac{\Delta \delta_{N}}{5}\right)^{2}+\left(\Delta \delta_{H}\right)^{2}}{2}}
$$

The decay of the protein signals in $1 \mathrm{D}{ }^{1} \mathrm{H}$ spectra was determined by integration of the protein signal in interval $0.8-0.55 \mathrm{ppm}$.

\section{STD NMR binding studies.}

STD NMR spectra were acquired on NMR spectrometers from Bruker AG (Karlsruhe, Germany) that are listed in the Table 4.1. STD was recorded using an excitation-sculpting water suppression pulse sequence (stddiffesgp. 2 in the Bruker library). The saturation was achieved by a train of Gaussian-shaped pulses. Total saturation time $2 \mathrm{~s}$ with $50 \mathrm{~ms}$ selective pulses. For all experiments, the off-resonance was set at $-50 \mathrm{ppm}$ and the on-resonance was set at a given resonance. 8-16 scans were acquired with 8 s relaxation delay between the scans. STD spectra were acquired at $25^{\circ} \mathrm{C}$. The STD amplification factors were calculated as described $^{457}$.

For binding STD studies with monomeric $\alpha$-synuclein, the concentrated $\alpha$-synuclein stock solution was filtered through a $0.22 \mu \mathrm{M}$ filter and ultracentrifuged at $40000 \mathrm{rpm}$ for $1 \mathrm{~h}$ at $4{ }^{\circ} \mathrm{C}$ (Optima L-80 XP, Rotor Ti70, Beckman Coulter). The supernatant was collected (approx. $95 \%$ of the volume) and concentration of $\alpha$-synuclein was determined with absorbance at $280 \mathrm{~nm}$ using an extinction coefficient of $5960 \mathrm{M}^{-1} \mathrm{~cm}^{-1}$. The concentrated stock of $\alpha$-synuclein was added to a solution of corresponding DPP compound in deuterated buffer $50 \mathrm{mM}$ sodium phosphate, $100 \mathrm{mM} \mathrm{NaCl}, \mathrm{pH} 7.4,5 \%$ DMSO-d6.

Composition of NMR samples for STD binding studies with monomeric $\alpha$-synuclein:

1) $\alpha$-synuclein $(3 \mu \mathrm{M})$, anle137c $(0,30,75,150,300 \mu \mathrm{M})$

2) $\alpha$-synuclein $(5 \mu \mathrm{M})$, sery139 $(0,50,125,250,500,1000 \mu \mathrm{M})$ 
3) $\alpha$-synuclein $(5 \mu \mathrm{M})$, sery166a $(0,50,125,250,500,750 \mu \mathrm{M})$

For binding STD studies with $\alpha$-synuclein aggregated in presence of sery166a, the aggregation assay was performed as described in 4.2.1.2. with samples containing $100 \mu \mathrm{M} \alpha-$ synuclein, $100 \mu \mathrm{M}$ sery166a in $50 \mathrm{mM}$ sodium phosphate, $100 \mathrm{mM} \mathrm{NaCl}$ buffer, $\mathrm{pH}$ 7.4, 0.01 $\% \mathrm{NaN}_{3}$ with $3 \%$ DMSO- $d 6$. During the course of aggregation the aliquots $(25 \mu \mathrm{l})$ were collected and added to $475 \mu \mathrm{l}$ of $1 \mathrm{mM}$ solution of sery 166a in deuterated buffer $50 \mathrm{mM}$ sodium phosphate, $100 \mathrm{mM} \mathrm{NaCl}, \mathrm{pH} \mathrm{7.4,5 \%} \mathrm{DMSO-d6.} \mathrm{The} \mathrm{collection} \mathrm{of} \mathrm{aliquots} \mathrm{for} \mathrm{STD}$ measurements were done at $0 \mathrm{~h}, 48 \mathrm{~h}, 96 \mathrm{~h}$, and $144 \mathrm{~h}$.

\subsubsection{Solid-State NMR Spectroscopy}

Solid-state NMR measurements were performed by Dr. Ashutosh Kumar in our department. Solid-state NMR experiments were conducted by on a 14.1 T NMR spectrometer (corresponding $1 \mathrm{H}$ resonance frequency: $600 \mathrm{MHz}$ ) equipped with a $4 \mathrm{~mm}$ triple-resonance $\left({ }^{1} \mathrm{H},{ }^{13} \mathrm{C}, 15 \mathrm{~N}\right)$ MAS probe (Bruker Biospin, Germany). All experiments were carried out under frozen conditions using a probe temperature of $-30^{\circ} \mathrm{C}$ and an MAS rate of $8.5 \mathrm{kHz}$. One-dimensional cross-polarization (CP) spectra of $\alpha$-synuclein monomers, toxic on-pathway $\alpha$-synuclein oligomers ${ }^{11}$, anle138c-stabilized off-pathway oligomers, and amyloid fibrils (kindly provided by Dr. Stefan Becker) were recorded using SPINAL64 heteronuclear decoupling with radio frequency (r.f.) amplitudes of $80-90 \mathrm{kHz}$. Two-dimensional $\left({ }^{13} \mathrm{C}-{ }^{13} \mathrm{C}\right)$ correlation spectra of anle138b-stabilized off-pathway oligomers and amyloid fibrils were recorded employing proton-driven spin-diffusion (PDSD) with mixing times of $50 \mathrm{~ms}$.

For solid-state NMR experiments, the samples $(3 \times 3.5 \mathrm{ml})$ containing $70 \mu \mathrm{M}{ }^{13} \mathrm{C},{ }^{15} \mathrm{~N}$ labelled $\alpha$-synuclein, $100 \mu \mathrm{M}$ anle138c in $50 \mathrm{mM}$ sodium phosphate, $100 \mathrm{mM} \mathrm{NaCl}$ buffer (pH 7.4) and 3\% DMSO were incubated as described in 4.2.1.2. for four days in $5 \mathrm{ml}$ sterile glass bottles. After ThioT, DLS and EM characterization, the aggregated samples were consecutively concentrated at $4{ }^{\circ} \mathrm{C}$ by using Amicon Centriplus YM-10 regenerated cellulose centrifugal filter (Millipore) up to approximately $4 \mathrm{mM}$ total $\alpha$-synuclein concentration. After filing the rotor, the samples were flash frozen in liquid $\mathrm{N}_{2}$.

\subsubsection{Other spectroscopic methods}

\subsubsection{Dynamic Light Scattering (DLS) Measurements}

DLS measurements were performed to characterize the oligomeric species in presence and absence of DPP compounds. During the course of aggregation the aliquots $(50 \mu \mathrm{l})$ were collected from the aggregated samples. After DLS recording the samples were added back to aggregation mixture. DLS measurements were performed on Zetasizer Nano ZS (Malvern) at $25^{\circ} \mathrm{C}$ in $45 \mu \mathrm{l}$ quartz cuvettes with a path-length of $0.3 \mathrm{~cm}$ (Hellma). DLS instrument was in the department of Dr. Tom Jovin. Nine different measurements were performed for each sample.

\subsubsection{Circular Dichroism (CD) Spectroscopy}

DLS measurements were performed to characterize the secondary structure of aggregated species in presence and absence of DPP compounds. Far UV-CD measurements were performed on a Chirascan (Applied Photophysics, UK) circular dichroism spectrometer (Department of Prof. Reinhard Jahn) in a quartz cuvette with $0.1 \mathrm{~cm}$ light-path. Measurement settings: window size $250-190 \mathrm{~nm}$, step $0.5 \mathrm{~nm}$; temperature $25^{\circ} \mathrm{C}$, number of repeats 3 and 
corrected for the buffer spectrum.

The aggregated samples for CD measurement were prepared as described in 4.2.1.2. Samples with sery166a: $100 \mu \mathrm{M} \alpha$-synuclein, $100 \mu \mathrm{M}$ sery166a, $50 \mathrm{mM}$ sodium phosphate, $100 \mathrm{mM} \mathrm{NaCl}, \mathrm{pH} \mathrm{7.4}$, and $2 \%$ ethanol. The control $\alpha$-synuclein samples were incubated under similar conditions without DPP compound. During the course of aggregation the aliquots were collected and after dilution in buffer $(50 \mathrm{mM}$ sodium phosphate, $100 \mathrm{mM} \mathrm{NaCl}$, $\mathrm{pH}$ 7.4) $\mathrm{CD}$ measurements were performed at $\alpha$-synuclein $5 \mu \mathrm{M}$ concentration.

\subsubsection{Electron microscopy (EM)}

Dr. Dietmar Riedel (Department of neurobiology, Max Planck Institute for biophysical chemistry) recorded electron microscopic images. For EM studies, the $\alpha$ synuclein aggregation in presence and absence of DPP compounds was done on samples containing $70 \mu \mathrm{M} \alpha$-synuclein, $100 \mu \mathrm{M}$ sery166a or $100 \mu \mathrm{M}$ anle138c, $50 \mathrm{mM}$ HEPES, 100 $\mathrm{mM} \mathrm{NaCl}$ buffer ( $\mathrm{pH} \mathrm{7.4)} \mathrm{and} \mathrm{3 \%} \mathrm{DMSO.} \mathrm{During} \mathrm{the} \mathrm{course} \mathrm{of} \mathrm{aggregation} \mathrm{the} \mathrm{aliquots} \mathrm{were}$ collected and samples were deposited on Formvar-coated 200 mesh copper grids (Electron Microscopy Sciences) at a concentration of 5-25 $\mu \mathrm{M}$. Grids were washed with two drops of water and stained with two drops of freshly prepared $0.75 \%(\mathrm{w} / \mathrm{v})$ uranyl acetate (Electron microscopy sciences). Specimens were inspected on a Philip CIME 12 electron microscope, operated at $80 \mathrm{kV}$. Digitized photographs were recorded with a slow scan CCD camera.

\subsubsection{Atomic force microscopy (AFM)}

AFM images were recorded with assistance of Dr. Avishay Pelah in department of Dr. Tom Jovin. For AFM, the samples containing $70 \mu \mathrm{M} \alpha$-synuclein, $100 \mu \mathrm{M}$ sery166a in 50 $\mathrm{mM}$ sodium phosphate, $100 \mathrm{mM} \mathrm{NaCl}$ buffer ( $\mathrm{pH} \mathrm{7.4)}$ ) and 3\% DMSO were incubated for three days as described in 4.2.1.2. The aggregated samples were diluted by a factor of ten. Small aliquots $(10 \mu \mathrm{l})$ were spin coated to dryness on a freshly cleaved mica surface. A Digital Instruments MultiMode scanning probe microscope IIIa (Veeco) was used. Samples were imaged in air at a $1 \mathrm{~Hz}$ scan rate by using silicon tips.

4.2.3.5. Screening and evaluation of DPP compounds in scanning for intensely fluorescent targets (SIFT) assay

The SIFT assays were performed in the laboratory of Prof. Armin Giese (LMU, München, Germany) according to published protocols ${ }^{10}$. During two lab visits, I did the SIFT assay on small libraries of DPP compounds.

\subsubsection{Electrical recording of planar lipid bilayer and LTP experiments}

\subsubsection{Electrical recording of planar lipid bilayer made of oxidized cholesterol/ $n$ -} decane

Electrical recordings of planar lipid bilayer were performed in the laboratory of Prof. Roland Benz (Jacobs University of Bremen, Bremen, Germany) according to the published procedures $^{11,490}$.

Planar lipid bilayers were prepared using the Müller-Rudin technique ${ }^{463}$. The membranes were formed from a $1 \%(\mathrm{w} / \mathrm{v})$ solution of diphytanoylphosphatidylcholine (DiphPC) dissolved in $n$-decane. The aqueous electrolyte solutions (1 $\mathrm{M} \mathrm{KCl}$ ) were unbuffered and had a $\mathrm{pH}$ of $\sim 6$ unless otherwise indicated. The lipid membranes were formed 
using a Teflon loop to spread the lipid solution across a hole with a surface area of approximately $0.3 \mathrm{~mm}^{2}$ in the wall dividing the two aqueous compartments in a Teflon cell. After the membrane had turned black, the $\alpha$-synuclein samples were added to the aqueous phase on one (cis) or both sides of the membrane at final concentrations of $2-10 \mu \mathrm{M} / \mathrm{ml}$.

The current across the lipid bilayer membranes was measured with a pair of $\mathrm{Ag} / \mathrm{AgCl}$ electrodes with salt bridges switched in series with a voltage source and a highly sensitive current amplifier Keithley 427 (Keithley Instruments, INC. Cleveland, OH). The output signal of the amplifier was recorded by a strip chart recorder (Rikadenki Electronics GmbH, Freiburg, Germany). The temperature was kept at $20^{\circ} \mathrm{C}$ throughout the experiment. The applied membrane potential was $+50,+100 \mathrm{mV},+150 \mathrm{mV},+200 \mathrm{mV}$.

For doping lipid bilayers with anle138b, the compound was dissolved in chloroform. The chloroform of a sample was then evaporated under vacuum. The lipid solution was added to the dry anle138b in such an amount that a final concentration of $1 \mathrm{mM}$ in the lipid solution was obtained. This lipid solution was used for the normal protocol of bilayer formation as described above.

Cold-induced $\alpha$-synuclein oligomers ${ }^{11,459}$ were treated overnight at room temperature with 10-20-fold excess of DPP compounds (sery166a or sery85 or anle138c), which were added in DMSO stock solution (10 volumes of oligomeric $\alpha$-synuclein preparation +1 volume of DPP compound solution in DMSO). The same measurement procedure was applied to these samples.

Compound-stabilized $\alpha$-synuclein oligomers were prepared as described in 4.2.1.2 under given conditions (see Table 2.6.1).

4.2.4.2. Electrophysiological studies of $\alpha$-synuclein oligomer $\left(F e^{3+}\right.$-induced $\alpha$ synuclein oligomers) pore-formation in planar lipid bilayers and effect of anle138b

These experiments were performed in the laboratory of Prof. Armin Giese (LMU, München, Germany) as described ${ }^{10}$.

\subsubsection{Electrophysiology on hippocampal slices, fEPSP recordings.}

These experiments were performed in the laboratory of Prof. Tiago Fleming Outeiro (University of Göttingen, Göttingen, Germany). I delivered the oligomeric samples.

The slice preparations, fEPSP recordings and LTP induction were performed according to published protocol ${ }^{8}$. Slices were incubated with compound-stabilized $\alpha-$ synuclein oligomers (compounds anle138c or anle145c) or without $\alpha$-synuclein for control. fEPSPs were recorded from control slices and slices treated with two different compoundstabilized $\alpha$-synuclein oligomeric species for 60 minutes. LTP was induced in control slices and in slices pre-treated for 60 minutes with two different compound-stabilized $\alpha$-synuclein oligomers $(500 \mathrm{nM})$ and the percentages of the increase of LTP compared.

For these experiments, the samples of compound-stabilized $\alpha$-synuclein oligomers were produced by incubation of $\alpha$-synuclein $(70 \mu \mathrm{M})$ with anle138c $(100 \mu \mathrm{M})$ or anle145c $(140 \mu \mathrm{M})$ in $50 \mathrm{mM}$ sodium phosphate, $100 \mathrm{mM} \mathrm{NaCl}$ buffer $(\mathrm{pH} \mathrm{7.4)}$ and 3\% DMSO for three days as described in 4.2.1.2. After preparation, the samples were kept on ice and the fEPSP recordings were conducted in the laboratory of Prof. Tiago Fleming Outeiro (University of Göttingen, Göttingen, Germany) over the next 8 hours.

\subsubsection{DPP compounds in in vivo models of Parkinson's disease}

\subsubsection{DPP compounds in Drosophila melanogaster model of Parkinson's disease.}


These experiments were performed by Dr. Aaron Voigt in the laboratory of Prof. Jörg Schulz (University of Göttingen, Göttingen, Germany). Transgenic flies expressing wild type $\alpha$-synuclein or A53T $\alpha$-synuclein mutant and control animals expressing lac Z were generated as described ${ }^{269,511}$.

Longevity Assay. Three DPP compounds, anle138b, anle138c and anle145c, were tested in the longevity assay. At 1-2 days post eclosion male flies expressing wild type $\alpha$ synuclein or A53T $\alpha$-synuclein mutant and control animals expressing lac $Z$ were transferred to vials at 20 flies per vial and maintained under a light-dark cycle (12 hours: 12 hours) at 25 ${ }^{\circ} \mathrm{C}$ with constant humidity. Each vial contained standard cornmeal food supplemented with the yeast containing DPP compound or DMSO. The yeast food supplement was prepared by mixing $24 \mathrm{~g}$ of yeast with $0.72 \mathrm{ml}$ of DMSO (control group) and $0.72 \mathrm{ml}$ of DMSO solution of DPP compound. Final concentrations of DPP compounds in the yeast food supplement were $250 \mu \mathrm{M}$ for anle138c and anle145c and $10 \mu \mathrm{M}$ for anle138b. Flies were transferred to fresh food on a weekly base, dead flies were counted twice per week. Survival curves were calculated and plotted using Kaplan-Meier statistics.

\subsubsection{DPP compounds in C. elegans model of Parkinson's disease.}

These experiments were performed by Prof. Stefan Eimer (ENI, Göttingen, Germany). Transgenic worms expressing A53T $\alpha$-synuclein mutant and control animals expressing egfp were generated and maintained as described ${ }^{269}$.

The test compounds were added into the nematode growth medium at final concentration of $50 \mu \mathrm{M}$ using $1 \%$ DMSO. The transgenic animals were imaged or assayed four days after reaching adulthood as described ${ }^{269}$. The images were scored for defects in neurite morphology of dopaminergic neurons.

4.2.5.3. Anle138b efficacy study in the oral rotenone in vivo mouse model of Parkinson's disease

Anle138b efficacy study in the oral rotenone in vivo mouse model was conducted by Dr. Francisco Pan-Montojo (TU-Dresden, Dresden, Germany) as described ${ }^{10}$.

4.2.5.4. Anle138b efficacy study in the MPTP in vivo mouse model of Parkinson's disease

Anle138b efficacy study in the MPTP in vivo mouse model was conducted in the laboratory of Prof. Jochen H. Weishaupt (University of Göttingen, Göttingen, Germany) as described $^{10}$.

4.2.5.5. Anle138b efficacy study in the transgenic A30P $\alpha$-synuclein in vivo mouse model of Parkinson's disease

Anle138b efficacy study in the transgenic A30P $\alpha$-synuclein in vivo mouse model was conducted in the laboratory of Prof. Armin Giese (LMU, München, Germany) as described ${ }^{10}$.

4.2.5.6. Anle138b efficacy study in the transgenic 1-120 $\alpha$-synuclein in vivo mouse model of Parkinson's disease 
Anle138b efficacy study in the transgenic 1-120 $\alpha$-synuclein in vivo mouse model was conducted in the laboratory of Prof. Maria Grazia Spillantini (University of Cambridge, Cambridge, UK) as described ${ }^{473}$.

dSTROM super-resolution microscopy measurements of the brain slices from transgenic 1-120 $\alpha$-synuclein mouse model were performed in the laboratory of Prof. Uri Ashery (Tel Aviv University, Tel Aviv, Israel) as described ${ }^{473}$.

\subsubsection{Pharmacokinetic studies with DPP compounds and prodrugs}

\subsubsection{Tissue homogenization}

Protocol 1: The tissues were defrosted at $4 \mathrm{C}$ prior to use. The tissue sample were transferred into dispersion Tube DT-20 (IKA-Werke GmbH, Staufen, Germany), weighed and homogenized twice in $5 \mathrm{ml}$ of acetonitrile at maximum speed for 3 minutes using a homogenizer IKA ULTRA-TURRAX Tube drive workstation (IKA-Werke GmbH, Staufen, Germany) at room temperature. After transferring into $15 \mathrm{ml}$ falcon tube, the homogenate was sonicated (Elmasonic S60, Elma Schmidbauer GmbH, Singen, Germany) at $30 \mathrm{C}$ for 5 minutes and centrifuged at 5000g (Eppendorf centrifuge 5804, Eppendorf, Germany) for 10 minutes. An aliquot $(100 \mathrm{ul})$ of supernatant was injected into HPLC system.

Protocol 2: The tissues were defrosted at $4 \mathrm{C}$ prior to use. The small tissue samples (tissue weight $<200 \mathrm{mg}$ ) were transferred into Precellys CKMix 2-mL tissue homogenizing tubes (Bertin Technologies SAS, Montigny-le-Bretonneux, France); the big tissue samples (tissue weight $>200 \mathrm{mg}$ ) were transferred into Precellys CKMix 7-mL tissue homogenizing tubes. After determination of the tissue weight, it was homogenized in $0.5-1.00 \mathrm{~mL}(2 \mathrm{ml}$ tubes) or $3.0-5.0 \mathrm{~mL}(7 \mathrm{ml}$ tubes $)$ of acetonitrile using Precellys Evolution Super homogenizer (Bertin Technologies SAS, Montigny-le-Bretonneux, France) at room temperature. Homogenization $(6,800 \times \mathrm{g}$, eight cycles for $30 \mathrm{~s}$ with a 30 -s pause between cycles) was repeated four times. Thereafter, the homogenate was sonicated (Elmasonic S60, Elma Schmidbauer GmbH, Singen, Germany) at $25 \mathrm{C}$ for 5 minutes and centrifuged at 8000 rpm for 2 minutes (Eppendorf centrifuge 5418, rotor FA-45-18-11). An aliquot (100 ul) of supernatant was injected into HPLC system.

Protocol 3: The serum and plasma samples were diluted with 2 volumes of acetonitrile, mixed in a vortex mixer to 5-10 minutes at room temperature. Thereafter, the homogenate was sonicated (Elmasonic S60, Elma Schmidbauer GmbH, Singen, Germany) at $25 \mathrm{C}$ for 10 minutes and centrifuged at $8000 \mathrm{rpm}$ for 2 minutes (Eppendorf centrifuge 5418, rotor FA-45-18-11). An aliquot (100 ul) of supernatant was injected into HPLC system.

\subsubsection{HPLC analysis of tissue homogenates}

An aliquot (100 ul) of the tissue homogenate was injected into an HPLC system. Analytical high performance liquid chromatography (HPLC) was performed using a Waters HPLC system with a Waters 996 Photodiode Array Detector. All separations involved a mobile phase of $0.1 \%$ trifluoroacetic acid (TFA) (v/v) in water (solvent A) and $0.1 \%$ TFA in acetonitrile (solvent B). HPLC was performed using reversed-phase (RP) column Eurospher RP $18,100 \AA, 5 \mu \mathrm{m}, 250 \times 4.6 \mathrm{~mm}$ at flow rates of $1 \mathrm{~mL} / \mathrm{min}$ with a gradient of solvent $\mathrm{B}$ from $0 \%$ to $100 \%$ in 50 minutes. The effluent was monitored for UV absorption at $260 \mathrm{~nm}$. Samples were quantified using peak area ratio of compounds to external standard. 


\subsubsection{Pharmacokinetic study of DPP compounds in mice}

The animal part of this PK experiments was performed by Dr. Jens Wagner in laboratory of Prof. Armin Giese (LMU, München, Germany). The tissue samples were sent to me (Max Planck Institute for biophysical chemistry) on dry ice for homogenization and HPLC analysis.

Study was performed in 8-10 weeks old female C57/BL6 mice ( $\mathrm{n}=2 /$ compound, weight range 19-21 g). DPP compound was administered orally at a single dose of $50 \mathrm{mg} / \mathrm{kg}$ in $\mathrm{DMSO} /$ peanut butter mixture. For preparation of a single dose, $1 \mathrm{mg}$ of DPP compound was dissolved in $10 \mu \mathrm{l}$ of DSMO solution and then mixed with $200 \mu \mathrm{l}$ peanut butter. This formulation was provided to single housed animal that ate it voluntarily in 5-10 minutes. 4 hours after dose administration the brains were collected from the euthanized animals, flash frozen in liquid nitrogen and stored at $-80{ }^{\circ} \mathrm{C}$. The tissue homogenates were prepared according to protocol 1 (see 4.2.6.1).

\subsubsection{Pharmacokinetic study of anle138b/olive oil in mice}

The animal part of this PK experiments was performed by Dr. Tobias Frank in laboratory of Prof. Jochen H. Weishaupt (University of Göttingen, Göttingen, Germany). The tissue samples were sent to me (Max Planck Institute for biophysical chemistry) on dry ice for homogenization and HPLC analysis.

Study was performed in 8-10 weeks old female C57/BL6 mice ( $\mathrm{n}=2$ /time point, weight range 19-21 g). Anle138b was administered orally via gavage at a single dose of 250 $\mathrm{mg} / \mathrm{kg}$ in olive oil (Aromaland, Röttingen, Germany) with 2.5\% DMSO. For preparation of a single dose, $5 \mathrm{mg}$ of DPP compound was dissolved in $12.5 \mu \mathrm{l}$ DMSO mixed with $475 \mu \mathrm{l}$ of olive oil. 2 mice per time point were sacrificed at 6,12 and 24 hours post dosing. The tissue samples (brain, liver, kidney) were collected from the euthanized animals, flash frozen in liquid nitrogen and stored at $-80{ }^{\circ} \mathrm{C}$. The tissue homogenates were prepared according to protocol 1 (see 4.2.6.1).

\subsubsection{Pharmacokinetic study of anle138b-containing diet in mice}

The animal part of this PK experiments was performed by Dr. Betina Göricke in laboratory of Prof. Jochen H. Weishaupt (University of Göttingen, Göttingen, Germany). The tissue samples were sent to me (Max Planck Institute for biophysical chemistry) on dry ice for homogenization and HPLC analysis.

Mice (B6SJLF1, Jackson Laboratory) were kept in groups of 4 animals in each cage in an inversed day/night-cycle for 2 weeks before starting and during the experiment (light 18:00 to 6:00, dark 6:00 to 18:00) with free access to food and water. Age ranged from $78 \mathrm{~d}$ to $96 \mathrm{~d}$. The normal food was exchanged to anle138b-containing food $(2 \mathrm{~g}$ anle138b/kg food; Ssniff, Soest, Germany) three days before killing animals to get a steady state. Animals were killed by cervical dislocation during their active phase on the fourth day at time points 12, 14, 16 and 20 hours. Brains were taken out and immediately frozen in liquid nitrogen. Samples were stored at $-80{ }^{\circ} \mathrm{C}$. The average daily food consumption was $5 \mathrm{~g}$ per animal, which translates to daily anle $138 \mathrm{~b}$ dose of approximately $500 \mathrm{mg} / \mathrm{kg}$. The tissue homogenates were prepared according to protocol 1 (see 4.2.6.1). 


\subsubsection{Pharmacokinetic study of anle138b/olive oil in rats}

The animal part of this PK experiments was performed by Dr. Sebastian Kügler (University of Göttingen, Göttingen, Germany). The tissue samples were sent to me (Max Planck Institute for biophysical chemistry) on dry ice for homogenization and HPLC analysis.

Study was performed in $8-10$ weeks old female Wistar rats $(n=1 /$ time point, weight range $250-290 \mathrm{~g}$ ). Anle $138 \mathrm{~b}$ was administered orally via gavage at a single dose of $250 \mathrm{mg} / \mathrm{kg}$ in olive oil (Aromaland, Röttingen, Germany) with 2.5\% DMSO. For preparation of a single dose, $62 \mathrm{mg}$ of anle $138 \mathrm{~b}$ was dissolved in a mixture of $750 \mu \mathrm{l}$ olive oil and $25 \mu \mathrm{l}$ of DMSO by heating to 70-90 $\quad$ C. Before administration the solution was cooled to 35-40 $\quad$ C. 1 rat per time point was sacrificed at 4,8 and 12 hours post dosing. The tissue samples (brain, liver, kidney, spleen, fat, lung, thymus) and blood samples were collected from the euthanized animals. Serum samples were prepared by centrifugation of the clotted blood samples. The tissue samples and serum were flash frozen in liquid nitrogen and stored at $-80{ }^{\circ} \mathrm{C}$. The tissue homogenates were prepared according to protocol 1 (see 4.2.6.1) and the serum samples were processed according to protocol 3 (see 4.2.6.1).

\subsubsection{Comparative pharmacokinetic study of anle138b in rats}

The animal part of this PK experiments was performed by Dr. Ulrike Teichmann (Animal facility, Max Planck Institute for biophysical chemistry, Göttingen). Dr. Ulrike Teichmann provided to me the blood samples. The solutions for the oral formulation V25, V32, V34 and for i.v. formulation were provided by company Aeterna Zentaris (Frankfurt am Main, Germany)

30 male wistar rats at 226-250 g body weight were ordered from a commercial supplier and kept under standard conventional housing conditions at $55 \%$ humidity, $21{ }^{\circ} \mathrm{C}$ room temperature and with ad libitum access to food and water until the start of the experiment. Anle138b was dissolved in the formulation solutions at $50{ }^{\circ} \mathrm{C}$ with gentle stirring during 1 hour. The animals were divided into five experimental groups of six rats each for the different routes of applications and formulations: i.v. application at a single anle138b dose of $2 \mathrm{mg} / \mathrm{kg}$, oral formulations $\mathrm{V} 34, \mathrm{~V} 32, \mathrm{~V} 25$ and olive oil with $2.5 \%$ DMSO at a single anle $138 \mathrm{~b}$ dose of $10 \mathrm{mg} / \mathrm{kg}$. The volume of all formulation was $2 \mathrm{ml} / \mathrm{kg}$. The exact dose for each animal was calculated according to the actual body weight that varied between 280 and $307 \mathrm{~g}$ at the day of the experiment. All rats were starved for one hour with free access to water for one hour before the application. Intravenous application was performed by injecting into the tail vein. Oral application was performed by gavaging using a blunt bended steel cannula. A volume of $350 \mu \mathrm{l}$ EDTA blood was withdrawn at the designated time points. The sampling was carried out under anesthesia with isoflurane (4\% for induction, $2 \%$ for maintenance) in oxygen via a nose mask. Each experimental group was divided into two subgroups for EDTA-blood sampling at $15 \mathrm{~min}, 60 \mathrm{~min}, 4$ hours and 24 hours or $30 \mathrm{~min}, 120$ min, and 8 hours after application respectively. From the latter subgroup, a blood sample before the application was taken to determine the baseline zero value.

To prepare the plasma samples, $350 \mu 1$ EDTA blood were centrifuged at $3000 \mathrm{rpm}$ for 5 minutes (Eppendorf centrifuge 5418, rotor FA-45-18-11). The collected plasma (150 $\mu \mathrm{l})$ samples were flash frozen in liquid nitrogen and stored at $-80{ }^{\circ} \mathrm{C}$. For HPLC analysis the plasma samples were processed according to protocol 3 (see 4.2.6.1). 


\subsubsection{Pharmacokinetic study of prodrugs in mice}

The animal part of this PK experiments was performed by Dr. Felix Schmidt in laboratory of Prof. Armin Giese (LMU, München, Germany). The tissue and plasma samples were sent to me (Max Planck Institute for biophysical chemistry) on dry ice for homogenization and HPLC analysis.

Study was performed in 11 weeks old female C57/BL6 mice $(n=2 /$ time point, weight range $18-20$ g). Sery433 or sery 453 were administered orally via gavage at a single dose of 70 $\mathrm{mg} / \mathrm{kg}$ in Milipore- $\mathrm{H}_{2} \mathrm{O}$. For preparation of a single dose, $1.45 \mathrm{mg}$ of sery 433 or sery 453 was dissolved in $50 \mu \mathrm{l}$ Milipore- $\mathrm{H}_{2} \mathrm{O} .2$ mice per time point were sacrificed at 1, 2, 4 and 8 hours post dosing. The brain and blood samples were collected from the euthanized animals. Serum samples were prepared by centrifugation of the clotted blood samples. The tissue samples and serum were flash frozen in liquid nitrogen and stored at $-80^{\circ} \mathrm{C}$. The tissue homogenates were prepared according to protocol 1 (see 4.2.6.1) and the serum samples were processed according to protocol 3 (see 4.2.6.1).

\subsubsection{Pharmacokinetic study of sery433 in rats}

The animal part of this PK experiments was performed by Dr. Sebastian Kügler (University of Göttingen, Göttingen, Germany). The tissue samples were sent to me (Max Planck Institute for biophysical chemistry) on dry ice for homogenization and HPLC analysis.

Study was performed in 8-10 weeks old female Wistar rats $(\mathrm{n}=2$ /time point, weight range $250-280 \mathrm{~g}$ ). Sery433 was administered orally via gavage at a single dose of $180 \mathrm{mg} / \mathrm{kg}$ in Milipore- $\mathrm{H}_{2} \mathrm{O}$. For preparation of a single dose, $7.25 \mathrm{mg}$ of sery433 was dissolved in 130 $\mu 1$ Milipore- $\mathrm{H}_{2} \mathrm{O} .2$ rat per time point was sacrificed at 2 and 4 hours post dosing. The brain and blood samples were collected from the euthanized animals. Serum samples were prepared by centrifugation of the clotted blood samples. The tissue samples and serum were flash frozen in liquid nitrogen and stored at $-80{ }^{\circ} \mathrm{C}$. The tissue homogenates were prepared according to protocol 1 (see 4.2.6.1) and the serum samples were processed according to protocol 3 (see 4.2.6.1).

\subsubsection{Comparative pharmacokinetic study of sery 433 in rats}

The corresponding studies were conducted by the company Aptuit, Italy

Briefly, anle138b was orally applied in excipient V34 at $10 \mathrm{mg} / \mathrm{kg}$ dose to three rats. During 24 hours after dosing, the blood samples were collected and later analysed. After one week of washout period, sery 433 in the aqueous solution was formulated orally at $10 \mathrm{mg} / \mathrm{kg}$ dose to the same animals. The advantage of this protocol is a low intraindividual variability due to the similar metabolism rate of a given animal in both experiments.

\subsubsection{Additional pharmacokinetic study of anle138b in mice}

Additional pharmacokinetic studies of anle138b formulated i.v. in DMSO or orally at different dose in the peanut butter and in the vegetable oil were performed by in the laboratories of Prof. Armin Giese (LMU, München, Germany) and Prof. Manfred Uhr (Max Planck Institute of Psychiatry, Munich, Germany) as described ${ }^{10}$. 


\subsubsection{Experiments with the prion protein}

\subsubsection{Inhibition of $P r P^{S c}$ accumulation in a cell based dot blot assay}

The DPP compound testing in SMB prion prion-infected cell was performed in the laboratory of Prof. Martin Groschup (FLI, Greifswald-Insel Riems, Germany) as described ${ }^{10}$.

\subsubsection{Inhibition of $P r P^{S c}$ amplification in PMCA assay}

The PMCA and screening of DPP compound in PMCA were performed in the laboratory of Prof. Armin Giese (LMU, München, Germany) as described ${ }^{10}$.

\subsubsection{Continuous sucrose-gradient assay}

Continuous sucrose-gradient assay was performed in the laboratory of Prof. Armin Giese (LMU, München, Germany) as described ${ }^{10}$.

4.2.7.4. Efficacy studies with anle138b and other DPP compounds in the RML prioninfected mouse model

The efficacy studies with anle138b and other DPP compounds in the RML prioninfected mouse model were conducted in the laboratory of Prof. Armin Giese (LMU, München, Germany) as described ${ }^{10}$.

\subsubsection{Solubility and stability tests of prodrugs}

\subsubsection{Water solubility test}

The solubility of compounds sery433 and sery453 in the water was evaluated by following protocol. Deionized water $(1 \mathrm{ml})$ was added into glass tube containing $10 \mathrm{mg}$ of compound. After mixing with vortex for 5 minutes the sample was visually inspected and formation of solution or suspension was documented.

\subsubsection{Stability test}

The stability of compounds sery433 and sery453 in the aqueous solution was determined by NMR. The sample containing a solution of compound $(5 \mathrm{mg})$ in $\mathrm{D}_{2} \mathrm{O}(0.5 \mathrm{ml})$ was incubated at room temperature for 1 day; ${ }^{1} \mathrm{H}$ NMR spectra were recorded at $0,12,24$ hour after sample preparation. The analysis of stability was done by overlying of ${ }^{1} \mathrm{H} 1 \mathrm{D}$ spectra. 


\subsection{Materials and Methods Chemistry}

\subsubsection{Materials and Methods}

All starting materials and solvents were purchased from ABCR, Acros, Alfa Aesar, Fluorochem, Sigma-Aldrich or Merck and used as such unless noted otherwise.

Melting points were determined on a Stuart Scientific (BIBBY, UK) melting point apparatus using open glass capillaries and are uncorrected.

Thin layer chromatography (TLC): Macherey-Nagel precoated sheets, $0.25 \mathrm{~mm}$ ALUGRAM ${ }^{\circledR}$ SIL G/UV254 plates, detection with UV and/or by charring with $10 \mathrm{wt} \%$ ethanolic phosphomolybdic acid reagent followed by heating at $200{ }^{\circ} \mathrm{C}$.

Flash column chromatography was performed by using Merck silica gel 60 (0.063$0.100 \mathrm{~mm})$.

Analytical and preparative high performance liquid chromatography (HPLC) were performed by using a Waters HPLC system with a Waters 996 Photodiode Array Detector. All separations involved a mobile phase of $0.1 \%$ trifluoroacetic acid (TFA) $(\mathrm{v} / \mathrm{v})$ in water (solvent A) and $0.1 \%$ TFA in acetonitrile (solvent B). HPLC was performed by using a reversed-phase (RP) column Eurospher RP 18, $100 \AA$, $5 \mu \mathrm{m}, 250 \times 4.6 \mathrm{~mm}$ (analytical) and $250 \times 16 \mathrm{~mm}$ (preparative) at flow rates of $1 \mathrm{~mL} \cdot \mathrm{min}^{-1}$ (analytical) and $7 \mathrm{~mL} \cdot \mathrm{min}^{-1}$ (preparative).

Electrospray ionization mass spectrometry (ESI-MS) and liquid chromatography/ mass spectrometry (LC-MS) analyses were obtained by using a Waters Micromass ZQ 4000 mass spectrometer in conjunction with the Waters HPLC apparatus described above. Highresolution mass spectra (HRMS) were recorded by using an Thermo Scientific LTQ Orbitrap XL hybrid FTMS mass spectrometer and are reported in $\mathrm{m} / \mathrm{z}$.

NMR spectra were recorded at a temperature of $298 \mathrm{~K}$ unless noted otherwise by using a $400 \mathrm{MHz}$ Bruker Avance spectrometer (Bruker AG, Rheinstetten, Germany) equipped with a TXI HCN z-gradient probe. All spectra were processed by using TOPSPIN2 (Bruker AG, Karlsruhe, Germany). ${ }^{1} \mathrm{H}$ NMR chemical shifts $(\delta)$ are reported in parts per million (ppm) relative to $\mathrm{CDCl}_{3}$ and $\mathrm{DMSO}\left[\mathrm{D}_{6}\right]$ as internal standards. Data are reported as follows: chemical shift, multiplicity $(\mathrm{s}=$ singlet, $\mathrm{d}=$ doublet, $\mathrm{t}=$ triplet, $\mathrm{dd}=$ doublet of doublets, $\mathrm{ddd}$ $=$ doublet of doublets of doublets, $\mathrm{dt}=$ doublet of triplet, $\mathrm{td}=$ triplet of doublets, $\mathrm{q}=$ quartet, bs $=$ broadened singlet, $\mathrm{m}=$ multiplet $)$, coupling constants $(J$, given in $\mathrm{Hz})$, integration. ${ }^{13} \mathrm{C}$ NMR chemical shifts $(\delta)$ are reported in parts per million (ppm) relative to $\mathrm{CDCl}_{3}$ and $\mathrm{DMSO}\left[\mathrm{D}_{6}\right]$ as internal standards. The following experiments were used to record the resonances of the compounds: ${ }^{1} \mathrm{H}-1 \mathrm{D},{ }^{13} \mathrm{C}-1 \mathrm{D}$ NMR spectra and ${ }^{13} \mathrm{C}-\mathrm{APT}$ (attached proton test with a single J-evolution time of $1 / 145 \mathrm{~s}$, spectra are processed such that quaternary and methylene groups have positive sign and methyl and methine groups negative sign). To resolve overlap of resonances and recover undetectable resonances in ${ }^{1} \mathrm{H}$ and APT spectra, 2D- $\left[{ }^{13} \mathrm{C},{ }^{1} \mathrm{H}\right]$-HSQC (heteronuclear single quantum coherence), 2D- $\left[{ }^{13} \mathrm{C},{ }^{1} \mathrm{H}\right]-\mathrm{HMBC}$ (heteronuclear multiple bond correlation) and 2D-NOESY were recorded for some compounds. 


\subsubsection{Experimental Procedures and characterisation data of compounds}

The synthesis, isolation and characterization of all compounds were done in the chemistry lab of department of NMR-based structural biology, Max Planck Institute. Compounds named sery were synthesized by Sergey Ryazanov, compounds named anle were synthesized by Dr. Andrei Leonov, compounds named ruli were synthesized by Ruth Linder,

\subsubsection{Synthesis of pyrazoles}

\section{General Experimental Procedure for the synthesis of 1,3-diarylpropane-1,3-diones (GEP} 1) ${ }^{393}$

\section{1,3-Bis(3,4-dimethoxyphenyl)propane-1,3-dione (sery148)}

This compound has been reported previously ${ }^{512}$ and is commercially available (CAS 52751$63-0)$. A $60 \%$ suspension of sodium hydride in mineral oil $(0.4 \mathrm{~g}, 10 \mathrm{mmol})$ was washed with petroleum benzine $(20 \mathrm{ml})$ two times, anhydrous DMSO $(10 \mathrm{ml})$ was added. After being stirred for $30 \mathrm{~min}$ at room temperature under argon, THF $(5 \mathrm{ml})$ was added, the flask was cooled down to $15^{\circ} \mathrm{C}$ and ethyl 3,4-dimethoxybenzoate $(2.1 \mathrm{~g}, 10 \mathrm{mmol})$ was added. The temperature was allowed to drop to $10^{\circ} \mathrm{C}$ and a solution of 3,4-dimethoxyacetophenone (1.08 $\mathrm{g}, 6 \mathrm{mmol})$ in DMSO $(4 \mathrm{ml})$ was added at such a rate that the temperature didn't rise above 15 ${ }^{\circ} \mathrm{C}$. Upon completion of addition the reaction mixture was stirred $15 \mathrm{~h}$ at room temperature, then poured slowly into crushed ice $(250 \mathrm{~g})$ containing $85 \%$ phosphoric acid $(1 \mathrm{ml})$. Resulting precipitate was collected by filtration, washed with water $(50 \mathrm{ml})$ and dried to provide sery148 $(2.1 \mathrm{~g}, 99 \%)$ as a yellow powder. m.p.: $128-130{ }^{\circ} \mathrm{C}\left(\operatorname{lit}^{512} 100.5-101{ }^{\circ} \mathrm{C}\right) .{ }^{1} \mathrm{H}$ NMR (400 MHz, DMSO[D 6$]) \delta=7.85(\mathrm{dd}, J=8.5,1.9 \mathrm{~Hz}, 2 \mathrm{H}), 7.61(\mathrm{~d}, J=1.9 \mathrm{~Hz}, 2 \mathrm{H})$, $7.21(\mathrm{~s}, 1 \mathrm{H}), 7.11(\mathrm{~d}, J=8.5 \mathrm{~Hz}, 2 \mathrm{H}), 3.88(\mathrm{~m}, 12 \mathrm{H})$ (in ${ }^{1} \mathrm{H}$ NMR spectrum $15 \%$ of diketone form is present). ${ }^{13} \mathrm{C}$ NMR (100.6 MHz, DMSO[D 6 ]) $\delta=184.2,152.9,148.7,127.2,121.7$, $111.2,110.0,91.8,55.7(2 \mathrm{C})$.

\section{General Experimental Procedure for the synthesis of 3,5-diaryl-1 $\mathrm{H}$-pyrazoles (GEP 2) ${ }^{394}$}

\section{1,3-Bis(3,4-dimethoxyphenyl)-1 $H$-pyrazole (sery149)}

This compound is commercially available (CAS 1159937-09-3). A solution of sery148 (1.0 g, $2.9 \mathrm{mmol})$ and hydrazine hydrate $(218 \mathrm{mg}, 4.4 \mathrm{mmol})$ in ethanol $(15 \mathrm{ml})$ was heated under reflux $3 \mathrm{~h}$ with stirring. The clear yellow solution was evaporated under reduced pressure, water was added and resulting precipitate was collected by filtration, washed with water and dried to provide sery149 $(960 \mathrm{mg}, 97 \%)$ as a beige powder. m.p.: $165-167{ }^{\circ} \mathrm{C} .{ }^{1} \mathrm{H}$ NMR (400 $\left.\mathrm{MHz}, \mathrm{DMSO}\left[\mathrm{D}_{6}\right]\right) \delta=13.07(\mathrm{~s}, 1 \mathrm{H}), 7.41(\mathrm{~s}, 2 \mathrm{H}), 7.36(\mathrm{~d}, J=8.2 \mathrm{~Hz}, 2 \mathrm{H}), 7.06(\mathrm{~s}, 1 \mathrm{H}), 7.01$ $(\mathrm{d}, J=8.2 \mathrm{~Hz}, 2 \mathrm{H}), 3.84(\mathrm{~s}, 6 \mathrm{H}), 3.79(\mathrm{~s}, 6 \mathrm{H}) .{ }^{13} \mathrm{C} \mathrm{NMR}\left(100.6 \mathrm{MHz}, \mathrm{DMSO}\left[\mathrm{D}_{6}\right]\right) \delta=148.9$, 148.6, 143.6, 122.1, 117.6, 112.1, 108.9, 98.6, $55.5(2 \mathrm{C})$. HRMS $(m / z)[\mathrm{M}+\mathrm{H}]^{+}$calcd for $\mathrm{M}=$ $\mathrm{C}_{19} \mathrm{H}_{20} \mathrm{~N}_{2} \mathrm{O}_{4}, 341.1501$; found, 341.1505.

\section{General Experimental Procedure for the $O$-deprotection of phenyl alkyl ethers (GEP $3)^{513}$}

\section{3,5-Bis(3,4-dihydroxyphenyl)-1 $H$-pyrazole hydrobromide (sery159a)}

This compound has been reported previously ${ }^{514}$ (CAS 1147315-28-3). A solution of sery149 (120 mg, $0.35 \mathrm{mmol})$ in dichloromethane $(5 \mathrm{ml})$ was cooled down to $-78{ }^{\circ} \mathrm{C}$, treated with boron tribromide $(0.34 \mathrm{ml}, 3.5 \mathrm{mmol})$, stirred at $-78{ }^{\circ} \mathrm{C}$ for $3 \mathrm{~h}$ and then overnight at room 
temperature. The mixture was cooled down to $-78^{\circ} \mathrm{C}$ and quenched with methanol $(5 \mathrm{ml})$. After stirring for $3 \mathrm{~h}$ at room temperature solvents were evaporated under reduced pressure, the residue was co-evaporated four times with methanol $(10 \mathrm{ml})$. The resulting precipitate was reflux in $5 \mathrm{ml}$ chloroform, after cooling the product was collected by filtration and dried to provide sery159a (108 mg, $85 \%)$ as a yellow powder. m.p.: $285-290{ }^{\circ} \mathrm{C}(\mathrm{dec}) .{ }^{1} \mathrm{H}$ NMR $(400$ MHz, DMSO[D 6$]) \delta=7.19(\mathrm{~d}, J=1.9 \mathrm{~Hz}, 2 \mathrm{H}), 7.08(\mathrm{dd}, J=8.2,1.9 \mathrm{~Hz}, 2 \mathrm{H}), 6.80(\mathrm{~d}, J=$ $8.2 \mathrm{~Hz}, 2 \mathrm{H}), 6.74(\mathrm{~s}, 1 \mathrm{H}) .{ }^{13} \mathrm{C} \mathrm{NMR}\left(100.6 \mathrm{MHz}, \mathrm{DMSO}\left[\mathrm{D}_{6}\right]\right) \delta=147.6,147.0,145.7,120.0$, $117.9,115.9,113.6,99.1$.

\section{General Experimental Procedure for the $\boldsymbol{O}$-deprotection of phenyl alkyl ethers with $\mathrm{AlBr}_{3}(\text { GEP 4) })^{515}$}

\section{3-(3-Bromophenyl)-5-(2-hydroxyphenyl)-1H-pyrazole (sery260a)}

To a solution of $\mathrm{AlBr}_{3}(4.00 \mathrm{~g}, 15 \mathrm{mmol})$ in ethanethiol $(8 \mathrm{ml})$ sery256b $(670 \mathrm{mg}, 2.04 \mathrm{mmol})$ was added in one portion. After stirring for $12 \mathrm{~h}$ at room temperature the reaction mixture was slowly poured in $200 \mathrm{ml}$ of $0.1 \mathrm{~N} \mathrm{HCl}$, extracted with ethyl acetate $(2 \times 50 \mathrm{ml})$, the combined extracts were washed with brine $(15 \mathrm{ml})$, dried $\left(\mathrm{Na}_{2} \mathrm{SO}_{4}\right)$ and evaporated. The resulting precipitate was purified by recrystallisation from petroleum benzine $\left(40-60{ }^{\circ} \mathrm{C}\right) /$ chloroform to provide sery260a as a white solid. Yield 97\%. m.p.: 147-149 ${ }^{\circ} \mathrm{C} .{ }^{1} \mathrm{H}$ NMR $(400 \mathrm{MHz}$, DMSO- $_{6}+1-2 \%$ conc. DCl) $\delta=10.50(\mathrm{~s}, 1 \mathrm{H}), 8.06(\mathrm{~s}, 1 \mathrm{H}), 7.85(\mathrm{~d}, J=7.8 \mathrm{~Hz}, 1 \mathrm{H}), 7.73$ $(\mathrm{d}, J=7.2 \mathrm{~Hz}, 1 \mathrm{H}), 7.54(\mathrm{~d}, J=7.2 \mathrm{~Hz}, 1 \mathrm{H}), 7.42(\mathrm{t}, J=7.8 \mathrm{~Hz}, 1 \mathrm{H}), 7.31(\mathrm{~s}, 1 \mathrm{H}), 7.20(\mathrm{t}, J=$ $7.8 \mathrm{~Hz}, 1 \mathrm{H}), 6.97(\mathrm{~d}, J=8.0 \mathrm{~Hz}, 1 \mathrm{H}), 6.91(\mathrm{t}, J=7.8 \mathrm{~Hz}, 1 \mathrm{H}) .{ }^{13} \mathrm{C}$ NMR $(100.6 \mathrm{MHz}$, DMSO-d $_{6}+1-2 \%$ conc. DCl) $\delta=154.7,147.0-145.0$ (2C), 134.0, 131.1, 130.8, 130.7, 129.3, $127.8,127.3,124.3,122.4,119.4,116.6,101.3$. HRMS $(m / z)[\mathrm{M}+\mathrm{H}]^{+}$calcd for $\mathrm{M}=$ $\mathrm{C}_{15} \mathrm{H}_{11}{ }^{79} \mathrm{BrN}_{2} \mathrm{O}, 315.0133$; found, 315.0138 .

General Experimental Procedure for the synthesis of 1,3-diaryl-2-propene-1-ones (GEP $5)^{516}$

\section{(E)-3-(3-Chlorophenyl)-1-(3-nitrophenyl)-2-propene-1-one (sery273a)}

A solution of 1-(3-nitrophenyl)ethanone (1.8 g, $10 \mathrm{mmol}), 3$-chlorobenzaldehyde $(1.4 \mathrm{~g}, 10$ $\mathrm{mmol}), \mathrm{NaOH}(50 \mathrm{mg}, 1.25 \mathrm{mmol})$ and $\mathrm{Ba}(\mathrm{OH})_{2} \bullet 8 \mathrm{H}_{2} \mathrm{O}(100 \mathrm{mg}, 0.32 \mathrm{mmol})$ in methanol (10 $\mathrm{ml}$ ) was stirred at room temperature for $24 \mathrm{~h}$. The reaction mixture was cooled to $+4{ }^{\circ} \mathrm{C}$, a resulting precipitate was collected by filtration and purified by recrystallisation from ethanol/EtOAc to provide sery273a $(2.22 \mathrm{~g})$ as a yellow powder. Yield $77 \%$. m.p.: 164-165 ${ }^{\circ} \mathrm{C} . \quad{ }^{1} \mathrm{H}$ NMR $\left(400 \mathrm{MHz}, \mathrm{DMSO}-\mathrm{d}_{6}\right) \delta=8.84(\mathrm{t}, J=1.9 \mathrm{~Hz}, 1 \mathrm{H}), 8.62(\mathrm{dt}, J=7.9,1.4 \mathrm{~Hz}$, $1 \mathrm{H}), 8.50$ (ddd, $J=8.1,2.3,0.9 \mathrm{~Hz}, 1 \mathrm{H}), 8.15-8.07(\mathrm{~m}, 2 \mathrm{H}), 7.91-7.84(\mathrm{~m}, 2 \mathrm{H}), 7.80(\mathrm{~d}, J=$ $15.6 \mathrm{~Hz}, 1 \mathrm{H}), 7.53-7.46(\mathrm{~m}, 2 \mathrm{H}) .{ }^{13} \mathrm{C}$ NMR $\left(100.6 \mathrm{MHz}, \mathrm{DMSO}-\mathrm{d}_{6}\right) \delta=187.4,148.2,143.8$, $138.5,136.7,134.8,133.8,130.6,130.4,128.3,128.2,128.1,127.5,123.0,122.9$. HRMS $(\mathrm{m} / z)[\mathrm{M}+\mathrm{H}]^{+}$calcd for $\mathrm{M}=\mathrm{C}_{15} \mathrm{H}_{10} \mathrm{ClNO}_{3}, 288.0427$; found, 288.0422.

\section{General Experimental Procedure for the synthesis of 1,3-diaryl-2,3-dibromopropane-1-} ones (GEP 6) ${ }^{397}$

\section{3-(3-Chlorophenyl)-2,3-dibromo-1-(3-nitrophenyl)propane-1-one (sery273b)}

To a stirred solution of sery273a $(1000 \mathrm{mg}, 3.5 \mathrm{mmol})$ in chloroform $(25 \mathrm{ml})$ was added dropwise a solution of bromine $(560 \mathrm{mg}, 3.5 \mathrm{mmol})$ in chloroform $(5 \mathrm{ml})$ at $0^{\circ} \mathrm{C}$. After being stirred for $2 \mathrm{~h}$ at $0^{\circ} \mathrm{C}$ the reaction mixture was diluted with petroleum benzine $(50 \mathrm{ml})$ and the 
mixture was refrigerated $\left(-24^{\circ} \mathrm{C}\right)$ for 10 hours, a resulting precipitate was collected by filtration, washed with $n$-hexane $(10 \mathrm{ml})$ and dried to provide sery273b $(1275 \mathrm{mg})$ as a white powder. Yield 82\%. m.p.: $167-170{ }^{\circ} \mathrm{C} .{ }^{1} \mathrm{H}$ NMR $\left(400 \mathrm{MHz}, \mathrm{DMSO}-\mathrm{d}_{6}\right) \delta=9.00(\mathrm{t}, J=2.0$ $\mathrm{Hz}, 1 \mathrm{H}), 8.72(\mathrm{~d}, J=8.0 \mathrm{~Hz}, 1 \mathrm{H}), 8.60(\mathrm{dd}, J=8.1,2.2 \mathrm{~Hz}, 1 \mathrm{H}), 8.05(\mathrm{~s}, 1 \mathrm{H}), 7.97(\mathrm{t}, J=8.0$ $\mathrm{Hz}, 1 \mathrm{H}), 7.82(\mathrm{dt}, J=6.7,2.1 \mathrm{~Hz}, 1 \mathrm{H}), 7.51-7.45(\mathrm{~m}, 2 \mathrm{H}), 6.91(\mathrm{~d}, J=11.2 \mathrm{~Hz}, 1 \mathrm{H}), 5.88(\mathrm{~d}$, $J=11.2 \mathrm{~Hz}, 1 \mathrm{H}) .{ }^{13} \mathrm{C}$ NMR $\left(100.6 \mathrm{MHz}, \mathrm{DMSO}-\mathrm{d}_{6}\right) \delta=190.0,148.5,140.8,135.1,134.7$, $133.3,131.1,130.5,129.1,128.9,128.6,127.7,123.4,48.7,46.0$.

\section{General Experimental Procedure for the synthesis of 3,5-diaryl-1H-pyrazoles from 1,3- diaryl-2,3-dibromopropane-1-ones (GEP 7) ${ }^{399}$}

\section{3-(3-Chlorophenyl)-5-(3-nitrophenyl)-1H-pyrazole (sery275a)}

A solution of sery273a (1.0 g, $2.2 \mathrm{mmol})$ and hydrazine hydrate $(773 \mathrm{mg}, 15.0 \mathrm{mmol})$ in ethanol $(20 \mathrm{ml})$ was heated under reflux $24 \mathrm{~h}$ with stirring. The clear solution was evaporated under reduced pressure, water was added and resulting precipitate was collected by filtration, washed with water and purified by recrystallisation from $\mathrm{MeOH}$ to provide sery275a (450 $\mathrm{mg})$ as a white powder. Yield $67 \%$. m.p.: $255^{\circ} \mathrm{C} .{ }^{1} \mathrm{H}$ NMR $\left(400 \mathrm{MHz}, \mathrm{DMSO}\left[\mathrm{D}_{6}\right]\right) \delta=13.72$ $(\mathrm{s}, 1 \mathrm{H}), 8.66(\mathrm{~s}, 1 \mathrm{H}), 8.28(\mathrm{~d}, J=7.5 \mathrm{~Hz}, 1 \mathrm{H}), 8.18(\mathrm{~d}, J=8.1 \mathrm{~Hz}, 1 \mathrm{H}), 7.93(\mathrm{~s}, 1 \mathrm{H}), 7.82(\mathrm{~d}, J$ $=7.8 \mathrm{~Hz}, 1 \mathrm{H}), 7.75(\mathrm{t}, J=8.0 \mathrm{~Hz}, 1 \mathrm{H}), 7.54(\mathrm{~s}, 1 \mathrm{H}), 7.50(\mathrm{t}, J=7.8 \mathrm{~Hz}, 1 \mathrm{H}), 7.41(\mathrm{~d}, J=7.8$ $\mathrm{Hz}, 1 \mathrm{H}) .{ }^{13} \mathrm{C}$ NMR $\left(100.6 \mathrm{MHz}, \mathrm{DMSO}\left[\mathrm{D}_{6}\right]\right) \delta=149.9,148.8,143.0,135.7,135.6,134.2$, $131.7,131.3,130.9,128.3,125.2,124.1,122.7,119.8,101.8$. HRMS $(\mathrm{m} / \mathrm{z})[\mathrm{M}+\mathrm{H}]^{+}$calcd for $\mathrm{M}=\mathrm{C}_{15} \mathrm{H}_{10} \mathrm{ClN}_{3} \mathrm{O}_{2}, 300.0540$; found, 300.0542 .

General Experimental Procedure for the synthesis of 3,5-diaryl-1 $H$-pyrazoles containing amino group (GEP 8) ${ }^{517}$

\section{3-(3-Aminophenyl)-5-(3-chlorophenyl)-1H-pyrazole (sery275b)}

The flask was charged with ethanol $(5 \mathrm{~mL})$ and iron powder $(186 \mathrm{mg}, 3.3 \mathrm{mmol})$ was added in portions under efficient stirring, followed by concentrated $\mathrm{HCl}(28 \mathrm{ul}, 0.3 \mathrm{mmol})$. The suspension was stirred at $60{ }^{\circ} \mathrm{C}$ for $2 \mathrm{~h}, 25 \%$ aqueous ammonium chloride solution $(0.5 \mathrm{~mL})$ was added followed by sery275a. The reaction mixture was stirred at $60{ }^{\circ} \mathrm{C}$ for an additional $3 \mathrm{~h}$ and was cooled down. Ethanol $(20 \mathrm{~mL})$ was added, the reaction mixture was filtered over a pad of Celite $(15 \mathrm{~g})$ with suction. The filter cake was washed with EtOH $(10 \mathrm{~mL})$ and the filtrate was concentrated under reduced pressure. A crude product was purified by recrystallization from $\mathrm{EtOH} /$ petroleum benzine $\left(40-60{ }^{\circ} \mathrm{C}\right)$ to provide sery275b $(96 \mathrm{mg})$ as a white powder. Yield 53\%. m.p.: 191-192 ${ }^{\circ} \mathrm{C} .{ }^{1} \mathrm{H}$ NMR $\left(400 \mathrm{MHz}, \mathrm{DMSO}\left[\mathrm{D}_{6}\right]\right) \delta=13.3$ (bs, $1 \mathrm{H}), 7.89(\mathrm{~s}, 1 \mathrm{H}), 7.80(\mathrm{~d}, J=7.0 \mathrm{~Hz}, 1 \mathrm{H}), 7.46(\mathrm{t}, J=7.7 \mathrm{~Hz}, 1 \mathrm{H}), 7.37(\mathrm{~d}, J=7.2 \mathrm{~Hz}, 1 \mathrm{H})$, 7.16-6.86 (m, 4H), $6.56(\mathrm{~d}, J=7.2 \mathrm{~Hz}, 1 \mathrm{H}), 5.16(\mathrm{~s}, 2 \mathrm{H}) .{ }^{13} \mathrm{C} \mathrm{NMR}\left(100.6 \mathrm{MHz}, \mathrm{DMSO}\left[\mathrm{D}_{6}\right]\right)$ $\delta=149.6,148.7,144.5,135.9,133.7,130.8,129.7,129.5,127.4,124.7,123.7,114.2,113.4$, 110.9, 99.9. HRMS $(\mathrm{m} / z)[\mathrm{M}+\mathrm{H}]^{+}$calcd for $\mathrm{M}=\mathrm{C}_{15} \mathrm{H}_{12} \mathrm{ClN}_{3}, 270.0798$; found, 270.0800.

\section{1-(1,3-Benzodioxol-5-yl)-3-phenylpropane-1,3-dione (anle125)}

The title compound has been reported previously ${ }^{518}$ and is commercially available (CAS 26036-40-8). It was prepared according to the GEP 1 using 1-(1,3-benzodioxol-5-yl)ethanone and methyl benzoate and purified by recrystallisation from ethanol/water to provide anle125 as a yellow solid. Yield 62\%. m.p.: 84-85 ${ }^{\circ} \mathrm{C} .{ }^{1} \mathrm{H}$ NMR (400 MHz, DMSO[D 6$\left.]\right) \delta=8.15(\mathrm{~d}, J$ $=7.5, \mathrm{~Hz}, 2 \mathrm{H}), 7.82(\mathrm{dd}, J=8.1,1.7 \mathrm{~Hz}, 1 \mathrm{H}), 7.73(\mathrm{~d}, J=1.3 \mathrm{~Hz}, 1 \mathrm{H}), 7.66-7.59(\mathrm{~m}, 1 \mathrm{H})$, $7.55(\mathrm{t}, J=7.5 \mathrm{~Hz}, 2 \mathrm{H}), 7.26(\mathrm{~s}, 1 \mathrm{H}), 7.09$ (d, $J=8.2 \mathrm{~Hz}, 1 \mathrm{H}), 6.16(\mathrm{~s}, 2 \mathrm{H})$ (in ${ }^{1} \mathrm{H} \mathrm{NMR}$ spectrum $9 \%$ of diketone form is present). 


\section{3-(1,3-Benzodioxol-5-yl)-5-phenyl-1 $H$-pyrazole (anle126)}

The title compound has been reported previously 398,519 (CAS 37060-27-8). It was prepared according to the GEP 2 starting from anle125. White solid. Yield $70 \%$. m.p.: $195-196{ }^{\circ} \mathrm{C}$

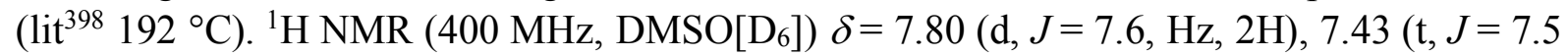
$\mathrm{Hz}, 2 \mathrm{H}), 7.37(\mathrm{~d}, J=1.3 \mathrm{~Hz}, 1 \mathrm{H}), 7.32$ (t, $J=7.5 \mathrm{~Hz}, 2 \mathrm{H}), 7.08(\mathrm{~s}, 1 \mathrm{H}), 6.98$ (d, $J=8.2 \mathrm{~Hz}, 1$ $\mathrm{H}), 6.05(\mathrm{~s}, 2 \mathrm{H})$.

\section{1-(3-Flurophenyl)-3-(3,4,5-trimethoxyphenyl)propane-1,3-dione (anle136a)}

The title compound was prepared according to the GEP 1 using 1-(3,4,5trimethoxyphenyl)ethanone and ethyl 3-fluorobenzoate and purified by recrystallisation from ethanol/water to provide anle136a as a yellow solid. Yield 95\%. m.p.: 95-96 ${ }^{\circ} \mathrm{C} .{ }^{1} \mathrm{H}$ NMR $\left(400 \mathrm{MHz}, \mathrm{DMSO}\left[\mathrm{D}_{6}\right]\right) \delta=8.05-7.95(\mathrm{~m}, 2 \mathrm{H}), 7.66-7.58(\mathrm{~m}, 1 \mathrm{H}), 7.49(\mathrm{td}, J=8.6,2.4 \mathrm{~Hz}$, $1 \mathrm{H}), 7.43(\mathrm{~s}, 2 \mathrm{H}), 7.31(\mathrm{~s}, 1 \mathrm{H}), 3.89(\mathrm{~s}, 6 \mathrm{H}), 3.76(\mathrm{~s}, 3 \mathrm{H})\left(\right.$ in ${ }^{1} \mathrm{H}$ NMR spectrum $7 \%$ of diketone form is present). ${ }^{13} \mathrm{C}$ NMR $(100.6 \mathrm{MHz}$, DMSO[D 6$\left.]\right) \delta=186.4,182.5,162.5$ (d, $J=$ $244.3 \mathrm{~Hz}), 153.2,142.2,137.7(\mathrm{~d}, J=7.2 \mathrm{~Hz}), 131.1(\mathrm{~d}, J=8.2 \mathrm{~Hz}), 130.0,123.7,119.8(\mathrm{~d}, J$ $=21.4 \mathrm{~Hz}), 114.2(\mathrm{~d}, J=23.0 \mathrm{~Hz}), 105.4,93.7,60.4,56.3$. HRMS $(\mathrm{m} / \mathrm{z})[\mathrm{M}+\mathrm{H}]^{+}$calcd for $\mathrm{M}$ $=\mathrm{C}_{18} \mathrm{H}_{17} \mathrm{FO}_{5}, 333.1138$; found, 333.1132.

\section{3-(3-Fluorophenyl)-5-(3,4,5-trimethoxyphenyl)-1H-pyrazole (anle136b)}

The title compound was prepared according to the GEP 2 starting from anle136a. White solid. Yield 88\%. m.p.: 110-113 ${ }^{\circ} \mathrm{C} .{ }^{1} \mathrm{H}$ NMR (400 MHz, DMSO[D 6$\left.]\right) \delta=7.68(\mathrm{~d}, J=7.8$, $\mathrm{Hz}, 1 \mathrm{H}), 7.63(\mathrm{dt}, J=10.6,2.0 \mathrm{~Hz}, 1 \mathrm{H}), 7.48(\mathrm{~m}, 1 \mathrm{H}), 7.29(\mathrm{~s}, 1 \mathrm{H}), 7.18-7.10(\mathrm{~m}, 3 \mathrm{H}), 3.85$ (s, 6H), $3.68(\mathrm{~s}, 3 \mathrm{H}) .{ }^{13} \mathrm{C}$ NMR (100.6 MHz, DMSO[D 6$\left.]\right) \delta=162.8(\mathrm{~d}, J=242.6 \mathrm{~Hz}), 153.5$, 147.6, 146.9, 137.6, 134.9, $131.1(\mathrm{~d}, J=8.5 \mathrm{~Hz}), 126.5,121.3,114.5(\mathrm{~d}, J=20.9 \mathrm{~Hz}), 111.9$ $(\mathrm{d}, J=22.8 \mathrm{~Hz}), 102.9,100.6,60.4,56.3$. HRMS $(\mathrm{m} / \mathrm{z})[\mathrm{M}+\mathrm{H}]^{+}$calcd for $\mathrm{M}=\mathrm{C}_{18} \mathrm{H}_{17} \mathrm{FN}_{2} \mathrm{O}_{3}$, 329.1301; found, 329.1295 .

\section{3-(3-Fluorophenyl)-5-(3,4,5-trihydroxyphenyl)-1 $H$-pyrazole (anle136c)}

The title compound was prepared according to the GEP 3 starting from anle136b. White solid. Yield 100\%. m.p.: 224-227 ${ }^{\circ} \mathrm{C}$. ${ }^{1} \mathrm{H}$ NMR (400 MHz, DMSO[D 6 ) $\delta=7.68$ (d, $J=7.9$ $\mathrm{Hz}, 1 \mathrm{H}), 7,64(\mathrm{dt}, J=10.5,2.1 \mathrm{~Hz}, 1 \mathrm{H}), 7.50-7.42(\mathrm{~m}, 1 \mathrm{H}), 7.14(\mathrm{td},=8.4,2.3 \mathrm{~Hz}, 1 \mathrm{H}), 7.00$ $(\mathrm{s}, 1 \mathrm{H}), 6.72(\mathrm{~s}, 2 \mathrm{H}) .{ }^{13} \mathrm{C}$ NMR $\left(100.6 \mathrm{MHz}, \mathrm{DMSO}\left[\mathrm{D}_{6}\right]\right) \delta=162.8(\mathrm{~d}, J=242.9 \mathrm{~Hz}), 147.4$, $147.0,146.5,134.6(\mathrm{~d}, J=9.0 \mathrm{~Hz}), 134.0,131.0(\mathrm{~d}, J=8.5 \mathrm{~Hz}), 121.4,120.9,114.6(\mathrm{~d}, J=$ $20.9 \mathrm{~Hz}), 111.9(\mathrm{~d}, J=22.7 \mathrm{~Hz}), 105.1,99.5$. HRMS $(\mathrm{m} / \mathrm{z})[\mathrm{M}+\mathrm{H}]^{+}$calcd for $\mathrm{M}=$ $\mathrm{C}_{15} \mathrm{H}_{11} \mathrm{FN}_{2} \mathrm{O}_{3}, 287.0832$; found, 287.0830.

\section{1-(3,5-Diflurophenyl)-3-(3,4,5-trimethoxyphenyl)propane-1,3-dione (anle137a)}

The title compound was prepared according to the GEP 1 using 1-(3,4,5trimethoxyphenyl)ethanone and methyl 3,5-difluorobenzoate and purified by recrystallisation from ethanol/water to provide anle137a as a yellow solid. Yield 70\%. m.p.: $125-126{ }^{\circ} \mathrm{C} .{ }^{1} \mathrm{H}$ NMR (400 MHz, DMSO[D 6 ) $\delta=7.91-7.83(\mathrm{~m}, 2 \mathrm{H}), 7.49$ (tt, $J=9.0,2.3 \mathrm{~Hz}, 1 \mathrm{H}), 7.43$ (s, 2 $\mathrm{H}), 7.32(\mathrm{~s}, 1 \mathrm{H}), 3.89(\mathrm{~s}, 6 \mathrm{H}), 3.76(\mathrm{~s}, 3 \mathrm{H})$ (in ${ }^{1} \mathrm{H}$ NMR spectrum $5 \%$ of diketone form is present). ${ }^{13} \mathrm{C}$ NMR (100.6 MHz, DMSO[D 6$\left.]\right) \delta=186.7,181.0,162.8$ (dd, $J=246.8,13.1$ $\mathrm{Hz}), 153.2,142.4,138.3,129.7,110.7$ (d, $J=26.6 \mathrm{~Hz}), 108.2(\mathrm{t}, J=26.7 \mathrm{~Hz}), 105.6,93.9$, 60.4, 56.5. HRMS $(m / z)[\mathrm{M}+\mathrm{H}]^{+}$calcd for $\mathrm{M}=\mathrm{C}_{18} \mathrm{H}_{16} \mathrm{~F}_{2} \mathrm{O}_{5}, 351.1044$; found, 351.1039.

\section{3-(3,5-Diflurophenyl)-5-(3,4,5-trimethoxyphenyl)-1 $H$-pyrazole (anle137b)}


The title compound was prepared according to the GEP 2 starting from anle137a. White solid. Yield 100\%. m.p.: 189-190 ${ }^{\circ} \mathrm{C} .{ }^{1} \mathrm{H}$ NMR (400 MHz, DMSO[D 6 ) $\delta=7.59-7.49$ (m, 2H), $7.36(\mathrm{~s}, 1 \mathrm{H}), 7.17(\mathrm{tt}, J=9.3,2.3 \mathrm{~Hz}, 1 \mathrm{H}), 7.12(\mathrm{~s}, 2 \mathrm{H}), 3.85(\mathrm{~s}, 6 \mathrm{H}), 3.68(\mathrm{~s}, 3 \mathrm{H}) .{ }^{13} \mathrm{C}$ NMR (100.6 MHz, DMSO[D 6 ) $\delta=163.1(\mathrm{dd}, J=245.2,13.6 \mathrm{~Hz}), 153.5,147.4,146.2$, 137.7, 132.9, 125.9, $108.2(\mathrm{~d}, J=26.4 \mathrm{~Hz}), 102.99$ (t, $J=26.0 \mathrm{~Hz}), 102.96,101.0,60.4,56.3$. HRMS $(m / z)[\mathrm{M}+\mathrm{H}]^{+}$calcd for $\mathrm{M}=\mathrm{C}_{18} \mathrm{H}_{16} \mathrm{~F}_{2} \mathrm{~N}_{2} \mathrm{O}_{3}, 347.1207$; found, 347.1203.

\section{3-(3,5-Difluorophenyl)-5-(3,4,5-trihydroxyphenyl)-1 H-pyrazole (anle137c)}

The title compound was prepared according to the GEP 3 starting from anle137b. White solid. Yield 100\%. m.p.: 265-270 ${ }^{\circ} \mathrm{C}(\mathrm{dec}) .{ }^{1} \mathrm{H}$ NMR (400 MHz, DMSO[D $\left.]\right) \delta=7.58-7.50$ $(\mathrm{m}, 2 \mathrm{H}), 7.14$ (tt, $J=9.3,2.3 \mathrm{~Hz}, 1 \mathrm{H}), 7.00(\mathrm{~s}, 1 \mathrm{H}), 6.70(\mathrm{~s}, 2 \mathrm{H}) .{ }^{13} \mathrm{C}$ NMR $(100.6 \mathrm{MHz}$, DMSO[D 6$]) \delta=163.0(\mathrm{dd}, J=244.9,13.5 \mathrm{~Hz}), 147.3,146.5(2 \mathrm{C}), 136.6,134.0,120.7,108.2$ $(\mathrm{d}, J=26.3 \mathrm{~Hz}), 105.0,102.8(\mathrm{t}, J=26.0 \mathrm{~Hz}), 99.7$. HRMS $(m / z)[\mathrm{M}+\mathrm{H}]^{+}$calcd for $\mathrm{M}=$ $\mathrm{C}_{15} \mathrm{H}_{10} \mathrm{~F}_{2} \mathrm{~N}_{2} \mathrm{O}_{3}, 305.0738$; found, 305.0736.

\section{1-(1,3-Benzodioxol-5-yl)-3-(3-bromophenyl)propane-1,3-dione (anle138a)}

The title compound has been reported previously ${ }^{519}$ (CAS 882696-95-9). It was prepared according to the GEP 1 using 1-(1,3-benzodioxol-5-yl)ethanone and methyl 3-bromobenzoate and purified by recrystallisation from ethanol/water to provide anle138a as a yellow solid. Yield 87\%. m.p.: $136-137{ }^{\circ} \mathrm{C} .{ }^{1} \mathrm{H}$ NMR $\left(400 \mathrm{MHz}, \mathrm{DMSO}\left[\mathrm{D}_{6}\right]\right) \delta=8.32(\mathrm{~s}, 1 \mathrm{H}), 8.1(\mathrm{~d}, J=$ $8.1 \mathrm{~Hz}, 1 \mathrm{H}), 7.82(\mathrm{dd}, J=8.1,1.6 \mathrm{~Hz}, 1 \mathrm{H}), 7.78(\mathrm{~d}, J=8.1 \mathrm{~Hz}, 1 \mathrm{H}), 7.73(\mathrm{~d}, J=1.6 \mathrm{~Hz}, 1 \mathrm{H})$, $7.50(\mathrm{t}, J=8.1 \mathrm{~Hz}, 1 \mathrm{H}), 7.27(\mathrm{~s}, 1 \mathrm{H}), 7.05(\mathrm{~d}, J=8.1 \mathrm{~Hz}, 1 \mathrm{H}), 6.15(\mathrm{~s}, 2 \mathrm{H})$ (in ${ }^{1} \mathrm{H} \mathrm{NMR}$ spectrum $6 \%$ of diketone form is present).

\section{3-(1,3-Benzodioxol-5-yl)-5-(3-bromophenyl)-1H-pyrazole (anle138b)}

The title compound has been reported previously ${ }^{519}$ (CAS 882697-00-9). It was prepared according to the GEP 2 starting from anle138a. White solid. Yield 89\%. m.p.: 192-193 ${ }^{\circ} \mathrm{C}$ $\left(\mathrm{lit}^{519} 128-130{ }^{\circ} \mathrm{C}\right) .{ }^{1} \mathrm{H}$ NMR $\left.\left(400 \mathrm{MHz}, \mathrm{DMSO}_{[} \mathrm{D}_{6}\right]\right) \delta=8.00(\mathrm{~s}, 1 \mathrm{H}), 7.81(\mathrm{~d}, J=8.1 \mathrm{~Hz}$, $1 \mathrm{H}), 7.50(\mathrm{~d}, J=8.0 \mathrm{~Hz}, 1 \mathrm{H}), 7.40(\mathrm{~d}, J=7.9 \mathrm{~Hz}, 1 \mathrm{H}), 7.37(\mathrm{~d}, J=1.5 \mathrm{~Hz}, 1 \mathrm{H}), 7.32(\mathrm{~d}, J=$ $8.0 \mathrm{~Hz}, 1 \mathrm{H}), 7.19(\mathrm{~s}, 1 \mathrm{H}), 7.00(\mathrm{~d}, \mathrm{~J}=8.0 \mathrm{~Hz}, 1 \mathrm{H}), 6.06(\mathrm{~s}, 2 \mathrm{H})$.

\section{3-(3-Bromophenyl)-5-(3,4-dihydroxyphenyl)-1H-pyrazole (anle138c)}

This compound was prepared according to the GEP 3 starting from sery255b. White solid. Yield 87\%. m.p.: $232-237{ }^{\circ} \mathrm{C} .{ }^{1} \mathrm{H}$ NMR (400 MHz, DMSO[D 6$\left.]\right) \delta=8.02$ (s, 1H), $7.83(\mathrm{~d}, J=$ $7.8 \mathrm{~Hz}, 1 \mathrm{H}), 7.50(\mathrm{~d}, J=7.8 \mathrm{~Hz}, 1 \mathrm{H}), 7.38(\mathrm{t}, J=7.8 \mathrm{~Hz}, 1 \mathrm{H}), 7.19(\mathrm{~d}, J=1.9 \mathrm{~Hz}, 1 \mathrm{H}), 7.08$ $(\mathrm{dd}, J=8.2,1.9 \mathrm{~Hz}, 1 \mathrm{H}), 7.02(\mathrm{~s}, 1 \mathrm{H}), 6.80(\mathrm{~d}, J=8.2 \mathrm{~Hz}, 1 \mathrm{H}) .{ }^{13} \mathrm{C}$ NMR $(100.6 \mathrm{MHz}$, DMSO[D 6$]) \delta=147.1,146.6,145.9,145.6,134.4,130.9,103.5,127.7,124.2,122.2,121.5$, 117.0, 115.9, 113.1, 99.3. HRMS $(\mathrm{m} / z)[\mathrm{M}+\mathrm{H}]^{+}$calcd for $\mathrm{M}=\mathrm{C}_{15} \mathrm{H}_{11}{ }^{79} \mathrm{BrN}_{2} \mathrm{O}_{2}, 331.0082$; found, 331.0088 .

\section{1-(3-Bromophenyl)-3-(3,4,5-trimethoxyphenyl)propane-1,3-dione (anle142a)}

The title compound was prepared according to the GEP 1 using 1-(3,4,5trimethoxyphenyl)ethanone and methyl 3-bromobenzoate and purified by recrystallisation from ethanol/water to provide anle142a as a yellow solid. Yield 99\%. m.p.: $109-111{ }^{\circ} \mathrm{C} .{ }^{1} \mathrm{H}$ NMR (400 MHz, DMSO[D $]) \delta=8.29(\mathrm{t}, J=1.8 \mathrm{~Hz}, 1 \mathrm{H}), 8.16(\mathrm{~d}, J=7.9 \mathrm{~Hz}, 1 \mathrm{H}), 7.83(\mathrm{~d}, J$ $=7.9 \mathrm{~Hz}, 1 \mathrm{H}), 7.53(\mathrm{t}, J=7.9 \mathrm{~Hz}, 1 \mathrm{H}), 7.43(\mathrm{~s}, 2 \mathrm{H}), 7.32(\mathrm{~s}, 1 \mathrm{H}), 3.90(\mathrm{~s}, 6 \mathrm{H}), 3.76(\mathrm{~s}, 3 \mathrm{H})$ (in ${ }^{1} \mathrm{H}$ NMR spectrum $7 \%$ of diketone form is present). $\left.\left.{ }^{13} \mathrm{C} \mathrm{NMR} \mathrm{(100.6} \mathrm{MHz,} \mathrm{DMSO[D} 6\right]\right) \delta$ $=186.8,182.7,153.5,142.8,137.3,135.9,131.5,130.3,130.2,126.9,122.8,105.9$, 94.1, 60.7, 56.8. HRMS $(\mathrm{m} / \mathrm{z})[\mathrm{M}+\mathrm{H}]^{+}$calcd for $\mathrm{M}=\mathrm{C}_{18} \mathrm{H}_{17}{ }^{79} \mathrm{BrO}_{5}, 393.0338$; found, 393.0337. 


\section{3-(3-Bromophenyl)-5-(3,4,5-trimethoxyphenyl)-1 $H$-pyrazole (anle142b)}

The title compound was prepared according to the GEP 2 starting from anle142a. White solid. Yield 92\%. m.p.: 114- $117^{\circ} \mathrm{C} .{ }^{1} \mathrm{H}$ NMR (400 MHz, DMSO[D 6$]+1-2 \%$ conc. DCl) $\delta=$ $8.02(\mathrm{~s}, 1 \mathrm{H}), 7.84(\mathrm{~d}, J=7.8 \mathrm{~Hz}, 1 \mathrm{H}), 7.51(\mathrm{~d}, J=8.0 \mathrm{~Hz}, 1 \mathrm{H}), 7.40(\mathrm{t}, J=7.8 \mathrm{~Hz}, 1 \mathrm{H}), 7.32$ $(\mathrm{s}, 1 \mathrm{H}), 7.14(\mathrm{~s}, 2 \mathrm{H}), 3.85(\mathrm{~s}, 6 \mathrm{H}), 3.68(\mathrm{~s}, 3 \mathrm{H}) .{ }^{13} \mathrm{C}$ NMR $\left(100.6 \mathrm{MHz}, \mathrm{DMSO}\left[\mathrm{D}_{6}\right]+1-2 \%\right.$ conc. DCl) $\delta=153.5,147.3,146.6,137.6,134.8,131.2,130.5,127.8,126.4,124.2,122.5$, 102.9, 100.6, 60.4, 56.5. HRMS $(m / z)[\mathrm{M}+\mathrm{H}]^{+}$calcd for $\mathrm{M}=\mathrm{C}_{18} \mathrm{H}_{17}{ }^{79} \mathrm{BrN}_{2} \mathrm{O}_{3}, 389.0501$; found, 389.0503.

\section{3-(3-Bromophenyl)-5-(3,4,5-trihydroxyphenyl)-1 $H$-pyrazole hydrobromide (anle142c)}

The title compound was prepared according to the GEP 3 starting from anle142b. Brown solid. Yield 68\%. m.p.: 221-224 ${ }^{\circ} \mathrm{C}$ (dec). ${ }^{1} \mathrm{H}$ NMR (400 MHz, DMSO[D 6$\left.]\right) \delta=8.02$ (t, $J=$ $1.7 \mathrm{~Hz}, 1 \mathrm{H}), 7.83(\mathrm{~d}, J=7.8 \mathrm{~Hz}, 1 \mathrm{H}), 7,49(\mathrm{~d}, J=8.0 \mathrm{~Hz}, 1 \mathrm{H}), 7.36(\mathrm{t}, J=7.8 \mathrm{~Hz}, 1 \mathrm{H}), 6.95$ $(\mathrm{s}, 1 \mathrm{H}), 6.72(\mathrm{~s}, 2 \mathrm{H}) .{ }^{13} \mathrm{C}$ NMR $\left(100.6 \mathrm{MHz}, \mathrm{DMSO}\left[\mathrm{D}_{6}\right]\right) \delta=147.2,146.8,135.3,134.2$, 131.4, 130.7, 128.0, 125.8, 124.6, 122.7, 121.4, 105.3, 99.6. HRMS $(\mathrm{m} / \mathrm{z})[\mathrm{M}+\mathrm{H}]^{+}$calcd for $\mathrm{M}=\mathrm{C}_{15} \mathrm{H}_{11}{ }^{79} \mathrm{BrN}_{2} \mathrm{O}_{3}, 347.0031$; found, 347.0035.

\section{1-(3,4-Dimethoxyphenyl)-3-(3,4,5-trimethoxyphenyl)propane-1,3-dione (anle143a)}

The title compound was prepared according to the GEP 1 using 1-(3,4,5trimethoxyphenyl)ethanone and ethyl 3,4-dimethoxybenzoate, and purified by column chromatography (hexane:EtOAc, 1:1 v/v) followed by recrystallisation from methanol/water to provide anle143a as a yellow solid. Yield 29\%. TLC (hexane:EtOAc, 1:1 v/v): RF $=0.57$.

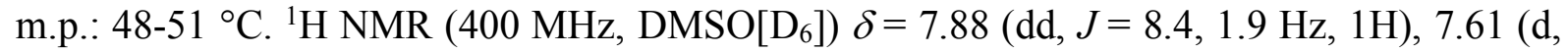
$J=1.9 \mathrm{~Hz}, 1 \mathrm{H}), 7.39(\mathrm{~s}, 2 \mathrm{H}), 7.21(\mathrm{~s}, 1 \mathrm{H}), 7.09(\mathrm{~d}, J=8.6 \mathrm{~Hz}, 1 \mathrm{H}), 3.89(\mathrm{~s}, 6 \mathrm{H}), 3.86(\mathrm{~s}, 3 \mathrm{H})$, $3.85(\mathrm{~s}, 3 \mathrm{H}), 3.75(\mathrm{~s}, 3 \mathrm{H})$ (in ${ }^{1} \mathrm{H}$ NMR spectrum $11 \%$ of diketone form is present). ${ }^{13} \mathrm{C}$ NMR (100.6 MHz, DMSO[D 6$]$ ) $\delta=185.5,183.6,153.3,153.1,148,9,141.7,130.2,127.4,122.2$, $111.4,110.3,105.1,92.7,60.4,56.4,56.0,55.9$. HRMS $(m / z)[\mathrm{M}+\mathrm{H}]^{+}$calcd for $\mathrm{M}=$ $\mathrm{C}_{20} \mathrm{H}_{22} \mathrm{O}_{7}, 375.1444$; found, 375.1442 .

\section{3-(3,4-Dimethoxyphenyl)-5-(3,4,5-trimethoxyphenyl)-1 $H$-pyrazole (anle143b)}

The title compound has been reported previously ${ }^{400}$ (CAS 861881-81-4). It was prepared according to the GEP 2 starting from anle143a. White solid. Yield 69\%. m.p.: 91-93 ${ }^{\circ} \mathrm{C}$ (lit. $\left.68{ }^{\circ} \mathrm{C}\right) .{ }^{1} \mathrm{H}$ NMR $\left(400 \mathrm{MHz}, \mathrm{DMSO}\left[\mathrm{D}_{6}\right]+1-2 \%\right.$ conc. DCl $) \delta=7.40(\mathrm{~d}, J=1.9 \mathrm{~Hz}, 1 \mathrm{H}), 7.36$ $(\mathrm{dd}, J=8.2,1.9 \mathrm{~Hz}, 1 \mathrm{H}), 7.12(\mathrm{~s}, 3 \mathrm{H}), 7.02(\mathrm{~d}, J=8.4 \mathrm{~Hz}, 1 \mathrm{H}), 3.85(\mathrm{~s}, 6 \mathrm{H}), 3.84(\mathrm{~s}, 3 \mathrm{H})$, $3.78(\mathrm{~s}, 3 \mathrm{H}), 3.68(\mathrm{~s}, 3 \mathrm{H})$.

\section{3-(3,4-Dihydroxyphenyl)-5-(3,4,5-trihydroxyphenyl)-1 $H$-pyrazole} (anle143c)

\section{hydrobromide}

The title compound was prepared according to the GEP 3 starting from anle143b. Brown solid. Yield 81\%. m.p.: 225-230 ${ }^{\circ} \mathrm{C}$ (dec). ${ }^{1} \mathrm{H}$ NMR (400 MHz, DMSO[D 6$]+1-2 \%$ conc. DCl) $\delta=9.57-8.57(\mathrm{~m}, 5 \mathrm{H}), 8.53-7.97(\mathrm{bs}, 1 \mathrm{H}), 7.17(\mathrm{~s}, 1 \mathrm{H}), 7.05(\mathrm{dd}, J=8.1,1.7 \mathrm{~Hz}, 1 \mathrm{H})$, $6.76(\mathrm{~d}, J=8.1 \mathrm{~Hz}, 1 \mathrm{H}), 6.70(\mathrm{~s}, 2 \mathrm{H}), 6.57(\mathrm{~s}, 1 \mathrm{H}) .{ }^{13} \mathrm{C} \mathrm{NMR}\left(100.6 \mathrm{MHz}, \mathrm{DMSO}\left[\mathrm{D}_{6}\right]+1-\right.$ $2 \%$ conc. DCl) $\delta=148.1,146.4,145.5,145.4,142.4,133.4,123.3,122.0,116.8,115.9$, 113.0, 104.8, 97.7. HRMS $(m / z)[\mathrm{M}+\mathrm{H}]^{+}$calcd for $\mathrm{M}=\mathrm{C}_{15} \mathrm{H}_{12} \mathrm{~N}_{2} \mathrm{O}_{5}, 301.0824$; found, 301.0823 .

\section{1-(3,5-Dibromophenyl)-3-(3,4,5-trimethoxyphenyl)propane-1,3-dione (anle145a)}

The title compound was prepared according to the GEP 1 using 1-(3,4,5trimethoxyphenyl)ethanone and methyl 3,5-dibromobenzoate and purified by recrystallisation 
from ethanol/water to provide anle145a as a yellow solid. Yield $90 \%$. m.p.: $126-129{ }^{\circ} \mathrm{C} .{ }^{1} \mathrm{H}$ NMR (400 MHz, DMSO[D 6$]) \delta=8.28(\mathrm{~d}, J=1.7 \mathrm{~Hz}, 2 \mathrm{H}), 8.10(\mathrm{t}, J=1.7 \mathrm{~Hz}, 1 \mathrm{H}), 7.44(\mathrm{~s}$, $2 \mathrm{H}), 7.33(\mathrm{~s}, 1 \mathrm{H}), 3.90(\mathrm{~s}, 6 \mathrm{H}), 3.77(\mathrm{~s}, 3 \mathrm{H})$ (in ${ }^{1} \mathrm{H}$ NMR spectrum $6 \%$ of diketone form is present). ${ }^{13} \mathrm{C}$ NMR (100.6 MHz, DMSO[D 6 ) $\delta=186.8,180.7,153.2,142.5,138.4,137.3$, $129.8,129.1,123.3,105.8,94.2,60.4,56.6$. HRMS $(m / z)[\mathrm{M}+\mathrm{H}]^{+}$calcd for $\mathrm{M}=$ $\mathrm{C}_{18} \mathrm{H}_{16}{ }^{79} \mathrm{Br}^{81} \mathrm{BrO}_{5}, 472.9422$; found, 472.9423 .

\section{3-(3,5-Dibromophenyl)-5-(3,4,5-trimethoxyphenyl)-1H-pyrazole (anle145b)}

The title compound was prepared according to the GEP 2 starting from anle145a. White solid. Yield 99\%. m.p.: $138-141{ }^{\circ} \mathrm{C} .{ }^{1} \mathrm{H}$ NMR (400 MHz, DMSO[D 6$]+1-2 \%$ conc. DCl) $\delta=$ $8.04(\mathrm{~d}, J=1.7 \mathrm{~Hz}, 2 \mathrm{H}), 7.77(\mathrm{t}, J=1.7 \mathrm{~Hz}, 1 \mathrm{H}), 7.42(\mathrm{~s}, 1 \mathrm{H}), 7.14(\mathrm{~s}, 2 \mathrm{H}), 3.85(\mathrm{~s}, 6 \mathrm{H}), 3.68$ $(\mathrm{s}, 3 \mathrm{H}) .{ }^{13} \mathrm{C}$ NMR $\left(100.6 \mathrm{MHz}, \mathrm{DMSO}\left[\mathrm{D}_{6}\right]+1-2 \%\right.$ conc. DCl) $\delta=153.8,147.3,137.9$, 133.4, 132.6, 131.6, 131.0, 127.0, 123.5, 103.1, 101.3, 60.6, 56.5. HRMS $(m / z)[\mathrm{M}+\mathrm{H}]^{+}$calcd for $\mathrm{M}=\mathrm{C}_{18} \mathrm{H}_{16}{ }^{79} \mathrm{Br}^{81} \mathrm{BrN}_{2} \mathrm{O}_{3}, 468.9585$; found, 468.9586 .

\section{3-(3,5-Dibromophenyl)-5-(3,4,5-trihydroxyphenyl)-1 $H$-pyrazole (anle145c)}

hydrobromide

The title compound was prepared according to the GEP 3 starting from anle145b. Beige solid. Yield 100\%. m.p.: 285-290 ${ }^{\circ} \mathrm{C}(\mathrm{dec}) .{ }^{1} \mathrm{H}$ NMR (400 MHz, DMSO[D 6$\left.]\right) \delta=8.04(\mathrm{~d}, J=$ $1.8 \mathrm{~Hz}, 2 \mathrm{H}), 7.74(\mathrm{t}, J=1.8 \mathrm{~Hz}, 1 \mathrm{H}), 7.06(\mathrm{~s}, 1 \mathrm{H}), 6.71(\mathrm{~s}, 2 \mathrm{H}) .{ }^{13} \mathrm{C}$ NMR $(100.6 \mathrm{MHz}$, DMSO[D $]$ ) $\delta=146.7-146.5$ (3C), 136.9, 134.1, 132.3, 126.9, 123.2, 120.7, 105.1, 100.0. $\operatorname{HRMS}(m / z)[\mathrm{M}+\mathrm{H}]^{+}$calcd for $\mathrm{M}=\mathrm{C}_{15} \mathrm{H}_{11}{ }^{79} \mathrm{Br}^{81} \mathrm{BrN}_{2} \mathrm{O}_{3}$, 426.9116; found, 426.9119.

\section{3-(3,5-Dibromophenyl)-5-(3,4,5-trihydroxyphenyl)-1 $H$-pyrazole (anle145d)}

Anle145c (171 mg, $0.34 \mathrm{mmol})$ was partitioned between ethyl acetate $(5 \mathrm{ml})$ and $0.2 \mathrm{M}$ phosphate buffer $\mathrm{pH}=7.5(10 \mathrm{ml})$. The organic layer was separated and the aqueous layer was extracted with ethyl acetate $(2 \times 5 \mathrm{ml})$. The combined organic layers were dried over $\mathrm{Na}_{2} \mathrm{SO}_{4}$ and concentrated in vacuo. The residue was stirred $30 \mathrm{~min}$ with water $(5 \mathrm{ml})$, the resulting precipitate was collected by filtration, washed with water $(2 \times 3 \mathrm{ml})$ and dried in vacuo to provide anle145d (106 mg, $73 \%$ ) as a brown solid. m.p.: 290-295 ${ }^{\circ} \mathrm{C}(\mathrm{dec})$. The observed characterisation data $\left({ }^{1} \mathrm{H}-,{ }^{13} \mathrm{C}-\mathrm{NMR}\right.$ in DMSO$\left[\mathrm{D}_{6}\right]+1-2 \%$ conc. DCl; HRMS) was consistent with that of compound anle145c.

\section{1-(1,3-Benzodioxol-5-yl)-3-(3-fluorophenyl)propane-1,3-dione (anle186a)}

The title compound was prepared according to the GEP 1 using 1-(1,3-benzodioxol-5yl)ethanone and ethyl 3-fluorobenzoate and purified by recrystallisation from ethanol/water to provide anle186a as a yellow solid. Yield 89\%. m.p.: 120-122 ${ }^{\circ} \mathrm{C} .{ }^{1} \mathrm{H}$ NMR $(400 \mathrm{MHz}$, DMSO[D 6 ] $\delta=8.05-7.93(\mathrm{~m}, 2 \mathrm{H}), 7.83(\mathrm{dd}, J=8.2,1.8 \mathrm{~Hz}, 1 \mathrm{H}), 7.75(\mathrm{~d}, J=1.7 \mathrm{~Hz}, 1 \mathrm{H})$, 7.64-7.55 (m, 1H), 7.50-7.42 (m, 1H), $7.29(\mathrm{~s}, 1 \mathrm{H}), 7.08(\mathrm{~d}, J=8.2 \mathrm{~Hz}, 1 \mathrm{H}), 6.16(\mathrm{~s}, 2 \mathrm{H})($ in ${ }^{1} \mathrm{H}$ NMR spectrum $3 \%$ of diketone form is present). ${ }^{13} \mathrm{C}$ NMR $\left(100.6 \mathrm{MHz}, \mathrm{DMSO}\left[\mathrm{D}_{6}\right]\right) \delta=$ 186.3, 181.6, 162.5 (d, $J=244.2 \mathrm{~Hz}), 151.9,148.2,137.1(\mathrm{~d}, J=7.3 \mathrm{~Hz}), 131.1$ (d, $J=8.2$ $\mathrm{Hz}), 129.0,124.0,123.5,119.6(\mathrm{~d}, J=21.2 \mathrm{~Hz}), 114.0(\mathrm{~d}, \mathrm{~J}=23.1 \mathrm{~Hz}), 108.6,107.4,102.4$, 93.3. HRMS $(m / z)[\mathrm{M}+\mathrm{H}]^{+}$calcd for $\mathrm{M}=\mathrm{C}_{16} \mathrm{H}_{11} \mathrm{FO}_{4}$, 287.0720; found, 287.0718.

\section{3-(1,3-Benzodioxol-5-yl)-5-(3-fluorophenyl)-1H-pyrazole (anle186b)}

The title compound was prepared according to the GEP 2 starting from anle186a. White solid. Yield 86\%. m.p.: 191-192 ${ }^{\circ} \mathrm{C} .{ }^{1} \mathrm{H}$ NMR (400 MHz, DMSO[D 6$]+1-2 \%$ conc. DCl) $\delta=$ $7.64(\mathrm{~d}, J=7.8 \mathrm{~Hz}, 1 \mathrm{H}), 7.63-7.58(\mathrm{~m}, 1 \mathrm{H}), 7.51-7.44(\mathrm{~m}, 1 \mathrm{H}), 7.36(\mathrm{~d}, J=1.6 \mathrm{~Hz}, 1 \mathrm{H}), 7.31$ 
$(\mathrm{dd}, J=8.1,1.6 \mathrm{~Hz}, 1 \mathrm{H}), 7.19-7.10(\mathrm{~m}, 1 \mathrm{H}), 7.16(\mathrm{~s}, 1 \mathrm{H}), 6.99(\mathrm{~d}, J=8.0 \mathrm{~Hz}, 1 \mathrm{H}), 6.05(\mathrm{~s}$, 2H). ${ }^{13} \mathrm{C}$ NMR (100.6 MHz, DMSO[D 6$]+1-2 \%$ conc. DCl) $\delta=162.8(\mathrm{~d}, J=242.7 \mathrm{~Hz})$, $148.0,147.3,135.0,131.0(\mathrm{~d}, J=8.6 \mathrm{~Hz}), 129.7,126.3,124.4,121.3,119.1,114.5(\mathrm{~d}, J=$ $21.2 \mathrm{~Hz}), 111.8(\mathrm{~d}, \mathrm{~J}=22.8 \mathrm{~Hz}), 108.9,105.8,101.4,100.0$. HRMS $(\mathrm{m} / \mathrm{z})[\mathrm{M}+\mathrm{H}]^{+}$calcd for $\mathrm{M}=\mathrm{C}_{16} \mathrm{H}_{11} \mathrm{FN}_{2} \mathrm{O}_{2}$, 283.0883; found, 283.0883.

\section{1-(1,3-Benzodioxol-5-yl)-3-(3-iodophenyl)propane-1,3-dione (anle197a)}

The title was prepared according to the GEP 1 using 1-(1,3-benzodioxol-5-yl)ethanone and methyl 3-iodobenzoate and purified by recrystallisation from ethanol/water to provide anle197a as a yellow solid. Yield $22 \%$. m.p.: 143-144 ${ }^{\circ} \mathrm{C} .{ }^{1} \mathrm{H}$ NMR (400 MHz, DMSO[D 6$\left.]\right) \delta$ $=8.49(\mathrm{t}, J=1.6 \mathrm{~Hz}, 1 \mathrm{H}), 8.14(\mathrm{~d}, J=7.9 \mathrm{~Hz}, 1 \mathrm{H}), 7.97(\mathrm{~d}, J=7.9 \mathrm{~Hz}, 1 \mathrm{H}), 7.84(\mathrm{dd}, J=8.2$, $1.8 \mathrm{~Hz}, 1 \mathrm{H}), 7.76(\mathrm{~d}, J=1.7 \mathrm{~Hz}, 1 \mathrm{H}), 7.34(\mathrm{t}, J=7.8 \mathrm{~Hz}, 1 \mathrm{H}), 7.28(\mathrm{~s}, 1 \mathrm{H}), 7.08(\mathrm{~d}, J=8.2$ $\mathrm{Hz}, 1 \mathrm{H}), 6.16(\mathrm{~s}, 2 \mathrm{H})$ (in ${ }^{1} \mathrm{H}$ NMR spectrum $7 \%$ of diketone form is present). ${ }^{13} \mathrm{C}$ NMR $(100.6$ $\left.\mathrm{MHz}, \mathrm{DMSO}\left[\mathrm{D}_{6}\right]\right) \delta=186.2,181.6,151.9,148.2,141.3,136.7,135.5,131.0,129.0,126.7$, 124.1, 108.6, 107.5, 102.3, 95.4, 93.2. HRMS $(\mathrm{m} / \mathrm{z})[\mathrm{M}+\mathrm{H}]^{+}$calcd for $\mathrm{M}=\mathrm{C}_{16} \mathrm{H}_{11} \mathrm{IO}_{4}$, 394.9780; found, 394.9788.

\section{3-(1,3-Benzodioxol-5-yl)-5-(3-iodophenyl)-1H-pyrazole (anle197b)}

The title compound was prepared according to the GEP 2 starting from anle197a. White solid. Yield 75\%. m.p.: 205-207 ${ }^{\circ} \mathrm{C} .{ }^{1} \mathrm{H}$ NMR (400 MHz, DMSO[D 6$]+1-2 \%$ conc. DCl) $\delta=$ $8.18(\mathrm{t}, J=1.6 \mathrm{~Hz}, 1 \mathrm{H}), 7.82(\mathrm{~d}, J=8.0 \mathrm{~Hz}, 1 \mathrm{H}), 7.67(\mathrm{~d}, J=8.2 \mathrm{~Hz}, 1 \mathrm{H}), 7.36(\mathrm{~d}, J=1.6 \mathrm{~Hz}$, 1H), $7.32(\mathrm{dd}, J=8.0,1.7 \mathrm{~Hz}, 1 \mathrm{H}), 7.23$ (t, $J=7.8 \mathrm{~Hz}, 1 \mathrm{H}), 7.16(\mathrm{~s}, 1 \mathrm{H}), 6.99(\mathrm{~d}, J=8.0 \mathrm{~Hz}$, $1 \mathrm{H}), 6.05$ (s, 2H). ${ }^{13} \mathrm{C}$ NMR (100.6 MHz, DMSO[D 6$]+1-2 \%$ conc. DCl) $\delta=148.0,147.3$, 146.8 (2C), 136.4, 134.4, 133.5, 131.1, 125.2, 124.5, 119.1, 108.9, 105.8, 101.4, 99.9, 95.5. $\operatorname{HRMS}(\mathrm{m} / z)[\mathrm{M}+\mathrm{H}]^{+}$calcd for $\mathrm{M}=\mathrm{C}_{16} \mathrm{H}_{11} \mathrm{IN}_{2} \mathrm{O}_{2}, 390.9944$; found, 390.9948 .

\section{1,3-Bis(1,3-benzodioxol-5-yl)-propane-1,3-dione (anle232a)}

The title compound has been reported previously ${ }^{520}$ (CAS 204397-06-8). It was prepared according to the GEP 1 using 1-(1,3-benzodioxol-5-yl)ethanone and methyl 1,3benzodioxole-5-carboxylate and purified by recrystallisation from ethanol/water to provide anle232a as a yellow solid. Yield 71\%. m.p.: 179-180 ${ }^{\circ} \mathrm{C}\left(1 \mathrm{it}{ }^{520} 185-186{ }^{\circ} \mathrm{C}\right) .{ }^{1} \mathrm{H}$ NMR $(400$ $\left.\mathrm{MHz}, \mathrm{DMSO}\left[\mathrm{D}_{6}\right]\right) \delta=7.78(\mathrm{dd}, J=8.2,1.7 \mathrm{~Hz}, 2 \mathrm{H}), 7.73(\mathrm{~d}, J=1.7 \mathrm{~Hz}, 2 \mathrm{H}), 7.17(\mathrm{~s}, 1 \mathrm{H})$, $7.07(\mathrm{~d}, J=8.2 \mathrm{~Hz}, 2 \mathrm{H}), 6.15(\mathrm{~s}, 4 \mathrm{H})$ (in ${ }^{1} \mathrm{H}$ NMR spectrum $11 \%$ of diketone form is present).

\section{3,5-Bis(1,3-benzodioxol-5-yl)-1H-pyrazole (anle232b)}

The title compound has been reported previously ${ }^{521}$ (CAS 1147315-26-1). It was prepared according to the GEP 2 starting from anle232a. Beige solid. Yield 71\%. m.p.: 228-229 ${ }^{\circ} \mathrm{C} .{ }^{1} \mathrm{H}$ NMR (400 MHz, DMSO[D $]$ ) $\delta=7.34$ (s, 2H), $7.30(\mathrm{~d}, J=8.0 \mathrm{~Hz}, 2 \mathrm{H}), 7.00(\mathrm{~s}, 1 \mathrm{H}), 6.98(\mathrm{~d}$, $J=8.0 \mathrm{~Hz}, 2 \mathrm{H}), 6.04(\mathrm{~s}, 4 \mathrm{H})$.

\section{1-(1,3-Benzodioxol-5-yl)-3-(3-methoxyphenyl)propane-1,3-dione (anle233a)}

The title compound has been reported previously ${ }^{519}$ (CAS 882696-96-0). It was prepared according to the GEP 1 using 1-(1,3-benzodioxol-5-yl)ethanone and ethyl 3-methoxybenzoate and purified by recrystallisation from ethanol/water to provide anle233a as a yellow solid. Yield 68\%. m.p.: 86-88 ${ }^{\circ} \mathrm{C}$. ${ }^{1} \mathrm{H}$ NMR (400 MHz, DMSO[D 6$) \delta=7.82$ (dd, $J=8.2,1.7 \mathrm{~Hz}$, $1 \mathrm{H}), 7.78-7.71(\mathrm{~m}, 2 \mathrm{H}), 7.63(\mathrm{t}, J=2.0 \mathrm{~Hz}, 1 \mathrm{H}), 7.46(\mathrm{t}, J=8.0 \mathrm{~Hz}, 1 \mathrm{H}), 7.24(\mathrm{~s}, 1 \mathrm{H}), 7.19$ $(\mathrm{dd}, J=8.0,2.4 \mathrm{~Hz}, 1 \mathrm{H}), 7.09(\mathrm{~d}, \mathrm{~J}=8.2 \mathrm{~Hz}, 1 \mathrm{H}), 6.16(\mathrm{~s}, 2 \mathrm{H}), 3.85(\mathrm{~s}, 3 \mathrm{H})$. 


\section{3-(1,3-Benzodioxol-5-yl)-5-(3-methoxyphenyl)-1H-pyrazole (anle233b)}

The title compound has been reported previously ${ }^{519}$ (CAS 882697-01-0). It was prepared according to the GEP 2 starting from anle233a. White solid. Yield 79\%. m.p.: $160-161{ }^{\circ} \mathrm{C}$ $\left(\mathrm{lit}^{519} 149{ }^{\circ} \mathrm{C}\right) .{ }^{1} \mathrm{H} \mathrm{NMR}\left(400 \mathrm{MHz}, \mathrm{DMSO}\left[\mathrm{D}_{6}\right]+1-2 \%\right.$ conc. DCl $) \delta=7.46-7.29(\mathrm{~m}, 5 \mathrm{H})$, $7.11(\mathrm{~s}, 1 \mathrm{H}), 6.98(\mathrm{~d}, J=8.0 \mathrm{~Hz}, 1 \mathrm{H}), 6.89$ ( br d, $J=7.3 \mathrm{~Hz}, 1 \mathrm{H}), 6.05$ (s, 2H), 3.81 (s, 3H).

\section{1-(1,3-Benzodioxol-5-yl)-3-(2-bromophenyl)propane-1,3-dione (anle234a)}

The title was prepared according to the GEP 1 using 1-(1,3-benzodioxol-5-yl)ethanone and methyl 2-bromobenzoate and purified by recrystallisation from ethanol/water to provide anle234a as a yellow solid. Yield 82\%. m.p.: $73-76{ }^{\circ} \mathrm{C} .{ }^{1} \mathrm{H}$ NMR $\left(400 \mathrm{MHz}, \mathrm{DMSO}\left[\mathrm{D}_{6}\right]\right) \delta=$ $7.76(\mathrm{dd}, J=7.9,1.0 \mathrm{~Hz}, 1 \mathrm{H}), 7.71(\mathrm{dd}, J=8.2,1.7 \mathrm{~Hz}, 1 \mathrm{H}), 7.67(\mathrm{dd}, J=7.6,1.7 \mathrm{~Hz}, 1 \mathrm{H})$, $7.59(\mathrm{~d}, J=1.7 \mathrm{~Hz}, 1 \mathrm{H}), 7.52(\mathrm{dt}, J=7.4,1.0 \mathrm{~Hz}, 1 \mathrm{H}), 7.45(\mathrm{dt}, J=7.6,1.7 \mathrm{~Hz}, 1 \mathrm{H}), 7.07$ (d, $J=8.2 \mathrm{~Hz}, 1 \mathrm{H}), 6.81(\mathrm{~s}, 1 \mathrm{H}), 6.15(\mathrm{~s}, 2 \mathrm{H})$ (in ${ }^{1} \mathrm{H}$ NMR spectrum $4 \%$ of diketone form is present). ${ }^{13} \mathrm{C}$ NMR (100.6 MHz, DMSO[D 6 ]) $\delta=186.7,184.6,151.9,148.3,137.6,133.8$, $132.5,130.4,128.4,128.1,123.9,119.7,108.7,107.1,102.4,97.6$. HRMS $(m / z)[\mathrm{M}+\mathrm{H}]^{+}$ calcd for $\mathrm{M}=\mathrm{C}_{16} \mathrm{H}_{11}{ }^{79} \mathrm{BrO}_{4}, 346.9919$; found, 346.9921 .

\section{3-(1,3-Benzodioxol-5-yl)-5-(2-bromophenyl)-1H-pyrazole (anle234b)}

The title compound was prepared according to the GEP 2 starting from anle234a. White solid. Yield 60\%. m.p.: $170{ }^{\circ} \mathrm{C} .{ }^{1} \mathrm{H}$ NMR (400 MHz, DMSO[D $]+1-2 \%$ conc. DCl) $\delta=7.73$ $(\mathrm{d}, J=7.9 \mathrm{~Hz}, 1 \mathrm{H}), 7.63(\mathrm{~d}, J=7.4 \mathrm{~Hz}, 1 \mathrm{H}), 7.46(\mathrm{t}, J=7.4 \mathrm{~Hz}, 1 \mathrm{H}), 7.38(\mathrm{~s}, 1 \mathrm{H}), 7.35-7.25$ $(\mathrm{m}, 2 \mathrm{H}), 7.05-6.90(\mathrm{~m}, 2 \mathrm{H}), 6.05(\mathrm{~s}, 2 \mathrm{H}) .{ }^{13} \mathrm{C} \mathrm{NMR}\left(100.6 \mathrm{MHz}, \mathrm{DMSO}\left[\mathrm{D}_{6}\right]+1-2 \%\right.$ conc. DCl) $\delta=148.0,147.2,146.0$ (2C), 133.6, 133.1, 131.3, 130.0, 128.0, 125.4, 121.6, 119.2, 108.9, 105.9, 103.0, 101.4. HRMS $(m / z)[\mathrm{M}+\mathrm{H}]^{+}$calcd for $\mathrm{M}=\mathrm{C}_{16} \mathrm{H}_{11}{ }^{79} \mathrm{BrN}_{2} \mathrm{O}_{2}, 343.0082$; found, 343.0087 .

\section{1-(1,3-Benzodioxol-5-yl)-3-[3-(trifluoromethyl)phenyl]propane-1,3-dione (anle236a)}

The title was prepared according to the GEP 1 using 1-(1,3-benzodioxol-5-yl)ethanone and methyl 3-(trifluoromethyl)benzoate and purified by recrystallisation from ethanol/water to provide anle236a as a yellow solid. Yield 83\%. m.p.: 97-99 ${ }^{\circ} \mathrm{C} .{ }^{1} \mathrm{H}$ NMR $(400 \mathrm{MHz}$, DMSO[D 6$]) \delta=8.50-8.39(\mathrm{~m}, 2 \mathrm{H}), 7.98(\mathrm{~d}, J=7.7 \mathrm{~Hz}, 1 \mathrm{H}), 7.86(\mathrm{dd}, J=8.2,1.7 \mathrm{~Hz}, 1 \mathrm{H})$, 7.83-7.74 (m, 2H), $7.39(\mathrm{~s}, 1 \mathrm{H}), 7.10(\mathrm{~d}, J=8.2 \mathrm{~Hz}, 1 \mathrm{H}), 6.16(\mathrm{~s}, 2 \mathrm{H})\left(\right.$ in ${ }^{1} \mathrm{H}$ NMR spectrum $6 \%$ of diketone form is present). ${ }^{13} \mathrm{C}$ NMR $\left(100.6 \mathrm{MHz}, \mathrm{DMSO}\left[\mathrm{D}_{6}\right]\right) \delta=186.6,181.2,152.0$, 148.2, 135.7. 131.3, 130.2, 129.9 (q, $J=32.3 \mathrm{~Hz}), 129.1$ (q, $J=3.6 \mathrm{~Hz}), 128.9,124.2,124.1$ $(\mathrm{q}, J=273.3 \mathrm{~Hz}), 123.8(\mathrm{q}, J=3.6 \mathrm{~Hz}), 108.6,107.5,102.4,93.5$. HRMS $(m / z)[\mathrm{M}+\mathrm{H}]^{+}$calcd for $\mathrm{M}=\mathrm{C}_{17} \mathrm{H}_{11} \mathrm{~F}_{3} \mathrm{O}_{4}, 337.0688$; found, 337.0688.

\section{3-(1,3-Benzodioxol-5-yl)-5-[3-(trifluoromethyl)phenyl]-1H-pyrazole (anle236b)}

The title compound was prepared according to the GEP 2 starting from anle236a. White solid. Yield 90\%. m.p.: 220-222 ${ }^{\circ} \mathrm{C} .{ }^{1} \mathrm{H}$ NMR (400 MHz, DMSO[D 6$]+1-2 \%$ conc. DCl) $\delta=$ $8.14(\mathrm{~s}, 1 \mathrm{H}), 8.11(\mathrm{t}, J=3.6 \mathrm{~Hz}, 1 \mathrm{H}), 7.75-7.60(\mathrm{~m}, 2 \mathrm{H}), 7.39(\mathrm{~d}, J=1.6 \mathrm{~Hz}, 1 \mathrm{H}), 7.34(\mathrm{dd}, J$ $=8.0,1.6 \mathrm{~Hz}, 1 \mathrm{H}), 7.27(\mathrm{~s}, 1 \mathrm{H}), 7.00(\mathrm{~d}, J=8.0 \mathrm{~Hz}, 1 \mathrm{H}), 6.06(\mathrm{~s}, 2 \mathrm{H}) .{ }^{13} \mathrm{C}$ NMR $(100.6 \mathrm{MHz}$, DMSO $\left[\mathrm{D}_{6}\right]+1-2 \%$ conc. DCl) $\delta=148.9,148.8,148.0,147.4,133.6,130.2,129.9(\mathrm{q}, J=$ $31.6 \mathrm{~Hz}), 129.1,124.9,124.5$ (q, $J=272.4 \mathrm{~Hz}), 124.3(\mathrm{q}, J=3.6 \mathrm{~Hz}), 121.5(\mathrm{q}, \mathrm{J}=3.7 \mathrm{~Hz})$, 119.2, 109.0, 105.8, 101.5, 100.2. HRMS $(\mathrm{m} / \mathrm{z})[\mathrm{M}+\mathrm{H}]^{+}$calcd for $\mathrm{M}=\mathrm{C}_{17} \mathrm{H}_{11} \mathrm{~F}_{3} \mathrm{~N}_{2} \mathrm{O}_{2}$, 333.0851; found, 333.0851.

\section{3-(3,4-dihydroxyphenyl)-5-[3-(trifluoromethyl)phenyl]-1H-pyrazole (anle236c)}

The title compound was prepared according to the GEP 3 starting from anle236b. Beige solid. Yield 100\%. m.p.: 270-280 ${ }^{\circ} \mathrm{C}(\mathrm{dec}) .{ }^{1} \mathrm{H}$ NMR (400 MHz, DMSO[D 6$]+1-2 \%$ conc. 
DCl) $\delta=8.15(\mathrm{~s}, 1 \mathrm{H}), 8.12(\mathrm{t}, J=4.3 \mathrm{~Hz}, 1 \mathrm{H}), 7.71-7.57(\mathrm{~m}, 2 \mathrm{H}), 7.20(\mathrm{~d}, J=2.0 \mathrm{~Hz}, 1 \mathrm{H})$, 7.14-7.05 (m, 2H), $6.81(\mathrm{~d}, J=8.1 \mathrm{~Hz}, 1 \mathrm{H}) .{ }^{13} \mathrm{C} \mathrm{NMR}\left(100.6 \mathrm{MHz}, \mathrm{DMSO}\left[\mathrm{D}_{6}\right]+1-2 \%\right.$ conc. DCl) $\delta=147.8,146.5,146.0,145.8,134.0,130.1,129.8(\mathrm{q}, J=31.6 \mathrm{~Hz}), 129.1,124.5(J=$ $272.6 \mathrm{~Hz}), 124.1,122.0,121.5,117.1,116.1,113.2,99.4$. HRMS $(m / z)[\mathrm{M}+\mathrm{H}]^{+}$calcd for $\mathrm{M}=$ $\mathrm{C}_{16} \mathrm{H}_{11} \mathrm{~F}_{3} \mathrm{~N}_{2} \mathrm{O}_{2}, 321.0851$; found, 321.0850 .

\section{1-(1,3-Benzodioxol-5-yl)-3-(3,5-dibromophenyl)propane-1,3-dione (anle246a)}

The title compound was prepared according to the GEP 1 using 1-(1,3-benzodioxol-5yl)ethanone and methyl 3,5-dibromobenzoate and purified by recrystallisation from ethanol/water to provide anle246a as a yellow solid. Yield 80\%. m.p.: $181-182{ }^{\circ} \mathrm{C} .{ }^{1} \mathrm{H}$ NMR $\left(400 \mathrm{MHz}, \mathrm{DMSO}\left[\mathrm{D}_{6}\right]\right) \delta=8.31(\mathrm{~d}, J=1.7 \mathrm{~Hz}, 2 \mathrm{H}), 8.08(\mathrm{t}, J=1.6 \mathrm{~Hz}, 1 \mathrm{H}), 7.85(\mathrm{dd}, J=$ 8.2, $1.7 \mathrm{~Hz}, 1 \mathrm{H}), 7.77(\mathrm{~d}, J=1.6 \mathrm{~Hz}, 1 \mathrm{H}), 7.33(\mathrm{~s}, J=8.2 \mathrm{~Hz}, 1 \mathrm{H}), 7.09$ (d, $J=\mathrm{Hz}, 1 \mathrm{H}), 6.17$ $\left(\mathrm{s}, 2 \mathrm{H}\right.$ ) (in ${ }^{1} \mathrm{H}$ NMR spectrum $5 \%$ of diketone form is present). ${ }^{13} \mathrm{C}$ NMR $(100.6 \mathrm{MHz}$, DMSO[D 6 ]) $\delta=186.8,180.1,152.4,148.5,138.7,137.5,129.3,129.1,124.7,123.6,108.9$, 107.9, 102.7, 94.0. HRMS $(m / z)[\mathrm{M}+\mathrm{H}]^{+}$calcd for $\mathrm{M}=\mathrm{C}_{16} \mathrm{H}_{10}{ }^{79} \mathrm{Br}^{81} \mathrm{BrO}_{4}, 426.9004$; found, 426.8998 .

\section{3-(1,3-Benzodioxol-5-yl)-5-(3,5-dibromophenyl)-1H-pyrazole (anle246b)}

The title compound was prepared according to the GEP 2 starting from anle246a. White solid. Yield 95\%. m.p.: 264-265 ${ }^{\circ} \mathrm{C} .{ }^{1} \mathrm{H}$ NMR (400 MHz, DMSO[D 6$]+1-2 \%$ conc. DCl) $\delta=$ $8.01(\mathrm{~d}, J=1.7 \mathrm{~Hz}, 2 \mathrm{H}), 7.75(\mathrm{t}, J=1.7 \mathrm{~Hz}, 1 \mathrm{H}), 7.35(\mathrm{~d}, J=1.6 \mathrm{~Hz}, 1 \mathrm{H}), 7.31(\mathrm{dd}, J=8.0$, $1.6 \mathrm{~Hz}, 1 \mathrm{H}), 7.28(\mathrm{~s}, 1 \mathrm{H}), 7.00(\mathrm{~d}, J=8.0 \mathrm{~Hz}, 1 \mathrm{H}), 6.05(\mathrm{~s}, 2 \mathrm{H}) .{ }^{13} \mathrm{C}$ NMR $(100.6 \mathrm{MHz}$, DMSO $\left[\mathrm{D}_{6}\right]+1-2 \%$ conc. DCl) $\delta=148.1,147.4,146.8-145.8$ (2C), 136.5, 132.3, 126.8, $124.6,123.3,119.2,109.0,105.8,101.5,100.6$. HRMS $(m / z)[\mathrm{M}+\mathrm{H}]^{+}$calcd for $\mathrm{M}=$ $\mathrm{C}_{16} \mathrm{H}_{10}{ }^{79} \mathrm{Br}^{81} \mathrm{BrN}_{2} \mathrm{O}_{2}, 422.9167$; found, 422.9159.

\section{1-(3-Bromophenyl)-3-[4-(dimethylamino)phenyl]propane-1,3-dione (anle253a)}

The title compound was prepared according to the GEP 1 using 1-[4(dimethylamino)phenyl]ethanone and methyl 3-bromobenzoate and purified by recrystallisation from ethanol/water to provide anle253a as a yellow solid. Yield 94\%. m.p.: 123-124 ${ }^{\circ} \mathrm{C} .{ }^{1} \mathrm{H}$ NMR $\left(400 \mathrm{MHz}, \mathrm{DMSO}\left[\mathrm{D}_{6}\right]\right) \delta=8.28(\mathrm{t}, J=1.7 \mathrm{~Hz}, 1 \mathrm{H}), 8.09(\mathrm{~d}, J=8.0$ $\mathrm{Hz}, 1 \mathrm{H}), 8.05(\mathrm{~d}, J=9.1 \mathrm{~Hz}, 2 \mathrm{H}), 7.77(\mathrm{~d}, J=8.0 \mathrm{~Hz}, 1 \mathrm{H}), 7.48(\mathrm{t}, J=7.9 \mathrm{~Hz}, 1 \mathrm{H}), 7.20$ (s, $1 \mathrm{H}), 6.76(\mathrm{~d}, J=9.1 \mathrm{~Hz}, 2 \mathrm{H}), 3.05(\mathrm{~s}, 6 \mathrm{H})$ (in ${ }^{1} \mathrm{H}$ NMR spectrum $6 \%$ of diketone form is present). ${ }^{13} \mathrm{C}$ NMR (100.6 MHz, DMSO[D 6 ]) $\delta=189.9,179.5,153.8,137.5,134.8,131.0$, $130.0,129.5,126.0,122.4,121.2,111.2,92.3,39.8$. HRMS $(m / z)[\mathrm{M}+\mathrm{H}]^{+}$calcd for $\mathrm{M}=$ $\mathrm{C}_{17} \mathrm{H}_{16}{ }^{79} \mathrm{BrNO}_{2}, 346.0443$; found, 346.0437.

\section{3-(3-Bromophenyl)-5-[4-(dimethylamino)phenyl]-1H-pyrazole (anle253b)}

The title compound was prepared according to the GEP 2 starting from anle253a. White solid. Yield 85\%. m.p.: 185-187 ${ }^{\circ} \mathrm{C} .{ }^{1} \mathrm{H}$ NMR (400 MHz, DMSO[D 6$\left.]\right) \delta=8.01(\mathrm{~s}, 1 \mathrm{H}), 7.83$ $(\mathrm{d}, J=7.2 \mathrm{~Hz}, 1 \mathrm{H}), 7.61(\mathrm{~d}, J=7.4 \mathrm{~Hz}, 2 \mathrm{H}), 7.48(\mathrm{~d}, J=7.2 \mathrm{~Hz}, 1 \mathrm{H}), 7.37(\mathrm{t}, J=7.2 \mathrm{~Hz}$, $1 \mathrm{H}), 7.04(\mathrm{~s}, 1 \mathrm{H}), 6.76(\mathrm{~d}, J=7.4 \mathrm{~Hz}, 2 \mathrm{H}), 2.92(\mathrm{~s}, 6 \mathrm{H}) .{ }^{13} \mathrm{C} \mathrm{NMR}\left(100.6 \mathrm{MHz}, \mathrm{DMSO}\left[\mathrm{D}_{6}\right]\right)$ $\delta=150.4,149.8,144.6,136.6,131.1,130.1,127.7,126.3,124.2,122.4,117.1,112.5,98.3$, 40.1. HRMS $(\mathrm{m} / \mathrm{z})[\mathrm{M}+\mathrm{H}]^{+}$calcd for $\mathrm{M}=\mathrm{C}_{17} \mathrm{H}_{16}{ }^{79} \mathrm{BrN}_{3}$, 342.0606; found, 342.0611 .

\section{1-(3-Chlorophenyl)-3-[4-(dimethylamino)phenyl]propane-1,3-dione (anle254a)}

The title compound was prepared according to the GEP 1 using 1-[4(dimethylamino)phenyl]ethanone and methyl 3-chlorobenzoate and purified by recrystallisation from ethanol/water to provide anle254a as a yellow solid. Yield $85 \%$. m.p.: 120-122 ${ }^{\circ} \mathrm{C} .{ }^{1} \mathrm{H}$ NMR $\left(400 \mathrm{MHz}, \mathrm{DMSO}\left[\mathrm{D}_{6}\right]\right) \delta=8.15(\mathrm{t}, J=1.6 \mathrm{~Hz}, 1 \mathrm{H}), 8.05$ (d, $J=9.0$ 
$\mathrm{Hz}, 3 \mathrm{H}), 7.64(\mathrm{~d}, J=7.9 \mathrm{~Hz}, 1 \mathrm{H}), 7.55$ (t, $J=7.9 \mathrm{~Hz}, 1 \mathrm{H}), 7.20(\mathrm{~s}, 1 \mathrm{H}), 6.77$ (d, $J=9.1 \mathrm{~Hz}$, $2 \mathrm{H}), 3.05(\mathrm{~s}, 6 \mathrm{H})$ (in ${ }^{1} \mathrm{H}$ NMR spectrum $6 \%$ of diketone form is present). ${ }^{13} \mathrm{C}$ NMR $(100.6$ $\left.\mathrm{MHz}, \mathrm{DMSO}\left[\mathrm{D}_{6}\right]\right) \delta=187.0,179.5,153.8,133.9,131.9,130.8,130.0,126.7,125.6,121.2$ (2C), 111.2, 92.3, 39.8. HRMS $(\mathrm{m} / \mathrm{z})[\mathrm{M}+\mathrm{Na}]^{+}$calcd for $\mathrm{M}=\mathrm{C}_{17} \mathrm{H}_{16} \mathrm{ClNO}_{2}, 324.0767$; found, 324.0774 .

\section{3-(3-Chlorophenyl)-5-[4-(dimethylamino)phenyl]-1H-pyrazole (anle254b)}

The title compound was prepared according to the GEP 2 starting from anle254a. White solid. Yield 85\%. m.p.: $184-186{ }^{\circ} \mathrm{C} .{ }^{1} \mathrm{H}$ NMR (400 MHz, DMSO[D 6$]+1-2 \%$ conc. DCl) $\delta=$ $7.87(\mathrm{t}, J=1.6 \mathrm{~Hz}, 1 \mathrm{H}), 7.79$ (d, $J=7.8 \mathrm{~Hz}, 1 \mathrm{H}), 7.63$ (br d, $J=8.8 \mathrm{~Hz}, 2 \mathrm{H}), 7.44$ (t, $J=7.8$ $\mathrm{Hz}, 1 \mathrm{H}), 7.35(\mathrm{~d}, J=7.8 \mathrm{~Hz}, 1 \mathrm{H}), 7.06(\mathrm{~s}, 1 \mathrm{H}), 6.80(\mathrm{~d}, J=8.8 \mathrm{~Hz}, 2 \mathrm{H}), 2.94(\mathrm{~s}, 6 \mathrm{H}) .{ }^{13} \mathrm{C}$ NMR (100.6 MHz, DMSO[D 6 + 1-2\% conc. DCl) $\delta=150.1,148.1,146.5,135.5,135.3$, $133.8,130.8,127.4,126.3,124.8,123.8,112.8,98.7,40.4$. HRMS $(m / z)[\mathrm{M}+\mathrm{H}]^{+}$calcd for $\mathrm{M}$ $=\mathrm{C}_{17} \mathrm{H}_{16} \mathrm{ClN}_{3}, 298.1111$; found, 298.1111.

\section{1-(3,4-Dimethoxyphenyl)-3-(3-fluorophenyl)propane-1,3-dione (sery157)}

The title compound was prepared according to the GEP 1 using 1-(3,4dimethoxyphenyl)ethanone and ethyl 3-fluorobenzoate and purified by recrystallisation from methanol to provide sery157 as a yellow solid. Yield 92\%. m.p.: 91-92 ${ }^{\circ} \mathrm{C} .{ }^{1} \mathrm{H}$ NMR (400 $\left.\mathrm{MHz}, \mathrm{DMSO}\left[\mathrm{D}_{6}\right]\right) \delta=8.03-7.95(\mathrm{~m}, 2 \mathrm{H}), 7.90(\mathrm{dd}, J=8.5,2.0 \mathrm{~Hz}, 1 \mathrm{H}), 7.66-7.57(\mathrm{~m}, 2 \mathrm{H})$, $7.48(\mathrm{td}, J=8.5,2.4 \mathrm{~Hz}, 1 \mathrm{H}), 7.31(\mathrm{~s}, 1 \mathrm{H}), 7.12(\mathrm{~d}, J=8.6 \mathrm{~Hz}, 1 \mathrm{H}), 3.87(\mathrm{~s}, 6 \mathrm{H})\left(\right.$ in ${ }^{1} \mathrm{H}$ NMR spectrum $7 \%$ of diketone form is present). ${ }^{13} \mathrm{C}$ NMR $\left(100.6 \mathrm{MHz}, \mathrm{DMSO}\left[\mathrm{D}_{6}\right]\right) \delta=186.7$, $181.2,162.3(\mathrm{~d}, J=244.3 \mathrm{~Hz}), 153.4,148.8,137.0(\mathrm{~d}, J=7.1 \mathrm{~Hz}), 130.9(\mathrm{~d}, J=8.1 \mathrm{~Hz})$, 127.1, 123.3, 122.3, 119.4 (d, $J=21.3 \mathrm{~Hz}), 113.8$ (d, $J=23.0 \mathrm{~Hz}), 111.3,110.2,93.0,55.8$, 55.75. HRMS $(m / z)[\mathrm{M}+\mathrm{H}]^{+}$calcd for $\mathrm{M}=\mathrm{C}_{17} \mathrm{H}_{15} \mathrm{FO}_{4}, 303.1033$; found, 303.1032 .

\section{3-(3,4-Dimethoxyphenyl)-5-(3-fluorophenyl)-1H-pyrazole (sery161)}

The title compound was prepared according to the GEP 2 starting from sery157. White solid. Yield 95\%. m.p.: $172-173{ }^{\circ} \mathrm{C} .{ }^{1} \mathrm{H}$ NMR (400 MHz, DMSO[D 6$]+1-2 \%$ conc. DCl) $\delta=13.30$ (bs, $1 \mathrm{H}), 7.69(\mathrm{~d}, J=7.8 \mathrm{~Hz}, 1 \mathrm{H}), 7.64(\mathrm{~d}, J=10.2 \mathrm{~Hz}, 1 \mathrm{H}), 7.51-7.44(\mathrm{~m}, 1 \mathrm{H}), 7.41(\mathrm{~d}, J=$ $1.7 \mathrm{~Hz}, 1 \mathrm{H}), 7.36(\mathrm{dd}, J=8.3,1.7 \mathrm{~Hz}, 1 \mathrm{H}), 7.20(\mathrm{~s}, 1 \mathrm{H}), 7.14(\mathrm{td}, J=8.6,2.3 \mathrm{~Hz}, 1 \mathrm{H}), 7.03$ $(\mathrm{d}, J=8.3 \mathrm{~Hz}, 1 \mathrm{H}), 3.84(\mathrm{~s}, 3 \mathrm{H}), 3.79(\mathrm{~s}, 3 \mathrm{H}) .{ }^{13} \mathrm{C}$ NMR $\left(100.6 \mathrm{MHz}, \mathrm{DMSO}\left[\mathrm{D}_{6}\right]+1-2 \%\right.$ conc. DCl) $\delta=162.7(\mathrm{~d}, J=242.7 \mathrm{~Hz}), 149.1,148.9,147.8,146.6,134.9,130.9$ (d, $J=8.5$ $\mathrm{Hz}), 123.4,121.2,117.8,114.3(\mathrm{~d}, J=21.2 \mathrm{~Hz}), 112.2,111.7(\mathrm{~d}, J=22.7 \mathrm{~Hz}), 109.1,99.8$, 55.7, 55.67. HRMS $(m / z)[\mathrm{M}+\mathrm{H}]^{+}$calcd for $\mathrm{M}=\mathrm{C}_{17} \mathrm{H}_{15} \mathrm{FN}_{2} \mathrm{O}_{2}, 299.1196$; found, 299.1197.

\section{3-(3,4-Dihydroxyphenyl)-5-(3-fluorophenyl)-1 H-pyrazole (sery85)}

The title compound was prepared according to the GEP 3 starting from sery161. White solid. Yield 85\%. m.p.: 231-233 ${ }^{\circ} \mathrm{C} .{ }^{1} \mathrm{H}$ NMR (400 MHz, DMSO[D 6$]+1-2 \%$ conc. DCl) $\delta=7.68$ $(\mathrm{d}, J=7.8 \mathrm{~Hz}, 1 \mathrm{H}), 7.66-7.60(\mathrm{~m}, 1 \mathrm{H}), 7.47(\mathrm{td}, J=8.2,6.2 \mathrm{~Hz}, 1 \mathrm{H}), 7.20(\mathrm{~d}, J=2.1 \mathrm{~Hz}, 1 \mathrm{H})$, $7.15(\mathrm{td}, J=8.2,2.3 \mathrm{~Hz}, 1 \mathrm{H}), 7.09(\mathrm{dd}, J=8.2,2.1 \mathrm{~Hz}, 1 \mathrm{H}), 7.04(\mathrm{~s}, 1 \mathrm{H}), 6.81(\mathrm{~d}, J=8.2 \mathrm{~Hz}$, 1H). ${ }^{13} \mathrm{C}$ NMR (100.6 MHz, DMSO[D $]+1-2 \%$ conc. DCl) $\delta=162.7(\mathrm{~d}, J=242.9 \mathrm{~Hz})$, 147.5, 146.7, 145.9, 145.6, $134.6(\mathrm{~d}, J=8.0 \mathrm{~Hz}), 130.9$ (d, $J=7.4 \mathrm{~Hz}), 121.7,121.4,117.0$, 116.0, $114.5(\mathrm{~d}, J=21.3 \mathrm{~Hz}), 113.1,111.8(\mathrm{~d}, J=23.7 \mathrm{~Hz}), 99.4$. HRMS $(m / z)[\mathrm{M}+\mathrm{H}]^{+}$calcd for $\mathrm{M}=\mathrm{C}_{15} \mathrm{H}_{11} \mathrm{FN}_{2} \mathrm{O}_{2}, 271.0883$; found, 271.0882.

\section{3-(3,4-Dihydroxyphenyl)-5-phenyl-1 $H$-pyrazole (sery106)}

The title compound was prepared according to the GEP 3 starting from anle126. White solid. Yield 90\%. m.p.: 224-229 ${ }^{\circ} \mathrm{C} .{ }^{1} \mathrm{H}$ NMR (400 MHz, DMSO[D $]$ ) $\delta=7.81(\mathrm{~d}, J=7.5 \mathrm{~Hz}, 2 \mathrm{H})$, 7.43 (t, $J=7.5 \mathrm{~Hz}, 2 \mathrm{H}), 7.32$ (t, $J=7.5 \mathrm{~Hz}, 1 \mathrm{H}), 7.20$ (d, $J=1.4 \mathrm{~Hz}, 1 \mathrm{H}), 7.09$ (dd, $J=8.1$, 
$1.4 \mathrm{~Hz}, 1 \mathrm{H}), 6.95(\mathrm{~s}, 1 \mathrm{H}), 6.79(\mathrm{~d}, J=8.1 \mathrm{~Hz}, 1 \mathrm{H}) .{ }^{13} \mathrm{C}$ NMR $\left(100.6 \mathrm{MHz}, \mathrm{DMSO}\left[\mathrm{D}_{6}\right]\right) \delta=$ $147.7,147.0,145.7,145.5,131.7,128.7,127.8,125.2,122.3,116.8,115.8,112.9,98.7$. HRMS $(m / z)[\mathrm{M}+\mathrm{H}]^{+}$calcd for $\mathrm{M}=\mathrm{C}_{15} \mathrm{H}_{12} \mathrm{~N}_{2} \mathrm{O}_{2}, 253.0977$; found, 253.0975 .

\section{1-(3,4-Dimethoxyphenyl)-3-(4-methoxyphenyl)propane-1,3-dione (sery150)}

The title compound has been reported previously ${ }^{522}$ (CAS 80370-28-1). It was prepared according to the GEP 1 using 1-(4-methoxyphenyl)ethanone and ethyl 3,4dimethoxybenzoate and purified by recrystallisation from acetonitrile/methanol to provide sery150 as a yellow solid. Yield 67\%. m.p.: 95-96 ${ }^{\circ} \mathrm{C}$. ( $\left(\mathrm{lit}^{522} 97{ }^{\circ} \mathrm{C}\right) .{ }^{1} \mathrm{H}$ NMR $(400 \mathrm{MHz}$, DMSO[D 6 ]) $\delta=8.14(\mathrm{~d}, J=8.9 \mathrm{~Hz}, 2 \mathrm{H}), 7.83(\mathrm{dd}, J=8.5,1.9 \mathrm{~Hz}, 1 \mathrm{H}), 7.62(\mathrm{~d}, J=1.9 \mathrm{~Hz}$, $1 \mathrm{H}), 7.19(\mathrm{~s}, 1 \mathrm{H}), 7.14-7.05(\mathrm{~m}, 3 \mathrm{H}), 3.87(\mathrm{~s}, 3 \mathrm{H}), 3.86(\mathrm{~s}, 6 \mathrm{H})\left(\right.$ in ${ }^{1} \mathrm{H}$ NMR spectrum $15 \%$ of diketone form is present).

\section{3-(3,4-Dimethoxyphenyl)-5-(4-methoxyphenyl)-1 $H$-pyrazole (sery156)}

The title compound was prepared according to the GEP 2 starting from sery150. White solid. Yield 90\%. m.p.: 82-85 ${ }^{\circ} \mathrm{C} .{ }^{1} \mathrm{H}$ NMR (400 MHz, DMSO[D 6$]+1-2 \%$ conc. DCl) $\delta=7.74$ (d, $J=8.7 \mathrm{~Hz}, 2 \mathrm{H}), 7.41(\mathrm{~d}, J=1.7 \mathrm{~Hz}, 1 \mathrm{H}), 7.36(\mathrm{dd}, J=8.3,1.7 \mathrm{~Hz}, 1 \mathrm{H}), 7.09-6.94(\mathrm{~m}, 4 \mathrm{H})$, $3.84(\mathrm{~s}, 3 \mathrm{H}), 3.79(\mathrm{~s}, 3 \mathrm{H}), 3.78(\mathrm{~s}, 3 \mathrm{H}) .{ }^{13} \mathrm{C} \mathrm{NMR}\left(100.6 \mathrm{MHz}, \mathrm{DMSO}\left[\mathrm{D}_{6}\right]\right) \delta=158.9,151.1$, 149.0, 148.6, 143.6, 126.4, 125.7, 122.6, 117.6, 114.2, 112.1, 109.0, 98.4, 55.6, 55.5, 55.1. $\operatorname{HRMS}(m / z)[\mathrm{M}+\mathrm{H}]^{+}$calcd for $\mathrm{M}=\mathrm{C}_{18} \mathrm{H}_{18} \mathrm{~N}_{2} \mathrm{O}_{3}, 311.1396$; found, 311.1397.

\section{1-(3,4-Dimethoxyphenyl)-3-(2-fluorophenyl)propane-1,3-dione (sery219b)}

The title compound was prepared according to the GEP 1 using 1-(3,4dimethoxyphenyl)ethanone and methyl 2-fluorobenzoate and purified by recrystallisation from ethanol/water to provide sery219b as a yellow solid. Yield 30\%. ${ }^{1} \mathrm{H}$ NMR $(400 \mathrm{MHz}$, DMSO[D 6$]) \delta=7.94(\mathrm{t}, J=7.6 \mathrm{~Hz}, 1 \mathrm{H}), 7.72(\mathrm{dd}, J=8.5,1.6 \mathrm{~Hz}, 1 \mathrm{H}), 7.68-7.60(\mathrm{~m}, 1 \mathrm{H})$, $7.54(\mathrm{~d}, J=1.6 \mathrm{~Hz}, 1 \mathrm{H}), 7.43-7.34(\mathrm{~m}, 2 \mathrm{H}), 7.11(\mathrm{~d}, J=8.5 \mathrm{~Hz}, 1 \mathrm{H}), 7.00(\mathrm{~s}, 1 \mathrm{H}), 3.86(\mathrm{~s}$, $3 \mathrm{H}), 3.85$ (s, $3 \mathrm{H})$ (in ${ }^{1} \mathrm{H}$ NMR spectrum $15 \%$ of diketone form is present). ${ }^{13} \mathrm{C}$ NMR $(100.6$ MHz, DMSO[D 6$]) \delta=186.6,179.6,160.2(\mathrm{~d}, J=254.3 \mathrm{~Hz}), 153.3,148.8,134.1(\mathrm{~d}, J=9.1$ $\mathrm{Hz}), 129.9,127.0,124.9,123.2(\mathrm{~d}, J=10.4 \mathrm{~Hz}), 121.9,116.8$ (d, $J=22.4 \mathrm{~Hz}), 111.3,109.8$, $96.8(\mathrm{~d}, J=7.9 \mathrm{~Hz}), 55.8,55.6$. ESI-MS $(\mathrm{m} / \mathrm{z})[\mathrm{M}+\mathrm{H}]^{+}$calcd for $\mathrm{M}=\mathrm{C}_{17} \mathrm{H}_{15} \mathrm{FO}_{4}, 303.10$; found, 303.24 .

\section{3-(3,4-Dimethoxyphenyl)-5-(2-fluorophenyl)-1H-pyrazole (sery221b)}

The title compound was prepared according to the GEP 2 starting from sery219b. White solid. Yield 87\%. ${ }^{1} \mathrm{H}$ NMR (400 MHz, DMSO[D 6$]+1-2 \%$ conc. DCl) $\delta=7.95$ (t, $J=7.8 \mathrm{~Hz}$, 1H), 7.46-7.24 (m, 5H), 7.07-6.97 (m, 2H), 3.84 (s, 3H), 3.79 (s, 3H). ${ }^{13} \mathrm{C}$ NMR $(100.6 \mathrm{MHz}$, DMSO[D 6$]) \delta=159.2(\mathrm{~d}, J=247.8 \mathrm{~Hz}), 149.0,148.9,145.7,143.3,137.1,129.1(\mathrm{~d}, J=22.4$ $\mathrm{Hz}$ ), 128.1, 124.6, 121.9, 117.7, 116.1 (d, $J=8.3 \mathrm{~Hz}$ ), 112.1, 109.1, 101.9, 55.7, 55.6 (main regioisomer is described). ESI-MS $(\mathrm{m} / \mathrm{z})[\mathrm{M}+\mathrm{H}]^{+}$calcd for $\mathrm{M}=\mathrm{C}_{17} \mathrm{H}_{15} \mathrm{FN}_{2} \mathrm{O}_{2}$, 299.32; found, 299.22 .

\section{1-(3,5-Difluoroxyphenyl)-3-(3,4-dimethoxyphenyl)propane-1,3-dione (sery219e)}

The title compound was prepared according to the GEP 1 using 1-(3,4dimethoxyphenyl)ethanone and methyl 3,5-difluorobenzoate and purified by recrystallisation from methanol to provide sery219e as a yellow solid. Yield $88 \%$. ${ }^{1} \mathrm{H}$ NMR $(400 \mathrm{MHz}$, DMSO[D 6$]) \delta=7.96-7.80(\mathrm{~m}, 3 \mathrm{H}), 7.63(\mathrm{~d}, J=1.9 \mathrm{~Hz}, 1 \mathrm{H}), 7.52(\mathrm{tt}, J=9.1,2.8 \mathrm{~Hz}, 1 \mathrm{H})$, $7.32(\mathrm{~s}, 1 \mathrm{H}), 7.11(\mathrm{~d}, J=8.6 \mathrm{~Hz}, 1 \mathrm{H}), 3.87(\mathrm{~m}, 6 \mathrm{H})$ (in ${ }^{1} \mathrm{H}$ NMR spectrum $5 \%$ of diketone form is present). ${ }^{13} \mathrm{C} \mathrm{NMR}\left(100.6 \mathrm{MHz}, \mathrm{DMSO}_{\left.\left[\mathrm{D}_{6}\right]\right)} \delta=187.0,179.6,162.7\right.$ (dd, $J=246.9$, $13.0 \mathrm{~Hz}), 153.5,148.8,138.2$ (d, $J=9.2 \mathrm{~Hz}), 126.9,122.5,111.2,110.6-110.5$ (m, 2C), 107.7 
$(\mathrm{t}, J=25.8 \mathrm{~Hz}), 93.3,55.8,55.75$. ESI-MS $(m / z)[\mathrm{M}+\mathrm{H}]^{+}$calcd for $\mathrm{M}=\mathrm{C}_{17} \mathrm{H}_{14} \mathrm{~F}_{2} \mathrm{O}_{4}, 321.09$; found, 321.19 .

\section{3-(3,5-Difluorophenyl)-5-(3,4-dimethoxyphenyl)-1H-pyrazole (sery221c)}

The title compound was prepared according to the GEP 2 starting from sery219e. White solid. Yield 81\%. ${ }^{1} \mathrm{H}$ NMR (400 MHz, DMSO[D 6$]+1-2 \%$ conc. DCl) $\delta=7.54(\mathrm{~d}, J=6.8 \mathrm{~Hz}$, 2H), $7.40(\mathrm{~d}, J=1.6 \mathrm{~Hz}, 1 \mathrm{H}), 7.34(\mathrm{dd}, J=8.4,1.7 \mathrm{~Hz}, 1 \mathrm{H}), 7.27(\mathrm{~s}, 1 \mathrm{H})), 7.17(\mathrm{~m}, 1 \mathrm{H}), 7.04$ $(\mathrm{d}, J=8.4 \mathrm{~Hz}, 1 \mathrm{H}) 3.84(\mathrm{~s}, 3 \mathrm{H}), 3.79(\mathrm{~s}, 3 \mathrm{H}) \cdot{ }^{13} \mathrm{C} \mathrm{NMR}\left(100.6 \mathrm{MHz}, \mathrm{DMSO}\left[\mathrm{D}_{6}\right]+1-2 \%\right.$ conc. DCl) $\delta=162.9(\mathrm{dd}, J=244.9,13.4 \mathrm{~Hz}), 149.2,149.0,148.95,144.0,137.5(\mathrm{t}, J=10.3$ $\mathrm{Hz}), 121.8,117.6,112.1,109.0,107.9$ (d, $J=24.8 \mathrm{~Hz}), 102.5$ (d, $J=25.3 \mathrm{~Hz}), 99.8$. 55.6, 55.55. ESI-MS $(m / z)[\mathrm{M}+\mathrm{H}]^{+}$calcd for $\mathrm{M}=\mathrm{C}_{17} \mathrm{H}_{14} \mathrm{~F}_{2} \mathrm{~N}_{2} \mathrm{O}_{2}$, 317.11; found, 317.11.

\section{1-(2,5-Difluoroxyphenyl)-3-(3,4-dimethoxyphenyl)propane-1,3-dione (sery220c)}

The title compound was prepared according to the GEP 1 using 1-(3,4dimethoxyphenyl)ethanone and methyl 2,5-difluorobenzoate and purified by recrystallisation from methanol to provide sery220c as a yellow solid. Yield $68 \% .{ }^{1} \mathrm{H}$ NMR $(400 \mathrm{MHz}$, DMSO[D 6$]) \delta=7.78-7.70(\mathrm{~m}, 2 \mathrm{H}), 7.54(\mathrm{~d}, J=2.1 \mathrm{~Hz}, 1 \mathrm{H}), 7.53-7.40(\mathrm{~m}, 2 \mathrm{H}), 7.11(\mathrm{~d}, J=$ $8.5 \mathrm{~Hz}, 1 \mathrm{H}), 7.02(\mathrm{~s}, 1 \mathrm{H}), 3,87(\mathrm{~s}, 3 \mathrm{H}), 3.85(\mathrm{~s}, 3 \mathrm{H})$ (in ${ }^{1} \mathrm{H}$ NMR spectrum 13\% of diketone form is present). ${ }^{13} \mathrm{C}$ NMR (100.6 MHz, DMSO[D 6$\left.]\right) \delta=186.8,178.3,158.0$ (d, $J=241.5$ $\mathrm{Hz}), 156.4(\mathrm{~d}, J=250.3 \mathrm{~Hz}), 153.5,148.8,126.8,124.6(\mathrm{~m}), 122.1,120.6(\mathrm{dd}, J=24.3,9.7$ $\mathrm{Hz}), 118.8(\mathrm{dd}, J=26.1,8.7 \mathrm{~Hz}), 115.9(\mathrm{~d}, J=25.3 \mathrm{~Hz}), 111.3,109.9,96.8,55.8,55.6$. ESIMS $(m / z)[\mathrm{M}+\mathrm{H}]^{+}$calcd for $\mathrm{M}=\mathrm{C}_{17} \mathrm{H}_{14} \mathrm{~F}_{2} \mathrm{O}_{4}, 321.09$; found, 321.19 .

\section{3-(2,5-Difluorophenyl)-5-(3,4-dimethoxyphenyl)-1H-pyrazole (sery221d)}

The title compound was prepared according to the GEP 2 starting from sery220c. White solid. Yield 20\%. ${ }^{1} \mathrm{H}$ NMR (400 MHz, 333K, DMSO[D $]+1-2 \%$ conc. DCl) $\delta=7.80-7.68$ $(\mathrm{m}, 1 \mathrm{H}), 7.45-7.31(\mathrm{~m}, 3 \mathrm{H}), 7.25-7.17(\mathrm{~m}, 1 \mathrm{H}), 7.06(\mathrm{~d}, J=3.4 \mathrm{~Hz}, 1 \mathrm{H}), 7.02(\mathrm{~d}, J=8.4 \mathrm{~Hz}$, $1 \mathrm{H}), 3.84(\mathrm{~s}, 3 \mathrm{H}), 3.78(\mathrm{~s}, 3 \mathrm{H}) .{ }^{13} \mathrm{C} \mathrm{NMR}\left(100.6 \mathrm{MHz}, \mathrm{DMSO}\left[\mathrm{D}_{6}\right]\right) \delta=158.3(\mathrm{~d}, J=239.3$ $\mathrm{Hz}), 155.4(\mathrm{~d}, J=243.9 \mathrm{~Hz}), 149.1,149.0,144.8,143.7,123.0(\mathrm{dd}, J=14.6,8.8 \mathrm{~Hz}), 121.8$, 117.8, $115.5(\mathrm{dd}, J=24.7,8.3 \mathrm{~Hz}), 113.6(\mathrm{~d}, J=23.8 \mathrm{~Hz}), 112.1,109.1(2 \mathrm{C}), 101.9,55.7$, 55.6 (main regioisomer is described). ESI-MS $(\mathrm{m} / \mathrm{z})[\mathrm{M}+\mathrm{H}]^{+}$calcd for $\mathrm{M}=\mathrm{C}_{17} \mathrm{H}_{14} \mathrm{~F}_{2} \mathrm{~N}_{2} \mathrm{O}_{2}$, 317.11; found, 317.25 .

\section{1-(3,4-Dimethoxyphenyl)-3-(2-fluorophenyl)propane-1,3-dione (sery230a)}

The title compound was prepared according to the GEP 1 using 1-(3-fluorophenyl)ethanone and ethyl 2,3-dimethoxybenzoate and purified by column chromatography on silica gel (hexane: EtOAc, 5:1 v/v) to provide sery230a as a yellow solid. Yield 67\%. TLC (hexane:EtOAc, $3: 1 \mathrm{v} / \mathrm{v}$ ): $\mathrm{RF}=0.72$. The isolated sery230a was used in next step without further purification.

\section{3-(2,3-Dimethoxyphenyl)-5-(3-fluorophenyl)-1 $H$-pyrazole (sery230b)}

The title compound was prepared according to the GEP 2 starting from sery230a and purified by column chromatography (hexane: EtOAc, 3:1 v/v) to provide sery230b as a white solid. Yield 79\%. TLC (hexane:EtOAc, 3:1 v/v): RF $=0.54 .{ }^{1} \mathrm{H}$ NMR $\left(400 \mathrm{MHz}, \mathrm{CDCl}_{3}\right) \delta=7.44$ $(\mathrm{d}, J=7.6 \mathrm{~Hz}, 1 \mathrm{H}), 7.33(\mathrm{~d}, J=10.0 \mathrm{~Hz}, 1 \mathrm{H}), 7.26(\mathrm{~m}, 1 \mathrm{H}), 7.21-7.12(\mathrm{~m}, 2 \mathrm{H}), 6.96(\mathrm{dt}, J=$ 8.4, 2.1 Hz, 1H), $6.79(\mathrm{~d}, J=8.7 \mathrm{~Hz}, 1 \mathrm{H}), 6.68(\mathrm{~s}, 1 \mathrm{H}), 3.88(\mathrm{~s}, 3 \mathrm{H}), 3.73(\mathrm{~s}, 3 \mathrm{H})$.

\section{3-(2,3-Dihydroxyphenyl)-5-(3-fluorophenyl)-1H-pyrazole hydrobromide (sery230c)}

The title compound was prepared according to the GEP 3 starting from sery230b. Beige solid. Yield 96\%. ${ }^{1} \mathrm{H}$ NMR (400 MHz, DMSO[D 6$\left.]\right) \delta=7.75-7.67(\mathrm{~m}, 2 \mathrm{H}), 7.55-7.47(\mathrm{~m}, 1 \mathrm{H})$, 
$7.34(\mathrm{~s}, 1 \mathrm{H}), 7.25-7.15(\mathrm{~m}, 2 \mathrm{H}), 6.82(\mathrm{dd}, J=7.9,1.5 \mathrm{~Hz}, 1 \mathrm{H}), 6.74(\mathrm{t}, J=7.9 \mathrm{~Hz}, 1 \mathrm{H}) .{ }^{13} \mathrm{C}$ NMR (100.6 MHz, DMSO[D 6$]) \delta=162.7(\mathrm{~d}, J=234.2 \mathrm{~Hz}), 147.3,145.9,145.0,143.6,132.7$ $(\mathrm{d}, J=9.1 \mathrm{~Hz}), 131.2(\mathrm{~d}, J=9.0 \mathrm{~Hz}), 121.7,119.4,117.7,116.6,115.5,115.3(\mathrm{~d}, J=21.5$ $\mathrm{Hz}), 112.3(\mathrm{~d}, J=23.3 \mathrm{~Hz}), 101.8$. ESI-MS $(\mathrm{m} / \mathrm{z})[\mathrm{M}+\mathrm{H}]^{+}$calcd for $\mathrm{M}=\mathrm{C}_{15} \mathrm{H}_{11} \mathrm{FN}_{2} \mathrm{O}_{2}$, 271.09; found, 271.20 .

\section{1-(3-Bromophenyl)-3-(3,4-dimethoxyphenyl)propane-1,3-dione (sery255a)}

The title compound was prepared according to the GEP 1 using 1-(3,4dimethoxyphenyl)ethanone and methyl 3-bromobenzoate and purified by recrystallisation from acetonitrile/methanol to provide sery255a as a yellow solid. Yield 74\%. m.p.: 131-132 ${ }^{\circ} \mathrm{C} .{ }^{1} \mathrm{H}$ NMR $\left(400 \mathrm{MHz}, \mathrm{DMSO}\left[\mathrm{D}_{6}\right]\right) \delta=8.31(\mathrm{t}, J=1.6 \mathrm{~Hz}, 1 \mathrm{H}), 8.14(\mathrm{~d}, J=8.0 \mathrm{~Hz}, 1 \mathrm{H})$, $7.91(\mathrm{dd}, J=8.6,2.0 \mathrm{~Hz}, 1 \mathrm{H}), 7.81(\mathrm{dd}, J=8.0,1.0 \mathrm{~Hz}, 1 \mathrm{H}), 7.64(\mathrm{~d}, J=2.0 \mathrm{~Hz}, 1 \mathrm{H}), 7.51(\mathrm{t}$, $J=8.0 \mathrm{~Hz}, 1 \mathrm{H}), 7.32(\mathrm{~s}, 1 \mathrm{H}), 7.11(\mathrm{~d}, J=8.6 \mathrm{~Hz}, 1 \mathrm{H}), 3.87(\mathrm{~s}, 6 \mathrm{H})\left(\right.$ in ${ }^{1} \mathrm{H}$ NMR spectrum $7 \%$ of diketone form is present). ${ }^{13} \mathrm{C}$ NMR $\left(100.6 \mathrm{MHz}, \mathrm{DMSO}\left[\mathrm{D}_{6}\right]\right) \delta=186.8,181.0,153.4$, $148.8,136.8,135.2,130.9,129.6,127.1,126.2,122.4,122.3,111.2,110.2,93.1,55.8(2 \mathrm{C})$. HRMS $(m / z)[\mathrm{M}+\mathrm{H}]^{+}$calcd for $\mathrm{M}=\mathrm{C}_{17} \mathrm{H}_{15}{ }^{79} \mathrm{BrO}_{4}, 363.0232$; found, 363.0237.

\section{3-(3-Bromophenyl)-5-(3,4-dimethoxyphenyl)-1H-pyrazole (sery255b)}

The title compound was prepared according to the GEP 2 starting from sery255a. White solid. Yield 98\%. m.p.: 164-165 ${ }^{\circ} \mathrm{C} .{ }^{1} \mathrm{H}$ NMR (400 MHz, DMSO[D 6$]+1-2 \%$ conc. DCl) $\delta=$ $8.03(\mathrm{t}, J=1.7 \mathrm{~Hz}, 1 \mathrm{H}), 7.85(\mathrm{dd}, J=7.9,0.9 \mathrm{~Hz}, 1 \mathrm{H}), 7.51$ (ddd, $J=7.9,1.7,0.9 \mathrm{~Hz}, 1 \mathrm{H})$, 7.44-7.33 (m, 3H), $7.22(\mathrm{~s}, 1 \mathrm{H}), 7.03(\mathrm{~d}, J=8.4 \mathrm{~Hz}, 1 \mathrm{H}), 3.84(\mathrm{~s}, 3 \mathrm{H}), 3.79(\mathrm{~s}, 3 \mathrm{H}) .{ }^{13} \mathrm{C} \mathrm{NMR}$ (100.6 MHz, DMSO[D 6$]+1-2 \%$ conc. DCl) $\delta=149.0,148.8,147.6,146.4,135.0,131.0$, $130.3,127.5,124.0,123.4,122.3,117.7,112.1,109.0,99.7,55.7,55.6$. HRMS $(m / z)[\mathrm{M}+\mathrm{H}]^{+}$ calcd for $\mathrm{M}=\mathrm{C}_{17} \mathrm{H}_{15}{ }^{79} \mathrm{BrN}_{2} \mathrm{O}_{2}, 359.0395$; found, 359.0402 .

\section{1-(3-Bromophenyl)-3-(2-methoxyphenyl)propane-1,3-dione (sery256a)}

The title compound was prepared according to the GEP 1 using 1-(3-bromophenyl)ethanone and methyl 2-methoxybenzoate and purified by column chromatography (hexane: $\mathrm{CH}_{2} \mathrm{Cl}_{2}, 2: 1$ $\mathrm{v} / \mathrm{v}$ ) to provide sery256a as a white solid. Yield 60\%. TLC (hexane:EtOAc, 5:1 v/v): RF = 0.52. m.p.: $84{ }^{\circ} \mathrm{C} .{ }^{1} \mathrm{H}$ NMR $\left(400 \mathrm{MHz}, \mathrm{CDCl}_{3}\right) \delta=8.09(\mathrm{t}, J=1.7 \mathrm{~Hz}, 1 \mathrm{H}), 7.94(\mathrm{dd}, J=7.8$, $1.8 \mathrm{~Hz}, 1 \mathrm{H}), 7.87(\mathrm{dt}, J=7.8,1.3 \mathrm{~Hz}, 1 \mathrm{H}), 7.65(\mathrm{ddd}, J=8.0,1.9,1.0 \mathrm{~Hz}, 1 \mathrm{H}), 7.52-7.45$ (m, $1 \mathrm{H}), 7.35(\mathrm{t}, J=7.9 \mathrm{~Hz}, 1 \mathrm{H}), 7.11-7.05(\mathrm{~m}, 2 \mathrm{H}), 7.01(\mathrm{~d}, J=8.4 \mathrm{~Hz}, 1 \mathrm{H}), 3.97(\mathrm{~s}, 3 \mathrm{H}) .{ }^{13} \mathrm{C}$ NMR (100.6 MHz, $\left.\mathrm{CDCl}_{3}\right) \delta=184.8,184.1,158.7,138.2,135.1,133.5,130.5,130.4,130.3$, $125.9,124.8,123.0,121.0,111.9,98.8,56.0$. HRMS $(m / z)[\mathrm{M}+\mathrm{H}]^{+}$calcd for $\mathrm{M}=$ $\mathrm{C}_{16} \mathrm{H}_{13}{ }^{79} \mathrm{BrO}_{3}, 333.0126$; found, 333.0126.

\section{3-(3-Bromophenyl)-5-(2-methoxyphenyl)-1H-pyrazole (sery256b)}

The title compound was prepared according to the GEP 2 starting from sery256a and purified by recrystallisation from ethanol to provide sery 256b as a white solid. Yield 63\%. m.p.: 113$114{ }^{\circ} \mathrm{C} .{ }^{1} \mathrm{H}$ NMR $\left(400 \mathrm{MHz}, \mathrm{DMSO}\left[\mathrm{D}_{6}\right]+1-2 \%\right.$ conc. DCl) $\delta=8.04(\mathrm{t}, J=1.7 \mathrm{~Hz}, 1 \mathrm{H}), 7.87$ $(\mathrm{d}, J=7.8 \mathrm{~Hz}, 1 \mathrm{H}), 7.78(\mathrm{~d}, J=7.0 \mathrm{~Hz}, 1 \mathrm{H}), 7.50(\mathrm{ddd}, J=8.1,1.7,0.8 \mathrm{~Hz}, 1 \mathrm{H}), 7.43-7.32$ $(\mathrm{m}, 2 \mathrm{H}), 7.23(\mathrm{~s}, 1 \mathrm{H}), 7.15(\mathrm{~d}, J=8.2 \mathrm{~Hz}, 1 \mathrm{H}), 7.05(\mathrm{td}, J=7.4,0.8 \mathrm{~Hz}, 1 \mathrm{H}), 3.92(\mathrm{~s}, 3 \mathrm{H}) .{ }^{13} \mathrm{C}$ NMR (100.6 MHz, DMSO[D 6 + 1-2\% conc. DCl) $\delta=155.8,147.6,141.9,135.6,130.9$, $130.0,129.4,127.7,127.4,124.0,122.2,120.7,118.3,111.9,102.2,55.5$. HRMS $(\mathrm{m} / \mathrm{z})$ $[\mathrm{M}+\mathrm{H}]^{+}$calcd for $\mathrm{M}=\mathrm{C}_{16} \mathrm{H}_{13}{ }^{79} \mathrm{BrN}_{2} \mathrm{O}$, 329.0290; found, 329.0294 .

\section{1-(3-Bromophenyl)-3-(5-chloro-2-methoxyphenyl)propane-1,3-dione (sery257a)}

The title compound was prepared according to the GEP 1 using 1-(3-bromophenyl)ethanone and methyl 5-chloro-2-methoxybenzoate and purified by recrystallisation from methanol 
(twice) to provide sery257a as a yellow solid. Yield 75\%. m.p.: 115-116 ${ }^{\circ} \mathrm{C} .{ }^{1} \mathrm{H}$ NMR (400 $\left.\mathrm{MHz}, \mathrm{CDCl}_{3}\right) \delta=8.08(\mathrm{t}, J=1.7 \mathrm{~Hz}, 1 \mathrm{H}), 7.90(\mathrm{~d}, J=2.7 \mathrm{~Hz}, 1 \mathrm{H}), 7.86(\mathrm{~d}, J=7.8 \mathrm{~Hz}, 1 \mathrm{H})$, $7.66(\mathrm{~m}, 1 \mathrm{H}), 7.42(\mathrm{dd}, J=8.9,2.7 \mathrm{~Hz}, 1 \mathrm{H}), 7.35(\mathrm{t}, J=7.9 \mathrm{~Hz}, 1 \mathrm{H}), 7.07(\mathrm{~s}, 1 \mathrm{H}), 6.94(\mathrm{~d}, J=$ $8.9 \mathrm{~Hz}, 1 \mathrm{H}), 3.96(\mathrm{~s}, 3 \mathrm{H}) .{ }^{13} \mathrm{C} \mathrm{NMR}\left(100.6 \mathrm{MHz}, \mathrm{CDCl}_{3}\right) \delta=184.5,183.0,157.2,137.9$, $135.3,132.9,130.5,130.3,130.1,126.4,126.0,125.9,123.1,113.3,98.8,56.4$. HRMS $(\mathrm{m} / \mathrm{z})$ $[\mathrm{M}+\mathrm{H}]^{+}$calcd for $\mathrm{M}=\mathrm{C}_{16} \mathrm{H}_{12}{ }^{79} \mathrm{BrClO}_{3}, 366.9737$; found, 366.9738 .

\section{3-(3-Bromophenyl)-5-(5-chloro-2-methoxyphenyl)-1H-pyrazole (sery257b)}

The title compound was prepared according to the GEP 2 starting from sery257a and purified by recrystallisation from ethanol to provide sery257b as a white solid. Yield 85\%. m.p.: 163$164{ }^{\circ} \mathrm{C} .{ }^{1} \mathrm{H}$ NMR $\left(400 \mathrm{MHz}, \mathrm{DMSO}\left[\mathrm{D}_{6}\right]+1-2 \%\right.$ conc. DCl) $\delta=8.05(\mathrm{t}, J=1.5 \mathrm{~Hz}, 1 \mathrm{H})$, 7.91-7.81 (m, 2H), $7.51(\mathrm{~d}, J=8.3 \mathrm{~Hz}, 1 \mathrm{H}), 7.44-7.36(\mathrm{~m}, 2 \mathrm{H}), 7.33(\mathrm{~s}, 1 \mathrm{H}), 7.17(\mathrm{~d}, J=8.9$ $\mathrm{Hz}, 1 \mathrm{H}), 3.93(\mathrm{~s}, 3 \mathrm{H}) .{ }^{13} \mathrm{C} \mathrm{NMR}\left(100.6 \mathrm{MHz}, \mathrm{DMSO}\left[\mathrm{D}_{6}\right]+1-2 \%\right.$ conc. DCl $) \delta=154.6$, 147.2 , 141.4, 135.1, 130.9, 130.2, 128.6, 127.5, 126.8, 124.5, 124.0, 122.2, 120.3, 113.9, 103.1, 55.9. HRMS $(\mathrm{m} / \mathrm{z})[\mathrm{M}+\mathrm{H}]^{+}$calcd for $\mathrm{M}=\mathrm{C}_{16} \mathrm{H}_{12}{ }^{79} \mathrm{BrClN}_{2} \mathrm{O}$, 362.9900; found, 362.9903.

\section{3-(3-Bromophenyl)-5-(5-chloro-2-hydroxyphenyl)-1H-pyrazole (sery260b)}

The title compound was prepared according to the GEP 4 starting from sery $257 \mathbf{b}$ and purified by recrystallisation from petroleum benzine $\left(40-60{ }^{\circ} \mathrm{C}\right) /$ chloroform and second recrystallisation from hexane/EtOAc to provide sery260b as a white solid. Yield 51\%. m.p.: 217-220 (dec.) ${ }^{\circ} \mathrm{C} .{ }^{1} \mathrm{H}$ NMR $\left(400 \mathrm{MHz}, \mathrm{DMSO}\left[\mathrm{D}_{6}\right]+1-2 \%\right.$ conc. DCl) $\delta=10.74$ (bs, $\left.1 \mathrm{H}\right)$, $8.04(\mathrm{~s}, 1 \mathrm{H}), 7.84(\mathrm{~d}, J=7.8 \mathrm{~Hz}, 1 \mathrm{H}), 7.80(\mathrm{~d}, J=2.6 \mathrm{~Hz}, 1 \mathrm{H}), 7.54(\mathrm{~d}, J=7.8 \mathrm{~Hz}, 1 \mathrm{H}), 7.47-$ $7.37(\mathrm{~m}, 2 \mathrm{H}), 7.22(\mathrm{dd}, J=8.7,2.6 \mathrm{~Hz}, 1 \mathrm{H}), 6.99(\mathrm{~d}, J=8.7 \mathrm{~Hz}, 1 \mathrm{H}) .{ }^{13} \mathrm{C}$ NMR $(100.6 \mathrm{MHz}$, DMSO $\left[\mathrm{D}_{6}\right]+1-2 \%$ conc. DCl) $\delta=153.6,145.2,144.5,133.7,131.2,130.8,128.6,127.8$, $126.5,124.3,123.1,122.4,118.5,118.2,102.0$. HRMS $(m / z)[\mathrm{M}+\mathrm{H}]^{+}$calcd for $\mathrm{M}=$ $\mathrm{C}_{15} \mathrm{H}_{10}{ }^{79} \mathrm{BrClN}_{2} \mathrm{O}$, 348.9743; found, 348.9748.

\section{1-(3-Fluorophenyl)-3-(2-methoxyphenyl)propane-1,3-dione (sery258)}

The title compound was prepared according to the GEP 1 using 1-(3-fluorophenyl)ethanone and methyl 2-methoxybenzoate and purified by recrystallisation (twice) from methanol to provide sery 258 as a yellow solid. Yield $73 \%$. m.p.: $95-96{ }^{\circ} \mathrm{C} .{ }^{1} \mathrm{H}$ NMR $\left(400 \mathrm{MHz}, \mathrm{CDCl}_{3}\right) \delta$ $=7.95(\mathrm{dd}, J=7.8,1.6 \mathrm{~Hz}, 1 \mathrm{H}), 7.74(\mathrm{~d}, J=7.8 \mathrm{~Hz}, 1 \mathrm{H}), 7.65(\mathrm{~d}, J=9.7 \mathrm{~Hz}, 1 \mathrm{H}), 7.53-7.40$ $(\mathrm{m}, 2 \mathrm{H}), 7.23(\mathrm{td}, J=8.4,2.3 \mathrm{~Hz}, 1 \mathrm{H}), 7.12(\mathrm{~s}, 1 \mathrm{H}), 7.07(\mathrm{t}, J=7.4 \mathrm{~Hz}, 1 \mathrm{H}), 7.01(\mathrm{~d}, J=8.4$ $\mathrm{Hz}, 1 \mathrm{H}), 3.97(\mathrm{~s}, 3 \mathrm{H}) .{ }^{13} \mathrm{C}$ NMR $\left(100.6 \mathrm{MHz}, \mathrm{CDCl}_{3}\right) \delta=184.7,184.2,163.0(\mathrm{~d}, J=246.5$ $\mathrm{Hz}), 158.7,138.5$ (d, $J=7.1 \mathrm{~Hz}), 133.5,130.5,130.3,124.7,123.0,121.0,119.1$ (d, $J=21.4$ $\mathrm{Hz}), 114.2(\mathrm{~d}, J=18.2 \mathrm{~Hz}), 111.8,98.8,96.0$. HRMS $(\mathrm{m} / \mathrm{z})[\mathrm{M}+\mathrm{H}]^{+}$calcd for $\mathrm{M}=\mathrm{C}_{16} \mathrm{H}_{13} \mathrm{FO}_{3}$, 273.0927; found, 273.0926.

\section{3-(3-Fluorophenyl)-5-(2-methoxyphenyl)-1H-pyrazole (sery261a)}

The title compound has been reported previously ${ }^{523}$ (CAS 939051-95-3). It was prepared according to the GEP 2 starting from sery 258 and purified by column chromatography (hexane:EtOAc, 4:1 v/v) to provide sery261a as a white solid. Yield $90 \%$. TLC (hexane:EtOAc, 3:1 v/v): RF $=0.51$. m.p.: $109{ }^{\circ} \mathrm{C} .{ }^{1} \mathrm{H}$ NMR $\left(400 \mathrm{MHz}, \mathrm{DMSO}\left[\mathrm{D}_{6}\right]+1-2 \%\right.$ conc. DCl) $\delta=7.78(\mathrm{~d}, J=7.4 \mathrm{~Hz}, 1 \mathrm{H}), 7.71(\mathrm{~d}, J=7.7 \mathrm{~Hz}, 1 \mathrm{H}), 7.65(\mathrm{~d}, J=10.4 \mathrm{~Hz}, 1 \mathrm{H})$, $7.47(\mathrm{q}, J=7.7 \mathrm{~Hz}, 1 \mathrm{H}), 7.35(\mathrm{t}, J=7.4 \mathrm{~Hz}, 1 \mathrm{H}), 7.21(\mathrm{~s}, 1 \mathrm{H}), 7.18-7.09(\mathrm{~m} .2 \mathrm{H}), 7.04$ (t, $J=$ $7.4 \mathrm{~Hz}, 1 \mathrm{H}), 3.91(\mathrm{~s}, 3 \mathrm{H})$. HRMS $(\mathrm{m} / \mathrm{z})[\mathrm{M}+\mathrm{H}]^{+}$calcd for $\mathrm{M}=\mathrm{C}_{16} \mathrm{H}_{13} \mathrm{FN}_{2} \mathrm{O}, 269.1090$; found, 269.1090 . 


\section{3-(3-Fluorophenyl)-5-(2-hydroxyphenyl)-1H-pyrazole (sery263a)}

The title compound has been reported previously ${ }^{524}$ (CAS 312314-31-1). It was prepared according to the GEP 4 starting from sery261a and purified by column chromatography (hexane:EtOAc, 5:1 v/v) to provide sery263a as a white solid. Yield 59\%. TLC (hexane:EtOAc, 6:1 v/v): RF $=0.28$. m.p.: $146{ }^{\circ} \mathrm{C} .{ }^{1} \mathrm{H}$ NMR $\left(400 \mathrm{MHz}, \mathrm{DMSO}\left[\mathrm{D}_{6}\right]+1-2 \%\right.$ conc. DCl) $\delta=10.56(\mathrm{~s}, 1 \mathrm{H}), 7.78-7.61(\mathrm{~m}, 3 \mathrm{H}), 7.50(\mathrm{~m}, 1 \mathrm{H}), 7.30(\mathrm{~s}, 1 \mathrm{H}), 7.23-7.13(\mathrm{~m}$, $2 \mathrm{H}), 6.97(\mathrm{dd}, J=8.2,0.9 \mathrm{~Hz}, 1 \mathrm{H}), 6.91(\mathrm{td}, J=7.6,1.0 \mathrm{~Hz}, 1 \mathrm{H})$. HRMS $(m / z)[\mathrm{M}+\mathrm{H}]^{+}$calcd for $\mathrm{M}=\mathrm{C}_{15} \mathrm{H}_{11} \mathrm{FN}_{2} \mathrm{O}$, 255.0934; found, 255.0932 .

\section{1-(4-Chlorophenyl)-3-(4-methoxyphenyl)propane-1,3-dione (sery269a)}

The title compound has been reported previously ${ }^{525}$ (CAS 37975-19-2). It was prepared according to the GEP 1 using 1-(4-methoxyphenyl)ethanone and ethyl 4-chlorobenzoate and purified by recrystallisation from methanol to provide sery269a as a white solid. Yield $84 \%$. m.p.: $116-117{ }^{\circ} \mathrm{C} .{ }^{1} \mathrm{H}$ NMR (400 MHz, DMSO[D 6$\left.]\right) \delta=8.15(\mathrm{~d}, J=8.3 \mathrm{~Hz}, 4 \mathrm{H}), 7.60(\mathrm{~d}, J=$ $8.6 \mathrm{~Hz}, 2 \mathrm{H}), 7.28(\mathrm{~s}, 1 \mathrm{H}), 7.08(\mathrm{~d}, J=8.9 \mathrm{~Hz}, 2 \mathrm{H}), 3.86(\mathrm{~s}, 3 \mathrm{H})\left(\right.$ in ${ }^{1} \mathrm{H}$ NMR spectrum $7.5 \%$ of diketone form is present).

\section{3-(4-Chlorophenyl)-5-(4-methoxyphenyl)-1 H-pyrazole (sery269b)}

The title compound has been reported previously ${ }^{526}$ (CAS 1204141-84-3). It was prepared according to the GEP 2 starting from sery269a and purified by recrystallisation from ethanol to provide sery269b as a white solid. Yield 83\%. m.p.: 201-202 ${ }^{\circ} \mathrm{C}$. (lit ${ }^{526} 221{ }^{\circ} \mathrm{C}$ ). ${ }^{1} \mathrm{H}$ NMR $\left(400 \mathrm{MHz}, \mathrm{DMSO}\left[\mathrm{D}_{6}\right]+1-2 \%\right.$ conc. DCl) $\delta=7.84(\mathrm{~d}, J=8.6 \mathrm{~Hz}, 2 \mathrm{H}), 7.74(\mathrm{~d}, J=8.8 \mathrm{~Hz}$, 2H), $7.49(\mathrm{~d}, J=8.6 \mathrm{~Hz}, 2 \mathrm{H}), 7.08(\mathrm{~s}, 1 \mathrm{H}), 7.02(\mathrm{~d}, J=8.8 \mathrm{~Hz}, 2 \mathrm{H}), 3.78(\mathrm{~s}, 3 \mathrm{H})$.

\section{3-(4-Chlorophenyl)-5-(4-hydroxyphenyl)- $1 H$-pyrazole hydrobromide (sery320a)}

This compound was prepared according to the GEP 3 starting from sery 269b. White solid.

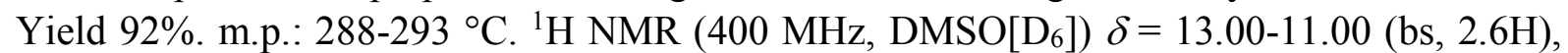
$7.87(\mathrm{~d}, J=8.5 \mathrm{~Hz}, 2 \mathrm{H}), 7.67(\mathrm{~d}, J=8.6 \mathrm{~Hz}, 2 \mathrm{H}), 7.51(\mathrm{~d}, J=8.5 \mathrm{~Hz}, 2 \mathrm{H}), 7.16(\mathrm{~s}, 1 \mathrm{H}), 6.87$ $(\mathrm{d}, J=8.6 \mathrm{~Hz}, 2 \mathrm{H}) .{ }^{13} \mathrm{C} \mathrm{NMR}\left(100.6 \mathrm{MHz}, \mathrm{DMSO}\left[\mathrm{D}_{6}\right]\right) \delta=158.0,147.3,146.5,132.7$, 130.2, 129.0, 128.9, 127.2, 127.0, 120.6, 115.8, 115.7, 99.3. HRMS $(\mathrm{m} / \mathrm{z})[\mathrm{M}+\mathrm{H}]^{+}$calcd for $\mathrm{M}=\mathrm{C}_{15} \mathrm{H}_{11} \mathrm{ClN}_{2} \mathrm{O}, 271.0638$; found, 271.0643.

\section{1-(4-Chlorophenyl)-3-(3-methoxyphenyl)propane-1,3-dione (sery287a)}

The title compound was prepared according to the GEP 1 using 1-(4-chlorophenyl)ethanone and ethyl 3-methoxybenzoate and purified by recrystallisation (twice) from methanol to provide sery287a as a yellow solid. Yield 34\%. m.p.: 77-79 ${ }^{\circ} \mathrm{C} .{ }^{1} \mathrm{H}$ NMR $(400 \mathrm{MHz}$, DMSO[D 6$]) \delta=8.19(\mathrm{~d}, J=8.5 \mathrm{~Hz}, 2 \mathrm{H}), 7.76(\mathrm{~d}, J=7.6 \mathrm{~Hz}, 1 \mathrm{H}), 7.70-7.57(\mathrm{~m}, 3 \mathrm{H}), 7.48(\mathrm{t}$, $J=8.0 \mathrm{~Hz}, 1 \mathrm{H}), 7.34(\mathrm{~s}, 1 \mathrm{H}), 7.22(\mathrm{dd}, J=8.0,1.9 \mathrm{~Hz}, 1 \mathrm{H}), 3.86(\mathrm{~s}, 3 \mathrm{H}) .{ }^{13} \mathrm{C} \mathrm{NMR}(100.6$ $\left.\mathrm{MHz}, \mathrm{DMSO}\left[\mathrm{D}_{6}\right]\right) \delta=185.3,184.0,159.6,137.9,135.9,133.4,130.0,129.3,128.9,119.9$, 119.0, 112.3, 93.6, 55.4. HRMS $(m / z)[\mathrm{M}+\mathrm{H}]^{+}$calcd for $\mathrm{M}=\mathrm{C}_{16} \mathrm{H}_{13} \mathrm{ClO}_{3}, 289.0631$; found, 289.0630 .

\section{3-(4-Chlorophenyl)-5-(3-methoxyphenyl)-1H-pyrazole (sery289)}

The title compound was prepared according to the GEP 2 starting from sery287a and purified by column chromatography (hexane:EtOAc, 3.5:1 v/v) to provide sery289 as a white solid. Yield 65\%. TLC (hexane:EtOAc, 3:1 v/v): RF $=0.40$. m.p.: 176-177 ${ }^{\circ} \mathrm{C} .{ }^{1} \mathrm{H} \mathrm{NMR}(400 \mathrm{MHz}$, DMSO $\left[\mathrm{D}_{6}\right]+1-2 \%$ conc. DCl) $\delta=7.86(\mathrm{~d}, J=8.6 \mathrm{~Hz}, 2 \mathrm{H}), 7.51(\mathrm{~d}, J=8.6 \mathrm{~Hz}, 2 \mathrm{H}), 7.43-$ $7.33(\mathrm{~m}, 3 \mathrm{H}), 7.23(\mathrm{~s}, 1 \mathrm{H}), 6.95-6.89(\mathrm{~m}, 1 \mathrm{H}), 3.82(\mathrm{~s}, 3 \mathrm{H}) .{ }^{13} \mathrm{C}$ NMR $(100.6 \mathrm{MHz}$, DMSO $\left[\mathrm{D}_{6}\right]+1-2 \%$ conc. DCl) $\delta=159.7,148.0-147.0$ (2C), 132.2, 131.9, 130.4, 130.1, 
128.9, 126.9, 117.5, 113.8, 110.5, 100.2, 55.4. HRMS $(m / z)[\mathrm{M}+\mathrm{H}]^{+}$calcd for $\mathrm{M}=$ $\mathrm{C}_{16} \mathrm{H}_{13} \mathrm{ClN}_{2} \mathrm{O}, 285.0795$; found, 285.0797.

\section{3-(4-Chlorophenyl)-5-(3-hydroxyphenyl)-1H-pyrazole hydrobromide (sery320b)}

This compound was prepared according to the GEP 3 starting from sery289. White solid. Yield 82\%. m.p.: 246-251 ${ }^{\circ} \mathrm{C}$ (dec). ${ }^{1} \mathrm{H}$ NMR (400 MHz, DMSO[D 6$)$ ) $\delta=9.0-8.0$ (bs, 4H), $7.87(\mathrm{~d}, J=8.6 \mathrm{~Hz}, 2 \mathrm{H}), 7.50(\mathrm{~d}, J=8.6 \mathrm{~Hz}, 2 \mathrm{H}), 7.30-7.18(\mathrm{~m}, 3 \mathrm{H}), 7.15(\mathrm{~s}, 1 \mathrm{H}), 6.78(\mathrm{~m}$, 1H). ${ }^{13} \mathrm{C}$ NMR (100.6 MHz, DMSO[D 6$\left.]\right) \delta=157.8,147.0,146.7,132.3,131.8,130.7,129.9$, $128.8,126.9,116.2,115.2,112.1,100.0$. $\operatorname{HRMS}(\mathrm{m} / \mathrm{z})[\mathrm{M}+\mathrm{H}]^{+}$calcd for $\mathrm{M}=\mathrm{C}_{15} \mathrm{H}_{11} \mathrm{ClN}_{2} \mathrm{O}$, 271.0638; found, 271.0642.

\section{1-(5-Chloro-2-methoxyphenyl)-3-(3-fluorophenyl)propane-1,3-dione (sery259)}

The title compound was prepared according to the GEP 1 using 1-(3-fluorophenyl)ethanone and methyl 5-chloro-2-methoxybenzoate and purified by recrystallisation (twice) from methanol to provide sery259 as a yellow solid. Yield 90\% m.p.: $98-99{ }^{\circ} \mathrm{C} .{ }^{1} \mathrm{H}$ NMR $(400$ $\left.\mathrm{MHz}, \mathrm{CDCl}_{3}\right) \delta=7.92(\mathrm{~d}, J=2.8 \mathrm{~Hz}, 1 \mathrm{H}), 7.73(\mathrm{~d}, J=7.9 \mathrm{~Hz}, 1 \mathrm{H}), 7.64$ (ddd, $J=9.7,2.3$, $1.7 \mathrm{~Hz}, 1 \mathrm{H}), 7.49-7.40(\mathrm{~m}, 2 \mathrm{H}), 7.24(\mathrm{tdd}, J=8.3,2.6,0.8 \mathrm{~Hz}, 1 \mathrm{H}), 7.09(\mathrm{~s}, 1 \mathrm{H}), 6.95(\mathrm{~d}, J=$ $8.9 \mathrm{~Hz}, 1 \mathrm{H}), 3.96(\mathrm{~s}, 3 \mathrm{H}) .{ }^{13} \mathrm{C} \mathrm{NMR}\left(100.6 \mathrm{MHz}, \mathrm{CDCl}_{3}\right) \delta=184.7,183.0,163.0(\mathrm{~d}, J=$ $247.2 \mathrm{~Hz}), 157.2,138.2(\mathrm{~d}, J=7.0 \mathrm{~Hz}), 132.9,130.4(\mathrm{~d}, J=7.9 \mathrm{~Hz}), 130.1,126.4,126.0$, 123.0, $119.4(\mathrm{~d}, J=21.4 \mathrm{~Hz}), 114.3(\mathrm{~d}, J=23.0 \mathrm{~Hz}), 113.3,98.8,56.4$. HRMS $(\mathrm{m} / \mathrm{z})[\mathrm{M}+\mathrm{H}]^{+}$ calcd for $\mathrm{M}=\mathrm{C}_{16} \mathrm{H}_{12} \mathrm{ClFO}_{3}, 307.0537$; found, 307.0537 .

\section{3-(5-Chloro-2-methoxyphenyl)-5-(3-fluorophenyl)-1H-pyrazole (sery261b)}

The title compound was prepared according to the GEP 2 starting from sery 259 and purified by column chromatography (hexane:EtOAc, 4:1 v/v) to provide sery261b as a white solid. Yield 98\%. TLC (hexane:EtOAc, 3:1 v/v): RF = 0.45. m.p.: $133{ }^{\circ} \mathrm{C} .{ }^{1} \mathrm{H}$ NMR $(400 \mathrm{MHz}$, DMSO $\left[\mathrm{D}_{6}\right]+1-2 \%$ conc. DCl $\delta=7.85(\mathrm{~s}, 1 \mathrm{H}), 7.76-7.61(\mathrm{~m}, 2 \mathrm{H}), 7.48(\mathrm{q}, J=6.8 \mathrm{~Hz}, 1 \mathrm{H})$, $7.38(\mathrm{~d}, J=7.7 \mathrm{~Hz}, 1 \mathrm{H}), 7.31(\mathrm{~s}, 1 \mathrm{H}), 7.21-7.10(\mathrm{~m}, 2 \mathrm{H}), 3.92(\mathrm{~s}, 3 \mathrm{H}) .{ }^{13} \mathrm{C}$ NMR $(100.6 \mathrm{MHz}$, DMSO $\left[\mathrm{D}_{6}\right]+1-2 \%$ conc. DCl) $\delta=162.7(\mathrm{~d}, J=242.6 \mathrm{~Hz}), 154.7,147.2,141.2,134.7,130.9$ $(\mathrm{d}, J=8.4 \mathrm{~Hz}), 128.7,126.9,124.6,121.2,120.6,114.4(\mathrm{~d}, J=21.0 \mathrm{~Hz}), 113.9,111.7(\mathrm{~d}, J=$ 22.6 Hz), 103.3, 56.0. HRMS $(\mathrm{m} / \mathrm{z})[\mathrm{M}+\mathrm{H}]^{+}$calcd for $\mathrm{M}=\mathrm{C}_{16} \mathrm{H}_{12} \mathrm{ClFN}_{2} \mathrm{O}$, 303.0700; found, 303.0704 .

\section{3-(5-Chloro-2-hydroxyphenyl)-5-(3-fluorophenyl)-1H-pyrazole (sery263b)}

The title compound was prepared according to the GEP 4 starting from sery $261 \mathbf{b}$ and purified by column chromatography (hexane:EtOAc, 6:1 v/v) to provide sery263b as a white solid. Yield 60\%. TLC (hexane:EtOAc, 6:1 v/v): RF = 0.38. m.p.: $202{ }^{\circ} \mathrm{C} .{ }^{1} \mathrm{H}$ NMR $(400 \mathrm{MHz}$, DMSO $\left[\mathrm{D}_{6}\right]+1-2 \%$ conc. DCl) $\delta=10.74(\mathrm{bs}, 1 \mathrm{H}), 7.80(\mathrm{~d}, J=2.6 \mathrm{~Hz}, 1 \mathrm{H}), 7.73-7.61(\mathrm{~m}$, $2 \mathrm{H}), 7.51(\mathrm{~m}, 1 \mathrm{H}), 7.40(\mathrm{~s}, 1 \mathrm{H}), 7.26-7.14(\mathrm{~m}, 2 \mathrm{H}), 6.98(\mathrm{~d}, J=8.7 \mathrm{~Hz}, 1 \mathrm{H}) .{ }^{13} \mathrm{C} \mathrm{NMR}(100.6$ $\mathrm{MHz}, \mathrm{DMSO}\left[\mathrm{D}_{6}\right]+1-2 \%$ conc. $\left.\mathrm{DCl}\right) \delta=162.6(\mathrm{~d}, J=243.0 \mathrm{~Hz}), 153.6,146.0-144.8(2 \mathrm{C})$, $133.2,131.1(\mathrm{~d}, J=8.5 \mathrm{~Hz}), 128.6,126.4,123.0,121.4,118.5,118.2,114.8(\mathrm{~d}, J=19.9 \mathrm{~Hz})$, $111.9(\mathrm{~d}, J=23.0 \mathrm{~Hz}), 102.0$. HRMS $(\mathrm{m} / z)[\mathrm{M}+\mathrm{H}]^{+}$calcd for $\mathrm{M}=\mathrm{C}_{15} \mathrm{H}_{10} \mathrm{ClFN}_{2} \mathrm{O}, 289.0544$ found, 289.0546.

\section{1-(3-Chlorophenyl)-3-(3-methoxyphenyl)propane-1,3-dione (sery287b)}

The title compound was prepared according to the GEP 1 using 1-(3-chlorophenyl)ethanone and ethyl 3-methoxybenzoate and purified by recrystallisation from methanol followed by column chromatography (hexane: $\mathrm{CHCl}_{3}, 3: 1 \mathrm{v} / \mathrm{v}$ ) to provide sery 287b as a white solid. Yield $30 \%$. TLC (hexane:EtOAc, 5:1 v/v): RF $=0.45$. m.p.: 56-57 ${ }^{\circ} \mathrm{C} .{ }^{1} \mathrm{H}$ NMR $\left(400 \mathrm{MHz}, \mathrm{CDCl}_{3}\right)$ $\delta=7.95(\mathrm{t}, J=1.9 \mathrm{~Hz}, 1 \mathrm{H}), 7.86(\mathrm{dt}, J=7.8,1.3 \mathrm{~Hz}, 1 \mathrm{H}), 7.56(\mathrm{~d}, J=7.8 \mathrm{~Hz}, 1 \mathrm{H}), 7.54-7.50$ 
(m, 2H), 7.46-7.38 (m, 2H), 7.11 (ddd, $J=8.2,2.6,0.7 \mathrm{~Hz}, 1 \mathrm{H}), 6.79$ (s, 1H), $3.89(\mathrm{~s}, 3 \mathrm{H})$. ${ }^{13} \mathrm{C}$ NMR $\left(100.6 \mathrm{MHz}, \mathrm{CDCl}_{3}\right) \delta=186.4,183.9,160.1,137.4,136.9,135.1,132.4,130.1$, $129.9,127.4,125.4,119.8,119.0,112.2,93.7,55.6$. HRMS $(m / z)[\mathrm{M}+\mathrm{H}]^{+}$calcd for $\mathrm{M}=$ $\mathrm{C}_{16} \mathrm{H}_{13} \mathrm{ClO}_{3}, 289.0631$; found, 289.0633 .

\section{3-(3-Chlorophenyl)-5-(3-methoxyphenyl)-1H-pyrazole (sery301)}

The title compound was prepared according to the GEP 2 starting from sery $287 \mathbf{b}$ and purified by recrystallisation from methanol to provide sery301 as a white solid. Yield 76\%. m.p.: 197$198{ }^{\circ} \mathrm{C} .{ }^{1} \mathrm{H}$ NMR $\left(400 \mathrm{MHz}, \mathrm{DMSO}\left[\mathrm{D}_{6}\right]+1-2 \%\right.$ conc. DCl) $\delta=7.90$ (s, 1H), 7.81 (d, $J=7.6$ $\mathrm{Hz}, 1 \mathrm{H}), 7.53-7.33(\mathrm{~m}, 5 \mathrm{H}), 7.31(\mathrm{~s}, 1 \mathrm{H}), 6.92(\mathrm{~d}, J=7.5 \mathrm{~Hz}, 1 \mathrm{H}), 3.82(\mathrm{~s}, 3 \mathrm{H}) .{ }^{13} \mathrm{C}$ NMR (100.6 MHz, DMSO[D 6 + 1-2\% conc. DCl) $\delta=159.7,147.2,146.3,134.2,133.7,132.0$, $130.8,130.1,127.5,124.7,123.7,117.5,113.8,110.5,100.5,55.2$. HRMS $(m / z)[\mathrm{M}+\mathrm{H}]^{+}$ calcd for $\mathrm{M}=\mathrm{C}_{16} \mathrm{H}_{13} \mathrm{ClN}_{2} \mathrm{O}$, 285.0795; found, 285.0797.

\section{3-(3-Chlorophenyl)-5-(3-hydroxyphenyl)-1H-pyrazole hydrobromide (sery320c)}

This compound was prepared according to the GEP 3 starting from sery301. White solid. Yield 67\%. m.p.: 244-250 ${ }^{\circ} \mathrm{C}$. ${ }^{1} \mathrm{H}$ NMR (400 MHz, DMSO[D 6$\left.\left.]\right) \delta=11.06 \mathrm{bs}, 1 \mathrm{H}\right), 7.91$ (t, $J$ $=1.7 \mathrm{~Hz}, 1 \mathrm{H}), 7.81(\mathrm{~d}, J=7.8 \mathrm{~Hz}, 1 \mathrm{H}), 7.47(\mathrm{t}, J=7.8 \mathrm{~Hz}, 1 \mathrm{H}), 7.38(\mathrm{~m}, 1 \mathrm{H}), 7.26-7.20(\mathrm{~m}$, $4 \mathrm{H}), 6.78(\mathrm{~m}, 1 \mathrm{H}) .{ }^{13} \mathrm{C} \mathrm{NMR}\left(100.6 \mathrm{MHz}, \mathrm{DMSO}\left[\mathrm{D}_{6}\right]\right) \delta=157.8,146.8,146.7,134.0,133.7$, $131.8,130.7,129.9,127.5,124.8,123.8,116.2,115.2,112.1,100.3$. HRMS $(m / z)[\mathrm{M}+\mathrm{H}]^{+}$ calcd for $\mathrm{M}=\mathrm{C}_{15} \mathrm{H}_{11} \mathrm{ClN}_{2} \mathrm{O}, 271.0638$; found, 271.0640.

\section{1-(3-Bromophenyl)-3-(naphthalen-2-yl)propane-1,3-dione (sery292a)}

The title compound was prepared according to the GEP 1 using 1-(naphthalen-2-yl)ethanone and ethyl 3-bromobenzoate and purified by recrystallisation from methanol to provide sery292a as a white solid. Yield 73\%. m.p.: $121-122{ }^{\circ} \mathrm{C} .{ }^{1} \mathrm{H}$ NMR (400 MHz, DMSO[D 6$\left.]\right) \delta$ $=8.88(\mathrm{~s}, 1 \mathrm{H}), 8.40(\mathrm{t}, J=1.6 \mathrm{~Hz}, 1 \mathrm{H}), 8.24(\mathrm{dd}, J=8.6,1.6 \mathrm{~Hz}, 1 \mathrm{H}), 8.20(\mathrm{~d}, J=7.9 \mathrm{~Hz}$, $1 \mathrm{H}), 8.13(\mathrm{~d}, J=7.9 \mathrm{~Hz}, 1 \mathrm{H}), 8.07(\mathrm{~d}, J=8.6 \mathrm{~Hz}, 1 \mathrm{H}), 8.02(\mathrm{~d}, J=7.7 \mathrm{~Hz}, 1 \mathrm{H}), 7.88-7.82(\mathrm{~m}$, $1 \mathrm{H}), 7.72-7.61(\mathrm{~m}, 2 \mathrm{H}), 7.58-7.51(\mathrm{~m}, 2 \mathrm{H})$, (in ${ }^{1} \mathrm{H}$ NMR spectrum $5.5 \%$ of diketone form is present). ${ }^{13} \mathrm{C}$ NMR (100.6 MHz, DMSO[D 6 ]) $\delta=185.7,183.3,136.9,135.6,135.1,132.3$, $131.7,131.0,129.8,129.4,129.0,128.6,128.4,127.7,127.0,126.4,123.4,122.3,94.0$. HRMS $(m / z)[\mathrm{M}+\mathrm{H}]^{+}$calcd for $\mathrm{M}=\mathrm{C}_{19} \mathrm{H}_{13}{ }^{79} \mathrm{BrO}_{2}, 353.0177$; found, 353.0178.

\section{3-(3-Bromophenyl)-5-(naphthalen-2-yl)-1H-pyrazole (sery292b)}

The title compound was prepared according to the GEP 2 starting from sery292a and purified by recrystallisation from ethanol to provide sery292b as a white solid. Yield 88\%. m.p.: 189$190{ }^{\circ} \mathrm{C} .{ }^{1} \mathrm{H}$ NMR $(400 \mathrm{MHz}$, DMSO[D 6$]+1-2 \%$ conc. DCl) $\delta=8.37(\mathrm{~s}, 1 \mathrm{H}), 8.09(\mathrm{t}, J=1.6$ $\mathrm{Hz}, 1 \mathrm{H}), 8.00(\mathrm{~s}, 2 \mathrm{H}), 7.97-7.91(\mathrm{~m}, 2 \mathrm{H}), 7.89$ (d, $J=7.8 \mathrm{~Hz}, 1 \mathrm{H}), 7.59-7.48(\mathrm{~m}, 3 \mathrm{H}), 7.46-$ $7.40(\mathrm{~m}, 2 \mathrm{H}) .{ }^{13} \mathrm{C}$ NMR $\left(100.6 \mathrm{MHz}, \mathrm{DMSO}\left[\mathrm{D}_{6}\right]+1-2 \%\right.$ conc. DCl) $\delta=147.5,146.9,130.5$, 134.1, 133.2, 132.6, 131.1, 130.5, 128.6, 128.0, 127.8, 127.6, 126.8, 126.3 (2C), 124.1, 123.6, 122.4, 100.8. HRMS $(m / z)[\mathrm{M}+\mathrm{H}]^{+}$calcd for $\mathrm{M}=\mathrm{C}_{19} \mathrm{H}_{13}{ }^{79} \mathrm{BrN}_{2}, 349.0340$; found, 349.0346.

\section{1-(1,3-Benzodioxol-5-yl)-3-(3,5-difluorophenyl)propane-1,3-dione (sery294a)}

The title compound was prepared according to the GEP 1 using 1-(1,3-benzodioxol-5yl)ethanone and methyl 3,5-difluorobenzoate and purified by recrystallisation from methanol to provide sery294a as a yellow solid. Yield 85\%. m.p.: $150-151{ }^{\circ} \mathrm{C} .{ }^{1} \mathrm{H}$ NMR $(400 \mathrm{MHz}$, DMSO[D 6$]) \delta=7.91(\mathrm{~m}, 3 \mathrm{H}), 7.76(\mathrm{~d}, J=1.7 \mathrm{~Hz}, 1 \mathrm{H}), 7.53(\mathrm{tt}, J=9.1,2.3 \mathrm{~Hz}, 1 \mathrm{H}), 7.32(\mathrm{~s}$, $1 \mathrm{H}), 7.10(\mathrm{~d}, J=8.3 \mathrm{~Hz}, 1 \mathrm{H}), 6.17(\mathrm{~s}, 2 \mathrm{H})$, (in ${ }^{1} \mathrm{H}$ NMR spectrum $4.5 \%$ of diketone form is present). ${ }^{13} \mathrm{C}$ NMR (100.6 MHz, DMSO[D 6$\left.]\right) \delta=186.4,179.9,162.6$ (dd, $J=246.8,12.7$ $\mathrm{Hz}), 151.9,148.1,138.1,128.6,124.1,110.4$ (d, $J=23.0 \mathrm{~Hz}), 108.4,107.9$ (t, $J=26.1 \mathrm{~Hz})$, 
107.3, 102.2, 93.4. HRMS $(\mathrm{m} / \mathrm{z})[\mathrm{M}+\mathrm{H}]^{+}$calcd for $\mathrm{M}=\mathrm{C}_{16} \mathrm{H}_{10} \mathrm{~F}_{2} \mathrm{O}_{4}, 305.0625$; found, 305.0626 .

\section{3-(1,3-Benzodioxol-5-yl)-5-(3,5-difluorophenyl)-1H-pyrazole (sery294b)}

The title compound was prepared according to the GEP 2 starting from sery294a and purified by recrystallisation from ethanol to provide sery294b as a white solid. Yield 81\%. m.p.: 222$223{ }^{\circ} \mathrm{C} .{ }^{1} \mathrm{H}$ NMR $\left(400 \mathrm{MHz}, \mathrm{DMSO}\left[\mathrm{D}_{6}\right]+1-2 \%\right.$ conc. DCl) $\delta=7.56-7.48(\mathrm{~m}, 2 \mathrm{H}), 7.35(\mathrm{~d}, J$ $=1.5 \mathrm{~Hz}, 1 \mathrm{H}), 7.31(\mathrm{dd}, J=8.1,1.5 \mathrm{~Hz}, 1 \mathrm{H}), 7.23(\mathrm{~s}, 1 \mathrm{H}), 7.16(\mathrm{tt}, J=9.3,2.2 \mathrm{~Hz}, 1 \mathrm{H}), 7.01$ $(\mathrm{d}, J=8.1 \mathrm{~Hz}, 1 \mathrm{H}), 6.07(\mathrm{~s}, 2 \mathrm{H}) .{ }^{13} \mathrm{C}$ NMR $\left(100.6 \mathrm{MHz}, \mathrm{DMSO}\left[\mathrm{D}_{6}\right]+1-2 \%\right.$ conc. DCl) $\delta=$ 162.8 (dd, $J=245.1,13.4 \mathrm{~Hz}$ ), 148.0, 147.3, 147.0, 146.1, 136.1, 124.5, 119.1, 108.9, 108.1 $(\mathrm{d}, J=26.1 \mathrm{~Hz}), 105.7,102.9(\mathrm{t}, J=26.0 \mathrm{~Hz}), 101.4,100.4$. HRMS $(\mathrm{m} / \mathrm{z})[\mathrm{M}+\mathrm{H}]^{+}$calcd for M $=\mathrm{C}_{16} \mathrm{H}_{10} \mathrm{~F}_{2} \mathrm{~N}_{2} \mathrm{O}_{2}, 301.0789$; found, 301.0789.

\section{1-(1,3-Benzodioxol-5-yl)-3-(3,5-dichlorophenyl)propane-1,3-dione (sery297a)}

The title compound was prepared according to the GEP 1 using 1-(1,3-benzodioxol-5yl)ethanone and methyl 3,5-dichlorobenzoate and purified by recrystallisation from methanol to provide sery297a as a yellow solid. Yield 98\%. m.p.: 160-161 ${ }^{\circ} \mathrm{C} .{ }^{1} \mathrm{H}$ NMR $(400 \mathrm{MHz}$, $\left.\mathrm{CDCl}_{3}\right) \delta=7.80(\mathrm{~d}, J=1.9 \mathrm{~Hz}, 2 \mathrm{H}), 7.60(\mathrm{dd}, J=8.2,1.8 \mathrm{~Hz}, 1 \mathrm{H}), 7.50(\mathrm{t}, J=1.9 \mathrm{~Hz}, 1 \mathrm{H})$, $7.46(\mathrm{~d}, J=1.8 \mathrm{~Hz}, 1 \mathrm{H}), 6.90(\mathrm{~d}, J=8.2 \mathrm{~Hz}, 1 \mathrm{H}), 6.65(\mathrm{~s}, 1 \mathrm{H}), 6.08(\mathrm{~s}, 2 \mathrm{H}) .{ }^{13} \mathrm{C}$ NMR $(100.6$ $\left.\mathrm{MHz}, \mathrm{CDCl}_{3}\right) \delta=186.7,180.7,152.1,148.5,138.5,135.7,131.9,129.7,125.5,123.5,108.4$, 107.4, 102.2, 93.0. HRMS $(m / z)[\mathrm{M}+\mathrm{H}]^{+}$calcd for $\mathrm{M}=\mathrm{C}_{16} \mathrm{H}_{10} \mathrm{Cl}_{2} \mathrm{O}_{4}, 337.0034$; found, 337.0033 .

\section{3-(1,3-Benzodioxol-5-yl)-5-(3,5-dichlorophenyl)-1H-pyrazole (sery297b)}

The title compound was prepared according to the GEP 2 starting from sery297a and purified by recrystallisation from methanol to provide sery297b as a white solid. Yield 81\%. m.p.: 257-259 ${ }^{\circ} \mathrm{C} .{ }^{1} \mathrm{H}$ NMR $\left(400 \mathrm{MHz}, \mathrm{DMSO}\left[\mathrm{D}_{6}\right]+1-2 \%\right.$ conc. DCl) $\delta=7.86(\mathrm{~s}, 2 \mathrm{H}), 7.52$ (s, $1 \mathrm{H}), 7.41-7.24(\mathrm{~m}, 3 \mathrm{H}), 7.01(\mathrm{~d}, J=8.0 \mathrm{~Hz}, 1 \mathrm{H}), 6.06(\mathrm{~s}, 2 \mathrm{H}) .{ }^{13} \mathrm{C}$ NMR $(100.6 \mathrm{MHz}$, DMSO[D 6$]) \delta=148.6,147.9,147.3,143.7,137.2,134.5,126.6,123.4,123.1,119.0,108.8$, 105.6, 101.3, 100.1. HRMS $(\mathrm{m} / \mathrm{z})[\mathrm{M}+\mathrm{H}]^{+}$calcd for $\mathrm{M}=\mathrm{C}_{16} \mathrm{H}_{10} \mathrm{Cl}_{2} \mathrm{~N}_{2} \mathrm{O}_{2}$, 333.0198; found, 333.0201 .

\section{1-(3-Bromophenyl)-3-(2,3-dihydrobenzofuran-5-yl)propane-1,3-dione (sery312a)}

The title compound was prepared according to the GEP 1 using 1-(2,3-dihydrobenzofuran-5yl)ethanone and ethyl 3-bromobenzoate and purified by recrystallisation from methanol to provide sery312a as a yellow solid. Yield 88\%. m.p.: 117-118 ${ }^{\circ} \mathrm{C}$. ${ }^{1} \mathrm{H}$ NMR $(400 \mathrm{MHz}$, $\left.\mathrm{CDCl}_{3}\right) \delta=8.08(\mathrm{t}, J=1.8 \mathrm{~Hz}, 1 \mathrm{H}), 7.92-7.86(\mathrm{~m}, 2 \mathrm{H}), 7.84(\mathrm{dd}, J=8.5,1.9 \mathrm{~Hz}, 1 \mathrm{H}), 7.65$ (ddd, $J=8.0,1.8,1.0 \mathrm{~Hz}, 1 \mathrm{H}), 7.35(\mathrm{t}, J=8.0 \mathrm{~Hz}, 1 \mathrm{H}), 6.86(\mathrm{~d}, J=8.5 \mathrm{~Hz}, 1 \mathrm{H}), 6.72(\mathrm{~s}, 1 \mathrm{H})$, 4.69 (t, $J=8.8 \mathrm{~Hz}, 2 \mathrm{H}), 3.29(\mathrm{t}, J=8.8 \mathrm{~Hz}, 2 \mathrm{H}) .{ }^{13} \mathrm{C} \mathrm{NMR}\left(100.6 \mathrm{MHz}, \mathrm{CDCl}_{3}\right) \delta=187.0$, 181.9, 164.6, 137.8, 135.0, 130.3, 130.1, 129.2, 128.3, 128.1, 125.6, 124.7, 123.0, 109.6, 92.7, 72.4, 29.3. HRMS $(m / z)[\mathrm{M}+\mathrm{H}]^{+}$calcd for $\mathrm{M}=\mathrm{C}_{17} \mathrm{H}_{13}{ }^{79} \mathrm{BrO}_{3}, 345.0126$; found, 345.0128.

\section{3-(3-Bromophenyl)-5-(2,3-dihydrobenzofuran-5-yl)-1H-pyrazole (sery312b)}

The title compound was prepared according to the GEP 2 starting from sery312a and purified by recrystallisation from ethanol/water to provide sery312b as a white solid. Yield $82 \%$. m.p.: $169-170{ }^{\circ} \mathrm{C} .{ }^{1} \mathrm{H}$ NMR $\left(400 \mathrm{MHz}, \mathrm{DMSO}\left[\mathrm{D}_{6}\right]+1-2 \%\right.$ conc. DCl) $\delta=8.02(\mathrm{~s}, 1 \mathrm{H}), 7.83(\mathrm{~d}, J$ $=7.5 \mathrm{~Hz}, 1 \mathrm{H}), 7.68(\mathrm{~s}, 1 \mathrm{H}), 7.55(\mathrm{~d}, J=8.1 \mathrm{~Hz}, 1 \mathrm{H}), 7.50(\mathrm{~d}, J=7.5 \mathrm{~Hz}, 1 \mathrm{H}), 7.39(\mathrm{t}, J=7.8$ $\mathrm{Hz}, 1 \mathrm{H}), 7.12(\mathrm{~s}, 1 \mathrm{H}), 6.84(\mathrm{~d}, J=8.2 \mathrm{~Hz}, 1 \mathrm{H}), 4.56(\mathrm{t}, J=8.5 \mathrm{~Hz}, 2 \mathrm{H}), 3.22(\mathrm{t}, J=8.5 \mathrm{~Hz}$, 2H). ${ }^{13} \mathrm{C}$ NMR (100.6 MHz, DMSO[D 6$]+1-2 \%$ conc. DCl) $\delta=159.8,147.0,146.2,134.8$, 
131.0, 130.3, 128.2, 127.6, 125.3, 124.1, 123.2, 122.3, 122.2, 109.3, 99.3, 71.3, 29.0. HRMS $(m / z)[\mathrm{M}+\mathrm{H}]^{+}$calcd for $\mathrm{M}=\mathrm{C}_{17} \mathrm{H}_{13}{ }^{79} \mathrm{BrN}_{2} \mathrm{O}, 341.0290$; found, 341.0294.

\section{1-(3-Bromophenyl)-3-(2,3-dihydro-1,4-benzodioxin-6-yl)propane-1,3-dione (sery315a)}

The title compound was prepared according to the GEP 1 using 1-(2,3-dihydro-1,4benzodioxin-6-yl)ethanone and ethyl 3-bromobenzoate and purified by recrystallisation from methanol to provide sery315a as a yellow solid. Yield 89\%. m.p.: 108-110 ${ }^{\circ} \mathrm{C}$. ${ }^{1} \mathrm{H}$ NMR $(400$ $\left.\mathrm{MHz}, \mathrm{DMSO}\left[\mathrm{D}_{6}\right]\right) \delta=8.35(\mathrm{~s}, 1 \mathrm{H}), 8.13(\mathrm{~d}, J=8.0 \mathrm{~Hz}, 1 \mathrm{H}), 7.80(\mathrm{~d}, J=8.0 \mathrm{~Hz}, 1 \mathrm{H}), 7.77-$ $7.70(\mathrm{~m}, 2 \mathrm{H}), 7.50(\mathrm{t}, J=8.0 \mathrm{~Hz}, 1 \mathrm{H}), 7.30(\mathrm{~s}, 1 \mathrm{H}), 7.01(\mathrm{~d}, J=8.3 \mathrm{~Hz}, 1 \mathrm{H}), 4.40-4.25(\mathrm{~m}$, $4 \mathrm{H}) .{ }^{13} \mathrm{C}$ NMR $\left(100.6 \mathrm{MHz}, \mathrm{DMSO}\left[\mathrm{D}_{6}\right]\right) \delta=186.1,181.5,148.1,143.6,136.8,135.2,130.9$, $129.7,127.7,126.2,122.3,121.7,117.3,116.7,93.1,64.6,63.9$. HRMS $(m / z)[\mathrm{M}+\mathrm{H}]^{+}$calcd for $\mathrm{M}=\mathrm{C}_{17} \mathrm{H}_{13}{ }^{79} \mathrm{BrO}_{4}, 361.0075$; found, 361.0077 .

\section{3-(3-Bromophenyl)-5-(2,3-dihydro-1,4-benzodioxin-6-yl)-1H-pyrazole (sery315b)}

The title compound was prepared according to the GEP 2 starting from sery315a and purified by recrystallisation from methanol to provide sery315b as a white solid. Yield $69 \%$. m.p.: $167-169{ }^{\circ} \mathrm{C} .{ }^{1} \mathrm{H}$ NMR $\left(400 \mathrm{MHz}, \mathrm{DMSO}\left[\mathrm{D}_{6}\right]+1-2 \%\right.$ conc. DCl) $\delta=8.02(\mathrm{~s}, 1 \mathrm{H}), 7.83(\mathrm{~d}, J$ $=7.8 \mathrm{~Hz}, 1 \mathrm{H}), 7.50(\mathrm{~d}, J=8.1 \mathrm{~Hz}, 1 \mathrm{H}), 7.39(\mathrm{t}, J=7.8 \mathrm{~Hz}, 1 \mathrm{H}), 7.33(\mathrm{~d}, J=1.8 \mathrm{~Hz}, 1 \mathrm{H})$, $7.28(\mathrm{dd}, J=8.4,1.8 \mathrm{~Hz}, 1 \mathrm{H}), 7.17(\mathrm{~s}, 1 \mathrm{H}), 6.93(\mathrm{~d}, J=8.4 \mathrm{~Hz}, 1 \mathrm{H}), 4.27(\mathrm{~s}, 4 \mathrm{H}) .{ }^{13} \mathrm{C} \mathrm{NMR}$ (100.6 MHz, DMSO[D 6$]+1-2 \%$ conc. DCl) $\delta=147.8,146.3,143.7,143.4,134.7,131.0$, 130.3, 127.6, $124.1(2 \mathrm{C}), 122.3,118.4,117.6,113.9,99.7,64.3,64.2 . \operatorname{HRMS}(\mathrm{m} / \mathrm{z})[\mathrm{M}+\mathrm{H}]^{+}$ calcd for $\mathrm{M}=\mathrm{C}_{17} \mathrm{H}_{13}{ }^{79} \mathrm{BrN}_{2} \mathrm{O}_{2}, 357.0239$; found, 357.0242 .

\section{1-(1,3-Benzodioxol-5-yl)-3-(2,5-difluorophenyl)propane-1,3-dione (sery316a)}

The title compound was prepared according to the GEP 1 using 1-(1,3-benzodioxol-5yl)ethanone and methyl 2,5-difluorobenzoate and purified by recrystallisation from methanol to provide sery316a as a yellow solid. Yield 89\%. m.p.: 131-133 ${ }^{\circ} \mathrm{C} .{ }^{1} \mathrm{H}$ NMR $(400 \mathrm{MHz}$, DMSO[D 6$]) \delta=7.79-7.68(\mathrm{~m}, 2 \mathrm{H}), 7.58(\mathrm{~d}, J=1.5 \mathrm{~Hz}, 1 \mathrm{H}), 7.54-7.40(\mathrm{~m}, 2 \mathrm{H}), 7.08(\mathrm{~d}, J=$ $8.2 \mathrm{~Hz}, 1 \mathrm{H}), 6.98(\mathrm{~s}, 1 \mathrm{H}), 6.17(\mathrm{~s}, 2 \mathrm{H}) .{ }^{13} \mathrm{C} \mathrm{NMR}\left(100.6 \mathrm{MHz}, \mathrm{DMSO}\left[\mathrm{D}_{6}\right]\right) \delta=186.2,178.7$, $158.0(\mathrm{~d}, J=241.6 \mathrm{~Hz}), 156.4(\mathrm{~d}, J=249.6 \mathrm{~Hz}), 151.9,148.1,128.5,124.4(\mathrm{~m}), 123.9,120.7$ $(\mathrm{dd}, J=23.5,9.7 \mathrm{~Hz}), 118.8(\mathrm{dd}, J=25.5,7.4 \mathrm{~Hz}), 115.9(\mathrm{dd}, J=25.5,7.4 \mathrm{~Hz}), 108.5,106.9$, 102.3, $96.8(\mathrm{dd}, J=8.7,3.7 \mathrm{~Hz})$. HRMS $(\mathrm{m} / \mathrm{z})[\mathrm{M}+\mathrm{H}]^{+}$calcd for $\mathrm{M}=\mathrm{C}_{16} \mathrm{H}_{10} \mathrm{~F}_{2} \mathrm{O}_{4}, 305.0625$; found, 305.0625 .

\section{3-(1,3-Benzodioxol-5-yl)-5-(2,5-difluorophenyl)-1 $H$-pyrazole (sery316b)}

The title compound was prepared according to the GEP 2 starting from sery316a and purified by recrystallisation from ethanol/EtOAc to provide sery316b as a white solid. Yield $83 \%$. m.p.: $225-226{ }^{\circ} \mathrm{C} .{ }^{1} \mathrm{H}$ NMR (400 MHz, DMSO[D $]+1-2 \%$ conc. DCl) $\delta=7.76-7.67(\mathrm{~m}, 1 \mathrm{H})$, 7.42-7.30 (m, 3H), 7.26-7.17 (m, 1H), $7.03(\mathrm{~d}, J=3.4 \mathrm{~Hz}, 1 \mathrm{H}), 6.99(\mathrm{~d}, J=8.1 \mathrm{~Hz}, 1 \mathrm{H}), 6.06$ (s, 2H). ${ }^{13} \mathrm{C}$ NMR (100.6 MHz, DMSO[D 6$\left.]\right) \delta=158.3(\mathrm{~d}, J=239.8 \mathrm{~Hz}), 155.2(\mathrm{~d}, J=244.6$ Hz), 147.9, 147.3, 146.4, 142.4, 124.6, 121.6, 119.2, 118.0 (dd, $J=25.3,8.9 \mathrm{~Hz}), 115.9$ (dd, $J$ $=24.2,8.8 \mathrm{~Hz}), 113.8(\mathrm{~m}), 108.8,105.8,102.3(\mathrm{~d}, J=8.4 \mathrm{~Hz}), 101.3$. HRMS $(\mathrm{m} / \mathrm{z})[\mathrm{M}+\mathrm{H}]^{+}$ calcd for $\mathrm{M}=\mathrm{C}_{16} \mathrm{H}_{10} \mathrm{~F}_{2} \mathrm{~N}_{2} \mathrm{O}_{2}, 301.0789$; found, 301.0789 .

5-(1,3-Benzodioxol-5-yl)-3-(3-bromophenyl)-1-(2-hydroxyethyl)-1 $H$-pyrazole and 3-(1,3Benzodioxol-5-yl)-5-(3-bromophenyl)-1-(2-hydroxyethyl)-1H-pyrazole (sery329)

The title compounds was prepared according to the GEP 2 starting from anle138b and 2hydroxyethylhydrazine and purified by column chromatography (hexane:EtOAc, 2.5:1 v/v) to provide sery329 as a white solid (mixture of isomers 1:1). Yield 50\%. TLC (hexane:EtOAc, 2.5:1 v/v): RF $=0.28$. m.p.: $90-120{ }^{\circ} \mathrm{C} .{ }^{1} \mathrm{H}$ NMR (400 MHz, DMSO[D 6 ] $\delta=8.08(\mathrm{t}, J=1.6$ 
$\mathrm{Hz}, 1 \mathrm{H}), 7.86(\mathrm{t}, J=1.6 \mathrm{~Hz}, 1 \mathrm{H}), 7.83(\mathrm{~d}, J=7.8 \mathrm{~Hz}, 1 \mathrm{H}), 7.69-7.63(\mathrm{~m}, 2 \mathrm{H}), 7.52-7.44(\mathrm{~m}$, $2 \mathrm{H}), 7.41-7.32(\mathrm{~m}, 3 \mathrm{H}), 7.23(\mathrm{~d}, J=1.5 \mathrm{~Hz}, 1 \mathrm{H}), 7.11(\mathrm{dd}, J=8.0,1.5 \mathrm{~Hz}, 1 \mathrm{H}), 7.05(\mathrm{~d}, J=$ $8.0 \mathrm{~Hz}, 1 \mathrm{H}), 6.95(\mathrm{~d}, J=8.0 \mathrm{~Hz}, 1 \mathrm{H}), 6.85(\mathrm{~s}, 1 \mathrm{H}), 6.83(\mathrm{~s}, 1 \mathrm{H}), 6.10(\mathrm{~s}, 2 \mathrm{H}), 6.04(\mathrm{~s}, 2 \mathrm{H})$, 5.04-4.97 (m, 2H), 4.18-4.09 (m, 4H), 3.88-3.80 (m, 4H). ${ }^{13} \mathrm{C}$ NMR (100.6 MHz, DMSO[D 6$\left.]\right)$ $\delta=149.3,147.7,147.6(2 \mathrm{C}), 147.5,146.8,145.2,143.6,135.7,132.5,131.4,131.3,130.85$, $130.8,130.1,127.9,127.5,127.4,124.0,123.6,123.0,122.1,121.9,118.7,109.3,108.5$, $108.45,105.5,103.35,103.3,101.4,101.0,60.0,59.9,51.5,51.4$. HRMS $(m / z)[\mathrm{M}+\mathrm{H}]^{+}$calcd for $\mathrm{M}=\mathrm{C}_{18} \mathrm{H}_{15}{ }^{79} \mathrm{BrN}_{2} \mathrm{O}_{3}, 387.0344$; found, 387.0345.

\section{1-(1,3-Benzodioxol-5-yl)-3-(3-chlorophenyl)propane-1,3-dione (sery335a)}

The title compound has been reported previously ${ }^{519}$ (CAS 749927-14-8). It was prepared according to the GEP 1 using 1-(1,3-benzodioxol-5-yl)ethanone and methyl 3-chlorobenzoate and purified by recrystallisation from methanol to provide sery335a as a yellow solid. Yield 87\%. m.p.: $127-128{ }^{\circ} \mathrm{C} .{ }^{1} \mathrm{H}$ NMR (400 MHz, DMSO[D 6$\left.]\right) \delta=8.20(\mathrm{~s}, 1 \mathrm{H}), 8.07$ (d, $J=7.8$ $\mathrm{Hz}, 1 \mathrm{H}), 7.82(\mathrm{~d}, J=8.2 \mathrm{~Hz}, 1 \mathrm{H}), 7.74(\mathrm{~s}, 1 \mathrm{H}), 7.66(\mathrm{~d}, J=7.8 \mathrm{~Hz}, 1 \mathrm{H}), 7.56$ (t, $J=7.8 \mathrm{~Hz}$, 1H), $7.27(\mathrm{~s}, 1 \mathrm{H}), 7.07(\mathrm{~d}, J=8.2 \mathrm{~Hz}, 1 \mathrm{H}), 6.16(\mathrm{~s}, 2 \mathrm{H})$ (in ${ }^{1} \mathrm{H}$ NMR spectrum $5.0 \%$ of diketone form is present). ${ }^{13} \mathrm{C}$ NMR $\left(100.6 \mathrm{MHz}, \mathrm{DMSO}\left[\mathrm{D}_{6}\right]\right) \delta=186.1,181.3,151.7,148.0$, $136.5,133.8,132.3,130.6,128.8,126.8,125.7,123.9,108.4,107.3,102.2,93.1$. HRMS $(m / z)$ $[\mathrm{M}+\mathrm{H}]^{+}$calcd for $\mathrm{M}=\mathrm{C}_{16} \mathrm{H}_{11} \mathrm{ClO}_{4}, 303.0424$; found, 303.0426.

\section{3-(1,3-Benzodioxol-5-yl)-5-(3-chlorophenyl)-1 $H$-pyrazole (sery335b)}

The title compound has been reported previously ${ }^{519}$ (CAS 749927-20-6). It was prepared according to the GEP 2 starting from sery335a and purified by recrystallisation from methanol to provide sery335b as a white solid. Yield 83\%. m.p.: 188-189 ${ }^{\circ} \mathrm{C}$. ${ }^{1} \mathrm{H}$ NMR (400 $\mathrm{MHz}, \mathrm{DMSO}\left[\mathrm{D}_{6}\right]+1-2 \%$ conc. $\left.\mathrm{DCl}\right) \delta=7.87(\mathrm{~s}, 1 \mathrm{H}), 7.78(\mathrm{~d}, J=7.2 \mathrm{~Hz}, 1 \mathrm{H}), 7.45(\mathrm{t}, J=$ $7.2 \mathrm{~Hz}, 1 \mathrm{H}), 7.4-7.25(\mathrm{~m}, 3 \mathrm{H}), 7.18(\mathrm{~s}, 1 \mathrm{H}), 7.00(\mathrm{~d}, J=6.9 \mathrm{~Hz}, 1 \mathrm{H}), 6.05(\mathrm{~s}, 2 \mathrm{H})$. HRMS $(\mathrm{m} / \mathrm{z})[\mathrm{M}+\mathrm{H}]^{+}$calcd for $\mathrm{M}=\mathrm{C}_{16} \mathrm{H}_{11} \mathrm{ClN}_{2} \mathrm{O}_{2}, 299.0587$; found, 299.0587.

\section{1-(4-Benzyloxyphenyl)-3-(3,4-dimethoxyphenyl)propane-1,3-dione (sery455a)}

The title compound was prepared according to the GEP 1 using 1-(4benzyloxyphenyl)ethanone and ethyl 3,4-dimethoxybenzoate and purified by recrystallisation from methanol/EtOAc to provide sery455a as a yellow solid. Yield 56\%. m.p.: 112-113 ${ }^{\circ} \mathrm{C}$. ${ }^{1} \mathrm{H}$ NMR (400 MHz, DMSO[D 6 ) $\delta=8.15(\mathrm{~d}, J=8.9 \mathrm{~Hz}, 2 \mathrm{H}), 7.83(\mathrm{dd}, J=8.5,2.0 \mathrm{~Hz}, 1 \mathrm{H})$, $7.61(\mathrm{~d}, J=2.0 \mathrm{~Hz}, 1 \mathrm{H}), 7.55-7.52(\mathrm{~m}, 2 \mathrm{H}), 7.44-7.34(\mathrm{~m}, 3 \mathrm{H}), 7.22-7.14(\mathrm{~m}, 3 \mathrm{H}), 7.10(\mathrm{~d}, J$ $=8.6 \mathrm{~Hz}, 1 \mathrm{H}), 5.23(\mathrm{~s}, 2 \mathrm{H}), 3.87(\mathrm{~s}, 3 \mathrm{H}), 3.86(\mathrm{~s}, 3 \mathrm{H})\left(\right.$ in ${ }^{1} \mathrm{H}$ NMR spectrum $12 \%$ of diketone form is present). ${ }^{13} \mathrm{C}$ NMR (100.6 MHz, DMSO[D 6 ]) $\delta=184.6,183.7,162.1,152.9,148.7$, $136.5,129.5,128.5,128.0,127.8,127.3(2 \mathrm{C}), 121.6,114.9,111.2,110.0,91.7,69.5,55.7$ (2C). HRMS $(m / z)[\mathrm{M}+\mathrm{H}]^{+}$calcd for $\mathrm{M}=\mathrm{C}_{24} \mathrm{H}_{22} \mathrm{O}_{5}, 391.1545$; found, 391.1542.

\section{3-(4-Benzyloxyphenyl)-5-(3,4-dimethoxyphenyl)-1 $H$-pyrazole (sery455b)}

The title compound was prepared according to the GEP 2 starting from sery455a and purified by recrystallisation from ethanol/water to provide sery $455 \mathrm{~b}$ as a white solid. Yield $76 \%$. m.p.: 131-134 ${ }^{\circ} \mathrm{C} .{ }^{1} \mathrm{H}$ NMR $\left(400 \mathrm{MHz}, \mathrm{DMSO}\left[\mathrm{D}_{6}\right]+1-2 \%\right.$ conc. DCl) $\delta=7.75(\mathrm{~d}, J=8.7 \mathrm{~Hz}, 2 \mathrm{H})$, 7.50-7.31 (m, 7H), $7.09(\mathrm{~d}, J=8.7 \mathrm{~Hz}, 2 \mathrm{H}), 7.04-6.99(\mathrm{~m}, 2 \mathrm{H}), 5.14(\mathrm{~s}, 2 \mathrm{H}), 3.84(\mathrm{~s}, 3 \mathrm{H})$, $3.79(\mathrm{~s}, 3 \mathrm{H}) .{ }^{13} \mathrm{C}$ NMR $\left(100.6 \mathrm{MHz}, \mathrm{DMSO}\left[\mathrm{D}_{6}\right]+1-2 \%\right.$ conc. DCl) $\delta=158.0,148.9,148.6$, 147.7-146.8 (2C), 137.0, 128.4, 127.9, 127.7, 126.4, 124.3, 124.1, 117.6, 115.1, 112.0, 109.0, 98.5, 69.3, 55.6 (2C). HRMS $(m / z)[\mathrm{M}+\mathrm{H}]^{+}$calcd for $\mathrm{M}=\mathrm{C}_{24} \mathrm{H}_{22} \mathrm{~N}_{2} \mathrm{O}_{3}, 387.1709$; found, 387.1710. 


\section{3-(3,4-Dimethoxyphenyl)-5-(4-hydroxyphenyl)-1H-pyrazole (sery279b)}

To a stirred suspension of palladium on activated carbon $(10 \mathrm{wt} \%, 40 \mathrm{mg})$ in acetic acid (2 $\mathrm{ml})$ under a hydrogen atmosphere a solution of sery $455 \mathrm{~b}(386 \mathrm{mg}, 1 \mathrm{mmol})$ in acetic acid (3 $\mathrm{ml}$ ) was added. After stirring for 3 hours under the hydrogen atmosphere at room temperature, the reaction mixture was filtered through Celite and concentrated under reduced pressure. A resulting precipitate was purified by recrystallisation from methanol/water to provide sery279b $(210 \mathrm{mg}, 0.71 \mathrm{mmol})$ as a white solid. Yield 71\%. m.p.: 232-233 ${ }^{\circ} \mathrm{C} .{ }^{1} \mathrm{H}$ NMR (400 $\mathrm{MHz}, \mathrm{DMSO}\left[\mathrm{D}_{6}\right]+1-2 \%$ conc. DCl) $\delta=9.63(\mathrm{bs}, 1 \mathrm{H}), 7.63(\mathrm{~d}, J=8.6 \mathrm{~Hz}, 2 \mathrm{H}), 7.41(\mathrm{~d}, J=$ $1.9 \mathrm{~Hz}, 1 \mathrm{H}), 7.34(\mathrm{dd}, J=8.4,1.9 \mathrm{~Hz}, 1 \mathrm{H}), 6.99(\mathrm{~d}, J=8.1 \mathrm{~Hz}, 1 \mathrm{H}), 6.95(\mathrm{~s}, 1 \mathrm{H}), 6.83(\mathrm{~d}, J=$ $8.6 \mathrm{~Hz}, 2 \mathrm{H}), 3.83(\mathrm{~s}, 3 \mathrm{H}), 3.78(\mathrm{~s}, 3 \mathrm{H}) .{ }^{13} \mathrm{C} \mathrm{NMR}\left(100.6 \mathrm{MHz}, \mathrm{DMSO}\left[\mathrm{D}_{6}\right]+1-2 \%\right.$ conc. DCl) $\delta=157.3,149.0,148.6,148.4-147.4$ (2C), 126.6, 125.0, 123.0, 117.6, 115.6, 112.1, 109.0, 98.2, 55.6 (2C). HRMS $(m / z)[\mathrm{M}+\mathrm{H}]^{+}$calcd for $\mathrm{M}=\mathrm{C}_{17} \mathrm{H}_{16} \mathrm{~N}_{2} \mathrm{O}_{3}, 297.1239$; found, 297.1234.

\section{1-(3-Benzyloxyphenyl)-3-(3,4-dimethoxyphenyl)propane-1,3-dione (sery456a)}

The title compound was prepared according to the GEP 1 using methyl 4-benzyloxybenzoate and 1-(3,4-dimethoxyphenyl)ethanone and purified by recrystallisation from methanol to provide sery456a as a yellow solid. Yield 63\%. m.p.: 109-111 ${ }^{\circ} \mathrm{C}$. ${ }^{1} \mathrm{H}$ NMR $(400 \mathrm{MHz}$, $\left.\mathrm{CDCl}_{3}\right) \delta=7.63-7.59(\mathrm{~m}, 2 \mathrm{H}), 7.59-7.54(\mathrm{~m}, 2 \mathrm{H}), 7.50-7.44(\mathrm{~m}, 2 \mathrm{H}), 7.44-7.32(\mathrm{~m}, 4 \mathrm{H}), 7.15$ $(\mathrm{dd}, J=8.2,2.6 \mathrm{~Hz}, 1 \mathrm{H}), 6.93(\mathrm{~d}, J=8.5 \mathrm{~Hz}, 1 \mathrm{H}), 6.76(\mathrm{~s}, 1 \mathrm{H}), 5.14(\mathrm{~s}, 2 \mathrm{H}), 3.98(\mathrm{~s}, 3 \mathrm{H})$, $3.96(\mathrm{~s}, 3 \mathrm{H})$ (in ${ }^{1} \mathrm{H}$ NMR spectrum $6 \%$ of diketone form is present). ${ }^{13} \mathrm{C}$ NMR $(100.6 \mathrm{MHz}$, $\left.\mathrm{CDCl}_{3}\right) \delta=186.7,183.3,159.2,153.1,149.3,137.1,136.7,129.8,128.8,128.7,128.3,127.7$, $121.5,119.8,119.0,113.3,110.6,109.9,92.8,70.4,56.2(2 \mathrm{C})$. HRMS $(\mathrm{m} / z)[\mathrm{M}+\mathrm{H}]^{+}$calcd for $\mathrm{M}=\mathrm{C}_{24} \mathrm{H}_{22} \mathrm{O}_{5}, 391.1545$; found, 391.1541.

\section{3-(4-Benzyloxyphenyl)-5-(3,4-dimethoxyphenyl)-1 $H$-pyrazole (sery456b)}

The title compound was prepared according to the GEP 2 starting from sery456a and purified by washing with water to provide sery456b as a white solid. Yield $86 \%$. m.p.: $74-75{ }^{\circ} \mathrm{C} .{ }^{1} \mathrm{H}$ NMR (400 MHz, DMSO[D 6$]+1-2 \%$ conc. DCl) $\delta=7.53-7.47(\mathrm{~m}, 3 \mathrm{H}), 7.45-7.31(\mathrm{~m}, 7 \mathrm{H})$, $7.15(\mathrm{~s}, 1 \mathrm{H}), 7.03(\mathrm{~d}, J=8.4 \mathrm{~Hz}, 1 \mathrm{H}), 6.98(\mathrm{dd}, J=8.1,1.8 \mathrm{~Hz}, 1 \mathrm{H}), 5.17(\mathrm{~s}, 2 \mathrm{H}), 3.84(\mathrm{~s}$, $3 \mathrm{H}), 3.79(\mathrm{~s}, 3 \mathrm{H}) .{ }^{13} \mathrm{C} \mathrm{NMR}\left(100.6 \mathrm{MHz}, \mathrm{DMSO}\left[\mathrm{D}_{6}\right]+1-2 \%\right.$ conc. DCl) $\delta=158.8,149.0$, $148.7,147.5,146.8,137.1,132.9,129.9,128.4,127.9,127.8,123.8,117.7,117.6,114.0$, 112.0, 111.5, 109.0, 99.4, 69.3, 55.6, 55.55. HRMS $(m / z)[\mathrm{M}+\mathrm{H}]^{+}$calcd for $\mathrm{M}=\mathrm{C}_{24} \mathrm{H}_{22} \mathrm{~N}_{2} \mathrm{O}_{3}$, 387.1709; found, 387.1708 .

\section{3-(3,4-Dimethoxyphenyl)-5-(3-hydroxyphenyl)-1 $H$-pyrazole (sery310b)}

The title compound was prepared using an analogous procedure to that described for sery 279b starting from sery456b and purified by recrystallisation from methanol/water to provide sery310b as a white solid. Yield 90\%. m.p.: $115{ }^{\circ} \mathrm{C}(\mathrm{dec}) .{ }^{1} \mathrm{H}$ NMR $(400 \mathrm{MHz}$, DMSO $\left[\mathrm{D}_{6}\right]+1-2 \%$ conc. DCl) $\delta=9.52(\mathrm{bs}, 1 \mathrm{H}), 7.41(\mathrm{~d}, J=1.6 \mathrm{~Hz}, 1 \mathrm{H}), 7.36(\mathrm{dd}, J=8.3$, $1.6 \mathrm{~Hz}, 1 \mathrm{H}), 7.27-7.19(\mathrm{~m}, 3 \mathrm{H}), 7.03(\mathrm{~s}, 1 \mathrm{H}), 7.01(\mathrm{~d}, J=8.4 \mathrm{~Hz}, 1 \mathrm{H}), 6.74(\mathrm{dt}, J=6.9,2.2$ $\mathrm{Hz}, 1 \mathrm{H}), 3.84$ (s, 3H), 3.78 (s, 3H). ${ }^{13} \mathrm{C}$ NMR (100.6 MHz, DMSO[D 6 + 1-2\% conc. DCl) $\delta$ $=157.8,149.1,148.8,147.9,146.9,133.2,129.9,124.2,117.8,116.3,114.9,112.15,112.1$, 109.1, 99.3, 55.7 (2C). HRMS $(m / z)[\mathrm{M}+\mathrm{H}]^{+}$calcd for $\mathrm{M}=\mathrm{C}_{17} \mathrm{H}_{16} \mathrm{~N}_{2} \mathrm{O}_{3}, 297.1239$; found, 297.1241.

\section{1-(1,3-Benzodioxol-5-yl)-3-(3-benzyloxyphenyl)propane-1,3-dione (sery336b)}

The title compound was prepared according to the GEP 1 using 1-(3benzyloxyphenyl)ethanone and methyl ester of 1,3-benzodioxole-5-carboxylic acid and purified by column chromatography $\left(\mathrm{CH}_{2} \mathrm{Cl}_{2}\right)$ followed by recrystallisation from methanol to provide sery336b as a yellow solid. Yield 46\%. TLC (hexane:EtOAc, 9:1 v/v): RF $=0.33$. 
m.p.: 102-103 ${ }^{\circ} \mathrm{C} .{ }^{1} \mathrm{H}$ NMR $\left.\left(400 \mathrm{MHz}, \mathrm{DMSO}^{\mathrm{D}} 6\right]\right) \delta=7.83(\mathrm{dd}, J=8.2,1.4 \mathrm{~Hz}, 1 \mathrm{H}), 7.79-$ $7.72(\mathrm{~m}, 3 \mathrm{H}), 7.54-7.31(\mathrm{~m}, 6 \mathrm{H}), 7.28(\mathrm{dd}, J=7.8,1.7 \mathrm{~Hz}, 1 \mathrm{H}), 7.25(\mathrm{~s}, 1 \mathrm{H}), 7.09(\mathrm{~d}, J=8.2$ $\mathrm{Hz}, 1 \mathrm{H}), 6.17(\mathrm{~s}, 2 \mathrm{H}), 5.21(\mathrm{~s}, 2 \mathrm{H}) .{ }^{13} \mathrm{C} \mathrm{NMR}\left(100.6 \mathrm{MHz}, \mathrm{DMSO}\left[\mathrm{D}_{6}\right]\right) \delta=185.7,183.0$, $158.6,151.5,148.0,136.8,135.9,129.9,128.9,128.4,127.9,127.8,123.7,119.8,119.2$, 113.2, 108.4, 107.2, 102.1, 92.9, 69.5. HRMS $(\mathrm{m} / \mathrm{z})[\mathrm{M}+\mathrm{H}]^{+}$calcd for $\mathrm{M}=\mathrm{C}_{23} \mathrm{H}_{18} \mathrm{O}_{5}$, 375.1232; found, 375.1234 .

\section{3-(1,3-Benzodioxol-5-yl)-5-(3-benzyloxyphenyl)-1 $H$-pyrazole (sery339a)}

The title compound was prepared according to the GEP 2 starting from sery336b and purified by recrystallisation from methanol (twice) to provide sery339a as a white solid. Yield $74 \%$. m.p.: $158-159{ }^{\circ} \mathrm{C} .{ }^{1} \mathrm{H}$ NMR (400 MHz, DMSO[D 6$]+1-2 \%$ conc. DCl) $\delta=7.52(\mathrm{~m}, 3 \mathrm{H})$, 7.43-7.31 (m, 7H), $7.12(\mathrm{~s}, 1 \mathrm{H}), 7.02-6.95(\mathrm{~m}, 2 \mathrm{H}), 6.05(\mathrm{~s}, 2 \mathrm{H}), 5.16(\mathrm{~s}, 2 \mathrm{H}) .{ }^{13} \mathrm{C} \mathrm{NMR}$ (100.6 MHz, DMSO[D $]+1-2 \%$ conc. DCl) $\delta=158.8,147.8,147.7-147.0$ (2C), 147.0, $137.1,132.9,130.0,128.5,127.9,127.8,125.8,118.9,117.8,114.2,111.5,108.7,105.6$, 101.2, 99.6, 69.3. HRMS $(\mathrm{m} / \mathrm{z})[\mathrm{M}+\mathrm{H}]^{+}$calcd for $\mathrm{M}=\mathrm{C}_{23} \mathrm{H}_{18} \mathrm{~N}_{2} \mathrm{O}_{3}, 371.1396$; found, 371.1391 .

\section{3-(1,3-Benzodioxol-5-yl)-5-(3-hydroxyphenyl)- $1 H$-pyrazole (sery339b)}

The title compound has been reported previously ${ }^{519}$ (CAS 882697-02-1). It was prepared using an analogous procedure to that described for sery 279b starting from sery339a and purified by recrystallisation from methanol/water to provide sery339b as a white solid. Yield 78\%. m.p.: 218-220 ${ }^{\circ} \mathrm{C} .{ }^{1} \mathrm{H}$ NMR (400 MHz, DMSO[D 6$]+1-2 \%$ conc. DCl) $\delta=9.51(\mathrm{~s}, 1 \mathrm{H})$, $7.39(\mathrm{~d}, J=1.6 \mathrm{~Hz}, 1 \mathrm{H}), 7.33(\mathrm{dd}, J=8.1,1.6 \mathrm{~Hz}, 1 \mathrm{H}), 7.24-7.19(\mathrm{~m}, 3 \mathrm{H}), 7.01(\mathrm{~s}, 1 \mathrm{H}), 6.98$ $(\mathrm{d}, J=8.1 \mathrm{~Hz}, 1 \mathrm{H}), 6.77-6.70(\mathrm{~m}, 1 \mathrm{H}), 6.05(\mathrm{~s}, 2 \mathrm{H})$. HRMS $(\mathrm{m} / \mathrm{z})[\mathrm{M}+\mathrm{H}]^{+}$calcd for $\mathrm{M}=$ $\mathrm{C}_{16} \mathrm{H}_{12} \mathrm{~N}_{2} \mathrm{O}_{3}, 281.0926$; found, 281.0927.

\section{Methyl 2-chloro-5-hydroxybenzoate (sery341)}

The title compound has been reported previously ${ }^{527}$ (CAS 247092-10-0). It was prepared by a modified method ${ }^{528}$. A mixture of 2-chloro-5-hydroxybenzoic acid (790 mg, $4.6 \mathrm{mmol}$ ), concentrated sulphuric acid $(0.3 \mathrm{ml})$ in methanol $(15 \mathrm{ml})$ was refluxed for $16 \mathrm{~h}$. The reaction mixture was then poured into cold water $(20 \mathrm{ml})$ and extracted with ether $(3 \times 20 \mathrm{ml})$. The combined ethereal extracts were washed with water $(20 \mathrm{ml}), 10 \%$ aqueous $\mathrm{NaHCO}_{3}(20 \mathrm{ml})$, dried with $\mathrm{Na}_{2} \mathrm{SO}_{4}$, and concentrated to provide sery341 (535 mg, $2.9 \mathrm{mmol}$ ) as a white solid. Yield 63\%. m.p.: $95-96{ }^{\circ} \mathrm{C} .{ }^{1} \mathrm{H}$ NMR (400 MHz, DMSO[D 6$\left.]\right) \delta=10.06(\mathrm{~s}, 1 \mathrm{H}), 7.34(\mathrm{~d}, J=$ $8.7 \mathrm{~Hz}, 1 \mathrm{H}), 7.16(\mathrm{~d}, J=3.0 \mathrm{~Hz}, 1 \mathrm{H}), 6.95(\mathrm{dd}, J=8.7,3.0 \mathrm{~Hz}, 1 \mathrm{H}), 3.83(\mathrm{~s}, 3 \mathrm{H})$.

\section{Methyl 5-benzyloxy-2-chlorobenzoate (sery342a)}

The title compound was prepared from sery341 according to the published protocol ${ }^{529}$ and purified by column chromatography (hexane: $\mathrm{CH}_{2} \mathrm{Cl}_{2}, 3: 2 \mathrm{v} / \mathrm{v}$ ) to provide sery342a as a white solid. Yield 98\%. TLC (hexane:EtOAc, 9:1 v/v): RF =0.44. m.p.: 68-70 ${ }^{\circ} \mathrm{C} .{ }^{1} \mathrm{H}$ NMR (400 $\mathrm{MHz}, \mathrm{DMSO}_{\left.\left[\mathrm{D}_{6}\right]\right)} \delta=7.48(\mathrm{~d}, J=8.8 \mathrm{~Hz}, 1 \mathrm{H}), 7.46-7.31(\mathrm{~m}, 6 \mathrm{H}), 7.22(\mathrm{dd}, J=8.8,3.1 \mathrm{~Hz}$, $1 \mathrm{H}), 5.15$ (s, 2H), 3.85 (s, 3H). ${ }^{13} \mathrm{C}$ NMR (100.6 MHz, DMSO[D 6$\left.]\right) \delta=165.3,156.9,136.4$, $131.6,131.0,128.5,128.0,127.8,122.9,119.7,116.7,69.8,52.6$. HRMS $(m / z)[\mathrm{M}+\mathrm{Na}]^{+}$ calcd for $\mathrm{M}=\mathrm{C}_{15} \mathrm{H}_{13} \mathrm{ClO}_{3}, 299.0451$; found, 299.0448.

\section{1-(1,3-Benzodioxol-5-yl)-3-(5-benzyloxy-2-chlorophenyl)propane-1,3-dione (sery342b)}

The title compound was prepared according to the GEP 1 using 1-(1,3-benzodioxol-5yl)ethanone and sery342a and purified by recrystallisation from methanol to provide sery342b as a yellow solid. Yield 87\%. m.p.: 94-96 ${ }^{\circ} \mathrm{C} .{ }^{1} \mathrm{H}$ NMR $\left(400 \mathrm{MHz}, \mathrm{DMSO}\left[\mathrm{D}_{6}\right]\right) \delta=$ $7.71(\mathrm{dd}, J=8.2,1.5 \mathrm{~Hz}, 1 \mathrm{H}), 7.60(\mathrm{~d}, J=1.5 \mathrm{~Hz}, 1 \mathrm{H}), 7.53-7.31(\mathrm{~m}, 7 \mathrm{H}), 7.20(\mathrm{dd}, J=8.8$, 
$3.0 \mathrm{~Hz}, 1 \mathrm{H}), 7.08(\mathrm{~d}, J=8.2 \mathrm{~Hz}, 1 \mathrm{H}), 6.83(\mathrm{~s}, 1 \mathrm{H}), 6.16(\mathrm{~s}, 2 \mathrm{H}), 5.19(\mathrm{~s}, 2 \mathrm{H})\left(\right.$ in ${ }^{1} \mathrm{H}$ NMR spectrum $8 \%$ of diketone form is present). ${ }^{13} \mathrm{C}$ NMR $\left(100.6 \mathrm{MHz}, \mathrm{DMSO}\left[\mathrm{D}_{6}\right]\right) \delta=184.9$, $184.7,157.1,151.8,148.1,136.5,136.0,131.5,128.5,128.3,128.0,127.8,123.7,122.0$, $118.8,116.3,108.5,106.9,102.2,97.4,69.8$. HRMS $(\mathrm{m} / z)[\mathrm{M}+\mathrm{H}]^{+}$calcd for $\mathrm{M}=$ $\mathrm{C}_{23} \mathrm{H}_{17} \mathrm{ClO}_{5}, 409.0843$; found, 409.0845 .

\section{3-(1,3-Benzodioxol-5-yl)-5-(5-benzyloxy-2-chlorophenyl)- $1 H$-pyrazole (sery342c)}

The title compound was prepared according to the GEP 2 starting from sery342b and purified by recrystallisation from methanol to provide sery342c as a white solid. Yield $84 \%$. m.p.: $122-123{ }^{\circ} \mathrm{C} .{ }^{1} \mathrm{H}$ NMR $\left(400 \mathrm{MHz}, \mathrm{DMSO}\left[\mathrm{D}_{6}\right]+1-2 \%\right.$ conc. DCl) $\delta=7.49-7.29(\mathrm{~m}, 9 \mathrm{H}), 7.09-$ $7.02(\mathrm{~m}, 2 \mathrm{H}), 6.99(\mathrm{~d}, J=8.1 \mathrm{~Hz}, 1 \mathrm{H}), 6.06(\mathrm{~s}, 2 \mathrm{H}), 5.16(\mathrm{~s}, 2 \mathrm{H}) .{ }^{13} \mathrm{C}$ NMR $(100.6 \mathrm{MHz}$, DMSO $\left[\mathrm{D}_{6}\right]+1-2 \%$ conc. DCl) $\delta=157.2,147.9,147.1,146.0,145.3,136.7,131.6,131.3$, $128.5,128.0,127.8,125.3,122.6,119.1,116.2$ (2C), 108.8, 105.7, 102.9, 101.3, 69.7. HRMS $(\mathrm{m} / \mathrm{z})[\mathrm{M}+\mathrm{H}]^{+}$calcd for $\mathrm{M}=\mathrm{C}_{23} \mathrm{H}_{17} \mathrm{ClN}_{2} \mathrm{O}_{3}, 405.1006$; found, 405.1013.

\section{3-(1,3-Benzodioxol-5-yl)-5-(2-chloro-5-hydroxyphenyl)-1H-pyrazole (sery344)}

The title compound was prepared using an analogous procedure to that described for sery279b starting from sery342c and purified by recrystallisation from methanol/water to provide sery344 as a white solid. Yield 93\%. m.p.: 193-195 ${ }^{\circ} \mathrm{C} .{ }^{1} \mathrm{H}$ NMR $(400 \mathrm{MHz}$, DMSO $\left[\mathrm{D}_{6}\right]+1-2 \%$ conc. DCl) $\delta=9.80(\mathrm{bs}, 1 \mathrm{H}), 7.39(\mathrm{~d}, J=1.6 \mathrm{~Hz}, 1 \mathrm{H}), 7.35-7.28(\mathrm{~m}, 2 \mathrm{H})$, $7.15(\mathrm{~d}, J=2.9 \mathrm{~Hz}, 1 \mathrm{H}), 7.02-6.96(\mathrm{~m}, 2 \mathrm{H}), 6.80(\mathrm{dd}, J=8.6,2.9 \mathrm{~Hz}, 1 \mathrm{H}), 6.06(\mathrm{~s}, 2 \mathrm{H}) .{ }^{13} \mathrm{C}$ NMR (100.6 MHz, DMSO[D $]+1-2 \%$ conc. DCl) $\delta=156.3,147.7,146.9,145.8,145.2$, $131.8,131.0,125.6,120.6,119.0,116.7,116.6,108.7,105.6,102.7,101.2$. HRMS $(\mathrm{m} / \mathrm{z})$ $[\mathrm{M}+\mathrm{H}]^{+}$calcd for $\mathrm{M}=\mathrm{C}_{16} \mathrm{H}_{11} \mathrm{ClN}_{2} \mathrm{O}_{3}, 315.0536$; found, 315.0539 .

\section{1-(1,3-Benzodioxol-5-yl)-3-(4-bromophenyl)propane-1,3-dione (sery378a)}

The title compound has been reported previously ${ }^{530}$ (CAS 87724-01-4). It was prepared according to the GEP 1 using 1-(1,3-benzodioxol-5-yl)ethanone and methyl 4-bromobenzoate and purified by recrystallisation from ethanol/EtOAc to provide sery378a as a yellow solid. Yield 76\%. m.p.: $158-160{ }^{\circ} \mathrm{C} .{ }^{1} \mathrm{H}$ NMR $\left(400 \mathrm{MHz}, \mathrm{CDCl}_{3}\right) \delta=7.82(\mathrm{~d}, J=8.6 \mathrm{~Hz}, 2 \mathrm{H}), 7.66-$ $7.55(\mathrm{~m}, 3 \mathrm{H}), 7.45$ (d, $J=1.5 \mathrm{~Hz}, 1 \mathrm{H}), 6.89$ (d, $J=8.2 \mathrm{~Hz}, 1 \mathrm{H}), 6.70(\mathrm{~s}, 1 \mathrm{H}), 6.07$ (s, 2H) (in ${ }^{1} \mathrm{H}$ NMR spectrum $2.5 \%$ of diketone form is present). ${ }^{13} \mathrm{C} \mathrm{NMR}\left(100.6 \mathrm{MHz}, \mathrm{CDCl}_{3}\right) \delta=$ $186.3,182.7,151.8,148.4,134.4,132.1,130.1,128.6,127.2,123.2,108.4,107.4$, 102.1, 92.6.

\section{3-(1,3-Benzodioxol-5-yl)-5-(4-bromophenyl)-1H-pyrazole (sery378b)}

The title compound has been reported previously ${ }^{530}$ (CAS 651717-21-4). It was prepared according to the GEP 2 starting from sery378a and purified by recrystallisation from ethanol/EtOAc to provide sery378b as a white solid. Yield 75\%. m.p.: 231-233 ${ }^{\circ} \mathrm{C} .{ }^{1} \mathrm{H}$ NMR (400 MHz, DMSO[D 6$]) \delta=13.29(\mathrm{bs}, 1 \mathrm{H}), 7.77(\mathrm{~d}, J=7.8 \mathrm{~Hz}, 2 \mathrm{H}), 7.63(\mathrm{~d}, J=7.7 \mathrm{~Hz}, 2 \mathrm{H})$, $7.37(\mathrm{~s}, 1 \mathrm{H}), 7.32$ (d, $J=7.8 \mathrm{~Hz}, 1 \mathrm{H}), 7.12(\mathrm{~s}, 1 \mathrm{H}), 7.00(\mathrm{~d}, J=7.9 \mathrm{~Hz}, 1 \mathrm{H}), 6.06(\mathrm{~s}, 2 \mathrm{H}) .{ }^{13} \mathrm{C}$ NMR (100.6 MHz, DMSO[D $]+1-2 \%$ conc. DCl) $\delta=148.0,147.4,147.3,146.7,131.9$, $131.4,127.3,125.1,120.9,119.2,108.9,105.8,101.4,99.7$.

\section{Methyl 3-(2-fluoroethoxy)benzoate (sery387)}

The title compound has been reported previously ${ }^{531}$ (CAS 132838-27-8). To a solution of methyl 3-hydroxybenzoate $(1520 \mathrm{mg}, 10 \mathrm{mmol})$ in DMF $(15 \mathrm{ml}) 60 \%$ suspension of sodium hydride in mineral oil $(420 \mathrm{mg}, 10.5 \mathrm{mmol})$ was added in small portions, resulting mixture was stirred for $15 \mathrm{~min}$ at room temperature under argon atmosphere and treated with 2fluoroethyl ester of 4-methylbenzenesulfonic acid (2.4 g, $11 \mathrm{mmol})$. After stirring overnight at room temperature solvent was evaporated under reduced pressure and a remaining oil was 
passed though a small pad of silica gel eluting with $\mathrm{CH}_{2} \mathrm{Cl}_{2}$ to provide sery387 (1550 mg, $78 \%$ ) as a colourless oil. ${ }^{1} \mathrm{H}$ NMR (400 MHz, DMSO[D 6$\left.]\right) \delta=7.57(\mathrm{~d}, J=7.7 \mathrm{~Hz}, 1 \mathrm{H}), 7.49$ $7.41(\mathrm{~m}, 2 \mathrm{H}), 7.27(\mathrm{dd}, J=8.2,2.2 \mathrm{~Hz}, 1 \mathrm{H}), 4.75(\mathrm{~m}, 2 \mathrm{H}), 4.30(\mathrm{~m}, 2 \mathrm{H}), 3.85(\mathrm{~s}, 3 \mathrm{H})$.

\section{1-(1,3-Benzodioxol-5-yl)-3-[3-(2-fluoroethoxy)phenyl]propane-1,3-dione (sery388a)}

The title compound was prepared according to the GEP 1 using 1-(1,3-benzodioxol-5yl)ethanone and sery387 and purified by recrystallisation from methanol to provide sery388a as a yellow solid. Yield 54\%. m.p.: $117-119{ }^{\circ} \mathrm{C} .{ }^{1} \mathrm{H}$ NMR $\left(400 \mathrm{MHz}, \mathrm{DMSO}\left[\mathrm{D}_{6}\right]\right) \delta=7.84$ (dd, $J=8.2,1.6 \mathrm{~Hz}, 1 \mathrm{H}), 7.79-7.73(\mathrm{~m}, 2 \mathrm{H}), 7.69(\mathrm{~s}, 1 \mathrm{H}), 7.48(\mathrm{t}, J=8.0 \mathrm{~Hz}, 1 \mathrm{H}), 7.27(\mathrm{~s}$, $1 \mathrm{H}), 7.24$ (dd, $J=8.2,2.4 \mathrm{~Hz}, 1 \mathrm{H}), 7.09$ (d, $J=8.2 \mathrm{~Hz}, 1 \mathrm{H}), 6.17(\mathrm{~s}, 2 \mathrm{H}), 4.79$ (ddd, $J=47.7$, $4.2,3.5 \mathrm{~Hz}, 2 \mathrm{H}$ ), 4.36 (ddd, $J=30.2,4.5,3.2 \mathrm{~Hz}, 2 \mathrm{H}$ ) (in ${ }^{1} \mathrm{H}$ NMR spectrum $4 \%$ of diketone form is present). ${ }^{13} \mathrm{C}$ NMR (100.6 MHz, DMSO[D 6 ] $\delta=185.8,182.9,158.5,151.6,148.0$, 136.0, 130.0, 129.0, 123.7, 120.0, 119.0, 112.8, 108.4, 107.2, 102.1, 92.9, 82.1 (d, $J=167.8$ $\mathrm{Hz}), 67.3(\mathrm{~d}, J=19.4 \mathrm{~Hz})$. HRMS $(\mathrm{m} / \mathrm{z})[\mathrm{M}+\mathrm{H}]^{+}$calcd for $\mathrm{M}=\mathrm{C}_{18} \mathrm{H}_{15} \mathrm{FO}_{5}, 331.0982$; found, 331.0976 .

\section{3-(1,3-Benzodioxol-5-yl)-5-[3-(2-fluoroethoxy)phenyl]-1 H-pyrazole (sery369)}

The title compound was prepared according to the GEP 2 starting from sery388a and purified by recrystallisation from ethanol to provide sery369 as a white solid. Yield 82\%. m.p.: 167$168{ }^{\circ} \mathrm{C} .{ }^{1} \mathrm{H}$ NMR $\left(400 \mathrm{MHz}, \mathrm{DMSO}\left[\mathrm{D}_{6}\right]+1-2 \%\right.$ conc. DCl) $\delta=13.22$ (bs, $\left.1 \mathrm{H}\right), 7.47-7.30$ $(\mathrm{m}, 5 \mathrm{H}), 7.13(\mathrm{~s}, 1 \mathrm{H}), 7.69(\mathrm{~d}, J=8.0 \mathrm{~Hz}, 1 \mathrm{H}), 6.93(\mathrm{~d}, J=7.9 \mathrm{~Hz}, 1 \mathrm{H}), 6.05(\mathrm{~s}, 2 \mathrm{H}), 4.77$ (ddd, $J=30.2,4.0,2.7 \mathrm{~Hz}, 2 \mathrm{H}), 4.30$ (ddd, $J=47.3,4.2,2.5 \mathrm{~Hz}, 2 \mathrm{H}) .{ }^{13} \mathrm{C}$ NMR $(100.6 \mathrm{MHz}$, DMSO $\left[\mathrm{D}_{6}\right]+1-2 \%$ conc. DCl) $\delta=158.7,147.9,147.4,147.2,147.0,133.0,130.2,125.8$, 119.0, 118.0, 114.0, 111.3, 108.8, 105.7, 101.3, 99.7, 82.3 (d, $J=166.4 \mathrm{~Hz}), 67.2$ (d, $J=18.4$ $\mathrm{Hz})$. HRMS $(\mathrm{m} / \mathrm{z})[\mathrm{M}+\mathrm{H}]^{+}$calcd for $\mathrm{M}=\mathrm{C}_{18} \mathrm{H}_{15} \mathrm{FN}_{2} \mathrm{O}_{3}$, 327.1145; found, 327.1144 .

\section{Methyl ester of imidazo[1,2-a]pyridine-7-carboxylic acid (sery391)}

The title compound has been reported previously ${ }^{532}$ (CAS 86718-01-6). It was prepared according to the published protocol ${ }^{532}$ and purified by column chromatography $\left(\mathrm{CHCl}_{3}: \mathrm{MeOH}, 100: 1 \mathrm{v} / \mathrm{v}\right)$ followed by recrystallisation from hexane/EtOAc to provide sery391 as a yellow solid. Yield 94\%. TLC $\left(\mathrm{CHCl}_{3}: \mathrm{MeOH}, 100: 1 \mathrm{v} / \mathrm{v}\right): \mathrm{RF}=0.22$. m.p.: 143 ${ }^{\circ} \mathrm{C} .{ }^{1} \mathrm{H}$ NMR $\left.\left(400 \mathrm{MHz}, \mathrm{DMSO}_{[\mathrm{D}}\right]\right) \delta=8.37(\mathrm{~s}, 1 \mathrm{H}), 8.17(\mathrm{~d}, J=7.1 \mathrm{~Hz}, 1 \mathrm{H}), 7.80(\mathrm{~s}, 1 \mathrm{H})$, $7.69(\mathrm{~s}, 1 \mathrm{H}), 7.40(\mathrm{~d}, J=7.1,1.4 \mathrm{~Hz}, 1 \mathrm{H}), 3.96(\mathrm{~s}, 3 \mathrm{H})$.

\section{1-(1,3-Benzodioxol-5-yl)-3-(6-bromopyridin-2-yl)propane-1,3-dione (sery393a)}

The title compound was prepared according to the GEP 1 using 1-(1,3-benzodioxol-5yl)ethanone and ethyl ester of 6-bromo-2-pyridinecarboxylic acid and by recrystallisation from mixture ethanol/EtOAc $(9 / 1 \mathrm{v} / \mathrm{v})$ to provide sery393a as a yellow solid. Yield $79 \%$. ${ }^{1} \mathrm{H}$ NMR (400 MHz, DMSO[D6]) $\delta=8.13(\mathrm{~d}, J=7.4 \mathrm{~Hz}, 1 \mathrm{H}), 7.99(\mathrm{t}, J=7.8 \mathrm{~Hz}, 1 \mathrm{H}), 7.89(\mathrm{~d}, J$ $=7.8 \mathrm{~Hz}, 1 \mathrm{H}), 7.73(\mathrm{dd}, J=8.3,1.5 \mathrm{~Hz}, 1 \mathrm{H}), 7.56(\mathrm{~d}, J=1.5 \mathrm{~Hz}, 1 \mathrm{H}), 7.33(\mathrm{~s}, 1 \mathrm{H}), 7.11(\mathrm{~d}, J$ $=8.3 \mathrm{~Hz}, 1 \mathrm{H}), 6.18(\mathrm{~s}, 2 \mathrm{H})$ (in ${ }^{1} \mathrm{H}$ NMR spectrum $15 \%$ of diketone form is present). ESI-MS $(\mathrm{m} / \mathrm{z})[\mathrm{M}+\mathrm{H}]^{+}$calcd for $\mathrm{M}=\mathrm{C}_{15} \mathrm{H}_{10}{ }^{79} \mathrm{BrNO}_{4}, 347.99$; found, 348.19.

\section{2-[3-(1,3-Benzodioxol-5-yl)-1H-pyrazol-5-yl]-6-bromopyridine (sery393b)}

The title compound was prepared according to the GEP 2 starting from sery393a and purified by refluxing in ethanol to provide sery393b as a yellow solid. Yield $85 \%$. m.p.: $250-252{ }^{\circ} \mathrm{C}$ ${ }^{1} \mathrm{H}$ NMR $\left(400 \mathrm{MHz}, \mathrm{DMSO}\left[\mathrm{D}_{6}\right]+1 \% \mathrm{DCl}\right) \delta=7.97(\mathrm{~d}, J=7.8 \mathrm{~Hz}, 1 \mathrm{H}), 7.80(\mathrm{t}, J=7.8 \mathrm{~Hz}$, $1 \mathrm{H}), 7.55(\mathrm{~d}, J=7.8 \mathrm{~Hz}, 1 \mathrm{H}), 7.45(\mathrm{~d}, J=1.6 \mathrm{~Hz}, 1 \mathrm{H}), 7.39(\mathrm{dd}, J=8.1,1.6 \mathrm{~Hz}, 1 \mathrm{H}), 7.20$ (s, $1 \mathrm{H}), 6.97(\mathrm{~d}, J=8.1 \mathrm{~Hz}, 1 \mathrm{H}), 6.04(\mathrm{~s}, 2 \mathrm{H}) .{ }^{13} \mathrm{C} \mathrm{NMR}\left(100.6 \mathrm{MHz}, \mathrm{DMSO}\left[\mathrm{D}_{6}\right]+1 \% \mathrm{DCl}\right) \delta=$ 
$151.8,148.0,147.5,147.4,146.2,141.3,140.5,127.1,124.3,119.5,119.2,108.9,106.1$, 101.4, 101.2. ESI-MS $(\mathrm{m} / z)[\mathrm{M}+\mathrm{H}]^{+}$calcd for $\mathrm{M}=\mathrm{C}_{15} \mathrm{H}_{10}{ }^{79} \mathrm{BrN}_{3} \mathrm{O}_{2}, 344.00$; found, 344.20.

\section{1-(1,3-Benzodioxol-5-yl)-3-(2-bromopyridin-4-yl)propane-1,3-dione (sery397a)}

The title compound was prepared according to the GEP 1 using 1-(1,3-benzodioxol-5yl)ethanone and methyl ester of 2-bromo-4-pyridinecarboxylic acid and by recrystallisation from mixture ethanol/EtOAc $(4 / 1 \mathrm{v} / \mathrm{v})$ to provide sery397a as a yellow solid. Yield $76 \% .{ }^{1} \mathrm{H}$ NMR (400 MHz, DMSO[D 6$] \delta=8.60(\mathrm{~d}, J=5.1 \mathrm{~Hz}, 1 \mathrm{H}), 8.33(\mathrm{~s}, 1 \mathrm{H}), 8.04(\mathrm{dd}, J=5.1,1.5$ $\mathrm{Hz}, 1 \mathrm{H}), 7.89$ (dd, $J=8.3,1.8 \mathrm{~Hz}, 1 \mathrm{H}), 7.78(\mathrm{~d}, J=1.8 \mathrm{~Hz}, 1 \mathrm{H}), 7.43(\mathrm{~s}, 1 \mathrm{H}), 7.12(\mathrm{~d}, J=8.3$ $\mathrm{Hz}, 1 \mathrm{H}), 6.19(\mathrm{~s}, 2 \mathrm{H})$ (in ${ }^{1} \mathrm{H}$ NMR spectrum $4 \%$ of diketone form is present). ESI-MS $(\mathrm{m} / \mathrm{z})$ $[\mathrm{M}+\mathrm{H}]^{+}$calcd for $\mathrm{M}=\mathrm{C}_{15} \mathrm{H}_{10}{ }^{79} \mathrm{BrNO}_{4}, 347.99$; found, 348.19.

\section{4-[3-(1,3-Benzodioxol-5-yl)-1H-pyrazol-5-yl]-2-bromopyridine (sery398a)}

The title compound was prepared according to the GEP 2 starting from sery397a and purified by purified by refluxing in ethanol to provide sery398a as a white powder. Yield $85 \%$. m.p.: 244-245 ${ }^{\circ} \mathrm{C}{ }^{1} \mathrm{H}$ NMR $(400 \mathrm{MHz}, 313 \mathrm{~K}$, DMSO[D 6$\left.]+1 \% \mathrm{DCl}\right) \delta=8.41(\mathrm{~d}, J=5.1 \mathrm{~Hz}, 1 \mathrm{H})$, $8.01(\mathrm{~d}, J=1.2 \mathrm{~Hz}, 1 \mathrm{H}), 7.84(\mathrm{dd}, J=5.1,1.5 \mathrm{~Hz}, 1 \mathrm{H}), 7.38(\mathrm{~d}, J=1.7 \mathrm{~Hz}, 1 \mathrm{H}), 7.34(\mathrm{~s}, 1 \mathrm{H})$, $7.33(\mathrm{dd}, J=8.1,1.7 \mathrm{~Hz}, 1 \mathrm{H}), 7.00(\mathrm{~d}, J=8.1 \mathrm{~Hz}, 1 \mathrm{H}), 6.06(\mathrm{~s}, 2 \mathrm{H}) .{ }^{13} \mathrm{C}$ NMR $(100.6 \mathrm{MHz}$, $\left.313 \mathrm{~K}, \mathrm{DMSO}\left[\mathrm{D}_{6}\right]+1 \% \mathrm{DCl}\right) \delta=150.7,147.8,147.3,145.8,145.5,142.8,142.1,123.8$, $123.0,119.1,119.0,108.6,105.6,101.2,101.0$. ESI-MS $(\mathrm{m} / \mathrm{z})[\mathrm{M}+\mathrm{H}]^{+}$calcd for $\mathrm{M}=$ $\mathrm{C}_{15} \mathrm{H}_{10}{ }^{79} \mathrm{BrN}_{3} \mathrm{O}_{2}, 344.00$; found, 344.10.

\section{1-(1,3-Benzodioxol-5-yl)-3-(5-bromopyridin-3-yl)propane-1,3-dione (sery397b)}

The title compound was prepared according to the GEP 1 using 1-(1,3-benzodioxol-5yl)ethanone and ethyl ester of 5-bromo-3-pyridinecarboxylic acid and by recrystallisation from mixture ethanol/EtOAc $(1 / 1 \mathrm{v} / \mathrm{v})$ to provide sery397b as a yellow solid. Yield $85 \%$. ${ }^{1} \mathrm{H}$ $\left.\operatorname{NMR}\left(400 \mathrm{MHz}, \mathrm{DMSO}_{[} \mathrm{D}_{6}\right]\right) \delta=9.24(\mathrm{~d}, J=1.8 \mathrm{~Hz}, 1 \mathrm{H}), 8.91(\mathrm{~d}, J=2.2 \mathrm{~Hz}, 1 \mathrm{H}), 8.74(\mathrm{t}, J$ $=2.0 \mathrm{~Hz}, 1 \mathrm{H}), 7.85(\mathrm{dd}, J=8.3,1.8 \mathrm{~Hz}, 1 \mathrm{H}), 7.76(\mathrm{~d}, J=1.8 \mathrm{~Hz}, 1 \mathrm{H}), 7.37(\mathrm{~s}, 1 \mathrm{H}), 7.11(\mathrm{~d}, J$ $=8.3 \mathrm{~Hz}, 1 \mathrm{H}), 6.18(\mathrm{~s}, 2 \mathrm{H})\left(\right.$ in ${ }^{1} \mathrm{H}$ NMR spectrum $5 \%$ of diketone form is present). ESI-MS $(m / z)[\mathrm{M}+\mathrm{H}]^{+}$calcd for $\mathrm{M}=\mathrm{C}_{15} \mathrm{H}_{10}{ }^{79} \mathrm{BrNO}_{4}, 347.99$; found, 348.05 .

\section{3-[3-(1,3-Benzodioxol-5-yl)-1H-pyrazol-5-yl]-5-bromopyridine (sery398b)}

The title compound was prepared according to the GEP 2 starting from sery397b and purified by recrystallisation from mixture ethanol/EtOAc $(1 / 1 \mathrm{v} / \mathrm{v})$ to provide sery398b as a grey powder. Yield 82\%. m.p.: 238-240 ${ }^{\circ} \mathrm{C} .{ }^{1} \mathrm{H}$ NMR (400 MHz, 313K, DMSO[D $\left.]+1 \% \mathrm{DCl}\right) \delta$ $=9.05(\mathrm{~d}, J=1.2 \mathrm{~Hz}, 1 \mathrm{H}), 8.67(\mathrm{~d}, J=2.0 \mathrm{~Hz}, 1 \mathrm{H}), 8.46(\mathrm{bs}, 1 \mathrm{H}), 7.37(\mathrm{~d}, J=2.0 \mathrm{~Hz}, 1 \mathrm{H})$, $7.32(\mathrm{dd}, J=8.1,1.5 \mathrm{~Hz}, 1 \mathrm{H}), 7.27(\mathrm{~s}, 1 \mathrm{H}), 6.99(\mathrm{~d}, J=8.1 \mathrm{~Hz}, 1 \mathrm{H}), 6.06(\mathrm{~s}, 2 \mathrm{H}) .{ }^{13} \mathrm{C} \mathrm{NMR}$ $\left(100.6 \mathrm{MHz}, 313 \mathrm{~K}, \mathrm{DMSO}\left[\mathrm{D}_{6}\right]+1 \% \mathrm{DCl}\right) \delta=148.1,147.7,147.2,145.7,144.6,144.0$, $135.0,130.3,124.0,120.5,119.0,108.6,105.6,101.2,100.3$. ESI-MS $(m / z)[\mathrm{M}+\mathrm{H}]^{+}$calcd for $\mathrm{M}=\mathrm{C}_{15} \mathrm{H}_{10}{ }^{79} \mathrm{BrN}_{3} \mathrm{O}_{2}, 344.00$; found, 344.10.

\section{1-(3-Bromophenyl)-3-(imidazo[1,2-a]pyridin-7-yl)propane-1,3-dione (sery401a)}

The title compound was prepared according to the GEP 1 using 1-(3-bromophenyl)ethanone and sery391 and purified by recrystallisation from methanol to provide sery388a as a yellow solid. Yield 49\%. m.p.: $188-189{ }^{\circ} \mathrm{C} .{ }^{1} \mathrm{H}$ NMR (400 MHz, DMSO[D $\left.]\right) \delta=8.73-8.65(\mathrm{~m}, 2 \mathrm{H})$, $8.44(\mathrm{~s}, 1 \mathrm{H}), 8.23-8.12(\mathrm{~m}, 2 \mathrm{H}), 7.88-7.79(\mathrm{~m}, 2 \mathrm{H}), 7.61-7.49(\mathrm{~m}, 3 \mathrm{H})\left(\right.$ in ${ }^{1} \mathrm{H}$ NMR spectrum $5 \%$ of diketone form is present). ${ }^{13} \mathrm{C}$ NMR $\left(100.6 \mathrm{MHz}, \mathrm{DMSO}\left[\mathrm{D}_{6}\right]\right) \delta=184.8,182.1,143.8$, $136.5,136.1,135.5,130.9,130.0,129.9,127.0,126.3,122.3,117.9,115.1,109.1$, 93.8 . HRMS $(m / z)[\mathrm{M}+\mathrm{H}]^{+}$calcd for $\mathrm{M}=\mathrm{C}_{16} \mathrm{H}_{11}{ }^{79} \mathrm{BrN}_{2} \mathrm{O}_{2}, 343.0082$; found, 343.0088 . 
7-[3-(3-bromophenyl)-1H-pyrazol-5-yl]imidazo[1,2-a]pyridine (sery401b)

The title compound was prepared according to the GEP 2 starting from sery401a and purified by refluxing in EtOAc/ethanol to provide sery401b as a white solid. Yield 78\%. m.p.: 282$283{ }^{\circ} \mathrm{C} .{ }^{1} \mathrm{H}$ NMR $\left(400 \mathrm{MHz}, \mathrm{DMSO}\left[\mathrm{D}_{6}\right]+1-2 \%\right.$ conc. DCl) $\delta=13.62(\mathrm{bs}, 1 \mathrm{H}), 8.62(\mathrm{~d}, J=$ $6.5 \mathrm{~Hz}, 1 \mathrm{H}), 8.06(\mathrm{~s}, 1 \mathrm{H}), 8.02(\mathrm{~s}, 1 \mathrm{H}), 7.96(\mathrm{~s}, 1 \mathrm{H}), 7.86(\mathrm{~d}, J=7.4 \mathrm{~Hz}, 1 \mathrm{H}), 7.61(\mathrm{~s}, 1 \mathrm{H})$, $7.54(\mathrm{~d}, J=7.8 \mathrm{~Hz}, 1 \mathrm{H}), 7.48(\mathrm{~s}, 1 \mathrm{H}), 7.46-7.34(\mathrm{~m}, 2 \mathrm{H}) .{ }^{13} \mathrm{C}$ NMR $\left(100.6 \mathrm{MHz}, \mathrm{DMSO}\left[\mathrm{D}_{6}\right]\right.$ $+1-2 \%$ conc. DCl) $\delta=149.9,144.6,142.3,138.1,134.0,132.8,131.1,130.6,127.6,127.2$, 124.1, 122.3, 113.4, 111.6, 109.8, 101.1. HRMS $(\mathrm{m} / \mathrm{z})[\mathrm{M}+\mathrm{H}]^{+}$calcd for $\mathrm{M}=\mathrm{C}_{16} \mathrm{H}_{11}{ }^{79} \mathrm{BrN}_{4}$, 339.0245; found, 339.0249.

1-(1-Methoxymethyl-1H-benzimidazol-6-yl)ethanone and 1-(1-methoxymethyl-1Hbenzimidazol-5-yl)ethanone (sery413)

The title compound was prepared analogously to the procedure for sery406 from $1-(1 \mathrm{H}-$ benzimidazole-6-yl)ethanone and purified by column chromatography on silica gel $\left(\mathrm{CHCl}_{3}:\right.$ methanol, 95:5 v/v) to provide sery413 as an inseparable mixture of 5- and 6-isomers in ratio 1:1 ( ${ }^{1} \mathrm{H}$ NMR). Colourless oil. Yield 69\%. TLC $\left(\mathrm{CHCl}_{3}: \mathrm{MeOH}, 95: 5 \mathrm{v} / \mathrm{v}\right): \mathrm{RF}=0.49$. ${ }^{1} \mathrm{H}$ NMR $\left(400 \mathrm{MHz}, \mathrm{CDCl}_{3}\right) \delta=8.44(\mathrm{~d}, J=1.3 \mathrm{~Hz}, 1 \mathrm{H}), 8.21(\mathrm{~d}, J=1.3 \mathrm{~Hz}, 1 \mathrm{H}), 8.12(\mathrm{~s}$, $1 \mathrm{H}), 8.07(\mathrm{~s}, 1 \mathrm{H}), 8.03(\mathrm{dd}, J=8.6,1.5 \mathrm{~Hz}, 1 \mathrm{H}), 7.95(\mathrm{dd}, J=8.5,1.5 \mathrm{~Hz}, 1 \mathrm{H}), 7.85(\mathrm{~d}, J=$ $8.6 \mathrm{~Hz}, 1 \mathrm{H}), 7.58(\mathrm{~d}, J=8.5 \mathrm{~Hz}, 1 \mathrm{H}), 5.56(\mathrm{~s}, 2 \mathrm{H}), 5.53(\mathrm{~s}, 2 \mathrm{H}), 3.32(\mathrm{~s}, 3 \mathrm{H}), 3.31(\mathrm{~s}, 3 \mathrm{H})$, $2.69(\mathrm{~s}, 3 \mathrm{H}), 2.68(\mathrm{~s}, 3 \mathrm{H})$.

1-(3-Chlorophenyl)-3-(1-methoxymethyl-1H-benzimidazol-6-yl)propane-1,3-dione and 1-(3-Chlorophenyl)-3-(1-methoxymethyl-1H-benzimidazol-5-yl)propane-1,3-dione (sery416a)

The title compound was prepared according to the GEP 1 using sery413 and methyl 3chlorobenzoate. The crude product after aqueous work up and extraction with EtOAc was concentrated under reduced pressure to provide sery416a as a yellow solid. Yield $75 \%$. The isolated sery416a was used directly for the next step without further purification.

6-[5-(3-Chlorophenyl)-1H-pyrazol-3-yl]-1-methoxymethyl- $1 H$-benzimidazole and 5-[5(3-chlorophenyl)-1H-pyrazol-3-yl]-1-methoxymethyl-1H-benzimidazole (sery416b)

The title compound was prepared according to the GEP 2 starting from sery416a and purified by column chromatography on silica gel $\left(\mathrm{CHCl}_{3}:\right.$ methanol, $\left.95: 5 \mathrm{v} / \mathrm{v}\right)$ to provide sery $416 \mathbf{b}$ as an inseparable mixture of 5- and 6-isomers. Beige solid. Yield 70\%. TLC $\left(\mathrm{CHCl}_{3}: \mathrm{MeOH}, 9: 1\right.$ $\mathrm{v} / \mathrm{v}): \mathrm{RF}=0.38$. The isolated sery416b was used directly for the next step without further purification. (400 MHz, 313K, DMSO[D 6$]+1 \% \mathrm{DCl}) \delta=10.00-9.80(\mathrm{~m}, 1 \mathrm{H}), 8.34(\mathrm{~s}, 1 \mathrm{H})$, 8.19-8.05 (m, 2H), $7.96(\mathrm{~s}, 1 \mathrm{H}), 7.86(\mathrm{~d}, J=7.8 \mathrm{~Hz}, 1 \mathrm{H}), 7.55-7.47(\mathrm{~m}, 2 \mathrm{H}), 7.41(\mathrm{~d}, J=8.0$ $\mathrm{Hz}, 1 \mathrm{H}), 5.90(\mathrm{~s}, 2 \mathrm{H}), 3.37(\mathrm{~s}, 3 \mathrm{H})$. ESI-MS $(\mathrm{m} / \mathrm{z})[\mathrm{M}+\mathrm{H}]^{+}$calcd for $\mathrm{M}=\mathrm{C}_{18} \mathrm{H}_{15} \mathrm{ClN}{ }_{4} \mathrm{O}$, 339.10; found, 339.21 .

\section{5-[5-(3-Chlorophenyl)-1H-pyrazol-3-yl]-1H-benzimidazole (sery418)}

The title compound was prepared according to the GEP 3 starting from sery416b and purified by column chromatography on silica gel $\left(\mathrm{CHCl}_{3}: \mathrm{MeOH}\right.$, gradient from 100:5 to $\left.100: 10 \mathrm{v} / \mathrm{v}\right)$ to provide sery418 as a white solid. Yield $60 \%$. TLC $\left(\mathrm{CHCl}_{3}: \mathrm{MeOH}, 10: 1 \mathrm{v} / \mathrm{v}\right): \mathrm{RF}=0.26 .{ }^{1} \mathrm{H}$ NMR (400 MHz, DMSO[D 6$]+1-2 \%$ conc. DCl) $\delta=9.68(\mathrm{~s}, 1 \mathrm{H}), 8.28(\mathrm{~s}, 1 \mathrm{H}), 8.08(\mathrm{dd}, J=$ 8.6, 1.6 Hz, 1H), 7.96-7.91 (m, 2H), $7.84(\mathrm{dt}, J=8.0,1.1 \mathrm{~Hz}, 1 \mathrm{H}), 7.48(\mathrm{t}, J=7.9 \mathrm{~Hz}, 1 \mathrm{H})$, $7.44(\mathrm{~s}, 1 \mathrm{H}), 7.38(\mathrm{ddd}, J=8.0,2.0,1.0 \mathrm{~Hz}, 1 \mathrm{H}) .{ }^{13} \mathrm{C} \mathrm{NMR}\left(100.6 \mathrm{MHz}, \mathrm{DMSO}\left[\mathrm{D}_{6}\right]+1-2 \%\right.$ conc. DCl) $\delta=147.7,146.0,140.8,133.8,133.1,131.1,130.9,130.1,129.9,127.7,124.9$, 123.9, 123.8, 115.0, 110.3, 101.0. ESI-MS $(m / z)[\mathrm{M}+\mathrm{H}]^{+}$calcd for $\mathrm{M}=\mathrm{C}_{16} \mathrm{H}_{11} \mathrm{ClN}_{4}, 295.07$; found, 295.21. 


\section{1-(Benzofuran-6-yl)-3-(3-bromophenyl)propane-1,3-dione (sery428a)}

The title compound was prepared according to the GEP 1 using 1-(benzofuran-6-yl)ethanone and methyl 3-bromobenzoate to provide sery428a. Yield $70 \%$. The isolated sery 428 a was used directly for the next step without further purification.

\section{3-(Benzofuran-6-yl)-5-(3-bromophenyl)-1H-pyrazole (sery428b)}

The title compound was prepared according to the GEP 2 starting from sery $428 \mathbf{b}$ and purified by recrystallisation from ethanol to provide sery $428 \mathrm{~b}$ as a white solid. Yield $55 \% .{ }^{1} \mathrm{H}$ NMR $\left(400 \mathrm{MHz}, \mathrm{DMSO}\left[\mathrm{D}_{6}\right]+1-2 \%\right.$ conc. $\left.\mathrm{DCl}\right) \delta=8.09-8.03(\mathrm{~m}, 3 \mathrm{H}), 7.88(\mathrm{dt}, J=7.8,1.1 \mathrm{~Hz}$, $1 \mathrm{H}), 7.78$ (dd, $J=8.1,1.4 \mathrm{~Hz}, 1 \mathrm{H}), 7.73$ (d, $J=8.2 \mathrm{~Hz}, 1 \mathrm{H}), 7.53(\mathrm{~m}, 1 \mathrm{H}), 7.42$ (d, $J=7.8$ $\mathrm{Hz}, 1 \mathrm{H}), 7.39$ (s, 1H), $6.99(\mathrm{dd}, J=2.2,0.9 \mathrm{~Hz}, 1 \mathrm{H}) .{ }^{13} \mathrm{C} \mathrm{NMR}\left(100.6 \mathrm{MHz}, \mathrm{DMSO}\left[\mathrm{D}_{6}\right]+1-\right.$ $2 \%$ conc. DCl) $\delta=154.8,147.0,146.8,146.7,134.1,131.1,130.5,127.7,127.2$ (2C), 124.2, $122.3,121.7,120.6,107.9,106.9$, 100.6. ESI-MS $(\mathrm{m} / \mathrm{z})[\mathrm{M}+\mathrm{H}]^{+}$calcd for $\mathrm{M}=$ $\mathrm{C}_{17} \mathrm{H}_{11}{ }^{79} \mathrm{BrN}_{2} \mathrm{O}, 339.01$; found, 339.12 .

\section{3-(3-Bromophenyl)-5-(2,2-difluoro-1,3-benzodioxol-5-yl)-1 $H$-pyrazole (anle270)}

The title compound was prepared by the modified published protocol ${ }^{395}$. To a mixture of 2,2difluoro-1,3-benzodioxole-5-carboxylic acid (606 $\mathrm{mg}, 3.0 \mathrm{mmol}$ ) and 2,3,4,5,6pentafluorophenol $(570 \mathrm{mg}, 3.1 \mathrm{mmol})$ in dry dioxane $(15 \mathrm{~mL})$ 1,3-dicyclohexylcarbodiimide (640 mg, $3.1 \mathrm{mmol}$ ) was added. The mixture was stirred for $15 \mathrm{~h}$ at room temperature. Dicyclohexylurea formed was filtered off, the solvent was evaporated under reduced pressure, the residue was mixed with dry toluene $(15 \mathrm{ml})$, the small amount of dicyclohexylurea was filtered off. The residue was concentrated under reduced pressure and redissolved in dry toluene $(8 \mathrm{ml})$ to provide a solution of 2,2-difluoro-1,3-benzodioxole-5-carboxylic acid 2,3,4,5,6-pentafluorophenyl ester. In a separate flask, hexamethyldisilazane $(1.251 \mathrm{ml}, 968$ $\mathrm{mg}, 6.0 \mathrm{mmol}$ ) was dissolved in $15 \mathrm{~mL}$ of dry cyclohexane in a screw cap vial (with septum), and then the solution was cooled to $0{ }^{\circ} \mathrm{C}$ under nitrogen. $n \mathrm{BuLi}(2.4 \mathrm{ml}, 2.5 \mathrm{M}$ in hexanes, 6.0 mmol) was added via syringe, the resulting mixture was stirred for $30 \mathrm{~min}$. 1-(3Bromophenyl)ethanone (797 $\mu \mathrm{l}, 1.194 \mathrm{~g}, 6.0 \mathrm{mmol})$ was added quickly via syringe, the resulting mixture was stirred at $0{ }^{\circ} \mathrm{C}$ for $15 \mathrm{~min}$, followed by the addition of 2,2-difluoro-1,3benzodioxole-5-carboxylic acid 2,3,4,5,6-pentafluorophenyl ester solution in toluene in one portion with stirring then allowed to stir at room temperature for $15 \mathrm{~min}$. AcOH $(6 \mathrm{ml})$ and EtOH $(30 \mathrm{ml})$ were added to form a homogeneous mixture, then hydrazine hydrate $(4.5 \mathrm{ml}$, $4.64 \mathrm{~g}, 92.7 \mathrm{mmol}$ ) was added, and the resulting mixture was heated under reflux for $30 \mathrm{~min}$ with stirring. After cooling the solvent was evaporated under reduced pressure, the residue was stirred 30 min with saturated sodium hydrogen carbonate solution $(60 \mathrm{ml})$, the resulting precipitate was collected by filtration, washed with water, dried and recrystallized from $\mathrm{CHCl}_{3} /$ cyclohexane to provide anle270 (733 mg, 64\%) as a white solid. m.p.: 202-203 ${ }^{\circ} \mathrm{C} .{ }^{1} \mathrm{H}$ NMR (400 MHz, DMSO[D 6$]+1-2 \%$ conc. DCl) $\delta=8.00(\mathrm{t}, J=1.7 \mathrm{~Hz}, 1 \mathrm{H}), 7.85-7.76(\mathrm{~m}$, 2H), $7.67(\mathrm{dd}, J=8.4,1.6 \mathrm{~Hz}, 1 \mathrm{H}), 7.52(\mathrm{~d}, J=7.8 \mathrm{~Hz}, 1 \mathrm{H}), 7.47$ (d, $J=8.4 \mathrm{~Hz}, 1 \mathrm{H}), 7.41$ (t, $J=7.8 \mathrm{~Hz}, 1 \mathrm{H}), 7.30(\mathrm{~s}, 1 \mathrm{H}) .{ }^{13} \mathrm{C} \mathrm{NMR}\left(100.6 \mathrm{MHz}, \mathrm{DMSO}\left[\mathrm{D}_{6}\right]+1-2 \%\right.$ conc. DCl) $\delta=$ 147.3, 146.9, 143.6, 142.5, 133.6, 131.5 (t, $J=253.1 \mathrm{~Hz}), 131.4,130.9,128.7,127.8,124.3$, 122.6, 121.6, 110.8, 107.1, 100.9. HRMS $(m / z)[\mathrm{M}+\mathrm{H}]^{+}$calcd for $\mathrm{M}=\mathrm{C}_{16} \mathrm{H}_{9}{ }^{79} \mathrm{BrF}_{2} \mathrm{~N}_{2} \mathrm{O}_{2}$, 378.9894; found, 378.9896 .

\section{3-(4-Chlorophenyl)-5-(2-nitrophenyl)-1H-pyrazole (sery309)}

The title compound was prepared analogously to the procedure for anle270 using 2nitrobenzoic acid and 1-(4-chlorophenyl)ethanone. The reaction mixture was stirred at reflux for $2 \mathrm{~h}$ after addition of hydrazine hydrate. The crude product after aqueous workup was 
purified by column chromatography on silica gel (hexane:EtOAc, 2:1 v/v) followed by recrystallisation from methanol/water to provide sery309 as a grey solid. Yield $48 \%$. TLC (hexane:EtOAc, 2:1 v/v): RF $=0.35$. m.p.: $135-137{ }^{\circ} \mathrm{C} .{ }^{1} \mathrm{H}$ NMR $\left(400 \mathrm{MHz}, \mathrm{DMSO}\left[\mathrm{D}_{6}\right]+1-\right.$ $2 \%$ conc. DCl) $\delta=7.85(\mathrm{~d}, J=8.0 \mathrm{~Hz}, 1 \mathrm{H}), 7.83-7.76(\mathrm{~m}, 3 \mathrm{H}), 7.72(\mathrm{dd}, J=7.5,1.0 \mathrm{~Hz}, 1 \mathrm{H})$, $7.58(\mathrm{dd}, J=8.0,1.3 \mathrm{~Hz}, 1 \mathrm{H}), 7.52(\mathrm{~d}, J=8.6 \mathrm{~Hz}, 2 \mathrm{H}), 7.01(\mathrm{~s}, 1 \mathrm{H}) .{ }^{13} \mathrm{C}$ NMR $(100.6 \mathrm{MHz}$, DMSO $\left[\mathrm{D}_{6}\right]+1-2 \%$ conc. DCl) $\delta=148.8,145.9,144.1,133.0,132.5,130.6,130.2,129.3$, $128.9,127.1,125.9,123.9,102.2$. $\operatorname{HRMS}(\mathrm{m} / \mathrm{z})[\mathrm{M}+\mathrm{H}]^{+}$calcd for $\mathrm{M}=\mathrm{C}_{15} \mathrm{H}_{10} \mathrm{ClN}_{3} \mathrm{O}_{2}$, 300.0540; found, 300.0542 .

1-Methoxymethyl-1 $H$-benzimidazole-5-carboxylic acid methyl ester and 1methoxymethyl-1H-benzimidazole-6-carboxylic acid methyl ester (sery406)

To a solution of methyl $1 H$-benzimidazole-6-carboxylate $(2.64 \mathrm{~g}, 15 \mathrm{mmol})$ in THF $(45 \mathrm{ml}) \mathrm{a}$ dispersion of sodium hydride $(60 \%$ in oil, $700 \mathrm{mg}, 17.5 \mathrm{mmol})$ was added in portions over a period of $15 \mathrm{~min}$ at $0{ }^{\circ} \mathrm{C}$ and stirred for $15 \mathrm{~min}$. Methoxymethyl chloride $(1.52 \mathrm{ml}, 1.61 \mathrm{~g}, 20$ mmol) was added, the mixture was stirred $1.5 \mathrm{~h}$ at room temperature and poured into cold saturated solution of $\mathrm{NaHCO}_{3}(150 \mathrm{ml})$. The solution was extracted with $\mathrm{Et}_{2} \mathrm{O}(2 \times 60 \mathrm{ml})$, the combined organic fractions were washed with brine $(50 \mathrm{ml})$, dried over $\mathrm{Na}_{2} \mathrm{SO}_{4}$ and evaporated under reduced pressure. The resulting oil was purified by column chromatography on silica gel $\left(\mathrm{CHCl}_{3}: \mathrm{MeOH}, 100: 5 \mathrm{v} / \mathrm{v}\right)$ to provide sery406 $(2.64 \mathrm{~g}, 80 \%)$ as a inseparable mixture of 5- and 6-isomers in ratio 1:1 ( ${ }^{1} \mathrm{H}$ NMR). Viscous red oil. TLC $\left(\mathrm{CHCl}_{3}: \mathrm{MeOH}\right.$, $100: 5 \mathrm{v} / \mathrm{v}): \mathrm{RF}=0.39 .{ }^{1} \mathrm{H}$ NMR $\left.\left(400 \mathrm{MHz}, \mathrm{DMSO}_{6}\right]\right) \delta=8.57(\mathrm{~s}, 1 \mathrm{H}), 8.53(\mathrm{~s}, 1 \mathrm{H}), 8.28$ $(\mathrm{s}, 2 \mathrm{H}), 7.92(\mathrm{dd}, J=8.4,1.5 \mathrm{~Hz}, 1 \mathrm{H}), 7.87(\mathrm{dd}, J=8.4,1.6 \mathrm{~Hz}, 1 \mathrm{H}), 7.77(\mathrm{~d}, J=5.6 \mathrm{~Hz}, 1 \mathrm{H})$, $7.76(\mathrm{~d}, J=5.6 \mathrm{~Hz}, 1 \mathrm{H}), 5.69(\mathrm{~s}, 2 \mathrm{H}), 5.64(\mathrm{~s}, 2 \mathrm{H}), 3.87(\mathrm{~s}, 3 \mathrm{H}), 3.86(\mathrm{~s}, 3 \mathrm{H}), 3.21(\mathrm{~s}, 3 \mathrm{H})$, $3.20(\mathrm{~s}, 3 \mathrm{H}) .{ }^{13} \mathrm{C}$ NMR (100.6 MHz, DMSO[D 6$\left.]\right) \delta=166.8,166.7,147.7,147.3,146.8$, $143.5,137.2$, 133.4, 124.4, 124.09, 124.05, 123.4, 121.4, 119.7, 113.0, 111.2, 75.53, 75.49, 55.9, 55.8, 52.26, 52.21. HRMS $(m / z)[\mathrm{M}+\mathrm{H}]^{+}$calcd for $\mathrm{M}=\mathrm{C}_{11} \mathrm{H}_{12} \mathrm{~N}_{2} \mathrm{O}_{3}, 221.0926$; found, 221.0921 .

1-Methoxymethyl-1H-benzimidazole-5-carboxylic acid and 1-methoxymethyl-1Hbenzimidazole-6-carboxylic acid (sery407)

To a solution of sery406 $(1.20 \mathrm{~g}, 5.45 \mathrm{mmol})$ in $\mathrm{MeOH}(2.5 \mathrm{ml})$ an aqueous $2 \mathrm{M} \mathrm{LiOH}$ solution $(2.73 \mathrm{ml}, 5.46 \mathrm{mmol})$ was added and stirred $15 \mathrm{~h}$ at room temperature. The solvent was evaporated under reduced pressure, the residue was acidified with $1 \mathrm{M} \mathrm{HCl}(5.5 \mathrm{ml}, 5.5$ mmol) at $0{ }^{\circ} \mathrm{C}$ and stirred for $30 \mathrm{~min}$. The product was collected by filtration and dried to provide sery407 $(960 \mathrm{mg}, 86 \%)$ as a inseparable mixture of 5 - and 6-isomers in ratio $1: 1\left({ }^{1} \mathrm{H}\right.$ NMR). Brown solid. m.p.: 153-157 ${ }^{\circ} \mathrm{C} .{ }^{1} \mathrm{H}$ NMR (400 MHz, DMSO[D 6 ) $\delta=12.83$ (br s, $2 \mathrm{H}), 8.54(\mathrm{~s}, 1 \mathrm{H}), 8.50(\mathrm{~s}, 1 \mathrm{H}), 8.26(\mathrm{~s}, 2 \mathrm{H}), 7.92(\mathrm{dd}, J=8.4,1.3 \mathrm{~Hz}, 1 \mathrm{H}), 7.86(\mathrm{dd}, J=8.4$, $1.4 \mathrm{~Hz}, 1 \mathrm{H}), 7.75(\mathrm{~d}, J=5.1 \mathrm{~Hz}, 1 \mathrm{H}), 7.73(\mathrm{~d}, J=5.1 \mathrm{~Hz}, 1 \mathrm{H}), 5.68(\mathrm{~s}, 2 \mathrm{H}), 5.64(\mathrm{~s}, 2 \mathrm{H}), 3.21$ (s, 3H), $3.20(\mathrm{~s}, 3 \mathrm{H}) .{ }^{13} \mathrm{C} \mathrm{NMR}\left(100.6 \mathrm{MHz}, \mathrm{DMSO}\left[\mathrm{D}_{6}\right]\right) \delta=167.9,167.8,147.4,147.1$, $146.6,143.4,136.9,133.4,125.5,125.2,124.3,123.6,121.5,119.5,113.1,110.9,75.49$, 75.46, 66.9, 55.8. HRMS $(\mathrm{m} / z)[\mathrm{M}+\mathrm{H}]^{+}$calcd for $\mathrm{M}=\mathrm{C}_{10} \mathrm{H}_{10} \mathrm{~N}_{2} \mathrm{O}_{3}, 207.0770$; found, 207.0760 .

6-[5-(3-Bromophenyl)-1H-pyrazol-3-yl]-1-methoxymethyl-1H-benzimidazole and 5-[5(3-bromophenyl)-1H-pyrazol-3-yl]-1-methoxymethyl-1H-benzimidazole (sery415)

The title compound was prepared analogously to the procedure for anle270 using sery407 and 1-(3-bromophenyl)ethanone. The crude product after aqueous work up was purified by column chromatography on silica gel $\left(\mathrm{CHCl}_{3}: \mathrm{MeOH}, 10: 1 \mathrm{v} / \mathrm{v}\right)$ to provide sery415 as a inseparable mixture of 5- and 6-isomers in ratio 7:3 $\left({ }^{1} \mathrm{H}\right.$ NMR). White solid. Yield 49\%. m.p. 189-198 ${ }^{\circ} \mathrm{C} . \mathrm{TLC}\left(\mathrm{CHCl}_{3}: \mathrm{MeOH}, 10: 1 \mathrm{v} / \mathrm{v}\right): \mathrm{RF}=0.45 .{ }^{1} \mathrm{H}$ NMR $\left(400 \mathrm{MHz}, \mathrm{DMSO}\left[\mathrm{D}_{6}\right]+1-\right.$ 
$2 \%$ conc. DCl $\delta=8.45(\mathrm{~s}, 1 \mathrm{H}), 8.16$ and $8.13(2 \times \mathrm{s}, 1 \mathrm{H}), 8.05(\mathrm{~s}, 1 \mathrm{H}), 7.86(\mathrm{~d}, J=7.6 \mathrm{~Hz}$, $1 \mathrm{H}), 7.83-7.69(\mathrm{~m}, 2 \mathrm{H}), 7.50(\mathrm{~d}, J=7.7 \mathrm{~Hz}, 1 \mathrm{H}), 7.40(\mathrm{t}, J=7.7 \mathrm{~Hz}, 1 \mathrm{H}), 7.33$ and $7.31(2 \times$ $\mathrm{s}, 1 \mathrm{H}), 5.65$ and $5.63(2 \times \mathrm{s}, 2 \mathrm{H}), 3.25$ and $3.23(2 \times \mathrm{s}, 3 \mathrm{H}) .{ }^{13} \mathrm{C} \mathrm{NMR}(100.6 \mathrm{MHz}$, DMSO $\left[\mathrm{D}_{6}\right]+1-2 \%$ conc. DCl) $\delta=146.5,145.5,144.5,143.7,143.1,134.8,134.0,133.5$, $131.1,130.3,127.6,124.1,122.3,121.0,120.2,119.8,116.0,111.5,107.6,100.4,100.1,75.3$, 55.8. HRMS $(m / z)[\mathrm{M}+\mathrm{H}]^{+}$calcd for $\mathrm{M}=\mathrm{C}_{18} \mathrm{H}_{15}{ }^{79} \mathrm{BrN}_{4} \mathrm{O}, 383.0507$; found, 383.0507.

\section{5-[5-(3-Bromophenyl)-1H-pyrazol-3-yl]-1H-benzimidazole (sery417)}

The title compound was prepared according to the GEP 3 starting from sery415 and purified by column chromatography on silica gel $\left(\mathrm{CHCl}_{3}: \mathrm{MeOH}, 10: 1 \mathrm{v} / \mathrm{v}\right)$. Yellow solid. Yield $54 \%$. TLC $\left(\mathrm{CHCl}_{3}: \mathrm{MeOH}, 10: 1 \mathrm{v} / \mathrm{v}\right): \mathrm{RF}=0.24$. m.p.: $164-166{ }^{\circ} \mathrm{C} .{ }^{1} \mathrm{H}$ NMR $(400 \mathrm{MHz}$, DMSO $\left[\mathrm{D}_{6}\right]+1-2 \%$ conc. DCl) $\delta=13.43(\mathrm{bs}, 1 \mathrm{H}), 8.30(\mathrm{~s}, 1 \mathrm{H}), 8.11-8.03(\mathrm{~m}, 2 \mathrm{H}), 7.87(\mathrm{~d}, J$ $=7.8 \mathrm{~Hz}, 1 \mathrm{H}), 7.81(\mathrm{~d}, J=7.8 \mathrm{~Hz}, 1 \mathrm{H}), 7.64-7.56(\mathrm{~m}, 2 \mathrm{H}), 7.40(\mathrm{t}, J=7.8 \mathrm{~Hz}, 1 \mathrm{H}), 7.28(\mathrm{~s}$, 1H). ${ }^{13} \mathrm{C}$ NMR (100.6 MHz, DMSO[D 6$]+1-2 \%$ conc. DCl) $\delta=150.0,144.9,143.1,138.5$, $138.0,136.2$, 131.0, 130.3, 127.6, 124.1, 123.4, 122.3, 120.0, 115.8, 112.0, 100.0. HRMS $(\mathrm{m} / z)[\mathrm{M}+\mathrm{H}]^{+}$calcd for $\mathrm{M}=\mathrm{C}_{16} \mathrm{H}_{11}{ }^{79} \mathrm{BrN}_{4}, 339.0245$; found, 339.0244.

\section{(E)-3-(4-Chlorophenyl)-1-(3-nitrophenyl)-2-propene-1-one (sery276a)}

The title compound has been reported previously ${ }^{533}$ (CAS 131497-20-6). It was prepared according to the GEP 5 using 1-(3-nitrophenyl)ethanone, 4-chlorobenzaldehyde and purified by refluxing in ethanol to provide sery276a as a yellow solid. Yield $98 \%$. m.p.: $197-198{ }^{\circ} \mathrm{C}$ $\left(\mathrm{lit}^{533} 197.5^{\circ} \mathrm{C}\right) .{ }^{1} \mathrm{H}$ NMR $\left.\left(400 \mathrm{MHz}, \mathrm{DMSO}_{[\mathrm{D}}\right]\right) \delta=8.85(\mathrm{t}, J=2.0 \mathrm{~Hz}, 1 \mathrm{H}), 8.61$ (dt, $J=$ $7.8,1.1 \mathrm{~Hz}, 1 \mathrm{H}), 8.50$ (ddd, $J=8.1,2.2,1.0 \mathrm{~Hz}, 1 \mathrm{H}), 8.07$ (d, $J=15.6 \mathrm{~Hz}, 1 \mathrm{H}), 7.99$ (d, $J=$ $8.6 \mathrm{~Hz}, 2 \mathrm{H}), 7.89$ (t, $J=8.0 \mathrm{~Hz}, 1 \mathrm{H}), 7.83(\mathrm{~d}, J=15.6 \mathrm{~Hz}, 1 \mathrm{H}), 7.56(\mathrm{~d}, J=8.6 \mathrm{~Hz}, 2 \mathrm{H})$.

\section{2,3-Dibromo-3-(4-chlorophenyl)-1-(3-nitrophenyl)propane-1-one (sery277a)}

The title compound has been reported previously ${ }^{534}$ (CAS 1032418-75-9). It was prepared according to the GEP 6 starting from sery276a to provide sery277a as a white solid. Yield 81\%. m.p.: 173-175 ${ }^{\circ} \mathrm{C} .{ }^{1} \mathrm{H}$ NMR (400 MHz, DMSO[D 6$\left.]\right) \delta=9.01(\mathrm{t}, J=2.0 \mathrm{~Hz}, 1 \mathrm{H}), 8.71$ $(\mathrm{d}, J=8.0 \mathrm{~Hz}, 1 \mathrm{H}), 8.59(\mathrm{ddd}, J=8.2,2.0,0.8 \mathrm{~Hz}, 1 \mathrm{H}), 7.96(\mathrm{t}, J=8.0 \mathrm{~Hz}, 1 \mathrm{H}), 7.90(\mathrm{~d}, J=$ $8.5 \mathrm{~Hz}, 2 \mathrm{H}), 7.53(\mathrm{~d}, J=8.5 \mathrm{~Hz}, 2 \mathrm{H}), 6.90(\mathrm{~d}, J=11.3 \mathrm{~Hz}, 1 \mathrm{H}), 5.89(\mathrm{~d}, J=11.3 \mathrm{~Hz}, 1 \mathrm{H})$.

\section{3-(4-Chlorophenyl)-5-(3-nitrophenyl)-1H-pyrazole (sery278a)}

The title compound was prepared according to the GEP 7 starting from sery 277a and purified by recrystallisation from methanol to provide sery278a as a white solid. Yield $60 \%$. m.p.: 240-241 ${ }^{\circ} \mathrm{C} .{ }^{1} \mathrm{H}$ NMR (400 MHz, DMSO[D 6$]+1-2 \%$ conc. DCl) $\delta=8.64(\mathrm{~s}, 1 \mathrm{H}), 8.26(\mathrm{~d}, J$ $=7.9 \mathrm{~Hz}, 1 \mathrm{H}), 8.16(\mathrm{~d}, J=7.9 \mathrm{~Hz}, 1 \mathrm{H}), 7.85(\mathrm{~d}, J=8.5 \mathrm{~Hz}, 2 \mathrm{H}), 7.74(\mathrm{t}, J=7.9 \mathrm{~Hz}, 1 \mathrm{H}), 7.52$ $(\mathrm{d}, J=8.5 \mathrm{~Hz}, 2 \mathrm{H}), 7.42(\mathrm{~s}, 1 \mathrm{H}) .{ }^{13} \mathrm{C}$ NMR $\left(100.6 \mathrm{MHz}, \mathrm{DMSO}\left[\mathrm{D}_{6}\right]+1-2 \%\right.$ conc. DCl $) \delta=$ $148.5,146.8,145.9,133.7,132.8,131.5,130.6,129.6,129.1,127.0,122.5,119.4,101.1$. $\operatorname{HRMS}(\mathrm{m} / \mathrm{z})[\mathrm{M}+\mathrm{H}]^{+}$calcd for $\mathrm{M}=\mathrm{C}_{15} \mathrm{H}_{10} \mathrm{ClN}_{3} \mathrm{O}_{2}, 300.0540$; found, 300.0544.

\section{3-(3-Aminophenyl)-5-(4-chlorophenyl)-1H-pyrazole (sery280a)}

The title compound was prepared according to the GEP 8 starting from sery 278a and purified by column chromatography $\left(\mathrm{CHCl}_{3}: \mathrm{MeOH}, 19: 1 \mathrm{v} / \mathrm{v}\right)$ to provide sery280a as a white solid. Yield 28\%. m.p.: $183{ }^{\circ} \mathrm{C}$. ${ }^{1} \mathrm{H}$ NMR (400 MHz, DMSO[D $\left.]\right) ~ \delta=13.25$ (bs, 1H), 7.85 (d, $J=$ $8.2 \mathrm{~Hz}, 2 \mathrm{H}), 7.49$ (d, $J=8.2 \mathrm{~Hz}, 2 \mathrm{H}), 7.09(\mathrm{t}, J=7.7 \mathrm{~Hz}, 1 \mathrm{H}), 7.04-6.86(\mathrm{~m}, 3 \mathrm{H}), 6.55(\mathrm{~d}, J=$ $7.7 \mathrm{~Hz}, 1 \mathrm{H}), 5.15(\mathrm{~s}, 2 \mathrm{H}) .{ }^{13} \mathrm{C} \mathrm{NMR}\left(100.6 \mathrm{MHz}, \mathrm{DMSO}\left[\mathrm{D}_{6}\right]+1-2 \%\right.$ conc. DCl) $\delta=147.3$, $145.3,133.4,132.5,132.4,130.3,129.5,128.9,126.9,124.8,122.6,119.8,100.4$. HRMS $(\mathrm{m} / \mathrm{z})[\mathrm{M}+\mathrm{H}]^{+}$calcd for $\mathrm{M}=\mathrm{C}_{15} \mathrm{H}_{12} \mathrm{ClN}_{3}, 270.0798$; found, 270.0799. 


\section{(E)-3-(4-Chlorophenyl)-1-(4-nitrophenyl)-2-propene-1-one (sery276b)}

The title compound has been reported previously ${ }^{535}$ (CAS 315180-19-9). It was prepared according to the GEP 5 using 1-(4-nitrophenyl)ethanone, 4-chlorobenzaldehyde and purified by refluxing in ethanol to provide sery276b as a yellow solid. Yield $87 \%$. m.p.: $163-165{ }^{\circ} \mathrm{C}$ (lit $\left.{ }^{535} 154-156{ }^{\circ} \mathrm{C}\right) .{ }^{1} \mathrm{H}$ NMR (400 MHz, DMSO[D 6$\left.]\right) \delta=8.37$ (bs, 4H), 8.03-7.93 (m, 3H), $7.8(\mathrm{~d}, J=15.6 \mathrm{~Hz}, 1 \mathrm{H}), 7.55(\mathrm{~d}, J=8.4 \mathrm{~Hz}, 2 \mathrm{H})$.

\section{2,3-Dibromo-3-(4-chlorophenyl)-1-(4-nitrophenyl)propane-1-one (sery277b)}

The title compound has been reported previously ${ }^{536}$ (CAS 24213-20-5). It was prepared according to the GEP 6 starting from sery 276b to provide sery277b as a white solid. Yield 78\%. m.p.: $154-156{ }^{\circ} \mathrm{C}\left(\right.$ lit $\left.^{536} 150-153{ }^{\circ} \mathrm{C}\right) .{ }^{1} \mathrm{H}$ NMR (400 MHz, DMSO[D 6 ] $\delta=8.53(\mathrm{~d}, J=$ $8.9 \mathrm{~Hz}, 2 \mathrm{H}), 8.45(\mathrm{~d}, J=8.9 \mathrm{~Hz}, 2 \mathrm{H}), 7.89(\mathrm{~d}, J=8.5 \mathrm{~Hz}, 2 \mathrm{H}), 7.53(\mathrm{~d}, J=8.5 \mathrm{~Hz}, 2 \mathrm{H}), 6.83$ (d, $J=11.3 \mathrm{~Hz}, 1 \mathrm{H}), 5.87(\mathrm{~d}, J=11.3 \mathrm{~Hz}, 1 \mathrm{H})$.

\section{3-(4-Chlorophenyl)-5-(4-nitrophenyl)-1H-pyrazole (sery278b)}

The title compound has been reported previously ${ }^{526}$ (CAS 106961-02-8). It was prepared according to the GEP 7 starting from sery $277 \mathbf{b}$ and purified by recrystallisation from methanol (twice) to provide sery278b as a white solid. Yield $50 \%$. m.p.: $271-272{ }^{\circ} \mathrm{C}\left(\mathrm{lit}^{526}\right.$ $\left.280{ }^{\circ} \mathrm{C}\right) .{ }^{1} \mathrm{H}$ NMR $\left.\left(400 \mathrm{MHz}, \mathrm{DMSO}_{[} \mathrm{D}_{6}\right]\right) \delta=13.77(\mathrm{~s}, 1 \mathrm{H}), 8.31(\mathrm{~d}, J=8.6 \mathrm{~Hz}, 2 \mathrm{H}), 8.10$ $(\mathrm{d}, J=8.6 \mathrm{~Hz}, 2 \mathrm{H}), 7.85(\mathrm{~d}, J=8.2 \mathrm{~Hz}, 2 \mathrm{H}), 7.55(\mathrm{~d}, J=8.2 \mathrm{~Hz}, 2 \mathrm{H}), 7.45(\mathrm{~s}, 1 \mathrm{H})$.

\section{3-(4-Aminophenyl)-5-(4-chlorophenyl)-1H-pyrazole (sery280b)}

The title compound was prepared according to the GEP 8 starting from sery $278 \mathbf{b}$ and purified by column chromatography $\left(\mathrm{CHCl}_{3}: \mathrm{MeOH}, 100: 1 \mathrm{v} / \mathrm{v}\right)$ to provide sery280b as a white solid. Yield 22\%. m.p.: 213-214 ${ }^{\circ} \mathrm{C} .{ }^{1} \mathrm{H}$ NMR (400 MHz, DMSO[D 6$\left.]\right) \delta=13.01(\mathrm{~s}, 1 \mathrm{H}), 7.84(\mathrm{~d}, J$ $=8.2 \mathrm{~Hz}, 2 \mathrm{H}), 7.46(\mathrm{~d}, J=5.1 \mathrm{~Hz}, 4 \mathrm{H}), 6.92(\mathrm{~s}, 1 \mathrm{H}), 6.62(\mathrm{~d}, J=8.5 \mathrm{~Hz}, 2 \mathrm{H}), 5.32(\mathrm{~s}, 2 \mathrm{H})$. ${ }^{13} \mathrm{C}$ NMR (100.6 MHz, DMSO[D 6 ) $\delta=149.8,148.8,144.8,133.0,131.8,128.6,126.7$, 126.2, 116.9, 113.8, 97.6. HRMS $(\mathrm{m} / z)[\mathrm{M}+\mathrm{H}]^{+}$calcd for $\mathrm{M}=\mathrm{C}_{15} \mathrm{H}_{12} \mathrm{ClN}_{3}, 270.0798$; found, 270.0798 .

\section{(E)-1-(1,3-Benzodioxol-5-yl)-3-(3-bromo-5-nitrophenyl)-2-propene-1-one (sery348)}

The title compound was prepared according to the GEP 5 using 1-(1,3-benzodioxol-5yl)ethanone, 3-bromo-5-nitrobenzaldehyde and purified by recrystallisation from mixture ethanol/EtOAc $(8 / 2 \mathrm{v} / \mathrm{v})$ to provide sery348 as a yellow solid. Yield $77 \%$. ${ }^{1} \mathrm{H}$ NMR (400 $\left.\mathrm{MHz}, \mathrm{DMSO}\left[\mathrm{D}_{6}\right]\right) \delta=8.76(\mathrm{~s}, 1 \mathrm{H}), 8.67(\mathrm{~s}, 1 \mathrm{H}), 8.40(\mathrm{t}, J=1.8 \mathrm{~Hz}, 1 \mathrm{H}), 8.20(\mathrm{~d}, J=15.6$ $\mathrm{Hz}, 1 \mathrm{H}), 7.95(\mathrm{dd}, J=8.2,1.6 \mathrm{~Hz}, 1 \mathrm{H}), 7.78(\mathrm{~d}, J=15.6 \mathrm{~Hz}, 1 \mathrm{H}), 7.73(\mathrm{~d}, J=1.6 \mathrm{~Hz}, 1 \mathrm{H})$, $7.12(\mathrm{~d}, J=8.2 \mathrm{~Hz}, 1 \mathrm{H}) 6.18(\mathrm{~s}, 2 \mathrm{H})$. ESI-MS $(\mathrm{m} / \mathrm{z})[\mathrm{M}+\mathrm{H}]^{+}$calcd for $\mathrm{M}=\mathrm{C}_{16} \mathrm{H}_{10}{ }^{79} \mathrm{BrNO}_{5}$, 375.98; found, 376.01 .

\section{1-(1,3-Benzodioxol-5-yl)-3-(3-bromo-5-nitrophenyl)-2,3-dibromopropane-1-one (sery351)}

The title compound was prepared according to the GEP 6 starting from sery348 to provide sery351 as a red solid. Yield 85\%. ${ }^{1} \mathrm{H}$ NMR $\left.\left(400 \mathrm{MHz}, \mathrm{DMSO} \mathrm{D}_{6}\right]\right) \delta=8.82(\mathrm{~s}, 1 \mathrm{H}), 8.65(\mathrm{~s}$, $1 \mathrm{H}), 8.40(\mathrm{t}, J=1.8 \mathrm{~Hz}, 1 \mathrm{H}), 7.99(\mathrm{dd}, J=8.3,1.7 \mathrm{~Hz}, 1 \mathrm{H}), 7.78(\mathrm{~d}, J=1.6 \mathrm{~Hz}, 1 \mathrm{H}), 7.18(\mathrm{~d}$, $J=8.2 \mathrm{~Hz}, 1 \mathrm{H}), 6.75(\mathrm{~d}, J=11.4 \mathrm{~Hz}, 1 \mathrm{H}), 6.21(\mathrm{~s}, 2 \mathrm{H}), 6.04(\mathrm{~d}, J=11.4 \mathrm{~Hz}, 1 \mathrm{H})$. ESI-MS $(\mathrm{m} / \mathrm{z})[\mathrm{M}+\mathrm{H}]^{+}$calcd for $\mathrm{M}=\mathrm{C}_{16} \mathrm{H}_{10}{ }^{79} \mathrm{Br}_{2}{ }^{81} \mathrm{BrNO}_{5}$, 535.82; found, 536.10. The isolated sery351 was used in next step without further purification. 


\section{3-(1,3-Benzodioxol-5-yl)-5-(3-bromo-5-nitrophenyl)-1H-pyrazole (sery352)}

The title compound was prepared according to the GEP 7 starting from sery351 and purified by refluxing in ethanol to provide sery352 as a yellow solid. Yield 55\%. ${ }^{1} \mathrm{H}$ NMR $(400 \mathrm{MHz}$, DMSO[D 6$]) \delta=13.53(\mathrm{bs}, 1 \mathrm{H}), 8.62(\mathrm{~s}, 1 \mathrm{H}), 8.45(\mathrm{~s}, 1 \mathrm{H}), 8.31(\mathrm{~s}, 1 \mathrm{H}), 7.44(\mathrm{~s}, 1 \mathrm{H}), 7.39(\mathrm{~s}$, $1 \mathrm{H}), 7.35(\mathrm{~d}, J=8.0 \mathrm{~Hz}, 1 \mathrm{H}), 7.04(\mathrm{~d}, J=8.0 \mathrm{~Hz}, 1 \mathrm{H}), 6.08(\mathrm{~s}, 2 \mathrm{H})$.

\section{3-(3-Amino-5-bromophenyl)-5-(1,3-benzodioxol-5-yl)-1 H-pyrazole (sery364)}

The title compound was prepared analogously to the procedure for sery383 from sery352 and purified by recrystallisation from mixture ethanol/petroleum ether $(1 / 2 \mathrm{v} / \mathrm{v})$ to provide sery364 as a grey solid. Yield 47\%. ${ }^{1} \mathrm{H}$ NMR (400 MHz, DMSO[D $]+1 \%$ DCl) $\delta=7.48(\mathrm{~s}$, $1 \mathrm{H}), 7.40(\mathrm{~s}, 1 \mathrm{H}), 7.37-7.30(\mathrm{~m}, 2 \mathrm{H}), 7.09(\mathrm{~s}, 1 \mathrm{H}), 7.05(\mathrm{~s}, 1 \mathrm{H}), 7.00(\mathrm{~d}, J=8.0 \mathrm{~Hz}, 1 \mathrm{H}), 6.06$ (s, 2H). ${ }^{13} \mathrm{C}$ NMR $\left(100.6 \mathrm{MHz}, \mathrm{DMSO}\left[\mathrm{D}_{6}\right]+1 \% \mathrm{DCl}\right) \delta=147.8,147.1,147.0,144.6,135.1$, 124.7, 122.5, 119.8, 119.0 (2C), 113.3, 113.0, 108.7, 105.7, 101.3, 99.7. ESI-MS $(\mathrm{m} / \mathrm{z})$ $[\mathrm{M}+\mathrm{H}]^{+}$calcd for $\mathrm{M}=\mathrm{C}_{16} \mathrm{H}_{12}{ }^{79} \mathrm{BrN}_{3} \mathrm{O}_{2}, 358.02$; found, 358.19.

\section{(E)-3-(3-Chlorophenyl)-1-(4-nitrophenyl)-2-propene-1-one (sery379a)}

The title compound has been reported previously ${ }^{537}$ (CAS 63831-41-4). It was prepared according to the GEP 5 using 1-(4-nitrophenyl)ethanone, 3-chlorobenzaldehyde and purified by recrystallisation from EtOAc/ethanol to provide sery379a as a yellow solid. Yield $81 \%$. m.p.: $144-146{ }^{\circ} \mathrm{C} .{ }^{1} \mathrm{H}$ NMR (400 MHz, DMSO[D $]$ ) $\delta=8.42-8.34(\mathrm{~m}, 4 \mathrm{H}), 8.10(\mathrm{~s}, 1 \mathrm{H}), 8.05$ $(\mathrm{d}, J=15.7 \mathrm{~Hz}, 1 \mathrm{H}), 7.85(\mathrm{~d}, J=7.1 \mathrm{~Hz}, 1 \mathrm{H}), 7.77(\mathrm{~d}, J=15.7 \mathrm{~Hz}, 1 \mathrm{H}), 7.55-7.46(\mathrm{~m}, 2 \mathrm{H})$.

\section{3-(3-Chlorophenyl)-2,3-dibromo-1-(4-nitrophenyl)-propane-1-one (sery380a)}

The title compound has been reported previously ${ }^{538}$ (CAS 649739-74-2). It was prepared according to the GEP 6 starting from sery379a to provide sery380a as a yellow solid. Yield

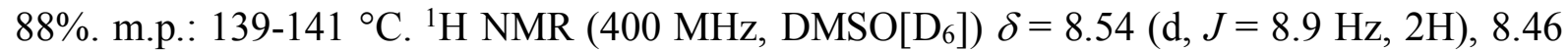
$(\mathrm{d}, J=8.9 \mathrm{~Hz}, 2 \mathrm{H}), 8.04(\mathrm{~s}, 1 \mathrm{H}), 7.80(\mathrm{dt}, J=6.5,2.0 \mathrm{~Hz}, 1 \mathrm{H}), 7.52-7.42(\mathrm{~m}, 2 \mathrm{H}), 6.84(\mathrm{~d}, J$ $=11.3 \mathrm{~Hz}, 1 \mathrm{H}), 5.87(\mathrm{~d}, J=11.3 \mathrm{~Hz}, 1 \mathrm{H})$.

\section{3-(3-Chlorophenyl)-5-(4-nitrophenyl)-1H-pyrazole (sery381a)}

The title compound was prepared according to the GEP 7 starting from sery380a and purified by recrystallisation from methanol to provide sery381a as a yellow solid. Yield $25 \%$. m.p.: $275-276{ }^{\circ} \mathrm{C} .{ }^{1} \mathrm{H}$ NMR $\left(400 \mathrm{MHz}, \mathrm{DMSO}\left[\mathrm{D}_{6}\right]+1-2 \%\right.$ conc. DCl) $\delta=8.30(\mathrm{~d}, J=8.6 \mathrm{~Hz}, 2 \mathrm{H})$, $8.09(\mathrm{~d}, J=8.6 \mathrm{~Hz}, 2 \mathrm{H}), 7.92(\mathrm{~s}, 1 \mathrm{H}), 7.81(\mathrm{~d}, J=7.7 \mathrm{~Hz}, 1 \mathrm{H}), 7.57-7.46(\mathrm{~m}, 2 \mathrm{H}), 7.42(\mathrm{~d}, J=$ $7.8 \mathrm{~Hz}, 1 \mathrm{H}) .{ }^{13} \mathrm{C}$ NMR $\left(100.6 \mathrm{MHz}, \mathrm{DMSO}\left[\mathrm{D}_{6}\right]+1-2 \%\right.$ conc. DCl $) \delta=149.4,146.9,146.5$, $138.1,133.7,132.1,130.9,127.9,125.9,124.8,124.3,123.7,102.0 . \operatorname{HRMS}(m / z)[\mathrm{M}+\mathrm{H}]^{+}$ calcd for $\mathrm{M}=\mathrm{C}_{15} \mathrm{H}_{10} \mathrm{ClN}_{3} \mathrm{O}_{2}, 300.0540$; found, 300.0543 .

\section{3-(4-Aminophenyl)-5-(3-chlorophenyl)-1H-pyrazole hydrochloride (sery383)}

To a suspension of sery381a $(650 \mathrm{mg}, 2.2 \mathrm{mmol})$ in acetic acid $(35 \mathrm{ml})$ iron powder $(186 \mathrm{mg}$, $3.3 \mathrm{mmol}$ ) was added in small portions under efficient stirring at $100{ }^{\circ} \mathrm{C}$. After addition the suspension was stirred at $100{ }^{\circ} \mathrm{C}$ for $30 \mathrm{~min}$ and poured in ice $(200 \mathrm{~g})$. A precipitate was filtered and aqueous filtrate was extracted with chloroform $(40 \mathrm{ml} \times 2)$. Resulting crystals were dissolved in methanol $(40 \mathrm{ml})$, solutions in chloroform and methanol were combined, filtered and concentrated under reduced pressure. A crude product was dissolved in THF (25 $\mathrm{ml})$ and conc. hydrochloric acid $(0.3 \mathrm{ml})$ was added. The mixture was cooled for $1 \mathrm{~h}$ at $4{ }^{\circ} \mathrm{C}$ and a white precipitate was collected to provide sery383 $(500 \mathrm{mg})$ as a white powder. Yield 75\%. m.p.: 265-268 ${ }^{\circ} \mathrm{C}$ (dec). ${ }^{1} \mathrm{H}$ NMR (400 MHz, DMSO[D 6$)$ ) $\delta=10.14$ (bs, 4H), 7.99-7.90 $(\mathrm{m}, 3 \mathrm{H}), 7.82(\mathrm{~d}, J=7.8 \mathrm{~Hz}, 1 \mathrm{H}), 7.53(\mathrm{~m}, 3 \mathrm{H}), 7.39(\mathrm{~d}, J=7.8 \mathrm{~Hz}, 1 \mathrm{H}), 7.33(\mathrm{~s}, 1 \mathrm{H}) .{ }^{13} \mathrm{C}$ NMR (100.6 MHz, DMSO[D 6 ) $\delta=146.5,146.1,133.7,133.3,131.6,130.9,130.8,127.7$, 
126.3, 124.8, 123.77, 123.75, 100.7. HRMS $(m / z)[\mathrm{M}+\mathrm{H}]^{+}$calcd for $\mathrm{M}=\mathrm{C}_{15} \mathrm{H}_{12} \mathrm{ClN}_{3}$, 270.0798; found, 270.0802 .

\section{(E)-3-(3-Bromophenyl)-1-(4-nitrophenyl)-2-propene-1-one (sery379b)}

The title compound has been reported previously ${ }^{539}$ (CAS 196082-55-0). It was prepared according to the GEP 5 using 1-(4-nitrophenyl)ethanone, 3-bromobenzaldehyde and purified by recrystallisation from EtOAc/ethanol to provide sery379b as a yellow solid. Yield $80 \%$. m.p.: $152-153{ }^{\circ} \mathrm{C}\left(\right.$ lit $\left.^{539} 130{ }^{\circ} \mathrm{C}\right) .{ }^{1} \mathrm{H}$ NMR $\left(400 \mathrm{MHz}, \mathrm{DMSO}\left[\mathrm{D}_{6}\right]\right) \delta=8.38(\mathrm{~s}, 4 \mathrm{H}), 8.24(\mathrm{~s}$, 1H), 8.04 (d, $J=15.7 \mathrm{~Hz}, 1 \mathrm{H}), 7.89(\mathrm{~d}, J=7.9 \mathrm{~Hz}, 1 \mathrm{H}), 7.77(\mathrm{~d}, J=15.7 \mathrm{~Hz}, 1 \mathrm{H}), 7.66(\mathrm{~d}, J$ $=7.9 \mathrm{~Hz}, 1 \mathrm{H}), 7.43(\mathrm{t}, J=7.9 \mathrm{~Hz}, 1 \mathrm{H})$.

\section{3-(3-Bromophenyl)-2,3-dibromo-1-(4-nitrophenyl)propane-1-one (sery380b)}

The title compound has been reported previously ${ }^{539}$ (CAS 577970-80-0). It was prepared according to the GEP 6 starting from sery379b to provide sery380b as a white solid. Yield 85\%. m.p.: $123-124{ }^{\circ} \mathrm{C}\left(1 i^{539} 180{ }^{\circ} \mathrm{C}\right) .{ }^{1} \mathrm{H}$ NMR (400 MHz, DMSO[D 6$\left.]\right) \delta=8.53(\mathrm{~d}, J=8.8$ $\mathrm{Hz}, 2 \mathrm{H}), 8.45$ (d, $J=8.8 \mathrm{~Hz}, 2 \mathrm{H}), 8.18(\mathrm{~s}, 1 \mathrm{H}), 7.84$ (d, $J=7.8 \mathrm{~Hz}, 1 \mathrm{H}), 7.59$ (d, $J=7.9 \mathrm{~Hz}$, $1 \mathrm{H}), 7.41(\mathrm{t}, J=7.9 \mathrm{~Hz}, 1 \mathrm{H}), 6.84(\mathrm{~d}, J=11.2 \mathrm{~Hz}, 1 \mathrm{H}), 5.85(\mathrm{~d}, J=11.2 \mathrm{~Hz}, 1 \mathrm{H})$.

\section{3-(3-Bromophenyl)-5-(4-nitrophenyl)-1H-pyrazole (sery381b)}

The title compound was prepared according to the GEP 7 starting from sery380b and purified by recrystallisation from methanol to provide sery381b as a yellow solid. Yield 27\%. m.p.: $272{ }^{\circ} \mathrm{C} .{ }^{1} \mathrm{H}$ NMR $\left(400 \mathrm{MHz}, \mathrm{DMSO}\left[\mathrm{D}_{6}\right]+1-2 \%\right.$ conc. DCl) $\delta=8.30(\mathrm{~d}, J=8.7 \mathrm{~Hz}, 2 \mathrm{H})$, $8.09(\mathrm{~d}, J=8.7 \mathrm{~Hz}, 2 \mathrm{H}), 8.05(\mathrm{~s}, 1 \mathrm{H}), 7.84(\mathrm{~d}, J=7.7 \mathrm{~Hz}, 1 \mathrm{H}), 7.56(\mathrm{~d}, J=7.8 \mathrm{~Hz}, 1 \mathrm{H}), 7.49$ $(\mathrm{s}, 1 \mathrm{H}), 7.43(\mathrm{t}, J=7.8 \mathrm{~Hz}, 1 \mathrm{H}) .{ }^{13} \mathrm{C} \mathrm{NMR}\left(100.6 \mathrm{MHz}, \mathrm{DMSO}\left[\mathrm{D}_{6}\right]+1-2 \%\right.$ conc. DCl $) \delta=$ $146.8,146.6,145.4,138.2,132.7,131.1,130.8,127.7,125.9,124.3,124.1,122.4,102.0$. HRMS $(m / z)[\mathrm{M}+\mathrm{H}]^{+}$calcd for $\mathrm{M}=\mathrm{C}_{15} \mathrm{H}_{10}{ }^{79} \mathrm{BrN}_{3} \mathrm{O}_{2}, 344.0035$; found, 344.0039.

\section{3-(4-Aminophenyl)-5-(3-bromophenyl)-1H-pyrazole hydrochloride (sery384)}

The title compound was prepared using an analogous procedure to that described for sery383 starting from sery381b to provide sery384 as a white solid. Yield $70 \%$. m.p.: $267-272{ }^{\circ} \mathrm{C}$ (dec). ${ }^{1} \mathrm{H}$ NMR (400 MHz, DMSO[D 6$\left.]\right) \delta=8.05(\mathrm{~s}, 1 \mathrm{H}), 7.89(\mathrm{~d}, J=8.5 \mathrm{~Hz}, 2 \mathrm{H}), 7.85(\mathrm{~d}, J=$ $7.8 \mathrm{~Hz}, 1 \mathrm{H}), 7.54(\mathrm{~d}, J=7.8 \mathrm{~Hz}, 1 \mathrm{H}), 7.42$ (t, $J=7.8 \mathrm{~Hz}, 1 \mathrm{H}), 7.37$ (d, $J=8.5 \mathrm{~Hz}, 2 \mathrm{H}), 7.28$ (s, 1H). ${ }^{13} \mathrm{C}$ NMR $\left(100.6 \mathrm{MHz}, \mathrm{DMSO}\left[\mathrm{D}_{6}\right]\right) \delta=146.5,146.0,133.5,131.5,131.1,131.0$, $130.7,127.7,126.4,124.2,124.0,122.4,100.8$. HRMS $(\mathrm{m} / z)[\mathrm{M}+\mathrm{H}]^{+}$calcd for $\mathrm{M}=$ $\mathrm{C}_{15} \mathrm{H}_{12}{ }^{79} \mathrm{BrN}_{3}, 314.0293$; found, 314.0297.

\section{(E)-1-(1,3-Benzodioxol-5-yl)-3-(3-nitrophenyl)-2-propene-1-one (sery302a)}

The title compound was prepared according to the GEP 5 using 1-(1,3-benzodioxol-5yl)ethanone, 3-nitrobenzaldehyde and purified by refluxing in ethanol to provide sery302a as a yellow solid. Yield 97\%. m.p.: 173-177 ${ }^{\circ} \mathrm{C} .{ }^{1} \mathrm{H}$ NMR $\left(400 \mathrm{MHz}, \mathrm{DMSO}\left[\mathrm{D}_{6}\right]\right) \delta=8.79$ (s, $1 \mathrm{H}), 8.33(\mathrm{~d}, J=7.8 \mathrm{~Hz}, 1 \mathrm{H}), 8.26(\mathrm{dd}, J=8.2,1.6 \mathrm{~Hz}, 1 \mathrm{H}), 8.15(\mathrm{~d}, J=15.6 \mathrm{~Hz}, 1 \mathrm{H}), 7.93$ $(\mathrm{dd}, J=8.2,1.6 \mathrm{~Hz}, 1 \mathrm{H}), 7.81(\mathrm{~d}, J=15.6 \mathrm{~Hz}, 1 \mathrm{H}), 7.77-7.70(\mathrm{~m}, 2 \mathrm{H}), 7.11(\mathrm{~d}, J=8.2 \mathrm{~Hz}$, 1H), $6.18(\mathrm{~s}, 2 \mathrm{H}) .{ }^{13} \mathrm{C}$ NMR (100.6 MHz, DMSO[D 6$\left.]\right) \delta=186.8,151.8,148.4,148.1,140.9$, $136.7,135.1,131.9,130.3,125.5,124.6,124.5,122.9,108.2,108.0,102.2$. HRMS $(\mathrm{m} / \mathrm{z})$ $[\mathrm{M}+\mathrm{H}]^{+}$calcd for $\mathrm{M}=\mathrm{C}_{16} \mathrm{H}_{11} \mathrm{~N}_{3} \mathrm{O}_{5}, 298.0715$; found, 298.0715.

\section{trans-1-(1,3-Benzodioxol-5-yl)-2,3-epoxy-3-(3-nitrophenyl)propane-1-one (sery302b)}

The title compound was prepared according to the published protocol ${ }^{398}$ starting from sery302a and purified by recrystallisation from ethanol/EtOAc to provide sery302b as a white solid. Yield 70\%. m.p.: 132-133 ${ }^{\circ} \mathrm{C} .{ }^{1} \mathrm{H}$ NMR (400 MHz, DMSO[D 6$\left.]\right) \delta=8.28(\mathrm{~s}, 1 \mathrm{H})$, 
$8.24(\mathrm{~d}, J=8.2 \mathrm{~Hz}, 1 \mathrm{H}), 7.90(\mathrm{~d}, J=7.7 \mathrm{~Hz}, 1 \mathrm{H}), 7.78-7.68(\mathrm{~m}, 2 \mathrm{H}), 7.53(\mathrm{~d}, J=1.6 \mathrm{~Hz}, 1 \mathrm{H})$, $7.10(\mathrm{~d}, J=8.2 \mathrm{~Hz}, 1 \mathrm{H}), 6.17(\mathrm{~s}, 1 \mathrm{H}), 6.16(\mathrm{~s}, 1 \mathrm{H}), 4.91(\mathrm{~d}, J=1.8 \mathrm{~Hz}, 1 \mathrm{H}), 4.34(\mathrm{~d}, J=1.8$ $\mathrm{Hz}, 1 \mathrm{H}) .{ }^{13} \mathrm{C}$ NMR (100.6 MHz, DMSO[D $\left.]\right) ~ \delta=190.4,152.4,148.1,148.0,138.3,133.1$, $130.1,129.9,125.5,123.6,121.2,108.4,107.4,102.3,59.2,57.3$. HRMS $(m / z)[\mathrm{M}+\mathrm{H}]^{+}$calcd for $\mathrm{M}=\mathrm{C}_{16} \mathrm{H}_{11} \mathrm{NO}_{6}, 314.0665$; found, 314.0664 .

\section{3-(1,3-Benzodioxol-5-yl)-5-(3-nitrophenyl)-1 H-pyrazole (sery302c)}

The title compound was prepared by a modified method ${ }^{400}$. A mixture of sery302b $(313 \mathrm{mg}$, $1 \mathrm{mmol})$, hydrazine hydrate $(150 \mathrm{mg}, 3 \mathrm{mmol})$ and $p$-toluenesulfonic acid monohydrate $(20$ $\mathrm{mg}$ ) in toluene $(5 \mathrm{ml})$ was heated under reflux $4 \mathrm{~h}$ with stirring. A reaction mixture was evaporated under reduced pressure and a resulting solid was purified by recrystallisation from ethanol to provide sery302c as a yellow solid. Yield $72 \%$. m.p.: $226-227{ }^{\circ} \mathrm{C}$ (dec). ${ }^{1} \mathrm{H}$ NMR $\left(400 \mathrm{MHz}, \mathrm{DMSO}\left[\mathrm{D}_{6}\right]+1-2 \%\right.$ conc. DCl) $\delta=8.63(\mathrm{~s}, 1 \mathrm{H}), 8.26(\mathrm{~d}, J=7.6 \mathrm{~Hz}, 1 \mathrm{H}), 8.16(\mathrm{~d}$, $J=7.6 \mathrm{~Hz}, 1 \mathrm{H}), 7.73(\mathrm{t}, J=7.9 \mathrm{~Hz}, 1 \mathrm{H}), 7.40(\mathrm{~s}, 1 \mathrm{H}), 7.38-7.26(\mathrm{~m}, 2 \mathrm{H}), 7.02(\mathrm{~d}, J=7.9 \mathrm{~Hz}$, 1H), 6.07 (s, 2H). ${ }^{13} \mathrm{C}$ NMR (100.6 MHz, DMSO[D 6$\left.]\right) \delta=149.1,148.3,147.9,147.3,143.8$, $135.4,131.2,130.3,123.1,122.0,119.2,119.1,108.8,105.6,101.4,99.9$. HRMS $(\mathrm{m} / \mathrm{z})$ $[\mathrm{M}+\mathrm{H}]^{+}$calcd for $\mathrm{M}=\mathrm{C}_{16} \mathrm{H}_{11} \mathrm{~N}_{3} \mathrm{O}_{4}, 310.0828$; found, 310.0831 .

\section{3-(3-Aminophenyl)-5-(1,3-benzodioxol-5-yl)-1H-pyrazole (sery308)}

The title compound was prepared according to the GEP 8 starting from sery302c and was purified by column chromatography $\left(\mathrm{CHCl}_{3}: \mathrm{MeOH}, 20: 1 \mathrm{v} / \mathrm{v}\right)$ to provide sery308 as a grey solid. Yield 31\%. TLC $\left(\mathrm{CHCl}_{3}: \mathrm{MeOH}, 10: 1 \mathrm{v} / \mathrm{v}\right): \mathrm{RF}=0.47$. m.p.: $200{ }^{\circ} \mathrm{C} .{ }^{1} \mathrm{H}$ NMR $(400$ $\left.\mathrm{MHz}, \mathrm{DMSO}\left[\mathrm{D}_{6}\right]\right) \delta=7.39(\mathrm{~s}, 1 \mathrm{H}), 7.34(\mathrm{~d}, J=8.1 \mathrm{~Hz}, 1 \mathrm{H}), 7.07(\mathrm{t}, J=7.5 \mathrm{~Hz}, 1 \mathrm{H}), 7.03-$ $6.91(\mathrm{~m}, 3 \mathrm{H}), 6.89(\mathrm{~s}, 1 \mathrm{H}), 6.54(\mathrm{~d}, J=7.5 \mathrm{~Hz}, 1 \mathrm{H}), 6.05(\mathrm{~s}, 2 \mathrm{H}), 5.16(\mathrm{~s}, 2 \mathrm{H}) .{ }^{13} \mathrm{C}$ NMR (100.6 MHz, DMSO[D 6$]$ ) $\delta=151.3,148.9,147.7,146.8,144.2,130.0,129.2,124.1,118.8$, $113.7,113.1,110.6,108.6,105.6,101.1,98.9$. HRMS $(\mathrm{m} / \mathrm{z})[\mathrm{M}+\mathrm{H}]^{+}$calcd for $\mathrm{M}=$ $\mathrm{C}_{16} \mathrm{H}_{13} \mathrm{~N}_{3} \mathrm{O}_{2}, 280.1086$; found, 280.1086.

\section{(E)-1-(1,3-Benzodioxol-5-yl)-3-(2,3-difluorophenyl)-2-propene-1-one (sery318a)}

The title compound was prepared according to the GEP 5 using 1-(1,3-benzodioxol-5yl)ethanone, 2,3-difluorobenzaldehyde and purified by recrystallisation from methanol to provide sery318a as a yellow solid. Yield 87\%. m.p.: 121-122 ${ }^{\circ} \mathrm{C}$. ${ }^{1} \mathrm{H}$ NMR $(400 \mathrm{MHz}$, DMSO[D 6$]) \delta=8.05-7.91(\mathrm{~m}, 2 \mathrm{H}), 7.86(\mathrm{dd}, J=8.2,1.5 \mathrm{~Hz}, 1 \mathrm{H}), 7.76(\mathrm{~d}, J=15.7 \mathrm{~Hz}, 1 \mathrm{H})$, $7.64(\mathrm{~d}, J=1.5 \mathrm{~Hz}, 1 \mathrm{H}), 7.56-7.26(\mathrm{~m} .2 \mathrm{H}), 7.10(\mathrm{~d}, J=8.2 \mathrm{~Hz}, 1 \mathrm{H}), 6.17(\mathrm{~s}, 2 \mathrm{H}) .{ }^{13} \mathrm{C} \mathrm{NMR}$ (100.6 MHz, DMSO[D 6$]$ ) $\delta=186.6,151.9,150.0(\mathrm{dd}, J=245.2,12.7 \mathrm{~Hz}), 148.5$ (dd, $J=$ $252.2,13.0 \mathrm{~Hz}), 148.1,133.1(\mathrm{t}, J=3.6 \mathrm{~Hz}), 131.8,125.4,125.3(\mathrm{~d}, J=3.9 \mathrm{~Hz}), 125.0(\mathrm{dd}, J$ $=7.0,4.8 \mathrm{~Hz}), 124.7(\mathrm{~d}, J=2.9 \mathrm{~Hz}), 124.1,119.0(\mathrm{~d}, J=17.0 \mathrm{~Hz}), 108.2,107.9,102.2$. HRMS $(m / z)[\mathrm{M}+\mathrm{H}]^{+}$calcd for $\mathrm{M}=\mathrm{C}_{16} \mathrm{H}_{10} \mathrm{~F}_{2} \mathrm{O}_{3}, 289.0676$; found, 289.0677.

\section{trans-1-(1,3-Benzodioxol-5-yl)-3-(2,3-difluorophenyl)-2,3-epoxypropane-1-one (sery318b)}

The title compound was prepared according to the published protocol ${ }^{398}$ starting from sery318a to provide sery318b as a white solid. Yield 82\%. m.p.: 92-93 ${ }^{\circ} \mathrm{C} .{ }^{1} \mathrm{H}$ NMR (400 $\left.\mathrm{MHz}, \mathrm{DMSO}\left[\mathrm{D}_{6}\right]\right) \delta=7.78(\mathrm{dd}, J=8.2,1.7 \mathrm{~Hz}, 1 \mathrm{H}), 7.54(\mathrm{~d}, J=1.7 \mathrm{~Hz}, 1 \mathrm{H}), 7.51-7.42(\mathrm{~m}$, $1 \mathrm{H}), 7.32-7.18(\mathrm{~m}, 2 \mathrm{H}), 7.10(\mathrm{~d}, J=8.2 \mathrm{~Hz}, 1 \mathrm{H}), 6.17(\mathrm{~s}, 2 \mathrm{H}), 4.84(\mathrm{~d}, J=1.9 \mathrm{~Hz}, 1 \mathrm{H}), 4.28$ $(\mathrm{d}, J=1.8 \mathrm{~Hz}, 1 \mathrm{H}) .{ }^{13} \mathrm{C}$ NMR $\left(100.6 \mathrm{MHz}, \mathrm{DMSO}\left[\mathrm{D}_{6}\right]\right) \delta=190.4,152.5,149.5$ (dd, $J=$ 246.1, $12.2 \mathrm{~Hz}), 148.8$ (dd, $J=248.1,13.8 \mathrm{~Hz}), 148.1,129.8,125.6,125.5$ (dd, $J=6.9,4.3$ 
Hz), 125.1, 122.4, $117.6(\mathrm{~d}, J=16.9 \mathrm{~Hz}), 108.4,107.4,102.3,57.8,52.9(\mathrm{t}, J=4.6 \mathrm{~Hz})$. HRMS $(\mathrm{m} / z)[\mathrm{M}+\mathrm{Na}]^{+}$calcd for $\mathrm{M}=\mathrm{C}_{16} \mathrm{H}_{10} \mathrm{~F}_{2} \mathrm{O}_{4}, 327.0045$; found, 327.0447 .

\section{3-(1,3-Benzodioxol-5-yl)-5-(2,3-difluorophenyl)-1H-pyrazole (sery319)}

The title compound was prepared using an analogous procedure to that described for sery302c starting from sery318b and purified by recrystallisation from methanol to provide sery319 as a brown solid. Yield 50\%. m.p.: 211-212 ${ }^{\circ} \mathrm{C}$ (dec). ${ }^{1} \mathrm{H}$ NMR $(400 \mathrm{MHz}$, DMSO $\left[\mathrm{D}_{6}\right]+1-2 \%$ conc. DCl) $\delta=13.47(\mathrm{bs}, 1 \mathrm{H}), 7.74(\mathrm{t}, J=7.4 \mathrm{~Hz}, 1 \mathrm{H}), 7.44-7.23(\mathrm{~m}$, 4H), $7.03(\mathrm{~d}, J=3.0 \mathrm{~Hz}, 1 \mathrm{H}), 6.99(\mathrm{~d}, J=8.1 \mathrm{~Hz}, 1 \mathrm{H}), 6.07(\mathrm{~s}, 2 \mathrm{H}) .{ }^{13} \mathrm{C}$ NMR $(100.6 \mathrm{MHz}$, DMSO $\left[\mathrm{D}_{6}\right]+1-2 \%$ conc. DCl) $\delta=150.4(\mathrm{~d}, J=244.6 \mathrm{~Hz}), 150.2(\mathrm{~d}, J=244.9 \mathrm{~Hz}), 147.8$, 147.2, 145.7, 142.3, 124.9, 124.4, 123.1, 122.2, 119.1, 116.3 (dd, $J=15.2,3.6 \mathrm{~Hz}$ ), 108.7, 105.7, $102.2(\mathrm{~d}, J=7.7 \mathrm{~Hz}), 101.2$. HRMS $(m / z)[\mathrm{M}+\mathrm{H}]^{+}$calcd for $\mathrm{M}=\mathrm{C}_{16} \mathrm{H}_{10} \mathrm{~F}_{2} \mathrm{NO}_{2} \mathrm{O}_{2}$, 301.0789; found, 301.0792 .

\section{2-Acetylphenyl-4-chlorobenzoate (sery281b)}

The title compound has been reported previously ${ }^{403}$ (CAS 84634-62-8). It was prepared according to the published protocol ${ }^{403}$ starting from 4-chlorobenzoyl chloride and 1-(2hydroxyphenyl)ethanone to provide sery281b as a white solid. Yield 75\%. m.p.: $93-94{ }^{\circ} \mathrm{C}$ (lit $\left.{ }^{403} 94{ }^{\circ} \mathrm{C}\right) .{ }^{1} \mathrm{H}$ NMR $\left(400 \mathrm{MHz}, \mathrm{DMSO}\left[\mathrm{D}_{6}\right]\right) \delta=8.12(\mathrm{~d}, J=8.7 \mathrm{~Hz}, 2 \mathrm{H}), 7.99$ (dd, $J=7.7$, $1.6 \mathrm{~Hz}, 1 \mathrm{H}), 7.73-7.66(\mathrm{~m}, 3 \mathrm{H}), 7.48(\mathrm{td}, J=7.7,1.1 \mathrm{~Hz}, 1 \mathrm{H}), 7.39(\mathrm{dd}, J=8.1,1.1 \mathrm{~Hz}, 1 \mathrm{H})$, $2.51(\mathrm{~s}, 3 \mathrm{H})$.

\section{1-(4-Chlorophenyl)-3-(2-hydroxyphenyl)propane-1,3-dione (sery282)}

The title compound has been reported previously ${ }^{403}$ (CAS 84634-62-8). It was prepared according to the published protocol ${ }^{540}$ starting from sery $\mathbf{2 8 1 b}$ and purified by recrystallisation from methanol to provide sery282 as a yellow solid. Yield $42 \%$. m.p.: $123-124{ }^{\circ} \mathrm{C}\left(\mathrm{lit}^{403} 120\right.$ $\left.{ }^{\circ} \mathrm{C}\right) .{ }^{1} \mathrm{H}$ NMR $\left(400 \mathrm{MHz}, \mathrm{CDCl}_{3}\right) \delta=12.01(\mathrm{~s}, 1 \mathrm{H}), 7.88(\mathrm{~d}, J=8.6 \mathrm{~Hz}, 2 \mathrm{H}), 7.77(\mathrm{dd}, J=$ 8.1, 1.4 Hz, 1H), 7.51-7.44 (m, 3H), $7.01(\mathrm{~d}, J=8.6 \mathrm{~Hz}, 1 \mathrm{H}), 6.93(\mathrm{t}, J=7.4 \mathrm{~Hz}, 1 \mathrm{H}), 6.81$ (s, $1 \mathrm{H})$.

\section{3-(4-Chlorophenyl)-5-(2-hydroxyphenyl)-1H-pyrazole (sery283)}

The title compound has been reported previously ${ }^{541}$ (CAS 38371-80-1). It was prepared according to the GEP 2 starting from sery282 and purified by recrystallisation from petroleum benzine $\left(40-60{ }^{\circ} \mathrm{C}\right) /$ methanol to provide sery283 as a white solid. Yield $46 \%$. m.p.: $192-194{ }^{\circ} \mathrm{C}\left(\right.$ lit $\left.^{541} 191{ }^{\circ} \mathrm{C}\right) .{ }^{1} \mathrm{H}$ NMR (400 MHz, DMSO[D $]+1-2 \%$ conc. DCl) $\delta=10.58$ (bs, $1 \mathrm{H}), 7.86(\mathrm{~d}, J=8.6 \mathrm{~Hz}, 2 \mathrm{H}), 7.72(\mathrm{dd}, J=7.7,1.4 \mathrm{~Hz}, 1 \mathrm{H}), 7.52(\mathrm{~d}, J=8.6 \mathrm{~Hz}, 2 \mathrm{H}), 7.26(\mathrm{~s}$, 1H), 7.22-7.15 (m, 1H), $6.97(\mathrm{~d}, J=8.2 \mathrm{~Hz}, 1 \mathrm{H}), 6.90$ (td, $J=7.7,1.0 \mathrm{~Hz}, 1 \mathrm{H})$.

\section{2-Acetylphenyl 3,4-dimethoxybenzoate (sery288b)}

The title compound has been reported previously ${ }^{403}$ (CAS 67858-33-7). It was prepared according to the published protocol ${ }^{403}$ starting from 3,4-dimethoxybenzoyl chloride and 1-(2hydroxyphenyl)ethanone and purified by recrystallisation from hexane/EtOAc to provide sery288b as a white solid. Yield 74\%. m.p.: $125-127{ }^{\circ} \mathrm{C}$ (lit $\left.{ }^{403} 126-127{ }^{\circ} \mathrm{C}\right) .{ }^{1} \mathrm{H}$ NMR (400 MHz, DMSO[D6]) $\delta=7.93(\mathrm{dd}, J=7.8,1.6 \mathrm{~Hz}, 1 \mathrm{H}), 7.78(\mathrm{dd}, J=8.4,2.0 \mathrm{~Hz}, 1 \mathrm{H}), 7.67(\mathrm{td}$, $J=7.8,1.6 \mathrm{~Hz}, 1 \mathrm{H}), 7.58(\mathrm{~d}, J=2.0 \mathrm{~Hz}, 1 \mathrm{H}), 7.45(\mathrm{td}, J=7.6,1.0 \mathrm{~Hz}, 1 \mathrm{H}), 7.34(\mathrm{dd}, J=8.1$, $1.0 \mathrm{~Hz}, 1 \mathrm{H}), 7.16(\mathrm{~d}, J=8.4 \mathrm{~Hz}, 1 \mathrm{H}), 3.88(\mathrm{~s}, 3 \mathrm{H}), 3.84(\mathrm{~s}, 3 \mathrm{H}), 2.50(\mathrm{~s}, 3 \mathrm{H})$.

\section{1-(3,4-Dimethoxyphenyl)-3-(2-hydroxyphenyl)propane-1,3-dione (sery290a)}

The title compound has been reported previously ${ }^{403}$ (CAS 4233-35-6). It was prepared according to the published protocol ${ }^{540}$ starting from sery $\mathbf{2 8 8 b}$ and purified by recrystallisation 
from methanol to provide sery290a as a yellow solid. Yield 73\%. m.p.: $129-130{ }^{\circ} \mathrm{C}\left(\mathrm{lit}^{403} 115\right.$ $\left.{ }^{\circ} \mathrm{C}\right) .{ }^{1} \mathrm{H}$ NMR $\left(400 \mathrm{MHz}, \mathrm{CDCl}_{3}\right) \delta=12.09(\mathrm{~s}, 1 \mathrm{H}), 7.75(\mathrm{dd}, J=8.1,1.4 \mathrm{~Hz}, 1 \mathrm{H}), 7.57(\mathrm{dd}, J$ $=8.4,2.0 \mathrm{~Hz}, 1 \mathrm{H}), 7.48-7.41(\mathrm{~m}, 2 \mathrm{H}), 6.99(\mathrm{dd}, J=8.4,0.9 \mathrm{~Hz}, 1 \mathrm{H}), 6.95-6.87(\mathrm{~m}, 2 \mathrm{H}), 6.75$ $(\mathrm{s}, 1 \mathrm{H}), 3.97(\mathrm{~s}, 3 \mathrm{H}), 3.95(\mathrm{~s}, 3 \mathrm{H})$.

\section{3-(3,4-Dimethoxyphenyl)-5-(2-hydroxyphenyl)-1H-pyrazole (sery290b)}

The title compound has been reported previously ${ }^{542}$ (CAS 38371-74-3). It was prepared according to the GEP 2 starting from sery290a and purified by recrystallisation from methanol to provide sery290b as a white solid. Yield 86\%. m.p.: $216-217^{\circ} \mathrm{C}\left(\operatorname{lit}^{542} 214{ }^{\circ} \mathrm{C}\right)$. ${ }^{1} \mathrm{H}$ NMR (400 MHz, DMSO[D 6$]+1-2 \%$ conc. DCl) $\delta=10.84(\mathrm{bs}, 1 \mathrm{H}), 7.75(\mathrm{~d}, J=7.3 \mathrm{~Hz}$, $1 \mathrm{H}), 7.44(\mathrm{~s}, 1 \mathrm{H}), 7.39(\mathrm{~d}, J=8.3 \mathrm{~Hz}, 1 \mathrm{H}), 7.23(\mathrm{~s}, 1 \mathrm{H}), 7.19(\mathrm{t}, J=8.1 \mathrm{~Hz}, 1 \mathrm{H}), 7.05(\mathrm{~d}, J=$ $8.3 \mathrm{~Hz}, 1 \mathrm{H}), 6.99-6.87(\mathrm{~m}, 2 \mathrm{H}), 3.85(\mathrm{~s}, 3 \mathrm{H}), 3.80(\mathrm{~s}, 3 \mathrm{H})$.

\section{1-Acetyl-3,5-bis(3,4-dimethoxyphenyl)-1H-pyrazole (sery153)}

The title compound was prepared according to the published protocol ${ }^{409}$. A mixture of sery149 (340 mg, $1 \mathrm{mmol})$, TEA (152 mg, $1.5 \mathrm{mmol})$ and acetyl chloride (118 mg, $1.5 \mathrm{mmol})$ in benzene $(5 \mathrm{ml})$ was heated under reflux $5 \mathrm{~h}$ with stirring. The reaction mixture was cooled down, quenched with water $(15 \mathrm{ml})$ and benzene $(10 \mathrm{ml})$. The organic layer was washed with $0.1 \mathrm{M} \mathrm{HCl}(10 \mathrm{ml}), 0.1 \mathrm{NaOH}(10 \mathrm{ml})$, brine $(15 \mathrm{ml})$, dried $\left(\mathrm{Na}_{2} \mathrm{SO}_{4}\right)$ and concentrated. The crude product was purified by column chromatography (hexane:EtOAc, 1:1 v/v) followed by recrystallisation from hexane to provide sery153 $(105 \mathrm{mg})$ as a white solid. Yield 28\%. m.p.: 113-115 ${ }^{\circ} \mathrm{C} .{ }^{1} \mathrm{H}$ NMR (400 MHz, DMSO[D 6$\left.]\right) \delta=7.55-7.48(\mathrm{~m}, 2 \mathrm{H}), 7.13(\mathrm{~d}, J=1.9 \mathrm{~Hz}$, $1 \mathrm{H}), 7.11(\mathrm{~s}, 1 \mathrm{H}), 7.08-7.03(\mathrm{~m}, 2 \mathrm{H}), 6.99(\mathrm{~d}, J=8.4 \mathrm{~Hz}, 1 \mathrm{H}), 3.85(\mathrm{~s}, 3 \mathrm{H}), 3.81(\mathrm{~s}, 3 \mathrm{H}), 3.80$ $(\mathrm{s}, 3 \mathrm{H}), 3.76(\mathrm{~s}, 3 \mathrm{H}), 2.73(\mathrm{~s}, 3 \mathrm{H}) .{ }^{13} \mathrm{C}$ NMR $\left(100.6 \mathrm{MHz}, \mathrm{DMSO}\left[\mathrm{D}_{6}\right]\right) \delta=170.0,152.5$, $149.8,149.2,149.0,147.9,146.5,124.1,123.3,121.6,118.9,113.1,111.9,110.9,109.7$, 109.4, 55.7, 55.6, $55.5(2 \mathrm{C}), 23.7$. HRMS $(\mathrm{m} / z)[\mathrm{M}+\mathrm{H}]^{+}$calcd for $\mathrm{M}=\mathrm{C}_{21} \mathrm{H}_{22} \mathrm{~N}_{2} \mathrm{O}_{5}$, 383.1607 ; found, 383.1608 .

\section{5-(1,3-Benzodioxol-5-yl)-3-(3-bromophenyl)-1-(2-fluoroethyl)-1H-pyrazole (sery363a) and 3-(1,3-benzodioxol-5-yl)-5-(3-bromophenyl)-1-(2-fluoroethyl)-1H-pyrazole (sery363b)}

A mixture of anle138b (1.5 g, $4.4 \mathrm{mmol}), 1 \mathrm{~N} \mathrm{NaOH}(4.4 \mathrm{ml})$ and DMF (40 ml) was heated for $10 \mathrm{~min}$ at $100{ }^{\circ} \mathrm{C}$ and a solution of 2-fluoroethyl ester of 4-methylbenzenesulfonic acid $(1.05 \mathrm{~g}, 4.8 \mathrm{mmol})$ in DMF $(5 \mathrm{ml})$ was added in one portion. After stirring for $5 \mathrm{~min}$ at $100{ }^{\circ} \mathrm{C}$ the reaction mixture was cooled down, the solvent was evaporated under reduced pressure. A remaining oil was diluted with water $(50 \mathrm{ml})$, extracted with EtOAc $(3 \times 50 \mathrm{ml})$, combined organic fractions were washed with brine $(20 \mathrm{ml})$, dried $\left(\mathrm{Na}_{2} \mathrm{SO}_{4}\right)$ and evaporated. A resulting oil was purified by column chromatography (hexane:EtOAc, $8: 1 \mathrm{v} / \mathrm{v})$ on fine silica gel (5-40 mesh) to provide sery363a (775 mg, $46 \%$ ) as a white solid. The second product was purified by additional column chromatography (gradient hexane: $\mathrm{CH}_{2} \mathrm{Cl}_{2}, 3: 1 \mathrm{v} / \mathrm{v}$ to hexane: $\mathrm{CH}_{2} \mathrm{Cl}_{2}$, $1: 4 \mathrm{v} / \mathrm{v})$ to provide sery363b (400 $\mathrm{mg}, 24 \%)$ as a white solid.

sery363a: TLC (hexane:EtOAc, 5:1 v/v): RF $=0.40$. m.p.: $94-95{ }^{\circ} \mathrm{C} .{ }^{1} \mathrm{H}$ NMR $(400 \mathrm{MHz}$, DMSO[D 6$]) \delta=8.02(\mathrm{t}, J=1.7 \mathrm{~Hz}, 1 \mathrm{H}), 7.84(\mathrm{~d}, J=7.9 \mathrm{~Hz}, 1 \mathrm{H}), 7.51(\mathrm{~d}, J=7.9 \mathrm{~Hz}, 1 \mathrm{H})$, $7.39(\mathrm{t}, J=7.9 \mathrm{~Hz}, 1 \mathrm{H}), 7.11(\mathrm{~d}, J=1.6 \mathrm{~Hz}, 1 \mathrm{H}), 7.07(\mathrm{~d}, J=8.0 \mathrm{~Hz}, 1 \mathrm{H}), 7.00(\mathrm{dd}, J=8.0$, $1.7 \mathrm{~Hz}, 1 \mathrm{H}), 6.90(\mathrm{~s}, 1 \mathrm{H}), 6.10(\mathrm{~s}, 2 \mathrm{H}), 4.84(\mathrm{dt}, J=47.0,4.7 \mathrm{~Hz}, 2 \mathrm{H}), 4.40(\mathrm{dt}, J=26.7,4.7$ $\mathrm{Hz}, 2 \mathrm{H}) .{ }^{13} \mathrm{C}$ NMR (100.6 MHz, DMSO[D $]$ ) $\delta=148.2,147.8,147.6,145.4,135.4,130.9$, $130.3,127.5,124.1,123.3,122.9,122.1,109.2,108.6,103.8,101.5,82.0$ (d, $J=167.4 \mathrm{~Hz})$, $49.5(\mathrm{~d}, J=20.4 \mathrm{~Hz})$. HRMS $(m / z)[\mathrm{M}+\mathrm{H}]^{+}$calcd for $\mathrm{M}=\mathrm{C}_{18} \mathrm{H}_{14}{ }^{79} \mathrm{BrFN}_{2} \mathrm{O}_{2}, 389.0301$; found, 389.0304 . 
sery363b: TLC (hexane:EtOAc, 5:1 v/v): RF $=0.36$. m.p.: 98-99 ${ }^{\circ} \mathrm{C} .{ }^{1} \mathrm{H}$ NMR $(400 \mathrm{MHz}$, DMSO[D 6$]) \delta=7.74(\mathrm{t}, J=1.6 \mathrm{~Hz}, 1 \mathrm{H}), 7.69(\mathrm{~d}, J=7.9 \mathrm{~Hz}, 1 \mathrm{H}), 7.54(\mathrm{~d}, J=7.9 \mathrm{~Hz}, 1 \mathrm{H})$, $7.49(\mathrm{t}, J=7.9 \mathrm{~Hz}, 1 \mathrm{H}), 7.4-7.34(\mathrm{~m}, 2 \mathrm{H}), 6.97(\mathrm{~d}, J=7.9 \mathrm{~Hz}, 1 \mathrm{H}), 6.88(\mathrm{~s}, 1 \mathrm{H}), 6.05(\mathrm{~s}, 2 \mathrm{H})$, $4.83(\mathrm{dt}, J=47.2,4.6 \mathrm{~Hz}, 2 \mathrm{H}), 4.40(\mathrm{dt}, J=26.7,4.6 \mathrm{~Hz}, 2 \mathrm{H}) .{ }^{13} \mathrm{C}$ NMR $(100.6 \mathrm{MHz}$, DMSO[D 6$]) \delta=149.8,147.65,146.9,143.8,132.2,131.5,131.3,130.9,127.8,127.2,122.0$, $118.8,108.5,105.5,103.8,101.0,82.2(\mathrm{~d}, J=169 \mathrm{~Hz}), 49.6(\mathrm{~d}, J=20 \mathrm{~Hz})$. HRMS $(\mathrm{m} / \mathrm{z})$ $[\mathrm{M}+\mathrm{H}]^{+}$calcd for $\mathrm{M}=\mathrm{C}_{18} \mathrm{H}_{14}{ }^{79} \mathrm{BrFN}_{2} \mathrm{O}_{2}, 389.0301$; found, 389.0303. A discrimination between two isomers was done based on NOESY spectrum.

\section{5-(1,3-Benzodioxol-5-yl)-3-(3-bromophenyl)-1-methyl-1H-pyrazole (sery392a) and 3- (1,3-benzodioxol-5-yl)-5-(3-bromophenyl)-1-methyl- $1 H$-pyrazole (sery392b)}

To a solution of anle138b $(2.0 \mathrm{~g}, 5.8 \mathrm{mmol})$ in anhydrous DMF $(20 \mathrm{ml}) 60 \%$ suspension of sodium hydride in mineral oil $(236 \mathrm{mg}, 5.9 \mathrm{mmol})$ was added in small portions, a resulting mixture was stirred for $1 \mathrm{~h}$ at room temperature under argon atmosphere and treated with iodomethane $(1.66 \mathrm{~g}, 11.7 \mathrm{mmol})$. After stirring for overnight at room temperature solvent was evaporated under reduced pressure and a remaining oil was diluted with water $(50 \mathrm{ml})$, extracted with EtOAc $(3 \times 50 \mathrm{ml})$, combined organic fractions were washed with brine $(20$ $\mathrm{ml})$, dried $\left(\mathrm{Na}_{2} \mathrm{SO}_{4}\right)$ and evaporated. A resulting mixture was purified by column chromatography (hexane:EtOAc, $10: 1 \mathrm{v} / \mathrm{v})$ on fine silica gel $(5-40 \mathrm{mesh})$ followed by recrystallisation from ethanol to provide two products: sery392a $(1.02 \mathrm{~g}, 49 \%)$ as a white solid and sery392b (625 mg, 30\%) as a white solid.

sery392a: TLC (hexane:EtOAc, 9:1 v/v): RF $=0.14$. m.p.: 96-97 ${ }^{\circ} \mathrm{C} .{ }^{1} \mathrm{H}$ NMR $(400 \mathrm{MHz}$, DMSO[D 6$]) \delta=7.99(\mathrm{t}, J=1.6 \mathrm{~Hz}, 1 \mathrm{H}), 7.82(\mathrm{~d}, J=7.8 \mathrm{~Hz}, 1 \mathrm{H}), 7.49(\mathrm{ddd}, J=7.9,1.6,0.8$ $\mathrm{Hz}, 1 \mathrm{H}), 7.37$ (t, $J=7.8 \mathrm{~Hz}, 1 \mathrm{H}), 7.17(\mathrm{~s}, 1 \mathrm{H}), 7.05$ (s, 2H), $6.90(\mathrm{~s}, 1 \mathrm{H}), 6.11(\mathrm{~s}, 2 \mathrm{H}), 3.87$ (s, $3 \mathrm{H}) .{ }^{13} \mathrm{C}$ NMR $\left(100.6 \mathrm{MHz}, \mathrm{DMSO}\left[\mathrm{D}_{6}\right]\right) \delta=147.6,147.6,147.2,144.4,135.6,130.9,130.0$, $127.4,123.9,123.5,122.5,122.1,108.9,108.6,103.3,101.4,37.7$. HRMS $(m / z)[\mathrm{M}+\mathrm{H}]^{+}$ calcd for $\mathrm{M}=\mathrm{C}_{17} \mathrm{H}_{13}{ }^{79} \mathrm{BrN}_{2} \mathrm{O}_{2}, 357.0239$; found, 357.0237.

sery392b: TLC (hexane:EtOAc, 9:1 v/v): RF =0.17. m.p.: 114-115 ${ }^{\circ} \mathrm{C} .{ }^{1} \mathrm{H}$ NMR $(400 \mathrm{MHz}$, DMSO[D 6$]) \delta=7.79(\mathrm{t}, J=1.6 \mathrm{~Hz}, 1 \mathrm{H}), 7.68-7.57(\mathrm{~m}, 2 \mathrm{H}), 7.48(\mathrm{t}, J=7.9 \mathrm{~Hz}, 1 \mathrm{H}), 7.39-$ $7.30(\mathrm{~m}, 2 \mathrm{H}), 6.95(\mathrm{~d}, J=8.0 \mathrm{~Hz}, 1 \mathrm{H}), 6.89(\mathrm{~s}, 1 \mathrm{H}), 6.04(\mathrm{~s}, 2 \mathrm{H}), 3.88(\mathrm{~s}, 3 \mathrm{H}) .{ }^{13} \mathrm{C} \mathrm{NMR}$ (100.6 MHz, DMSO[D 6$]) \delta=148.9,147.6,146.8,142.7,132.3,131.3,130.9,130.9,127.4$, $123.3,122.0,118.6,108.5,105.4,103.3,101.0,37.3$. HRMS $(m / z)[\mathrm{M}+\mathrm{H}]^{+}$calcd for $\mathrm{M}=$ $\mathrm{C}_{17} \mathrm{H}_{13}{ }^{79} \mathrm{BrN}_{2} \mathrm{O}_{2}, 357.0239$; found, 357.0241. A discrimination between two isomers was done based on NOESY spectrum.

\section{3-(1,3-Benzodioxol-5-yl)-4-bromo-5-phenyl-1 $H$-pyrazole (anle134)}

The title compound was prepared by the published protocol ${ }^{408}$ with minor modifications. A mixture of anle126 (264 mg, $1.00 \mathrm{mmol})$ and $N$-bromosuccinimide (180 mg, $1.01 \mathrm{mmol})$ in $\mathrm{CHCl}_{3}(6 \mathrm{ml})$ was stirred $2 \mathrm{~h}$ at room temperature. The solvent was evaporated under reduced pressure, the residue was co-evaporated two times with $\mathrm{MeOH}(20 \mathrm{ml})$. The resulting precipitate was stirred $2 \mathrm{~h}$ with water $(40 \mathrm{ml})$, the product was collected by filtration and dried to provide anle134 $(286 \mathrm{mg}, 83 \%)$ as a white solid. m.p.: $183-185{ }^{\circ} \mathrm{C} .{ }^{1} \mathrm{H}$ NMR $(400 \mathrm{MHz}$, DMSO[D $]+1-2 \%$ conc. DCl) $\delta=7.77(\mathrm{~d}, J=7.2 \mathrm{~Hz}, 2 \mathrm{H}), 7.50(\mathrm{t}, J=7.2 \mathrm{~Hz}, 2 \mathrm{H}), 7.43(\mathrm{t}, J$ $=7.2 \mathrm{~Hz}, 1 \mathrm{H}), 7.33-7.23(\mathrm{~m}, 2 \mathrm{H}), 7.05(\mathrm{~d}, J=8.6 \mathrm{~Hz}, 1 \mathrm{H}), 6.08(\mathrm{~s}, 2 \mathrm{H}) .{ }^{13} \mathrm{C}$ NMR $(100.6$ $\mathrm{MHz}, \mathrm{DMSO}\left[\mathrm{D}_{6}\right]+1-2 \%$ conc. DCl) $\delta=147.8,147.7,145.1$ (2C), 130.7, 128.9, 128.8, $127.8,124.3,121.9,108.8,108.1,101.7,89.6$. HRMS $(\mathrm{m} / \mathrm{z})[\mathrm{M}+\mathrm{H}]^{+}$calcd for $\mathrm{M}=$ $\mathrm{C}_{16} \mathrm{H}_{11}{ }^{79} \mathrm{BrN}_{2} \mathrm{O}_{2}, 343.0082$; found, 343.0084. 


\section{3-(1,3-Benzodioxol-5-yl)-4-bromo-5-(3-bromophenyl)-1H-pyrazole (sery330)}

The title compound was prepared analogously to the procedure for anle134 starting from anle138b. White solid. Yield 93\%. m.p.: 204-205 ${ }^{\circ} \mathrm{C} .{ }^{1} \mathrm{H}$ NMR $\left(400 \mathrm{MHz}, \mathrm{DMSO}\left[\mathrm{D}_{6}\right]+1-\right.$ $2 \%$ conc. DCl) $\delta=13.75(\mathrm{bs}, 1 \mathrm{H}), 7.97(\mathrm{~s}, 1 \mathrm{H}), 7.83(\mathrm{~d}, J=6.8 \mathrm{~Hz}, 1 \mathrm{H}), 7.63(\mathrm{~d}, J=7.2 \mathrm{~Hz}$, $1 \mathrm{H}), 7.46(\mathrm{t}, J=7.6 \mathrm{~Hz}, 1 \mathrm{H}), 7.27(\mathrm{~s}, 2 \mathrm{H}), 7.06(\mathrm{~d}, J=8.0 \mathrm{~Hz}, 1 \mathrm{H}), 6.09(\mathrm{~s}, 2 \mathrm{H}) .{ }^{13} \mathrm{C} \mathrm{NMR}$ (100.6 MHz, DMSO[D 6$]+1-2 \%$ conc. DCl) $\delta=147.8,147.6,144.2$ (2C), 133.3, 131.2, $130.9,129.9,126.5,123.3,121.8,121.8,108.6,107.9,101.5,89.6$. HRMS $(\mathrm{m} / \mathrm{z})[\mathrm{M}+\mathrm{H}]^{+}$ calcd for $\mathrm{M}=\mathrm{C}_{16} \mathrm{H}_{10}{ }^{79} \mathrm{Br}^{81} \mathrm{BrN}_{2} \mathrm{O}_{2}, 422.9167$; found, 422.9168 .

\subsubsection{Synthesis of isoxazoles}

\section{(E)-1-(1,3-Benzodioxol-5-yl)-3-phenyl-2-propene-1-one (sery89)}

The title compound has been reported previously ${ }^{543}$ (CAS 204450-46-4). It was prepared according to the GEP 5 using 1-(1,3-benzodioxol-5-yl)ethanone, benzaldehyde and purified by recrystallisation from methanol to provide sery89 as a yellow solid. Yield 87\%. m.p.: 99$100{ }^{\circ} \mathrm{C}\left(\mathrm{lit}^{543} 97{ }^{\circ} \mathrm{C}\right) .{ }^{1} \mathrm{H}$ NMR $\left(400 \mathrm{MHz}, \mathrm{DMSO}\left[\mathrm{D}_{6}\right]\right) \delta=7.97-7.83(\mathrm{~m}, 4 \mathrm{H}), 7.70(\mathrm{~d}, J=$ $15.6 \mathrm{~Hz}, 1 \mathrm{H}), 7.66(\mathrm{~d}, J=1.4 \mathrm{~Hz}, 1 \mathrm{H}), 7.50-7.40(\mathrm{~m}, 3 \mathrm{H}), 7.08$ (d, $J=8.2 \mathrm{~Hz}, 1 \mathrm{H}), 6.16$ (s, $2 \mathrm{H})$.

\section{1-(1,3-Benzodioxol-5-yl)-2,3-dibromo-3-phenylpropane-1-one (sery93a)}

The title compound has been reported previously ${ }^{544}$ (CAS 25856-08-0). It was prepared according to the GEP 6 starting from sery89 to provide sery93a as a red solid. Yield $60 \%$. m.p.: $159-160{ }^{\circ} \mathrm{C}\left(\mathrm{lit}^{544} 160-162{ }^{\circ} \mathrm{C}\right) .{ }^{1} \mathrm{H}$ NMR (400 MHz, DMSO[D 6$) \delta=7.99(\mathrm{dd}, J=8.3$, $1.4 \mathrm{~Hz}, 1 \mathrm{H}), 7.85-7.75(\mathrm{~m}, 3 \mathrm{H}), 7.48-7.33(\mathrm{~m}, 3 \mathrm{H}), 7.16(\mathrm{~d}, J=8.3 \mathrm{~Hz}, 1 \mathrm{H}), 6.62(\mathrm{~d}, J=11.3$ $\mathrm{Hz}, 1 \mathrm{H}), 6.20(\mathrm{~s}, 2 \mathrm{H}), 5.73(\mathrm{~d}, J=11.3 \mathrm{~Hz}, 1 \mathrm{H})$.

\section{General Experimental Procedure for the synthesis of 3,5-diarylisoxazoles (GEP 9) ${ }^{397}$}

\section{3-(1,3-Benzodioxol-5-yl)-5-phenylisoxazole (sery93b)}

The title compound has been reported previously ${ }^{544}$ (CAS 25856-13-7). A mixture of sery93a (150 mg, $0.36 \mathrm{mmol})$, hydroxylamine hydrochloride $(110 \mathrm{mg}, 1.6 \mathrm{mmol})$ and water $(500 \mathrm{mg})$ in ethanol $(2 \mathrm{ml})$ was heated under reflux $2 \mathrm{~h}$ with stirring. The reaction mixture was treated with water $(0.5 \mathrm{ml})$ and cooled in ice bath for $2 \mathrm{~h}$. A resulting precipitate was collected by filtration and dried to provide sery93b as a white solid. Yield $53 \%$. m.p.: $134-135{ }^{\circ} \mathrm{C}\left(\mathrm{lit}^{544}\right.$ $\left.130{ }^{\circ} \mathrm{C}\right) .{ }^{1} \mathrm{H}$ NMR $\left(400 \mathrm{MHz}, \mathrm{DMSO}_{\left.\left[\mathrm{D}_{6}\right]\right)} \delta=7.89(\mathrm{~d}, J=7.2 \mathrm{~Hz}, 2 \mathrm{H}), 7.63-7.40(\mathrm{~m}, 6 \mathrm{H})\right.$, $7.08(\mathrm{~d}, J=8.3 \mathrm{~Hz}, 1 \mathrm{H}), 6.12(\mathrm{~s}, 2 \mathrm{H}) .{ }^{13} \mathrm{C} \mathrm{NMR}\left(100.6 \mathrm{MHz}, \mathrm{DMSO}\left[\mathrm{D}_{6}\right]\right) \delta=169.5,162.2$, $148.9,148.0,130.4,129.3,126.9,125.5,122.4,121.1,108.9,106.4,101.6,98.5$.

\section{(E)-3-(3,4-Dimethoxyphenyl)-1-(4-methoxyphenyl)-2-propene-1-one (sery96)}

The title compound has been reported previously ${ }^{545}$ (CAS 144309-49-9). It was prepared according to the GEP 5 using 1-(4-methoxyphenyl)ethanone, 3,4-dimethoxybenzaldehyde and purified by recrystallisation from methanol to provide sery96 as a yellow solid. Yield $77 \%$. m.p.: $92-93{ }^{\circ} \mathrm{C}\left(\mathrm{lit}^{545} 93{ }^{\circ} \mathrm{C}\right) .{ }^{1} \mathrm{H}$ NMR $\left(400 \mathrm{MHz}, \mathrm{DMSO}\left[\mathrm{D}_{6}\right]\right) \delta=8.16(\mathrm{~d}, J=8.9 \mathrm{~Hz}, 2 \mathrm{H})$, $7.82(\mathrm{~d}, J=15.5 \mathrm{~Hz}, 1 \mathrm{H}), 7.67(\mathrm{~d}, J=15.5 \mathrm{~Hz}, 1 \mathrm{H}), 7.57(\mathrm{~d}, J=2.0 \mathrm{~Hz}, 1 \mathrm{H}), 7.37(\mathrm{dd}, J=$ 8.2, $2.0 \mathrm{~Hz}, 1 \mathrm{H}), 7.08(\mathrm{~d}, J=8.9 \mathrm{~Hz}, 2 \mathrm{H}), 7.01(\mathrm{~d}, J=8.2 \mathrm{~Hz}, 1 \mathrm{H}), 3.86(\mathrm{~s}, 6 \mathrm{H}), 3.81(\mathrm{~s}, 3 \mathrm{H})$.

\section{2,3-Dibromo-3-(3,4-dimethoxyphenyl)-1-(4-methoxyphenyl)propane-1-one (sery102)}

The title compound was prepared according to the GEP 6 starting from sery96 to provide sery102 as a white solid. Yield 79\%. m.p.: $136{ }^{\circ} \mathrm{C} .{ }^{1} \mathrm{H}$ NMR $\left(400 \mathrm{MHz}, \mathrm{CDCl}_{3}\right) \delta=8.09(\mathrm{~d}, J$ 
$=8.9 \mathrm{~Hz}, 2 \mathrm{H}), 7.11(\mathrm{dd}, J=8.3,2.0 \mathrm{~Hz}, 1 \mathrm{H}), 7.05-6.98(\mathrm{~m}, 3 \mathrm{H}), 6.89(\mathrm{~d}, J=8.3 \mathrm{~Hz}, 1 \mathrm{H})$, $5.76(\mathrm{~d}, J=11.3 \mathrm{~Hz}, 1 \mathrm{H}), 5.65(\mathrm{~d}, J=11.3 \mathrm{~Hz}, 1 \mathrm{H}), 3.95(\mathrm{~s}, 3 \mathrm{H}), 3.91$ (s, 3H), $3.90(\mathrm{~s} 3 \mathrm{H})$. ${ }^{13} \mathrm{C}$ NMR (100.6 MHz, DMSO[D 6 ) $\delta=189.8,164.6,149.9,149.2,131.5,130.9,127.4$, 121.3, 114.4, 111.4, 111.1, 56.2, 56.1, 55.8, 51.0, 47.2. HRMS $(m / z)[\mathrm{M}-2 \mathrm{HBr}+\mathrm{H}]^{+}$calcd for $\mathrm{M}=\mathrm{C}_{18} \mathrm{H}_{18}{ }^{79} \mathrm{Br}_{2} \mathrm{O}_{4}, 297.1127$; found, 297.1122.

\section{5-(3,4-Dimethoxyphenyl)-3-(4-methoxyphenyl)isoxazole (sery103)}

The title compound was prepared according to the GEP 9 starting from sery102 to provide sery103 as a white solid. Yield 43\%. m.p.: 115-118 ${ }^{\circ} \mathrm{C} .{ }^{1} \mathrm{H}$ NMR (400 MHz, DMSO[D 6$\left.]\right)$ $7.84(\mathrm{~d}, J=8.8 \mathrm{~Hz}, 2 \mathrm{H}), 7.50-7.42(\mathrm{~m}, 3 \mathrm{H}), 7.13(\mathrm{~d}, J=8.4 \mathrm{~Hz}, 1 \mathrm{H}), 7.10(\mathrm{~d}, J=8.8 \mathrm{~Hz}, 2 \mathrm{H})$, $3.87(\mathrm{~s}, 3 \mathrm{H}), 3.83(\mathrm{~s}, 6 \mathrm{H}) .{ }^{13} \mathrm{C} \mathrm{NMR}\left(100.6 \mathrm{MHz}, \mathrm{DMSO}\left[\mathrm{D}_{6}\right]\right) \delta=169.6,162.1,160.7,150.6$, $149.1,128.0,121.1,119.7,118.6,114.5,112.0,108.9,97.1,55.7,55.6,55.3$. HRMS $(\mathrm{m} / \mathrm{z})$ $[\mathrm{M}+\mathrm{Na}]^{+}$calcd for $\mathrm{M}=\mathrm{C}_{18} \mathrm{H}_{17} \mathrm{NO}_{4}, 334.1055$; found, 334.1053 .

\section{5-(3,4-Dihydroxyphenyl)-3-(4-hydroxyphenyl)isoxazole (sery118)}

The title compound was prepared according to the GEP 3 starting from sery103 to provide sery118 as a white solid. Yield 68\%. m.p.: $>300{ }^{\circ} \mathrm{C} .{ }^{1} \mathrm{H}$ NMR $\left(400 \mathrm{MHz}, \mathrm{DMSO}\left[\mathrm{D}_{6}\right]\right) \delta=$ $9.89(\mathrm{~s}, 1 \mathrm{H}), 9.58(\mathrm{~s}, 1 \mathrm{H}), 9.35(\mathrm{~s}, 1 \mathrm{H}), 7.72(\mathrm{~d}, J=8.6 \mathrm{~Hz}, 2 \mathrm{H}), 7.25(\mathrm{~d}, J=2.0 \mathrm{~Hz}, 1 \mathrm{H}), 7.20$ $(\mathrm{dd}, J=8.2,2.0 \mathrm{~Hz}, 1 \mathrm{H}), 7.15(\mathrm{~s}, 1 \mathrm{H}), 6.92-6.84(\mathrm{~m}, 3 \mathrm{H}) .{ }^{13} \mathrm{C}$ NMR $(100.6 \mathrm{MHz}$, DMSO[D 6$]) \delta=169.8,162.2,159.1,147.8,145.7,128.1,119.6,118.5,117.6,116.1,115.8$, 112.7, 96.0. HRMS $(\mathrm{m} / \mathrm{z})[\mathrm{M}+\mathrm{H}]^{+}$calcd for $\mathrm{M}=\mathrm{C}_{15} \mathrm{H}_{11} \mathrm{NO}_{4}, 270.0766$; found, 270.0761.

\section{(E)-1-(3,4-Dimethoxyphenyl)-3-(3-fluorophenyl)-2-propene-1-one (sery97)}

The title compound prepared according to the GEP 5 using 1-(3,4dimethoxyphenyl)ethanone, 3-fluorobenzaldehyde and purified by recrystallisation from methanol to provide sery97 as a white solid. Yield 58\%. m.p.: 105-107 ${ }^{\circ} \mathrm{C}$. ${ }^{1} \mathrm{H}$ NMR (400 $\left.\mathrm{MHz}, \mathrm{DMSO}\left[\mathrm{D}_{6}\right]\right) \delta=8.01(\mathrm{~d}, J=15.6 \mathrm{~Hz}, 1 \mathrm{H}), 7.94(\mathrm{dd}, J=8.4,1.8 \mathrm{~Hz}, 1 \mathrm{H}), 7.85(\mathrm{~d}, J=$ $10.3 \mathrm{~Hz}, 1 \mathrm{H}), 7.74-7.65(\mathrm{~m}, 2 \mathrm{H}), 7.61(\mathrm{~d}, J=1.8 \mathrm{~Hz}, 1 \mathrm{H}), 7.53-7.45(\mathrm{~m}, 1 \mathrm{H}), 7.27(\mathrm{td}, J=$ 8.5, $2.1 \mathrm{~Hz}, 1 \mathrm{H}), 7.11(\mathrm{~d}, J=8.5 \mathrm{~Hz}, 1 \mathrm{H}), 3.88(\mathrm{~s}, 3 \mathrm{H}), 3.86(\mathrm{~s}, 3 \mathrm{H}) .{ }^{13} \mathrm{C} \mathrm{NMR}(100.6 \mathrm{MHz}$, DMSO[D 6 ] $\delta=187.2,162.5(\mathrm{~d}, J=243.3 \mathrm{~Hz}), 153.4,148.8,141.6,137.4(\mathrm{~d}, J=8.0 \mathrm{~Hz})$, $130.8(\mathrm{~d}, J=8.0 \mathrm{~Hz}), 130.3,125.5,123.6,123.4,117.0(\mathrm{~d}, J=21.4 \mathrm{~Hz}), 114.5(\mathrm{~d}, J=21.8$ $\mathrm{Hz}), 110.9$, 110.8, 55.8, 55.6. HRMS $(\mathrm{m} / \mathrm{z})[\mathrm{M}+\mathrm{H}]^{+}$calcd for $\mathrm{M}=\mathrm{C}_{17} \mathrm{H}_{15} \mathrm{FO}_{3}, 287.1083$; found, 287.1083 .

\section{2,3-Dibromo-1-(3,4-dimethoxyphenyl)-3-(3-fluorophenyl)propane-1-one (sery107)}

The title compound was prepared according to the GEP 6 starting from sery97 to provide sery107 as a white solid. Yield 70\%. m.p.: $174{ }^{\circ} \mathrm{C} .{ }^{1} \mathrm{H}$ NMR (400 MHz, DMSO[D $\left.]\right) \delta=8.09$ (dd, $J=8.5,2.0 \mathrm{~Hz}, 1 \mathrm{H}) .7 .83(\mathrm{~d}, J=10.2 \mathrm{~Hz}, 1 \mathrm{H}), 7.70-7.60(\mathrm{~m}, 2 \mathrm{H}), 7.53-7.43(\mathrm{~m}, 1 \mathrm{H})$, 7.22 (td, $J=8.5,2.3 \mathrm{~Hz}, 1 \mathrm{H}), 7.18(\mathrm{~d}, J=8.5 \mathrm{~Hz}, 1 \mathrm{H}), 6.67(\mathrm{~d}, J=11.3 \mathrm{~Hz}, 1 \mathrm{H}), 5.81(\mathrm{~d}, J=$ $11.3 \mathrm{~Hz}, 1 \mathrm{H}), 3.91(\mathrm{~s}, 3 \mathrm{H}), 3.88(\mathrm{~s}, 3 \mathrm{H}) .{ }^{13} \mathrm{C} \mathrm{NMR}\left(100.6 \mathrm{MHz}, \mathrm{DMSO}\left[\mathrm{D}_{6}\right]\right) \delta=189.7,162.2$ $(\mathrm{d}, J=245.5 \mathrm{~Hz}), 154.4,149.1,141.4(\mathrm{~d}, J=7.9 \mathrm{~Hz}), 130.6(\mathrm{~d}, J=8.5 \mathrm{~Hz}), 126.4,125.2$, $124.5,116.0(\mathrm{~d}, J=21.0 \mathrm{~Hz}), 115.6(\mathrm{~d}, J=23.0 \mathrm{~Hz}), 111.1,111.0,56.0,55.7,49.7,45.8$. HRMS $(m / z)[\mathrm{M}-2 \mathrm{HBr}+\mathrm{H}]^{+}$calcd for $\mathrm{M}=\mathrm{C}_{17} \mathrm{H}_{15}{ }^{79} \mathrm{Br}^{81} \mathrm{Br} \mathrm{FO}_{3}, 285.0927$; found, 285.0924.

\section{3-(3,4-Dimethoxyphenyl)-5-(3-fluorophenyl)isoxazole (sery109)}

The title compound was prepared according to the GEP 9 starting from sery107 to provide sery109 as a white solid. Yield 60\%. m.p.: $116{ }^{\circ} \mathrm{C} .{ }^{1} \mathrm{H}$ NMR $\left(400 \mathrm{MHz}, \mathrm{DMSO}\left[\mathrm{D}_{6}\right]\right) \delta=$ 7.80-7.71 (m, 2H), $7.70(\mathrm{~s}, 1 \mathrm{H}), 7.66-7.58(\mathrm{~m}, 1 \mathrm{H}), 7.50-7.42(\mathrm{~m}, 2 \mathrm{H}), 7.37(\mathrm{td}, J=8.6,2.1$ $\mathrm{Hz}, 1 \mathrm{H}), 7.11(\mathrm{~d}, J=8.1 \mathrm{~Hz}, 1 \mathrm{H}), 3.86(\mathrm{~s}, 3 \mathrm{H}), 3.83(\mathrm{~s}, 3 \mathrm{H}) .{ }^{13} \mathrm{C}$ NMR $(100.6 \mathrm{MHz}$, DMSO[D 6$]) \delta=168.0,162.5,162.4(\mathrm{~d}, J=244.5 \mathrm{~Hz}), 150.6,149.1,131.6(\mathrm{~d}, J=8.5 \mathrm{~Hz})$, 
$129.0(\mathrm{~d}, J=8.7 \mathrm{~Hz}), 121.6,120.8,119.6,117.3(\mathrm{~d}, J=20.9 \mathrm{~Hz}), 112.4(\mathrm{~d}, J=23.7 \mathrm{~Hz})$, 112.0, 109.6, 99.6, 55.6 (2C). HRMS $(\mathrm{m} / \mathrm{z})[\mathrm{M}+\mathrm{Na}]^{+}$calcd for $\mathrm{M}=\mathrm{C}_{17} \mathrm{H}_{14} \mathrm{FNO}_{3}, 300.1036$; found, 300.1034 .

\section{3-(3,4-Dihydroxyphenyl)-5-(3-fluorophenyl)isoxazole (sery117)}

The title compound was prepared according to the GEP 3 starting from sery109 to provide sery117 as a white solid. Yield 65\%. m.p.: 190-200 ${ }^{\circ} \mathrm{C}(\mathrm{dec}) .{ }^{1} \mathrm{H}$ NMR $(400 \mathrm{MHz}$, DMSO[D 6$]) \delta=9.46(\mathrm{~s}, 1 \mathrm{H}), 9.29(\mathrm{~s}, 1 \mathrm{H}), 7.78-7.70(\mathrm{~m}, 2 \mathrm{H}), 7.65-7.56(\mathrm{~m}, 1 \mathrm{H}), 7.52(\mathrm{~s}$, $1 \mathrm{H}), 7.31(\mathrm{td}, J=8.6,2.5 \mathrm{~Hz}, 1 \mathrm{H}), 7.32(\mathrm{~d}, J=2.0 \mathrm{~Hz}, 1 \mathrm{H}), 7.18(\mathrm{dd}, J=8.2,2.0 \mathrm{~Hz}, 1 \mathrm{H})$, $6.87(\mathrm{~d}, J=8.2 \mathrm{~Hz}, 1 \mathrm{H}) .{ }^{13} \mathrm{C}$ NMR $\left(100.6 \mathrm{MHz}, \mathrm{DMSO}\left[\mathrm{D}_{6}\right]\right) \delta=167.8,162.7,162.4(\mathrm{~d}, J=$ $244.4 \mathrm{~Hz}), 147.7,145.8,131.5(\mathrm{~d}, J=8.5 \mathrm{~Hz}), 129.0(\mathrm{~d}, J=8.6 \mathrm{~Hz}), 121.7,119.4,118.6$, $117.2(\mathrm{~d}, J=20.5 \mathrm{~Hz}), 115.9,113.5,112.4(\mathrm{~d}, J=21.2 \mathrm{~Hz}), 99.4$. HRMS $(m / z)[\mathrm{M}+\mathrm{H}]^{+}$calcd for $\mathrm{M}=\mathrm{C}_{15} \mathrm{H}_{10} \mathrm{FNO}_{3}, 272.0723$; found, 272.0719.

\section{3,5-Bis(3,4-Dimethoxyphenyl)isoxazole (sery137)}

The title compound has been reported previously ${ }^{546}$ (CAS 52789-39-6). A mixture of sery149 (772 $\mathrm{mg}, 2.25 \mathrm{mmol})$, hydroxylamine hydrochloride $(165 \mathrm{mg}, 2.36 \mathrm{mmol})$ and pyridine $(2 \mathrm{ml})$ in ethanol $(8 \mathrm{ml})$ was heated under reflux $2 \mathrm{~h}$ with stirring. The reaction mixture was evaporated under reduced pressure, treated with water $(20 \mathrm{ml})$. A resulting precipitate was collected by filtration and purified by recrystallisation from ethanol to provide sery137 as a white solid. Yield 89\%. m.p.: $134{ }^{\circ} \mathrm{C} .{ }^{1} \mathrm{H}$ NMR $\left(400 \mathrm{MHz}, \mathrm{DMSO}\left[\mathrm{D}_{6}\right]\right) \delta=7.50-7.41$ (m, 5H), 7.15-7.07 (m, 2H), $3.88(\mathrm{~s}, 3 \mathrm{H}), 3.86(\mathrm{~s}, 3 \mathrm{H}), 3.85-3.80(\mathrm{~m}, 6 \mathrm{H}) .{ }^{13} \mathrm{C}$ NMR $(100.6 \mathrm{MHz}$, DMSO[D $\left.\left.\mathrm{D}_{6}\right]\right) \delta=169.6,162.3,150.6,150.4,149.1,149.0,121.2,119.7,119.5,118.6,112.0$, $111.9,109.6,108.9,97.3,55.6(4 \mathrm{C})$.

\section{3,5-Bis(3,4-Dihydroxyphenyl)isoxazole hydrobromide (sery158b)}

The title compound was prepared according to the GEP 3 starting from sery137 to provide sery158b as a grey solid. Yield 93\%. m.p.: $277{ }^{\circ} \mathrm{C}$ (dec). ${ }^{1} \mathrm{H}$ NMR (400 MHz, DMSO[D 6$\left.]\right) \delta$ $=7.29(\mathrm{~d}, J=2.1 \mathrm{~Hz}, 1 \mathrm{H}), 7.24(\mathrm{~d}, J=2.1 \mathrm{~Hz}, 1 \mathrm{H}), 7.22-7.14(\mathrm{~m}, 2 \mathrm{H}), 7.09(\mathrm{~s}, 1 \mathrm{H}), 6.87(\mathrm{~d}, J$ $=8.2 \mathrm{~Hz}, 1 \mathrm{H}), 6.84(\mathrm{~d}, J=8.2 \mathrm{~Hz}, 1 \mathrm{H}) .{ }^{13} \mathrm{C} \mathrm{NMR}\left(100.6 \mathrm{MHz}, \mathrm{DMSO}\left[\mathrm{D}_{6}\right]\right) \delta=169.7,162.4$, $147.7,147.4,145.7,145.6,120.0,118.6,118.5,117.6,116.1,115.8,113.5,112.8,96.1$. HRMS $(m / z)[\mathrm{M}+\mathrm{H}]^{+}$calcd for $\mathrm{M}=\mathrm{C}_{15} \mathrm{H}_{11} \mathrm{NO}_{5}, 286.0715$; found, 286.0716.

\section{(E)-1-(1,3-Benzodioxol-5-yl)-3-(3-bromophenyl)-2-propene-1-one (sery298a)}

The title compound was prepared according to the GEP 5 using 1-(1,3-benzodioxol-5yl)ethanone, 3-bromobenzaldehyde and purified by washing on filter with methanol to provide sery298a as a white solid. Yield 91\%. m.p.: 121-122 ${ }^{\circ} \mathrm{C} .{ }^{1} \mathrm{H}$ NMR $(400 \mathrm{MHz}$, $\left.\operatorname{DMSO}\left[\mathrm{D}_{6}\right]\right) \delta=8.21(\mathrm{t}, J=1.6 \mathrm{~Hz}, 1 \mathrm{H}), 8.00(\mathrm{~d}, J=15.6 \mathrm{~Hz}, 1 \mathrm{H}), 7.90(\mathrm{dd}, J=8.2,1.8 \mathrm{~Hz}$, $1 \mathrm{H}), 7.84(\mathrm{~d}, J=7.8 \mathrm{~Hz}, 1 \mathrm{H}), 7.70-7.60(\mathrm{~m}, 3 \mathrm{H}), 7.40$ (d, $J=7.8 \mathrm{~Hz}, 1 \mathrm{H}), 7.09$ (d, $J=8.2 \mathrm{~Hz}$, $1 \mathrm{H}), 6.17(\mathrm{~s}, 2 \mathrm{H}) .{ }^{13} \mathrm{C}$ NMR $\left(100.6 \mathrm{MHz}, \mathrm{DMSO}\left[\mathrm{D}_{6}\right]\right) \delta=186.8,151.7,148.0,141.6,137.3$, $132.9,132.0,130.9,130.7,128.3,125.3,123.4,122.4,108.2,108.0,102.1$. HRMS $(\mathrm{m} / \mathrm{z})$ $[\mathrm{M}+\mathrm{H}]^{+}$calcd for $\mathrm{M}=\mathrm{C}_{16} \mathrm{H}_{11}{ }^{79} \mathrm{BrO}_{3}, 330.9970$; found, 330.9972 .

\section{1-(1,3-Benzodioxol-5-yl)-3-(3-bromophenyl)-2,3-dibromopropane-1-one (sery338a)}

The title compound was prepared according to the GEP 6 starting from sery298a to provide sery338a as a red solid. Yield $88 \%$. m.p.: $122{ }^{\circ} \mathrm{C} .{ }^{1} \mathrm{H}$ NMR $\left(400 \mathrm{MHz}, \mathrm{CDCl}_{3}\right) \delta=7.71(\mathrm{dd}, J$ $=8.2,1.8 \mathrm{~Hz}, 1 \mathrm{H}), 7.68(\mathrm{~d}, J=1.8 \mathrm{~Hz}, 1 \mathrm{H}), 7.56(\mathrm{~d}, J=1.8 \mathrm{~Hz}, 1 \mathrm{H}), 7.51$ (ddd, $J=7.9,1.8$, $1.1 \mathrm{~Hz}, 1 \mathrm{H}), 7.44(\mathrm{dt}, J=7.9,1.2 \mathrm{~Hz}, 1 \mathrm{H}), 7.31(\mathrm{t}, J=7.9 \mathrm{~Hz}, 1 \mathrm{H}), 6.94(\mathrm{~d}, J=8.2 \mathrm{~Hz}, 1 \mathrm{H})$, $6.11(\mathrm{~s}, 2 \mathrm{H}), 5.68(\mathrm{~d}, J=11.3 \mathrm{~Hz}, 1 \mathrm{H}), 5.56(\mathrm{~d}, J=11.3 \mathrm{~Hz}, 1 \mathrm{H}) .{ }^{13} \mathrm{C}$ NMR $(100.6 \mathrm{MHz}$, $\left.\mathrm{CDCl}_{3}\right) \delta=189.1,153.1,148.8,140.7,132.5,131.5,130.5,129.0,127.3,125.6,122.9,108.8$, 
108.4, 102.4, 48.8, 46.6. HRMS $(m / z)[\mathrm{M}+\mathrm{H}]^{+}$calcd for $\mathrm{M}=\mathrm{C}_{16} \mathrm{H}_{11}{ }^{79} \mathrm{Br}_{2}{ }^{81} \mathrm{BrO}_{3}, 490.8316$; found, 490.8321 .

\section{3-(1,3-Benzodioxol-5-yl)-5-(3-bromophenyl)isoxazole (sery338b)}

The title compound was prepared according to the GEP 9 starting from sery338a to provide sery338b as a white solid. Yield $62 \%$. m.p.: $158-160{ }^{\circ} \mathrm{C} .{ }^{1} \mathrm{H}$ NMR (400 MHz, DMSO[D 6$\left.]\right) \delta$ $=8.08(\mathrm{t}, J=1.7 \mathrm{~Hz}, 1 \mathrm{H}), 7.89(\mathrm{dt}, J=7.9,1.1 \mathrm{~Hz}, 1 \mathrm{H}), 7.73(\mathrm{ddd}, J=7.9,1.7,1.1 \mathrm{~Hz}, 1 \mathrm{H})$, $7.67(\mathrm{~s}, 1 \mathrm{H}), 7.53(\mathrm{t}, J=7.9 \mathrm{~Hz}, 1 \mathrm{H}), 7.46-7.39(\mathrm{~m}, 2 \mathrm{H}), 7.09(\mathrm{~d}, J=8.2 \mathrm{~Hz}, 1 \mathrm{H}), 6.12(\mathrm{~s}$, 2H). ${ }^{13} \mathrm{C}$ NMR (100.6 MHz, DMSO[D 6$\left.]\right) \delta=167.8,162.3,149.0,148.0,133.1,131.5,128.9$, 128.0, 124.4, 122.5, 122.1, 121.1, 108.9, 106.4, 101.6, 99.7. HRMS $(\mathrm{m} / \mathrm{z})[\mathrm{M}+\mathrm{H}]^{+}$calcd for $\mathrm{M}=\mathrm{C}_{16} \mathrm{H}_{10}{ }^{79} \mathrm{BrNO}_{3}, 343.9922$; found, 343.9926.

\subsubsection{Synthesis of 3,5-diaryl-1,2,4-oxadiazoles}

\section{General Experimental Procedure for the synthesis of 3,5-diaryl-1,2,4-oxadiazoles (GEP} $10)^{547}$

\section{3-(1,3-Benzodioxol-5-yl)-5-phenyl-1,2,4-oxadiazole (anle128b)}

The title compound was prepared according to the published protocol ${ }^{547}$. To a solution of 1,3benzodioxole-5-carboximidamide $(360 \mathrm{mg}, 2.0 \mathrm{mmol})$ and methyl benzoate $(300 \mathrm{mg}, 2.2$ $\mathrm{mmol})$ in ethanol $(5 \mathrm{ml})$ potassium tert-butoxide $(230 \mathrm{mg}, 3.79 \mathrm{mmol})$ was added and the reaction mixture was heated under reflux for $12 \mathrm{~h}$. After cooling a precipitate was collected by filtration, washed with hot ethanol followed by cold water and dried to provide anle128b (266 $\mathrm{mg})$ as a white powder. Yield 50\%. m.p.: $144-145{ }^{\circ} \mathrm{C} .{ }^{1} \mathrm{H}$ NMR $\left(400 \mathrm{MHz}, \mathrm{DMSO}\left[\mathrm{D}_{6}\right]\right) \delta=$ $8.16(\mathrm{~d}, J=7.4 \mathrm{~Hz}, 2 \mathrm{H}), 7.73(\mathrm{t}, J=7.4 \mathrm{~Hz}, 1 \mathrm{H}), 7.69-7.62(\mathrm{~m}, 3 \mathrm{H}), 7.52(\mathrm{~d}, J=1.5 \mathrm{~Hz}, 1 \mathrm{H})$, $7.11(\mathrm{~d}, J=8.1 \mathrm{~Hz}, 1 \mathrm{H}), 6.14(\mathrm{~s}, 2 \mathrm{H}) .{ }^{13} \mathrm{C} \mathrm{NMR}\left(100.6 \mathrm{MHz}, \mathrm{DMSO}\left[\mathrm{D}_{6}\right]\right) \delta=175.3,168.1$, $150.3,148.2,133.5,129.7,1278.1,123.5,122.4,120.0,109.1,106.8,102.1$. HRMS $(\mathrm{m} / \mathrm{z})$ $[\mathrm{M}+\mathrm{H}]^{+}$calcd for $\mathrm{M}=\mathrm{C}_{15} \mathrm{H}_{10} \mathrm{~N}_{2} \mathrm{O}_{3}, 267.0770$; found, 267.0765.

\section{3-(3,4-Dihydroxyphenyl)-5-phenyl-1,2,4-oxadiazole (sery95)}

The title compound was prepared according to the GEP 3 starting from anle128b and purified by recrystallisation from chloroform/methanol to provide sery95 as a white solid. Yield $54 \%$. m.p.: $194-196{ }^{\circ} \mathrm{C} .{ }^{1} \mathrm{H}$ NMR $\left.\left(400 \mathrm{MHz}, \mathrm{DMSO}_{6} \mathrm{D}_{6}\right]\right) \delta=8.15(\mathrm{~d}, J=7.9 \mathrm{~Hz}, 2 \mathrm{H}), 7.75-7.61$ $(\mathrm{m}, 3 \mathrm{H}), 7.50(\mathrm{~d}, J=2.0 \mathrm{~Hz}, 1 \mathrm{H}), 7.42(\mathrm{dd}, J=8.2,2.0 \mathrm{~Hz}, 1 \mathrm{H}), 6.91(\mathrm{~d}, J=8.2 \mathrm{~Hz}, 1 \mathrm{H}) .{ }^{13} \mathrm{C}$ NMR (100.6 MHz, DMSO[D 6$]) \delta=174.8,168.3,148.8,145.8,133.2,129.6,127.8,123.6$, 119.2, 117.0, 116.1, 114.1. HRMS $(\mathrm{m} / z)[\mathrm{M}+\mathrm{H}]^{+}$calcd for $\mathrm{M}=\mathrm{C}_{14} \mathrm{H}_{10} \mathrm{~N}_{2} \mathrm{O}_{3}, 255.0770$; found, 255.0765 .

\section{3-(1,3-Benzodioxol-5-yl)-5-(3-fluorophenyl)-1,2,4-oxadiazole (sery128)}

The title compound was prepared according to the GEP 10 starting from 1,3-benzodioxole-5carboximidamide and ethyl 3-fluorobenzoate and purified by recrystallisation from ethanol (twice) to provide sery128 as a white solid. Yield 25\%. m.p.: $161{ }^{\circ} \mathrm{C} .{ }^{1} \mathrm{H}$ NMR $(400 \mathrm{MHz}$, DMSO[D 6$]) \delta=8.03(\mathrm{~d}, J=7.7 \mathrm{~Hz}, 1 \mathrm{H}), 7.97(\mathrm{dt}, J=9.3,2.0 \mathrm{~Hz}, 1 \mathrm{H}), 7.78-7.69(\mathrm{~m}, 1 \mathrm{H})$, $7.67(\mathrm{dd}, J=8.1,1.6 \mathrm{~Hz}, 1 \mathrm{H}), 7.61(\mathrm{td}, J=8.5,2.0 \mathrm{~Hz}, 1 \mathrm{H}), 7.54(\mathrm{~d}, J=1.6 \mathrm{~Hz}, 1 \mathrm{H}), 7.14(\mathrm{~d}$, $J=8.1 \mathrm{~Hz}, 1 \mathrm{H}), 6.16(\mathrm{~s}, 2 \mathrm{H}) .{ }^{13} \mathrm{C} \mathrm{NMR}\left(100.6 \mathrm{MHz}, \mathrm{DMSO}\left[\mathrm{D}_{6}\right]\right) \delta=174.1,168.0,162.2(\mathrm{~d}$, $J=244.4 \mathrm{~Hz}), 150.2,148.1,132.0(\mathrm{~d}, J=8.2 \mathrm{~Hz}), 125.3(\mathrm{~d}, J=8.7 \mathrm{~Hz}), 124.2(\mathrm{~d}, J=2.8$ $\mathrm{Hz}), 122.3,120.3(\mathrm{~d}, J=21.0 \mathrm{~Hz}), 119.6,114.6(\mathrm{~d}, J=24.1 \mathrm{~Hz}), 109.0,106.6,102.0$. HRMS $(\mathrm{m} / \mathrm{z})[\mathrm{M}+\mathrm{H}]^{+}$calcd for $\mathrm{M}=\mathrm{C}_{15} \mathrm{H}_{9} \mathrm{FN}_{2} \mathrm{O}_{3}, 285.0675$; found, 285.0672 . 


\section{3-(3,4-Dihydroxyphenyl)-5-(3-fluorophenyl)-1,2,4-oxadiazole (sery140)}

The title compound was prepared according to the GEP 3 starting from sery128 to provide sery140 as a grey solid. Yield $60 \%$. m.p.: 206-207 ${ }^{\circ} \mathrm{C} .{ }^{1} \mathrm{H}$ NMR $\left(400 \mathrm{MHz}, \mathrm{DMSO}\left[\mathrm{D}_{6}\right]\right) \delta=$ $9.64(\mathrm{~s}, 1 \mathrm{H}), 9.45(\mathrm{~s}, 1 \mathrm{H}), 8.00(\mathrm{dt}, J=7.8,1.1 \mathrm{~Hz}, 1 \mathrm{H}), 7.92(\mathrm{ddd}, J=9.3,2.4,1.4 \mathrm{~Hz}, 1 \mathrm{H})$, 7.75-7.67 (m, 1H), 7.59 (td, $J=8.4,2.2 \mathrm{~Hz}, 1 \mathrm{H}), 7.49$ (d, $J=2.0 \mathrm{~Hz}, 1 \mathrm{H}), 7.42(\mathrm{dd}, J=8.2$, $2.0 \mathrm{~Hz}, 1 \mathrm{H}), 6.91(\mathrm{~d}, J=8.2 \mathrm{~Hz}, 1 \mathrm{H}) .{ }^{13} \mathrm{C}$ NMR $\left(100.6 \mathrm{MHz}, \mathrm{DMSO}\left[\mathrm{D}_{6}\right]\right) \delta=173.8,168.4$, $162.2(\mathrm{~d}, J=245.7 \mathrm{~Hz}), 148.9,145.8,132.0(\mathrm{~d}, J=8.3 \mathrm{~Hz}), 125.5(\mathrm{~d}, J=8.8 \mathrm{~Hz}), 124.2$, $120.2(\mathrm{~d}, J=21.1 \mathrm{~Hz}), 119.3,116.8,116.1,114.5(\mathrm{~d}, J=24.1 \mathrm{~Hz}), 114.1$. HRMS $(\mathrm{m} / \mathrm{z})$ $[\mathrm{M}+\mathrm{H}]^{+}$calcd for $\mathrm{M}=\mathrm{C}_{14} \mathrm{H}_{9} \mathrm{FN}_{2} \mathrm{O}_{3}, 273.0675$; found, 273.0669 .

\section{3,5-Bis(1,3-benzodioxol-5-yl)-1,2,4-oxadiazole (sery129)}

The title compound has been reported previously ${ }^{548}$ (CAS 861350-99-4). It was prepared according to the GEP 10 starting from 1,3-benzodioxole-5-carboximidamide and methyl 1,3benzodioxole-5-carboxylate and purified by washing with hot ethanol followed by hot water to provide sery129 as a white solid. Yield 38\%. m.p.: 208-209 ${ }^{\circ} \mathrm{C} .{ }^{1} \mathrm{H}$ NMR $(400 \mathrm{MHz}$, DMSO[D 6$]) \delta=7.76(\mathrm{dd}, J=8.1,2.1 \mathrm{~Hz}, 1 \mathrm{H}), 7.67-7.60(\mathrm{~m}, 2 \mathrm{H}), 7.5(\mathrm{~d}, J=1.6 \mathrm{~Hz}, 1 \mathrm{H})$, $7.18(\mathrm{~d}, J=8.1 \mathrm{~Hz}, 1 \mathrm{H}), 7.12(\mathrm{~d}, J=8.1 \mathrm{~Hz}, 1 \mathrm{H}), 6.2(\mathrm{~s}, 2 \mathrm{H}), 6.15(\mathrm{~s}, 2 \mathrm{H}) .{ }^{13} \mathrm{C}$ NMR $(100.6$ $\left.\mathrm{MHz}, \mathrm{DMSO}\left[\mathrm{D}_{6}\right]\right) \delta=174.9,167.8,151.6,150.1,148.2,148.0,123.8,122.1,119.0,117.0$, 109.2, 109.0, 107.3, 106.6, 102.4, 101.9.

\section{3,5-Bis(3,4-Dihydroxyphenyl)-1,2,4-oxadiazole hydrobromide (sery139)}

The title compound was prepared according to the GEP 3 starting from sery129 to provide sery139 as a grey solid. Yield $90 \%$. m.p.: $235-240{ }^{\circ} \mathrm{C} .{ }^{1} \mathrm{H}$ NMR $\left(400 \mathrm{MHz}, \mathrm{DMSO}\left[\mathrm{D}_{6}\right]\right) \delta=$ $7.55-7.43(\mathrm{~m}, 3 \mathrm{H}), 7.37(\mathrm{dd}, J=8.2,1.8 \mathrm{~Hz}, 1 \mathrm{H}), 6.94(\mathrm{~d}, J=8.2 \mathrm{~Hz}, 1 \mathrm{H}), 6.88(\mathrm{~d}, J=8.2$ $\mathrm{Hz}, 1 \mathrm{H}) .{ }^{13} \mathrm{C}$ NMR (100.6 MHz, DMSO[D $]$ ) $\delta=175.1,168.0,150.5,148.6,145.9,145.7$, $120.4,119.1,117.4,116.3,116.1,114.6,114.5,114.1$. HRMS $(m / z)[\mathrm{M}+\mathrm{H}]^{+}$calcd for $\mathrm{M}=$ $\mathrm{C}_{14} \mathrm{H}_{10} \mathrm{~N}_{2} \mathrm{O}_{5}, 287.0668$; found, 287.0663.

\section{3-(3,4-Dimethoxyphenyl)-5-(3,4-dimethoxyphenyl)-1,2,4-oxadiazole (sery180)}

The title compound has been reported previously (CAS 421583-83-7). It was prepared according to the GEP 10 starting from 3,4-dimethoxybenzamidoxime and ethyl 3,4dimethoxybenzoate and purified by refluxing in small volume of ethanol to provide sery180 as a white solid. Yield 44\%. ${ }^{1} \mathrm{H}$ NMR (400 MHz, DMSO[D 6$\left.]\right) \delta=7.80$ (dd, $J=8.5,2.0 \mathrm{~Hz}$, $1 \mathrm{H}), 7.69(\mathrm{dd}, J=8.4,2.0 \mathrm{~Hz}, 1 \mathrm{H}), 7.63(\mathrm{~d}, J=2.0 \mathrm{~Hz}, 1 \mathrm{H}), 7.56(\mathrm{~d}, J=2.0 \mathrm{~Hz}, 1 \mathrm{H}), 7.21(\mathrm{~d}$, $J=8.5 \mathrm{~Hz}, 1 \mathrm{H}), 7.15(\mathrm{~d}, J=8.5 \mathrm{~Hz}, 1 \mathrm{H}), 3.90(\mathrm{~s}, 3 \mathrm{H}), 3.88(\mathrm{~s}, 3 \mathrm{H}), 3.87(\mathrm{~s}, 3 \mathrm{H}), 3.85(\mathrm{~s}, 3 \mathrm{H})$. ${ }^{13} \mathrm{C}$ NMR (100.6 MHz, DMSO[D 6 ) $\delta=175.1,168.1,152.9,151.5,149.1,149.0,121.9$, $120.6,118.6,115.7,112.0,111.9,110.3,109.7,55.8,55.75,55.6(2 \mathrm{C})$. ESI-MS $(\mathrm{m} / \mathrm{z})[\mathrm{M}+\mathrm{H}]^{+}$ calcd for $\mathrm{M}=\mathrm{C}_{18} \mathrm{H}_{18} \mathrm{~N}_{2} \mathrm{O}_{5}, 343.13$; found, 343.16.

\section{3-(3,4-Dimethoxyphenyl)-5-(3-fluorophenyl)-1,2,4-oxadiazole (sery182)}

The title compound was prepared according to the GEP 10 starting from 3,4dimethoxybenzamidoxime and ethyl 3-fluorobenzoate and purified by recrystallisation from ethanol to provide sery180 as a white solid. Yield 34\%. ${ }^{1} \mathrm{H}$ NMR (400 MHz, DMSO[D 6$\left.]\right)$ $8.03(\mathrm{dt}, J=7.9,1.2 \mathrm{~Hz}, 1 \mathrm{H}), 7.98-7.93(\mathrm{~m}, 1 \mathrm{H}), 7.75-7.66(\mathrm{~m}, 2 \mathrm{H}), 7.60(\mathrm{td}, J=8.8,2.7 \mathrm{~Hz}$, $1 \mathrm{H}), 7.55(\mathrm{~d}, J=1.9 \mathrm{~Hz}, 1 \mathrm{H}), 7.15(\mathrm{~d}, J=8.4 \mathrm{~Hz}, 1 \mathrm{H}), 3.87(\mathrm{~s}, 3 \mathrm{H}), 3.85(\mathrm{~s}, 3 \mathrm{H}) .{ }^{13} \mathrm{C}$ NMR (100.6 MHz, DMSO[D 6$]) \delta=174.1,168.2,162.2(\mathrm{~d}, J=245.7 \mathrm{~Hz}), 151.6,149.0,131.9(\mathrm{~d}, J$ $=8.4 \mathrm{~Hz}), 125.4(\mathrm{~d}, J=8.4 \mathrm{~Hz}), 124.2,120.6,120.3(\mathrm{~d}, J=21.4 \mathrm{~Hz}), 118.5,114.6(\mathrm{~d}, J=$ $24.2 \mathrm{~Hz}), 111.9,109.7$, 55.7, 55.6. ESI-MS $(\mathrm{m} / \mathrm{z})[\mathrm{M}+\mathrm{H}]^{+}$calcd for $\mathrm{M}=\mathrm{C}_{16} \mathrm{H}_{13} \mathrm{FN}_{2} \mathrm{O}_{3}$, 301.10; found, 301.26 . 


\section{3-(3,4-Dimethoxyphenyl)-5-phenyl-1,2,4-oxadiazole (sery266)}

The title compound was prepared according to the GEP 10 starting from 3,4dimethoxybenzamidoxime and methyl benzoate to provide sery266 as a white solid. Yield 46\%. ${ }^{1} \mathrm{H}$ NMR (400 MHz, DMSO[D 6$\left.]\right) \delta=8.19(\mathrm{~d}, J=7.6 \mathrm{~Hz}, 2 \mathrm{H}), 7.79-7.61(\mathrm{~m}, 4 \mathrm{H}), 7.57$ $(\mathrm{d}, J=1.4 \mathrm{~Hz}, 1 \mathrm{H}), 7.17(\mathrm{~d}, J=8.4 \mathrm{~Hz}, 1 \mathrm{H}), 3.87(\mathrm{~s}, 3 \mathrm{H}), 3.85(\mathrm{~s}, 3 \mathrm{H}) .{ }^{13} \mathrm{C}$ NMR $(100.6$ $\left.\mathrm{MHz}, \mathrm{DMSO}\left[\mathrm{D}_{6}\right]\right) \delta=175.1,168.1,151.5,149.0,133.2,129.5,127.9,123.4,120.5,118.4$, 111.9, 109.6, 55.6, 55.5. ESI-MS $(m / z)[\mathrm{M}+\mathrm{H}]^{+}$calcd for $\mathrm{M}=\mathrm{C}_{16} \mathrm{H}_{14} \mathrm{~N}_{2} \mathrm{O}_{3}$, 283.11; found, 283.24 .

\subsubsection{Synthesis of 2,4-diaryl-1H-imidazoles}

\section{General Experimental Procedure for the synthesis of 2,4-diaryl-1H-imidazoles (GEP 11) $\mathbf{4 0 6}^{406}$}

\section{4-(1,3-Benzodioxol-5-yl)-2-phenyl-1H-imidazole (anle131b)}

The title compound was prepared according to the published protocol ${ }^{406}$ with minor modifications. A mixture of benzamidine hydrochloride $(313 \mathrm{mg}, 2.0 \mathrm{mmol})$ and sodium bicarbonate $(672 \mathrm{mg}, 8.0 \mathrm{mmol})$ in THF $(8 \mathrm{ml})$ and water $(2 \mathrm{ml})$ was heated under reflux. A solution of 1-(1,3-benzodioxol-5-yl)-2-bromoethanone $(486 \mathrm{mg}, 2.0 \mathrm{mmol})$ in THF $(2 \mathrm{ml})$ was added over a period of $30 \mathrm{~min}$, while keeping the reaction under reflux. After addition, the reaction was heated under reflux for $2 \mathrm{~h}$, THF was evaporated under reduced pressure. Ethyl acetate $(20 \mathrm{ml})$ was added to the mixture, organic phase was separated, washed with the brine $(10 \mathrm{ml})$, dried over $\mathrm{Na}_{2} \mathrm{SO}_{4}$ and evaporated under reduced pressure. The resulting crude product was purified by column chromatography on silica gel $\left(\mathrm{CHCl}_{3}: \mathrm{MeOH}, 50: 1 \mathrm{v} / \mathrm{v}\right)$ to provide anle131b $(376 \mathrm{mg}, 71 \%)$ as a white solid. TLC $\left(\mathrm{CHCl}_{3}: \mathrm{MeOH}, 50: 1 \mathrm{v} / \mathrm{v}\right): \mathrm{RF}=0.28$. m.p.: $168{ }^{\circ} \mathrm{C} .{ }^{1} \mathrm{H}$ NMR $\left(400 \mathrm{MHz}, \mathrm{DMSO}\left[\mathrm{D}_{6}\right]+1-2 \%\right.$ conc. DCl) $\delta=7.98(\mathrm{~d}, J=7.2 \mathrm{~Hz}$, 2H), $7.61(\mathrm{~s}, 1 \mathrm{H}), 7.45$ (t, $J=7.6 \mathrm{~Hz}, 2 \mathrm{H}), 7.40(\mathrm{~d}, J=1.5 \mathrm{~Hz}, 1 \mathrm{H}), 7.39-7.30(\mathrm{~m}, 2 \mathrm{H}), 6.93$ $(\mathrm{d}, J=8.0 \mathrm{~Hz}, 1 \mathrm{H}), 6.01(\mathrm{~s}, 2 \mathrm{H}) \cdot{ }^{13} \mathrm{C}$ NMR $\left(100.6 \mathrm{MHz}, \mathrm{DMSO}\left[\mathrm{D}_{6}\right]+1-2 \%\right.$ conc. DCl $) \delta=$ 147.8, 146.1, 145.9, 139.5, 130.7, 128.9, 128.3, 125.2, 118.1, 116.2 (2C), 108.7, 105.3, 101.0. HRMS $(m / z)[\mathrm{M}+\mathrm{H}]^{+}$calcd for $\mathrm{M}=\mathrm{C}_{16} \mathrm{H}_{12} \mathrm{~N}_{2} \mathrm{O}_{2}, 265.0977$; found, 265.0976.

\section{4-(3,4-Dihydroxyphenyl)-2-phenyl- $1 \mathrm{H}$-imidazole hydrobromide (anle132)}

The title compound was prepared according to the GEP 3 starting from anle131b. Grey solid. Yield 75\%. m.p.: $240-245{ }^{\circ} \mathrm{C}$ (dec). ${ }^{1} \mathrm{H}$ NMR (400 MHz, DMSO[D 6$\left.]\right) \delta=9.54$ (bs, $\left.1 \mathrm{H}\right), 9.19$ (bs, $1 \mathrm{H}), 8.40-7.93(\mathrm{~m}, 3 \mathrm{H}), 7.65(\mathrm{~s}, 3 \mathrm{H}), 7.27(\mathrm{~s}, 1 \mathrm{H}), 7.20(\mathrm{~d}, J=8.0 \mathrm{~Hz}, 1 \mathrm{H}), 6.90(\mathrm{~d}, J=$ $8.0 \mathrm{~Hz}, 1 \mathrm{H}) .{ }^{13} \mathrm{C}$ NMR $\left(100.6 \mathrm{MHz}, \mathrm{DMSO}\left[\mathrm{D}_{6}\right]\right) \delta=147.1,146.0,143.9,134.7,132.2,129.6$, $127.4,123.4,118.2,118.0,116.2,115.1,113.9$. HRMS $(\mathrm{m} / z)[\mathrm{M}+\mathrm{H}]^{+}$calcd for $\mathrm{M}=$ $\mathrm{C}_{15} \mathrm{H}_{12} \mathrm{~N}_{2} \mathrm{O}_{2}, 253.0977$; found, 253.0972.

\section{2,4-Bis(1,3-benzodioxol-5-yl)-1 $\mathrm{H}$-imidazole (anle237)}

The title compound has been reported previously ${ }^{514}$ (CAS 1147315-16-9). It was prepared according to the GEP 11 using 1,3-benzodioxole-5-carboximidamide hydrochloride and 1(1,3-benzodioxol-5-yl)-2-bromoethanone and purified by recrystallisation from ethanol/water to provide anle237 as a pale brown solid. Yield 73\%. m.p.: $136-138{ }^{\circ} \mathrm{C} .{ }^{1} \mathrm{H}$ NMR $(400 \mathrm{MHz}$, DMSO[D 6$]) \delta=12.37(\mathrm{bs}, 1 \mathrm{H}), 7.54(\mathrm{bs}, 1 \mathrm{H}), 7.52-7.46(\mathrm{~m}, 2 \mathrm{H}), 7.36(\mathrm{~d}, J=1.6 \mathrm{~Hz}, 1 \mathrm{H})$, $7.33(\mathrm{dd}, J=8.0,1.5 \mathrm{~Hz}, 1 \mathrm{H}), 7.00(\mathrm{~d}, J=8.5 \mathrm{~Hz}, 1 \mathrm{H}), 6.91(\mathrm{~d}, J=8.0 \mathrm{~Hz}, 1 \mathrm{H}), 6.06(\mathrm{~s}, 2 \mathrm{H})$, 6.00 (s, 2H). 


\section{4-(3,4-Dimethoxyphenyl)-2-phenyl-1H-imidazole (sery152)}

The title compound has been reported previously ${ }^{549}$ (CAS 1204141-45-6). It was prepared according to the GEP 11 using benzamidine hydrochloride and 2-bromo-1-(3,4dimethoxyphenyl)ethanone and purified by column chromatography on silica gel $\left(\mathrm{CHCl}_{3}: \mathrm{MeOH}, 100: 1 \mathrm{v} / \mathrm{v}\right)$. Yield $84 \%$. m.p. $95-98^{\circ} \mathrm{C}$. TLC $\left(\mathrm{CHCl}_{3}: \mathrm{MeOH}, 100: 1 \mathrm{v} / \mathrm{v}\right): \mathrm{RF}=$ 0.31. ${ }^{1} \mathrm{H}$ NMR (400 MHz, DMSO[D $\left.]\right) \delta=12.57(\mathrm{~s}, 1 \mathrm{H}), 8.00(\mathrm{~d}, \mathrm{~J}=7.5 \mathrm{~Hz}, 2 \mathrm{H}), 7.62(\mathrm{~s}$, $1 \mathrm{H}), 7.47(\mathrm{t}, J=7.5 \mathrm{~Hz}, 2 \mathrm{H}), 7.43(\mathrm{~d}, J=1.8 \mathrm{~Hz}, 1 \mathrm{H}), 7.41-7.32(\mathrm{~m}, 2 \mathrm{H}), 6.97(\mathrm{~d}, J=8.3 \mathrm{~Hz}$, $1 \mathrm{H}), 3.84(\mathrm{~s}, 3 \mathrm{H}), 3.77(\mathrm{~s}, 3 \mathrm{H})$.

\section{2-(1,3-Benzodioxol-5-yl)-4-(3,4-dimethoxyphenyl)-1H-imidazole (sery165)}

The title compound was prepared according to the GEP 11 using 1,3-benzodioxole-5carboximidamide hydrochloride and 2-bromo-1-(3,4-dimethoxyphenyl)ethanone and purified by column chromatography on silica gel $\left(\mathrm{CHCl}_{3}: \mathrm{MeOH}, 95: 5 \mathrm{v} / \mathrm{v}\right)$ followed by recrystallisation from methanol/water to provide sery165 as a pale yellow solid. Yield $82 \%$. TLC $\left(\mathrm{CHCl}_{3}: \mathrm{MeOH}, 95: 5 \mathrm{v} / \mathrm{v}\right): \mathrm{RF}=0.55$. m.p.: $107-110{ }^{\circ} \mathrm{C} .{ }^{1} \mathrm{H}$ NMR $(400 \mathrm{MHz}$, DMSO $\left[\mathrm{D}_{6}\right]+1-2 \%$ conc. DCl) $\delta=7.58(\mathrm{~s}, 1 \mathrm{H}), 7.55-7.51(\mathrm{~m}, 2 \mathrm{H}), 7.39(\mathrm{~d}, J=1.8 \mathrm{~Hz}, 1 \mathrm{H})$, 7.34 (dd, $J=8.2,1.8 \mathrm{~Hz}, 1 \mathrm{H}), 7.03(\mathrm{~d}, J=8.4 \mathrm{~Hz}, 1 \mathrm{H}), 6.97$ (d, $J=8.4 \mathrm{~Hz}, 1 \mathrm{H}), 6.08(\mathrm{~s}, 2 \mathrm{H})$, $3.83(\mathrm{~s}, 3 \mathrm{H}), 3.76(\mathrm{~s}, 3 \mathrm{H}) .{ }^{13} \mathrm{C}$ NMR $\left(100.6 \mathrm{MHz}, \mathrm{DMSO}\left[\mathrm{D}_{6}\right]+1-2 \%\right.$ conc. DCl) $\delta=149.0$, 147.9 (2C), 147.3, 145.7, 138.9, 126.6, 124.9, 119.1, 116.8, 116.1, 112.1, 108.7, 108.6, 105.5, 101.3, 55.6 (2C). HRMS $(\mathrm{m} / \mathrm{z})[\mathrm{M}+\mathrm{H}]^{+}$calcd for $\mathrm{M}=\mathrm{C}_{18} \mathrm{H}_{16} \mathrm{~N}_{2} \mathrm{O}_{4}, 325.1188$; found, 325.1192 .

\section{2,4-Bis(3,4-dihydroxyphenyl)-1 $H$-imidazole hydrobromide (sery166a)}

This compound has been reported previously ${ }^{514}$ (CAS $1147315-18-1$ as a free base). It was prepared according to the GEP 3 starting from sery165. Pale brown solid. Yield 89\%. m.p.: $>300{ }^{\circ} \mathrm{C} .{ }^{1} \mathrm{H}$ NMR $\left(400 \mathrm{MHz}, \mathrm{DMSO}\left[\mathrm{D}_{6}\right]\right) \delta=14.27$ (bs, $\left.1 \mathrm{H}\right), 13.99$ (bs, $\left.1 \mathrm{H}\right), 10.40-8.60$ (m, $4 \mathrm{H}), 7.92(\mathrm{~s}, 1 \mathrm{H}), 7.46(\mathrm{~d}, J=2.3 \mathrm{~Hz}, 1 \mathrm{H}), 7.41(\mathrm{dd}, J=8.2,2.3 \mathrm{~Hz}, 1 \mathrm{H}), 7.23(\mathrm{~d}, J=2.2 \mathrm{~Hz}$, $1 \mathrm{H}), 7.16(\mathrm{dd}, J=8.2,2.2 \mathrm{~Hz}, 1 \mathrm{H}), 6.98(\mathrm{~d}, J=8.3 \mathrm{~Hz}, 1 \mathrm{H}), 6.88(\mathrm{~d}, J=8.2 \mathrm{~Hz}, 1 \mathrm{H})$.

\section{2,4-Bis(3,4-dimethoxyphenyl)-1H-imidazole (sery267a)}

The title compound was prepared according to the GEP 11 using 3,4-dimethoxybenzamidine hydrochloride and 2-bromo-1-(3,4-dimethoxyphenyl)ethanone and purified by column chromatography on silica gel $\left(\mathrm{CHCl}_{3}: \mathrm{MeOH}, 100: 1 \mathrm{v} / \mathrm{v}\right)$ to provide sery267a as a solid. Yield $72 \%$. TLC $\left(\mathrm{CHCl}_{3}: \mathrm{MeOH}, 100: 1 \mathrm{v} / \mathrm{v}\right): \mathrm{RF}=0.24 .{ }^{1} \mathrm{H}$ NMR $\left(400 \mathrm{MHz}, \mathrm{DMSO}\left[\mathrm{D}_{6}\right]\right) \delta=12.38$ (bs, 1H), 7.63-7.52 (m, 3H), $7.40(\mathrm{~s}, 1 \mathrm{H}), 7.37(\mathrm{~d}, J=8.4 \mathrm{~Hz}, 1 \mathrm{H}), 7.04(\mathrm{~d}, J=8.4 \mathrm{~Hz}, 1 \mathrm{H})$, $6.96(\mathrm{~d}, J=8.3 \mathrm{~Hz}, 1 \mathrm{H}), 3.85(\mathrm{~s}, 3 \mathrm{H}), 3.83(\mathrm{~s}, 3 \mathrm{H}), 3.80(\mathrm{~s}, 3 \mathrm{H}), 3.77$ (s, 3H). ${ }^{13} \mathrm{C}$ NMR $\left(100.6 \mathrm{MHz}, \mathrm{CDCl}_{3}\right) \delta=149.8,149.5,149.4,148.4,147.3,132.5,123.4,117.9-117.4$ (3C), $111.6,111.3,109.1,109.0,108.6,56.1(2 \mathrm{C}), 55.9(2 \mathrm{C})$. ESI-MS $(\mathrm{m} / \mathrm{z})[\mathrm{M}+\mathrm{H}]^{+}$calcd for $\mathrm{M}=$ $\mathrm{C}_{19} \mathrm{H}_{20} \mathrm{~N}_{2} \mathrm{O}_{4}, 341.15$; found, 341.32 .

\section{2-(1,3-Benzodioxol-5-yl)-4-(3-bromophenyl)-1 $H$-imidazole (sery345)}

The title compound was prepared according to the GEP 11 using 1,3-benzodioxole-5carboximidamide hydrochloride and 2-bromo-1-(3-bromophenyl)ethanone and purified by column chromatography on silica gel $\left(\mathrm{CHCl}_{3}: \mathrm{MeOH}, 100: 1 \mathrm{v} / \mathrm{v}\right)$ to provide sery345 as a white solid. Yield $85 \%$. TLC $\left(\mathrm{CHCl}_{3}: \mathrm{MeOH}, 100: 1 \mathrm{v} / \mathrm{v}\right): \mathrm{RF}=0.33$. m.p.: $236-238{ }^{\circ} \mathrm{C} .{ }^{1} \mathrm{H}$ NMR (400 MHz, DMSO[D $]+1-2 \%$ conc. DCl) $\delta=12.57(\mathrm{bs}, 1 \mathrm{H}), 8.04(\mathrm{~s}, 1 \mathrm{H}), 7.83(\mathrm{~d}, J=$ $7.6 \mathrm{~Hz}, 1 \mathrm{H}), 7.80(\mathrm{~s}, 1 \mathrm{H}), 7.58-7.49(\mathrm{~m}, 2 \mathrm{H}), 7.38(\mathrm{~d}, J=8.0 \mathrm{~Hz}, 1 \mathrm{H}), 7.33(\mathrm{t}, J=7.0 \mathrm{~Hz}$, $1 \mathrm{H}), 7.02(\mathrm{~d}, J=8.6 \mathrm{~Hz}, 1 \mathrm{H}), 6.08(\mathrm{~s}, 2 \mathrm{H}) .{ }^{13} \mathrm{C} \mathrm{NMR}\left(100.6 \mathrm{MHz}, \mathrm{DMSO}\left[\mathrm{D}_{6}\right]+1-2 \%\right.$ conc. DCl) $\delta=147.7,147.4,146.2,138.3,136.6,130.8,128.7,126.6,124.7,123.1,122.1,119.1$, 
117.0, 108.6, 105.4, 101.3. HRMS $(m / z)[\mathrm{M}+\mathrm{H}]^{+}$calcd for $\mathrm{M}=\mathrm{C}_{16} \mathrm{H}_{11}{ }^{79} \mathrm{BrN}_{2} \mathrm{O}_{2}, 343.0082$; found, 343.0090 .

\subsubsection{Synthesis of 1-acetyl-3,5-diaryl-4,5-dihydro-1H-pyrazoles}

General Experimental Procedure for the synthesis of 1-acetyl-3,5-diaryl-4,5-dihydro$1 H$-pyrazoles (GEP 12)

\section{1-Acetyl-3-(1,3-benzodioxol-5-yl)-4,5-dihydro-5-phenyl-1 $H$-pyrazole (anle129)}

The title compound was prepared according to the published protocol ${ }^{550}$. To a solution of sery89 $(504 \mathrm{mg}, 2.0 \mathrm{mmol})$ in $\mathrm{AcOH}(12 \mathrm{~mL})$ was added dropwise hydrazine hydrate $(243 \mu \mathrm{l}$, $250 \mathrm{mg}, 5.0 \mathrm{mmol}$ ) and kept under stirring at $120^{\circ} \mathrm{C}$ for $24 \mathrm{~h}$. The mixture was then poured in ice-water at stirring, the resulting precipitate was collected by filtration and dried. The resulting crude product was purified by recrystallisation from ethanol to provide anle129 (458 mg, 74\%) as a white solid. m.p.: 190-192 ${ }^{\circ} \mathrm{C} .{ }^{1} \mathrm{H}$ NMR (400 MHz, DMSO[D $\left.]\right) \delta=7.35(\mathrm{~d}, J$ $=1.6 \mathrm{~Hz}, 1 \mathrm{H}), 7.31(\mathrm{t}, J=7.4 \mathrm{~Hz}, 2 \mathrm{H}), 7.28-7.20(\mathrm{~m}, 2 \mathrm{H}), 7.20-7.13(\mathrm{~m}, 2 \mathrm{H}), 6.97(\mathrm{~d}, J=8.1$ $\mathrm{Hz}, 1 \mathrm{H}), 6.08(\mathrm{~s}, 2 \mathrm{H}), 5.50(\mathrm{dd}, J=11.8,4.4 \mathrm{~Hz}, 1 \mathrm{H}), 3.80$ and $3.76(2 \times \mathrm{d}, J=11.8 \mathrm{~Hz}, 1 \mathrm{H})$, 3.09 and $3.05(2 \times \mathrm{d}, J=4.5 \mathrm{~Hz}, 1 \mathrm{H}), 2.28(\mathrm{~s}, 3 \mathrm{H}) .{ }^{13} \mathrm{C} \mathrm{NMR}\left(100.6 \mathrm{MHz}, \mathrm{DMSO}\left[\mathrm{D}_{6}\right]\right) \delta=$ 167.4, 154.0, 149.3, 148.0, 142.6, 128.8, 127.3, 125.6, 125.4, 122.0, 108.6, 106.1, 101.8, 59.6, 42.4, 21.8. HRMS $(m / z)[\mathrm{M}+\mathrm{H}]^{+}$calcd for $\mathrm{M}=\mathrm{C}_{18} \mathrm{H}_{16} \mathrm{~N}_{2} \mathrm{O}_{3}, 309.1239$; found, 309.1241.

1-Acetyl-4,5-dihydro-3-(3,4-dimethoxyphenyl)-5-(3-fluorophenyl)-1 H-pyrazole (sery115) The title compound was prepared according to the GEP 12 starting from sery97 and purified by recrystallisation from methanol to provide sery115 as a white solid. Yield 70\%. m.p.: 161 ${ }^{\circ} \mathrm{C} .{ }^{1} \mathrm{H}$ NMR $\left(400 \mathrm{MHz}, \mathrm{DMSO}\left[\mathrm{D}_{6}\right]\right) \delta=7.42-7.32(\mathrm{~m}, 2 \mathrm{H}), 7.28(\mathrm{dd}, J=8.3,1.8 \mathrm{~Hz}, 1 \mathrm{H})$, 7.08 (td, $J=9.0,1.8 \mathrm{~Hz}, 1 \mathrm{H}), 7.04-6.97(\mathrm{~m}, 3 \mathrm{H}), 5.55(\mathrm{dd}, J=11.8,4.5 \mathrm{~Hz}, 1 \mathrm{H}), 3.87-3.74$ $(\mathrm{m}, 7 \mathrm{H}), 3.17(\mathrm{dd}, J=18.1,4.7 \mathrm{~Hz}, 1 \mathrm{H}), 2.31(\mathrm{~s}, 3 \mathrm{H}) .{ }^{13} \mathrm{C}$ NMR $\left(100.6 \mathrm{MHz}, \mathrm{DMSO}\left[\mathrm{D}_{6}\right]\right) \delta=$ $167.3,162.3(\mathrm{~d}, J=243.6 \mathrm{~Hz}), 154.2,150.8,148.8,145.3$ (d, $J=6.7 \mathrm{~Hz}), 130.7$ (d, $J=8.1$ $\mathrm{Hz}), 123.5,121.4,120.4,113.9$ (d, $J=21.0 \mathrm{~Hz}), 112.4$ (d, $J=21.7 \mathrm{~Hz}), 111.4,109.2,58.8$, 55.6, 55.5, 42.1, 21.7. HRMS $(m / z)[\mathrm{M}+\mathrm{Na}]^{+}$calcd for $\mathrm{M}=\mathrm{C}_{19} \mathrm{H}_{19} \mathrm{FN}_{2} \mathrm{O}_{3}, 365.1277$; found, 365.1276 .

\section{1-Acetyl-4,5-dihydro-5-(3,4-dimethoxyphenyl)-3-(4-methoxyphenyl)-1H-pyrazole (sery108)}

The title compound was prepared according to the GEP 12 starting from sery96 and purified by recrystallisation from ethanol to provide sery108 as a white solid. Yield 58\%. m.p.: 128$129{ }^{\circ} \mathrm{C} .{ }^{1} \mathrm{H}$ NMR $\left.\left(400 \mathrm{MHz}, \mathrm{DMSO}_{[} \mathrm{D}_{6}\right]\right) \delta=7.72(\mathrm{~d}, J=8.7 \mathrm{~Hz}, 2 \mathrm{H}), 7.01(\mathrm{~d}, J=8.7 \mathrm{~Hz}$, $2 \mathrm{H}), 6.87(\mathrm{~d}, J=8.3 \mathrm{~Hz}, 1 \mathrm{H}), 6.8(\mathrm{~s}, 1 \mathrm{H}), 6.65(\mathrm{~d}, J=8.3 \mathrm{~Hz}, 1 \mathrm{H}), 5.45(\mathrm{dd}, J=11.6,4.0 \mathrm{~Hz}$, $1 \mathrm{H}), 3.86-3.75(\mathrm{~m}, 4 \mathrm{H}), 3.72(\mathrm{~s}, 3 \mathrm{H}), 3.71(\mathrm{~s}, 3 \mathrm{H}), 3.10(\mathrm{dd}, J=17.8,4.0 \mathrm{~Hz}, 1 \mathrm{H}), 2.29$ (s, $3 \mathrm{H}) .{ }^{13} \mathrm{C}$ NMR (100.6 MHz, DMSO[D 6$\left.]\right) \delta=167.1,160.9,153.9,148.8,147.9,135.0,128.3$, $123.7,117.0,114.2,112.0,109.6,59.0,55.6,55.5,55.3,42.2,21.7$. HRMS $(m / z)[\mathrm{M}+\mathrm{H}]^{+}$ calcd for $\mathrm{M}=\mathrm{C}_{20} \mathrm{H}_{22} \mathrm{~N}_{2} \mathrm{O}_{4}, 355.1658$; found, 355.1656.

\section{1-Acetyl-4,5-dihydro-3-(3,4-dihydroxyphenyl)-5-phenyl-1H-pyrazole (sery105)}

The title compound was prepared according to the GEP 3 starting from anle129 and purified by column chromatography $\left(\mathrm{CHCl}_{3}: \mathrm{MeOH}, 19: 1 \mathrm{v} / \mathrm{v}\right)$ to provide sery105 as a yellow solid. Yield $37 \%$. TLC $\left(\mathrm{CHCl}_{3}: \mathrm{MeOH}, 19: 1 \mathrm{v} / \mathrm{v}\right): \mathrm{RF}=0.27$. m.p.: $100-102{ }^{\circ} \mathrm{C}(\mathrm{dec}) .{ }^{1} \mathrm{H}$ NMR $\left(400 \mathrm{MHz}, \mathrm{CDCl}_{3}\right) \delta=7.55$ (bs, $\left.1 \mathrm{H}\right), 7.32-7.17(\mathrm{~m}, 7 \mathrm{H}), 7.06(\mathrm{dd}, J=8.3,2.0 \mathrm{~Hz}, 1 \mathrm{H}), 6.77$ $(\mathrm{d}, J=8.3 \mathrm{~Hz}, 1 \mathrm{H}), 5.55(\mathrm{dd}, J=11.6,4.4 \mathrm{~Hz}, 1 \mathrm{H}), 3.66(\mathrm{dd}, J=17.7,11.6 \mathrm{~Hz}, 1 \mathrm{H}), 3.09$ $(\mathrm{dd}, J=17.7,4.4 \mathrm{~Hz}, 1 \mathrm{H}), 2.46$ (s, 3H). ${ }^{13} \mathrm{C} \mathrm{NMR}\left(100.6 \mathrm{MHz}, \mathrm{DMSO}\left[\mathrm{D}_{6}\right]\right) \delta=166.9,154.3$, 
148.1, 145.5, 142.6, 128.6, 127.1, 125.4, 122.4, 119.3, 115.5, 113.1, 59.1, 42.2, 21.7. HRMS $(\mathrm{m} / \mathrm{z})[\mathrm{M}+\mathrm{H}]^{+}$calcd for $\mathrm{M}=\mathrm{C}_{17} \mathrm{H}_{16} \mathrm{~N}_{2} \mathrm{O}_{3}, 297.1239$; found, 297.1241.

\subsubsection{Synthesis of 3,5-diaryl-4,5-dihydro-1H-pyrazoles}

\section{(E)-1,3-Bis(3,4-dimethoxyphenyl)-2-propene-1-one (sery112)}

The title compound has been reported previously ${ }^{551}$ (CAS 130768-85-3). It was prepared according to the GEP 5 using 1-(3,4-dimethoxyphenyl)ethanone, 3,4-dimethoxybenzaldehyde to provide sery112 as a yellow solid. Yield 82\%. m.p.: 91-92 ${ }^{\circ} \mathrm{C} .{ }^{1} \mathrm{H}$ NMR $(400 \mathrm{MHz}$, DMSO[D 6$]) \delta=7.92(\mathrm{dd}, J=8.4,1.8 \mathrm{~Hz}, 1 \mathrm{H}), 7.82(\mathrm{~d}, J=15.5 \mathrm{~Hz}, 1 \mathrm{H}), 7.67(\mathrm{~d}, J=15.5$ $\mathrm{Hz}, 1 \mathrm{H}), 7.60(\mathrm{~d}, J=1.8 \mathrm{~Hz}, 1 \mathrm{H}), 7.52(\mathrm{~d}, J=1.7 \mathrm{~Hz}, 1 \mathrm{H}), 7.40(\mathrm{dd}, J=8.5,1.7 \mathrm{~Hz}, 1 \mathrm{H})$, $7.10(\mathrm{~d}, J=8.5 \mathrm{~Hz}, 1 \mathrm{H}), 7.02$ (d, $J=8.4 \mathrm{~Hz}, 1 \mathrm{H}), 3.88(\mathrm{~s}, 3 \mathrm{H}), 3.87-3.84(\mathrm{~m}, 6 \mathrm{H}), 3.82(\mathrm{~s}$, $3 \mathrm{H})$.

\section{General Experimental Procedure for the synthesis of 3,5-diaryl-4,5-dihydro-1 $\mathrm{H}$ - pyrazoles (GEP 13)}

\section{4,5-Dihydro-3,5-bis(3,4-dimethoxyphenyl)-1 $H$-pyrazole (sery132)}

A suspension of sery112 $(330 \mathrm{mg}, 1 \mathrm{mmol})$ and hydrazine hydrate $(2.5 \mathrm{ml}, 50 \mathrm{mmol})$ in water $(0.7 \mathrm{ml})$ was heated at $100^{\circ} \mathrm{C}$ for $1.5 \mathrm{~h}$ with stirring. The reaction mixture was cooled down, water $(0.2 \mathrm{ml})$ was added and a resulting precipitate was collected by filtration, washed with water and dried to provide sery132 $(310 \mathrm{mg})$ as a white solid. Yield $90 \%$. m.p.: $54-57{ }^{\circ} \mathrm{C} .{ }^{1} \mathrm{H}$ NMR (400 MHz, DMSO[D 6$) \delta=7.42(\mathrm{~s}, 1 \mathrm{H}), 7.03(\mathrm{~d}, J=8.2 \mathrm{~Hz}, 1 \mathrm{H}), 6.95(\mathrm{~s}, 1 \mathrm{H}), 6.90$ (d, $J=8.2 \mathrm{~Hz}, 1 \mathrm{H}), 6.87-6.79(\mathrm{~m}, 2 \mathrm{H}), 4.86(\mathrm{t}, J=9.9 \mathrm{~Hz}, 1 \mathrm{H}), 3.99-3.81(\mathrm{~m}, 12 \mathrm{H}), 3.43(\mathrm{dd}, J=$ $16.2,10.5 \mathrm{~Hz}, 1 \mathrm{H}), 3.00(\mathrm{dd}, J=16.2,9.4 \mathrm{~Hz}, 1 \mathrm{H}) .{ }^{13} \mathrm{C}$ NMR $\left(100.6 \mathrm{MHz}, \mathrm{DMSO}\left[\mathrm{D}_{6}\right]\right) \delta=$ $151.7,150.1,149.4,149.3,148.7,135.4,126.0,119.5,118.8,111.3,110.7,109.5,108.3,64.4$, 56.2-55.9 (4C), 41.8. HRMS $(\mathrm{m} / z)[\mathrm{M}+\mathrm{H}]^{+}$calcd for $\mathrm{M}=\mathrm{C}_{19} \mathrm{H}_{22} \mathrm{~N}_{2} \mathrm{O}_{4}, 343.1658$; found, 343.1657 .

\section{4,5-Dihydro-5-(3,4-dimethoxyphenyl)-3-(4-methoxyphenyl)-1 $\mathrm{H}$-pyrazole (sery133)}

The title compound has been reported previously ${ }^{552}$ (CAS 130768-85-3). It was prepared according to the GEP 13 starting from sery96 to provide sery133 as a white solid. Yield $85 \%$. m.p.: $60-65{ }^{\circ} \mathrm{C}\left(\operatorname{lit}^{552} 100-105^{\circ} \mathrm{C}\right) .{ }^{1} \mathrm{H}$ NMR $\left(400 \mathrm{MHz}, \mathrm{DMSO}\left[\mathrm{D}_{6}\right]\right) \delta=7.56(\mathrm{~d}, J=8.1 \mathrm{~Hz}$, $2 \mathrm{H}), 7.29(\mathrm{~s}, 1 \mathrm{H}), 7.06-6.82(\mathrm{~m}, 5 \mathrm{H}), 4.74(\mathrm{t}, J=10.7 \mathrm{~Hz}, 1 \mathrm{H}), 3.76(\mathrm{~s}, 3 \mathrm{H}), 3.74(\mathrm{~s}, 3 \mathrm{H}), 3.73$ $(\mathrm{s}, 3 \mathrm{H}), 3.36(\mathrm{dd}, J=15.7,10.7 \mathrm{~Hz}, 1 \mathrm{H}), 2.80(\mathrm{dd}, J=15.7,11.5 \mathrm{~Hz}, 1 \mathrm{H}) .{ }^{13} \mathrm{C}$ NMR $(100.6$ $\left.\mathrm{MHz}, \mathrm{DMSO}\left[\mathrm{D}_{6}\right]\right) \delta=159.3,148.8,148.7,147.9,135.4,126.8,126.0,118.6,113.9,111.7$, $110.4,63.5,55.6,55.4,55.1,40.8$.

\section{(E)-3-(3,4-Dimethoxyphenyl)-1-phenyl-2-propene-1-one (sery113)}

The title compound has been reported previously ${ }^{543}$ (CAS 53744-27-7). It was prepared according to the GEP 5 using 1-phenylethanone, 3,4-dimethoxybenzaldehyde to provide sery113 as a yellow solid. Yield 90\%. m.p.: 85-87 ${ }^{\circ} \mathrm{C}\left(\operatorname{lit}^{543} 84{ }^{\circ} \mathrm{C}\right) .{ }^{1} \mathrm{H}$ NMR $(400 \mathrm{MHz}$, DMSO[D 6$]) \delta=8.15(\mathrm{~d}, J=8.3 \mathrm{~Hz}, 2 \mathrm{H}), 7.83(\mathrm{~d}, J=15.5 \mathrm{~Hz}, 1 \mathrm{H}), 7.72(\mathrm{~d}, J=15.5 \mathrm{~Hz}, 1 \mathrm{H})$, $7.66(\mathrm{~d}, J=7.4 \mathrm{~Hz}, 1 \mathrm{H}), 7.6-7.53(\mathrm{~m}, 3 \mathrm{H}), 7.39(\mathrm{dd}, J=8.4,1.9 \mathrm{~Hz}, 1 \mathrm{H}), 7.02(\mathrm{~d}, J=8.4 \mathrm{~Hz}$, $1 \mathrm{H}), 3.87(\mathrm{~s}, 3 \mathrm{H}), 3.82(\mathrm{~s}, 3 \mathrm{H})$.

\section{4,5-Dihydro-5-(3,4-dimethoxyphenyl)-3-phenyl-1 $H$-pyrazole (sery135)}

The title compound has been reported previously ${ }^{552}$ (CAS 399031-89-1). It was prepared according to the GEP 13 starting from sery113 to provide sery135 as a white solid. Yield 
82\%. m.p.: 59-64 ${ }^{\circ} \mathrm{C}\left(\right.$ lit $\left.^{552} 85-88{ }^{\circ} \mathrm{C}\right) .{ }^{1} \mathrm{H}$ NMR (400 MHz, DMSO[D 6$\left.]\right) \delta=7.63(\mathrm{~d}, J=7.4$ $\mathrm{Hz}, 2 \mathrm{H}), 7.50(\mathrm{~s}, 1 \mathrm{H}), 7.37(\mathrm{t}, J=7.4 \mathrm{~Hz}, 2 \mathrm{H}), 7.30(\mathrm{t}, J=7.2 \mathrm{~Hz}, 1 \mathrm{H}), 7.00(\mathrm{~s}, 1 \mathrm{H}), 6.90(\mathrm{~s}$, $2 \mathrm{H}), 4.79(\mathrm{t}, J=10.7 \mathrm{~Hz}, 1 \mathrm{H}), 3.74(\mathrm{~s}, 3 \mathrm{H}), 3.73(\mathrm{~s}, 3 \mathrm{H}), 3.40(\mathrm{dd}, J=16.2,10.7 \mathrm{~Hz}, 1 \mathrm{H})$, $2.84(\mathrm{dd}, J=16.2,11.1 \mathrm{~Hz}, 1 \mathrm{H}) .{ }^{13} \mathrm{C}$ NMR $\left(100.6 \mathrm{MHz}, \mathrm{DMSO}\left[\mathrm{D}_{6}\right]\right) \delta=148.7,148.6,148.0$, $135.3,133.3,128.5,128.0,125.3,118.6,111.7,110.4,63.6,55.6,55.4,40.5$.

\section{3-(1,3-Benzodioxol-5-yl)-4,5-dihydro-5-phenyl-1H-pyrazole (sery136)}

The title compound was prepared according to the GEP 13 starting from sery89 to provide sery136 as a white solid. Yield 85\%. m.p.: 108-110 ${ }^{\circ} \mathrm{C} .{ }^{1} \mathrm{H}$ NMR $\left(400 \mathrm{MHz}, \mathrm{CDCl}_{3}\right) \delta=$ 7.44-7.23 (m, 6H), $7.05(\mathrm{~d}, J=7.9 \mathrm{~Hz}, 1 \mathrm{H}), 6.81(\mathrm{~d}, J=8.0 \mathrm{~Hz}, 1 \mathrm{H}), 6.10-5.82(\mathrm{~m}, 3 \mathrm{H}), 4.92$ $(\mathrm{t}, J=9.4 \mathrm{~Hz}, 1 \mathrm{H}), 3.44(\mathrm{dd}, J=16.2,10.6 \mathrm{~Hz}, 1 \mathrm{H}), 3.02(\mathrm{dd}, J=16.2,8.6 \mathrm{~Hz}, 1 \mathrm{H}) .{ }^{13} \mathrm{C}$ NMR $\left(100.6 \mathrm{MHz}, \mathrm{CDCl}_{3}\right) \delta=151.2,148.4,148.1,142.9,129.0,127.9,127.4,126.4,120.6$, 108.2, 106.1, 101.4, 64.4, 41.7. HRMS $(m / z)[\mathrm{M}+\mathrm{H}]^{+}$calcd for $\mathrm{M}=\mathrm{C}_{16} \mathrm{H}_{14} \mathrm{~N}_{2} \mathrm{O}_{2}, 267.1134$; found, 267.1133.

\section{(E)-3-(3,4-Dihydroxyphenyl)-1-(3,4-dimethoxyphenyl)-2-propene-1-one (sery131)}

The title compound has been reported previously ${ }^{53}$ (CAS 152809-90-0) and prepared according to the published protocol ${ }^{553}$ using 1-(3,4-dimethoxyphenyl)ethanone, 3,4Di(tetrahydropyran-2-oxy)benzaldehyde to provide sery131 as a yellow solid. Yield $40 \%$. m.p.: $142-144{ }^{\circ} \mathrm{C}\left(\right.$ lit $\left.^{553} 132-137{ }^{\circ} \mathrm{C}\right) .{ }^{1} \mathrm{H}$ NMR (400 MHz, DMSO[D $\left.]\right) \delta=9.38$ (bs, $\left.1,6 \mathrm{H}\right)$, $7.83(\mathrm{dd}, J=8.5,1.8 \mathrm{~Hz}, 1 \mathrm{H}), 7.67-7.53(\mathrm{~m}, 3 \mathrm{H}), 7.26(\mathrm{~d}, J=1.8 \mathrm{~Hz}, 1 \mathrm{H}), 7.18(\mathrm{dd}, J=8.2$, $1.8 \mathrm{~Hz}, 1 \mathrm{H}), 7.08(\mathrm{~d}, J=8.5 \mathrm{~Hz}, 1 \mathrm{H}), 7.81(\mathrm{~d}, J=8.2 \mathrm{~Hz}, 1 \mathrm{H}), 3.86(\mathrm{~s}, 3 \mathrm{H}), 3.85(\mathrm{~s}, 3 \mathrm{H}) .{ }^{13} \mathrm{C}$ NMR (100.6 MHz, DMSO[D 6$]) \delta=187.2,152.9,148.7,148.5,145.6,144.0,130.9,126.4$, $122.9,122.0,118.3,115.7,115.5,110.9,110.7,55.7,55.6$.

\section{4,5-Dihydro-5-(3,4-dihydroxyphenyl)-3-(3,4-dimethoxyphenyl)-1H-pyrazole (sery144)}

The title compound was prepared according to the GEP 13 starting from sery131 to provide sery144 as a white solid. Yield 53\%. m.p.: 190-191 ${ }^{\circ} \mathrm{C} .{ }^{1} \mathrm{H}$ NMR $\left(400 \mathrm{MHz}, \mathrm{DMSO}\left[\mathrm{D}_{6}\right]\right) \delta=$ $8.80(\mathrm{~s}, 1.9 \mathrm{H}), 7.26(\mathrm{~d}, J=1.3 \mathrm{~Hz}, 1 \mathrm{H}), 7.19(\mathrm{~d}, J=2.5 \mathrm{~Hz}, 1 \mathrm{H}), 7.06(\mathrm{dd}, J=8.2,1.3 \mathrm{~Hz}$, $1 \mathrm{H}), 6.94(\mathrm{~d}, J=8.2 \mathrm{~Hz}, 1 \mathrm{H}), 6.76(\mathrm{~d}, J=1.5 \mathrm{~Hz}, 1 \mathrm{H}), 6.66(\mathrm{~d}, J=8.1 \mathrm{~Hz}, 1 \mathrm{H}), 6.59(\mathrm{dd}, J=$ $8.1,1.5 \mathrm{~Hz}, 1 \mathrm{H}), 4.62(\mathrm{td}, J=10.3,2.5 \mathrm{~Hz}, 1 \mathrm{H}), 3.77-3.76(6 \mathrm{H}), 3.40-3.24(\mathrm{~m}, 1 \mathrm{H}$, overlapping with water), $2.74(\mathrm{dd}, J=16.2,10.3 \mathrm{~Hz}, 1 \mathrm{H}) .{ }^{13} \mathrm{C}$ NMR $(100.6 \mathrm{MHz}$, DMSO[D 6 ]) $\delta=149.1,148.7,148.6,145.2,144.4,134.1,126.3,118.6,117.4,115.3,113.8$, 111.4, 108.2, 63.3, 55.5, 55.3, 40.8. HRMS $(\mathrm{m} / \mathrm{z})[\mathrm{M}+\mathrm{H}]^{+}$calcd for $\mathrm{M}=\mathrm{C}_{17} \mathrm{H}_{18} \mathrm{~N}_{2} \mathrm{O}_{4}$, 315.1345; found, 315.1346 .

\section{4,5-Dihydro-3-(3,4-dimethoxyphenyl)-5-(3-fluorophenyl)-1H-pyrazole (sery145)}

The title compound was prepared according to the GEP 13 starting from sery97 to provide sery145 as a white solid. Yield $62 \%$. m.p.: $119-122{ }^{\circ} \mathrm{C} .{ }^{1} \mathrm{H}$ NMR $\left(400 \mathrm{MHz}, \mathrm{CDCl}_{3}\right) \delta=7.41$ $(\mathrm{d}, J=1.6 \mathrm{~Hz}, 1 \mathrm{H}), 7.34-7.27(\mathrm{~m}, 1 \mathrm{H}), 7.17-7.08(\mathrm{~m}, 2 \mathrm{H}), 7.02(\mathrm{dd}, J=8.3,1.7 \mathrm{~Hz}, 1 \mathrm{H}), 6.97$ (td, $J=8.3,2.0 \mathrm{~Hz}, 1 \mathrm{H}), 6.84(\mathrm{~d}, J=8.3 \mathrm{~Hz}, 1 \mathrm{H}), 5.92(\mathrm{bs}, 1 \mathrm{H}), 4.90$ (t, $J=9.8 \mathrm{~Hz}, 1 \mathrm{H}), 3.92$ $(\mathrm{s}, 3 \mathrm{H}), 3.90(\mathrm{~s}, 3 \mathrm{H}), 3.47(\mathrm{dd}, J=16.3,10.6 \mathrm{~Hz}, 1 \mathrm{H}), 3.00(\mathrm{~d}, J=16.3,9.0 \mathrm{~Hz}, 1 \mathrm{H}) .{ }^{13} \mathrm{C}$ NMR $\left(100.6 \mathrm{MHz}, \mathrm{CDCl}_{3}\right) \delta=163.2(\mathrm{~d}, J=246.7 \mathrm{~Hz}), 151.5,150.2,149.3,145.6(\mathrm{~d}, J=6.5$ $\mathrm{Hz}), 130.5(\mathrm{~d}, J=8.2 \mathrm{~Hz}), 125.8,122.1(\mathrm{~d}, J=2.6 \mathrm{~Hz}), 119.5,114.7(\mathrm{~d}, J=21.2 \mathrm{~Hz}), 113.4$ $(\mathrm{d}, J=21.9 \mathrm{~Hz}), 110.7,108.4,63.9,56.05,56.0,41.7$. HRMS $(m / z)[\mathrm{M}+\mathrm{H}]^{+}$calcd for $\mathrm{M}=$ $\mathrm{C}_{17} \mathrm{H}_{17} \mathrm{FN}_{2} \mathrm{O}_{2}, 301.1352$; found, 301.1350. 


\subsubsection{Synthesis of 2,4-diaryl-1H-pyrroles ${ }^{405}$}

\section{1-(1,3-Benzodioxol-5-yl)-4-nitro-3-phenylbutane-1-one (anle130a)}

The title compound was prepared according to the published protocol ${ }^{405}$. A solution of sery89 $(757 \mathrm{mg}, 3 \mathrm{mmol})$ in $\mathrm{MeOH}(30 \mathrm{~mL})$ was treated with $\mathrm{Et}_{2} \mathrm{NH}(2.07 \mathrm{ml}, 1.46 \mathrm{~g}, 20 \mathrm{mmol})$ and $\mathrm{CH}_{3} \mathrm{NO}_{2}(1.08 \mathrm{ml}, 1.22 \mathrm{~g}, 20 \mathrm{mmol})$ and heated under reflux for $15 \mathrm{~h}$. The solvent was evaporated under reduced pressure, the residue was partitioned between $\mathrm{CH}_{2} \mathrm{Cl}_{2}(30 \mathrm{ml})$ and water $(30 \mathrm{ml})$, and acidified with $1 \mathrm{M} \mathrm{HCl}$. The aqueous layer was extracted with a further portion of $\mathrm{CH}_{2} \mathrm{Cl}_{2}(30 \mathrm{ml})$, and the combined organics were washed with water $(30 \mathrm{ml})$, brine $(30 \mathrm{ml})$ and dried over $\mathrm{Na}_{2} \mathrm{SO}_{4}$. The solvent was removed under reduced pressure, and the resulting oil was purified by column chromatography on silica gel (hexane:EtOAc, 2:1 v/v) to provide anle130a $(750 \mathrm{mg}, 80 \%)$ as a yellow oil. TLC (hexane:EtOAc, $2: 1 \mathrm{v} / \mathrm{v})$ : RF $=0.57$. ${ }^{1} \mathrm{H}$ NMR $\left(400 \mathrm{MHz}, \mathrm{CDCl}_{3}\right) \delta=7.52(\mathrm{dd}, J=8.2,1.7 \mathrm{~Hz}, 1 \mathrm{H}), 7.39(\mathrm{~d}, J=1.7 \mathrm{~Hz}, 1 \mathrm{H}), 7.37-$ $7.31(\mathrm{~m}, 2 \mathrm{H}), 7.31-7.24(\mathrm{~m}, 3 \mathrm{H}), 6.84(\mathrm{~d}, J=8.2 \mathrm{~Hz}, 1 \mathrm{H}), 6.04(\mathrm{~s}, 2 \mathrm{H}), 4.83$ and $4.82(2 \times \mathrm{d}$, $J=12.5 \mathrm{~Hz}, 1 \mathrm{H}), 4.69$ and $4.67(2 \times \mathrm{d}, J=12.5 \mathrm{~Hz}, 1 \mathrm{H}), 4.20(\mathrm{q}, J=7.1 \mathrm{~Hz}, 1 \mathrm{H}), 3.45-3.29$ $(\mathrm{m}, 2 \mathrm{H}) .{ }^{13} \mathrm{C}$ NMR $\left(100.6 \mathrm{MHz}, \mathrm{CDCl}_{3}\right) \delta=194.8,152.1,148.3,139.2,131.2,129.0,127.8$, $127.4,124.4,107.9,107.8,101.9,79.6,41.2,39.4$. HRMS $(\mathrm{m} / \mathrm{z})[\mathrm{M}+\mathrm{Na}]^{+}$calcd for $\mathrm{M}=$ $\mathrm{C}_{17} \mathrm{H}_{15} \mathrm{NO}_{5}, 336.0848$; found, 336.0847 .

\section{2-(1,3-Benzodioxol-5-yl)-4-phenyl-1H-pyrrole (anle130b)}

The title compound was prepared according to the published protocol ${ }^{405}$. A stirred solution of anle130a (707 $\mathrm{mg}, 2.25 \mathrm{mmol})$ in $\mathrm{MeOH}(20 \mathrm{ml})$ and THF $(40 \mathrm{ml})$ at room temperature was treated with $\mathrm{KOH}(633 \mathrm{mg}, 11.3 \mathrm{mmol})$. After $1 \mathrm{~h}$ the reaction mixture was added dropwise to a solution of $\mathrm{H}_{2} \mathrm{SO}_{4}(4 \mathrm{ml})$ in $\mathrm{MeOH}(20 \mathrm{ml})$ at $0{ }^{\circ} \mathrm{C}$ and stirred $1.5 \mathrm{~h}$ at room temperature. Water $(50 \mathrm{ml})$ and ice $(50 \mathrm{ml})$ were added, and the mixture was neutralized with aqueous $4 \mathrm{M}$ $\mathrm{NaOH}$ and extracted with $\mathrm{CH}_{2} \mathrm{Cl}_{2}(2 \times 50 \mathrm{ml})$. The combined organic fractions were washed with water $(50 \mathrm{ml})$ and brine $(50 \mathrm{ml})$, dried over $\mathrm{Na}_{2} \mathrm{SO}_{4}$ and evaporated under reduced pressure. The resulting oil was treated with $\mathrm{AcOH}(10 \mathrm{ml})$ and $\mathrm{NH}_{4} \mathrm{OAc}(870 \mathrm{mg}, 11.3$ mmol), the solution was heated at $100{ }^{\circ} \mathrm{C}$ for $1 \mathrm{~h}$. The reaction mixture was cooled down, ice $(50 \mathrm{ml})$ was added, and the mixture was neutralized with aqueous $4 \mathrm{M} \mathrm{NaOH}$. The solution was extracted with $\mathrm{CH}_{2} \mathrm{Cl}_{2}(2 \times 50 \mathrm{ml})$, the combined organic fractions were washed with brine $(50 \mathrm{ml})$, dried over $\mathrm{Na}_{2} \mathrm{SO}_{4}$ and evaporated under reduced pressure. The crude product was purified by column chromatography on silica gel $\left(\mathrm{CH}_{2} \mathrm{Cl}_{2}\right)$ followed by recrystallisation from $\mathrm{CHCl}_{3} /$ petroleum benzine $40-60{ }^{\circ} \mathrm{C}$ to provide anle130b $(321 \mathrm{mg}, 54 \%)$ as a pink solid. TLC $\left(\mathrm{CH}_{2} \mathrm{Cl}_{2}\right): \mathrm{RF}=0.7$. m.p.: $188-190{ }^{\circ} \mathrm{C}(\mathrm{dec}) .{ }^{1} \mathrm{H}$ NMR $\left(400 \mathrm{MHz}, \mathrm{CDCl}_{3}\right) \delta=8.32(\mathrm{bs}$, $1 \mathrm{H}), 7.56(\mathrm{dd}, J=8.1,1.0 \mathrm{~Hz}, 2 \mathrm{H}), 7.36(\mathrm{t}, J=7.7 \mathrm{~Hz}, 2 \mathrm{H}), 7.20(\mathrm{t}, J=7.4 \mathrm{~Hz}, 1 \mathrm{H}), 7.10$ (t, $J$ $=1.9 \mathrm{~Hz}, 1 \mathrm{H}), 7.01(\mathrm{~d}, J=1.5 \mathrm{~Hz}, 1 \mathrm{H}), 6.98(\mathrm{dd}, J=8.0,1.6 \mathrm{~Hz}, 1 \mathrm{H}), 6.84(\mathrm{~d}, J=8.0 \mathrm{~Hz}$, $1 \mathrm{H}), 6.69(\mathrm{t}, J=2.2 \mathrm{~Hz}, 1 \mathrm{H}), 5.99(\mathrm{~s}, 2 \mathrm{H}) .{ }^{13} \mathrm{C} \mathrm{NMR}\left(100.6 \mathrm{MHz}, \mathrm{CDCl}_{3}\right) \delta=148.2,146.5$, $135.5,133.1,128.6,127.1,126.5,125.7,125.2,117.3,115.0,108.7,105.1,103.4,101.1$. HRMS $(m / z)[\mathrm{M}+\mathrm{H}]^{+}$calcd for $\mathrm{M}=\mathrm{C}_{17} \mathrm{H}_{13} \mathrm{NO}_{2}, 264.1025$; found, 264.1023.

\section{3-(3,4-Dimethoxyphenyl)-4-nitro-1-phenylbutane-1-one (sery155)}

The title compound was prepared analogously to the procedure for anle130a using sery113 and purified by column chromatography on silica gel (hexane:EtOAc, 1:1 v/v) to provide sery155 as a white solid. Yield 79\%. TLC (hexane:EtOAc, 1:1 v/v): RF = 0.7. m.p.: 98-100 ${ }^{\circ} \mathrm{C} .{ }^{1} \mathrm{H}$ NMR $\left(400 \mathrm{MHz}, \mathrm{DMSO}\left[\mathrm{D}_{6}\right]\right) \delta=7.94(\mathrm{~d}, J=7.6 \mathrm{~Hz}, 2 \mathrm{H}), 7.63(\mathrm{t}, J=7.6 \mathrm{~Hz}, 1 \mathrm{H})$, $7.52(\mathrm{t}, J=7.6 \mathrm{~Hz}, 2 \mathrm{H}), 7.00(\mathrm{~s}, 1 \mathrm{H}), 6.84(\mathrm{~s}, 2 \mathrm{H}), 4.95(\mathrm{dd}, J=12.8,5.8 \mathrm{~Hz}, 1 \mathrm{H}), 4.83(\mathrm{dd}, J$ $=12.8,9.5 \mathrm{~Hz}, 1 \mathrm{H}), 3.99(\mathrm{~m}, 1 \mathrm{H}), 3.73(\mathrm{~s}, 3 \mathrm{H}), 3.70(\mathrm{~s}, 3 \mathrm{H}), 3.55(\mathrm{dd}, J=17.7,7.1 \mathrm{~Hz}, 1 \mathrm{H})$, $3.46(\mathrm{dd}, J=17.7,7.0 \mathrm{~Hz}, 1 \mathrm{H}) .{ }^{13} \mathrm{C} \mathrm{NMR}\left(100.6 \mathrm{MHz}, \mathrm{DMSO}\left[\mathrm{D}_{6}\right]\right) \delta=197.6,148.7,147.9$, 
136.5, 133.4, 132.2, 128.7, 127.9, 119.9, 111.6, 111.4, 79.8, 55.5, 55.4, 41.2, 39.0. HRMS $(m / z)[\mathrm{M}+\mathrm{H}]^{+}$calcd for $\mathrm{M}=\mathrm{C}_{18} \mathrm{H}_{19} \mathrm{NO}_{5}, 330.1341$; found, 330.1341 .

\section{4-(3,4-Dimethoxyphenyl)-2-phenyl-1 $H$-pyrrole (sery160)}

The title compound was prepared analogously to the procedure for anle130b starting from sery155 and purified by column chromatography on silica gel (hexane:EtOAc, 3:1 v/v) followed by recrystallisation from hexane/EtOAc to provide sery155 as a white solid. Yield 45\%. TLC (hexane:EtOAc, 3:1 v/v): RF $=0.25$. m.p.: $167-169{ }^{\circ} \mathrm{C} .{ }^{1} \mathrm{H}$ NMR $(400 \mathrm{MHz}$, DMSO[D 6$]) \delta=11.34(\mathrm{~s}, 1 \mathrm{H}), 7.68(\mathrm{~d}, J=7.5 \mathrm{~Hz}, 2 \mathrm{H}), 7.36(\mathrm{t}, J=7.5 \mathrm{~Hz}, 2 \mathrm{H}), 7.26(\mathrm{~s}, 1 \mathrm{H})$, 7.20-7.14 (m, 2H), 7.12 (dd, $J=8.2,1.9 \mathrm{~Hz}, 1 \mathrm{H}), 6.94-6.87(\mathrm{~m}, 2 \mathrm{H}), 3.82(\mathrm{~s}, 3 \mathrm{H}), 3.74(\mathrm{~s}$, $3 \mathrm{H}) .{ }^{13} \mathrm{C}$ NMR $\left(100.6 \mathrm{MHz}, \mathrm{DMSO}\left[\mathrm{D}_{6}\right]\right) \delta=149.0,146.7,132.8,131.9,128.9,128.7,125.5$, $124.9,123.3,116.5,116.0,112.3,108.9,103.3,55.6,55.5$. HRMS $(m / z)[\mathrm{M}+\mathrm{H}]^{+}$calcd for $\mathrm{M}$ $=\mathrm{C}_{18} \mathrm{H}_{17} \mathrm{NO}_{2}, 280.1338$; found, 330.1337 .

\section{4-(3,4-Dihydroxyphenyl)-2-phenyl-1H-pyrrole (sery166b)}

The title compound was prepared according to the GEP 3 starting from anle160 and purified by column chromatography on silica gel $\left(\mathrm{CHCl}_{3}: \mathrm{MeOH}, 95: 5 \mathrm{v} / \mathrm{v}\right)$ followed by recrystallisation from $\mathrm{CHCl}_{3} / \mathrm{CH}_{3} \mathrm{CN}$. Brown solid. Yield 50\%. TLC $\left(\mathrm{CHCl}_{3}: \mathrm{MeOH}, 95: 5\right.$ v/v): RF = 0.4. m.p.: $>300{ }^{\circ} \mathrm{C} .{ }^{1} \mathrm{H}$ NMR (400 MHz, DMSO[D $\left.]\right) \delta=11.24(\mathrm{~s}, 1 \mathrm{H}), 8.69(\mathrm{~s}$, 2H), $7.65(\mathrm{~d}, J=7.4 \mathrm{~Hz}, 2 \mathrm{H}), 7.35(\mathrm{t}, J=7.4 \mathrm{~Hz}, 2 \mathrm{H}), 7.15(\mathrm{t}, J=7.4 \mathrm{~Hz}, 1 \mathrm{H}), 7.07(\mathrm{~s}, 1 \mathrm{H})$, $6.95(\mathrm{~d}, J=2.0 \mathrm{~Hz}, 1 \mathrm{H}), 6.85(\mathrm{dd}, J=8.1,2.0 \mathrm{~Hz}, 1 \mathrm{H}), 6.74(\mathrm{~s}, 1 \mathrm{H}), 6.69(\mathrm{~d}, J=8.1 \mathrm{~Hz}, 1 \mathrm{H})$. ${ }^{13} \mathrm{C}$ NMR (100.6 MHz, DMSO[D 6 ) $\delta=145.3,143.1,132.8,131.7,128.7,127.4,125.5$, $125.3,123.3,115.8,115.7,115.2,112.4,102.9$. HRMS $(\mathrm{m} / z)[\mathrm{M}+\mathrm{H}]^{+}$calcd for $\mathrm{M}=$ $\mathrm{C}_{16} \mathrm{H}_{13} \mathrm{NO}_{2}, 252.1025$; found, 252.1023.

\section{1,3-Bis(3,4-dimethoxyphenyl)-4-nitrobutane-1-one (sery154)}

The title compound was prepared analogously to the procedure for anle130a using sery112 and purified by column chromatography on silica gel (hexane:EtOAc, 1:1 v/v) followed by recrystallisation from $\mathrm{CH}_{2} \mathrm{Cl}_{2} / \mathrm{Et}_{2} \mathrm{O}$ to provide sery154 as a white solid. Yield $73 \%$. TLC (hexane:EtOAc, 1:1 v/v): RF =0.33. m.p.: $134{ }^{\circ} \mathrm{C} .{ }^{1} \mathrm{H}$ NMR $\left(400 \mathrm{MHz}, \mathrm{DMSO}\left[\mathrm{D}_{6}\right]\right) \delta=7.63$ (dd, $J=8.4,1.8 \mathrm{~Hz}, 1 \mathrm{H}), 7.40(\mathrm{~d}, J=1.8 \mathrm{~Hz}, 1 \mathrm{H}), 7.05(\mathrm{~d}, J=8.4 \mathrm{~Hz}, 1 \mathrm{H}), 7.00(\mathrm{~s}, 1 \mathrm{H}), 6.83$ $(\mathrm{s}, 2 \mathrm{H}), 4.98-4.78(\mathrm{~m}, 2 \mathrm{H}), 3.98(\mathrm{~m}, 1 \mathrm{H}), 3.83(\mathrm{~s}, 3 \mathrm{H}), 3.79(\mathrm{~s}, 3 \mathrm{H}), 3.73(\mathrm{~s}, 3 \mathrm{H}), 3.69(\mathrm{~s}, 3 \mathrm{H})$, $3.52(\mathrm{dd}, J=17.4,7.3 \mathrm{~Hz}, 1 \mathrm{H}), 3.34(\mathrm{dd}, J=17.4,6.9 \mathrm{~Hz}, 1 \mathrm{H}) .{ }^{13} \mathrm{C}$ NMR $(100.6 \mathrm{MHz}$, DMSO[D 6 ]) $\delta=196.0,153.2,148.6,148.5,147.9,132.3,129.4,122.8,119.9,111.6,111.5$, $110.8,110.2,79.8,55.8,55.6,55.5,55.4,40.7,39.3$. HRMS $(m / z)[\mathrm{M}+\mathrm{Na}]^{+}$calcd for $\mathrm{M}=$ $\mathrm{C}_{20} \mathrm{H}_{23} \mathrm{NO}_{7}, 412.1372$; found, 412.1365 .

\section{2,4-Bis(3,4-dimethoxyphenyl)-1H-pyrrole (sery167)}

The title compound was prepared analogously to the procedure for anle130b starting from sery154 and purified by column chromatography on silica gel (hexane:EtOAc, 1:1 v/v) to provide sery167 as a white solid. Yield 39\%. TLC (hexane:EtOAc, 1:1 v/v): RF $=0.39$. m.p.: 181-182 ${ }^{\circ} \mathrm{C} .{ }^{1} \mathrm{H}$ NMR $\left(400 \mathrm{MHz}, \mathrm{CDCl}_{3}\right) \delta=8.42(\mathrm{bs}, 1 \mathrm{H}), 7.12(\mathrm{dd}, J=8.2,2.0 \mathrm{~Hz}, 1 \mathrm{H})$, $7.08(\mathrm{~d}, J=2.0 \mathrm{~Hz}, 1 \mathrm{H}), 7.06(\mathrm{~d}, J=2.0 \mathrm{~Hz}, 1 \mathrm{H}), 7.05-6.99(\mathrm{~m}, 2 \mathrm{H}), 6.88(\mathrm{~d}, J=8.2 \mathrm{~Hz}, 2 \mathrm{H})$, $6.65(\mathrm{dd}, J=2.6,1.7 \mathrm{~Hz}, 1 \mathrm{H}), 3.94(\mathrm{~s}, 3 \mathrm{H}), 3.93(\mathrm{~s}, 3 \mathrm{H}), 3.90(\mathrm{~s}, 3 \mathrm{H}), 3.89(\mathrm{~s}, 3 \mathrm{H}) .{ }^{13} \mathrm{C} \mathrm{NMR}$ $\left(100.6 \mathrm{MHz}, \mathrm{CDCl}_{3}\right) \delta=149.3,149.1,148.0,147.4,133.1,128.9,126.4,126.0,117.4,116.2$, $114.4,111.6(2 \mathrm{C}), 109.0,108.0,103.1,56.0(3 \mathrm{C}), 55.9$. HRMS $(\mathrm{m} / \mathrm{z})[\mathrm{M}+\mathrm{H}]^{+}$calcd for $\mathrm{M}=$ $\mathrm{C}_{20} \mathrm{H}_{21} \mathrm{NO}_{4}, 340.1549$; found, 340.1553 . 


\subsubsection{Synthesis of other compounds}

\section{3-(1,3-Benzodioxol-5-yl)-4,5-dihydro-4-hydroxy-5-phenyl-1 $H$-pyrazole (anle127b)}

The title compound was prepared according to the published protocol ${ }^{398}$. A mixture of trans1-(1,3-benzodioxol-5-yl)-2,3-epoxy-3-phenylpropane-1-one s54 $\left.^{587} \mathrm{mg}, 2.19 \mathrm{mmol}\right)$ and hydrazine hydrate $(181 \mu 1,186 \mathrm{mg}, 3.72 \mathrm{mmol})$ in ethanol $(10 \mathrm{ml})$ was heated under reflux for $6 \mathrm{~h}$. Solvent was evaporated and the residue crystallised from $\mathrm{MeOH}$ to provide anle127b (348 mg, 56\%) as a pale yellow solid. m.p.: $175-177{ }^{\circ} \mathrm{C} .{ }^{1} \mathrm{H}$ NMR (400 MHz, DMSO[D 6$\left.]\right) \delta$ $=7.56(\mathrm{~d}, J=3.3 \mathrm{~Hz}, 1 \mathrm{H}), 7.38-7.29(\mathrm{~m}, 4 \mathrm{H}), 7.28-7.19(\mathrm{~m}, 3 \mathrm{H}), 6.90(\mathrm{~d}, J=8.0 \mathrm{~Hz}, 1 \mathrm{H})$, $6.01(\mathrm{~s}, 2 \mathrm{H}), 5.95(\mathrm{~d}, J=7.8 \mathrm{~Hz}, 1 \mathrm{H}), 4.91(\mathrm{t}, J=7.3 \mathrm{~Hz}, 1 \mathrm{H}), 4.49(\mathrm{dd}, \mathrm{J}=7.0,3.3 \mathrm{~Hz}, 1 \mathrm{H})$. ${ }^{13} \mathrm{C}$ NMR (100.6 MHz, DMSO[D6]) $\delta=149.2$, 147.5, 147.2, 141.7, 128.6, 127.4, 127.1, $126.7,120.0,108.3,105.7,101.1,82.3,72.3$. HRMS $(m / z)[\mathrm{M}+\mathrm{H}]^{+}$calcd for $\mathrm{M}=$ $\mathrm{C}_{16} \mathrm{H}_{14} \mathrm{~N}_{2} \mathrm{O}_{3}, 283.1083$; found, 283.1084 .

\section{3-(1,3-Benzodioxol-5-yl)-4-hydroxy-5-phenyl-1H-pyrazole (anle127c)}

The title compound was prepared according to the published protocol ${ }^{555}$. A solution of bromine $(62 \mu \mathrm{l}, 192 \mathrm{mg}, 1.20 \mathrm{mmol})$ in $\mathrm{CHCl}_{3}(2 \mathrm{ml})$ was added to a suspension of anle127b (340 mg, $1.20 \mathrm{mmol}$ ) in $\mathrm{CHCl}_{3}(5 \mathrm{ml})$. The mixture was stirred for $30 \mathrm{~min}$, the resulting precipitate was filtered off, washed with $\mathrm{CHCl}_{3}(5 \mathrm{ml})$, and dried $10 \mathrm{~min}$ on the air. The solid was dissolved in DMSO (3 ml), the solution was added slowly to a ice water $(30 \mathrm{ml})$ at stirring, the resulting precipitate was collected by filtration and dried. The resulting crude product was purified by column chromatography on silica gel (hexane:EtOAc, 2:1 v/v) to provide anle127c $(70 \mathrm{mg}, 20 \%)$ as a white solid. TLC (hexane:EtOAc, $2: 1 \mathrm{v} / \mathrm{v}$ ): RF $=0.21$. m.p.: $218-219^{\circ} \mathrm{C} .{ }^{1} \mathrm{H}$ NMR $\left(400 \mathrm{MHz}, \mathrm{DMSO}\left[\mathrm{D}_{6}\right]+1-2 \%\right.$ conc. DCl) $\delta=8.23$ (bs, $\left.1 \mathrm{H}\right), 7.89$ $(\mathrm{d}, J=7.6 \mathrm{~Hz}, 2 \mathrm{H}), 7.53-7.36(\mathrm{~m}, 4 \mathrm{H}), 7.28(\mathrm{t}, J=7.4 \mathrm{~Hz}, 1 \mathrm{H}), 6.99(\mathrm{~d}, J=8.6 \mathrm{~Hz}, 1 \mathrm{H}), 6.04$ (s, 2H). ${ }^{13} \mathrm{C}$ NMR $\left(100.6 \mathrm{MHz}, \mathrm{DMSO}\left[\mathrm{D}_{6}\right]+1-2 \%\right.$ conc. DCl) $\delta=147.6,146.5,135.8(2 \mathrm{C})$, $134.9,131.7,128.8,127.2,125.9,125.7,119.5,108.8,106.1,101.2$. HRMS $(m / z)[\mathrm{M}+\mathrm{H}]^{+}$ calcd for $\mathrm{M}=\mathrm{C}_{16} \mathrm{H}_{12} \mathrm{~N}_{2} \mathrm{O}_{3}, 281.0926$; found, 281.0928.

\section{trans-1-(1,3-Benzodioxol-5-yl)-3-(3-bromophenyl)-2,3-epoxypropane-1-one (sery298b)}

The title compound was prepared according to the published protocol ${ }^{398}$ starting from sery298a to provide sery298b as a white solid. Yield 93\%. m.p.: 82-83 ${ }^{\circ} \mathrm{C} .{ }^{1} \mathrm{H}$ NMR $(400$ $\left.\mathrm{MHz}, \mathrm{DMSO}\left[\mathrm{D}_{6}\right]\right) \delta=7.74(\mathrm{dd}, J=8.2,1.3 \mathrm{~Hz}, 1 \mathrm{H}), 7.63(\mathrm{~s}, 1 \mathrm{H}), 7.58(\mathrm{~d}, J=8.0 \mathrm{~Hz}, 1 \mathrm{H})$, $7.51(\mathrm{~d}, J=1.3 \mathrm{~Hz}, 1 \mathrm{H}), 7.46(\mathrm{~d}, J=7.7 \mathrm{~Hz}, 1 \mathrm{H}), 7.37(\mathrm{t}, J=7.7 \mathrm{~Hz}, 1 \mathrm{H}), 7.08(\mathrm{~d}, J=8.2 \mathrm{~Hz}$, $1 \mathrm{H}), 6.16(\mathrm{~s}, 1 \mathrm{H}), 6.15(\mathrm{~s}, 1 \mathrm{H}), 4.83(\mathrm{~d}, J=1.4 \mathrm{~Hz}, 1 \mathrm{H}), 4.13(\mathrm{~d}, J=1.4 \mathrm{~Hz}, 1 \mathrm{H}) .{ }^{13} \mathrm{C}$ NMR (100.6 MHz, DMSO[D 6$]) \delta=190.6,152.3,148.1,138.6,131.6,130.6,130.0,129.0,125.7$, $125.4,121.9,108.4,107.3,102.3,59.1,57.5$. HRMS $(\mathrm{m} / \mathrm{z})[\mathrm{M}+\mathrm{Na}]^{+}$calcd for $\mathrm{M}=$ $\mathrm{C}_{16} \mathrm{H}_{11}{ }^{79} \mathrm{BrO}_{4}, 368.9738$; found, 368.9741.

\section{3-(1,3-Benzodioxol-5-yl)-5-(3-bromophenyl)-4,5-dihydro-4-hydroxy-1H-pyrazole (sery300a)}

The title compound was prepared using an analogous procedure to that described for anle127b starting from sery $298 \mathrm{~b}$ and purified by recrystallisation from methanol to provide sery300a as a white solid. Yield 51\%. m.p.: 180-182 ${ }^{\circ} \mathrm{C} .{ }^{1} \mathrm{H}$ NMR $\left(400 \mathrm{MHz}, \mathrm{DMSO}\left[\mathrm{D}_{6}\right]\right) \delta$ $=7.62(\mathrm{~d}, J=3.4 \mathrm{~Hz}, 1 \mathrm{H}), 7.49-7.44(\mathrm{~m}, 2 \mathrm{H}), 7.34-7.29(\mathrm{~m}, 2 \mathrm{H}), 7.26-7.21(\mathrm{~m}, 2 \mathrm{H}), 6.90(\mathrm{~d}$, $J=8.0 \mathrm{~Hz}, 1 \mathrm{H}), 6.04-5.98(\mathrm{~m}, 3 \mathrm{H}), 4.92(\mathrm{t}, J=7.4 \mathrm{~Hz}, 1 \mathrm{H}), 4.51(\mathrm{dd}, J=7.0,3.4 \mathrm{~Hz}, 1 \mathrm{H})$. ${ }^{13} \mathrm{C}$ NMR (100.6 MHz, DMSO[D 6 ) $\delta=149.2,147.3,147.1,144.4,130.7,130.0,129.2$, $126.7,125.6,121.7,119.9,108.2,105.6,101.0,82.0,71.3$. HRMS $(m / z)[\mathrm{M}+\mathrm{H}]^{+}$calcd for $\mathrm{M}$ $=\mathrm{C}_{16} \mathrm{H}_{13}{ }^{79} \mathrm{BrN}_{2} \mathrm{O}_{3}, 361.0188$; found, 361.0191. 


\section{$\mathrm{N}$-[4-[5-(3-Bromohenyl)-1H-pyrazol-3-yl]phenyl]formamide (ruli16)}

The title compounds was prepared according to published protocol ${ }^{410}$. Sery384 (2g, 6.4 mmol) was dissolved in formic acid $(8.0 \mathrm{~mL}, 9.76 \mathrm{~g}, 212 \mathrm{mmol}, 33 \mathrm{Eq}$.$) and stirred for 10 \mathrm{~h}$ at $80^{\circ} \mathrm{C}$. After completion of the reaction, the reaction mixture was diluted with water $(20 \mathrm{~mL})$, the resulting precipitate was collected by filtration, washed with water $(10 \mathrm{~mL})$ and dried. Recrystallization from EtOH / THF (150 mL, 2 / 1) provided the title compound ruli16 (1.20 g, $3.51 \mathrm{mmol}, 55 \%)$ as a yellow solid. m.p.: $260{ }^{\circ} \mathrm{C}$. ${ }^{1} \mathrm{H}$ NMR $\left(400 \mathrm{MHz}, \mathrm{DMSO}\left[\mathrm{D}_{6}\right]+1-2 \%\right.$ conc. DCl) $\delta=8.87(\mathrm{~d}, J=11.2 \mathrm{~Hz}, 0.3 \mathrm{H}), 8.31(\mathrm{~s}, 0.7 \mathrm{H}, \mathrm{CHO}), 8.04(\mathrm{~s}, 1 \mathrm{H}), 7.84(\mathrm{~d}, J=7.5$ $\mathrm{Hz}, 1 \mathrm{H}), 7.78(\mathrm{~d}, J=7.8 \mathrm{~Hz}, 2 \mathrm{H}), 7.67(\mathrm{~d}, J=7.3 \mathrm{~Hz}, 1.5 \mathrm{H}), 7.52(\mathrm{~d}, J=7.7 \mathrm{~Hz}, 1 \mathrm{H}), 7.41$ $(\mathrm{t}, J=7.8 \mathrm{~Hz}, 1 \mathrm{H}), 7.29(\mathrm{~d}, J=8.0 \mathrm{~Hz}, 0.5 \mathrm{H}), 7.22(\mathrm{~s}, 1 \mathrm{H}) .{ }^{13} \mathrm{C}$ NMR $(100.6 \mathrm{MHz}$, DMSO $\left[\mathrm{D}_{6}\right]+1-2 \%$ conc. DCl) $\delta=162.9,160.1,138.8,131.5,130.7,128.0,126.7,126.2$, $124.5,122.7,119.9,118.1$ (2C), 100.3 (pyrazole signals we not detected). ESI-MS $(\mathrm{m} / \mathrm{z})$ $[\mathrm{M}+\mathrm{H}]^{+}$calcd for $\mathrm{M}=\mathrm{C}_{16} \mathrm{H}_{12}{ }^{79} \mathrm{BrN}_{3} \mathrm{O}, 342.02$; found, 341.95 .

\section{3-(3-Bromophenyl)-5-[4-(methylamino)phenyl]-1H-pyrazole (ruli22)}

The title compounds was prepared according to published protocol ${ }^{411}$. Borane dimethylsulfide (5.55 mL, $4.44 \mathrm{~g}, 58.4 \mathrm{mmol}, 2.00 \mathrm{Eq}$.) was added dropwise over $10 \mathrm{~min}$ at $0{ }^{\circ} \mathrm{C}$ to a suspension of ruli16 (10.0 g, $29.2 \mathrm{mmol}, 1.00 \mathrm{Eq}$.) in THF. The solution was stirred for 30 min at $0{ }^{\circ} \mathrm{C}$ and for $2 \mathrm{~h}$ at room temperature. An additional amount of borane dimethylsulfide $(1 \mathrm{~mL}, 1.25 \mathrm{~g}, 16.5 \mathrm{mmol}, 0.56 \mathrm{Eq}$.$) and THF (15 \mathrm{~mL})$ were added and the reaction mixture was stirred for $2 \mathrm{~h}$ at room temperature. After addition of $\mathrm{MeOH}(80 \mathrm{~mL})$ and aq. $\mathrm{HCl}(1 \mathrm{~N}$, $250 \mathrm{~mL}$ ) the solution was stirred overnight at room temperature. A $10 \%$ aq. $\mathrm{NaOH}$ solution $(150 \mathrm{~mL})$ was added and the mixture was stirred for $30 \mathrm{~min}$. The mixture was extracted with EtOAc $(150 \mathrm{~mL})$, the organic phase was washed with aq. $\mathrm{NaHCO}_{3}(150 \mathrm{~mL})$ and brine $(150$ $\mathrm{mL})$ and dried over $\mathrm{Na}_{2} \mathrm{SO}_{4}$. Recrystallization a mixture EtOH$/ \mathrm{H}_{2} \mathrm{O}=1 / 1(400 \mathrm{~mL})$ provided the title compound ruli22 (7.05 g, $74 \%)$ as a white solid. m.p.: $217{ }^{\circ} \mathrm{C} .{ }^{1} \mathrm{H}$ NMR $(400 \mathrm{MHz}$, DMSO $\left[\mathrm{D}_{6}\right]+1-2 \%$ conc. DCl) $\delta=8.07(\mathrm{~s}, 1 \mathrm{H}), 7.98(\mathrm{~d}, J=8.4 \mathrm{~Hz}, 2 \mathrm{H}), 7.87(\mathrm{~d}, J=8.4 \mathrm{~Hz}$, $1 \mathrm{H}), 7.67$ (d, $J=7.3 \mathrm{~Hz}, 2 \mathrm{H}), 7.54$ (d, $J=7.9 \mathrm{~Hz}, 1 \mathrm{H}), 7.43$ (t, $J=7.9 \mathrm{~Hz}, 1 \mathrm{H}), 7.37$ (s, 1H), $2.92(\mathrm{~s}, 3 \mathrm{H})$. 


\subsubsection{Experimental Procedures and characterisation data of prodrug compounds}

\subsubsection{Synthesis sery 432}

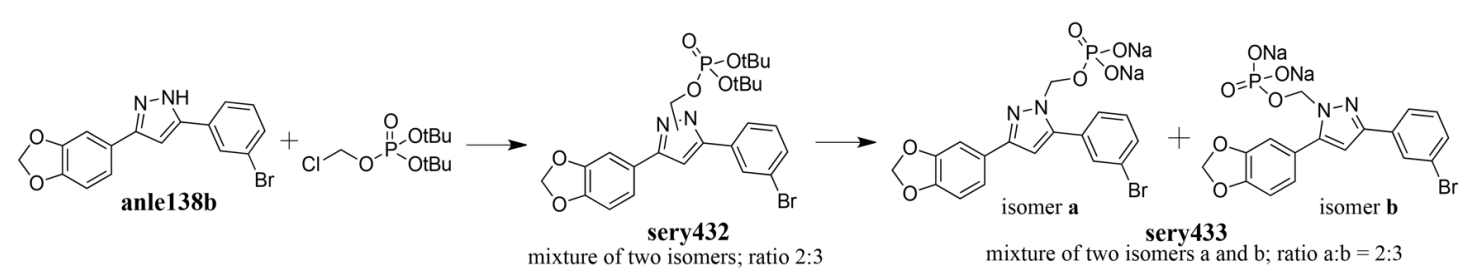

[3-(1,3-Benzodioxol-5-yl)-5-(3-bromophenyl)-1H-pyrazol-1-yl]methyl di-tert-butyl phosphate and [5-(1,3-benzodioxol-5-yl)-3-(3-bromophenyl)-1H-pyrazol-1-yl]methyl ditert-butyl phosphate (sery432)

To a mixture of anle138b (25 g, $72.8 \mathrm{mmol}), \mathrm{Cs}_{2} \mathrm{CO}_{3}(35.6 \mathrm{~g}, 109 \mathrm{mmol})$ in DMSO $(200 \mathrm{ml})$ di-tert-butyl chloromethyl phosphate ${ }^{497}(28.1 \mathrm{~g}, 109 \mathrm{mmol})$ was added in one portion. After stirring at room temperature for $5 \mathrm{~h}$ the reaction mixture was diluted with water $(1200 \mathrm{ml})$ and extracted with diethyl ether $(500+200+100 \mathrm{ml})$. Combined organic fractions were washed with water $(500 \mathrm{ml})$, brine $(100 \mathrm{ml})$ and dried over $\mathrm{Na}_{2} \mathrm{SO}_{4}$. After sodium sulphate was filtered the solution was concentrated under a reduced pressure to provide the sery 432 as oil $(40.8 \mathrm{~g}, 82 \%)$ as a mixture of two isomers in ratio $2: 3$. The resulting product also contains $10.5 \%$ of di-tert-butyl chloromethyl phosphate and $7.1 \% \mathrm{Bu}_{3} \mathrm{~N}$ based on ${ }^{1} \mathrm{H}$ NMR spectrum. The product was used in next step without further purification. ${ }^{1} \mathrm{H}$ NMR $\left(400 \mathrm{MHz}, \mathrm{CDCl}_{3}\right) \delta$ $=8.02(\mathrm{t}, J=1.8 \mathrm{~Hz}, 1 \mathrm{H}), 7.77(\mathrm{dt}, J=7.9,1.2 \mathrm{~Hz}, 1 \mathrm{H}), 7.75(\mathrm{t}, J=1.7 \mathrm{~Hz}, 0.7 \mathrm{H}), 7.60-7.54$ (m, 1.4H), $7.45(\mathrm{ddd}, J=8.0,1.9,0.9 \mathrm{~Hz}, 1 \mathrm{H}), 7.39-7.28(\mathrm{~m}, 2.1 \mathrm{H}), 7.26(\mathrm{~m}, 1 \mathrm{H}), 7.11-7.06$ $(\mathrm{m}, 2 \mathrm{H}), 6.91(\mathrm{~d}, J=7.9 \mathrm{~Hz}, 1 \mathrm{H}), 6.86(\mathrm{~d}, J=8.1 \mathrm{~Hz}, 0.7 \mathrm{H}), 6.60(\mathrm{~s}, 1.4 \mathrm{H}), 6.04(\mathrm{~s}, 2 \mathrm{H}), 6.00$ $(\mathrm{s}, 1.4 \mathrm{H}), 5.87-5.82(\mathrm{~m}, 3.4 \mathrm{H}), 1.51-1.48(\mathrm{~m}, 31 \mathrm{H})$. ESI-MS $(\mathrm{m} / \mathrm{z})[\mathrm{M}+\mathrm{H}]^{+}$calcd for $\mathrm{M}=$ $\mathrm{C}_{25} \mathrm{H}_{30}{ }^{79} \mathrm{BrN}_{2} \mathrm{O}_{6} \mathrm{P}, 565.11$; found, 565.21.

[3-(1,3-Benzodioxol-5-yl)-5-(3-bromophenyl)-1H-pyrazol-1-yl]methyl disodium phosphate and [5-(1,3-benzodioxol-5-yl)-3-(3-bromophenyl)-1H-pyrazol-1-yl]methyl disodium phosphate (sery433)

To a cooled solution of sery432 $(34.4 \mathrm{~g}, 60.9 \mathrm{mmol})$ in DCM $(400 \mathrm{ml})$ TFA $(23.5 \mathrm{ml})$ was added in 5 minutes with continuous vigorous stirring at $0^{\circ} \mathrm{C}$. After $2 \mathrm{~h}$ an additional portion of TFA $(23 \mathrm{ml})$ was added and the reaction mixtures was stirred at $0^{\circ} \mathrm{C}$ for $8 \mathrm{~h}$. After dilution with cold toluene $(300 \mathrm{ml})$ the reaction mixture was concentrated under a reduced pressure at $0^{\circ} \mathrm{C}$, the residue was mixed with cold toluene $(300 \mathrm{ml})$ and concentrated once again at $0^{\circ} \mathrm{C}$. (DCM was evaporated at 50-100 mbar, TFA and toluene were evaporated with a high vacuum rotor evaporator). The resulting mixture was diluted with cold acetonitrile $(300 \mathrm{ml})$, stirred $1 \mathrm{~h}$ at $0^{\circ} \mathrm{C}$. A white precipitate was filtered off and dried under reduced pressure to provide sery433 in acid form (31.0 g total, $29.0 \mathrm{~g}$ of sery433 in acid form and $2 \mathrm{~g}$ acetonitrile based on NMR). To the crude sery433 $1 \mathrm{M}$ aqueous $\mathrm{NaOH}$ solution $(129 \mathrm{ml}, 129 \mathrm{mmol}, 2$ eq) and water $(80 \mathrm{ml})$ were slowly added, the resulting solution was filtered off (Millipore Express Plus filter) and the filtrate was lyophilized to provide disodium salt sery433 $(30.0 \mathrm{~g}, 99 \%$, a mixture of two isomers with ratio $2: 3)$ as a white powder. ${ }^{1} \mathrm{H}$ NMR $\left(400 \mathrm{MHz}, \mathrm{D}_{2} \mathrm{O}\right) \delta=7.63$ $(\mathrm{s}, 1 \mathrm{H}), 7.50(\mathrm{~d}, J=7.8 \mathrm{~Hz}, 1 \mathrm{H}), 7.45(\mathrm{~m}, 1.3 \mathrm{H}), 7.40(\mathrm{~d}, J=8.3 \mathrm{~Hz}, 0.65 \mathrm{H}), 7.33-7.25(\mathrm{~m}$, $1.65 \mathrm{H}), 7.16(\mathrm{t}, J=7.9 \mathrm{~Hz}, 1 \mathrm{H}), 6.98(\mathrm{~d}, J=8.2 \mathrm{~Hz}, 0.65 \mathrm{H}), 6.90(\mathrm{~s}, 0.65 \mathrm{H}), 6.86(\mathrm{~d}, J=8.5$ $\mathrm{Hz}, 1 \mathrm{H}), 6.76(\mathrm{~d}, J=8.0 \mathrm{~Hz}, 1 \mathrm{H}), 6.73-6.66(\mathrm{~m}, 1.65 \mathrm{H}), 6.21-6.15(\mathrm{~m}, 1.65 \mathrm{H}), 5.81(\mathrm{~s}, 2 \mathrm{H})$, 5.77 (s, 1.3H), 5.48 (bs, 3.3H). ${ }^{13} \mathrm{C}$ NMR $\left(100.6 \mathrm{MHz}, \mathrm{D}_{2} \mathrm{O}\right) \delta=151.5,150.1,147.7,147.3$, $147.25,147.2,146.1,144.7,133.7,131.9,131.0$ (2C), 130.7, 130.5, 130.3, 128.4, 127.0, 
125.7, 124.1, 122.7, 122.3, 122.2, 122.0, 119.6, 108.6 (2C), 105.9, 105.8, 103.8, 103.5, 101.5, 101.2, 72.5 (2C). ESI-MS (m/z) $[\mathrm{M}-\mathrm{H}]^{-}$calcd for $\mathrm{M}=\mathrm{C}_{17} \mathrm{H}_{14}{ }^{79} \mathrm{BrN}_{2} \mathrm{O}_{6} \mathrm{P}, 450.97$; found, 450.95 .

\subsubsection{Synthesis sery 453}
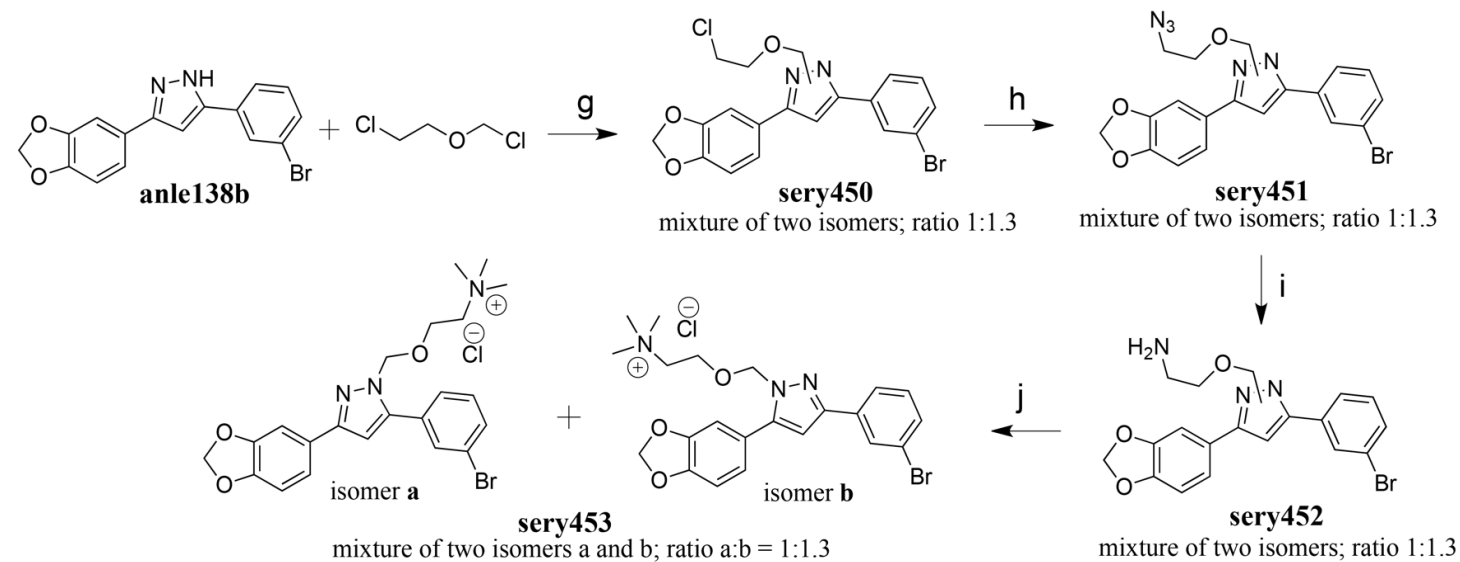

\section{5-(1,3-Benzodioxol-5-yl)-3-(3-bromophenyl)-1-[(2-chloroethoxy)methyl]-1H-pyrazole and \\ 3-(1,3-benzodioxol-5-yl)-5-(3-bromophenyl)-1-[(2-chloroethoxy)methyl]-1H-pyrazole (sery450)}

To a suspension of sodium hydride $(220 \mathrm{mg}, 5.5 \mathrm{mmol} ; 60 \%$ suspension in mineral oil) in anhydrous DMF $(10 \mathrm{ml})$ a solution of anle138b $(1.71 \mathrm{~g}, 5 \mathrm{mmol})$ in anhydrous DMF $(5 \mathrm{ml})$ was added in 10 minutes with continuous vigorous stirring at room temperature. The mixture was stirred for $30 \mathrm{~min}$ at room temperature and then 2-chloroethyl chloroformate $(0.7 \mathrm{~g}, 5.5$ mmol) was added drop wise. After incubation for $30 \mathrm{~min}$ at room temperature followed by evaporation of DMF under reduced pressure, the residue was dissolved in EtOAc $(60 \mathrm{ml})$, and the solution was washed with water $(50 \mathrm{ml})$, brine $(25 \mathrm{ml})$ and concentrated under reduced pressure. The crude product was purified by column chromatography on silica gel (EtOAc:hexane, $1: 3 \mathrm{v} / \mathrm{v})$ to provide sery450 $(2.1 \mathrm{~g}, 96 \%$, a mixture of two isomers with ratio $1: 1.3)$ as an oil. TLC (EtOAc:hexane, 1:3 v/v): RF $=0.58 .{ }^{1} \mathrm{H}$ NMR $\left.\left(400 \mathrm{MHz}, \mathrm{DMSO}^{\mathrm{D}} 6\right]\right) \delta$ $=8.05(\mathrm{t}, J=1.7 \mathrm{~Hz}, 1 \mathrm{H}), 7.91(\mathrm{t}, J=1.7 \mathrm{~Hz}, 0.8 \mathrm{H}), 7.86(\mathrm{~d}, J=7.8 \mathrm{~Hz}, 1 \mathrm{H}), 7.73-7.64(\mathrm{~m}$, $1.6 \mathrm{H}), 7.56-7.45(\mathrm{~m}, 1.8 \mathrm{H}), 7.43-7.36(\mathrm{~m}, 2.6 \mathrm{H}), 7.26(\mathrm{~d}, J=1.7 \mathrm{~Hz}, 1 \mathrm{H}), 7.19(\mathrm{dd}, J=8.0$, $1.7 \mathrm{~Hz}, 1 \mathrm{H}), 7.10-7.03(\mathrm{~m}, 2.8 \mathrm{H}), 6.98(\mathrm{~d}, J=7.9 \mathrm{~Hz}, 0.8 \mathrm{H}), 6.11(\mathrm{~s}, 2 \mathrm{H}), 6.06(\mathrm{~s}, 1.6 \mathrm{H}), 5.51$ $(\mathrm{s}, 3.6 \mathrm{H}), 3.93-3.86(\mathrm{~m}, 3.6 \mathrm{H}), 3.78-3.70(\mathrm{~m}, 3.6 \mathrm{H}) .{ }^{13} \mathrm{C}$ NMR $\left(100.6 \mathrm{MHz}, \mathrm{DMSO}\left[\mathrm{D}_{6}\right]\right) \delta=$ $150.1,148.5,147.9,147.8,147.7,147.2,145.5,143.7,135.1,131.8,131.6,131.0,130.9$, $130.6,127.7,127.4,126.8,124.3,123.0,122.6,122.2,122.1,119.2,119.1,108.8,108.7$, 108.5, 105.7, 104.9, 104.5, 101.5, 101.1, 78.1 (2C), 68.8, 68.7, 43.45, 43.4. ESI-MS $(\mathrm{m} / \mathrm{z})$ $[\mathrm{M}+\mathrm{H}]^{+}$calcd for $\mathrm{M}=\mathrm{C}_{19} \mathrm{H}_{16}{ }^{81} \mathrm{BrClN}_{2} \mathrm{O}_{3}, 437.00$; found, 437.16 .

\section{1-[(2-Azidoethoxy)methyl]-5-(1,3-benzodioxol-5-yl)-3-(3-bromophenyl)-1H-pyrazole and 1-[(2-azidoethoxy)methyl]-3-(1,3-benzodioxol-5-yl)-5-(3-bromophenyl)-1H-pyrazole (sery451)}

The title compounds was prepared according to published protocol ${ }^{501}$. A mixture of sery 450 (1.87 g, $4.3 \mathrm{mmol}), \mathrm{NaN}_{3}(2.79 \mathrm{~g}, 43 \mathrm{mmol}), \mathrm{NaI}(0.1 \mathrm{~g})$ in DMF $(25 \mathrm{ml})$ was stirred for $15 \mathrm{~h}$ at $80^{\circ} \mathrm{C}$ and concentrated under a reduced pressure. The residue was dissolved in EtOAc (90 $\mathrm{ml})$, the organic phase was washed with water $(2 \times 50 \mathrm{ml})$, brine and dried over $\mathrm{Na}_{2} \mathrm{SO}_{4}$. After 
sodium sulfate was filtered the solution was concentrated under a reduced pressure and the resulting mixture was purified by column chromatography on silica gel (EtOAc:hexane, 1:4 $\mathrm{v} / \mathrm{v})$ to provide sery451 (1.81 g, 95\%, a mixture of two isomers with ratio 1:1.3) as an oil. TLC (EtOAc:hexane, 1:5 v/v): RF $=0.36 .{ }^{1} \mathrm{H}$ NMR (400 MHz, DMSO[D 6$\left.]\right) \delta=8.05(\mathrm{t}, J=$ $1.7 \mathrm{~Hz}, 1 \mathrm{H}), 7.90-7.84(\mathrm{~m}, 1.8 \mathrm{H}), 7.72-7.65(\mathrm{~m}, 1.6 \mathrm{H}), 7.53(\mathrm{ddd}, J=8.0,2.0,1.0 \mathrm{~Hz}, 1 \mathrm{H})$, $7.48(\mathrm{t}, J=7.9 \mathrm{~Hz}, 0.8 \mathrm{H}), 7.43-7.37(\mathrm{~m}, 2.6 \mathrm{H}), 7.24(\mathrm{~d}, J=1.7 \mathrm{~Hz}, 1 \mathrm{H}), 7.18(\mathrm{dd}, J=8.0,1.7$ $\mathrm{Hz}, 1 \mathrm{H}), 7.10-7.05(\mathrm{~m}, 2.8 \mathrm{H}), 6.98(\mathrm{~d}, J=8.0 \mathrm{~Hz}, 0.8 \mathrm{H}), 6.11(\mathrm{~s}, 2 \mathrm{H}), 6.06(\mathrm{~s}, 1.6 \mathrm{H}), 5.51(\mathrm{~s}$, $3.6 \mathrm{H}), 3.84-3.75(\mathrm{~m}, 3.6 \mathrm{H}), 3.49-3.40(\mathrm{~m}, 3.6 \mathrm{H}) .{ }^{13} \mathrm{C}$ NMR $\left(100.6 \mathrm{MHz}, \mathrm{DMSO}\left[\mathrm{D}_{6}\right]\right) \delta=$ $151.1,148.6,148.5,147.9,147.7,147.2,145.4,143.7,135.1,131.8,131.6,131.0,130.9$, $130.8,130.6,127.7,127.3,126.8,124.3,123.1,122.5,122.2,122.1,119.2,108.7$ (2C), 108.6, 105.7, 104.8, 104.5, 101.5, 101.1, 78.2 (2C), 67.6, 67.5, $50.0(2 \mathrm{C})$. ESI-MS $(\mathrm{m} / \mathrm{z})[\mathrm{M}+\mathrm{H}]^{+}$ calcd for $\mathrm{M}=\mathrm{C}_{19} \mathrm{H}_{16}{ }^{81} \mathrm{BrN}_{5} \mathrm{O}_{3}, 444.05$; found, 444.12 .

\section{2-[[5-(1,3-Benzodioxol-5-yl)-3-(3-bromophenyl)-1H-pyrazol-1-yl]methoxy]ethanamine and}

2-[[3-(1,3-benzodioxol-5-yl)-5-(3-bromophenyl)-1H-pyrazol-1-yl]methoxy]ethanamine (sery452)

The title compounds was prepared according to published protocol ${ }^{502}$. A mixture of sery451 (1.67 g, $3.78 \mathrm{mmol}), \mathrm{Ph}_{3} \mathrm{P}(1.49 \mathrm{~g}, 5.67 \mathrm{mmol})$, water $(2 \mathrm{ml})$ in THF $(25 \mathrm{ml})$ was stirred for $18 \mathrm{~h}$ at room temperature and then concentrated under a reduced pressure. The resulting mixture was purified by column chromatography on silica gel with a gradient elution $\left(\mathrm{CHCl}_{3}: \mathrm{MeOH}, 30: 1 \mathrm{v} / \mathrm{v}\right.$ to $\left.9: 1 \mathrm{v} / \mathrm{v}\right)$ to provide sery452 $(0.38 \mathrm{~g}, 96 \%$, a mixture of isomers in ration $1: 1.3)$ as an oil. TLC $\left(\mathrm{CHCl}_{3}: \mathrm{MeOH}, 9: 1 \mathrm{v} / \mathrm{v}\right): \mathrm{RF}=0.19 .{ }^{1} \mathrm{H}$ NMR $(400 \mathrm{MHz}$, DMSO[D 6$]) \delta=8.04(\mathrm{t}, J=1.7 \mathrm{~Hz}, 1 \mathrm{H}), 7.91-7.84(\mathrm{~m}, 1.8 \mathrm{H}), 7.72-7.65(\mathrm{~m}, 1.6 \mathrm{H}), 7.56-7.47$ $(\mathrm{m}, 1.8 \mathrm{H}), 7.43-7.36(\mathrm{~m}, 2.6 \mathrm{H}), 7.24(\mathrm{~d}, J=1.7 \mathrm{~Hz}, 1 \mathrm{H}), 7.18(\mathrm{dd}, J=8.0,1.7 \mathrm{~Hz}, 1 \mathrm{H}), 7.10-$ $7.04(\mathrm{~m}, 2.8 \mathrm{H}), 6.98(\mathrm{~d}, J=8.0 \mathrm{~Hz}, 0.8 \mathrm{H}), 6.11(\mathrm{~s}, 2 \mathrm{H}), 6.06(\mathrm{~s}, 1.6 \mathrm{H}), 5.50-5.42(\mathrm{~m}, 3.6 \mathrm{H})$, 3.58-3.50 (m, 3.6H), 2.69-2.61 (m, 3.6H). ${ }^{13} \mathrm{C}$ NMR (100.6 MHz, DMSO[D $\left.]\right) \delta=149.8$, $148.3,147.8,147.7,147.65,147.1,145.3,143.5,135.2,131.9,131.5,131.0,130.9,130.8$, $130.5,127.7,127.3,126.9,124.2,123.2,122.5,122.2,122.1,119.1,108.7$ (2C), 108.5, 105.6, 104.7, 104.4, 101.5, 101.1, 78.4 (2C), 71.4, 71.2, 41.1 (2C). ESI-MS $(\mathrm{m} / \mathrm{z})[\mathrm{M}+\mathrm{H}]^{+}$calcd for $\mathrm{M}=\mathrm{C}_{19} \mathrm{H}_{18}{ }^{79} \mathrm{BrN}_{3} \mathrm{O}_{3}, 416.06$; found, 416.18 .

2-[[5-(1,3-benzodioxol-5-yl)-3-(3-bromophenyl)-1H-pyrazol-1-yl]methoxy]- $N, N, N$ trimethylethanaminium chloride and 2-[[3-(1,3-benzodioxol-5-yl)-5-(3-bromophenyl)$\mathbf{1 H}$-pyrazol-1-yl]methoxy]- $\mathrm{N}, \mathrm{N}, \mathrm{N}$-trimethylethanaminium chloride (sery453)

A mixture of sery453 (1.45 g, $3.48 \mathrm{mmol})$, MeI (2.97 g, $20.9 \mathrm{mmol}), \mathrm{KHCO}_{3}(2.1 \mathrm{~g}, 21$ mmol) in acetonitrile $(25 \mathrm{ml})$ was stirred for $24 \mathrm{~h}$ at room temperature. Insoluble material was filtered off and the filtrate was concentrated under a reduced pressure to provide sery 453 with iodine as a counterion. Using anion-exchange resin iodine counterion was exchanged to chlorine counterion to provide sery453 $(0.88 \mathrm{mg}, 51 \%$, a mixture of isomers in ration 1:1.3) as a white solid. TLC $\left(\mathrm{CHCl}_{3}: \mathrm{MeOH}, 9: 1 \mathrm{v} / \mathrm{v}\right): \mathrm{RF}=0.2$. LC MS (RP18-100 $\mathrm{A}$, gradient $100 \% \mathrm{H}_{2} \mathrm{O} \rightarrow 100 \% \mathrm{CH}_{3} \mathrm{CN}$ in $\left.50 \mathrm{~min}\right)$ isomer $1 \mathrm{RT} 31.23$ and ESI-MS $(\mathrm{m} / \mathrm{z})[\mathrm{M}]^{+}$calcd for $\mathrm{M}=\mathrm{C}_{22} \mathrm{H}_{25}{ }^{79} \mathrm{BrN}_{3} \mathrm{O}_{3}$, 458.11; found, 458.16; isomer 2 RT $32.07 \mathrm{~min}$ ESI-MS $(\mathrm{m} / \mathrm{z})[\mathrm{M}]^{+}$ calcd for $\mathrm{M}=\mathrm{C}_{22} \mathrm{H}_{25}{ }^{81} \mathrm{BrN}_{3} \mathrm{O}_{3}, 460.11$; found, 460.16 . 


\subsubsection{Synthesis sery 474}

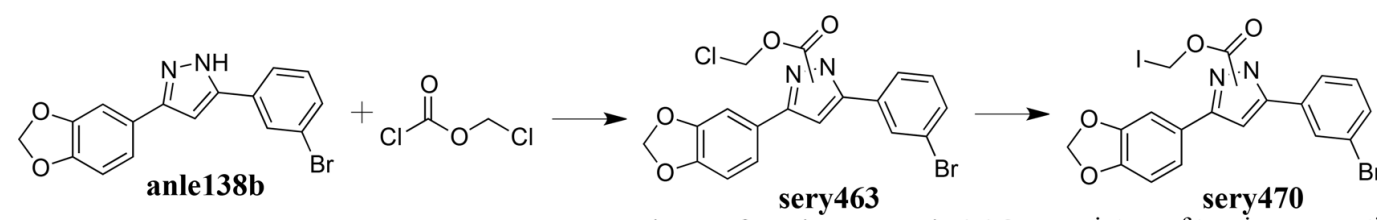

mixture of two isomers; ratio 1:1.2 mixture of two isomers; ratio 1:1.2

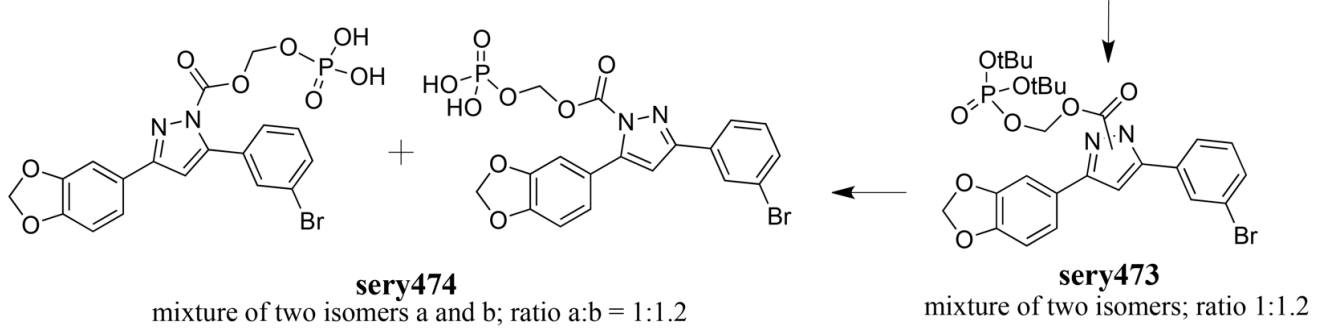

5-(1,3-Benzodioxol-5-yl)-3-(3-bromophenyl)-1H-pyrazole-1-carboxylic acid chloromethyl ester and 3-(1,3-benzodioxol-5-yl)-5-(3-bromophenyl)-1H-pyrazole-1carboxylic acid chloromethyl ester (sery463)

To a mixture of anle138b ( $1 \mathrm{~g}, 2.9 \mathrm{mmol})$ and pyridine $(0.276 \mathrm{~g}, 3.5 \mathrm{mmol})$ in DCM $(10 \mathrm{ml}) \mathrm{a}$ solution of chloromethyl chloroformate $(0.452 \mathrm{~g}, 3.5 \mathrm{mmol})$ in DCM $(5 \mathrm{ml})$ was added in 10 minutes with continuous vigorous stirring at room temperature. After stirring at room temperature for $2 \mathrm{~h}$ reaction mixture was diluted with DCM $(20 \mathrm{ml})$, the organic phase was washed with water $(2 \times 30 \mathrm{ml})$, brine and concentrated under a reduced pressure. The resulting residue was recrystallized from acetonitrile to provide sery463 $(0.85 \mathrm{~g}, 67 \%$, a mixture of isomers in ratio 2:3) as a white solid. ${ }^{1} \mathrm{H}$ NMR $\left(400 \mathrm{MHz}, \mathrm{CDCl}_{3}\right) \delta=8.13(\mathrm{t}, J=1.7 \mathrm{~Hz}$, $0.65 \mathrm{H}), 7.96(\mathrm{~d}, J=7.8 \mathrm{~Hz}, 0.65 \mathrm{H}), 7.80(\mathrm{t}, J=1.7 \mathrm{~Hz}, 1 \mathrm{H}), 7.71-7.63(\mathrm{~m}, 1.6 \mathrm{H}), 7.57(\mathrm{~d}, J=$ $7.9 \mathrm{~Hz}, 1 \mathrm{H}), 7.52-7.46(\mathrm{~m}, 2 \mathrm{H}), 7.43(\mathrm{t}, J=7.9 \mathrm{~Hz}, 1 \mathrm{H}), 7.28(\mathrm{~s}, 0.65 \mathrm{H}), 7.27(\mathrm{~s}, 1 \mathrm{H}), 7.18(\mathrm{~d}$, $J=1.5 \mathrm{~Hz}, 0.65 \mathrm{H}), 7.07-6.99(\mathrm{~m}, 2.3 \mathrm{H}), 6.14-6.07(\mathrm{~m}, 6.4 \mathrm{H})$. ESI-MS $(\mathrm{m} / \mathrm{z})[\mathrm{M}+\mathrm{H}]^{+}$calcd for $\mathrm{M}=\mathrm{C}_{18} \mathrm{H}_{12}{ }^{81} \mathrm{BrClN}_{2} \mathrm{O}_{4}, 436.97$; found, 436.90 .

5-(1,3-Benzodioxol-5-yl)-3-(3-bromophenyl)-1 $H$-pyrazole-1-carboxylic acid iodomethyl ester and 3-(1,3-benzodioxol-5-yl)-5-(3-bromophenyl)-1 $H$-pyrazole-1-carboxylic acid iodomethyl ester (sery470)

The title compounds was prepared according to published protocol ${ }^{498}$. A mixture of sery463 $(0.8 \mathrm{~g}, 1.84 \mathrm{mmol}), \mathrm{NaI}(0.413 \mathrm{~g}, 2.76 \mathrm{mmol})$ in acetone $(15 \mathrm{ml})$ was stirred for 3 days at $40^{\circ} \mathrm{C}$ and then concentrated under a reduced pressure. The residue was dissolved in DCM (30 $\mathrm{ml})$, the organic phase was washed with water $(20 \mathrm{ml})$, aqueous $1 \mathrm{M} \mathrm{Na}_{2} \mathrm{~S}_{2} \mathrm{O}_{3}$ solution $(20 \mathrm{ml})$, brine and dried over $\mathrm{Na}_{2} \mathrm{SO}_{4}$. After sodium sulphate was filtered the solution was concentrated under a reduced pressure and the resulting mixture was purified by column chromatography on silica gel (EtOAc:hexane, 1:3 v/v) to provide sery470 $(0.73 \mathrm{~g}, 75 \%$, a mixture of isomers in ration 1:1.2). sery470 has a limited stability in solution, the isolated sery470 was used directly for the next step without further purification. TLC (EtOAc:hexane, $1: 3 \mathrm{v} / \mathrm{v}): \mathrm{RF}=0.55$. ESI-MS $(\mathrm{m} / \mathrm{z})[\mathrm{M}+\mathrm{H}]^{+}$calcd for $\mathrm{M}=\mathrm{C}_{18} \mathrm{H}_{12}{ }^{81} \mathrm{BrIN}_{2} \mathrm{O}_{4}, 528.91$; found, 528.90 .

3-(1,3-Benzodioxol-5-yl)-5-(3-bromophenyl)-1H-pyrazole-1-carboxylic acid [(di-tertbutoxyphosphinyl)oxy]methyl ester and 5-(1,3-benzodioxol-5-yl)-3-(3-bromophenyl)-1Hpyrazole-1-carboxylic acid [(di-tert-butoxyphosphinyl)oxy]methyl ester (sery473)

The title compounds was prepared according to published method ${ }^{499}$. A mixture of sery470 (53 mg, $0.1 \mathrm{mmol}$ ), di-tert-butylphosphate potassium salt $\left(42 \mathrm{mg}, 0.2 \mathrm{mmol}\right.$ ) and $\mathrm{Ag}_{2} \mathrm{O}$ (24 
$\mathrm{mg}, 0.1 \mathrm{mmol})$ in acetonitrile $(3 \mathrm{ml})$ was stirred for 2 hours at room temperature. Then insoluble material was filtered off and the solid was washed on the filter with acetonitrile (2 $\mathrm{ml})$. The combined organic solution was concentrated under reduced pressure and a resulting oil was purified by column chromatography on silica gel (EtOAc:hexane, 1:2 v/v) to provide sery 473 (45 $\mathrm{mg}, 74 \%$, a mixture of isomers in ration 1:1.2) as an oil. TLC (EtOAc:hexane, $1: 2 \mathrm{v} / \mathrm{v}): \mathrm{RF}=0.24 .{ }^{1} \mathrm{H} \mathrm{NMR}\left(400 \mathrm{MHz}, \mathrm{CDCl}_{3}\right) \delta=8.08(\mathrm{t}, J=1.7 \mathrm{~Hz}, 0.8 \mathrm{H}), 7.79(\mathrm{~d}, J=$ $7.8 \mathrm{~Hz}, 0.8 \mathrm{H}), 7.61(\mathrm{t}, J=1.6 \mathrm{~Hz}, 1 \mathrm{H}), 7.59-7.50(\mathrm{~m}, 1.8 \mathrm{H}), 7.44-7.38(\mathrm{~m}, 2 \mathrm{H}), 7.35(\mathrm{dd}, J=$ 8.1, 1.7 Hz, 1H), 7.33-7.27 (m, 1.8H), 6.96-6.91 (m, 1.8H), 6.89-6.83 (m, 1.8H), $6.67(\mathrm{~s}$, $0.8 \mathrm{H}), 6.66(\mathrm{~s}, 1 \mathrm{H}), 6.03(\mathrm{~s}, 1.6 \mathrm{H}), 6.02(\mathrm{~s}, 2 \mathrm{H}), 5.85-5.76(\mathrm{~m}, 3.8 \mathrm{H}), 1.50(\mathrm{~s}, 15 \mathrm{H}), 1.49$ (18H). ESI-MS $(m / z)\left[\mathrm{M}+\mathrm{H}+\mathrm{Et}_{3} \mathrm{~N}\right]^{+}$calcd for $\mathrm{M}=\mathrm{C}_{18} \mathrm{H}_{12}{ }^{81} \mathrm{BrClN}_{2} \mathrm{O}_{4}, 710.22$; found, 710.20.

5-(1,3-Benzodioxol-5-yl)-3-(3-bromophenyl)-1H-pyrazole-1-carboxylic

acid (phosphonooxy)methyl ester and 3-(1,3-benzodioxol-5-yl)-5-(3-bromophenyl)-1Hpyrazole-1-carboxylic acid (phosphonooxy)methyl ester (sery474)

A mixture of sery473 $(10 \mathrm{mg})$, TFA $(20 \mathrm{mg})$ in deuterated chloroform $(0.7 \mathrm{ml})$ was placed in the NMR tube and the reaction progression was monitored by NMR. After $6 \mathrm{~h}$ incubation at room temperature the ${ }^{1} \mathrm{H}$ spectrum demonstrated the reaction completion, the aqueous workout under neutral conditions resulted in decomposition of sery474 leading to formation of anle138b. ${ }^{1} \mathrm{H}$ NMR of reaction mixture $\left(400 \mathrm{MHz}, \mathrm{CDCl}_{3}+\mathrm{TFA}\right) \delta=7.90(\mathrm{~s}, 0.8 \mathrm{H}), 7.76-$ $7.67(\mathrm{~m}, 2.8 \mathrm{H}), 7.68(\mathrm{~s}, 1 \mathrm{H}), 7.50(\mathrm{~d}, J=7.9 \mathrm{~Hz}, 1 \mathrm{H}), 7.43-7.34(\mathrm{~m}, 2.6 \mathrm{H}), 7.26(\mathrm{~d}, J=1.7$ $\mathrm{Hz}, 1 \mathrm{H}), 7.04(\mathrm{~d}, J=8.1,1.8 \mathrm{~Hz}, 0.8 \mathrm{H}), 7.00-6.90(\mathrm{~m}, 2.8 \mathrm{H}), 6.09(\mathrm{~s}, 2 \mathrm{H}), 6.08(\mathrm{~s}, 1.6 \mathrm{H})$, 5.96-5.87 $(\mathrm{m}, 3.6 \mathrm{H})$. Due to significant amount of impurities, the integral values are approximated. 


\section{Bibliography}

1. Chen, J. J.; Swope, D. M. Pharmacotherapy for Parkinson's disease. Pharmacotherapy 27, 161S-173S (2007).

2. Roberts, H. L.; Brown, D. R. Seeking a mechanism for the toxicity of oligomeric $\alpha-$ synuclein. Biomolecules 5, 282-305 (2015).

3. Martinez-Vicente, M.; Vila, M. Alpha-synuclein and protein degradation pathways in Parkinson's disease: a pathological feed-back loop. Exp. Neurol. 247, 308-313 (2013).

4. Auluck, P. K. et al. $\alpha$-Synuclein: membrane interactions and toxicity in Parkinson's disease. Annu. Rev. Cell Dev. Biol. 26, 211-233 (2010).

5. Lashuel, H. A. et al. The many faces of $\alpha$-synuclein: from structure and toxicity to therapeutic target. Nat. Rev. Neurosci. 2013, 38-48 (2013).

6. Schmidt, F. et al. Single-channel electrophysiology reveals a distinct and uniform pore complex formed by $\alpha$-synuclein oligomers in lipid membranes. PLoS One 7, e42545 (2012).

7. Bertsch, U. et al. Systematic identification of antiprion drugs by high-throughput screening based on scanning for intensely fluorescent targets. J. Virol. 79, 7785-7791 (2005).

8. Diógenes, M. J. et al. Extracellular alpha-synuclein oligomers modulate synaptic transmission and impair LTP via NMDA-receptor activation. J. Neurosci. 32, 11750-11762 (2012).

9. Jiang, Z. et al. Molecular details of $\alpha$-synuclein membrane association revealed by neutrons and photons. J. Phys. Chem. B 119, 4812-4823 (2015).

10. Wagner, J. et al. Anle138b: a novel oligomer modulator for disease-modifying therapy of neurodegenerative diseases such as prion and Parkinson's disease. Acta Neuropathol. 125, 795-813 (2013).

11. Kim HY, C. M., Kumar A, Maier E, Siebenhaar C, Becker S, Fernandez CO, Lashuel HA, Benz R, Lange A, Zweckstetter M. Structural properties of pore-forming oligomers of alpha-synuclein. J. Am. Chem. Soc. 131, 17482-17489 (2009).

12. Ferreon, A. C. et al. Interplay of alpha-synuclein binding and conformational switching probed by single-molecule fluorescence. Proc. Natl. Acad. Sci. U. S. A. 106, 56455650 (2009).

13. Fusco, G. et al. Direct observation of the three regions in $\alpha$-synuclein that determine its membrane-bound behaviour. Nat. Commun. 5, 3827 (2014).

14. Dikiy, I.; Eliezer, D. Folding and misfolding of alpha-synuclein on membranes. Biochem. Biophys. Acta 1818, 1013-1018 (2012).

15. Factor, S. A.; Weiner, W. J., Parkinson's disease: diagnosis and clinical management. 2nd Edition ed.; Demos Medical Publishing: New York, 2008.

16. Marttila, R. J., Epidemiology. In: Koller W.C., ed. Handbook of Parkinson's Disease. 1st Edition ed.; Marcel Dekker Inc.: New York, 1987.

17. Rajput, A. H.; Birdi, S. Epidemiology of Parkinson's disease. Parkinsonism Relat. Disord. 3, 175-186 (1997).

18. de Rijk, M. C. et al. Prevalence of Parkinson's disease in the elderly: the Rotterdam Study. Neurology 45, 2143-2146 (1995).

19. Elbaz, A. et al. Risk tables for parkinsonism and Parkinson's disease. J. Clin. Epidemiol. 55, 25-31 (2002).

20. Klockgether, T. Parkinson's disease: clinical aspects. Cell Tissue Res. 318, 115-120 (2004). 
21. Park, A.; Stacy, M. Non-motor symptoms in Parkinson's disease. J. Neurol. 256, Suppl 3, 293-298 (2009).

22. Braak, H. et al. Staging of brain pathology related to sporadic Parkinson's disease. Neurobiol. Aging 24, 197-211 (2003).

23. Fearnley, J. M.; Lees, A. J. Ageing and Parkinson's disease: substantia nigra regional selectivity. Brain 114, 2283-2301 (1991).

24. Poewe, W. The natural history of Parkinson's disease. J. Neurol. 253, Suppl 7, 2-6 (2006).

25. Samii, A. et al. Parkinson's disease. Lancet 363, 1783-1793 (2004).

26. Jankovic, J. Parkinson's disease: clinical features and diagnosis. J. Neurol., Neurosurg. Psychiatry 79, 368-376 (2008).

27. Forno, L. S. Neuropathology of Parkinson's disease. J. Neuropathol. Exp. Neurol. 55, 259-272 (1996).

28. Jellinger, K. A. Neuropathology of sporadic Parkinson's disease: Evaluation and changes of concepts. Mov. Disord. 27, 8-30 (2011).

29. Forster, E.; Lewy, F. H., "Paralysis agitans". In Pathologische Anatomie. Handbuch der Neurologie. 1st Edition ed.; Springer: Berlin, 1912.

30. Tretiakoff, M. C. Contribution a L'étude de L'anatomie Pathologique de Locus Niger de Soemmerlingnerves. Université de Paris, Paris, 1919.

31. Wakabayashi, K. et al. The Lewy body in Parkinson's disease: molecules implicated in the formation and degradation of alpha-synuclein aggregates. Neuropathology 27, 494-506 (2007).

32. Spillantini, M. G. et al. Alpha-synuclein in Lewy bodies. Nature 388, 839-840 (1997).

33. Polymeropoulos, M. H. et al. Mutation in the alpha-synuclein gene identified in families with Parkinson's disease. Science 276, 2045-2047 (1997).

34. Moore, D. J. et al. Molecular pathophysiology of Parkinson's disease. Annu. Rev. Neurosci. 28, 57-87 (2005).

35. Gupta, A. et al. What causes cell death in Parkinson's disease? Ann. Neurol. 64, 3-15 (2008).

36. Glickman, M. H.; Ciechanover, A. The ubiquitin-proteasome proteolytic pathway: destruction for the sake of construction. Physiol. Rev. 82, 373-428 (2002).

37. McNaught, K. S. et al. Altered proteasomal function in sporadic Parkinson's disease. Exp. Neurol. 179, 38-46 (2003).

38. Tofaris, G. K. et al. Ubiquitination of alpha-synuclein in Lewy bodies is a pathological event not associated with impairment of proteasome function. J. Biol. Chem. 278, 44405-44411 (2003).

39. Grünblatt, E. et al. Gene expression profiling of parkinsonian substantia nigra pars compacta; alterations in ubiquitin-proteasome, heat shock protein, iron and oxidative stress regulated proteins, cell adhesion/cellular matrix and vesicle trafficking genes. J. Neural Transm. 111, 1543-1573 (2004).

40. Kitada, T. et al. Mutations in the parkin gene cause autosomal recessive juvenile parkinsonism. Nature 392, 605-608 (1998).

41. Leroy, E. et al. The ubiquitin pathway in Parkinson's disease. Nature 395, 451-452 (1998).

42. Shimura, H. et al. Familial Parkinson disease gene product, parkin, is a ubiquitinprotein ligase. Nat. Genet. 25, 302-305 (2000).

43. Wilkinson, K. D. et al. The neuron-specific protein PGP 9.5 is a ubiquitin carboxylterminal hydrolase. Science 246, 4930 (1989). 
44. Dong, Z. et al. Dopamine-dependent neurodegeneration in rats induced by viral vector-mediated overexpression of the parkin target protein, CDCrel-1. Proc. Natl. Acad. Sci. U. S. A. 100, 12438-12443 (2003).

45. Imai, Y. et al. An unfolded putative transmembrane polypeptide, which can lead to endoplasmic reticulum stress, is a substrate of Parkin. Cell 105, 891-902 (2001).

46. Corti, O. et al. The p38 subunit of the aminoacyl-tRNA synthetase complex is a Parkin substrate: linking protein biosynthesis and neurodegeneration. Hum. Mol. Genet. 12, 1427-1437 (2003).

47. Engelender, S. et al. Synphilin-1 associates with alpha-synuclein and promotes the formation of cytosolic inclusions. Nat. Genet. 22, 110-114 (1999).

48. Petrucelli, L. et al. Parkin protects against the toxicity associated with mutant alphasynuclein: proteasome dysfunction selectively affects catecholaminergic neurons. Neuron 36, 1007-1019 (2002).

49. Greene, J. C. et al. Mitochondrial pathology and apoptotic muscle degeneration in Drosophila parkin mutants. Proc. Natl. Acad. Sci. U. S. A. 100, 4078-4083 (2003).

50. Liu, Y. et al. The UCH-L1 gene encodes two opposing enzymatic activities that affect alpha-synuclein degradation and Parkinson's disease susceptibility. Cell 111, 209-218 (2002).

51. Nishikawa, K. et al. Alterations of structure and hydrolase activity of parkinsonismassociated human ubiquitin carboxyl-terminal hydrolase L1 variants. Biochem. Biophys. Res. Commun. 304, 176-183 (2003).

52. McNaught, K. S. et al. Proteasome inhibition causes nigral degeneration with inclusion bodies in rats. Neuroreport 13, 1437-1441 (2002).

53. Bedford, L. et al. Depletion of $26 \mathrm{~S}$ proteasomes in mouse brain neurons causes neurodegeneration and Lewy-like inclusions resembling human pale bodies. J. Neurosci. 28, 8189-8198 (2008).

54. Winklhofer, K. F.; Haass, C. Mitochondrial dysfunction in Parkinson's disease. Biochim. Biophys. Acta 1802, 29-44 (2010).

55. Banerjee, R. et al. Mitochondrial dysfunction in the limelight of Parkinson's disease pathogenesis. Biochim. Biophys. Acta 1792, 651-663 (2009).

56. Murphy, M. P. How mitochondria produce reactive oxygen species. Biochem. J. 417, 1-13 (2009).

57. Richter, C. et al. Oxidants in mitochondria: from physiology to diseases. Biochim. Biophys. Acta 1271, 67-74 (1995).

58. Tsang, A. H.; Chung, K. K. Oxidative and nitrosative stress in Parkinson's disease. Biochim. Biophys. Acta 1792, 643-650 (2009).

59. Schapira, A. H. et al. Mitochondrial complex I deficiency in Parkinson's disease. $J$. Neurochem. 54, 823-827 (1990).

60. Mann, V. M. et al. Brain, skeletal muscle and platelet homogenate mitochondrial function in Parkinson's disease. Brain 115, 333-342 (1992).

61. DiDonato, S. et al. Respiratory chain and mitochondrial DNA in muscle and brain in Parkinson's disease patients. Neurology 43, 2262-2268 (1993).

62. Mizuno, Y. et al. Deficiencies in complex I subunits of the respiratory chain in Parkinson's disease. Biochem. Biophys. Res. Commun. 163, 1450-1455 (1989).

63. Davis, G. C. et al. Chronic Parkinsonism secondary to intravenous injection of meperidine analogues. Psychiatry Res. 1, 249-254 (1979).

64. Langston, J. W. et al. Chronic Parkinsonism in humans due to a product of meperidine-analog synthesis. Science 219, 979-980 (1983).

65. Hantraye, P. et al. Stable parkinsonian syndrome and uneven loss of striatal dopamine fibres following chronic MPTP administration in baboons. Neuroscience 53, 169-178 (1993). 
66. Hisahara, S.; Shimohama, S. Toxin-induced and genetic animal models of Parkinson's disease. Parkinsons Dis. 2011, id951709 (2010).

67. Dauer, W.; Przedborski, S. Parkinson's disease: mechanisms and models. Neuron 39, 889-909 (2003).

68. Hatcher, J. M. et al. Parkinson's disease and pesticides: a toxicological perspective. Trends Pharmacol. Sci. 29, 322-329 (2008).

69. Hertzman, C. et al. Parkinson's disease: a case-control study of occupational and environmental risk factors. Am. J. Ind. Med. 17, 349-355 (1990).

70. Meco, G. et al. Parkinsonism after chronic exposure to the fungicide maneb (manganese ethylene-bis-dithiocarbamate). Scand. J. Work, Environ. Health 20, 301-305 (1994).

71. Tawara, T. et al. Effects of paraquat on mitochondrial electron transport system and catecholamine contents in rat brain. Arch. Toxicol. 70, 585-589 (1996).

72. Sherer, T. B. et al. Subcutaneous rotenone exposure causes highly selective dopaminergic degeneration and alpha-synuclein aggregation. Exp. Neurol. 179, 9-16 (2003).

73. Zhang, J. et al. Manganese ethylene-bis-dithiocarbamate and selective dopaminergic neurodegeneration in rat: a link through mitochondrial dysfunction. J. Neurochem. 84, 336346 (2003).

74. Bové, J. et al. Toxin-induced models of Parkinson's disease. NeuroRx 2, 484-494 (2005).

75. Valente, E. M. et al. Hereditary early-onset Parkinson's disease caused by mutations in PINK1. Science 304, 1158-1160 (2004).

76. Lin, W.; Kang, U. J. Characterization of PINK1 processing, stability, and subcellular localization. J. Neurochem. 106, 464-474 (2008).

77. Silvestri, L. et al. Mitochondrial import and enzymatic activity of PINK1 mutants associated to recessive parkinsonism. Hum. Mol. Genet. 14, 3477-3492 (2005).

78. Petit, A. et al. Wild-type PINK1 prevents basal and induced neuronal apoptosis, a protective effect abrogated by Parkinson disease-related mutations. J. Biol. Chem. 280, 34025-34032 (2005).

79. Clark, I. E. et al. Drosophila pink1 is required for mitochondrial function and interacts genetically with parkin. Nature 441, 1162-1166 (2006).

80. Park, J. et al. Mitochondrial dysfunction in Drosophila PINK1 mutants is complemented by parkin. Nature 441, 1157-1161 (2006).

81. Gautier, C. A. et al. Loss of PINK1 causes mitochondrial functional defects and increased sensitivity to oxidative stress. Proc. Natl. Acad. Sci. U. S. A. 105, 11364-11369 (2008).

82. Haque, M. E. et al. Cytoplasmic Pink1 activity protects neurons from dopaminergic neurotoxin MPTP. Proc. Natl. Acad. Sci. U. S. A. 105, 1716-1721 (2008).

83. Pridgeon, J. W. et al. PINK1 protects against oxidative stress by phosphorylating mitochondrial chaperone TRAP1. PloS Biol. 5, e172 (2007).

84. Plun-Favreau, H. et al. The mitochondrial protease HtrA2 is regulated by Parkinson's disease-associated kinase PINK1. Nat. Cell Biol. 9, 1243-1252 (2007).

85. Martins, L. M. et al. Neuroprotective role of the Reaper-related serine protease HtrA2/Omi revealed by targeted deletion in mice. Mol. Cell Biol. 24, 9848-9862 (2004).

86. Bonifati, V. et al. Mutations in the DJ-1 gene associated with autosomal recessive early-onset parkinsonism. Science 299, 256-259 (2003).

87. Moore, D. J. et al. Association of DJ-1 and parkin mediated by pathogenic DJ-1 mutations and oxidative stress. Hum. Mol. Genet. 14, 71-84 (2005).

88. Bandopadhyay, R. et al. The expression of DJ-1 (PARK7) in normal human CNS and idiopathic Parkinson's disease. Brain 127, 420-430 (2004). 
89. Li, H. M. et al. Association of DJ-1 with chaperones and enhanced association and colocalization with mitochondrial Hsp70 by oxidative stress. Free Radical Res. 39, 10911099 (2005).

90. Lev, N. et al. Oxidative insults induce DJ-1 upregulation and redistribution: implications for neuroprotection. Neurotoxicology 29, 397-405 (2008).

91. Canet-Avilés, R. M. et al. The Parkinson's disease protein DJ-1 is neuroprotective due to cysteine-sulfinic acid-driven mitochondrial localization. Proc. Natl. Acad. Sci. U. S. A. 101, 9103-9108 (2004).

92. Martinat, C. et al. Sensitivity to oxidative stress in DJ-1-deficient dopamine neurons: an ES- derived cell model of primary Parkinsonism. PloS Biol. 2, e327 (2004).

93. Taira, T. et al. DJ-1 has a role in antioxidative stress to prevent cell death. EMBO Rep. 5, 213-218 (2004).

94. Yokota, T. et al. Down regulation of DJ-1 enhances cell death by oxidative stress, ER stress, and proteasome inhibition. Biochem. Biophys. Res. Commun. 312, 1342-1348 (2003).

95. Kim, R. H. et al. Hypersensitivity of DJ-1-deficient mice to 1-methyl-4-phenyl1,2,3,6-tetrahydropyrindine (MPTP) and oxidative stress. Proc. Natl. Acad. Sci. U. S. A. 102, 5215-5220 (2005).

96. Yang, W. et al. Paraquat induces dopaminergic dysfunction and proteasome impairment in DJ-1-deficient mice. Hum. Mol. Genet. 16, 2900-2910 (2007).

97. Goldberg, M. S. et al. Nigrostriatal dopaminergic deficits and hypokinesia caused by inactivation of the familial Parkinsonism-linked gene DJ-1. Neuron 45, 489-496 (2005).

98. Junn, E. et al. Interaction of DJ-1 with Daxx inhibits apoptosis signal-regulating kinase 1 activity and cell death. Proc. Natl. Acad. Sci. U. S. A. 102, 9691-9696 (2005).

99. Zimprich, A. et al. Mutations in LRRK2 cause autosomal-dominant parkinsonism with pleomorphic pathology. Neuron 44, 601-607 (2004).

100. Biskup, S. et al. Localization of LRRK2 to membranous and vesicular structures in mammalian brain. Ann. Neurol. 60, 557-569 (2006).

101. Giasson, B. I. et al. Biochemical and pathological characterization of Lrrk2. Ann. Neurol. 59, 315-322 (2006).

102. West, A. B. et al. Parkinson's disease-associated mutations in leucine-rich repeat kinase 2 augment kinase activity. Proc. Natl. Acad. Sci. U. S. A. 102, 16842-16847 (2005).

103. Maroteaux, L. et al. Synuclein: a neuron-specific protein localized to the nucleus and presynaptic nerve terminal. J. Neurosci. 8, 2804-2815 (1988).

104. Uversky, V. N. Neuropathology, biochemistry, and biophysics of alpha-synuclein aggregation. J. Neurochem. 103, 17-37 (2007).

105. Wales, P. et al. Limelight on alpha-synuclein: pathological and mechanistic implications in neurodegeneration. J. Parkinson's Dis. 3, 415-459 (2013).

106. Silva, B. A. et al. Agrochemicals, $\alpha$-Synuclein, and Parkinson's Disease. Mol. Neurobiol., (2012).

107. George, J. M. The synucleins. Genome Biol. 3, reviews3002 (2002).

108. Lavedan, C. The synuclein family. Genome Res. 8, 871-880 (1998).

109. Breydo, L. et al. A-synuclein misfolding and Parkinson's disease. Biochim. Biophys. Acta 1822, 261-285 (2012).

110. Sung, Y. H.; Eliezer, D. Residual structure, backbone dynamics, and interactions within the synuclein family. J. Mol. Biol. 372, 689-707 (2007).

111. Uversky, V. N. et al. Biophysical properties of the synucleins and their propensities to fibrillate: inhibition of alpha-synuclein assembly by beta- and gamma-synucleins. J. Biol. Chem. 277, 11970-11978 (2002).

112. Kim, W. S. et al. Alpha-synuclein biology in Lewy body diseases. Alzheimer's Res. Ther. 6, 73 (2014). 
113. Garland, E. M. et al. Pure autonomic failure. Handb. Clin. Neurol. 117, 243-257 (2013).

114. Rochet, J. C. et al. Molecular insights into Parkinson's disease. Prog. Mol. Biol. Tranls. Sci. 107, 125-188 (2012).

115. Uversky, V. N.; Eliezer, D. Biophysics of Parkinson's disease: structure and aggregation of alpha-synuclein. Curr. Protein Pept. Sci. 10, 483-499 (2009).

116. Guerrero-Ferreira, R. et al. Cryo-EM structure of alpha-synuclein fibrils. eLife 7, e36402 (2018).

117. Bertoncini, C. W. et al. Release of long-range tertiary interactions potentiates aggregation of natively unstructured alpha-synuclein. Proc. Natl. Acad. Sci. U. S. A. 102, 1430-1435 (2005).

118. Burré, J. et al. Properties of native brain $\alpha$-synuclein. Nature 498, e4-6 (2013).

119. Fauvet, B. et al. a-Synuclein in central nervous system and from erythrocytes, mammalian cells, and Escherichia coli exists predominantly as disordered monomer. J. Biol. Chem. 287, 15345-15364 (2012).

120. Uversky, V. N. Intrinsically disordered proteins may escape unwanted interactions via functional misfolding. Biochem. Biophys. Acta 1814, 693-712 (2011).

121. Theillet, F. X. et al. Structural disorder of monomeric $\alpha$-synuclein persists in mammalian cells. Nature 530, 45-50 (2016).

122. Weinreb, P. H. et al. NACP, a protein implicated in Alzheimer's disease and learning, is natively unfolded. Biochemistry 35, 13709-13715

123. Eliezer, D. et al. Conformational properties of alpha-synuclein in its free and lipidassociated states. J. Mol. Biol. 307, 1061-1073 (2001).

124. Dedmon, M. M. et al. Mapping long-range interactions in alpha-synuclein using spinlabel NMR and ensemble molecular dynamics simulations. J. Am. Chem. Soc. 127, 476-477 (2005).

125. Conway, K. A. et al. Accelerated in vitro fibril formation by a mutant alpha-synuclein linked to early-onset Parkinson disease. Nat. Med. 4, 1318-1320 (1998).

126. Stefani, M. Protein Folding and Misfolding on Surfaces. Int. J. Mol. Sci. 9, 25152542 (2008).

127. Kalia, L. V. et al. $\alpha$-Synuclein oligomers and clinical implications for Parkinson disease. Ann. Neurol. 73, 155-169 (2013).

128. Fink, A. L. The aggregation and fibrillation of alpha-synuclein. Acc. Chem. Res. 39, 628-634 (2006).

129. Uversky, V. N. et al. Evidence for a partially folded intermediate in alpha-synuclein fibril formation. J. Biol. Chem. 276, 10737-10744 (2001).

130. Wood, S. J. et al. alpha-synuclein fibrillogenesis is nucleation-dependent. Implications for the pathogenesis of Parkinson's disease. J. Biol. Chem. 274, 19509-19512 (1999).

131. Buell, A. K. et al. Solution conditions determine the relative importance of nucleation and growth processes in $\alpha$-synuclein aggregation. Proc. Natl. Acad. Sci. U. S. A. 111, 76717676 (2014).

132. Esposito, L. A. Targeting $\alpha$-Synuclein as a Parkinson's Disease Therapeutic. Top. Med. Chem., book chapter (2014).

133. Beyer, K.; Ariza, A. $\alpha$-Synuclein posttranslational modification and alternative splicing as a trigger for neurodegeneration. Mol. Neurobiol. 47, 509-524 (2013).

134. Chavarría, C.; Souza, J. M. Oxidation and nitration of $\alpha$-synuclein and their implications in neurodegenerative diseases. Arch. Biochem. Biophys. 533, 25-32 (2013).

135. Paleologou, K. E.; El-Agnaf, O. M. $\alpha$-Synuclein Aggregation and Modulating Factors. Protein Aggregation and Fibrillogenesis in Cerebral and Systemic Amyloid Disease, Harris, J. R. (Ed.) Chapter 6, 109-164 (2012). 
136. Zhou, J. et al. Changes in the solubility and phosphorylation of $\alpha$-synuclein over the course of Parkinson's disease. Acta Neuropathol. 121, 695-704 (2011).

137. Anderson, J. P. et al. Phosphorylation of Ser-129 is the dominant pathological modification of alpha-synuclein in familial and sporadic Lewy body disease. J. Biol. Chem. 281, 29739-29752 (2006).

138. Foulds, P. G. et al. A longitudinal study on $\alpha$-synuclein in blood plasma as a biomarker for Parkinson's disease. Sci. Rep. 3, 2540 (2013).

139. Paleologou, K. E. et al. Phosphorylation at S87 is enhanced in synucleinopathies, inhibits alpha-synuclein oligomerization, and influences synuclein-membrane interactions. $J$. Neurosci. 30, 3184-3198 (2010).

140. Tenreiro, S. et al. Protein phosphorylation in neurodegeneration: friend or foe? Front. Mol. Neurosci. 7, 42 (2014).

141. Mahul-Mellier, A. L. et al. c-Abl phosphorylates $\alpha$-synuclein and regulates its degradation: implication for $\alpha$-synuclein clearance and contribution to the pathogenesis of Parkinson's disease. Hum. Mol. Genet. 23, 2858-2879

142. Paleologou, K. E. et al. Phosphorylation at Ser-129 but not the phosphomimics S129E/D inhibits the fibrillation of alpha-synuclein. J. Biol. Chem. 283, 16895-16905 (2008).

143. Sato, H. et al. The role of Ser 129 phosphorylation of $\alpha$-synuclein in neurodegeneration of Parkinson's disease: a review of in vivo models. Rev. Neurosci. 24, 115-123 (2012).

144. Lewis, K. A. et al. Accelerated formation of alpha-synuclein oligomers by concerted action of the 20S proteasome and familial Parkinson mutations. J. Bioenerg. Biomembr. 42, 85-95 (2010).

145. Dufty, B. M. et al. Calpain-cleavage of alpha-synuclein: connecting proteolytic processing to disease-linked aggregation. Am. J. Pathol. 170, 1725-1738 (2007).

146. Choi, D. H. et al. Role of matrix metalloproteinase 3-mediated alpha-synuclein cleavage in dopaminergic cell death. J. Biol. Chem. 286, 14168-14177 (2011).

147. Lee, J. T. et al. Ubiquitination of alpha-synuclein by Siah-1 promotes alpha-synuclein aggregation and apoptotic cell death. Hum. Mol. Genet. 17, 906-917 (2008).

148. Rott, R. et al. Monoubiquitylation of alpha-synuclein by seven in absentia homolog (SIAH) promotes its aggregation in dopaminergic cells. J. Biol. Chem. 283, 3316-3328 (2008).

149. Meier, F. et al. Semisynthetic, site-specific ubiquitin modification of $\alpha$-synuclein reveals differential effects on aggregation. J. Am. Chem. Soc. 134, 5468-5471 (2012).

150. Jones, L. H. Chemistry and biology of biomolecule nitration. Chem. Biol. 19, 10861092 (2012).

151. Burai, R. et al. Elucidating the Role of Site-Specific Nitration of $\alpha$-Synuclein in the Pathogenesis of Parkinson's Disease via Protein Semisynthesis and Mutagenesis. J. Am. Chem. Soc. 137, 5041-5052 (2015).

152. Yu, Z. et al. Nitrated alpha-synuclein induces the loss of dopaminergic neurons in the substantia nigra of rats. PLoS One 5, e9956 (2010).

153. Uversky, V. N. et al. Effects of nitration on the structure and aggregation of alphasynuclein. Mol. Brain Res. 134, 84-102 (2005).

154. Vijayakumaran, S. et al. Direct and/or Indirect Roles for SUMO in Modulating AlphaSynuclein Toxicity. Biomolecules 5, 1697-1716 (2015).

155. Krumova, P. et al. Sumoylation inhibits alpha-synuclein aggregation and toxicity. $J$. Cell Biol. 194, 49-60 (2011).

156. Guerrero, E. et al. Recent advances in $\alpha$-synuclein functions, advanced glycation, and toxicity: implications for Parkinson's disease. Mol. Neurobiol. 47, 525-536 (2013). 
157. Beyer, K. Alpha-synuclein structure, posttranslational modification and alternative splicing as aggregation enhancers. Acta Neuropathol. 112, 237-251 (2006).

158. Junn, E. et al. Tissue transglutaminase-induced aggregation of alpha-synuclein: Implications for Lewy body formation in Parkinson's disease and dementia with Lewy bodies. Proc. Natl. Acad. Sci. U. S. A. 100, 2047-2052 (2003).

159. Bussell, R. J. et al. Helix periodicity, topology, and dynamics of membrane-associated alpha-synuclein. Protein Sci. 14, 862-872 (2005).

160. Chandra, S. et al. A broken alpha-helix in folded alpha-Synuclein. J. Biol. Chem. 278, 15313-15318 (2003).

161. Zhu, M. et al. The association of alpha-synuclein with membranes affects bilayer structure, stability, and fibril formation. J. Biol. Chem. 278, 40186-40197 (2003).

162. Ulmer, T. S. et al. Structure and dynamics of micelle-bound human alpha-synuclein. J. Biol. Chem. 280, 9595-9603 (2005).

163. Bodner, C. R. et al. Differential phospholipid binding of alpha-synuclein variants implicated in Parkinson's disease revealed by solution NMR spectroscopy. Biochemistry 49, 862-871 (2010).

164. Beyer, K. Mechanistic aspects of Parkinson's disease: alpha-synuclein and the biomembrane. Cell Biochem. Biophys. 47, 285-299 (2007).

165. Pandey, A. P. et al. $\alpha$-Synuclein-induced tubule formation in lipid bilayers. J. Phys. Chem. B 115, 5886-5893 (2011).

166. Zhu, M.; Fink, A. L. Lipid binding inhibits alpha-synuclein fibril formation. J. Biol. Chem. 278, 16873-16877 (2003).

167. Sharon, R. et al. The formation of highly soluble oligomers of alpha-synuclein is regulated by fatty acids and enhanced in Parkinson's disease. Neuron 37, 583-595 (2003).

168. Jo, E. et al. $\alpha$-Synuclein-synaptosomal membrane interactions: implications for fibrillogenesis. Eur. J. Biochem. 271, 3180-3189 (2004).

169. Burré, J. et al. $\alpha$-Synuclein assembles into higher-order multimers upon membrane binding to promote SNARE complex formation. Proc. Natl. Acad. Sci. U. S. A. 111, E42744283 (2014).

170. Haque, F. et al. Adsorption of alpha-synuclein on lipid bilayers: modulating the structure and stability of protein assemblies. J. Phys. Chem. B 114, 4070-4081 (2010).

171. Hähl, H. et al. $\alpha$-Synuclein insertion into supported lipid bilayers as seen by in situ Xray reflectivity. ACS Chem. Neurosci. 6, 374-379 (2015).

172. Lee, H. J. et al. Membrane-bound alpha-synuclein has a high aggregation propensity and the ability to seed the aggregation of the cytosolic form. J. Biol. Chem. 277, 671-678 (2002).

173. Ruipérez, V. et al. Alpha-synuclein, lipids and Parkinson's disease. Prog. Lipid Res. 49, 420-428 (2010).

174. Vilar, M. et al. The fold of alpha-synuclein fibrils. Proc. Natl. Acad. Sci. U. S. A. 105, 8637-8642 (2008).

175. Heise, H. et al. Molecular-level secondary structure, polymorphism, and dynamics of full-length alpha-synuclein fibrils studied by solid-state NMR. Proc. Natl. Acad. Sci. U. S. A. 102, 15871-15876 (2005).

176. Serpell, L. C. et al. Fiber diffraction of synthetic alpha-synuclein filaments shows amyloid-like cross-beta conformation. Proc. Natl. Acad. Sci. U. S. A. 97, 4897-4902 (2000).

177. Der-Sarkissian, A. et al. Structural organization of alpha-synuclein fibrils studied by site-directed spin labeling. J. Biol. Chem. 278, 37530-37535 (2003).

178. Pornsuwan, S. et al. Long-range distances in amyloid fibrils of $\alpha$-synuclein from PELDOR spectroscopy. Angew. Chem., Int. Ed. Engl. 52, 10290-10294 (2013). 
179. Hwang, S. et al. Comparison of the 3D structures of mouse and human $\alpha$-synuclein fibrils by solid-state NMR and STEM. J. Struct. Biol. 206, 43-48 (2019).

180. Crowther, R. A. et al. Characterisation of isolated alpha-synuclein filaments from substantia nigra of Parkinson's disease brain. Neurosci. Lett. 292, 128-130 (2000).

181. Lv, G. et al. Structural comparison of mouse and human $\alpha$-synuclein amyloid fibrils by solid-state NMR. J. Mol. Biol. 420, 99-111 (2012).

182. Tuttle, M. D. et al. Solid-state NMR structure of a pathogenic fibril of full-length human $\alpha$-synuclein. Nat. Struct. Mol. Biol. 23, 409-415 (2016).

183. Li, B. et al. Cryo-EM of full-length $\alpha$-synuclein reveals fibril polymorphs with a common structural kernel. Nat. Commun. 9, 3609 (2018).

184. Lorenzen, N. et al. The role of stable $\alpha$-synuclein oligomers in the molecular events underlying amyloid formation. J. Am. Chem. Soc. 136, 3859-3868 (2014).

185. Paslawski, W. et al. High stability and cooperative unfolding of $\alpha$-synuclein oligomers. Biochemistry 53, 6252-6263 (2014).

186. van Rooijen, B. D. et al. Lipid bilayer disruption by oligomeric alpha-synuclein depends on bilayer charge and accessibility of the hydrophobic core. Biochem. Biophys. Acta 1788, 1271-1278 (2009).

187. van Rooijen, B. D. et al. Tryptophan fluorescence reveals structural features of alphasynuclein oligomers. J. Mol. Biol. 394, 826-833 (2009).

188. Mysling, S. et al. Characterizing the dynamics of $\alpha$-synuclein oligomers using hydrogen/deuterium exchange monitored by mass spectrometry. Biochemistry 52, 9097-9103 (2013).

189. Apetri, M. M. et al. Secondary structure of alpha-synuclein oligomers: characterization by raman and atomic force microscopy. J. Mol. Biol. 355, 63-71 (2006).

190. Celej, M. S. et al. Toxic prefibrillar $\alpha$-synuclein amyloid oligomers adopt a distinctive antiparallel $\beta$-sheet structure. Biochem. J. 443, 719-726 (2012).

191. Gallea, J. I.; Celej, M. S. Structural insights into amyloid oligomers of the Parkinson disease-related protein $\alpha$-synuclein. J. Biol. Chem. 289, 26733-26742 (2014).

192. Danzer, K. M. et al. Different species of alpha-synuclein oligomers induce calcium influx and seeding. J. Neurosci. 27, 9220-9232 (2007).

193. Illes-Toth, E. et al. Distinct higher-order $\alpha$-synuclein oligomers induce intracellular aggregation. Biochem. J. 468, 485-493 (2015).

194. Kostka, M. et al. Single particle characterization of iron-induced pore-forming alphasynuclein oligomers. J. Biol. Chem. 283, 10992-11003 (2008).

195. Cremades, N. et al. Direct observation of the interconversion of normal and toxic forms of $\alpha$-synuclein. Cell 149, 1048-1059 (2012).

196. Luth, E. S. et al. Soluble, prefibrillar $\alpha$-synuclein oligomers promote complex Idependent, Ca2+-induced mitochondrial dysfunction. J. Biol. Chem. 289, 21490-21507 (2014).

197. Giehm, L. et al. Low-resolution structure of a vesicle disrupting \&alpha;-synuclein oligomer that accumulates during fibrillation. Proc. Natl. Acad. Sci. U. S. A. 108, 3246-3251 (2011).

198. Hinault, M. P. et al. Stable alpha-synuclein oligomers strongly inhibit chaperone activity of the Hsp70 system by weak interactions with J-domain co-chaperones. J. Biol. Chem. 285, 38173-38182 (2010).

199. Quist, A. et al. Amyloid ion channels: a common structural link for protein-misfolding disease. Proc. Natl. Acad. Sci. U. S. A. 102, 10427-10432 (2005).

200. Varkey, J. et al. $\alpha$-Synuclein oligomers with broken helical conformation form lipoprotein nanoparticles. J. Biol. Chem. 288, 17620-17630 (2013). 
201. Villar-Piqué, A. et al. Structure, function and toxicity of alpha-synuclein: the Bermuda triangle in synucleinopathies. J. Neurochem. 139, 240-255 (2015).

202. Volles, M. J.; Lansbury, P. T. J. Vesicle permeabilization by protofibrillar alphasynuclein is sensitive to Parkinson's disease-linked mutations and occurs by a pore-like mechanism. Biochemistry 41, 4595-4602 (2002).

203. Lashuel, H. A. et al. Neurodegenerative disease: amyloid pores from pathogenic mutations. Nature 418, 291 (2002).

204. Feng, L. R. et al. Alpha-synuclein mediates alterations in membrane conductance: a potential role for alpha-synuclein oligomers in cell vulnerability. Eur. J. Neurosci. 32, 10-17 (2010).

205. Stöckl, M. T. et al. $\alpha$-Synuclein Oligomers: an Amyloid Pore? : Insights into Mechanisms of $\alpha$-Synuclein Oligomer-Lipid Interactions. Mol. Neurobiol. 47, 613-621 (2012).

206. Stöckl, M. et al. Kinetic measurements give new insights into lipid membrane permeabilization by $\alpha$-synuclein oligomers. Mol. BioSyst., in press (2011).

207. Stefanovic, A. N. et al. $\alpha$-Synuclein oligomers distinctively permeabilize complex model membranes. FEBS J. 281, 2838-2850 (2014).

208. Camilleri, A. et al. Mitochondrial membrane permeabilisation by amyloid aggregates and protection by polyphenols. Biochim. Biophys. Acta 1828, 2532-2543 (2013).

209. Choubey, V. et al. Mutant A53T alpha-synuclein induces neuronal death by increasing mitochondrial autophagy. J. Biol. Chem. 286, 10814-10824 (2011).

210. Kamp, F. et al. Inhibition of mitochondrial fusion by $\alpha$-synuclein is rescued by PINK1, Parkin and DJ-1. EMBO J. 29, 3571-3589 (2010).

211. Plotegher, N. et al. Number and Brightness analysis of alpha-synuclein oligomerization and the associated mitochondrial morphology alterations in live cells. Biochem. Biophys. Acta 1840, 2014-2024 (2014).

212. Olanow, C. W.; McNaught, K. Parkinson's disease, proteins, and prions: milestones. Mov. Disord. 26, 1056-1071 (2011).

213. Martinez-Vicente, M. et al. Dopamine-modified alpha-synuclein blocks chaperonemediated autophagy. J. Clin. Invest. 118, 777-788 (2008).

214. Chen, L. et al. Proteasome dysfunction in aged human alpha-synuclein transgenic mice. Neurobiol. Dis. 23, 120-126 (2006).

215. Tanik, S. A. et al. Lewy body-like $\alpha$-synuclein aggregates resist degradation and impair macroautophagy. J. Biol. Chem. 288, 15194-15210 (2013).

216. Emmanouilidou, E. et al. Cell-produced alpha-synuclein oligomers are targeted to, and impair, the 26S proteasome. Neurobiol. Aging 31, 953-968 (2010).

217. Snyder, H. et al. Aggregated and monomeric alpha-synuclein bind to the S6' proteasomal protein and inhibit proteasomal function. J. Biol. Chem. 278, 11753-11759 (2003).

218. Colla, E. et al. Accumulation of toxic $\alpha$-synuclein oligomer within endoplasmic reticulum occurs in $\alpha$-synucleinopathy in vivo. J. Neurosci. 32, 3301-3305 (2012).

219. Su, L. J. et al. Compounds from an unbiased chemical screen reverse both ER-toGolgi trafficking defects and mitochondrial dysfunction in Parkinson's disease models. Dis. Models \& Mech. 3, 194-208 (2010).

220. Colla, E. et al. Endoplasmic reticulum stress is important for the manifestations of $\alpha-$ synucleinopathy in vivo. J. Neurosci. 32, 3306-3320 (2012).

221. Chen, S. W. et al. Structural characterization of toxic oligomers that are kinetically trapped during $\alpha$-synuclein fibril formation. Proc. Natl. Acad. Sci. U. S. A. 112, E1994-2003 (2015). 
222. Poewe, W. et al. Levodopa in the treatment of Parkinson's disease: an old drug still going strong. Clin. Interventions Aging 5, 229-238 (2010).

223. Schapira, A. H. V. et al. Levodopa in the treatment of Parkinson. Eur. J. Neurol. 16, 982-989 (2009).

224. Hauser, R. A. Levodopa: Past, Present, and Future. Eur. Neurol. 62, 1-8 (2009).

225. Olanow, C. W. et al. Continuous dopamine-receptor treatment of Parkinson's disease: scientific rationale and clinical implications. Lancet Neurol. 5, 677-687 (2006).

226. Nutt, J. G.; Fellman, J. H. Pharmacokinetics of levodopa. Clin. Neuropharmacol. 7, 35-49 (1984).

227. Sasahara, K. et al. Dosage form design for improvement of bioavailability of levodopa $\mathrm{V}$ : Absorption and metabolism of levodopa in intestinal segments of dogs. J. Pharm. Sci. 70, 1157-1160 (1981).

228. Jorga, K. et al. The effect of COMT inhibition by tolcapone on tolerability and pharmacokinetics of different levodopa/benserazide formulations. Eur. Neurol. 38, 59-67 (1997).

229. Senek, M.; Nyholm, D. Continuous drug delivery in Parkinson's disease. CNS Drugs 28, 19-27 (2014).

230. Edmondson, D. E. et al. Structure and mechanism of monoamine oxidase. Curr. Med. Chem. 11, 1983-1993 (2004).

231. Shih, J. C. et al. Monoamine oxidase: from genes to behavior. Annu. Rev. Neurosci. 22, 197-217 (1999).

232. Youdim, M. B. et al. The therapeutic potential of monoamine oxidase inhibitors. Nat. Rev. Neurosci. 7, 295-309 (2006).

233. LeWitt, P. A.; Taylor, D. C. Protection against Parkinson's disease progression: clinical experience. Neurotherapeutics 5, 210-225 (2008).

234. Tintner, R.; Jankovic, J. Dopamine agonists in Parkinson's disease. Expert Opin. Invest. Drugs 12, 1803-1820 (2003).

235. Beaulieu, J. M.; Gainetdinov, R. R. The physiology, signaling, and pharmacology of dopamine receptors. Pharmacol. Rev. 63, 182-217 (2011).

236. Diaz, N. L.; Waters, C. H. Current strategies in the treatment of Parkinson's disease and a personalized approach to management. Expert Rev. Neurother. 9, 1781-1789 (2009).

237. Mendis, T. et al. Management of Parkinson's disease: a review of current and new therapies. Can. J. Neurol. Sci. 26, 89-103 (1999).

238. Muller, T. Drug therapy in patients with Parkinson's disease. Transl. Neurodegener. 1, 10 (2012).

239. Schwab, S. T. et al. Amantadine in the Treatment of Parkinson's Disease. JAMA 208, 1168-1170 (1969).

240. No authors listed. Amantadine and other antiglutamate agents: management of Parkinson's disease. Mov. Disord. 17, Suppl 4, S13-S22 (2002).

241. Kornhuber, J. et al. Effects of the 1-amino-adamantanes at the MK-801-binding site of the NMDA-receptor-gated ion channel: a human postmortem brain study. Eur. J. Pharmacol. 206, 297-300 (1991).

242. Löhle, M.; Reichmann, H. Clinical neuroprotection in Parkinson's disease - still waiting for the breakthrough. J. Neurol. Sci. 289, 104-114 (2010).

243. No authors listed. Anticholinergic therapies in the treatment of Parkinson's disease. Mov. Disord. 17, Suppl 4, S7-S12 (2002).

244. Abdel-Salam, O. M. Drugs used to treat Parkinson's disease, present status and future directions. CNS Neurol. Disord.: Drug Targets 7, 321-342 (2008).

245. Chaudhuri, K. R. et al. Non-motor symptoms of Parkinson's disease: diagnosis and management. Lancet Neurol. 5, 235-245 (2006). 
246. Duker, A. P.; Espay, A. J. Surgical Treatment of Parkinson Disease : Past, Present, and Future. Neurol. Clin. 31, 799-808 (2013).

247. Hauser, R. A. et al. Long-term evaluation of bilateral fetal nigral transplantation in Parkinson disease. Arch. Neurol. 56, 179-187 (1999).

248. Le, W. et al. Animal models of Parkinson's disease: a gateway to therapeutics? Neurotherapeutics 11, 92-110 (2014).

249. Pienaar, I. S. et al. Parkinson's disease: insights from non-traditional model organisms. Prog. Neurobiol. 92, 558-571 (2010).

250. Dawson, T. M. et al. Genetic animal models of Parkinson's disease. Neuron 66, 646$661(2010)$.

251. Emborg, M. E. Evaluation of animal models of Parkinson's disease for neuroprotective strategies. J. Neurosci. Merhods 139, 121-143 (2004).

252. Luk, K. C.; Lee, V. M. Modeling Lewy pathology propagation in Parkinson's disease. Parkinsonism Relat. Disord. 20, Suppl 1, S85-S87 (2014).

253. Meredith, G. E. et al. Animal models of Parkinson's disease progression. Acta Neuropathol. 115, 385-398 (2008).

254. Blesa, J. et al. Classic and new animal models of Parkinson's disease. J. Biomed. Biotechnol. 2012, ID 845618 (2012).

255. Bezard, E. et al. Animal models of Parkinson's disease: Limits and relevance to neuroprotection studies. Mov. Disord. 28, 61-70 (2013).

256. Betarbet, R. et al. Chronic systemic pesticide exposure reproduces features of Parkinson's disease. Nat. Neurosci. 3, 1301-1306 (2000).

257. Coulom, H.; Birman, S. Chronic exposure to rotenone models sporadic Parkinson's disease in Drosophila melanogaster. J. Neurosci. 24, 10993-10998 (2004).

258. Zhou, S. et al. Caenorhabditis elegans neuron degeneration and mitochondrial suppression caused by selected environmental chemicals. Int. J. Biochem. Mol. Biol. 4, 191200 (2013).

259. Inden, M. et al. Parkinsonian rotenone mouse model: reevaluation of long-term administration of rotenone in C57BL/6 mice. Biol. Pharm. Bull. 34, 92-96 (2011).

260. Pan-Montojo, F. et al. Progression of Parkinson's disease pathology is reproduced by intragastric administration of rotenone in mice. PLoS One 5, e8762 (2010).

261. Eslamboli, A. Marmoset monkey models of Parkinson's disease: Which model, when and why? Brain Res. Bull. 68, 140-149 (2005).

262. Alvarez-Fischer, D. et al. Characterization of the striatal 6-OHDA model of Parkinson's disease in wild type and alpha-synuclein-deleted mice. Exp. Neurol. 210, 182193 (2008).

263. Chaudhuri, A. et al. Interaction of genetic and environmental factors in a Drosophila parkinsonism model. J. Neurosci. 27, 2457-2467 (2007).

264. Muthukumaran, K. et al. Orally delivered water soluble Coenzyme Q10 (Ubisol-Q10) blocks on-going neurodegeneration in rats exposed to paraquat: potential for therapeutic application in Parkinson's disease. BMC Neurosci. 15, 21 (2014).

265. Czerniczyniec, A. et al. Paraquat induces behavioral changes and cortical and striatal mitochondrial dysfunction. Free Radic. Biol. Med. 51, 1428-1436 (2011).

266. Dutta, G. et al. The lipopolysaccharide Parkinson's disease animal model: mechanistic studies and drug discovery. Fundam. Clin. Pharmacol. 22, 453-464 (2008).

267. Chege, P. M.; McColl, G. Caenorhabditis elegans: a model to investigate oxidative stress and metal dyshomeostasis in Parkinson's disease. Front Aging Neurosci. 6, Article 89 (2014).

268. Springer, W.; Kahle, P. J. Mechanisms and models of alpha-synuclein-related neurodegeneration. Curr. Neurol. Neurosci. Rep. 6, 432-436 (2006). 
269. Karpinar, D. P. et al. Pre-fibrillar alpha-synuclein variants with impaired betastructure increase neurotoxicity in Parkinson's disease models. EMBO J. 28, 3256-3268 (2009).

270. Botella, J. A. et al. Modelling Parkinson's disease in Drosophila. NeuroMol. Med. 11, 268-280 (2009).

271. Muñoz-Soriano, V.; Paricio, N. Drosophila models of Parkinson's disease: discovering relevant pathways and novel therapeutic strategies. Parkinsons Dis. 2011, id520640 (2011).

272. Chen, L.; Feany, M. B. Alpha-synuclein phosphorylation controls neurotoxicity and inclusion formation in a Drosophila model of Parkinson disease. Nat. Neurosci. 8, 657-663 (2005).

273. O'Donnell, K. C. et al. Axon degeneration and PGC-1 $\alpha$-mediated protection in a zebrafish model of $\alpha$-synuclein toxicity. Dis. Models \& Mech. 7, 571-582 (2014).

274. Prabhudesai, S. et al. A novel "molecular tweezer" inhibitor of $\alpha$-synuclein neurotoxicity in vitro and in vivo. Neurotherapeutics 9, 464-476 (2012).

275. Crabtree, D. M.; Zhang, J. Genetically engineered mouse models of Parkinson's disease. Brain Res. Bull. 88, 13-32 (2012).

276. Hashimoto, M. et al. Transgenic models of alpha-synuclein pathology: past, present, and future. Ann. N. Y. Acad. Sci. 991, 171-188 (2003).

277. Chesselet, M. F. In vivo alpha-synuclein overexpression in rodents: a useful model of Parkinson's disease? Exp. Neurol. 209, 22-27 (2008).

278. Masliah, E. et al. Dopaminergic loss and inclusion body formation in alpha-synuclein mice: implications for neurodegenerative disorders. Science 287, 1265-1269 (2000).

279. Rockenstein, E. et al. Differential neuropathological alterations in transgenic mice expressing alpha-synuclein from the platelet-derived growth factor and Thy-1 promoters. $J$. Neurosci. Res. 68, 568-578 (2002).

280. Gispert, S. et al. Transgenic mice expressing mutant A53T human alpha-synuclein show neuronal dysfunction in the absence of aggregate formation. Mol. Cell Neurosci. 24, 419-429 (2003).

281. Giasson, B. I. et al. Neuronal alpha-synucleinopathy with severe movement disorder in mice expressing A53T human alpha-synuclein. Neuron 34, 521-533 (2002).

282. Lee, M. K. et al. Human alpha-synuclein-harboring familial Parkinson's disease-linked Ala-53 --> Thr mutation causes neurodegenerative disease with alpha-synuclein aggregation in transgenic mice. Proc. Natl. Acad. Sci. U. S. A. 99, 8968-8973 (2002).

283. Neumann, M. et al. Misfolded proteinase K-resistant hyperphosphorylated alphasynuclein in aged transgenic mice with locomotor deterioration and in human alphasynucleinopathies. J. Clin. Invest. 110, 1429-1439 (2002).

284. van der Putten, H. et al. Neuropathology in mice expressing human alpha-synuclein. $J$. Neurosci. 20, 6021-6029 (2000).

285. Tofaris, G. K. et al. Pathological changes in dopaminergic nerve cells of the substantia nigra and olfactory bulb in mice transgenic for truncated human alpha-synuclein(1-120): implications for Lewy body disorders. J. Neurosci. 26, 3942-3950 (2006).

286. Nuber, S. et al. Neurodegeneration and motor dysfunction in a conditional model of Parkinson's disease. J. Neurosci. 28, 2471-2484 (2008).

287. Schneider, B. et al. Viral vectors, animal models and new therapies for Parkinson's disease. Parkinsonism Relat. Disord. 14, S169-S171 (2008).

288. Welchko, R. M. et al. Genetic Rat Models of Parkinson's Disease. Parkinsons Dis. 2012, ID 128356 (2012).

289. Taschenberger, G. et al. Aggregation of $\alpha$ Synuclein promotes progressive in vivo neurotoxicity in adult rat dopaminergic neurons. Acta Neuropathol. 123, 671-683 (2012). 
290. Shulman, J. M. et al. Parkinson's Disease: Genetics and Pathogenesis. Annu. Rev. Pathol.: Mech. Dis. 6, 193-222 (2011).

291. Daniel, G.; Moore, D. J. Modeling LRRK2 Pathobiology in Parkinson's Disease: From Yeast to Rodents. Curr. Top. Behav. Neurosci. 22, 331-368 (2014).

292. Whitworth, A. J. et al. Increased glutathione S-transferase activity rescues dopaminergic neuron loss in a Drosophila model of Parkinson's disease. Proc. Natl. Acad. Sci. U. S. A. 102, 8024-8029 (2005).

293. Xi, Y. et al. Modeling neurodegeneration in zebrafish. Curr. Neurol. Neurosci. Rep. 11, 274-282 (2011).

294. Fleming, S. M. et al. Genetic mouse models of parkinsonism: strengths and limitations. NeuroRx 2, 495-503 (2005).

295. Jones, J. M. et al. Loss of Omi mitochondrial protease activity causes the neuromuscular disorder of mnd2 mutant mice. Nature 425, 721-727 (2003).

296. Setsuie, R. et al. Dopaminergic neuronal loss in transgenic mice expressing the Parkinson's disease-associated UCH-L1 I93M mutant. Neurochem. Int. 50, 119-129 (2007).

297. Decressac, M. et al. NURR1 in Parkinson disease - from pathogenesis to therapeutic potential. Nat. Rev. Neurol. 9, 629-636 (2013).

298. Jiang, C. et al. Age-dependent dopaminergic dysfunction in Nurr1 knockout mice. Exp. Neurol. 191, 154-162 (2005).

299. Li, J. et al. The role of transcription factor Pitx3 in dopamine neuron development and Parkinson's disease. Curr. Top. Med. Chem. 9, 855-859 (2009).

300. Luk, K. C. et al. Pathological $\alpha$-synuclein transmission initiates Parkinson-like neurodegeneration in nontransgenic mice. Science 338, 949-953 (2012).

301. Luk, K. C. et al. Intracerebral inoculation of pathological $\alpha$-synuclein initiates a rapidly progressive neurodegenerative $\alpha$-synucleinopathy in mice. J. Exp. Med. 209, 975-986 (2012).

302. Recasens, A. et al. Lewy body extracts from Parkinson disease brains trigger $\alpha-$ synuclein pathology and neurodegeneration in mice and monkeys. Ann. Neurol. 75, 351-362 (2014).

303. Recasens, A.; Dehay, B. Alpha-Synuclein spreading in Parkinson disease. Front. Neuroanat. 8, 159 (2014).

304. Olanow, C. W.; Schapira, A. H. Therapeutic prospects for Parkinson disease. Ann. Neurol. 74, 337-347 (2013).

305. Yacoubian, T. A.; Standaert, D. G. Targets for neuroprotection in Parkinson's disease. Biochim. Biophys. Acta 1792, 676-687 (2009).

306. Savitt, D.; Jankovic, J. Targeting $\alpha$-Synuclein in Parkinson's Disease: Progress Towards the Development of Disease-Modifying Therapeutics. Drugs 79, 797-810 (2019).

307. Perry, D. et al. Building a roadmap for developing combination therapies for Alzheimer's disease. Expert Rev. Neurother. 15, 327-333 (2015).

308. Sardi, S. P. et al. Targeted Therapies for Parkinson's Disease: From Genetics to the Clinic. Mov. Disord. 33, 684-696 (2018).

309. Stoker, T. B. et al. Emerging Treatment Approaches for Parkinson's Disease. Front. Neurosci. 12, 693 (2018).

310. Elkouzi, A. et al. Emerging therapies in Parkinson disease - repurposed drugs and new approaches. Nat. Rev. Neurol. 15, 204-223 (2019).

311. Brundin, P. et al. Therapeutic approaches to target alpha-synuclein pathology. Exp. Neurol. 298, 225-235 (2017).

312. Junn, E. et al. Repression of alpha-synuclein expression and toxicity by microRNA-7.

Proc. Natl. Acad. Sci. U. S. A. 106, 13052-13057 (2009). 
313. Kabaria, S. et al. Inhibition of miR-34b and miR-34c enhances $\alpha$-synuclein expression in Parkinson's disease. FEBS Lett. 589, 319-325 (2015).

314. Kalia, L. V. et al. Disease-modifying strategies for Parkinson's disease. Mov. Disord. 30, 1442-1450 (2015).

315. Bae, E. J. et al. Antibody-aided clearance of extracellular $\alpha$-synuclein prevents cell-tocell aggregate transmission. J. Neurosci. 32, 13454-13469 (2012).

316. Lynch, S. M. et al. An scFv intrabody against the nonamyloid component of alphasynuclein reduces intracellular aggregation and toxicity. J. Mol. Biol. 377, 136-147 (2008).

317. Spencer, B. et al. ESCRT-mediated Uptake and Degradation of Brain-targeted $\alpha-$ synuclein Single Chain Antibody Attenuates Neuronal Degeneration In Vivo. Mol. Ther. 22, 1753-1767 (2014).

318. Kalia, S. K. et al. Molecular chaperones as rational drug targets for Parkinson's disease therapeutics. CNS Neurol. Disord.: Drug Targets 9, 741-753 (2010).

319. Cullen, V. et al. Cathepsin D expression level affects alpha-synuclein processing, aggregation, and toxicity in vivo. Mol. Brain 2, 5 (2009).

320. Spencer, B. et al. Lentivirus mediated delivery of neurosin promotes clearance of wild-type $\alpha$-synuclein and reduces the pathology in an $\alpha$-synuclein model of LBD. Mol. Ther. 21, 31-41 (2013).

321. Ono, K. et al. Effects of antiparkinsonian agents on $\beta$-amyloid and $\alpha$-synuclein oligomer formation in vitro. J. Neurosci. Res. 91, 1371-1381 (2013).

322. Li, H. T. et al. Inhibition of alpha-synuclein fibrillization by dopamine analogs via reaction with the amino groups of alpha-synuclein. Implication for dopaminergic neurodegeneration. FEBS J. 272, 3661-3672 (2005).

323. Herrera, F. E. et al. Inhibition of alpha-synuclein fibrillization by dopamine is mediated by interactions with five C-terminal residues and with $\mathrm{E} 83$ in the NAC region. PLoS One 3, e3394 (2008).

324. Leong, S. L. et al. Formation of dopamine-mediated alpha-synuclein-soluble oligomers requires methionine oxidation. Free Radical Biol. Med. 46, 1328-1337 (2009).

325. Cappai, R. et al. Dopamine promotes alpha-synuclein aggregation into SDS-resistant soluble oligomers via a distinct folding pathway. FASEB J. 19, 1377-1379 (2005).

326. Norris, E. H. et al. Reversible inhibition of alpha-synuclein fibrillization by dopaminochrome-mediated conformational alterations. J. Biol. Chem. 280, 21212-21219 (2005).

327. Conway, K. A. et al. Kinetic stabilization of the alpha-synuclein protofibril by a dopamine-alpha-synuclein adduct. Science 294, 1346-1349 (2001).

328. Li, J. et al. Dopamine and L-dopa disaggregate amyloid fibrils: implications for Parkinson's and Alzheimer's disease. FASEB J. 18, 962-964 (2004).

329. Mazzulli, J. R. et al. Cytosolic catechols inhibit alpha-synuclein aggregation and facilitate the formation of intracellular soluble oligomeric intermediates. J. Neurosci. 26, 10068-10078 (2006).

330. Caruana, M. et al. Inhibition and disaggregation of $\alpha$-synuclein oligomers by natural polyphenolic compounds. FEBS Lett. 585, 1113-1120 (2011).

331. Hong, D. P. et al. Structural characteristics of alpha-synuclein oligomers stabilized by the flavonoid baicalein. J. Mol. Biol. 383, 214-223 (2008).

332. Zhu, M. et al. The flavonoid baicalein inhibits fibrillation of alpha-synuclein and disaggregates existing fibrils. J. Biol. Chem. 279, 26846-26857 (2004).

333. Meng, X. et al. Molecular mechanisms underlying the flavonoid-induced inhibition of alpha-synuclein fibrillation. Biochemistry 48, 8206-8224 (2009). 
334. Lu, J. H. et al. Baicalein inhibits formation of $\alpha$-Synuclein oligomers within living cells and prevents $A \beta$ peptide fibrillation and oligomerisation. Chembiochem. 12, 615-624 (2011).

335. Latawiec, D. et al. Modulation of alpha-synuclein aggregation by dopamine analogs. PLoS One 5, e9234 (2010).

336. Leong, S. L. et al. Modulation of alpha-synuclein aggregation by dopamine: a review. Neurochem. Res. 34, 1838-1846 (2009).

337. Yamakawa, K. et al. Dopamine facilitates alpha-synuclein oligomerization in human neuroblastoma SH-SY5Y cells. Biochem. Biophys. Res. Commun. 391, 129-134 (2010).

338. Shaltiel-Karyo, R. et al. A blood-brain barrier (BBB) disrupter is also a potent $\alpha-$ synuclein ( $\alpha$-syn) aggregation inhibitor: a novel dual mechanism of mannitol for the treatment of Parkinson disease (PD). J. Biol. Chem. 288, 17579-17588 (2013).

339. Price, D. L. et al. The small molecule alpha-synuclein misfolding inhibitor, NPT20011, produces multiple benefits in an animal model of Parkinson's disease. Sci. Rep. 8, 16165 (2018).

340. Wrasidlo, W. et al. A de novo compound targeting $\alpha$-synuclein improves deficits in models of Parkinson's disease. Brain 139, 3217-3236 (2016).

341. Di Giovanni, S. et al. Entacapone and tolcapone, two catechol O-methyltransferase inhibitors, block fibril formation of alpha-synuclein and beta-amyloid and protect against amyloid-induced toxicity. J. Biol. Chem. 285, 14941-14954 (2010).

342. Ono, K.; Yamada, M. Antioxidant compounds have potent anti-fibrillogenic and fibril-destabilizing effects for alpha-synuclein fibrils in vitro. J. Neurochem. 97, 105-115 (2006).

343. Ruzza, P. et al. Ceftriaxone Blocks the Polymerization of $\alpha$-Synuclein and Exerts Neuroprotective Effects in Vitro. ACS Chem. Neurosci. 5, 30-38 (2014).

344. Maltsev, A. S. et al. Monomeric $\alpha$-synuclein binds Congo Red micelles in a disordered manner. Biochemistry 51, 631-642 (2012).

345. Lendel, C. et al. On the mechanism of nonspecific inhibitors of protein aggregation: dissecting the interactions of alpha-synuclein with Congo red and Lacmoid. Biochemistry 48, 8322-8334 (2009).

346. Rao, J. N. et al. Characterization of alpha-synuclein interactions with selected aggregation-inhibiting small molecules. Biochemistry 47, 4651-4656 (2008).

347. Morshedi, D. et al. Cuminaldehyde as the Major Component of Cuminum cyminum, a Natural Aldehyde with Inhibitory Effect on Alpha-Synuclein Fibrillation and Cytotoxicity. $J$. Food Sci. 80, 2336-2345 (2015).

348. Marchiani, A. et al. Small molecules interacting with $\alpha$-synuclein: antiaggregating and cytoprotective properties. Amino Acids 45, 327-338 (2013).

349. Pandey, N. et al. Curcumin inhibits aggregation of alpha-synuclein. Acta Neuropathol. 115, 479-489 (2008).

350. Takahashi, R. et al. Phenolic compounds prevent the oligomerization of $\alpha$-synuclein and reduce synaptic toxicity. J. Neurochem. 134, 943-955 (2015).

351. Gadad, B. S. et al. Curcumin-glucoside, a novel synthetic derivative of curcumin, inhibits $\alpha$-synuclein oligomer formation: relevance to Parkinson's disease. Curr. Pharm. Des. 18, 76-84 (2012).

352. Zhou, W. et al. At low concentrations, 3,4-dihydroxyphenylacetic acid (DOPAC) binds non-covalently to alpha-synuclein and prevents its fibrillation. J. Mol. Biol. 288, 597610 (2009).

353. Bieschke, J. et al. EGCG remodels mature alpha-synuclein and amyloid-beta fibrils and reduces cellular toxicity. Proc. Natl. Acad. Sci. U. S. A. 107, 7710-7715 (2010). 
354. Ehrnhoefer, D. E. et al. EGCG redirects amyloidogenic polypeptides into unstructured, off-pathway oligomers. Nat. Struct. Mol. Biol. 15, 558-566 (2008).

355. Masuda, M. et al. Inhibition of alpha-synuclein fibril assembly by small molecules: analysis using epitope-specific antibodies. FEBS Lett. 583, 787-791 (2009).

356. Yamaguchi, Y. et al. Characterization of inhibitor-bound alpha-synuclein dimer: role of alpha-synuclein N-terminal region in dimerization and inhibitor binding. J. Mol. Biol. 395, 445-456 (2010).

357. Horvath, I. et al. Mechanisms of protein oligomerization: inhibitor of functional amyloids templates $\alpha$-synuclein fibrillation. J. Am. Chem. Soc. 134, 3439-3444 (2012).

358. Ardah, M. T. et al. Structure activity relationship of phenolic acid inhibitors of $\alpha-$ synuclein fibril formation and toxicity. Front Aging Neurosci. 6, 197 (2014).

359. Meng, X. et al. Effects of various flavonoids on the $\alpha$-Synuclein fibrillation process. Parkinsons Dis. 2010, 650794 (2010).

360. Masuda, M. et al. Small molecule inhibitors of alpha-synuclein filament assembly. Biochemistry 45, 6085-6094 (2006).

361. Liu, Y. et al. Hemin as a generic and potent protein misfolding inhibitor. Biochem. Biophys. Res. Commun. 454, 295-300 (2014).

362. Dedmon, M. M. et al. Heat shock protein 70 inhibits alpha-synuclein fibril formation via preferential binding to prefibrillar species. J. Biol. Chem. 280, 14733-14740 (2005).

363. Hong, D. P. et al. Smoking and Parkinson's disease: does nicotine affect alphasynuclein fibrillation? Biochim. Biophys. Acta 1794, 282-290 (2009).

364. Lee, D. et al. The modification of alpha-synuclein by dicarbonyl compounds inhibits its fibril-forming process. Biochim. Biophys. Acta 1794, 421-430 (2009).

365. Rekas, A. et al. PAMAM dendrimers as potential agents against fibrillation of alphasynuclein, a Parkinson's disease-related protein. Macromol. Biosci. 9, 230-238 (2009).

366. Milowska, K. et al. PAMAM G4 dendrimers affect the aggregation of $\alpha$-synuclein. Int. J. Biol. Macromol. 48, 742-746 (2011).

367. Scherzer-Attali, R. et al. Generic inhibition of amyloidogenic proteins by two naphthoquinone-tryptophan hybrid molecules. Proteins 80, 1962-1973 (2012).

368. Lee, E. N. et al. Phthalocyanine tetrasulfonates affect the amyloid formation and cytotoxicity of alpha-synuclein. Biochemistry 43, 3704-3715 (2004).

369. Lamberto, G. R. et al. Structural and mechanistic basis behind the inhibitory interaction of PcTS on alpha-synuclein amyloid fibril formation. Proc. Natl. Acad. Sci. U. S. A. 106, 21057-21062 (2009).

370. Cheruvara, H. et al. Intracellular screening of a peptide library to derive a potent peptide inhibitor of $\alpha$-synuclein aggregation. J. Biol. Chem. 290, 7426-7435 (2015).

371. El-Agnaf, O. M. et al. A strategy for designing inhibitors of alpha-synuclein aggregation and toxicity as a novel treatment for Parkinson's disease and related disorders. FASEB J. 18, 1315-1317 (2004).

372. Kobayashi, M. et al. Pyrroloquinoline quinone (PQQ) prevents fibril formation of alpha-synuclein. Biochem. Biophys. Res. Commun. 349, 1139-1144 (2006).

373. Li, J. et al. Rifampicin inhibits alpha-synuclein fibrillation and disaggregates fibrils. Chem. Biol. 11, 1513-1521 (2004).

374. Emadi, S. et al. Inhibiting aggregation of alpha-synuclein with human single chain antibody fragments. Biochemistry 43, 2871-2878 (2004).

375. Ibrahim, T.; McLaurin, J. $\alpha$-Synuclein aggregation, seeding and inhibition by scylloinositol. Biochem. Biophys. Res. Commun. 469, 529-534 (2016).

376. Ji, K. et al. Inhibition effects of tanshinone on the aggregation of $\alpha$-synuclein. Food Funct. 7, 409-416 (2016). 
377. Grelle, G. et al. Black tea theaflavins inhibit formation of toxic amyloid- $\beta$ and $\alpha-$ synuclein fibrils. Biochemistry 50, 10624-10636 (2011).

378. Sivanesam, K. et al. Binding Interactions of Agents That Alter $\alpha$-Synuclein Aggregation. RSC Adv. 5, 11577-11590 (2015).

379. Rekas, A. et al. Interaction of the molecular chaperone alphaB-crystallin with alphasynuclein: effects on amyloid fibril formation and chaperone activity. J. Mol. Biol. 340, 1167-1183 (2004).

380. Stocchi, F.; Olanow, C. W. Neuroprotection in Parkinson's disease: clinical trials. Ann. Neurol. 53, S87-S97 (2003).

381. Allain, H. et al. Disease-modifying drugs and Parkinson's disease. Prog. Neurobiol. 84, 25-39 (2008).

382. Meissner, W. et al. Neuroprotective strategies for Parkinson's disease: conceptual limits of animal models and clinical trials. Trends Pharmacol. Sci. 25, 249-253 (2004).

383. Ravina, B. M. et al. Neuroprotective agents for clinical trials in Parkinson's disease. Neurology 60, 1234-1240 (2003).

384. Park, A.; Stacy, M. Disease-Modifying Drugs in Parkinson's Disease. Drugs 75 , 2065-2071 (2015).

385. Petit, G. H. et al. The future of cell therapies and brain repair: Parkinson's disease leads the way. Neuropathol. Appl. Neurobiol. 40, 60-70 (2014).

386. Dehay, B. et al. Targeting $\alpha$-synuclein: Therapeutic options. Mov Disord. 31, 882-888 (2016).

387. Bieschke, J. et al. Ultrasensitive detection of pathological prion protein aggregates by dual-color scanning for intensely fluorescent targets. Proc. Natl. Acad. Sci. U. S. A. 97, 5468$5473(2000)$.

388. Vilette, D. Cell models of prion infection. Vet. Res. 39, 10 (2008).

389. Venda, L. L. et al. $\alpha$-Synuclein and dopamine at the crossroads of Parkinson's disease.

Trends Neurosci. 33, 559-568 (2010).

390. Karakaya, S. Bioavailability of phenolic compounds. Crit. Rev. Food Sci. Nutr. 44, 453-464 (2004).

391. Bulic, B. et al. Progress and developments in tau aggregation inhibitors for Alzheimer disease. J. Med. Chem. 56, 4135-4155 (2013).

392. de la Torre, R. et al. MDMA, methamphetamine, and CYP2D6 pharmacogenetics: what is clinically relevant? Front. Genet. 3, 235 (2012).

393. Anselme, J. P. Convenient and practical preparation of dibenzoylmethane. J. Org. Chem. 32, 3716-3718 (1967).

394. Hauser, C. R.; Eby, C. J. N- and C-benzoylation of p-aminoacetophenone with methyl benzoate by sodium amide. Synthesis of $\beta$-diketones having p-acylamino and p-hydroxy groups. J. Org. Chem. 22, 909-912 (1957).

395. Heller, S. T.; Natarajan, S. R. 1,3-Diketones from acid chlorides and ketones: a rapid and general one-pot synthesis of pyrazoles. Org. Lett. 8, 2675-2678 (2006).

396. Nam, N. H. et al. Synthesis and cytotoxicity of 2,5-dihydroxychalcones and related compounds. Arch. Pharmacal Res. 27, 581-588 (2004).

397. Harris, R. L. N.; Huppatz, J. L. Synthetic plant growth regulators. The synthesis of Co-Carboxyphenyl derivatives of some five-membered heterocycles. Aust. J. Chem. 30, $2225-$ 2240 (1977).

398. Bhat, B. A. et al. Synthesis of 3,5-diphenyl-1H-pyrazoles. Synth. Commun. 35, 11351142 (2005).

399. Pinto, D. C. G. A. et al. New bis(chalcones) and their transformation into bis(pyrazoline) and bis(pyrazole) derivatives. Eur. J. Org. Chem., 747-755 (2003). 
400. LeBlanc, R. et al. Synthesis and cytotoxicity of epoxide and pyrazole analogs of the combretastatins. Bioorg. Med. Chem. 13, 6025-6034 (2005).

401. Sayed, G. H.; Kjoesen, H. Syntheses and spectroscopic characterization of some new 3,5-bisaryl-2-pyrazoline derivatives. II. J. Prakt. Chem. (Leipzig) 322, 716-722 (1980).

402. Agrawal, K. Oxidation of pyrazolines with manganese dioxide. J. Ind. Chem. Soc. 64, 408-410 (1987).

403. Cunningham, B. D. M. et al. Structural studies on bioactive compounds. Part 12. Tautomerism and conformation of aryl-substituted 1-(2-hydroxyphenyl)-3-phenylpropane1,3-diones in the solid phase and in solution. J. Chem. Soc., Perkin Trans. 2, 1275-1283 (1989).

404. Muthukumar, V. A. et al. Synthesis characterization and biological evaluation of isoxazole and pyrazole derivatives from $\beta$-diketones. J. Pharm. Res. 4, 4654-4657 (2011).

405. Hall, M. J. et al. A modular synthesis of unsymmetrical tetraarylazadipyrromethenes. J. Org. Chem. 70, 5571-5578 (2005).

406. Li, B. et al. An optimized process for formation of 2,4-disubstituted imidazoles from condensation of amidines and $\alpha$-haloketones. Org. Process Res. Dev. 6, 682-683 (2002).

407. Barrett, A. G. M. et al. Chromatography-Free Synthesis of 1,2,4-Oxadiazoles Using ROMPGEL-Supported Acylating Reagents. Comb. Chem. High Throughput Screening 3, 131-138 (2000).

408. Zhao, Z.-G.; Wang, Z.-X. Halogenation of pyrazoles using $N$-halosuccinimides in $\mathrm{CCl}_{4}$ and in Water. Synth. Commun. 37, 137-147 (2007).

409. Kashima, C. et al. The preparation of N-acylpyrazoles and their behavior toward alcohols. Synthesis, 61-65 (1994).

410. Dhake, K. P. et al. An efficient, catalyst- and solvent-free N-formylation of aromatic and aliphatic amines. Green Chem. Lett. Rev. 4, 151-157 (2011).

411. Okamoto, I. et al. Acid-induced conformational alteration of cis-preferential aromatic amides bearing N-methyl-N-(2-pyridyl) moiety. Tetrahedron 67, 8536-8543 (2011).

412. Dua, R. et al. Pharmacological Significance of Synthetic Heterocycles Scaffold: A Review. Adv. Biol. Res. 5, 120-144 (2011).

413. Birkett, C. R. et al. Scrapie strains maintain biological phenotypes on propagation in a cell line in culture. EMBO J. 20, 3351-3358 (2001).

414. Saborio, G. P. et al. Sensitive detection of pathological prion protein by cyclic amplification of protein misfolding. Nature 411, 810-813 (2001).

415. Saá, P.; Cervenakova, L. Protein misfolding cyclic amplification (PMCA): Current status and future directions. Virus Res. 207, 47-61 (2015).

416. Mallucci, G. R. et al. Targeting cellular prion protein reverses early cognitive deficits and neurophysiological dysfunction in prion-infected mice. Neuron 53, 325-335 (2007).

417. Collinge, J. Prion diseases of humans and animals: their causes and molecular basis. Annu. Rev. Neurosci. 24, 519-550 (2001).

418. Prusiner, S. B. et al. Experimental scrapie in the mouse: electrophoretic and sedimentation properties of the partially purified agent. J. Neurochem. 35, 574-582 (1980).

419. Giles, K. et al. Different 2-Aminothiazole Therapeutics Produce Distinct Patterns of Scrapie Prion Neuropathology in Mouse Brains. J. Pharmacol. Exp. Ther. 355, 2-12 (2015).

420. Giles, K. et al. Optimization of Aryl Amides that Extend Survival in Prion-Infected Mice. J. Pharmacol. Exp. Ther. 358, 537-547 (2016).

421. Kawasaki, Y. et al. Orally administered amyloidophilic compound is effective in prolonging the incubation periods of animals cerebrally infected with prion diseases in a prion strain-dependent manner. J. Virol. 81, 12889-12898 (2007).

422. Demaimay, R. et al. Pharmacological studies of a new derivative of amphotericin B, MS-8209, in mouse and hamster scrapie. J. Gen. Virol. 75, 2499-2503 (1994). 
423. Demaimay, R. et al. Late treatment with polyene antibiotics can prolong the survival time of scrapie-infected animals. J. Virol. 71, 9685-9689 (1997).

424. Riemer, C. et al. Evaluation of drugs for treatment of prion infections of the central nervous system. J. Gen. Virol. 89, 594-597 (2008).

425. Priola, S. A. et al. Prophylactic and therapeutic effects of phthalocyanine tetrasulfonate in scrapie-infected mice. J. Infect. Dis. 188, 699-705 (2003).

426. Doh-ura, K. et al. Treatment of transmissible spongiform encephalopathy by intraventricular drug infusion in animal models. J. Virol. 78, 4999-5006 (2004).

427. Chung, E. et al. Styryl-based and tricyclic compounds as potential anti-prion agents. PLoS One 6, e24844 (2011).

428. Vetrugno, V. et al. Oral pravastatin prolongs survival time of scrapie-infected mice. $J$. Gen. Virol. 90, 1775-1780 (2009).

429. Haviv, Y. et al. Induced neuroprotection independently from PrPSc accumulation in a mouse model for prion disease treated with simvastatin. Arch. Neurol. 65, 762-775 (2008).

430. Brazier, M. W. et al. Manganese chelation therapy extends survival in a mouse model of M1000 prion disease. J. Neurochem. 114, 440-451 (2010).

431. Herrmann, U. S. et al. Structure-based drug design identifies polythiophenes as antiprion compounds. Sci. Transl. Med. 7, 299 ra123 (2015).

432. Karapetyan, Y. E. et al. Unique drug screening approach for prion diseases identifies tacrolimus and astemizole as antiprion agents. Proc. Natl. Acad. Sci. U. S. A. 110, 7044-7049 (2013).

433. Mukherjee, A. et al. Calcineurin inhibition at the clinical phase of prion disease reduces neurodegeneration, improves behavioral alterations and increases animal survival. PLoS Pathog. 6, e1001138 (2010).

434. Kraus, A. et al. Prions and the potential transmissibility of protein misfolding diseases. Annu. Rev. Microbiol. 67, 543-564 (2013).

435. Aguzzi, A. et al. Toward Therapy of Human Prion Diseases. Annu. Rev. Pharmacol. Toxicol. 58, 331-351 (2018).

436. Kretzschmar, H.; Tatzelt, J. Prion disease: a tale of folds and strains. Brain Pathol. 23, 321-332 (2013).

437. Prusiner, S. B. Prion encephalopathies of animals and humans. Dev. Biol. Stand. 80, 31-44 (1993).

438. Colby, D. W.; Prusiner, S. B. Prions. Cold Spring Harbor Perspect. Biol. 3, a006833 (2011).

439. Trevitt, C. R.; Collinge, J. A systematic review of prion therapeutics in experimental models. Brain 129, 2241-2265 (2006).

440. Giles, K. et al. Developing Therapeutics for PrP Prion Diseases. Cold Spring Harbor Perspect. Med. 7, a023747 (2017).

441. Ghaemmaghami, S. et al. Successes and challenges in phenotype-based lead discovery for prion diseases. J. Med. Chem. 57, 6919-6929 (2014).

442. Moda, F. et al. Novel screening approaches for human prion diseases drug discovery. Expert Opin. Drug Discov. 14, 983-993 (2019).

443. Qin, K. et al. Anle138b prevents PrP plaque accumulation in $\operatorname{Tg}(\mathrm{PrP}-\mathrm{A} 116 \mathrm{~V})$ mice but does not mitigate clinical disease. J. Gen. Virol. 100, 1027-1037 (2019).

444. Hegde, R. S. et al. A transmembrane form of the prion protein in neurodegenerative disease. Science 279, 827-834 (1998).

445. Giese, A. et al. Single particle detection and characterization of synuclein coaggregation. Biochem. Biophys. Res. Commun. 333, 1202-1210 (2005).

446. Naiki, H. et al. Fluorometric determination of amyloid fibrils in vitro using the fluorescent dye, thioflavin T1. Anal. Biochem. 177, 244-249 (1989). 
447. Stetefeld, J. et al. Dynamic light scattering: a practical guide and applications in biomedical sciences. Biophys. Rev. 8, 409-427 (2016).

448. Stephens, A. D. et al. Different Structural Conformers of Monomeric $\alpha$-Synuclein Identified after Lyophilizing and Freezing. Anal. Chem. 90, 6975-6983 (2018).

449. Arndt, C. et al. Native polyacrylamide gels. Methods Mol. Biol. 869, 49-53 (2012).

450. Uversky, V. N. et al. Methionine oxidation inhibits fibrillation of human alphasynuclein in vitro. FEBS Lett. 517, 239-244 (2002).

451. Glaser, C. B. et al. Methionine oxidation, alpha-synuclein and Parkinson's disease. Biochem. Biophys. Acta 1703, 157-169 (2005).

452. Williamson, M. P. Using chemical shift perturbation to characterise ligand binding. Prog. Nucl. Magn. Reson. Spectrosc. 73, 1-16 (2013).

453. Pellecchia, M. et al. NMR in drug discovery. Nat. Rev. Drug Discov. 1, 211-219 (2002).

454. Pellecchia, M. Solution nuclear magnetic resonance spectroscopy techniques for probing intermolecular interactions. Chem. Biol. 12, 961-971 (2005).

455. Meyer, B.; Peters, T. NMR spectroscopy techniques for screening and identifying ligand binding to protein receptors. Angew. Chem., Int. Ed. Engl. 42, 864-890 (2003).

456. Meyer, B. et al. Screening mixtures for biological activity by NMR. Eur. J. Biochem. 246, 705-709 (1997).

457. Mayer, M.; Meyer, B. Characterization of Ligand Binding by Saturation Transfer Difference NMR Spectroscopy. Angew. Chem., Int. Ed. Engl. 38, 1784-1788 (1999).

458. Angulo, J. et al. Ligand-receptor binding affinities from saturation transfer difference (STD) NMR spectroscopy: the binding isotherm of STD initial growth rates. Chemistry 16, 7803-7812 (2010).

459. Kim, H. Y. et al. Dissociation of amyloid fibrils of alpha-synuclein in supercooled water. Angew. Chem., Int. Ed. Engl. 47, 5046-5048 (2008).

460. Deeg, A. A. et al. Anle138b and related compounds are aggregation specific fluorescence markers and reveal high affinity binding to $\alpha$-synuclein aggregates. Biochim. Biophys. Acta 1850, 1884-1890 (2015).

461. Reiner, A. M. et al. Photophysics of diphenyl-pyrazole compounds in solutions and $\alpha-$ synuclein aggregates. Biochim. Biophys. Acta, Gen. Subj. 1862, 800-807 (2018).

462. Arispe, N. et al. Alzheimer disease amyloid beta protein forms calcium channels in bilayer membranes: blockade by tromethamine and aluminum. Proc. Natl. Acad. Sci. U. S. A. 90, 567-571 (1993).

463. Benz, R. et al. Formation of large, ion-permeable membrane channels by the matrix protein (porin) of Escherichia coli. Biochem. Biophys. Acta 511, 305-319 (1978).

464. Bliss, T. V.; Collingridge, G. L. A synaptic model of memory: long-term potentiation in the hippocampus. Nature 361, 31-39 (1993).

465. Martin, S. J. et al. Synaptic plasticity and memory: an evaluation of the hypothesis. Annu. Rev. Neurosci. 23, 649-711 (2000).

466. Puzzo, D. et al. LTP and memory impairment caused by extracellular A $\beta$ and Tau oligomers is APP-dependent. eLife 6, e26991 (2017).

467. Gelman, S. et al. Differences in Synaptic Dysfunction Between rTg4510 and APP/PS1 Mouse Models of Alzheimer's Disease. J. Alzheimer's Dis. 61, 195-208 (2018).

468. Bieschke, J. et al. Small-molecule conversion of toxic oligomers to nontoxic $\beta$-sheetrich amyloid fibrils. Nat. Chem. Biol. 8, 93-101 (2011).

469. Lu, B.; Vogel, H. Drosophila models of neurodegenerative diseases. Annu. Rev. Pathol. 4, 315-342 (2009). 
470. Kashima, N. et al. Development of a method for oral administration of hydrophobic substances to Caenorhabditis elegans: pro-longevity effects of oral supplementation with lipid-soluble antioxidants. Biogerontology 13, 337-344 (2012).

471. Jucker, M.; Walker, L. C. Self-propagation of pathogenic protein aggregates in neurodegenerative diseases. Nature 501, 45-51 (2013).

472. Kaufman, S. K.; Diamond, M. I. Prion-like propagation of protein aggregation and related therapeutic strategies. Neurotherapeutics 10, 371-382 (2013).

473. Wegrzynowicz, M. et al. Depopulation of dense $\alpha$-synuclein aggregates is associated with rescue of dopamine neuron dysfunction and death in a new Parkinson's disease model. Acta Neuropathol. 138, 575-595 (2019).

474. Cannon, J. R. et al. A highly reproducible rotenone model of Parkinson's disease. Neurobiol. Dis. 34, 279-290 (2009).

475. Pan-Montojo, F. et al. Environmental toxins trigger PD-like progression via increased alpha-synuclein release from enteric neurons in mice. Sci. Rep. 2, 898 (2012).

476. Tolosa, E. et al. The diagnosis of Parkinson's disease. Lancet Neurol. 5, 75-86 (2006). 477. Sorrentino, Z. A. et al. Physiological C-terminal truncation of $\alpha$-synuclein potentiates the prion-like formation of pathological inclusions. J. Biol. Chem. 293, 18914-18932 (2018).

478. Liu, C. W. et al. A precipitating role for truncated alpha-synuclein and the proteasome in alpha-synuclein aggregation: implications for pathogenesis of Parkinson disease. J. Biol. Chem. 280, 22670-22678 (2005).

479. Sigal, Y. M. et al. Visualizing and discovering cellular structures with superresolution microscopy. Science 361, 880-887 (2018).

480. Fellner, L. et al. Anle138b Partly Ameliorates Motor Deficits Despite Failure of Neuroprotection in a Model of Advanced Multiple System Atrophy. Front. Neurosci. 10, 99 (2016).

481. Heras-Garvin, A. et al. Anle138b modulates $\alpha$-synuclein oligomerization and prevents motor decline and neurodegeneration in a mouse model of multiple system atrophy. Mov Disord. 34, 255-263 (2019).

482. Burré, J. et al. Alpha-synuclein promotes SNARE-complex assembly in vivo and in vitro. Science 329, 1663-1667 (2010).

483. Ghiglieri, V. et al. Alpha-Synuclein: From Early Synaptic Dysfunction to Neurodegeneration. Front. Neurol. 9, 295 (2018).

484. Goedert, M. Alpha-synuclein and neurodegenerative diseases. Nat. Rev. Neurosci. 2, 492-501 (2001).

485. Ubeda-Bañon, I. et al. $\alpha$-Synuclein in the olfactory system in Parkinson's disease: role of neural connections on spreading pathology. Brain Struct. Funct., in press (2013).

486. Levin, J. et al. The oligomer modulator anle138b inhibits disease progression in a Parkinson mouse model even with treatment started after disease onset. Acta Neuropathol. 127, 779-780 (2014).

487. Karpowicz, R. J. J. et al. Transmission of $\alpha$-synuclein seeds in neurodegenerative disease: recent developments. Lab. Invest. 99, 971-981 (2019).

488. Li, J. Y. et al. Lewy bodies in grafted neurons in subjects with Parkinson's disease suggest host-to-graft disease propagation. Nat. Med. 14, 5501-503 (2008).

489. Wagner, J. et al. Reducing tau aggregates with anle $138 \mathrm{~b}$ delays disease progression in a mouse model of tauopathies. Acta Neuropathol. 130, 619-631 (2015).

490. Martinez Hernandez, A. et al. The diphenylpyrazole compound anle138b blocks A $\beta$ channels and rescues disease phenotypes in a mouse model for amyloid pathology. EMBO Mol. Med. 10, 32-47 (2018).

491. Strickley, R. G. Solubilizing excipients in oral and injectable formulations. Pharm. Res. 21, 201-230 (2004). 
492. Walther, R. et al. Prodrugs in medicinal chemistry and enzyme prodrug therapies. Adv. Drug Deliv. Rev. 118, 65-77 (2017).

493. Rautio, J. et al. Prodrugs: design and clinical applications. Nat. Rev. Drug Discov. 7, 255-270 (2008).

494. Giang, I. et al. Prodrug applications for targeted cancer therapy. AAPS J. 16, 899-913 (2014).

495. Pavan, B. et al. Progress in drug delivery to the central nervous system by the prodrug approach. Molecules 13, 1035-1065 (2008).

496. Müller, C. E. Prodrug approaches for enhancing the bioavailability of drugs with low solubility. Chem. Biodivers. 6, 2071-2083 (2009).

497. Sato, K. et al. Composition containing chloromethyl phosphate derivative with improved stability and method for producing the same. EP2133355A1 (2009).

498. Baughman, T. W. et al. The facile preparation of alkenyl metathesis synthons. Tetrahedron 60, 10943-10948 (2004).

499. Furuta, T. et al. Direct Esterification of Phosphates with Various Halides and its Application to Synthesis of cAMP Alkyl Triesters. J. Chem. Soc., Perkin Trans. 1, 3139-3142 (1993).

500. Kirby, A. J. et al. Intramolecular general acid catalysis of phosphate transfer. nucleophilic attack by oxyanions on the PO3 2- group. J. Am. Chem. Soc. 127, 7033-7040 (2005).

501. Ni, J. et al. Synthesis of Maleimide-Activated Carbohydrates as Chemoselective Tags for Site-Specific Glycosylation of Peptides and Proteins. Bioconjugate Chem. 14, 232-238 (2003).

502. Klein, E. et al. New chemical tools for investigating human mitotic kinesin Eg5. Bioorg. Med. Chem. 15, 6474-6488 (2007).

503. Giese, A. et al. Water-soluble derivatives of 3,5-diphenyl-diazole compounds. PCT Int. Appl. WO2017102893A1 (2017).

504. Camilleri, A. et al. Tau-induced mitochondrial membrane perturbation is dependent upon cardiolipin. Biochim. Biophys. Acta, Biomembr., in press (2019).

505. Brendel, M. et al. Late-stage Anle138b treatment ameliorates tau pathology and metabolic decline in a mouse model of human Alzheimer's disease tau. Alzheimer's Res. Ther. 11, 67 (2019).

506. Turriani, E. et al. Treatment with diphenyl-pyrazole compound anle $138 \mathrm{~b} / \mathrm{c}$ reveals that $\alpha$-synuclein protects melanoma cells from autophagic cell death. Proc. Natl. Acad. Sci. U. S. A. 114, E4971-E4977 (2017).

507. Becker, D. et al. Phenyl-heterocycle-phenyl derivatives for use in the treatment or prevention of melanoma. PCT Int. Appl. WO2018206778A1 (2018).

508. Hoyer, W. et al. Dependence of alpha-synuclein aggregate morphology on solution conditions. J. Mol. Biol. 322, 383-393 (2002).

509. Delaglio, F. et al. MRPipe: a multidimensional spectral processing system based on UNIX pipes. J. Biomol. NMR. 6, 277-293 (1995).

510. Craik, D. J.; Wilce, J. A. Studies of protein-ligand interactions by NMR. Methods Mol. Biol. 60, 195-232 (1997).

511. Butler, E. K. et al. The mitochondrial chaperone protein TRAP1 mitigates $\alpha$-Synuclein toxicity. PLoS Genet. 8, e1002488 (2012).

512. Choshi, T. et al. Synthesis of dibenzoylmethane derivatives and inhibition of mutagenicity in Salmonella typhimurium. Chem. Pharm. Bull. 40, 1047-1049 (1992).

513. Vanelle, P. et al. Functional derivatives of 5-benzo[1,3]dioxol-5-yl-1-methyl-1Himidazole-2-carbaldehyde and evaluation of leishmanicidal activity. Eur. J. Med. Chem. 35, 157-162 (2000). 
514. Esposito, L. A. et al. Compounds, compositions and methods for the treatment of $\beta$ amyloid diseases and synucleinopathies. U.S. Pat. Appl. Publ. US 2009111863 A1 (2009).

515. Hu, Y.-Z.; Clive, D. L. J. Synthesis of the aromatic unit of calicheamicin $\gamma 1$ I. J. Chem. Soc., Perkin Trans. 1 9, 1421-1424 (1997).

516. Nam, N.-H. et al. Synthesis and cytotoxicity of 2,5-dihydroxychalcones and related compounds. Arch. Pharm. Res. 27, 581-588 (2004).

517. Liu, Y. et al. A practical and chemoselective reduction of nitroarenes to anilines using activated iron. Adv. Synth. Catal. 347, 217-219 (2005).

518. Bhagwan, J. et al. Synthesis and spectral studies of some potential antibacterial diaryl 1,3-diketones. J. Inst. Chemists (India) 54, 266-268 (1982).

519. Chauhan, S. S. et al. Solid phase synthesis of novel pyrazole derivatives from diaryl 1,3-diketones under microwave irradiation. J. Ind. Chem. Soc. 82, 1016-1018 (2005).

520. Lopez, J. A. et al. Galiposin A new $\beta$-hydroxychalcone from Galipea granulosa. Planta Medica 64, (1998).

521. Lake, T.; Snow, A. D. Dihydroxy aryl compounds, compositions and methods for the treatment of islet amyloid polypeptide (IAPP) accumulation in diabetes. U.S. Pat. Appl. Publ. WO 2009111611 A2 (2009).

522. Bradley, W.; Robinson, R. Hydrolytic fission of some substituted dibenzoylmethanes. J. Chem. Soc., 2356-2367 (1926).

523. Cao, S. X. et al. Pyrazole and triazole derivatives as glucokinase activators and their preparation, pharmaceutical compositions and use in the treatment of diseases. PCT Int. Appl. WO 2007061923 A2 (2007).

524. Vangoor, F. F. et al. Preparation of substituted pyrazoles as modulators of ATPbinding cassette transporters. PCT Int. Appl. WO 2004080972 A1 (2004).

525. Hubaud, J.-C. et al. Synthesis and spectroscopic examination of various substituted 1,3-dibenzoylmethane, active agents for UVA/UVB photoprotection. J. Photochem. Photobiol., B 92, 103-109 (2008).

526. Shaw, A. Y. et al. 3,5-Diaryl-1H-pyrazole as a molecular scaffold for the synthesis of apoptosis-inducing agents. Bioorg. Med. Chem. 18, 3270-3278 (2010).

527. Lal, B. et al. Preparation of pyrrolidinylchromenones as inhibitors of cyclin-dependent kinases. U.S. Pat. Appl. US 20070015802 A1 (2007).

528. Winkle, M. R.; Ronald, R. C. Regioselective metalation reactions of some substituted (methoxymethoxy)arenes. J. Org. Chem. 47, 2101-2108 (1982).

529. Li, Y.-X. et al. Design and syntheses of novel phthalazin-1(2H)-one derivatives as acetohydroxyacid synthase inhibitors. J. Agric. Food Chem. 54, 9135-9139 (2006).

530. Nigam, S. et al. Synthesis of novel pyrazole derivatives from diaryl 1,3-diketones. Part-I. Heterocycl. Commun. 9, 405-410 (2003).

531. Roush, D. M. et al. Preparation of aryl 2-fluoroethyl ethers as pesticides. US 4960884 A (1990).

532. Saxty, G. et al. Bicyclic heterocyclic compounds as selective protein tyrosine kinase inhibitors, their preparation and use in treating cancer and other diseases mediated by FGFR kinase. PCT Int. Appl. WO 2009047506 A1 (2009).

533. Zhong, Q. et al. Organotellurium ylide reactions. Part I. Synthesis of $\alpha, \beta$-unsaturated ketones via telluronium ylides. Youji Huaxue 10, 459-463 (1990).

534. Mahmoodi, N. O. et al. Synthesis and photochromic properties of new heterocyclic derivatives of 1,3-diazabicyclo[3.1.0]hex-3-ene. J. Chin. Chem. Soc. (Taipei, Taiwan) 54, 635-641 (2007).

535. Okunrobo, L. O. et al. Anti-inflammatory and gastroprotective properties of some chalcones. Acta Pol. Pharm. 63, 195-199 (2006). 
536. Weber, F. G. Preparation, N.M.R. and IR spectra of 2,3-dibromo- and 2-iodo-3chloro-1,3-diphenyl-1-propanones. Tetrahedron 25, 4283-4289 (1969).

537. Popat, K. H. et al. Synthesis of some new cyanopyrans and cyanopyridines and their biological activities. J. Ind. Chem. Soc. 81, 157-159 (2004).

538. Popat, K. H. et al. Synthesis and biological activities of some quinoxaline derivatives bearing aromatic halogen nucleus. J. Ind. Chem. Soc. 80, 709-710 (2003).

539. Nimavat, K. S. et al. 2-(3-Bromophenyl)-3-(substituted benzyl)quinoxalines as anticancer, antitubercular and antimicrobial agents. J. Inst. Chem. (India) 74, 171-174 (2002).

540. Beutner, S. et al. Carotenylflavonoids, a novel group of potent, dual-functional antioxidants. ARKIVOC (Gainesville, FL, U. S.), 279-295 (2007).

541. Sharma, T. C. et al. Oxidation of some hydroxyarylpyrazolines with manganese dioxide. Acta Chim. Acad. Sci. Hung. 93, 415-419 (1977).

542. FERLUX. Pharmacologically active 5-(o-hydroxyphenyl)pyrazoles. FR 2104932 A1 (1972).

543. Silva, V. D. et al. Efficient chemoselective biohydrogenation of 1,3-diaryl-2-propen1-ones catalyzed by Saccharomyces cerevisiae yeasts in biphasic system. J. Mol. Catal. B: Enzym. 63, 157-163 (2010).

544. Larkin, J. et al. Reactions of $\beta$-diketone with hydroxylamine hydrochloride; synthesis of 3,5-disubstituted isoxazoles. J. Chem. Soc. C 7, 947-949 (1970).

545. Shettigar, V. et al. Crystal growth and characterization of new nonlinear optical chalcone derivative: 1-(4-Methoxyphenyl)-3-(3,4-dimethoxyphenyl)-2-propen-1-one. $J$. Cryst. Growth 295, 44-49 (2006).

546. Zicmanis, A. et al. Alkoxydibenzoylmethanes. Latv. PSR Zinat. Akad. Vestis, Kim. Ser. 2, 229-234 (1974).

547. Korbonits, D. et al. Ring transformation of 3-(2-aminoaryl)-1,2,4-oxadiazoles into 3acylaminoindazoles; extension of the Boulton-Katritzky scheme. J. Chem. Soc., Perkin Trans. 1, 759-766 (1982).

548. Dallacker, F. Preparation of 1,2,4,5-tetrazines from pyridine and methylenedioxybenzene. Monatsh. Chem. 91, 294-304 (1960).

549. Naruse, S. et al. Novel imidazole compounds as surface treatment agents used in manufacture of printed circuit boards. Jpn. Kokai Tokkyo Koho (2010).

550. Chimenti, F. et al. Synthesis and delective inhibitory activity of 1-Acetyl-3,5diphenyl-4,5-dihydro-(1H)-pyrazole derivatives against Monoamine Oxidase. J. Med. Chem. 47, 2071-2074 (2004).

551. Yamakoshi, H. et al. Structure-activity relationship of C5-curcuminoids and synthesis of their molecular probes thereof. Bioorg. Med. Chem. 18, 1083-1092 (2010).

552. Palaska, E. et al. Synthesis and antidepressant activities of some 3,5-diphenyl-2pyrazolines. Eur. J. Med. Chem. 36, 539-543 (2001).

553. Sogawa, S. et al. 3,4-Dihydroxychalcones as potent 5-lipoxygenase and cyclooxygenase inhibitors. J. Med. Chem. 36, 3904-3909 (1993).

554. Lygo, B.; Wainwright, P. G. Phase-transfer catalysed asymmetric epoxidation of enones using $N$-Anthracenylmethyl-substituted cinchona alkaloids. Tetrahedron 55, 62896300 (1999).

555. Sabate-Alduy, C. et al. Synthesis of 2-pyrazolin-4-ones (4-hydroxypyrazoles). Bull. Soc. Chim. Fr. (Chim. Mol.), 1841-1842 (1976). 


\section{List of Figures and Tables}

\section{List of Figures}

Figure 1.1. 6

Figure 1.2. Scientific Literature

Figure 1.3. Lewy bodies and Lewy neurites

Figure 1.4. Progression of neuronal Lewy pathology $\ldots$

Figure 1.5. Ubiquitin-Proteasomal System $\ldots$

Figure 1.6. Mitochondrial toxins

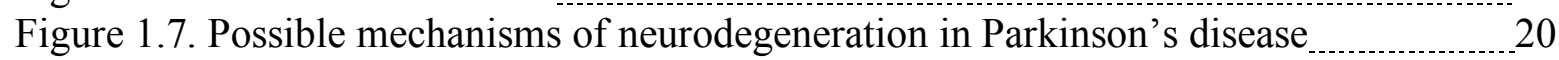

Figure 1.8. Synuclein sequences $\quad 21$

Figure 1.9. Human synuclein domain organization $\ldots$

Figure 1.10. Overview of synucleinopathies and key hallmark _ $\quad 24$

Figure 1.11. Spectral properties of $\alpha$-synuclein $\ldots 27$

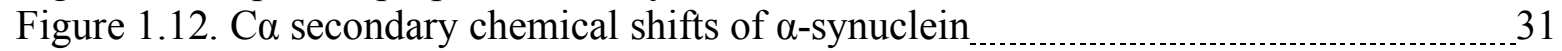

Figure 1.13. PRE data on MTSL-labeled $\alpha$-synuclein and its native state conformations 34

Figure 1.14. Schematic energy landscape for protein folding and aggregation _................. 36

Figure 1.15. $\alpha$-Synuclein aggregation pathways 38

Figure 1.16. Oxidative and nitrative modifications $\quad 49$

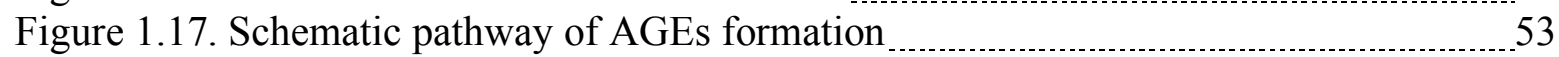

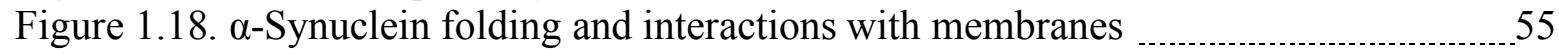

Figure 1.19. Lipids 56

Figure 1.20. $\alpha$-Synuclein subcellular localization in yeast $\quad 61$

Figure 1.21. Structure of $\alpha$-synuclein fibrils

Figure 1.22. Putative toxicity pathways induced by misfolded $\alpha$-synuclein $\quad 70$

Figure 1.23. Degradation of monomeric and misfolded $\alpha$-synuclein $\ldots 75$

Figure 1.24. L-Dopa and dopamine biosynthesis

Figure 1.25. Change in levodopa response over the course of Parkinson's disease $\quad 81$

Figure 1.26. Main L-dopa metabolic pathways

Figure 1.27. Drugs for concomitant therapy

Figure 1.28. Monoamino oxidases

Figure 1.29. Simplified model of basal ganglia-thalamocortical circuit $\ldots$

Figure 1.30. Dopamine agonists

Figure 1.31. Non-dopaminergic drugs

Figure 1.32. Management of Parkinson's disease $\quad 91$

Figure 1.33. Pathogenic mechanisms and corresponding therapeutic strategies $\ldots 125$

Figure 2.2.1. Principle of aggregation analysis by dual-color SIFT 149

Figure 2.2.2. N-benzylidene-benzohydrazide scaffold $\ldots$

Figure 2.2.3. Structure-Activity-Relationships (SAR)-Map and DPP scaffold $\ldots \ldots \ldots \ldots \ldots \ldots \ldots \ldots . . . . . . . . . . . . . . . .152$

Figure 2.3.1. Markush structure of DPP-related compounds $\ldots 153$

Figure 2.3.2. Overview and classification DPP-related compounds $\ldots$

Figure 2.3.3. Main synthetic scheme

Figure 2.3.4. General synthesis of DPP compounds bearing hydroxy or amino groups $\ldots 158$

Figure 2.3.5. Methods of synthesis of pyrazole-containing DPP compounds _..................... 160

Figure 2.3.6. Methods of synthesis of DPP analogs bearing isoxazole, pyrrole, imidazole and 1,2,4-oxadiazole heterocycles 166

Figure 2.3.7. Synthesis of 3,4,5-substituted pyrazoles and isoxazole 169

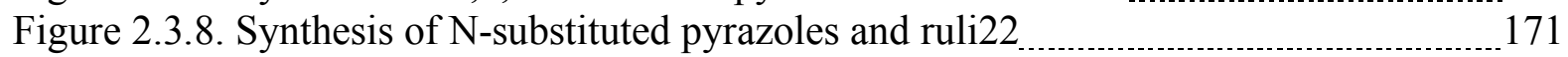


Figure 2.4.1. Schematic representation of the SIFT assay on prion protein 173

Figure 2.4.2. Results of primary SIFT screening of DPP compounds

Figure 2.4.3. Concentration-dependent inhibitory activity of DPP compounds in

SIFT assay

Figure 2.4.4. Inhibition of PrPSc accumulation in SMB cells infected with RML

scrapie strain

Figure 2.4.5. Structures of DPP compounds tested in SMB cells

Figure 2.4.6. Schematic representation of the PMCA assay

Figure 2.4.7. Inhibition of prion propagation by DPP compounds in PMCA assay

Figure 2.4.8. Inhibition of propagation of different prion strains by anle138b PMCA assay

Figure 2.4.9. Structure-activity relationship for selected DPP-derivatives from "Prion mouse" library.

Figure 2.4.10. Correlation between anti-prion activity in vitro and in vivo 189

Figure 2.4.11. Influence of daily anle138b treatment on PrPSc accumulation and prion pathology of mice infected with RML scrapie

Figure 2.4.12. Size modulation of PrPSc oligomers by anle138b and inhibition of various human and non-human prion strains in PMCA

Figure 2.4.13. Drug-discovery strategy against prion disease

Figure 2.5.1. Examples of two-dimensional intensity distribution histograms recorded in molecular SIFT assay

Figure 2.5.2. Inhibition of $\alpha$-synuclein aggregation in SIFT assay

Figure 2.5.3. Chemical structures of DPP compounds tested in ThioT assay $\quad 205$

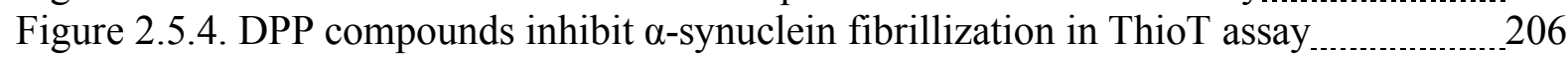

Figure 2.5.5. anle138b moderately inhibits $\alpha$-synuclein fibrillization in ThioT assay .............207

Figure 2.5.6. Representative TEM images $\quad 208$

Figure 2.5.7. AFM images

Figure 2.5.8. DLS measurements $\quad 212$

Figure 2.5.9. Far-UV CD spectral data $\ldots \ldots \ldots$

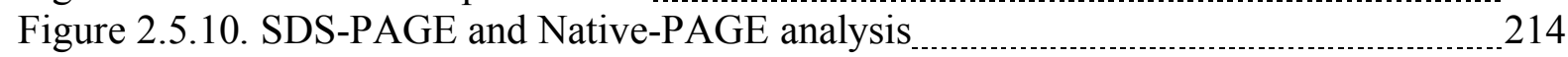

Figure 2.5.11. NMR investigation of $\alpha$-synuclein aggregation in presence of anle $138 \mathrm{c} \ldots \ldots . . .217$

Figure 2.5.12. NMR investigation of $\alpha$-synuclein aggregation in presence of sery166a $\ldots . . .218$

Figure 2.5.13. NMR investigation of $\alpha$-synuclein aggregation in presence of anle145c $\quad 220$

Figure 2.5.14. Inhibition of $\alpha$-synuclein aggregation by DPP compounds under reducing conditions

Figure 2.5.15. NMR investigation of $\alpha$-synuclein aggregation in presence of anle145c under reducing conditions

Figure 2.5.16. 1H-15N HSQC spectra of $\alpha$-synuclein in the presence of sery166a and sery 139

Figure 2.5.17. 1H-15N HSQC-NMR titration experiments of $\alpha$-synuclein with anle $137 \mathrm{c}$ and sery 139

Figure 2.5.18. 1H-15N HSQC-NMR experiments with $\alpha$-synuclein and selected DPP compounds

Figure 2.5.19. Motional and relaxation properties of small molecular weight ligands, big macromolecules and corresponding complexes

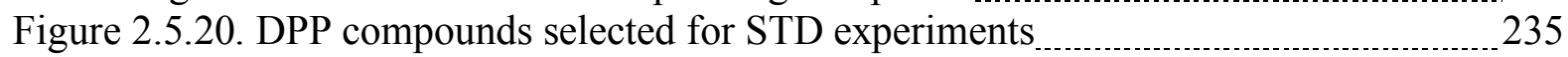

Figure 2.5.21. Characterization of anle137c- $\alpha$-synuclein interactions by STD NMR $\quad 236$

Figure 2.5.22. Characterization of sery139- $\alpha$-synuclein interactions by STD NMR _........... 237

Figure 2.5.23. Characterization of sery166a- $\alpha$-synuclein interactions by STD NMR _......... 238 
Figure 2.5.24. Characterization of sery166a- $\alpha$-synuclein interactions by STD NMR over the course of $\alpha$-synuclein aggregation in presence of sery166a.

Figure 2.5.25. Characterization of anle138c-stabilized $\alpha$-synuclein oligomers by SSNMR 245

Figure 2.5.26. Comparison of various oligomeric $\alpha$-synuclein species $\quad 248$

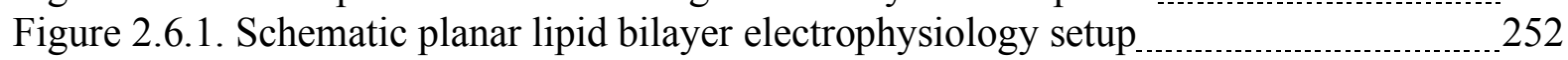

Figure 2.6.2. Pore formation by oligomeric forms of $\alpha$-synuclein in planar lipid bilayers 253

Figure 2.6.3. Lack of pore formation activity for sery166a-stabilized oligomers $\quad \ldots \quad 255$

Figure 2.6.4. Complete or partial inhibition of oligomer-induced pore formation by DPP compounds 256

Figure 2.6.5. Effect of various $\alpha$-synuclein forms on LTP 260

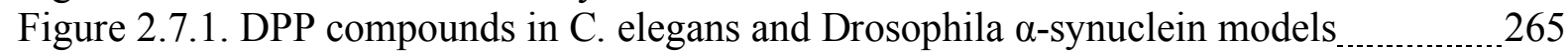

Figure 2.7.2. Effect of anle138b in low-dose intragastric mice model of

Parkinson's disease $\quad 268$

Figure 2.7.3. Effect of anle138b in A30P-hum- $\alpha$-synuclein transgenic mice model of

Parkinson's disease $\quad 269$

Figure 2.7.3. Effect of anle138b in transgenic 1-120 haSyn MI2 mice _ $\quad 271$

Figure 2.8.1. PK study of DPP compounds in mice $\quad 279$

Figure 2.8.2. Pharmacokinetic analysis of anle138b in mice $\ldots$

Figure 2.8.3. Pharmacokinetic analysis of anle138b in mice _ _ 282

Figure 2.8.4. Pharmacokinetic analysis of anle138b in rats _.................................................. 284

Figure 2.8.5. Comparative pharmacokinetic analysis of anle $138 \mathrm{~b}$ formulations in rats 286

Figure 2.9.1. Simplified illustration of the prodrug concept

Figure 2.9.2. Common functional groups and types of chemical modifications

with promoiety 289

Figure 2.9.3. Examples of developed prodrugs $\quad 290$

Figure 2.9.4. Design of anle138b prodrug 292

Figure 2.9.5. Anle138b prodrug candidates $\ldots$

Figure 2.9.6. Synthesis of prodrug candidates $\ldots$

Figure 2.9.7. HPLC chromatogram of sery $433 \ldots \ldots \ldots \ldots$

Figure 2.9.8. Putative mechanism of sery474 hydrolysis $\ldots \ldots \ldots \ldots$

Figure 2.9.9. PK study of prodrugs in mice 298

Figure 2.9.10. PK study of sery433 in rat 299

Figure 2.9.11. The comparative PK study of sery433 and anle138b in rats $\quad 300$ 


\section{List of Tables}

Table 1.1. Motor and non-motor symptoms of Parkinson's disease $\ldots$

Table 1.2. Genetic Forms of Parkinson's disease $\quad 12$

Table 1.3. $\alpha$-Synuclein phosphorylation and kinases

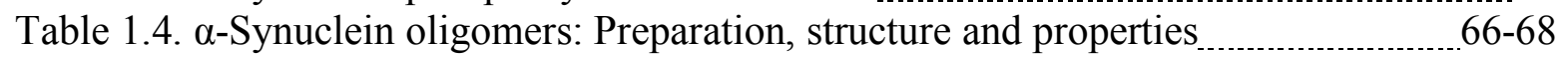

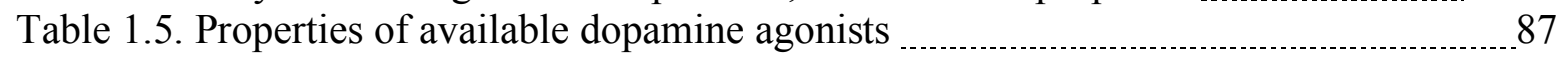

Table 1.6. Properties of available MPTP-based animal models $\ldots$

Table 1.7. Properties of available rotenone-based animal models $\quad 97$

Table 1.8. Properties of available 6-OHDA-based animal models _.................................. 99

Table 1.9. Properties of available paraquat-based animal models $\quad 100$

Table 1.10. Properties of available $\alpha$-synuclein non-mammalian models $\ldots$

Table 1.11. Properties of available $\alpha$-synuclein transgenic mouse models $\ldots$

Table 1.12. Properties of available LRRK2 animal models 115

Table 1.13. Major animal models of autosomal recessive Parkinson's disease $\ldots$

Table 1.14. Pharmacological activation of protein degradation systems $\quad 130$

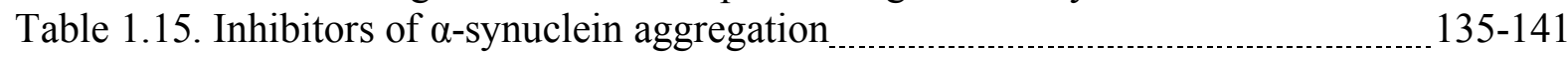

Table 1.16. Chemical structures of inhibitors listed in table $1.15 \ldots$

Table 1.17. Assessment of potential neuroprotective drug candidates

Table 2.3.1. Fragment-based design of DPP compounds $\ldots$

Table 2.3.2. Synthesized 1,3-diketones and 3,5-substituted pyrazoles _ 162-164

Table 2.3.3. Synthesized chalcones, dibromides and epoxides

Table 2.3.4. List of synthesized 2-pyrazoline-based compounds $\ldots 165$

Table 2.3.5. Synthesized DPP compounds bearing isoxazole, 1,2,4-oxadiazole, imidazole and pyrrole heterocycles 168

Table 2.4.1. Comparison of in vivo activity of anle138b with other published compounds

Table 2.5.1. Activity of DPP compounds in SIFT assay $\quad 204$

Table 2.6.1. DPP-stabilized oligomers in BLM $\ldots$

Table 2.6.2. Inhibition of pore formation by DPP compounds in solution or in membrane 


\section{Abbreviations}

$\begin{array}{ll}\text { 3-OMD } & \text { 3-O-Methyldopa } \\ \text { 4E-BP } & \text { Eukaryotic initiation factor 4E binding protein } \\ \text { 6-OHDA } & \text { 6-Hydroxydopamine } \\ \text { aa } & \text { Amino acid } \\ \text { AAAD } & \text { Aromatic L-amino acid decarboxylase } \\ \text { AADC } & \text { Aromatic L-amino acid decarboxylase } \\ \text { AAV } & \text { Adeno-associated virus } \\ \text { ACN } & \text { Acetonitrile } \\ \text { AD } & \text { Alzheimer's disease } \\ \text { ADME } & \text { Absorption, distribution, metabolism, and excretion } \\ \text { AFM } & \text { Atomic force microscope } \\ \text { AGEs } & \text { Advanced glycation end products } \\ \text { ALS } & \text { Amyotrophic lateral sclerosis } \\ \text { ANS } & \text { 1-Anilinonaphthalene- 8-sulfonic acid } \\ \text { ATP } & \text { Adenosine triphosphate } \\ \text { ATP13A2 } & \text { Probable cation-transporting ATPase 13A2 } \\ \text { ATPase } & \text { Adenylpyrophosphatase } \\ \text { AUC } & \text { Area Under the Curve } \\ \text { BBB } & \text { Blood-brain barrier } \\ \text { Bcl-2 } & \text { B-cell lymphoma 2 } \\ \text { BiFC } & \text { Bimolecular fluorescence complementation } \\ \text { BLM } & \text { Black lipid membrane } \\ \text { c-Abl } & \text { Abelson tyrosine kinase } \\ \text { C. elegans } & \text { Caenorhabditis elegans } \\ \text { calcd } & \text { Calculated } \\ \text { CaMKII } \alpha & \text { Calcium/calmodulin-dependent protein kinase type II subunit } \\ \text { cAMP } & \text { alpha } \\ \text { CAS } & \text { Cyclic adenosine monophosphate } \\ \text { CD } & \text { Chemical Abstracts Service } \\ \text { CHIP } & \text { Circular dichroism } \\ \text { CK } & \text { Co-chaperone Hsp70-interacting protein } \\ \text { CMA } & \text { Casein kinase } \\ \text { C } \text { max } & \text { Chaperone-mediated autophagy } \\ \text { CNS } & \text { Maximum concentration } \\ & \text { Central nervous system } \\ & \end{array}$




\begin{tabular}{|c|c|}
\hline COMT & Catechol-O-methyl transferase \\
\hline COX-2 & Cyclooxygenase 2 \\
\hline cryo-EM & Cryogenic electron microscopy \\
\hline Ctm-PrP & Transmembrane version of the prion protein \\
\hline CYP2D6 & Cytochrome P450 2D6 \\
\hline CytC & Cytochrome complex \\
\hline DA & Dopamine \\
\hline DAT & Dopamine transporter \\
\hline DBS & Deep brain stimulation \\
\hline DBU & 1,8-Diazabicyclo[5.4.0]undec-7-ene \\
\hline DCC & N,N'-Dicyclohexylcarbodiimide \\
\hline DCM & Dichloromethane \\
\hline dec. & Decomposition \\
\hline DLB & Dementia with Lewy bodies \\
\hline DLS & Dynamic light scattering \\
\hline DMAP & 4-Dimethylaminopyridine \\
\hline DMF & Dimethylformamide \\
\hline DMSO & Dimethylsulfoxide \\
\hline DNA & Deoxyribonucleic acid \\
\hline DOPAC & 3,4-Dihydroxyphenylacetic acid \\
\hline dpi & Days Post Infection \\
\hline DPP & Diphenyl Pentocycle \\
\hline dSTORM & Direct stochastic optical reconstruction microscopy \\
\hline DTT & Dithiothreitol \\
\hline Dyrk1A & Dual specificity tyrosine-phosphorylation-regulated kinase $1 \mathrm{~A}$ \\
\hline E. Coli & Escherichia coli \\
\hline EDTA & Ethylenediaminetetraacetic acid \\
\hline EGCG & Epigallocatechin gallate \\
\hline ELISA & Enzyme-linked immunosorbent assay \\
\hline EM & Electron microscopy \\
\hline ENS & Enteric nervous system \\
\hline EPR & Electron paramagnetic resonance \\
\hline $\mathbf{E R}$ & Endoplasmic reticulum \\
\hline ESI-MS & Electrospray ionisation mass spectrometry \\
\hline ETC & Electron transport chain \\
\hline FAD & Flavin adenine dinucleotide \\
\hline FDA & U.S. Food and Drug Administration \\
\hline fEPSP & Field excitatory postsynaptic potential \\
\hline
\end{tabular}


FRET

FTIR

Fyn

GABA

GCase

GdmCl

GDNF

GEP

GFP

Grk

HEPES

HIV

HMBC

HMDS

HNE

HPLC

HRMS

Hsp

HSQC

HtrA2/Omi

i.c.

i.p.

IMS-MS

iNOS

IPTG

KI

KO

L-DOPA

LAMP 2A

LAT1

LB(s)

LC-MS

LDH

LN(s)

LPS

LRRK-2

LTP

LUV
Förster resonance energy transfer

Fourier-transform infrared spectroscopy

Proto-oncogene tyrosine-protein kinase

gamma-Aminobutyric acid

Glucocerebrosidase

Guanidinium chloride

Glial cell-derived neurotrophic factor

General experimental procedure

Green fluorescent protein

$G$ protein-coupled receptor kinase

4-(2-hydroxyethyl)-1-piperazineethanesulfonic acid

Human immunodeficiency viruses

Heteronuclear Multiple Bond Correlation

Hexamethyldisilazane

4-Hydroxynonenal

High-performance liquid chromatography

High resolution mass spectrometry

Heat shock proteins

Heteronuclear single quantum coherence

Serine protease HTRA2

Intracerebral infection

Intraperitoneal injection

Ion-mobility spectrometry-mass spectrometry

Inducible nitric oxide synthase

Isopropyl $\beta$-d-1-thiogalactopyranoside

Knock in

Knockout

L-3,4-dihydroxyphenylalanine

Lysosomal-associated membrane protein 2

Large neutral amino acid transporter

Lewy body

Liquid chromatography-mass spectrometry

Lactate Dehydrogenase

Lewy neurites

Lipopolysaccharide

Leucine-rich repeat kinase 2

Long-term potentiation

Large unilamellar vesicle 


\begin{tabular}{|c|c|}
\hline Lyn & Lck/Yes novel tyrosine kinase \\
\hline m.p. & Melting point \\
\hline МАО-А & Monoamine oxidase A \\
\hline MCT1 & Monocarboxylate transporter 1 \\
\hline MD & Molecular dynamics \\
\hline MDA & Methylenedioxylated phenethylamine \\
\hline MDMA & Methylenedioxy-methylamphetamine \\
\hline miRNA & microRNA \\
\hline MOM & Methoxymethyl ether \\
\hline MPP+ & 1-Methyl-4-phenylpyridinium \\
\hline MPTP & 1-Methyl-4-phenyl-1,2,3,6-tetrahydropyridine \\
\hline mRNA & Messenger RNA \\
\hline MS & Mass spectrometry \\
\hline MSA & Multiple system atrophy \\
\hline mTOR & Mammalian target of rapamycin \\
\hline MTSL & $\begin{array}{l}\text { S-(1-oxyl-2,2,5,5-tetramethyl-2,5-dihydro-1H-pyrrol-3-yl)methyl } \\
\text { methanesulfonothioate }\end{array}$ \\
\hline MTT & $\begin{array}{l}\text { 3-(4,5-Dimethylthiazol-2-yl)-2,5-diphenyltetrazolium bromide for } \\
\text { assay }\end{array}$ \\
\hline MWCO & Molecular weight cut-off \\
\hline $\mathrm{Na}_{2} \mathrm{CaCDTA}$ & cyclohexanediaminetetraacetic acid di-sodium calcium \\
\hline $\mathbf{N A C}$ & Non Amyloid- $\beta$ component \\
\hline NADPH & Nicotinamide adenine dinucleotide phosphate \\
\hline NBS & N-Bromosuccinimide \\
\hline NBT & Nitrotetrazolium blue chloride \\
\hline ND & Not determined \\
\hline NeuN & Neuronal nuclei \\
\hline NMDA & N-methyl-D-aspartate \\
\hline NMP & N-Methyl-2-pyrrolidone \\
\hline NMR & Nuclear magnetic resonance \\
\hline NOE & Nuclear Overhauser effect \\
\hline NUB1 & NEDD8 ultimate buster 1 \\
\hline Nurr1 & Nuclear receptor related 1 \\
\hline p38 & Mitogen activated protein kinase p38 \\
\hline PA & Phosphatidic acids \\
\hline PA700 & ATP-dependent activator of the $20 \mathrm{~S}$ proteasome \\
\hline Pael-R & Parkin-associated endothelin receptor-like receptor \\
\hline PAF & Platelet-activating factor \\
\hline PAMAM & Poly(amidoamine) \\
\hline
\end{tabular}




\begin{tabular}{|c|c|}
\hline PBS & Phosphate buffered saline \\
\hline PC & Phosphatidylcholine \\
\hline PcTS & Phthalocyanine tetrasulfonate \\
\hline PD & Parkinson's disease \\
\hline PDGF $\beta$ & Platelet-derived growth factor $\beta$ \\
\hline PDSD & Proton-driven spin diffusion spectrum \\
\hline PE & Phosphatidylethanolamine \\
\hline PEG & Polyethylene glycol \\
\hline PERK & Protein kinase R-like endoplasmic reticulum kinase \\
\hline PET & Positron-emission tomography \\
\hline PFP & Pentafluorophenyl \\
\hline PG & Phosphatidylglycerol \\
\hline PI & Phosphatidylinositol \\
\hline PiB & Pittsburgh compound B \\
\hline PICUP & Photo-Induced Cross-Linking of Unmodified Proteins \\
\hline PINK1 & PTEN-induced putative kinase 1 \\
\hline PITX3 & Paired-like homeodomain transcription factor 3 \\
\hline PK & Proteinase $\mathrm{K}$ \\
\hline PK study & Pharmacokinetics study \\
\hline PLK-1 & Polo like kinase 1 \\
\hline PLP & Pyridoxal phosphate \\
\hline PMCA & Protein misfolding cyclic amplification \\
\hline PMSF & Phenylmethylsulfonyl fluoride \\
\hline POPG & Palmitoyloleoylphosphatidylglycerol \\
\hline ppm & Parts per million \\
\hline PQQ & Pyrroloquinoline quinone \\
\hline PRE & Paramagnetic Relaxation Enhancement \\
\hline PrP & Prion protein \\
\hline $\operatorname{PrP}^{\mathrm{C}}$ & Cellular prion protein \\
\hline PrPes & PK resistant prion protein \\
\hline $\operatorname{PrP} \mathbf{P}^{\mathrm{Sc}}$ & Scrapie prion protein \\
\hline PS & Phosphatidylserine \\
\hline PTNE & Phosphatase and tensin homolog \\
\hline PTSA & p-Toluenesulfonic acid \\
\hline PUFA & Polyunsaturated fatty acid \\
\hline rAAV & Recombinant adeno-associated virus \\
\hline RDC & Residual dipolar coupling \\
\hline RF & Retention factor \\
\hline
\end{tabular}




\begin{tabular}{|c|c|}
\hline RML & Rocky Mountain Laboratory \\
\hline RNA & Ribonucleic acid \\
\hline ROS & Reactive oxygen species \\
\hline $\mathbf{R P}$ & Reversed phase \\
\hline rpm & Revolutions per minute \\
\hline rPrP & Recombinant prion protein \\
\hline RT & Room temperature \\
\hline SANS & Small-angle neutron scattering \\
\hline SAR & Structure-activity relationship \\
\hline SAXS & Small-angle X-ray scattering \\
\hline scFv & Single-chain variable fragment \\
\hline sCJD & Sporadic Creutzfeldt-Jakob disease \\
\hline SDS & Sodium dodecyl sulphate \\
\hline SEC & Size exclusion chromatography \\
\hline shRNA & Small hairpin RNA \\
\hline SIAH-1 & Seven-in-absentia homolog protein 1 \\
\hline SIFT & Scanning for intensely fluorescent targets \\
\hline SiRNA & Small interfering RNA \\
\hline SLS & Sodium lauryl sulphate \\
\hline SMB & Scrapie mouse brain \\
\hline SN & Substantia nigra \\
\hline SNARE & $\begin{array}{l}\text { Soluble N-ethylmaleimide sensitive factor attachment protein } \\
\text { receptor }\end{array}$ \\
\hline SNCA & Synuclein alpha \\
\hline SNCB & Synuclein beta \\
\hline SNCG & Synuclein gamma \\
\hline SNpc & Substantia nigra the pars compacta \\
\hline Src & Proto-oncogene tyrosine-protein kinase Src \\
\hline SSNMR & Solid-state NMR \\
\hline STD & Saturation-Transfer Difference \\
\hline SUMO & Small Ubiquitin-like Modifier \\
\hline SUV & Small unilamellar vesicle \\
\hline Syk & Spleen tyrosine kinase \\
\hline TBAF & Tetra-n-butylammonium fluoride \\
\hline TEA & Triethylamine \\
\hline TEM & Transmission electron microscopy \\
\hline TFA & Trifluoroacetic acid \\
\hline THF & Tetrahydrofuran \\
\hline Thiot & Thioflavin $\mathrm{T}$ \\
\hline
\end{tabular}




$\begin{array}{ll}\text { TLC } & \text { Thin-layer chromatography } \\ \text { T }_{\text {max }} & \text { Time of Cmax } \\ \text { TNF } & \text { Tumor necrosis factor } \\ \text { TRAP1 } & \text { Tumor Necrosis Factor Receptor-Associated Protein 1 } \\ \text { Tris-HCl } & \text { Tris-(hydroxymethyl)-aminomethanhydrochlorid } \\ \text { tRNA } & \text { Transfer RNA } \\ \text { trNOE } & \text { Transferred nuclear Overhauser effect } \\ \text { UCH-L1 } & \text { Ubiquitin carboxyl-terminal esterase L1 } \\ \text { UPDRS } & \text { Unified Parkinson's Disease Rating Scale } \\ \text { UPS } & \text { Ubiquitin proteasome system } \\ \text { UV } & \text { Ultraviolet } \\ \text { vCJD } & \text { Variant Creutzfeldt-Jakob disease } \\ \text { VMAT2 } & \text { Vesicular monoamine transporter 2 } \\ \text { WT } & \text { Wild type } \\ \boldsymbol{\alpha S} & \alpha \text {-synuclein } \\ \boldsymbol{\alpha S y n} & \alpha \text {-synuclein }\end{array}$

20 Main Amino Acids and Their Abbreviations

\begin{tabular}{lcc}
\hline Amino acid & Three letter code & One letter code \\
\hline Alanine & Ala & $\mathrm{A}$ \\
Arginine & Arg & $\mathrm{R}$ \\
Asparagine & Asn & $\mathrm{N}$ \\
Aspartate & Asp & $\mathrm{D}$ \\
Cysteine & Cys & $\mathrm{C}$ \\
Glutamate & Glu & $\mathrm{E}$ \\
Glutamine & Gln & $\mathrm{Q}$ \\
Glycine & Gly & $\mathrm{G}$ \\
Histidine & His & $\mathrm{H}$ \\
Isoleucine & Ile & $\mathrm{I}$ \\
Leucine & Leu & $\mathrm{L}$ \\
Lysine & Lys & $\mathrm{K}$ \\
Methionine & Met & $\mathrm{M}$ \\
Phenylalanine & Phe & $\mathrm{F}$ \\
Proline & Pro & $\mathrm{P}$ \\
Serine & Ser & $\mathrm{S}$ \\
Threonine & Thr & $\mathrm{T}$ \\
Tryptophan & Trp & $\mathrm{W}$ \\
Tyrosine & Tyr & $\mathrm{Y}$ \\
Valine & Val & $\mathrm{V}$ \\
\hline
\end{tabular}




\section{Appendix}

Table 8.1. "Prion mice" library of DPP compounds tested for anti-prion activity in vivo.

Table is adopted from J. Wagner, S. Ryazanov, et al. Acta Neuropathol. 125, 795 (2013). Copyright (C) 2013

\begin{tabular}{|c|c|c|c|c|}
\hline No. & compound & structure & $\%$ inhibition $\#$ & $\Delta$ survival $^{+}$ \\
\hline 1 & Anle138b & & $78^{a}, 57^{b}, 108^{c}, 70^{d}$ & $\begin{array}{c}\text { 15/14 }{ }^{\mathrm{f}}, 22 / 24^{\mathrm{g}}, 51 / 53^{\mathrm{i}} \\
74 / 88^{\mathrm{j}}, 76 / 82^{\mathrm{k}}, 198 / 179^{\mathrm{l}}\end{array}$ \\
\hline 2 & Sery335b & & $68^{a}, 46^{b}$ & \\
\hline 3 & Anle253b & & $59^{a}$ & \\
\hline 4 & Sery149 & & $<10^{b}$ & $12 / 14^{f}$ \\
\hline 5 & 10353F11 & & & $11 / 14^{f}$ \\
\hline 6 & Sery378b & & $39^{a}$ & \\
\hline 7 & Sery313b & & $37^{b}$ & \\
\hline 8 & Sery363a & & $35^{a}$ & \\
\hline 9 & Sery338b & & $35^{b}$ & \\
\hline 10 & Anle233b & & $33^{a}$ & \\
\hline 11 & Sery392b & & $33^{a}$ & \\
\hline 12 & Sery383 & & $31^{a}$ & \\
\hline 13 & Anle186b & & $30^{c}$ & $4 / 249$ \\
\hline 14 & Anle237 & & $26^{a}$ & \\
\hline 15 & Sery255b & & $25^{a},<10^{d}$ & \\
\hline 16 & Anle232b & & $23^{a}$ & \\
\hline 17 & Sery344 & & $21^{b}$ & \\
\hline 18 & Sery392a & & $14^{a}$ & \\
\hline 19 & Sery312b & & $13^{a}$ & \\
\hline 20 & Sery85 & & $13^{a}$ & \\
\hline 21 & Anle236b & & $12^{a}$ & \\
\hline 22 & Anle143b & & & $3 / 14^{f}$ \\
\hline 23 & Sery106 & & & $3 / 14^{f}$ \\
\hline
\end{tabular}




\begin{tabular}{|c|c|c|c|c|}
\hline No. & compound & structure & $\%$ inhibition" & $\Delta$ survival $^{+}$ \\
\hline 24 & Sery166a & & $<10^{\mathrm{e}}$ & $2 / 85^{h}$ \\
\hline 25 & Sery $158 b$ & & $<10^{e}$ & \\
\hline 26 & Sery159a & & $<10^{e}$ & \\
\hline 27 & Sery294b & & $<10^{b}$ & $12 / 14^{f}$ \\
\hline 28 & Anle197b & & $<10^{b}$ & $11 / 14^{f}$ \\
\hline 29 & Anle138c & & $<10^{a}$ & \\
\hline 30 & Sery345 & & $<10^{a}$ & \\
\hline 31 & Anle234b & & $<10^{a}$ & \\
\hline 32 & Sery384 & & $<10^{a}$ & \\
\hline 33 & Sery401b & & $<10^{a}$ & \\
\hline 34 & Anle270 & & $<10^{\mathrm{a}}$ & \\
\hline 35 & Sery417 & & $<10^{a}$ & \\
\hline 36 & Sery363b & & $n / a^{*}$ & \\
\hline 37 & Anle143c & & & toxic \\
\hline 38 & Sery369 & & toxic & \\
\hline
\end{tabular}

\# relative inhibition of $\mathrm{PrP}^{\mathrm{Sc}}$ accumulation normalized to DMSO-treated group ( $0 \%$ inhibition) and $\mathrm{PrP}^{\mathrm{Sc}}$ level at start of treatment (100\% inhibition).

${ }^{+} \Delta$ survival (prolongation of survival in days/treatment days up to mean survival of controls)

* mice refused to eat peanut butter pellets

${ }^{\text {a }} \mathrm{PrP}^{\mathrm{Sc}}$ level in brain 120 days after i.c. infection and treatment for 40 days with $1 \mathrm{mg}$ compound (oral, in peanut butter).

${ }^{\mathrm{b}} \mathrm{PrP}^{\mathrm{Sc}}$ level in spleen determined 35 days after i.p. infection followed by 34 days of treatment with $1 \mathrm{mg}$ compound (oral, in peanut butter).

${ }^{\mathrm{c}} \mathrm{PrP}^{\mathrm{Sc}}$ level in brain at 106 days after i.c. infection and treatment for 24 days (14 days i.p. ( $0.84 \mathrm{mg}$ compound); 2 x 5 days oral by gavage $(1 \mathrm{mg})$ ).

${ }^{\mathrm{d}} \mathrm{PrP}^{\mathrm{Sc}}$ level in spleen at 35 days after i.p. infection and treatment for 24 days (14 days i.p. (0.84 mg compound); 2 x 5 days oral by gavage $(1 \mathrm{mg})$ ).

${ }^{\text {e }} \mathrm{PrP}^{\mathrm{Sc}}$ level in spleen at 35 days after i.p. infection and treatment for 14 days (14 days i.p. (50 $\mu 1100 \mu \mathrm{M}$ compound). 
${ }^{\mathrm{f}}$ Prolongation of survival time (days) after i.p. infection and treatment for 14 days starting at 80 dpi (14 days i.p. (50 $\mu 1100 \mu \mathrm{M}$ compound).

$\mathrm{g}$ Prolongation of survival time (days) after i.c. infection and treatment for 24 days starting at 80 dpi (14 days i.p. ( $0.84 \mathrm{mg}$ compound); 2 x 5 days oral by gavage $(1 \mathrm{mg})$ ).

${ }^{\mathrm{h}}$ Prolongation of survival time (days) after i.c. infection and application of the compounds via an osmotic pump that was implanted at $80 \mathrm{dpi}$ ( $60 \mathrm{nmol}$ compound/d).

${ }^{i}$ Prolongation of survival time (days) after i.c. infection and daily treatment starting at 120 dpi with $5 \mathrm{mg}$ compound (oral, in peanut butter) until terminal disease.

j Prolongation of survival time (days) after i.c. infection and daily treatment starting at $80 \mathrm{dpi}$ with $5 \mathrm{mg}$ compound (oral, in peanut butter) until terminal disease.

${ }^{\mathrm{k}}$ Prolongation of survival time (days) after i.c. infection and $2 \mathrm{x}$ daily treatment starting at 80 dpi with $5 \mathrm{mg}$ compound (oral, in peanut butter) until terminal disease.

${ }^{1}$ Prolongation of survival time (days) after i.c. infection and $2 \mathrm{x}$ daily treatment starting at 0 dpi with $5 \mathrm{mg}$ compound (oral, in peanut butter) until terminal disease. 


\subsection{Backbone assignment of wt $\alpha$-synuclein at $15^{\circ} \mathrm{C}$, pH 7.4, PBS}

In the following table, assigned ${ }^{1} \mathrm{H}$ and ${ }^{15} \mathrm{~N}$ resonances in ppm are shown.

\begin{tabular}{|c|c|c|c|}
\hline & Residue & ${ }^{15} \mathbf{N}$ & ${ }^{1} \mathbf{H}$ \\
\hline 1 & $\mathrm{M}$ & - & - \\
\hline 2 & $\mathrm{D}$ & - & - \\
\hline 3 & $\mathrm{~V}$ & 120.1 & 8.221 \\
\hline 4 & $\mathrm{~F}$ & 123.2 & 8.329 \\
\hline 5 & M & 122.0 & 8.213 \\
\hline 6 & $\mathrm{~K}$ & - & - \\
\hline 7 & $\mathrm{G}$ & 109.8 & 8.416 \\
\hline 8 & $\mathrm{~L}$ & 121.5 & 8.054 \\
\hline 9 & $\mathrm{~S}$ & 116.6 & 8.315 \\
\hline 10 & $\mathrm{~K}$ & - & - \\
\hline 11 & $\mathrm{~A}$ & - & - \\
\hline 12 & K & 120.8 & 8.335 \\
\hline 13 & $\mathrm{E}$ & 122.0 & 8.439 \\
\hline 14 & $\mathrm{G}$ & 109.9 & 8.434 \\
\hline 15 & V & 120.1 & 7.970 \\
\hline 16 & $\mathrm{~V}$ & 125.1 & 8.292 \\
\hline 17 & $\mathrm{~A}$ & 128.3 & 8.442 \\
\hline 18 & A & 123.6 & 8.313 \\
\hline 19 & A & 123.0 & 8.277 \\
\hline 20 & $\mathrm{E}$ & 120.0 & 8.328 \\
\hline 21 & $\mathrm{~K}$ & 122.2 & 8.326 \\
\hline 22 & $\mathrm{~T}$ & 115.2 & 8.139 \\
\hline 23 & $\mathrm{~K}$ & 123.7 & 8.348 \\
\hline 24 & $\mathrm{Q}$ & - & - \\
\hline 25 & $\mathrm{G}$ & 110.4 & 8.481 \\
\hline 26 & $\mathrm{~V}$ & 119.7 & 8.017 \\
\hline 27 & $\mathrm{~A}$ & 127.2 & 8.421 \\
\hline 28 & $\mathrm{E}$ & 120.5 & 8.405 \\
\hline 29 & A & 124.8 & 8.292 \\
\hline 30 & A & - & - \\
\hline 31 & $\mathrm{G}$ & 107.7 & 8.313 \\
\hline 32 & $\mathrm{~K}$ & 120.6 & 8.112 \\
\hline 33 & $\mathrm{~T}$ & 115.5 & 8.241 \\
\hline 34 & $\mathrm{~K}$ & - & - \\
\hline 35 & $\mathrm{E}$ & - & - \\
\hline 36 & $\mathrm{G}$ & - & - \\
\hline 37 & V & 119.5 & 7.900 \\
\hline 38 & $\mathrm{~L}$ & 125.7 & 8.283 \\
\hline 39 & $\mathrm{Y}$ & 122.3 & 8.265 \\
\hline 40 & V & 123.2 & 8.088 \\
\hline 41 & $\mathrm{G}$ & 112.1 & 8.046 \\
\hline 42 & $\mathrm{~S}$ & - & - \\
\hline 43 & $\mathrm{~K}$ & - & - \\
\hline 44 & $\mathrm{~T}$ & 1154 & 8.199 \\
\hline
\end{tabular}


45

46

47

48

49

50

51

52

53

54

55

56

57

58

59

60

61

62

63

64

65

66

67

68

69

70

71

72

73

74

75

76

77

78

79

80

81

82

83

84

85

86

87

88

89

90

91

92

93

94
K

E

G

V

V

$\mathrm{H}$

G

V

A

$\mathrm{T}$

V

A

E

K

$\mathrm{T}$

K

E

Q

V

$\mathrm{T}$

$\mathrm{N}$

V

G

G

A

V

V

$\mathrm{T}$

G

V

$\mathrm{T}$

A

V

A

Q

K

$\mathrm{T}$

V

E

G

A

G

S

I

A

A

A

$\mathrm{T}$

G

F
123.7

$-$

109.8

119.9

125.1

124.9

110.4

119.5

128.1

114.7

122.9

128.0

120.8

122.7

115.9

123.6

-

121.6

121.8

118.0

120.6

112.5

108.7

123.7

120.4

125.3

118.5

111.3

119.4

118.8

127.3

119.9

128.0

120.2

123.2

116.8

122.9

125.2

110.6

123.9

108.1

115.6

122.7

127.9

123.2

123.3

112.5

110.6

120.2
8.480

8.416

7.903

8.292

8.474

8.413

8.033

8.495

8.217

8.234

8.415

8.374

8.435

8.217

8.386

8.413

8.283

8.292

8.240

8.562

8.235

8.162

8.213

8.389

8.301

8.444

8.085

8.294

8.366

8.139

8.415

8.389

8.433

8.289

8.298

8.576

8.517

8.270

8.505

8.148

8.193

8.347

8.207

8.288

8.085

8.308

8.073 


\begin{tabular}{|c|c|c|c|}
\hline 95 & $\mathrm{~V}$ & 123.6 & 8.063 \\
\hline 96 & $\mathrm{~K}$ & 126.4 & 8.397 \\
\hline 97 & $\mathrm{~K}$ & 123.7 & 8.480 \\
\hline 98 & D & 121.2 & 8.405 \\
\hline 99 & Q & 120.1 & 8.337 \\
\hline 100 & L & 122.7 & 8.295 \\
\hline 101 & $\mathrm{G}$ & 109.7 & 8.487 \\
\hline 102 & $\mathrm{~K}$ & 120.7 & 8.214 \\
\hline 103 & $\mathrm{~N}$ & 119.9 & 8.625 \\
\hline 104 & $\mathrm{E}$ & 121.3 & 8.484 \\
\hline 105 & $\mathrm{E}$ & 122.0 & 8.470 \\
\hline 106 & $\mathrm{G}$ & 110.1 & 8.431 \\
\hline 107 & A & 124.9 & 8.128 \\
\hline 108 & $\mathrm{P}$ & - & - \\
\hline 109 & $\mathrm{Q}$ & 121.1 & 8.592 \\
\hline 110 & $\mathrm{E}$ & 122.5 & 8.532 \\
\hline 111 & $\mathrm{G}$ & 110.1 & 8.487 \\
\hline 112 & I & 120.1 & 7.997 \\
\hline 113 & $\mathrm{~L}$ & 126.9 & 8.408 \\
\hline 114 & $\mathrm{E}$ & 122.2 & 8.426 \\
\hline 115 & $\mathrm{D}$ & 121.4 & 8.354 \\
\hline 116 & $\mathrm{M}$ & 122.0 & 8.254 \\
\hline 117 & $\mathrm{P}$ & - & - \\
\hline 118 & V & 120.8 & 8.300 \\
\hline 119 & D & 126.0 & 8.522 \\
\hline 120 & $\mathrm{P}$ & - & - \\
\hline 121 & $\mathrm{D}$ & 119.2 & 8.376 \\
\hline 122 & $\mathrm{~N}$ & 119.1 & 8.097 \\
\hline 123 & $\mathrm{E}$ & 121.7 & 8.384 \\
\hline 124 & A & 124.3 & 8.230 \\
\hline 125 & $Y$ & 119.9 & 8.020 \\
\hline 126 & $\mathrm{E}$ & 123.8 & 8.128 \\
\hline 127 & $\mathrm{M}$ & 123.9 & 8.423 \\
\hline 128 & $\mathrm{P}$ & - & - \\
\hline 129 & $\mathrm{~S}$ & 116.7 & 8.490 \\
\hline 130 & $\mathrm{E}$ & 123.2 & 8.595 \\
\hline 131 & $\mathrm{E}$ & 121.8 & 8.469 \\
\hline 132 & $\mathrm{G}$ & 109.9 & 8.434 \\
\hline 133 & $Y$ & 120.2 & 8.073 \\
\hline 134 & $\mathrm{Q}$ & 122.7 & 8.235 \\
\hline 135 & D & 121.6 & 8.235 \\
\hline 136 & $\mathrm{Y}$ & 120.4 & 8.036 \\
\hline 137 & $\mathrm{E}$ & 125.4 & 8.257 \\
\hline 138 & $\mathrm{P}$ & - & - \\
\hline 139 & $\mathrm{E}$ & 121.5 & 8.526 \\
\hline 140 & A & 130.9 & 7.98 \\
\hline
\end{tabular}


